\title{
Organic Analysis of AW-101 and AN-107 Tank Waste
}

VOA and SVOA

G. S. Klinger

M. W. Urie

PCB/Pesticides, Dioxins \& Furans, Headspace, and Organic Anions

J. A. Campbell

S. A. Clauss

T. W. Clauss

E. W. Hoppe

G. M. Mong

A. K. Sharma

August 2000

Prepared for

BNFL, Inc.

Under Contract W375-LC-98-4168

Battelle, Pacific Northwest Division

Richland, Washington 99352 


\section{DISCLAIMER}

This report was prepared as an account of work sponsored by an agency of the United States Government. Neither the United States Government nor any agency thereof, nor any of their employees, make any warranty, express or implied, or assumes any legal liability or responsibility for the accuracy, completeness, or usefulness of any information, apparatus, product, or process disclosed, or represents that its use would not infringe privately owned rights. Reference herein to any specific commercial product, process, or service by trade name, trademark, manufacturer, or otherwise does not necessarily constitute or imply its endorsement, recommendation, or favoring by the United States Government or any agency thereof. The views and opinions of authors expressed herein do not necessarily state or reflect those of the United States Government or any agency thereof. 


\section{DISCLAIMER}

Portions of this document may be illegible in electronic image products. Images are produced from the best available original document. 


\section{Summary}

Thirty jars of waste material from tank AW-101 and seventeen jars of waste material from tank AN-107 were received by Battelle. The contents of all jars of AW-101 were mixed to provide a single composite, as were the contents of all jars of AN-107. Each composite was homogenized and representative sub-samples extracted for organic, radiochemical and inorganic regulatory analyses. The representative sub-samples were analyzed for inorganic, radiochemical, and organic analyses for analytes of interest as defined in Test Plan BNFL-29953-2. This report presents the organic results. The inorganic (including TCLP metals) and radiochemical results are reported in report BNFL-RPT-008 (PNWD-2462).

The organic characterization analyses of the as received material for AW-101 and AN-107 include:

- Volatile Organic Analysis

- Semi-volatile Organic Analysis

- Polychlorinated biphenyls and pesticides

- Polychlorinated dibenzo-p-dioxins and polychlorinated dibenzofurans

- Oxalate, formate, acetate, and acrylate by ion chromatography

- Ethanol, methanol, 2-propanol, 1-propanol, n-butanol, triethylamine, 2-methyl-2-propanol, and 2-butanol by headspace analysis

Except for a very few cases, the characterization results met or exceeded the quality control requirements established by the governing quality assurance plan, and met or exceeded the minimum reportable quantity requirements specified by BNFL. Whenever possible the analyses were performed to SW-846 protocols so that the results can be used to support permit application, as well as provide feed envelope characterization data.

Tables S.a and S.b summarize the results for the BNFL target analytes that produced measurable results. All other BNFL target analytes were non-detects. Also, numerous non-BNFL target analytes were measured and are presented in this report. 
Table S.a. AW-101 Summary Results - BNFL Target Analytes Detected

\begin{tabular}{|c|c|c|c|c|c|c|c|}
\hline \multirow[b]{3}{*}{ CAS\# } & \multirow[b]{3}{*}{ BNFL Target Compound } & \multicolumn{3}{|c|}{ AW-101 Supernatant } & \multicolumn{3}{|c|}{ AW-101 Centrifuged Solids } \\
\hline & & Blank & Sample & Duplicate & Blank & Sample & Duplicate \\
\hline & & $\mu g / L$ & $\mu \mathrm{g} / \mathrm{L}$ & $\mu \mathrm{g} / \mathrm{L}$ & $\mu \mathrm{g} / \mathrm{Kg}$ & $\mu \mathrm{g} / \mathrm{Kg}$ & $\mu \mathrm{g} / \mathrm{Kg}$ \\
\hline \multicolumn{8}{|c|}{ VOA Compounds } \\
\hline $106-35-4$ & 3-Heptanone & $\overline{\mathrm{U}}$ & $\bar{U}$ & $\mathrm{U}$ & $\overline{\mathrm{U}}$ & 59 & $32 \mathrm{~J}$ \\
\hline $106-97-8$ & Butane & $\bar{U}$ & $\mathrm{U}$ & $\bar{U}$ & $\mathrm{U}$ & 860 & 920 \\
\hline $108-10-1$ & 4-Methyl-2-pentanone & $\bar{U}$ & $\mathrm{U}$ & $\bar{U}$ & $\bar{U}$ & $10 \mathrm{~J}$ & $\mathrm{U}$ \\
\hline $109-66-0$ & Pentane & $\bar{U}$ & $\bar{U}$ & $\mathrm{U}$ & $\bar{U}$ & 150 & $80 \mathrm{~J}$ \\
\hline $109-99-9$ & Tetrahydrofuran & $\bar{U}$ & 140 & 110 & $\bar{U}$ & 180 & $89 \mathrm{~J}$ \\
\hline $110-43-0$ & 2-Heptanone & $\overline{\mathrm{U}}$ & $\mathrm{U}$ & $\bar{U}$ & $\bar{U}$ & 59 & $36 \mathrm{~J}$ \\
\hline $110-54-3$ & Hexane & $\bar{U}$ & $\overline{\mathrm{U}}$ & $\bar{U}$ & $\overline{\mathrm{U}}$ & 200 & $80 \mathrm{~J}$ \\
\hline $111-65-9$ & Octane & $\overline{\mathrm{U}}$ & $\overline{\mathrm{U}}$ & $\overline{\mathrm{U}}$ & $\overline{\mathrm{U}}$ & 200 & 99 \\
\hline $111-84-2$ & Nonane & $\bar{U}$ & $\bar{U}$ & $\overline{\mathrm{U}}$ & $\bar{U}$ & 250 & 180 \\
\hline $123-19-3$ & 4-Heptanone & $\bar{U}$ & $\bar{U}$ & $\bar{U}$ & $\bar{U}$ & $8 \mathrm{~J}$ & $\mathrm{U}$ \\
\hline $123-91-1$ & 1,4-Dioxane & $\bar{U}$ & 180 & 150 & $\bar{U}$ & 97 & $\bar{U}$ \\
\hline $142-82-5$ & Heptane & $\overline{\mathrm{U}}$ & $\mathrm{U}$ & $\mathrm{U}$ & $\bar{U}$ & 210 & $66 \mathrm{~J}$ \\
\hline $563-80-4$ & 3-Methyl-2-butanone & $\bar{U}$ & $\overline{\mathrm{U}}$ & $\bar{U}$ & $\bar{U}$ & $34 \mathrm{~J}$ & $20 \mathrm{~J}$ \\
\hline $591-78-6$ & 2-Hexanone & $\overline{\mathrm{U}}$ & $\bar{U}$ & $\bar{U}$ & $\bar{U}$ & $40 \mathrm{~J}$ & $24 \mathrm{~J}$ \\
\hline $67-64-1$ & Acetone & $\mathrm{U}$ & 330 & 360 & $6 \mathrm{JB}$ & $620 \mathrm{~B}$ & $360 \bar{B}$ \\
\hline $71-43-2$ & Benzene & $\overline{\mathrm{U}}$ & $\overline{\mathrm{U}}$ & $\overline{\mathrm{U}}$ & $\mathrm{U}$ & $10 \mathrm{~J}$ & $\mathrm{U}$ \\
\hline $75-05-8$ & Acetonitrile & $\mathrm{U}$ & $\overline{\mathrm{U}}$ & $\bar{U}$ & $\mathrm{U}$ & $41 \mathrm{~J}$ & $\bar{U}$ \\
\hline $75-45-6$ & Chlorodifluoromethane & $5 \mathrm{JB}$ & $\bar{U}$ & $\bar{U}$ & $7 \mathrm{JB}$ & $\bar{U}$ & $\mathrm{U}$ \\
\hline $78-93-3$ & 2-Butanone & $\mathrm{U}$ & $\bar{U}$ & $55 \mathrm{~J}$ & $\mathrm{U}$ & 160 & 110 \\
\hline \multicolumn{8}{|c|}{ SVOA Compounds } \\
\hline $126-73-8$ & Tributyl phosphate & $\mathrm{U}$ & $\bar{U}$ & $\overline{\mathrm{U}}$ & $\bar{U}$ & $\bar{U}$ & $1800 \mathrm{~J}$ \\
\hline $62-75-9$ & N-Nitrosodimethylamine & $\overline{\mathrm{U}}$ & $160 \mathrm{~J}$ & 390 & $\overline{\mathrm{U}}$ & 12000 & $\mathrm{U}$ \\
\hline \multicolumn{8}{|l|}{ Pesticides } \\
\hline $319-84-6$ & alpha-BHC & $\overline{\mathrm{U}}$ & $\overline{\mathrm{U}}$ & $\overline{\mathrm{U}}$ & $\bar{U}$ & 18.1 & $\overline{\mathrm{U}}$ \\
\hline $76-44-8$ & Heptachlor & $\mathrm{U}$ & $\bar{U}$ & 6.5 & $\bar{U}$ & 73.4 & $\overline{\mathrm{U}}$ \\
\hline \multicolumn{8}{|l|}{ PCBS } \\
\hline $11097-69-1$ & AROCLOR 1260 & $\overline{\mathrm{U}}$ & $\mathrm{U}$ & $\overline{\mathrm{U}}$ & $\bar{U}$ & $23 \mathrm{~J}$ & $\mathrm{U}$ \\
\hline \multicolumn{8}{|c|}{ Dioxin/Furans } \\
\hline (1) & $\mathrm{xx}$-Pentachlorodibenzofuran & $\overline{\mathrm{U}}$ & $\bar{U}$ & $\overline{\mathrm{U}}$ & $0.56 \mathrm{JB}$ & $\bar{U}$ & $\bar{U}$ \\
\hline $35822-39-4$ & $1,2,3,4,6,7,8$-Heptachlorodibenzo-p-dioxin & $\bar{U}$ & $\overline{\mathrm{U}}$ & $\overline{\mathrm{U}}$ & $0.68 \mathrm{JB}$ & $0.87 \mathrm{JB}$ & $\mathrm{U}$ \\
\hline (1) & xx-Heptachlorodibenzofuran & $\overline{\mathrm{U}}$ & $\bar{U}$ & $\bar{U}$ & $0.78 \mathrm{JB}$ & $0.72 \mathrm{JB}$ & U \\
\hline \multirow[t]{2}{*}{$39001-02-0$} & $1,2,3,4,6,7,8,9-$-Octachlorodibenzofuran & $\bar{U}$ & $\bar{U}$ & $\bar{U}$ & $2.90 \mathrm{~B}$ & $2.94 \mathrm{~B}$ & $0.18 \mathrm{JB}$ \\
\hline & & $\mu \mathrm{g} / \mathrm{ml}$ & $\mu \mathrm{g} / \mathrm{ml}$ & $\mu \mathrm{g} / \mathrm{ml}$ & $\mu \mathrm{g} / \mathrm{g}$ & $\mu g / g$ & $\mu g / g$ \\
\hline \multicolumn{8}{|c|}{ Organic Anions } \\
\hline $144-62-7$ & Oxalate & $\overline{\mathrm{U}}$ & $600 \mathrm{~J}$ & $500 \mathrm{~J}$ & $\bar{U}$ & 9700 & 11100 \\
\hline $64-18-6$ & Formate & $\bar{U}$ & 2200 & 2100 & $\overline{\mathrm{U}}$ & 3400 & 2800 \\
\hline $79-10-7$ & Acrylate & $\bar{U}$ & $\mathrm{U}$ & $\mathrm{U}$ & $\bar{U}$ & $\overline{\mathrm{U}}$ & $\bar{U}$ \\
\hline $64-19-7$ & Acetate (2) & $\bar{U}$ & $1200 \mathrm{~J}$ & $1200 \mathrm{~J}$ & $\mathbf{U}$ & $\mathrm{U}$ & $\bar{U}$ \\
\hline \multicolumn{8}{|c|}{ Headspace Analysis } \\
\hline $67-65-1$ & Methanol & $\mathrm{U}$ & $1.1 \mathrm{~J}$ & $\mathrm{U}$ & $n / a$ & $n / a$ & $n / a$ \\
\hline $64-17-5$ & Ethanol & $2.5 \mathrm{~B}$ & $2.6 \mathrm{~B}$ & $2.6 \bar{B}$ & $\mathrm{n} / \mathrm{a}$ & $\mathrm{n} / \mathrm{a}$ & $\mathrm{n} / \mathrm{a}$ \\
\hline
\end{tabular}

1) Multiple isomers possible in retention time window; each isomer has unique CAS \#. Component represents sum of dioxins or furans meeting retention time window and ion abundance ratio criteria.

2) Acetate and glycolate are not resolved; results represent bounding upper concentration

$\mathrm{U}$ flag = not detected; results less than MDL

$\mathrm{J}$ flag $=$ detected and quantified, but results less than $\mathrm{MDL}$.

$\mathrm{B}$ flag $=$ Compound was present in the method blank 
Table S.b. AN-107 Summary Results - BNFL Target Analytes Detected

\begin{tabular}{|c|c|c|c|c|c|c|c|}
\hline \multirow[b]{3}{*}{ CAS\# } & \multirow[b]{3}{*}{ BNFL Target Compound } & \multicolumn{3}{|c|}{ AN-107 Supernatant } & \multicolumn{3}{|c|}{ AN-107 Centrifuged Solids } \\
\hline & & Blank & Sample & Duplicate & Blank & Sample & Duplicate \\
\hline & & $\mu g / \mathbf{L}$ & $\mu g / L$ & $\mu g / L$ & $\mu \mathrm{g} / \mathrm{Kg}$ & $\mu \mathrm{g} / \mathbf{K g}$ & $\mu \mathrm{g} / \mathrm{Kg}$ \\
\hline \multicolumn{8}{|c|}{ VOA Compounds } \\
\hline $108-87-2$ & Methylcyclohexane & $\mathrm{U}$ & $\overline{\mathrm{U}}$ & $\overline{\mathrm{U}}$ & $\mathrm{U}$ & $40 \mathrm{~J}$ & $26 \mathrm{~J}$ \\
\hline $109-99-9$ & Tetrahydrofuran & $\overline{\mathrm{U}}$ & $31 \mathrm{~J}$ & $26 \mathrm{~J}$ & $\mathrm{U}$ & $43 \mathrm{~J}$ & $36 \mathrm{~J}$ \\
\hline $110-43-0$ & 2-Heptanone & $\overline{\mathrm{U}}$ & $\mathrm{U}$ & $\mathrm{U}$ & $\overline{\mathrm{U}}$ & $\overline{\mathrm{U}}$ & $15 \mathrm{~J}$ \\
\hline $67-64-1$ & Acetone & $4 \mathrm{JB}$ & $140 \mathrm{~B}$ & $100 \mathrm{~B}$ & $6 \mathrm{JB}$ & $100 \mathrm{JB}$ & $370 \mathrm{~B}$ \\
\hline $75-05-8$ & Acetonitrile & $\mathrm{U}$ & 94 & 70 & $\mathrm{U}$ & $\mathrm{U}$ & 89 \\
\hline $75-45-6$ & Chlorodifluoromethane & $5 \mathrm{JB}$ & $37 \mathrm{JB}$ & $30 \mathrm{JB}$ & $7 \mathrm{JB}$ & $80 \mathrm{JB}$ & $29 \mathrm{JB}$ \\
\hline $78-93-3$ & 2-Butanone & $\overline{\mathrm{U}}$ & $16 \mathrm{~J}$ & $13 \mathrm{~J}$ & $\mathrm{U}$ & $\mathrm{U}$ & $58 \mathrm{~J}$ \\
\hline \multicolumn{8}{|c|}{ SVOA Compounds } \\
\hline $110-86-1$ & Pyridine & $\overline{\mathrm{U}}$ & $\bar{U}$ & $\overline{\mathrm{U}}$ & $\overline{\mathrm{U}}$ & $\overline{\mathrm{U}}$ & $1400 \mathrm{~J}$ \\
\hline $126-73-8$ & Tributyl phosphate & $\overline{\mathrm{U}}$ & $\mathrm{U}$ & $100 \mathrm{~J}$ & $\overline{\mathrm{U}}$ & $\overline{\mathrm{U}}$ & $\mathrm{U}$ \\
\hline $62-75-9$ & N-Nitrosodimethylamine & $\overline{\mathrm{U}}$ & $170 \mathrm{~J}$ & $260 \mathrm{~J}$ & $\mathrm{U}$ & $\mathrm{U}$ & $2600 \mathrm{~J}$ \\
\hline \multicolumn{8}{|l|}{ Pesticides } \\
\hline $309-00-2$ & Aldrin & $\overline{\mathrm{U}}$ & 1.2 & 3.7 & $\overline{\mathrm{U}}$ & $\bar{U}$ & $\bar{U}$ \\
\hline $319-84-6$ & alpha-BHC & $\overline{\mathrm{U}}$ & $\mathrm{U}$ & $\bar{U}$ & $\overline{\mathrm{U}}$ & 11.8 & 35.1 \\
\hline $76-44-8$ & Heptachlor & $\mathrm{U}$ & $\overline{\mathrm{U}}$ & $\bar{U}$ & $\bar{U}$ & $\overline{\mathrm{U}}$ & 18.4 \\
\hline \multicolumn{8}{|l|}{ PCBs } \\
\hline $11097-69-1$ & AROCLOR 1260 & $0.8 \mathrm{JB}$ & $1.7 \mathrm{JB}$ & $1.8 \mathrm{JB}$ & $\overline{\mathrm{U}}$ & $\overline{\mathrm{U}}$ & $17 \mathrm{~J}$ \\
\hline \multicolumn{8}{|c|}{ Dioxins/Furans } \\
\hline $1746-01-6$ & $2,3,7,8$-Tetrachlorodibenzo-p-dioxin & $\overline{\mathrm{U}}$ & $\bar{U}$ & $\mathrm{U}$ & $\overline{\mathrm{U}}$ & $\overline{\mathrm{U}}$ & $0.05 \mathrm{~J}$ \\
\hline $51207-31-9$ & $2,3,7,8$-Tetrachlorodibenzofuran & $\overline{\mathrm{U}}$ & $\bar{U}$ & $\bar{U}$ & $\overline{\mathrm{U}}$ & $\mathrm{U}$ & $0.06 \mathrm{~J}$ \\
\hline $40321-76-4$ & $1,2,3,7,8$-Pentachlorodibenzo-p-dioxin & $\overline{\mathrm{U}}$ & $\overline{\mathrm{U}}$ & $\bar{U}$ & $\overline{\mathrm{U}}$ & $\overline{\mathrm{U}}$ & $0.46 \mathrm{~J}$ \\
\hline (1) & xx-Pentachlorodibenzofuran & $\overline{\mathrm{U}}$ & $\bar{U}$ & $\overline{\mathrm{U}}$ & $\overline{\mathrm{U}}$ & $\overline{\mathrm{U}}$ & $0.45 \mathrm{~J}$ \\
\hline (1) & $\mathrm{xx}$-Hexachlorodibenzo-p-dioxin & $\overline{\mathrm{U}}$ & $\overline{\mathrm{U}}$ & $\bar{U}$ & $\bar{U}$ & $\overline{\mathrm{U}}$ & $0.06 \mathrm{~J}$ \\
\hline (1) & xx-Hexachlorodibenzofuran & $\overline{\mathrm{U}}$ & $\overline{\mathrm{U}}$ & $\overline{\mathrm{U}}$ & $\overline{\mathrm{U}}$ & $\overline{\mathrm{U}}$ & $0.43 \mathrm{~J}$ \\
\hline $35822-39-4$ & $1,2,3,4,6,7,8$-Heptachlorodibenzo-p-dioxin & $\overline{\mathrm{U}}$ & $\overline{\mathrm{U}}$ & $\overline{\mathrm{U}}$ & $0.63 \mathrm{JB}$ & $\overline{\mathrm{U}}$ & $0 . \overline{43} \mathrm{JB}$ \\
\hline (1) & $\mathrm{xx}$-Heptachlorodibenzofuran & $\bar{U}$ & $\bar{U}$ & $\overline{\mathrm{U}}$ & $\bar{U}$ & $\overline{\mathrm{U}}$ & $0.43 \mathrm{~J}$ \\
\hline $3268-87-9$ & $1,2,3,4,6,7,8,9-$ Octachlorodibenzo-p- & $\mathrm{U}$ & $\overline{\mathrm{U}}$ & $\overline{\mathrm{U}}$ & $\overline{\mathrm{U}}$ & $\overline{\mathrm{U}}$ & $1.65 \mathrm{~J}$ \\
\hline \multirow[t]{2}{*}{$39001-02-0$} & $1,2,3,4,6,7,8,9-$ Octachlorodibenzofuran & $\bar{U}$ & $\overline{\mathrm{U}}$ & $\overline{\mathrm{U}}$ & $\mathrm{U}$ & $\bar{U}$ & $1.94 \mathrm{~J}$ \\
\hline & & $\mu \mathrm{g} / \mathrm{ml}$ & $\mu \mathrm{g} / \mathrm{ml}$ & $\mu \mathrm{g} / \mathrm{ml}$ & $\mu \mathrm{g} / \mathrm{g}$ & $\mu g / g$ & $\mu g / g$ \\
\hline \multicolumn{8}{|c|}{ Organic Anions } \\
\hline $144-62-7$ & Oxalate & $\bar{U}$ & $200 \mathrm{~J}$ & $500 \mathrm{~J}$ & $\overline{\mathrm{U}}$ & 22000 & 22700 \\
\hline $64-18-6$ & Formate & $\bar{U}$ & 1700 & 2000 & $\overline{\mathrm{U}}$ & $600 \mathrm{~J}$ & $600 \mathrm{~J}$ \\
\hline $79-10-7$ & Acrylate & $\bar{U}$ & $\mathrm{U}$ & $\mathrm{U}$ & $\overline{\mathrm{U}}$ & $\mathrm{U}$ & $\mathrm{U}$ \\
\hline $64-19-7$ & Acetate (2) & $\bar{U}$ & $4000 \mathrm{~J}$ & $4000 \mathrm{~J}$ & $\overline{\mathrm{U}}$ & $\overline{\mathrm{U}}$ & $\overline{\mathrm{U}}$ \\
\hline \multicolumn{8}{|c|}{ Headspace Analysis } \\
\hline $67-65-1$ & Methanol & $\bar{U}$ & $4.6 \mathrm{~J}$ & $4.4 \mathrm{~J}$ & $\mathrm{n} / \mathrm{a}$ & $n / a$ & $\mathrm{n} / \mathrm{a}$ \\
\hline $64-17-5$ & Ethanol & $2.7 \mathrm{~B}$ & $3.0 \mathrm{~B}$ & $2.4 \mathrm{~B}$ & $\mathbf{n} / \mathbf{a}$ & $\mathrm{n} / \mathrm{a}$ & $\mathrm{n} / \mathrm{a}$ \\
\hline $121-44-8$ & Triethylamine & $\mathrm{U}$ & $5.8 \mathrm{~J}$ & $1.0 \mathrm{~J}$ & $\mathrm{n} / \mathrm{a}$ & $\mathrm{n} / \mathrm{a}$ & $\mathrm{n} / \mathrm{a}$ \\
\hline
\end{tabular}

1) Multiple isomers possible in retention time window; each isomer has unique CAS \#. Component represents sum of dioxins or furans meeting retention time window and ion abundance ratio criteria.

2) Acetate and glycolate are not resolved; results represent bounding upper concentration

$\mathrm{U}$ flag = not detected; results less than MDL

$\mathrm{J}$ flag = detected and quantified, but results less than MDL.

$B$ flag $=$ Compound was present in the method blank 



\section{Terms and Abbreviations}

$\begin{array}{ll}\text { ASR } & \text { analytical service request } \\ \text { BNFL } & \text { BNFL, Inc. (subsidiary of British Nuclear Fuels, Ltd.) } \\ \text { CAS\# } & \text { chemical Abstracts Service Registry Number } \\ \text { CCC } & \text { Calibration check compound } \\ \text { CLP } & \text { Contract Laboratory Program } \\ \text { COC } & \text { chain of custody } \\ \text { K-D } & \text { Kuderna-Danish } \\ \text { ECD } & \text { electron capture detector } \\ \text { GC/ECD } & \text { gas chromatography/electron capture detection } \\ \text { GC/MS } & \text { gas chromatography/mass spectrometry } \\ \text { HLRF } & \text { High Level Radiation Facility } \\ \text { IC } & \text { ion chromatography } \\ \text { LCS } & \text { Laboratory Control Standard } \\ \text { MDL } & \text { method detection limit } \\ \text { MRQ } & \text { minimum reportable quantity } \\ \text { MS } & \text { matrix spike } \\ \text { MSD } & \text { matrix spike duplicate } \\ \text { M\&TE } & \text { measuring and test equipment } \\ \% D & \text { percent difference } \\ \text { PCB } & \text { polychlorinated biphenyl } \\ \text { QC } & \text { quality control } \\ \text { RSD } & \text { relative standard deviation } \\ \text { SAL } & \text { Shielded Analytical Laboratory } \\ \text { SPCC } & \text { system performance check compound } \\ \text { SVOA } & \text { semi-volatile organic analysis } \\ \text { TCLP } & \text { Toxicity characteristic leaching procedure } \\ \text { TIC } & \text { tentatively identified compound } \\ \text { USEPA } & \text { United States Environmental Protection Agency } \\ \text { VOA } & \text { volatile organic analysis } \\ & \end{array}$




\section{Units}

$\begin{array}{ll}{ }^{\circ} \mathrm{C} & \begin{array}{l}\text { degree Centigrade } \\ \text { degree Fahrenheit } \\ { }^{\circ} \mathrm{F}\end{array} \\ \mathrm{g} & \text { gram } \\ \mathrm{Kg} & \text { kilogram } \\ \mathrm{L} & \text { liter } \\ \mu \mathrm{g} & \text { microgram } \\ \mathrm{mL} & \text { milliliter } \\ \mathrm{mM} & \text { millimolar } \\ \mathrm{min} & \text { minute } \\ \mathrm{ng} & \text { nanogram } \\ \text { Vol\% } & \text { volume percent } \\ \mathrm{Wt} \% & \text { weight percent }\end{array}$

viii 


\section{Contents}

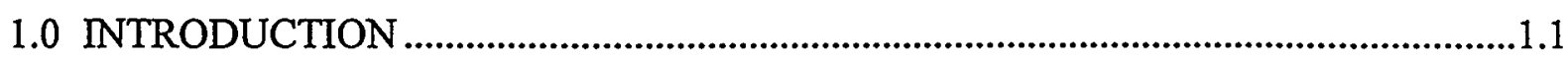

2.0 SAMPLE RECEIPT AND ANALYTICAL SUB-SAMPLING .................................2.1

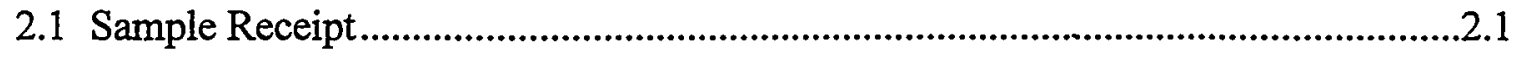

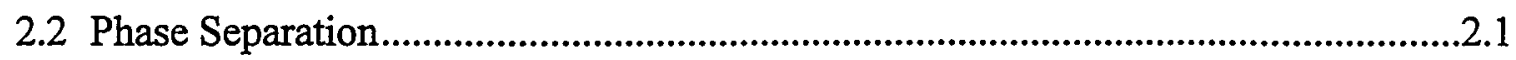

2.3 Supernatant Density Measurements .....................................................................2.1

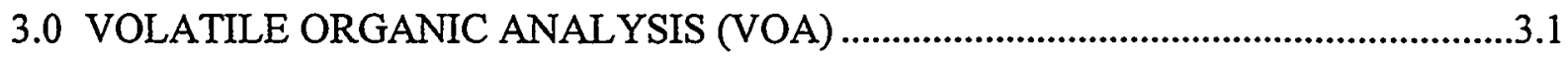

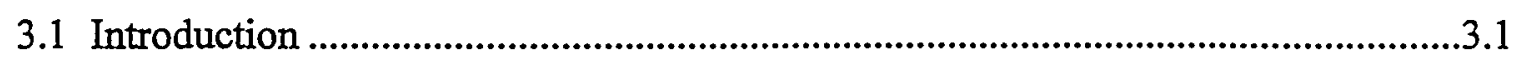

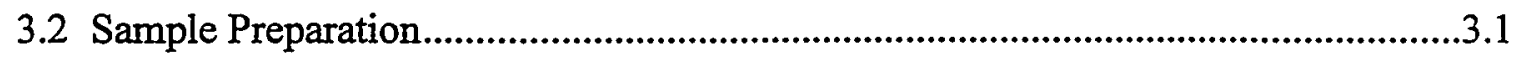

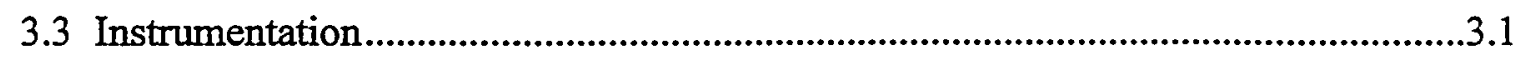

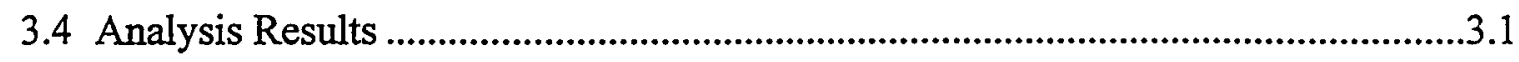

3.4.1 Results for Calibrated/Regulatory Analytes of Interest...............................3.2

3.4.2 QC Evaluation.......................................................................................11

3.4.3 Other Observations (or Deviations/Concerns/Issues) ...................................3.13

4.0 SEMI-VOLATILE ORGANIC ANALYSIS (SVOA) .............................................4.1

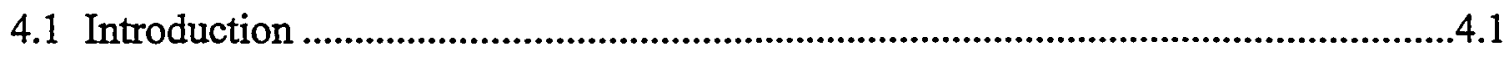

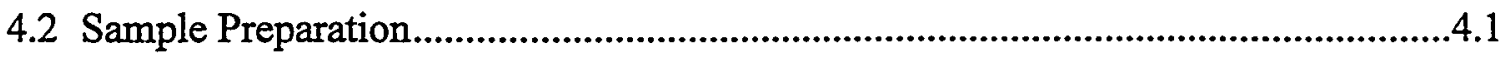

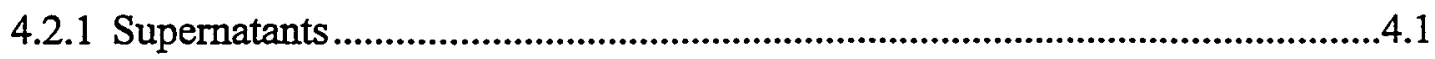

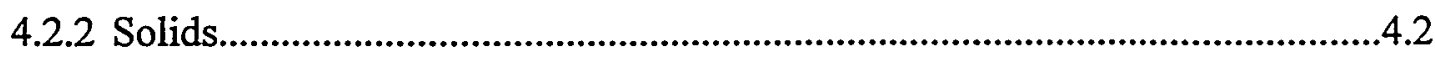

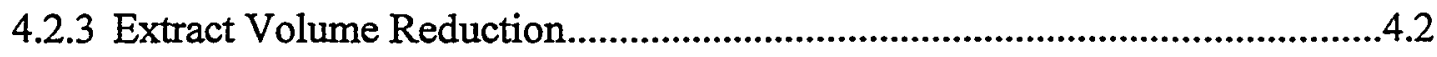

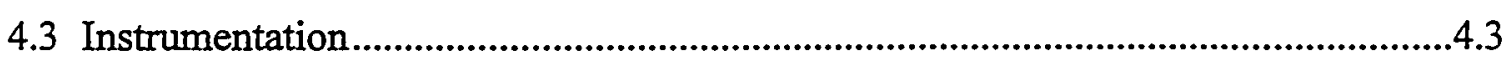

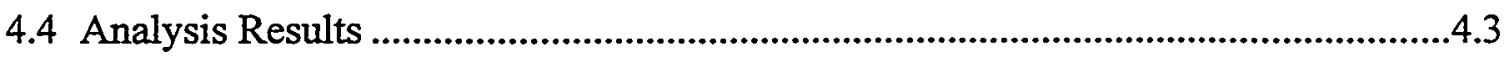

4.4.1 Results for Calibrated/Regulatory Analytes of Interest .................................4.3

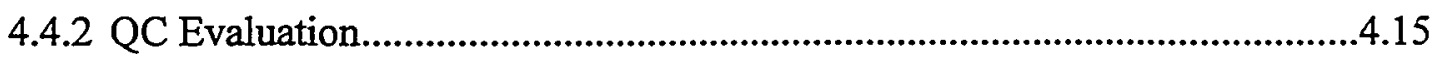

4.4.3 Other Observations (or Deviations/Concerns/Issues) ..............................4.18

5.0 POLYCHLORINATED BIPHENYLS/PESTICIDES ANALYSIS ............................5.1

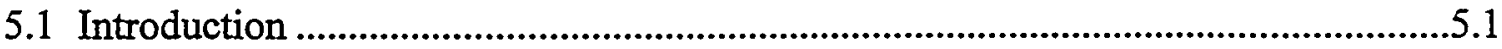

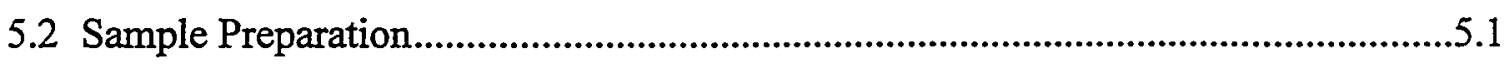

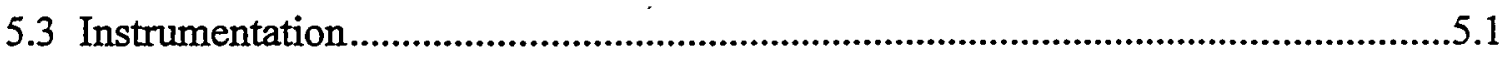

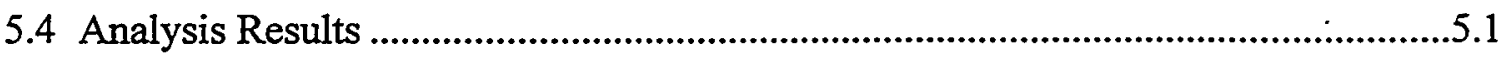

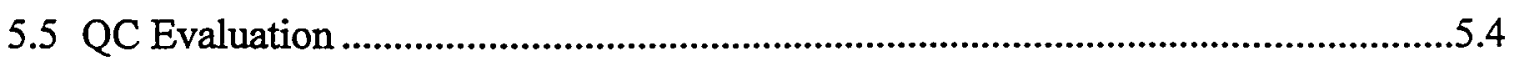

6.0 POLYCHLORINATED DIBENZO-P-DIOXINS AND DIBENZOFURANS ANALYSIS... 


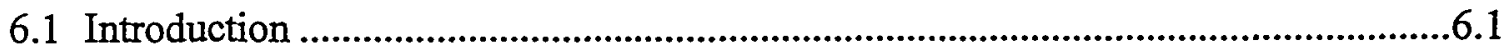

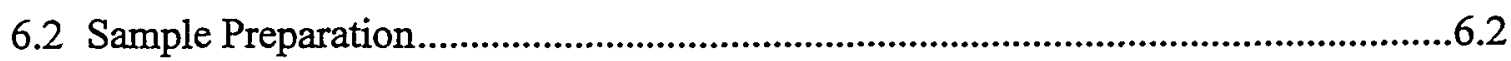

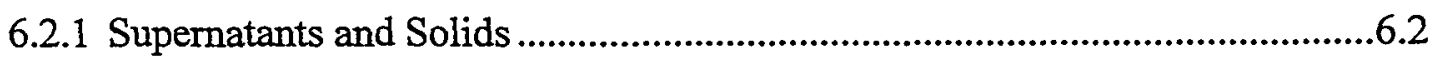

6.2.2 Laboratory Control Standard and Glassware Blank ........................................6.2

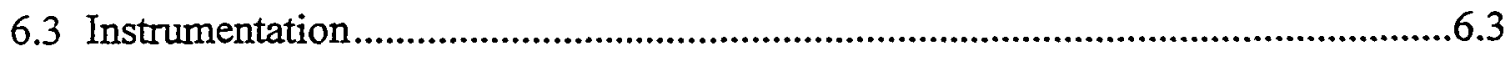

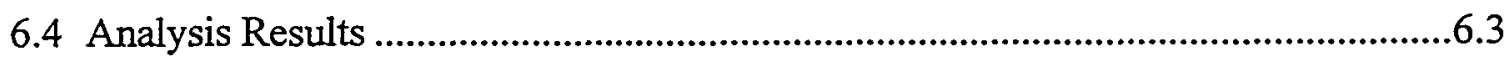

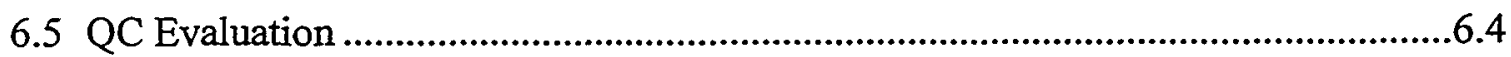

6.5.1 Ion Abundance Ratios and Response Factors --.- Standards ...........................6.4

6.5.2 Ion Abundance Ratios - Samples, Duplicates, and Matrix Spikes..................6.5

6.5.3 Dioxins and Furans Matrix Spike Recoveries .................................................6.7

6.5.4 Other Observations (Deviations/Concerns/Issues) ..........................................6.8

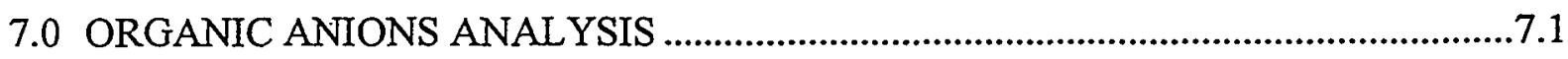

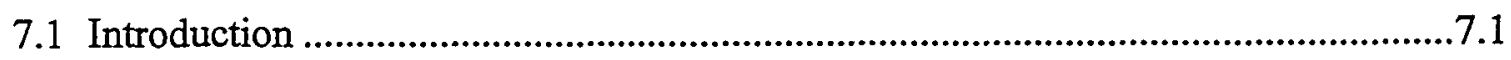

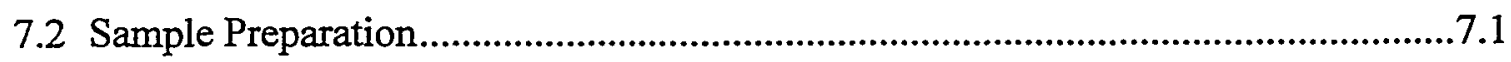

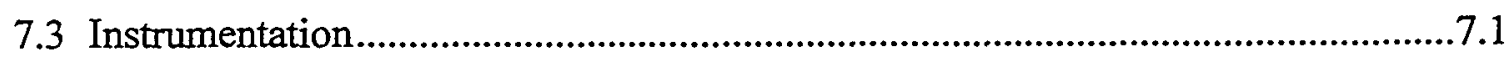

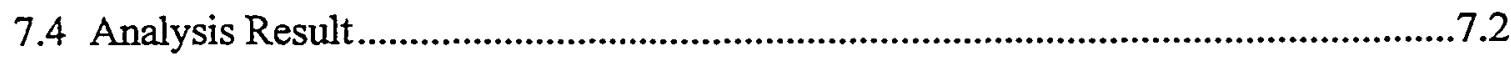

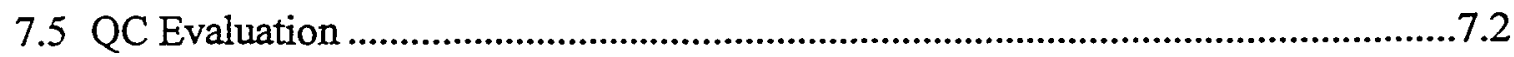

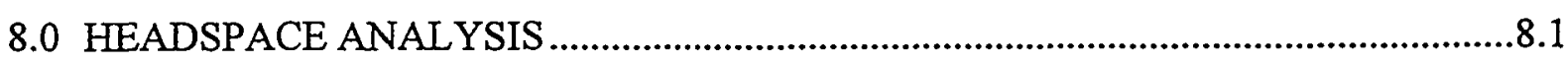

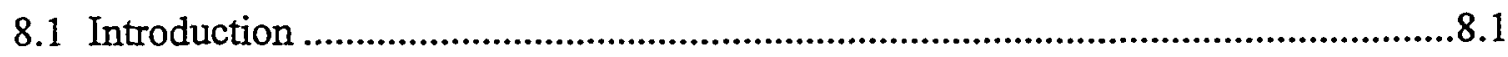

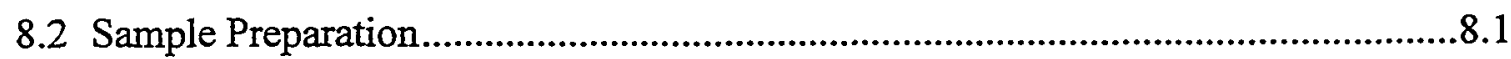

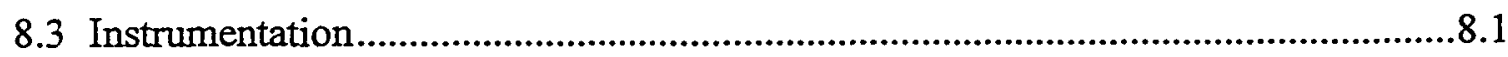

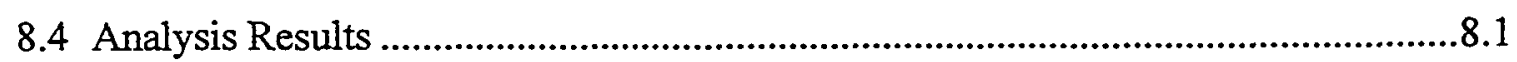

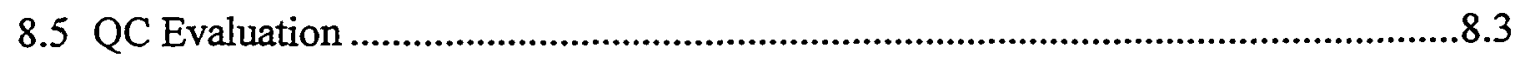

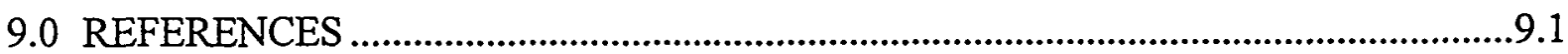

APPENDIX A: VOLATILE ORGANIC ANALYSIS RESULT FORMS ............................1

APPENDIX B: SEMI-VOLATILE ORGANIC ANALYSIS RESULT FORMS...................1

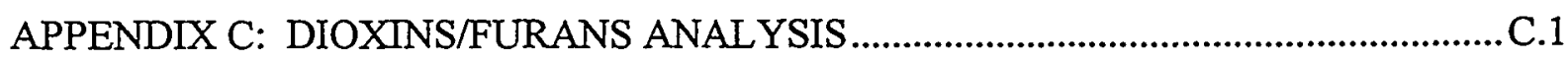




\section{Figures}

Figure 1.1. Flow Diagram for AW-101 As Received Composite Analytical Samples .........1.3

Figure 1.2 Flow Diagram for AN-107 As Received Composite Analytical Samples ............1.4

Figure 4.1 Phosphoric Acid Titration Curve.........................................................................4.2

\section{Tables}

Table S.a. AW-101 Summary Results - BNFL Target Analytes Detected ............................. iv

Table S.b. AN-107 Summary Results - BNFL Target Analytes Detected..................................

Table 2.a. Supernatant and Solids following Phase Separation.............................................2.2

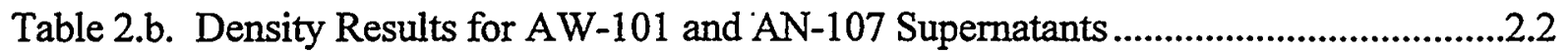

Table 3.a. VOA Instrumentation............................................................................................

Table 3.b. AW-101 VOA Results - BNFL \& SW846 8260B Target Analyte List.................3.3

Table 3.c. AN-107 VOA Results - BNFL \& SW846 8260B Target Analyte List....................5

Table 3.d. AW-101 \& AN-107 VOA Tentatively Identified Compounds ...............................3.8

Table 3.e. Target VOA Minimum Reportable Quantities..........................................................

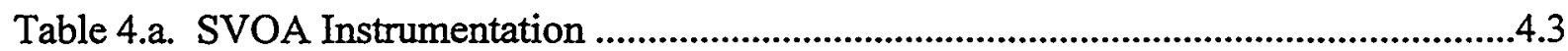

Table 4.b. AW-101 SVOA Results - BNFL \& SW846 8270C Target Analyte List .............4.4

Table 4.c. AN-107 SVOA Results - BNFL \& SW846 8270C Target Analyte List ..............4.7

Table 4.d. AW-101 Supernatant SVOA Tentatively Identified Compounds .......................4.11

Table 4.e. AW-101 Solids SVOA Tentatively Identified Compounds....................................12

Table 4.f. AN-107 Supernatant SVOA Tentatively Identified Compounds...........................13

Table 4.g. AN-107 Solids SVOA Tentatively Identified Compounds ...................................4.14

Table 4.h. Target SVOA Minimum Reportable Quantities ....................................................15

Table 5.a. PCB/Pesticides Analysis Instrumentation..............................................................

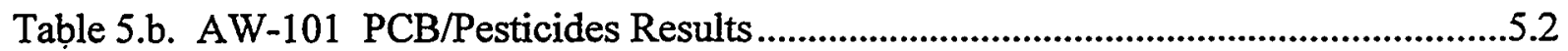

Table 5.c. AN-107 PCB/Pesticides Results .......................................................................

Table 5.d. Target PCB/Pesticides Minimum Reportable Quantities ......................................5.4

Table 5.e. AW-101 and AN-107 Supernatant -- Spike Recoveries .........................................5.4

Table 5.f. AW-101 and AN-107 Wet Centrifuged Solids - Spike Recoveries........................5.5

Table 6.a. Standard Compounds Measured Using HRGC/LRMS.........................................6.1

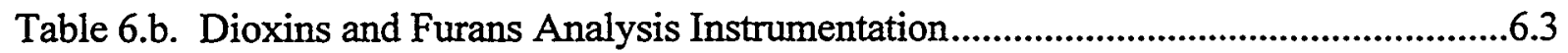

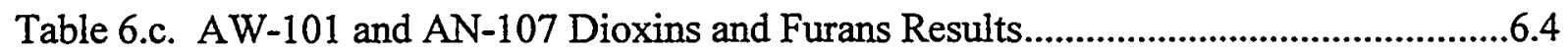

Table 6.d. Theoretical Ion Abundance Ratios and Control Limits ...........................................6.5

Table 6.e. Acceptance Criteria Summary of Ion Abundance Ratios ......................................6.6 
Table 6.f. AW-101 and AN-107 Supernatant - Dioxins and Furans Spike Recoveries........6.7

Table 6.g. AW-101 and AN-107 Solids -.- Dioxins and Furans Spike Recoveries ...............6.8

Table 7.a. Ion Chromatography Instrumentation ..............................................................

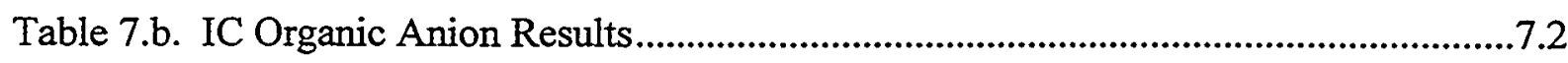

Table 7.c. IC Organic Anions Matrix Spike Recoveries .........................................................

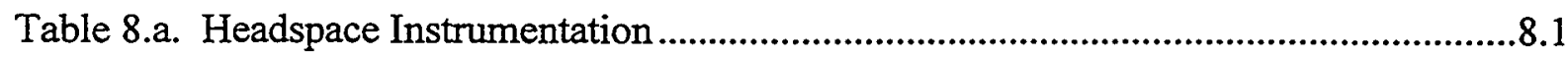

Table 8.b. AW-101 and AN-107 Results - Headspace Analysis ...........................................

Table 8.c. Target Headspace Minimum Reportable Quantities................................................8.2

Table 8.d. AW-101 and AN-107 Headspace Matrix Spike Recoveries ...................................

Table C.1. Ion Abundance Ratios and Response Factors for Standards................................

Table C.2. Ion Abundance Ratios for Supernatant Matrix Spikes.........................................

Table C.3. Ion Abundance Ratios for Solids Matrix Spikes.................................................. .5

Table C.4. Ion Abundance Ratios for Supernatant Samples, Duplicates, and Process

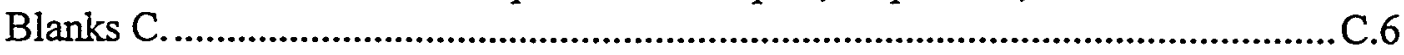

Table C.5. Ion Abundance Ratios for Solids Samples, Duplicates, and Process Blanks......C.6 


\subsection{Introduction}

This report presents the organic analytical results for "as received" AW-101 and AN-107 tank waste materials. The organic analyses were conducted in support of the BNFL Proposal No. 29274/30406 Task 5.0. The organic analysis results obtained from the "as received" tank waste materials may be used to support permitting activities, as well as to provide limited characterization information for subsequent process testing (Tasks 2 through 4). SW-846 sampling, preservation, and hold time protocols are difficult to address for highly radioactive Hanford tank wastes and have historically not been implemented when sampling, transporting, and processing these materials. Based on the sampling and storage history of the samples, preservation or refrigeration of the "as received" samples was not performed, and hold times specified by SW-846 protocols had expired well prior to receiving the samples.

The method detection limits (MDL) for the analytes of interest were significantly impacted by the limited quantity of sample available for analysis. However, wherever possible the analytical protocols follow SW-846 guidelines. The concentrations of spiking solutions and choice of extraction solvents are based on SW-846 methods.

For all of the organic analyses (i.e., VOA, SVOA, PCB/Pesticides, Dioxins and Furans, Headspace, and organic anion) SW-846 methodology was followed as closely as possible. The organic analyses were conducted per approve Battelle analytical methods (procedures or test plans). Due to the sample matrices and radiological condition of the samples, the Battelle procedures deviate slightly from their SW-846 counterpart. Due to time and funding limitations, no effort has been made to validate these deviations. However, analytical quality control samples (e.g., matrix spike, matrix spike duplicates, and laboratory control samples) have been used to ensure that the methods used meet the quality objective for the task. Deviations considered significant are noted in the appropriate sections. The organic analytes of interest (target compounds) and recommended methods are defined in the BNFL Proposal No. 29274/30406 and Test Plan BNFL-29953-2 Revision 1. Except where noted in this report, all organic target compounds defined by these documents are reported, with estimated MDLs provided where target compounds are not detected. Where detected, non-target compounds are identified, reported and quantified to the extent possible.

Composites of both $\mathrm{AW}-101$ and $\mathrm{AN}-107$ as received material were prepared per Test Plan BNFL-29953-1, Revision 0. A composite of the AN-107 as received material was prepared by combining the contents from the $17 \mathrm{AN}-107$ shipping jars in a four-liter glass kettle. A composite of the AW-101 as received material was prepared similarly from the contents from the $30 \mathrm{AW}-101$ shipping jars. Once in the four-liter glass kettle, a bladed impeller was used to homogenize each of the composites. While each composite was being stirred, a $1 / 4$-inch (outside diameter) stainless steel probe was used to vacuum transfer sub-samples of the homogenized composite slurry to a minimum of five $125-\mathrm{mL}$ glass jars. The composite sub-samples transferred to each $125-\mathrm{mL}$ glass jar were extracted from different elevations and radial locations within the kettles during mixing. These sub-samples were allowed to settle for approximately 12 hours. After this settling period, the volume percent of settled solids in each of the 125 -mL glass jars were similar (i.e., within $\pm 10 \%$ ), providing indication that the sub-samples were representative of the composite.

Figures 1.1 and 1.2 provide sample flow diagrams for the preparation of the AW-101 and AN-107 as received analytical characterization sub-samples. Six $125-\mathrm{mL}$ glass jars of AN-107 and five 125-mL jars of AW-101 were sub-sampled for characterization analysis. The compositing and sub-sampling operations were conducted in the High Level Radiation Facility (HLRF). The sub-samples were 
transferred under chain-of-custody (COC) to the Shielded Analytical Laboratory (SAL) for phase separation and analytical preparations.

The organic results for the analytes of interest for the AW-101 and AN-107 as received materials are typically reported in " $\mu \mathrm{g} / \mathrm{L}$ supernatant" or " $\mu \mathrm{g} / \mathrm{Kg}$ centrifuged wet solids". However, in some cases where the analyte concentrations are high or the method sensitivity is low, the results are reported in $\mu \mathrm{g} / \mathrm{mL}$ or $\mu \mathrm{g} / \mathrm{g}$. Although the supernatants were processed by weight, the density of the supernatants has been used to provide the results in $\mu \mathrm{g} / \mathrm{L}$ or $\mu \mathrm{g} / \mathrm{mL}$, as appropriate.

To evaluate the concentration of analytes of interest in the as received slurry material, estimates of the slurry concentration have been calculated from the analyte concentrations measured in the supernatant and in the wet centrifuged solids. To provide a conservative total slurry concentration, the highest measured concentration from either the sample or the duplicate for each phase is used in the calculation. Where no analyte concentration is measured (i.e., results less than MDL), the lowest MDL is used in the calculation. The "maximum" slurry concentration is calculated by Equation (1).

$$
C_{m}=\left(\left(C_{1} / D_{1}\right) * W_{1}\right)+\left(C_{s} * W_{s}\right)
$$

Where: $\quad C_{m} \quad=$ Maximum slurry concentration in $\mu \mathrm{g} / \mathrm{Kg}$

$\mathrm{C}_{\mathrm{l}} \quad=$ Concentration of supernatant in $\mu \mathrm{g} / \mathrm{L}$

$D_{1} \quad=$ Density of supernatant in $\mathrm{Kg} / \mathrm{L}$

$\mathrm{W}_{1}=$ Weight fraction of supernatant (i.e., 0.838 for $\mathrm{AW}-101,0.886$ for $\mathrm{AN}-107$ )

$\mathrm{C}_{\mathrm{s}} \quad=$ Concentration of solids in $\mu \mathrm{g} / \mathrm{Kg}$

$\mathrm{W}_{\mathrm{s}} \quad=$ Weight fraction of solids (i.e., 0.162 for $\mathrm{AW}-101,0.114$ for $\mathrm{AN}-107$ ) 


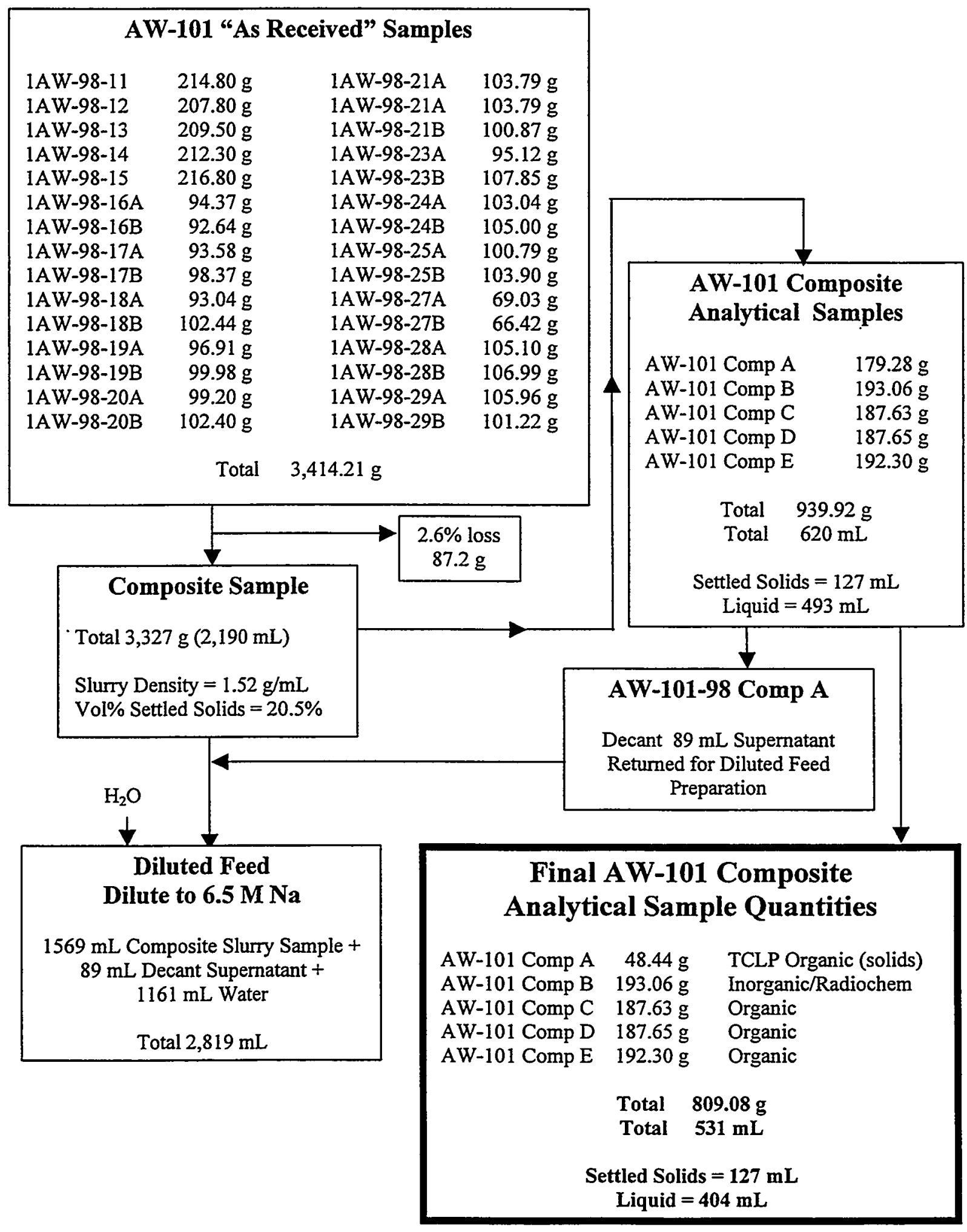

Figure 1.1. Flow Diagram for AW-101 As Received Composite Analytical Samples 


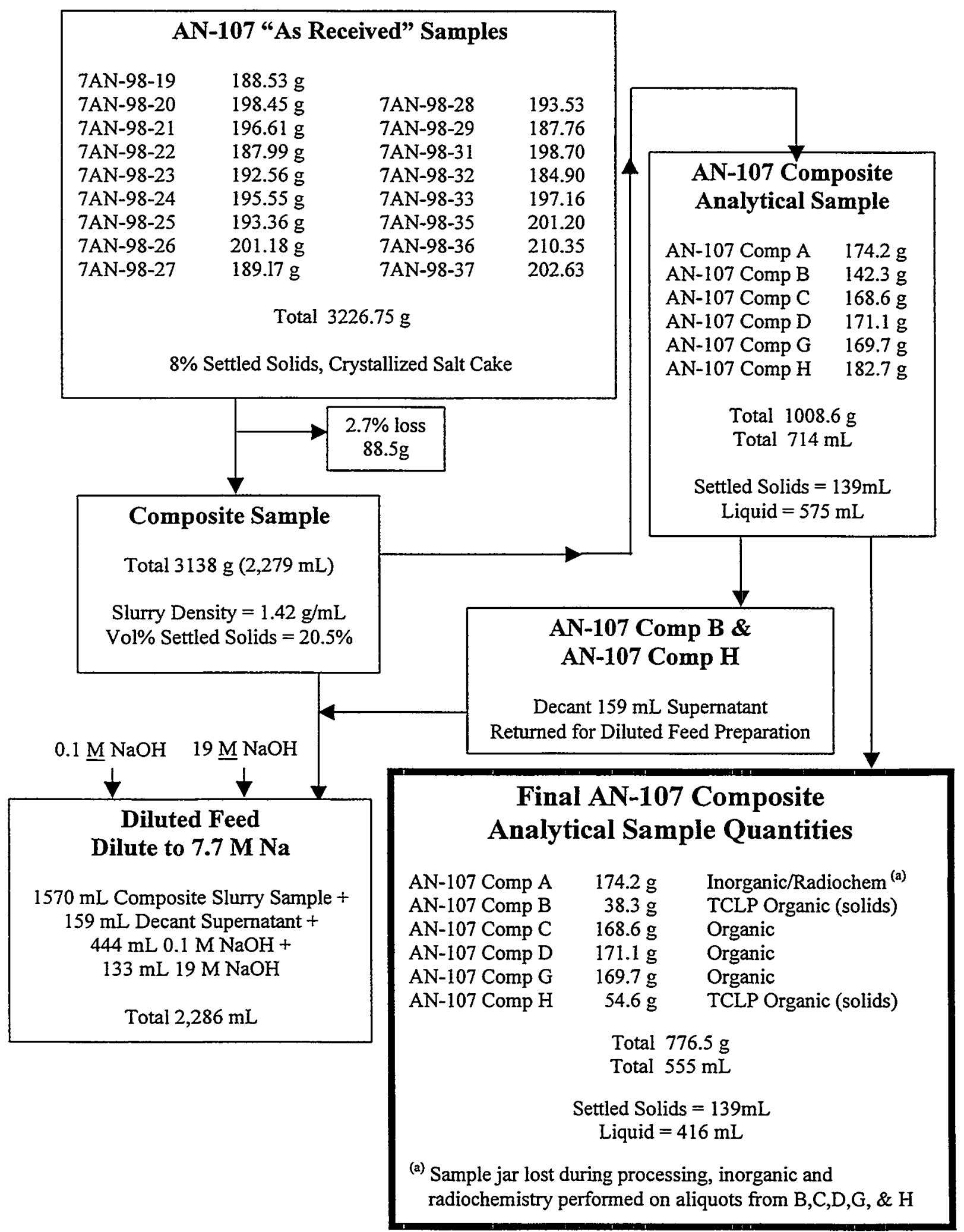

Figure 1.2 Flow Diagram for AN-107 As Received Composite Analytical Samples 


\subsection{Sample Receipt and Analytical Sub-sampling}

\subsection{Sample Receipt}

A total of five 125-mL glass jars of AW-101 and six 125-mL glass jars of $A N-107$ were received by the SAL following compositing, homogenizing, and sub-sampling of the "as received" composite material in the HLRF. Of these jars, three from each composite were allocated for organic analysis and one from each composite was allocated for inorganic and radiochemical analysis. The remaining jar of AW-101 and two jars of AN-107 were allocated for solids toxic characteristic leaching procedure (TCLP) analysis and contained only wet solids (i.e., the majority of supernatant had been decanted for use in process testing tasks in HLRF).

Due to a jar failure during magnetic stirring, the entire AN-107 composite originally allocated for inorganic and radiochemical analysis was lost. Therefore, sub-samples from each of the AN-107 jars allocated for organic analysis were taken to support the inorganic and radiochemical analyses; thus reducing the quantity available for the organic analysis. The phase separation and sub-sampling activities are fully detailed in the Analytical Service Request (ASR) 5188.02 Special Instructions submit for the inorganic, radiochemical, and organic analysis. The sub-sampling quantities defined in the ASR 5188.02 supersede the sub-sampling quantities estimated in Test Plan BNFL-29953-2. For all organic analyses, the duplicate, matrix spikes (MS), and matrix spike duplicates (MSD) for the supernatants and wet centrifuged solids are sub-sampled prior to any preparation, extraction processing, or direct analysis.

\subsection{Phase Separation}

Following receipt of the samples in the SAL, the contents of each of jar was phase separated so that organic analyses could be performed on each phase (i.e., supernatant and wet solids). The phase separation was performed by centrifuging and decanting the supernatant. Centrifuging and decanting was chosen because initial filtering attempts in the HLRF indicated that filtering of the samples would be extremely difficult (i.e., hours to days to filter small quantities of material) and there is an associated risk of loosing analytes of interest due to filtering. Each sample was centrifuged in its original jar, and following centrifuging the supernatant was decanted to pre-labeled Teflon bottles (i.e., the solids remained in the original jar). Table 2.a details the quantity of supernatant and solids collected from each sub-sample. The weight percent supernatant and solids values are used to recombine the results from the supernatant and solids phases into the estimated maximum organic results for the original tank waste slurry.

\subsection{Supernatant Density Measurements}

Due to the viscous nature of the tank waste material, all supernatant samples were processed by weight (i.e., samples aliquots taken by weight instead of by volume). Therefore, the density of each of the tank waste samples was determined using method PNL-ALO-501. The resulting densities are used to report the analytical results on a per volume basis. Table $2 . b$ provide the density results obtained by the SAL on AW-101 and AN-107 supernatants following phase separation. For both AW-101 and AN-107 supernatant, the density was measured in duplicate from the jars labeled "C". It is assumed that the supernatants in the other jars have the same densities, respectively. 
Table 2.a. Supernatant and Solids following Phase Separation

\begin{tabular}{|c|c|c|c|c|c|c|}
\hline Sample ${ }^{(1)}$ & Lab ID & $\begin{array}{c}\text { Total } \\
\text { Mass }^{(2)}\end{array}$ & $\begin{array}{l}\text { Supernatant } \\
\text { Mass (g) }\end{array}$ & $\begin{array}{c}\text { Wt\% } \\
\text { Supernatant }\end{array}$ & $\begin{array}{l}\text { Wet Solids } \\
\text { Mass (g) }\end{array}$ & $\begin{array}{l}\text { Wt\% Wet } \\
\text { Solids }\end{array}$ \\
\hline $\begin{array}{l}\text { AW-101 } \\
\text { Comp (C) }\end{array}$ & \multirow{3}{*}{$\begin{array}{l}99-0648 \\
\text { Supernatant } \\
99-0650 \\
\text { Solids }\end{array}$} & 188.2 & 157.6 & 83.7 & 30.6 & 16.3 \\
\hline $\begin{array}{l}\text { AW-101 } \\
\text { Comp (D) }\end{array}$ & & 190.7 & 160.0 & 83.9 & 30.7 & 16.1 \\
\hline $\begin{array}{l}\text { AW-101 } \\
\text { Comp (E) }\end{array}$ & & 190.1 & 159.3 & 83.8 & 30.8 & 16.2 \\
\hline $\begin{array}{l}\text { AN-107 } \\
\text { Comp (C) }\end{array}$ & \multirow{3}{*}{$\begin{array}{l}99-0649 \\
\text { Supernatant } \\
99-0651 \\
\text { Solids }\end{array}$} & 170.4 & 151.2 & 88.8 & 19.2 & 11.2 \\
\hline $\begin{array}{l}\text { AN-107 } \\
\text { Comp (D) }\end{array}$ & & 170.8 & 151.4 & 88.6 & 19.4 & 11.4 \\
\hline $\begin{array}{l}\text { AN-107 } \\
\text { Comp (G) }\end{array}$ & & 162.5 & 143.4 & 88.2 & 19.1 & 11.8 \\
\hline
\end{tabular}

1) Suffix () represents individual bottle/jar identification number

2) Total mass remaining following phase separation activities in the SAL.

Table 2.b. Density Results for AW-101 and AN-107 Supernatants

\begin{tabular}{|l|l|c|c|c|}
\hline Sample $^{(\prime)}$ & Lab ID & $\begin{array}{c}\text { Sample } \\
\text { Density (g/mL) }\end{array}$ & $\begin{array}{c}\text { Duplicate } \\
\text { Density (g/mL) }\end{array}$ & $\begin{array}{c}\text { Average } \\
\text { Density (g/mL) }\end{array}$ \\
\hline \hline AW-101 Comp (C) & $99-0648$ & 1.487 & 1.480 & 1.484 \\
\hline AN-107 Comp (C) & $99-0649$ & 1.421 & 1.418 & 1.420 \\
\hline \multicolumn{2}{|l|}{ 1) Suffix () } & \multicolumn{2}{l}{} \\
\hline
\end{tabular}

1) Suffix () represents individual bottle identification. 


\subsection{Volatile Organic Analysis (VOA)}

\subsection{Introduction}

Volatile organic analyses were performed on both the supernatant and solids from samples AW-101 and AN-107 following phase separation. The VOA samples were aliquotted and prepared for analysis following phase separation and were removed from the SAL hot cells prior to introducing any organic solvents (e.g., methylene chloride) into the hot cells.

The samples, both supernatants and solids, were diluted with organic-free water from 3-fold to 10 -fold to a final volume of approximately $5-\mathrm{mL}$ in special disposable dual septa-sealed purge vessels. The volatile compounds were purged from the samples with helium onto a multi-bed absorbent trap using a commercial purge and trap sample concentrator and auto-sampler. The trapped volatile compounds were then thermally desorbed onto a 75 -meter by 0.45 -millimeter DB-624 column (2.55-micron film) that was directly interfaced to the mass spectrometer. The samples were analyzed using VOA method PNL-ALO-335 (per SW-846 8260B protocols).

\subsection{Sample Preparation}

Supernatant and solids from AW-101 and AN-107 were prepared in the SAL by accurately weighing 0.3 to $1.4 \mathrm{~g}$ of sample into pre-cleaned, disposable, $40-\mathrm{mL}$ purge vessels and adding 4 to $5 \mathrm{~mL}$ of water diluent. Sample, duplicate, MS, MSD and blank samples were prepared in this manner in the SAL. Following transfer under COC from the SAL to the analytical laboratories, all samples were refrigerated to ensure that sample integrity was maintained. Internal standards and surrogate compounds were added to each sample (including the MS and MSD) and target spike compounds are added to the MS and MSD. Once the spikes and standards were added, the samples were loaded into the VOA auto-sampler for purging.

\subsection{Instrumentation}

The analytical instrumentation used for VOA consists of an auto-sampler, purge and trap system, and gas chromatograph mass spectrometer system. Detailed description of the VOA system is provided in Table 3.a.

Table 3.a. VOA Instrumentation

\begin{tabular}{|l|l|c|c|}
\hline \hline System/Instrument & Manufacturer & Model Number & M\&TE $^{(\text {()) }}$ Number \\
\hline \hline Auto-sampler & Dynatech & PTA-30 & WD25729 \\
\hline Purge \& Trap & OI & 4560 & WD25728 \\
\hline GC/MS & Hewlett Packard & $589011 / 5989 \mathrm{~A}$ & WC22547/WC28119 \\
\hline
\end{tabular}

1) Measuring and Test Equipment

\subsection{Analysis Results}

The VOA target (calibrated analytes) results for AW-101 and AN-107 supernatant and solids phases are given in Tables 3.b and 3.c. Additionally, the results for any VOA tentatively identified compounds (TIC) that were detected for both AW-101 and AN-107 supernatant and solids phases are given in Table 3.d. For both target compounds and TICs, the results are given in units of $\mu \mathrm{g} / \mathrm{L}$ for the supernatant and $\mu \mathrm{g} / \mathrm{Kg}$ for the wet solid phase. 
The MDLs provided are based upon instrument detection limits and the weight or volume of the sample used for the analysis. The MDLs are nominal, and are not based upon performance of the method on these specific sample matrices. In all cases the MDLs for the wet centrifuged solids and the supernatants (after adjusting for density) meet the BNFL VOA minimum reportable quantity (MRQ) requirements as detailed in Table 3.e.

VOA results below the MDL are quantified by the data analysis system; however, only those results greater then $20 \%$ of the MDL are reported. Results less than $20 \%$ of the MDL, while detected, are consider too low to accurately quantify.

\subsubsection{Results for Calibrated/Regulatory Analytes of Interest}

\section{AW-101 Results}

As seen in Table 3.b, few target compounds were detected in the AW-101 supernatant. Only 1,4-dioxane, acetone, and tetrahydrofuran were found at concentrations greater than the MDL. 2-Butanone was detected in the duplicate and chlorodifluoromethane was detected in the blank at concentrations slightly below the MDL. Chlorodifluoromethane was detected in the storage blank (used as the method blank in the analysis sequence), and is likely due to contamination from the walk-in refrigeration unit that the samples were stored in after removal from SAL. 1-Butanol and 2-ethyl-1-hexanol were detected in the sample and sample duplicate and measured as TICs. These compounds are likely decomposition products from other compounds, such as tributylphosphate and bis(2-ethylhexyl)phosphate used in the PUREX and B-Plant extraction processes.

The AW-101 solids samples contained a greater number of target analytes. 1,4-Dioxane, acetone, tetrahydrofuran, 2-butanone, 3-heptanone, butane, heptane, hexane, nonane, octane, pentane were all found at concentrations greater than the MDL. 2-Hexanone, 3-methyl-2-butanone, 4-heptanone, 4-methyl-2-pentanone, acetonitrile, benzene, and chlorodifluoromethane were detected at concentrations slightly below the MDL. The AW-101 solids had a large number of TICs, including 1-butanol, 2-ethyl-1-hexanol, undecane, dodecane, tridecane, and various other alkanes, alkenes and alcohols. It is interesting to note that the TIC data for AW-101 solids contain 1-butene and 2-methyl1-propene. Like butane, these compounds are gases at room temperature, and not likely to be retained in the original tank material given its storage history. And, it is conceivable that these compounds are generated continuously from the breakdown of dodecane, tridecane, tetradecane, tributylphosphate, or 1-butanol.

\section{AN-107 Results}

As seen in Table 3.c, five target compounds were detected in the supernatant. Only acetone and acetonitrile were found at concentrations greater than the MDL. 2-Butanone, tetrahydrofuran and chlorodifluoromethane were detected at concentration less than the MDL. As noted above, chlorodifluoromethane was detected in the storage blank and is most likely from contamination following removal of the samples from the SAL (i.e., the presence of chlorodifluoromethane in the samples is unlikely). Butenal and 1-butanol, were detected in the sample and sample duplicate and measured as TICs.

AN-107 solids sample results are similar to the supernatant result, but also include 2-heptanone and methylcyclohexane (all found at concentrations below the MDL). Tridecane, tetradecane, and 5-tridecanone were detected and measured as TICs in the AN-107 solids. 
Table 3.b. AW-101 VOA Results - BNFL \& SW846 8260B Target Analyte List

\begin{tabular}{|c|c|c|c|c|c|c|c|c|}
\hline & \multicolumn{3}{|c|}{ AW-101 Supernatant } & \multicolumn{3}{|c|}{ AW-101 Solids ${ }^{(1)}$} & \multirow{3}{*}{$\begin{array}{l}\text { Maximum } \\
\mu g / K^{(2)} \\
\text { of Slurry }\end{array}$} \\
\hline 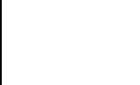 & Sample ID & $\begin{array}{l}99-0648-b l \\
\text { VBLK01 }\end{array}$ & $\begin{array}{l}99-0648 \\
\text { Sample }\end{array}$ & $\begin{array}{l}\text { 99-0648-d } \\
\text { Duplicate }\end{array}$ & \begin{tabular}{c|}
$99-0650-b l$ \\
VBLK03
\end{tabular} & $\begin{array}{l}99-0650 \\
\text { Sample }\end{array}$ & $\begin{array}{l}99-0650-d \\
\text { Duplicate }\end{array}$ & \\
\hline \multirow{2}{*}{\multicolumn{2}{|c|}{$\begin{array}{r}\text { Units } \\
M D L^{(3)}\end{array}$}} & $\mu \mathrm{g} / \mathrm{L}$ & $\mu \mathrm{g} / \mathrm{L}$ & $\mu g / L$ & $\mu \mathrm{g} / \mathbf{K g}$ & $\mu g / \mathrm{Kg}$ & $\mu \mathrm{g} / \mathrm{Kg}$ & \\
\hline & & 10 & 51 & 56 & 10 & 42 & 94 & 36 \\
\hline \multicolumn{9}{|c|}{ BNFL Target Analyte List } \\
\hline $100-41-4$ & Ethylbenzene & $\bar{U}$ & $\bar{U}$ & $\overline{\mathrm{U}}$ & $\mathbf{U}$ & $\overline{\mathrm{U}}$ & $\bar{U}$ & $\bar{U}$ \\
\hline $100-42-5$ & Styrene & $\mathrm{U}$ & $\bar{U}$ & $\overline{\mathrm{U}}$ & $\mathrm{U}$ & $\overline{\mathrm{U}}$ & $\bar{U}$ & $\bar{U}$ \\
\hline 10061-01- & cis-1,3-Dichloropropene & $\mathrm{U}$ & $\overline{\mathrm{U}}$ & $\overline{\mathrm{U}}$ & $\mathrm{U}$ & $\bar{U}$ & $\overline{\mathrm{U}}$ & $\bar{U}$ \\
\hline 10061-02- & trans-1,3-Dichloropropene & $\bar{U}$ & $\bar{U}$ & $\bar{U}$ & $\bar{U}$ & $\overline{\mathrm{U}}$ & $\overline{\mathrm{U}}$ & $\bar{U}$ \\
\hline $106-35-4$ & 3-Heptanone & $\mathrm{U}$ & $\overline{\mathrm{U}}$ & $\overline{\mathrm{U}}$ & $\bar{U}$ & 59 & $32 \mathrm{~J}$ & 10 \\
\hline $106-42-3$ & Xylene (m \& p) & $\overline{\mathrm{U}}$ & $\bar{U}$ & $\overline{\mathrm{U}}$ & $\bar{U}$ & $\mathrm{U}$ & $\bar{U}$ & $U$ \\
\hline $106-46-7$ & 1,4-Dichlorobenzene & $\mathrm{U}$ & U & $\bar{U}$ & $\mathrm{U}$ & $\mathrm{U}$ & $\mathrm{U}$ & $U$ \\
\hline $106-93-4$ & 1,2-Dibromoethane & $\bar{U}$ & $\mathrm{U}$ & $\mathrm{U}$ & $\mathrm{U}$ & $\bar{U}$ & $\overline{\mathrm{U}}$ & $U$ \\
\hline $106-97-8$ & Butane & $\mathrm{U}$ & $\mathrm{U}$ & $\mathrm{U}$ & $\mathrm{U}$ & 860 & 920 & 149 \\
\hline $106-99-0$ & 1,3-Butadiene & $\bar{U}$ & $\overline{\mathrm{U}}$ & $\mathrm{U}$ & $\bar{U}$ & $\mathrm{U}$ & $\mathrm{U}$ & $U$ \\
\hline $107-02-8$ & Acrolein & $\bar{U}$ & $\bar{U}$ & $\bar{U}$ & $\overline{\mathrm{U}}$ & $\mathrm{U}$ & $\mathrm{U}$ & $\bar{U}$ \\
\hline $107-05-1$ & 3-Chloropropene & $\bar{U}$ & $\bar{U}$ & $\overline{\mathrm{U}}$ & $\overline{\mathrm{U}}$ & $\bar{U}$ & $\overline{\mathrm{U}}$ & $\bar{U}$ \\
\hline $107-06-2$ & 1,2-Dichloroethane & $\bar{U}$ & $\bar{U}$ & $\overline{\mathrm{U}}$ & $\mathrm{U}$ & $\bar{U}$ & $\mathrm{U}$ & $\bar{U}$ \\
\hline $107-13-1$ & Acrylonitrile & $\bar{U}$ & $\bar{U}$ & $\overline{\mathrm{U}}$ & $\bar{U}$ & $\overline{\mathrm{U}}$ & $\overline{\mathrm{U}}$ & $\bar{U}$ \\
\hline $107-87-9$ & 2-Pentanone & $\overline{\mathrm{U}}$ & $\bar{U}$ & $\overline{\mathrm{U}}$ & $\bar{U}$ & $\mathrm{U}$ & $\bar{U}$ & $U$ \\
\hline $108-10-1$ & 4-Methyl-2-pentanone & $\bar{U}$ & $\bar{U}$ & $\bar{U}$ & $\mathrm{U}$ & $10 \mathrm{~J}$ & $\overline{\mathrm{U}}$ & $\overline{2}$ \\
\hline $108-87-2$ & Methylcyclohexane & $\mathrm{U}$ & $\mathrm{U}$ & $\mathrm{U}$ & $\mathrm{U}$ & $\mathrm{U}$ & $\mathrm{U}$ & $\bar{U}$ \\
\hline $108-88-3$ & Toluene & $\mathrm{U}$ & $U$ & $\mathrm{U}$ & $\mathrm{U}$ & $\mathrm{U}$ & $\bar{U}$ & $\bar{U}$ \\
\hline $108-90-7$ & Chlorobenzene & $\mathrm{U}$ & $\bar{U}$ & $\bar{U}$ & $\mathrm{U}$ & $\mathrm{U}$ & $\overline{\mathrm{U}}$ & $\bar{U}$ \\
\hline $109-66-0$ & Pentane & $\mathrm{U}$ & $\mathrm{U}$ & $\mathrm{U}$ & $\overline{\mathrm{U}}$ & 150 & $80 \mathrm{~J}$ & 24 \\
\hline $109-99-9$ & Tetrahydrofuran & $\overline{\mathrm{U}}$ & 140 & 110 & $\mathrm{U}$ & 180 & $89 \mathrm{~J}$ & 108 \\
\hline $110-12-3$ & 5-Methyl-2-hexanone & $\overline{\mathrm{U}}$ & $\mathrm{U}$ & $\mathrm{U}$ & $\bar{U}$ & $\mathrm{U}$ & $\bar{U}$ & $\bar{U}$ \\
\hline $110-43-0$ & 2-Heptanone & $\bar{U}$ & $\overline{\mathrm{U}}$ & $\bar{U}$ & $\bar{U}$ & 59 & $36 \mathrm{~J}$ & 10 \\
\hline $110-54-3$ & Hexane & $\overline{\mathrm{U}}$ & $\bar{U}$ & $\overline{\mathrm{U}}$ & $\overline{\mathrm{U}}$ & 200 & $80 \mathrm{~J}$ & 32 \\
\hline $110-82-7$ & Cyclohexane & $\bar{U}$ & $\overline{\mathrm{U}}$ & $\mathrm{U}$ & $\bar{U}$ & $\mathrm{U}$ & $\mathrm{U}$ & $U$ \\
\hline $110-83-8$ & Cyclohexene & $\overline{\mathrm{U}}$ & $\overline{\mathrm{U}}$ & $\bar{U}$ & $\bar{U}$ & $\bar{U}$ & $\bar{U}$ & $U$ \\
\hline $111-65-9$ & Octane & $\mathrm{U}$ & $\mathrm{U}$ & $\mathrm{U}$ & $\mathbf{U}$ & 200 & 99 & 32 \\
\hline $111-84-2$ & Nonane & $\mathrm{U}$ & $\mathrm{U}$ & $\mathrm{U}$ & $\mathrm{U}$ & 250 & 180 & 41 \\
\hline $123-19-3$ & 4-Heptanone & $\mathrm{U}$ & $\mathrm{U}$ & $\mathrm{U}$ & $\mathrm{U}$ & $8 \mathrm{~J}$ & $\mathrm{U}$ & $I$ \\
\hline $123-38-6$ & Propionaldehyde & $\mathrm{U}$ & $\mathrm{U}$ & U & $\mathrm{U}$ & $\mathrm{U}$ & $\mathrm{U}$ & $U$ \\
\hline $123-86-4$ & Butylacetate & $\mathrm{U}$ & $\mathrm{U}$ & $\mathrm{U}$ & $\mathrm{U}$ & $\mathrm{U}$ & $\bar{U}$ & $\bar{U}$ \\
\hline $123-91-1$ & 1,4-Dioxane & $\mathrm{U}$ & 180 & 150 & $\overline{\mathrm{U}}$ & 97 & $\bar{U}$ & 117 \\
\hline $126-98-7$ & 2-Methyl-2-propenenitrile & $\bar{U}$ & $\mathrm{U}$ & $\mathrm{U}$ & $\overline{\mathrm{U}}$ & $\mathrm{U}$ & $\bar{U}$ & $U$ \\
\hline $127-18-4$ & Tetrachloroethene & $\mathbf{U}$ & $\overline{\mathrm{U}}$ & $\bar{U}$ & $\bar{U}$ & $\bar{U}$ & $\overline{\mathrm{U}}$ & $\bar{U}$ \\
\hline $141-78-6$ & Ethyl acetate & $\mathrm{U}$ & $\mathrm{U}$ & $\mathrm{U}$ & $\mathrm{U}$ & $\mathrm{U}$ & $\mathrm{U}$ & $U$ \\
\hline $142-82-5$ & Heptane & $\mathrm{U}$ & $\mathrm{U}$ & $\bar{U}$ & $\bar{U}$ & 210 & $66 \mathrm{~J}$ & 34 \\
\hline $287-92-3$ & Cyclopentane & $\mathrm{U}$ & $\mathrm{U}$ & $\mathrm{U}$ & $\bar{U}$ & $\mathrm{U}$ & $\mathrm{U}$ & $U$ \\
\hline $4170-30-3$ & 2-Butenal & $\bar{U}$ & $\overline{\mathrm{U}}$ & $\overline{\mathrm{U}}$ & $\bar{U}$ & $\overline{\mathrm{U}}$ & $\bar{U}$ & $\bar{U}$ \\
\hline $541-73-1$ & 1,3-Dichlorobenzene & $\bar{U}$ & $\overline{\mathrm{U}}$ & $\bar{U}$ & $\overline{\mathrm{U}}$ & $\overline{\mathrm{U}}$ & $\bar{U}$ & $\bar{U}$ \\
\hline $56-23-5$ & Carbon Tetrachloride & $\overline{\mathrm{U}}$ & $\bar{U}$ & $\overline{\mathrm{U}}$ & $\mathrm{U}$ & $\mathrm{U}$ & $\mathrm{U}$ & $\bar{U}$ \\
\hline $563-80-4$ & 3-Methyl-2-butanone & $\bar{U}$ & $\mathrm{U}$ & $\bar{U}$ & $\overline{\mathrm{U}}$ & $34 \mathrm{~J}$ & $20 \mathrm{~J}$ & 6 \\
\hline $591-78-6$ & 2-Hexanone & $\bar{U}$ & $\mathrm{U}$ & $\overline{\mathrm{U}}$ & $\mathrm{U}$ & $40 \mathrm{~J}$ & $24 \mathrm{~J}$ & 6 \\
\hline $627-13-4$ & Propyl nitrate & $\overline{\mathrm{U}}$ & $\mathrm{U}$ & $\mathrm{U}$ & $\mathrm{U}$ & $\mathrm{U}$ & $\mathrm{U}$ & $U$ \\
\hline $67-64-1$ & Acetone & $\mathrm{U}$ & 330 & 360 & $6 \mathrm{JB}$ & $620 \mathrm{~B}$ & $360 \mathrm{~B}$ & 304 \\
\hline $67-66-3$ & Chloroform & $\mathrm{U}$ & $\bar{U}$ & $\mathrm{U}$ & $\mathrm{U}$ & $\bar{U}$ & $\mathrm{U}$ & $U$ \\
\hline $71-23-8$ & 1-Propanol & $\bar{U}$ & $\bar{U}$ & $\bar{U}$ & $\mathrm{U}$ & $\bar{U}$ & $\bar{U}$ & $U$ \\
\hline
\end{tabular}




\begin{tabular}{|c|c|c|c|c|c|c|c|c|}
\hline \multirow[b]{4}{*}{ CAS \# } & \multirow{2}{*}{$\begin{array}{r}\text { Tank Material } \\
\text { Sample ID }\end{array}$} & \multicolumn{3}{|c|}{ AW-101 Supernatant } & \multicolumn{3}{|c|}{ AW-101. Solids ${ }^{(1)}$} & \multirow{3}{*}{$\begin{array}{l}\text { Maximum } \\
\mu g / K g(2) \\
\text { of Slurny }\end{array}$} \\
\hline & & \begin{tabular}{|c|}
$\begin{array}{c}99-0648-b \mid \\
\text { VBLK01 }\end{array}$ \\
\end{tabular} & \begin{tabular}{|l|}
$99-0648$ \\
Sample \\
\end{tabular} & \begin{tabular}{|l|}
$99-0648-d$ \\
Duplicate \\
\end{tabular} & \begin{tabular}{|c|}
$99-0650-b \mid$ \\
VBLK03 \\
\end{tabular} & $\begin{array}{l}99-0650 \\
\text { Sample } \\
\end{array}$ & \begin{tabular}{|l|}
$99-0650-d$ \\
Duplicate
\end{tabular} & \\
\hline & Units & $\mu \mathrm{g} / \mathrm{L}$ & $\mu \mathrm{g} / \mathrm{L}$ & $\mu \mathrm{g} / \mathrm{L}$ & $\mu \mathrm{g} / \mathrm{Kg}$ & $\mu \mathrm{g} / \mathrm{Kg}$ & $\mu \mathrm{g} / \mathrm{Kg}$ & \\
\hline & & 10 & 51 & 56 & 10 & 42 & 94 & 36 \\
\hline $71-43-2$ & Benzene & $\mathrm{U}$ & $U$ & $\overline{\mathrm{U}}$ & $\overline{\mathrm{U}}$ & $10 \mathrm{~J}$ & $\overline{\mathrm{U}}$ & 2 \\
\hline $71-55-6$ & 1,1,1-Trichloroethane & $\bar{U}$ & $\bar{U}$ & $\bar{U}$ & $\bar{U}$ & $\mathrm{U}$ & $\overline{\mathrm{U}}$ & $\bar{U}$ \\
\hline $74-83-9$ & Bromomethane & $\mathrm{U}$ & $\mathrm{U}$ & $\bar{U}$ & $\overline{\mathrm{U}}$ & $\overline{\mathrm{U}}$ & $\bar{U}$ & $\bar{U}$ \\
\hline $74-87-3$ & Chloromethane & $\overline{\mathrm{U}}$ & $\bar{U}$ & $\overline{\mathrm{U}}$ & $\overline{\mathrm{U}}$ & $\bar{U}$ & $\mathrm{U}$ & $\bar{U}$ \\
\hline $75-00-3$ & Chloroethane & $\bar{U}$ & $\bar{U}$ & $\bar{U}$ & $\overline{\mathrm{U}}$ & $\mathrm{U}$ & $\mathrm{U}$ & $\bar{U}$ \\
\hline $75-01-4$ & Vinyl Chloride & $\bar{U}$ & $\mathrm{U}$ & $\overline{\mathrm{U}}$ & $\overline{\mathrm{U}}$ & $\mathrm{U}$ & $\bar{U}$ & $\bar{U}$ \\
\hline $75-05-8$ & Acetonitrile & $\overline{\mathrm{U}}$ & $\bar{U}$ & $\mathrm{U}$ & $\bar{U}$ & $41 \mathrm{~J}$ & $\mathrm{U}$ & 7 \\
\hline $75-09-2$ & Methylene Chloride & $\overline{\mathrm{U}}$ & $\bar{U}$ & $\overline{\mathrm{U}}$ & $\bar{U}$ & $\mathrm{U}$ & $\bar{U}$ & $\bar{U}$ \\
\hline $75-15-0$ & Carbon Disulfide & $\bar{U}$ & $\mathrm{U}$ & $\bar{U}$ & $\bar{U}$ & $\mathrm{U}$ & $\mathrm{U}$ & $\bar{U}$ \\
\hline $75-34-3$ & 1,1-Dichloroethane & $\overline{\mathrm{U}}$ & $\bar{U}$ & $\bar{U}$ & $\overline{\mathrm{U}}$ & $\bar{U}$ & $\bar{U}$ & $\bar{U}$ \\
\hline $75-35-4$ & 1,1-Dichloroethene & $\overline{\mathrm{U}}$ & $\bar{U}$ & $\bar{U}$ & $\bar{U}$ & $\mathrm{U}$ & $\overline{\mathrm{U}}$ & $\bar{U}$ \\
\hline $75-43-4$ & Dichlorofluoromethane & $\mathrm{U}$ & $\bar{U}$ & $\bar{U}$ & $\overline{\mathrm{U}}$ & U & $\bar{U}$ & $\bar{U}$ \\
\hline $75-45-6$ & Chlorodifluoromethane & $5 \mathrm{JB}$ & $\overline{\mathrm{U}}$ & $\overline{\mathrm{U}}$ & $7 \sqrt{\mathrm{B}}$ & $\mathrm{U}$ & $\bar{U}$ & $\bar{U}$ \\
\hline $75-69-4$ & Trichlorofluoromethane & $\mathrm{U}$ & $\mathrm{U}$ & $\mathrm{U}$ & $\mathrm{U}$ & $\overline{\mathrm{U}}$ & $\bar{U}$ & $\bar{U}$ \\
\hline $75-71-8$ & Dichlorodifluoromethane & $\bar{U}$ & $\mathrm{U}$ & $\mathrm{U}$ & $\bar{U}$ & $\mathrm{U}$ & $\mathrm{U}$ & $\bar{U}$ \\
\hline $76-13-1$ & 1,2,2-Trichloro-1,1,2-trifluoroethane & $\bar{U}$ & $\mathrm{U}$ & $\overline{\mathrm{U}}$ & $\bar{U}$ & $\mathrm{U}$ & $\mathrm{U}$ & $\bar{U}$ \\
\hline $76-14-2$ & 1,2-Dichloro-1,1,2,2- & $\mathbf{U}$ & $\mathrm{U}$ & $\mathrm{U}$ & $\overline{\mathrm{U}}$ & $\mathrm{U}$ & $\bar{U}$ & $\bar{U}$ \\
\hline 78-87-5 & 1,2-Dichloropropane & $\mathrm{U}$ & $\bar{U}$ & $\mathbf{U}$ & $\bar{U}$ & $\mathrm{U}$ & $\bar{U}$ & $\bar{U}$ \\
\hline 78-93-3 & 2-Butanone & $\mathrm{U}$ & $\mathrm{U}$ & $55 \mathrm{~J}$ & $\mathrm{U}$ & 160 & 110 & 57 \\
\hline $79-00-5$ & 1,1,2-Trichloroethane & $\mathbf{U}$ & $\bar{U}$ & $\mathrm{U}$ & $\bar{U}$ & $\mathrm{U}$ & $\mathrm{U}$ & $U$ \\
\hline $79-01-6$ & Trichloroethene & $\mathbf{U}$ & $\bar{U}$ & $\bar{U}$ & $\overline{\mathrm{U}}$ & $\overline{\mathrm{U}}$ & $\overline{\mathrm{U}}$ & $\bar{U}$ \\
\hline $79-34-5$ & 1,1,2,2-Tetrachloroethane & $\mathrm{U}$ & $\overline{\mathrm{U}}$ & $\bar{U}$ & $\bar{U}$ & $\bar{U}$ & $\bar{U}$ & $\bar{U}$ \\
\hline $95-47-6$ & Xylene (o) & $\mathrm{U}$ & $\mathrm{U}$ & $\overline{\mathrm{U}}$ & $\bar{U}$ & $\overline{\mathrm{U}}$ & $\overline{\mathrm{U}}$ & $\bar{U}$ \\
\hline $95-50-1$ & 1,2-Dichlorobenzene & $\overline{\mathrm{U}}$ & $\overline{\mathrm{U}}$ & $\overline{\mathrm{U}}$ & $\bar{U}$ & $\bar{U}$ & $\bar{U}$ & $\bar{U}$ \\
\hline $96-22-0$ & 3-Pentanone & $\bar{U}$ & $\overline{\mathrm{U}}$ & $\bar{U}$ & $\overline{\mathrm{U}}$ & $\bar{U}$ & $\bar{U}$ & $\bar{U}$ \\
\hline \multicolumn{9}{|c|}{ SW-846 8260B Target Analyte List } \\
\hline $103-65-1$ & Propylbenzene & $\mathrm{U}$ & $\bar{U}$ & $\mathrm{U}$ & $\overline{\mathrm{U}}$ & $\mathrm{U}$ & $\bar{U}$ & $\bar{U}$ \\
\hline $104-51-8$ & Butylbenzene & $\bar{U}$ & $\bar{U}$ & $\bar{U}$ & $\overline{\mathrm{U}}$ & $\overline{\mathrm{U}}$ & $\bar{U}$ & $\bar{U}$ \\
\hline $106-43-4$ & 4-Chlorotoluene & $\bar{U}$ & $\mathrm{U}$ & $\bar{U}$ & $\overline{\mathrm{U}}$ & $\overline{\mathrm{U}}$ & $\overline{\mathrm{U}}$ & $\bar{U}$ \\
\hline $108-67-8$ & 1,2,3-Trimethylbenzene & $\overline{\mathrm{U}}$ & $\bar{U}$ & $\bar{U}$ & $\bar{U}$ & $\overline{\mathrm{U}}$ & $\bar{U}$ & $\bar{U}$ \\
\hline $108-86-1$ & Bromobenzene & $\mathbf{U}$ & $\bar{U}$ & $\bar{U}$ & $\bar{U}$ & $\bar{U}$ & $\bar{U}$ & $\bar{U}$ \\
\hline $110-57-6$ & trans-1,4-Dichloro-2-butene & $\overline{\mathrm{U}}$ & $\overline{\mathrm{U}}$ & $\bar{U}$ & $\bar{U}$ & $\bar{U}$ & $\bar{U}$ & $\bar{U}$ \\
\hline $120-82-1$ & 1,2,4-Trichlorobenzene & $\mathrm{U}$ & $\bar{U}$ & $\bar{U}$ & $\overline{\mathrm{U}}$ & $\overline{\mathrm{U}}$ & $\bar{U}$ & $\bar{U}$ \\
\hline $124-48-1$ & Dibromochloromethane & $\bar{U}$ & $\mathrm{U}$ & $\bar{U}$ & $\overline{\mathrm{U}}$ & $\overline{\mathrm{U}}$ & $\bar{U}$ & $\bar{U}$ \\
\hline $135-98-8$ & sec-Butylbenzene & $\mathrm{U}$ & $\mathbf{U}$ & $\mathbf{U}$ & $\mathrm{U}$ & $\bar{U}$ & $\bar{U}$ & $\bar{U}$ \\
\hline $142-28-9$ & 1,3-Dichloropropane & $\mathrm{U}$ & $\overline{\mathrm{U}}$ & $\dot{U}$ & $\mathrm{U}$ & $\bar{U}$ & $\mathrm{U}$ & $\bar{U}$ \\
\hline $156-59-2$ & cis-1,2-Dichloroethene & $\mathrm{U}$ & $\mathrm{U}$ & $\mathrm{U}$ & $\overline{\mathrm{U}}$ & $\overline{\mathrm{U}}$ & $\overline{\mathrm{U}}$ & $\bar{U}$ \\
\hline $156-60-5$ & trans-1,2-Dichloroethene & $\bar{U}$ & $\mathbf{U}$ & $\mathrm{U}$ & $\bar{U}$ & $\overline{\mathrm{U}}$ & $\bar{U}$ & $\bar{U}$ \\
\hline $563-58-6$ & 1,1-Dichloropropene & $\mathrm{U}$ & $\mathrm{U}$ & $\mathrm{U}$ & $\mathrm{U}$ & $\overline{\mathrm{U}}$ & $\mathrm{U}$ & $\bar{U}$ \\
\hline $594-20-7$ & 2,2-Dichloropropane & $\mathrm{U}$ & $\mathrm{U}$ & $\mathrm{U}$ & $\overline{\mathrm{U}}$ & $\mathrm{U}$ & $\mathrm{U}$ & $U$ \\
\hline $74-88-4$ & Iodomethane & $\mathrm{U}$ & $\mathrm{U}$ & $\mathrm{U}$ & $\mathrm{U}$ & $\mathbf{U}$ & $\mathrm{U}$ & $U$ \\
\hline 74-95-3 & Dibromomethane & $\mathbf{U}$ & $\mathrm{U}$ & $\mathrm{U}$ & $\mathrm{U}$ & $\mathbf{U}$ & $\bar{U}$ & $U$ \\
\hline 74-97-5 & Bromochloromethane & $\mathrm{U}$ & $\mathrm{U}$ & $\mathrm{U}$ & $\mathrm{U}$ & $\mathrm{U}$ & $\mathrm{U}$ & $U$ \\
\hline $75-25-2$ & Bromoform & $\mathrm{U}$ & $\mathrm{U}$ & $\mathrm{U}$ & $\mathrm{U}$ & $\mathrm{U}$ & $\mathrm{U}$ & $\bar{U}$ \\
\hline $75-27-4$ & Bromodichloromethane & $\mathrm{U}$ & $\mathrm{U}$ & $\mathrm{U}$ & $\mathrm{U}$ & $\mathrm{U}$ & $\mathrm{U}$ & $U$ \\
\hline $87-61-6$ & 1,2,3-Trichlorobenzene & $\overline{\mathrm{U}}$ & $\bar{U}$ & $\mathrm{U}$ & $\mathrm{U}$ & $\mathrm{U}$ & $\mathrm{U}$ & $\bar{U}$ \\
\hline $87-68-3$ & Hexachloro-1,3-butadiene & $\mathrm{U}$ & $\overline{\mathrm{U}}$ & $\mathrm{U}$ & $\overline{\mathrm{U}}$ & $\mathrm{U}$ & $\bar{U}$ & $U$ \\
\hline $91-20-3$ & Nathphalene & $\mathrm{U}$ & $\bar{U}$ & $\mathrm{U}$ & $\bar{U}$ & $\mathrm{U}$ & $\bar{U}$ & $\bar{U}$ \\
\hline
\end{tabular}




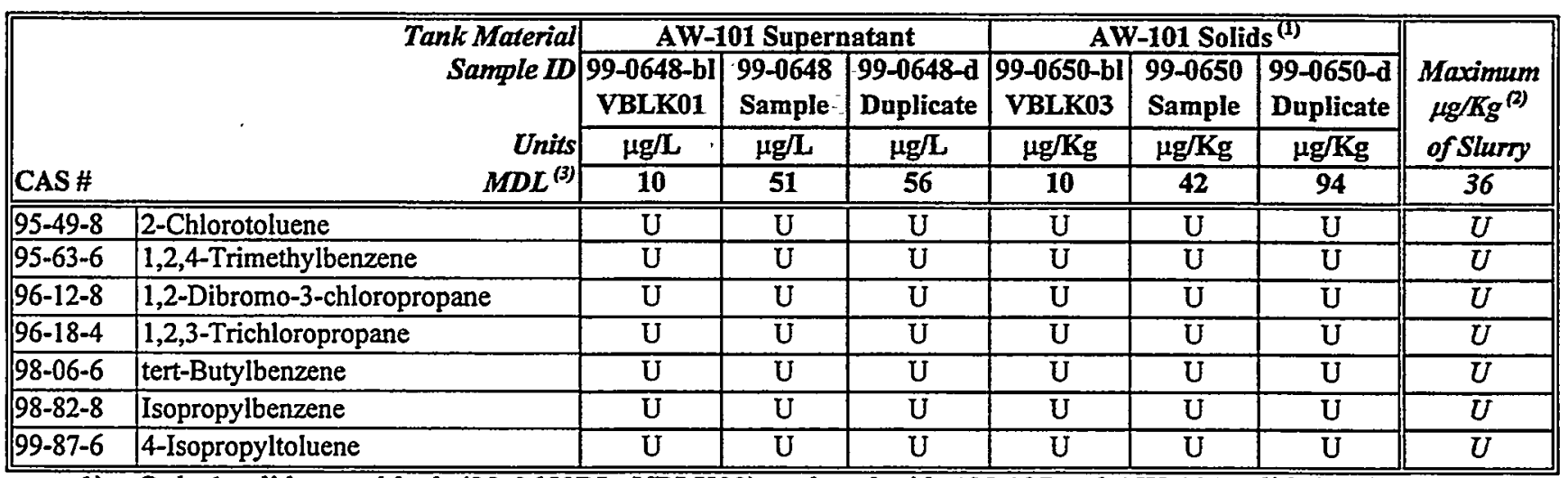

1) Only 1 solids prep blank (99-0650BL, VBLK03) analyzed with AN-107 and AW-101 solids batch

2) Maximum slurry $\mu \mathrm{g} / \mathrm{Kg}$ calculated using results of Tables $2 . \mathrm{a}$ (weight fractions) and $2 . \mathrm{b}$ (supernatant density)-See Section 1.0

3) $\mathrm{MDL}=$ Method detection limit based on instrument detection limit and sample quantity

$\mathrm{U}$ flag = Compound not detected; Compound concentration less than the MDL

$\mathrm{J}$ flag = Compound detected, but concentration is less than the MDL

$\mathrm{B}$ flag $=$ Compound was present in the method blank

Table 3.c. AN-107 VOA Results - BNFL \& SW846 8260B Target Analyte List

\begin{tabular}{|c|c|c|c|c|c|c|c|c|}
\hline \multirow[b]{3}{*}{ CAS \# } & $\begin{array}{l}\text { Tank Material } \\
\text { Sample DD }\end{array}$ & \begin{tabular}{|r|} 
AN-1 \\
99-0649-bl \\
VBLK02
\end{tabular} & $\begin{array}{l}7 \text { Supert } \\
99-0649 \\
\text { Sample }\end{array}$ & $\begin{array}{l}\text { atant } \\
\text { D9-0649-d } \\
\text { Duplicate }\end{array}$ & \begin{tabular}{|r|} 
Al \\
$99-0650-b l$ \\
VBLK03
\end{tabular} & $\begin{array}{l}107 \text { Solic } \\
99-0651 \\
\text { Sample }\end{array}$ & $\begin{array}{l}s^{(1)} \\
\text { D9-0651-d } \\
\text { Duplicate }\end{array}$ & \multirow{2}{*}{$\begin{array}{l}\text { Maximum } \\
\mu g / \mathrm{Kg}^{(2)} \\
\text { of Slumy }\end{array}$} \\
\hline & Units & $\mu \mathrm{g} / \mathrm{L}$ & $\mu \mathrm{g} / \mathrm{L}$ & $\mu \mathrm{g} / \mathrm{L}$ & $\mu \mathrm{g} / \mathrm{Kg}$ & $\mu g / \mathrm{Kg}$ & $\mu \mathrm{g} / \mathrm{Kg}$ & \\
\hline & & 10 & 66 & 56 & 10 & 110 & 74 & 43 \\
\hline \multicolumn{9}{|c|}{ BNFL Target Analyte List } \\
\hline $100-41-4$ & Ethylbenzene & $\mathrm{U}$ & $\mathrm{U}$ & $U$ & $\mathrm{U}$ & $U$ & $\overline{\mathrm{U}}$ & $U$ \\
\hline $100-42-5$ & Styrene & $\bar{U}$ & $\overline{\mathrm{U}}$ & $\bar{U}$ & $\bar{U}$ & $\bar{U}$ & $\bar{U}$ & $\bar{U}$ \\
\hline $10061-01-$ & cis-1,3-Dichloropropene & $\bar{U}$ & $\bar{U}$ & $\bar{U}$ & $\bar{U}$ & $\bar{U}$ & $\bar{U}$ & $\bar{U}$ \\
\hline 10061-02- & trans-1,3-Dichloropropene & $\bar{U}$ & $\bar{U}$ & $\mathrm{U}$ & $\bar{U}$ & $\bar{U}$ & $\bar{U}$ & $\bar{U}$ \\
\hline $106-35-4$ & 3-Heptanone & $\bar{U}$ & $\bar{U}$ & $\bar{U}$ & $\bar{U}$ & $\bar{U}$ & $\bar{U}$ & $\bar{U}$ \\
\hline $106-42-3$ & Xylene (m \& p) & $\mathrm{U}$ & $\bar{U}$ & $\bar{U}$ & $\mathrm{U}$ & $\mathrm{U}$ & $\mathrm{U}$ & $\bar{U}$ \\
\hline $106-46-7$ & 1,4-Dichlorobenzene & $\bar{U}$ & $\overline{\mathrm{U}}$ & $\overline{\mathrm{U}}$ & $\overline{\mathrm{U}}$ & $\overline{\mathrm{U}}$ & $\overline{\mathrm{U}}$ & $\bar{U}$ \\
\hline $106-93-4$ & 1,2-Dibromoethane & $\bar{U}$ & $\overline{\mathrm{U}}$ & $\overline{\mathrm{U}}$ & $\overline{\mathrm{U}}$ & $\bar{U}$ & $\bar{U}$ & $\bar{U}$ \\
\hline $106-97-8$ & Butane & $\bar{U}$ & $\overline{\mathrm{U}}$ & $\overline{\mathrm{U}}$ & $\overline{\mathrm{U}}$ & $\bar{U}$ & $\bar{U}$ & $\bar{U}$ \\
\hline $106-99-0$ & 1,3-Butadiene & $\bar{U}$ & $\bar{U}$ & $\overline{\mathrm{U}}$ & $\overline{\mathrm{U}}$ & $\overline{\mathrm{U}}$ & $\overline{\mathrm{U}}$ & $\bar{U}$ \\
\hline $107-02-8$ & Acrolein & $\bar{U}$ & $\overline{\mathrm{U}}$ & $\overline{\mathrm{U}}$ & $\overline{\mathrm{U}}$ & $\overline{\mathrm{U}}$ & $\bar{U}$ & $\bar{U}$ \\
\hline $107-05-1$ & 3-Chloropropene & $\bar{U}$ & $\bar{U}$ & $\overline{\mathrm{U}}$ & $\bar{U}$ & $\bar{U}$ & $\overline{\mathrm{U}}$ & $\bar{U}$ \\
\hline $107-06-2$ & 1,2-Dichloroethane & $\bar{U}$ & $\bar{U}$ & $\overline{\mathrm{U}}$ & $\bar{U}$ & $\bar{U}$ & $\bar{U}$ & $\bar{U}$ \\
\hline $107-13-1$ & Acrylonitrile & $\bar{U}$ & $\overline{\mathrm{U}}$ & $\overline{\mathrm{U}}$ & $\overline{\mathrm{U}}$ & $\bar{U}$ & $\overline{\mathrm{U}}$ & $\bar{U}$ \\
\hline $107-87-9$ & 2-Pentanone & $\bar{U}$ & $\overline{\mathrm{U}}$ & $\bar{U}$ & $\bar{U}$ & $\overline{\mathrm{U}}$ & $\overline{\mathrm{U}}$ & $\bar{U}$ \\
\hline $108-10-1$ & 4-Methyl-2-pentanone & $\bar{U}$ & $\bar{U}$ & $\bar{U}$ & $\overline{\mathrm{U}}$ & $\overline{\mathrm{U}}$ & $\bar{U}$ & $\bar{U}$ \\
\hline $108-87-2$ & Methylcyclohexane & $\overline{\mathrm{U}}$ & $\overline{\mathrm{U}}$ & $\overline{\mathrm{U}}$ & $\overline{\mathrm{U}}$ & $40 \mathrm{~J}$ & $26 \mathrm{~J}$ & 5 \\
\hline $108-88-3$ & Toluene & $\overline{\mathrm{U}}$ & $\bar{U}$ & $\overline{\mathrm{U}}$ & $\overline{\mathrm{U}}$ & $\mathrm{U}$ & $U$ & $\bar{U}$ \\
\hline $108-90-7$ & Chlorobenzene & $\overline{\mathrm{U}}$ & $\bar{U}$ & $\bar{U}$ & $\overline{\mathrm{U}}$ & $\overline{\mathrm{U}}$ & $\bar{U}$ & $\bar{U}$ \\
\hline $109-66-0$ & Pentane & $\bar{U}$ & $\overline{\mathrm{U}}$ & $\overline{\mathrm{U}}$ & $\overline{\mathrm{U}}$ & $\overline{\mathrm{U}}$ & $\overline{\mathrm{U}}$ & $\bar{U}$ \\
\hline $109-99-9$ & Tetrahydrofuran & $\overline{\mathrm{U}}$ & $31 \mathrm{~J}$ & $26 \mathrm{~J}$ & $\overline{\mathrm{U}}$ & $43 \mathrm{~J}$ & $36 \mathrm{~J}$ & 24 \\
\hline $110-12-3$ & 5-Methyl-2-hexanone & $\bar{U}$ & $\mathrm{U}$ & $\overline{\mathrm{U}}$ & $\overline{\mathrm{U}}$ & $\bar{U}$ & $\bar{U}$ & $\bar{U}$ \\
\hline $110-43-0$ & 2-Heptanone & $\overline{\mathrm{U}}$ & $\bar{U}$ & $\overline{\mathrm{U}}$ & $\bar{U}$ & $\bar{U}$ & $15 \mathrm{~J}$ & 2 \\
\hline
\end{tabular}




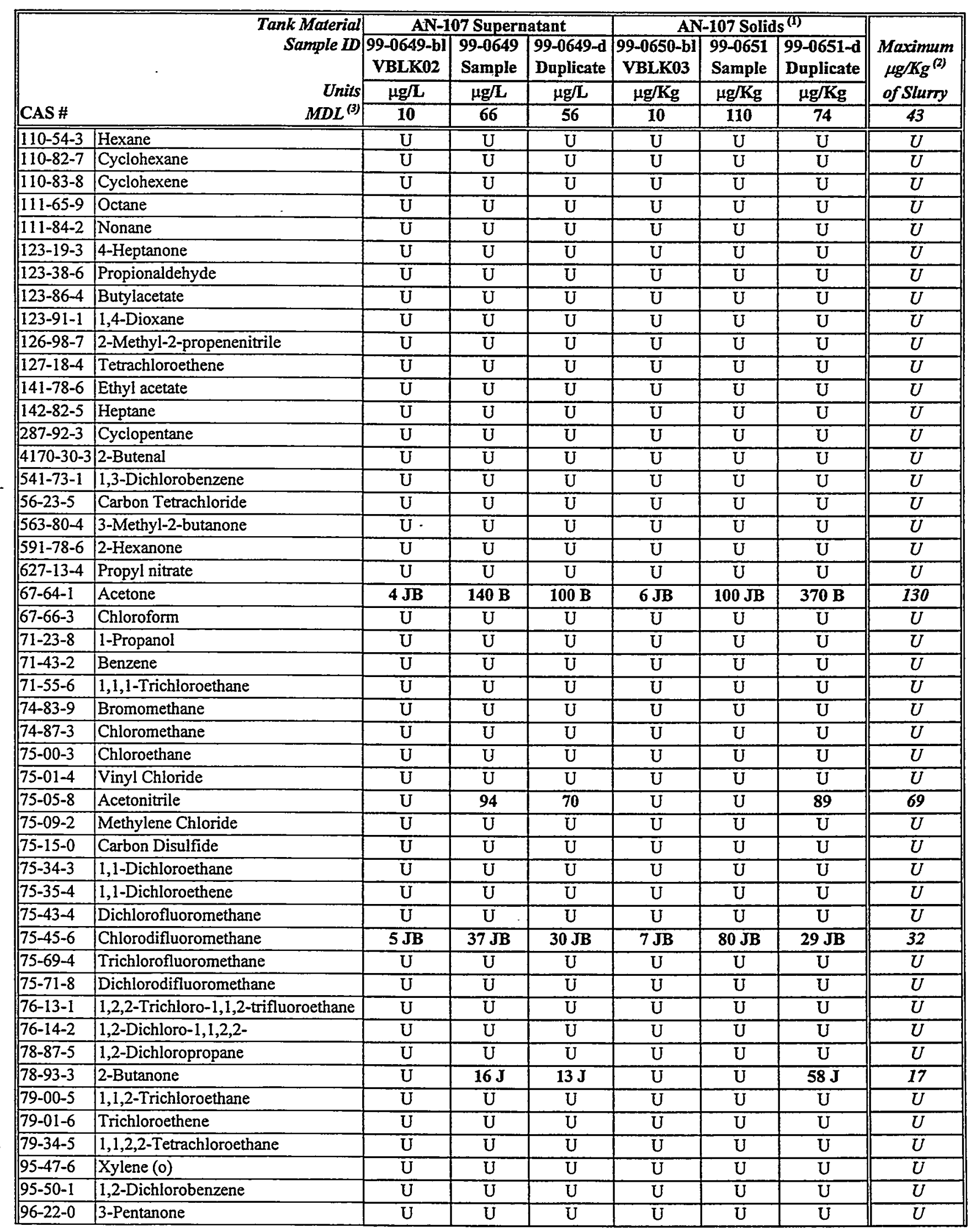




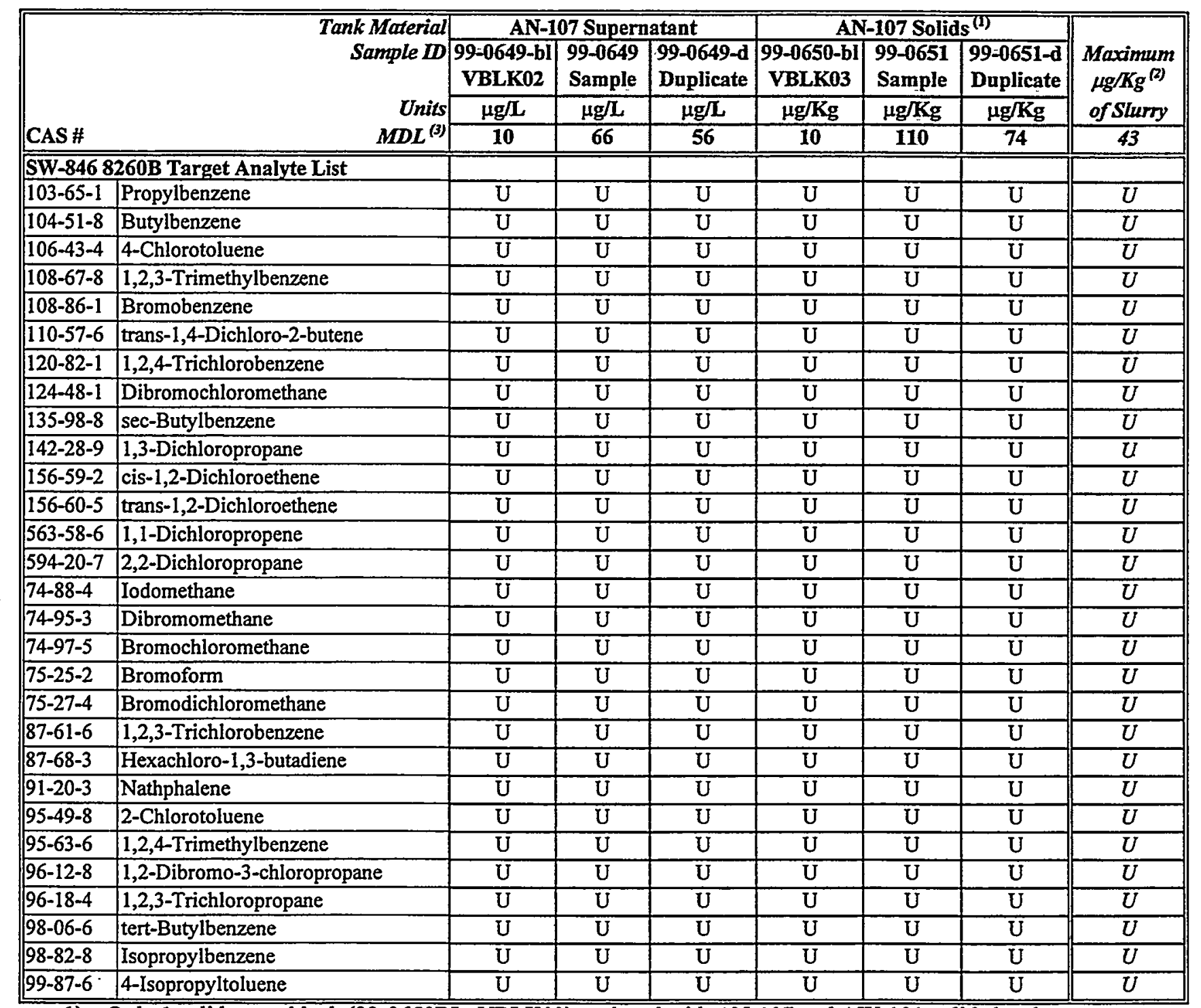

1) Only 1 solids prep blank (99-0650BL, VBLK03) analyzed with $\mathrm{AN}-107$ and $\mathrm{AW}-101$ solids batch

2) Maximum slurry $\mu \mathrm{g} / \mathrm{Kg}$ calculated using results of Tables $2 . \mathrm{a}$ (weight fractions) and $2 . \mathrm{b}$ (supernatant density)-See Section 1.0

3) $\mathrm{MDL}=$ Method detection limit based on instrument detection limit and sample quantity

$\mathrm{U}$ flag = Compound not detected; Compound concentration less than the MDL

$\mathrm{J}$ flag = Compound detected, but concentration is less than the $\mathrm{MDL}$

$\mathrm{B}$ flag $=$ Compound was present in the method blank 
Table 3.d. AW-101 \& AN-107 VOA Tentatively Identified Compounds

\begin{tabular}{|c|c|c|c|c|c|}
\hline \multicolumn{3}{|c|}{ AW-101 Supernatant 99-0648 } & \multirow{2}{*}{$\frac{\text { Sample }}{\mu \mathrm{g} / \mathrm{L}}$} & \multirow{2}{*}{$\begin{array}{c}\text { Duplicate } \\
\mu \mathrm{g} / \mathrm{L}\end{array}$} & \multirow{2}{*}{$\frac{\text { Blank }}{\mu \mathrm{g} / \mathrm{L}}$} \\
\hline CAS\# & TIC & Ret. Time & & & \\
\hline $71-23-8$ & 1-Propanol & 8.37 & 2 & 2 & $\ldots$ \\
\hline $598-32-3$ & 3-Buten-2-ol & 9.00 & 1 & $\cdots$ & $\ldots$ \\
\hline $71-36-3$ & 1-Butanol & 11.53 & 96 & 64 & ---- \\
\hline $111-70-6$ & 1-Heptanol & 19.37 & 2 & 2 & $\cdots$ \\
\hline $100-52-7$ & Benzaldehyde & 19.66 & 1 & 1 & $\ldots$ \\
\hline $104-76-7$ & 1-Hexanol, 2-ethyl- & 20.68 & 6 & 4 & $\cdots$ \\
\hline \multicolumn{3}{|c|}{ AW-101 Solids 99-0650 } & Sample & Duplicate & Blank \\
\hline CAS \# & TIC & Ret. Time & $\mu \mathrm{g} / \mathrm{Kg}$ & $\mu \mathrm{g} / \mathrm{Kg}$ & $\mu \mathrm{g} / \mathrm{Kg}$ \\
\hline $115-17$ & 1-Propene, 2-methyl- & 3.71 & 70 & 56 & $-\cdots$ \\
\hline $106-98-9$ & 1-Butene & 3.91 & 45 & 41 & $\cdots$ \\
\hline $592-41-6$ & 1-Hexene & 7.87 & 34 & 34 & $\cdots$ \\
\hline $592-76-7$ & 1-Heptene & 11.05 & 38 & 19 & $-\cdots$ \\
\hline $71-36-3$ & 1-Butanol & 11.74 & 520 & 170 & $\cdots$ \\
\hline $111-66-0$ & 1-Octene & 13.99 & 62 & 33 & - \\
\hline $124-11-8$ & 1-Nonene & $\overline{16.66}$ & 49 & 22 & ---- \\
\hline $872-05-9$ & 1-Decene & 19.07 & 59 & 48 & $-\overline{--\infty}$ \\
\hline $124-18-5$ & Decane & 19.16 & 278 & 227 & $\cdots$ \\
\hline $111-70-6$ & 1-Heptanol & 19.57 & 79 & 58 & $\cdots$ \\
\hline $104-76-7$ & 1-Hexanol, 2-ethyl- & 20.90 & 209 & 149 & $\cdots$ \\
\hline $4941-53-1$ & 5-Undecene & 21.29 & 70 & 59 & -a- \\
\hline $1120-21-4$ & Undecane & 21.38 & 1224 & 1772 & $\cdots$ \\
\hline $111-87-5$ & 1-Octanol & 21.76 & 62 & --- & $\cdots$ \\
\hline $7239-23-8$ & 3-Dodecene, (Z)- & 23.31 & 122 & 163 & $\cdots$ \\
\hline $112-40-3$ & Dodecane & 23.46 & 2308 & 5616 & $\cdots$ \\
\hline $24949-38-0$ & 6-Tridecene & 25.19 & 41 & 102 & $-\cdots$ \\
\hline $629-50-5$ & Tridecane & 25.34 & 1034 & 3590 & $\cdots$ \\
\hline $629-59-4$ & Tetradecane & 27.12 & 22 & 55 & - \\
\hline \multicolumn{3}{|c|}{ AN-107 Supernatant 99-0649 } & Sample & Duplicate & Blank \\
\hline CAS\# & TIC & Ret. Time & $\mu g / L$ & $\mu \mathrm{g} / \mathrm{L}$ & $\mu \mathrm{g} / \mathrm{L}$ \\
\hline $123-72-8$ & Butanal & 9.03 & 4 & 4 & 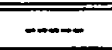 \\
\hline $71-36-3$ & 1-Butanol & 11.76 & 1 & 1 & $-\infty$ \\
\hline $66-25-1$ & Hexanal & 15.26 & 4 & $\cdots$ & $\cdots$ \\
\hline $107-31-1$ & Butanal, 3-hydroxy- & 17.88 & 1 & $\cdots$ & $\cdots$ \\
\hline $16747-32-3$ & Pentane, 3-ethyl-2,2-dimethyl- & 20.94 & $\cdots$ & 2 & $\cdots$ \\
\hline $112-31-2$ & Decanal & 24.52 & 2 & $\cdots$ & $\cdots$ \\
\hline \multicolumn{3}{|c|}{ AN-107 Solids 99-0651 } & Sample & Duplicate & Blank \\
\hline CAS \# & TIC & Ret. Time & $\mu \mathrm{g} / \mathrm{Kg}$ & $\mu \mathrm{g} / \mathrm{Kg}$ & $\mu \mathrm{g} / \mathrm{Kg}$ \\
\hline $629-50-5$ & Tridecane & 25.23 & 358 & 101 & $\cdots$ \\
\hline $629-59-4$ & Tetradecane & 27.02 & 603 & 119 & $-\cdots$ \\
\hline $30692-16-1$ & 5-Tridecanone & 29.30 & 307 & 29 & - \\
\hline
\end{tabular}


Table 3.e. Target VOA Minimum Reportable Quantities

\begin{tabular}{|c|c|c|c|}
\hline CAS \# & VOA Compounds & $\begin{array}{c}\text { Solids Target } \\
\mathrm{MRQ}^{(1)} \\
\mu \mathrm{g} / \mathrm{Kg} \\
\end{array}$ & $\begin{array}{c}\text { Supernatant Target } \\
\text { (Density }=1.45 \text { ) } \\
\mu \mathrm{g} / \mathrm{L}\end{array}$ \\
\hline $100-41-4$ & Ethylbenzene & 3300 & 4800 \\
\hline $100-42-5$ & Styrene & $\overline{-\cdots}$ & $\ldots$ \\
\hline 10061-01- & cis-1,3-Dichloropropene & 6000 & 8700 \\
\hline 10061-02- & trans-1,3-Dichloropropene & 6000 & 8700 \\
\hline $106-35-4$ & 3-Heptanone & $\ldots$ & 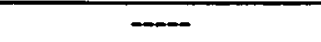 \\
\hline $106-42-3$ & p-Xylene & 3300 & 4800 \\
\hline $106-93-4$ & 1,2-Dibromoethant & 5000 & 7300 \\
\hline $106-97-8$ & Butane & - & $=$ \\
\hline $106-99-0$ & $\overline{1,3-\text { Butadiene }}$ & $\overline{-}$ & 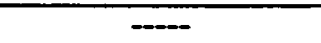 \\
\hline $107-02-8$ & Acrolein & $\overline{-}$ & $\cdots$ \\
\hline $107-05-1$ & 3-Chloropropene & 10000 & 14500 \\
\hline $107-06-2$ & 1,2-Dichloroethane & 2000 & 2900 \\
\hline $107-12-0$ & Propionitrile & 120000 & 174000 \\
\hline $107-13-1$ & Acrylonitrile & 28000 & 41000 \\
\hline $107-87-9$ & 2-Pentanone & $=$ & - \\
\hline $108-10-1$ & 4-Methyl-2-pentanone & 11000 & 16000 \\
\hline $108-38-3$ & m-Xylene & 3300 & 4800 \\
\hline $108-87-2$ & Methylcyclohexane & $-\cdots$ & $m$ \\
\hline $108-88-3$ & Toluene & 3300 & 4800 \\
\hline $108-90-7$ & Chlorobenzene & 2000 & 2900 \\
\hline $108-94-1$ & Cyclohexanone & $\cdots$ & - \\
\hline $109-66-0$ & Pentane & $\overline{-}$ & $\ldots$ \\
\hline $109-99-9$ & Tetrahydrofuran & $\overline{-}$ & $\ldots$ \\
\hline $110-12-3$ & 5-Methyl-2-hexanone & $\overline{-}$ & $\overline{-\ldots}$ \\
\hline $110-43-0$ & 2-Heptanone & $=$ & $\ldots$ \\
\hline $110-54-3$ & Hexane & 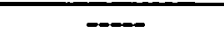 & $\overline{-}$ \\
\hline $110-82-7$ & Cyclohexane & $\cdots$ & $=$ \\
\hline $110-83-8$ & Cyclohexene & $\overline{-}$ & - \\
\hline $111-65-9$ & Octane & $\cdots$ & - \\
\hline $111-84-2$ & Nonane & $\cdots$ & $-\cdots$ \\
\hline $123-19-3$ & 4-Heptanone & 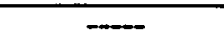 & $=$ \\
\hline $123-38-6$ & Propionaldehyde & $\overline{-\infty}$ & - \\
\hline $123-86-4$ & Butylacetate & $\overline{-}$ & {[} \\
\hline $123-91-1$ & 1,4-Dioxane & - & $\overline{-}$ \\
\hline $126-98-7$ & 2-Methyl-2-propenenitrile & 28000 & 41000 \\
\hline $127-18-4$ & Tetrachloroethene & 2000 & 2900 \\
\hline $141-78-6$ & Ethylacetate & 11000 & 16000 \\
\hline $142-82-5$ & Heptane & $\cdots$ & + \\
\hline $287-92-3$ & Cyclopentane & $\overline{-\infty}$ & $\cdots$ \\
\hline $3825-26-1$ & Ammonium perfluorooctanoate & - & $\overline{-}$ \\
\hline $4170-30-3$ & 2-Butenal & $\overline{-\cdots}$ & - \\
\hline $56-23-5$ & Carbon tetrachloride & 2000 & 2900 \\
\hline $563-80-4$ & 3-Methyl-2-butanone & $\cdots$ & - \\
\hline 57-14-7 & 1,1-Dimethylhydrazine & - & $\ldots$ \\
\hline $591-78-6$ & 2-Hexanone & $-\cdots$ & $\ldots$ \\
\hline
\end{tabular}




\begin{tabular}{|c|c|c|c|}
\hline CAS\# & VOA Compounds & $\begin{array}{c}\text { Solids Target } \\
\text { MRQ }^{(1)} \\
\mu \mathrm{g} / \mathrm{Kg}\end{array}$ & $\begin{array}{c}\text { Supernatant Target } \\
\text { (Density }=1.45 \text { ) } \\
\mu \mathrm{g} / \mathbf{L}\end{array}$ \\
\hline $60-34-4$ & Methylhydrazine & $\cdots$ & $-\ldots$ \\
\hline $624-83-9$ & Methyl isocyanate & $\cdots$ & $\cdots$ \\
\hline $627-13-4$ & Propyl nitrate & - & $\ldots$ \\
\hline $64-17-5$ & Ethyl alcohol & $\cdots$ & $\ldots$ \\
\hline $67-56-1$ & Methanol & $\overline{-m}$ & - \\
\hline $67-63-0$ & Isopropanol & $\ldots$ & - \\
\hline $67-64-1$ & Acetone & 53300 & 77300 \\
\hline $67-66-3$ & Chloroform & 2000 & 2900 \\
\hline $684-16-2$ & Hexafluoroacetone & $\ldots$ & $-\cdots$ \\
\hline $71-23-8$ & 1-propanol & $\ldots$ & $\ldots$ \\
\hline $71-36-3$ & n-Butyl alcohol & 900 & 1300 \\
\hline $71-43-2$ & Benzene & 3300 & 4800 \\
\hline $71-55-6$ & $1,1,1$-Trichloroethane & 2000 & 2900 \\
\hline $74-83-9$ & Bromomethane & 5000 & 7300 \\
\hline $74-87-3$ & Chloromethane & 10000 & 14500 \\
\hline $75-00-3$ & Chloroethane & $-\infty$ & $\cdots$ \\
\hline $75-01-4$ & Vinyl chloride & 2000 & 2900 \\
\hline $75-05-8$ & Acetonitrile & 12700 & 18400 \\
\hline $75-09-2$ & methylene chloride & 10000 & 14500 \\
\hline $75-15-0$ & Carbon disulfide & 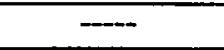 & $-\cdots$ \\
\hline $75-21-8$ & Oxirane & - & $\cdots$ \\
\hline $75-34-3$ & 1,1-Dichloroethane & 2000 & 2900 \\
\hline $75-35-4$ & 1,1-Dichloroethene & 2000 & 2900 \\
\hline $75-43-4$ & Dichlorofluoromethane & $=$ & $\cdots$ \\
\hline $75-45-6$ & Chlorodifluoromethane & $\cdots$ & $\cdots$ \\
\hline $75-65-0$ & 2-Methyl-2-propanol & $\cdots$ & - \\
\hline $75-69-4$ & Trichlorofluoromethane & 10000 & 14500 \\
\hline $75-71-8$ & Dichlorodifluoromethane & 2400 & 3500 \\
\hline $76-13-1$ & 1,2,2-Trichloro-1,1,2-trifluoroethane & 10000 & 14500 \\
\hline $76-14-2$ & 1,2-Dichloro-1,1,2,2-tetrafluoroethane & $\cdots$ & $\cdots$ \\
\hline $78-87-5$ & 1,2-Dichloropropane & $\ldots$ & $=$ \\
\hline $78-92-2$ & 2-butanol & $-\cdots$ & $\cdots$ \\
\hline $78-93-3$ & 2-Butanone & 12000 & 17400 \\
\hline $79-00-5$ & 1,1,2-Trichloroethane & 2000 & 2900 \\
\hline $79-01-6$ & 1,1,2-Trichloroethylene & 2000 & 2900 \\
\hline $79-34-5$ & 1,1,2,2-Tetrachloroethane & 2000 & 2900 \\
\hline $95-47-6$ & o-Xylene & 3300 & 4800 \\
\hline $96-22-0$ & 3-Pentanone & -- & $-\cdots$ \\
\hline
\end{tabular}

(1) $\mathrm{MRQ}=$ Minimum Reportable Quantity. Values provided through BNFL private communication from L. Bostic (BNFL) to G. Klinger (Battelle)

"...." = No MRQ target provided in communication. 


\subsubsection{QC Evaluation}

Instrument tuning check criteria and 12-hour calibration clock window criteria were met (USEPA CLP 3/90 SOW) for all initial calibration and sample analysis sequences as seen in the " $5 \mathrm{~A}$ " Forms in the Appendix A. The initial calibration met the criteria of USEPA SW-846 method $8260 \mathrm{~B}$, as seen in the " $6 \mathrm{~A}$ " Form in the Appendix A. All five-system performance check compounds (SPCC) met the criteria for minimum response factor, and all six calibration check compounds (CCC) met the maximum relative standard deviation (RSD) criteria. Six of the target analytes of interest have RSDs greater than the recommended 15\%; however, none exceeded $25 \%$. The target compounds failing to meet the RSD of $15 \%$ were acrylonitrile (20\%), 2-butenal (18\%), xylene-m\&p (16\%), nonane (16\%), propyl nitrate (18\%), and 1-propanol (24\%).

The continuing calibration check standard met the criteria of USEPA SW-846 method 8260B, as seen in the " $7 \mathrm{~A}$ " Forms in the Appendix A. All calibration check standards met the SPCC and CCC criteria. However, in comparison of the results for the continuing calibration standards to the initial calibration, a few target analytes of interest exceed the recommended percent difference (\%D) of $15 \%$. The target analytes failing to meet the $\% \mathrm{D}$ of $15 \%$ were propionaldehyde $(17 \%), 1,3$-butadiene $(17 \%)$, butane $(20 \%)$, dichlorodifluoromethane $(22 \%)$, 1,4-dioxane $(33 \%)$, acrolein $(33 \% \& 33 \%)$, 1,2-dichloro-1,1,2,2-tetrachloroethane (31\%, 26\%, \& 34\%).

The internal standards used in this study were difluorobenzene, pentafluorbenzene, chorobenzene- $\mathrm{D}_{5}$, and 1,4-dichorobenzene- $\mathrm{D}_{4}$. The surrogate compounds used were dibromofluoromethane, toluene$\mathrm{D}_{8}$, and bromofluorobenzene. These seven compounds were added to each sample, duplicate, MS, MSD, and blank sample analyzed.

Evaluation of surrogate recoveries are somewhat difficult in that performance based recovery limits have not been established for this type of sample matrix. In the absences of documented agreement between BNFL, WDOE, and Battelle, the Contract Laboratory Program (CLP) limits for low-level soil samples were used as a guide and are included on the " $2 A$ \& $2 B$ " Forms in Appendix A.

Dibromofluoromethane was poorly recovered from all of the samples, especially the AW-101 samples. This may be due to the reaction of this compound with the sample matrix. One possible reaction of this compound is the reaction with an alcohol in a Williamson synthesis type of reaction:

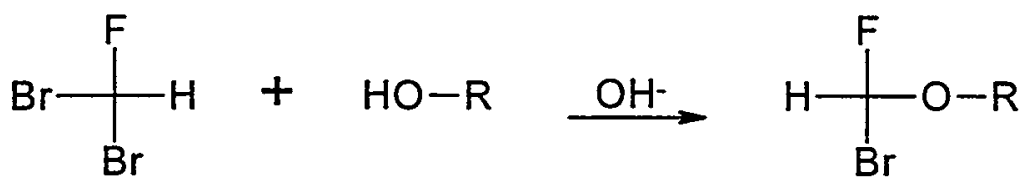

The fluoro-bromo ether product was not detected in the VOA as a TIC by the data analysis system. However, manual evaluation of the spectral data indicates the possible presence of this compound type but that the compound closely coelutes with methanol making it impossible to detect as a TIC.

The recoveries of the toluene- $D_{8}$ and bromofluorobenzene surrogates are somewhat high in all the samples and in one of the storage blanks. The reason for this is not clearly understood, but may be due to the way the spiking solution was handled within the limitations of permissible practices in contamination area fume hoods. Specifically, it is possible some methanol in the vial containing the methanolic surrogate spiking solution may have evaporated before the samples were spiked. This may be another reason for the poor dibromofluoromethane recoveries exhibited on all of the samples, 
especially the AW-101 samples, since dibromofluoromethane has a boiling point similar to that of methanol. Alternate methods for handling and introducing the spiking solutions into highly radioactive samples needs to be evaluated, since the high velocity air movement and the restricted handling of the radioactive samples most likely contributed to the observed high variability in spike recoveries. The surrogate recovery data are summarized on the each of the CLP-type " $2 A$ \& $2 B$ " Forms in the Appendix A.

The CLP criterion for internal standard response was used $( \pm 50 \%$ of the calibration check standard response). Internal standard response met the criteria for all of the AW-101 supernatant samples spike samples and the blank. The internal standard response for the AN-107 supernatant samples was consistently low for all of the samples and spike samples; however, the blank met the criteria. This may be a matrix effect. Due to the limited quantity of sample available, it was not possible to reanalyze the samples to confirm this effect.

The same effect was observed for the AN-107 solids samples. The AW-101 solids samples met the internal standard response criteria. The internal standard data are summarized on the each of the CLP-type "8A" Forms in the Appendix A.

Matrix spiking was performed by adding the methanolic calibration solution to the samples at a level of 500 nanograms per compound. The MSs and MSDs revealed some interesting trends. The polar compounds such as acetone, 2-butanone, tetrahydrofuran, 2-pentanone, 3-methyl-2-butanone, 1,4-dioxane, 3-pentanone, 4-methyl-2-pentanone, 2-hexanone, propionaldehyde, 4-heptanone, 3-heptanone, 2-heptanone had high recoveries in the samples. Butylacetate and ethylacetate had variable recoveries. The high recoveries may be due to the salting-out effect of the sample matrix, relative to the calibration standard. The calibration standard is prepared using carbon filtered deionized water. Compounds that are completely or partially soluble in water are not completely purged from the aqueous calibration standard solution. Ordinarily this is not a problem as their partial recoveries are reasonably linear with respect to concentration in the solution. Since the samples are a concentrated salt solution $\left(>20 \% \mathrm{Na}^{+},>20 \% \mathrm{NO}_{3}{ }^{-}\right)$, the solubility of partially soluble organic compounds is greatly reduced, increasing their recovery by sparging. The sparging was conducted at a temperature of about $30^{\circ} \mathrm{C}$; no other sparging temperatures were evaluated for potentially improving the recoveries of some polar compounds. Also, considering the high salt content of the samples, alternate calibration matrices with higher ionic strength (e.g., $1 \mathrm{~N} \mathrm{NaOH}$ ) should be considered for the preparation of the calibration standards.

Some chloroalkanes (such as 1,1,2,2-tetrachloroethane and 1,1,2-trichloroethane) that were in the matrix spike solution had poor recoveries. Some chloroalkene compounds (such as 1,1-dichloroethene and 1,1,2-trichloroethene) that were in the matrix spike solution had high recoveries. These high recoveries may be due to textbook dehydrohalogenation reactions such as: 

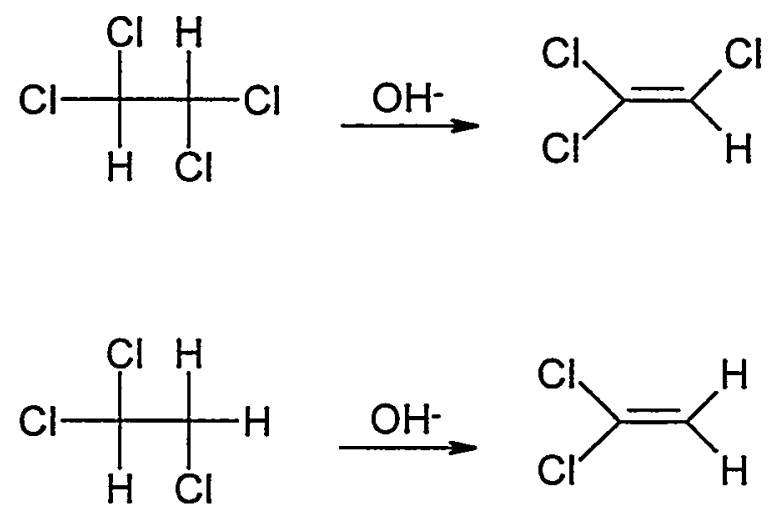

Acrolein, acrylonitrile, 2-butenal, carbon disulfide, and chloroform had somewhat low and variable recoveries, possibly due to reactivity with the matrix. Bromomethane had low recovery in some spiked samples possibly due to a Williamson synthesis type reaction or from losses due to volatilization during preparation of the matrix spikes, as described above. Cis- and trans1,3-dichloropropene had low and variable recoveries. Butylacetate and ethylacetate had variable recoveries, sometimes greater than $100 \%$ and sometimes very low. While the salting-out effect can increase the apparent recoveries of water-soluble compounds, a strong base and catalyst in the matrix may cause these esters to hydrolyze to alcohols and acids.

\subsubsection{Other Observations (or Deviations/Concerns/lssues)}

Where compounds were detected in the sample and the duplicate (for both the AW-101 and AN-107 supernatant and solids), the reproducibility was generally poor; i.e., results within a factor of 2 to 3 . However, it is noted that most of the results fall within 5 times the MDL and at this concentration the precision is anticipated to be poor. In part, the poor precision can be attributed to the complexity of the sample handling and sub-sampling in the SAL, as discussed below.

Several compounds specified in BNFL Proposal No. 29274/30406 Task 5.0 were not included in the VOA calibration. Specifically, ammonium perfluorooctanoate and oxirane (ethylene oxide) were deleted from the list as per personal communication with Lee Bostic on September 18, 1998. Hexafluoroacetone could not be procured from any vendor in a timeframe that permitted completion of the work by the March 1, 1999 milestone; however, if present, this compound would be identified as a TIC. Methylhydrazine, 1,1-dimethylhydrazine and methyl isocyanate were included in the calibration stock solution, however their recovery from the standard was extremely poor and variable. The poor recovery is possibly due to the high susceptibility to oxidation for the hydrazines or to hydrolysis for the isocyanate. Consequently, these compounds are not reported on the list of target analytes in the analysis results, and additional work on these analytes is.recommended.

The vial containing the MS sample for the $\mathrm{AN}-107$ solids was found to be leaking, so it was not analyzed in order to avoid problems with airborne radiological contamination. The leaking is most likely the results from the difficulties experienced in crimping the vial/lid in the shielded hot cell facility. The loss of the MS for AN-107 resulted in not being able to establish precision for the AN-107 solids samples. The AW-101 solids MS and MSD, which were processed in the same batch, were used to evaluate analytical precision for both AW-101 and AN-107 solids. 
2-Methyl-2-propanol, 1-propanol, 1-propanol, 1-butanol were included in the calibration VOA standard and spike, however they are not reported as VOA target analytes since they will be reported in the headspace analysis.

Finally, it can not be stress strongly enough that the reported VOA results are for the sample composited and sub-sampled under the HLRF and SAL environmental conditions (e.g., high velocity airflow and slightly elevated temperature). The processing of highly radioactive tank waste in shielded facilities cannot, at this time, ensure that zero headspace is maintained for VOA samples and sub-samples. The VOA results presented in this report most assuredly do not represent the VOA compound concentrations resident in the waste tanks. It is inevitable that VOA compound losses are experienced at nearly every major processing point (i.e., tank sub-sampling, compositing, phase separation, and analytical sub-sampling). 


\subsection{Semi-Volatile Organic Analysis (SVOA)}

\subsection{Introduction}

Semi-volatile organic analyses were performed on both the supernatant and solids from samples AW-101 and AN-107 following phase separation. Supernatants and solids were extracted with methylene chloride (or methylene chloride and acetone) per Test Plan BNFL-29953-5, Revision 0. The extracted samples were reduced in volume using a Kuderna-Danish concentrator. Following volume reduction, an aliquot was prepared and analyzed for semi-volatile compounds by method PNL-ALO-345.

Due to limited sample availability, a single extraction method was developed to provide samples for SVOA, PCB/pesticides, and dioxins and furans analysis. This extraction approach is fully detailed in Test Plan BNFL-29953-5, Revision 0. This Test Plan included dilution steps in an attempt to dissolve the bulk of the solids (i.e., leaving less than $0.5 \%$ ) and thus be able to use only liquid-liquid extraction processing. However, during preliminary processing of the solids phases it was determined that a high fraction of the solids were insoluble following dilution with water. Therefore, the water dissolution step detailed in the Test Plan was eliminated and the solids were subjected directly to an ultra-sonication extraction using a methylene chloride and acetone mixture with desiccant.

\subsection{Sample Preparation}

Prior to performing the single extraction process for the SVOA, PCB/pesticides, and dioxin and furans, the aliquots of the supernatants from AW-101 and AN-107 and the solids (mixed with deionized water) were titrated with phosphoric acid. The resulting titration curves are used to establish the quantity of phosphoric acid required to adjust the extracting $\mathrm{pH}$ to level defined by the procedure (approximately 6.5). See Figure 4.1. It should be noted that the solids were not subjected to a water dissolution/dilution as detailed in the Test Plan; however, the solids titration curve was used to $\mathrm{pH}$-adjust the solids fraction following centrifuging.

\subsubsection{Supernatants}

For each supernatant sample of AW-101 or AN-107 extracted, a known quantity (45 to $145 \mathrm{~g}$ ) of sample was transferred into a Teflon separatory funnel. Appropriate spikes, internal standards, and surrogates were added to the samples prior to subjecting the samples to the extraction process. Each supernatant sample was extracted with three $25-\mathrm{mL}$ portions of methylene chloride by subjecting the separatory funnel to mechanical shaking. Following this initial extraction, the supernatant was chilled in ice and stirred while the $\mathrm{pH}$ was adjusted with a predetermined quantity of phosphoric acid. Samples of AW-101 formed significant precipitates that were separated from the supernatant by centrifuging and decanting. The extraction process was repeated on the $\mathrm{pH}$-adjusted supernatant. The solids from AW-101 were ultra-sonicated three $25-\mathrm{mL}$ portions of methylene chloride. The methylene chloride-only extraction (versus methylene chloride-acetone) for these solids should be adequate, since only a moderate quantity of interstitial liquid remained following the centrifuging and decanting process. However, the use of methylene chloride-only for these precipitated solids has not been validated. All extracts from the supernatant sample, including the extracts from the solids formed during $\mathrm{pH}$ adjustment, were combined and passed through a column containing an anhydrous sodium sulfate desiccant to complete the supernatant extraction process. 


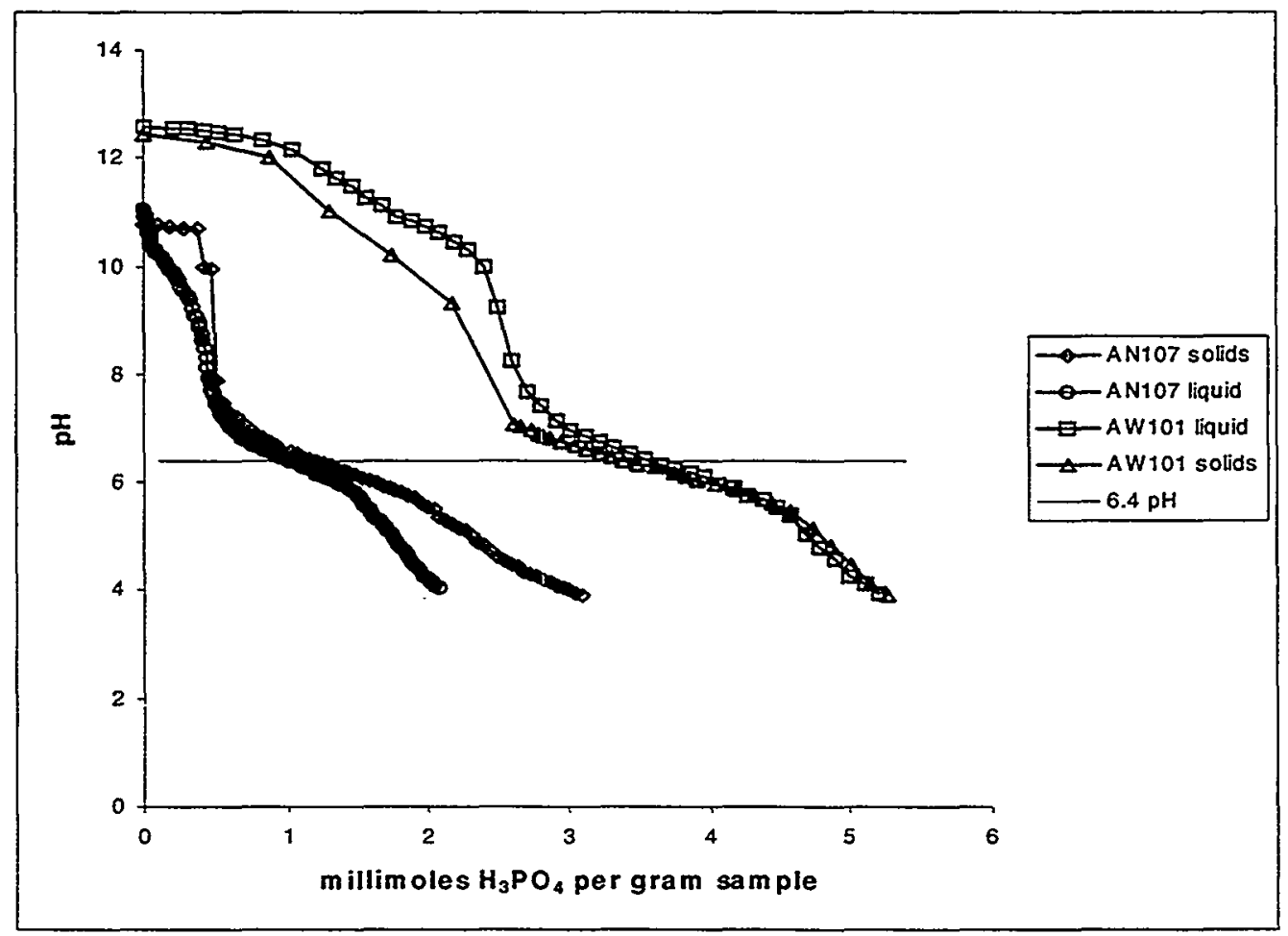

Figure 4.1 Phosphoric Acid Titration Curve

\subsubsection{Solids}

For each solids sample of AW-101 or AN-107 extracted, a known quantity ( 2.6 to $4.8 \mathrm{~g}$ ) of sample was transferred to small Teflon bottle and anhydrous sodium sulfate (pre-dried in a muffle furnace) desiccant added. Appropriate spikes, internal standards, and surrogates were added to the samples prior to subjecting the samples to the ultra-sonication extraction process. Each sample was ultrasonicated with three $25-\mathrm{mL}$ portions of a 1:1 methylene chloride to acetone mixture. Following this initial extraction, the $\mathrm{pH}$ of the solids was adjusted with a predetermined quantity of phosphoric acid and the ultra-sonication extraction process repeated. All extracts from the solids sample were combined and passed through a column containing an anhydrous sodium sulfate desiccant to complete the solids extraction process.

\subsubsection{Extract Volume Reduction}

Once the extraction processes were completed in the SAL, the supernatant extracts and the solids extracts were transferred under COC from the SAL to the analytical laboratories and refrigerated prior to subsequent volume reduction processing. During the volume reduction processing, each extract was reduced in volume to $10 \mathrm{~mL}$ using a Kuderna-Danish (K-D) apparatus. Following removal of a 5-mL aliquot for PCB/Pesticides and dioxins and furans (See Sections 5.0 and 6.0), the SVOA aliquot $(5 \mathrm{~mL}$ ) was further reduced in volume to $1 \mathrm{~mL}$, and refrigerated until analysis was performed. 


\subsection{Instrumentation}

The analytical instrumentation used for SVOA consists of an auto-sampler-injector and gas chromatograph mass spectrometer system. Detailed description of the SVOA system is provided in Table 4.a.

Table 4.a. SVOA Instrumentation

\begin{tabular}{|l|l|c|c|}
\hline System/Instrument & Manufacturer & Model Number & M\&TE $^{(\text {() }}$ Number \\
\hline \hline Auto-sampler & Hewlett Packard & $7673 \mathrm{~A}$ & N/A \\
\hline GC/MS & Hewlett Packard & $5890 \mathrm{II} / 5972$ & WB47238/WD25623 \\
\hline
\end{tabular}

1) Measuring and Test Equipment

\subsection{Analysis Results}

The SVOA target (calibrated analytes) results for AW-101 and AN-107 supernatant and solids phases are given in Tables 4.b and 4.c. Additionally, the results for any SVOA TICs that were detected for both AW-101 and AN-107 supernatant and solids phases are given in Tables 4.d through 4.g. For both target compounds and TICs, the results are given in units of $\mu \mathrm{g} / \mathrm{L}$ for the supernatant and $\mu \mathrm{g} / \mathrm{Kg}$ for the wet solid phase.

The MDLs provided are based upon instrument detection limits and the weight or volume of the sample used for the analysis. The MDLs are nominal, and are not based upon performance of the method on these specific sample matrices. In all cases, the MDLs for the supernatants (after adjusting for density) meet the BNFL SVOA MRQ requirements as detailed in Table 4.h. However, the MDLs for the solids met the BNFL SVOA MRQ requirements only for N,N-diphenylamine, nitrobenzene, and pyridine. The MDLs for the solids exceeded the BNFL SVOW MRQ requirement for all other compounds in the solids by a factor of 1.3 to 5 , the most significant being for 1,4dinitrobenzene and $\mathrm{N}$-nitrosodimethlylamine.

SVOA results below the MDL are quantified by the data analysis system; however, only those results greater then $20 \%$ of the MDL are reported. Results less than $20 \%$ of the MDL, while detected, are consider too low to accurately quantify.

\subsubsection{Results for Calibrated/Regulatory Analytes of Interest}

\section{AW-101 Results}

A few target compounds were detected in the AW-101 supernatant. Only N-nitrosodimethylamine and N-nitrosomethylethylamine were found at levels greater than the MDL.

Results for nonane in the TIC data should not be interpreted as an indication the presence of nonane in the samples. Nonane was used as the solvent in the dioxins and furans spiking stock solutions that was added to all blanks, spikes, and samples prior to the single extraction processing for SVOA, $\mathrm{PCB} /$ pesticides, and dioxins and furans. The VOA results should be used for the determination of the nonane concentration in the samples. 
Chloroform and 1,1,2-trichloroethane are reported in the TIC results, but are likely to be contaminants of the methylene chloride extraction solvent since neither of these compounds were detected in the VOA results. Tetramethyl silane was found in the samples and blank, and were likely leached from the Teflon lined, silcone rubber septum used in the I-Chem bottles that held the sample extracts prior to removal from the SAL. A number of organic acids, alcohols, ether-alcohols, and nitric acid esters were also detected as TICs in the AW-101 supernatant samples.

The AW-101 solids samples also contained N-nitrosodimethylamine at a concentration greater than the MDL. N-Nitrosomethylethylamine, tributylphosphate, and 1-naphthalamine were detected at concentrations less than the MDL.

Tridecane, tetradecane and pentadecane (used in the PUREX and B-Plant extraction processes) were among the TICs reported in the AW-101 solids (Tables 4.d and 4e). A number of artifact compounds such as aldol condensation products were reported in the data; these results are discussed in the quality control (QC) evaluation section.

For the AN-107 results, three target compounds were detected in the supernatant. None were found at levels greater than the MDL. N-nitrosodimethylamine, tributylphosphate, and diethylphthalate were detected at concentrations less than the MDL.

As with AW-101 supernatant, chloroform and 1,1,2-trichloroethane are reported in the TIC results and are likely contaminants of the methylene chloride extraction process, as discussed above. AN107 supernatant TIC results contain a large number of unknowns and $\mathrm{N}$-nitrosoamines. It is not clear whether these $\mathrm{N}$-nitrosoamines are present in the original tank material or formed in the extraction process. Also, as noted previously, nonane was detected in all samples and blanks; however, nonane was used as a solvent for the dioxins and furans spike solution and was added to all samples, spikes, and blanks.

Both AN-107 supernatant and solids samples TIC results contain a large number of nitrogen containing compounds, such as $\mathrm{N}$-nitroso substituted morpholine, piperizine, and piperidine. It is conceivable that these TICs are ring closure products of HEPTA, ED3A or some other EDTA-related chelators or their degradation products. AN-107 TIC results also contain various nitrate ester compounds, $\mathrm{N}$-nitrosoamines, carboxylic acids, unknown amines, and unknowns.

AN-107 solids sample results are similar to the supernatant results. Only 1-naphthayamine was detected at a concentration than the MDL. Pyridine was detected at a concentration slightly below the MDL.

AN-107 solids samples TIC results contain some nitration products of surrogate spike compounds. These nitration products are discussed in the QC evaluation section below. 
Table 4.b. AW-101 SVOA Results - BNFL \& SW846 8270C Target Analyte List

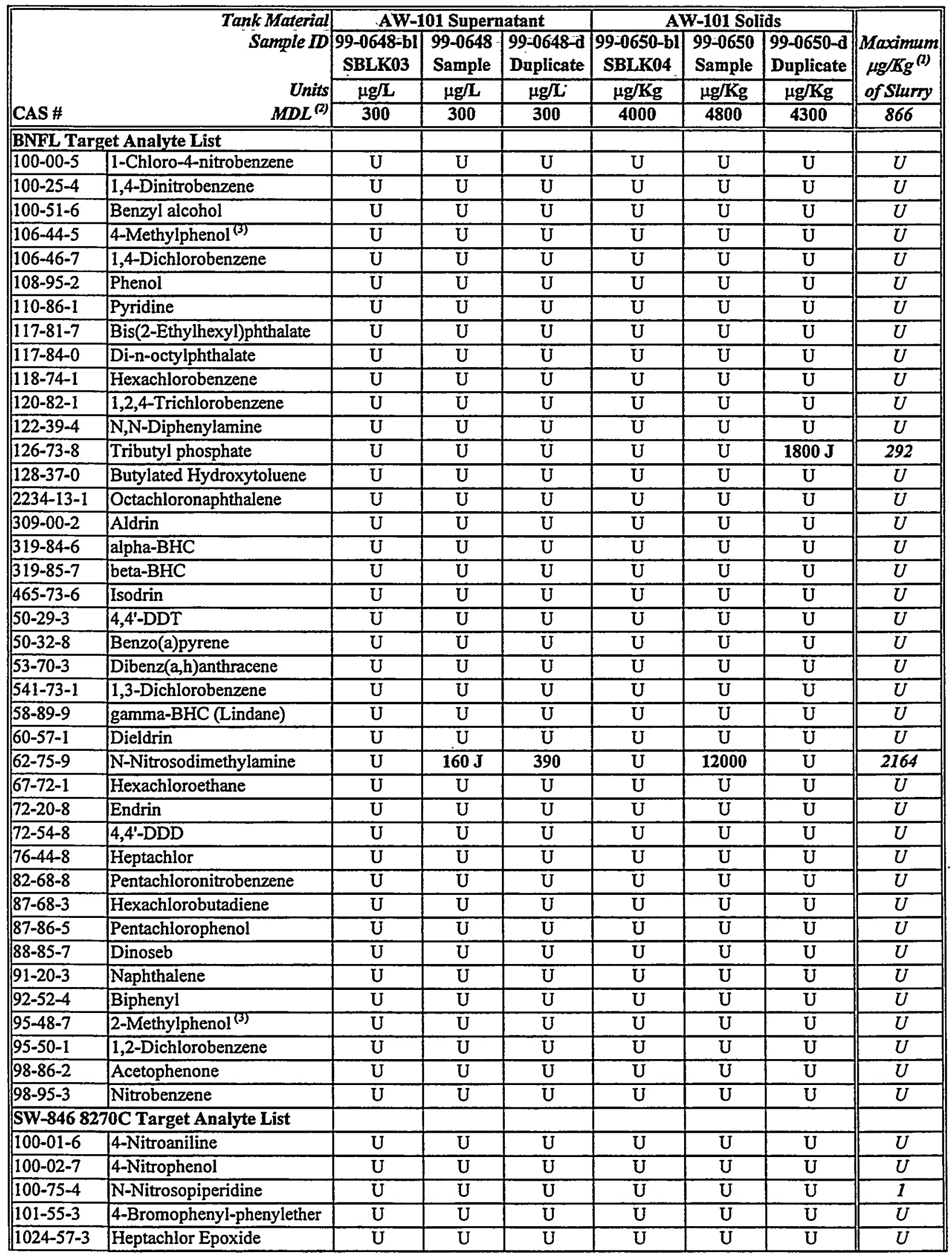




\begin{tabular}{|c|c|c|c|c|c|c|c|c|}
\hline & \multicolumn{3}{|c|}{ AW-101 Supernatant } & \multicolumn{3}{|c|}{ AW-101 Solids } & \multirow{3}{*}{$\mid \begin{array}{l}\text { Maximun } \\
\mu g / \mathrm{Kg}^{(l)} \\
\text { of Slurry }\end{array}$} \\
\hline & Sample ID & $\begin{array}{c}\text { 99-0648-bl } \\
\text { SBLK03 }\end{array}$ & $\begin{array}{l}99-0648 \\
\text { Sample }\end{array}$ & $\begin{array}{l}\text { 99-0648-d } \\
\text { Duplicate }\end{array}$ & $\begin{array}{c}\text { 99-0650-bl } \\
\text { SBLK04 }\end{array}$ & $\begin{array}{l}99-0650 \\
\text { Sample }\end{array}$ & $\begin{array}{l}\text { 99-0650-d } \\
\text { Duplicate }\end{array}$ & \\
\hline \multirow{2}{*}{\multicolumn{2}{|c|}{$\begin{array}{r}\text { Units } \\
M D L^{(2)}\end{array}$}} & $\mu \mathrm{g} / \mathrm{L}$ & $\mu g / L$ & $\mu g / \mathrm{L}$ & $\mu \mathrm{g} / \mathrm{Kg}$ & $\mu \mathrm{g} / \mathrm{Kg}$ & $\mu \mathrm{g} / \mathrm{Kg}$ & \\
\hline & & 300 & 300 & $\mathbf{3 0 0}$ & 4000 & 4800 & 4300 & 866 \\
\hline $1031-07-8$ & Endosulfan Sulfate & $\mathrm{U}$ & $\mathrm{U}$ & $\mathrm{U}$ & $\mathrm{U}$ & $\mathrm{U}$ & $\mathrm{U}$ & $U$ \\
\hline $103-33-3$ & Azeobenzene & $\overline{\mathrm{U}}$ & $\overline{\mathrm{U}}$ & $\overline{\mathrm{U}}$ & $\overline{\mathrm{U}}$ & $\overline{\mathrm{U}}$ & $\bar{U}$ & $\bar{U}$ \\
\hline $105-67-9$ & 2,4-Dimethylphenol & $\bar{U}$ & $\overline{\mathrm{U}}$ & $\overline{\mathrm{U}}$ & $\overline{\mathrm{U}}$ & $\overline{\mathrm{U}}$ & $\overline{\mathrm{U}}$ & $\bar{U}$ \\
\hline $10595-95-6$ & N-Nitrosomethylethylamine & $\bar{U}$ & $160 \mathrm{~J}$ & $\mathbf{5 8 0}$ & $\overline{\mathrm{U}}$ & $\overline{1300 \mathrm{~J}}$ & $\overline{\mathrm{U}}$ & 538 \\
\hline $106-47-8$ & 4-Chloroaniline & $\overline{\mathrm{U}}$ & $\mathrm{U}$ & $\overline{\mathrm{U}}$ & $\overline{\mathrm{U}}$ & $\mathrm{U}$ & $\overline{\mathrm{U}}$ & $U$ \\
\hline $108-60-1$ & 2,2'-oxybis(1-Chloropropane) & $\bar{U}$ & $\bar{U}$ & $\bar{U}$ & $\overline{\mathrm{U}}$ & $\overline{\mathrm{U}}$ & $\overline{\mathrm{U}}$ & $\bar{U}$ \\
\hline $109-06-8$ & 2-Methylpyridine & $\bar{U}$ & $\bar{U}$ & $\bar{U}$ & $\overline{\mathrm{U}}$ & $\overline{\mathrm{U}}$ & $\overline{\mathrm{U}}$ & $\bar{U}$ \\
\hline $111-44-4$ & bis(2-Chloroethyl)ether & $\bar{U}$ & $\bar{U}$ & $\bar{U}$ & $\overline{\mathrm{U}}$ & $\overline{\mathrm{U}}$ & $\overline{\mathrm{U}}$ & $\bar{U}$ \\
\hline $111-91-1$ & bis(2-Chloroethoxy)methane & $\bar{U}$ & $\overline{\mathrm{U}}$ & $\bar{U}$ & $\overline{\mathrm{U}}$ & $\bar{U}$ & $\overline{\mathrm{U}}$ & $\bar{U}$ \\
\hline $119-93-7$ & 3,3'-Dimethylbenzidine & $\bar{U}$ & $\bar{U}$ & $\overline{\mathrm{U}}$ & $\bar{U}$ & $\bar{U}$ & $\bar{U}$ & $\bar{U}$ \\
\hline $120-12-7$ & Anthracene & $\overline{\mathrm{U}}$ & $\overline{\mathrm{U}}$ & $\overline{\mathrm{U}}$ & $\overline{\mathrm{U}}$ & $\overline{\mathrm{U}}$ & $\overline{\mathrm{U}}$ & $\bar{U}$ \\
\hline $120-58-1$ & Isosafrole & $\overline{\mathrm{U}}$ & $\overline{\mathrm{U}}$ & $\overline{\mathrm{U}}$ & $\overline{\mathrm{U}}$ & $\bar{U}$ & $\overline{\mathrm{U}}$ & $\bar{U}$ \\
\hline $120-83-2$ & 2,4-Dichlorophenol & $\overline{\mathrm{U}}$ & $\overline{\mathrm{U}}$ & $\bar{U}$ & $\overline{\mathrm{U}}$ & $\overline{\mathrm{U}}$ & $\overline{\mathrm{U}}$ & $\bar{U}$ \\
\hline $121-14-2$ & 2,4-Dinitrotoluene & $\bar{U}$ & $\overline{\mathrm{U}}$ & $\overline{\mathrm{U}}$ & $\overline{\mathrm{U}}$ & $\overline{\mathrm{U}}$ & $\overline{\mathrm{U}}$ & $\bar{U}$ \\
\hline $129-00-0$ & Pyrene & $\bar{U}$ & $\overline{\mathrm{U}}$ & $\bar{U}$ & $\overline{\mathrm{U}}$ & $\overline{\mathrm{U}}$ & $\overline{\mathrm{U}}$ & $\bar{U}$ \\
\hline $130-15-4$ & 1,4-Naphthoquinone & $\bar{U}$ & $\bar{U}$ & $\overline{\mathrm{U}}$ & $\overline{\mathrm{U}}$ & $\overline{\mathrm{U}}$ & $\bar{U}$ & $\bar{U}$ \\
\hline $131-11-3$ & Dimethylphthalate & $\overline{\mathrm{U}}$ & $\mathrm{U}$ & $\overline{\mathrm{U}}$ & $\overline{\mathrm{U}}$ & $\overline{\mathrm{U}}$ & $\overline{\mathrm{U}}$ & $\bar{U}$ \\
\hline $132-64-9$ & Dibenzofuran & $\bar{U}$ & $\overline{\mathrm{U}}$ & $\bar{U}$ & $\overline{\mathrm{U}}$ & $\overline{\mathrm{U}}$ & $\bar{U}$ & $\bar{U}$ \\
\hline $134-32-7$ & 1-Naphthylamine & $\overline{\mathrm{U}}$ & $\overline{\mathrm{U}}$ & $\overline{\mathrm{U}}$ & $\overline{\mathrm{U}}$ & $1400 \mathrm{~J}$ & $\overline{\mathrm{U}}$ & 227 \\
\hline $143-50-0$ & Kepone & $\overline{\mathrm{U}}$ & $\overline{\mathrm{U}}$ & $\overline{\mathrm{U}}$ & $\overline{\mathrm{U}}$ & $\mathrm{U}$ & $\bar{U}$ & $U$ \\
\hline $1888-71-7$ & Hexachloropropene & $\bar{U}$ & $\bar{U}$ & $\bar{U}$ & $\bar{U}$ & $\overline{\mathrm{U}}$ & $\bar{U}$ & $\bar{U}$ \\
\hline $191-24-2$ & Benzo(g,h,i)perylene & $\overline{\mathrm{U}}$ & $\mathrm{U}$ & $\bar{U}$ & $\overline{\mathrm{U}}$ & $\bar{U}$ & $\bar{U}$ & $\bar{U}$ \\
\hline $193-39-5$ & Indeno(1,2,3-cd)pyrene & $\overline{\mathrm{U}}$ & $\overline{\mathrm{U}}$ & $\bar{U}$ & $\overline{\mathrm{U}}$ & $\overline{\mathbf{U}}$ & $\bar{U}$ & $U$ \\
\hline $205-99-2$ & Benzo(b)fluoranthene & $\bar{U}$ & $\overline{\mathrm{U}}$ & $\bar{U}$ & $\overline{\mathrm{U}}$ & $\overline{\mathrm{U}}$ & $\overline{\mathrm{U}}$ & $\bar{U}$ \\
\hline $206-44-0$ & Fluoranthene & $\bar{U}$ & $\bar{U}$ & $\overline{\mathrm{U}}$ & $\bar{U}$ & $\overline{\mathbf{U}}$ & $\overline{\mathrm{U}}$ & $\bar{U}$ \\
\hline $207-08-9$ & Benzo(k)fluoranthene & $\bar{U}$ & $\overline{\mathrm{U}}$ & $\overline{\mathrm{U}}$ & $\overline{\mathrm{U}}$ & $\overline{\mathrm{U}}$ & $\bar{U}$ & $\bar{U}$ \\
\hline $208-96-8$ & Acenaphthylene & $\overline{\mathrm{U}}$ & $\bar{U}$ & $\bar{U}$ & $\overline{\mathrm{U}}$ & $\overline{\mathrm{U}}$ & $\bar{U}$ & $\bar{U}$ \\
\hline $218-01-9$ & Chrysene & $\bar{U}$ & $\mathrm{U}$ & $\overline{\mathrm{U}}$ & $\bar{U}$ & $\overline{\mathrm{U}}$ & $\overline{\mathrm{U}}$ & $\bar{U}$ \\
\hline $2303-16-4$ & Diallate (cis) & $\overline{\mathrm{U}}$ & $\overline{\mathrm{U}}$ & $\bar{U}$ & $\overline{\mathrm{U}}$ & $\overline{\mathrm{U}}$ & $\overline{\mathrm{U}}$ & $\bar{U}$ \\
\hline $2303-16-4$ & Diallate (trans) & $\overline{\mathrm{U}}$ & $\bar{U}$ & $\overline{\mathrm{U}}$ & $\bar{U}$ & $\overline{\mathrm{U}}$ & $\overline{\mathrm{U}}$ & $\bar{U}$ \\
\hline $23950-58-5$ & Pronamine & $\mathrm{U}$ & $\overline{\mathrm{U}}$ & $\bar{U}$ & $\bar{U}$ & $\bar{U}$ & $\bar{U}$ & $\bar{U}$ \\
\hline $319-86-8$ & delta-BHC & $\mathrm{U}$ & $\overline{\mathrm{U}}$ & $\overline{\mathrm{U}}$ & $\mathrm{U}$ & $\overline{\mathrm{U}}$ & $\overline{\mathrm{U}}$ & $\bar{U}$ \\
\hline $33213-65-9$ & Endosulfan II & $\mathrm{U}$ & $\overline{\mathrm{U}}$ & $\mathrm{U}$ & $\bar{U}$ & $\overline{\mathrm{U}}$ & $\mathrm{U}$ & $\bar{U}$ \\
\hline $510-15-6$ & Chlorobenzilate & $\mathrm{U}$ & $\mathrm{U}$ & $\overline{\mathrm{U}}$ & $\overline{\mathrm{U}}$ & $\bar{U}$ & $\bar{U}$ & $\bar{U}$ \\
\hline $51-28-5$ & 2,4-Dinitrophenol & $\mathrm{U}$ & $\overline{\mathrm{U}}$ & $\bar{U}$ & $\overline{\mathrm{U}}$ & $\overline{\mathrm{U}}$ & $\mathrm{U}$ & $\bar{U}$ \\
\hline $534-52-1$ & 4,6-Dinitro-2-methylphenol & $\mathrm{U}$ & $\overline{\mathrm{U}}$ & $\overline{\mathrm{U}}$ & $\bar{U}$ & $\bar{U}$ & $\bar{U}$ & $\bar{U}$ \\
\hline $53494-70-5$ & Endrin Ketone & $\mathrm{U}$ & $\bar{U}$ & $\overline{\mathrm{U}}$ & $\overline{\mathrm{U}}$ & $\overline{\mathrm{U}}$ & $\mathrm{U}$ & $U$ \\
\hline $53-96-3$ & 2-Acetylaminofluorene & $\mathrm{U}$ & $\bar{U}$ & $\mathrm{U}$ & $\bar{U}$ & $\bar{U}$ & $\mathrm{U}$ & $U$ \\
\hline $55-18-5$ & N-Nitrosodiethylamine & $\mathrm{U}$ & $\overline{\mathrm{U}}$ & $\bar{U}$ & $\bar{U}$ & $\overline{\mathrm{U}}$ & $\vec{U}$ & $\bar{U}$ \\
\hline $56-49-5$ & 3-Methylcholanthrene & $\mathrm{U}$ & $\mathbf{U}$ & $\mathbf{U}$ & $\bar{U}$ & $\bar{U}$ & $\mathrm{U}$ & $\bar{U}$ \\
\hline $56-55-3$ & Benzo(a)anthracene & $\mathrm{U}$ & $\bar{U}$ & $\bar{U}$ & $\bar{U}$ & $\overline{\mathrm{U}}$ & $\overline{\mathrm{U}}$ & $U$ \\
\hline $57-74-9$ & Chlordane (alpha) & $\mathrm{U}$ & $\overline{\mathrm{U}}$ & $\bar{U}$ & $\overline{\mathrm{U}}$ & $\overline{\mathrm{U}}$ & $\overline{\mathrm{U}}$ & $U$ \\
\hline $57-74-9$ & Chlordane (gamma) & $\mathrm{U}$ & $\mathrm{U}$ & $\bar{U}$ & $\mathrm{U}$ & $\overline{\mathrm{U}}$ & $\bar{U}$ & $U$ \\
\hline $58-90-2$ & 2,3,4,6-Tetrachlorophenol & $\mathrm{U}$ & $\mathrm{U}$ & $\overline{\mathrm{U}}$ & $\bar{U}$ & $\overline{\mathrm{U}}$ & $\bar{U}$ & $\bar{U}$ \\
\hline $59-50-7$ & 4-Chloro-3-methylphenol & $\mathrm{U}$ & $\mathbf{U}$ & $\mathbf{U}$ & $\mathrm{U}$ & $\mathrm{U}$ & $\bar{U}$ & $U$ \\
\hline $60-11-7$ & p-Dimethylaminoazobenzene & $\bar{U}$ & $\bar{U}$ & $\overline{\mathrm{U}}$ & $\overline{\mathrm{U}}$ & $\overline{\mathrm{U}}$ & $\overline{\mathrm{U}}$ & $\bar{U}$ \\
\hline $606-20-2$ & 2,6-Dinitrotoluene & $\bar{U}$ & $\overline{\mathrm{U}}$ & $\overline{\mathrm{U}}$ & $\overline{\mathrm{U}}$ & $\bar{U}$ & $\bar{U}$ & $\bar{U}$ \\
\hline $608-93-5$ & Pentachlorobenzene & $\mathrm{U}$ & $\mathbf{U}$ & $\mathrm{U}$ & $\mathrm{U}$ & $\overline{\mathrm{U}}$ & $\mathbf{U}$ & $U$ \\
\hline
\end{tabular}




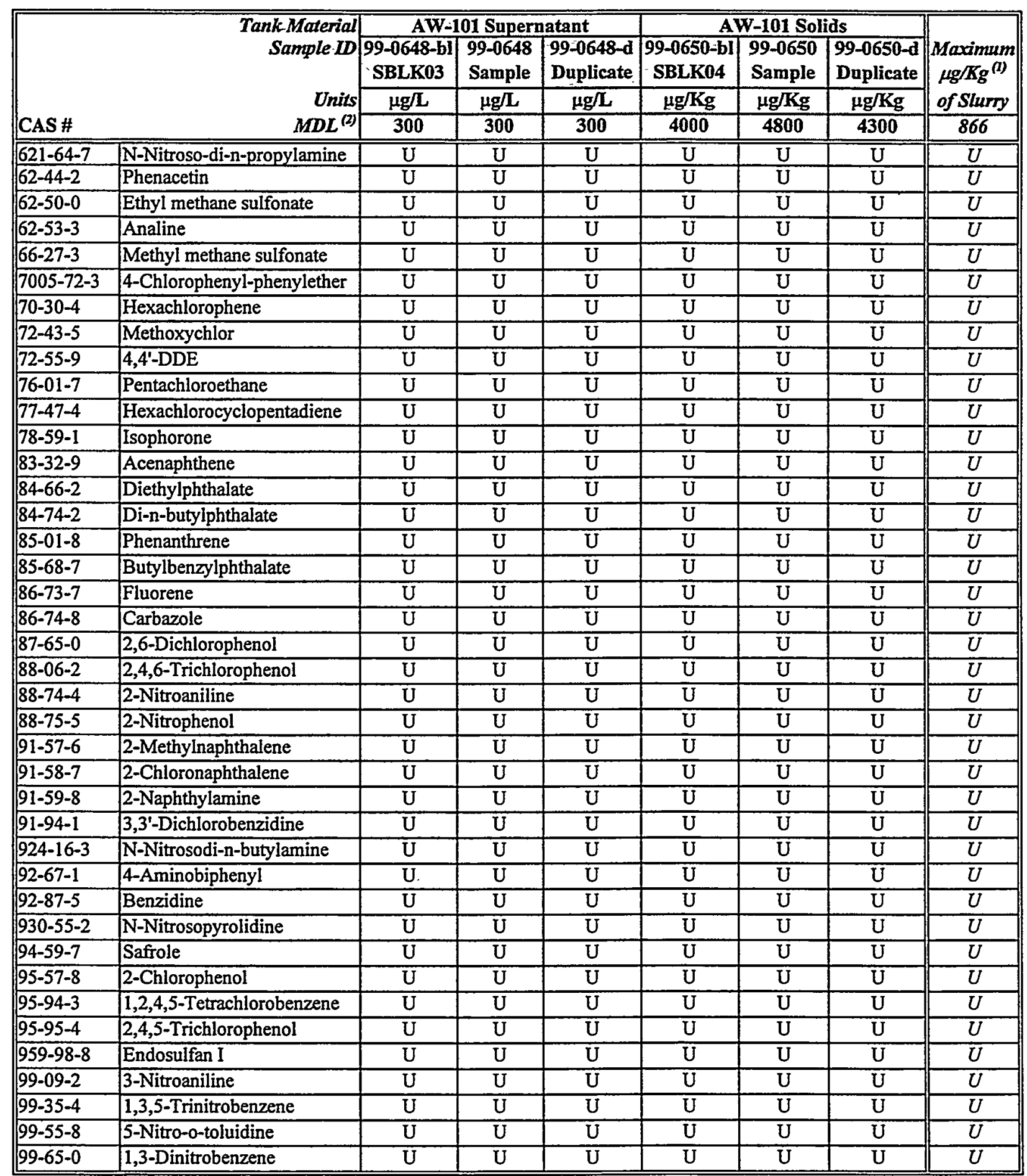

1) Maximum slurry $\mu \mathrm{g} / \mathrm{Kg}$ calculated using results of Tables $2 . a$ (weight fractions) and 2.b (supernatant density)-See Section 1.0

2) $\mathrm{MDL}=$ Method detection limit based on instrument detection limit and sample quantity

3) Cresol reported as individual isomers, 3- and 4-methyl phenols coelute

$\mathrm{U}$ flag = Compound not detected; Compound concentration less than the MDL

$\mathrm{J}$ flag $=$ Compound detected, but concentration less than the MDL 
Table 4.c. AN-107 SVOA Results - BNFL \& SW846 8270C Target Analyte List

\begin{tabular}{|c|c|c|c|c|c|c|c|c|}
\hline \multirow{4}{*}{ 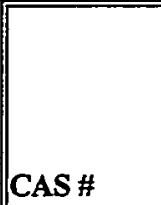 } & \multirow{2}{*}{$\begin{array}{r}\text { Tank Material } \\
\text { Sample ID }\end{array}$} & \multicolumn{3}{|c|}{ AN-107 Supernatant } & \multicolumn{3}{|c|}{ AN-107 Solids } & \multirow{3}{*}{$\begin{array}{l}\text { Maximum } \\
\mu g / \mathrm{Kg}^{(l)} \\
\text { of Slurry }\end{array}$} \\
\hline & & $\begin{array}{l}\text { 99-0649-b] } \\
\text { SBLK01 }\end{array}$ & $\begin{array}{l}99-0649 \\
\text { Sample }\end{array}$ & $\begin{array}{l}\text { 99-0649-d } \\
\text { Duplicate }\end{array}$ & $\begin{array}{c}\text { 99-0651-bl } \\
\text { SBLK02 }\end{array}$ & $\begin{array}{l}99-0651 \\
\text { Sample }\end{array}$ & $\begin{array}{l}99-0651-d \\
\text { Duplicate }\end{array}$ & \\
\hline & Units & $\mu \mathrm{g} / \mathrm{L}$ & $\mu \mathrm{g} / \mathrm{L}$ & $\mu \mathrm{g} / \mathrm{L}$ & $\mu \mathrm{g} / \mathrm{Kg}$ & $\mu \mathrm{g} / \mathrm{Kg}$ & $\mu \mathrm{g} / \mathrm{Kg}$ & \\
\hline & $M D L^{(2)}$ & 200 & $\mathbf{3 0 0}$ & 300 & 4000 & 4300 & 4100 & 657 \\
\hline \multicolumn{9}{|c|}{ BNFL Target Analyte List } \\
\hline $100-00-5$ & 1-Chloro-4-nitrobenzene & $\mathrm{U}$ & $\mathrm{U}$ & $U$ & $\mathrm{U}$ & $\mathbf{U}$ & $\mathrm{U}$ & $U$ \\
\hline $100-25-4$ & 1,4-Dinitrobenzene & $\overline{\mathrm{U}}$ & $\overline{\mathrm{U}}$ & $\bar{U}$ & $\overline{\mathrm{U}}$ & $\overline{\mathrm{U}}$ & $\bar{U}$ & $\bar{U}$ \\
\hline $100-51-6$ & Benzyl alcohol & $\bar{U}$ & $\overline{\mathrm{U}}$ & $\bar{U}$ & $\overline{\mathrm{U}}$ & $\bar{U}$ & $\bar{U}$ & $\bar{U}$ \\
\hline $106-44-5$ & 4-Methylphenol ${ }^{(3)}$ & $\bar{U}$ & $\overline{\mathrm{U}}$ & $\bar{U}$ & $\overline{\mathrm{U}}$ & $\bar{U}$ & $\bar{U}$ & $\bar{U}$ \\
\hline $106-46-7$ & 1,4-Dichlorobenzene & $\overline{\mathrm{U}}$ & $\overline{\mathrm{U}}$ & $\bar{U}$ & $\overline{\mathrm{U}}$ & $\overline{\mathrm{U}}$ & $\overline{\mathrm{U}}$ & $\bar{U}$ \\
\hline $108-95-2$ & Phenol & $\overline{\mathrm{U}}$ & $\bar{U}$ & $\bar{U}$ & $\overline{\mathrm{U}}$ & $\bar{U}$ & $\overline{\mathrm{U}}$ & $\bar{U}$ \\
\hline $110-86-1$ & Pyridine & $\overline{\mathrm{U}}$ & $\bar{U}$ & $\bar{U}$ & $\overline{\mathrm{U}}$ & $\overline{\mathrm{U}}$ & $1400 \mathrm{~J}$ & 161 \\
\hline $117-81-7$ & Bis(2-Ethylhexyl)phthalate & $\bar{U}$ & $\bar{U}$ & $\bar{U}$ & $\overline{\mathrm{U}}$ & $\overline{\mathrm{U}}$ & $\mathrm{U}$ & $\bar{U}$ \\
\hline $117-84-0$ & Di-n-octylphthalate & $\overline{\mathrm{U}}$ & $\bar{U}$ & $\bar{U}$ & $\overline{\mathrm{U}}$ & $\overline{\mathrm{U}}$ & $\bar{U}$ & $\bar{U}$ \\
\hline $118-74-1$ & Hexachlorobenzene & $\bar{U}$ & $\overline{\mathrm{U}}$ & $\bar{U}$ & $\bar{U}$ & $\overline{\mathrm{U}}$ & $\overline{\mathrm{U}}$ & $U$ \\
\hline $120-82-1$ & 1,2,4-Trichlorobenzene & $\mathrm{U}$ & $\bar{U}$ & $\bar{U}$ & $\overline{\mathrm{U}}$ & $\bar{U}$ & $\bar{U}$ & $\bar{U}$ \\
\hline $122-39-4$ & $\mathrm{~N}, \mathrm{~N}$-Diphenylamine & $\bar{U}$ & $\bar{U}$ & $\bar{U}$ & $\bar{U}$ & $\bar{U}$ & $\overline{\mathrm{U}}$ & $\bar{U}$ \\
\hline $126-73-8$ & Tributyl phosphate & $\mathrm{U}$ & $\overline{\mathrm{U}}$ & $100 \mathrm{~J}$ & $\overline{\mathrm{U}}$ & $\bar{U}$ & $\bar{U}$ & 62 \\
\hline $128-37-0$ & Butylated Hydroxytoluene & $\mathrm{U}$ & $\mathrm{U}$ & $\mathrm{U}$ & $\bar{U}$ & $\bar{U}$ & $\overline{\mathrm{U}}$ & $\bar{U}$ \\
\hline $2234-13-1$ & Octachloronaphthalene & $\mathrm{U}$ & $\mathrm{U}$ & $\mathrm{U}$ & $\mathrm{U}$ & $\mathrm{U}$ & $\bar{U}$ & $\bar{U}$ \\
\hline $309-00-2$ & Aldrin & $\mathrm{U}$ & $\mathrm{U}$ & $U$ & $\mathrm{U}$ & $\mathrm{U}$ & $\overline{\mathrm{U}}$ & $\bar{U}$ \\
\hline $319-84-6$ & alpha-BHC & $\bar{U}$ & $\overline{\mathrm{U}}$ & $\overline{\mathrm{U}}$ & $\overline{\mathrm{U}}$ & $\overline{\mathrm{U}}$ & $\overline{\mathrm{U}}$ & $\bar{U}$ \\
\hline $319-85-7$ & beta-BHC & $\overline{\mathrm{U}}$ & $\bar{U}$ & $\bar{U}$ & $\bar{U}$ & $\overline{\mathrm{U}}$ & $\overline{\mathrm{U}}$ & $\bar{U}$ \\
\hline $465-73-6$ & Isodrin & $\bar{U}$ & $\bar{U}$ & $\overline{\mathrm{U}}$ & $\overline{\mathrm{U}}$ & $\overline{\mathrm{U}}$ & $\overline{\mathrm{U}}$ & $\bar{U}$ \\
\hline $50-29-3$ & $4,4^{\prime}$-DDT & $\mathrm{U}$ & $\mathrm{U}$ & $\mathrm{U}$ & $\mathrm{U}$ & $\bar{U}$ & $\overline{\mathrm{U}}$ & $\bar{U}$ \\
\hline $50-32-8$ & Benzo(a)pyrene & $\mathrm{U}$ & $\mathrm{U}$ & $\mathrm{U}$ & $\overline{\mathrm{U}}$ & $\bar{U}$ & $\overline{\mathrm{U}}$ & $\bar{U}$ \\
\hline $53-70-3$ & Dibenz $(\mathrm{a}, \mathrm{h})$ anthracene & $\mathrm{U}$ & $\mathrm{U}$ & $\mathrm{U}$ & $\bar{U}$ & $\bar{U}$ & $\overline{\mathrm{U}}$ & $U$ \\
\hline 541-73-1 & 1,3-Dichlorobenzene & $\mathrm{U}$ & $\mathrm{U}$ & $U$ & $\mathbf{U}$ & $\mathbf{U}$ & $\mathrm{U}$ & $\bar{U}$ \\
\hline 58-89-9 & gamma-BHC (Lindane) & $\mathrm{U}$ & $\mathrm{U}$ & $\mathrm{U}$ & $\mathrm{U}$ & $\overline{\mathrm{U}}$ & $\bar{U}$ & $\bar{U}$ \\
\hline $60-57-1$ & Dieldrin & $\mathrm{U}$ & $\mathrm{U}$ & $\mathrm{U}$ & $\mathbf{U}$ & $\bar{U}$ & $\overline{\mathrm{U}}$ & $\bar{U}$ \\
\hline $62-75-9$ & N-Nitrosodimethylamine & $\overline{\mathrm{U}}$ & $170 \mathrm{~J}$ & $260 \mathrm{~J}$ & $\bar{U}$ & $\overline{\mathrm{U}}$ & $2600 \mathrm{~J}$ & 460 \\
\hline $67-72-1$ & Hexachloroethane & $\mathrm{U}$ & $\mathrm{U}$ & $\mathrm{U}$ & $\mathbf{U}$ & $\overline{\mathrm{U}}$ & $\mathrm{U}$ & $U$ \\
\hline $72-20-8$ & Endrin & $\mathrm{U}$ & $\mathrm{U}$ & $\mathbf{U}$ & $\mathrm{U}$ & $\mathrm{U}$ & $\bar{U}$ & $U$ \\
\hline $72-54-8$ & 4,4'-DDD & $\mathrm{U}$ & $\mathrm{U}$ & $\mathrm{U}$ & $\mathrm{U}$ & $\mathrm{U}$ & $\overline{\mathrm{U}}$ & $\bar{U}$ \\
\hline $76-44-8$ & Heptachlor & $\mathrm{U}$ & U & U & $\mathrm{U}$ & $\mathbf{U}$ & $\overline{\mathrm{U}}$ & $\bar{U}$ \\
\hline $82-68-8$ & Pentachloronitrobenzene & $\mathrm{U}$ & $\mathrm{U}$ & $\mathrm{U}$ & $\mathbf{U}$ & $\mathbf{U}$ & $\mathrm{U}$ & $U$ \\
\hline $87-68-3$ & Hexachlorobutadiene & $\mathrm{U}$ & $\mathrm{U}$ & $\mathbf{U}$ & $\mathbf{U}$ & $\mathrm{U}$ & $\mathrm{U}$ & $U$ \\
\hline $87-86-5$ & Pentachlorophenol & $\mathrm{U}$ & $\mathrm{U}$ & U & $\mathbf{U}$ & $\mathrm{U}$ & $\mathrm{U}$ & $\bar{U}$ \\
\hline $88-85-7$ & Dinoseb & $\mathrm{U}$ & $\mathrm{U}$ & $\mathrm{U}$ & $\mathrm{U}$ & $\mathrm{U}$ & $\overline{\mathrm{U}}$ & $\bar{U}$ \\
\hline $91-20-3$ & Naphthalene & U & $\mathrm{U}$ & $\mathrm{U}$ & $\mathbf{U}$ & $\mathbf{U}$ & $\mathrm{U}$ & $U$ \\
\hline $92-52-4$ & Biphenyl & $\mathrm{U}$ & $\mathrm{U}$ & $\mathrm{U}$ & $\mathbf{U}$ & $\mathbf{U}$ & $\mathrm{U}$ & $U$ \\
\hline $95-48-7$ & 2-Methylphenol ${ }^{(3)}$ & $\mathrm{U}$ & $\mathrm{U}$ & $\mathrm{U}$ & $\mathrm{U}$ & $\mathrm{U}$ & $\mathrm{U}$ & $U$ \\
\hline $95-50-1$ & 1,2-Dichlorobenzene & $\mathrm{U}$ & $\overline{\mathrm{U}}$ & $\overline{\mathrm{U}}$ & $\overline{\mathrm{U}}$ & $\bar{U}$ & $\bar{U}$ & $\bar{U}$ \\
\hline $98-86-2$ & Acetophenone & $\mathrm{U}$ & $\mathrm{U}$ & $\mathrm{U}$ & $\mathrm{U}$ & $\mathrm{U}$ & $\mathbf{U}$ & $U$ \\
\hline $98-95-3$ & Nitrobenzene & $\mathrm{U}$ & $\mathrm{U}$ & $\mathrm{U}$ & $\mathrm{U}$ & $\mathrm{U}$ & $\mathrm{U}$ & $U$ \\
\hline \multicolumn{9}{|c|}{ SW-846 8270C Target Analyte List } \\
\hline $100-01-6$ & 4-Nitroaniline & $\mathrm{U}$ & $\mathrm{U}$ & $\mathrm{U}$ & $\mathrm{U}$ & $\mathrm{U}$ & $\bar{U}$ & $\bar{U}$ \\
\hline $100-02-7$ & 4-Nitrophenol & $\mathrm{U}$ & $\mathrm{U}$ & $\mathrm{U}$ & $\mathrm{U}$ & $\mathrm{U}$ & $\mathrm{U}$ & $U$ \\
\hline $100-75-4$ & N-Nitrosopiperidine & $\mathrm{U}$ & $\mathrm{U}$ & $\mathrm{U}$ & $\mathrm{U}$ & $\mathrm{U}$ & $\mathrm{U}$ & $U$ \\
\hline $101-55-3$ & 4-Bromophenyl-phenylether & $\mathrm{U}$ & $\mathrm{U}$ & $\mathrm{U}$ & $\mathrm{U}$ & $\mathrm{U}$ & $\mathrm{U}$ & $U$ \\
\hline 1024-57-3 & Heptachlor Epoxide & $\mathrm{U}$ & $\mathrm{U}$ & $\mathrm{U}$ & $\mathrm{U}$ & $\mathrm{U}$ & $\mathbf{U}$ & $\bar{U}$ \\
\hline
\end{tabular}




\begin{tabular}{|c|c|c|c|c|c|c|c|c|}
\hline & \multicolumn{3}{|c|}{ AN-107 Supernatant } & \multicolumn{3}{|c|}{ AN-107 Solids } & \multirow{3}{*}{$\begin{array}{l}\text { Moximum } \\
\mu g / K g^{(I)} \\
\text { of Slurry }\end{array}$} \\
\hline 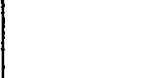 & Sample ID & $\begin{array}{c}\text { 99:0649-bl } \\
\text { SBLK01 }\end{array}$ & $\begin{array}{l}99-0649 \\
\text { Sample }\end{array}$ & $\begin{array}{l}\text { 99:0649-d } \\
\text { Duplicate }\end{array}$ & $\begin{array}{c}\text { 99-0651-b1 } \\
\text { SBLK02 }\end{array}$ & $\begin{array}{l}\text { 99-0651 } \\
\text { Sample }\end{array}$ & $\begin{array}{l}\text { 99-0651-d } \\
\text { Duplicate }\end{array}$ & \\
\hline & Units & $\mu \mathrm{g} / \mathbf{L}$ & $\mu \mathrm{g} / \mathrm{L}$ & $\mu g / L$ & $\mu \mathrm{g} / \mathrm{Kg}$ & $\mu \mathrm{g} / \mathrm{Kg}$ & $\mu g / \mathrm{Kg}$ & \\
\hline CAS \# & $M D L^{(2)}$ & 200 & 300 & 300 & 4000 & 4300 & 4100 & 657 \\
\hline $1031-07-8$ & Endosulfan Sulfate & $\mathrm{U}$ & $\mathrm{U}$ & $\overline{\mathrm{U}}$ & $\overline{\mathrm{U}}$ & $\overline{\mathrm{U}}$ & $\overline{\mathrm{U}}$ & $\overline{U U}$ \\
\hline $103-33-3$ & Azeobenzene & $\bar{U}$ & $\bar{U}$ & $\bar{U}$ & $\overline{\mathrm{U}}$ & $\overline{\mathrm{U}}$ & $\overline{\mathrm{U}}$ & $\bar{U}$ \\
\hline $105-67-9$ & 2,4-Dimethylphenol & $\bar{U}$ & $\bar{U}$ & $\bar{U}$ & $\overline{\mathrm{U}}$ & $\overline{\mathrm{U}}$ & $\overline{\mathrm{U}}$ & $\bar{U}$ \\
\hline $10595-95-6$ & N-Nitrosomethylethylamine & $\bar{U}$ & $\overline{\mathrm{U}}$ & $\overline{\mathrm{U}}$ & $\bar{U}$ & $\bar{U}$ & $\overline{\mathrm{U}}$ & $\bar{U}$ \\
\hline $106-47-8$ & 4-Chloroaniline & $\overline{\mathrm{U}}$ & $\overline{\mathrm{U}}$ & $\bar{U}$ & $\bar{U}$ & $\overline{\mathrm{U}}$ & $\bar{U}$ & $U$ \\
\hline $108-60-1$ & $2,2^{\prime}-$ oxybis $(1-$ & $\bar{U}$ & $\mathrm{U}$ & $\bar{U}$ & $\overline{\mathrm{U}}$ & $\overline{\mathrm{U}}$ & $\overline{\mathrm{U}}$ & $\bar{U}$ \\
\hline $109-06-8$ & 2-Methylpyridine & $\bar{U}$ & $\mathrm{U}$ & $\mathrm{U}$ & $\overline{\mathrm{U}}$ & $\overline{\mathrm{U}}$ & $\bar{U}$ & $\bar{U}$ \\
\hline $111-44-4$ & bis(2-Chloroethyl)ether & $\overline{\mathrm{U}}$ & $\overline{\mathrm{U}}$ & $\bar{U}$ & $\bar{U}$ & $\bar{U}$ & $\mathrm{U}$ & $\bar{U}$ \\
\hline $111-91-1$ & bis(2-Chloroethoxy)methane & $\bar{U}$ & $\overline{\mathrm{U}}$ & $\bar{U}$ & $\overline{\mathrm{U}}$ & U & $\bar{U}$ & $\bar{U}$ \\
\hline $119-93-7$ & 3,3'-Dimethylbenzidine & $U$ & $\bar{U}$ & $\bar{U}$ & $\bar{U}$ & $\mathrm{U}$ & $\mathrm{U}$ & $\bar{U}$ \\
\hline $120-12-7$ & Anthracene & $\mathrm{U}$ & $\bar{U}$ & $\mathrm{U}$ & $\bar{U}$ & $\bar{U}$ & $\mathrm{U}$ & $\bar{U}$ \\
\hline $120-58-1$ & Isosafrole & $\bar{U}$ & $\bar{U}$ & $\overline{\mathrm{U}}$ & $\mathrm{U}$ & $\mathrm{U}$ & $\bar{U}$ & $\bar{U}$ \\
\hline$\overline{120-83-2}$ & 2,4-Dichlorophenol & $\bar{U}$ & $\bar{U}$ & $\bar{U}$ & $\overline{\mathrm{U}}$ & $\mathrm{U}$ & $\overline{\mathrm{U}}$ & $\bar{U}$ \\
\hline $121-14-2$ & 2,4-Dinitrotoluene & $\bar{U}$ & $\overline{\mathrm{U}}$ & $\bar{U}$ & $\bar{U}$ & $\bar{U}$ & $\bar{U}$ & $U$ \\
\hline $129-00-0$ & Pyrene & $\bar{U}$ & $\bar{U}$ & $\bar{U}$ & $\bar{U}$ & $\bar{U}$ & $\overline{\mathrm{U}}$ & $\bar{U}$ \\
\hline $130-15-4$ & 1,4-Naphthoquinone & $\overline{\mathrm{U}}$ & $\overline{\mathrm{U}}$ & $\bar{U}$ & $\overline{\mathrm{U}}$ & $\bar{U}$ & $\overline{\mathrm{U}}$ & $\bar{U}$ \\
\hline $131-11-3$ & Dimethylphthalate & $\bar{U}$ & $\bar{U}$ & $\bar{U}$ & $\overline{\mathrm{U}}$ & $\mathrm{U}$ & $\overline{\mathrm{U}}$ & $\bar{U}$ \\
\hline 132-64-9 & Dibenzofuran & $\bar{U}$ & $\overline{\mathrm{U}}$ & $\overline{\mathrm{U}}$ & $\overline{\mathrm{U}}$ & $\bar{U}$ & $\overline{\mathrm{U}}$ & $\bar{U}$ \\
\hline $134-32-7$ & 1-Naphthylamine & $\overline{\mathrm{U}}$ & $\bar{U}$ & $\bar{U}$ & $\bar{U}$ & 5400 & $2000 \mathrm{~J}$ & 619 \\
\hline $143-50-0$ & Kepone & $\overline{\mathrm{U}}$ & $\mathrm{U}$ & $\overline{\mathrm{U}}$ & $\overline{\mathrm{U}}$ & $\mathrm{U}$ & $\mathrm{U}$ & $U$ \\
\hline $1888-71-7$ & Hexachloropropene & $\bar{U}$ & $\overline{\mathrm{U}}$ & $\bar{U}$ & $\overline{\mathrm{U}}$ & $\bar{U}$ & $\bar{U}$ & $\bar{U}$ \\
\hline $191-24-2$ & Benzo(g,h,i)perylene & $\bar{U}$ & $\overline{\mathrm{U}}$ & $\overline{\mathrm{U}}$ & $\bar{U}$ & $\overline{\mathrm{U}}$ & $\overline{\mathrm{U}}$ & $\bar{U}$ \\
\hline 193-39-5 & Indeno(1,2,3-cd)pyrene & $\bar{U}$ & $\overline{\mathrm{U}}$ & $\overline{\mathrm{U}}$ & $\bar{U}$ & $\overline{\mathrm{U}}$ & $\bar{U}$ & $\bar{U}$ \\
\hline $205-99-2$ & Benzo(b)fluoranthene & $\overline{\mathrm{U}}$ & $\overline{\mathrm{U}}$ & $\bar{U}$ & $\overline{\mathrm{U}}$ & $\bar{U}$ & $\mathrm{U}$ & $\bar{U}$ \\
\hline $206-44-0$ & Fluoranthene & $\bar{U}$ & $\overline{\mathrm{U}}$ & $\overline{\mathrm{U}}$ & $\overline{\mathrm{U}}$ & $\bar{U}$ & $\mathrm{U}$ & $\bar{U}$ \\
\hline 207-08-9 & Benzo(k)fluoranthene & $\overline{\mathrm{U}}$ & $\overline{\mathrm{U}}$ & $\bar{U}$ & $\overline{\mathrm{U}}$ & $\bar{U}$ & $\bar{U}$ & $\bar{U}$ \\
\hline $208-96-8$ & Acenaphthylene & $\bar{U}$ & $\mathrm{U}$ & $\overline{\mathrm{U}}$ & $\bar{U}$ & $\overline{\mathrm{U}}$ & $\mathrm{U}$ & $\bar{U}$ \\
\hline $218-01-9$ & Chrysene & $\bar{U}$ & $\bar{U}$ & $\overline{\mathrm{U}}$ & $\bar{U}$ & $\bar{U}$ & $\bar{U}$ & $\bar{U}$ \\
\hline $2303-16-4$ & Diallate (cis) & $\mathrm{U}$ & $\mathrm{U}$ & $\mathrm{U}$ & $\mathbf{U}$ & $\overline{\mathrm{U}}$ & $\bar{U}$ & $\bar{U}$ \\
\hline $2303-16-4$ & Diallate (trans) & $\mathrm{U}$ & $\bar{U}$ & $\mathrm{U}$ & $\mathbf{U}$ & $\mathrm{U}$ & $\mathbf{U}$ & $\bar{U}$ \\
\hline $23950-58-5$ & Pronamine & $\mathrm{U}$ & $\mathrm{U}$ & $\mathrm{U}$ & $\mathrm{U}$ & $\mathrm{U}$ & $\mathrm{U}$ & $U$ \\
\hline $319-86-8$ & delta-BHC & $\mathrm{U}$ & $\mathrm{U}$ & $\mathrm{U}$ & $\mathrm{U}$ & $\mathrm{U}$ & $\mathrm{U}$ & $U$ \\
\hline $33213-65-9$ & Endosulfan II & $\mathrm{U}$ & $\mathrm{U}$ & $\mathrm{U}$ & $\mathrm{U}$ & $\mathrm{U}$ & $\mathbf{U}$ & $U$ \\
\hline $510-15-6$ & Chlorobenzilate & $\mathrm{U}$ & $\mathrm{U}$ & $\mathrm{U}$ & $\mathrm{U}$ & $\mathrm{U}$ & $\mathrm{U}$ & $U$ \\
\hline $51-28-5$ & 2,4-Dinitrophenol & $\bar{U}$ & $\mathrm{U}$ & $\mathrm{U}$ & $\mathrm{U}$ & $\overline{\mathrm{U}}$ & $\overline{\mathrm{U}}$ & $\bar{U}$ \\
\hline $534-52-1$ & 4,6-Dinitro-2-methylphenol & $\mathrm{U}$ & $\bar{U}$ & $\mathbf{U}$ & $\mathrm{U}$ & $\overline{\mathrm{U}}$ & $\bar{U}$ & $\bar{U}$ \\
\hline $53494-70-5$ & Endrin Ketone & $\mathrm{U}$ & $\mathrm{U}$ & $\mathrm{U}$ & $\mathrm{U}$ & $\mathrm{U}$ & $\mathbf{U}$ & $\bar{U}$ \\
\hline $53-96-3$ & 2-Acetylaminofluorene & $\mathrm{U}$ & $\bar{U}$ & $\mathbf{U}$ & $\mathrm{U}$ & $\mathrm{U}$ & $\mathrm{U}$ & $\bar{U}$ \\
\hline $55-18-5$ & N-Nitrosodiethylamine & $\mathrm{U}$ & $\bar{U}$ & $\mathrm{U}$ & $\mathrm{U}$ & $\bar{U}$ & $\overline{\mathrm{U}}$ & $\bar{U}$ \\
\hline $56-49-5$ & 3-Methylcholanthrene & $\bar{U}$ & $\overline{\mathrm{U}}$ & $\overline{\mathrm{U}}$ & $\overline{\mathrm{U}}$ & $\bar{U}$ & $\overline{\mathrm{U}}$ & $\bar{U}$ \\
\hline $56-55-3$ & Benzo(a)anthracene & $\bar{U}$ & $\bar{U}$ & $\bar{U}$ & $\bar{U}$ & $\bar{U}$ & $\overline{\mathrm{U}}$ & $\bar{U}$ \\
\hline $57-74-9$ & Chlordane (alpha) & $\mathrm{U}$ & $\mathrm{U}$ & $\bar{U}$ & $\bar{U}$ & $\bar{U}$ & $\bar{U}$ & $\bar{U}$ \\
\hline $57-74-9$ & Chlordane (gamma) & $\overline{\mathrm{U}}$ & $\mathrm{U}$ & $\bar{U}$ & $\bar{U}$ & $\overline{\mathrm{U}}$ & $\bar{U}$ & $\bar{U}$ \\
\hline $58-90-2$ & 2,3,4,6-Tetrachlorophenol & $\bar{U}$ & $\bar{U}$ & $\bar{U}$ & $\bar{U}$ & $\overline{\mathrm{U}}$ & $\overline{\mathrm{U}}$ & $U$ \\
\hline $59-50-7$ & 4-Chloro-3-methylphenol & $\mathrm{U}$ & $\overline{\mathrm{U}}$ & $\mathrm{U}$ & $\bar{U}$ & $\overline{\mathrm{U}}$ & $\bar{U}$ & $\bar{U}$ \\
\hline $60-11-7$ & $\mathrm{p}-$ & $\overline{\mathrm{U}}$ & $\mathrm{U}$ & $\bar{U}$ & $\overline{\mathrm{U}}$ & $\overline{\mathrm{U}}$ & $\bar{U}$ & $U$ \\
\hline $606-20-2$ & 2,6-Dinitrotoluene & $\overline{\mathrm{U}}$ & $\mathrm{U}$ & $\mathrm{U}$ & $\mathrm{U}$ & $\bar{U}$ & $\bar{U}$ & $U$ \\
\hline 608-93-5 & Pentachlorobenzene & $\bar{U}$ & $\overline{\mathrm{U}}$ & $\bar{U}$ & $\bar{U}$ & $\bar{U}$ & $\mathrm{U}$ & $U$ \\
\hline
\end{tabular}




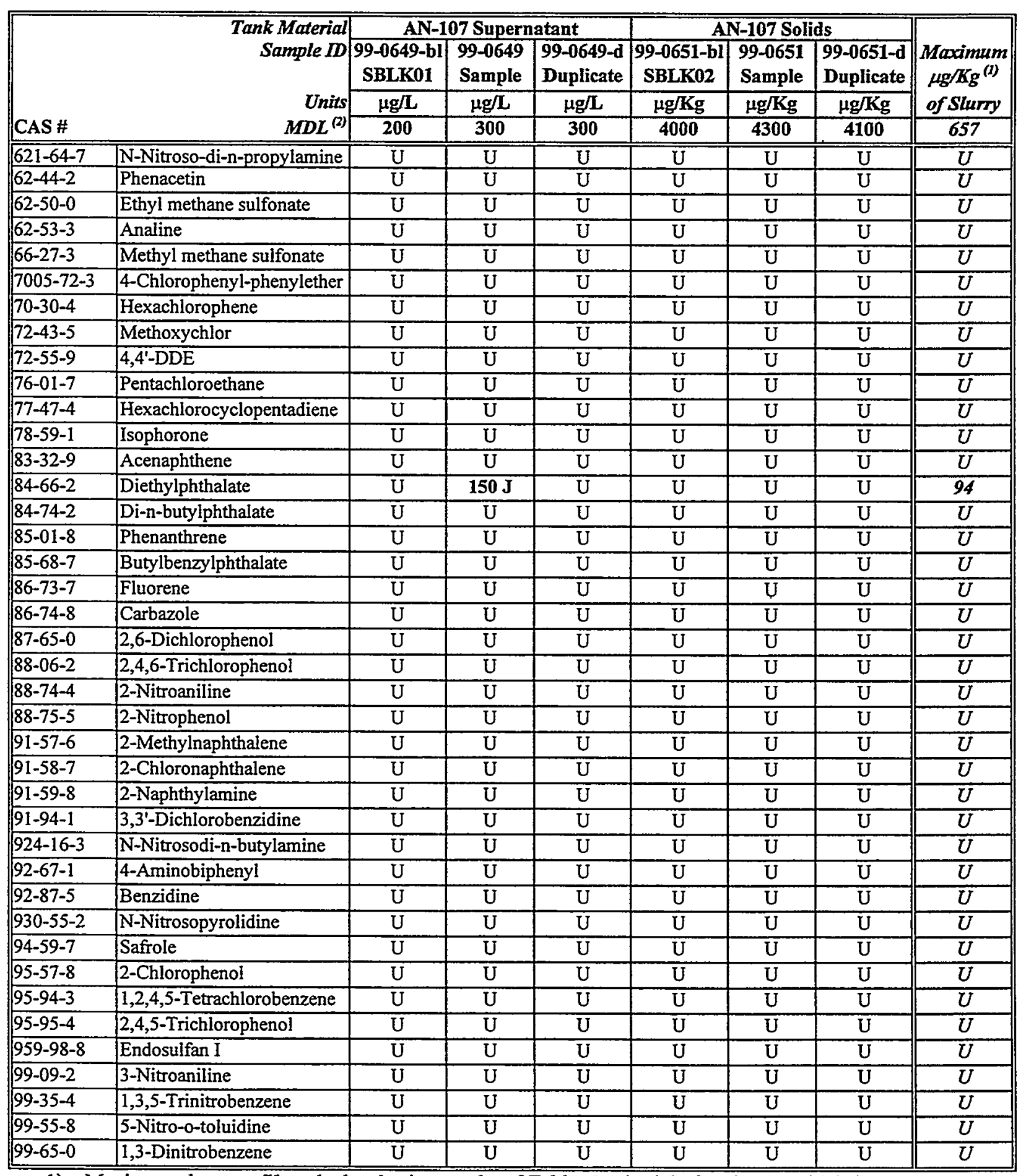

1) Maximum slurry $\mu \mathrm{g} / \mathrm{Kg}$ calculated using results of Tables 2.a (weight fractions) and 2.b (supernatant density)-See Section 1.0

2) $\mathrm{MDL}=$ Method detection limit based on instrument detection limit and sample quantity

3) Cresol reported as individual isomers, 3- and 4-methyl phenols coelute

$\mathrm{U}$ flag = Compound not detected; Compound concentration less than the MDL

$\mathrm{J}$ flag = Compound detected, but concentration less than the MDL 
Table 4.d. AW-101 Supernatant SVOA Tentatively Identified Compounds

\begin{tabular}{|c|c|c|c|c|c|}
\hline \multirow[b]{3}{*}{ CAS \# } & \multirow[b]{3}{*}{ TIC } & \multirow[b]{3}{*}{ Ret. Time } & \multicolumn{3}{|c|}{ AW-101 Supernatant 99-0648 } \\
\hline & & & Sample & Duplicate & Blank \\
\hline & & & $\mu \mathrm{g} / \mathrm{L}$ & $\mu \mathrm{g} / \mathrm{L}$ & $\mu \mathrm{g} / \mathrm{L}$ \\
\hline 67-66-3 & Chloroform & 4.82 & 430 & $\ldots$ & $\ldots$ \\
\hline $79-00-5$ & Ethane, 1,1,2-trichloro- & 5.45 & 160 & $\overline{-\cdots}$ & - \\
\hline $107-92-6$ & Butanoic acid & 6.85 & 2000 & $\ldots$ & - \\
\hline 75-98-9 & Propanoic acid, 2,2-dimethyl- & 7.08 & 410 & - & - \\
\hline $75-76-3$ & Silane, tetramethyl- & 7.31 & 3300 & 2300 & 7200 \\
\hline $123-42-2$ & 2-Pentanone, 4-hydroxy-4-methyl & 7.40 & $2000 \mathrm{~A}$ & $5200 \mathrm{~A}$ & $-\cdots$ \\
\hline $141-78-6$ & Ethyl Acetate & 7.88 & 1100 & 1100 & - \\
\hline $4245-37-8$ & 2-Propenoic acid, 2-methyl-, ethenyl & 8.04 & 440 & - & 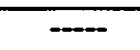 \\
\hline $156-87-6$ & 1-Propanol, 3-amino- & $\overline{8.11}$ & 390 & $=$ & $\cdots$ \\
\hline$\overline{111-84-2}$ & Nonane & 9.28 & 89000 & 80000 & 130000 \\
\hline $616-40-0$ & Hydrazine, 1,1-diethyl- & 9.60 & 96 & - & - \\
\hline \begin{tabular}{|l|}
$53778-73-7$ \\
\end{tabular} & 2-Butanol, 1-methoxy- & 9.97 & 2100 & - & $=$ \\
\hline \multirow[t]{2}{*}{$3970-62-5$} & 3-Pentanol, 2,2-dimethyl- & 9.97 & -- & 3000 & - \\
\hline & Unknown & 10.07 & 460 & $\ldots$ & - \\
\hline $13861-97-7$ & 2(3H)-Furanone, dihydro-4,4-dimethyl- & 11.11 & 580 & - & $\overline{-}$ \\
\hline \multirow[t]{8}{*}{$78-42-2$} & Phosphoric acid, tris(2-ethylhexyl) & 13.57 & 240 & $\cdots$ & - \\
\hline & Unknown & 14.25 & 350 & $\overline{-\cdots}$ & - \\
\hline & Unknown nitric acid ester & 16.24 & 170 & 130 & - \\
\hline & Unknown nitric acid ester & 16.52 & 240 & 200 & $\cdots$ \\
\hline & Unknown nitric acid ester & 16.89 & 380 & 300 & - \\
\hline & Unknown nitric acid ester & 17.32 & 48 & 66 & $=$ \\
\hline & Unknown nitric acid ester & 18.04 & 180 & 150 & - \\
\hline & Unknown & 23.04 & 170 & 170 & - \\
\hline
\end{tabular}

". TIC not detected

$A=$ Suspected aldol condensation product 
Table 4.e. AW-101 Solids SVOA Tentatively Identified Compounds

\begin{tabular}{|c|c|c|c|c|c|}
\hline \multirow[b]{3}{*}{ CAS\# } & \multirow[b]{3}{*}{ TIC } & \multirow[b]{3}{*}{ Ret. Time } & \multicolumn{3}{|c|}{ AW-101 Solids 99-0650 } \\
\hline & & & Sample & Duplicate & Blank \\
\hline & & & $\mu \mathrm{g} / \mathrm{Kg}$ & $\mu \mathrm{g} / \mathrm{Kg}$ & $\mu \mathrm{g} / \mathrm{Kg}$ \\
\hline & Unknown Aldol Condensate & 6.64 & 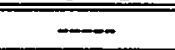 & $-\cdots$ & $20000 \mathrm{~A}$ \\
\hline $141-79-7$ & 3-Penten-2-one, 4-methyl- & 6.56 & $980000 \mathrm{~A}$ & $11000 \mathrm{~A}$ & $\ldots$ \\
\hline $109-49-9$ & 5-Hexen-2-one & 4.67 & ---- & $17000 \mathrm{~A}$ & $-\cdots$ \\
\hline $141-79-7$ & 3-Penten-2-one, 4-methyl- & 6.72 & $-\cdots$ & $770000 \mathrm{~A}$ & - \\
\hline $123-42-2$ & 2-Pentanone, 4-hydroxy-4-methyl & 8.75 & $2600000 \mathrm{~A}$ & $4600000 \mathrm{~A}$ & $430000 \mathrm{~A}$ \\
\hline $111-84-2$ & Nonane & 9.40 & 690000 & 1500000 & 1600000 \\
\hline $627-08-7$ & Propane, 1-(1-methylethoxy) & 10.11 & $76000 \mathrm{~A}$ & --- & $\overline{-\infty}$ \\
\hline & Unknown Aldol Condensate & 10.24 & - & $59000 \mathrm{~A}$ & $\ldots$ \\
\hline $758-21-4$ & Silane, ethyldimethyl- & 10.39 & 15000 & $-\cdots$ & $\overline{----}$ \\
\hline $3970-62-5$ & 3-Pentanol, 2,2-dimethyl- & 10.52 & $\cdots$ & 22000 & $\ldots$ \\
\hline & Unknown & 13.36 & 56000 & 41000 & $\ldots$ \\
\hline $591-76-4$ & Hexane, 2-methyl- & 13.48 & 9400 & --- & - \\
\hline $504-20-1$ & 2,5-Heptadien-4-one, 2,6-dimethyl- & 14.04 & $-\cdots$ & $19000 \mathrm{~A}$ & - \\
\hline $73583-56-9$ & 2,6-Dimethyl-6-nitro-2-hepten-4-one & 14.78 & 32000 & $-\cdots$ & $-\cdots$ \\
\hline $638-10-8$ & 2-Butenoic acid, 3-methyl-, ethyl ester & 14.87 & $\cdots$ & 99000 & $=$ \\
\hline $565-61-7$ & 2-Pentanone, 3-methyl- & 14.99 & 36000 & $\cdots$ & $-\cdots$ \\
\hline $112-40-3$ & Dodecane & 15.74 & ---- & 73000 & $=$ \\
\hline $629-50-5$ & Tridecane & 15.75 & 39000 & $\cdots$ & $\ldots$ \\
\hline $18720-66-6$ & 3-Heptanol, 6-methyl- & 15.85 & $-\cdots$ & 34000 & - \\
\hline $3973-27-1$ & p-Dioxin, 2,3-dihydro-2,5,6-trimethyl & 16.05 & 110000 & $\cdots$ & - \\
\hline & Unknown & 16.09 & $\cdots$ & 210000 & - \\
\hline & Unknown & 16.44 & 19000 & 44000 & 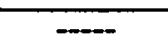 \\
\hline $629-50-5$ & Tridecane & 17.63 & 170000 & 190000 & - \\
\hline $629-59-4$ & Tetradecane & 19.30 & 160000 & 210000 & $-\ldots$ \\
\hline $110-13-4$ & 2,5-Hexanedione & 20.31 & $\cdots$ & 17000 & $\ldots$ \\
\hline $629-62-9$ & Pentadecane & 20.86 & 14000 & 20000 & - \\
\hline
\end{tabular}

$A=$ Suspected aldol condensation product 
Table 4.f. AN-107 Supernatant SVOA Tentatively Identified Compounds

\begin{tabular}{|c|c|c|c|c|c|}
\hline \multirow[b]{3}{*}{ CAS\# } & \multirow[b]{3}{*}{ TIC } & \multirow[b]{3}{*}{ Ret. Time } & \multicolumn{3}{|c|}{ AN-107 Supernatant 99-0649 } \\
\hline & & & Sample & Duplicate & $\overline{\text { Blank }}$ \\
\hline & & & $\mu \mathrm{g} / \mathrm{L}$ & $\mu \mathrm{g} / \mathrm{L}$ & $\mu \mathrm{g} / \mathrm{L}$ \\
\hline $67-66-3$ & Chloroform & 4.78 & 160 & 380 & ב-...- \\
\hline $79-00-5$ & Ethane, 1,1,2-trichloro- & $\overline{5.42}$ & 380 & 290 & $\cdots$ \\
\hline $994-05-8$ & Butane, 2-methoxy-2-methyl- & $\overline{7.23}$ & 1200 & 1300 & $\overline{-\infty}$ \\
\hline$\longdiv { 1 1 2 0 - 6 4 - 5 }$ & Oxazole, 4,5-dihydro-2-methyl- & 7.48 & 360 & 450 & $-\cdots--$ \\
\hline $34075-28-0$ & Butane, 2,3-dimethyl-2-nitro- & 7.98 & $\ldots$ & 1200 & $-\ldots$ \\
\hline $625-58-1$ & Nitric acid, ethyl ester & $\overline{8.76}$ & 770 & 2200 & -.-- \\
\hline$\longdiv { 1 1 1 - 8 4 - 2 }$ & Nonane & 9.41 & 110000 & 140000 & 70000 \\
\hline $79-34-5$ & Ethane, 1,1,2,2-tetrachloro- & 9.65 & 170 & $\cdots$ & -- \\
\hline $9224-46-9$ & 1-Propanamine, N-methyl-N-nitroso- & 10.59 & 2400 & 3600 & $\ldots$ \\
\hline 39884-53-2 & N-nitroso-2-methyl-oxazolidine & 11.67 & - & 740 & $-\ldots$ \\
\hline $59-89-2$ & Morpholine, 4-nitroso- & 13.14 & 1800 & 3900 & $\cdots$ \\
\hline $34419-76-6$ & 1-Propanamine, N,2-dimethy]- & 13.25 & 2100 & - & $\cdots$ \\
\hline$\overline{111-14-8}$ & Heptanoic acid & 13.45 & $\ldots$ & 9500 & $\ldots$ \\
\hline \multirow[t]{5}{*}{$149-57-5$} & Hexanoic acid, 2-ethyl- & 14.25 & 290 & 740 & 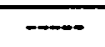 \\
\hline & Unknown & $\overline{14.44}$ & $\overline{-1}$ & 800 & $\overline{-}$ \\
\hline & Unknown & 15.97 & 570 & 630 & -- \\
\hline & Unknown & 16.69 & 380 & 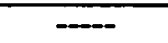 & $\ldots$ \\
\hline & Unknown amine & 17.77 & 2900 & 3300 & $\ldots$ \\
\hline \multirow[t]{2}{*}{$1116-54-7$} & Ethanol, 2,2'-(nitrosoimino) bis- & 19.43 & 290 & 1000 & $\cdots$ \\
\hline & Unknown Amine & 19.58 & 760 & $\cdots$ & $\cdots$ \\
\hline $3034-41-1$ & 1H-Imidazole, 1-methyl-4-nitro- & 19.64 & $\cdots$ & 840 & ---- \\
\hline \multirow[t]{3}{*}{$140-79-4$} & Piperazine, 1,4-dinitroso- & 19.77 & 550 & 1500 & $\ldots$ \\
\hline & Unknown & 20.76 & 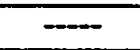 & 750 & $-\ldots$ \\
\hline & Unknown & 20.96 & 1800 & 3200 & $\ldots$ \\
\hline \multirow[t]{7}{*}{$7247-89-4$} & Piperidine, 2-methyl-1-nitroso- & 21.21 & 160 & - & $-\cdots$ \\
\hline & Unknown & 21.38 & 120 & - & $-\ldots$ \\
\hline & Unknown & 21.87 & 3500 & $\overline{-\cdots}$ & $\overline{---}$ \\
\hline & Unknown & 22.31 & 540 & 1000 & $=$ \\
\hline & Unknown amine & 22.53 & 470 & $\cdots$ & $-\cdots$ \\
\hline & Unknown amine & 22.65 & 1400 & $\overline{-}$ & 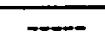 \\
\hline & Unknown & 23.51 & 710 & $\cdots$ & - \\
\hline $55556-93-9$ & 4-Piperidinol, 1-nitroso- & 25.61 & 8100 & 16000 & $=$ \\
\hline 15104-03-7 & Piperidine, 4-methyl-1-nitroso- & 26.57 & 230 & - & $\overline{-}$ \\
\hline $55556-86-0$ & Pyrrolidine, 2,5-dimethyl-1-nitroso- & 26.68 & $\overline{-}$ & 820 & $\Longrightarrow$ \\
\hline
\end{tabular}


Table 4.g. AN-107 Solids SVOA Tentatively Identified Compounds

\begin{tabular}{|c|c|c|c|c|c|}
\hline \multirow[b]{3}{*}{ CAS \# } & \multirow[b]{3}{*}{ TIC } & \multirow[b]{3}{*}{ Ret. Time } & \multicolumn{3}{|c|}{ AN-107 Solids 99-0651 } \\
\hline & & & Sample & Duplicate & Blank \\
\hline & & & $\mu \mathrm{g} / \mathrm{Kg}$ & $\mu \mathrm{g} / \mathrm{Kg}$ & $\mu \mathrm{g} / \mathrm{Kg}$ \\
\hline $67-66-3$ & Chloroform & 4.85 & 3200 & 3300 & $\ldots$ \\
\hline $106-94-5$ & Propane, 1-bromo- & 5.04 & 1400 & $\cdots$ & 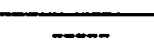 \\
\hline $141-78-6$ & Ethyl Acetate & 5.66 & 2500 & --- & $\cdots$ \\
\hline $141-79-7$ & 3-Penten-2-one, 4-methyl- & 6.02 & $5100 \mathrm{~A}$ & $19000 \mathrm{~A}$ & - \\
\hline \multirow[t]{2}{*}{$108-64-5$} & Butanoic acid, 3-methyl-, ethyl ester & 6.46 & $-\ldots$ & 7300 & $\ldots$ \\
\hline & Unknown aldol condensate & 6.61 & $-\cdots$ & $11000 \mathrm{~A}$ & $23000 \mathrm{~A}$ \\
\hline $108-21-4$ & Acetic acid, 1-methylethyl ester & 6.62 & 23000 & $\cdots$ & $\ldots$ \\
\hline \multirow[t]{2}{*}{$123-42-2$} & 2-Pentanone, 4-hydroxy-4-methyl- & 7.50 & $390000 \mathrm{~A}$ & $260000 \mathrm{~A}$ & $450000 \mathrm{~A}$ \\
\hline & Unknown aldol condensate & 8.55 & 18000 & 110000 & $\ldots$ \\
\hline \multirow[t]{2}{*}{$111-84-2$} & Nonane & 9.54 & 2000000 & 1700000 & 860000 \\
\hline & Unknown aldol condensate & 9.57 & $-\cdots$ & $12000 \mathrm{~A}$ & ---- \\
\hline $3970-62-5$ & 3-Pentanol, 2,2-dimethyl- & 9.95 & - & 18000 & --- \\
\hline $625-50-3$ & Acetamide, N-ethyl- & 11.72 & - & 11000 & $\cdots$ \\
\hline $5343-96-4$ & 2-Butanol, 3-methyl-, acetate & 11.73 & 36000 & $-\cdots$ & $\cdots$ \\
\hline $107-87-9$ & 2-Pentanone & 12.58 & 68000 & 75000 & $\cdots$ \\
\hline $59-89-2$ & Morpholine, 4-nitroso- & 13.12 & 3800 & $\cdots$ & $-\cdots$ \\
\hline $107-92-6$ & Butanoic acid & 13.19 & 5300 & $\cdots$ & - \\
\hline $1721-93-3$ & Isoquinoline, 1-methyl- & 14.37 & $\cdots$ & 4200 & --- \\
\hline $91-63-4$ & Quinoline, 2-methyl- & 14.38 & 12000 & - & $\cdots$ \\
\hline $1526-17-6$ & 2-Fluoro-6-nitrophenol & 14.74 & 7500 & $\ldots$ & $\ldots$ \\
\hline \multirow[t]{2}{*}{$3973-27-1$} & p-Dioxin, 2,3-dihydro-2,5,6-trimethyl & 15.87 & $\ldots$ & 13000 & $\ldots$ \\
\hline & 2-Chloro-6-nitrophenol-d3 & 18.02 & 7400 & 2700 & - \\
\hline $403-19-0$ & 2-Fluoro-4-nitrophenol & 18.11 & 5400 & 1800 & $\overline{-}$ \\
\hline \multirow[t]{4}{*}{$1116-54-7$} & Ethanol, 2,2'-(nitrosoimino) bis- & 19.07 & 5800 & 3800 & - \\
\hline & 2-Chloro-4-nitrophenol-d3 & 19.94 & 5400 & 2300 & $\ldots$ \\
\hline & Unknown & 20.06 & 5900 & $\ldots$ & - \\
\hline & Unknown & 20.27 & 1900 & 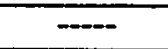 & $-\cdots$ \\
\hline $55556-93-9$ & 4-Piperidinol, 1-nitroso- & 25.26 & 26000 & 19000 & $\cdots$ \\
\hline
\end{tabular}

"-...." = TIC not detected

$A=$ Suspected aldol condensation product 
Table 4.h. Target SVOA Minimum Reportable Quantities

\begin{tabular}{|c|c|c|c|}
\hline CAS\# & SVOA Compounds & $\begin{array}{c}\text { Solids Target } \\
\text { MRQ }^{(\mathbf{1})} \\
\mu \mathrm{g} / \mathrm{Kg}\end{array}$ & $\begin{array}{c}\text { Supernatant Target } \\
\text { (Density }=1.45 \mathrm{~g} / \mathrm{mL}) \\
\mu \mathrm{g} / \mathrm{L}\end{array}$ \\
\hline $100-00-5$ & 1-Chloro-4-nitrobenzene & $\cdots$ & $\ldots$ \\
\hline $100-25-4$ & 1,4-Dinitrobenzene & 800 & 1200 \\
\hline $100-51-6$ & Benzyl alcohol & $\overline{-\infty}$ & - \\
\hline $106-46-7$ & 1,4-Dichlorobenzene & $\cdots$ & $\ldots$ \\
\hline $108-95-2$ & Phenol & 2100 & 3000 \\
\hline $110-86-1$ & Pyridine & 5300 & 7700 \\
\hline $117-81-7$ & Bis (2-Ethylhexyl)phthalate & $\cdots$ & 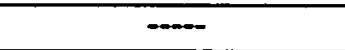 \\
\hline $117-84-0$ & Di-n-octylphthalate & $\cdots$ & $\cdots$ \\
\hline $118-74-1$ & Hexachlorobenzene & 3300 & 4800 \\
\hline $120-82-1$ & $1,2,4$-Trichlorobenzene & $-\cdots$ & $\cdots$ \\
\hline $122-39-4$ & N,N-Diphenylamine & 4300 & 6200 \\
\hline $126-73-8$ & Tributyl phosphate & $-\infty$ & $\cdots$ \\
\hline $128-37-0$ & Butylated & $-\cdots$ & $\cdots$ \\
\hline $1319-77-3$ & Cresol & $\overline{-\cdots}$ & $\cdots$ \\
\hline $1321-64-8$ & Pentachloronaphthalene & $\cdots$ & $\cdots$ \\
\hline $1335-87-1$ & Hexachloronaphthalene & $\cdots$ & $\cdots$ \\
\hline $1335-88-2$ & Tetrachloronaphthalene & $\cdots$ & $\cdots$ \\
\hline $2234-13-1$ & Octachloronaphthalene & $\cdots$ & $-\infty$ \\
\hline $50-32-8$ & Benzo(a)pyrene & 1100 & 1600 \\
\hline $53-70-3$ & Dibenz $[a, h]$ anthracene & 2700 & 3900 \\
\hline $541-73-1$ & 1,3-Dichlorobenzene & - & $=$ \\
\hline $62-75-9$ & $\mathrm{~N}$-nitrosodimethylamine & 800 & 1200 \\
\hline $67-72-1$ & Hexachloroethane & $\overline{--}$ & $\ldots$ \\
\hline $82-68-8$ & Pentachloronitrobenzene (PCNB) & 1600 & 2300 \\
\hline $87-68-3$ & Hexachlorobutadiene & 1900 & 2800 \\
\hline $87-86-5$ & Pentachlorophenol & + & $\cdots$ \\
\hline $88-85-7$ & Dinoseb (Dinoseb) & $-\cdots$ & 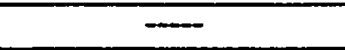 \\
\hline $91-20-3$ & Naphthalene & - & $\cdots$ \\
\hline $92-52-4$ & Biphenyl & $\cdots$ & $\cdots$ \\
\hline $95-50-1$ & 1,2-Dichlorobenzene & 2000 & 2900 \\
\hline $98-86-2$ & Acetophenone & 3200 & 4600 \\
\hline $98-95-3$ & Nitrobenzene & 4700 & 6800 \\
\hline
\end{tabular}

1) $\mathrm{MRQ}=$ Minimum Reportable Quantity. Values provided through BNFL private communication from L. Bostic (BNFL) to G. Klinger (Battelle)

"-..." = No MRQ target provided in communication. 


\subsubsection{QC Evaluation}

Instrument tuning check criteria and 12-hour calibration clock window criteria were met (USEPA CLP 3/90 SOW) for all initial calibration and sample analysis sequences as seen in the " $5 \mathrm{~A}$ " Forms in Appendix B. The initial calibration met the criteria of USEPA SW-846 method $8270 \mathrm{C}$, as seen in the "6B \& 6C" Form in the Appendix B. All four SPCCs met the criteria for minimum response factor, and all 13 calibration CCCs met the maximum RSD criteria. Four of the target analytes of interest have RSDs greater than the recommended $15 \%$. Hexachlorophene had a low response $(0.032)$ factor and high RSD (81.9\%). This compound was measured only because it was included in the commercial $8270 \mathrm{C}$ calibration mixture. The other three targets which had $>15 \%$ RSDs were 2-naphthylamine (18\%), 3,3'-dimethylbenzidine (28\%), and 2,4-dinitrophenol (20\%).

The continuing calibration check standard met the criteria of USEPA SW-846 method $8270 \mathrm{C}$, as seen in the "7A \& 7B" Forms in the Appendix B. All calibration check standards met the SPCC and CCC criteria. However, in comparison of the results for the continuing calibration standards to the initial calibration, several target analytes of interest exceed the recommended $\% \mathrm{D}$ of $15 \%$, but with the exception of Hexachlorophene, all were below $30 \%$.

The internal standards used in this study were 1,4-dichlorobenzene- $D_{4}$, naphthalene- $D_{8}$, acenaphthene- $D_{10}$, phenanthrene- $D_{10}$, chrysene- $D_{12}$, and perylene- $D_{12}$. Each target compound was quantified using the relative response calculated from the most closely eluting internal standard. Acetophenone, nitrobenzene and nitrobenzene- $\mathrm{D}_{5}$ where quantified using the first internal standard. An additional internal standard, pyridine- $\mathrm{D}_{5}$, added to each sample, spike, blank and calibration standard to quantify the earliest eluting peaks. The internal standard area criteria of $-50 \%$ and $+100 \%$ were not met for $A W-101$ supernatant, $A W-101$ supernatant duplicate, $A W-101$ solids sample, AW-101 solids duplicate, AW-101 solids MS, and AN-107 solids sample. In each case the perylene- $D_{12}$ area was low, and in some cases the pyridine- $D_{5}$ was low or not detected. These sample extracts were reanalyzed on March 3,1999 and in four cases the results were the same. In the two cases where the perylene- $D_{12}$ area met the criteria, it was low and followed the trend of the original analysis. All of these extracts appeared slightly cloudy, possibly indicating the presence of suspended water. The low pyridine- $D_{5}$ area is likely the result of the solubility of the pyridine in the water residue, which is slightly acidic following the $\mathrm{pH}$-adjustment. The low perylene- $\mathrm{D}_{12}$ area may be the result of loss of this material due to adsorption by ultra-fine particulate in the sample extracts. These fine particulate may have been produced by ultra-sonication. Ultra-sonication was used during the extraction of AW-101 supernatant, since the $\mathrm{pH}$ adjustment formed a considerable quantity of solids. Ultra-sonication was also used in the extraction of AW-101 and AN-107 solids. Internal standard area data are found on the " $8 \mathrm{~B} \& 8 \mathrm{C}$ " Forms in Appendix B.

The surrogate compounds used were 2-fluorophenol, phenol- $\mathrm{D}_{5}$, nitrobenzene- $\mathrm{D}_{5}, 2$-fluorobiphenyl, 2,4,6-tribromophenol, and terphenyl- $\mathrm{D}_{14}$. Two additional surrogates, 2-chlorophenol- $\mathrm{D}_{4}$ and 1,4-dichorobenzene- $\mathrm{D}_{4}$ were also added to each of the samples, spikes and blanks. These two additional surrogates have only advisory recoveries and were not included in the calibration mixture. However, to provide estimate of concentration and advisory recoveries for these additional surrogates, response factors from previous analyses (i.e., non-BNFL sample analyses) were used.

Evaluation of surrogate recoveries are somewhat difficult in that performance based recovery limits have not been established for this type of sample matrix. In the absences of documented agreement between BNFL, WDOE, and Battelle, the CLP limits for low-level soil samples were used as a guide and are included on the " $2 \mathrm{C} \& 2 \mathrm{D}$ " Forms in Appendix B. 
All phenolic surrogates were poorly recovered from all of the samples, especially the AW-101 samples (supernatant and solids). It is believed this is due to the reaction of these compounds with the sample matrix. One possible reaction of these compounds is the reaction nitrous acid to form nitration products. Nitrous acid is a relatively weak acid, and some was likely formed when the $\mathrm{pH}$ of the samples was adjusted to 6.4 with the addition of phosphoric acid. Nitration products of 2-fluorophenol and 2-chlorophenol- $\mathrm{D}_{4}$ were identified in the $\mathrm{AN}-107$ solids samples TIC data (Table 4.f). These nitration products could be the results of localized areas of low pH generated during the $\mathrm{pH}$-adjustment of the samples; these localized areas of low $\mathrm{pH}$ may result from inadequate mixing during the $\mathrm{pH}$-adjustment. The base neutral surrogate compounds all have acceptable recoveries.

Matrix spike recovery data are found on the " $3 C$ \& 3D" Forms in Appendix B. The phenolic MS compounds exhibited poor recovery in both the AW-101 solids and supernatant MSs. The AW-101 solids MS data exhibits the greatest number of MS failures. However, performance based recovery limits for these spike compounds need to be established to access these spike recoveries. AN-107 MS recoveries for both the supernatant and solids were generally higher than those of AW-101. Dinoseb was poorly recovered in the supernatant, and had higher recovery from the solids spike; the reason for this difference is unknown. The phenolic MS compounds exhibited the opposite trend, with higher recoveries in the supernatant. Some of the inconsistencies noted in phenolic matrix spike recoveries may be attributed to inadequate mixing during the $\mathrm{pH}$ adjustment, resulting from nitration of the phenolic compound.

Several N-nitrosoamines are reported in the AN-107 supernatant and solids data, such as $\mathrm{N}$-nitrosodimethylamine, $\mathrm{N}$-nitrosomethylethylamine, $\mathrm{N}$-nitrosodiethylamine, 1,4-dinitrosopiperazine, etc. Both $\mathrm{AN}-107$ and $\mathrm{AW}-101$ samples contain large quantities of nitrite, $5.8 \%$ and $10 \%$, respectively. Adjustment of the sample $\mathrm{pH}$ to 6.4 with phosphoric acid in the second part of the extraction procedure was performed in order to protonate phenolic compounds so they were extractable in the solvent. As discussed above, this $\mathrm{pH}$ adjustment can produce some nitrous acid. Secondary amines, both aliphatic and aromatic, react with nitrous acid to produce Nnitrosoamines:

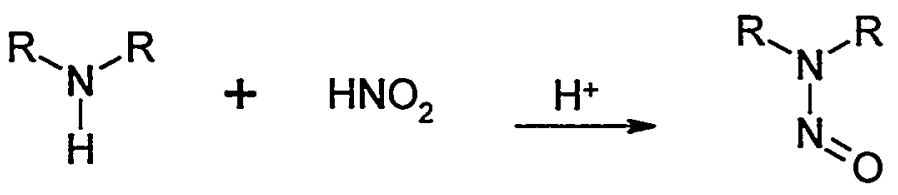

Primary amines react with $\mathrm{HNO}_{2}$ to form diazonium salts, however these tend to be unstable and produce alkenes, alcohols and nitrogen gas. It is not entirely clear as how to interpret the presence of $\mathrm{N}$-nitroso- compounds in the samples. It is conceivable that $\mathrm{N}$-nitroso- compounds may be present in the native tank material; however, it is equally as possible that the $\mathrm{N}$-nitroso compounds are formed during the $\mathrm{pH}$ adjustment conducted as part of the solvent extraction process. Further investigation into N-nitroso- compounds is warranted.

A 1:1 methylene chloride to acetone solution was used to extract the solids samples. This watercutting solvent mixture was used to ensure that all the aqueous-wetted solids particles were wetted with the extraction solvent. Unfortunately, acetone undergoes some reactions in a hydroxide containing solutions, to produce aldol condensation products, notably 4-methyl-4-hydroxy-2pentanone. 
<smiles>CC(=O)CC(C)(C)O</smiles>

Also, 4-Methyl-4-hydroxy-2-pentanone undergoes further dehydration to 4-methyl-3-pentenone. This occurs only in the samples and not the extraction blank.<smiles>CC(=O)CC(C)(C)O</smiles><smiles>CC(=O)C=C(C)C</smiles>

The presence of these aldol condensation products in the AW-101 solids sample data should not be interpreted as a positive indication of their presence in the tank sample.

\subsubsection{Other Observations (or Deviations/Concerns/Issues)}

Test Plan BNFL-TP-29953-5 specified a 32-component SVOA MS solution. Several of these compounds were not included in the matrix spiking solution for various reasons. A commercial source of the various isomers of pentachloronaphthalene, hexachloro-naphthalene, and heptachloronaphthalene could not be found, however octachloro-naphthalene was included in the spiking solution. Equal amounts of 2-, 3-, and 4-methylphenol were used to represent cresol [CAS 1319-77-3]. Some difficulties were encountered in preparing the multi-component spiking solution. The solvent initially used to prepare the spiking solution was methanol, which is completely miscible with the aqueous sample matrix. Unfortunately, several of the spike compounds have limited solubility or are insoluble in methanol. Other solvents were added, and solvent "cocktail" consisting of methanol, methylene chloride, diethyl ether, and acetone was used to dissolve the various compounds. After the additions of each octachloro-naphthalene, pentachloronitrobenzene and dinoseb, crystallization occurred. The relative amounts of the various solvents used were adjusted in order to get the crystals back into solution. A decision was made to limit the number of components in this spiking solution in order to avoid further problems with recrystallizations from the solution. The samples were spiked with 16 of the analytes specified in the test plan, plus an additional seven that were part of the commercially available acid and base/neutral matrix spiking solutions.

As stated earlier, SVOA results for nonane in the TIC data should not be interpreted as an indication of a true nonane concentration or the presence of nonane in the samples. nonane was used as the solvent in the dioxins and furans spiking stock solutions that were added to all blanks, spikes, and samples prior to the single extraction processing for SVOA, PCB/pesticides, and dioxin and furans. The VOA results should be used for the determination of the nonane concentration in the samples.

The precision between duplicates for both the AW-101 and AN-107 supernatant and solids was poorer than expected. For example, a sample may be reported at 3 to 5 times the $M D L$ and the duplicate shows a non-detect. As was noted in the preparation sections, reasonably small sample sizes were used for the extractions, and an attempt was made to use a common extraction for the SVOA, PCB/Pesticides, and dioxins and furans due to the limited quantity of sample available and the complexity of processing the extractions in the SAL. 


\subsection{Polychlorinated Biphenyls/Pesticides Analysis}

\subsection{Introduction}

The supernatants and solids samples were prepared in the SAL by the procedure outlined in Section 4.2. Following extraction, $5 \mathrm{~mL}$ of the of the resulting methylene chloride residue were transferred under $\mathrm{COC}$ to the laboratory for analysis of $\mathrm{PCB} /$ pesticides and dioxins and furans. Of this $5 \mathrm{~mL}$ of extraction residue, $2 \mathrm{~mL}$ were used for the $\mathrm{PCB} /$ pesticides analysis by gas chromatography/electron capture detection (GC/ECD), and the remaining $3 \mathrm{~mL}$ were used for dioxins and furans analysis.

\subsection{Sample Preparation}

Additional cleanup of the 2-mL aliquot of the extract residue was performed following exchange into hexane. When residues to be analyzed by this method are splits from semi-volatile extractions or are samples suspected of containing substantial interferences, then additional cleanup is performed. These are typically columns employing silica gel, alumina, or Florisil. In this case, Florisil (SW-846 Method 3620) cleanups were used. Florisil cleanup was selected because of the ease of use. Batch to batch variation in the composition of the Florisil or overloading the column may cause a change in the distribution patterns of the organochlorine pesticides. The lot number of cartridges used for this cleanup was evaluated for recovery of pesticides and PCBs and removal of unwanted polar materials before processing samples. The resulting Florisil cleaned residues were concentrated to $1 \mathrm{~mL}$ using a micro-Snyder Kuderna-Danish (K-D) apparatus.

\subsection{Instrumentation}

The instrumentation used for the analysis of pesticides and PCBs consists of a gas chromatograph equipped with two electron capture detectors (ECD). The analytical instrumentation is identified in Table 5.a. Both of the detectors were operated at $300^{\circ} \mathrm{C}$. Injections were made on-column onto a 5 $\mathrm{m}$ fused silica retention gap, which was split between two analytical columns: a) $0.32 \mathrm{~mm} \times 30 \mathrm{~m}$ DB-17 (0.25 $\mu \mathrm{m}$ phase) and b) $0.32 \mathrm{~mm}$ X $30 \mathrm{~m}$ DB-1701 (0.25 $\mu \mathrm{m}$ film thickness).

Table 5.a. PCB/Pesticides Analysis Instrumentation

\begin{tabular}{|l|l|c|c|}
\hline System/Instrument & Manufacturer & Model Number & M\&TE ${ }^{(1)}$ Number \\
\hline \hline Gas Chromatograph & Hewlett-Packard & 5890 & WD 11127 \\
\hline
\end{tabular}

1) Measuring and Test Equipment

\subsection{Analysis Results}

Polychlorinated biphenyl and pesticide results are presented in Tables 5.b and 5.c. As seen in Table 5.d, the BNFL target MRQs have been met for both the wet centrifuged solids and supernatant (after adjusting for density). 
Table 5.b. AW-101 PCB/Pesticides Results

\begin{tabular}{|c|c|c|c|c|c|c|c|c|c|}
\hline \multirow{3}{*}{\multicolumn{2}{|c|}{\begin{tabular}{||rr|} 
& Tank Material \\
& Sample ID \\
CAS \# & \\
& Units
\end{tabular}}} & \multicolumn{4}{|c|}{ AW-101 Supernatant } & \multicolumn{4}{|c|}{ AW-101 Wet Centrifuged Solids } \\
\hline & & \multirow{2}{*}{$\begin{array}{l}\mathrm{MDL} \\
\mu \mathrm{g} / \mathrm{L}\end{array}$} & \multirow{2}{*}{$\begin{array}{c}\begin{array}{c}99- \\
\text { Proc Blk }\end{array} \\
\mu \mathrm{g} / \mathrm{L}\end{array}$} & \multirow{2}{*}{$\begin{array}{l}99-0648 \\
\text { Sample } \\
\mu g / L\end{array}$} & \multirow{2}{*}{\begin{tabular}{|c|}
$\begin{array}{c}99-0648 \mathrm{~d} \\
\text { Duplicate }\end{array}$ \\
$\mu \mathrm{g} / \mathrm{L}$ \\
\end{tabular}} & \multirow{2}{*}{$\begin{array}{c}\text { MDL } \\
\mu \mathrm{g} / \mathrm{Kg}\end{array}$} & \multirow{2}{*}{$\begin{array}{c}99- \\
\text { Proc Blk } \\
\mu \mathrm{g} / \mathrm{Kg}\end{array}$} & \multirow{2}{*}{$\begin{array}{l}\overline{99-0650} \\
\text { Sample } \\
\mu \mathrm{g} / \mathrm{Kg}\end{array}$} & \multirow{2}{*}{\begin{tabular}{|c|}
$\begin{array}{c}99-0650 \mathrm{~d} \\
\text { Duplicate }\end{array}$ \\
$\mu \mathrm{g} / \mathrm{Kg}$ \\
\end{tabular}} \\
\hline & & & & & & & & & \\
\hline \multicolumn{10}{|c|}{ BNFL Pesticide Analyte List } \\
\hline $309-00-2$ & Aldrin & 1.0 & $\bar{U}$ & $\bar{U}$ & $\overline{\mathrm{U}}$ & 10 & $\bar{U}$ & $\overline{\mathrm{U}}$ & $\bar{U}$ \\
\hline $319-84-6$ & alpha-BHC & 1.0 & $\overline{\mathrm{U}}$ & $\overline{\mathrm{U}}$ & $\bar{U}$ & 10 & $\bar{U}$ & 18.1 & $\bar{U}$ \\
\hline $319-85-7$ & beta-BHC & 1.0 & $\overline{\mathrm{U}}$ & $\overline{\mathrm{U}}$ & $\bar{U}$ & 10 & $\bar{U}$ & $\mathrm{U}$ & $\bar{U}$ \\
\hline $465-73-6$ & Isodrin & 2.0 & $\overline{\mathrm{U}}$ & $\overline{\mathrm{U}}$ & $\bar{U}$ & 20 & $\bar{U}$ & $\overline{\mathrm{U}}$ & $\bar{U}$ \\
\hline $50-29-3$ & $4,4^{\prime}-\mathrm{DDT}$ & 2.0 & $\bar{U}$ & $\overline{\mathrm{U}}$ & $\overline{\mathrm{U}}$ & 20 & $\bar{U}$ & $\overline{\mathrm{U}}$ & $\overline{\mathrm{U}}$ \\
\hline $58-89-9$ & gamma-BHC & 1.0 & $\bar{U}$ & $\overline{\mathrm{U}}$ & $\overline{\mathrm{U}}$ & 10 & $\overline{\mathrm{U}}$ & $\bar{U}$ & $\overline{\mathrm{U}}$ \\
\hline $60-57-1$ & Dieldrin & 2.0 & $\bar{U}$ & $\bar{U}$ & $\bar{U}$ & 20 & $\overline{\mathrm{U}}$ & $\overline{\mathrm{U}}$ & $\overline{\mathrm{U}}$ \\
\hline $72-20-8$ & Endrin & 2.0 & $\overline{\mathrm{U}}$ & $\bar{U}$ & $\overline{\mathrm{U}}$ & 20 & $\bar{U}$ & $\bar{U}$ & $\overline{\mathrm{U}}$ \\
\hline $72-54-8$ & $4,4^{\prime}-\mathrm{DDD}$ & 2.0 & $\overline{\mathrm{U}}$ & $\overline{\mathrm{U}}$ & $\overline{\mathrm{U}}$ & 20 & $\overline{\mathrm{U}}$ & $\overline{\mathrm{U}}$ & $\overline{\mathrm{U}}$ \\
\hline $76-44-8$ & Heptachlor & 1.0 & $\overline{\mathrm{U}}$ & $\overline{\mathrm{U}}$ & 6.5 & 10 & $\overline{\mathrm{U}}$ & 73.4 & $\bar{U}$ \\
\hline $8001-35-2$ & Toxaphene & 2.1 & $\bar{U}$ & $\overline{\mathrm{U}}$ & $\overline{\mathrm{U}}$ & 36 & $\bar{U}$ & $\overline{\mathrm{U}}$ & $\overline{\mathrm{U}}$ \\
\hline \multicolumn{10}{|c|}{ SW-846 8081A Pesticide Analyte List } \\
\hline $1024-57-3$ & Heptachlor & 1.0 & $\bar{U}$ & $\bar{U}$ & $\bar{U}$ & 10 & $\bar{U}$ & $\overline{\mathrm{U}}$ & $\overline{\mathrm{U}}$ \\
\hline $1031-07-8$ & Endosulfan & 2.0 & $\overline{\mathrm{U}}$ & $\overline{\mathrm{U}}$ & $\overline{\mathrm{U}}$ & 20 & $\overline{\mathbf{U}}$ & $\bar{U}$ & $\bar{U}$ \\
\hline $319-86-8$ & delta-BHC & 1.0 & $\overline{\mathrm{U}}$ & $\overline{\mathrm{U}}$ & $\overline{\mathrm{U}}$ & 10 & $\overline{\mathrm{U}}$ & $\bar{U}$ & 76.9 \\
\hline $33213-65-$ & Endosulfan II & 2.0 & $\bar{U}$ & $\overline{\mathrm{U}}$ & $\overline{\mathrm{U}}$ & 20 & $\overline{\mathbf{U}}$ & $\overline{\mathrm{U}}$ & $\mathrm{U}$ \\
\hline $5103-74-2$ & gamma- & 1.0 & $\overline{\mathrm{U}}$ & $\bar{U}$ & $\overline{\mathrm{U}}$ & 10 & $\overline{\mathrm{U}}$ & $\overline{\mathrm{U}}$ & $\overline{\mathrm{U}}$ \\
\hline $5103-71-9$ & alpha-Chlordane & 1.0 & $\mathrm{U}$ & $\bar{U}$ & $\overline{\mathrm{U}}$ & 10 & $\overline{\mathrm{U}}$ & $\overline{\mathrm{U}}$ & $\overline{\mathrm{U}}$ \\
\hline 53494-70- & Endrin Ketone & 2.0 & $\overline{\mathrm{U}}$ & $\overline{\mathrm{U}}$ & $\bar{U}$ & 20 & $\overline{\mathrm{U}}$ & $\overline{\mathrm{U}}$ & $\overline{\mathrm{U}}$ \\
\hline $72-43-5$ & Methoxychlor & 10 & $\mathrm{U}$ & $\overline{\mathrm{U}}$ & $\overline{\mathrm{U}}$ & 100 & $\overline{\mathrm{U}}$ & $\overline{\mathrm{U}}$ & $\overline{\mathrm{U}}$ \\
\hline $72-55-9$ & $4,4^{\prime}-\mathrm{DDE}$ & 2.0 & $\bar{U}$ & $\bar{U}$ & $\overline{\mathrm{U}}$ & 20 & $\overline{\mathrm{U}}$ & $\bar{U}$ & $\overline{\mathrm{U}}$ \\
\hline $7421-93-4$ & Endrin Aldehyde & 2.0 & $\mathrm{U}$ & $\overline{\mathrm{U}}$ & $\bar{U}$ & 20 & $\overline{\mathrm{U}}$ & $\overline{\mathrm{U}}$ & $\overline{\mathrm{U}}$ \\
\hline $959-98-8$ & Endosulfan I & 1.0 & $\bar{U}$ & $\overline{\mathrm{U}}$ & $\overline{\mathrm{U}}$ & 10 & $\overline{\mathrm{U}}$ & $\overline{\mathrm{U}}$ & $\overline{\mathrm{U}}$ \\
\hline \multicolumn{10}{|c|}{ BNFL Polychlorinated Biphenyl's Analyte List } \\
\hline 12674-11- & AROCLOR 1016 & 2.1 & $\mathrm{U}$ & $\bar{U}$ & $\bar{U}$ & 36 & $\overline{\mathbf{U}}$ & $\overline{\mathrm{U}}$ & $\bar{U}$ \\
\hline $11104-28-$ & AROCLOR 1221 & 2.1 & $\overline{\mathrm{U}}$ & $\overline{\mathrm{U}}$ & $\overline{\mathrm{U}}$ & 36 & $\overline{\mathrm{U}}$ & $\bar{U}$ & $\overline{\mathrm{U}}$ \\
\hline $11141-16-$ & AROCLOR 1232 & 2.1 & $\overline{\mathrm{U}}$ & $\overline{\mathrm{U}}$ & $\overline{\mathrm{U}}$ & 36 & $\overline{\mathrm{U}}$ & $\overline{\mathrm{U}}$ & $\overline{\mathrm{U}}$ \\
\hline $53469-21-$ & AROCLOR 1242 & 2.1 & $\overline{\mathrm{U}}$ & $\overline{\mathrm{U}}$ & $\bar{U}$ & 36 & $\overline{\mathrm{U}}$ & $\overline{\mathrm{U}}$ & $\overline{\mathrm{U}}$ \\
\hline $12672-29-$ & AROCLOR 1248 & 2.1 & $\bar{U}$ & $\overline{\mathrm{U}}$ & $\bar{U}$ & 36 & $\overline{\mathrm{U}}$ & $\overline{\mathrm{U}}$ & $\overline{\mathrm{U}}$ \\
\hline $11097-69-$ & AROCLOR 1254 & 2.1 & $\overline{\mathrm{U}}$ & $\overline{\mathrm{U}}$ & $\overline{\mathrm{U}}$ & 36 & $\overline{\mathrm{U}}$ & $23 \mathrm{~J}$ & $\overline{\mathrm{U}}$ \\
\hline \multirow[t]{4}{*}{$11096-82-$} & AROCLOR 1260 & 2.1 & $\bar{U}$ & $\bar{U}$ & $\overline{\mathrm{U}}$ & 36 & $\overline{\mathrm{U}}$ & $\bar{U}$ & $\overline{\mathrm{U}}$ \\
\hline & & & \% Rec & \% Rec & \% Rec & & \% Rec & \% Rec & \% Rec \\
\hline & TCX (surrogate) & & 11 & 45 & 95 & & 9 & 73 & 0 \\
\hline & $\overline{\mathrm{DCB} \text { (surrogate) }}$ & & 24 & 8.8 & 20 & & 15 & 20 & 19 \\
\hline
\end{tabular}

U flag = Not detected; results less than MDL

$\mathrm{J}$ flag = Detected and quantified, but result is less than MDL. 
Table 5.c. AN-107 PCB/Pesticides Results

\begin{tabular}{|c|c|c|c|c|c|c|c|c|c|}
\hline \multirow[b]{3}{*}{ CAS \# } & \multirow{2}{*}{$\begin{array}{r}\text { Tank Material } \\
\text { Sample ID }\end{array}$} & \multicolumn{4}{|c|}{ AN-107 Supernatant } & \multicolumn{4}{|c|}{ AN-107 Wet Centrifuged Solids } \\
\hline & & \multirow{2}{*}{$\begin{array}{l}\mathrm{MDL} \\
\mu \mathrm{g} / \mathrm{L}\end{array}$} & $\begin{array}{c}99- \\
\text { Proc Blk }\end{array}$ & $\begin{array}{l}\text { 99-0649 } \\
\text { Sample }\end{array}$ & $\begin{array}{l}99-0649 d \\
\text { Duplicate }\end{array}$ & \multirow{2}{*}{$\frac{\mathrm{MDL}}{\mu \sigma / \mathrm{Ka}}$} & \multirow{2}{*}{$\begin{array}{c}99- \\
\text { Proc Blk } \\
\mu \mathrm{g} / \mathrm{Kg} \\
\end{array}$} & \multirow{2}{*}{$\begin{array}{l}\text { trifuged } \\
99-0651 \\
\text { Sample } \\
\mu \mathrm{g} / \mathrm{Kg}\end{array}$} & \multirow{2}{*}{\begin{tabular}{|c|}
$\begin{array}{c}99-0651 d \\
\text { Duplicate }\end{array}$ \\
$\mu \mathrm{g} / \mathrm{Kg}$ \\
\end{tabular}} \\
\hline & Units & & $\mu \mathrm{g} / \mathrm{L}$ & $\mu \mathrm{g} / \mathrm{L}$ & $\mu \mathrm{g} / \mathbf{L}$ & & & & \\
\hline \multicolumn{10}{|c|}{ BNFL Pesticide Analyte List } \\
\hline $309-00-2$ & Aldrin & 1.0 & $\overline{\mathrm{U}}$ & 1.2 & 3.7 & 10 & $\bar{U}$ & $\overline{\mathrm{U}}$ & $\overline{\mathrm{U}}$ \\
\hline $319-84-6$ & alpha-BHC & 1.0 & $\overline{\mathrm{U}}$ & $\bar{U}$ & $\mathrm{U}$ & 10 & $\overline{\mathrm{U}}$ & 11.8 & 35.1 \\
\hline $319-85-7$ & beta-BHC & 1.0 & $\bar{U}$ & $\overline{\mathrm{U}}$ & $\overline{\mathrm{U}}$ & 10 & $\overline{\mathrm{U}}$ & $\mathrm{U}$ & $\overline{\mathrm{U}}$ \\
\hline $465-73-6$ & Isodrin & 2.0 & $\bar{U}$ & $\overline{\mathrm{U}}$ & $\overline{\mathrm{U}}$ & 20 & $\bar{U}$ & $\overline{\mathrm{U}}$ & $\overline{\mathrm{U}}$ \\
\hline $50-29-3$ & $4,4^{\prime}$-DDT & 2.0 & $\bar{U}$ & $\overline{\mathrm{U}}$ & $\overline{\mathrm{U}}$ & 20 & $\bar{U}$ & $\overline{\mathrm{U}}$ & $\bar{U}$ \\
\hline $58-89-9$ & gamma-BHC & 1.0 & $\overline{\mathrm{U}}$ & $\overline{\mathrm{U}}$ & $\overline{\mathrm{U}}$ & 10 & $\bar{U}$ & $\overline{\mathrm{U}}$ & $\overline{\mathrm{U}}$ \\
\hline $60-57-1$ & Dieldrin & 2.0 & $\overline{\mathrm{U}}$ & $\overline{\mathrm{U}}$ & $\overline{\mathrm{U}}$ & 20 & $\overline{\mathrm{U}}$ & $\overline{\mathrm{U}}$ & $\overline{\mathrm{U}}$ \\
\hline $72-20-8$ & Endrin & 2.0 & $\overline{\mathrm{U}}$ & $\overline{\mathrm{U}}$ & $\overline{\mathrm{U}}$ & 20 & $\overline{\mathrm{U}}$ & $\overline{\mathrm{U}}$ & $\overline{\mathrm{U}}$ \\
\hline $72-54-8$ & $4,4^{\prime}-\mathrm{DDD}$ & 2.0 & $\overline{\mathrm{U}}$ & $\overline{\mathrm{U}}$ & $\overline{\mathrm{U}}$ & 20 & $\overline{\mathrm{U}}$ & $\overline{\mathrm{U}}$ & $\overline{\mathrm{U}}$ \\
\hline $76-44-8$ & Heptachlor & 1.0 & $\overline{\mathrm{U}}$ & $\bar{U}$ & $\overline{\mathrm{U}}$ & 10 & $\overline{\mathrm{U}}$ & $\overline{\mathrm{U}}$ & 18.4 \\
\hline $8001-35-2$ & Toxaphene & 2.3 & $\overline{\mathrm{U}}$ & $\overline{\mathrm{U}}$ & $\overline{\mathrm{U}}$ & 33 & $\overline{\mathrm{U}}$ & $\overline{\mathrm{U}}$ & $\bar{U}$ \\
\hline \multicolumn{10}{|c|}{ SW-846 8081A Pesticide Analyte List } \\
\hline $1024-57-3$ & Heptachlor & 1.0 & $\overline{\mathrm{U}}$ & $\bar{U}$ & $\overline{\mathrm{U}}$ & 10 & $\overline{\mathrm{U}}$ & $\bar{U}$ & $\overline{\mathrm{U}}$ \\
\hline $1031-07-8$ & Endosulfan & 2.0 & $\overline{\mathrm{U}}$ & $\overline{\mathrm{U}}$ & $\overline{\mathrm{U}}$ & 20 & $\bar{U}$ & $\overline{\mathrm{U}}$ & $\overline{\mathbf{U}}$ \\
\hline $319-86-8$ & delta-BHC & 1.0 & $\overline{\mathrm{U}}$ & $\overline{\mathrm{U}}$ & $\overline{\mathrm{U}}$ & 10 & $\overline{\mathrm{U}}$ & $\overline{\mathrm{U}}$ & $\overline{\mathrm{U}}$ \\
\hline $33213-65-$ & Endosulfan II & 2.0 & $\overline{\mathrm{U}}$ & $\overline{\mathrm{U}}$ & $\overline{\mathrm{U}}$ & 20 & $\bar{U}$ & $\bar{U}$ & $\overline{\mathrm{U}}$ \\
\hline $5103-74-2$ & gamma- & 1.0 & $\overline{\mathrm{U}}$ & $\overline{\mathrm{U}}$ & $\overline{\mathrm{U}}$ & 10 & $\overline{\mathrm{U}}$ & $\overline{\mathrm{U}}$ & $\overline{\mathrm{U}}$ \\
\hline $5103-71-9$ & alpha-Chlordane & 1.0 & $\overline{\mathrm{U}}$ & $\bar{U}$ & $\overline{\mathrm{U}}$ & 10 & $\overline{\mathrm{U}}$ & $\overline{\mathrm{U}}$ & $\bar{U}$ \\
\hline $53494-70-$ & Endrin Ketone & $\overline{2.0}$ & $\overline{\mathrm{U}}$ & $\bar{U}$ & $\overline{\mathrm{U}}$ & 20 & $\overline{\mathrm{U}}$ & $\overline{\mathrm{U}}$ & $\overline{\mathrm{U}}$ \\
\hline $72-43-5$ & Methoxychlor & 10 & $\overline{\mathrm{U}}$ & $\bar{U}$ & $\overline{\mathrm{U}}$ & 100 & $\overline{\mathrm{U}}$ & $\overline{\mathrm{U}}$ & $\overline{\mathrm{U}}$ \\
\hline $72-55-9$ & $4,4^{4}-\mathrm{DDE}$ & 1.0 & $\bar{U}$ & $\mathrm{U}$ & $\overline{\mathrm{U}}$ & 20 & $\mathrm{U}$ & $\overline{\mathrm{U}}$ & $\mathrm{U}$ \\
\hline $7421-93-4$ & Endrin Aldehyde & 1.0 & $\overline{\mathrm{U}}$ & $\overline{\mathrm{U}}$ & $\overline{\mathrm{U}}$ & 20 & $\bar{U}$ & $\overline{\mathrm{U}}$ & $\overline{\mathrm{U}}$ \\
\hline $959-98-8$ & Endosulfan I & 1.0 & $\mathrm{U}$ & $\bar{U}$ & $\overline{\mathrm{U}}$ & 10 & $\bar{U}$ & $\overline{\mathrm{U}}$ & $\overline{\mathrm{U}}$ \\
\hline \multicolumn{10}{|c|}{ BNFL Polychlorinated Biphenyl's Analyte List } \\
\hline $12674-11-$ & AROCLOR 1016 & 2.3 & $\mathrm{U}$ & $\overline{\mathrm{U}}$ & $\overline{\mathrm{U}}$ & 33 & $\bar{U}$ & $\bar{U}$ & $\overline{\mathrm{U}}$ \\
\hline $11104-28-$ & AROCLOR 1221 & $\overline{2.3}$ & $\overline{\mathrm{U}}$ & $\overline{\mathrm{U}}$ & $\overline{\mathrm{U}}$ & $\overline{33}$ & $\overline{\mathrm{U}}$ & $\overline{\mathrm{U}}$ & $\overline{\mathrm{U}}$ \\
\hline $11141-16$ & AROCLOR 1232 & 2.3 & $\overline{\mathrm{U}}$ & $\bar{U}$ & $\overline{\mathrm{U}}$ & 33 & $\overline{\mathrm{U}}$ & $\overline{\mathrm{U}}$ & $\overline{\mathrm{U}}$ \\
\hline 53469-21- & AROCLOR 1242 & 2.3 & $\overline{\mathrm{U}}$ & $\overline{\mathrm{U}}$ & $\bar{U}$ & 33 & $\overline{\mathrm{U}}$ & $\overline{\mathrm{U}}$ & $\overline{\mathrm{U}}$ \\
\hline $12672-29-$ & AROCLOR 1248 & 2.3 & $\overline{\mathrm{U}}$ & $\overline{\mathrm{U}}$ & $\overline{\mathrm{U}}$ & 33 & $\overline{\mathrm{U}}$ & $\overline{\mathrm{U}}$ & $\overline{\mathrm{U}}$ \\
\hline 11097-69- & AROCLOR 1254 & 2.3 & $0.8 \mathrm{JB}$ & $1.7 \mathrm{JB}$ & $1.8 \mathrm{JB}$ & 33 & $\overline{\mathrm{U}}$ & $\overline{\mathrm{U}}$ & $17 \mathrm{~J}$ \\
\hline \multirow[t]{4}{*}{ 11096-82- } & AROCLOR 1260 & 2.3 & $\mathrm{U}$ & $\mathrm{U}$ & $\mathrm{U}$ & 33 & $\overline{\mathrm{U}}$ & $\overline{\mathrm{U}}$ & $\mathrm{U}$ \\
\hline & & & $\%$ Rec & $\% \operatorname{Rec}$ & $\%$ Rec & & $\% \operatorname{Rec}$ & \% Rec & \% Rec \\
\hline & TCX (surrogate) & & 44 & 17 & 40 & & 38 & 48 & 79 \\
\hline & $\overline{\mathrm{DCB}}$ (surrogate) & & 72 & 54 & 55 & & 65 & 95 & 80 \\
\hline
\end{tabular}


Table 5.d. Target PCB/Pesticides Minimum Reportable Quantities

\begin{tabular}{|c|c|c|c|}
\hline CAS \# & Compound & $\begin{array}{c}\text { Solids Target } \\
\text { MRQ }^{(\mathbf{1})} \\
\mu \mathrm{g} / \mathbf{K g}\end{array}$ & $\begin{array}{c}\text { Supernatant Target MRQ } \\
\text { (Density }=1.45 \mathrm{~g} / \mathrm{mL}) \\
\mu \mathrm{g} / \mathrm{L}\end{array}$ \\
\hline All & Polychlorinated Biphenyls & 3300 & 4800 \\
\hline $309-00-2$ & Aldrin & 22 & 32 \\
\hline $319-84-6$ & alpha-BHC & 22 & 32 \\
\hline $319-85-7$ & beta-BHC & 22 & 32 \\
\hline $465-73-6$ & Isodrin & 22 & 32 \\
\hline $50-29-3$ & $4,4^{\prime}-\mathrm{DDT}$ & $\overline{--}$ & -- \\
\hline $58-89-9$ & gamma-BHC & $\cdots$ & - \\
\hline $60-57-1$ & Dieldrin & 43 & 62 \\
\hline $72-20-8$ & Endrin & 43 & 62 \\
\hline $72-54-8$ & $4,4^{\prime}-\mathrm{DDD}$ & --- & - \\
\hline $76-44-8$ & Heptachlor & 22 & 32 \\
\hline $8001-35-2$ & Toxaphene & 900 & 1300 \\
\hline
\end{tabular}

\subsection{QC Evaluation}

Surrogate and spike compounds were added to the supernatants and solids at levels that did not take into account adequately the residue splitting operation. Therefore, the amounts of these compounds were present at levels that were below the quantitation limits and resulted in erratic recoveries. If these materials were not known to be present as spikes, quantitation would not likely have been performed.

Table 5.e. AW-101 and AN-107 Supernatant -- Spike Recoveries

\begin{tabular}{|c|c|c|c|c|c|c|}
\hline \multirow[b]{4}{*}{ CAS \# } & \multirow[t]{2}{*}{$\begin{array}{r}\text { Tank Material } \\
\text { Sample ID }\end{array}$} & \multicolumn{2}{|c|}{$\begin{array}{c}\mathbf{A W}-101 \\
99-0648\end{array}$} & \multicolumn{2}{|c|}{$\begin{array}{l}\text { AN-107 } \\
99-0649\end{array}$} & \multirow[b]{2}{*}{ LCS } \\
\hline & & MS & MSD & MS & MSD & \\
\hline & Units & $\% \operatorname{Rec}(\mu \mathrm{g} / \mathrm{L})$ & $\% \operatorname{Rec}(\mu \mathrm{g} / \mathrm{L})$ & $\% \operatorname{Rec}(\mu \mathrm{g} / \mathrm{L})$ & $\% \operatorname{Rec}(\mu \mathrm{g} / \mathrm{L})$ & $\% \operatorname{Rec}(\mu \mathrm{g} / \mathrm{L})$ \\
\hline & $M D L$ & 2.9 & 3.3 & 4.8 & 4.8 & 0.3 \\
\hline $8001-35-2$ & Toxaphene & $\mathrm{n} / \mathrm{a}(\mathrm{U})$ & $\mathrm{n} / \mathrm{a}(\mathrm{U})$ & $\mathrm{n} / \mathrm{a}(\mathrm{U})$ & $\mathrm{n} / \mathrm{a}(\mathrm{U})$ & $\mathrm{n} / \mathrm{a}(\mathrm{U})$ \\
\hline $12674-11-$ & AROCLOR 1016 & $\mathrm{n} / \mathrm{a}(\mathrm{U})$ & $\mathrm{n} / \mathrm{a}(\mathrm{U})$ & $\mathrm{n} / \mathrm{a}(\mathrm{U})$ & $\mathrm{n} / \mathrm{a}(\mathrm{U})$ & $\mathrm{n} / \mathrm{a}(\mathrm{U})$ \\
\hline 11104-28- & AROCLOR 1221 & $\mathrm{n} / \mathrm{a}(\mathrm{U})$ & $\mathrm{n} / \mathrm{a}(\mathrm{U})$ & $\mathrm{n} / \mathrm{a}(\mathrm{U})$ & $\mathrm{n} / \mathrm{a}(\mathrm{U})$ & $\mathrm{n} / \mathrm{a}(\mathrm{U})$ \\
\hline 11141-16- & AROCLOR 1232 & $\mathrm{n} / \mathrm{a}(\mathrm{U})$ & $\mathrm{n} / \mathrm{a}(\mathrm{U})$ & $\mathrm{n} / \mathrm{a}(\mathrm{U})$ & $\mathrm{n} / \mathrm{a}(\mathrm{U})$ & $\mathrm{n} / \mathrm{a}(\mathrm{U})$ \\
\hline $53469-21-$ & AROCLOR 1242 & $\mathrm{n} / \mathrm{a}(\mathrm{U})$ & $\mathrm{n} / \mathrm{a}(\mathrm{U})$ & $\mathrm{n} / \mathrm{a}(\mathrm{U})$ & $\mathrm{n} / \mathrm{a}(\mathrm{U})$ & $\mathrm{n} / \mathrm{a}(\mathrm{U})$ \\
\hline $12672-29-$ & AROCLOR 1248 & $\mathrm{n} / \mathrm{a}(\mathrm{U})$ & $\mathrm{n} / \mathrm{a}(\mathrm{U})$ & $\mathrm{n} / \mathrm{a}(\mathrm{U})$ & $n / a(\bar{U})$ & $\mathrm{n} / \mathrm{a}(\mathrm{U})$ \\
\hline $11097-69-$ & AROCLOR 1254 & $77(1.5 \mathrm{~J})$ & $129(2.8 \mathrm{~J})$ & $139(6.2)$ & $145(6.4)$ & $97(0.97)$ \\
\hline $11096-82-$ & AROCLOR 1260 & $\mathrm{n} / \mathrm{a}(\mathrm{U})$ & $\mathrm{n} / \mathrm{a}(\mathrm{U})$ & $\mathrm{n} / \mathrm{a}(\mathrm{U})$ & $\mathrm{n} / \mathrm{a}(\mathrm{U})$ & $\mathrm{n} / \mathrm{a}(\mathrm{U})$ \\
\hline & & \% Rec & \% Rec & $\% \operatorname{Rec}$ & \% Rec & \% Rec \\
\hline & TCX (surrogate) & 206 & 495 & 0 & 41 & 76 \\
\hline & DCB (surrogate) & 73000 & 100 & 0 & 71 & 114 \\
\hline
\end{tabular}

$\mathrm{n} / \mathrm{a}=$ Not applicable, since only AROCLOR 1254 spike added to the MS and MSD.

$\mathrm{U}$ flag = Not detected; results less than MDL

$\mathrm{J}$ flag $=$ Detected and quantified, but result is less than MDL. 
Table 5.f. AW-101 and AN-107 Wet Centrifuged Solids - Spike Recoveries

\begin{tabular}{|c|c|c|c|c|c|}
\hline \multirow[b]{4}{*}{ CAS \# } & $\begin{array}{r}\text { Tank Material } \\
\text { Sample ID }\end{array}$ & \multicolumn{2}{|c|}{$\begin{array}{l}\text { AW-101 } \\
99-0650\end{array}$} & \multicolumn{2}{|c|}{$\frac{\mathrm{AN}-107}{99-0651}$} \\
\hline & & MS & MSD & MS & MSD \\
\hline & Units & $\% \operatorname{Rec}(\mu \mathrm{g} / \mathrm{Kg})$ & $\% \operatorname{Rec}(\mu \mathrm{g} / \mathrm{Kg})$ & $\% \operatorname{Rec}(\mu \mathrm{g} / \mathrm{Kg})$ & $\% \operatorname{Rec}(\mu \mathrm{g} / \mathrm{Kg})$ \\
\hline & $M D L$ & 54 & 59 & 55 & 46 \\
\hline $8001-35-2$ & Toxaphene & $\mathrm{n} / \mathrm{a}(U)$ & $\mathrm{n} / \mathrm{a}(\mathrm{U})$ & $\mathrm{n} / \mathrm{a}(\mathrm{U})$ & $\mathrm{n} / \mathrm{a}(\mathrm{U})$ \\
\hline $12674-11-2$ & AROCLOR 1016 & $\mathrm{n} / \mathrm{a}(\mathrm{U})$ & $\mathrm{n} / \mathrm{a}(\mathrm{U})$ & $\mathrm{n} / \mathrm{a}(\mathrm{U})$ & $\mathrm{n} / \mathrm{a}(\mathrm{U})$ \\
\hline $11104-28-2$ & AROCLOR 1221 & $\mathrm{n} / \mathrm{a}(\mathrm{U})$ & $\mathrm{n} / \mathrm{a}(\mathrm{U})$ & $\mathrm{n} / \mathrm{a}(\bar{U})$ & $\mathrm{n} / \mathrm{a}(\mathrm{U})$ \\
\hline $11141-16-5$ & AROCLOR 1232 & $\mathrm{n} / \mathrm{a}(\mathrm{U})$ & $\mathrm{n} / \mathrm{a}(\mathrm{U})$ & $\mathrm{n} / \mathrm{a}(\mathrm{U})$ & $\mathrm{n} / \mathrm{a}(\mathrm{U})$ \\
\hline $53469-21-6$ & AROCLOR 1242 & $\mathrm{n} / \mathrm{a}(\mathrm{U})$ & $\mathrm{n} / \mathrm{a}(\mathrm{U})$ & $\mathrm{n} / \mathrm{a}(\mathrm{U})$ & $\mathrm{n} / \mathrm{a}(\mathrm{U})$ \\
\hline $12672-29-6$ & AROCLOR 1248 & $\mathrm{n} / \mathrm{a}(\mathrm{U})$ & $\mathrm{n} / \mathrm{a}(\mathrm{U})$ & $\mathbf{n} / \mathbf{a}(\mathrm{U})$ & $\mathrm{n} / \mathrm{a}(\mathrm{U})$ \\
\hline $11097-69-1$ & AROCLOR 1254 & $42(38 \mathrm{~J})$ & $87(57 \mathrm{~J})$ & $46(33 \mathrm{~J})$ & $38(28 \mathrm{~J})$ \\
\hline $11096-82-5$ & AROCLOR 1260 & $\mathrm{n} / \mathrm{a}(\mathrm{U})$ & $\mathrm{n} / \mathrm{a}(\mathrm{U})$ & n/a (U) & $\mathrm{n} / \mathrm{a}(\mathrm{U})$ \\
\hline & & $\% \operatorname{Rec}$ & \% Rec & \% Rec & $\% \operatorname{Rec}$ \\
\hline & TCX (surrogate) & 740 & 810 & 0 & 0 \\
\hline & DCB (surrogate) & 0 & 0 & $\overline{0}$ & 0 \\
\hline
\end{tabular}

$\mathrm{n} / \mathrm{a}=$ Not applicable, since only AROCLOR 1254 spike added to the MS and MSD.

$\mathrm{U}$ flag = Not detected; results less than MDL

$\mathrm{J}$ flag = Detected and quantified, but result is less than MDL.

Chromatographic resolution suffered noticeably following a number of sample residue analyses, indicating some column degradation had occurred. The observed peak broadening may have adversely impacted the retention time windows. The high surrogate recoveries for the $\mathrm{PCB} /$ pesticide analysis can be attributed in part to these interferences. However, as mentioned above, quantitation of the surrogates was performed at abnormally low levels, making their quantitation highly susceptible to even minor interference. In some cases, a well-defined chromatographic peak could not be identified. In these cases, the surrogate recovery was reported as $0 \%$.

The AW-101 solids, AN-107 solids, and AW-101 supernatant were spiked with Aroclor 1254 at levels lower than desired as well. However, the recovery in these samples is satisfactory and compares well to the results obtained for the AN-107 supernatant which was spiked at a higher level. Therefore, because of the low concentrations that the surrogates were added for this sample set, low surrogate recovery does not equate to poor recovery of analytes. This conclusion is supported because, unlike the surrogate recoveries, the MSs are satisfactory. This is likely due to the multicompound quantitation of the Aroclors producing an average result and is therefore less susceptible to interference.

The surrogates and single component pesticides rely on a single peak for quantitation, producing less reliable results at these concentration levels. Since the concentration of the surrogates was below the MDL for the single component pesticides, erratic surrogate recovery does not equate to poor recovery of analytes here as well.

For both the solids and supernatant samples, some variation was observed for duplicate samples of the single component pesticide results. However, in all cases, the results were well below ten times the MDL. This is where the greatest variation is expected to occur. In addition, the erratic surrogate recoveries also demonstrate there is significant matrix interferences at this low concentration level. In addition to the analytes of interest specified by BNFL, additional analytes normally analyzed utilizing this method have been reported. 


\subsection{Polychlorinated Dibenzo-p-Dioxins and Dibenzofurans Analysis}

\subsection{Introduction}

The supernatants and solids samples were prepared in the SAL by the procedure outlined in Section 4.2. Following extraction, $5 \mathrm{~mL}$ of the of the resulting methylene chloride extraction residue was transferred under $\mathrm{COC}$ to the laboratory for analysis of PCBs/pesticides and dioxins and furans. Of this $5 \mathrm{~mL}$ of extraction residue, $3 \mathrm{~mL}$ was used for the dioxins and furans analysis and the remaining $2 \mathrm{~mL}$ was used for the $\mathrm{PCB} /$ pesticide analysis.

The dioxins and furans portion was exchanged into hexane and passed through several column cleanup procedures including alumina and silica gel to remove interfering components. After column cleanup, the resulting solutions were further concentrated and were to be analyzed using high resolution gas chromatography/high resolution mass spectrometry (HRGC/HRMS) as per Test Plan BNFL-TP-29953-018 and SW-846 Method 8290. However, due to a problem with the peak adjustment feature, the samples were ultimately screened for dioxins and furans using high-resolution gas chromatography/low-resolution mass spectrometry (HRGC/LRMS) per SW-846, Method 8280. A resolution of 1000 was utilized for the dioxins and furans analyses.

For both dioxins and furans, there are multiple isomers. Table 6.a lists the dioxin and furan standards analyzed to establish retention time windows and use as the basis for the determination of response factors.

Table 6.a. Standard Compounds Measured Using HRGC/LRMS

\begin{tabular}{|c|c|c|}
\hline$\overline{\text { CAS \# }}$ & Dioxin Compounds & Report ID ${ }^{(I)}$ \\
\hline $1746-01-6$ & 2,3,7,8-Tetrachlorodibenzo-p-dioxin & TCDD \\
\hline $40321-76-4$ & $1,2,3,7,8$-Pentachlorodibenzo-p-dioxin & $\overline{\mathrm{PeCDD}}$ \\
\hline $57653-85-7$ & $1,2,3,6,7,8$-Hexachlorodibenzo-p-dioxin & $\overline{\mathrm{HxCDD}}$ \\
\hline $39227-28-6$ & $1,2,3,4,7,8-$ Hexachlorodibenzo-p-dioxin & HxCDD \\
\hline $19408-74-3$ & $1,2,3,7,8,9-$ Hexachlorodibenzo-p-dioxin & HxCDD \\
\hline $35822-39-4$ & $1,2,3,4,6,7,8$-Heptachlorodibenzo-p-dioxin & HpCDD \\
\hline $3268-87-9$ & $1,2,3,4,6,7,8,9$-Octachlorodibenzo-p-dioxin & OCDD \\
\hline$\overline{\mathbf{C A S} \#}$ & Furan Compounds & Report ID $^{\text {(I) }}$ \\
\hline $51207-31-9$ & 2,3,7,8-Tetrachlorodibenzofuran & TCDF \\
\hline $57117-41-6$ & $1,2,3,7,8$-Pentachlorodibenzofuran & PeCDF \\
\hline $57117-31-4$ & $2,3,4,7,8$-Pentachlorodibenzofuran & PeCDF \\
\hline $57117-44-9$ & $1,2,3,6,7,8$-Hexachlorodibenzofuran & $\overline{\mathrm{HxCDF}}$ \\
\hline $72918-21-9$ & $1,2,3,7,8,9-$ Hexachlorodibenzofuran & $\mathrm{HxCDF}$ \\
\hline $70648-26-9$ & $1,2,3,4,7,8$-Hexachlorodibenzofuran & HxCDF \\
\hline $60851-34-5$ & $2,3,4,6,7,8$-Hexachlorodibenzofuran & $\mathrm{HxCDF}$ \\
\hline $67562-39-4$ & $1,2,3,4,6,7,8$-Heptachlorodibenzofuran & $\overline{\mathrm{HpCDF}}$ \\
\hline $55673-89-7$ & $1,2,3,4,7,8,9-$ Heptachlorodibenzofuran & HpCDF \\
\hline $39001-02-0$ & $1,2,3,4,6,7,8,9-$-octachlorodibenzofuran & OCDF \\
\hline
\end{tabular}

1) Report ID is shorthand notation for dioxins and furans for use in results tables 
No previous documented analysis of dioxins and furans in high level waste has been identified. The data presented in this report was obtained on a best effort basis, utilizing procedures, instrumentation, and data systems for the first time for dioxins and furans analysis of radioactive materials. 1

\subsection{Sample Preparation}

\subsubsection{Supernatants and Solids}

Following methylene chloride extraction of the supernatant samples and methylene chloride-acetone extraction of the solids samples and the volume reduction and sub-sampling of the methylene chloride extracts (See Section 4.2), the sub-sample fractions for dioxins and furans analysis were exchanged in hexane. The hexane residues from both the supernatant samples and the solids samples were then processed through an extensive cleanup procedure to remove potential interfering components, primarily PCBs.

The hexane extracts were first washed with concentrated sulfuric acid, $20 \% \mathrm{KOH}$, and $5 \% \mathrm{NaCl}$ in a separatory funnel. The washed hexane extract was passed through a column of anhydrous sodium sulfate. Following this water removal step, the hexane extract was applied to the top of a silica gel column and eluted with hexane. The eluate was concentrated to approximately $1 \mathrm{~mL}$ using a K-D apparatus, and added to an alumina column. The concentrated solution was then eluted with $60 \%$ methylene chloride in hexane $(\mathrm{v} / \mathrm{v})$ and collected. The resulting eluate was concentrated to approximately $1 \mathrm{~mL}$ using a K-D apparatus, and then reduced to a final volume of $200 \mu \mathrm{L}$ using nitrogen blow-down techniques for subsequent analysis using HRGC/LRMS.

\subsubsection{Laboratory Control Standard and Glassware Blank}

For the laboratory control standard (LCS), $100 \mathrm{~mL}$ of double distilled water was spiked with pre-measured volume of spiking materials and extracted with six $25-\mathrm{mL}$ portions of methylene chloride. The LCS was extracted in like manner to the supernatants and solids, except that the LCS was extracted in the laboratory instead of the SAL hot cells. Following extraction, the combined volumes were transferred to a K-D flask, exchanged into hexane, and reduced in volume to approximately $2 \mathrm{~mL}$. The hexane extract was transferred to a separatory funnel and washed with concentrated sulfuric acid, $20 \% \mathrm{KOH}$, and $5 \% \mathrm{NaCl}$. The hexane extract was then eluted through a column consisting of anhydrous sodium sulfate. The separatory funnel was then rinsed twice with $5 \mathrm{~mL}$ of hexane and the rinsates passed through a sodium sulfate column. The hexane fractions were combined and evaporated with a rotary evaporator to a final volume of $2 \mathrm{~mL}$, and then put through the cleanup procedure discussed above.

A glassware blank was prepared to demonstrate that it was free of interferants under the conditions of analysis. The blank was prepared by rinsing various glassware associated with sample preparation and analysis including separatory funnels, beakers, graduated cylinders, K-D flasks, glass columns, and storage vials with $50 \mathrm{~mL}$ of methylene chloride. The rinsates were transferred to a K-D flask, exchanged into hexane, and reduced in volume to approximately $2 \mathrm{~mL}$. The hexane extract was transferred to a separatory funnel and washed with concentrated sulfuric acid, $20 \% \mathrm{KOH}$, and $5 \% \mathrm{NaCl}$. The washed hexane extract was eluted through a column consisting of anhydrous sodium sulfate. The separatory funnel was rinsed twice with $5 \mathrm{~mL}$ of hexane and rinsates passed through a sodium sulfate column. The hexane fractions were combined and evaporated with a rotary evaporator to a final volume of $2 \mathrm{~mL}$. The hexane fractions were further concentrated to approximately $200 \mu \mathrm{L}$ using nitrogen blow-down techniques, and analyzed by HRGC/LRMS. The purpose of a glassware blank is to demonstrate that there is no contamination resulting from the glassware, therefore, the glassware blank was not put through the column cleanup procedure. 


\subsection{Instrumentation}

The analytical instrumentation used for the analysis of dioxins and furans consists of a gas chromatograph equipped with a $5 \mathrm{~m}$ X $0.32 \mathrm{~mm} \mathrm{HP}$ retention gap (uncoated and deactivated) column followed by a RTX -5 ( $60 \mathrm{~m} \mathrm{X} 0.25 \mathrm{~mm}, 0.25 \mu \mathrm{m}$ film thickness, Restek) column. Analyses were performed using on-column injection techniques and auto sampler injections. The JEOL highresolution mass spectrometer (HRMS) system was operated in the low-resolution mode. A description of the instrumentation is shown in Table 6.b.

Table 6.b. Dioxins and Furans Analysis Instrumentation

\begin{tabular}{|l|l|c|c|}
\hline System/Instrument & Manufacturer & Model Number & M\&TE Number $^{\text {(I) }}$ \\
\hline \hline GC & Hewlett-Packard & 5890 & WD11062 \\
\hline HRMS & JEOL & SX-102/SX-102 & WD11061 \\
\hline
\end{tabular}

1) Measuring and Test Equipment

\subsection{Analysis Results}

The dioxins and furans results for AW-101 and AN-107 are presented in Tables 6.c. The CAS numbers have been omitted since there are multiple dioxin and furan isomers that are not included as standards. For example, if a peak is detected in the TCDD retention time window with a slightly different retention time than the standard, the exact TCDD isomer cannot be identified since it does not match the standard. Per SW-846, Method 8280 protocol, if multiple isomers are detected in the appropriate retention time window, the multiple isomers are summed to provide single dioxin or furan result. However, there were no cases for either AN-107 or AW-101 supernatant or solids where multiple isomers were detected.

Dioxins and furans were detected in some of the solid samples, e.g., AN-107 solids duplicate. For the AN-107 solids duplicate, the retention times matched with standards and the ion ratios were within the control limits. However, the criteria (See Section 6.5.2) were not met with the AN-107 solids sample; therefore, no dioxins or furans results were reported. It is believed that the cross contamination may have occurred during the MS and sample preparation. An estimate of the contamination is approximately $1 \%$, based on percent recovery. A third analysis may have resolved the MS contamination issue; however, this analysis could not be performed due to lack of additional sample material for testing.

For both the AW-101 wet centrifuged solids process blank and sample, OCDF was detected at $2.90 \mu \mathrm{g} / \mathrm{Kg}$ and $2.94 \mu \mathrm{g} / \mathrm{Kg}$, respectively. The results of the AW-101 wet centrifuged solids duplicate indicates that OCDF is well below the MDL, which may indicate that the sample and blank were contaminated during handling. The OCDF results for the sample and blank are only slightly above the MDL and are considered qualitative. 
Table 6.c. AW-101 and AN-107 Dioxins and Furans Results

\begin{tabular}{|c|c|c|c|c|c|c|c|c|c|}
\hline \multirow{3}{*}{ CAS \# } & \multirow{3}{*}{$\begin{array}{r}\text { Tank Material } \\
\text { Sample ID } \\
\text { Units }\end{array}$} & \multicolumn{4}{|c|}{ AW-101 Supernatant } & \multicolumn{4}{|c|}{ AW-101 Wet Centrifuged Solids } \\
\hline & & \multirow{2}{*}{$\begin{array}{l}\text { MDL } \\
\mu \mathrm{g} / \mathrm{L}\end{array}$} & $\begin{array}{l}99-0648 \mathrm{bl} \\
\text { Proc Blk }\end{array}$ & $\begin{array}{c}99-0648 \\
\text { Sample }\end{array}$ & $\begin{array}{l}\text { 99-0648d } \\
\text { Duplicate }\end{array}$ & \multirow{2}{*}{$\begin{array}{c}\mathrm{MDL} \\
\mu \mathrm{g} / \mathrm{Kg}\end{array}$} & \multirow{2}{*}{$\begin{array}{c}\frac{-101 \text { Wet C }}{99-0650 b l} \\
\text { Proc Blk } \\
\mu \mathrm{g} / \mathrm{Kg}\end{array}$} & \multirow{2}{*}{$\begin{array}{c}99-0650 \\
\text { Sample } \\
\mu \mathrm{g} / \mathrm{Kg}\end{array}$} & \multirow{2}{*}{$\begin{array}{c}\begin{array}{c}99-0650 d \\
\text { Duplicate }\end{array} \\
\mu \mathrm{g} / \mathrm{Kg}\end{array}$} \\
\hline & & & $\mu \mathrm{g} / \mathrm{L}$ & $\mu \mathrm{g} / \mathrm{L}$ & $\mu \mathrm{g} / \mathrm{L}$ & & & & \\
\hline $1746-01-6$ & TCDD & 0.02 & $\mathrm{U}$ & $\mathrm{U}$ & $\overline{\mathrm{U}}$ & 0.4 & $\mathrm{U}$ & $\mathrm{U}$ & $\mathrm{U}$ \\
\hline $51207-31-9$ & TCDF & 0.02 & $\bar{U}$ & $\overline{\mathrm{U}}$ & $\bar{U}$ & 0.4 & $\bar{U}$ & $\mathrm{U}$ & $\bar{U}$ \\
\hline $40321-76-4$ & PeCDD & $\overline{0.06}$ & $\overline{\mathrm{U}}$ & $\overline{\mathrm{U}}$ & $\overline{\mathrm{U}}$ & 1.0 & $\overline{\mathrm{U}}$ & $\bar{U}$ & $\overline{\mathrm{U}}$ \\
\hline (1) & PeCDF & 0.06 & $\overline{\mathrm{U}}$ & $\bar{U}$ & $\bar{U}$ & 1.0 & $\overline{\mathrm{U}}$ & $\overline{\mathrm{U}}$ & $\overline{\mathrm{U}}$ \\
\hline$(1)$ & $\mathrm{HxCDD}$ & 0.06 & $\mathrm{U}$ & $\mathrm{U}$ & $\overline{\mathrm{U}}$ & 1.0 & $\mathrm{U}$ & $\overline{\mathrm{U}}$ & $\overline{\mathrm{U}}$ \\
\hline (1) & HxCDF & 0.06 & $\overline{\mathrm{U}}$ & $\overline{\mathrm{U}}$ & $\overline{\mathrm{U}}$ & 1.0 & $0.56 \sqrt{\mathrm{JB}}$ & $\mathrm{U}$ & $\overline{\mathrm{U}}$ \\
\hline $35822-39-4$ & HpCDD & 0.06 & $\overline{\mathrm{U}}$ & $\bar{U}$ & $\overline{\mathrm{U}}$ & 1.0 & $0.68 \mathrm{JB}$ & $0.87 \mathrm{JB}$ & $\mathrm{U}$ \\
\hline (1) & $\mathrm{HpCDF}$ & 0.06 & $\overline{\mathrm{U}}$ & $\overline{\mathrm{U}}$ & $\overline{\mathrm{U}}$ & 1.0 & $0.78 \mathrm{JB}$ & $0.72 \mathrm{JB}$ & $\bar{U}$ \\
\hline $3268-87-9$ & OCDD & 0.11 & $\bar{U}$ & $\bar{U}$ & $\bar{U}$ & 2.1 & $\mathrm{U}$ & $\mathrm{U}$ & U \\
\hline 39001-02-0 & OCDF & 0.11 & $\overline{\mathrm{U}}$ & $\mathrm{U}$ & $\bar{U}$ & 2.1 & $2.90 \mathrm{~B}$ & $2.94 \mathrm{~B}$ & $0.18 \mathrm{JB}$ \\
\hline \multirow[b]{3}{*}{ CAS \# } & \multirow{2}{*}{$\begin{array}{r}\text { Tank Material } \\
\text { Sample ID }\end{array}$} & \multicolumn{4}{|c|}{ AN-107 Supernatant } & \multicolumn{4}{|c|}{ AN-107 Wet Centrifuged Solids } \\
\hline & & \multirow{2}{*}{$\begin{array}{l}M D L \\
\mu \mathrm{g} / \mathrm{L}\end{array}$} & $\begin{array}{l}\text { 99-0649bl } \\
\text { Proc Blk }\end{array}$ & $\begin{array}{l}99-0649 \\
\text { Sample }\end{array}$ & $\begin{array}{l}\text { 99-0649d } \\
\text { Duplicate }\end{array}$ & \multirow{2}{*}{$\begin{array}{c}\mathrm{MDL} \\
\mu \mathrm{g} / \mathrm{Kg}\end{array}$} & $\begin{array}{l}\text { 99-0651bl } \\
\text { Proc Blk }\end{array}$ & $\begin{array}{l}99-0651 \\
\text { Sample }\end{array}$ & $\begin{array}{l}\text { 99-0651d } \\
\text { Duplicate }\end{array}$ \\
\hline & Units & & $\mu \mathrm{g} / \mathrm{L}$ & $\mu \mathrm{g} / \mathrm{L}$ & $\mu g / \mathbf{L}$ & & $\mu \mathrm{g} / \mathrm{Kg}$ & $\mu \mathrm{g} / \mathrm{Kg}$ & $\mu \mathrm{g} / \mathrm{Kg}$ \\
\hline 1746-01-6 & TCDD & 0.03 & $\mathrm{U}$ & $\overline{\mathrm{U}}$ & $\overline{\mathrm{U}}$ & 0.4 & $\bar{U}$ & $\mathrm{U}$ & $0.05 \mathrm{~J}$ \\
\hline $51207-31-9$ & TCDF & 0.03 & $\mathrm{U}$ & $\overline{\mathrm{U}}$ & $\bar{U}$ & 0.4 & $\overline{\mathrm{U}}$ & $\overline{\mathrm{U}}$ & $0.06 \mathrm{~J}$ \\
\hline $40321-76-4$ & PeCDD & 0.07 & $\overline{\mathrm{U}}$ & $\bar{U}$ & $\overline{\mathrm{U}}$ & 1.0 & $\bar{U}$ & $\overline{\mathrm{U}}$ & $0.46 \mathrm{~J}$ \\
\hline (1) & PeCDF & 0.07 & $\mathrm{U}$ & $\bar{U}$ & $\bar{U}$ & 1.0 & $\overline{\mathrm{U}}$ & $\bar{U}$ & $0.45 \mathrm{~J}$ \\
\hline$(1)$ & $\mathrm{HxCDD}$ & 0.07 & $\overline{\mathrm{U}}$ & $\overline{\mathrm{U}}$ & $\overline{\mathrm{U}}$ & 1.0 & $\overline{\mathrm{U}}$ & $\overline{\mathrm{U}}$ & $0.06 \mathrm{~J}$ \\
\hline (1) & $\mathrm{HxCDF}$ & 0.07 & $\overline{\mathrm{U}}$ & $\bar{U}$ & $\overline{\mathrm{U}}$ & 1.0 & $\mathrm{U}$ & $\bar{U}$ & $0.43 \mathrm{~J}$ \\
\hline $35822-39-4$ & HpCDD & 0.07 & $\overline{\mathrm{U}}$ & $\overline{\mathrm{U}}$ & $\overline{\mathrm{U}}$ & 1.0 & $0.62 \mathrm{JB}$ & $\overline{\mathrm{U}}$ & $0.43 \mathrm{JB}$ \\
\hline$(1)$ & HpCDF & 0.07 & $\bar{U}$ & $\bar{U}$ & $\bar{U}$ & 1.0 & U & $\mathrm{U}$ & $0.43 \mathrm{~J}$ \\
\hline $3268-87-9$ & OCDD & 0.14 & $\overline{\mathrm{U}}$ & $\overline{\mathrm{U}}$ & $\bar{U}$ & 2.1 & $\overline{\mathrm{U}}$ & $\overline{\mathrm{U}}$ & $1.65 \mathrm{~J}$ \\
\hline $39001-02-0$ & OCDF & 0.14 & $\bar{U}$ & $\overline{\mathrm{U}}$ & $\bar{U}$ & 2.1 & $\overline{\mathrm{U}}$ & $\bar{U}$ & $1.94 \mathrm{~J}$ \\
\hline
\end{tabular}

1) Multiple isomers possible in retention time window; each isomer has unique CAS \#. Component represents sum of dioxins or furans meeting retention time window and ion abundance ratio criteria.

$\mathrm{U}$ flag = not detected; results less than MDL

$\mathrm{J}$ flag $=$ detected and quantified, but results less than MDL. Analysis met ion abundance ratio and retention time criteria.

$B$ flag $=$ Compound was present in the method blank

\subsection{QC Evaluation}

The QC evaluation focuses on a) ion abundance ratios and response factors for the standards, $b$ ) ion abundance ratios for the samples, duplicates, MSs and MSDs, c) the spike recovery results from the analyses of the MSs for both liquids and solids, and d) the use of LRMS instead of HRMS for dioxins and furans analysis.

\subsubsection{Ion Abundance Ratios and Response Factors -- Standards}

The theoretical ion abundance ratios and control limits are listed in Table 6.d. These ratios are applicable to both the LRMS and HRMS. The matrix spiking solutions contain both ${ }^{13} \mathrm{C}$-labeled and unlabeled standards. All samples, including MSs, were spiked with labeled internal standards. The ion ratios for both the native (unlabeled) and the labeled components must meet the criteria shown in 
Table 6.d. The letter designations $M, M+2$, and $M+4$ refer to the parent ion (M) and the corresponding additional masses associated with the chlorine isotope pattern.

Table 6.d. Theoretical Ion Abundance Ratios and Control Limits

\begin{tabular}{|c|c|c|c|c|}
\hline \multirow{2}{*}{$\begin{array}{l}\text { Number of } \\
\mathrm{Cl} \text { atoms }\end{array}$} & \multirow[b]{2}{*}{ Ion Type } & \multirow{2}{*}{$\begin{array}{l}\text { Theoretical } \\
\text { Abundance }\end{array}$} & \multicolumn{2}{|c|}{ Control Limits } \\
\hline & & & Lower & Upper \\
\hline 4 & $\mathrm{M} / \mathrm{M}+2$ & $0 . \overline{77}$ & 0.65 & 0.89 \\
\hline 5 & $\mathrm{M}+2 / \mathrm{M}+4$ & 1.55 & 1.32 & 1.78 \\
\hline 6 & $M+2 / M+4$ & 1.24 & 1.05 & 1.43 \\
\hline 7 & $\bar{M}+2 / \mathrm{M}+4$ & 1.04 & 0.88 & 1.20 \\
\hline$\overline{8}$ & $\bar{M}+2 / M+4$ & 0.89 & 0.76 & 1.02 \\
\hline
\end{tabular}

Appendix $\mathrm{C}$ contains the ion abundance ratio $\mathrm{QC}$ data for the native and labeled components of the standards. The only failures of the ion abundance ratios for the standards were associated with the highest concentration standards. The concentration of the standards ranged from 0.5 to $200 \mu \mathrm{g} / \mathrm{L}$ for TCDD and TCDF, 5.0 to $2000 \mu \mathrm{g} / \mathrm{L}$ for OCDF and OCDD, and 2.5 to $1000 \mu \mathrm{g} / \mathrm{L}$ for all other compounds. The values for the highest concentration standards were not used for calculating the average response factor due to the fact that the low-resolution mode of the HRMS was used for the analysis. In the low-resolution mode the concentration versus peak area response was not linear over the entire concentration range. In all instances except for TCDD, the highest concentration standards produced the lowest response factor, since it was outside the linear calibration range of the system. In the cases of TCDD and TCDF, the response factor obtained for the highest concentrations were not used in the determination of the average response factor.

The analyses could not be performed with HRMS due to a peak adjustment problem. During analysis certain lock masses are assigned per group. Prior to actual sample analysis, peak adjustment is performed where the centroid of the peak are located. If certain criteria are met, the peak adjustment succeeds. However, in the high-resolution mode the peak adjustment failed numerous times and the decision was made to perform the analysis in the low resolutions screening mode. The percent relative standard deviations for the average response factors are less than $15 \%$ as required by the LRMS method, except for components:

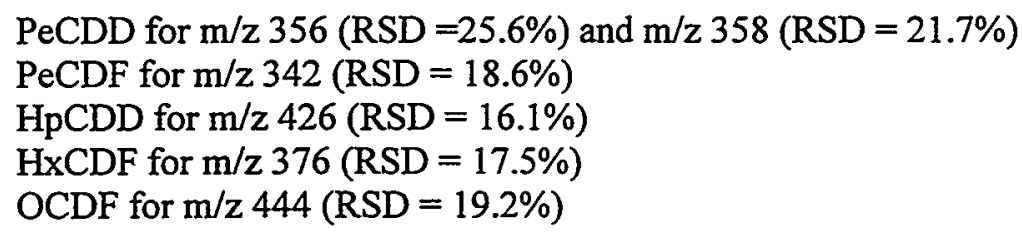

\subsubsection{Ion Abundance Ratios - Samples, Duplicates, and Matrix Spikes}

The ion abundance ratios for both the native and labeled components for all samples, duplicates, MSs, MSDs, and process blanks are listed in Appendix C. The ion abundance ratio is a major criterion for identifying dioxins and furans. If the ion abundance ratio is not within the control limits listed in Table 6.d, the component is not identified as a dioxin or furan even though the retention times may be consistent with dioxins and furans. Table 6.e provides a summary of those compounds tentatively identified as dioxins and furans by retention time and whether they meet the ion abundance ratio control limit criteria. For example, for TCDD in the AW-101 wet centrifuged solids sample a peak was detected within the retention time window but did not meet the ion abundance ratio criteria; therefore was assigned an " $f$ " (fail). The corresponding labeled TCDD was detected and met the ion abundance ratio criteria, and was assigned a " $p$ " (pass). 
Table 6.e. Acceptance Criteria Summary of Ion Abundance Ratios

\begin{tabular}{|c|c|c|c|c|c|c|c|c|c|c|c|c|c|c|c|c|c|c|c|c|c|}
\hline Sample ID & 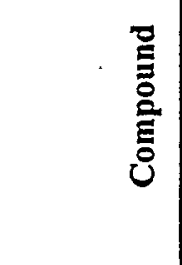 & 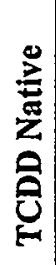 & 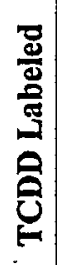 & 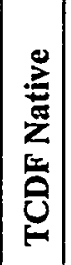 & 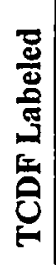 & 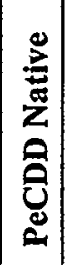 & 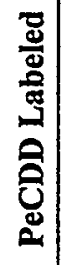 & 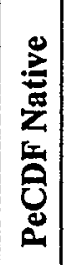 & 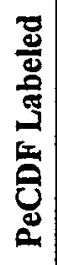 & 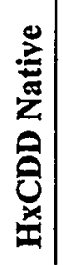 & 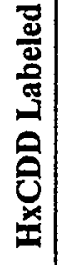 & 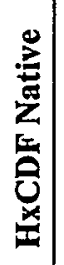 & 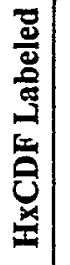 & 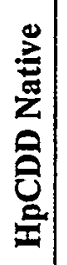 & 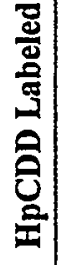 & 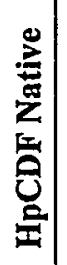 & 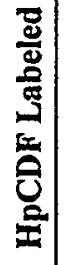 & 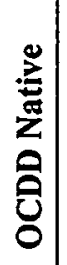 & 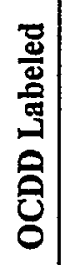 & 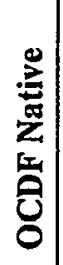 & 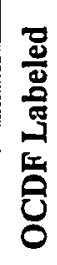 \\
\hline \multirow{5}{*}{$\begin{array}{l}\text { AW-101 } \\
\text { Supernatant }\end{array}$} & Proc & $\overline{-}$ & $\mathrm{p}$ & - & $\bar{p}$ & - & f & - & $\bar{p}$ & - & $\bar{p}$ & - & $p$ & - & $\mathrm{p}$ & - & p & - & $p$ & - & $\bar{p}$ \\
\hline & Sample & - & $\mathrm{p}$ & - & $\mathrm{p}$ & - & $\mathrm{p}$ & - & $\mathrm{p}$ & - & $f$ & - & $\mathrm{p}$ & - & $\mathrm{p}$ & - & $\mathrm{p}$ & - & $\mathrm{p}$ & - & $\mathrm{p}$ \\
\hline & Duplicate & - & p & - & $\bar{p}$ & - & $\mathbf{f}$ & - & $\mathrm{p}$ & - & $\mathrm{p}$ & - & $\mathrm{p}$ & - & $\mathrm{p}$ & - & $\mathrm{p}$ & - & $p$ & - & $\mathrm{p}$ \\
\hline & MS & $\mathrm{p}$ & $\mathrm{p}$ & $p$ & $p$ & $p$ & $\mathrm{p}$ & $p$ & $\mathrm{p}$ & $\mathrm{p}$ & $\mathrm{p}$ & $\mathrm{p}$ & $\mathrm{p}$ & $\mathrm{p}$ & $p$ & p & $\mathrm{p}$ & $\mathrm{p}$ & $\mathrm{p}$ & $\mathrm{p}$ & $\mathrm{p}$ \\
\hline & MSD & $p$ & $\mathrm{p}$ & $\mathrm{p}$ & $\mathrm{p}$ & $\mathrm{p}$ & $\mathrm{p}$ & $\bar{p}$ & $\mathrm{p}$ & $\mathrm{p}$ & $\mathrm{p}$ & $\bar{p}$ & $\bar{p}$ & $\bar{p}$ & $\mathrm{p}$ & $\bar{p}$ & $\mathrm{p}$ & $\mathrm{p}$ & $\mathrm{p}$ & $\mathrm{p}$ & $\mathrm{p}$ \\
\hline \multirow{5}{*}{$\begin{array}{l}\text { AW-101 } \\
\text { Wet } \\
\text { Centrifuged } \\
\text { Solids }\end{array}$} & Proc & - & $\mathrm{f}$ & $\mathrm{f}$ & - & $f$ & $p$ & $\mathrm{f}$ & $p$ & $f$ & $p$ & $p$ & $p$ & $p$ & $p$ & $p$ & $p$ & f & $p$ & $\mathrm{p}$ & $\mathrm{p}$ \\
\hline & Sample & $f$ & $\mathrm{p}$ & $f$ & $\mathbf{f}$ & $\mathrm{p}$ & $\mathrm{p}$ & $f$ & $\mathrm{p}$ & f & f & $\mathrm{p}$ & $f$ & $\mathrm{p}$ & $\mathrm{p}$ & $p$ & p & $f$ & $\mathrm{p}$ & $\mathrm{p}$ & p \\
\hline & Duplicate & - & $\mathrm{p}$ & - & $\mathrm{p}$ & - & $\mathrm{p}$ & - & $p$ & f & f & - & $\mathrm{p}$ & $f$ & $\mathrm{p}$ & - & $\mathrm{p}$ & $f$ & $\mathrm{p}$ & $\mathrm{p}$ & $\mathrm{p}$ \\
\hline & MS & $p$ & $\mathrm{p}$ & $p$ & $\mathrm{p}$ & $\mathrm{p}$ & $\mathrm{p}$ & $\mathrm{p}$ & $\mathrm{p}$ & $\mathrm{p}$ & $\mathrm{p}$ & $\mathrm{p}$ & $\mathrm{p}$ & $\mathrm{p}$ & $\mathrm{p}$ & $\mathrm{p}$ & $\mathrm{p}$ & $\bar{p}$ & $\bar{p}$ & $\mathrm{p}$ & $\mathrm{p}$ \\
\hline & MSD & $\mathrm{p}$ & $\mathrm{p}$ & $\mathrm{p}$ & p & p & $\mathrm{p}$ & $\mathrm{p}$ & $\mathrm{p}$ & $\mathrm{p}$ & $\mathrm{p}$ & $\mathrm{p}$ & $\mathrm{p}$ & $\mathrm{p}$ & $\mathrm{p}$ & $\mathrm{p}$ & $\mathrm{p}$ & $\mathrm{p}$ & $\bar{p}$ & $\mathrm{p}$ & $\mathrm{p}$ \\
\hline \multirow{5}{*}{$\begin{array}{l}\text { AN-107 } \\
\text { Supernatant }\end{array}$} & Proc & $-\cdot$ & $\mathrm{p}$ & - & f & - & f & - & $p$ & - & $p$ & - & $p$ & - & $\mathrm{p}$ & - & $\mathrm{p}$ & - & $p$ & - & $\mathrm{p}$ \\
\hline & Sample & - & $\mathrm{p}$ & - & $\mathrm{p}$ & - & $p$ & - & $\mathrm{p}$ & - & $\mathrm{p}$ & - & $\mathrm{p}$ & - & $\mathrm{p}$ & - & p & - & $\mathrm{p}$ & - & $\hat{\mathrm{p}}$ \\
\hline & Duplicate & - & $\mathrm{p}$ & - & $\mathrm{p}$ & - & $\mathrm{p}$ & - & $\mathrm{p}$ & - & $\mathrm{p}$ & - & $\mathrm{p}$ & - & $\mathrm{p}$ & - & $\mathrm{p}$ & - & $\mathrm{p}$ & - & $\mathrm{p}$ \\
\hline & MS & $\mathrm{p}$ & $\mathrm{p}$ & $p$ & $\mathrm{p}$ & $\mathrm{p}$ & $\mathrm{p}$ & $\mathrm{p}$ & $\mathrm{p}$ & $\mathrm{p}$ & $p$ & $\mathrm{p}$ & $\mathrm{p}$ & $\mathrm{p}$ & $p$ & $\mathrm{p}$ & $\mathrm{p}$ & $\mathrm{p}$ & p & $\mathrm{p}$ & $\mathrm{p}$ \\
\hline & $\overline{\mathrm{MSD}}$ & $p$ & $p$ & $\mathrm{p}$ & $\mathrm{p}$ & $\mathrm{p}$ & $\mathrm{p}$ & $p$ & $p$ & $\mathrm{p}$ & $\mathrm{p}$ & $\mathrm{p}$ & $\mathrm{p}$ & $\mathrm{p}$ & $p$ & $p$ & $\mathrm{p}$ & $\mathrm{p}$ & $\mathrm{p}$ & $\mathrm{p}$ & $\mathrm{p}$ \\
\hline \multirow{5}{*}{$\begin{array}{l}\text { AN-107 } \\
\text { Wet } \\
\text { Centrifuged } \\
\text { Solids }\end{array}$} & Proc & - & $p$ & $f$ & $p$ & - & f & - & $p$ & - & $p$ & $\mathbf{f}$ & $p$ & $\mathrm{p}$ & $p$ & f & $p$ & $\mathrm{p}$ & $p$ & - & $p$ \\
\hline & Sample & - & $\mathrm{p}$ & - & $\mathrm{p}$ & - & $\mathrm{p}$ & - & $\mathrm{p}$ & $f$ & $\mathrm{p}$ & - & $\mathrm{p}$ & $f$ & $\mathrm{p}$ & - & p & - & $\mathrm{p}$ & - & p \\
\hline & Duplicate & $\mathrm{p}$ & $\mathrm{p}$ & $\mathrm{p}$ & $p$ & $\mathrm{p}$ & $p$ & $\mathrm{p}$ & $\mathrm{p}$ & $\mathrm{p}$ & $p$ & $\mathrm{p}$ & $p$ & $\mathrm{p}$ & $\mathrm{p}$ & $\mathrm{p}$ & $\mathrm{p}$ & $\mathrm{p}$ & $\mathrm{p}$ & $\mathrm{p}$ & $\mathrm{p}$ \\
\hline & $\mathrm{MS}$ & $\mathrm{p}$ & $\mathrm{p}$ & $p$ & $\mathrm{p}$ & $\mathrm{p}$ & $p$ & $\mathrm{p}$ & $\mathrm{p}$ & $\mathrm{p}$ & $p$ & $\mathrm{p}$ & $p$ & $\mathrm{p}$ & $\mathrm{p}$ & $\mathrm{p}$ & $\mathrm{p}$ & $\mathrm{p}$ & $\mathrm{p}$ & $\mathrm{p}$ & $\mathrm{p}$ \\
\hline & MSD & $\mathrm{p}$ & $\mathrm{p}$ & $\mathrm{p}$ & p & $p$ & $\mathrm{p}$ & $\mathrm{p}$ & $\mathrm{p}$ & $\mathrm{p}$ & $\mathrm{p}$ & $\mathrm{p}$ & $\mathrm{p}$ & $\mathrm{p}$ & $\mathrm{p}$ & $\mathrm{p}$ & p & $\mathrm{p}$ & $\mathrm{p}$ & $\mathrm{p}$ & $\mathrm{p}$ \\
\hline $\mathrm{LCS}$ & Standard & $\mathrm{p}$ & f & $p$ & $p$ & $p$ & $p$ & $\mathrm{p}$ & $\mathrm{p}$ & $p$ & $p$ & $p$ & $p$ & $\mathrm{p}$ & $p$ & $p$ & $p$ & $p$ & $p$ & $p$ & $p$ \\
\hline
\end{tabular}

For the AW-101 and AN-107 supernatant MS and MSD analysis and the LCS analysis, all native and labeled compounds, except TCDD on the LCS, met the ion abundance ratio acceptance criteria. For the process blanks, samples and duplicates, only five of the 110 labeled compounds analyses failed to meet the ion abundance ratio acceptance criteria; these being PeCDD on the AW-101 process blank and duplicate, HxCDD on the AW-101 sample, PeCDD and TCDF on the AN-107 process blank, and TCDD on the LCS.

For AN-107 solids analyses, the only labeled compound to fail the acceptance criteria was PeCDD for the process blank. However, TCDF, HxCDF and $\mathrm{HpCDF}$ native compounds for the process blank and $\mathrm{HxCDD}$ and $\mathrm{HpCDD}$ native compounds for the sample also failed to meet the ion abundance ratio acceptance criteria. In the case of AW-101 solids, five ion abundance ratios for the labeled components failed including TCDD in the process blank, TCDF in the sample, HxCDD in the sample and duplicate, and $\mathrm{HxCDF}$ in the sample. There were numerous failures of the native components for AW-101 solids samples including TCDD in the sample, TCDF in the process blank and sample, PeCDF in the process blank and sample, HxCDD in the process blank, sample, and duplicate, $\mathrm{HpCDD}$ in the duplicate, and OCDD in the process blank, sample, and duplicate. All of the native 
and labeled components in both the MS and duplicates for both AN-107 solids and AW-101 solids met the ion abundance criteria.

As detailed in Table 6.e, numerous compounds for the AW-101and AN-107 process blanks, samples and duplicates failed to meet the ion abundance ratio acceptance criteria. The ion abundance ratios were established for labeled components at concentrations approximately seven times greater than the spike concentrations in the prepared samples. The low spike concentrations, as well as the measured concentrations of the native compounds (i.e., typically below the MDLs), significant impact the ability to meet the ion abundance ratio criteria.

\subsubsection{Dioxins and Furans Matrix Spike Recoveries}

The recoveries for the MSs and MSDs for the AW-101 and AN-107 supernatants are detailed in Table 6.f and the recoveries for solids are detailed in Table 6.g. The CAS numbers are included to identify the components in the matrix spiking solution. All ion abundance ratios for both the native and labeled components in the MSs and MSDs met the criteria listed in Table 6.d. The only failure related to ion abundance ratios was observed in the laboratory control sample for the labeled TCDD. There are several instances where the percent recovery is $>130 \%$. This may be due in part to the low spike level for the internal standards. Using manual integration of the labeled components, the peak areas were typically less than 100 . In addition, the ratio of the peak areas of the native/labeled was approximately 10. In those cases, a small error in the peak area of the internal standards can make a relatively large difference in the calculated concentrations and ion abundance ratios.

Table 6.f. AW-101 and AN-107 Supernatant-Dioxins and Furans Spike Recoveries

\begin{tabular}{|c|c|c|c|c|c|c|}
\hline \multirow[b]{3}{*}{ CAS \# } & \multirow{3}{*}{$\begin{array}{r}\text { Tank Material } \\
\text { Sample WD } \\
\text { Units }\end{array}$} & \multicolumn{2}{|c|}{$\frac{\text { AW-101 Supernatant }}{99-0648}$} & \multicolumn{2}{|c|}{$\frac{\text { AN-107 Supernatant }}{99-0649}$} & \multirow[t]{2}{*}{$\begin{array}{l}\text { Laboratory } \\
\text { Control }\end{array}$} \\
\hline & & MS & MSD & MS & MSD & \\
\hline & & \%Rec & \%Rec & $\% \operatorname{Rec}$ & \%Rec & \%Rec \\
\hline $1746-01-6$ & $\overline{\overline{T C D D}}$ & 87 & $\overline{85}$ & $\overline{74}$ & $\overline{988}$ & $104^{*}$ \\
\hline $51207-31-9$ & TCDF & 129 & 126 & 130 & 149 & 132 \\
\hline $40321-76-4$ & PeCDD & 100 & 110 & 127 & 133 & 84 \\
\hline $57117-41-6$ & $\overline{\mathrm{PeCDF}}$ & 113 & 93 & 88 & 98 & 73 \\
\hline $57653-85-7$ & HXCDD & 121 & $\overline{92}$ & 106 & 148 & 114 \\
\hline $57117-44-9$ & $\overline{\mathrm{HxCDF}}$ & 115 & 114 & 103 & 134 & 116 \\
\hline $35822-39-4$ & $\overline{\mathrm{HpCDD}}$ & 115 & 103 & 112 & 167 & 85 \\
\hline $67562-39-4$ & $\mathrm{HpCDF}$ & $\overline{111}$ & 95 & 105 & 135 & 85 \\
\hline $3268-87-9$ & OCDD & $\overline{121}$ & 114 & 110 & 136 & 22 \\
\hline $39001-02-0$ & OCDF & 115 & 105 & 97 & 122 & 86 \\
\hline
\end{tabular}

* = Failed to meet Table 6.e ion abundance ratio QC criteria. 
Table 6.g. AW-101 and AN-107 Solids --- Dioxins and Furans Spike Recoveries

\begin{tabular}{|c|c|c|c|c|c|}
\hline \multirow[b]{3}{*}{ CAS \# } & $\begin{array}{r}\text { Tank Material } \\
\text { Sample } \mathbf{W}\end{array}$ & \multicolumn{2}{|c|}{$\frac{\text { AW-101 Wet Centrifuged Solids }}{99-0650}$} & \multicolumn{2}{|c|}{$\frac{\mathrm{N}-107 \text { Wet Centrifuged Solids }}{99-0651}$} \\
\hline & & MS & MSD & MS & MSD \\
\hline & Units & \%Rec & \%Rec & \%Rec & \%Rec \\
\hline $1746-01-6$ & TCDD & 70 & 77 & 73 & 73 \\
\hline $51207-31-9$ & TCDF & 97 & 123 & 119 & 120 \\
\hline $40321-76-4$ & PeCDD & 82 & 110 & 98 & 93 \\
\hline $57117-41-6$ & PeCDF & 51 & 74 & 87 & 78 \\
\hline $57653-85-7$ & HxCDD & 96 & 118 & 107 & 89 \\
\hline $57117-44-9$ & HxCDF & 68 & 122 & 104 & 96 \\
\hline $35822-39-4$ & HpCDD & 86 & 109 & 112 & 89 \\
\hline $67562-39-4$ & $\overline{\mathrm{HpCDF}}$ & 61 & 106 & 103 & 89 \\
\hline $3268-87-9$ & OCDD & 67 & 96 & 127 & 90 \\
\hline $39001-02-0$ & $\overline{O C D F}$ & 52 & 84 & 111 & 83 \\
\hline
\end{tabular}

\subsubsection{Other Observations (Deviations/Concerns/lssues)}

Due to the peak adjustment difficulty, the dioxins and furans analyses were performed by LRMS rather than HRMS. At lower resolution, the mass spectrometer has higher sensitivity but less specificity (i.e., resolving power). The resolving power of a mass spectrometer is a measure of its ability to separate two ions of any defined mass difference. Basically, for two overlapping peaks $M_{1}$ and $M_{2}$, the resolution may be defined in terms of the mass difference $(\Delta M)$ between them. The resolution is then defined as $M_{1} / \triangle M$. The HRMS method requires a resolution of 10,000 . At 10,000 resolution a mass at $\mathrm{m} / \mathrm{z} 300.00$ is separated from a mass at $\mathrm{m} / \mathrm{z} 300.03$. For each group of ions, a lock mass is assigned in high resolution. If the peak adjustment fails, the lock mass may not be adequately tracked during the analysis; ultimately affecting quantitation. In high-resolution mode, the peak adjustment failed repeatedly. Therefore, the analyses were performed in low-resolution mode. In low-resolution mode the resolution was set at 1,000 . At 1,000 resolution a mass at $\mathrm{m} / \mathrm{z}$ of 300.00 is distinguished from a mass at $\mathrm{m} / \mathrm{z} 300.30$.

The concentration levels of the internal standard spikes were significantly low as to affect the quantitation and recovery. In most cases, manual integration of the peak areas resulted in areas less than 100. At these minimal areas, a small error in the peak area may make a relatively large difference in the calculated concentrations. Typically, for an internal standard at $100 \mu \mathrm{g} / \mathrm{L}$, the peak areas were in the range of $4000-6000$.

One possible explanation for the presence of dioxins and furans components in AN-107 solids duplicate may be contamination due to a) sample carryover from analysis of standards or b) contamination incurred during the sample preparation and spiking of internal standards. The dioxins and furans pattern observed in the AN-107 solids duplicate was consistent with the spiking solution and not the standard solutions. The spiking solution contains less components than the standard solution. As an example, the spiking solution contains one compound for each dioxin and furan from tetra to octa-substituted. The standard solution, however, contains three HxCDDs and four HxCDFs. The pattern observed in the sample is consistent with the pattern for the matrix spiking solution. In addition, there is no evidence to indicate the contamination was the result of carryover from one injection to the next. The concentrations of the components in the AN-107 solids duplicate are so low that it is certainly possible that the components would not have been detected with HRMS. The presence of dioxins and furans in the other samples at low levels may also be due to contamination during sample preparation. 


\subsection{Organic Anions Analysis}

\subsection{Introduction}

Analyses of organic anions were performed on both the supernatant and solids from samples of tank waste material from Tanks AW-101 and AN-107. The supernatant samples were passed through cation exchange resin material to reduce the radioactivity to a sufficient level to be safely handled in the laboratory. The solid samples were leached at ambient temperature with water, filtered, and the resulting solution passed through cation exchange resin to reduce the radioactivity levels. The supernatant and solids were aliquotted and prepared in the SAL and transferred under COC to the analytical laboratory for organic anion analysis by ion chromatography (IC).

\subsection{Sample Preparation}

Tank samples from AW-101 and AN-107 were prepared in the SAL. For the supernatants, a 1-mL sample was accurately weighed and then passed through a column of cation exchange resin to reduce the radioactivity level. Similarly for the solids, an approximate 1-g sample was accurately weighed, leached at ambient temperature with approximately $5 \mathrm{~mL}$ of distilled water for 12 hours, and then filtered prior to passing the resulting solution through a cation exchange column. The activity reduction was performed per Test Plan BNFL-29953-014, "Activity Reduction Via Cation Exchange for Carboxylate Analysis". The treatment within the SAL resulted in an approximate 10-fold to 12-fold dilution (weight/weight) into a dilute caustic matrix that is not significantly different from the caustic matrix of the original sample. An additional 300-fold dilution was performed to dilute the major inorganic ions (nitrate and nitrite) to levels that prevent overloading the capacity of the analytical column. Previous experience (Campbell 1997) has demonstrated that ion-exchange sites within the IC column apparently do not recover quickly from an overload of these inorganic species, which results in a non-uniform elution of the weakly retained analytes (e.g. acetate and formate). Blank spikes, MSs and MSDs were prepared in the laboratory after elution through the cation exchange resin. Previous studies on samples from these two tanks have shown that organic material was neither introduced nor removed with the use of cation exchange resin. Spiking solutions were prepared using oxalic acid, sodium formate, and sodium acrylate in deionized water.

\subsection{Instrumentation}

The analytical instrumentation utilized for the analysis of low molecular weight organic acids consisted of an IC unit equipped with a conductivity detector. A Dionex AS-11 column and AG-11 guard column were used at ambient temperature with a $25-\mu \mathrm{L}$ sample loop. An anion suppressor was used. The flow rate of the mobile phase was $2.0 \mathrm{~mL} / \mathrm{min}$. A description of the $\mathrm{IC}$ system is provided in Table 7.a.

Table 7.a. Ion Chromatography Instrumentation

\begin{tabular}{|l|l|c|c|}
\hline \hline System/Instrument & Manufacturer & Model Number & M\&TE ${ }^{(I)}$ Number \\
\hline \hline IC System & Dionex & 500 DX & WD 24293 \\
\hline Conductivity Detector & Dionex & CD20 & WD 24295 \\
\hline
\end{tabular}

1) Measuring and Test Equipment

The IC gradient conditions were: (a) $0.0 \mathrm{~min} 0 \% 100 \mathrm{mM} \mathrm{NaOH}, 98.1 \%$ deionized water and $1.9 \% 5$ $\mathrm{mM} \mathrm{NaOH}$, (b) $6.4 \mathrm{~min} 0 \% 100 \mathrm{mM} \mathrm{NaOH}, 0 \%$ deionized water and $100 \% 5 \mathrm{mM} \mathrm{NaOH}$, and (c) $18.4 \mathrm{~min} 35 \% 100 \mathrm{mM} \mathrm{NaOH}, 0 \%$ deionized water and $65 \% 5 \mathrm{mM} \mathrm{NaOH}$. The mobile phase contained a gradient of deionized water and a weak solution of $\mathrm{NaOH}$. 


\subsection{Analysis Result}

The results of the analysis of the AW-101 and AN-107 supernatant and wet centrifuged solids samples are listed below in Table 7.b.

Although acetate is reported, acetate coelutes with glycolate and requires the use of an alternate column for separation from glycolate. Without additional separation, one can not unequivocally state that the observed peak contains only acetate, only glycolate, a combination of both anions, or a possible contaminant. From tank waste and solubility studies, the results are dependent on tank waste type. In other words, for tanks with different fill histories, the analytical results may be primarily glycolate, primarily acetate, or a combination of both anions (Sharma et al. 1998, Camaioni et al. 1998, Barney 1997, Ashby et al. 1994.)

For AW-101 and AN-107 analyses, no acetate/glycolate peak was detected in the supernatants or solids above the MDL, and the reported acetate MDL can be used to bound the upper acetate/glycolate concentration. However, acetate/glycolate peaks were detected below the MDL for both AW-101 and AN-107 supernatants, with the "estimated" AW-101 and AN-107 acetate/glycolate concentrations being $1200 \mu \mathrm{g} / \mathrm{mL}$ and $4000 \mu \mathrm{g} / \mathrm{mL}$, respectively.

Table 7.b. IC Organic Anion Results

\begin{tabular}{|c|c|c|c|c|c|c|c|c|c|c|}
\hline \multirow{2}{*}{\multicolumn{2}{|c|}{$\begin{array}{r}\text { Tank Material } \\
\text { Sample Do }\end{array}$}} & \multicolumn{4}{|c|}{ AW-101 Supernatant } & \multicolumn{4}{|c|}{ AW-101 Wet Centrifuged Solids } & \multirow{3}{*}{$\begin{array}{l}\text { Maximum } \\
\mu g / g^{(2)} \\
\text { of Slurry }\end{array}$} \\
\hline & & \multirow{2}{*}{$\begin{array}{l}M \mathbf{M L}^{(1)} \\
\mu \mathrm{g} / \mathrm{mL}\end{array}$} & \multirow{2}{*}{$\begin{array}{c}\begin{array}{c}99- \\
\text { Proc Blk }\end{array} \\
\mu \mathrm{g} / \mathbf{m L}\end{array}$} & \multirow{2}{*}{$\begin{array}{l}\begin{array}{l}99-0648 \\
\text { Sample }\end{array} \\
\mu \mathrm{g} / \mathrm{mL} \\
\end{array}$} & \multirow{2}{*}{$\begin{array}{l}99-0648 d \\
\text { Duplicate } \\
\mu g / m L \\
\end{array}$} & \multirow{2}{*}{$\begin{array}{c}\mathrm{MDL}^{(1)} \\
\mu \mathrm{g} / \mathrm{g}\end{array}$} & \multirow{2}{*}{\begin{tabular}{|c|}
$\begin{array}{c}99- \\
\text { Proc Blk }\end{array}$ \\
$\mu \mathrm{g} / \mathrm{g}$ \\
\end{tabular}} & \multirow{2}{*}{\begin{tabular}{|c|}
$99-0650$ \\
Sample \\
$\mu \mathrm{g} / \mathrm{g}$ \\
\end{tabular}} & \multirow{2}{*}{\begin{tabular}{|c|}
$\begin{array}{c}99-0650 \mathrm{~d} \\
\text { Duplicate }\end{array}$ \\
$\mu \mathrm{g} / \mathrm{g}$
\end{tabular}} & \\
\hline CAS \# & Units & & & & & & & & & \\
\hline $144-62-7$ & Oxalate & 1300 & $\mathrm{U}$ & $600 \mathrm{~J}$ & $500 \mathrm{~J}$ & 900 & $\mathrm{U}$ & 9700 & 11100 & 1600 \\
\hline $64-18-6$ & Formate & 1700 & $\mathrm{U}$ & 2200 & 2100 & 1200 & $\bar{U}$ & 3400 & 2800 & 2300 \\
\hline $79-10-7$ & Acrylate & 1300 & $\overline{\mathrm{U}}$ & $\overline{\mathrm{U}}$ & $\mathrm{U}$ & 900 & $\bar{U}$ & $\mathrm{U}$ & $\bar{U}$ & $\bar{U}$ \\
\hline 64-19-7 & Acetate ${ }^{(3)}$ & 4400 & $\mathrm{U}$ & $1200 \mathrm{~J}$ & $1200 \mathrm{~J}$ & 1000 & $\mathrm{U}$ & $\mathrm{U}$ & $\overline{\mathrm{U}}$ & $\bar{U}$ \\
\hline \multirow[b]{3}{*}{ CAS \# } & \multirow{2}{*}{$\begin{array}{r}\text { Tank Material } \\
\text { Sample ID }\end{array}$} & \multicolumn{4}{|c|}{ AN-107 Supernatant } & \multicolumn{4}{|c|}{ AN-107 Wet Centrifuged Solids } & \multirow{3}{*}{$\begin{array}{c}\text { Maximum } \\
\mu g / g^{(2)} \\
\text { of Slurry }\end{array}$} \\
\hline & & \multirow{2}{*}{\begin{tabular}{|l|}
$\mathrm{MLL}^{(1)}$ \\
$\mu \mathrm{g} / \mathrm{mL}$
\end{tabular}} & $\begin{array}{c}99- \\
\text { Proc Blk }\end{array}$ & $\begin{array}{l}99-0649 \\
\text { Sample }\end{array}$ & $\begin{array}{l}\text { 99-0649d } \\
\text { Duplicate }\end{array}$ & \multirow{2}{*}{$\begin{array}{c}\mathrm{MDL}^{(1)} \\
\mu \mathrm{g} / \mathrm{g} \\
\end{array}$} & $\begin{array}{c}99- \\
\text { Proc Blk }\end{array}$ & $\begin{array}{l}99-0651 \\
\text { Sample }\end{array}$ & \multirow{2}{*}{\begin{tabular}{|c|}
$\begin{array}{c}99-0651 d \\
\text { Duplicate }\end{array}$ \\
$\mu \mathrm{g} / \mathrm{g}$ \\
\end{tabular}} & \\
\hline & Units & & $\mu \mathrm{g} / \mathbf{m L}$ & $\mu \mathrm{g} / \mathbf{m L}$ & $\mu \mathrm{g} / \mathrm{mL}$ & & $\mu \mathrm{g} / \mathrm{g}$ & $\mu \mathrm{g} / \mathrm{g}$ & & \\
\hline $144-62-7$ & Oxalate & 1300 & $\mathrm{U}$ & $200 \mathrm{~J}$ & $500 \mathrm{~J}$ & 900 & $\mathrm{U}$ & 22000 & 22700 & 2900 \\
\hline $64-18-6$ & Formate & 1700 & $\overline{\mathrm{U}}$ & 1700 & 2000 & 1200 & $\overline{\mathrm{U}}$ & $600 \mathrm{~J}$ & $600 \mathrm{~J}$ & 1800 \\
\hline $79-10-7$ & Acrylate & 1300 & $\mathrm{U}$ & $\bar{U}$ & $\mathrm{U}$ & 900 & $\overline{\mathrm{U}}$ & $\mathrm{U}$ & $\overline{\mathrm{U}}$ & $\bar{U}$ \\
\hline 64-19-7 & Acetate $^{(3)}$ & 4400 & $\bar{U}$ & $4000 \mathrm{~J}$ & $4000 \mathrm{~J}$ & 1000 & $\bar{U}$ & $\bar{U}$ & $\mathrm{U}$ & $U$ \\
\hline
\end{tabular}

1) $\mathrm{MDL}=$ Method detection limit based on instrument detection limit and sample quantity

2) Maximum slurry $\mu \mathrm{g} / \mathrm{g}$ calculated using results of Tables $2 . \mathrm{a}$ (weight fractions) and 2.b (supernatant density)-See Section 1.0

3) Acetate and glycolate are not resolved; results or MDL represent bounding upper concentration $\mathrm{U}$ flag = Anion not detected above the MDL; the MDL is based on the lowest calibration standard adjusted for analytical dilution factors.

$\mathrm{J}$ flag $=$ Anion detected, but concentration is less than MDL

\subsection{QC Evaluation}

No organic anions of interest were detected in the blank samples. The MDLs stated in Table 7.b are assumed to be adequate, since no MRQs were established by BNFL for the organic anions measured. 
Table 7.c presents the average MS and MSD recoveries and blank spike recovery results for the AW-101 and AN-107 supernatants and wet centrifuged solids. Spike recoveries for blank spikes, MSs, and MSDs ranged from $77 \%$ to $165 \%$ and in most cases meet the acceptance criteria of the governing quality assurance plan. Three spikes failed to meet the acceptance criteria of $75 \%$ to 125\%; oxalate for the AN-107 solids MS and formate for the AW-101 solids MS and MSD.

Table 7.c. IC Organic Anions Matrix Spike Recoveries

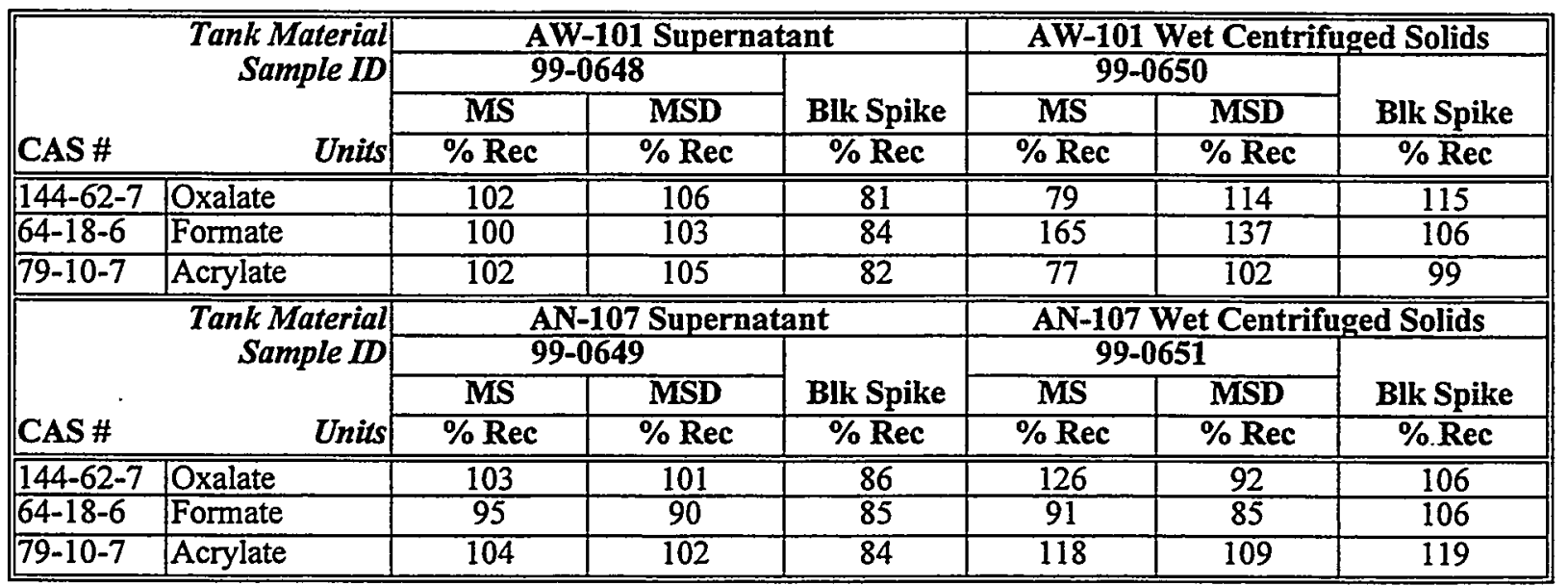




\subsection{Headspace Analysis}

\subsection{Introduction}

Headspace analyses were performed on the supernatant of tank waste material from Tanks AW-101 and $A N-107$. The samples were prepared in the SAL and transferred under COC to the laboratory for analyses. Analyses were performed using an automated headspace sampler interfaced to a gas chromatographic/mass spectrometric system. Test Plan BNFL-29953-026 Rev. 0 "Analysis for Volatile Constituents using Headspace Gas Chromatography Mass Spectrometry" provides details of the procedure used for this analysis. Analytes of interest include methanol, ethanol, 1-propanol, 2-propanol, n-butanol, 2-methyl-2-propanol, and triethylamine.

\subsection{Sample Preparation}

Tank samples from AW-101 and AN-107 were prepared in the SAL. Sample aliquots of $1 \mathrm{~mL}$ were placed in 10-mL headspace vials and immediately sealed using crimp-top septa. The samples were then transported to the laboratory for subsequent analysis. Immediately prior to analysis, internal standards and surrogate compounds were added through the septa.

\subsection{Instrumentation}

The analytical instrumentation utilized for the analysis of volatile components by headspace consists of an automated headspace analyzer interfaced to a GC/MS system. A description of the system is provided in Table 8.a.

Table 8.a. Headspace Instrumentation

\begin{tabular}{||l|l|c|c|}
\hline \hline System//nstrument & Manufacturer & Model Number & M\&TE (') Number \\
\hline \hline Headspace Sampler & Hewlett-Packard & 7964 & WD 25715 \\
\hline Gas Chromatograph & Hewlett-Packard & 5890 & WD 14120 \\
\hline Mass Spectrometer & Hewlett-Packard & 5972 & WD 17020 \\
\hline
\end{tabular}

1) Measuring and Test Equipment

\subsection{Analysis Results}

The headspace analysis results are presented in Tables 8.b. Headspace analysis for alcohols and triethylamine was performed on the supernatants only. As seen in Table 8.c, the target MRQs (after adjusting for density) for $\mathrm{n}$-butanol and triethylamine were not met. 
Table 8.b. AW-101 and AN-107 Results - Headspace Analysis

\begin{tabular}{|c|c|c|c|c|c|c|c|c|}
\hline \multirow{3}{*}{\multicolumn{2}{|c|}{$\begin{array}{r}\text { Tank Material } \\
\text { Sample ID } \\
\text { Units }\end{array}$}} & \multirow{3}{*}{$\begin{array}{r}\mathrm{MDL} \\
\mu \mathrm{g} / \mathrm{mL}\end{array}$} & \multicolumn{3}{|c|}{ AW-101 Supernatant } & \multicolumn{3}{|c|}{ AN-107 Supernatant } \\
\hline & & & $\begin{array}{c}99-0648 \\
\text { Proc Blank }\end{array}$ & $\begin{array}{l}\text { 99-0648 } \\
\text { Sample }\end{array}$ & $\begin{array}{c}99-0648 \\
\text { Duplicate }\end{array}$ & $\begin{array}{c}99-0649 \\
\text { Proc Blank }\end{array}$ & $\begin{array}{l}99-0649 \\
\text { Sample }\end{array}$ & $\begin{array}{c}99-0649 \\
\text { Duplicate }\end{array}$ \\
\hline & & & $\mu \mathrm{g} / \mathrm{mL}$ & $\mu \mathrm{g} / \mathrm{mL}$ & $\mu \mathrm{g} / \mathrm{mL}$ & $\mu \mathrm{g} / \mathrm{mL}$ & $\mu \mathrm{g} / \mathrm{mL}$ & $\mu \mathrm{g} / \mathrm{mL}$ \\
\hline CAS\# & BNFL Compound Lis & & & & & & & \\
\hline $67-56-1$ & Methanol & 30 & $\overline{\mathrm{U}}$ & $1.1 \mathrm{~J}$ & $\bar{U}$ & $\bar{U}$ & $4.6 \mathrm{~J}$ & $4.4 \mathrm{~J}$ \\
\hline $64-17-5$ & Ethanol & 1 & $2.5 \mathrm{~B}$ & $2.6 \mathrm{~B}$ & $2.6 \mathrm{~B}$ & $2.7 \mathrm{~B}$ & $3.0 \mathrm{~B}$ & $2.4 \mathrm{~B}$ \\
\hline $67-63-0$ & 2-Propanol & 1 & $\mathrm{U}$ & $\bar{U}$ & $\mathrm{U}$ & $\mathrm{U}$ & $\bar{U}$ & $\overline{\mathrm{U}}$ \\
\hline $75-65-0$ & 2-Methyl-2-propanol & $\mathrm{I}$ & $\overline{\mathrm{U}}$ & $\bar{U}$ & $\overline{\mathrm{U}}$ & $\overline{\mathrm{U}}$ & $\overline{\mathrm{U}}$ & $\bar{U}$ \\
\hline $71-23-8$ & 1-Propanol & 1 & $\overline{\mathrm{U}}$ & $\bar{U}$ & $\bar{U}$ & $\bar{U}$ & $\overline{\mathrm{U}}$ & $\bar{U}$ \\
\hline $78-92-2$ & 2-Butanol & 1 & $\overline{\mathrm{U}}$ & $\overline{\mathrm{U}}$ & $\overline{\mathrm{U}}$ & $\overline{\mathrm{U}}$ & $\overline{\mathrm{U}}$ & $\overline{\mathrm{U}}$ \\
\hline $71-36-3$ & n-Butanol & 1 & $\overline{\mathrm{U}}$ & $\overline{\mathrm{U}}$ & $\overline{\mathrm{U}}$ & $\overline{\mathrm{U}}$ & $\bar{U}$ & $\overline{\mathrm{U}}$ \\
\hline $121-44-8$ & Triethylamine & 20 & $\bar{U}$ & $\bar{U}$ & $\overline{\mathrm{U}}$ & $\overline{\mathrm{U}}$ & $5.8 \mathrm{~J}$ & $\mathbf{1 . 0 \mathrm { J }}$ \\
\hline CAS \# & Other Compounds & & & & & & & \\
\hline $78-93-3$ & Butanone & 1 & $\overline{\mathrm{U}}$ & $\overline{\mathrm{U}}$ & $\overline{\mathrm{U}}$ & $\bar{U}$ & $\bar{U}$ & $\bar{U}$ \\
\hline $109-99-9$ & Tetrahydrofuran & 1 & $\bar{U}$ & $\overline{\mathrm{U}}$ & $\overline{\mathrm{U}}$ & $\overline{\mathrm{U}}$ & $\overline{\mathrm{U}}$ & $\overline{\mathrm{U}}$ \\
\hline $107-87-9$ & 2-Pentanone & 1 & $\overline{\mathrm{U}}$ & $\mathrm{U}$ & $\overline{\mathrm{U}}$ & $\bar{U}$ & $\bar{U}$ & $\overline{\mathrm{U}}$ \\
\hline 108-10-1 & 4-Methyl-2-pentanone & $\overline{1}$ & $\overline{\mathrm{U}}$ & $\overline{\mathrm{U}}$ & $\overline{\mathrm{U}}$ & $\overline{\mathrm{U}}$ & $\bar{U}$ & $\overline{\mathrm{U}}$ \\
\hline $591-78-6$ & 2-Hexanone & 1 & $\overline{\mathrm{U}}$ & $\bar{U}$ & $\bar{U}$ & $\overline{\mathrm{U}}$ & $\bar{U}$ & $\bar{U}$ \\
\hline & & & $\% \operatorname{Rec}$ & \% Rec & \% Rec & \% Rec & \% Rec & $\% \operatorname{Rec}$ \\
\hline & Methanol-d4 & & 119.5 & 35.8 & 35.7 & 120.5 & 61.8 & 61.4 \\
\hline
\end{tabular}

$\mathrm{J}$ flag = Compound was detected below the level of quantitation

$\mathrm{B}$ flag $=$ Compound was present in the method blank

Table 8.c. Target Headspace Minimum Reportable Quantities

\begin{tabular}{|c|c|c|c|}
\hline CAS \# & BNFL Compound List & $\begin{array}{c}\text { Solids Target } \\
\text { MRQ }^{(1)} \\
\mu \mathrm{g} / \mathrm{Kg}\end{array}$ & $\begin{array}{c}\text { Supernatant Target MRQ } \\
\text { (Density }=1.45 \mathrm{~g} / \mathrm{mL} \text { ) } \\
\mu \mathrm{g} / \mathrm{mL}\end{array}$ \\
\hline $67-56-1$ & Methanol & $-\cdots$ & -1 \\
\hline $64-17-5$ & Ethanol & $\cdots$ & $-\cdots$ \\
\hline $67-63-0$ & 2-Propanol & $\cdots$ & $\overline{----}$ \\
\hline $75-65-0$ & 2-Methyl-2-propanol & $-\cdots$ & $\cdots$ \\
\hline $71-23-8$ & 1-Propanol & $-\cdots$ & - \\
\hline $78-92-2$ & 2-Butanol & ---- & ---- \\
\hline $71-36-3$ & n-Butanol & 900 & 1.3 \\
\hline $121-44-8$ & Triethylamine & 500 & $\overline{0.7}$ \\
\hline
\end{tabular}




\subsection{QC Evaluation}

Response for the internal standard, ethanol- $\mathrm{d}_{6}$, varied substantially (nearly an order of magnitude) throughout the analytical batch. This effected the detection limits, particularly for methanol and triethylamine. In addition, the surrogate recoveries for methanol- $\mathrm{d}_{4}$ were effected in the later portion of the run. This appears primarily due to the internal standard response as well. Matrix spike and MSD recoveries are presented in Table 8.d.

Table 8.d. AW-101 and AN-107 Headspace Matrix Spike Recoveries

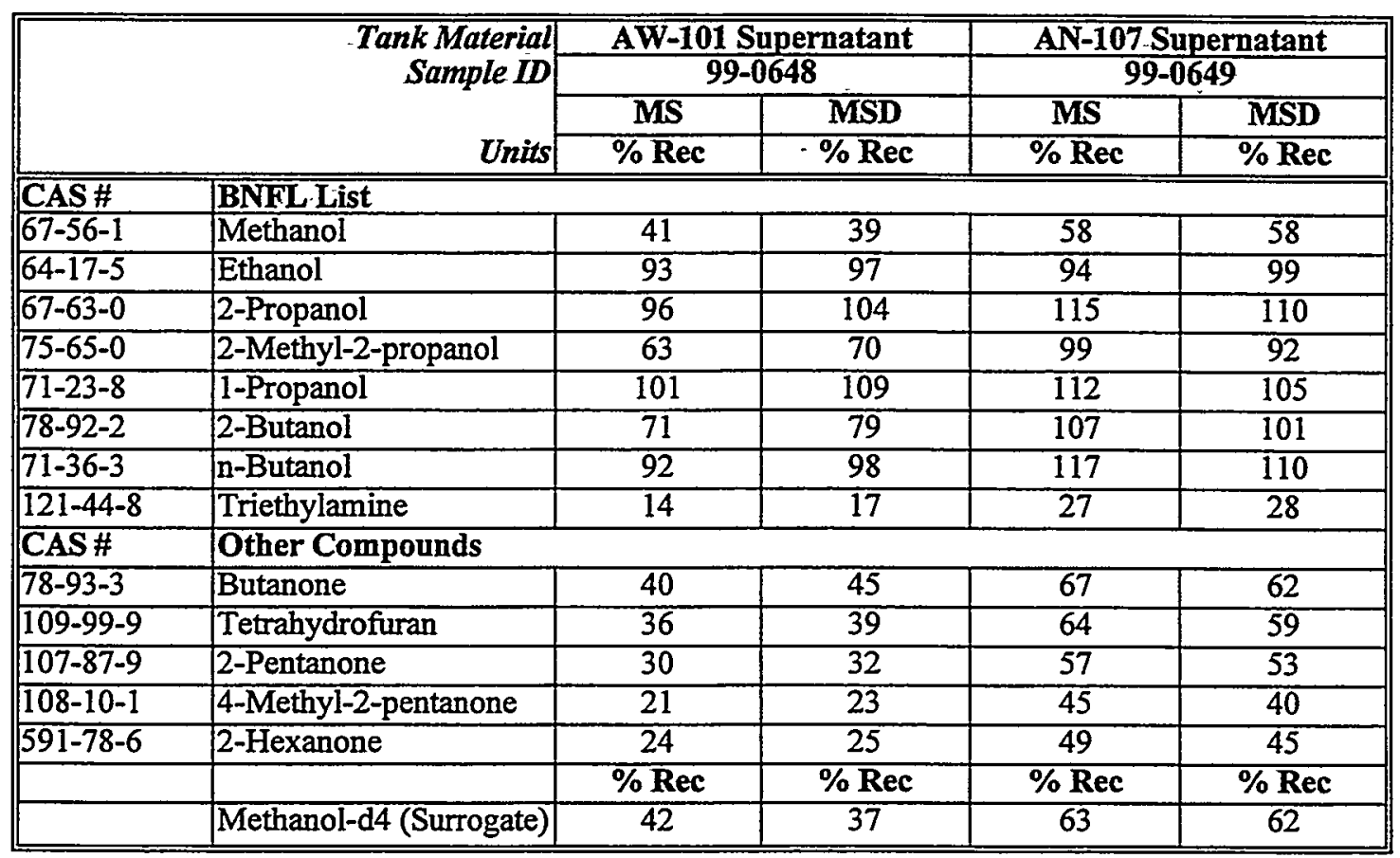

This method has typically performed well for methanol and shows promise as an effective technique for the analysis of triethylamine. Additional analytes that are normally analyzed utilizing this method, not requested by BNFL, were also included in the tables. Further development and evaluation will be necessary to determine the cause of the erratic performance for this technique. 


\subsection{REFERENCES}

Ashby, E.C. et al. 1994. Synthetic Waste Chemical Mechanism Studies, WHC-EP-0823, Westinghouse Hanford Company, Richland, Washington.

Barney, G.L. 1996. Solubilities of Significant Organic Compounds in HLW Tank Supernate Solutions-FY 1996 Progress Report, WHC-EP-0899, Westinghouse Hanford Company, Richland, Washington.

Camaioni, D.M., W.D. Samuels, J.C. Linehan, S.A. Clauss, A.K. Sharma, K.L. Wahl, J.A. Campbell. 1996. Organic Tanks Safety Program, FY 96 Waste Aging Studies, PNNL-11312, Pacific Northwest National Laboratory, Richland, Washington.

Campbell, J.A. 1997. Organic Analysis Progress Report FY 1997. PNNL-1738, Pacific Northwest National Laboratory, Richland, Washington.

Sharma, A.K., S.A. Clauss, G.M. Mong, K.L. Wahl, J.A. Campbell. 1998. Analysis And Quantification of Organic Acids in Simulated Hanford Tank Waste and Hanford Tank Waste. J. of Chromatogr, 805, 101-107. 


\section{APPENDIX A}

\section{VOLATILE ORGANIC ANALYSIS RESULTS}

1

AW-101 Supernatant - Forms 1A through 8A

AN-107 Supernatant - Forms 1A through 8A

AW-101 and AN-107 Solids - Forms 1A through 8A 
Lab Name: PNNL

Lab Code: PNNL

Matrix: (soil/water) SUPERNATAN

Sample. wt/vol: $\quad 5.000(\mathrm{~g} / \mathrm{mL}) \mathrm{ML}$

Level: (low/med) LOW

$\div$ Moisture: not dec.

GC Column: $\mathrm{DB}-62475 \mathrm{M} \times 2.55 \mathrm{UM}$ ID: 0.45 (mm)

Soil Extract Volume:

(UI)
Contract: BNEL

SAS NO.:
EPA SAMPLE NO.

AW-101

SDG No.: 990205

Lab Sample ID: 99-0648

Lab File ID: $\quad 99021207$

Date Received:

Date Analyzed: 02/12/99 (mm) Dilution Factor: 1.0

Soil Aliquot Volume: (uI) CONCENTRATION UNITS:

CAS NO. COMPOUND (ug/I or ug/Kg) UG/I

Q

\begin{tabular}{|c|}
\hline 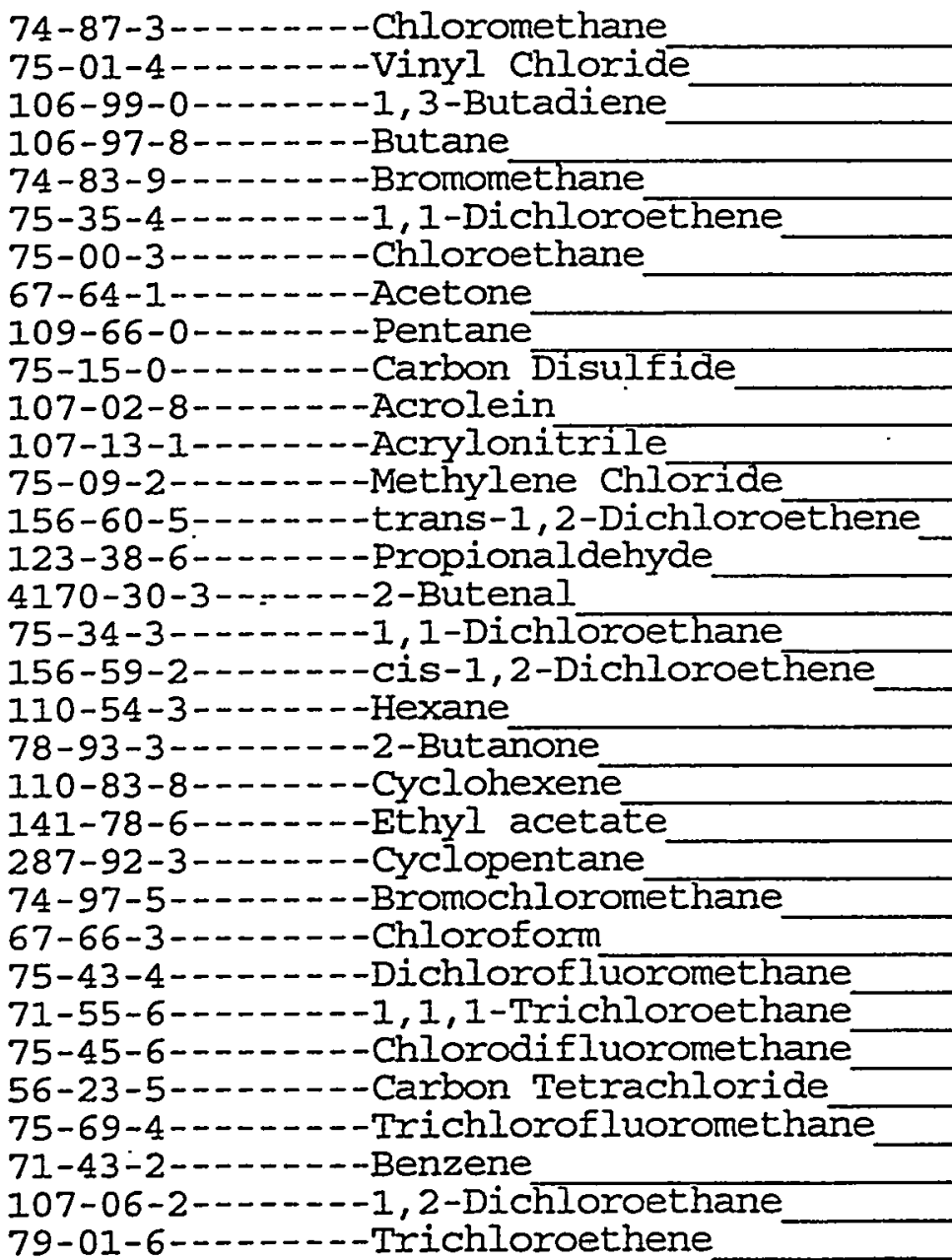 \\
\hline
\end{tabular}


Lab Name: PNNL

Iab Code: PNNL

Case No.:

Matrix: (soil/water) SUPERNATAN

Sample wt/vol:

$5.000(\mathrm{~g} / \mathrm{mL}) \mathrm{ML}$

Level: (low/med) IOW

$\div$ Moisture: not dec.

GC Column: DB-624 75M X.2.55UM ID: 0.45 (mm) (UI)
Contract: BNFL.

SAS NO. :
AW-101

SDG No.: 990205
Lab Sample ID: 99-0648

Lab File ID: $\quad 99021207$

Date Received:

Date Analyzed: 02/12/99
Soil Extract Volume:

CAS NO.
COMPOUND
Dilution Factor: 1.0

Soil Aliquot Volume: (uL)

\begin{tabular}{|c|}
\hline 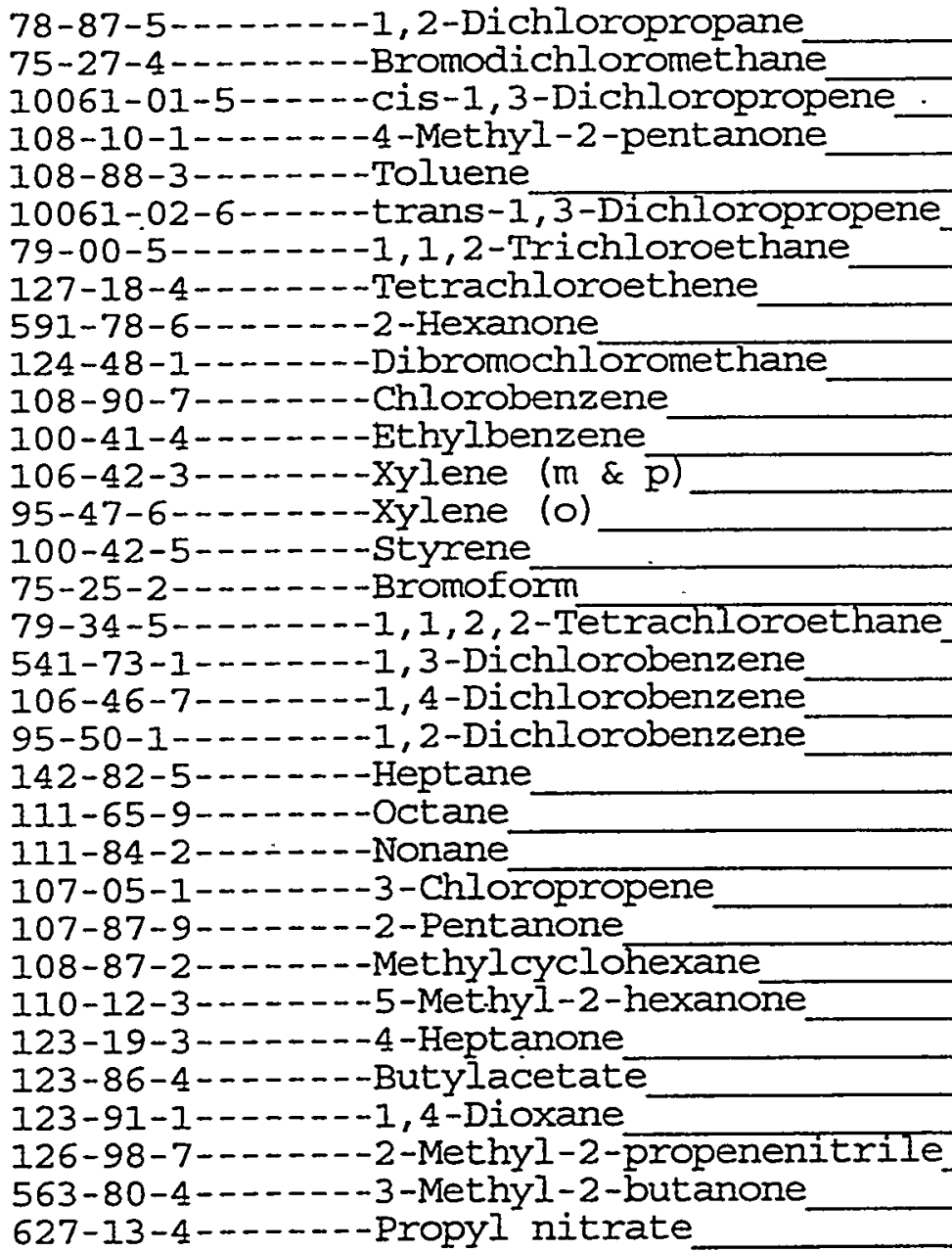 \\
\hline
\end{tabular}

CONCENTRATION UNITS: (ug/L or ug/Kg) UG/L

Q

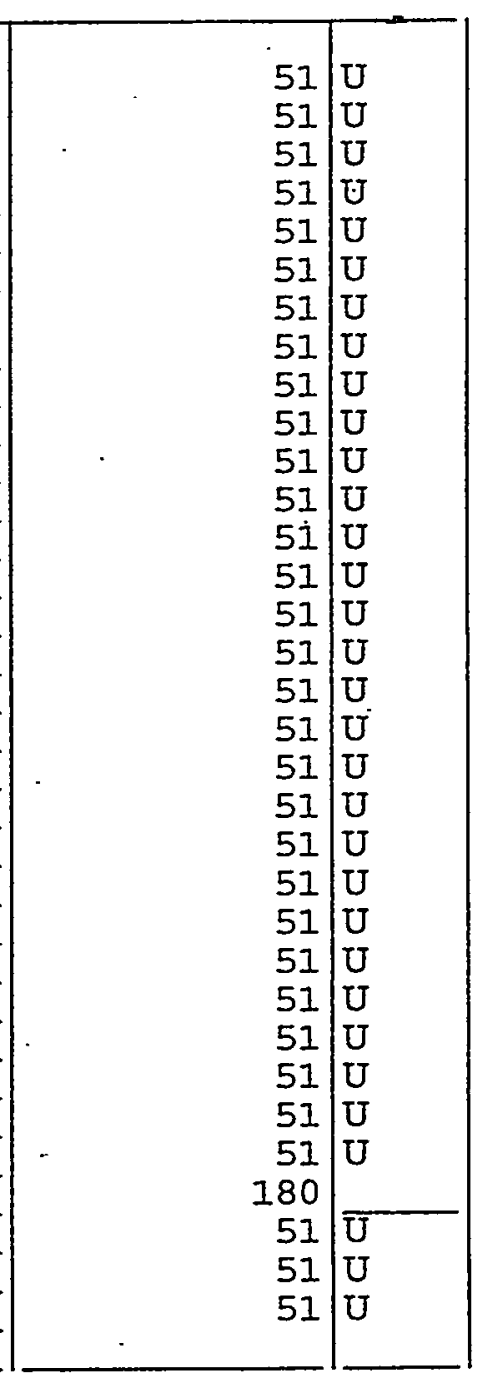


Lab Name: PNNL

Lab Code: PNNL

Case No.:

Matrix: (soil/water) SUPERNATAN

Sample wt/vol:

$5.000(\mathrm{~g} / \mathrm{mL}) \mathrm{ML}$

Level: (low/med) LOW

$\%$ Moisture: not dec.

GC Column: DB-624 75M X 2.55UM ID: 0.45 (mm)

Soil Extract Volume:

(uI)
Contract: BNFL

SAS NO: :
AW-101

SDG No.: 990205

Lab Sample ID: 99-0648

Lab File ID: 99021207

Date Received:

Date Analyzed: 02/12/99

(mm) Dilution Factor: 1.0

Soil Aliquot Volume: (Ut)

CONCENTRATION UNITS:

CAS NO. COMPOUND (ug/L or ug/Kg) UG/L

$51 \mathrm{U}$

$51 \mathrm{U}$

$51 \mathrm{U}$

$51 \mathrm{U}$

51 U

$51 \mathrm{U}$

51 U

$5 \mathrm{I} \mathrm{U}$

51 U

$5 I U$

51 U

$51 \mathrm{U}$

$51 \mathrm{U}$

$51 \mathrm{U}$

51 U

$51 \mathrm{U}$

$51 \mathrm{U}$

$5 I U$

51 U

51 U

$51 \mathrm{U}$

$51 \mathrm{U}$

51 U

51 U

51 U

51 U

51 U

$51 \mathrm{U}$

51 U

51 U

51 U

$51 \mathrm{U}$

140

109-99-9-...-- Tetrahydrofuran 
VOLATILE ORGANICS. ANALYSIS DATA SHEET

Lab Name: PNNL Contract: BNFL

AW-101.

Lab Code: PNNL Case No.:

SAS NO.:

SDG NO.: 990205

Matrix: (soil/water) SUPERNATAN

Lab Sample ID: 99-0648

Sample wt/vol: $\quad 5.000(\mathrm{~g} / \mathrm{mL}) \mathrm{ML}$

Lab File ID: $\quad 99021207$

Level: (low/med) LOW

Date Received:

$\because$ Moisture: not dec.

Date Analyzed: 02/12/99 .

GC Column: DB-624 75M X 2.55UM ID: 0.45 (mm)

Dilution Factor: 1.0

Soil Extract Volume: (uI)

Soil Aliquot Volume: (UT.)

CAS NO.

COMPOUND CONCENTRATION UNITS: (ug/I or ug/Kg) UG/L

$Q$

74-95-3--...-Dibromomethane 
Lab Name: PNNL

Lab Code: PNNL

Case No.:

Matrix: (soil/water) SUPERNATAN

Sample wt/vol:

$5.000(\mathrm{~g} / \mathrm{mL}) \mathrm{ML}$

Level:' (low/med) LOW

$\div$ Moisture: not dec.

GC Column: DB-624 75M X 2.55UM ID: 0.45 (mm)

Soil Extract Volume: (uL)
Contract : BNFL

SAS NO. :
$A W-101 D$

SDG No.: 990205

Lab Sample ID: 99-0648D

Lab File ID: 99021208

Date Received:

Date Analyzed: 02/12/99

Dilution Factor: 1:0

Soil Aliquot Volume:

(uW)

CAS NO. COMPOUND (ug/I or ug/Kg) UG/L \&

74-87-3--------Chloromethane

75-01-4------vinyl Chloride

106-99-0-...--1, 3-Butadiene

106-97-8--.-.--Butane

74-83-9--.-.---Bromomethane

75-35-4-- - - - 1, 1-Dichloroethene

75-00-3-...-.-Chloroethane

67-64-1-...-.-Acetone

109-66-0-...-.-Pentane

75-15-0-...--.-Carbon Disulfide

107-02-8------Acrolein

107-13-1-...-Acrylonitrile

75-09-2-...--.-Methylene Chloride

156-60-5--..--trans-1, 2-Dichloroethene

123-38-6-- - - - Propionaldehyde

4170-30-3----- -2-Butenal

75-34-3-...-. 1, 1-Dichloroethane

156-59-2-...-cis-1, 2-Dichloroethene

110-54-3---.-- Hexane

78-93-3-------2-Butanone

110-83-8--..--Cyclohexene

141-78-6-...--Ethyl acetate

287-92-3-..---Cyclopentane

74-97-5-.....-Bromochloromethane

67-66-3-...-.-Chloroform

75-43-4--.-.-Dichlorofluoromethane

71-55-6--..--1, 1, 1-Trichloroethane

75-45-6-....-Chlorodifluoromethane

56-23-5-...-.-Carbon Tetrachloride

75-69-4-...-- Trichlorofluoromethane

$71-43-2------$-Benzene

107-06-2--..--1, 2-Dichloroethane

79-01-6-_..-- Trichloroethene

\begin{tabular}{|r|l|l|}
56 & $\mathrm{U}$ \\
56 & $\mathrm{U}$ \\
56 & $\mathrm{U}$ \\
56 & $\mathrm{U}$ \\
56 & $\mathrm{U}$ \\
56 & $\mathrm{U}$ \\
56 & $\mathrm{U}$ \\
360 & \\
56 & $\mathrm{U}$ \\
56 & $\mathrm{U}$ \\
56 & $\mathrm{U}$ \\
56 & $\mathrm{U}$ \\
56 & $\mathrm{U}$ \\
56 & $\mathrm{U}$ \\
56 & $\mathrm{U}$ \\
3 & $\mathrm{~J}$ \\
56 & $\mathrm{U}$ \\
56 & $\mathrm{U}$ \\
56 & $\mathrm{U}$ \\
55 & $\mathrm{~J}$ \\
56 & $\mathrm{U}$ \\
56 & $\mathrm{U}$ \\
56 & $\mathrm{U}$ \\
56 & $\mathrm{U}$ \\
56 & $\mathrm{U}$ \\
56 & $\mathrm{U}$ \\
56 & $\mathrm{U}$ \\
7 & $\mathrm{JB}$ \\
56 & $\mathrm{U}$ \\
56 & $\mathrm{U}$ \\
56 & $\mathrm{U}$ \\
56 & $\mathrm{U}$ \\
56 & $\mathrm{U}$ \\
& \\
\hline
\end{tabular}


Lab Name : PNNL

Lab Code: PNNL

Matrix: (soil/water) SUPERNATAN

Sample wt/vol:

$5.000(\mathrm{~g} / \mathrm{mL}) \mathrm{ML}$.

Level: (low/med) LOW

$\%$ Moisture: not dec.

GC Column: $\mathrm{DB}-62475 \mathrm{M} \times 2.55 \mathrm{UM}$ ID: 0.45 (mm)

Soil Extract Volume:

(uI)
Contract : BNFL

SAS NO. :
AW-101D

Lab Sample ID: 99-0648D

Lab File ID: ' 99021208

Date Received:

Date Analyzed: 02/12/99 (mm) Dilution Factor: 1.0

Soil Aliquot Volume: (uL) CONCENTRATION UNITS:

CAS NO.

COMPOUND (ug/L or $u g / \mathrm{kg}) \mathrm{UG} / \mathrm{I}$

Q 
Lab Name: PNNL

Lab Code: PNNL

Matrix: (soil/water) SUPERNATAN

Sample wt/vol:

$5.000(\mathrm{~g} / \mathrm{mL}) \mathrm{ML}$

Level: (low $/ \mathrm{med}$ ) IOW

$\%$ Moisture: not dec.

GC Column: $\mathrm{DB}-62475 \mathrm{M} \times 2.55 \mathrm{UM}$ ID: 0.45 (mm) (uI)
Contract: BNFL

SÁS No. :
AW-101D

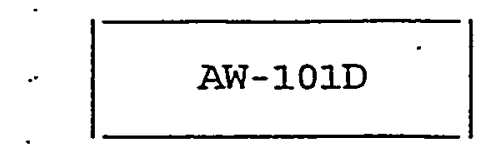

SDG No.: 990205
Lab Sample ID: 99-0648D

Lab File ID: $\quad 99021208$

Date Received:

Date Analyzed: 02/12/99
Soil Extract Volume:

CAS NO.

COMPOUND

Dilution Factor: 1.0

Soil Aliquot Volume: (UI) CONCENTRATION UNITS: (ug/L or $u g / \mathrm{Kg}$ ) UG/L

Q

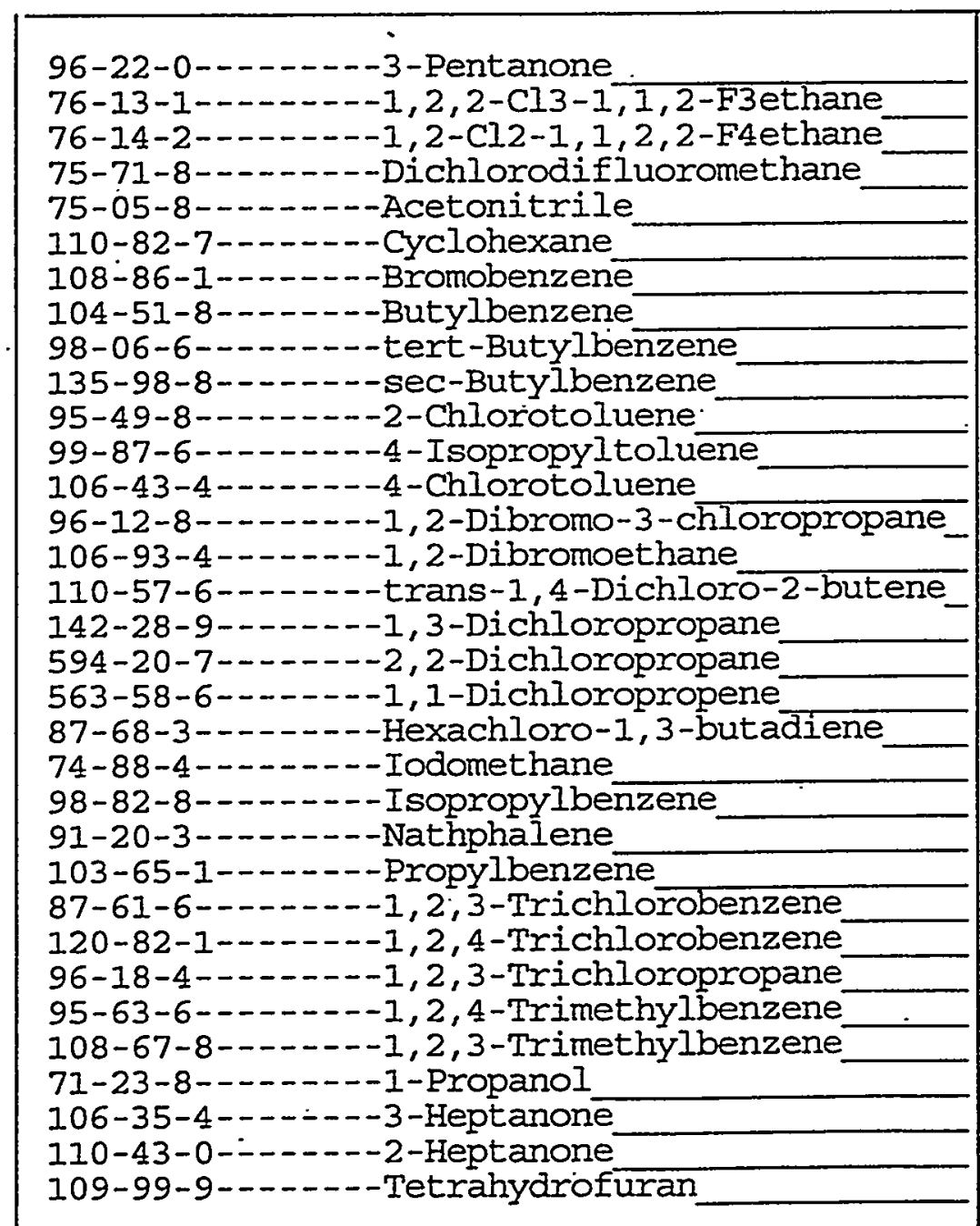

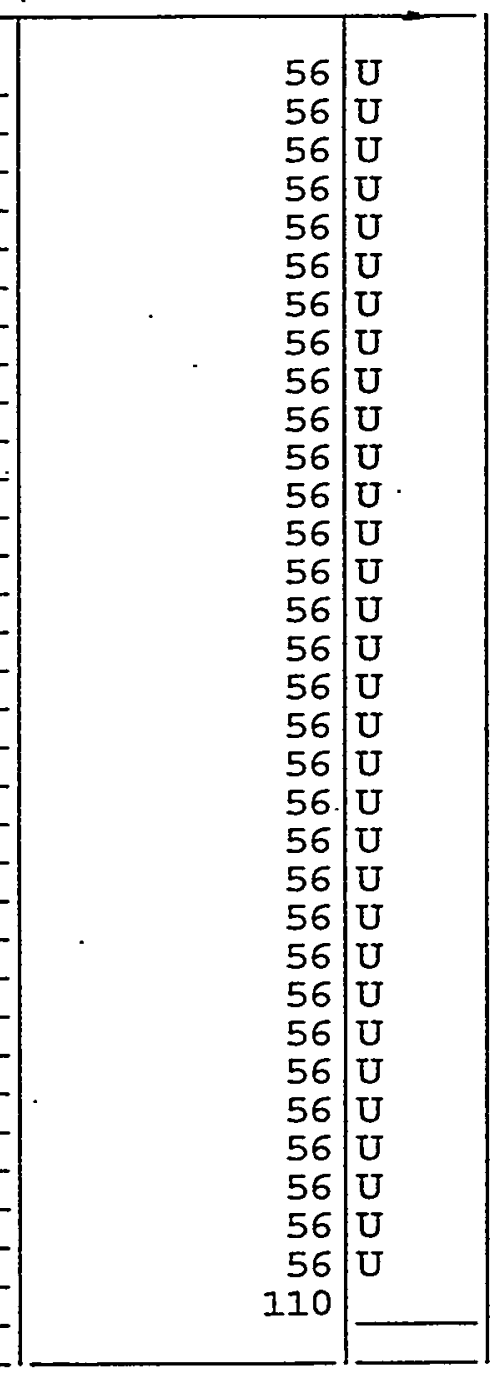


$1 \mathrm{~A}$

VOIATILE ORGAN̈ICS ANALYSIS DATA SHEET

Lab Name: PNNL

Contract: BNFL

Case No.:

SAS NO. :

Lab Sample ID: 99-0648D

Lab.File ID: 99021208

Level: (low/med) Low

Date Received:

$\%$ Moisture: not dec.

Date Analyzed: 02/12/99

GC Column: DB-624 75M X 2.55UM ID: 0.45

Soil Extract Volume: (uI)

(mm)

Soil Aliquot Volume: CONCENTRATION UNITS:

CAS NO.

COMPOUND

(ug/L or $\mathrm{ug} / \mathrm{Kg}$ ) UG/L (uL)

AW-101D

SDG No.: 990205

Dilution Factor: 1.0

74-95-3--...--Dibromomethane 560 
Lab Name: PNNL

Lab Code: PNNL

Matrix: (soil/water) SUPERNATAN

Sample wt/vol: $\quad 5.000(\mathrm{~g} / \mathrm{mL}) \mathrm{ML}$

Level: (low/med) Iow

$\%$ Moisture: not dec.

GC Column: DB-62475M X 2.55UM ID: 0.45 (mm)

Soil Extract Volume:

(uI)
Contract: BNFL

SAS NO.:
EPA SAMPLE NO.

AW-101MS

SDG No. : 990205

Lab Sample ID: 99-0648MS

Lab File ID: 99021209

Date Received:

Date Analyzed: 02/12/99

(mm) Dilution Factor: 1.0

Soil Aliquot Volume:

(uL)

CAS NO. COMPOUND $(u g / L$ or $u g / K g) ~ U G / L$

$Q$

\begin{tabular}{|c|}
\hline 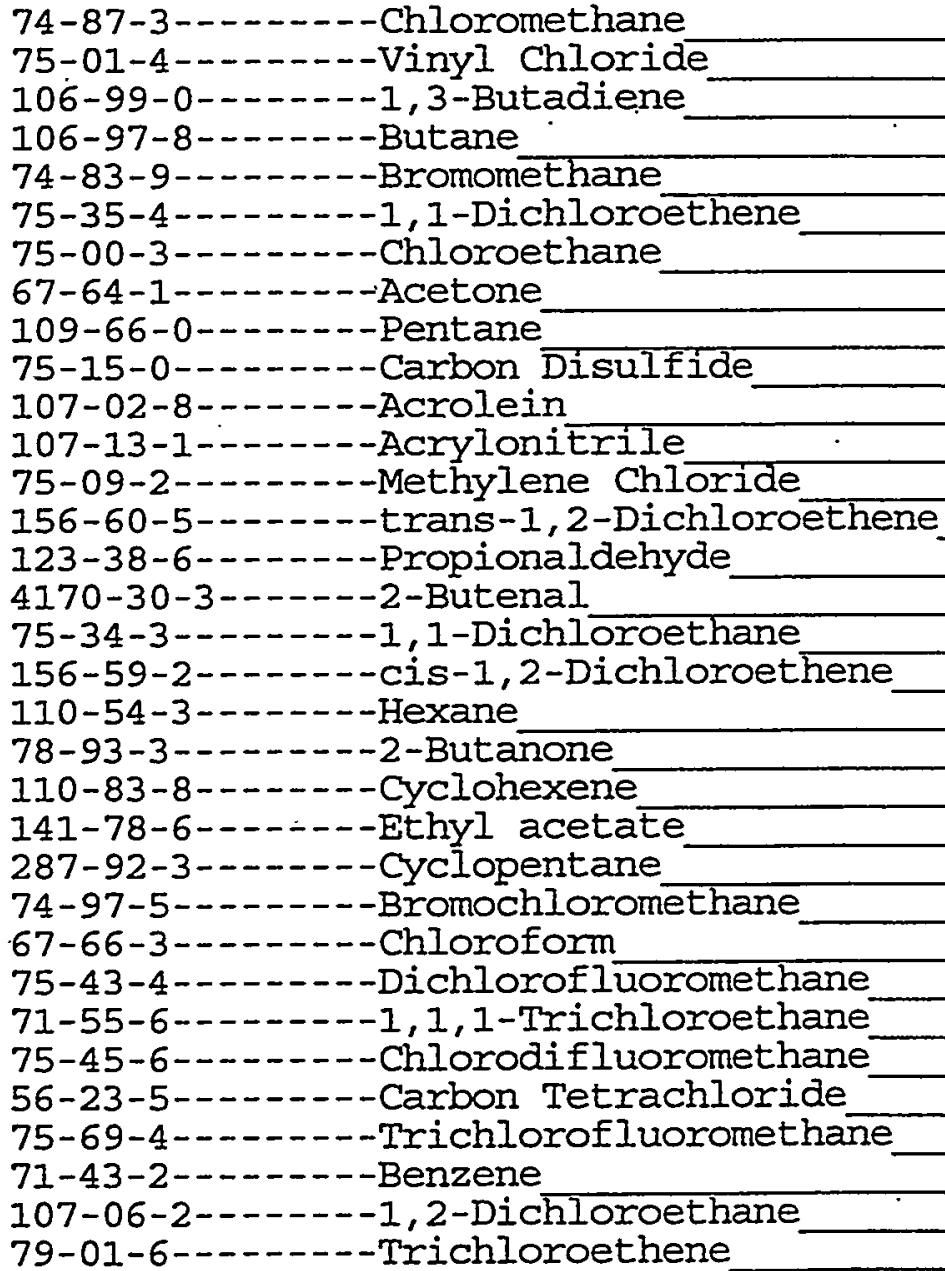 \\
\hline
\end{tabular}

\begin{tabular}{rr|l|}
89 & \\
100 & \\
90 & \\
110 & \\
30 & $J$ \\
170 & \\
87 & \\
330 & $\mathrm{E}$ \\
120 & \\
41 & $\mathrm{~J}$ \\
11 & $\mathrm{~J}$ \\
13 & $\mathrm{~J}$ \\
96 & \\
440 & \\
340 & $\mathrm{E}$ \\
20 & $\mathrm{~J}$ \\
100 & \\
480 & \\
98 & \\
340 & $\mathrm{E}$ \\
97 & \\
140 & \\
97 & \\
520 & \\
52 & $\mathrm{~J}$ \\
40 & $\mathrm{~J}$ \\
110 & \\
52 & $\mathrm{JB}$ \\
100 & \\
95 & \\
94 & \\
100 & \\
180 & - \\
\hline
\end{tabular}


Lab Name: PNNL

Lab Code: PNNL
Contract : BNFL

SAS NO: :
EPA SAMPLE NO.

AW-101MS
Matrix: (soil/water) SUPERNATAN

Sample wt/vol: $\quad 5.000(\mathrm{~g} / \mathrm{mL}) \mathrm{ML}$

Level: (low/med) LOW

$\%$ Moisture: not dec.

GC Column: $\mathrm{DB}-62475 \mathrm{M} \times 2.55 \mathrm{UM}$ ID: 0.45 (mm) (uL)
Soil Extract Volume:
Lab Sample ID: 99-0648MS

Lab File ID: 99021209

Date Received:

Date Analyzed: 02/12/99
Dilution Factor: 1.0

Soil Aliquot Volume: (uL)

CAS NO.

COMPOUND

CONCENTRATION UNITS:

(ug/L or ug/Kg) UG/L

\begin{tabular}{|c|}
\hline 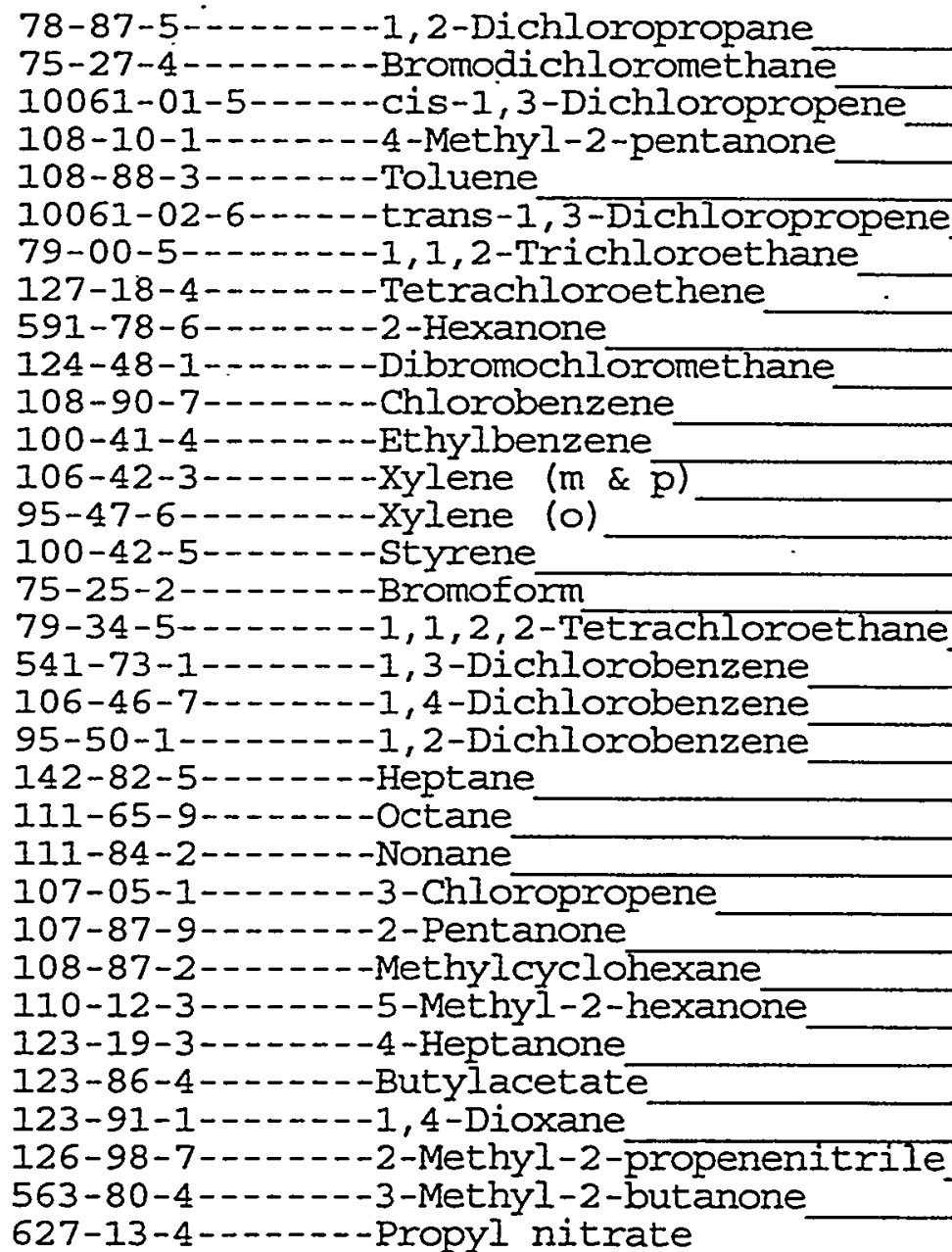 \\
\hline
\end{tabular}

\begin{tabular}{rr|l}
100 & \\
11 & $J$ \\
15 & $J$ \\
330 & $\mathrm{E}$ \\
100 & $\overline{27}$ & $\mathrm{~J}$ \\
0.9 & $\mathrm{~J}$ \\
87 & \\
390 & $\mathrm{E}$ \\
3 & $\mathrm{~J}$ \\
84 & \\
80 & \\
84 & \\
74 & \\
77 & \\
2 & $\mathrm{~J}$ \\
0.3 & $\mathrm{~J}$ \\
400 & \\
350 & \\
410 & \\
96 & \\
96 & \\
78 & \\
70 & \\
300 & $\mathrm{E}$ \\
100 & \\
140 & \\
250 & $\mathrm{E}$ \\
170 & \\
270 & $\mathrm{E}$ \\
140 & \\
280 & $\mathrm{E}$ \\
110 & \\
\hline
\end{tabular}


Lab Name: PNNL

Iab Code: PNNL

Case No.:

Matrix: (soil/water) SUPERNATAN

Sample wt/vol:

$5.000(\mathrm{~g} / \mathrm{mL}) \mathrm{ML}$

Ievel: (low/med) LOW

$\div$ Moisture: not dec.

GC Column: $\mathrm{DB}-62475 \mathrm{M} \times 2.55 \mathrm{UM}$ ID: 0.45 (mm)

Soil Extract Volume: (uL)
Contract: BNFL

SAS NO.:
EPA SAMPLE NO.

AW-10IMS

SDG NO.: 990205

Lab Sample ID: 99-0648MS

Lab File ID: 99021209

Date Received:

Date Analyzed: 02/12/99

(mm) Dilution Factor: 1.0

Soil Aliquot Volume: (uL)

CONCENTRATION UNITS:

CAS NO. COMPOUND (ug/L or $\mathrm{ug} / \mathrm{Kg}$ ) UG/L

\begin{tabular}{r|l|}
\multicolumn{2}{|l}{$Q$} \\
310 & $\mathrm{E}$ \\
110 & \\
98 & \\
73 & $\square$ \\
100 & \\
510 & \\
290 & \\
430 & \\
390 & \\
400 & \\
330 & \\
360 & \\
52 & $\mathrm{U}$ \\
47 & $\mathrm{~J}$ \\
9 & $\mathrm{~J}$ \\
680 & \\
610 & \\
480 & \\
270 & \\
290 & \\
380 & \\
740 & \\
400 & \\
380 & \\
320 & - \\
65 & \\
350 & \\
390 & \\
1000 & \\
290 & $\mathrm{E}$ \\
320 & $\mathrm{E}$ \\
290 & $\mathrm{E}$ \\
\hline & \\
\hline
\end{tabular}

96-22-0-- - - - - 3-Pentanone

76-13-1-..---1,2,2-C13-1, 1,2-F3ethane

76-14-2--.---1,2-Cl2-1, 1,2,2-F4ethane

75-71-8-....-Dichlorodifluoromethane

75-05-8-.----Acetonitrile

110-82-7--.----Cyclohexane

108-86-1-..----Bromobenzene

104-51-8--.----Butylbenzene

98-06-6-...--tert-Butylbenzene

135-98-8--.---sec-Butylbenzene

95-49-8-..-- 2-Chlorotoluene

99-87-6.....-4-Isopropyltoluene

106-43-4-..----4-Chlorotoluene

96-12-8-...--1, 2-Dibromo-3-chloropropane

106-93-4-------1,2-Dibromoethane

110-57-6-...--trans-1, 4-Dichloro-2-butene

142-28-9--.--1, 3-Dichloropropane

594-20-7--.---2,2-Dichloropropane

563-58-6--.---1,1-Dichloropropene

87-68-3-...--Hexachloro-1, 3-butadiene

74-88-4-...-.--Iodomethane

98-82-8-.-.---Isopropylbenzene

91-20-3-...--Nathphalene

103-65-1-.-.---Propylbenzene

87-61-6-...--1,2,3-Trichlorobenzene

120-82-1-...-.-1,2,4-Trichlorobenzene

96-18-4-...--1, 2, 3-Trichloropropane

95-63-6--.----1, 2,4-Trimethylbenzene

108-67-8-.---1, 2,3-Trimethylbenzene

71-23-8-...--1-Propanol

106-35-4-..---3-Heptanone

110-43-0------2-Heptanone

109-99-9---.---Tetrahydrofuran

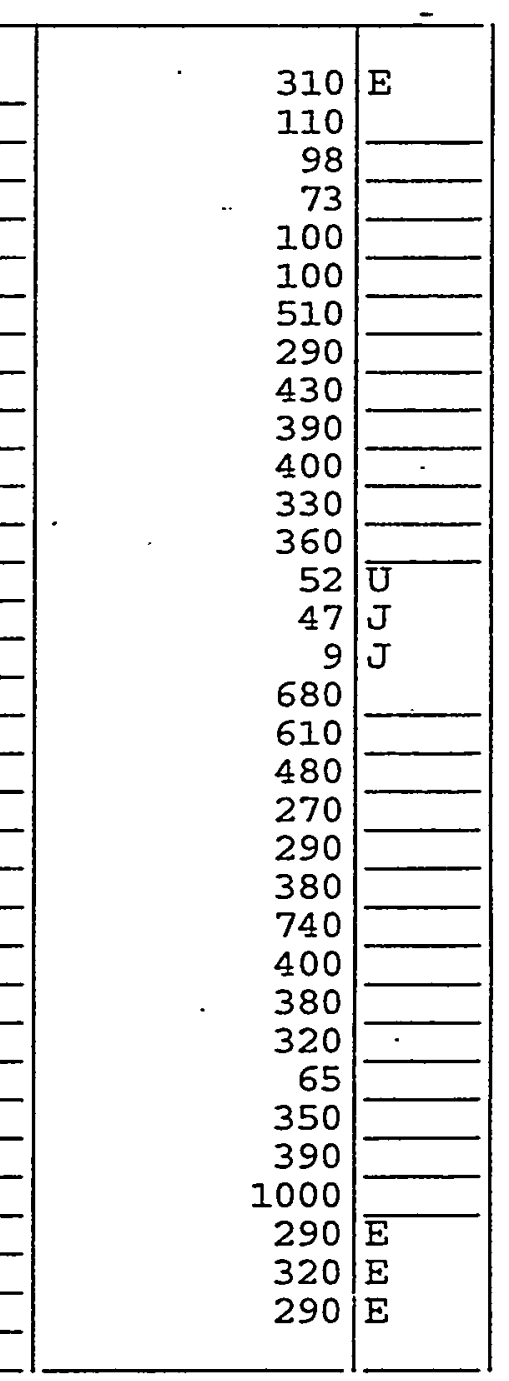


VOLATILE ORGANICS ANALYSIS DATA SHEET

Lab Name: PNNLi

Lab Code: PNNL

Case No.:

Matrix: (soil/water) SUPERNATAN

Sample wt/vol:

$5.000(\mathrm{~g} / \mathrm{mL}) \mathrm{ML}$

Level: (low/med) LOW

$\div$ Moisture: not dec.

GC Column: DB-624 75M X 2.55UM ID: 0.45 (mm) Soil Extract Volume: (uI)
Contract: BNFL

SAS NO.:
EPA SAMPLE NO.

AW-101MS

SDG NO.: 990205

Lab Sample ID: 99-0648MS

Lab File ID: 99021209

Date Received:

Date Analyzed: 02/12/99

Dilution Factor: 1.0

Soil Aliquot Volume: (uL) CONCENTRATION UNITS:

CAS NO. COMPOUND (ug/L or $\mathrm{ug} / \mathrm{Kg}$ ) UG/I

Q

74-95-3-------Dibromomethane 
Lab Name: PNNL

Lab Code: PNNL

Matrix: (soil/water) SUPERNATAN

Sample wt/vol: $\quad 5.000(\mathrm{~g} / \mathrm{mL}) \mathrm{ML}$

Level: (low/med) IOW

$\%$ Moisture: not dec. _GC Column: DB-624 75M X 2.55UM ID: 0.45 (mm)

Soil Extract Volume:

(UI)
Case No.: SAS No.:
Contract: BNFL
EPA SAMPLE NO.

AW-101MSD
Lạb Sample ID: 99-0648MSD

Lab File ID: $\quad 99021210$

Date Received:

Date Analyzed: 02/12/99

Dilution Factor: 1.0

CAS NO.

COMPOUND

(ug/I or $u g / \mathrm{Kg}$ ) UG/L

74-87-3-...--Chloromethane

75-01-4-..----Vinyl Chloride

106-99-0-..---1,3-Butadiene

106-97-8-...--Butane

74-83-9-....-. Bromomethane

75-35-4-...--1, 1-Dichloroethene

75-00-3-..-.--Chloroethane

67-64-1......-Acetone

109-66-0-..---Pentane

75-15-0-...---Carbon Disulfide

107-02-8-...--Acrolein

107-13-1-...--Acrylonitrile

75-09-2-...--Methylene Chloride

156-60-5--..--trans-1,2-Dichloroethene

123-38-6--.----Propionaldehyde

4170-30-3-..--2-Butenal

75-34-3-...--1, I-Dichloroethane

156-59-2-..---Cis-1, 2-Dichloroethene

$110-54-3-\cdots---H e x a n e$

78-93-3--.----2-Butanone

110-83-8-..---Cyclohexene

141-78-6-...-.-Ethyl acetate

287-92-3-.----Cyclopentane

74-97-5-...---Bromochloromethane

67-66-3-....-.-Chloroform

75-43-4-...--Dichlorofluoromethane

71-55-6-...--1,1,1-Trichloroethane

75-45-6-..-.-.-Chlorodifluoromethane

56-23-5-...--.-Carbon Tetrachloride

75-69-4-...-- - Trichlorofluoromethane

71-43-2--.----Benzene

107-06-2--.--1,2-Dichloroethane

79-01-6-...-.-Trichloroethene
OLM03.0

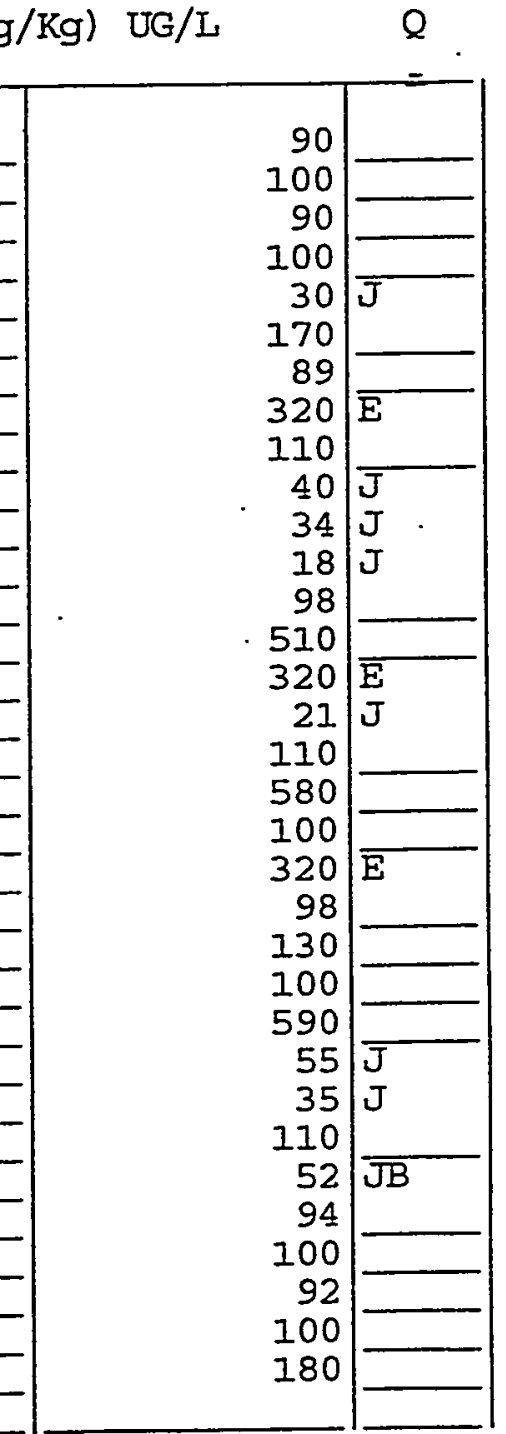


Lab Name: PNNL

Lab Code: PNNL
Contract: BNFL

SAS NO.:

AW-101MSD .

Case No. :

Matrix: (soil/water) SUPERNATAN

Sample wt/vol: $\quad 5.000(\mathrm{~g} / \mathrm{mL})$ ML

Level: (low/med) LOW

$\because$ Moisture: not dec.

GC Column: $\mathrm{DB}-62475 \mathrm{M} \times 2.55 \mathrm{UM}$ ID: 0.45 (mm) (UL)
Lab Sample ID: 99-0648MSD

Lab File ID: 99021210

Date Received:

Date Analyzed: 02/12/99
Soil Extract Volume:

CAS NO.
COMPOUND
Dilution Factor: 1.0

Soil Aliquot Volume: (UL)

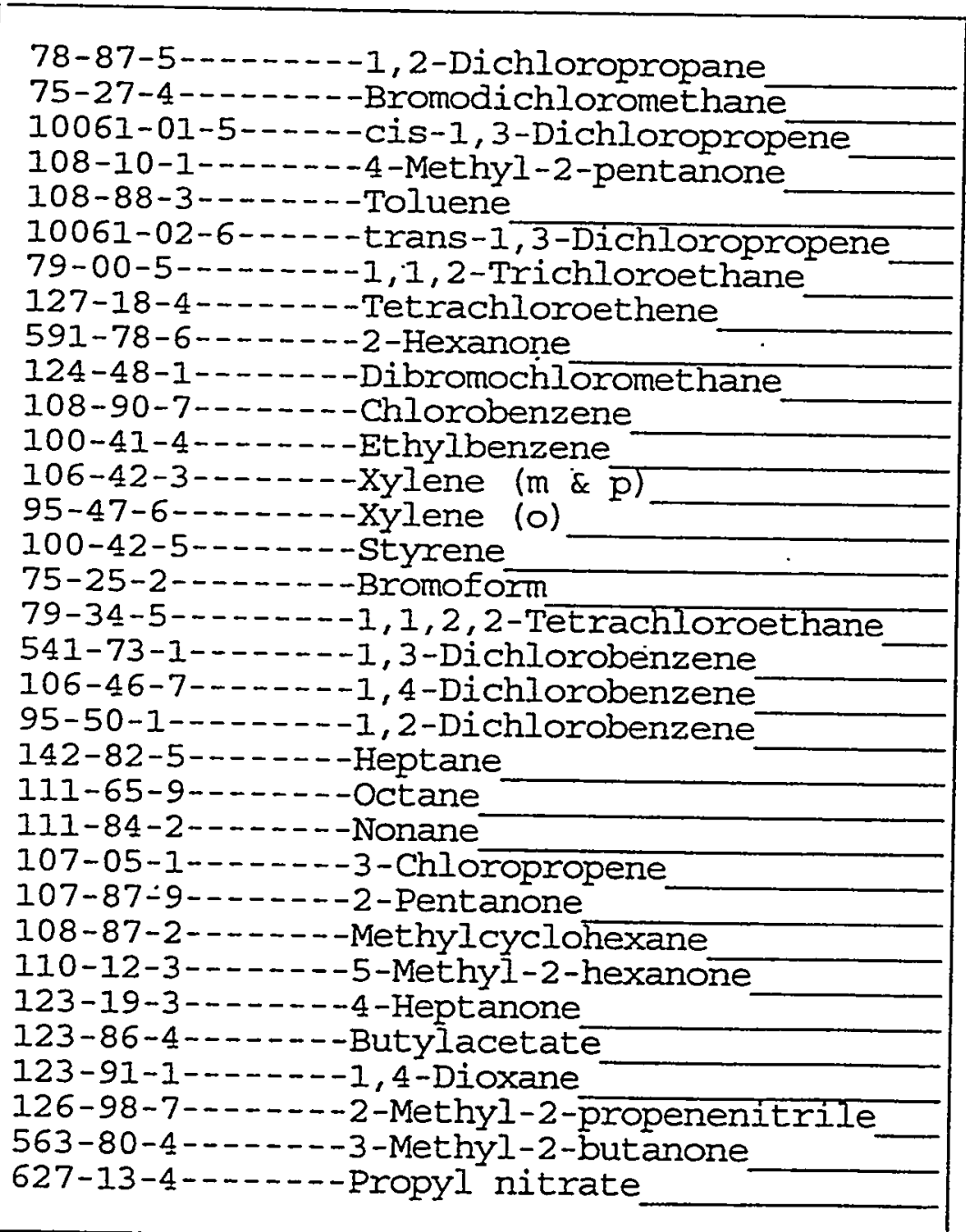
CONCENTRATION UNITS: (ug/L or ug/Kg) UG/L

-

\begin{tabular}{r|l}
\multicolumn{2}{|l}{$Q$} \\
100 & \\
7 & $\mathrm{~J}$ \\
14 & $\mathrm{~J}$ \\
290 & $\mathrm{E}$ \\
100 & \\
29 & $\mathrm{~J}$ \\
0.9 & $\mathrm{~J}$ \\
85 & \\
340 & $\mathrm{E}$ \\
7 & $\mathrm{~J}$ \\
88 & \\
86 & \\
85 & \\
77 & \\
80 & \\
2 & $\mathrm{~J}$ \\
4 & $\mathrm{~J}$ \\
520 & \\
460 & \\
530 & \\
97 & \\
97 & \\
80 & \\
73 & \\
260 & $\mathrm{E}$ \\
90 & \\
120 & \\
240 & \\
150 & \\
230 & $\mathrm{E}$ \\
130 & \\
250 & $\mathrm{E}$ \\
110 & \\
\hline & \\
\hline
\end{tabular}


Lab Name: PNNL

Lab Code: PNNL

Case No.:

Matrix: (soil/water) SUPERNATAN

Sample wt/vol: $\quad 5.000(\mathrm{~g} / \mathrm{mL}) \mathrm{ML}$

Level: (low/med) IOW

\% Moisture: not dec.

GC Column: $\mathrm{DB}-62475 \mathrm{M} \times 2.55 \mathrm{UM}$ ID: 0.45 (mm) (UI)

Soil Extract Volume:
Contract: BNFL

SAS NO.:
AW-I0IMSD

SDG No.: 990205

Lab Sample ID: 99-0648MSD

Lab File ID: 99021210

Date Received:

Date Analyzed: 02/12/99

Dilution Factor: 1.0

Soil Aliquot Volume:

CAS NO.

COMPOUND CONCENIRATION UNITS: (ug/L or $u g / \mathrm{kg}$ ) UG/L

\begin{tabular}{r|l|}
\multicolumn{2}{|l}{$Q$} \\
260 & $\mathrm{E}$ \\
110 & \\
100 & \\
89 & \\
110 & \\
100 & \\
630 & \\
380 & \\
570 & \\
510 & \\
540 & \\
450 & \\
490 & \\
59 & $\mathrm{U}$ \\
49 & $\mathrm{~J}$ \\
28 & $\mathrm{~J}$ \\
730 & \\
700 & \\
570 & \\
350 & \\
330 & \\
470 & \\
900 & \\
540 & \\
460 & \\
390 & \\
85 & \\
480 & \\
520 & \\
1000 & \\
260 & $\mathrm{Z}$ \\
300 & $\mathrm{E}$ \\
250 & $\mathrm{E}$ \\
& \\
\hline
\end{tabular}


$1 A$

VOLATILE ORGANICS ANALYSIS DATA SHEET

Lab Name: PNNL

Iab code: PNNL

Case No.:

Matrix: (soil/water) SUPERNATAN

Sample wt/vol:

$5.000(\mathrm{~g} / \mathrm{mL}) \mathrm{ML}$

Level: (low/med) IOW

\% Moisture: not dec.

GC Column: DB-624 75M X 2.55UM ID: 0.45 (mm)

Soil Extract Volume: (UI)

CAS NO.

COMPOUND
EPA SAMPLE NO.

AW-101MSD

SDG NO.: 990205

Lab Sample ID: 99-0648MSD

Lab File ID: 99021210

Date Received:

Date Analyzed: 02/12/99

Dilution Factor: 1.0

Soil Aliquot Volume: (uL) CONCENTRATION UNITS: (ug/I or ug/Kg) UG/L

Q

660 
Lab Name: PNNL

Lab Code: PNNL Case No.:

Matrix: (soil/water) SUPERNATAN

Sample wt/vol:

$5.000(\mathrm{~g} / \mathrm{mL}) \mathrm{ML}$

Level: (low/med) LOW

Contract: BNFI

SAS NO: :

Lab Sample ID: VBLKOI

Lab File ID: 99021204

Date Received:

Date Analyzed: 02/12/99

$\%$ Moisture: not dec.

GC Column: DB-624 75M X 2.55UM ID: 0.45 (mm)

Dilution Factor: 1.0

Soil Extract Volume: (uL)

Soil Aliquot Volume: (UI)
CAS NO. COMPOUND

$(\mathrm{ug} / \mathrm{L}$ or $\mathrm{ug} / \mathrm{Kg}) \mathrm{UG} / \mathrm{L}$
METHOD BLANK

SDG No.: 990205

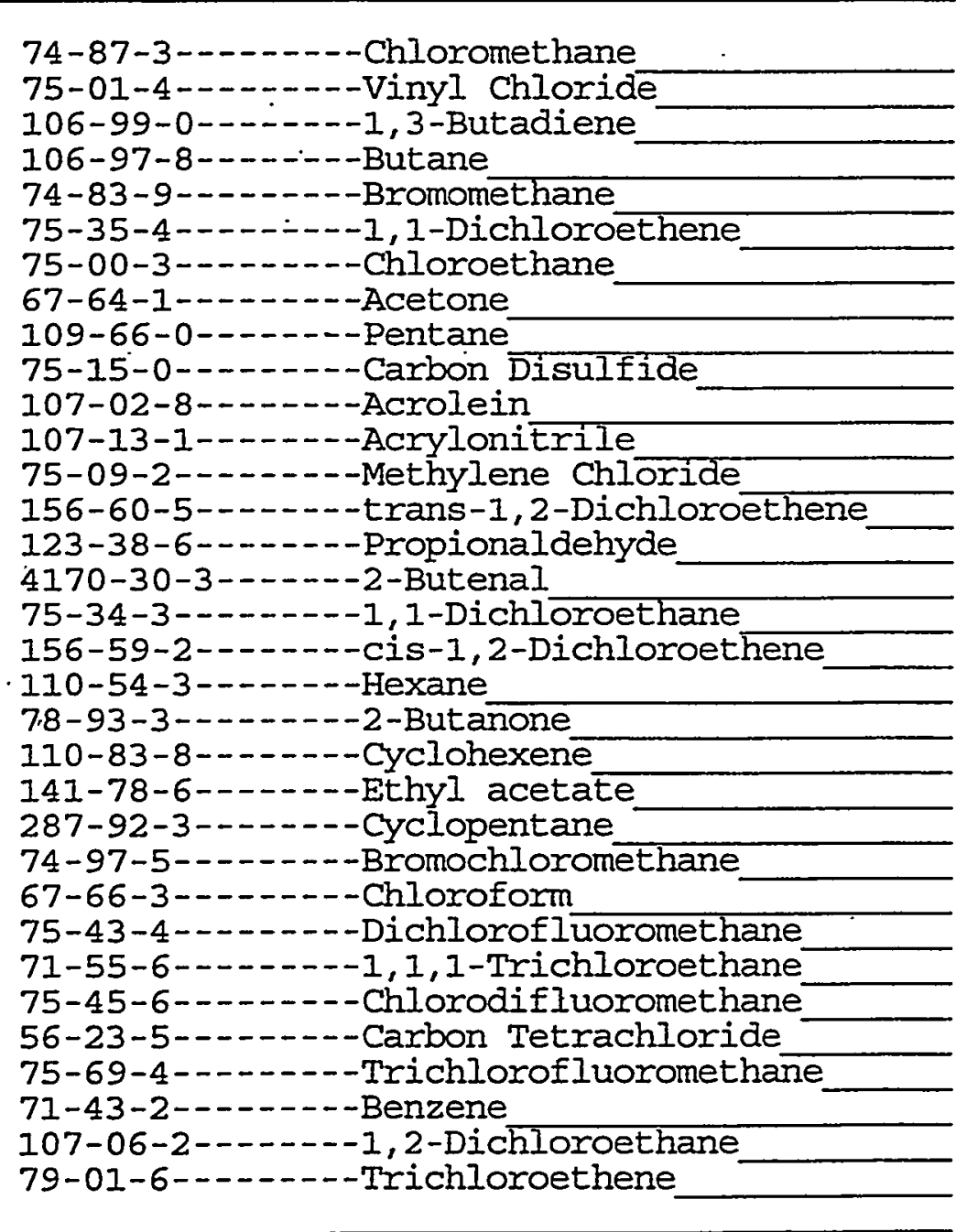

$10 \mathrm{U}$

$10 \mathrm{U}$

$10 \mathrm{U}$

$10 \mathrm{U}$

$10 \mathrm{U}$

$10 \mathrm{U}$

$10 \mathrm{U}$

$10 \mathrm{U}$

$10 \mathrm{U}$

$10 \mathrm{U}$

$10 \mathrm{U}$

$10 \mathrm{U}$

$10 \mathrm{U}$

$10 \mathrm{U}$

$10 \mathrm{U}$

$10 \mathrm{U}$

$10 \mathrm{U}$

$10 \mathrm{U}$

$10 \mathrm{U}$

$10 \mathrm{U}$

$10 \mathrm{U}$

$10 \mathrm{U}$

$10 \mathrm{U}$

$10 \mathrm{U}$

$10 \mathrm{U}$

$10 \mathrm{U}$

$10 \mathrm{U}$

$5 \mathrm{~J}$

$10 \mathrm{U}$

$10 \mathrm{U}$

$10 \mathrm{U}$

$10 \mathrm{U}$

$10 \mathrm{U}$ 
Lab Name: PNNL,

Lab Code: PNNL

Matrix: (soil/water) SUPERNATAN

Sample wt/vol: $\quad 5.000(\mathrm{~g} / \mathrm{mL}) \mathrm{ML}$

Level: (low/med) LOW

\% Moisture: not dec.

GC Column: DB-624 75M X 2.55UM ID: 0.45 (mm) Soil Extract Volume: (UL)

CAS NO.
Contract : BNFL

SAS NO.:

SDG No. : 990205

Lab Sample ID: VBLKOI

Lab File ID: 99021204

Date Received:

Date Analyzed: 02/12/99

Dilution Factor: 1.0

Soil Aliquot Volume: (uL)

CONCENTRATION UNITS:

(ug/L or ug/Kg) UG/L

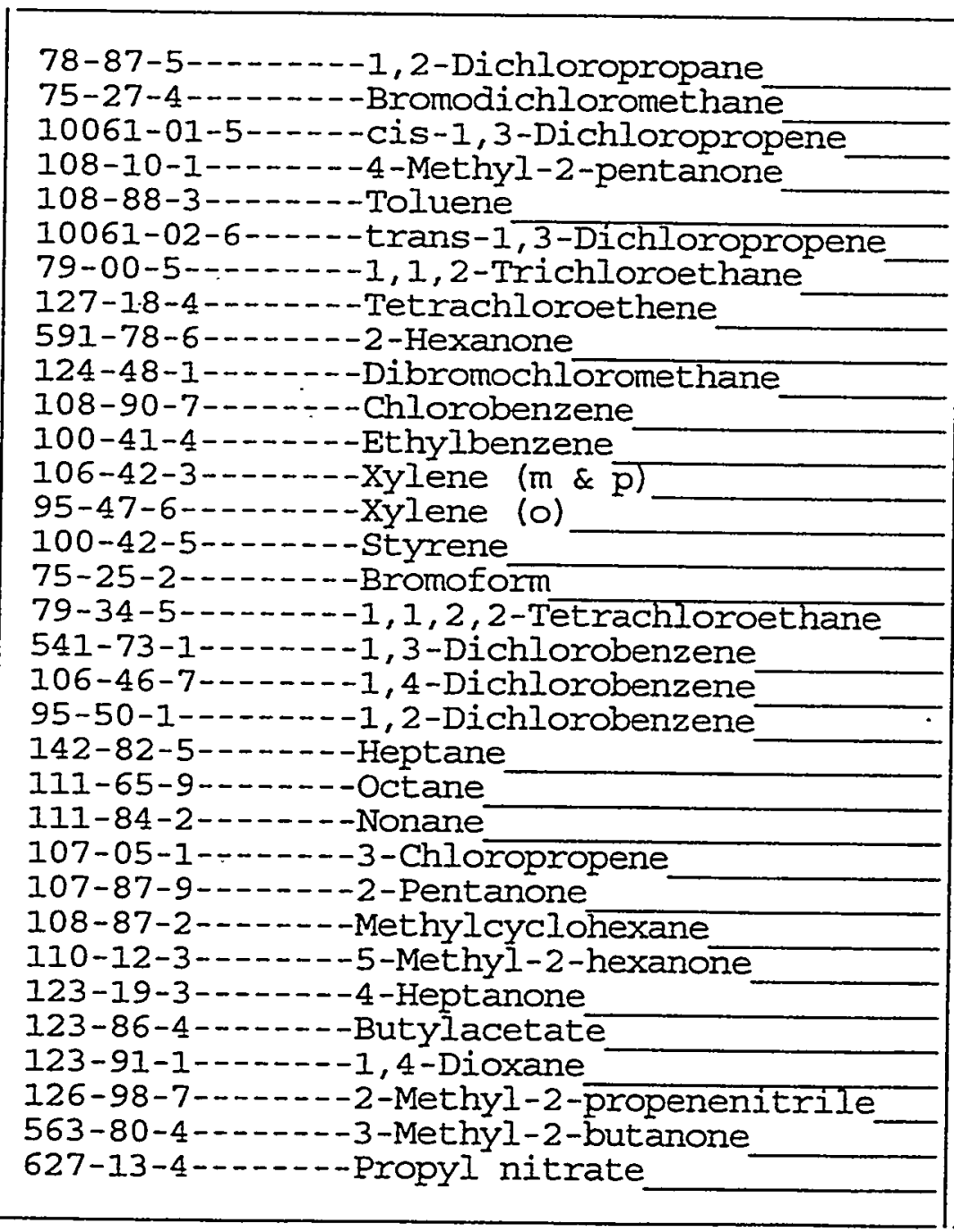


Lab Name: PNNL

Lab Code: PNNL

Case No.:

Matrix: (soil/water) SUPERNATAN

Sample wt/vol:

$5.000(\mathrm{~g} / \mathrm{mL}) \mathrm{ML}$

Ievel: (low/med) LOW

$\div$ Moisture: not dec.

GC Column: $\mathrm{DB}-62475 \mathrm{M} \times 2.55 \mathrm{UM}$ ID: 0.45 (mm) (uL)

Soil Extract Volume:
Contract: BNFL

SAS NO.:
EPA SAMPLE NO.

METHOD BLANK

CONCENTRATION UNITS:

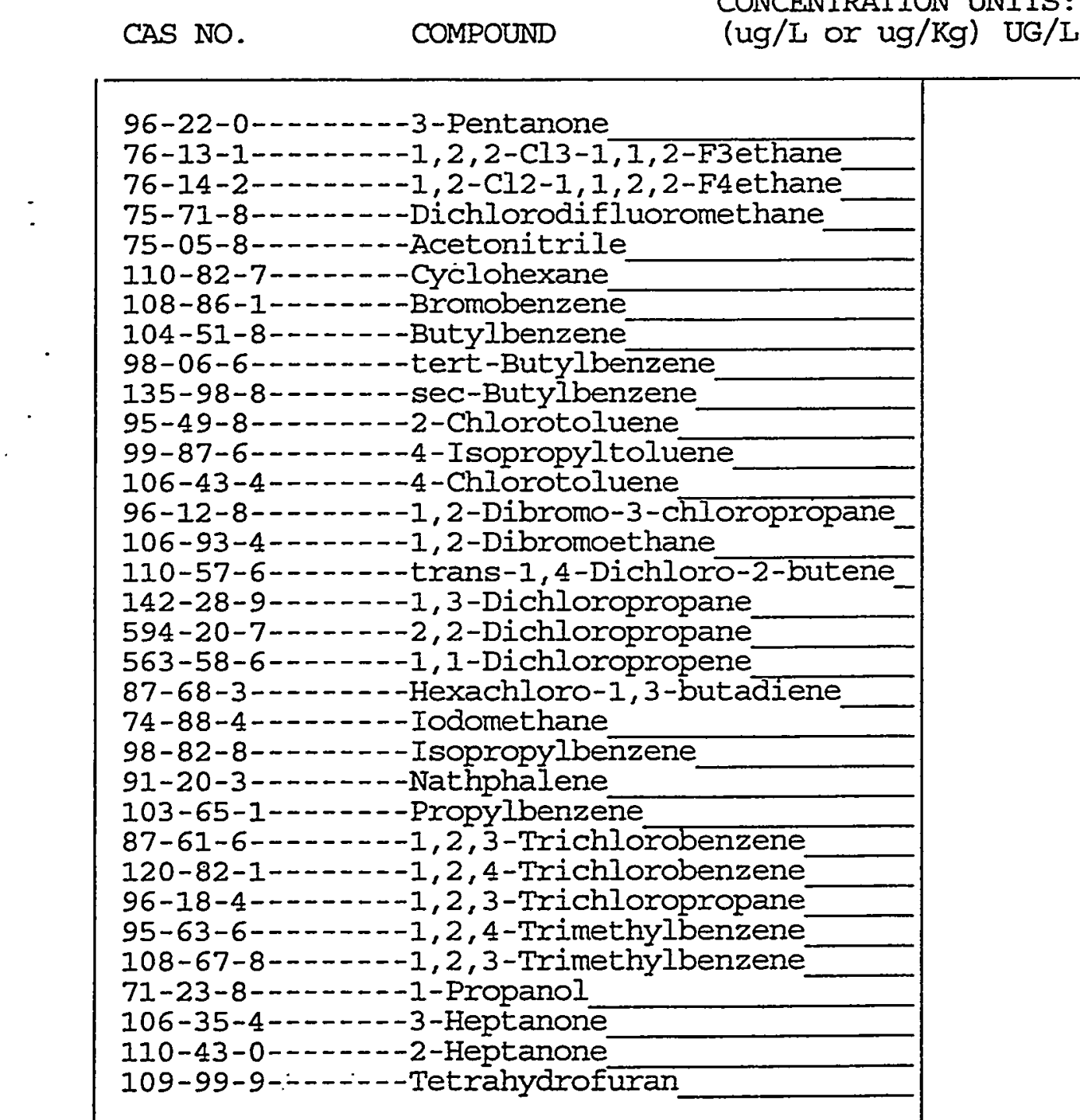

Lab Sample ID: VBLK01

Lab File ID: 99021204

Date Received:

Date Analyzed: 02/12/99

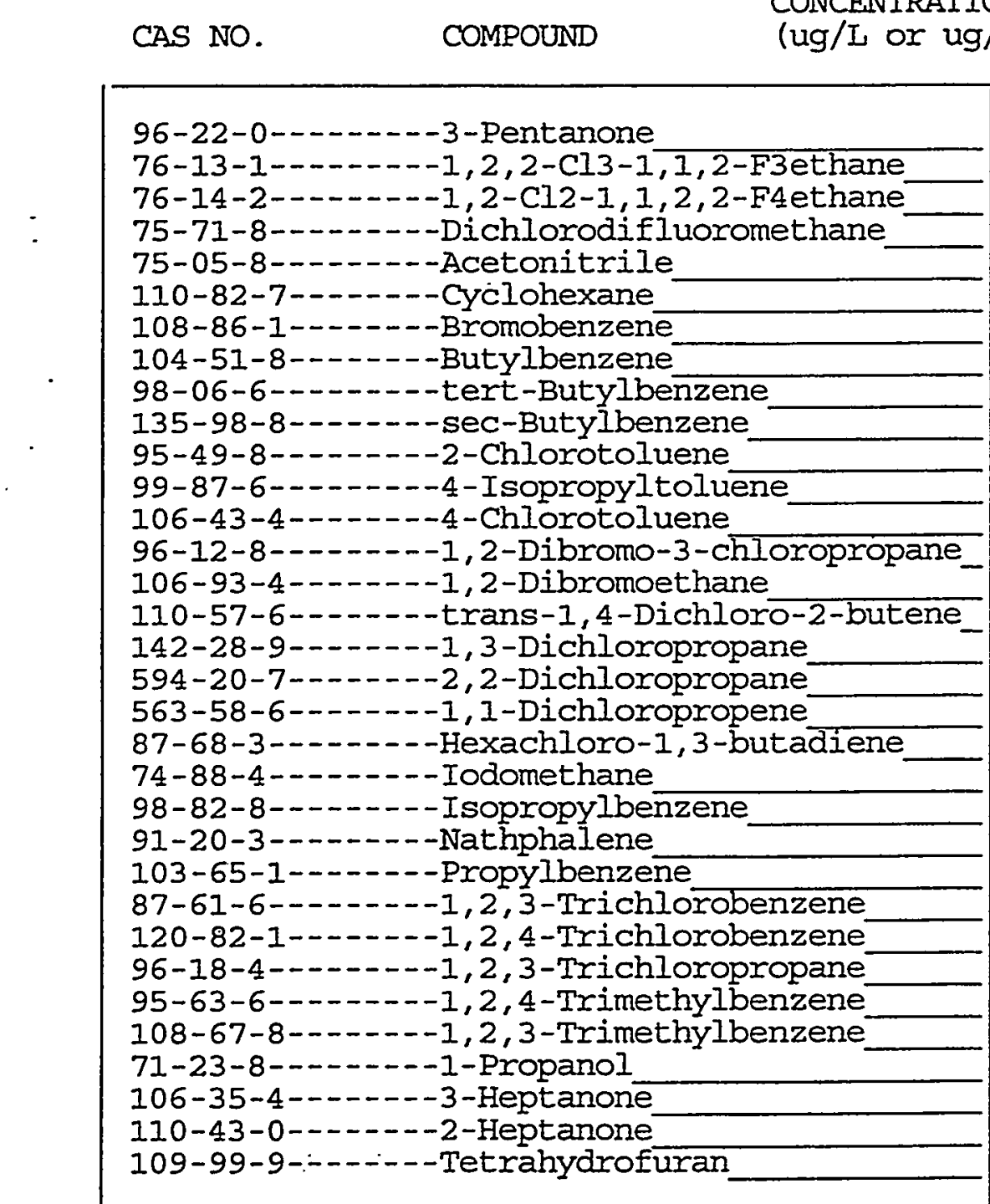

Dilution Factor: 1.0

Soil Aliquot Volume: (uI) 
$I \dot{A}$

VOLATILE ORGANICS ANALYSIS DATA SHEET

Lab Name: PNNL

Contract: BNFL

EPA SAMPLE NO.

Lab Code: PNNL

Case No.:

SAS NO.:

METHOD BLANK

Matrix: (soil/water) SUPERNATAN

Iab Sample ID: VBLKOI

Sample wt/vol:

$5.000(\mathrm{~g} / \mathrm{mL}) \mathrm{ML}$

Lab File ID: '99021204

Level: (low/med) Low

Date Received:

$\%$ Moisture: not dec.

Date Analyzed: 02/12/99

.GC Column: DB-624 75M X 2.55UM ID: 0.45 (mm)

Dilution Factor: 1.0

Soil Extract Volume: (UI)

Soil Aliquot Volume: (uL)

CAS NO.

COMPOUND CONCENTRATION UNITS:

(ug/L or ug/Kg) UG/L

Q

74-95-3--------Dibromomethane

OLM03. 0 
Lab Name: PNNL

ILab Code: PNNL

Case No.:

Matrix: (soil/water) SUPERNATAN

Sample wt/vol:

$5.000(\mathrm{~g} / \mathrm{mL}) \mathrm{ML}$

Level: (low/med) IOW

\% Moisture: not dec.

GC Column: DB-624 75M X 2.55UM ID: $0.45 \cdot(\mathrm{mm})$

Contract: BNFL

AW-101DIL
SAS NO. :

SDG NO.: 990205 (uIs)

Soil Extract Volume:
Dilution Factor: 1.0

Soil Aliquot Volume:

CAS NO. COMPOUND (ug/L or ug/Kg) UG/L
(us)

74-87-3-...--Chloromethane

75-01-4-....--Vinyl Chloride

106-99-0--.---1,3-Butadiene

106-97-8-..----Butane

74-83-9-...--Bromomethane

75-35-4-.----1, 1-Dichloroethene

75-00-3-...--Chloroethane

67-64-1-....-Acetone

109-66-0-..----Pentane

75-15-0--.----Carbon Disulfide

107-02-8-...-Acrolein

107-13-1-...--Acrylonitrile

75-09-2-..----Methylene Chloride

156-60-5-..---trans-1,2-Dichloroethene

123-38-6-...--Propionaldehyde

4170-30-3-.----2-Butenal

75-34-3-...-1, 1-Dichloroethane

156-59-2------cis-1,2-Dichloroethene

$110-54-3-1----H e x a n e$

78-93-3-..---2-Butanone

110-83-8-...--Cyclohexene

141-78-6-..----Ethyl acetate

287-92-3-..----Cyclopentane

74-97-5---.----Bromochloromethane

67-66-3-..-.-.-Chloroform

75-43-4-2...-Dichlorofluoromethane

71-55-6-...---1, 1, 1-Trichloroethane

75-45-6-..-.---Chlorodifluoromethane

56-23-5-...--Carbon Tetrachloride

75-69-4-...- - Trichlorofluoromethane

71-43-2-...---Benzene

107-06-2-.---1, 2-Dichloroethane

79-01-6-..----Trichloroethene 
Lab Name: PNNL

Lab Code: PNNL
Contract : BNFL SAS NO.:
AW-10IDII

SDG NO.: 990205
Matrix: (soil/water) SUPERNATAN

Sample wt/vol: $5.000(\mathrm{~g} / \mathrm{mL}) \mathrm{ML}$

Level: (low/med) LOW

$\div$ Moisture: not dec.

GC Column: $\mathrm{DB}-62475 \mathrm{M} \times 2.55 \mathrm{UM}$ ID: 0.45 (mm) Soil Extract Volume: (uI)
Lab Sample ID: 99-0648DIL Lab File ID: $\quad 99021205$

Date Received:

Date Analyzed: 02/12/99

Dilution Factor: 1.0

Soil Aliquot Volume: CONCENTRATION UNITS :

CAS NO.

COMPOUND (ug/L or $u g / K g$ ) UG/L

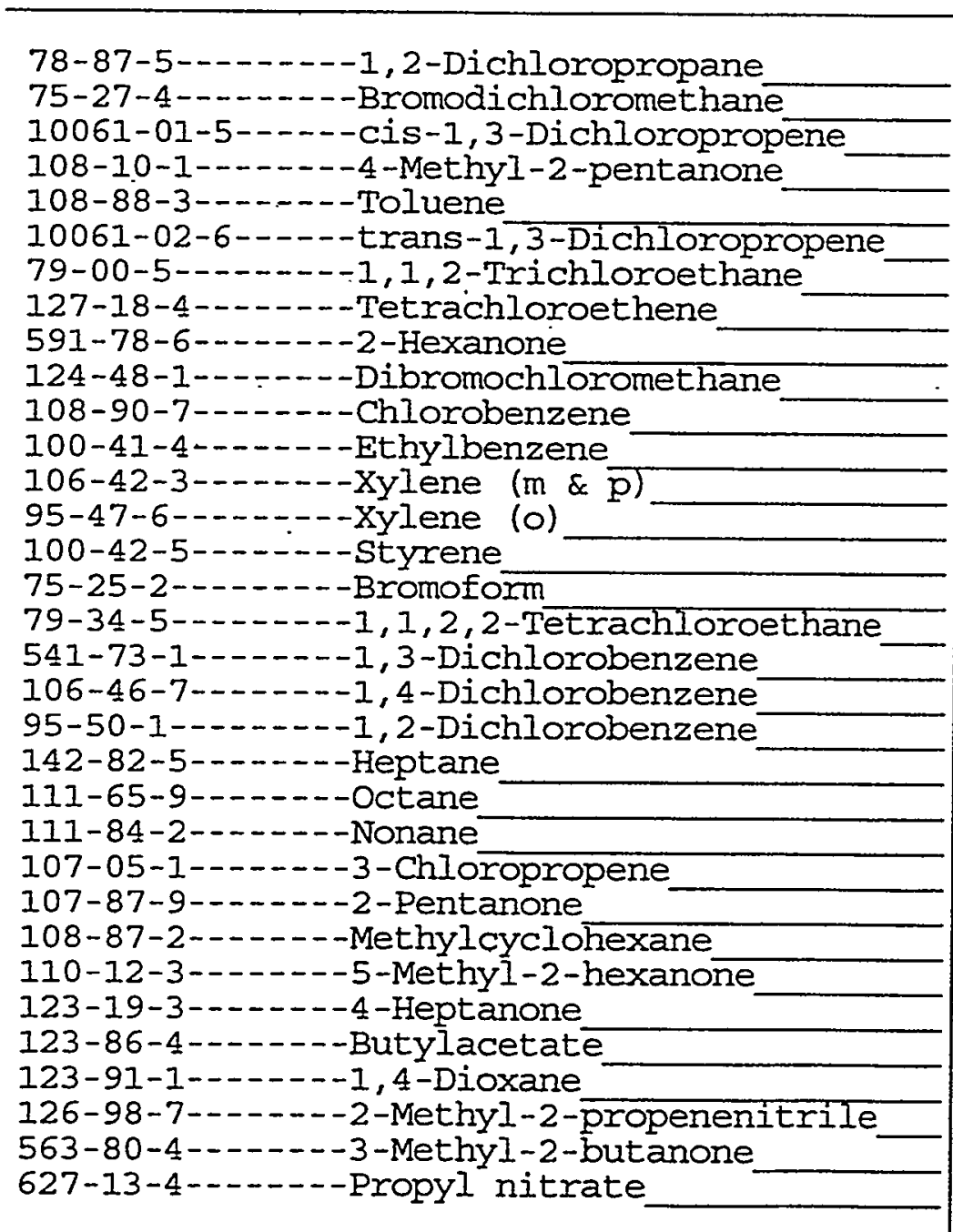

\begin{tabular}{l|l}
\multicolumn{2}{l}{} \\
\hline 310 \\
\hline 310 & $U$ \\
310 & $U$ \\
310 & $U$ \\
310 & $U$ \\
310 & $U$ \\
310 & $U$ \\
310 & $U$ \\
310 & $U$ \\
310 & $U$ \\
310 & $U$ \\
310 & $U$ \\
310 & $U$ \\
310 & $U$ \\
310 & $U$ \\
310 & $U$ \\
310 & $U$ \\
310 & $U$ \\
310 & $U$ \\
310 & $U$ \\
310 & $U$ \\
310 & $U$ \\
310 & $U$ \\
310 & $U$ \\
310 & $U$ \\
310 & $U$ \\
310 & $U$ \\
310 & $U$ \\
310 & $U$ \\
310 & $U$ \\
310 & $U$ \\
310 & $U$ \\
310 & $U$ \\
& \\
& \\
\hline
\end{tabular}


Lab Name: PNNL

Lab Code: PNNL . Case No.:
Contract: BNEI

SAS NO. :

AW-101DIL

Matrix: (soil/water) SUPERNATAN

Sample wt/vol:

$5.000(\mathrm{~g} / \mathrm{mL}) \mathrm{ML}$

Level: (low/med) IOW

$\%$ Moisture: not dec.

GC Column: DB-624 75M X 2.55UM ID: 0.45 (mm)

Soil Extract Volume: (uU)
Lab Sample ID: 99-0648DIL

Lab File ID: 99021205

Date Received:

Date Analyzed: 02/12/99

Dilution Factor: 1.0

Soil Aliquot Volume: (ut)
CAS NO. CONCENIRATION UNITS: (ug/L or $\mathrm{ug} / \mathrm{Kg}$ ) UG/I

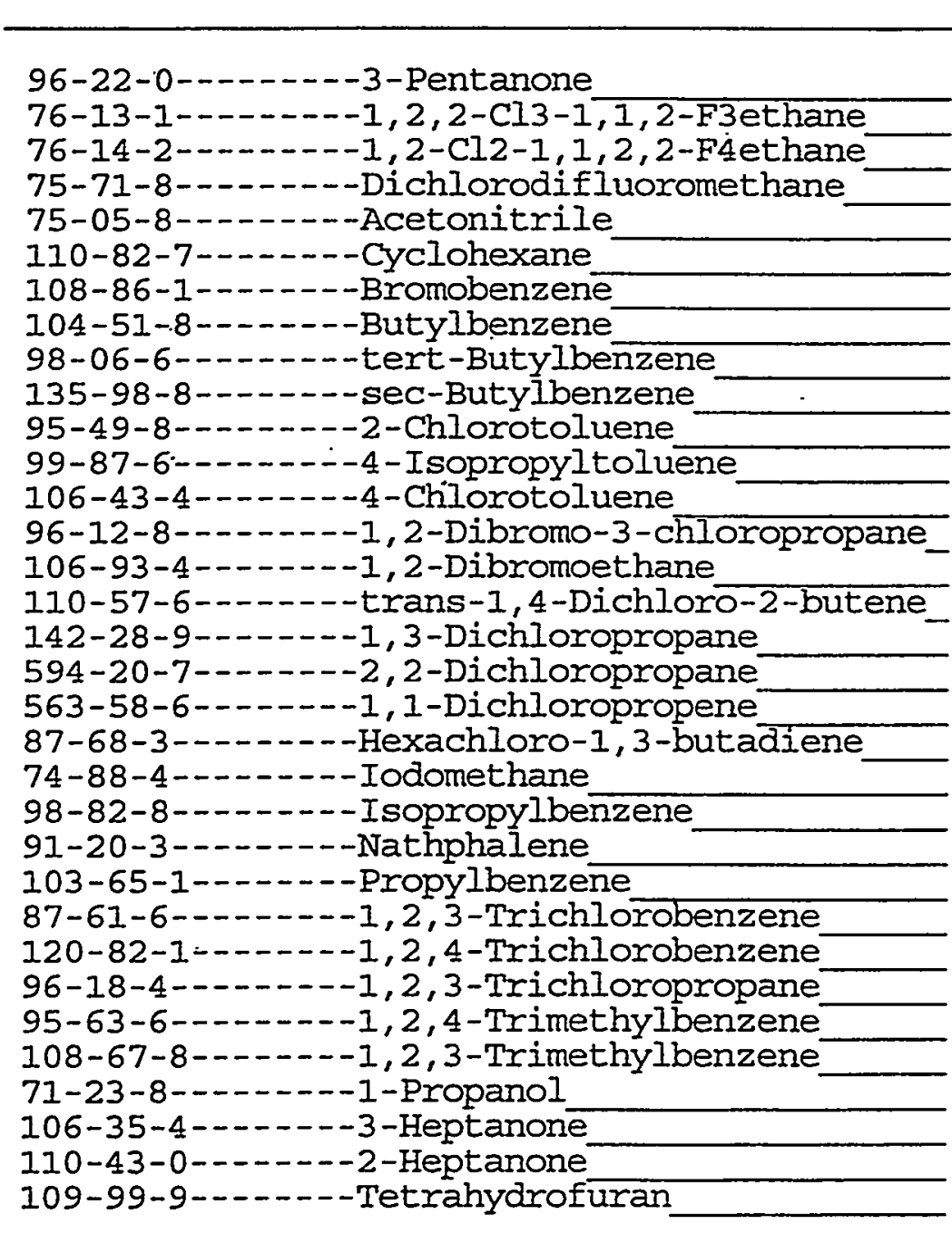

76-13-1-...--1, 2, 2-C13-1, 1, 2-F3ethane

76-14-2--..-- 1, 2-Cl2-1,1,2, 2-FÁ ethane

75-71-8-...-. Dichlorodifluoromethane

75-05-8-...-Acetonitrile

110-82-7-...--Cyclohexane

$104-51-8-1--1-0--B u t y 1$ benzene

98-06-6--..---tert-Butylbenzene

135-98-8--.---sec-Butylbenzene

99-87-6-- - - - -4-Isopropyltoluene

106-43-4--.---4-Chilorotoluene

96-12-8-- - - - 1, 2-Dibromo-3-chloropropane

110-57-6------trans-1, 4-Dichloro-2-butene

142-28-9------ I, 3-Dichloropropane

563-58-6-....-1, 1-Dichloropropene

87-68-3-.-.--Hexachloro-1, 3-butadiene

74-88-4--.----Iodomethane

91-20-3-...--Nathphalene

103-65-1-...---Propylbenzene

87-61-6--...- - 1, 2, 3-Trichlorobenzene

120-82-1--.-.- - 1, 2, 4-Trichlorobenzene

96-18-4--.---1, 2, 3-Trichloropropane

95-63-6-...--1, 2, 4-Trimethylbenzene

108-67-8--.---1, 2, 3-Trimethylbenzene

71-23-8-.-.--1-Propanol

110-43-0-...--2-Heptanone

109-99-9-..---Tetrahydrofuran 
Lab Name: PNNL

Contract: BNFL

AW-101DIL

Iab Code: PNNL

Case No.:

SAS NO'.:

SDG NO.: 990205

Matrix: (soil/water) SUPERNATAN

Lab Sample ID: 99-0648DIL

Sample wt/vol:

$5.000(\mathrm{~g} / \mathrm{mL}) \mathrm{ML}$

Lab File ID: 99021205

Level: (low/med) LOW

Date Received:

$\div$ Moisture: not dec.

Date Analyzed: 02/12/99

GC Column: DB-62475M X 2.55UM ID: 0.45 (mm)

Dilution Factor: 1.0

Soil Extract Volume: (uL)

Soil Aliquot Volume: (uL)

\section{CAS NO.}

COMPOUND CONCENTRATION UNITS:

(ug/L or ug/Kg) UG/L

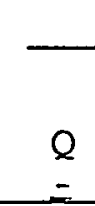

74-95-3--.-----Dibromomethane 
Lab Name: PNNL

Irab Code: PNNL

Case No.:

Matrix: (soil/water) SUPERNATAN

Sample wt/vol:

$5.000(\mathrm{~g} / \mathrm{mL}) \mathrm{ML}$

Irevel: (low/med) LoW

$\%$ Moisture: not dec.

GC Column: DB-624 75M X $2.55 U M$ ID: 0.45 (mm)

Soil Extract Volume: (UI)

Contract: BNFL

SAS NO.:

Lab Sample ID: 99-0648DIID

Lab File ID: 99021206

Date Received:

Date Analyzed: 02/12/99

Dilution Factor: 1.0 .

Soil Aliquot Volume: (UI) $\begin{array}{ll}\text { CAS NO. COMPOUND } & \text { CONCENTRATION UNITS: } \\ & (\mathrm{ug} / \mathrm{L} \text { or } \mathrm{ug} / \mathrm{Kg}) \mathrm{UG} / \mathrm{L}\end{array}$
EPA SAMPLE :NO.

AW-10IDIID

SDG NO.: 990205

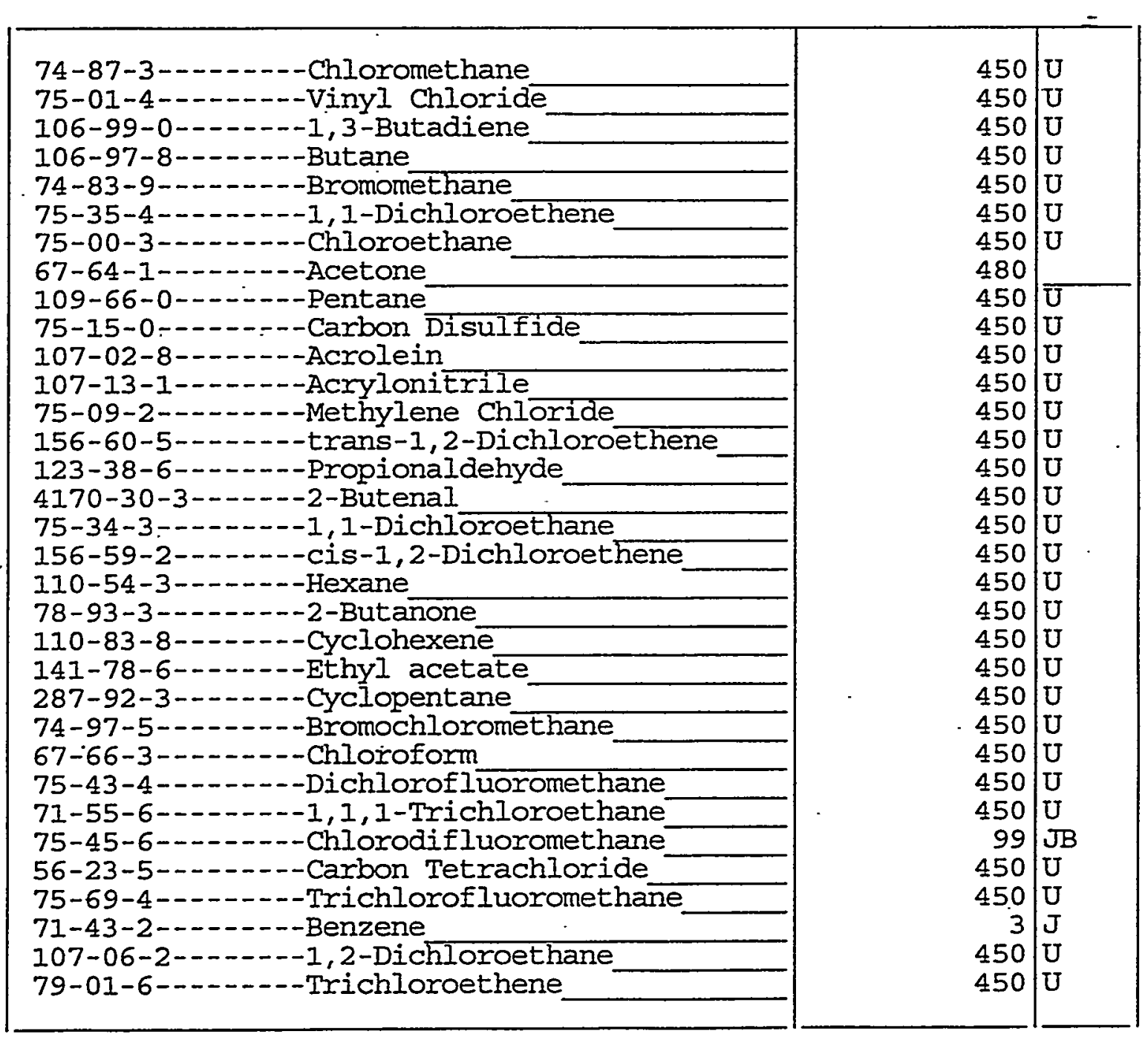


Lab Name: PNNL

Lab Code: PNNL

Case No.:

Matrix: (soil/water) SUPERNATAN

Sample wt/vol:

$5.000(\mathrm{~g} / \mathrm{mL}) \mathrm{ML}$

Level: (low/med) LOW

$\%$ Moisture: not dec.

GC. Column: DB-624 75M X 2.55UM ID: 0.45 (mm) (UL)

Soil Extract Volume:
Contract: BNFI

SAS NO. :
EPA SAMPLE NO.

AW-101DTLD
Lab Sample ID: 99-0648DIID

Lab File ID: 99021206

Date Received:

Date Analyzed: 02/12/99

Dilution Factor: 1.0

Soil Aliquot Volume: (uI) CONCENTRATION UNITS :

CAS NO. COMPOUND (ug/L or $\mathrm{ug} / \mathrm{Kg}$ ) UG/I

\begin{tabular}{l|l}
\multicolumn{2}{c}{$Q$} \\
\hline 450 & $\mathrm{U}$ \\
450 & $\mathrm{U}$ \\
450 & $\mathrm{U}$ \\
74 & $\mathrm{~J}$ \\
450 & $\mathrm{U}$ \\
450 & $\mathrm{U}$ \\
450 & $\mathrm{U}$ \\
450 & $\mathrm{U}$ \\
450 & $\mathrm{U}$ \\
450 & $\mathrm{U}$ \\
450 & $\mathrm{U}$ \\
450 & $\mathrm{U}$ \\
450 & $\mathrm{U}$ \\
450 & $U$ \\
450 & $\mathrm{U}$ \\
450 & $\mathrm{U}$ \\
450 & $\mathrm{U}$ \\
450 & $\mathrm{U}$ \\
450 & $\mathrm{U}$ \\
450 & $\mathrm{U}$ \\
450 & $\mathrm{U}$ \\
450 & $\mathrm{U}$ \\
450 & $\mathrm{U}$ \\
450 & $\mathrm{U}$ \\
450 & $\mathrm{U}$ \\
450 & $\mathrm{U}$ \\
450 & $\mathrm{U}$ \\
450 & $\mathrm{U}$ \\
450 & $\mathrm{U}$ \\
450 & $\mathrm{U}$ \\
450 & $U$ \\
450 & $\mathrm{U}$ \\
450 & $\mathrm{U}$ \\
& \\
\hline & \\
\hline
\end{tabular}

78-87-5-1-2--1, 2-Dichloropropane

75-27-4-...---Bromodichloromethane

10061-01-5-----cis-1,3-Dichloropropene

108-10-1-..--4-Methyl-2-pentanone

108-88-3-...-.--Toluene

10061-02-6--.--trans-1, 3-Dichloropropene

79-00-5-...-1,1,2-Trichloroethane

127-18-4-..--Tetrachloroethene

591-78-6--.---2-Hexanone

124-48-1--.---Dibromochloromethane

108-90-7-..-.--Chlorobenzene

100-41-4--.----Ethylbenzene

I06-42-3-..--.Xylene $(m \&$ p)

95-47-6--.---xylene (0)

$100-42-5-1----$-Styrene

75-25-2-..----Bromoform

79-34-5-..-.-1,1,2,2-Tetrachloroethane

541-73-1-...--1, 3-Dichlorobenzene

106-46-7-...--1,4-Dichlorobenzene

95-50-1-...-1, 2-Dichlorobenzene

142-82-5-.-.---Heptane

1II-65-9-....--Octane

111-84-2....--Nonane

107-05-1-...---3-Chloropropene

107-87-9--.----2-Pentanone

108-87-2-----Methylcyclohexane

110-12-3-.-.--5-Methyl-2-hexanone

123-19-3--.---4-Heptanone

123-86-4------Butylacetate

123-91-1-..-.-1,-1, 4ioxane

126-98-7-....-2-Methyl-2-propenenitrile

563-80-4---.--3-Methyl-2-butanone

627-13-4--.---Propyl nitrate 
Lab Name: PNNL

Lab Code: PNNL

Case No.:
Contract: BNFL

SAS NO.:

AW-101DILD

Matrix: (soil/water) SUPERNATAN

Sample wt/vol:

$5.000(\mathrm{~g} / \mathrm{mL}) \mathrm{ML}$

Level: (low/med) LOW

$\%$ Moisture: not dec.

.. GC Column: DB-624 75M X 2.55UM ID: 0.45 (mm)

Soil Extract Volume:

(uI)

Iab Sample ID: 99-0648DILD

Iab File ID: 99021206

Date Received:

Date Analyzed: 02/12/99

(mm) Dilution Factor: 1:0

Soil Aliquot Volume:

(uL)

CONCENTRATION UNITS:

CAS NO. COMPOUND (ug/L or ug/Kg) UG/L

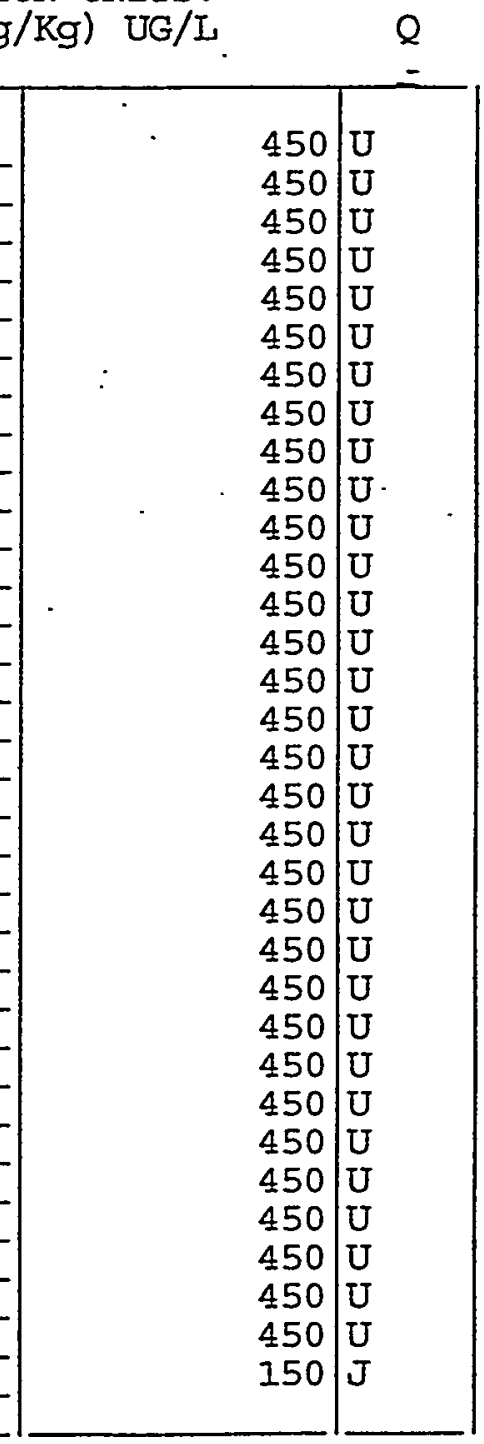

96-22-0--.-.--3-Pentanone

76-13-1-...--1, 2, 2-Cl3-1, 1, 2-F3ethane

76-14-2-...--1,2-Cl2-I, 1, 2, 2-F4ethane

75-71-8------Dichlorodifluoromethane

75-05-8-..---Acetonitrile

110-82-7-..---Cyclohexane

108-86-1--.---Bromobenzene

104-51-8-..----Butylbenzene

98-06-6-...--tert-Butylbenzene

135-98-8-------sec-Butylbenzene

95-49-8-...-.-2-Chlorotoluene

99-87-6--.---4-Isopropyltoluene

106-43-4-.-.--4-Chlorotoluene

96-12-8-...---1, 2-Dibromo-3-chloropropane

106-93-4-...--1,2-Dibromoethane

110-57-6--.---trans-1,4-Dichloro-2-butene

142-28-9-...--1,3-Dichloropropane

594-20-7--.---2, 2-Dichloropropane

563-58-6------1, 1-Dichloropropene

87-68-3-...---Hexachloro-1,3-butadiene.

74-88-4--.----- Iodomethane

98-82-8-..-----Isopropylbenzene

91-20-3-....--Nathphalene

103-65-1-..----Propylbenzene

87-61-6-..---1, 2,3-Trichlorobenzene

120-82-1-....-1, 2,4-Trichlorobenzene

96-18-4-...--1,2,3-Trichloropropane

95-63-6-..----1, 2,4-Trimethylbenzene

108-67-8-----1, 2, 3-Trimethylbenzene

71-23-8-------1-Propanol

106-35-4-1.---3-Heptanone

110-43-0--.----2-Heptanone

109-99-9-.----Tetrahydrofuran 
$I A$

VOLATILE ORGANICS ANALYSIS DATA SHEET
Lab Name: PNNL

Contract: BNFL,
EPA SAMPLE NO.

AW-IOIDIID
Lab Code: PNIL Case No.:

Matrix: (soil/water) SUPERNATAN

Sample wt/vol: $\quad 5.000(\mathrm{~g} / \mathrm{mL}) \mathrm{ML}$

Level: (low $/ \mathrm{med}$ ) IOW

$\%$ Moisture: not dec.

GC Column: DB-624 75M X 2.55UM ID: 0.45 (mm)

Soil Extract Volume: (UL)

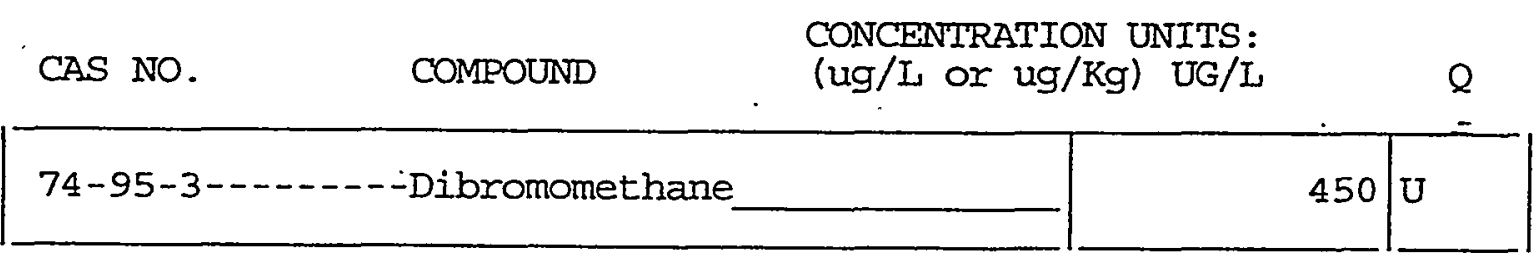

Lab Sample ID: 99-0648DILD

Lab File ID: 99021206

Date Received:

Date Analyzed: 02/12/99

Dilution Factor: 1.0

Soil Aliquot Volume: (UI) 
Lab Name: PNNL

Contract: BNFI

Lab Code: PNNL

Case No.:

Matrix: (soil/water) SUPERNATANT

Sample wt/vol:

$5.000(\mathrm{~g} / \mathrm{mL}) \mathrm{ML}$

Level: (low/med) IOW

\% Moisture: not dec.

GC Column: DB-624 75M X 2.55UM ID: 0.45 (mm)

Soil Extract Volume: (uI)
SAS NO.:
EPA SAMPLE NO.

AW-101

SDG NO.: 990205

Lab File ID: 99021207

Date Received:

Date Analyzed: 02/12/99

Dilution Factor: 1.0

Soil Aliquot Volume: (uL)

CONCENTRATION UNITS:

Number TICs found: 6 (ug/L or $\mathrm{ug} / \mathrm{Kg}$ ) ug/L

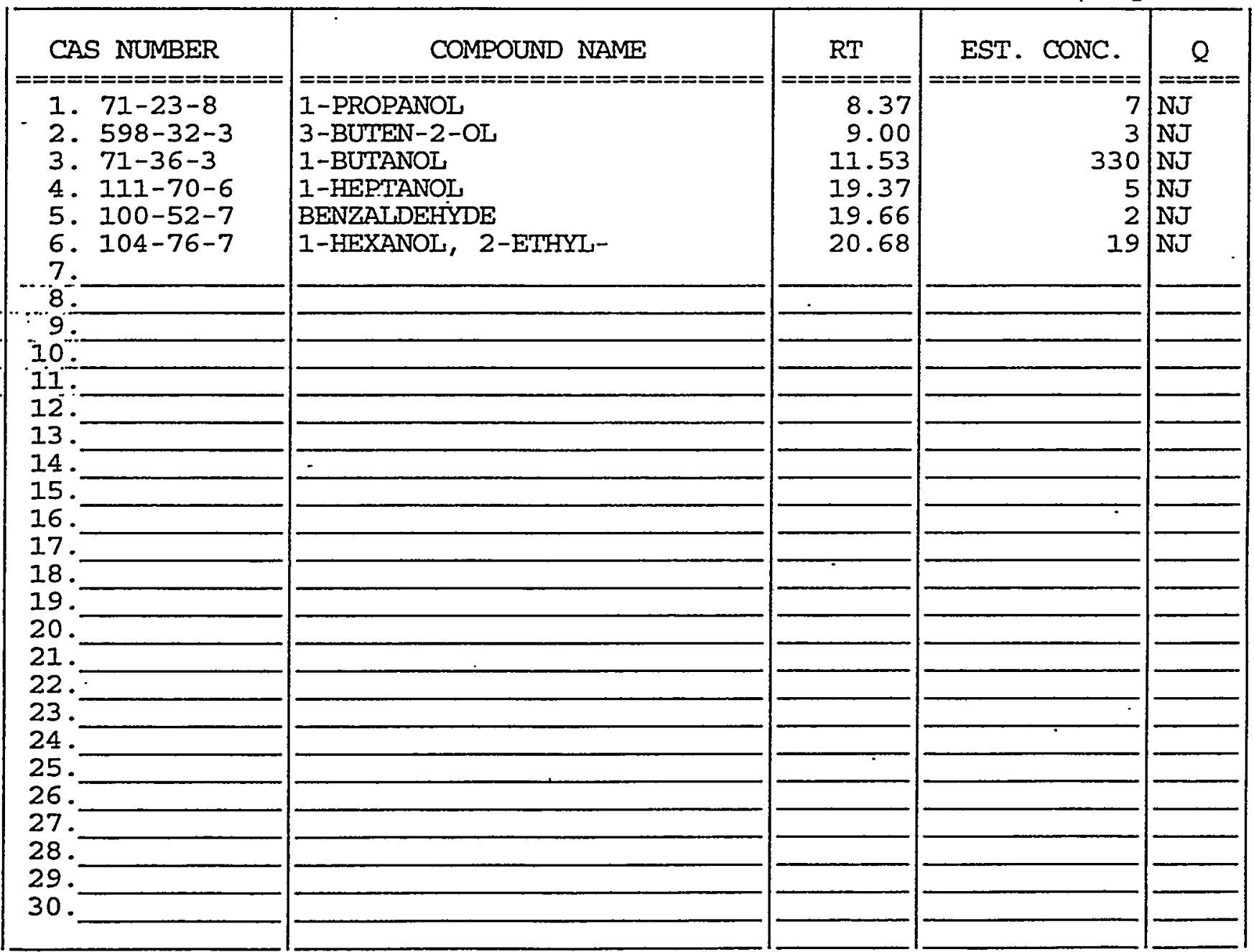


Lab Name: PNIL

Lab Code: PNNL

Case No.:

Matrix: (soil/water) SUPERNATANT

Sample wt/vol:

$5.000(\mathrm{~g} / \mathrm{mU}) \mathrm{ML}$

Level: (low/med) LOW

$\%$ Moisture: not dec.

GC Column: $\mathrm{DB}-62475 \mathrm{M} \times 2.55 \mathrm{UM}$ ID: 0.45 (mm) Soil Extract Volume: (UT)

Number TICs found: 5
Contract: BNFL

SAS NO.:
EPA SAMPLE NO.

AW-101D

SDG NO.: 990205

Iab Sample ID: 99-0648D

Lab File ID: 99021208

Date Received:

Date Analyzed: 02/12/99

Dilution Factor: 1.0

Soil Aliquot Volume: (uI)

CONCENTRATION UNITS:

(ug/I or $u g / \mathrm{Kg}$ ) ug/L

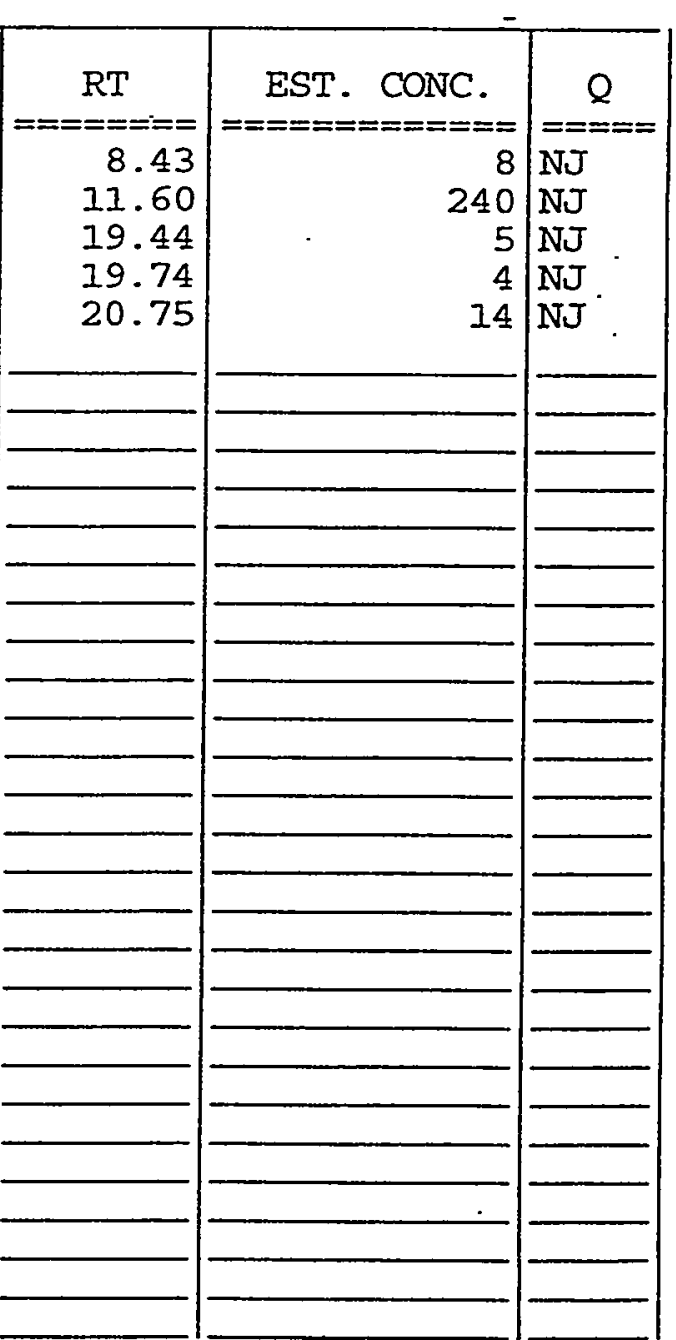


Lab Name: PNNL

Contract: BNFL

Lab Code: PNNL

Case No.:

Matrix: (soil/water) SUPERNATANT

Sample wt/vol:. $\quad 5.000(\mathrm{~g} / \mathrm{mL}) \mathrm{ML}$

Level: (low/med) LOW

$\%$ Moisture: not dec.

GC Column: DB-624 75M X 2.55UM ID: 0.45 (mm)

Soil Extract Volume:

(ut)

Number TICs found: 0
SAS NO: :

\section{METHOD BI_ANK}

SDG No.: 990205

Iab Sample ID: VBLK0I

Lab File ID: 99021204

Date Received:

Date Analyzed: 02/12/99

Dilution Factor: 1.0

Soil Aliquot Volume: (uL)

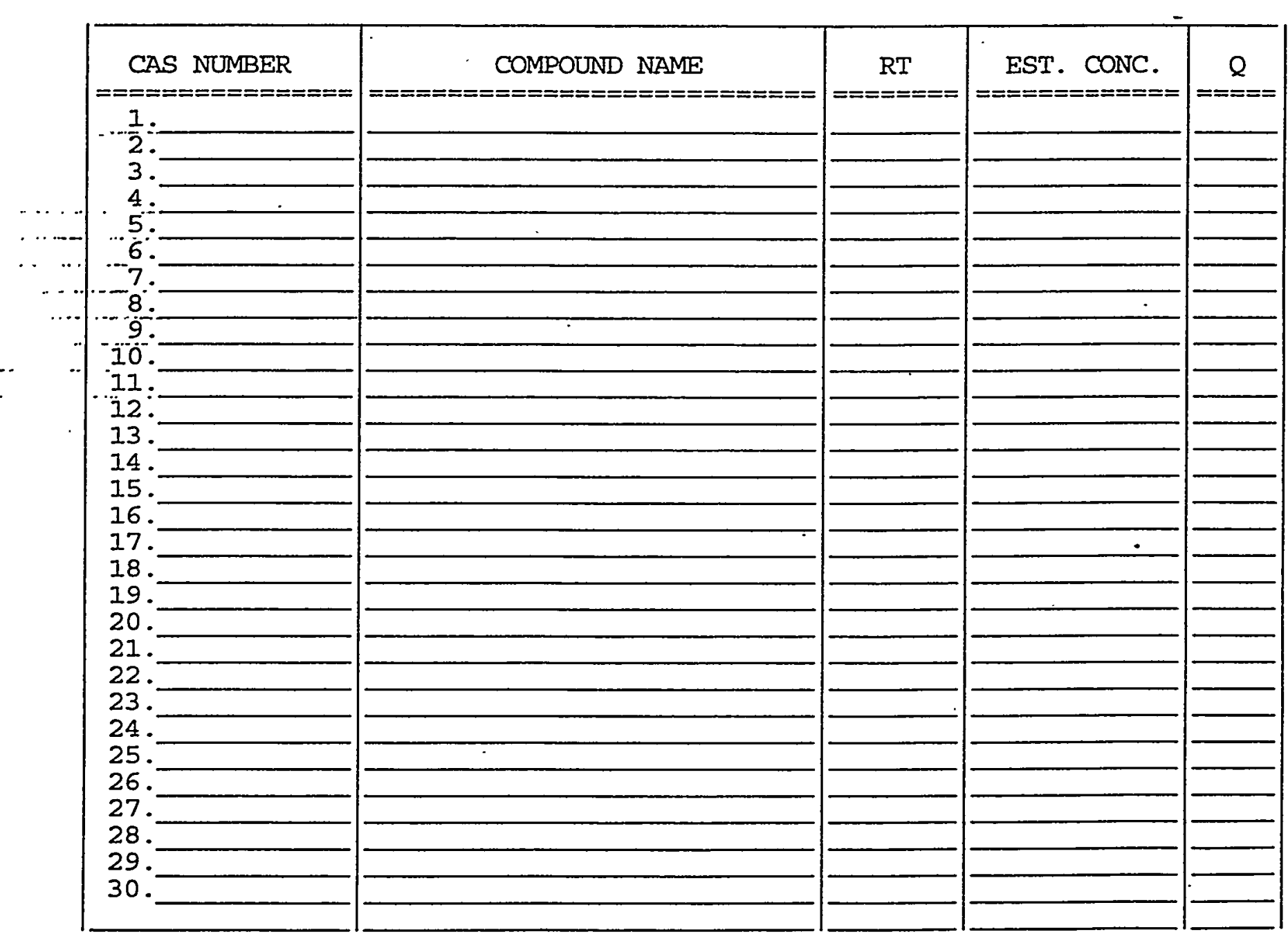

CONCENTRATION UNITS:

\section{(ug/L or $u g / \mathrm{Kg}) \mathrm{ug} / \mathrm{I}$}


$1 E$

VOLATILE ORGANICS ANAIYSIS DATA SHEET

TENTATIVELY IDENTIFIED COMPOUNDS

Lab Name: PNNL

Contract: BNFL

Lab Code: PNNL

Case No.:

SAS NO. :
EPA SAMPLE NO.

AW-IOIDIL

SDG No.: 990205

Lab Sample ID: 99-0648DIL

Lab File ID: 99021205

Date Received:

Date Analyzed: 02/12/99

(mm)

Dilution Factor: 1.0

Soil Aliquot Volume: (uL)

Number TICs found: 2 (uL)

CONCENTRATION UNITS :

(ug/L or $u g / \mathrm{Kg}$ ) ug/L
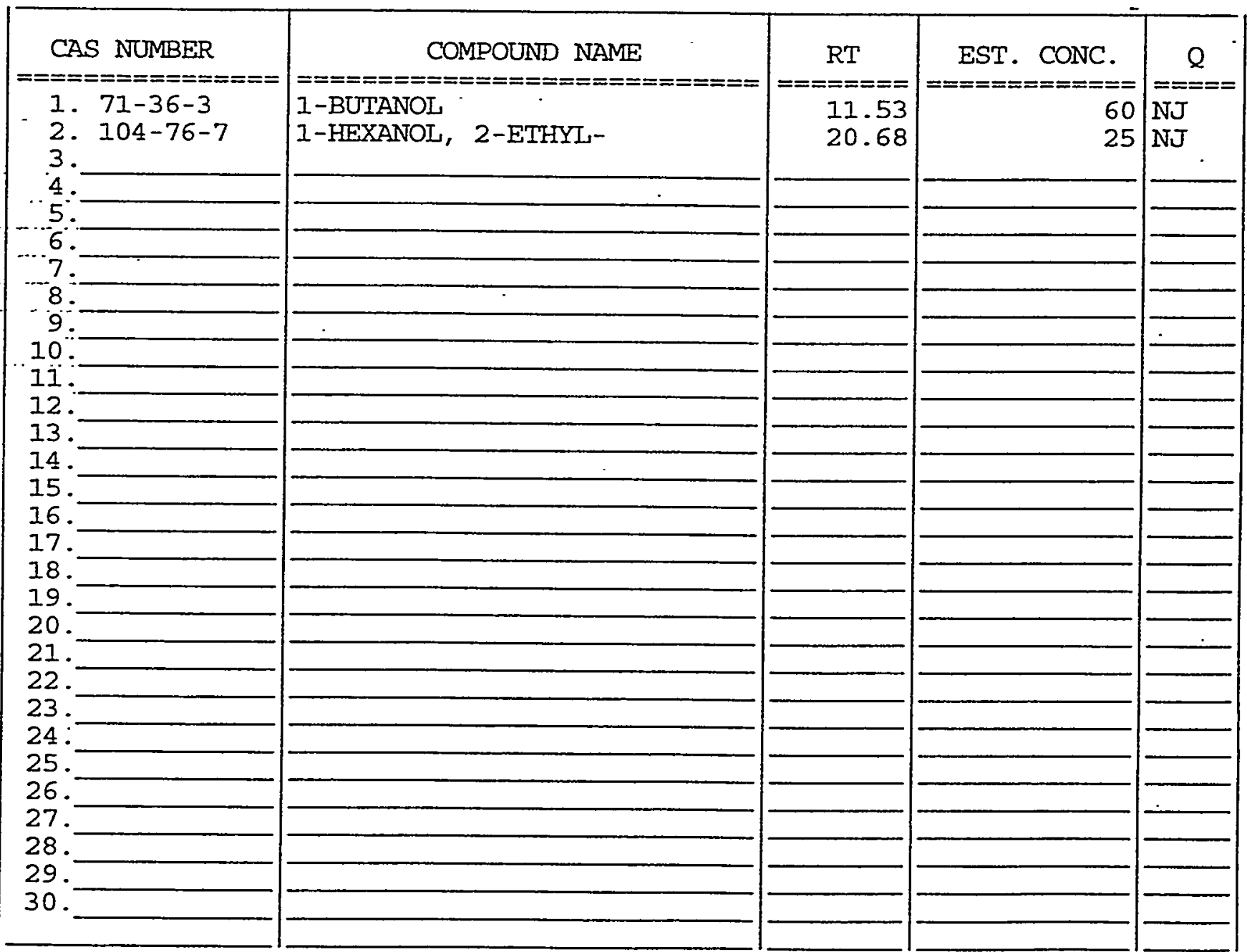
Lab Name: PNNL

Iab Code: PNNL

Case No.:

Matrix: (soil/water) SUPERNATANT

Sample wt/vol:

$5.000(\mathrm{~g} / \mathrm{mL}) \mathrm{ML}$

Ievel: (low/med) IOW

$\%$ Moisture: not dec.

GC Column: DB-624 75M X 2.55UM ID: 0.45 (mm)

Soil Extract Volume: (uL)
Contract: BNFL

SAS NO.:
AW-101DILD

SDG No.: 990205

Lab Sample ID: 99-0648DILD

Lab File ID: 99021206

Date Received:

Date Analyzed: 02/12/99

Dilution Factor: 1.0

Soil Aliquot Volume:

(UI)

CONCENTRATION UNITS

Number TICs found: 2 (ug/L or $\mathrm{ug} / \mathrm{Kg}$ ) ug/L

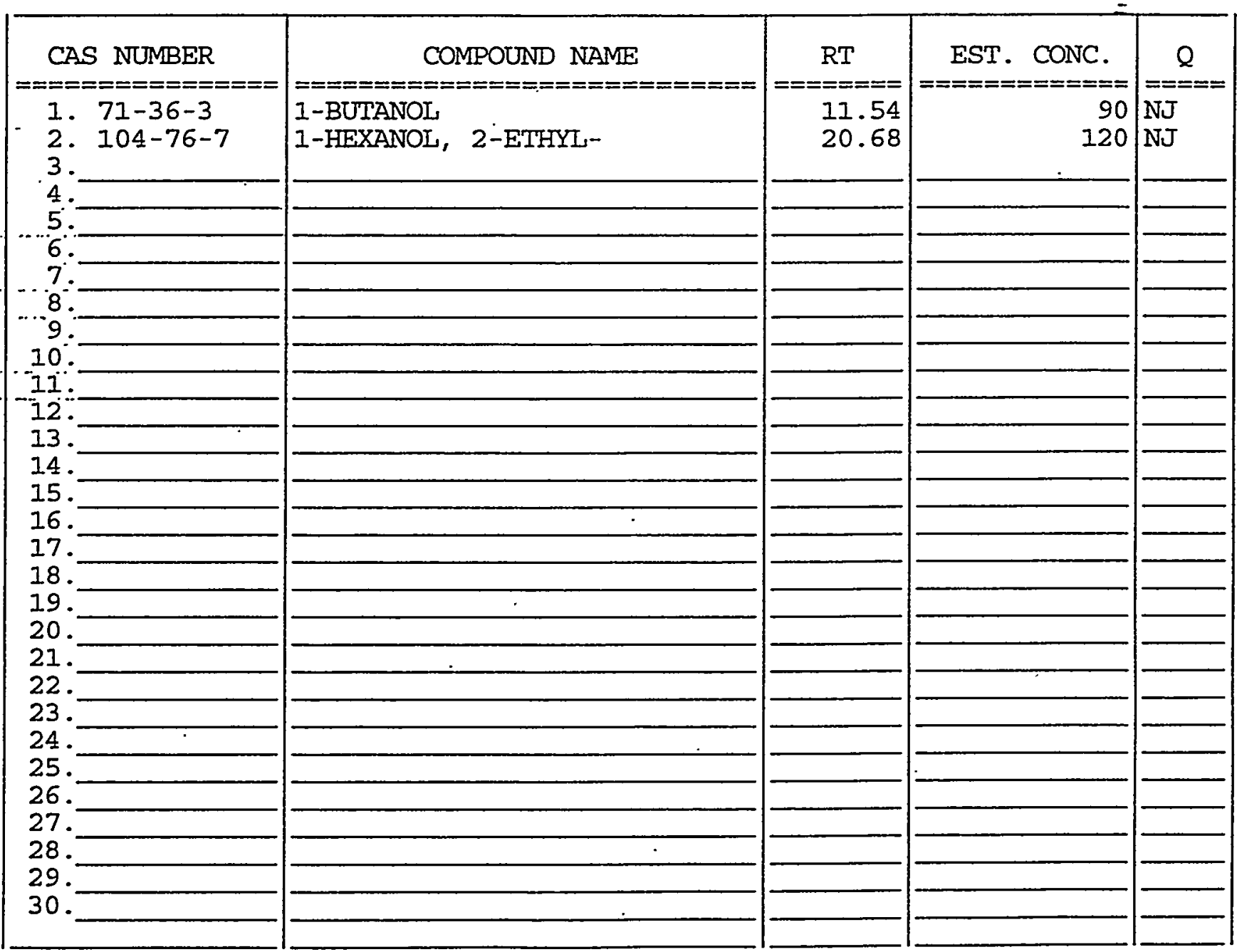


SUPERNATANT VOLATIIE SYSTEM MONITORING COMPOUND RECOVERY

Lab Name: PNNL

Lab Code: PNNL
Contract: BNFL

Case No.:
SAS NO.:

SDG No.: 990205

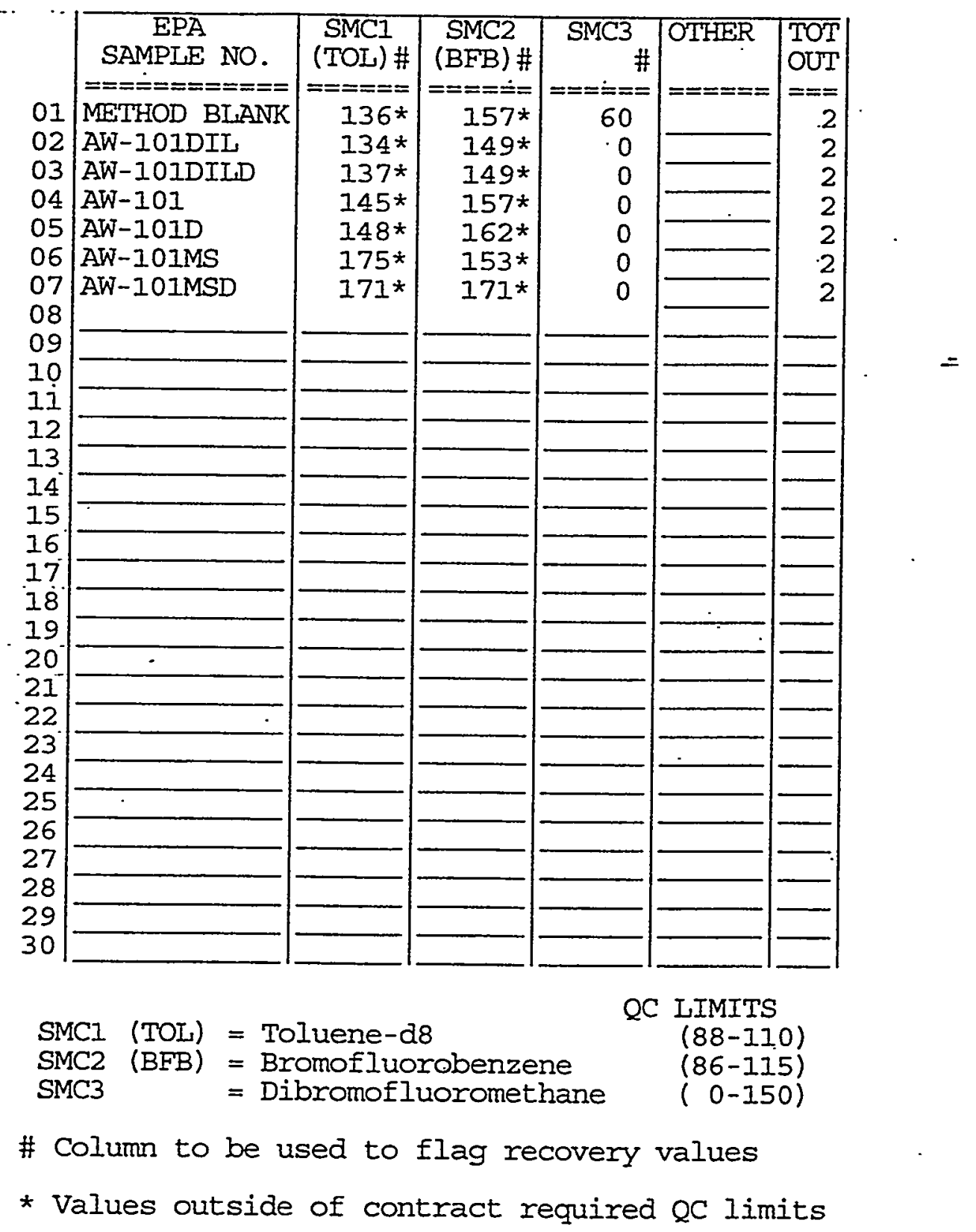


Lab Name: PNNL

Lab Code: PNNL
Contract: BNFL

SAS NO. :
SDG No.: 990205

Matrix Spike - EPA Sample No.: AW-101

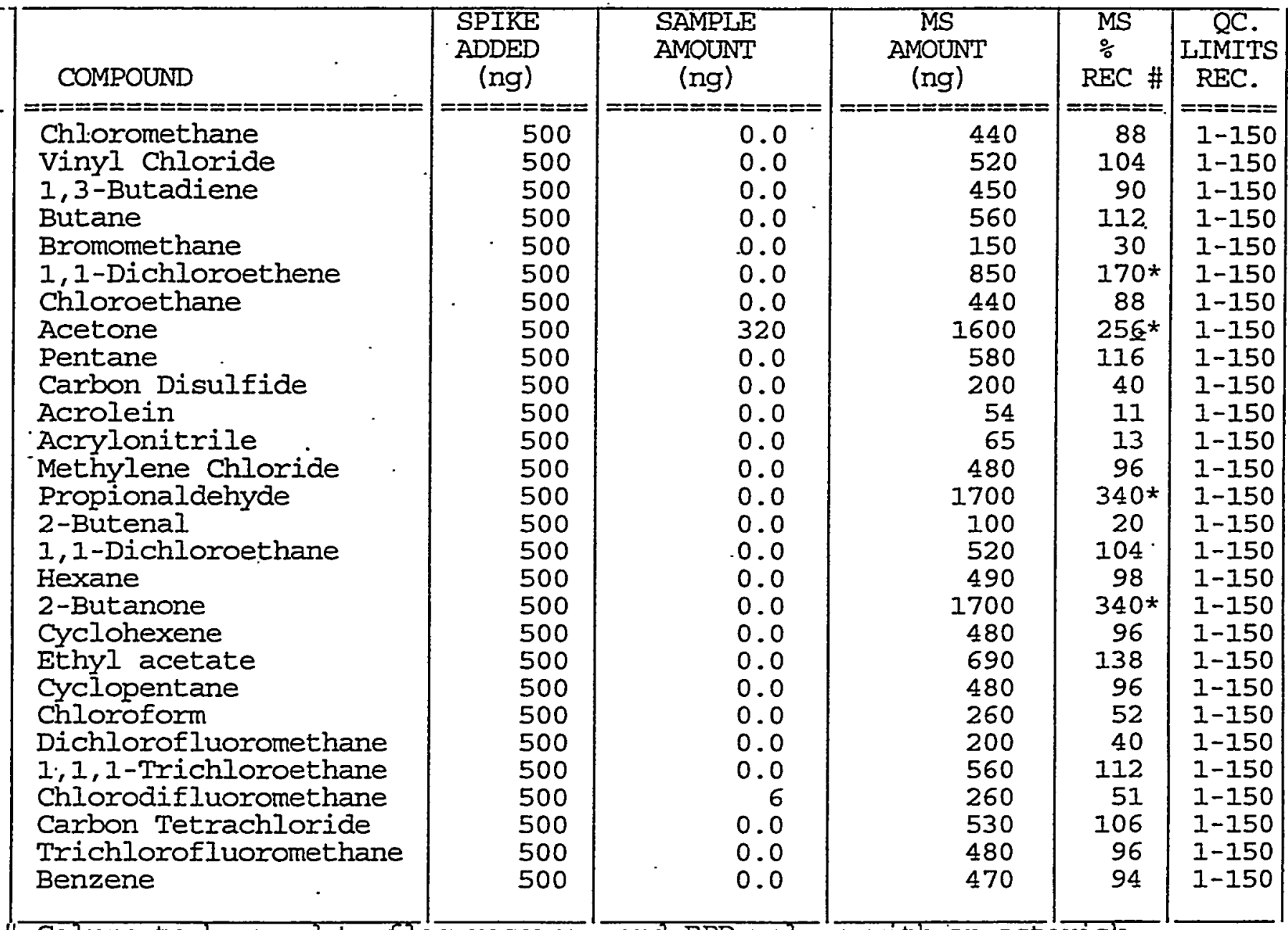

Column to be used to flag recovery and RPD values with an asterisk

* Values outside of QC limits

COMMENTS : 
Lab Name: PNNL

Contract: BNFL

Lab Code: PNNL

Case No.:

SAS NO.:

SDG No. : 990205

Matrix Spike - EPA Sample No.: AW-101

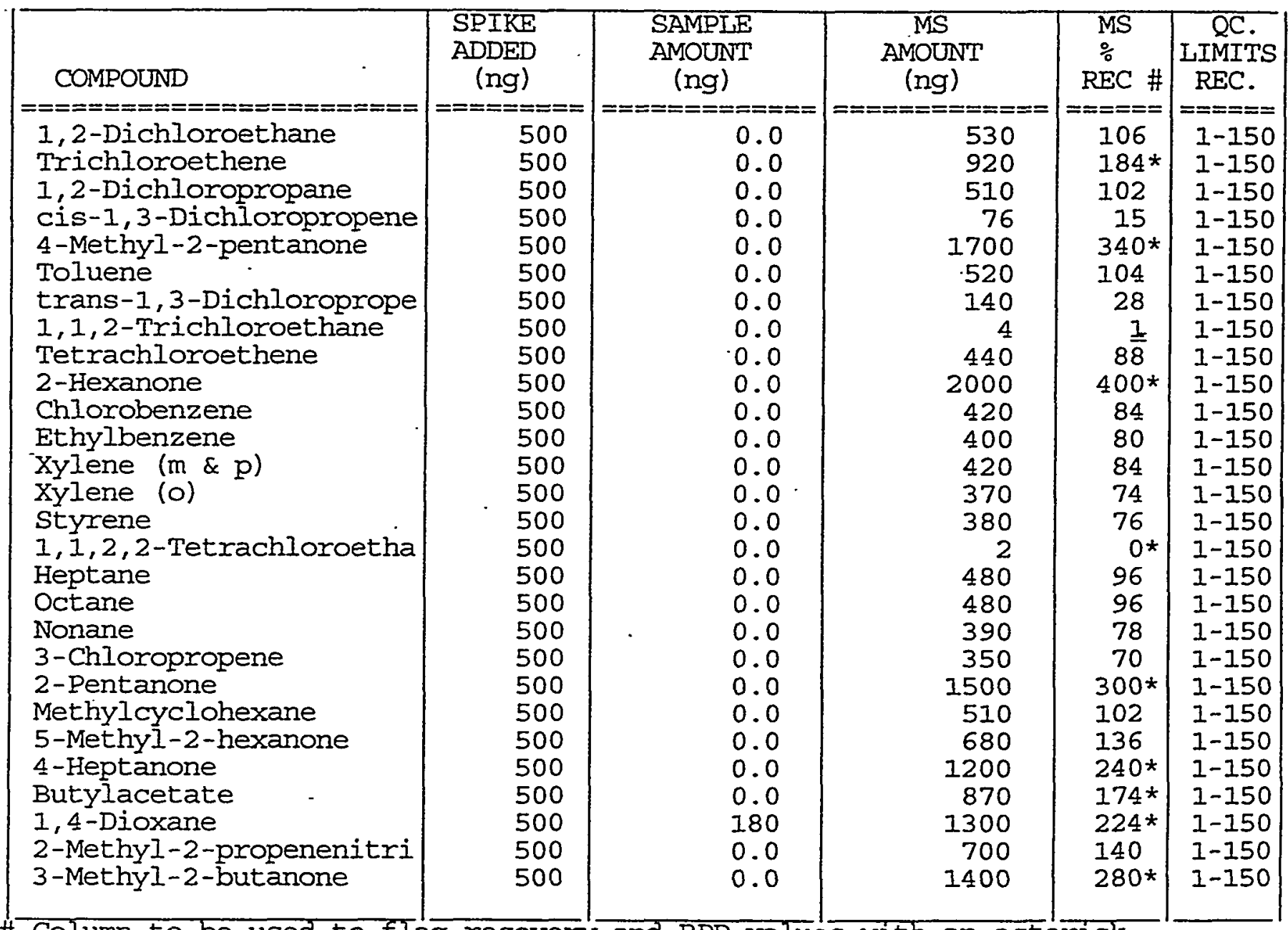

\# Column to be used to flag recovery and RPD values with an asterisk

* Values outside of QC Iimits

COMMENTS: 
SUPERNATANT VOLATILE MATRIX SPIKE/MATRIX SPIKE DÜPLICATE RECOVERY

Lab Name: PNNL

Contract: BNEL

Lab Code: PNNL

Case No.:

SAS NO.:

SDG No. : 990205

Matrix Spike - EPA Sample No.: AW-101

\begin{tabular}{|c|c|c|c|c|c|}
\hline 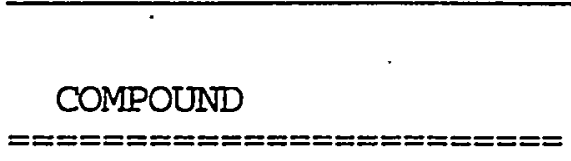 & $\begin{array}{l}\text { SPIKE } \\
\text { ADDED } \\
\text { (ng) } \\
=========\end{array}$ & $\begin{array}{c}\text { SAMPLE } \\
\text { AMOUNT } \\
\text { (ng) } \\
============\end{array}$ & $\begin{array}{c}\text { MS } \\
\text { AMOUNT } \\
(\mathrm{ng}) \\
============\end{array}$ & $\begin{array}{c}\text { MS } \\
\% \\
\text { REC \# } \\
====\equiv\end{array}$ & $\begin{array}{l}\text { QC. } \\
\text { IIMITS } \\
\text { REC. } \\
=====\end{array}$ \\
\hline $\begin{array}{l}\text { Propyl nitrate } \\
\text { 3-Pentanone } \\
1,2,2-C 13-1,1,2-\text { F3ethan } \\
1,2-C 12-1,1,2,2-\text { F4ethan } \\
\text { Dichlorodifluoromethane } \\
\text { Acetonitrile } \\
\text { Cyclohexane } \\
1,2 \text {-Dibromoethane } \\
\text { 3-Heptanone } \\
\text { 2-Heptanone } \\
\text {. Tetrahydrofuran }\end{array}$ & $\begin{array}{l}500 \\
500 \\
500 \\
500 \\
500 \\
500 \\
500 \\
500 \\
500 \\
500 \\
500\end{array}$ & $\begin{array}{l}0.0 \\
0.0 \\
0.0 \\
0.0 \\
0.0 \\
0.0 \\
0.0 \\
0.0 \\
0.0 \\
0.0 \\
140\end{array}$ & $\begin{array}{r}550 \\
1500 \\
540 \\
490 \\
370 \\
510 \\
510 \\
240 \\
1400 \\
1600 \\
1400\end{array}$ & $\begin{array}{l}110 \\
300 * \\
108 \\
98 \\
74 \\
102 \\
102 \\
48 \\
280 * \\
320 * \\
252 *\end{array}$ & $\begin{array}{l}1-150 \\
1-150 \\
1-150 \\
1-150 \\
1-150 \\
1-150 \\
1-150 \\
1-150 \\
1-150 \\
1-150 \\
1-150\end{array}$ \\
\hline
\end{tabular}

\# Column to be used to flag recovery and RPD values with an asterisk

* Values outside of QC limits

COMMENTS:

page 3 of 6

FORM III VOA-I

OTM03.0 
Lab Name: PNNL

Lab Code: PNNL
Contract: BNEL

SAS NO: :
SDG NO.: 990205

Matrix Spike - EPA Sample No.: AW-I0I

\begin{tabular}{|c|c|c|c|c|c|c|}
\hline & $\begin{array}{l}\text { SPIKE } \\
\text { ADDED }\end{array}$ & $\begin{array}{c}\text { MSD } \\
\text { AMOUNT }\end{array}$ & $\begin{array}{c}\text { MSD } \\
\%\end{array}$ & $\%$ & \multicolumn{2}{|c|}{ QC IIMITS } \\
\hline COMPOUND & (ng) & (ng) & REC \# & RPD \# & $\mathrm{RPD}$ & REC. \\
\hline 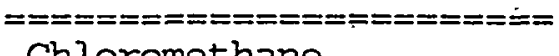 & $========$ & $=============$ & $======$ & $======$ & $======$ & $=====$ \\
\hline Chloromethane & 500 & 450 & 90 & 2 & 40 & $1-150$ \\
\hline Vinyl Chloride & 500 & 530 & 106 & 2 & 40 & $1-150$ \\
\hline 1,3-Butadiene & 500 & 450 & 90 & 0 & 40 & $1-150$ \\
\hline Butane & 500 & 500 & 100 & 11 & 40 & $1-150$ \\
\hline Bromomethane & 500 & 150 & 30 & 0 & 40 & $1-150$ \\
\hline I,I-Dichloroethene & 500 & 860 & $172 *$ & 1 & 40 & $1-150$ \\
\hline Chloroethane & 500 & 450 & 90 & 2 & 40 & $1-150$ \\
\hline Acetone & 500 & 1600 & $256 *$ & 0 & 40 & $1-150$ \\
\hline Pentane & 500 & 560 & 112 & 4 & 40 & $1-150$ \\
\hline Carbon Disulfide & 500 & 200 & 40 & 0 & 40 & $1-150$ \\
\hline Acrolein & 500 & 170 & 34 & $102 *$ & 40 & $1-150$ \\
\hline Acrylonitrile & 500 & 92 & 18 & 32 & 40 & $1-150$ \\
\hline Methylene Chloride & 500 & 490 & 98 & 2 & 40 & $1-150$ \\
\hline Propionaldehyde & 500 & 1600 & $320 *$ & 6 & 40 & $1-150$ \\
\hline 2-Butenal & 500 & 100 & 20 & 0 & 40 & $1-150$ \\
\hline 1,1-Dichloroethane & 500 & 530 & 106 & 2 & 40 & $1-150$ \\
\hline Hexane & 500 & 500 & 100 & 2 & 40 & $1-150$ \\
\hline 2-Butanone & 500 & 1600 & $320 *$ & 6 & 40 & $1-150$ \\
\hline Cyclohexene & 500 & 490 & 98 & 2 & 40 & $1-150$ \\
\hline Ethyl acetate & 500 & 640 & 128 & 8 & 40 & $1-150$ \\
\hline Cyclopentane & 500 & 520 & 104 & 8 & 40 & $1-150$ \\
\hline Chloroform & 500 & 270 & 54 & 4 & 40 & $1-150$ \\
\hline Dichlorofluoromethane & 500 & 180 & 36 & 10 & 40 & $1-150$ \\
\hline 1,1,1-Trichloroethane & 500 & 570 & 114 & 2 & 40 & $1-150$ \\
\hline Chlorodifluoromethane & 500 & 260 & 51 & 0 & 40 & $1-150$ \\
\hline Carbon Tetrachloride & 500 & 470 & 94 & 12 & 40 & $1-150$ \\
\hline Trichlorofluoromethane & 500 & 510 & 102 & 6 & 40 & $1-150$ \\
\hline Benzene & 500 & 460 & 92 & 2 & 40 & $1-150$ \\
\hline
\end{tabular}

\# Column to be used to flag recovery and RPD values with an asterisk

* Values outside of QC Iimits.

COMMENTS: 
SUPERNATANT VOLATILE MATRIX SPIKE/MATRIX SPIKE DUPLICATE RECOVERY

Lab Name: PNNL

Lab Code: PNNL
Contract : BNFL

SAS NO.:

SDG NO.: 990205

Matrix Spike - EPA Sample No.: AW-101

\begin{tabular}{|c|c|c|c|c|c|c|}
\hline & $\begin{array}{l}\text { SPIKE } \\
\text { ADDED }\end{array}$ & $\begin{array}{c}\text { MSD } \\
\text { AMOUNT }\end{array}$ & $\begin{array}{c}\text { MSD } \\
\%\end{array}$ & $\frac{0}{\sigma}$ & \multicolumn{2}{|c|}{ QC IIMITS } \\
\hline COMPOUND & (ng) & (ng) & REC \# & RPD \# & RPD & REC. \\
\hline$=0=0=0=0=$ & $========$ & $=========\approx===$ & $======$ & $======$ & $======$ & $======$ \\
\hline 1,2-Dichloroethane & 500 & 520 & 104 & 2 & 40 & $1-150$ \\
\hline Trichloroethene & 500 & 910 & $182 *$ & 1 & 40 & $1-150$ \\
\hline 1,2-Dichloropropane & 500 & 510 & 102 & 0 & 40 & $1-150$ \\
\hline cis-1,3-Dichloropropene & 500 & 72 & 14 & 7 & 40 & $1-150$ \\
\hline 4-Methyl-2-pentanone & 500 & 1400 & $280 *$ & 19 & 40 & $1-150$ \\
\hline Toluene & 500 & 510 & 102 & 2 & 40 & $1-150$ \\
\hline trans-1,3-Dichloroprope & 500 & 140 & 28 & 0 & 40 & $I-150$ \\
\hline 1,1,2-Trichloroethane & 500 & 5 & 1 & 0 & 40 & $1-150$ \\
\hline Tetrachloroethene & 500 & 430 & 86 & 2 & 40 & $1-150$ \\
\hline 2-Hexanone & 500 & 1700 & $340 *$ & 16 & 40 & $1-150$ \\
\hline Chlorobenzene & 500 & 440 & 88 & 5 & 40 & $1-150$ \\
\hline Ethylbenzene & 500 & 430 & 86 & 7 & 40 & $1-150$ \\
\hline Xylene $(m \& p)$ & 500 & 420 & 84 & 0 & 40 & $1-150$ \\
\hline xylene (o) & 500 & 380 & 76 & 3 & 40 & $1-150$ \\
\hline Styrene & 500 & 400 & 80 & 5 & 40 & $1-150$ \\
\hline $1,1,2,2$-Tetrachloroetha & 500 & 20 & 4 & $200 *$ & 40 & $1-150$ \\
\hline Heptane & 500 & 490 & 98 & 2 & 40 & $1-150$ \\
\hline Octane & 500 & 490 & 98 & 2 & 40 & $1-150$ \\
\hline Nonane & 500 & 400 & 80 & 2 & 40 & $1-150$ \\
\hline 3-Chloropropene & 500 & 360 & 72 & 3 & 40 & $1-150$ \\
\hline 2-Pentanone & 500 & 1300 & $260 *$ & 14 & 40 & $1-150$ \\
\hline Methylcyclohexane & 500 & 450 & 90 & 12 & 40 & $1=150$ \\
\hline 5-Methyl-2-hexanone & 500 & 630 & 126 & 8 & 40 & $1-150$ \\
\hline 4-Heptanone & 500 & 1200 & $240 *$ & 0 & 40 & $1-150$ \\
\hline Butylacetate & 500 & 750 & 150 & 15 & 40 & $1-150$ \\
\hline I,4-Dioxane & 500 & 1100 & $184 *$ & 20 & 40 & $1-150$ \\
\hline 2-Methyl-2-propenenitri & 500 & 640 & 128 & 9 & 40 & $1-150$ \\
\hline 3-Methyl-2-butanone & 500 & 1300 & $260 *$ & 7 & 40 & $1-150$ \\
\hline
\end{tabular}

\# Column to be used to flag recovery and RPD values with an asterisk

* Values outside of QC limits

COMMENTS: 
SUPERNATANT VOLATIIE MATRIX,SPIKE/MATRIX.SPIKE DUPLICATE RECOVVERY

Lab Name: PNNL

Contract : BNEL

Lab Code: PNNL

Case No.:

SAS NO. :

SDG No.: 990205

Matrix Spike - EPA Sample No.: AW-101

\begin{tabular}{|c|c|c|c|c|c|c|}
\hline & $\begin{array}{l}\text { SPIKE } \\
\text { ADDED }\end{array}$ & $\begin{array}{c}\text { MSD } \\
\text { AMOUNT }\end{array}$ & $\begin{array}{c}\text { MSD } \\
\div\end{array}$ & $\div$ & \multicolumn{2}{|c|}{ QC IIMITS } \\
\hline COMPOUND & (ng) & (ng) & REC \# & RPD \# & RPD & REC. \\
\hline 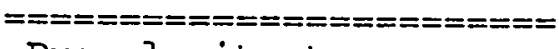 & $========$ & $=============$ & $=====$ & $======$ & $===\equiv=$ & $=====$ \\
\hline $\begin{array}{l}\text { Propyl nitrate } \\
\text { 3-Pentanone }\end{array}$ & $\begin{array}{l}500 \\
500\end{array}$ & $\begin{array}{r}560 \\
1300\end{array}$ & $\begin{array}{l}112 \\
260 *\end{array}$ & $\begin{array}{r}2 \\
74\end{array}$ & $\begin{array}{l}4 \overline{0} \\
40\end{array}$ & $\begin{array}{l}1-150 \\
1-150\end{array}$ \\
\hline $1,2,2-C 13-1,1,2-F 3$ ethan & 500 & 560 & 112 & $\begin{array}{r}14 \\
4\end{array}$ & $\begin{array}{l}40 \\
40\end{array}$ & $\begin{array}{l}1-150 \\
1-150\end{array}$ \\
\hline $1,2-\mathrm{Cl} 2-1,1,2,2-\mathrm{F} 4$ ethan & 500 & 520 & 104 & 6 & 40 & $1-150$ \\
\hline Dichlorodifluoromethane & 500 & 450 & 90 & 20 & 40 & $1-150$ \\
\hline Acetonitrile & 500 & 530 & 106 & 4 & 40 & $1-150$ \\
\hline Cyclohexane & 500 & 520 & 104 & 2 & 40 & $1-150$ \\
\hline 1,2-Dibromoethane & 500 & 240 & 48 & 0 & 40 & $1-150$ \\
\hline 3-Heptanone & 500 & 1300 & $260 *$ & 7 & 40 & $1-150$ \\
\hline 2-Heptanone & 500 & 1500 & $300 *$ & 6 & 40 & $1-150$ \\
\hline Tetrahydrofuran & 500 & 1300 & $232 *$ & 8 & 40 & $I-150$ \\
\hline
\end{tabular}

\# Column to be used to flag recovery and RPD values with an asterisk

* Values outside of QC limits

RPD: 2 out of 67 outside limits

Spike Recovery: 32 out of 134 outside limits

COMMENTS : 
Lab: Name: PNNL

Lab Code: PNNL

Lab File ID: 99021204

Date Analyzed: 02/12/99

GC Column: DB-624 75M X 2.55UMID: 0.45 (mm)

Instrument ID: HP1
Case No.:
Contract: BNFL

SAS NO.:
EPA SAMPLE NO.

METHOD BLANK
Lab Sample ID: VBLK0I

Time Analyzed: 1543

SDG NO.: 990205

........THIS. METHOD BLANK APPLIES TO THE FOLIOWING SAMPLES, MS and MSD:

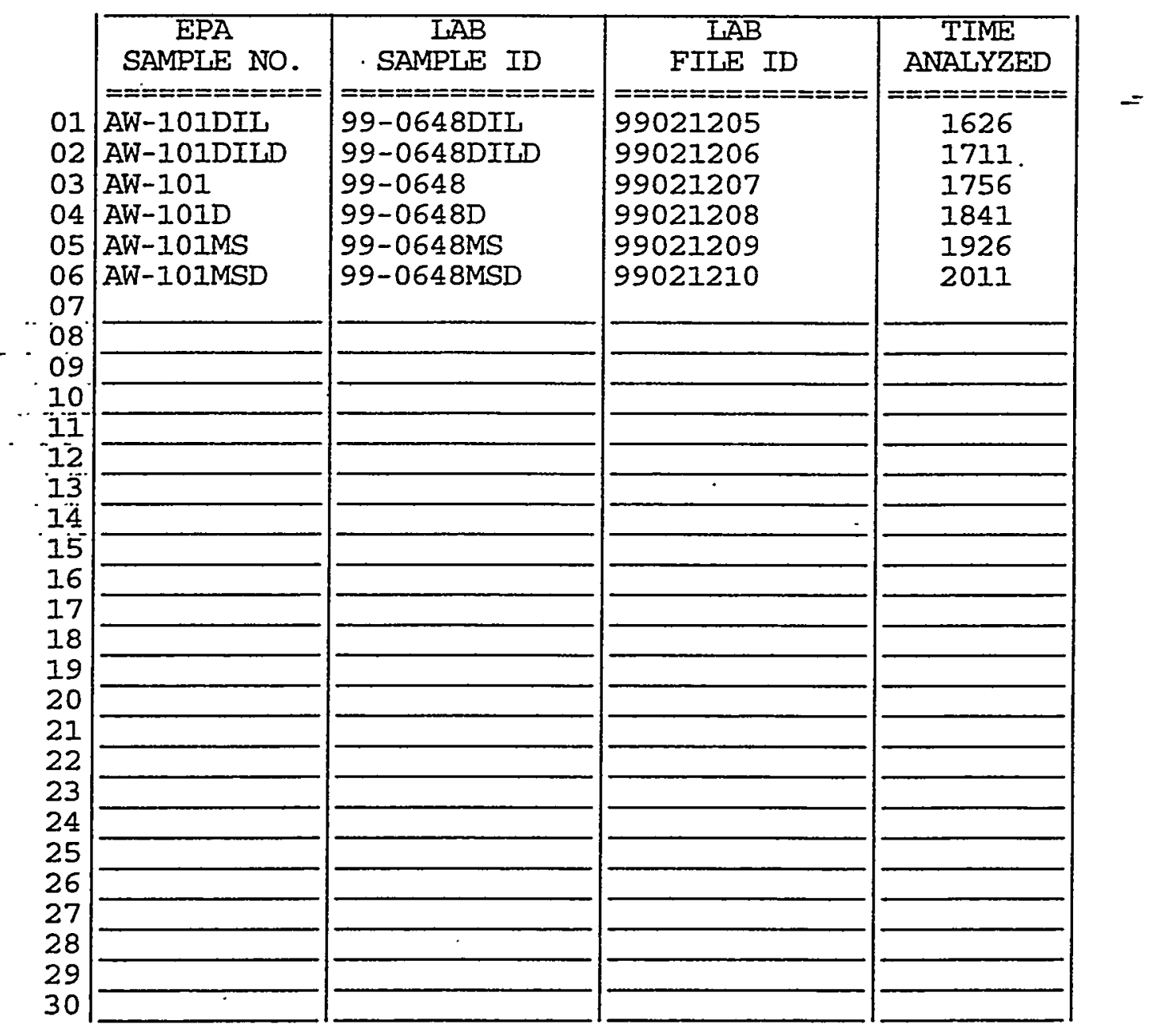

COMMENTS :

Heated Purge: $(Y / N) N$ 
VOLATILE ORGANIC INSTRUMENT PERFORMANCE CHECK BROMOFLUOROBENZENE (BFB)

Lab Name: PNNL

Lab Code: PNNL

Iab File ID: 99021201

Instrument ID: HPI

GC Column:
Contract: BNFL

SAS NO. :
SDG NO. : 990205

BFB Injection Date: 02/12/99

BFB Injection Time: 1313
Case No.:

ID: $2.00(\mathrm{~mm})$
Heated Purge: $(Y / N) N$

\begin{tabular}{|c|c|c|}
\hline \begin{tabular}{r|}
$\mathrm{m} / \mathrm{e}$ \\
$=====$ \\
50 \\
75 \\
95 \\
96 \\
173 \\
174 \\
175 \\
176 \\
177
\end{tabular} & $\begin{array}{l}\text { ION ABUNDANCE CRITERIA } \\
================================================== \\
8.0-40.0 \% \text { of mass } 95 \\
30.0-66.0 \% \text { of mass } 9 \overline{5} \\
\text { Base Peak, } 100 \% \text { relative abundance } \\
5.0-9.0 \% \text { of mass } 95 \\
\text { Less than } 2.0 \% \text { of mass } 174 \\
50.0-120.0 \% \text { of mass } 95 \\
4.0-9.0 \% \text { of mass } 174 \\
93.0-101.0 \% \text { of mass } 174 \\
5.0-9.0 \% \text { of mass } 176\end{array}$ & 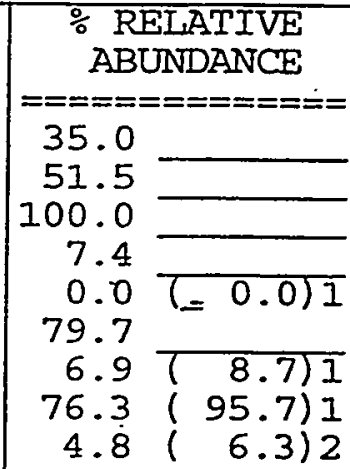 \\
\hline
\end{tabular}

THIS :CHECK APPLIES TO THE FOLIOWING SAMPLES, MS, MSD, BIANKS, AND STANDARDS:

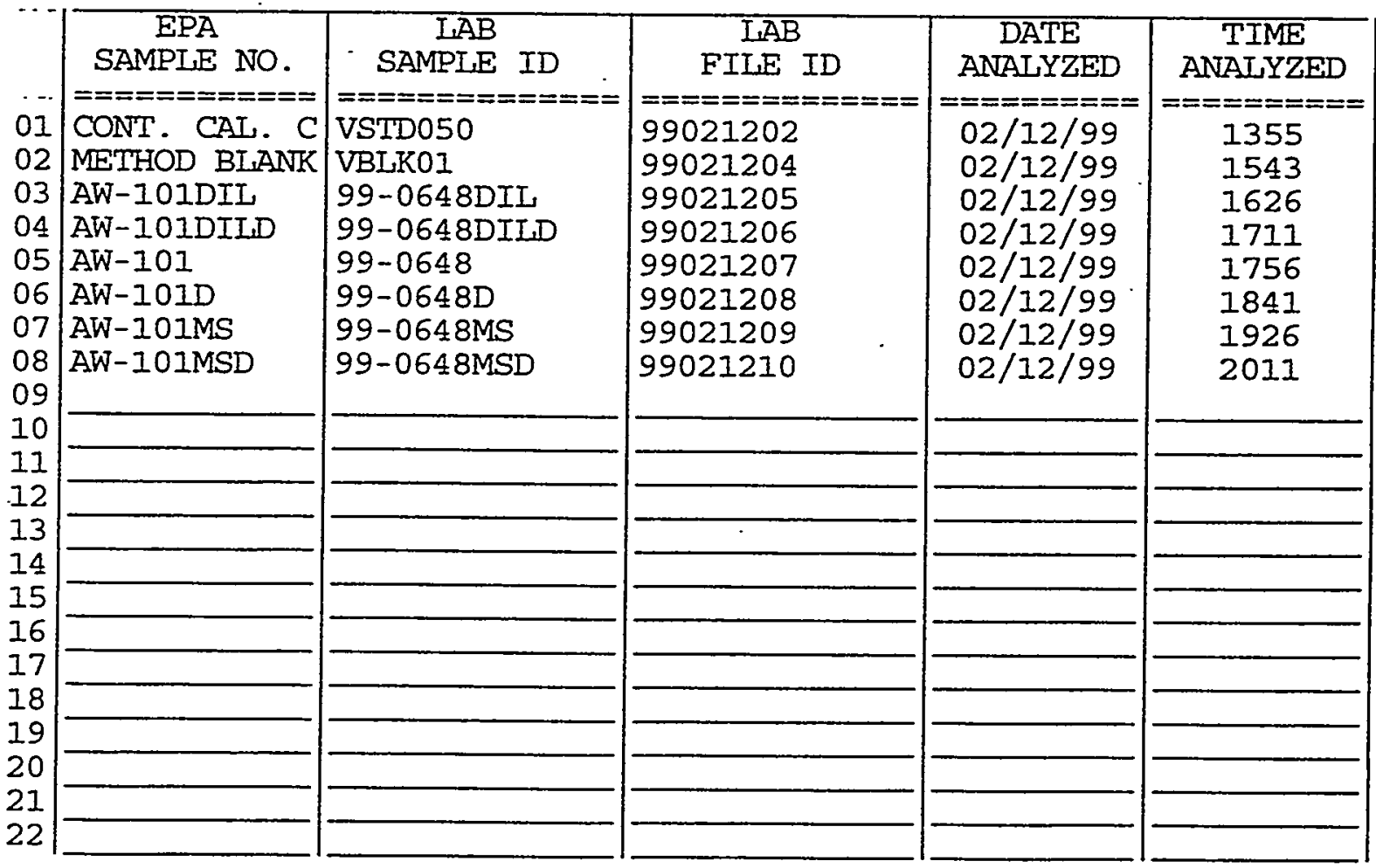

page 1 of 1 
VOLATILE ORGANIC INSTRUMENT PERFORMANCE CHECK BROMOFLUOROBENZENE (BFB)

Iub Name: PNNL

Iuab Code: PNNL . Case No.:

Lab File ID: 99021101

Instrument ID: HPI

GC Column:
Contract : BNFL

SAS NO.:
SDG NO.: 990205

BFB Injection Date: 02/11/99

BFB Injection Time: 1206

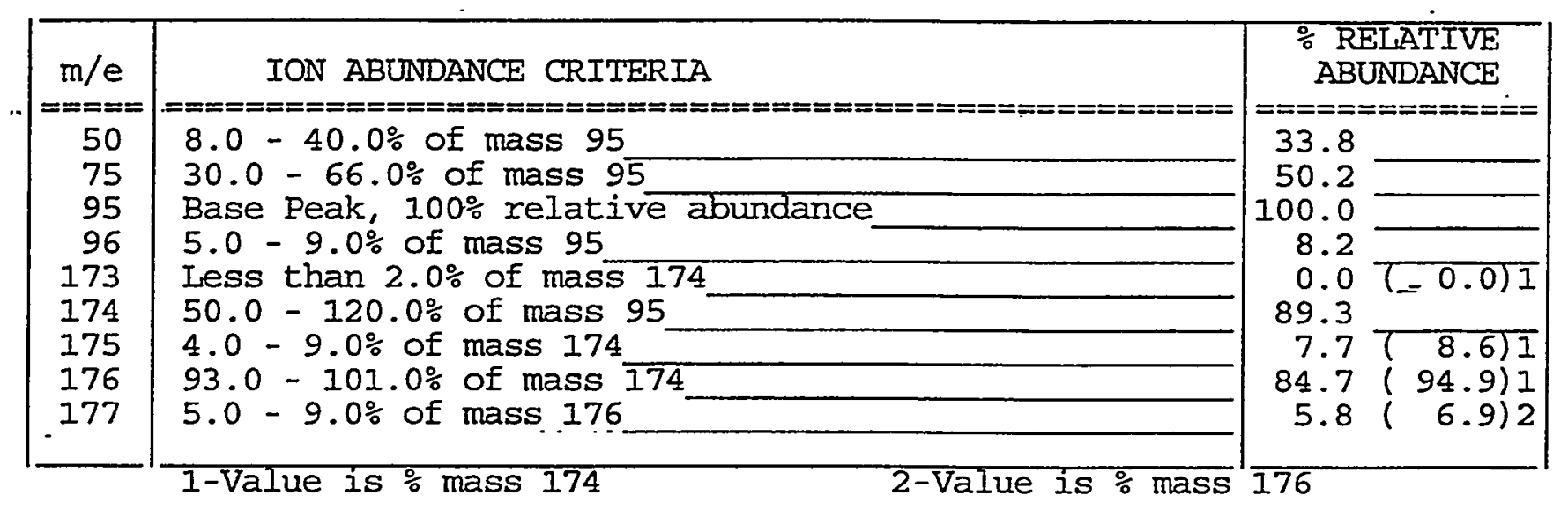

THIS: CHECK APPLIES TO THE“FOLIOWING SAMPLES, MS, MSD, BLANKS, AND STANDARDS:

\begin{tabular}{|c|c|c|c|c|c|}
\hline & $\begin{array}{c}\text { EPA } \\
\text { SAMPLE NO. } \\
==========\end{array}$ & $\begin{array}{c}\text { IAAB } \\
\text { SAMPIE ID } \\
============\end{array}$ & $\begin{array}{c}\text { IAAB } \\
\text { FILE ID } \\
============\end{array}$ & $\begin{array}{c}\text { DATE } \\
\text { ANAIYZED } \\
==========\end{array}$ & $\begin{array}{c}\text { TIME } \\
\text { ANALYZED } \\
=========\end{array}$ \\
\hline $\begin{array}{l}01 \\
02 \\
03 \\
04 \\
05 \\
06\end{array}$ & $\begin{array}{l}\text { VSTD020 } \\
\text { VSTD050 } \\
\text { VSTD100 } \\
\text { VSTD150 } \\
\text { VSTD200 }\end{array}$ & $\begin{array}{l}\text { VSTD020 } \\
\text { VSTD050 } \\
\text { VSTD100 } \\
\text { VSTD150 } \\
\text { VSTD200 }\end{array}$ & $\begin{array}{l}99021102 \\
99021103 \\
99021104 \\
99021105 \\
99021106\end{array}$ & $\begin{array}{l}02 / 11 / 99 \\
02 / 11 / 99 \\
02 / 11 / 99 \\
02 / 11 / 99 \\
02 / 11 / 99\end{array}$ & $\begin{array}{l}1249 \\
1334 \\
1419 \\
1504 \\
1549\end{array}$ \\
\hline $\begin{array}{l}07 \\
08\end{array}$ & & & & & \\
\hline 09 & & & & & \\
\hline $\begin{array}{l}10 \\
11\end{array}$ & & & & & \\
\hline 12 & & $\cdot$ & & & \\
\hline 13 & & & & & \\
\hline 14 & & & & & \\
\hline 15 & & & & & \\
\hline 16 & & & & & \\
\hline 11 & & & & & \\
\hline 19 & & & & & \\
\hline 20 & & & & & \\
\hline & & & & & \\
\hline 2 & & & & & \\
\hline
\end{tabular}

page 1 of 1 
VOLATILE ORGANICS INITIAL CALIBRATION DATA

Irab Name: PNNL

Lab Code: PNNL Case No.:
Contract: BNFL

SAS NO.:

SDG No.: 990205
Instrument ID: HP1

Heated Purge: (Y/N) N
Calibration Date (s) : 02/11/99

Calibration Time(s): 1249
$02 / 11 / 99$

1549

GC Column: DB-624 75M X 2.55UM ID: 0.45 (mm)

\begin{tabular}{|c|c|c|c|c|c|c|c|}
\hline $\begin{array}{l}\text { LAAB FILE ID: } \\
\text { RRF500=99021104 }\end{array}$ & $\begin{array}{l}0=99021 \\
0=99021\end{array}$ & $\begin{array}{l}102 \\
105\end{array}$ & $\begin{array}{l}\text { RRF2 } \\
\text { RRF1 }\end{array}$ & $\begin{array}{l}50=9902 \\
000=990\end{array}$ & $\begin{array}{l}1103 \\
21106\end{array}$ & & \\
\hline 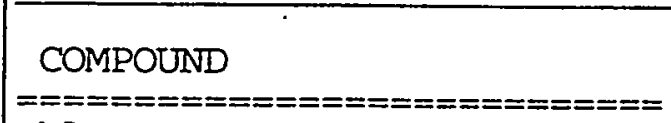 & $\begin{array}{l}\text { RRF100 } \\
======\end{array}$ & $\begin{array}{l}\text { RRF250 } \\
======\end{array}$ & $\begin{array}{l}\text { RRF500 } \\
======\end{array}$ & $\begin{array}{l}\text { RRF750 } \\
======\end{array}$ & $\begin{array}{l}\text { RRF } \\
1000 \\
=====\end{array}$ & $\begin{array}{c}\overline{R R F} \\
======\end{array}$ & $\begin{array}{c}\frac{0}{0} \\
\text { RSD } \\
=====\end{array}$ \\
\hline Chloromethane & 0.595 & 0.460 & 0.515 & 0.567 & 0.561 & 0.540 & $9.8 *$ \\
\hline Vinyl Chloride & 0.402 & 0.379 & 0.412 & 0.474 & 0.471 & 0.428 & 10.0 \\
\hline 1,3-Butadiene & 1.167 & 0.910 & 0.960 & 1.093 & 1.094 & 1.045 & 10.1 \\
\hline Butane & 0.141 & 0.118 & 0.154 & 0.160 & 0.169 & 0.148 & 13.2 \\
\hline Bromomethane & 0.096 & 0.125 & 0.113 & 0.111 & 0.093 & $0 . \overline{1} 08$ & 12.0 \\
\hline 1,1-Dichloroethene & 0.359 & 0.320 & 0.401 & 0.460 & 0.438 & 0.396 & 14.4 \\
\hline Chloroethane & 0.138 & 0.136 & 0.124 & 0.117 & 0.102 & 0.119 & 10.2 \\
\hline Acetone & 0.300 & 0.238 & 0.240 & 0.255 & 0.238 & 0.254 & 10.6 \\
\hline Pentane & 0.593 & 0.637 & 0.575 & $0.68 i$ & 0.715 & 0.640 & 9.1 \\
\hline Carbon Disulfide & 1.023 & 1.057 & 1.093 & 1.248 & 1.196 & 1.123 & 8.5 \\
\hline Acrolein & 0.010 & 0.010 & 0.008 & 0.009 & 0.008 & 0.009 & $9.5^{\star}$ \\
\hline Acrylonitrile & 0.194 & 0.174 & 0.224 & .0 .265 & 0.282 & 0.228 & 20.0 \\
\hline Methylene Chloride & 0.411 & 0.358 & 0.395 & 0.494 & 0.503 & 0.432 & 14.8 \\
\hline Propionaldehyde & 0.072 & 0.052 & 0.054 & 0.060 & 0.057 & 0.059 & 13.1 \\
\hline trans-1,2-Dichloroethene & 0.278 & 0.318 & 0.282 & 0.310 & 0.295 & 0.297 & 5.8 \\
\hline 2-Butenal & 0.049 & 0.047 & 0.054 & 0.066 & 0.071 & 0.057 & 18.3 \\
\hline 1,1-Dichloroethane & 0.801 & 0.819 & 0.880 & 1.063 & 1.075 & 0.928 & $14.3 *$ \\
\hline cis-1,2-Dichloroethene & $0.4 I I$ & 0.442 & 0.453 & 0.529 & 0.531 & 0.473 & 11.4 \\
\hline Hexane & 0.490 & 0.490 & 0.538 & 0.651 & 0.654 & 0.565 & 14.7 \\
\hline 2-Butanone & 0.271 & 0.299 & 0.344 & 0.346 & 0.397 & .331 & 14.6 \\
\hline Cyclohexene & 0.162 & 0.165 & 0.164 & 0.191 & 0.179 & 0.172 & 7.2 \\
\hline Ethyl acetate & 0.705 & 0.741 & 0.800 & 0.931 & 0.968 & 0.829 & 14.0 \\
\hline Cyclopentane & 1.373 & 1.352 & 1.358 & 1.746 & 1.754 & 1.517 & 14.0 \\
\hline $\begin{array}{l}\text { Bromochloromethane } \\
\text { 1, 1-Dimethyl hydrazine }\end{array}$ & 0.182 & 0.146 & 0.195 & 0.209 & 0.206 & 0.188 & 13.6 \\
\hline $\begin{array}{l}1,1-\text { Dimethy inydrazine } \\
\text { Chloroform }\end{array}$ & 0.742 & 0.034 & 0.834 & 0.982 & 0.984 & $\begin{array}{l}0.034 \\
0.849\end{array}$ & $\begin{array}{r}0.0 \\
15.4\end{array}$ \\
\hline $1,1,1$-Trichloroethane & 0.063 & 0.073 & 0.067 & 0.074 & 0.073 & 0.070 & 7.0 \\
\hline Dichlorofluoromethane & 0.541 & 0.642 & 0.638 & 0.717 & 0.700 & 0.648 & 10.7 \\
\hline Chlorodifluoromethane & 0.316 & 0.281 & 0.332 & 0.388 & 0.398 & 0.343 & 14.4 \\
\hline Carbon Tetrachloride & 0.475 & 0.470 & 0.430 & 0.463 & 0.444 & 0.456 & 4.2 \\
\hline Trichlorofluoromethane & 0.287 & 0.285 & 0.331 & 0.296 & 0.375 & 0.315 & 12.3 \\
\hline Benzene & 0.761 & 0.713 & 0.771 & 0.738 & 0.662 & 0.729 & 6.0 \\
\hline 1,2-Dichloroethane & 0.376 & 0.319 & 0.406 & 0.396 & 0.379 & 0.375 & 9.0 \\
\hline Trichloroethene & 0.190 & 0.194 & 0.186 & 0.188 & 0.169 & 0.185 & 5.4 \\
\hline 1,2-Dichloropropane & 0.220 & 0.202 & 0.236 & 0.248 & 0.227 & 0.227 & 7.6 \\
\hline Bromodichloromethane & 0.290 & 0.260 & 0.308 & 0.33 & 0.314 & & 9.1 \\
\hline cis-1,3-Dichloroprope & 0.340 & 0.294 & 0.358 & 0.3 & 0.352 & 0.345 & 9.1 \\
\hline
\end{tabular}

Compounds with required minimum $R R F$ and maximim $: \overline{R S D}$ values

All other compounds must meet a minimim RRF of 0.010 . 
- Lab Name: PNNL

Lab Code: PNNL
Contract: BNFL

SAS NO.:

SDG NO.: 990205

Instrument ID: HP1 Calibration Date(s): 02/11/99 02/11/99

Heated Purge: (Y/N) N Calibration Time(s) : $1249 \quad 1549$

GC Column: DB-62475M X 2.55UM ID: 0.45 (mm)

\begin{tabular}{|c|c|c|c|c|c|c|c|}
\hline $\begin{array}{l}\text { LAB FILE ID: } \\
\text { RRF500=99021104 }\end{array}$ & $\begin{array}{l}0=99021 \\
0=99021\end{array}$ & $\begin{array}{l}102 \\
105\end{array}$ & $\begin{array}{l}\text { RRF2 } \\
\text { RRF1 }\end{array}$ & $\begin{array}{l}0=9902 \\
00=990\end{array}$ & $\begin{array}{l}.103 \\
1106\end{array}$ & & \\
\hline $\begin{array}{l}\text { COMPOUND } \\
=========1\end{array}$ & $\begin{array}{l}\text { RRF100 } \\
======\end{array}$ & $\begin{array}{l}\text { RRF250 } \\
======\end{array}$ & $\begin{array}{l}\text { RRF500 } \\
======\end{array}$ & $\begin{array}{l}\text { RRF750 } \\
======\end{array}$ & $\begin{array}{c}\text { RRF } \\
1000 \\
======\end{array}$ & $\begin{array}{c}\overline{R R F} \\
======\end{array}$ & $\begin{array}{c}\frac{0}{\circ} \\
R S D \\
=====\end{array}$ \\
\hline 4-Methyl-2-pentanone & 0.400 & 0.414 & 0.438 & 0.492 & 0.491 & 0.447 & 9.6 \\
\hline Toluene & & 0.564 & 0.583 & & 0.582 & 0.580 & 4.3 \\
\hline trans-1,3-Dichloropropene & 0.386 & 0.348 & 0.436 & 0.475 & 0.460 & 0.421 & 12.6 \\
\hline 1,1,2-Trichlorc & 0.179 & 0.155 & 0.195 & & 0.206 & 0.190 & 12.3 \\
\hline etrachloroetl & & 0.184 & 0.141 & & 0.129 & & 14.4 \\
\hline none & 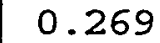 & 0.252 & 0.299 & & 0.350 & 0.303 & 14.6 \\
\hline Dibromochloromet & 0.222 & 0.198 & 0.239 & 0 . & 0.242 & 0.231 & 9.1 \\
\hline Chlorobenzene & 49 & 0.5 & 0.534 & & 02 & 0.533 & $3.4 *$ \\
\hline thylbenzene - & 0.2 & 0.319 & 0.282 & & 0.2 & 0.2 & 5.8 \\
\hline lene $(m \& \bar{p})$ & 0.778 & 0.7 & 0.602 & & & 0.6 & 16.2 \\
\hline Xylene (o) & 0.367 & 0.369 & 0.316 & & $0: 285$ & 0.332 & 10.9 \\
\hline Styrene & 0.603 & 0.576 & 0.545 & & 0.478 & 0.548 & .8 .6 \\
\hline Bromoform & 0.128 & 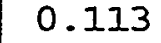 & 0.138 & & & & $8.6 *$ \\
\hline $1,1,2,2$-Tetrachloroethane & 0.373 & 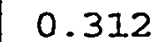 & 0.370 & & & & $7.8 *$ \\
\hline 1,3-Dichlo] & 0.556 & 0.5 & 0.453 & & & & 14.9 \\
\hline 1,4-Dichlorobenzene & 0.564 & 0.5 & 0.474 & & & & 10.4 \\
\hline 1,2-Dichloroben & 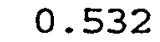 & 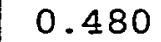 & 0.451 & & & & 10.8 \\
\hline Heptane & $0 . c$ & 0.0 & 0.056 & & & & .8 .9 \\
\hline & 0.039 & 0. & 0.036 & & & & 12.0 \\
\hline ne & 0.035 & 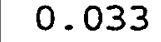 & 0.027 & & & & 16.4 \\
\hline loropropene & & 0. & 0.619 & & & & 10.3 \\
\hline anone & & 0 . & 0.028 & & & & 11.3 \\
\hline ylcyclohexane & 0.3 & 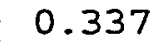 & 0.293 & & & & 7.7 \\
\hline lethyl-2-hexanone & & 0. & 0.547 & & & & 11.0 \\
\hline Heptanone & & & 0.472 & & & & 14.9 \\
\hline ylacetate & 0.6 & 0. & 0.681 & & & & 12.0 \\
\hline ine & 0.003 & 0.002 & 0.002 & & 0. & & $7.5 \star$ \\
\hline 2-Methyl-2-propenenitrile & 0.215 & 0.177 & 0.224 & & 0.225 & & 10.6 \\
\hline 3-Methyl-2-butanone & & & 0.036 & & & & 14.2 \\
\hline nyl- nitrate & & 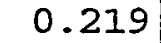 & 0 . & & & & 17.5 \\
\hline מר & .72 & 0.15 & 0.189 & & & & 13.2 \\
\hline $1,2,2-C 13-1,1,2-F 3$ ethane & 0.2 & 0.3 & 0.321 & & & 0.3 & 12.2 \\
\hline $1,2-\mathrm{Cl} 2-1,1,2,2-\mathrm{F} 4$ ethane & 76 & 0.1 & 0.134 & & 0. & 0.164 & 13.4 \\
\hline Dichlorodifluoromethane & & 0.5 & 0.543 & & & & 6.2 \\
\hline etonitrile & & 0.1 & 0.092 & & & & 0.6 \\
\hline & & & & & & & \\
\hline Bromol & & 0.272 & & & 0.200 & 0.249 & 14.7 \\
\hline
\end{tabular}

* Compounds with required minimum RRF and maximim $: \overline{R S D}$ values.

Al1 other compounds must meet a minimim RRF of 0.010 .

page 2 of 3 
VOLATILE ORGANICS INITIAL CAIIBRÁTION DATA

Lab Name: PNNL

Lab Code: PNNL
Contract: BNFL

SAS NO. :
SDG NO.: 990205

$02 / 11 / 99$

Heated Purge: ( $Y / N) N \quad$ Calibration Time(s) : $1249 \quad 1549$

GC Column: DB-624 75M X 2.55UM ID: 0.45 (mm)

\begin{tabular}{|c|c|c|c|c|c|c|c|}
\hline \multicolumn{3}{|l|}{$\begin{array}{l}\text { LAB FILE ID: } \\
\text { RRF500 }=99021104\end{array}$} & \multicolumn{3}{|c|}{$\begin{array}{l}\text { RRF250 }=99021103 \\
\text { RRF1000 }=99021106\end{array}$} & \multirow[b]{2}{*}{$\overline{\mathrm{RRF}}$} & \multirow[b]{2}{*}{$\begin{array}{c}\% \\
\mathrm{RSD} \\
====\end{array}$} \\
\hline $\begin{array}{l}\text { COMPOUND } \\
=============1 .\end{array}$ & \multirow{29}{*}{\begin{tabular}{|c} 
RRFI00 \\
$======$ \\
0.263 \\
0.886 \\
1.283 \\
0.919 \\
1.066 \\
1.069 \\
0.065 \\
0.195 \\
0.158 \\
0.372 \\
0.298 \\
0.579 \\
0.135 \\
0.434 \\
0.840 \\
0.876 \\
1.539 \\
0.300 \\
0.316 \\
0.529 \\
1.010 \\
1.002 \\
0.046 \\
0.338 \\
0.409 \\
0.203 \\
0.130 \\
$======$
\end{tabular}} & $\begin{array}{l}\text { RRF250 } \\
======\end{array}$ & $\begin{array}{l}\text { RRF500 } \\
======\end{array}$ & $\begin{array}{l}\text { RRF750 } \\
======\end{array}$ & \begin{tabular}{|l}
$R R F$ \\
$I 000$ \\
$=====$
\end{tabular} & & \\
\hline Butylbenzene & & 0.283 & 0.215 & 0.236 & 0.204 & 0.240 & 13.7 \\
\hline tert-Butylbenzene & & 0.944 & 0.736 & 0.826 & 0.727 & 0.824 & 11.4 \\
\hline sec-Butylbenzene & & 1.382 & 1.078 & 1.167 & 1.009 & 1.184 & 12.7 \\
\hline 2-Chlorotoluene - & & 0.915 & 0.836 & 0.898 & 0.776 & 0.869 & 7.1 \\
\hline 4-Isopropyltoluene & & 1.059 & 0.854 & 0.863 & 0.765 & 0.921 & 14.6 \\
\hline 4-Chlorotoluene & & 1.059 & 0.973 & 1.017 & 0.881 & 1.000 & 7.7 \\
\hline 1,2-Dibromo-3-chloropropane & & 0.052 & 0.067 & 0.072 & 0.068 & 0.065 & 11.5 \\
\hline 1,2-Dibromoethane & & 0.168 & 0.210 & 0.230 & 0.223 & 0.205 & 12.1 \\
\hline Erans-1,4-Dichloro-2-butene & & 0.122 & 0.164 & 0.179 & 0.162 & 0.157 & 13.3 \\
\hline 1,3-Dichloropropane & & 0.330 & 0.396 & 0.399 & 0.377 & 0.375 & 7.4 \\
\hline 2,2-Dichloropropane & & 0.368 & 0.309 & 0.343 & 0.342 & 0.332 & 8.5 \\
\hline 1,1-Dichloropropene & & 0.549 & 0.642 & 0.729 & 0.701 & 0.640 & 12.0 \\
\hline Hexachloro-1,3-butadiene & & 0.126 & 0.106 & 0.107 & 0.098 & 0.114 & 13.3 \\
\hline Iodomethane & & 0.437 & 0.488 & 0.534 & 0.528 & 0.484 & 9.9 \\
\hline Isopropylbenzene & & 0.906 & 0.774 & 0.843 & 0.755 & 0.824 & 7.3 \\
\hline Nathphalene & & 0.668 & 0.773 & 0.798 & 0.705 & 0.764 & 10.6 \\
\hline Propylbenzene & & 1.489 & 1.263 & 1.278 & 1.167 & 1.347 & 11.8 \\
\hline $1,2,3$-Trichlorobenzene & & 0.247 & 0.233 & 0.246 & 0.214 & 0.248 & 13.0 \\
\hline 1,2,4-Trichlorobenzene & & 0.281 & 0.250 & 0.255 & 0.224 & 0.265 & 13.1 \\
\hline 1,2,3-Trichloropropane & & 0.431 & 0.544 & 0.583 & 0.537 & 0.525 & 10.7 \\
\hline $1,2,4$-Trimethylbenzene & & 1.023 & 0.874 & 0.923 & 0.804 & 0.927 & 10.0 \\
\hline I, 2,3-Trimethylbenzene & & 1.043 & 0.848 & 0.874 & 0.749 & 0.903 & 13.2 \\
\hline 1-Propanol & & 0.041 & 0.055 & 0.070 & 0.071 & 0.057 & 23.9 \\
\hline 3-Heptanone & & 0.280 & 0.330 & 0.315 & 0.288 & 0.310 & 8.2 \\
\hline 2-Heptanone & & 0.353 & 0.458 & 0.518 & 0.512 & 0.450 & 15.6 \\
\hline Tetrahydrofuran & & 0.176 & 0.208 & 0.240 & 0.250 & 0.215 & 13.8 \\
\hline Dibromomethane & & 0.110 & 0.139 & 0.149 & 0.141 & 0.134 & 11.1 \\
\hline 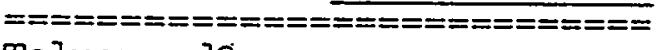 & & $=====0$ & $=====$ & $======$ & $======$ & $===$ & $=====$ \\
\hline Toluene-d8 & 0.586 & 0.594 & 0.583 & 0.619 & 0.580 & 0.592 & 2.7 \\
\hline Bromofluorobenzene & 0.382 & 0.352 & 0.356 & 0.393 & 0.355 & 0.368 & 5.1 \\
\hline Dibromofluoromethane & 0.336 & 0.284 & 0.358 & 0.384 & 0.379 & 0.348 & 11.7 \\
\hline & & & & & & & \\
\hline & & & & & & & \\
\hline & & & & & & & \\
\hline & & & & & & & \\
\hline & & & & & & & \\
\hline
\end{tabular}

Compounds with required minimum $R R F$ and maximim $\div \overline{R S D}$ values.

All other compounds must meet a minimim RRF of 0.010 .

page 3 of 3 
$7 A$

VOLATILE CONTINUING CALIBRATION CHECK

Lab Name: PNNL

Contract: BNFL

Lab Code: PNNL

Case No. :

SAS NO.:

SDG NO.: 990205

Instrument ID: $\mathrm{HPI}$

Calibration Date: 02/11/99 Time: 1334

Lab File ID: 99021103

Init. Calib. Date (s) : 02/11/99

$02 / 11 / 99$

Heated Purge: $(Y / N) N$

Init. Calib. Times: 1249

1549

GC Column: DB-624 75M X 2.55UM ID: 0.45 (mm)

\begin{tabular}{|c|c|c|c|c|c|}
\hline $\begin{array}{l}\text { COMPOUND } \\
=========================\end{array}$ & $\begin{array}{c}\overline{\mathrm{RRF}} \\
======\end{array}$ & RRF250 & $\begin{array}{l}\text { MIN } \\
\text { RRF } \\
=====\end{array}$ & $====$ & $\begin{array}{c}\text { MAX } \\
\frac{\circ D}{2} \\
====\end{array}$ \\
\hline Chloromethane & 0.540 & 0.460 & 0.100 & 14.8 & 100 \\
\hline Vinyl Chloride & 0.428 & 0.379 & & 11.4 & \\
\hline 1,3-Butadiene & 1.045 & 0.910 & & 12.9 & \\
\hline Butane & 0.148 & 0.118 & & 20.3 & \\
\hline Bromomethane & 0.108 & 0.125 & & -15.7 & \\
\hline 1,1-Dichloroethene & 0.396 & 0.320 & & 19.2 & \\
\hline Chloroethane & 0.119 & 0.136 & . & $\cdot-14.3$ & \\
\hline Acetone & 0.254 & 0.238 & & 6.3 & \\
\hline Pentane & 0.640 & 0.637 & & 0.5 & \\
\hline Carbon Disulfide & 1.123 & 1.057 & & 5.9 & \\
\hline Acrolein & .0 .009 & 0.010 & 0.001 & -11.1 & 100 \\
\hline Acrylonitrile & 0.228 & 0.174 & & 23.7 & \\
\hline Methylene Chloride & 0.432 & 0.358 & & 17.1 & \\
\hline Propionaldehyde & 0.059 & 0.052 & & 11.9 & \\
\hline trans-1,2-Dichloroethene & 0.297 & 0.318 & & -7.1 & . \\
\hline 2-Butenal & 0.057 & 0.047 & & 17.5 & \\
\hline 1,1-Dichloroethane & 0.928 & 0.819 & 0.100 & 11.7 & 100 \\
\hline cis-I,2-Dichloroethene & 0.473 & 0.442 & & .6 .6 & \\
\hline Hexane & 0.565 & 0.490 & & 13.3 & \\
\hline 2-Butanone & 0.331 & 0.299 & & 9.7 & \\
\hline Cyclohexene & 0.172 & 0.165 & & 4.1 & \\
\hline Ethyl acetate & 0.829 & 0.741 & & 10.6 & \\
\hline Cyclopentane & 1.517 & 1.352 & & 10.9 & \\
\hline Bromochloromethane & 0.188 & 0.146 & & 22.3 & \\
\hline 1,1-Dimethylhydrazine & 0.034 & 0.034 & & 0.0 & \\
\hline Chloroform & 0.849 & 0.705 & & 17.0 & \\
\hline $1,1,1$-Trichloroethane & 0.070 & 0.073 & & $-4 \cdot 3$ & \\
\hline Dichlorofluoromethane & 0.648 & 0.642 & & 0.9 & \\
\hline Chlorodifluoromethane & 0.343 & 0.281 & & 18.1 & \\
\hline Carbon Tetrachloride - & 0.456 & 0.470 & & $-3 \cdot 1$ & \\
\hline Trichlorofluoromethane & 0.315 & 0.285 & & 9.5 & \\
\hline Benzene & 0.729 & 0.713 & & 2.2 & \\
\hline 1,2-Dichloroethane & 0.375 & 0.319 & & 14.9 & \\
\hline Trichloroethene & 0.185 & 0.194 & & -4.9 & \\
\hline 1,2-Dichloropropane & 0.227 & 0.202 & & 11.0 & \\
\hline Bromodichloromethane & 0.301 & 0.260 & & 13.6 & \\
\hline cis-1,3-Dichloropropene & 0.345 & 0.294 & & 14.8 & \\
\hline
\end{tabular}

All other compounds must meet a minimum RRF of 0.010 .

page 1 of 3

FORM VII VOA

OLMO3. 0 
VOLATILE: CONTINUING CALIBRATION CHECK

Lab Name: PNNL

Lab Code: PNNL
Contract: BNFL

SAS NO.:
SDG No.: 990205

Time: 1334

$02 / 11 / 99$

Heated Purge: (Y/N) N Init. Calib. Times: $1249 \quad 1549$

GC Column: DB-624 75M X 2.55UM ID: 0.45 (mm)

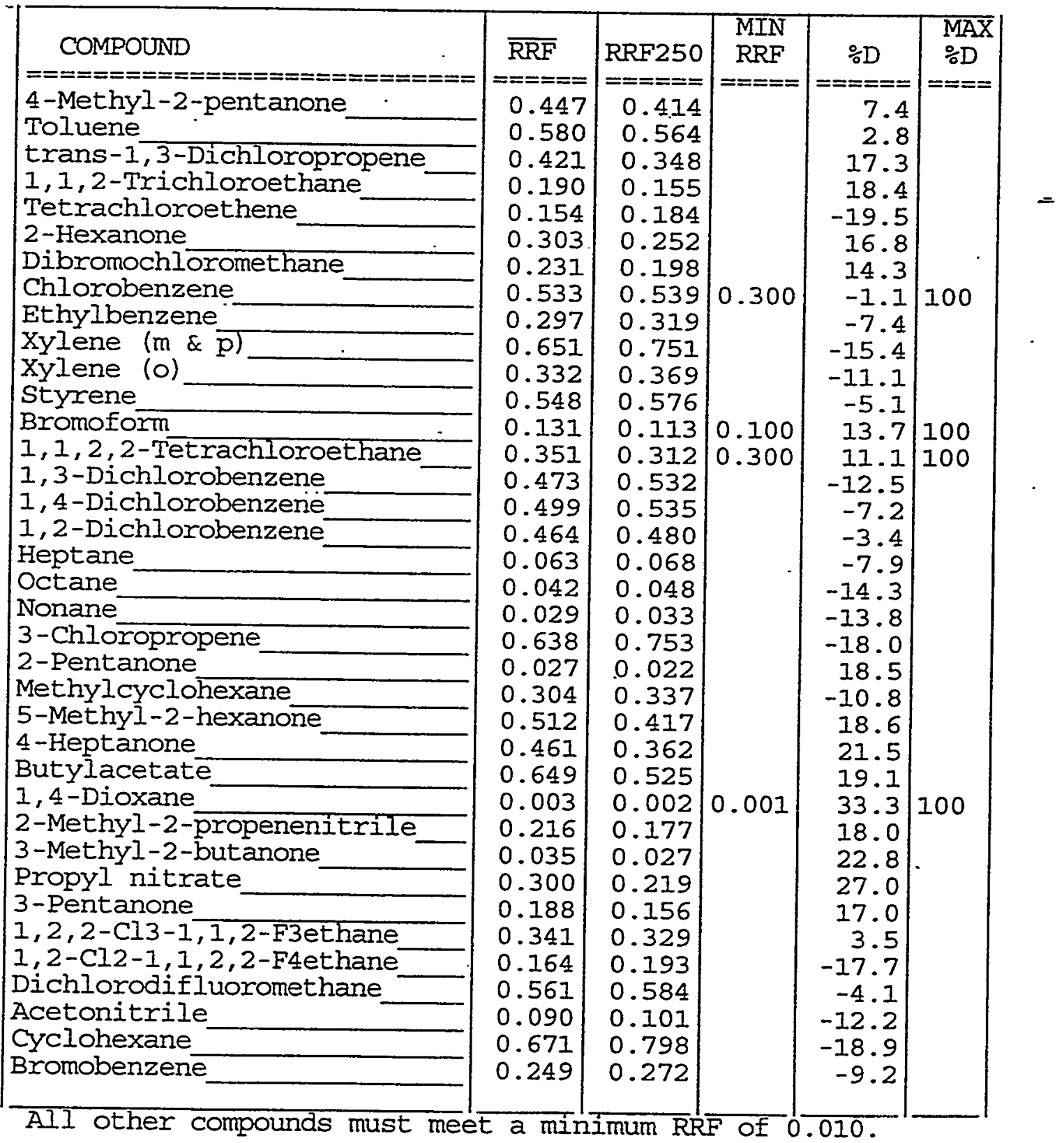

page 2 of 3 


\section{VOLATILE CONTINUING CALIBRATION CHECK}

Lab Name: PNNL

Lab Code: PNNL

Instrument ID: HPI

Lab File ID: 99021103

Heated Purge: ( $\mathrm{Y} / \mathrm{N}) \mathrm{N}$

GC Column: $\mathrm{DB}-62475 \mathrm{M} \times 2.55 \mathrm{UM}$ ID: 0.45 (mm)
Contract: BNFL

SAS NO.:

SDG NO.: 990205

Calibration Date: 02/11/99 Time: 1334

Init. Calib. Date(s) : 02/11/99 02/11/99

Init. Calib. Times: 1249

1549

\begin{tabular}{|c|}
\hline 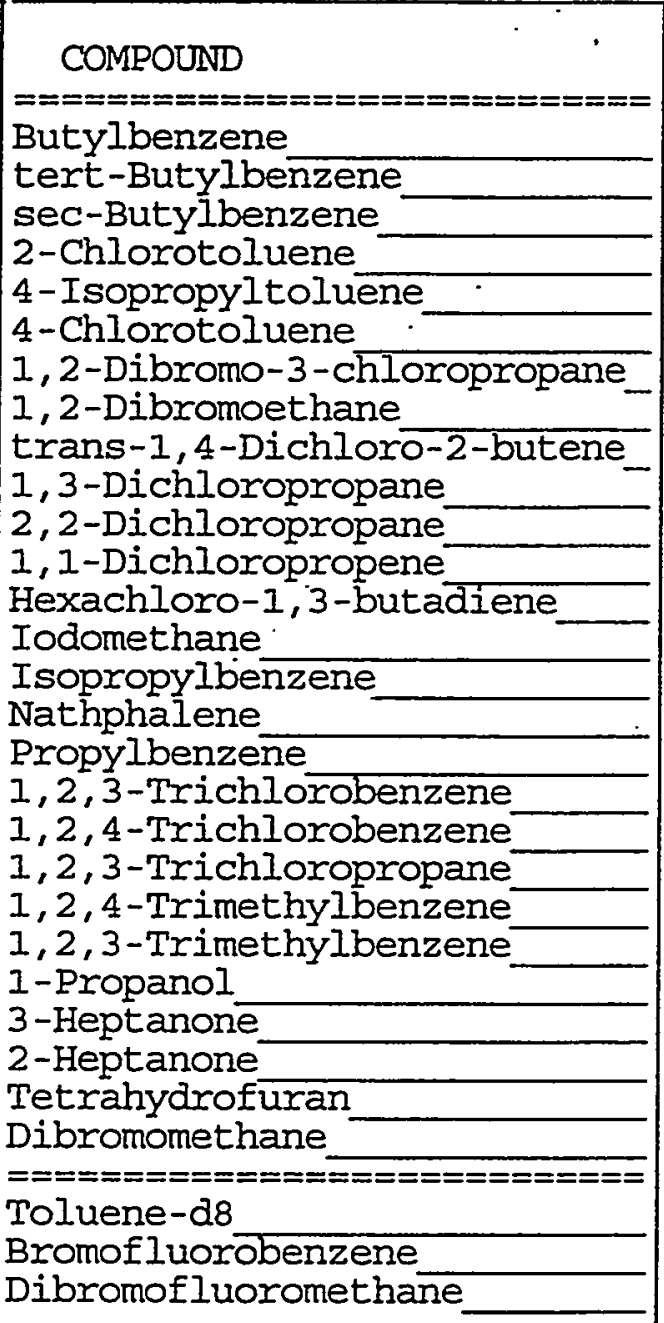 \\
\hline
\end{tabular}

\begin{tabular}{|c|c|c|c|c|}
\hline$\overline{\mathrm{RRF}}$ & RRF250 & $\begin{array}{l}\text { MIN } \\
\text { RRF }\end{array}$ & $\div D$ & $\begin{array}{l}\text { MAX } \\
\because D\end{array}$ \\
\hline$===-=$ & $=====$ & $====$ & $======$ & $===$ \\
\hline $0: 240$ & 0.283 & & -17.9 & \\
\hline 0.824 & 0.944 & & -14.6 & \\
\hline 1.184 & 1.382 & & -16.7 & \\
\hline 0.869 & 0.915 & & -5.3 & \\
\hline 0.921 & 1.059 & & -15.0 & \\
\hline 1.000 & 1.059 & & -5.9 & \\
\hline 0.065 & 0.052 & & 20.0 & \\
\hline 0.205 & 0.168 & & 18.0 & \\
\hline 0.157 & 0.122 & & 22.3 & \\
\hline 0.375 & 0.330 & & 12.0 & \\
\hline 0.332 & 0.368 & & -10.8 & \\
\hline 0.640 & 0.549 & & 14.2 & \\
\hline 0.114 & 0.126 & & -10.5 & \\
\hline 0.484 & 0.437 & & 9.7 & \\
\hline 0.824 & 0.906 & & -10.0 & \\
\hline 0.764 & 0.668 & & 12.6 & \\
\hline 1.347 & 1.489 & & -10.5 & \\
\hline 0.248 & 0.247 & & 0.4 & \\
\hline 0.265 & 0.281 & & -6.0 & \\
\hline 0.525 & 0.431 & & 17.9 & \\
\hline 0.927 & 1.023 & & -10.4 & \\
\hline 0.903 & 1.043 & & -15.5 & \\
\hline 0.057 & 0.041 & & 28.1 & \\
\hline 0.310 & 0.280 & & 9.7 & \\
\hline 0.450 & 0.353 & & 21.6 & \\
\hline 0.215 & 0.176 & & 18.1 & \\
\hline 0.134 & 0.110 & & 17.9 & \\
\hline$======$ & $======$ & $=====$ & $======$ & \\
\hline 0.592 & 0.594 & & -0.3 & \\
\hline 0.368 & 0.352 & & 4.3 & \\
\hline 0.348 & 0.284 & & 18.4 & \\
\hline
\end{tabular}

All other compounds must meet a minimum $R R F$ of 0.010 . 
$7 A$

VOLATILE CONTINUING CAIIBRATION CHECK

Lab Name: PNNL

Lab Code: PNNL Case No.:
Contract $: \cdot$ BNFL

SAS NO.:

SDG NO.: 990205
Instrument ID: HP1

Lab File ID: 99021202

Heated Purge: $(\mathrm{Y} / \mathrm{N}) \mathrm{N}$

GC Column: DB-624 75M X 2.55UM ID: 0.45 (mm)
Calibration Date: 02/12/99 Time: 1355

Init. Calib. Date(s) : 02/11/99 02/11/99

Init. Calib. Times: $1249 \quad 1549$

\begin{tabular}{|c|c|c|c|c|c|}
\hline $\begin{array}{l}\text { COMPOUND } \\
=================:==========1\end{array}$ & $\begin{array}{c}\overline{R R F} \\
======\end{array}$ & $\begin{array}{l}R R F 250 \\
======\end{array}$ & 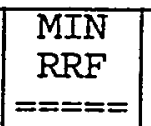 & $\stackrel{\circ D}{=====}$ & $\begin{array}{l}\text { MAX } \\
\therefore D \\
====\end{array}$ \\
\hline Chloromethane & 0.540 & 0.467 & 0.100 & 13.5 & 100 \\
\hline Vinyl Chloride & 0.428 & 0.370 & & 13.6 & \\
\hline $\begin{array}{l}\text { 1,3-Butadiene } \\
\text { Butane }\end{array}$ & $\begin{array}{l}1.045 \\
0.148\end{array}$ & $\begin{array}{l}0.942 \\
0.148\end{array}$ & & $\begin{array}{l}9.8 \\
0.0\end{array}$ & \\
\hline Bromomethane & 0.108 & 0.111 & & -2.8 & - \\
\hline 1,1-Dichloroethene & 0.396 & 0.390 & & 1.5 & \\
\hline Chloroethane & 0.119 & 0.114 & & 4.2 & \\
\hline Acetone & 0.254 & 0.256 & & -0.8 & \\
\hline $\begin{array}{l}\text { Pentane } \\
\text { Carbon Disulfide }\end{array}$ & 0.640 & 0.551 & & 13.9 & \\
\hline & 1.123 & 1.046 & & 6.8 & \\
\hline $\begin{array}{l}\text { Acrolein } \\
\text { Acrylonitrile }\end{array}$ & 0.009 & $\begin{array}{l}0.006 \\
0.222\end{array}$ & 0.001 & $\begin{array}{r}33.3 \\
2.6\end{array}$ & 100 \\
\hline Methylene Chloride & $\begin{array}{l}0.228 \\
0.432\end{array}$ & $\begin{array}{l}0.222 \\
0.459\end{array}$ & & $\begin{array}{r}2.0 \\
-6.2\end{array}$ & \\
\hline trans-1,2-Dichloroethene & 0.297 & 0.275 & & 7.4 & \\
\hline Propionaldehyde & 0.059 & 0.059 & & 0.0 & \\
\hline 2-Butenal & 0.057 & 0.057 & & 0.0 & \\
\hline 1,1-Dichloroethane & 0.928 & 0.857 & 0.100 & 7.6 & 100 \\
\hline cis-1,2-Dichloroethene & 0.473 & 0.452 & & 4.4 & \\
\hline Hexane & 0.565 & 0.507 & & 10.3 & \\
\hline 2-Butañone & 0.331 & 0.312 & & 5.7 & \\
\hline Cyclohexene & 0.172 & 0.166 & & 3.5 & \\
\hline Ethyl acetate & 0.829 & 0.769 & & 7.2 & \\
\hline Cyclopentane & 1.517 & 1.381 & & 9.0 & \\
\hline Bromochloromethane & 0.188 & 0.194 & & -3.2 & \\
\hline Chloroform & 0.849 & 0.833 & & 1.9 & \\
\hline $\begin{array}{l}\text { Dichlorofluoromethane } \\
1,1,1 \text {-Trichloroethane }\end{array}$ & 0.648 & 0.626 & & 3.4 & \\
\hline Chlorodifluoromethane & $\begin{array}{l}0.070 \\
0.343\end{array}$ & 0.064 & & $\begin{array}{r}8.6 \\
8\end{array}$ & \\
\hline Carbon Tetrachloride & 0.456 & 0.449 & & $\begin{array}{r}10.8 \\
1.5\end{array}$ & \\
\hline Trichlorofluoromethane & 0.315 & 0.313 & & 0.6 & \\
\hline Benzene & 0.729 & 0.783 & & -7.4 & \\
\hline 1,2-Dichloroethane & 0.375 & 0.399 & & -6.4 & \\
\hline Trichloroethene & 0.185 & 0.193 & & -4.3 & \\
\hline 1,2-Dichloropropane & 0.227 & 0.232 & & -2.2 & \\
\hline Bromodichloromethane & 0.301 & 0.314 & & -4.3 & \\
\hline cis-I, 3-Dichloropropene & 0.345 & 0.359 & & -4.0 & \\
\hline 4-Methyl-2-pentanone & 0.447 & 0.442 & & 1.1 & \\
\hline
\end{tabular}

page 1 of 3 
$7 A$

VOLATIIE CONTINUING CALIBRATION CHECK

Lab Name: PNNL.

Contract: BNFL

Lab code: PNNL

Case No.:

SAS NO.:

SDG NO.: 990205

Instrument ID: HPI

Calibration Date: 02/12/99 Time: 1355

Lab File ID: 99021202

Init. Calib. Date(s) : 02/11/99

$02 / 11 / 99$

Heated Purge: ( $Y / N)$ N

Init. Calib. Times: 1249

1549

GC Column: DB-624 75M X 2.55UM ID: 0.45 (mm)

\begin{tabular}{|c|c|c|c|c|c|}
\hline 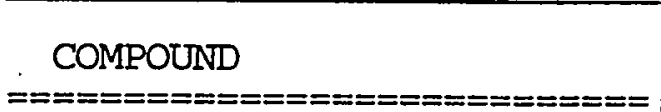 & $\begin{array}{l}\overline{\mathrm{RRF}} \\
======\end{array}$ & $\begin{array}{l}\text { RRF250 } \\
======\end{array}$ & $\begin{array}{l}\text { MIN } \\
\text { RRF } \\
=====\end{array}$ & $\begin{array}{c}\because D \\
=====\end{array}$ & $\begin{array}{c}\text { MAX } \\
\% D \\
===\end{array}$ \\
\hline Toluene $\cdot$. & 0.580 & 0.595 & & -2.6 & \\
\hline trans-1,3-Dichloropropene & 0.421 & 0.415 & & 1.4 & \\
\hline $\begin{array}{l}\text { 1, 1,2-Trichloroethane } \\
\text { Tetrachloroethene }\end{array}$ & 0.190 & 0.195 & & $\begin{array}{l}-2.6 \\
-9.7\end{array}$ & \\
\hline $\begin{array}{l}\text { Tetracnloroethene } \\
\text { 2-Hexanone }\end{array}$ & 0.154 & $\begin{array}{l}0.168 \\
0.309\end{array}$ & & $\begin{array}{l}-9.1 \\
-2.0\end{array}$ & \\
\hline Dibromochloromethane & 0.231 & 0.240 & & -3.9 & \\
\hline Chlorobenzene & 0.533 & 0.568 & 0.300 & -6.6 & 100 \\
\hline Ethylbenzene & 0.297 & 0.313 & & -5.4 & \\
\hline Xylene $(m \& \bar{p})$ & 0.651 & 0.679 & & -4.3 & \\
\hline Xylene (0) & 0.332 & 0.370 & & -11.4 & \\
\hline Styrene & 0.548 & 0.587 & & -7.1 & \\
\hline Bromoform & 0.131 & 0.142 & 0.100 & -8.4 & 100 \\
\hline $\begin{array}{l}1,1 ; 2,2 \text {-Tetrachloroethane } \\
1,3-\text { Dichlorobenzene }\end{array}$ & $\begin{array}{l}0.351 \\
0.473\end{array}$ & $\begin{array}{l}0.376 \\
0.492\end{array}$ & 0.300 & $\begin{array}{l}-7.1 \\
-4.0\end{array}$ & 100 \\
\hline I, 4-Dichlorobenzene & 0.499 & 0.561 & & -12.4 & \\
\hline 1,2-Dichlorobenzene & 0.464 & 0.522 & & -12.5 & \\
\hline Heptane & 0.063 & 0.060 & & 4.8 & \\
\hline Octane & 0.042 & 0.041 & & 2.4 & \\
\hline Nonane & 0.029 & 0.032 & & -10.3 & \\
\hline 3-Chloropropene & 0.638 & 0.592 & & 7.2 & \\
\hline & 0.027 & 0.029 & & -7.4 & \\
\hline $\begin{array}{l}\text { Methylcyclohexane } \\
\text { 5-Methyl-2-hexanone }\end{array}$ & 0.304 & 0.313 & & $\begin{array}{r}-3.0 \\
-11.3\end{array}$ & \\
\hline 4-Heptanone & 0.461 & 0.476 & & $\begin{array}{r}-11.3 \\
-3.2\end{array}$ & \\
\hline Butylacetate & 0.649 & 0.701 & & -8.0 & \\
\hline 1,4-Dioxane & 0.003 & 0.003 & 0.001 & 0.0 & 100 \\
\hline 2-Methyl-2-propenenitrile & 0.216 & 0.228 & & -5.6 & \\
\hline 3-Methyl-2-butanone & 0.035 & 0.038 & & $\begin{aligned} &-8-6 \\
& 7\end{aligned}$ & \\
\hline Propyl nitrate & 0.300 & 0.296 & & $\begin{array}{r}1.3 \\
-1.1\end{array}$ & \\
\hline & & $\begin{array}{l}0.190 \\
0.316\end{array}$ & & $\frac{1}{7.3}$ & \\
\hline $\begin{array}{l}1,2,2-C 13-1,1,2-F 3 \text { ethane } \\
1,2-\mathrm{Cl} 2-1,1,2,2-\mathrm{F} 4 \text { ethane }\end{array}$ & $\begin{array}{l}0.341 \\
0.164\end{array}$ & $\begin{array}{l}0.316 \\
0.114\end{array}$ & & 30.5 & \\
\hline Dichlorodifluoromethane & 0.561 & 0.493 & & 12.1 & \\
\hline Acetonitrile & 0.090 & 0.097 & & -7.8 & \\
\hline Cyclohexane & 0.671 & 0.600 & & 10.6 & \\
\hline Bromobenzene & 0.249 & 0.284 & & -14.0 & \\
\hline Butylbenzene & 0.240 & 0.253 & & -5.4 & \\
\hline
\end{tabular}

page 2 of 3 
$7 A$

VOLATILE CONTINUING CALIBRATION CHECK

Lab Name: PNNL

Contract: BNFL

Lab Code: PNNL

Case No.:

SAS NO.:

SDG No. : 990205

Instrument ID: HPI

Calibration Date: 02/12/99 Time: 1355

Lab File ID: 99021202

Init. Calib. Date(s) : 02/11/99 02/11/99

Heated Purge: $(\mathrm{Y} / \mathrm{N}) \mathrm{N}$

Init. Calib. Times: 1249

1549

GC Column: DB-624 75M X 2.55UM ID: 0.45 (mm)

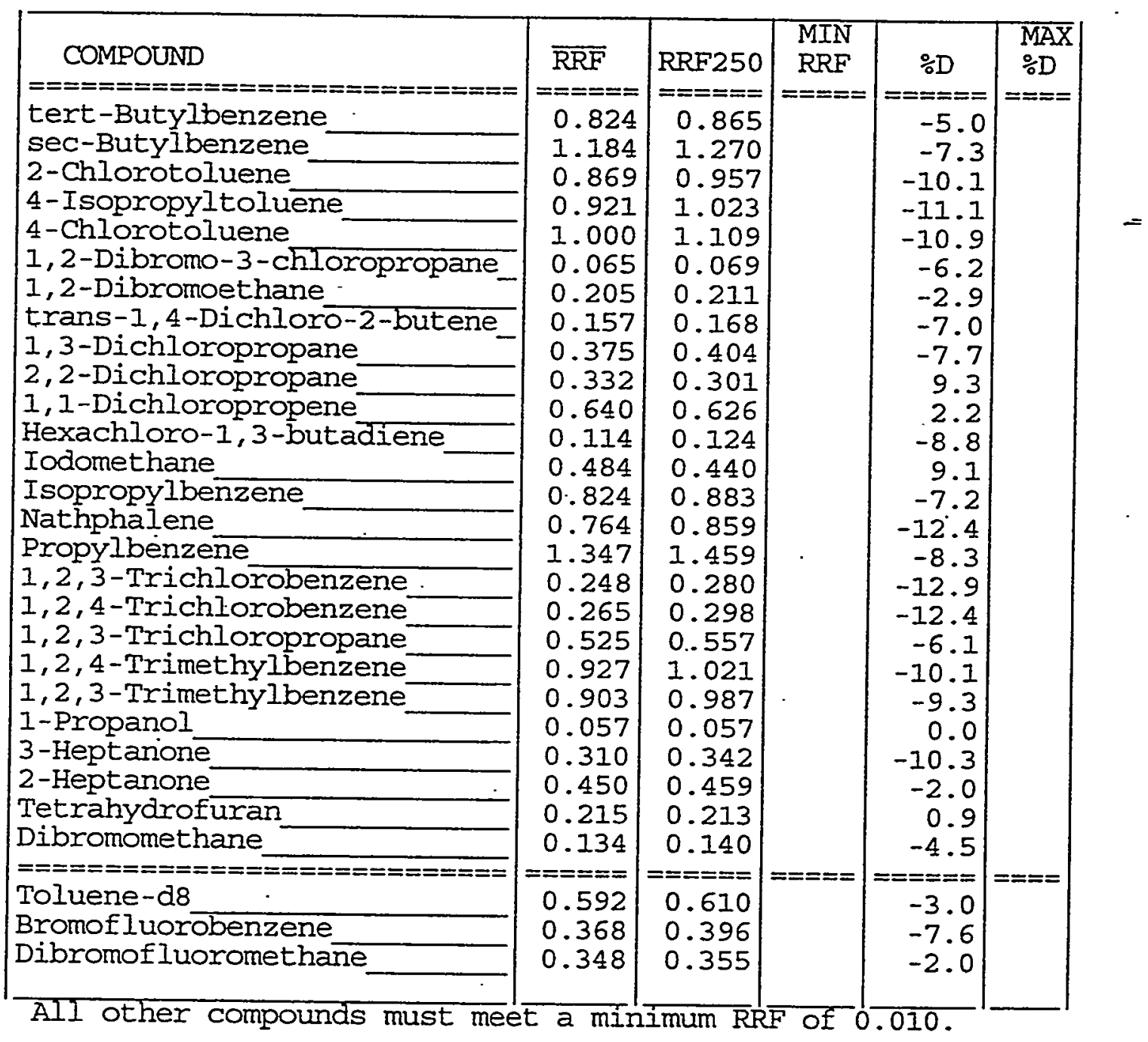

page 3 of 3 
Lab Name: PNNL

Lab Code: PNNL Case No.:

Lab File ID (Standard): 99021202

Instrument ID: HPI

GC Column: DB-624 75M X 2.55UM ID: $0.45(\mathrm{~mm}) \quad$ Heated Purge: (Y/N) N
Contract : BNFL

SAS NO.:

SDG NO.: 990205

Date Analyzed: 02/12/99

Time Analyzed: 1355

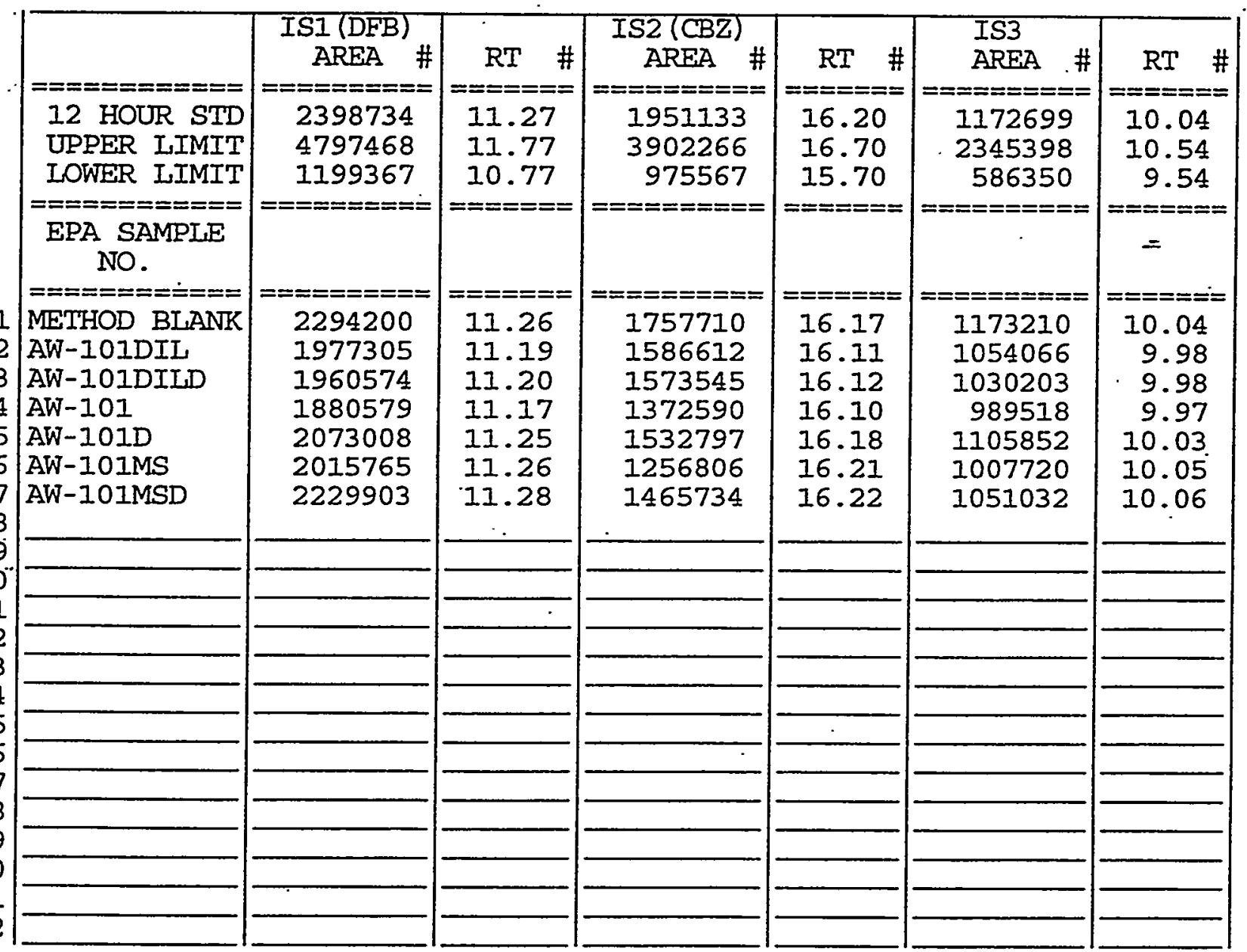

\begin{abstract}
AREA UPPER LIMIT $=+100 \%$ of internal standard area AREA IOWER IIMIT $=-50 \%$ of internal standard area RT UPPER IIMIT $=+0.50$ minutes of internaI standard RT RT LOWER LIMIT $=-0.50$ minutes. of internal standard RT

\# Column used to flag values outside QC limits with an asterisk. * Values outside of QC limits.
\end{abstract}

page 1 of 2
OLM03. 0 
$8 \mathrm{~A}$

VOLATILE INTERNAL STANDARD AREA AND RT SUMMARY

Lab Name: PNNL

Iab Code: PNNL.
Contract: BNFL

Case No.:

SAS NO.:
SDG NO.: 990205

Date Analyzed: 02/12/99

Time Analyzed: 1355

Instrument ID: HP1

GC Column: $\mathrm{DB}-62475 \mathrm{M} \times 2.55 \mathrm{UM}$ ID: $0.45(\mathrm{~mm}) \quad$ Heated Purge: (Y/N) N

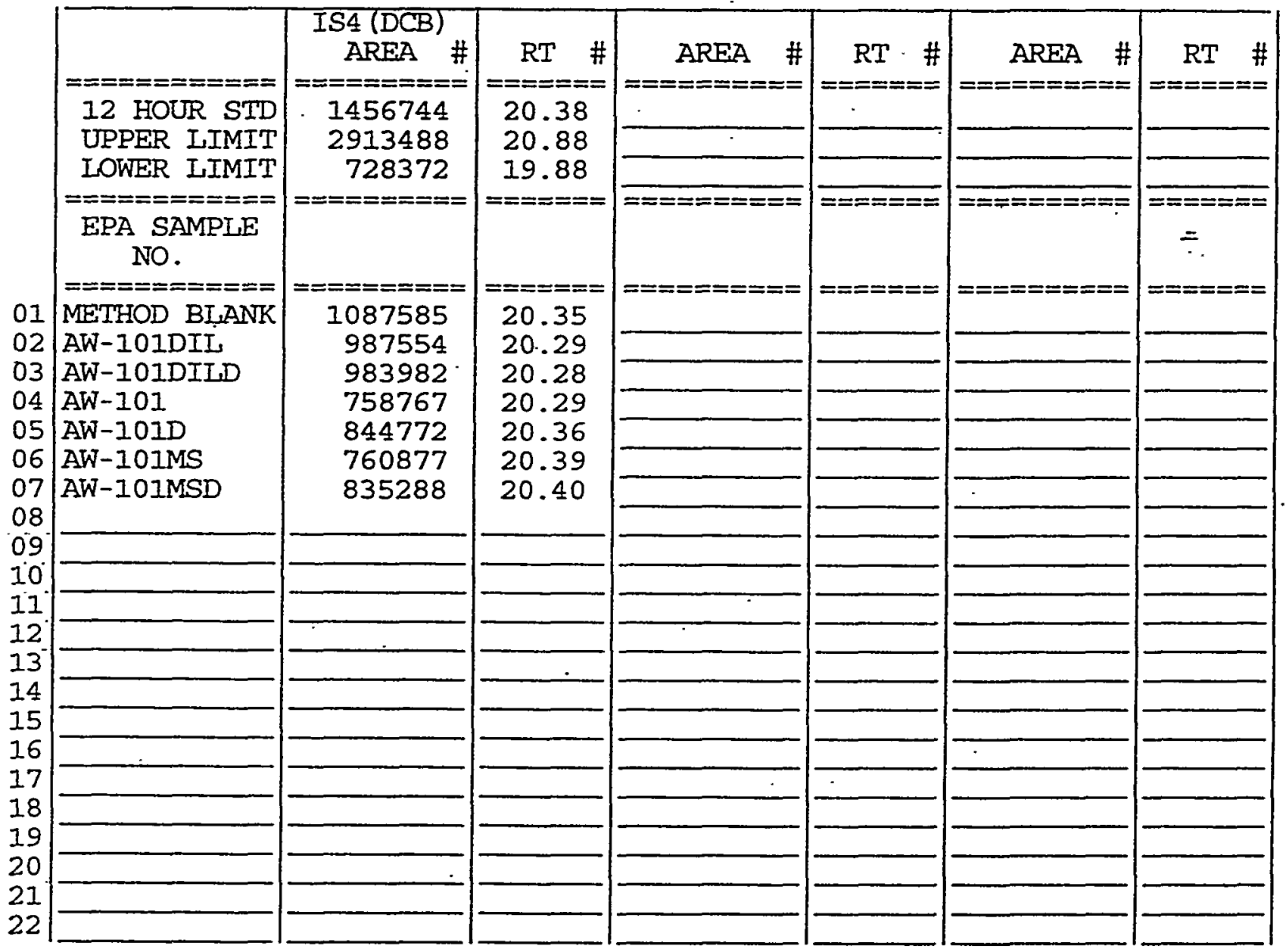

IS4 $(D C B)=1,4-D i c h l o r o b e n z e n e-d 4$

AREA UPPER LIMIT $=+100 \%$ of internal standard area AREA LOWER IIMIT $=-50 \%$ of internal standard area RT UPPER IIMIT $=+0.50$ minutes of internal standard RT RT LOWER IIMIT $=-0.50$ minutes of internal standard RT

\# Column used to flag values outside QC limits with an asterisk. * Values outside of QC Iimits. 
Lab Name: PNNL

Lab Code: PNNL

Case No.:
Contract: BNFL

SAS NO.:
AN-107
Matrix: (soil/water) SUPERNATAN

Sample wt/vol:

$5.000(\mathrm{~g} / \mathrm{mL}) \mathrm{ML}$

Level: (low/med) IOW

\% Moisture: not dec.

GC.Column: $\mathrm{DB}-62475 \mathrm{M} \times 2.55 \mathrm{UM}$ ID: 0.45 (mm)

Soil Extract Volume: (UL)
Lab Sample ID: 99-0649

Lab File ID: $\quad 99021217$

Date Received:

Date Analyzed: 02/13/99

Dilution Factor: 1.0

Soil Aliquot Volume: (UI)

CONCENTRATION UNITS:

CAS NO. COMPOUND $\quad(u g / L$ or $u g / K g) ~ U G / L$

\begin{tabular}{|c|c|}
\hline 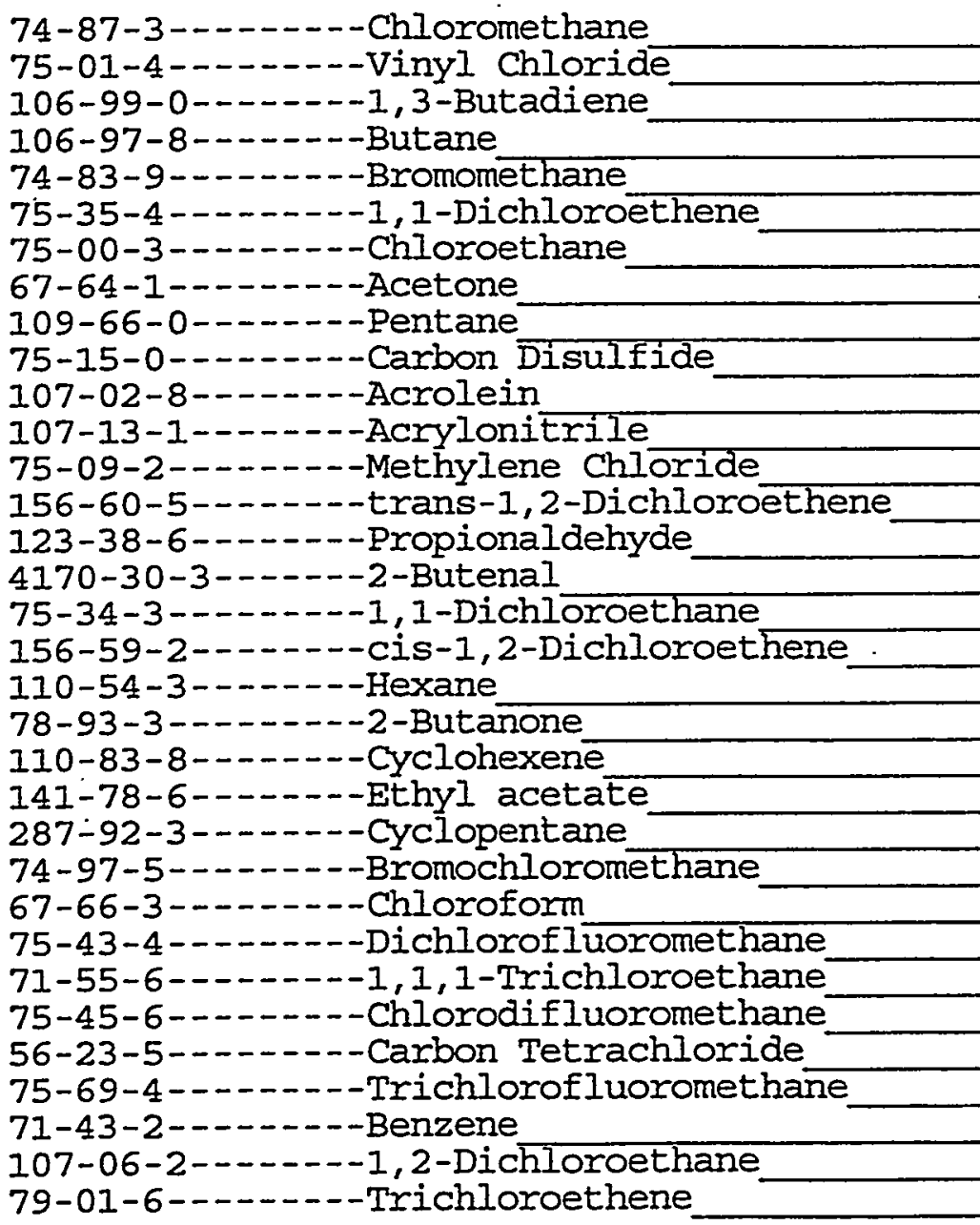 & $\begin{array}{r}66 \\
66 \\
66 \\
66 \\
66 \\
66 \\
66 \\
140 \\
66 \\
66 \\
66 \\
66 \\
66 \\
66 \\
66 \\
66 \\
66 \\
66 \\
66 \\
16 \\
66 \\
66 \\
66 \\
66 \\
66 \\
66 \\
66 \\
37 \\
66 \\
66 \\
66 \\
66 \\
66\end{array}$ \\
\hline
\end{tabular}


Lab Name: PNNL

Lab Code: PNNL

Matrix: (soil/water) SUPERNATAN

Sample wt/vol:

$5.000(\mathrm{~g} / \mathrm{mL}) \mathrm{ML}$

Level: (low/med) IOW

$\because$ Moisture: not dec.

GC Column: DB-624 75M X 2.55UM ID: 0.45 (mm)

Soil Extract Volume: (UI)

CAS NO. COMPOUND (ug/L or ug/Kg) UG/L

Contract: BNFL

SAS NO.:
AN-107

SDG NO.: 99021202

Lab Sample ID: 99-0649

Lab File ID: 99021217

Date Received:

Date Analyzed: 02/13/99

Dilution Factor: 1.0

Soil Aliquot Volume:

(uI)

CONCENTRATION UNITS :

Q

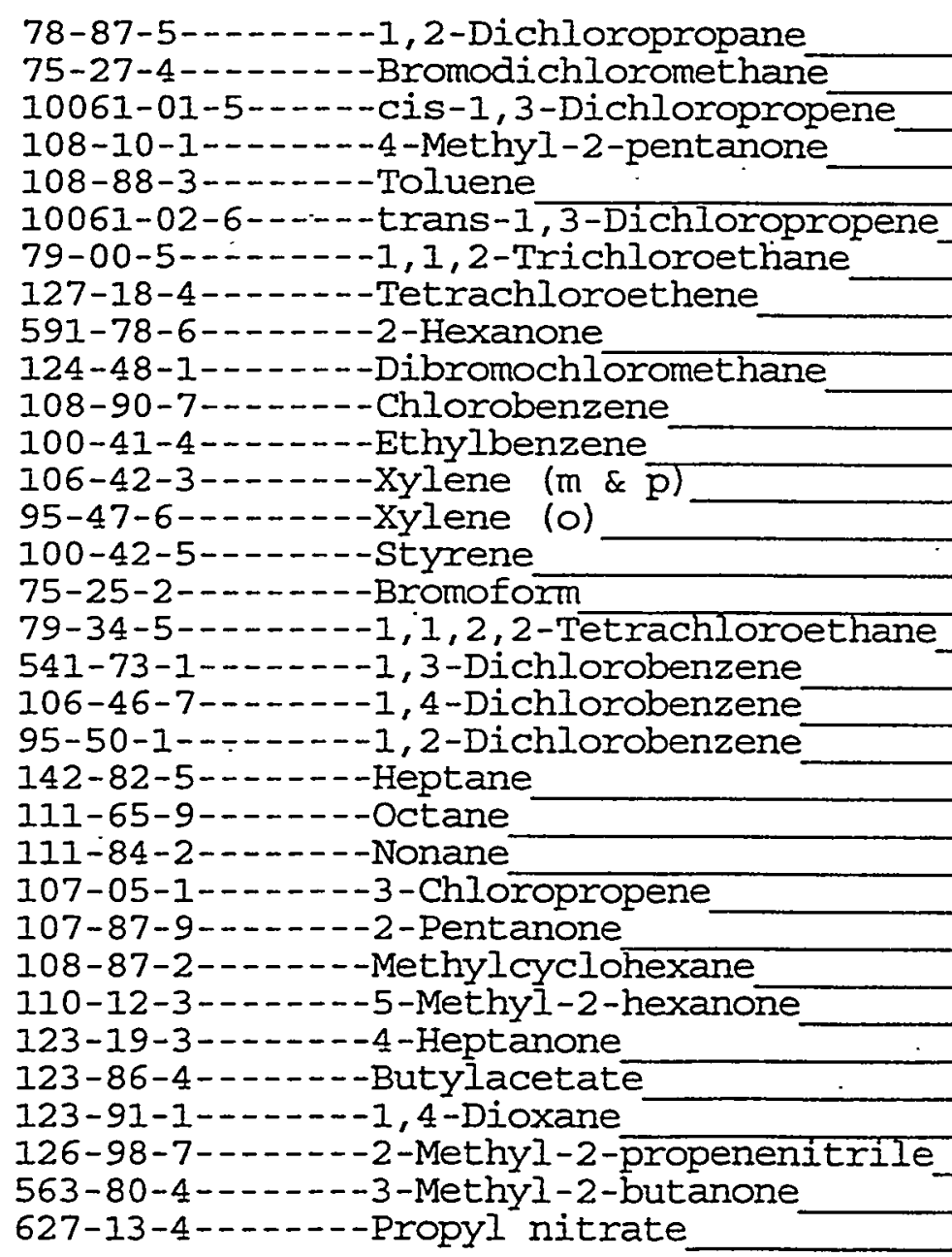


Lab Name: PNNL

Lab Code: PNNL

Case No.: contract: BNFL

SAS NO.:
EPA SAMPLE NO.

AN-107
Matrix: (soil/water) SUPERNATAN

Sample wt/vol: $\quad 5.000(\mathrm{~g} / \mathrm{mL}) \mathrm{MI}$

Level: (low/med) LOW

\% Moisture: not dec.

GC Column:' DB-624 75M X 2.55UM ID: 0.45 (mm)

Soil Extract Volume: (UI)
Lab Sample ID: 99-0649

Lab File ID: 99021217

Date Received:

Date Analyzed: 02/13/99

Dilution Factor: 1.0

Soil Aliquot Volume: (UL)

(ug/L or ug/Kg) UG/L $Q$

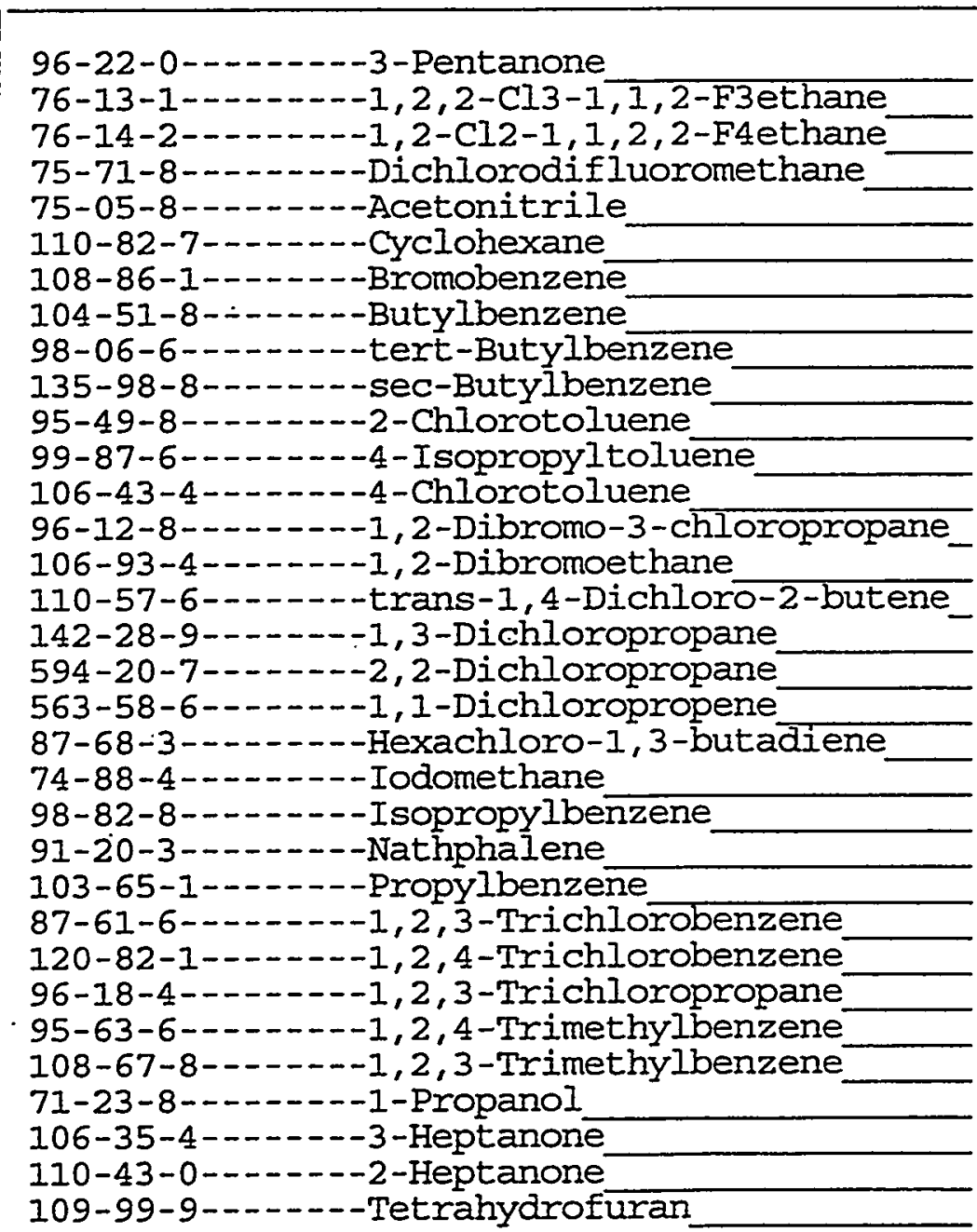

\begin{tabular}{|l|l|l|}
\hline 66 & $U$ \\
66 & $U$ \\
66 & $U$ \\
66 & $U$ \\
94 & \\
66 & $U$ \\
66 & $U$ \\
66 & $U$ \\
66 & $U$ \\
66 & $U$ \\
66 & $U$ \\
66 & $U$ \\
66 & $U$ \\
66 & $U$ \\
66 & $U$ \\
66 & $U$ \\
66 & $U$ \\
66 & $U$ \\
66 & $U$ \\
66 & $U$ \\
66 & $U$ \\
66 & $U$ \\
66 & $U$ \\
66 & $U$ \\
66 & $U$ \\
66 & $U$ \\
66 & $U$ \\
66 & $U$ \\
66 & $U$ \\
66 & $U$ \\
7 & $J$ \\
66 & $U$ \\
31 & $J$ \\
& & \\
\hline
\end{tabular}


: $1 A$

VOLATILE ORGANICS ANALYSIS DATA SHEET

Lab Name: PNNL Contract: BNFL

Lab Code: PNNL Case No.:

Matrix: (soil/water) SUPERNATAN

Sample wt/vol: $\quad 5.000(\mathrm{~g} / \mathrm{mL}) \mathrm{ML}$

Level: (low/med) LOW

SAS NO.:

SDG No.: 99021202

Lab Sample ID: 99-0649

Iab File ID: 99021217

Date Received:

Date Analyzed: 02/13/99

GC.Column: DB-62475M X 2.55UM ID: 0.45 (mm)

Soil Extract Volume: (uI)

Soil Aliquot Volume: (uL)
Dilution Factor: 1.0
CAS NO.

COMPOUND
CONCENTRATION UNITS:

(ug/L or $\mathrm{ug} / \mathrm{Kg}$ ) UG/L

$Q$

74-95-3-----Dibromomethane 
Lab Name: PNNL.

Lab Code: PNNL

Case No.:

Matrix: (soil/water) SUPERNATAN

Sample wt/vol: $\quad 5.000(\mathrm{~g} / \mathrm{mL}) \mathrm{MI}$

Level: (low/med) IOW

$\div$ Moisture: not dec.

GC Column: DB-624 75M X 2.55UM ID: 0.45 (mm) (UL)

Soil Extract Volume:
Contract: BNFL

SAS NO.:
EPA SAMPLE NO.

AN-107DUP

Soil Aliquot Volume: (uL) CONCENTRATION UNITS:

CAS NO. COMPOUND (ug/I or $u g / K g$ ) UG/L

SDG No.: 99021202

Lab Sample ID: 99-0649D

Lab File ID: 99021218

Date Received:

Date Analyzed: 02/13/99.

\begin{tabular}{|c|}
\hline 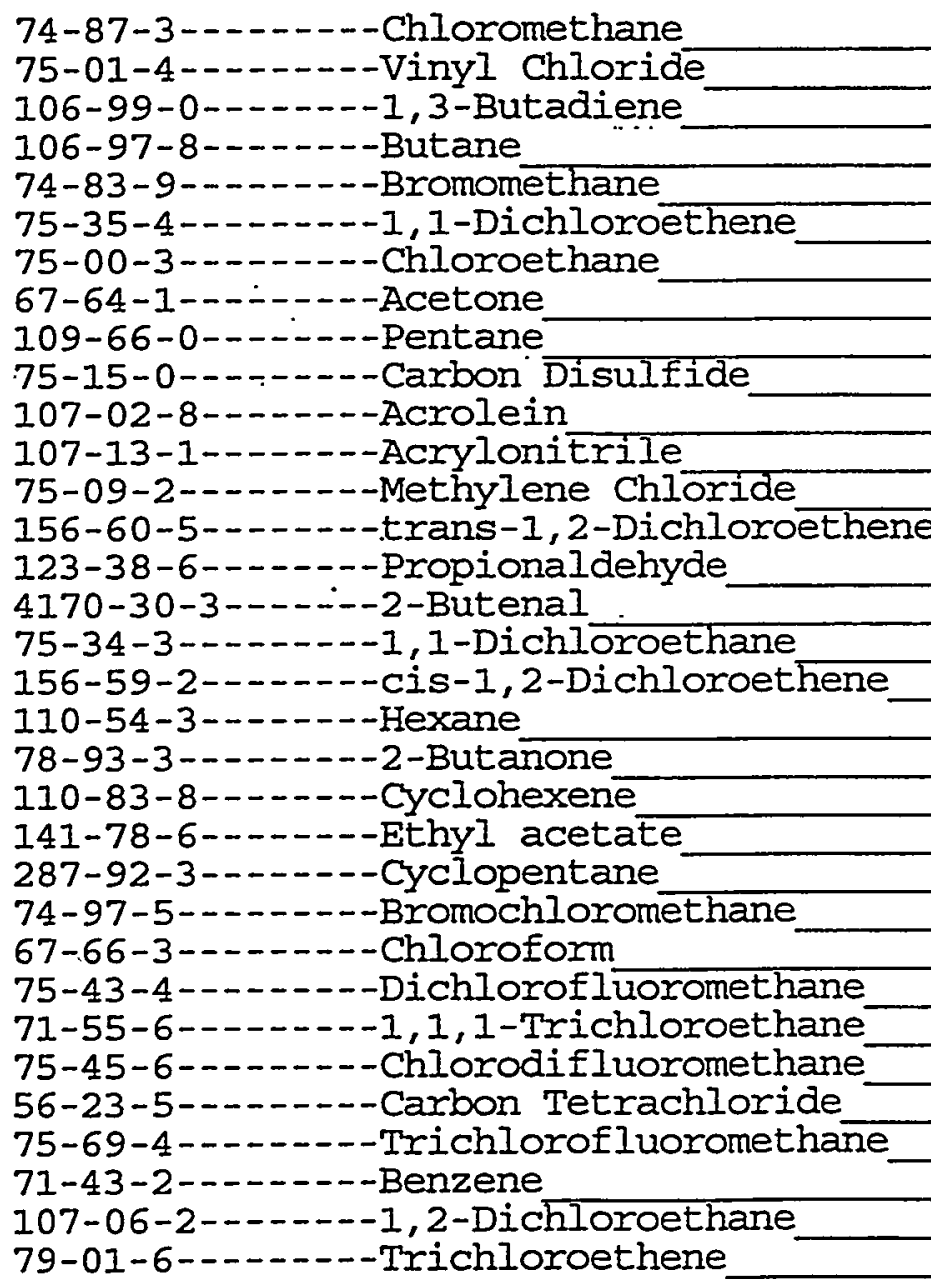 \\
\hline
\end{tabular}

Dilution Factor: 1.0

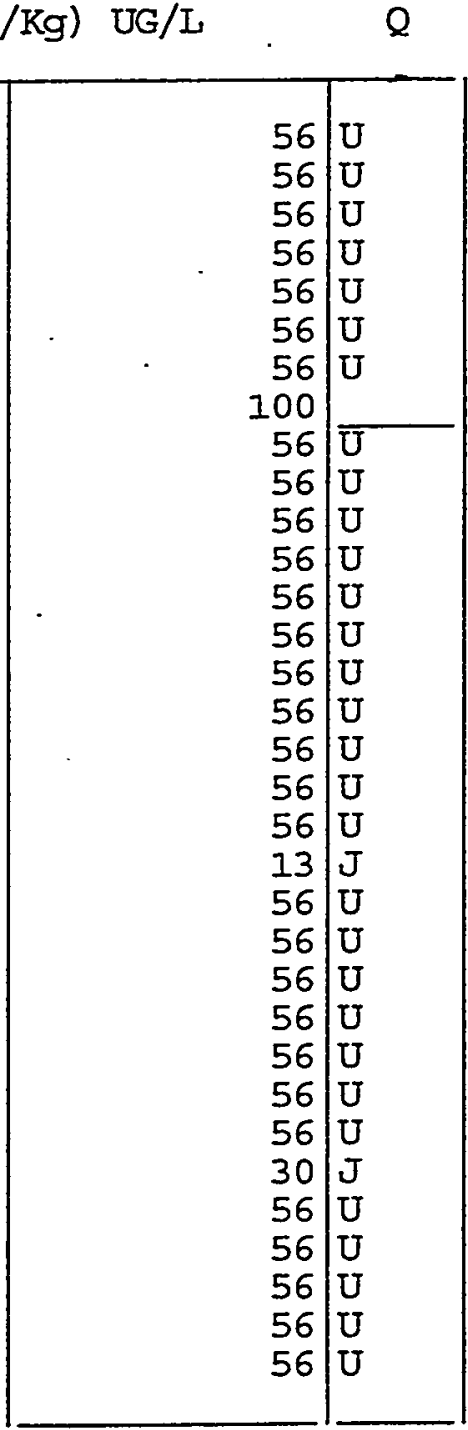


Lab Name: PNNL

Lab Code: PNNL

Case No.:

Matrix: (soil/water) SUPERNATAN

Sample wt/vol:

$5.000(\mathrm{~g} / \mathrm{mL}) \mathrm{ML}$

Level: (low/med) IOW

\% Moisture: not dec.

GC Column: DB-624 75M X 2.55UM ID: 0.45 (mm)

Soil Extract Volume: (UI)
Contract: BNFL

SAS No. :
EPA SAMPLE NO.

AN-I07DUP

SDG NO.: 99021202

Lab Sample ID: 99-0649D

Lab File ID: 99021218

Date Received:

Date Analyzed: 02/13/99

(mm) Dilution Factor: 1.0

Soil Aliquot Volume:

(uL)

CONCENTRATION UNITS:

CAS NO. COMPOUND (ug/L or $\mathrm{ug} / \mathrm{Kg}$ ) UG/L

Q

\begin{tabular}{|c|}
\hline 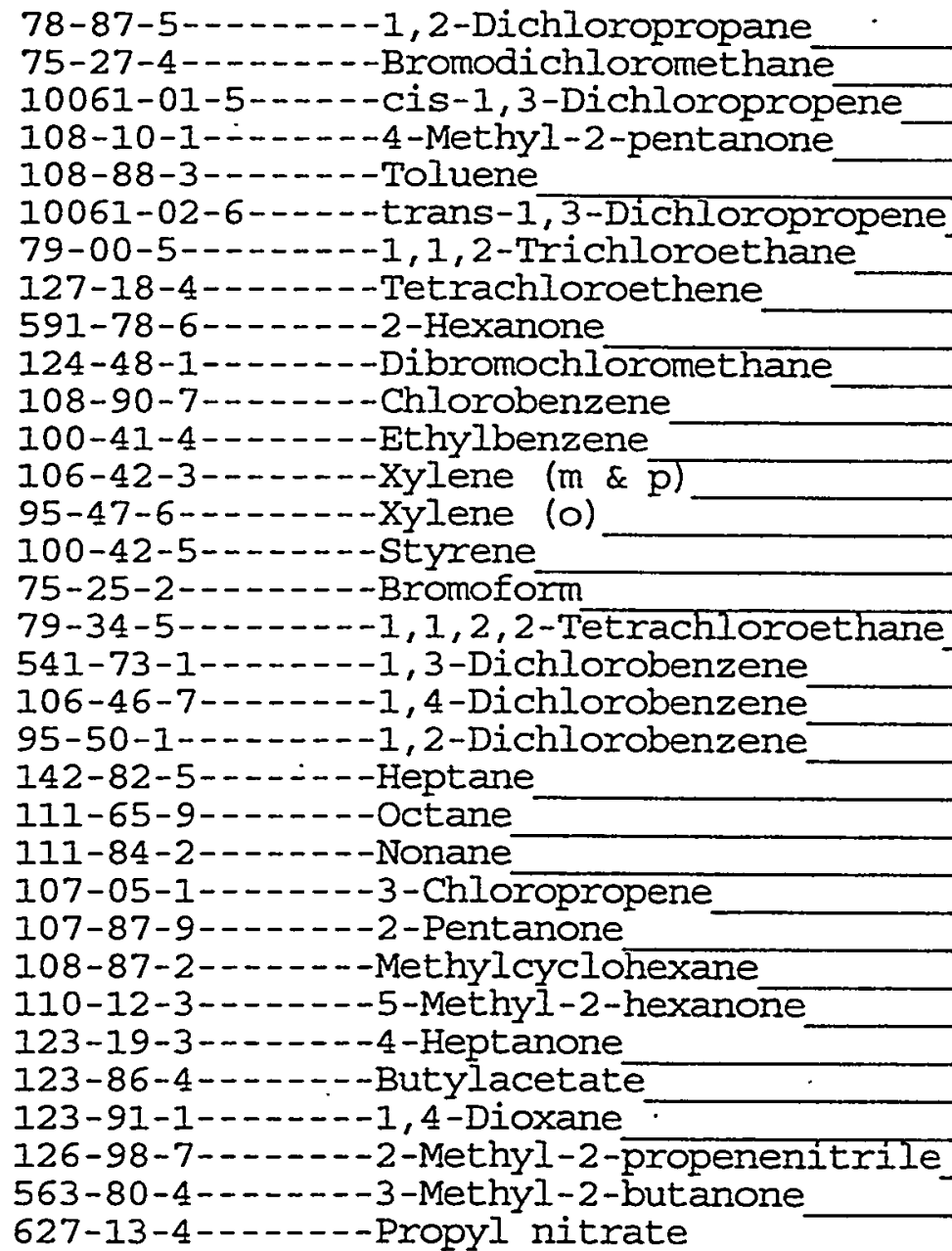 \\
\hline
\end{tabular}


Lab Name: PNNL

Lab Code: PNNL

Case No.:

Matrix: (soil/water) SUPERNATAN

Sample wt/vol:

$5.000(\mathrm{~g} / \mathrm{mL}) \mathrm{ML}$

Level: (low/med) IOW

$\%$ Moisture: not dec.

GC Column: DB-624 75M X 2.55UM ID: 0.45 (mm)

(uL)

Soil Extract Volume:
Contract: BNEL

SAS NO. :
AN-107DUP

SDG NO.: 99021202

Lab Sample ID: 99-0649D

Lab File ID: 99021218

Date Received:

Date Analyzed: 02/13/99

Dilution Factor: 1.0

Soil Aliquot Volume:

(uL)

CONCENTRATION UNITS:

CAS NO.

COMPOUND

(ug/L or ug/Kg) UG/L

Q

96-22-0-- - - - -3-Pentanone

76-13-1-.----1, 2,2-C13-1, 1,2-F3ethane

76-14-2--.---1,2-Cl2-1,1,2,2-F4ethane

75-71-8-....-Dichlorodifluoromethane

75-05-8--.---Acetonitrile

110-82-7--.--Cyclohexane

108-86-1-....--Bromobenzene

104-51-8-.....-Butylbenzene

98-06-6--.----tert-Butylbenzene

135-98-8--.----sec-Butylbenzene

95-49-8-..---2-Chlorotoluene

99-87-6-..---4-Isopropyltoluene

106-43-4-...-4-Chlorotoluene

96-12-8-------1, 2-Dibromo-3-chloropropane

106-93-4-...-1,2-Dibromoethane

110-57-6-...-.-trans-1, 4-Dichloro-2-butene

142-28-9--.--1, 3-Dichloropropane

594-20-7--.---2, 2-Dichloropropane

563-58-6-...-1, 1-Dichloropropene

87-68-3-...-.--Hexachloro-1, 3-butadiene

74-88-4-..---- Iodomethane

98-82-8-..-----Isopropylbenzene

91-20-3--------Nathphalene

103-65-1-...-.-Propylbenzene

87-61-6_.....-1, 2,3-Trichlorobenzene

120-82-1-..---1, 2,4-Trichlorobenzene

96-18-4--.---1, 2, 3-Trichloropropane

95-63-6-...--1, 2,4-Trimethylbenzene

108-67-8-...--1, 2,3-Trimethylbenzene

71-23-8-...--1-Propanol

106-35-4-..---3-Heptanone

110-43-0-...--2-Heptanone

109-99-9-..---. Tetrahydrofuran

\begin{tabular}{|l|l|l}
\hline 56 & $\mathrm{U}$ \\
56 & $\mathrm{U}$ \\
56 & $\mathrm{U}$ \\
56 & $\mathrm{U}$ \\
70 & \\
56 & $\mathrm{U}$ \\
56 & $\mathrm{U}$ \\
56 & $\mathrm{U}$ \\
56 & $\mathrm{U}$ \\
56 & $\mathrm{U}$ \\
56 & $\mathrm{U}$ \\
56 & $\mathrm{U}$ \\
56 & $\mathrm{U}$ \\
56 & $\mathrm{U}$ \\
56 & $\mathrm{U}$ \\
56 & $\mathrm{U}$ \\
56 & $\mathrm{U}$ \\
56 & $\mathrm{U}$ \\
56 & $\mathrm{U}$ \\
56 & $\mathrm{U}$ \\
56 & $\mathrm{U}$ \\
56 & $\mathrm{U}$ \\
56 & $\mathrm{U}$ \\
56 & $\mathrm{U}$ \\
56 & $\mathrm{U}$ \\
56 & $\mathrm{U}$ \\
56 & $\mathrm{U}$ \\
56 & $\mathrm{U}$ \\
56 & $\mathrm{U}$ \\
56 & $\mathrm{U}$ \\
6 & $\mathrm{~J}$ \\
56 & $\mathrm{U}$ \\
26 & $\mathrm{~J}$ \\
& & \\
\hline & \\
& \\
& \\
& \\
& \\
& \\
& \\
& \\
& \\
&
\end{tabular}

FORM I VOA

OLMO3. 0 
IA

VOLATILE ORGANICS ANALYSIS DATA SHEET

Lab Name: PNNL

Lab Code: PNNL Case No.:

Matrix: (soil/water) SUPERNATAN

Sample wt/vol: $\quad 5.000(\mathrm{~g} / \mathrm{mL}) \mathrm{ML}$

Level: (low/med) Iow

\% Moisture: not dec.

GC Column: DB-624 75M X 2.55UM ID: 0.45 (mm)

Soil Extract Volume: (uI)

CAS NO.

COMPOUND
Contract: BNFI SAS NO. :
EPA SAMPLE NO.

AN-107DUP

SDG No.: 99021202

Iab Sample ID: 99-0649D

Lab File ID: . 99021218

Date Received:

Date Analyzed: 02/13/99

Dilution Factor: 1.0

Soil Aliquot Volume: (uL). CONCENTRATION UNITS: (ug/L or $\mathrm{ug} / \mathrm{Kg}$ ) UG/L

Q 
Lab Name: PNNL

Lab Code: PNNL
Contract: BNFL
AN-107MS

SDG No.: 99021202 .

Matrix: (soil/water) SUPERNATAN

Sample wt/vol: $\quad 5.000(\mathrm{~g} / \mathrm{mL}) \mathrm{MU}$

Level: (low/med) Low

$\because$ Moisture: not dec.

GC Column: DB-624 75M X 2.55UM ID: 0.45 (mm)

Soil Extract Volume:

(UI)
Lab Sample ID: 99-0649MS

Lab File ID: 99021219

Date Received:

Date Analyzed: 02/13/99

. Dilution Factor: 1.0

Soil Aliquot Volume: (uL)
CAS NO. COMPOUND $\quad$ (ug/L or ug/Kg) UG/L

(ug/L or ug/Kg) UG/L

\begin{tabular}{|c|}
\hline 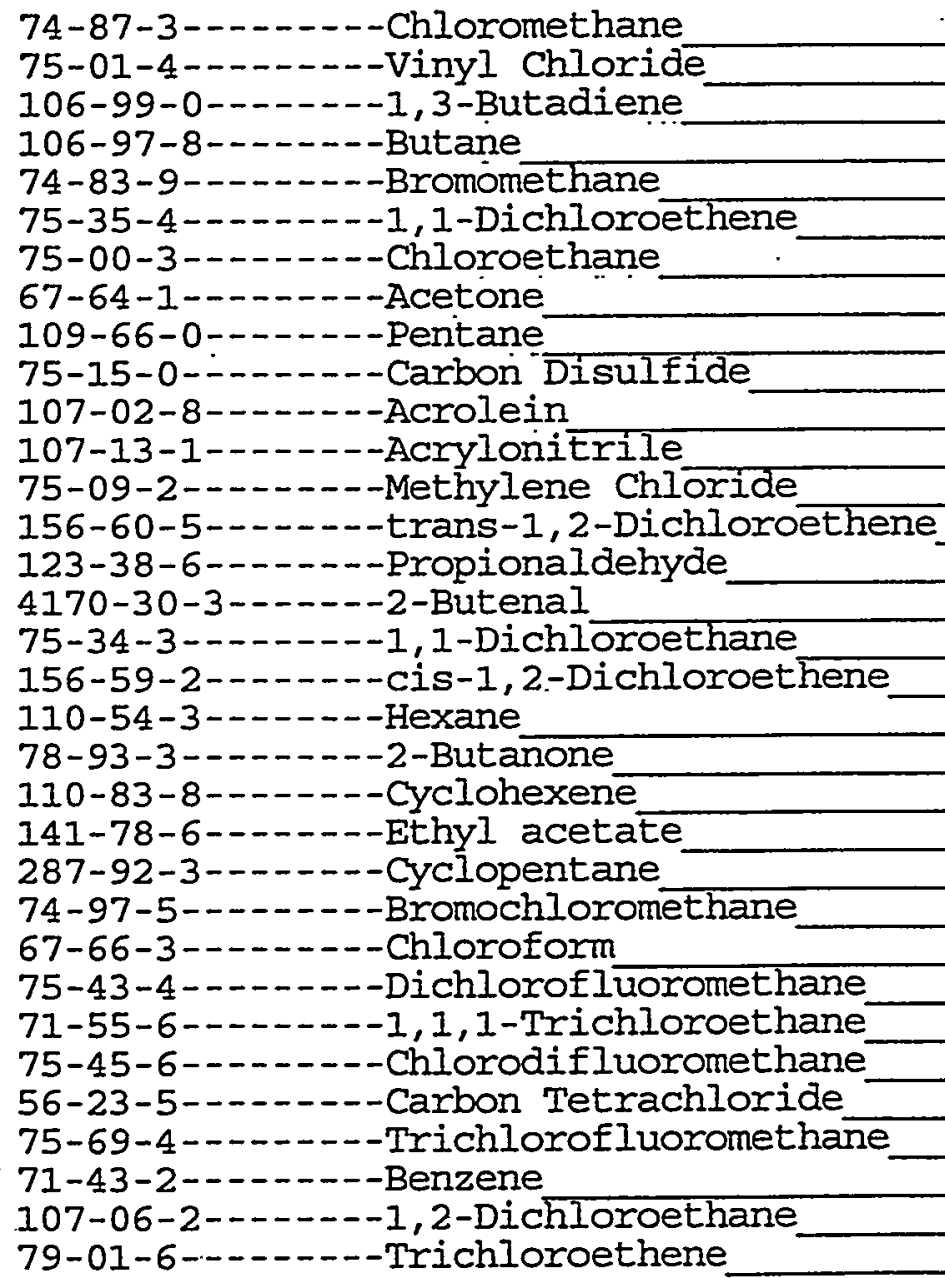 \\
\hline
\end{tabular}

OLM03.0 
Lab Name: PNNL

Lab Code: PNNL

Case No.:

Matrix: (soil/water) SUPERNATAN

Sample wt/vol:

$5.000(\mathrm{~g} / \mathrm{mL}) \mathrm{ML}$

Level: (low/med) LOW

\% Moisture: not dec.

GC Column: $\mathrm{DB}-62475 \mathrm{M} \times 2.55 \mathrm{UM}$ ID: 0.45 (mm)

Soil Extract Volume: (UL)
Contract: BNFL

SAS NO. :
AN-107MS

SDG No.: 99021202

Iab Sample ID: 99-0649MS

Lab File ID: 99021219

Date Received:

Date Analyzed: 02/13/99

(mm) Dilution Factor: 1.0

Soil Aliquot Volume:

(UI)

CONCENTRATION UNITS:

CAS NO. COMPOUND $\quad(u g / L$ or $u g / K g) ~ U G / L$

$Q$

78-87-5-------1, 2-Dichloropropane

75-27-4-...-.-Bromodichloromethane

10061-01-5----cis-1,3-Dichloropropene

108-10-1--.---4-Methyl-2-pentanone

108-88-3-...-.-Toluene

10061-02-6-----trans-1,3-Dichloropropene

79-00-5-...--1, 1,2-Trichloroethane

127-18-4-...--Tetrachloroethene

591-78-6--.---2-Hexanone

124-48-1-...--Dibromochloromethane

108-90-7-...---Chlorobenzene

100-41-4-.-.---Ethylbenzene

106-42-3-...--Xylene (m \& p)

95-47-6-.....-Xylene (o)

100-42-5-.-.---styrene

75-25-2-.-.----Bromoform

79-34-5--..--1, 1, 2, 2-Tetrachloroethane

541-73-1-...-1, 3-Dichlorobenzene

106-46-7-...-1, 4-Dichlorobenzene

95-50-1-...-1, 2-Dichlorobenzene

142-82-5-------Heptane

$111-65-9-\ldots-0-$ Octane

111-84-2-...--Nonane

107-05-1-...--3-Chloropropene

107-87-9-.-.--2-Pentanone

108-87-2-..---Methylcyclohexane

110-12-3-..--5-Methyl-2-hexanone

123-19-3-..---4-Heptanone

123-86-4-..----Butylacetate

123-91-1--..--1,4-Dioxane

126-98-7--..--2-Methyl-2-propenenitrile

563-80-4------3-Methyl-2-butanone

627-13-4-..---Propyl nitrate

\begin{tabular}{r|l|}
130 & \\
540 & \\
6 & $\mathrm{~J}$ \\
520 & $\mathrm{E}$ \\
100 & \\
19 & $\mathrm{~J}$ \\
170 & \\
87 & \\
560 & $\mathrm{E}$ \\
880 & \\
87 & \\
68 & \\
67 & \\
72 & \\
75 & \\
1100 & $\overline{\mathrm{E}}$ \\
10 & $\mathrm{~J}$ \\
390 & \\
390 & \\
500 & \\
84 & \\
79 & \\
63 & \\
77 & \\
380 & $\mathrm{E}$ \\
100 & \\
170 & \\
420 & $\bar{E}$ \\
7 & $\mathrm{~J}$ \\
310 & $\mathrm{E}$ \\
270 & $\mathrm{E}$ \\
290 & $\mathrm{E}$ \\
150 & \\
\hline
\end{tabular}


Lab Name: PNNL

Lab Code: PNNL

Matrix: (soil/water) SUPERNATAN

Sample wt/vol: $\quad 5.000(\mathrm{~g} / \mathrm{mL}) \mathrm{ML}$

Level: (low/med) IOW

$\%$ Moisture: not dec. GC Column: DB-624 75M X 2.55UM ID: 0.45 (mm) (uL)

Soil Extract Volume:
Contract: BNFL

SAS NO.:
AN-I07MS

SDG No.: 99021202

Lab Sample ID: 99-0649MS

Lab File ID: 99021219

Date Received:

Date Analyzed: 02/13/99

Dilution Factor: 1.0

Soil Aliquot Volume: (uL) CONCENTRATION UNITS:

CAS NO.

COMPOUND (ug/L or $\mathrm{ug} / \mathrm{Kg}$ ) UG/L

$Q$

96-22-0--- - - - 3-Pentanone

76-13-1......-1,2,2-C13-1,1,2-F3ethane

76-14-2-..--.-1,2-Cl2-1, 1, 2, 2-F4ethane

75-71-8--..--Dichlorodifluoromethane

75-05-8-.-.-.-Acetonitrile

110-82-7-...--.-Cyclohexane

108-86-1-------Bromobenzene

104-51-8-.-.---Butylbenzene

98-06-6------tert-Butylbenzene

135-98-8-.-.--sec-Butylbenzene

95-49-8-...--2-Chlorotoluene

99-87-6-1-----4-Isopropyltoluene

106-43-4-...-.-4-Chlorotoluene

96-12-8--...-1, 2-Dibromo-3-chloropropane

106-93-4-..---1,2-Dibromoethane

110-57-6-..-.-trans-1, 4-Dichloro-2-butene

142-28-9-..---1,3-Dichloropropane

594-20-7--.---2,2-Dichloropropane

563-58-6--.--1, 1-Dichloropropene

87-68-3-....--Hexachloro-1, 3-butadiene

74-88-4---------Iodomethane

98-82-8-....--.--Isopropylbenzene

91-20-3-....--Nathphalene

103-65-1--.-.--Propylbenzene

87-61-6-...--1, 2, 3-Trichlorobenzene

120-82-1-...---1,2,4-Trichlorobenzene

96-18-4-.----1, 2, 3-Trichloropropane

95-63-6--.----1,2,4-Trimethylbenzene

108-67-8-...-.-1,2,3-Trimethylbenzene

71-23-8-..-.-.-1-Propanol

106-35-4-1----3-Heptanone

110-43-0------2-Heptanone

109-99-9-----Tetrahydrofuran 
: IA

VOLATIIE ORGANICS ANAIYSIS DATA SHEET

Lab Name: PNNL Contract: BNFI,

Lab Code: PNNI

Case No.:

SAS No: :
EPA SAMPLE NO.

AN-107MS

SDG No.: 99021202 .
Matrix: (soil/water) SUPERNATAN

Sample wt/vol: $\quad 5.000(\mathrm{~g} / \mathrm{mL}) \mathrm{ML}$

Level: (low/med) IOW

\% Moisture: not dec.

GC Column: DB-624 75M X 2.55UM ID: 0.45 (mm)

Soil Extract Volume: (u山)

COMPOUND (ug/L or $u g / \mathrm{Kg}$ ) UG/L

Q

Lab Sample ID: 99-0649MS

Lab File ID: 99021219

Date Received:

Date Analyzed: 02/13/99

CAS NO.

74-95-3------Dibromomethane 
Lab Name: PNNL

Iab code: PNNL

Case No.:

Matrix: (soil/water) SUPERNATAN

Sample wt/vol:

$5.000(\mathrm{~g} / \mathrm{mL}) \mathrm{ML}$

Level: (low/med) IOW

$\div$ Moisture: not dec.

GC Column: $\mathrm{DB}-62475 \mathrm{M} \times 2.55 \mathrm{UM}$ ID: 0.45 (mm) (UI)
Contract: BNFL

SAS NO.:
EPA SAMPLE NO.

$\cdot A N-107 M S D$
Soil Extract Volume:

CAS NO.

COMPOUND

CONCENTTRATION UNITS:

(ug/I or ug/Kg) UG/L

SDG No.: 99021202

Iab Sample ID: 99-0649MSD

Lab File ID: 99021220

Date Received:

Date Analyzed: 02/13/99

\begin{tabular}{|c|c|c|}
\hline 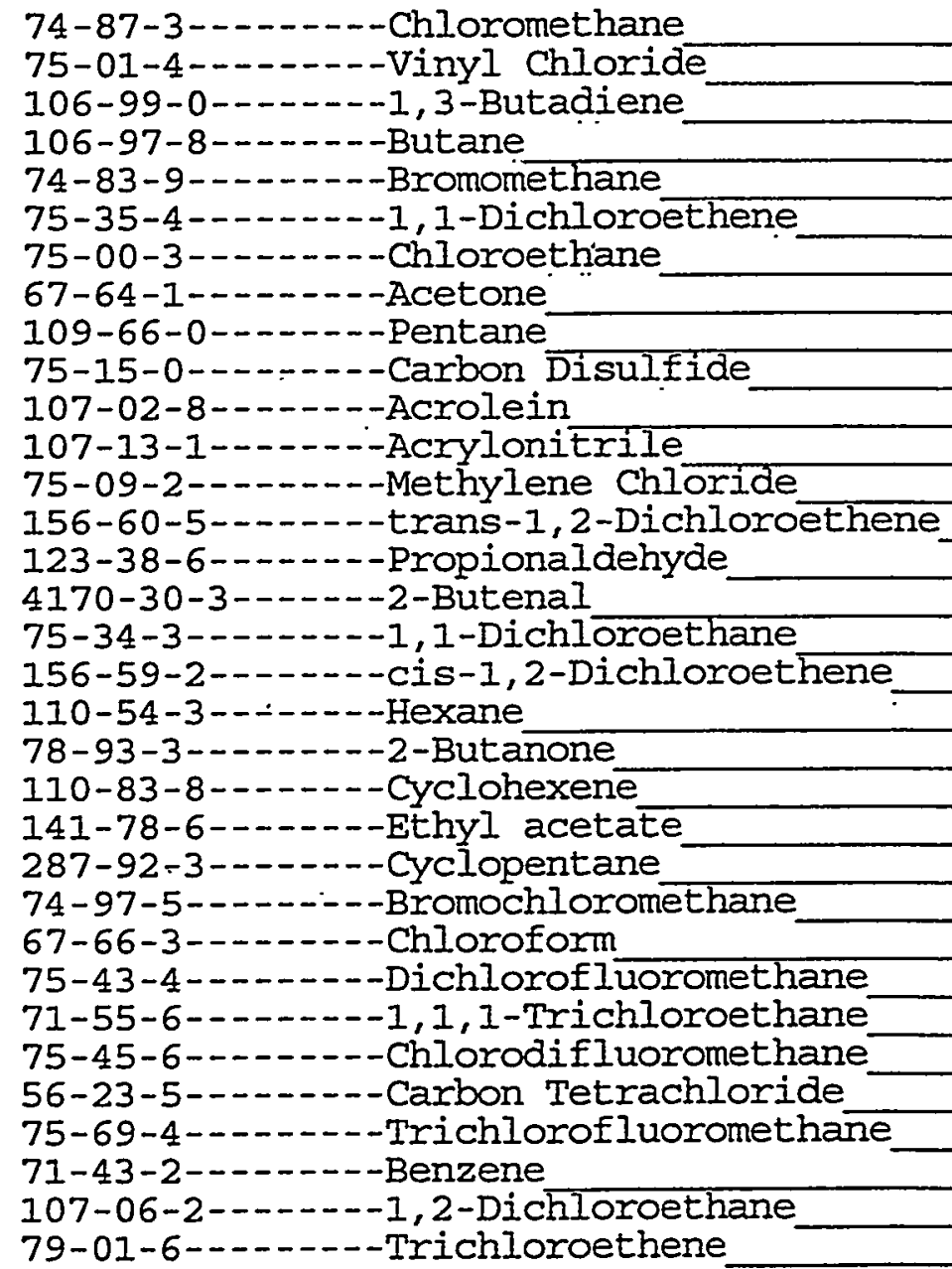 & $\begin{array}{r}110 \\
120 \\
100 \\
160 \\
24 \\
130 \\
110 \\
360 \\
100 \\
12 \\
13 \\
21 \\
110 \\
520 \\
360 \\
21 \\
110 \\
550 \\
95 \\
310 \\
100 \\
18 \\
100 \\
600 \\
110 \\
120 \\
120 \\
150 \\
110 \\
110 \\
110 \\
150 \\
160\end{array}$ & $\begin{array}{l} \\
\\
\mathrm{J} \\
\mathrm{E} \\
\mathrm{J} \\
\mathrm{J} \\
\mathrm{J} \\
\mathrm{E} \\
\mathrm{J} \\
\\
\mathrm{E} \\
\mathrm{J} \\
\\
\\
\\
\\
\\
\\
\end{array}$ \\
\hline
\end{tabular}


Lab Name: PNNL

Lab Code: PNNL

Case No.:

Matrix: (soil/water) SUPERNATAN

Sample wt/vol:

$5.000(\mathrm{~g} / \mathrm{mL}) \mathrm{ML}$

Level: (low/med) LOW

$\%$ Moisture: not dec.

GC Column: DB-624 75M X 2.55UM ID: 0.45 (mm)

Soil Extràct Volume: (uU)
Contract : BNFL

SAS NO.:
EPA SAMPLE NO.

AN-107MSD

SDG No.: 99021202

Lab Sample ID: 99-0649MSD

Lab File ID: $\quad 99021220$

Date Received:

Date Analyzed: 02/13/99

(mm) Dilution Factor: 1.0

Soil Aliquot Volume: (uL)

CONCENTRATION UNITS:

CAS NO.

COMPOUND

(ug/L or $\mathrm{ug} / \mathrm{Kg}$ ) UG/L

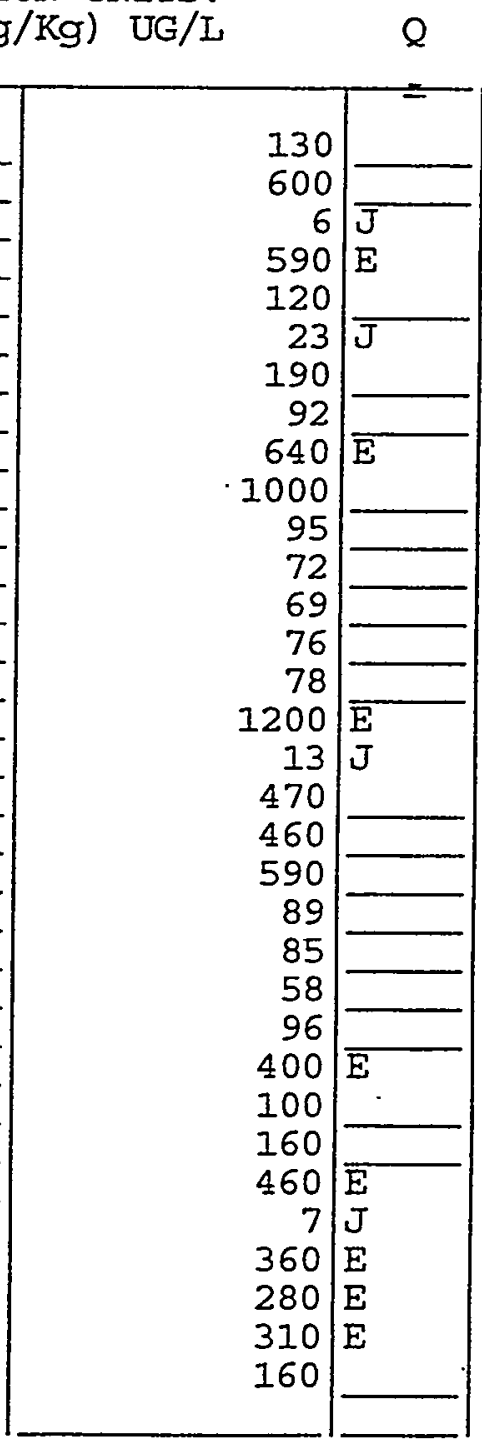

78-87-5-...---1, 2-Dichloropropane

75-27-4-1.--- Bromodichloromethane

10061-01-5-----cis-1,3-Dichloropropene

108-10-1-..--4-Methyl-2-pentanone

108-88-3-...--Toluene

10061-02-6----trans-1, 3-Dichloropropene

79-00-5-...--1, 1,2-Trichloroethane

127-18-4-...--Tetrachloroethene

591-78-6-..---2-Hexanone

124-48-1-..---Dibromochloromethane

108-90-7-....-.-Chlorobenzene

100-41-4-..---Ethylbenzene

106-42-3-..--Xylene (m \& p)

95-47-6-...-XXlene (o)

100-42-5-------styrene

75-25-2-..----Bromoform

79-34-5--.--1, 1,2,2-Tetrachloroethane

541-73-1--.---1;3-Dichlorobenzene

106-46-7--.---1,4-Dichlorobenzene

95-50-1-...-.-1, 2-Dichlorobenzene

142-82-5-------Heptane

$111-65-9-\ldots-.-0 c t a n e$

$111-84-2-\ldots-$-Nonane

107-05-1-..--3-Chloropropene

107-87-9--.----2-Pentanone

108-87-2-...--Methylcyclohexane

110-12-3--.---5-Methyl-2-hexanone

123-19-3--.----4-Heptanone

123-86-4-.-----Butylacetate

123-91-1-..---1,4-Dioxane

126-98-7--.--2-Methyl-2-propenenitrile

563-80-4-------3-Methyl-2-butanone

627-13-4-------Propyl nitrate 
- Lab Name: PNNL

Lab Code:' PNNL
Contract: BNFL

SAS NO.:
EPA SAMPLE NO.

AN-107MSD
Matrix: (soil/water) SUPERNATAN

Sample wt/vol: $\quad 5.000(\mathrm{~g} / \mathrm{mL}) \mathrm{ML}$

Level: (low/med) IOW

\% Moisture: not dec.

GC Column: DB-.624 75M X 2.55UM ID: 0.45 (mm)

Soil Extract Volume:
(uI)
Lab Sample ID: 99-0649MSD

Lab File ID: 99021220

Date Received:

Date Analyzed: 02/13/99

SDG No.: 99021202

Dilution Factor: 1.0

Soil Aliquot Volume: (UT)

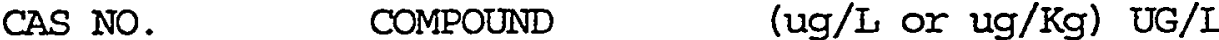

CONCENTRATION UNITS:
$(u g / L$ or $u g / \mathrm{Kg})$ UG/L

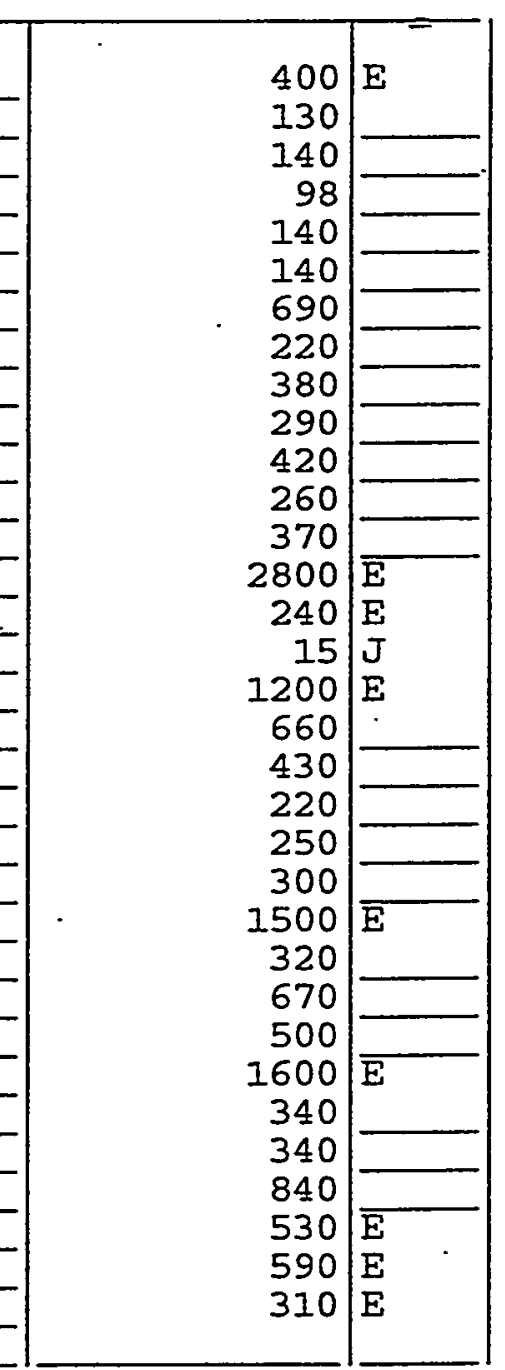


Lab Name: PNNL Contract: BNFL

Lab Code: PNNL

Case No.:

SAS NO.:

SDG No.: 99021202

Matrix: (soil/water) SUPERNATAN

- Lab Sample ID: 99-0649MSD

Sample wt/vol:

$5.000(\mathrm{~g} / \mathrm{mL}) \mathrm{ML}$

Lab File ID: $\quad 99021220$

Level: (low/med) IOW

Date Received:

$\%$ Moisture: not dec.

Date Analyzed: 02/13/99

GC Column: $\mathrm{DB}-624^{\circ} 75 \mathrm{M} \times 2.55 \mathrm{UM}$ ID: 0.45 (mm)

Soil Extract Volume: (uL)

Dilution Factor: 1.0

Soil Aliquot Volume: (UL)

CAS NO.

COMPOUND CONCENTRATION UNITS: (ug/L or ug/Kg) UG/L

Q.

\begin{tabular}{|c|c|}
\hline 74-95-3---.-Dibromomethane & 840 \\
\hline
\end{tabular}


Lab Name: PNNL

Lab Code: PNNL

Case No.:

Matrix: (soil/water) SUPERNATAN

Sample wt/vol:

$5.000(\mathrm{~g} / \mathrm{mL}) \mathrm{ML}$

Ievel: (low/med) IOW

$\%$ Moisture: not dec.

:GC Column: DB-624 75M X 2.55UM ID: 0.45 (mm)

Soil Extract Volume: (ut)
Contract: BNFL

SAS NO.:

\section{METHOD BLANK}

SDG No.: 99021202

Lab Sample ID: VBLK02

Lab File ID: 99021214

Date Received:

Date Analyzed: 02/12/99

Dilution Factor: 1.0

Soil Aliquot Volume: (UI)

CONCENTRATION UNITS:

CAS NO. COMPOUND (ug/I or ug/Kg) UG/L

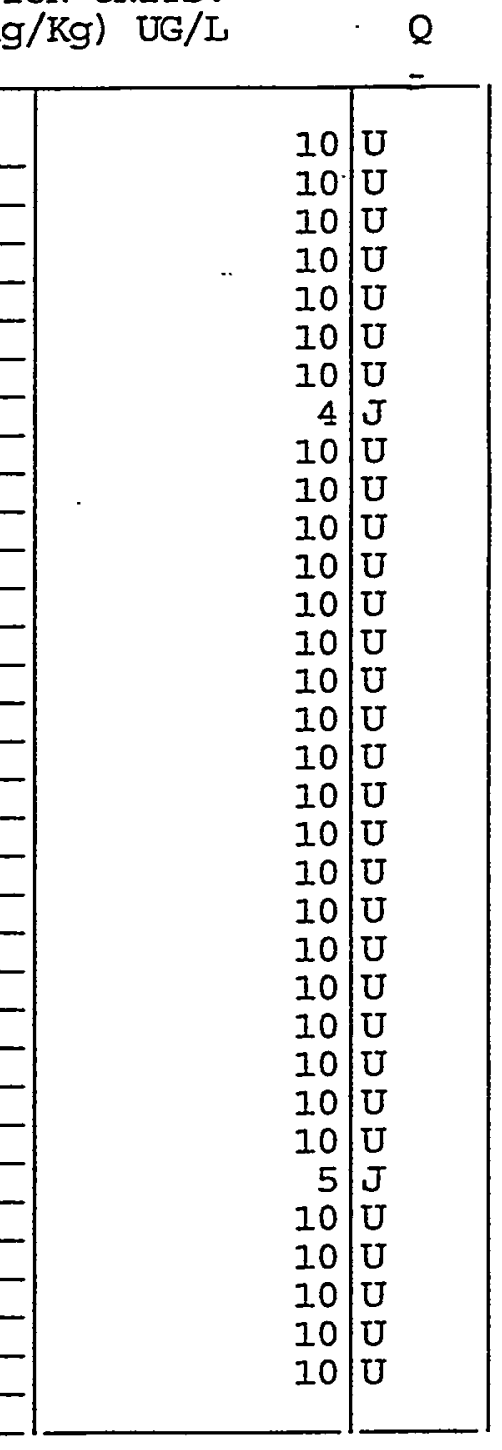

74-87-3-...-.-Chloromethane

75-01-4-..---.-Vinyl Chloride

106-99-0------1,3-Butadiene

106-97-8-..----Butane

74-83-9-...---Bromomethane

75-35-4-_----1, 1-Dichloroethene

75-00-3-...-.-Chloroethane

67-64-1......-Acetone

109-66-0-...-.-Pentane

75-15-0.....--Carbon Disulfide

107-02-8------Acrolein

107-13-1-...--Acrylonitrile

75-09-2-....-.-Methylene Chloride

156-60-5--.----trans-1,2-Dichloroethene

123-38-6-...-.-Propionaldehyde

4170-30-3-..---2-Butenal

75-34-3-.----1,1-Dichloroethane

156-59-2-....-cis-1, 2-Dichloroethene

$110-54-3-\ldots-.-$ Hexane

78-93-3--.---2-Butanone

110-83-8-.....-. Cyclohexene

141-78-6-...-.-Ethyl acetate

287-92-3-..---Cyclopentane

74-97-5-...--Bromochloromethane

67-66-3--.---Chloroform

75-43-4-1.----Dichlorofluoromethane

71-55-6-....-1, 1, 1-Trichloroethane

75-45-6-...---Chlorodifluoromethane

56-23-5--------Carbon Tetrachloride

75-69-4-...-.-Trichlorofluoromethane

71-43-2-...---Benzene

107-06-2--.--1,2-Dichloroethane

79-01-6--.---Trichloroethene 
Lab Name: PNNL

Contract: BNFL

Lab Code: PNNL

Case No.:

Matrix: (soil/water) SUPERNATAN

Sample wt/vol:

$5.000(\mathrm{~g} / \mathrm{mL}) \mathrm{ML}$

Level: (low/med) LOW

$\%$ Moisture: not dec.

GC Column: DB-624 75M X 2.55UM ID: 0.45 (mm)

Soil Extract Volume: (uI)
SAS NO.:
EPA SAMPLE NO.

METHOD BLANK
SDG No.: 99021202

Lab Sample ID: VBLKO2

Lab File ID: 99021214

Date Received:

Date Analyzed: 02/12/99

Dilution Factor: 1.0

Soil Aliquot Volume: (uI)

CAS NO. COMPOUND (ug/I or ug./Kg) UG/L

CONCENTRATION UNITS:

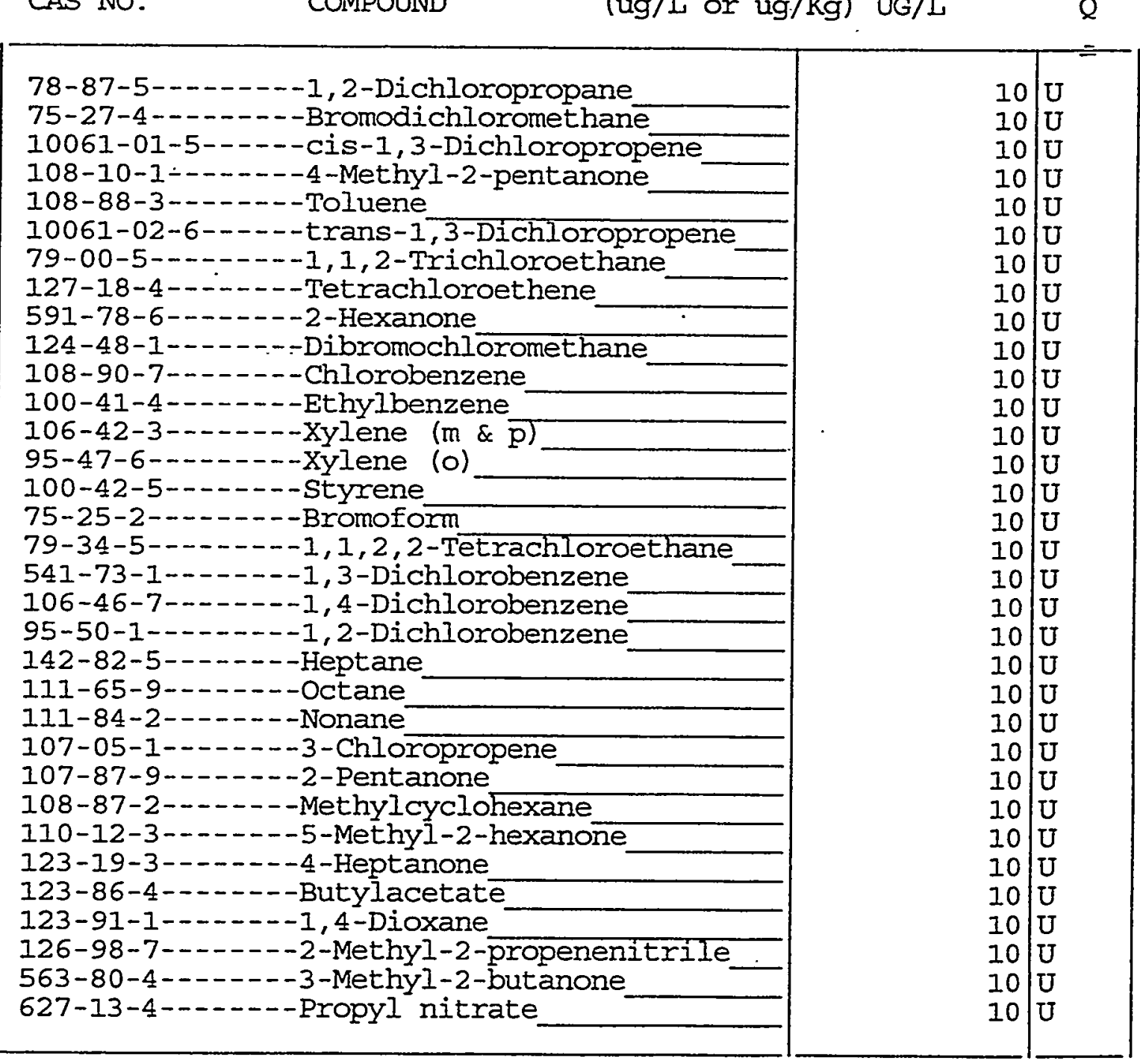


Lab Name: PNNL

Iab Code: PNNL

Matrix: (soil/water) SUPERNATAN

Sample wt/vol:

$5.000(\mathrm{~g} / \mathrm{mL}) \mathrm{ML}$

Level: (low/med) LOW

\% Moisture: not dec.

GC Column: DB-624 75M X 2.55UM ID: 0.45 (mm)

Soil Extract Volume: (UI)
Contract: BNEL

SAS NO. :
EPA SAMPLE NO.

METHOD BLANK

SDG No.: 99021202

Lab Sample ID: VBLKO2

Lab File ID: $\quad 99021214$

Date Received:

Date Analyzed: 02/12/99

(min) Dilution Factor: 1.0

Soil Aliquot Volume: (UL) CONCENTRATION UNITS : CAS NO. COMPOUND (ug/L or. ug/Kg) UG/L \& 96-22-0------- - - Pentanone

$76-13-1-\ldots--1,2,2-C 13-1,1,2-$ F3ethane

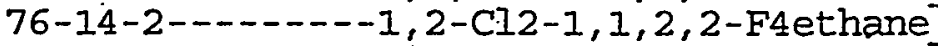

75-71-8-...-D-Dichlorodifluoromethane

75-05-8--.----Acetonitrile

110-82-7-...-.-Cyclohexane

108-86-1-...--Bromobenzene

104-51-8--.----Butylbenzene

98-06-6-..---tert-Butylbenzene

135-98-8-..---sec-Butylbenzene

95-49-8-...-.-2-Chlorotoluene

99-87-6--.----4-Isopropyltoluene

106-43-4-1----4-Chlorotoluene

96-12-8-....-1, 2-Dibromo-3-chloropropane

106-93-4-...--1,2-Dibromoethane

110-57-6-...--trans-1,4-Dichloro-2-butene

142-28-9-..--1,3-Dichloropropane

594-20-7------2, 2-Dichloropropane

563-58-6------1,1-Dichloropropene

87-68-3-.....-Hexachloro-1, 3-butadiene

74-88-4---------Iodomethane

98-82-8-...---Isopropylbenzene

91-20-3--.----Nathphalene

103-65-1-.-.---Propylbenzene

87-61-6-...--1, 2,3-Trichlorobenzene

120-82-1-....-1, 2,4-Trichlorobenzene

96-18-4-------1, 2,3-Trichloropropane

95-63-6--.---1,2,4-Trimethylbenzene

108-67-8-.----1, 2,3-Trimethylbenzene

71-23-8-...-.--1-Propanol

106-35-4-.-----3-Heptanone

110-43-0--.---2-Heptanone

109-99-9-...-.-Tetrahydrofuran

\begin{tabular}{|l|l|l|}
10 & $\mathrm{U}$ \\
10 & $\mathrm{U}$ \\
10 & $\mathrm{U}$ \\
10 & $\mathrm{U}$ \\
10 & $\mathrm{U}$ \\
10 & $\mathrm{U}$ \\
10 & $\mathrm{U}$ \\
10 & $\mathrm{U}$ \\
10 & $\mathrm{U}$ \\
10 & $\mathrm{U}$ \\
10 & $\mathrm{U}$ \\
10 & $\mathrm{U}$ \\
10 & $\mathrm{U}$ \\
10 & $\mathrm{U}$ \\
10 & $\mathrm{U}$ \\
10 & $\mathrm{U}$ \\
10 & $\mathrm{U}$ \\
10 & $\mathrm{U}$ \\
10 & $\mathrm{U}$ \\
10 & $\mathrm{U}$ \\
10 & $\mathrm{U}$ \\
10 & $\mathrm{U}$ \\
10 & $\mathrm{U}$ \\
10 & $\mathrm{U}$ \\
10 & $\mathrm{U}$ \\
10 & $\mathrm{U}$ \\
10 & $\mathrm{U}$ \\
10 & $\mathrm{U}$ \\
10 & $\mathrm{U}$ \\
10 & $\mathrm{U}$ \\
10 & $\mathrm{U}$ \\
10 & $\mathrm{U}$ \\
10 & $\mathrm{U}$ \\
& \\
\hline
\end{tabular}

FORM I VOA

OLMO3.0 
Lab Name: PNNL Contract: BNFL METHOD BLANK

Lab Code: PNNL

Case No.:

SAS NO.:

SDG No.: 99021202

Matrix: (soil/water) SUPERNATAN

Lab Sample ID: VBIKO2

Sample wt/vol: $\quad 5.000(\mathrm{~g} / \mathrm{mL}) \mathrm{ML}$

Lab File ID: 99021214

Level: (low/med) LOW

Date Received:

$\%$ Moisture: not dec.

Date Analyzed: 02/12/99

GC Column: DB-624 75M X 2.55UM ID: 0.45 (mm)

Dilution Factor: 1.0

Soil Extract Volume: (uL)

Soil Aliquot Volume: (uL)

CAS NO.

COMPOUND CONCENTRATION UNITS: (ug/L or ug/kg) UG/L

Q

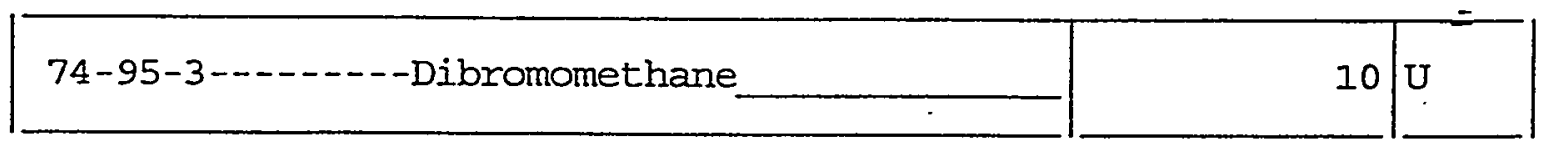


Lab Name: PNNL

Lab Code: PNNL

Case No.:

Matrix: (soil/water) SUPERNATAN

Sample wt/vol:

$5.000(\mathrm{~g} / \mathrm{mL}) \mathrm{ML}$

Level: (low/med) LOW

$\%$ Moisture: not dec.

GC Column: DB-624 75M X 2.55UM ID: 0.45 (mm) (UI)

Soil Extract Volume:
Contract: BNFL

SAS NO.:
AN-107DIL

SDG No.: 99021202

Lab Sample ID: 99-0649DII

Lab File ID: 99021215

Date Received:

Date Analyzed: 02/12/99 .

Dilution Factor: 1.0

Soil Aliquot Volume:

CAS NO.

COMPOUND CONCENTRATION UNITS: (ug/I or $\mathrm{ug} / \mathrm{Kg}$ ) UG/L

\begin{tabular}{|c|}
\hline 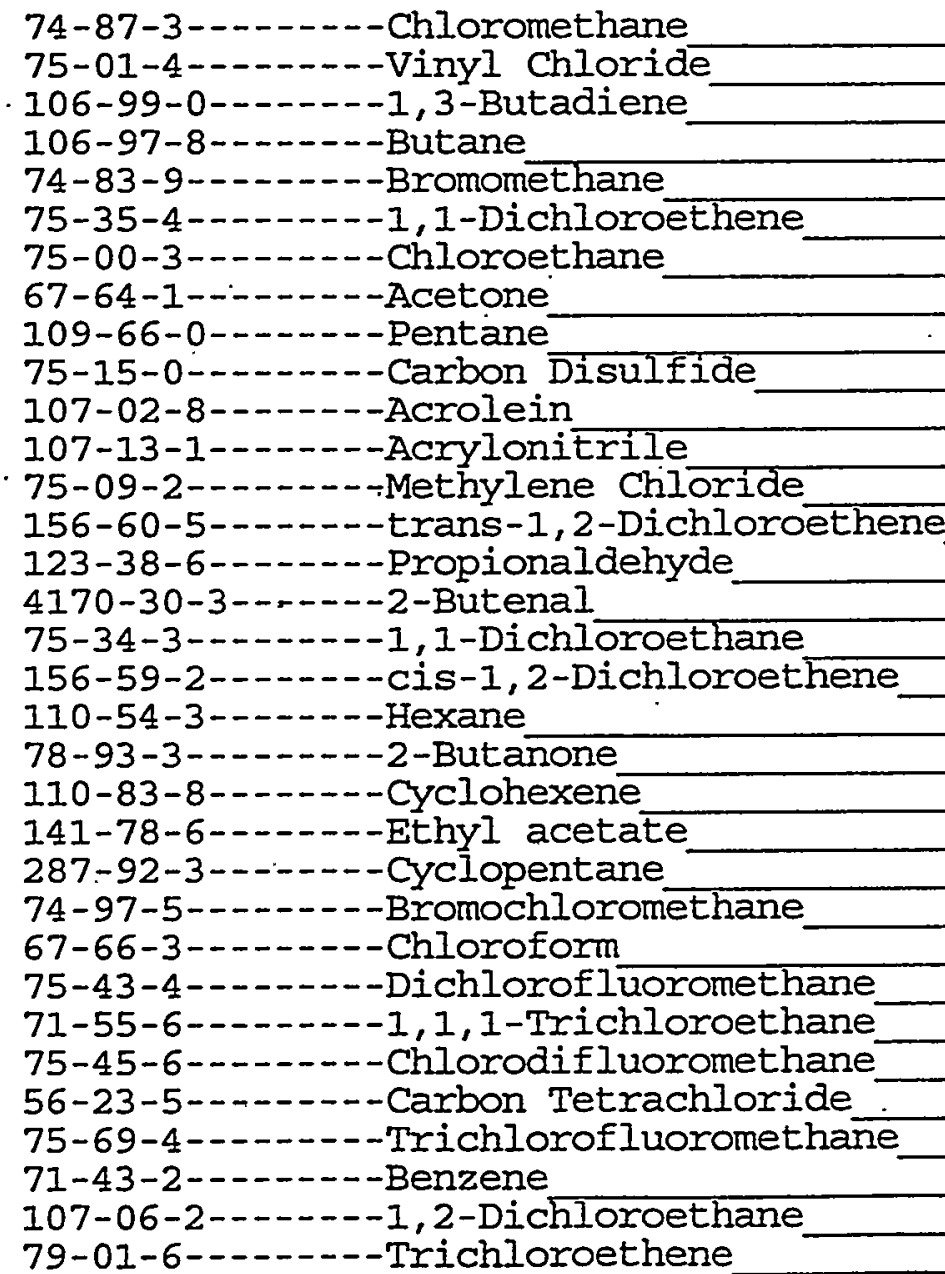 \\
\hline
\end{tabular}

\begin{tabular}{|l|l|l|}
270 & $\mathrm{U}$ \\
270 & $\mathrm{U}$ \\
270 & $\mathrm{U}$ \\
270 & $\mathrm{U}$ \\
270 & $\mathrm{U}$ \\
270 & $\mathrm{U}$ \\
270 & $\mathrm{U}$ \\
690 & \\
\hline 270 & $\mathrm{U}$ \\
270 & $\mathrm{U}$ \\
270 & $\mathrm{U}$ \\
270 & $\mathrm{U}$ \\
270 & $\mathrm{U}$ \\
270 & $\mathrm{U}$ \\
270 & $\mathrm{U}$ \\
270 & $\mathrm{U}$ \\
270 & $\mathrm{U}$ \\
270 & $\mathrm{U}$ \\
270 & $\mathrm{U}$ \\
38 & $\mathrm{~J}$ \\
270 & $\mathrm{U}$ \\
270 & $\mathrm{U}$ \\
270 & $\mathrm{U}$ \\
270 & $\mathrm{U}$ \\
270 & $\mathrm{U}$ \\
270 & $\mathrm{U}$ \\
270 & $\mathrm{U}$ \\
160 & $\mathrm{~J}$ \\
270 & $\mathrm{U}$ \\
270 & $\mathrm{U}$ \\
270 & $\mathrm{U}$ \\
270 & $\mathrm{U}$ \\
270 & $\mathrm{U}$ \\
& \\
\hline
\end{tabular}


Irab Name: PNNL

Lab Code: PNNL

Case No.:

Matrix: (soil/water) SUPERNATAN

Sample wt/vol:

$5.000(\mathrm{~g} / \mathrm{mL}) \mathrm{ML}$

Level: (low/med) IOW

$\because$ Moisture: not dec.

GC Column: DB-624 75M X 2.55UM ID: 0.45 (mm)

Soil Extract Volume: (uL)
Contract: BNFL

SAS NO.:
EPA SAMPLE NO.

AN-107DIL
Lab Sample ID: 99-0649DIL

Lab File ID: $\quad 99021215$

Date Received:

Date Analyzed: 02/12/99

Dilution Factor: 1.0

Soil Aliquot Volume: (UI)

CAS NO. COMPOUND $(u g / L$ or $u g / K g) ~ U G / L \quad Q$

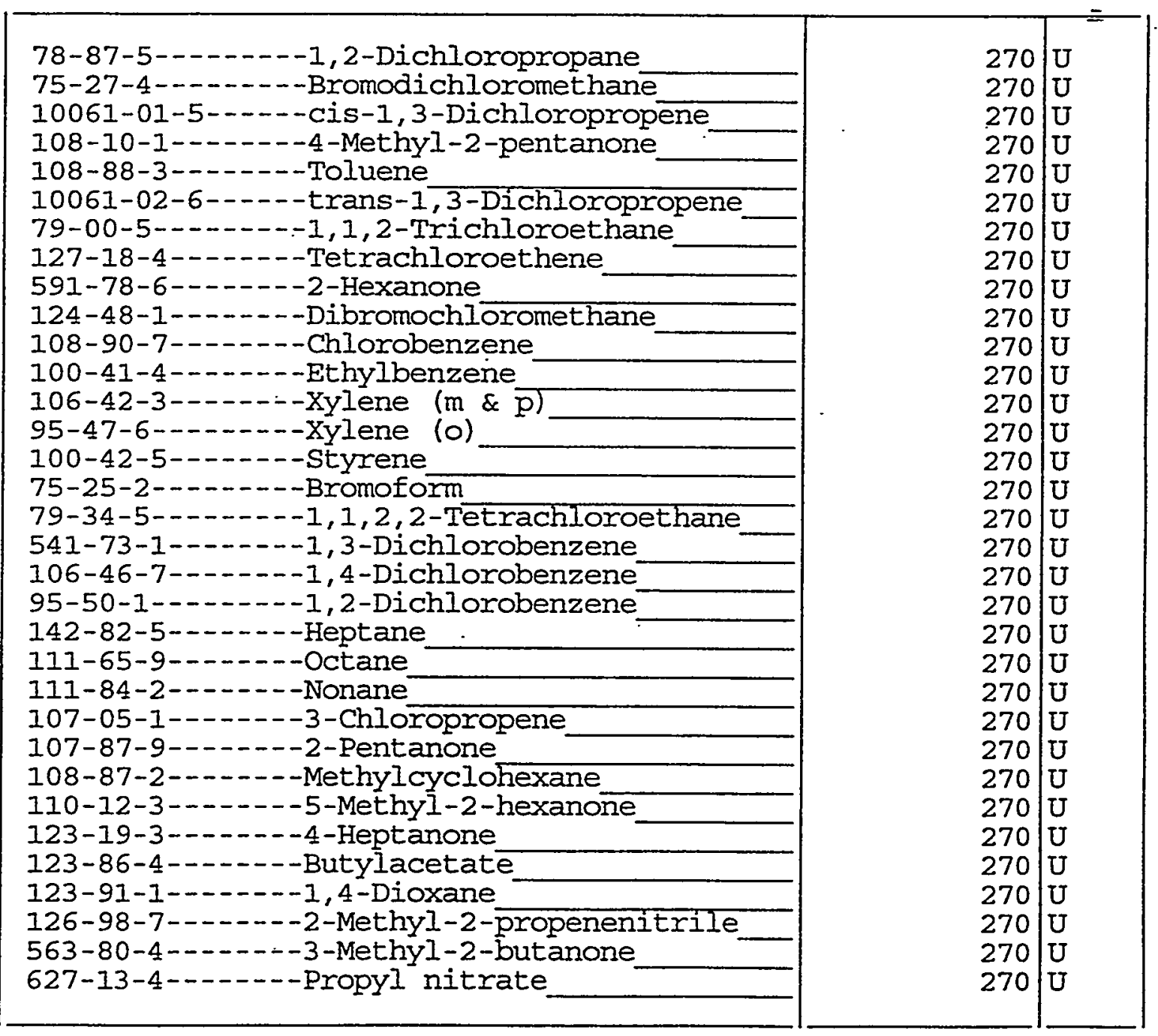


Lab Name: PNNL

Lab Code: PNNL

Case No.:

Matrix: (soil/water) SUPERNATAN

Sample wt/vol:

$5.000(\mathrm{~g} / \mathrm{mL}) \mathrm{ML}$

Ievel: (low/med) IOW

$\%$ Moisture: not dec.

GC Column: DB-624 75M X 2.55UM ID: 0.45 (mm)

Soil Extract Volume: (uI)
Contract: BNFL

SAS NO. :
AN-107DIL

SDG No.: 99021202

Lab Sample ID: 99-0649DIL

Lab File ID: 99021215

Date Received:

Date Analyzed: 02/12/99

Dilution Factor: 1.0

Soil Aliquot Volume:

(u) CONCENTRATION UNITS:

CAS NO. COMPOUND (ug/L or ug/Kg) UG/L

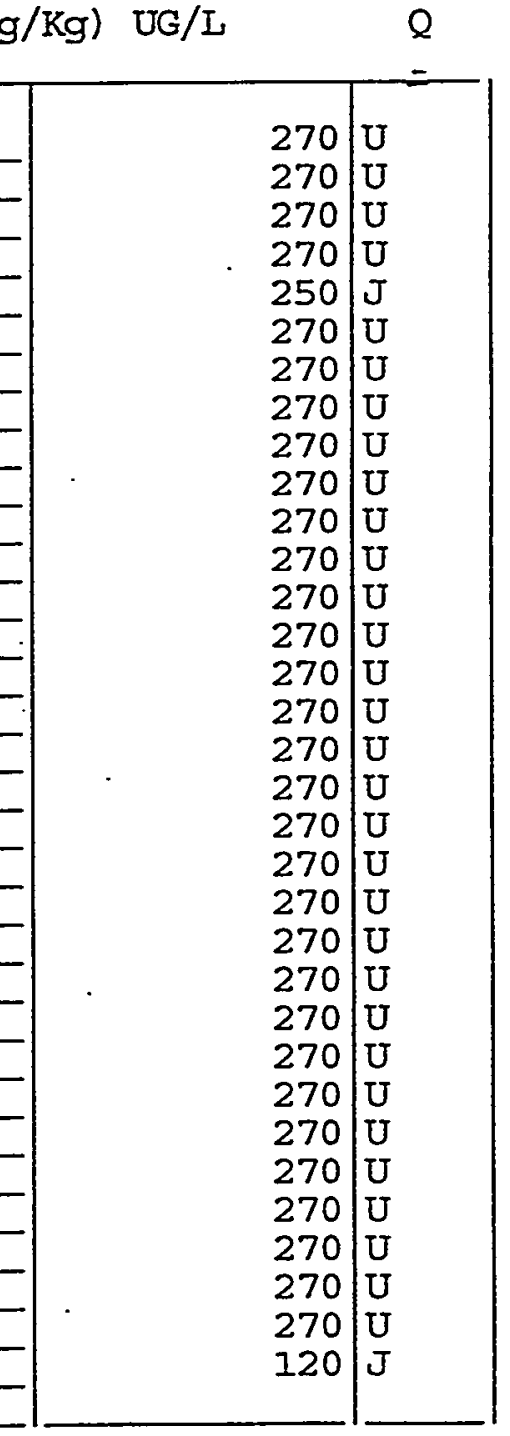

96-22-0-1-- - - 3-Pentanone

76-13-1--.---1,2,2-Cl3-1, 1, 2-F3ethane

76-14-2--..--1,2-Cl2-1,1,2,2-F4ethane

75-71-8-....-Dichlorodifluoromethane

75-05-8-...--Acetonitrile

110-82-7-..---Cyclohexane

108-86-1-.....-Bromobenzene

104-51-8-.-.--Butylbenzene

98-06-6-....-tert-Butylbenzene

135-98-8-------sec-Butylbenzene

95-49-8--.---2-Chlorotoluene

99-87-6-...-.-4-Isopropyltoluene

106-43-4-------4-Chlorotoluene

96-12-8-...--1, 2-Dibromo-3-chloropropane

106-93-4----1, 2-Dibromoethane

110-57-6-..---trans-1, 4-Dichloro-2-butene

142-28-9-_..--1, 3-Dichloropropane

594-20-7--.---2,2-Dichloropropane

563-58-6--.---1,1-Dichloropropene

87-68-3-.....-Hexachloro-1, 3-butadiene

74-88-4-2.----Iodomethane

98-82-8-....-.-Isopropylbenzene

91-20-3-...---Nathphalene

103-65-1-------Propylbenzene

87-61-6-...--1,2,3-Trichlorobenzene

120-82-1.....-1, 2,4-Trichlorobenzene

96-18-4-...--1, 2,3-Trichloropropane

95-63-6-..----1,2,4-Trimethylbenzene

108-67-8 .....-1,2,3-Trimethylbenzene

71-23-8-....-1-Propanol

106-35-4-.-.--3-Heptanone

110-43-0--.---2-Heptanone

109-99-9-..---Tetrahydrofuran 
Lab Name: PNNL

Lab Code: PNNL

Case No.:

SAS NO.:

SDG No.: 99021202

Matrix: (soil/water) SUPERNATAN

Lab Sample ID: 99-0649DIL

Sample wt/vol:

$5.000(\mathrm{~g} / \mathrm{mL}) \mathrm{ML}$

Lab File ID: 99021215

Level: (low/med) LOW

Date Received:

$\div$ Moisture: not dec.

Date Analyzed: 02/12/99

GC Column: DB-624 75M X 2.55UM ID: 0.45 (mm)

Dilution Factor: 1.0

Soil Extract Volume: (uL)

Soil Aliquot Volume: (UI)

CAS NO.

COMPOUND CONCENTRATION UNITS: (ug/I or $\mathrm{ug} / \mathrm{kg}$ ) UG/I

Q

\begin{tabular}{|c|c|c|}
\hline 74-95-3-----Dibromomethane & 270 & $\mathrm{U}$ \\
\hline
\end{tabular}


Iab Name: PNNL

Lab Code: PNNL

Case No.:

Matrix: (soil/water) SUPERNATAN

Sample wt/vol: $\quad 5.000(\mathrm{~g} / \mathrm{mL}) \mathrm{ML}$

Ievel: (low/med) LOW

$\%$ Moisture: not dec.

GC Column: $\mathrm{DB}-62475 \mathrm{M} \times 2.55 \mathrm{UM}$ ID: 0.45 (mm)

Soil Extract Volume: (uIs)
Contract: BNFL

SAS NO.:
EPA SAMPLE NO .

AN-107DIIDUP .

SDG No.: 99021202

Lab Sample ID: 99-0649DILD

Iab File ID: 99021216

Date Received:

Date Analyzed: 02/13/99 (mm) Dilution Factor: 1.0

Soil Aliquot Volume: (UL) CONCENTRATION UNITS:

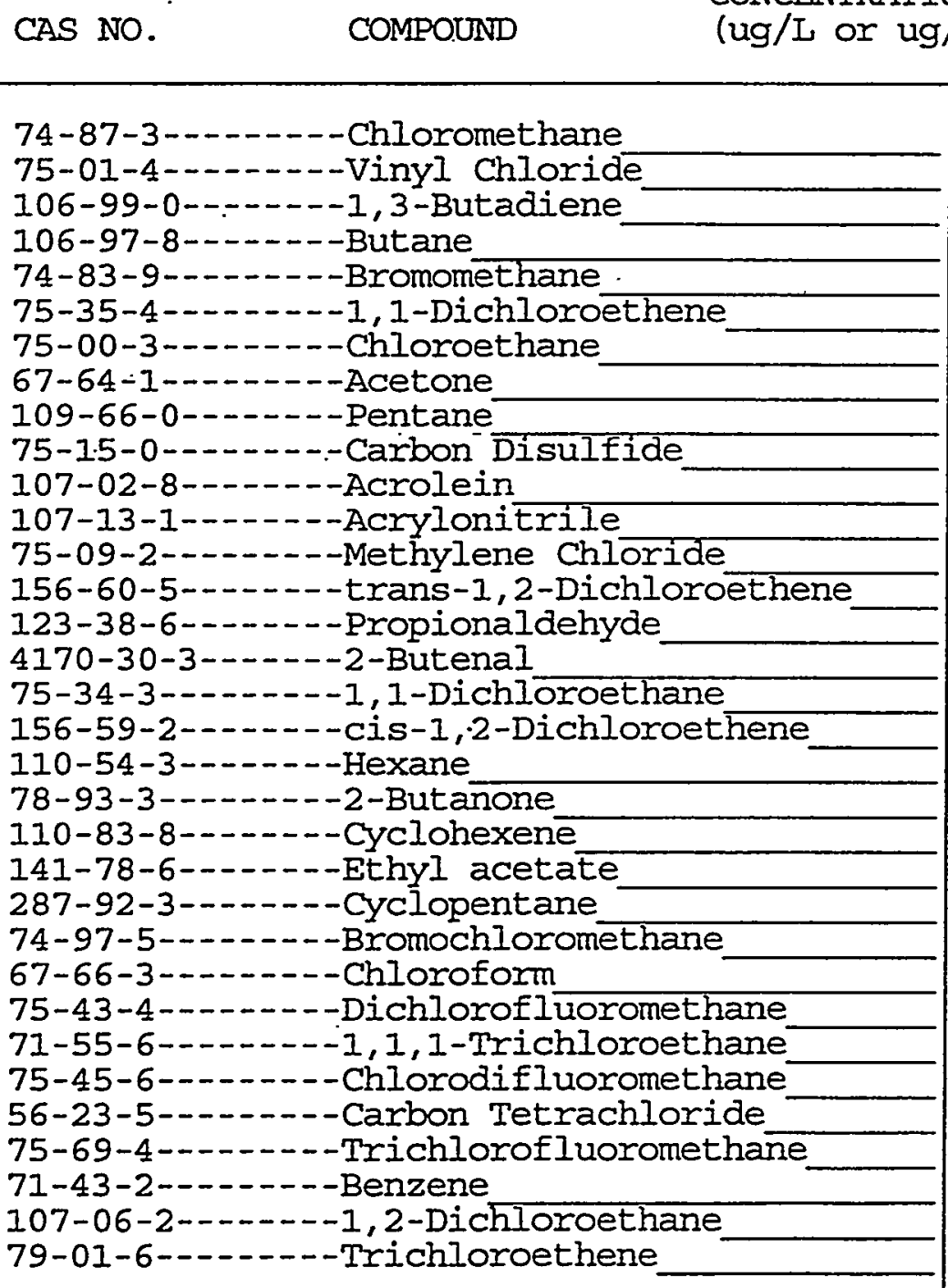
g/Kg) UG/L

\begin{tabular}{r|l}
\multicolumn{2}{l|}{} \\
\multicolumn{1}{l|}{} \\
\hline 180 & $\mathrm{U}$ \\
180 & $\mathrm{U}$ \\
180 & $\mathrm{U}$ \\
180 & $\mathrm{U}$ \\
180 & $\mathrm{U}$ \\
180 & $\mathrm{U}$ \\
180 & $\mathrm{U}$ \\
4300 & $\mathrm{E}$ \\
180 & $\mathrm{U}$ \\
180 & $\mathrm{U}$ \\
180 & $\mathrm{U}$ \\
180 & $\mathrm{U}$ \\
180 & $\mathrm{U}$ \\
180 & $\mathrm{U}$ \\
180 & $\mathrm{U}$ \\
180 & $\mathrm{U}$ \\
180 & $\mathrm{U}$ \\
180 & $\mathrm{U}$ \\
180 & $\mathrm{U}$ \\
230 & \\
180 & $\mathrm{U}$ \\
180 & $\mathrm{U}$ \\
180 & $\mathrm{U}$ \\
180 & $\mathrm{U}$ \\
180 & $\mathrm{U}$ \\
180 & $\mathrm{U}$ \\
180 & $\mathrm{U}$ \\
4000 & $\mathrm{E}$ \\
180 & $\mathrm{U}$ \\
180 & $\mathrm{U}$ \\
180 & $\mathrm{U}$ \\
180 & $\mathrm{U}$ \\
180 & $\mathrm{U}$ \\
& \\
\hline & \\
\hline
\end{tabular}


Lab Name: PNNL

Contract: BNFL

Iab code: PNiNL
SAS NO: :
EPA SAMPLE NO.

AN-107DILDUP
Matrix: (soil/water) SUPERNATAN

Sample wt/vol: $\quad 5.000(\mathrm{~g} / \mathrm{mL}) \mathrm{ML}$

Level: (low/med) IOW

\% Moisture: not dec.

GC Column: DB-624 75M X 2.55UM ID: 0.45 (mm)

Soil Extract Volume: (UI)
SDG No.: 99021202
Lab Sample ID: 99-0649DIID

Lab File ID: 99021216

Date Received:

Date Analyzed: 02/13/99

Dilution Factor: 1.0

Soil Aliquot Volume: (uL)
COMPOUND
CAS NO.

\begin{tabular}{|c|}
\hline 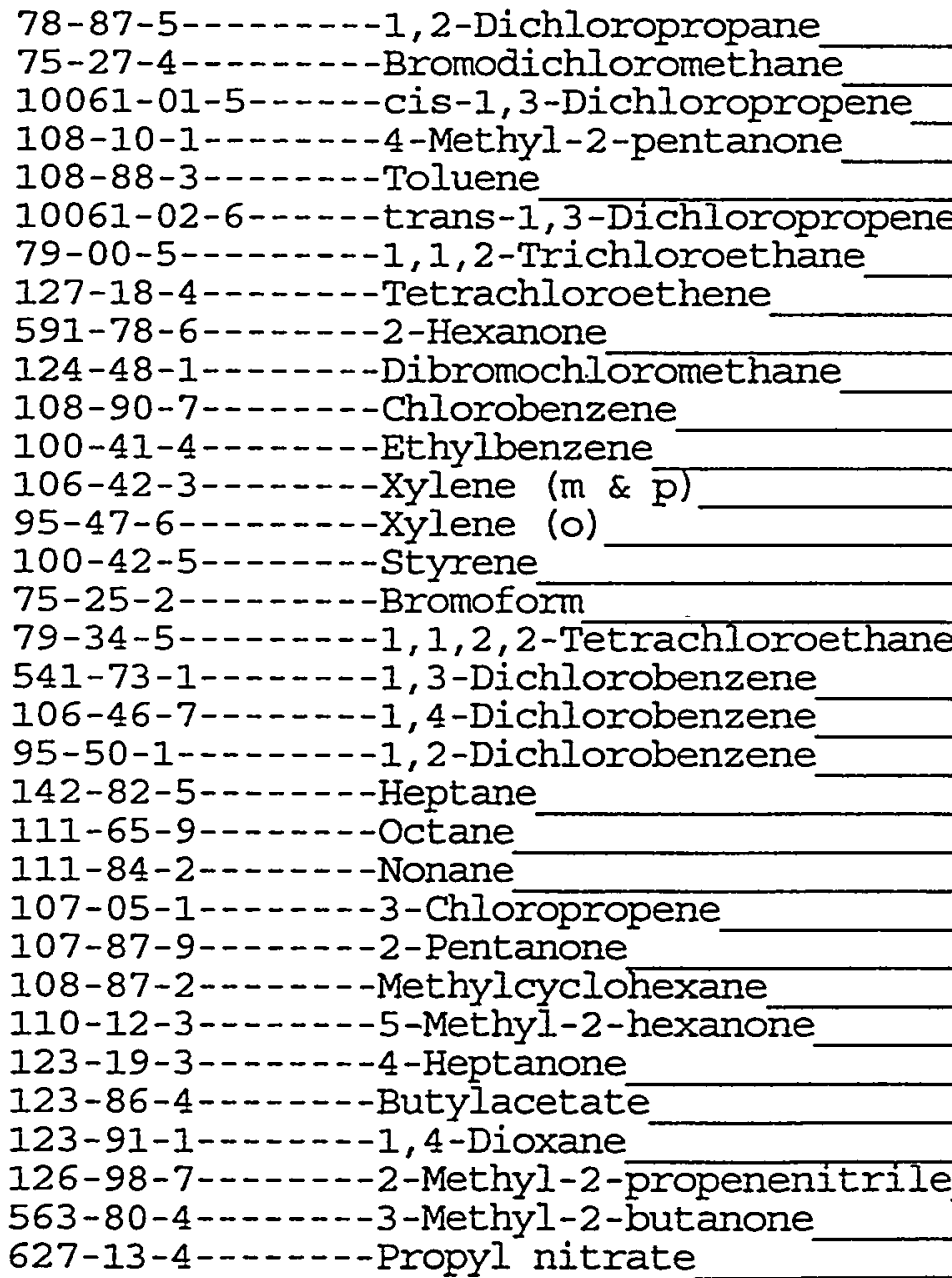 \\
\hline
\end{tabular}

CONCENTRATION UNITS:

(ug/I or $u g / \mathrm{kg}) \mathrm{UG} / \mathrm{I}$

Q

\begin{tabular}{|l|l|l|}
180 & $\mathrm{U}$ \\
180 & $\mathrm{U}$ \\
180 & $\mathrm{U}$ \\
180 & $\mathrm{U}$ \\
180 & $\mathrm{U}$ \\
180 & $\mathrm{U}$ \\
180 & $\mathrm{U}$ \\
180 & $\mathrm{U}$ \\
180 & $\mathrm{U}$ \\
180 & $\mathrm{U}$ \\
180 & $\mathrm{U}$ \\
180 & $\mathrm{U}$ \\
180 & $\mathrm{U}$ \\
180 & $\mathrm{U}$ \\
180 & $\mathrm{U}$ \\
180 & $\mathrm{U}$ \\
180 & $\mathrm{U}$ \\
180 & $\mathrm{U}$ \\
180 & $\mathrm{U}$ \\
180 & $\mathrm{U}$ \\
180 & $\mathrm{U}$ \\
180 & $\mathrm{U}$ \\
180 & $\mathrm{U}$ \\
180 & $\mathrm{U}$ \\
180 & $\mathrm{U}$ \\
180 & $\mathrm{U}$ \\
180 & $\mathrm{U}$ \\
180 & $\mathrm{U}$ \\
180 & $\mathrm{U}$ \\
180 & $\mathrm{U}$ \\
180 & $\mathrm{U}$ \\
180 & $\mathrm{U}$ \\
180 & $\mathrm{U}$ \\
& & \\
\hline & \\
\hline
\end{tabular}


Lab Name: PNNL

Lab Code: PNNL

Case No. :

Matrix: (soil/water) SUPERNATAN

Sample wt/vol:

$5.000(\mathrm{~g} / \mathrm{mL}) \mathrm{ML}$

Level: (low/med) LOW

\% Moisture: not dec.

GC Column: $\mathrm{DB}-62475 \mathrm{M} \times 2.55 \mathrm{UM}$ ID: 0.45 (mm) (UU)

Soil Extract Volume:
Contract: BNFL

SAS NO.:

AN-107DIIDUP

SDG No.: 99021202

Iab Sample ID: 99-0649DIID

Lab File ID: 99021216

Date Received:

Date Analyzed: 02/13/99

Dilution Factor: 1.0

Soil Aliquot Volume: (uL) CONCENIRATION UNITS :

CAS NO.

COMPOUND (ug/L or $\mathrm{ug} / \mathrm{Kg}$ ) UG/L

\begin{tabular}{l|l}
\multicolumn{2}{l}{} \\
\multicolumn{1}{l|}{} \\
\hline 180 & $U$ \\
180 & $U$ \\
180 & $U$ \\
180 & $U$ \\
120 & $U$ \\
180 & $U$ \\
180 & $U$ \\
180 & $U$ \\
180 & $U$ \\
180 & $U$ \\
180 & $U$ \\
180 & $U$ \\
180 & $U$ \\
180 & $U$ \\
180 & $U$ \\
180 & $U$ \\
180 & $U$ \\
180 & $U$ \\
180 & $U$ \\
180 & $U$ \\
180 & $U$ \\
180 & $U$ \\
180 & $U$ \\
180 & $U$ \\
180 & $U$ \\
180 & $U$ \\
180 & $U$ \\
180 & $U$ \\
180 & $U$ \\
180 & $U$ \\
180 & $U$ \\
180 & $U$ \\
290 & \\
& \\
\end{tabular}

96-22-0--------3-Pentanone

76-13-1-..---1,2,2-C13-1, 1, 2-F3ethane

76-14-2-.-.--1,2-Cl2-1,1,2, 2-F4ethane

75-71-8-...-Dichlorodifluoromethane

75-05-8------Acetonitrile

110-82-7--.---Cyclohexane

108-86-1-.----Bromobenzene

104-51-8-.-.---Butylbenzene

98-06-6-....--tert-Butylbenzene

135-98-8--.----sec-Butylbenzene

95-49-8-...--2-Chlorotoluene

99-87-6-...--4-Isopropyltoluene

106-43-4--.---4-Chlorotoluene

96-12-8-...--1, 2-Dibromo-3-chloropropane

106-93-4------1,2-Dibromoethane

110-57-6-...--trans-1,4-Dichloro-2-butene

142-28-9-...-1, 3-Dichloropropane

594-20-7-------2,2-Dichloropropane

563-58-6--.-.-1,1-Dichloropropene

87-68-3-.....-Hexachloro-1,3-butadiene

74-88-4--.-----Iodomethane

98-82-8--.-----Isopropylbenzene

91-20-3-..---Nathphalene

103-65-1-...--Propylbenzene

87-61-6-...--1, 2, 3-Trichlorobenzene

120-82-1-...--1,2,4-Trichlorobenzene

96-18-4--.----1,2,3-Trichloropropane

95-63-6--.----1, 2,4-Trimethylbenzene

108-67-8-....-1, 2, 3-Trimethylbenzene

71-23-8-....-1-Propanol

106-35-4-.-----3-Heptanone

110-43-0--.----2-Heptanone

109-99-9-...--Tetrahydrofuran 
$I A$

VOLATILE ORGANICS ANALYSIS DATA SHEET

Lab Name: PNNL

Contract: BNFL

Iab code: PNNL

Case No.:

Matrix: (soil/water) SUPERNATAN

Sample wt/vol:

$5.000(\mathrm{~g} / \mathrm{mL}) \mathrm{ML}$

Level: (low/med) IOW

SAS NO.:

Lab Sample ID: 99-0649DILD

Lab File ID: 99021216

Date Received:

Date Analyzed: 02/13/99

$\%$ Moisture: not dec.

GC Column: DB-624 75M X 2.55UM ID: 0.45 (mm) Soil Extract Volume: (uI)
AN-107DILDUP

SDG No. : 99021202

\begin{tabular}{|c|c|c|c|c|}
\hline CAS NO. & COMPOUND & $\begin{array}{l}\text { CONCENTRATION UNITS: } \\
\text { (ug/L or } \mathrm{ug} / \mathrm{Kg} \text { ) UG/L }\end{array}$ & & $Q$ \\
\hline $74-95-3$ & -Dibromomethane & & 180 & U \\
\hline
\end{tabular}


VOLATILE ORGANICS ANAIYSIS DATA SHEET TENTATIVELY IDENTIFIED COMPOUNDS

Lab Name: PNNL.

Contract: BNFL

Lab Code: PNNL

Case No.:

SAS NO. :
EPA SAMPIE NO.

AN-107

SDG No.: 99021202
Matrix: (soil/water) SUPERNATANT

Sample wt/vol:

$5.000(\mathrm{~g} / \mathrm{mL}) \mathrm{ML}$

Level: (low/med) LOW

\% Moisture: not dec.

GC Column: $\mathrm{DB}-62475 \mathrm{M} \times 2.55 \mathrm{UM}$ ID: 0.45 (mm)

Soil Extract Volume: (uL)
Lab Sample ID: 99-0649

Lab File ID: 99021217

Date Received:

Date Analyzed: 02/13/99

Dilution Factor: 1.0

Soil Aliquot Volume:

(UI)

Number TICs found: 6

CONCENTRATION UNITS:

(ug/L or $\mathrm{ug} / \mathrm{Kg}$ ) ug/L

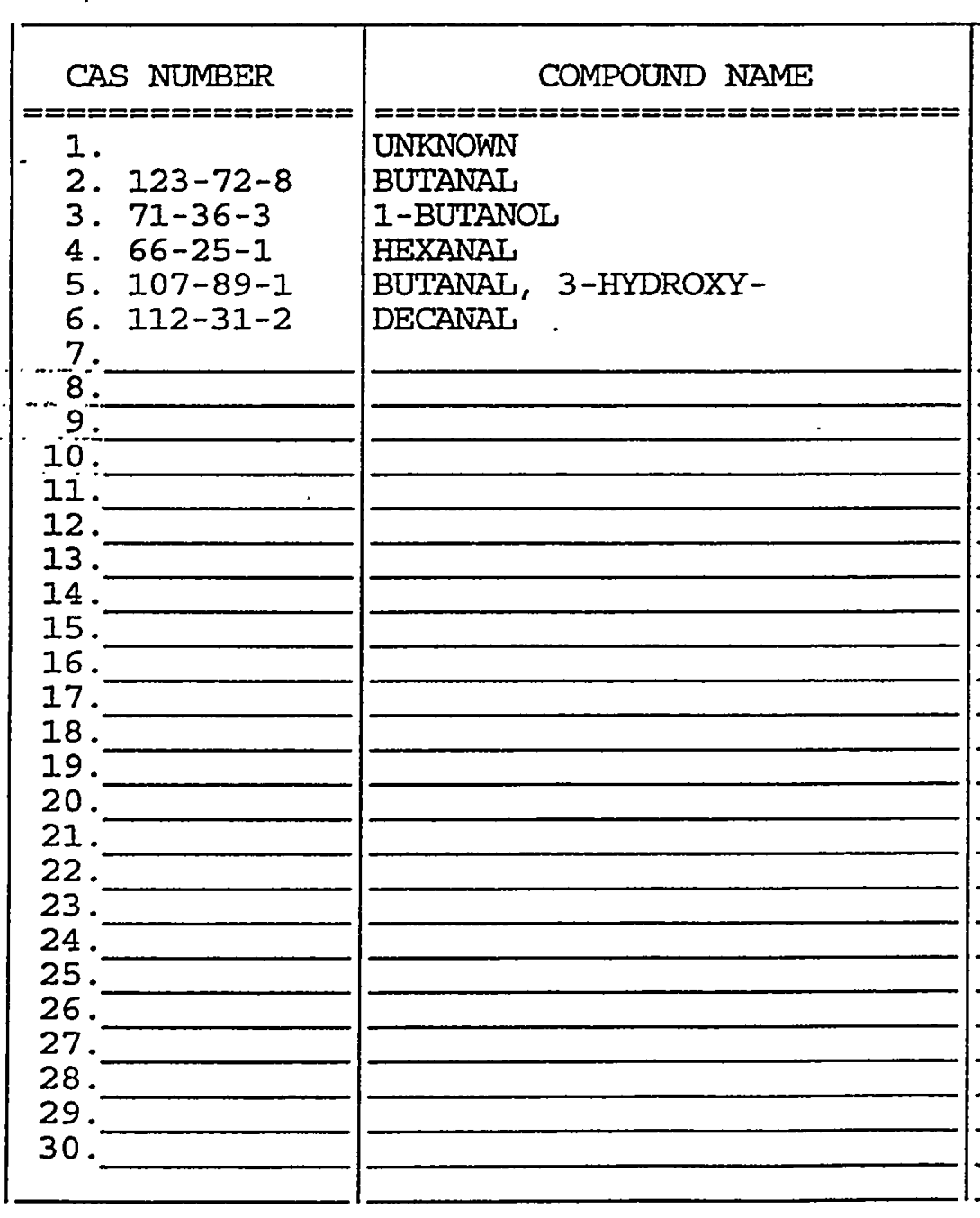

FORM I VOA-TIC

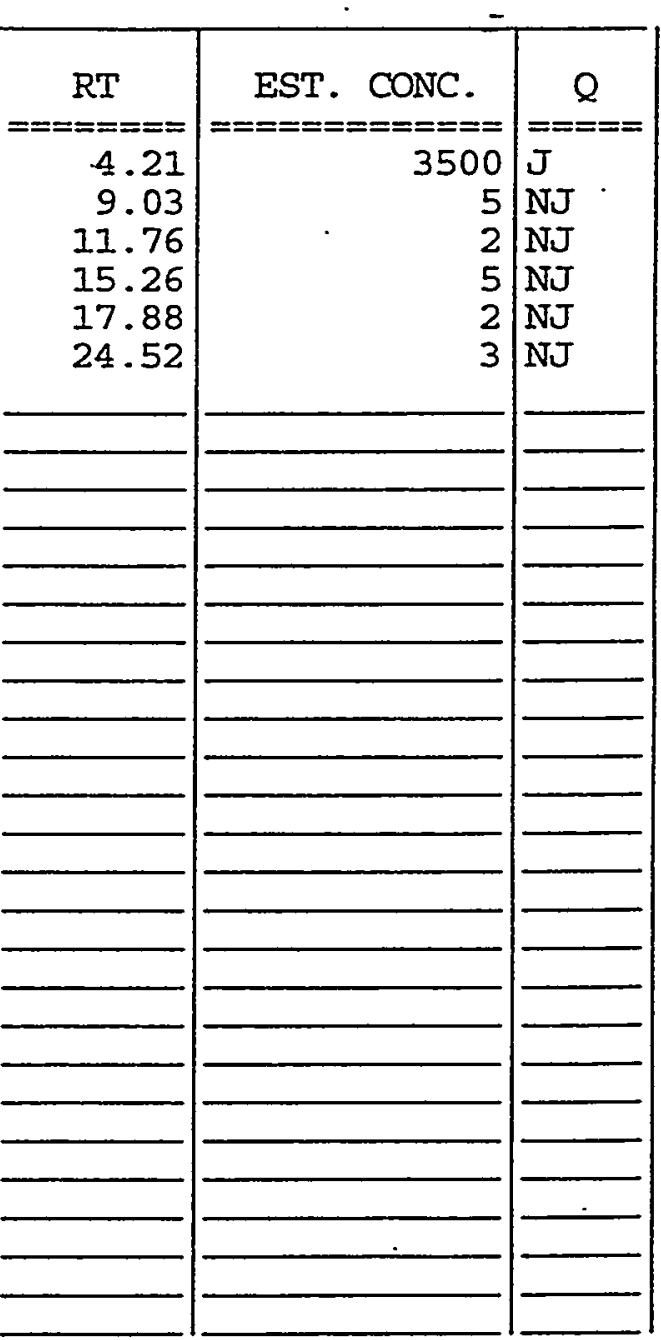

OLM03. 0 
Lab Name: PNNL

Contract : BNFL

Lab Code: PNNL
SAS NO. :
EPA SAMPLE NO.
Matrix: (soil/water) SUPERNATANT

Sample wt/vol:

$5.000(\mathrm{~g} / \mathrm{mL}) \mathrm{ML}$

Level: (low/med) IOW

$\%$ Moisture: not dec.

GC Column: $\mathrm{DB}-62475 \mathrm{M} \times 2.55 \mathrm{UM}$ ID: 0.45 (mm)

Soil Extract Volume: (uI)
AN-I07DUP

SDG No.: $99021202^{\circ}$

Lab Sample ID: 99-0649D

Lab File ID: $\quad 99021218$

Date Received:

Date Analyzed: 02/13/99

Dilution Factor: 1.0

Soil Aliquot Volume: (uI) CONCENTRATION UNITS:

Number TICs found: 3 (ug/L or ug/Kg) ug/L

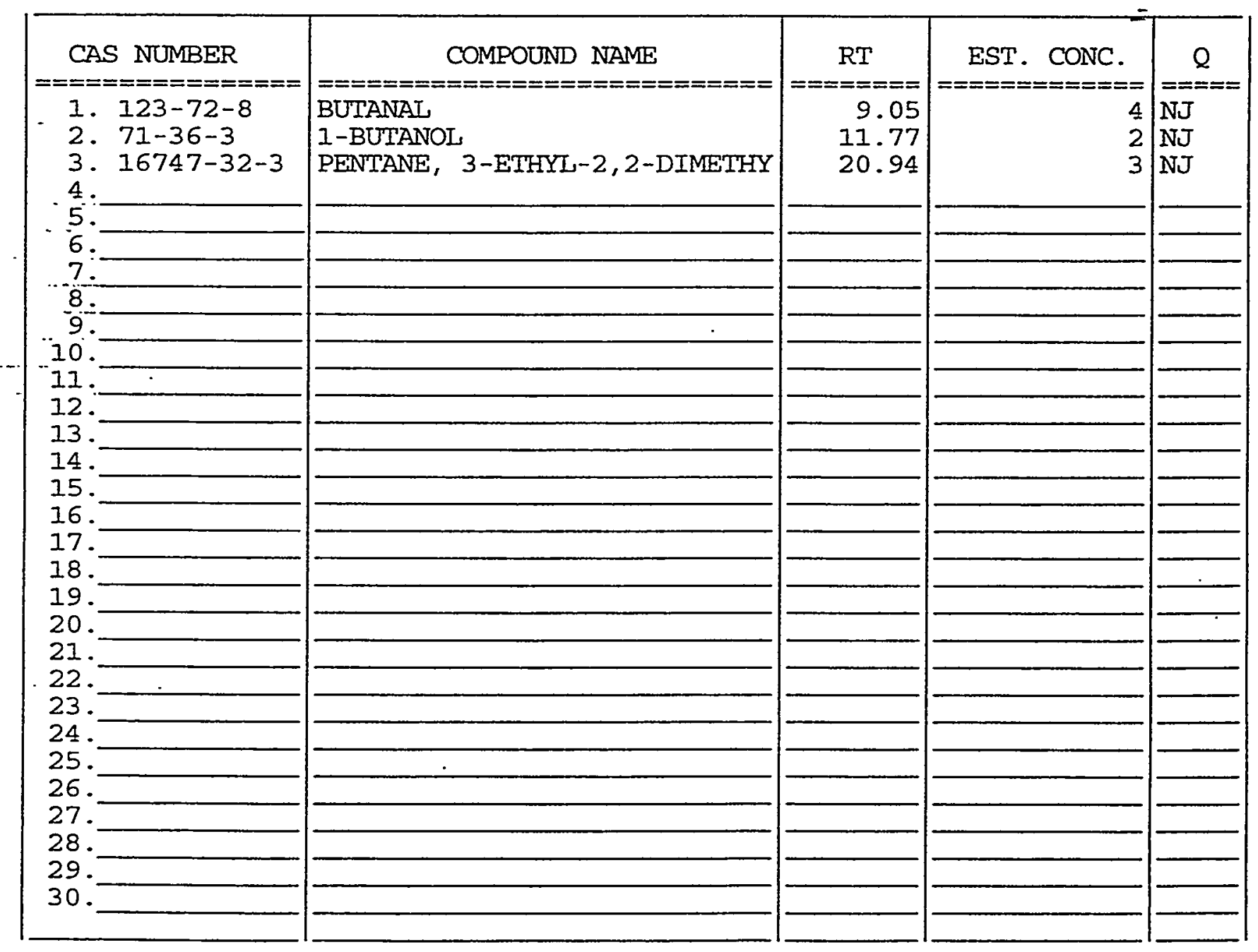


Lab Name: PNNL

Lab Code: PNNL

Case No.:

Matrix: (soil/water) SUPERNATANT

Sample wt/vol: $\quad 5.000(\mathrm{~g} / \mathrm{mL}) \mathrm{MU}$

Level: (low/med) IOW

$\%$ Moisture: not dec.

GC Column: $\mathrm{DB}-62475 \mathrm{M} \times 2.55 \mathrm{UM}$ ID: 0.45 (mm)

Soil Extract Volume:

(UU)

Number TICs found: 0
Contract: BNFL

SAS NO. :
EPA SAMPLE NO.

METHOD BLANK

SDG No.: 99021202

Lab Sample ID: VBLKO2

Lab File ID: 99021214

Date Received:

Date Analyzed: 02/12/99

Dilution Factor: 1.0

Soil Aliquot Volume: (uL)

CONCENTRATION UNITS :

(ug/L or $\mathrm{ug} / \mathrm{Kg}$ ) ug/L
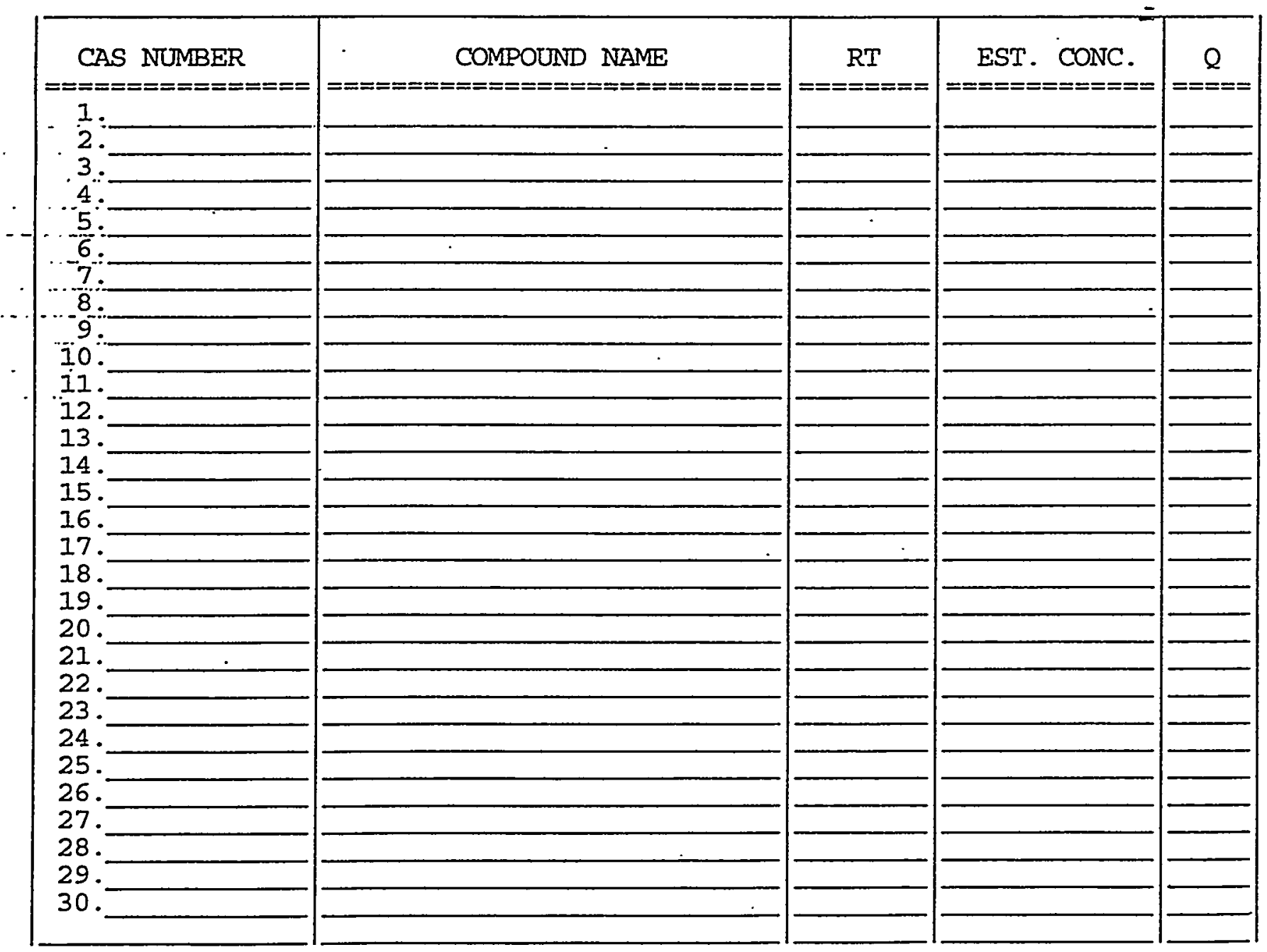
Lab Name: PNNL

contract: BNFL

Lab Code: PNNL

Case No.:

Matrix: (soil/water) SUPERNATANT

Sample wt/vol: $\quad 5.000(\mathrm{~g} / \mathrm{mL}) \mathrm{ML}$

Level: (low/med) IOW

$\%$ Moisture: not dec.

GC Column: DB-624 75M X 2.55UM ID: 0.45 (mm)

Soil Extract Volume:

(uL)
EPA: SAMPLE NO.

AN-107DIL

SAS NO.:

SDG No.: 99021202

Lab Sample ID: 99-0649DII

Lab File ID: 99021215

Date Received:

Date Analyzed: 02/12/99

Dilution Factor: 1.0

Soil Aliquot Volume: (uL)

Numbex TICs found: 3

CONCENIRATION UNITS :

(ug/L or $\mathrm{ug} / \mathrm{Kg}$ ) ug/I
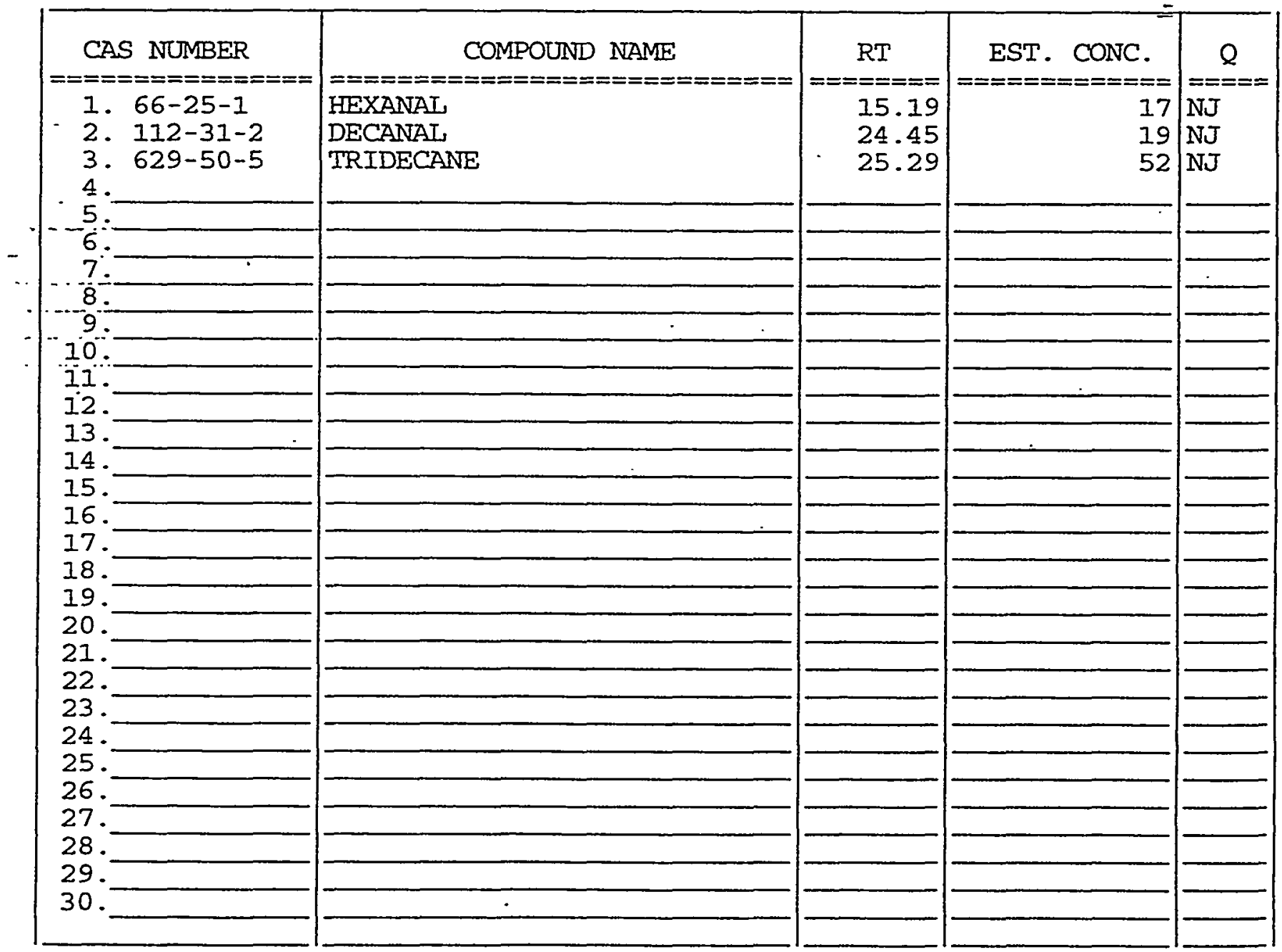
EPA SAMPLE NO.

VOLATILE ORGANICS ANAIYYSIS DATA SHEET

TENTATIVELY IDENTIFIED COMPOUNDS

Lab Name: PNNL

Contract: BNFL

Lab Code: PNNL

Case No.:

SAS NO.:

AN-107DILDUP.

Matrix: (soil/water) SUPERNATANT

Lab Sample ID: 99-0649DIID

Sample wt/vol:

$5.000(\mathrm{~g} / \mathrm{mL}) \mathrm{ML}$

Iab File ID: 99021216

Level: (low/med) LOW

Date Received:

\% Moisture: not dec.

Date Analyzed: 02/13/99

GC Column: $\mathrm{DB}-62475 \mathrm{M} \times 2.55 \mathrm{UM}$ ID: 0.45 (mm)

Dilution Factor: 1.0

Soil Extract Volume: (uL)

Soil Aliquot Volume: (UI)

Number TICs found: 0

CONCENTRATION UNITS :

(ug/L or $\mathrm{ug} / \mathrm{Kg}$ ) ug/L

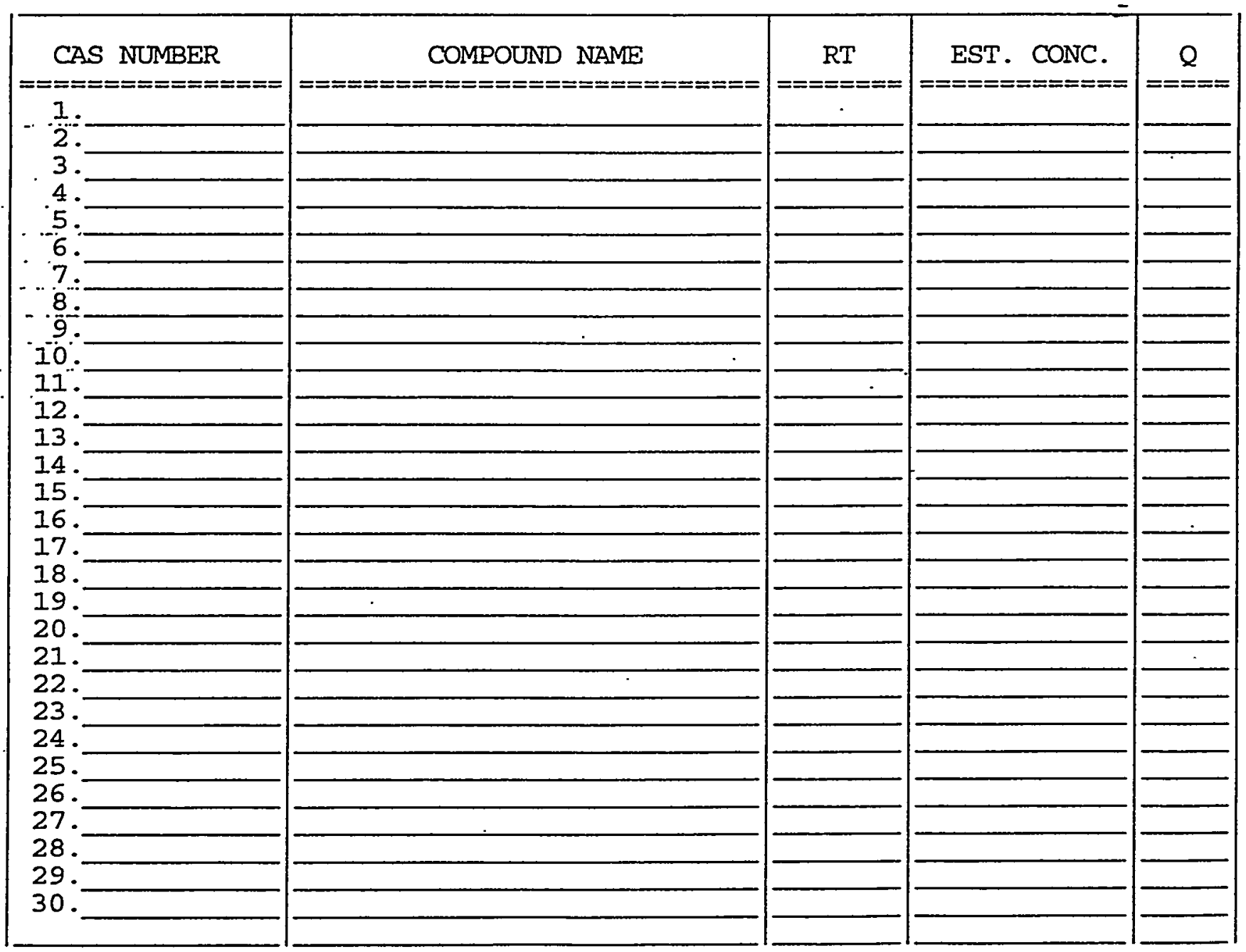


Lab Name: PNNL

Lab Code: PNNL,
Contract: BNFL

Case No.:
SAS NO.:
SDG No.: 99021202

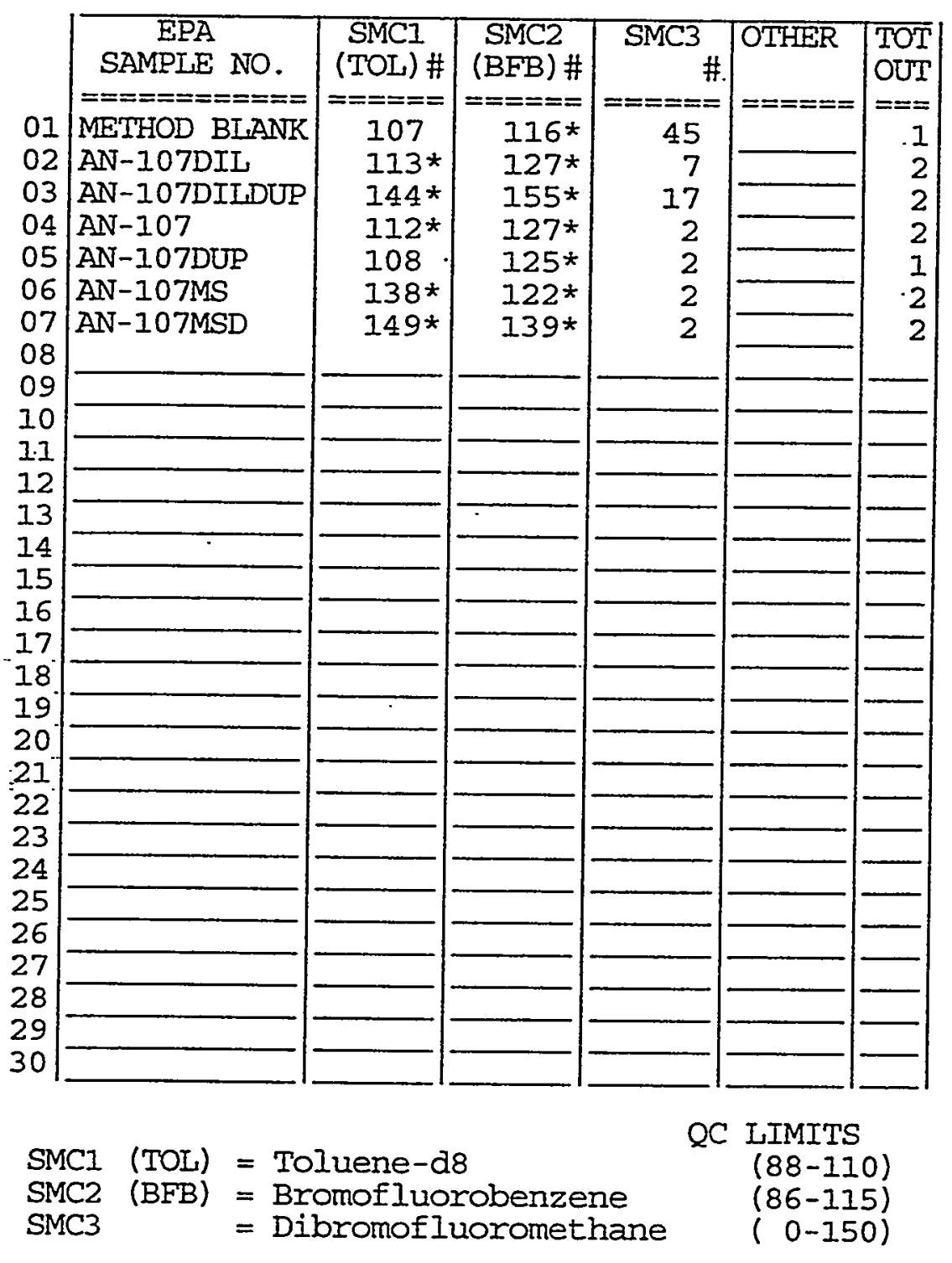

\# Column to be used to flag recovery values

* Values outside of contract required QC limits 
3A

SUPERNATANT VOLATILE MATRIX SPIKE/MATRIX SPIKE DUPLICATE RECOVERY

Lab Name: PNNL

Contract: BNFI

Iab code: PNINL

Case No.:

SAS NO.:

SDG No.: 99021202

Matrix Spike - EPA Sample No.: AN-I07

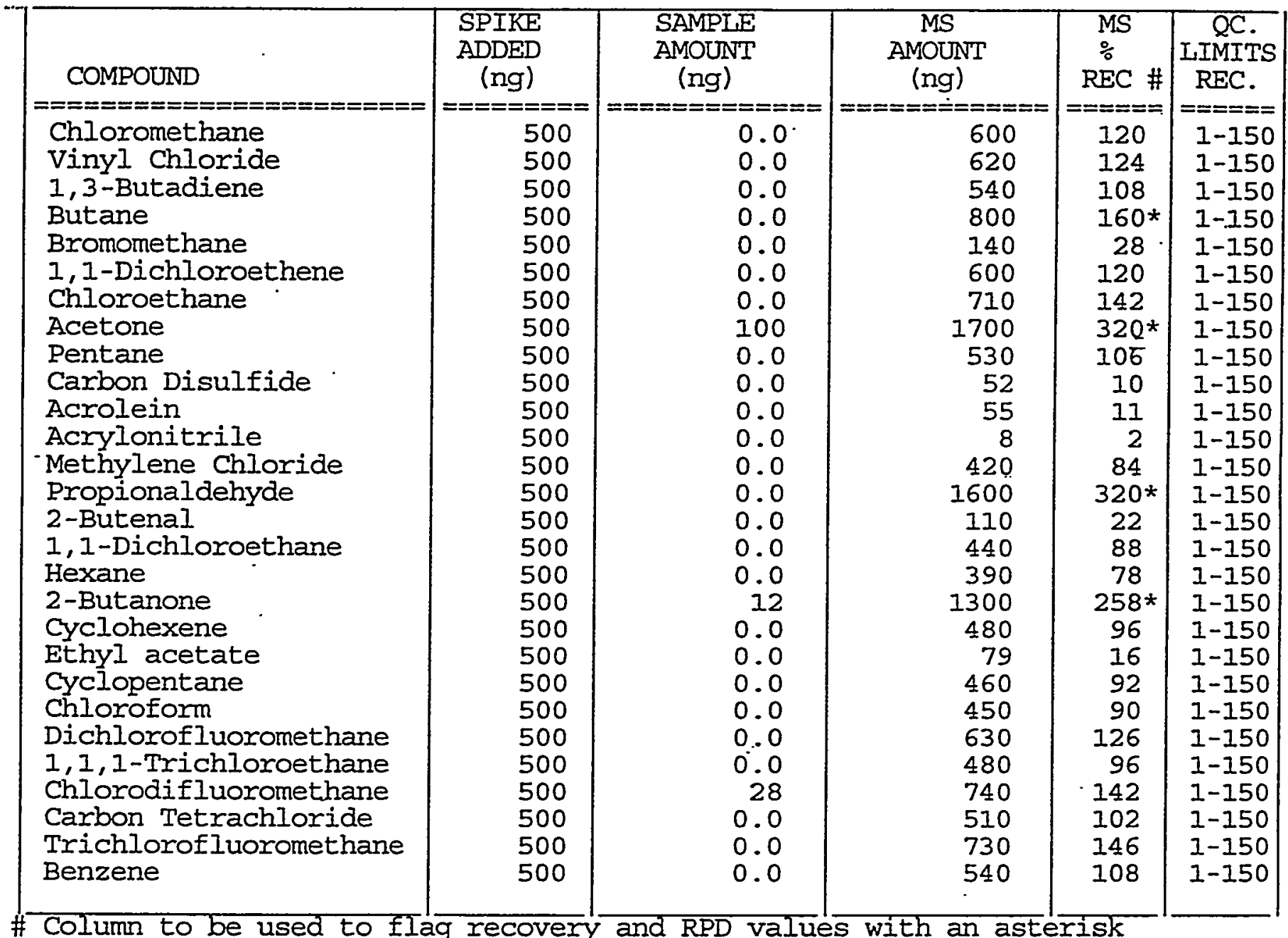

* Values outside of QC limits

COMMENTS : 
$3 A$

SUPERNATANT VOLATILE MATRIX SPIKE/MATRIX SPIKE DUPLICATE RECOVERY

Lab Name: PNNL

Contract: BNFL

Lab Code: PNNL

Case No.:

SAS NO.:

SDG NO.: 99021202

Matrix Spike - EPA Sample No.: AN-107

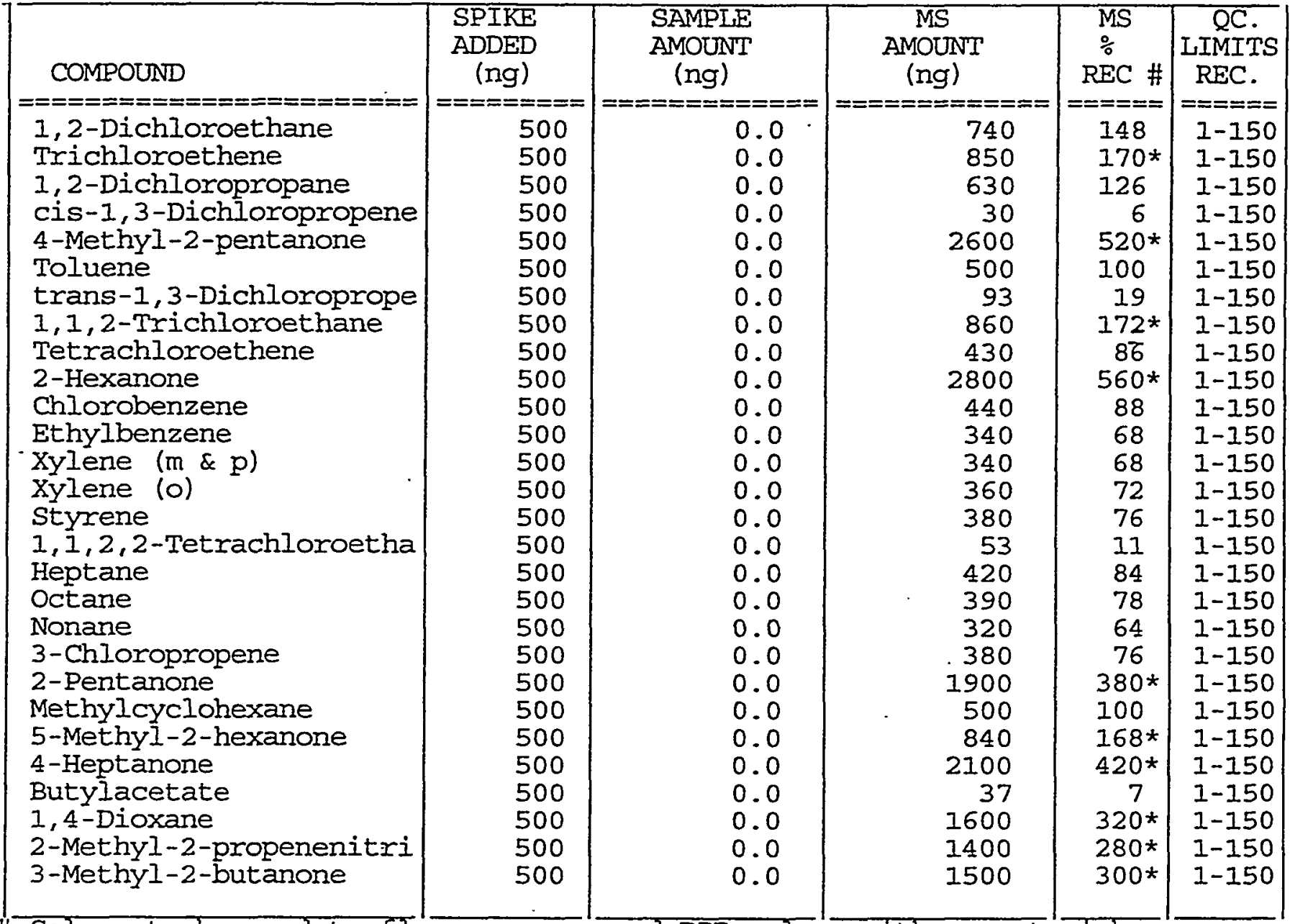

Column to be used to flag recovery and RPD values with an asterisk

* Values outside of QC Iimits

COMMENTS : 
$3 A$

SUPERNATANT VOLATILE MATRIX SPIKE/MATRIX SPIKE DUULICATE RECOVERY

Lab Name: PNNL

Contract: BNFL

Lab Code: PNNL

Case No.:

SAS NO: :

SDG No.: 99021202

Matrix Spike - EPA Sample No. : AN-107

\begin{tabular}{|c|c|c|c|c|c|}
\hline 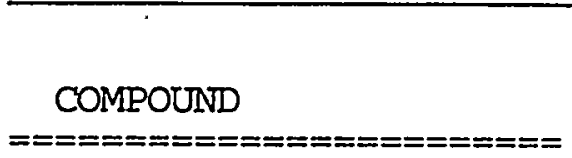 & $\begin{array}{l}\text { SPIKE } \\
\text { ADDED } \\
\text { (ng) } \\
========\end{array}$ & $\begin{array}{c}\text { SAMPLE } \\
\text { AMOUNT } \\
(\mathrm{ng}) \\
============\end{array}$ & $\begin{array}{c}\text { MS } \\
\text { AMOUNT } \\
(\mathrm{ng}) \\
=============\end{array}$ & $\begin{array}{c}\text { MS } \\
\frac{\circ}{0} \\
\text { REC \# } \\
=====\end{array}$ & $\begin{array}{l}\text { QC. } \\
\text { IIMITS } \\
\text { REC. } \\
======\end{array}$ \\
\hline $\begin{array}{l}\text { Propyl nitrate } \\
\text { 3-Pentanone } \\
1,2,2-C 13-1,1,2-\text { F3ethan } \\
1,2-C 12-1,1,2,2-F 4 \text { ethan } \\
\text {-Dichlorodifluoromethane } \\
\text { Acetonitrile } \\
\text { Cyclohexane. } \\
\text { 1,2-Dibromoethane } \\
\text { 3-Heptanone } \\
\text { 2-Heptanone } \\
\text {.Tetrahydrofuran }\end{array}$ & $\begin{array}{l}500 \\
500 \\
500 \\
500 \\
500 \\
500 \\
500 \\
500 \\
500 \\
500 \\
500\end{array}$ & $\begin{array}{r}0.0 \\
0.0 \\
0.0 \\
0.0 \\
0.0 \\
71 \\
0.0 \\
0.0 \\
5 \\
0.0 \\
24\end{array}$ & $\begin{array}{r}750 \\
1900 \\
580 \\
700 \\
580 \\
600 \\
580 \\
-1100 \\
2300 \\
2600 \\
1300\end{array}$ & $\begin{array}{l}150 \\
380 * \\
116 \\
140 \\
116 \\
106 \\
116 \\
220 * \\
459 * \\
520 * \\
255 *\end{array}$ & $\begin{array}{l}1-150 \\
1-150 \\
1-150 \\
1-150 \\
1-150 \\
1-150 \\
1-150 \\
1-150 \\
1-150 \\
1-150 \\
1-150\end{array}$ \\
\hline
\end{tabular}

\# Column to be used to flag recovery and RPD values with an asterisk

* Values outside of QC limits

COMMENTS: 
$3 A$

SUPERNATANT VOLATILE MATRIX SPIKE/MATRIX SPIKE DUPLICATE .RECOVERY

Lab Name: PNNL

Contract: BNFL

Iab Code: PNNL

Case No.:

SAS NO.:

SDG No.: 99021202

Matrix Spike - EPA Sample No.: AN-107.

\begin{tabular}{|c|c|c|c|c|c|c|}
\hline & $\begin{array}{l}\text { SPIKE } \\
\text { ADDED }\end{array}$ & $\begin{array}{c}\text { MSD } \\
\text { AMOUNT }\end{array}$ & $\begin{array}{c}\text { MSD } \\
\%\end{array}$ & $\%$ & \multicolumn{2}{|c|}{ OC IIMITS } \\
\hline COMPOUND & (ng) & (ng) & REC \# & RPD \# & RPD & REC. \\
\hline 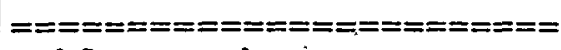 & $=======$ & $=============$ & $=====$ & $=====$ & $=====$ & $======$ \\
\hline Chloromethane & 500 & 570 & 114 & 5 & 40 & $1-150$ \\
\hline Vinyl Chloride & 500 & 610 & 122 & 2 & 40 & $1-150$ \\
\hline 1,3-Butadiene & 500 & 510 & 102 & 6 & 40 & $1-150$ \\
\hline Butane & 500 & 800 & $160 *$ & 0 & 40 & $1-150$ \\
\hline Bromomethane & 500 & 120 & 24 & 15 & 40 & $I-150$ \\
\hline 1,1-Dichloroethene & 500 & 660 & 132 & 10 & 40 & $1-150$ \\
\hline Chloroethane & 500 & 560 & 112 & 24 & 40 & $1-150$ \\
\hline Acetone & 500 & 1800 & $340 *$ & 6 & 40 & $1-150$ \\
\hline Pentane & 500 & 510 & 102 & 4 & 40 & $1-150$ \\
\hline Carbon Disulfide & 500 & 62 & 12 & 18 & 40 & $1-150$ \\
\hline Acrolein & 500 & 66 & 13 & 17 & 40 & $1-150$ \\
\hline Acrylonitrile & 500 & 100 & 20 & $164 *$ & 40 & $1-150$ \\
\hline Methylene Chloride & 500 & 570 & 114 & 30 & 40 & $1-150$ \\
\hline Propionaldehyde & 500 & 1800 & $360 *$ & 12 & 40 & $1-150$ \\
\hline 2-Butenal & 500 & 100 & 20 & 10 & 40 & $1-150$ \\
\hline 1, 1-Dichloroethane & 500 & 560 & 112 & 24 & 40 & $1-150$ \\
\hline Hexane & 500 & 470 & 94 & 19 & 40 & $1-150$ \\
\hline 2-Butanone & 500 & 1500 & $298 *$ & 14 & 40 & $1-150$ \\
\hline Cyclohexene & 500 & 530 & 106 & 10 & 40 & $1-150$ \\
\hline Ethyl acetate & 500 & 93 & 19 & 17 & 40 & $1-150$ \\
\hline Cyclopentane & 500 & 520 & 104 & 12 & 40 & $1-150$ \\
\hline Chloroform & 500 & 540 & 108 & 18 & 40 & $1-150$ \\
\hline Dichlorofluoromethane & 500 & 610 & 122 & 3 & 40 & $1-150$ \\
\hline $1,1,1$-Trichloroethane & 500 & 600 & 120 & 22 & 40 & $1-150$ \\
\hline Chlorodifluoromethane & 500 & 770 & 148 & 4 & 40 & $1-150$ \\
\hline Carbon Tetrachloride. & 500 & 550 & 110 & 8 & 40 & $1-150$ \\
\hline Trichlorofluoromethane & 500 & 560 & 112 & 26 & 40 & $1-150$ \\
\hline Benzene & 500 & 570 & 114 & 5 & 40 & $1-150$ \\
\hline
\end{tabular}

\# Column to be used to flag recovery and RPD values with an asterisk

* Values outside of QC Iimits

COMMENTS : 
Lab Name: PNNL

Lab Code: PNNL
Contract : BNFL

SAS NO.:
SDG No.: 99021202

Matrix Spike - EPA Sample No.: AN-107

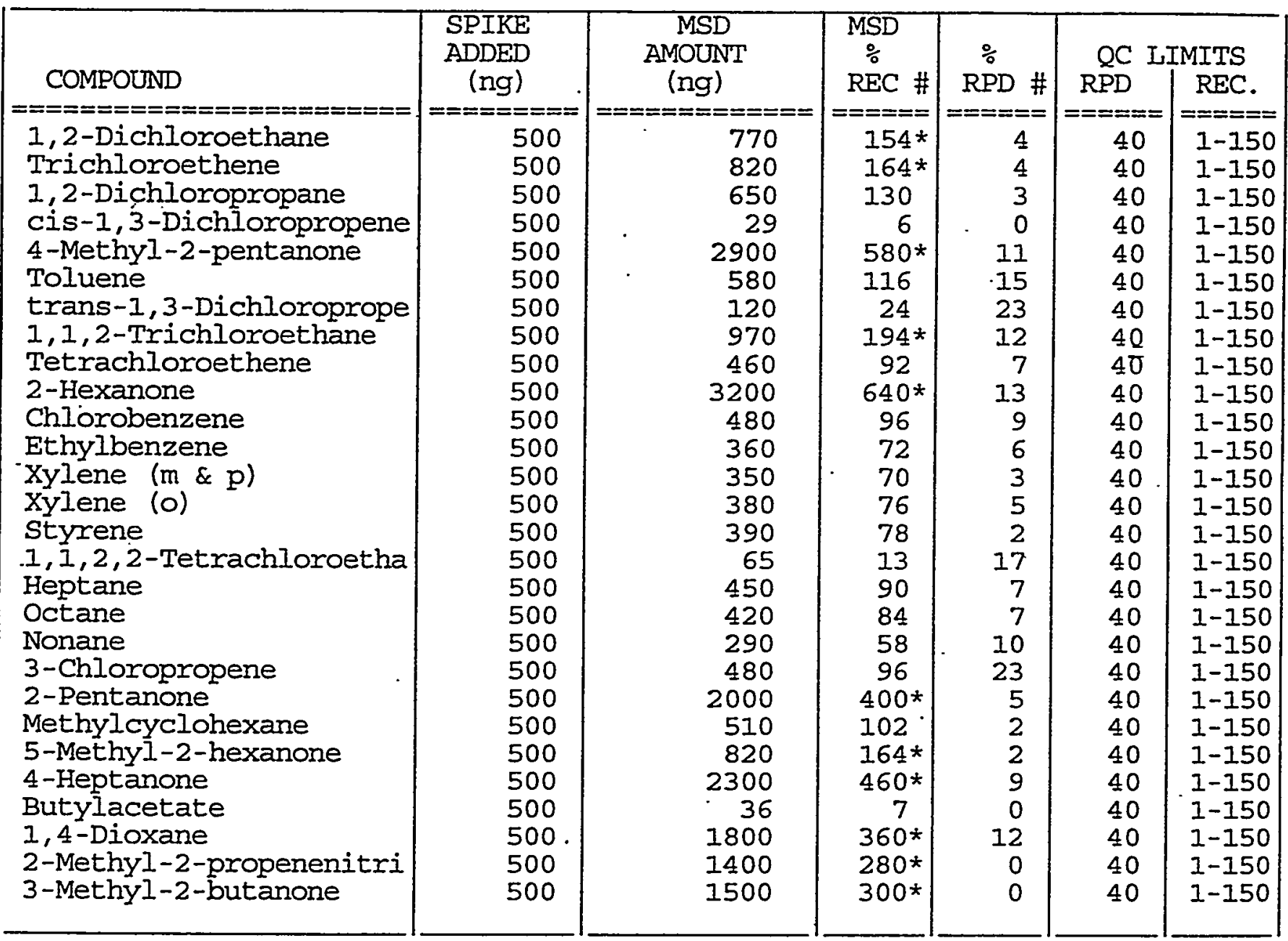

\# Column to be used to flag recovery and RPD values with an asterisk

* Values outside of QC limits

COMIMENTS: 
SUPERNATANT VOLATILE MATRIX SPIKE/MATRIX SPIKE DUPLICATE RECOVERY

Lab Name: PNNL

Lab Code: PNNI

Case No. :

Matrix Spike - EPA Sample No.: AN-107
Contract: BNFL

SAS NO: :
SDG No.: 99021202

\begin{tabular}{|c|c|c|c|c|c|c|}
\hline & $\begin{array}{l}\text { SPIKE } \\
\text { ADDED }\end{array}$ & $\begin{array}{c}\text { MSD } \\
\text { AMOUNT }\end{array}$ & $\begin{array}{c}\text { MSD } \\
\%\end{array}$ & $\%$ & \multicolumn{2}{|c|}{ QC LIMITS } \\
\hline COMPOUND & (ng) & (ng) & REC \# & RPD \# & RPD & REC. \\
\hline 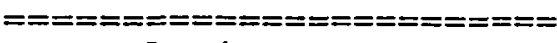 & 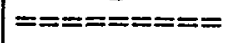 & 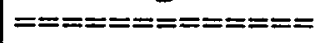 & $=====$ & $======$ & $====\equiv=$ & $======$ \\
\hline $\begin{array}{l}\text { Propyl nitrate } \\
\text { 3-Pentanone }\end{array}$ & $\begin{array}{l}500 \\
500\end{array}$ & $\begin{array}{r}780 \\
2000\end{array}$ & $\begin{array}{l}156 * \\
400 *\end{array}$ & $\begin{array}{l}4 \\
5\end{array}$ & $\begin{array}{l}4 \overline{0} \\
40\end{array}$ & $\begin{array}{l}1-150 \\
1-150\end{array}$ \\
\hline$I, 2,2-C 13-I, I, 2-F 3 e t h a n$ & 500 & 660 & 132 & 13 & 40 & $1-150$ \\
\hline $1,2-C 12-1,1,2,2-F 4$ ethan & 500 & 690 & 138 & 1 & 40 & $1-150$ \\
\hline Dichlorodifluoromethane & 500 & 490 & 98 & 17 & 40 & $1-150$ \\
\hline Acetonitrile & 500 & 700 & 126 & 17 & 40 & $1-150$ \\
\hline Cyclohexane & 500 & 680 & 136 & 16 & 40 & $1-150$ \\
\hline 1,2-Dibromoethane & 500 & 1200 & $240 *$ & .9 & 40 & $1-150$ \\
\hline 3-Heptanone & 500 & .2600 & $519 *$ & 12 & 40 & $1-150$ \\
\hline 2-Heptanone & 500 & 2900 & $580 *$ & II & 40 & $1-150$ \\
\hline Tetrahydrofuran & 500 & 1600 & $315 *$ & 21 & 40 & $1-150$ \\
\hline
\end{tabular}

\# Column to be used to flag recovery and RPD values with an asterisk

* Values outside of QC limits

RPD: 1 out of 67 outside limits

Spike Recovery: 40 out of 134 outside limits

COMMENTS: 
Lab Name: PNNL

Lab Code: PNNL

Case No.:

Lab File ID: 99021214

Date Analyzed: 02/12/99

GC Column: DB-624 75M X 2.55UMID: 0.45 (mm)

Instrument ID: HPI
EPA SAMPIE NO.

METHOD BLANK
Contract : BNFL

SAS NO.:

SDG No.: 99021202

Lab Sample ID: VBLío2

Time Analyzed: 2311

Heated Purge: ( $\mathrm{Y} / \mathrm{N}) \mathrm{N}$

THIS.METHOD..BLANK .APPLIES TO THE FOILOWING SAMPIES, MS and MSD:

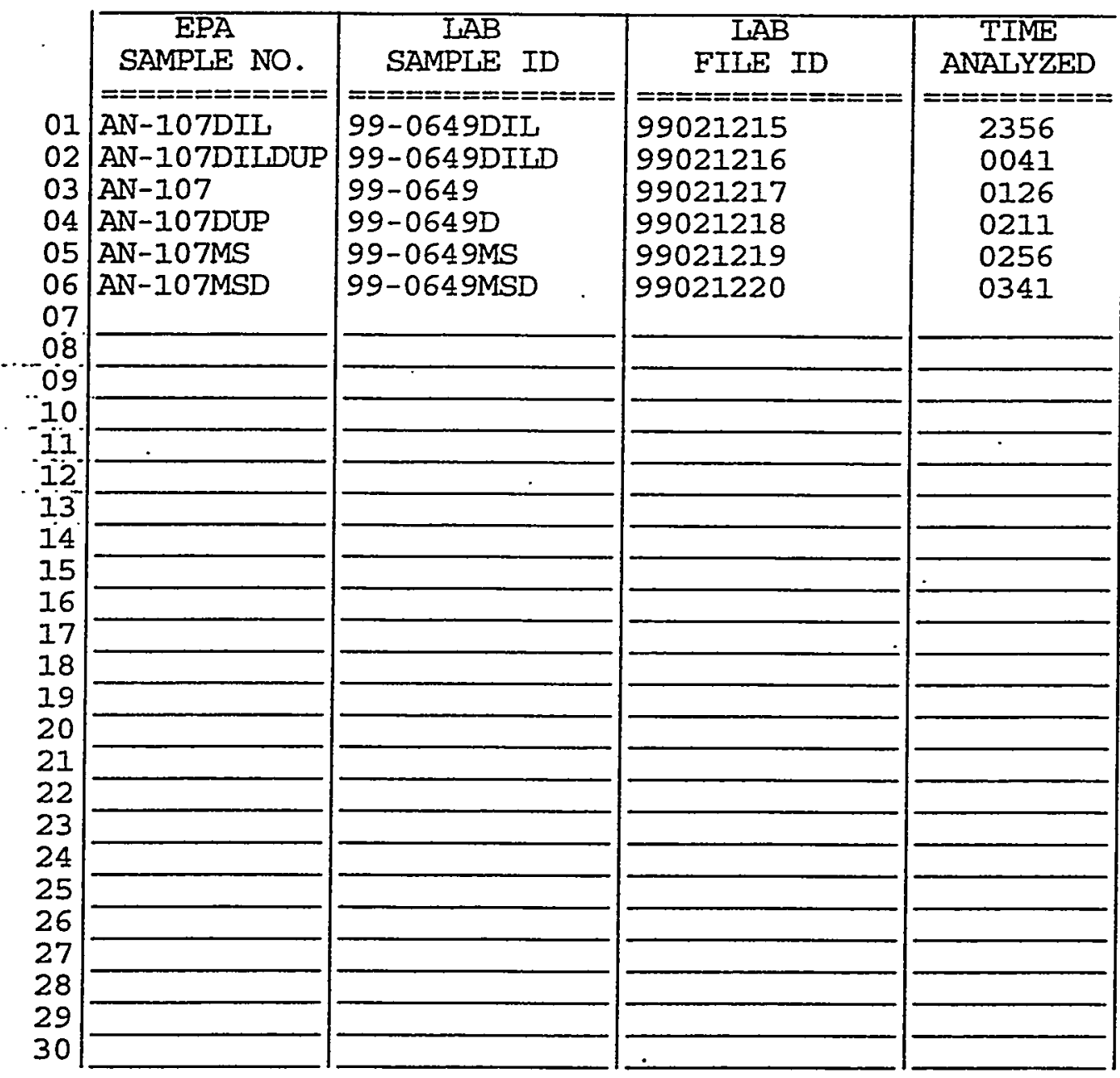

COMMENTS:

page $I$ of $I$ 
Lab Name: PNNL

Lab Code: PNNL

Lab File ID: 99021211

Instrument ID: HP1

GC Column:
Contract: BNFL

SAS NO.:
SDG NO.: 99021202

BFB Injection Time: 2056

Heated Purge: $(\mathrm{Y} / \mathrm{N}) \mathrm{N}$

\begin{tabular}{|c|c|c|}
\hline $\begin{array}{c}\mathrm{m} / \mathrm{e} \\
=====\end{array}$ & 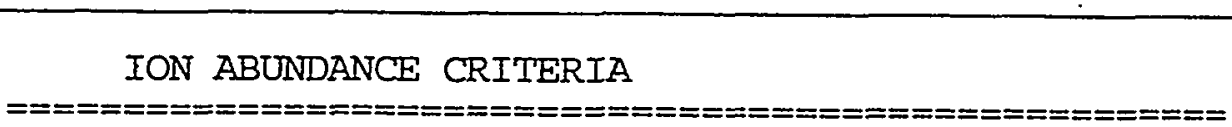 & $\begin{array}{c}\text { \% RELATIVE } \\
\text { ABUNDANCE } \\
=============\end{array}$ \\
\hline 50 & $8.0-40.0 \%$ of mass 95 & 39.2 \\
\hline 75 & $30.0-66.0 \%$ of mass $9 \overline{5}$ & 61.1 \\
\hline 95 & Base Peak, $100 \%$ relative abundance & 100.0 \\
\hline 96 & $5.0-9.0 \%$ of mass 95 & $6.8 \longrightarrow$ \\
\hline 173 & Iess than $2.0 \%$ of mass 174 & $0.0 T-0.0) I$ \\
\hline 174 & $50.0 .-120.0 \%$ of mass 95 & $73.3-$ \\
\hline 175 & $4.0-9.0 \%$ of mass 174 & 5.9 \\
\hline 176 & 93.0 - $101.0 \%$ of mass $\overline{174}$ & $71.3(97.4) 1$ \\
\hline 177 & $5.0-9.0 \%$ of mass 176 & $4.7(6.6) 2$ \\
\hline
\end{tabular}

'THIS CHECK APPLIES.TO THE FOLIOWING SAMPLES, MS, MSD, BLANKS, AND STANDARDS:

\begin{tabular}{|c|c|c|c|c|c|}
\hline & $\begin{array}{c}\text { EPA } \\
\text { SAMPLE NO. } \\
===========\end{array}$ & $\begin{array}{c}\text { IAAB } \\
\text { SAMPLE. ID } \\
============\end{array}$ & $\begin{array}{c}I \overline{I A B} \\
\text { FILE ID } \\
=============\end{array}$ & $\begin{array}{c}\text { DATE } \\
\text { ANALYZED } \\
==========\end{array}$ & $\begin{array}{c}\text { TIME } \\
\text { ANAIYYZED } \\
=========\end{array}$ \\
\hline 01 & CONT. CAI. C & VSTD050 & 99021212 & $02 / 12 / 99$ & 2141 \\
\hline 02 & METHOD BLANK & VBLKO2 & 99021214 & $02 / 12 / 99$ & 2311 \\
\hline 03 & AN-107DIL & 99-0649DIL & 99021215 & $02 / 12 / 99$ & 2356 \\
\hline 04 & AN-107DILDUP & 99-0649DILD & 99021216 & $02 / 13 / 99$ & 0041 \\
\hline 05 & $A N-107$ & $99-0649$ & 99021217 & $02 / 13 / 99$ & 0126 \\
\hline 06 & AN-107DUP & 99-0649D & 99021218 & $02 / 13 / 99$ & 0211 \\
\hline 07 & AN-I07MS & 99-0649MS & 99021219 & $02 / 13 / 99$ & 0256 \\
\hline 08 & AN-I07MSD & 99-0649MSD & 99021220 & $02 / 13 / 99$ & 0341 \\
\hline 10 & & & & & \\
\hline 11 & & & & & \\
\hline 12 & & & & & \\
\hline 13 & & & & & \\
\hline 14 & & & & & \\
\hline 16 & & & & & \\
\hline 17 & & & & & \\
\hline 18 & & & & & \\
\hline & & & & & \\
\hline 27 & & & & & \\
\hline 22 & & & & & \\
\hline & & & & & \\
\hline
\end{tabular}

page 1 of 1 
VOLATILE CONTINUING CALIBRATION CHECK

Lab Name: PNNL

Contract: BNFL

Lab Code: PNNL

Case No.:

SAS NO.:

SDG Nó. : 99021202

Instrument ID: HPI

Calibration Date: 02/12/99 Time: 2141

Lab File ID: 99021212

Init. Calib. Date (s) : 02/11/99

$02 / 11 / 99$

Heated Purge: $(Y / N) N$

Init. Calib. Times: 1249

1549

GC Column: $\mathrm{DB}-62475 \mathrm{M} \times 2.55 \mathrm{UM}$ ID: 0.45 (mm)

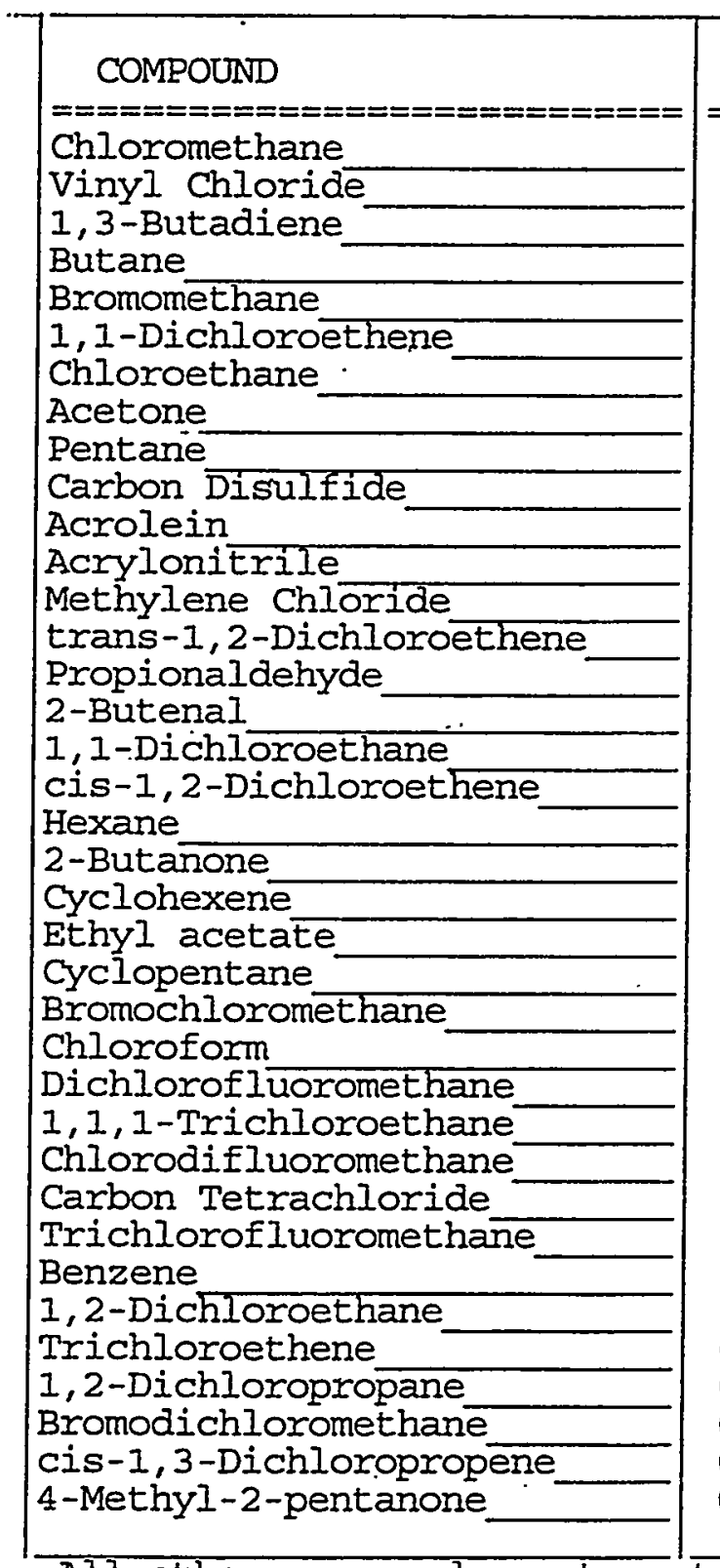

\begin{tabular}{|c|c|c|c|c|}
\hline $\begin{array}{c}\overline{R R F} \\
=====\end{array}$ & $\begin{array}{l}\text { RRF250 } \\
=====\end{array}$ & $\begin{array}{l}\text { MIN } \\
\text { RRF } \\
=====\end{array}$ & $\begin{array}{c}\circ \mathrm{D} \\
=====\end{array}$ & $\begin{array}{l}\text { MAX } \\
\frac{\circ D}{} \\
====\end{array}$ \\
\hline 0.540 & 0.467 & 0.100 & 13.5 & 100 \\
\hline 0.428 & 0.401 & & 6.3 & \\
\hline 1.045 & 0.969 & & 7.3 & \\
\hline 0.148 & 0.147 & & 0.7 & \\
\hline 0.108 & 0.104 & & 3.7 & \\
\hline 0.396 & 0.366 & & 7.6 & \\
\hline 0.119 & 0.114 & & 4.2 & \\
\hline 0.254 & 0.239 & & 5.9 & \\
\hline 0.640 & 0.587 & & $\begin{array}{l}8.3 \\
0.3\end{array}$ & \\
\hline $\begin{array}{l}1.123 \\
0.009\end{array}$ & $\begin{array}{l}1.030 \\
0.006\end{array}$ & 0 & $\begin{array}{r}8.3 \\
33.3\end{array}$ & 100 \\
\hline 0.228 & 0.197 & 0.001 & $\begin{array}{l}33.3 \\
13.6\end{array}$ & \\
\hline 0.432 & 0.420 & & 2.8 & \\
\hline 0.297 & 0.267 & & 10.1 & \\
\hline 0.059 & 0.054 & & 8.5 & \\
\hline 0.057 & 0.050 & & 12.3 & \\
\hline 0.928 & 0.874 & 0.100 & 5.8 & 100 \\
\hline 0.473 & 0.422 & & 10.8 & \\
\hline 0.565 & 0.504 & & 10.8 & \\
\hline 0.331 & 0.338 & & -2.1 & \\
\hline 0.172 & 0.149 & & $\begin{array}{r}13.4 \\
9\end{array}$ & \\
\hline $\begin{array}{l}0.829 \\
1.517\end{array}$ & $\begin{array}{l}0.753 \\
1.368\end{array}$ & & $\begin{array}{l}9.2 \\
9.8\end{array}$ & \\
\hline 0.188 & 0.188 & & 0.0 & \\
\hline 0.849 & 0.774 & & 8.8 & \\
\hline 0.648 & 0.622 & & 4.0 & \\
\hline 0.070 & 0.060 & & 14.3 & \\
\hline 0.343 & 0.308 & & 10.2 & \\
\hline 0.456 & 0.428 & & 6.1 & \\
\hline 0.315 & 0.311 & & 1.3 & \\
\hline 0.729 & 0.694 & & 4.8 & \\
\hline 0.375 & 0.359 & & 4.3 & \\
\hline 0.185 & 0.177 & & 4.3 & \\
\hline 0.227 & 0.205 & & 9.7 & \\
\hline 0.301 & 0.280 & & 7.0 & \\
\hline $\begin{array}{l}0.345 \\
0.447\end{array}$ & $\begin{array}{l}0.319 \\
0.391\end{array}$ & & $\begin{array}{r}7.5 \\
12.5\end{array}$ & \\
\hline & & & & \\
\hline
\end{tabular}

All other compounds must meet a minimum RRF of 0.010 .

page 1 of 3

FORM VII VOA

OLM03.0 
$7 A$

VOLATILE CONTINUING CALIBRATION CHECK

Lab Name: PNNL,

Lab Coide: PNNL

Instrument ID: HP1

Lab File ID: 99021212

Heated Purge: $(Y / N)$ N

Case No.:
Contract: BNFL

SAS NO.:

SDG No.: 99021202
Calibration Date: 02/12/99 Time: 2141

Init. Calib. Date(s) : 02/11/99 02/11/99

Init. Calib. Times: 1249

1549

GC Column: DB-624 75M X $2.55 U M$ ID: 0.45 (mm)

\begin{tabular}{|c|c|c|c|c|c|}
\hline 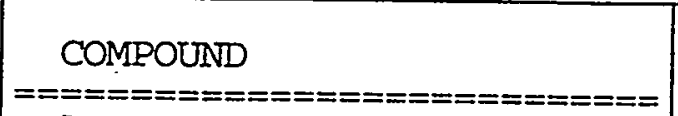 & $\begin{array}{c}\overline{\mathrm{RRF}} \\
======\end{array}$ & $\begin{array}{l}\text { RRF250 } \\
======\end{array}$ & $\begin{array}{c}\text { MIN } \\
\text { RRF } \\
=====\end{array}$ & $\begin{array}{c}\% \mathrm{D} \\
=====\end{array}$ & \begin{tabular}{c|} 
MAX \\
$\because D$ \\
$=====$
\end{tabular} \\
\hline Toluene & 0.580 & 0.546 & & 5.9 & \\
\hline & 0.421 & 0.379 & & 10.0 & \\
\hline $\begin{array}{l}\text { 1, I, } 2 \text {-Trichloroethane } \\
\text { Tetrachloroethene }\end{array}$ & $\begin{array}{l}0.190 \\
0.154\end{array}$ & $\begin{array}{l}0.178 \\
0.155\end{array}$ & & $\begin{array}{r}6.3 \\
-0.6\end{array}$ & \\
\hline 2-Hexanone & 0.303 & 0.272 & & 10.2 & \\
\hline Dibromochloromethane & 0.231 & 0.226 & & 2.2 & \\
\hline Chlorobenzene & 0.533 & 0.527 & 0.300 & 1.1 & 100 \\
\hline Ethylbenzene - & 0.297 & 0.292 & & 1.7 & \\
\hline Xylene (m\& $\&$ ) & 0.651 & 0.705 & & $-8 \cdot 3$ & \\
\hline $\begin{array}{l}\text { Xylene (0) } \\
\text { Styrene }\end{array}$ & $\begin{array}{l}0.332 \\
0.548\end{array}$ & 0.346 & & $-4 \cdot 2$ & \\
\hline $\begin{array}{l}\text { Styrene } \\
\text { Bromoform }\end{array}$ & $\begin{array}{l}0.548 \\
0.131\end{array}$ & $\begin{array}{l}0.583 \\
0.132\end{array}$ & 10.700 & $\begin{array}{l}-6.4 \\
-0.8\end{array}$ & 700 \\
\hline $1,1,2,2$-Tetrachloroethane & $0.35 I$ & 0.349 & 0.300 & $\begin{array}{r}-0.8 \\
0.6\end{array}$ & 100 \\
\hline $\begin{array}{l}\text { 1,3-Dichlorobenzene } \\
\text { 1,4-Dichlorobenzene }\end{array}$ & $\begin{array}{l}0.473 \\
0.499\end{array}$ & $\begin{array}{l}0.508 \\
0.531\end{array}$ & & -7.4 & \\
\hline 1,2-Dichlorobenzene & 0.464 & 0.500 & & $\begin{array}{l}-0.4 \\
-7.8\end{array}$ & \\
\hline Heptane & 0.063 & 0.059 & & 6.3 & \\
\hline Octane & 0.042 & 0.040 & & 4.8 & \\
\hline Nonane & 0.029 & 0.032 & & -10.3 & \\
\hline 3-Chloropropene & 0.638 & 0.593 & & 7.0 & \\
\hline 2-Pentanone & 0.027 & 0.025 & & 7.4 & \\
\hline Methylcyclohexane & 0.304 & 0.298 & & 2.0 & \\
\hline 5-Methyl-2-hexanone & 0.512 & 0.503 & & 1.8 & \\
\hline 4-Heptanone & 0.461 & 0.430 & & 6.7 & \\
\hline Butylacetate & 0.649 & 0.625 & & $\begin{array}{r}3.7 \\
23 \\
2\end{array}$ & \\
\hline 2 -Methyl-2-propenenitrile & 0.003 & $\begin{array}{l}0.002 \\
0.205\end{array}$ & 0.001 & $\begin{array}{r}33.3 \\
5.1\end{array}$ & 100 \\
\hline 3-Methyl-2-butanone & 0.035 & $\begin{array}{l}0.205 \\
0.033\end{array}$ & & 5.7 & \\
\hline Propyl nitrate & 0.300 & 0.268 & & 10.7 & \\
\hline 3-Pentanone & 0.188 & 0.164 & & 12.8 & \\
\hline $1,2,2-\mathrm{C} 13-1,1,2-\mathrm{F} 3$ ethane & 0.341 & 0.325 & & 4.7 & \\
\hline $1,2-\mathrm{Cl} 2-1,1,2,2-\mathrm{F} 4$ ethane & 0.164 & 0.122 & & 25.6 & \\
\hline Dichlorodifluoromethane & 0.561 & 0.508 & & 9.4 & \\
\hline Acetonitrile & 0.090 & 0.096 & & -6.7 & \\
\hline Cyclohexane & 0.671 & 0.610 & & 9.1 & \\
\hline Bromobenzene & 0.249 & 0.274 & & -10.0 & \\
\hline Butylbenzene & 0.240 & 0.248 & & -3.3 & \\
\hline
\end{tabular}

page 2 of 3 
$7 A$

VOLATILE CONTINUING CALIBRATION CHECK

Lab Name: PNNL

Contract: BNFL

Lab Code: PNNL

Case No.:

SAS NO.:

SDG No.: 99021202

Instrument ID: HP1

Calibration Date: 02/12/99 Time: 2141

Lab File ID: 99021212

Init. Calib. Date(s) : 02/11/99 02/11/99

Heated Purge: $(Y / N) N$

Init. Calib. Times: 1249

1549

GC Column: $\mathrm{DB}-62475 \mathrm{M} \times 2.55 \mathrm{UM}$ ID: 0.45 (mm)

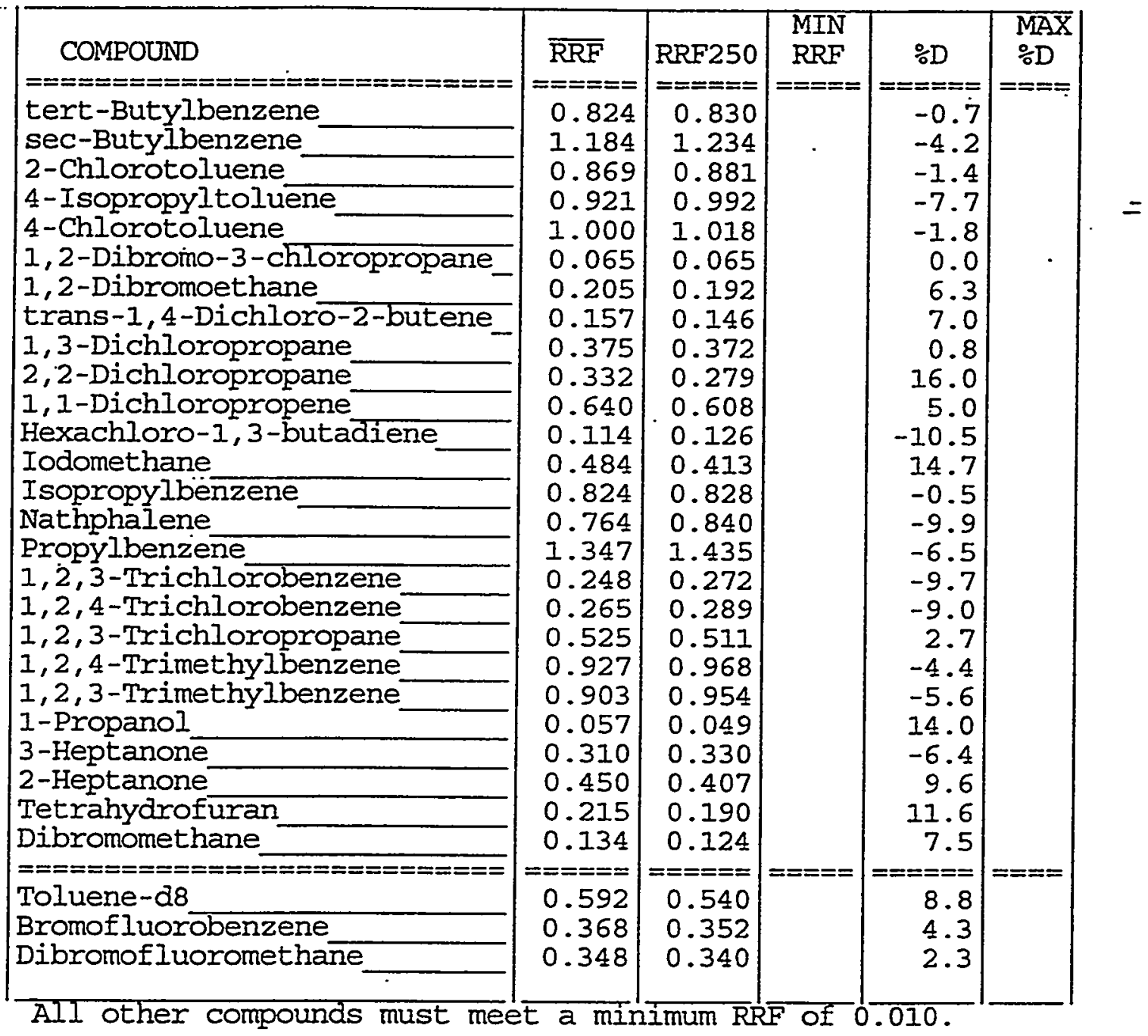

page 3 of 3

FORM VII VOA

OLMO3. 0 
Lab Name: PNNL

Lab code: PNNL

Lab File ID (Standard): 99021212

Instrument ID: HPI

GC Column: $\mathrm{DB}-62475 \mathrm{M} \times 2.55 \mathrm{UM}$ ID: $0.45(\mathrm{~mm}) \quad$ Heated Purge: (Y/N) $\mathrm{N}$
Contract: BNFL

SAS NO.:

SDG No.: 99021202

Date Analyzed: 02/12/99

Time Analyzed: 2141

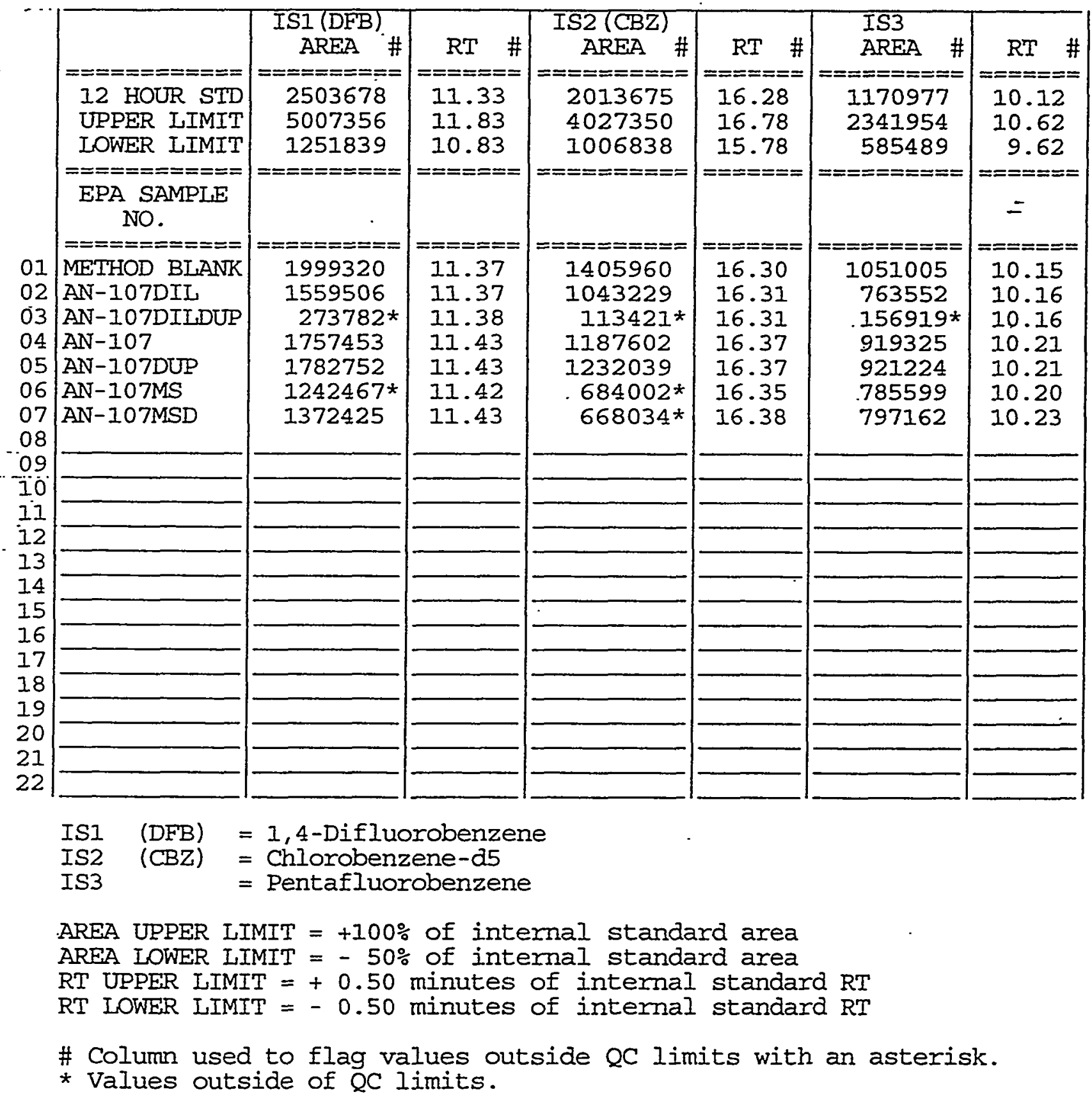

page 1 of 2 
Lab Name: PNNL.

Lab Code: PNNL Case No.:

Lab File ID (Standard): 99021212

Instrument ID: HPI

GC.Column: $\mathrm{DB}-62475 \mathrm{M} \times 2.55 \mathrm{UM}$ ID: 0.45 (mm)
Contract: BNFL

SAS NO. :

SDG No.: 99021202
Date Analyzed: 02/12/99

Time Analyzed: 2141

Heated Purge: $(Y / N) N$

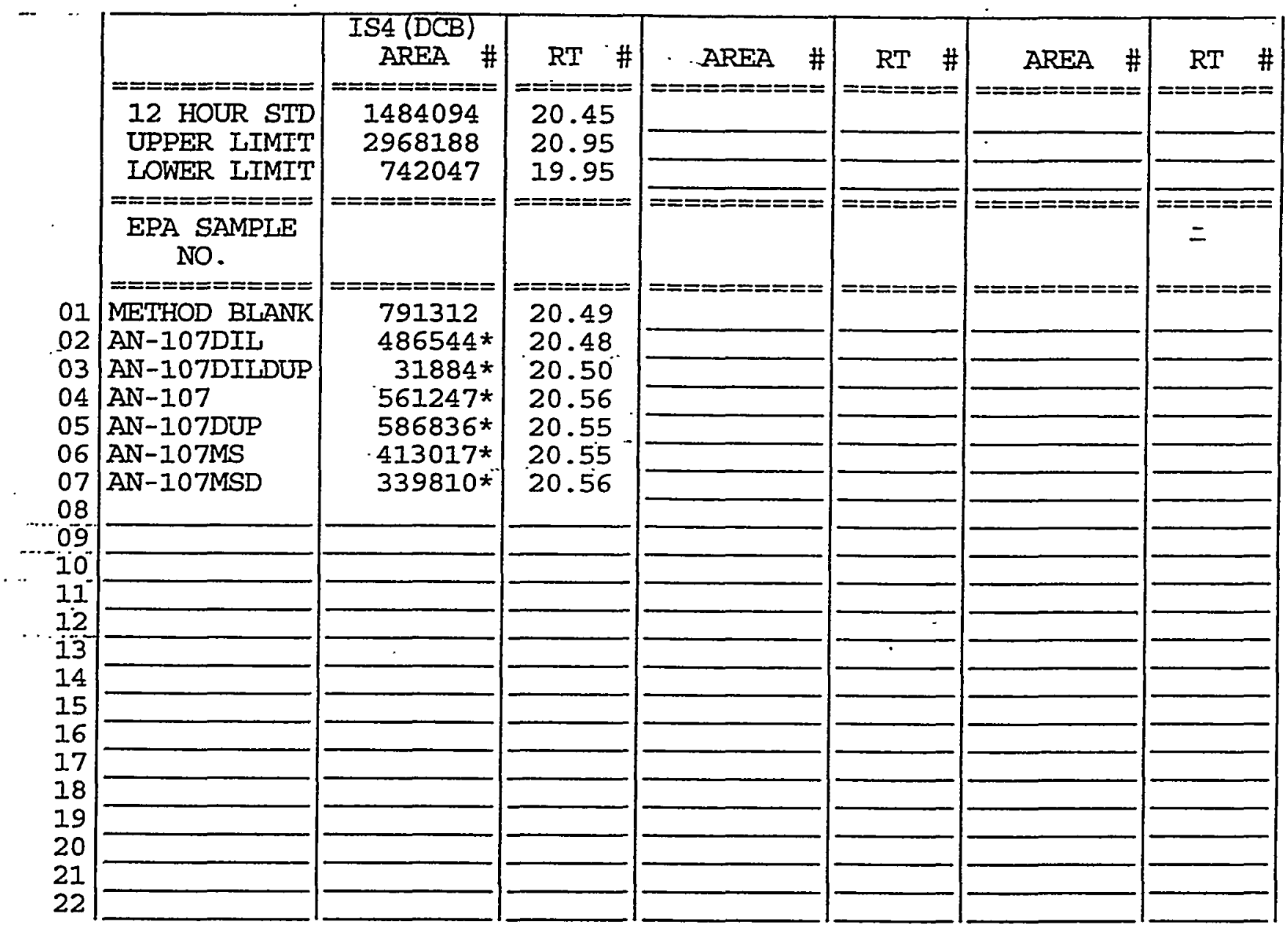

IS4 $(D C B)=1,4-D i c h l o r o b e n z e n e-d 4$

AREA UPPER IIMIT $=+100 \%$ of internal standard area

AREA LOWER LIMIT $=-50 \%$ of internal standard area

RT UPPER LIMIT $=+0.50$ minutes of internal standard RT

RT LOWER IIMIT $=-0.50$ minutes of internal standard RT

\# Column used to flag values outside QC limits with an asterisk.

* Values outside of QC limits. 
Lab Name: PNNL

Lab code: PNNL

Case No.:

Matrix: (soil/water) SOLID

Sample wt/vol:

$1.2(\mathrm{~g} / \mathrm{mU}) \mathrm{G}$

Level: (low/med) LOW

$\%$ Moisture: not dec:

GC Column: $\mathrm{DB}-62475 \mathrm{M} \times 2.55 \mathrm{UM}$ ID: 0.45 (mm)

Soil Extract Volume:

(uL)
Contract: BNFL

SAS NO.:
$-A W-101$

SDG NO.: 99021601
Lab Sample ID: 99-0650

Lab File ID: 99021605

Date Received:

Date Analyzed: 02/16/99

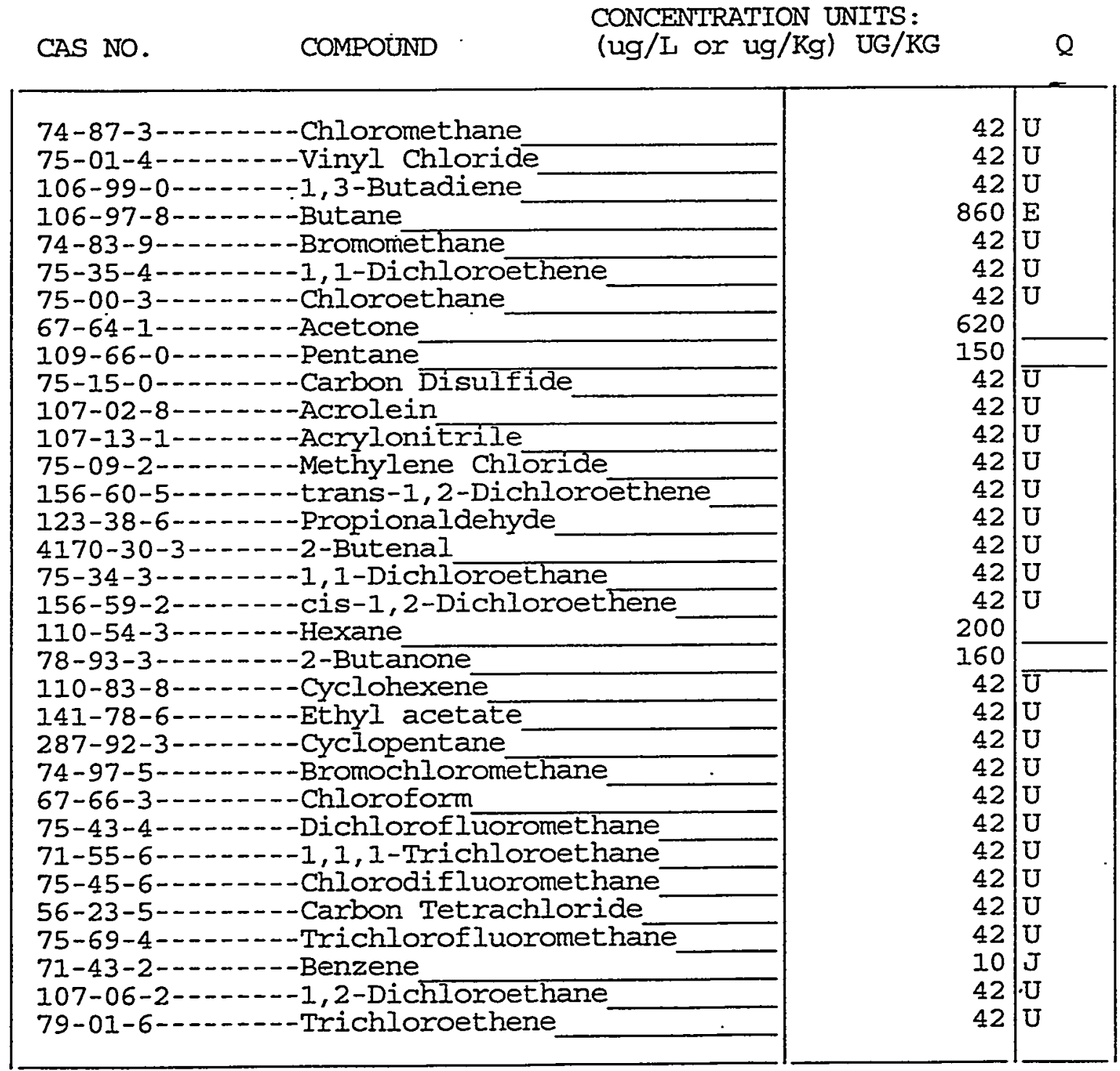

Dilution Factor: 1.0

Soil Aliquot Volume: (UL) CONCENTRATION UNITS: 
Lab Name: PNNL

Lab Code: PNNL

Case No.:

Matrix: (soil/water) SOLID

Sample wt/vol:

$1.2(\mathrm{~g} / \mathrm{mL}) \mathrm{G}$

Level: (low/med) IOW

$\%$ Moisture: not dec.

GC Column: DB-62475M X 2.55UM ID: 0.45 (mm)

Soil Extract Volume: (uL)
Contract: BNFL,

SAS NO.:
EPA SAMPLE NO.

AW-101

SDG No.: 99021601

Lab Sample ID: 99-0650

Lab File ID: 99021605

Date Received:

Date Analyzed: 02/16/99

Dilution Factor: 1.0

Soil Aliquot Volume: (uL)

CONCENTRATION UNITS:

CAS NO.

COMPOUND

(ug/L or $\mathrm{ug} / \mathrm{Kg}$ ) UG/KG

Q

78-87-5--.---1, 2-Dichloropropane

75-27-4 -....--Bromodichloromethane

10061-01-5-..--cis-1,3-Dichloropropene

108-10-1-.---4-Methyl-2-pentanone

108-88-3-..---Toluene

10061-02-6-----trans-1,3-Dichloropropene

79-00-5-...--1, 1,2-Trichloroethane

127-18-4-1.-.-Tetrachloroethene

591-78-6-....-2-Hexanone

124-48-1-...--Dibromochloromethane

108-90-7--.---Chlorobenzene

100-41-4--1----Ethylbenzene

106-42-3-...--Xylene (m \& p)

95-47-6-.----Xylene (o)

100-42-5-------Styrene

75-25-2-1.----Bromoform

79-34-5-.....-1, 1, 2, 2-Tetrachloroethane

541-73-1------1,3-Dichlorobenzene

106-46-7--.--1,4-Dichlorobenzene

95-50-1--.---1, 2-Dichlorobenzene

142-82-5-..----Heptane

$111-65-9-. .-.-$-Octane

111-84-2-...--Nonane

107-05-1--.---3-Chloropropene

107-87-9-..--2-2-Pentanone

108-87-2-....--Methylcyclohexane

110-12-3--.----5-Methyl-2-hexanone

123-19-3-.-.---4-Heptanone

123-86-4-..---Butylacetate

123-91-1-.......-1,4-Dioxane

126-98-7-.-.--2-Methyl-2-propenenitrile

563-80-4-.-.--3-Methyl-2-butanone

627-13-4-...-.-Propyl nitrate

\begin{tabular}{|r|l|l|}
42 & $\mathrm{U}$ \\
42 & $\mathrm{U}$ \\
42 & $\mathrm{U}$ \\
10 & $\mathrm{~J}$ \\
42 & $\mathrm{U}$ \\
42 & $\mathrm{U}$ \\
42 & $\mathrm{U}$ \\
42 & $\mathrm{U}$ \\
40 & $\mathrm{~J}$ \\
42 & $\mathrm{U}$ \\
42 & $\mathrm{U}$ \\
2 & $\mathrm{~J}$ \\
6 & $\mathrm{~J}$ \\
2 & $\mathrm{~J}$ \\
42 & $\mathrm{U}$ \\
42 & $\mathrm{U}$ \\
42 & $\mathrm{U}$ \\
42 & $\mathrm{U}$ \\
42 & $\mathrm{U}$ \\
42 & $\mathrm{U}$ \\
210 & \\
200 & \\
250 & \\
42 & $\mathrm{U}$ \\
42 & $\mathrm{U}$ \\
42 & $\mathrm{U}$ \\
42 & $\mathrm{U}$ \\
8 & $\mathrm{~J}$ \\
42 & $\mathrm{U}$ \\
97 & \\
42 & $\mathrm{U}$ \\
34 & $\mathrm{~J}$ \\
42 & $\mathrm{U}$ \\
& \\
\hline & \\
\hline
\end{tabular}


Liab Name: PNNL

Irab Code: PNNL

Case No.:
Contract: BNFL

SAS NO.:
$A W-101 \cdot$

SDG No.: 99021601
Matrix: (soil/water) SOLID

Sample wt/vol: $\quad 1.2(\mathrm{~g} / \mathrm{mL}) \mathrm{G}$

Level: (low/med) IOW

\% Moisture: not dec.

GC.Column: DB-624 75M X 2.55UM ID: 0.45 (mm)

Soil Extract Volume: (UL)
Lab Sample ID: 99-0650

Iab File ID: $\quad 99021605$

Date Received:

Date Analyzed: 02/16/99

(milution Factor: 1.0

Soil Aliquot Volume: (uL)
CAS NO.

COMPOUND
CONCENTRATION UNITS:

(ug/L or $\mathrm{ug} / \mathrm{Kg}$ ) UG/KG

Q

\section{6-22-0-...-.--3-Pentanone}

76-13-1-....-1,2,2-Cl3-1, 1, 2-F3ethane

76-I4-2---.---I,2-Cl2-I, I, 2,2-F4ethane

75-71-8-..---Dichlorodifluoromethane

75-05-8-.-.--Acetonitrile

110-82-7-...---Cyclohexane

108-86-1-..---Bromobenzene

104-51-8-...---Butylbenzene

98-06-6-..----tert-Butylbenzene

135-98-8--.----sec-Butylbenzene

95-49-8-...-.-2-Chlorotoluene

99-87-6--.---4-Isopropyltoluene

106-43-4-.-.--4-Chlorotoluene

96-12-8-...-1, 2-Dibromo-3-chloropropane

106-93-4-.---1, 2-Dibromoethane

110-57-6--..--trans-1, 4-Dichloro-2-butene

142-28-9------1,3-Dichloropropane

594-20-7-..---2, 2-Dichloropropane

563-58-6------1,1-Dichloropropene

87-68-3-...---Hexachloro-1, 3-butadiene

74-88-4-...--. Iodomethane

98-82-8-...----Isopropylbenzene

91-20-3-...--Nathphalene

103-65-1-.-----Propylbenzene

87-61-6-.....-1, 2,3-Trichlorobenzene

120-82-1-....-1, 2, 4-Trichlorobenzene

96-18-4-...--1,2, 3-Trichloropropane

95-63-6-...---1, 2,4-Trimethylbenzene

108-67-8--.---1,2,3-Trimethylbenzene

71-23-8-..-.--1-Propanol

106-35-4-..---3-Heptanone

110-43-0-..---2-Heptanone

109-99-9-..--.-Tetrahydrofuran

\begin{tabular}{|l|l|l|}
42 & $U$ \\
42 & $U$ \\
42 & $U$ \\
42 & $U$ \\
41 & $\mathrm{U}$ \\
42 & $\mathrm{U}$ \\
42 & $\mathrm{U}$ \\
42 & $\mathrm{U}$ \\
42 & $\mathrm{U}$ \\
42 & $\mathrm{U}$ \\
42 & $\mathrm{U}$ \\
42 & $\mathrm{U}$ \\
42 & $\mathrm{U}$ \\
42 & $\mathrm{U}$ \\
42 & $\mathrm{U}$ \\
42 & $\mathrm{U}$ \\
42 & $\mathrm{U}$ \\
42 & $\mathrm{U}$ \\
42 & $\mathrm{U}$ \\
42 & $\mathrm{U}$ \\
42 & $\mathrm{U}$ \\
42 & $\mathrm{U}$ \\
42 & $\mathrm{U}$ \\
42 & $\mathrm{U}$ \\
42 & $\mathrm{U}$ \\
42 & $\mathrm{U}$ \\
42 & $\mathrm{U}$ \\
42 & $\mathrm{U}$ \\
42 & $\mathrm{U}$ \\
42 & $\mathrm{U}$ \\
59 & \\
59 & - \\
180 & - \\
\hline & & \\
\hline
\end{tabular}


Lab Name: PNNL Contract: BNFI:

$A W-101$

Iab Code: PNNL

Case No.:

SAS NO.:

SDG No.: 99021601 .

Matrix: (soil/water) SOLID

Lab Sample ID: 99-0650

Sample wt/vol:

$1.2(\mathrm{~g} / \mathrm{mL}) \mathrm{G}$

Lab File ID: 99021605

Level: (low/med) LOW

Date Received:

$\%$ Moisture: not dec.

Date Analyzed: 02/16/99

GC Column: .DB-624 75M X 2.55UM ID: 0.45 (mm) Dilution Factor: 1.0

Soil Extract Volume: (uI)

Soil Aliquot Volume: (uI)

CAS NO.

COMPOUND CONCENTRATION UNITS: (ug/L or $u g / K g)$ UG/KG Q

74-95-3-..--Dibromomethane

$42 \mathrm{U}$ 
Irab Name: PNNL

Iab Code: PNNL

Case No.:
Contract: BNEL

SAS NO.:
AW-101D

SDG No.: 99021601
Matrix: (soil/water) SOLID

Sample wt/vol:

$0.5(\mathrm{~g} / \mathrm{mI}) \mathrm{G}$
Lab Sample ID: 99-0650D

Lab File ID: 99021606
Level: (low/med) LOW

$\%$ Moisture: not dec.

GC Column: DB-624 75M X 2.55UM ID: 0.45 (mm) (UL)
Date Received:

Date Analyzed: 02/16/99

Soil Extract Volume:

Dilution Factor: 1.0

Soil Aliquot Volume: (uI) CONCENTRATION UNITS:

CAS NO. COMPOUND $(\mathrm{ug} / \mathrm{L}$ or $\mathrm{ug} / \mathrm{Kg})$ UG/KG

\begin{tabular}{r|r|l}
94 & $\mathrm{U}$ \\
94 & $\mathrm{U}$ \\
94 & $\mathrm{U}$ \\
920 & \\
94 & $\mathrm{U}$ \\
94 & $\mathrm{U}$ \\
94 & $\mathrm{U}$ \\
360 & \\
80 & $\mathrm{~J}$ \\
94 & $\mathrm{U}$ \\
94 & $\mathrm{U}$ \\
94 & $\mathrm{U}$ \\
94 & $\mathrm{U}$ \\
94 & $\mathrm{U}$ \\
94 & $\mathrm{U}$ \\
94 & $\mathrm{U}$ \\
94 & $\mathrm{U}$ \\
94 & $\mathrm{U}$ \\
80 & $\mathrm{~J}$ \\
110 & \\
94 & $\mathrm{U}$ \\
94 & $\mathrm{U}$ \\
94 & $\mathrm{U}$ \\
94 & $\mathrm{U}$ \\
94 & $\mathrm{U}$ \\
94 & $\mathrm{U}$ \\
94 & $\mathrm{U}$ \\
94 & $\mathrm{U}$ \\
94 & $\mathrm{U}$ \\
94 & $\mathrm{U}$ \\
7 & $\mathrm{~J}$ \\
94 & $\mathrm{U}$ \\
94 & $\mathrm{U}$ \\
& \\
\hline
\end{tabular}

74-87-3-...--Chloromethane

75-01-4-....-.-Vinyl chloride

106-99-0-...--1,3-Butadiene

106-97-8-..---Butane

74-83-9-...---Bromomethane

75-35-4-...-1, 1-Dichloroethene

75-00-3-...---Chloroethane

67-64-1-....-Acetone

109-66-0-....-.-Pentane

75-15-0_....-.-Carbon Disulfide

107-02-8-...--Acrolein

107-13-1-..---Acrylonitrile

75-09-2-....--Methylene Chloride

156-60-5-...--trans-1,2-Dichloroethene

123-38-6-.....-.-Propionaldehyde

4170-30-3--.---2-Butenal

75-34-3-..---1,1-Dichloroethane

156-59-2-...-Cis-1, 2-Dichloroethene

110-54-3-.-----Hexane

78-93-3-..-.--2-Butanone

110-83-8-..---Cyclohexene

141-78-6-...--Ethyl acetate

287-92-3-...---Cyclopentane

74-97-5-...---Bromochloromethane

67-66-3-...---Chloroform

75-43-4--.----Dichlorofluoromethane

71-55-6-....-1, 1, I-Trichloroethane

75-45-6-...--.-Chlorodifluoromethane

56-23-5-1.----Carbon Tetrachloride

75-69-4-....- Trichlorofluoromethane

71-43-2-...----Benzene

107-06-2-..---1,2-Dichloroethane

79-01-6-...-.-Trichloroethene 
Lab Name: PNNL

Lab Code: PNNL

Case No.:

Matrix: (soil/water) SOLID

Sample wt/vol:

$0.5(\mathrm{~g} / \mathrm{mL}) \mathrm{G}$

Level: (low/med) LOW

$\%$ Moisture: not dec.

GC Column: DB-624 75M X 2.55UM ID: 0.45 (mm) (uI)

Soil Extract Volume:
Contract: BNFL

SAS NO.:
$A W-101 D$

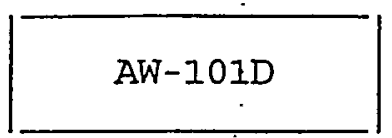

SDG No.: 99021601

Lab Sample ID: 99-0650D

Lab File ID: 99021606

Date Received:

Date Analyzed: 02/16/99.

Soil Aliquot Volume:

Dilution Factor: 1.0

CONCENTRATION UNITS:

CAS NO.

COMPOUND (ug/L or $\mathrm{ug} / \mathrm{Kg}$ ) UG/KG (UI)

\begin{tabular}{|c|}
\hline 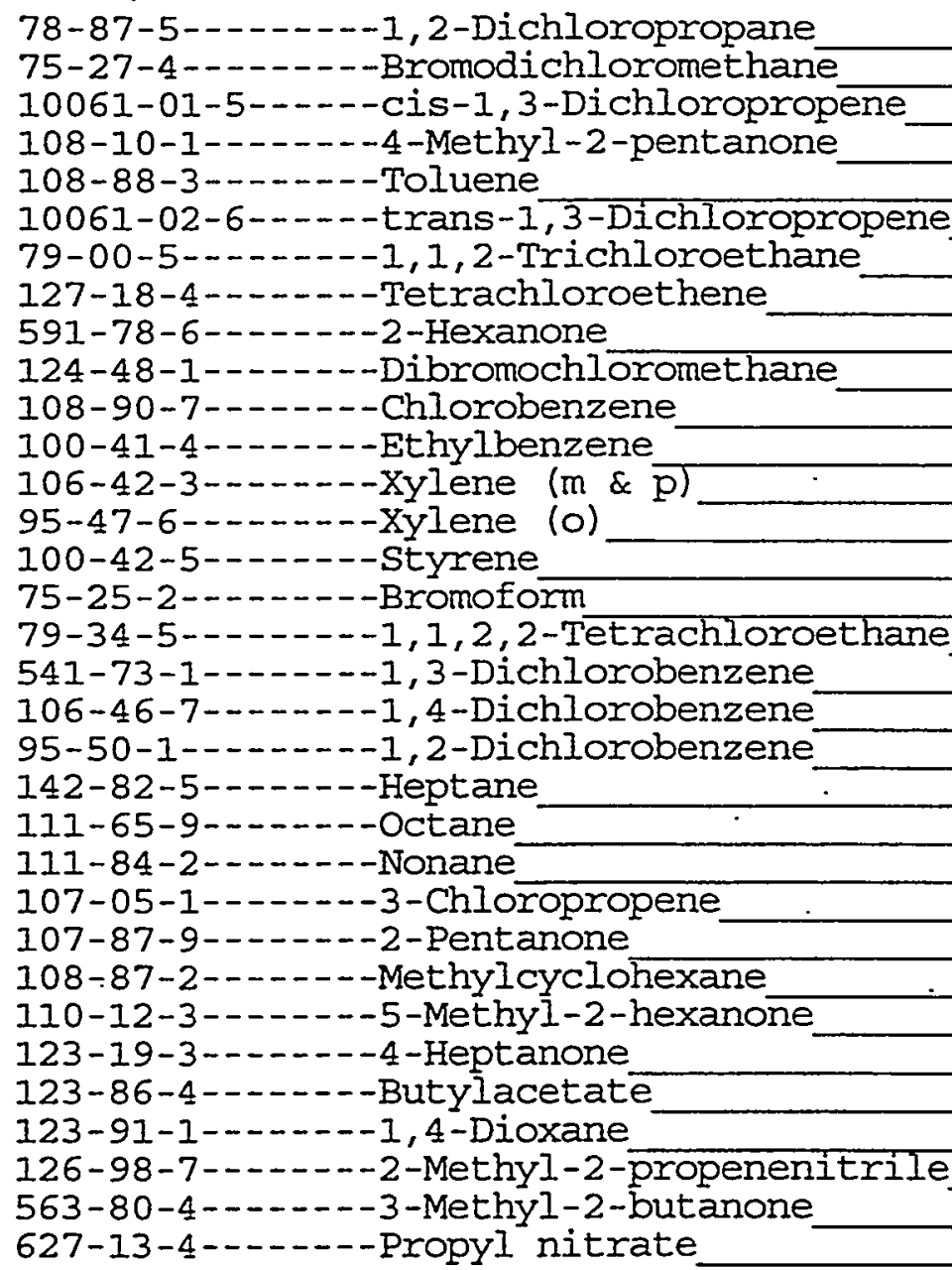 \\
\hline
\end{tabular}


Lab Name: PNNL

Lab Code: PNNL

Case No.:

Matrix: (soil/water) SOIID

Sample wt/vol:

$0.5(\mathrm{~g} / \mathrm{mL}) \mathrm{G}$

Level: (low/med) LOW

\% Moisture: not dec.

GC Column: $\mathrm{DB}-62475 \mathrm{M} \times 2.55 \mathrm{UM}$ ID: 0.45 (mm)

Soil Extract Volume:

(uL)
Contract: BNEL

SAS No.:
EPA SAMPLE NO.

$A W-101 D$

SDG No.: 99021601

Lab Sample ID: 99-0650D

Lab File ID: 99021606

Date Received:

Date Analyzed: 02/16/99

Dilution Factor: 1.0

Soil Aliquot Volume:

(uL)

CONCENTRATION UNITS :

CAS NO: COMPOUND (ug/L or ug/Kg) UG/KG

Q

96-22-0--------3-Pentanone

76-13-1-....--1,2,2-Cl3-1, 1, 2-F3ethane

76-14-2--...- 1, 2-C12-1, 1, 2, 2-F4ethane

75-71-8-..---Dichlorodifluoromethane

75-05-8------Acetonitrile

110-82-7-...--Cyclohexane

108-86-1--.-.--Bromobenzene

104-51-8-....-Butylbenzene

98-06-6-..----tert-Butylbenzene

135-98-8---.---sec-Butylbenzene

95-49-8--...--2-Chlorotoluene

99-87-6-- - - - 4-Isopropyltoluene

106-43-4-..--4-Chlorotoluene

96-12-8--..--1, 2-Dibromo-3-chloropropane

106-93-4--.---1,2-Dibromoethane

110-57-6-..---trans-1, 4-Dichloro-2-butene

142-28-9------1, 3-Dichloropropane

594-20-7-..... 2, 2-Dichloropropane

563-58-6-..---1, 1-Dichloropropene

87-68-3-...---Hexachloro-1,3-butadiene

74-88-4-.....-. Iodomethane

98-82-8--.---- Isopropylbenzene

91-20'3--...--Nathphalene

103-65-1-..---Propylbenzene

87-61-6-..-.--1, 2,3-Trichlorobenzene

120-82-1-...--1, 2,4-Trichlorobenzene

96-18-4-...-- - 1, 2, 3-Trichloropropane

95-63-6-- - - - - 1, 2, 4-Trimethylbenzene

108-67-8--.---1, 2,3-Trimethylbenzene

71-23-8-..-.-.1-Propanol

106-35-4-------3-Heptanone

110-43-0-.-.--2-Heptanone

109-99-9-...--Tetrahydrofuran

\begin{tabular}{l|l|}
94 & $U$ \\
94 & $U$ \\
94 & $U$ \\
94 & $U$ \\
94 & $U$ \\
94 & $U$ \\
94 & $U$ \\
94 & $U$ \\
94 & $U$ \\
94 & $U$ \\
94 & $U$ \\
94 & $U$ \\
94 & $U$ \\
94 & $U$ \\
94 & $U$ \\
94 & $U$ \\
94 & $U$ \\
94 & $U$ \\
94 & $U$ \\
94 & $U$ \\
94 & $U$ \\
94 & $U$ \\
94 & $U$ \\
94 & $U$ \\
94 & $U$ \\
94 & $U$ \\
94 & $U$ \\
94 & $U$ \\
94 & $U$ \\
94 & $U$ \\
32 & $J$ \\
36 & $J$ \\
89 & $J$ \\
\hline & \\
\hline
\end{tabular}

OLM03. 0 
Lab Name: . PNNL

Lab Code: PNNL

Case No.:

Matrix: (soil/water) SOLID

Sample wt/vol:

$0.5(\mathrm{~g} / \mathrm{mL}) \mathrm{G}$

Level: (low/med) LOW

\% Moisture: not dec.

GC Column: DB-62475M X 2.55UM ID: 0.45 (mm)

Soil Extract Volume: (نì)

CAS NO.

COMPOUND

74-95-3--------Dibromomethane
Contract: BNFL

SAS NO.:
EPA SAMPLE NO.

AW-101D

SDG No.: 99021601

Lab Sample ID: 99-0650D

Iab File ID: 99021606

Date Received:

Date Analyzed: 02/16/99

Soil Aliquot Volume: (uL) CONCENTRATION UNITS:

(ug/I or $u g / K g$ ) UG/KG Q 
Lab Name: PNNL

Lab Code: PNNL

Matrix: (soil/water) SOLID

Sample wt/vol:

$0.4(\mathrm{~g} / \mathrm{mL}) \mathrm{G}$

Level: (low/med) LOW

$\%$ Moisture: not. dec.

GC Column: DB-624 75M X 2.55UM ID: 0.45 (mm)

Soil Extract Volume:
Contract: BNFL

SAS NO.:
EPA SAMPLE NO.

AW-101MS

SDG No.: 99021601

Iab Sample ID: 99-0650MS

Lab File ID: 99021607

Date Received:

Date Analyzed: 02/16/99

Dilution Factor: 1.0

Soil Aliquot Volume: (uT)

CAS NO. COMPOUND (ug/L or ug/Kg) UG/KG Q

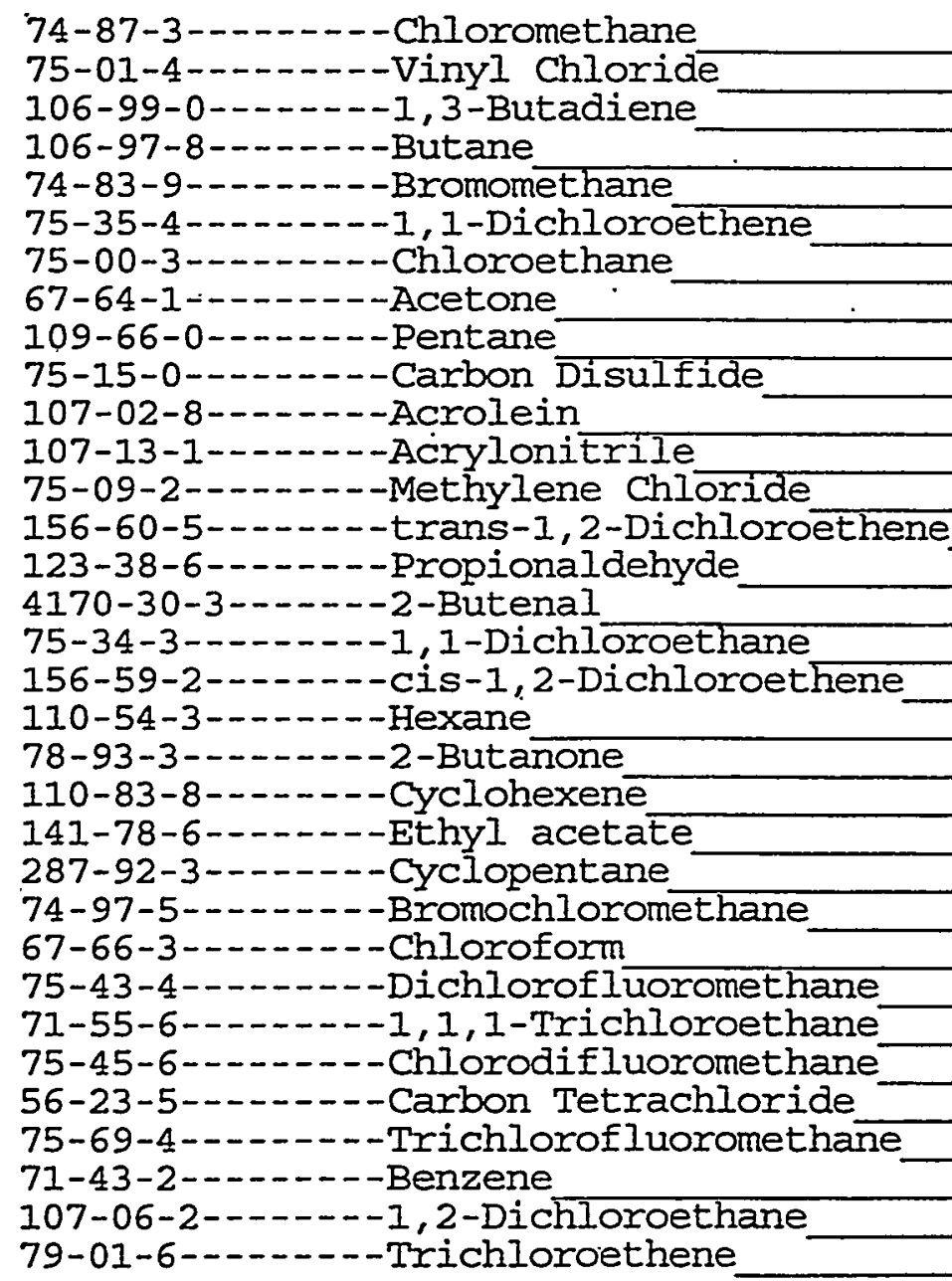


Lab Name: PNNL

Iab Code: PNNL
Contract: BNFI

SAS NO. :
EPA SAMPLE NO.

AW-10IMS
Matrix: (soil/water) SOLID

Sample wt/vol:

$0.4(\mathrm{~g} / \mathrm{mL}) \mathrm{G}$

Level: (low/med) LOW

\% Moisture: not dec.

GC Column: DB-624 75M X 2.55UM ID: 0.45 (mm)

Soil Extract Volume: (uL)
Lab Sample ID: 99-0650MS

Lab File ID: 99021607

Date Received:

Date Analyzed: 02/16/99

CAS NO.

COMPOUND

TNTTS :

(ug/L or $u g / K g$ ) UG/KG

Dilution Factor: 1.0

Soil Aliquot Volume: (uL)

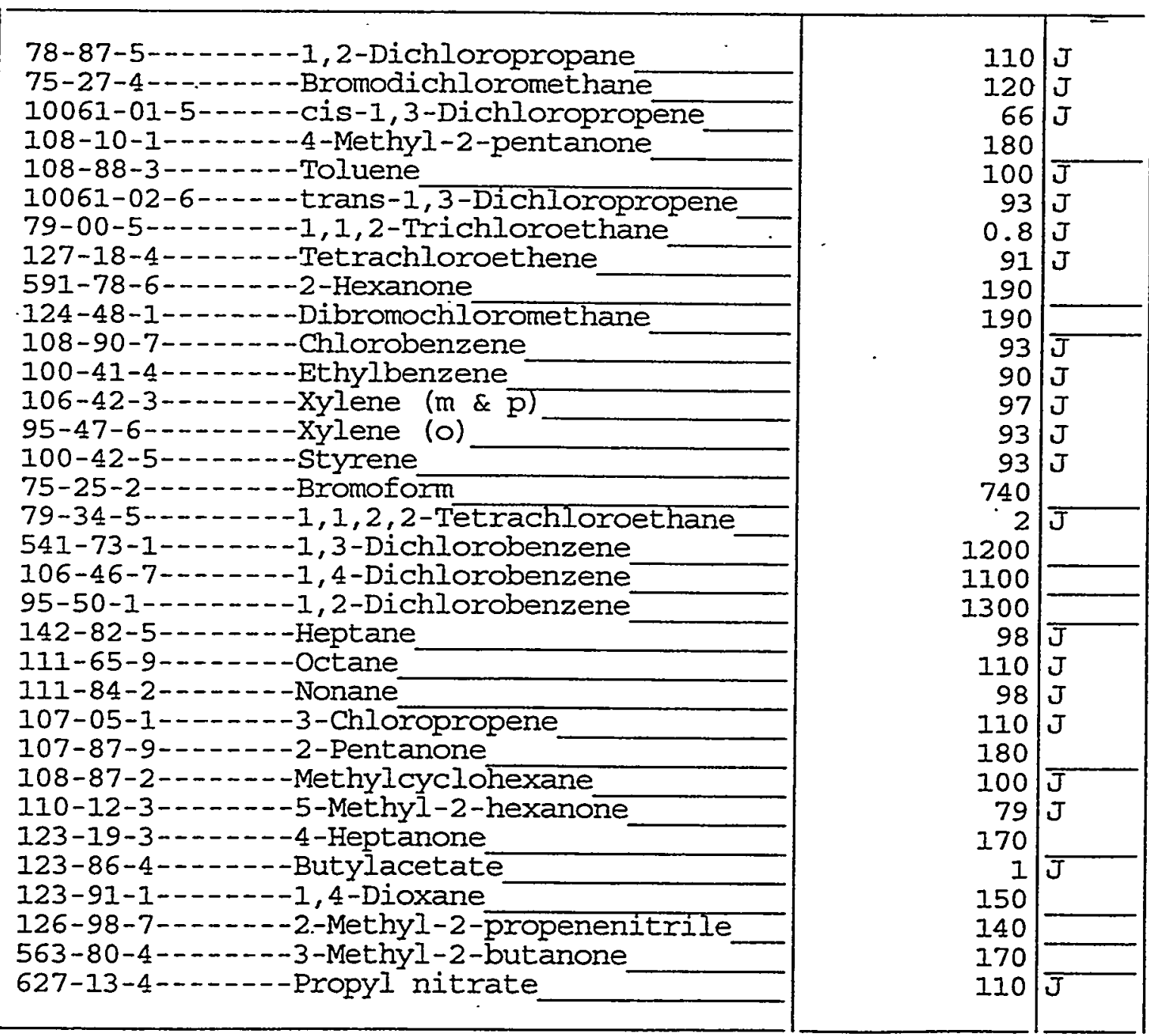


Lab Name: PNNL

Lab code: PNNL
Contract: BNEL

SAS NO.:
EPA SAMPLE NO.

AW-101MS
Matrix: (soil/water) SOLID

Sample wt/vol: $\quad 0.4(\mathrm{~g} / \mathrm{mL}) \mathrm{G}$

Level: (low/med) LOW

\% Moisture: not dec.

GC Column: DB-624 75M X 2.55UM ID: 0.45 (mm)

Soil Extract Volume: (ut)
Lab Sample ID: 99-0650MS

Lab File ID: 99021607

Date Received:

Date Analyzed: 02/16/99

Dilution Factor: 1.0 (uI) CONCENTRATION UNITS:

CAS NO. COMPOUND (ug/L or ug/Kg) UG/KG

Q

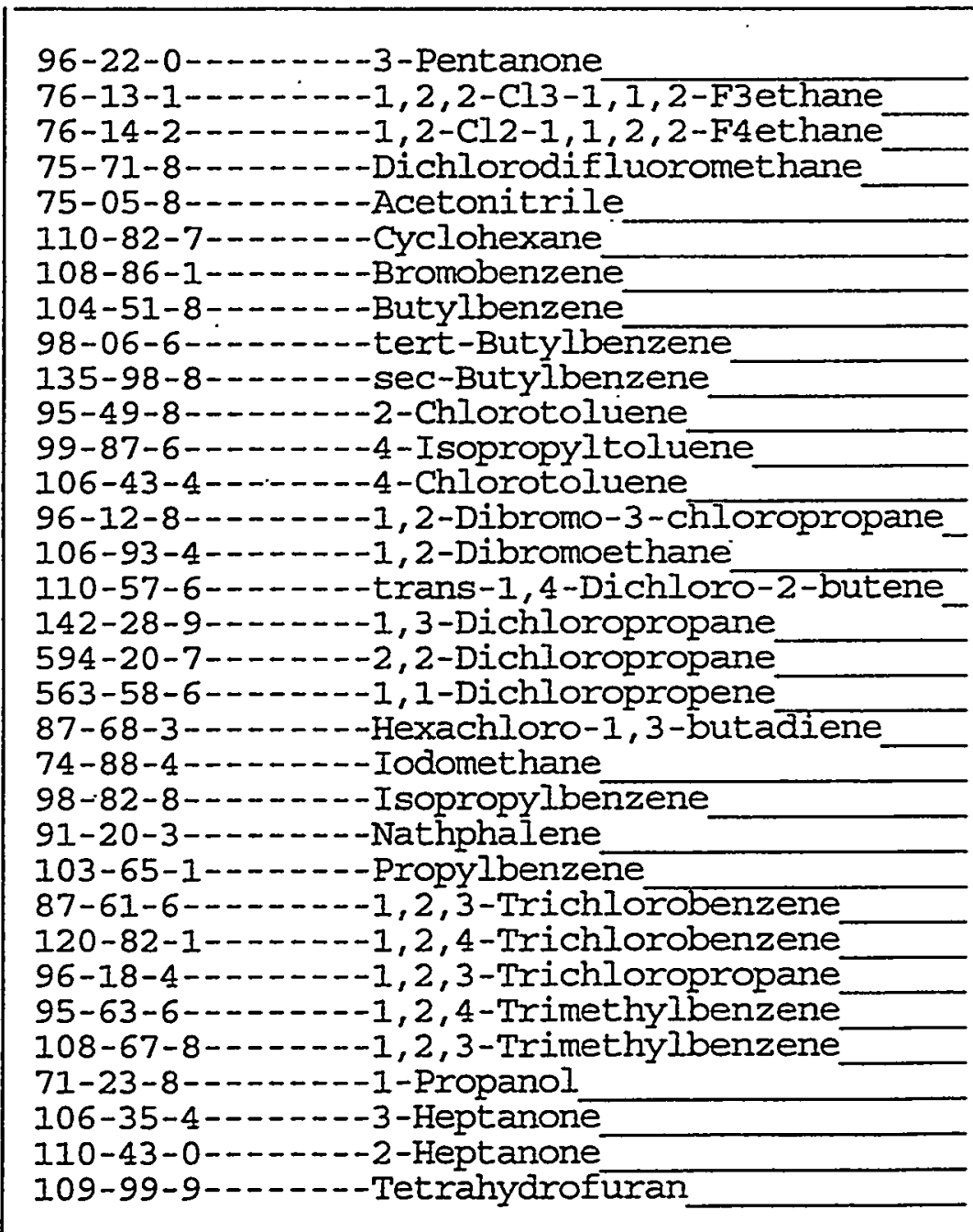


Lab Name: PNNL

Lab Code: PNNL

Case No.:

SAS NO.:

AW-101MS

Matrix: (soil/water) SOLID

Iab Sample ID: 99-0650MS

Sample wt/vol:

$0.4(\mathrm{~g} / \mathrm{mL}) \mathrm{G}$

Lab File ID: $\quad 99021607$

Irevel: (low/med) IOW

Date Received:

$\div$ Moisture: not dec.

Date Analyzed: 02/16/99

GC Column: DB-624 75M X 2.55UM ID: 0.45 (mm)

Dilution Factor: 1.0

Soil Extract Volume: (uL)

Soil Aliquot Volume: (uL)

CAS NO.

COMPOUND CONCENIRATION UNITS: (ug/L or $\mathrm{ug} / \mathrm{Kg}$ ) UG/KG

$Q$ 74-95-3--.---Dibromomethane 1400 
Lab Name: PNNL

Lab Code: PNNL

Case No.:
Contract: BNFL

SAS NO.:
EPA SAMPLE NO.

AW-101MSD
Matrix: (soil/water) SOLID

Sample wt/vol:

$0.5(\mathrm{~g} / \mathrm{mL}) \mathrm{G}$

Level: (low/med) IOW

$\%$ Moisture: not dec.

GC Column: DB-624 75M X 2.55UM ID: 0.45 (mm)

Soil Extract Volume: (ut)

Lab Sample ID: 99-0650MSD

Lab File ID: 99021608

Date Received:

Date Analyzed: 02/16/99

(mm) Dilution Factor: 1.0

Soil Aliquot Volume:

(uL)

CONCENTRATION UNITS:

CAS NO. COMPOUND (ug/L or $\mathrm{ug} / \mathrm{Kg}) \mathrm{UG} / \mathrm{KG}$

Q

\begin{tabular}{|c|}
\hline 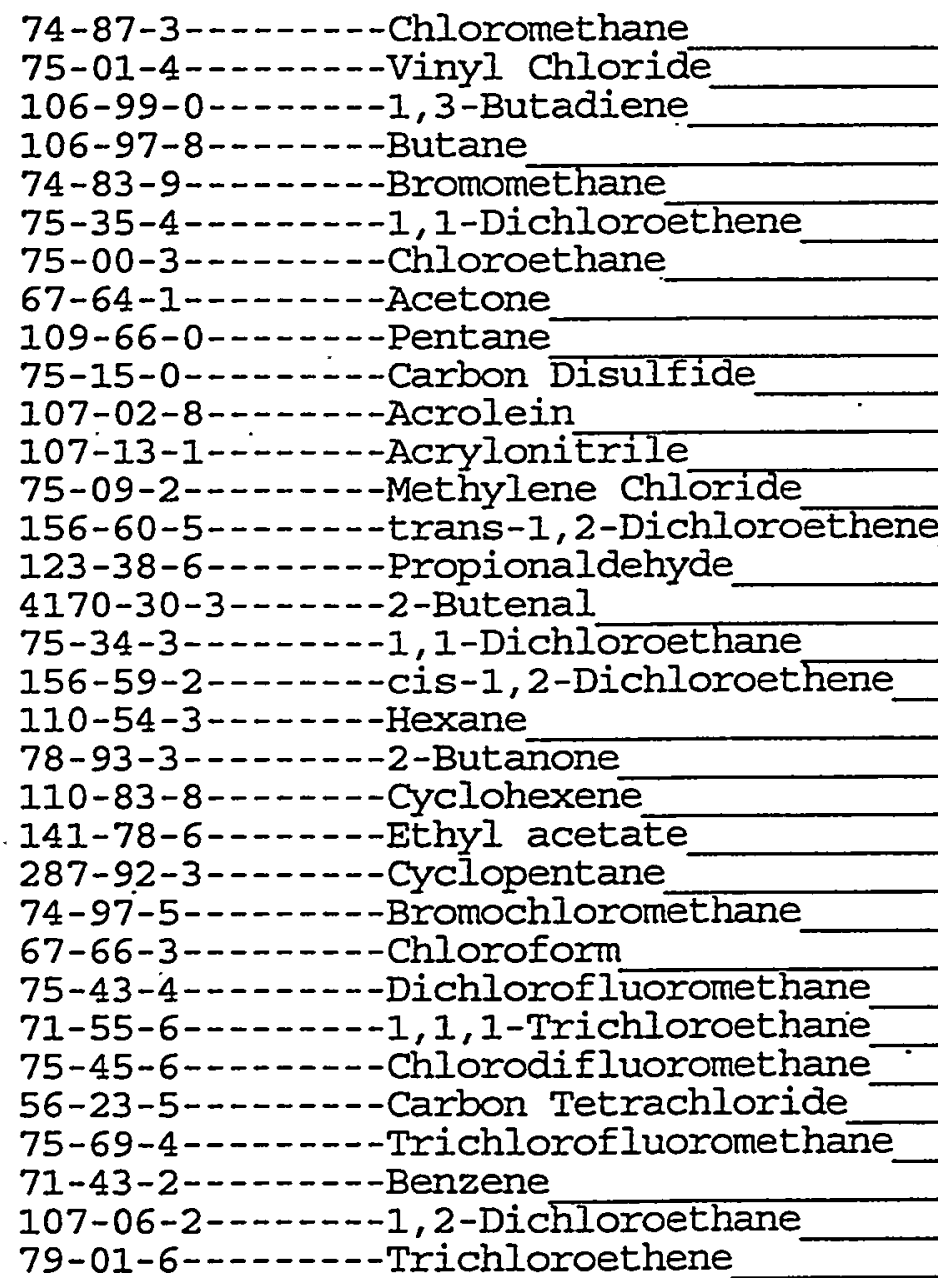 \\
\hline
\end{tabular}

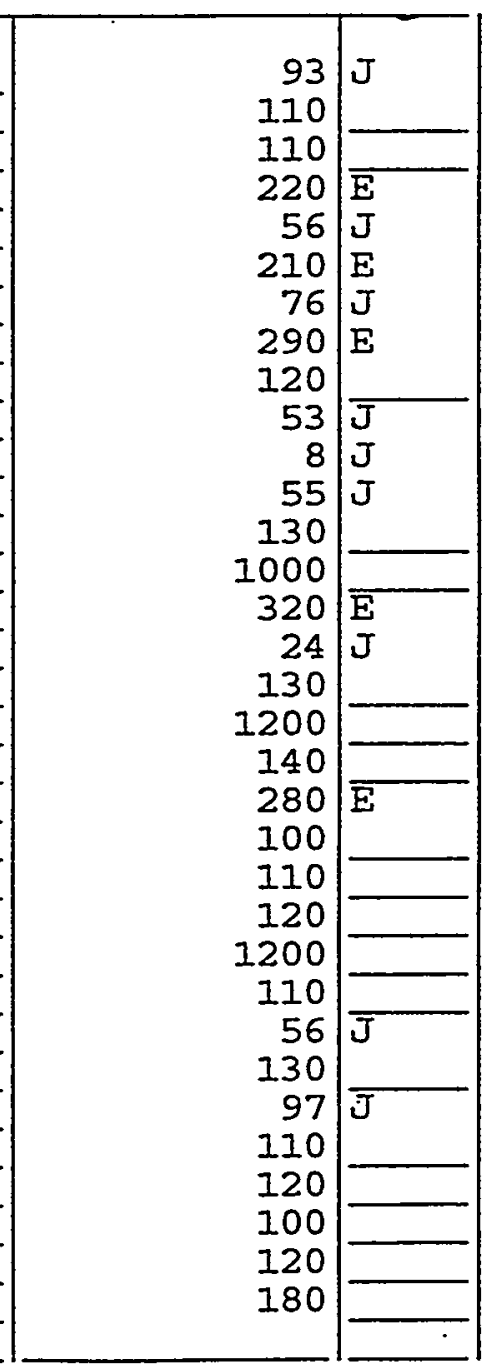


IA

VOLATILE ORGANICS ANAIYSIS DATA SHEET
Lab Name: PNNL

Lab Code: PNNL
Contract: BNFL

SAS NO.:
EPA SAMPLE NO.

AW-101MSD
Matrix: (soil/water) SOLID

Sample wt/vol:

$0.5(\mathrm{~g} / \mathrm{mL}) \mathrm{G}$

Level: (low/med) IOW

\% Moisture: not dec.

GC Column: DB-624 75M X 2.55UM ID: 0.45 (mm) (ut)

Soil Extract Volume:
Lab Sample ID: 99-0650MSD

Lab File ID: 99021608

Date Received:

Date Analyzed: 02/16/99

Dilution Factor: 1.0

Soil Aliquot Volume: (uI) CONCENTRATION UNITS:

CAS NO.

COMPOUND (ug/L or $\mathrm{ug} / \mathrm{Kg}$ ) UG/KG

78-87-5------1, 2-Dichloropropane

75-27-4-...---Bromodichloromethane 10061-01-5-..--cis-1, 3-Dichloropropene 108-10-1--.---4-Methyl-2-pentanone 108-88-3--.---Toluene

10061-02-6-----trans-1,3-Díchloropropene

79-00-5--.----1, 1,2-Trichloroethane

127-18-4--.---Tetrachloroethene

591-78-6-..---2-Hexanone

124-48-1--.---Dibromochloromethane

108-90-7-....-Chlorobenzene

100-41-4-..----Ethylbenzene

106-42-3-...-.-Xylene (m \& p)

95-47-6-.......-Xylene (o)

100-42-5-------Styrene

75-25-2--.----Bromoform

79-34-5-.-.-1, 1, 2, 2-Tetrachloroethane

541-73-1-...---1,3-Dichlorobenzene

106-46-7--.--1, 4-Dichlorobenzene

95-50-1-.....-1, 2-Dichlorobenzene

142-82-5--.-.--Heptane

111-65-9-...--Octane

111-84-2------Nonane

107-05-1-...--3-Chloropropene

107-87-9--..---2-Pentanone

108-87-2-.....-Methyl cyclohexane

110-12-3-...---5-Methyl-2-hexanone

123-19-3-------4-Heptanone

123-86-4-..---.-Butylacetate

123-91-1-....-1,4-Dioxane

126-98-7-..---2-Methyl-2-propenenitrile

563-80-4-------3-Methyl-2-butanone

627-13-4--.---Propyl nitrate

\begin{tabular}{r|l}
\multicolumn{2}{|l}{} \\
\hline 110 & \\
48 & $\mathrm{~J}$ \\
55 & $\mathrm{~J}$ \\
220 & $\mathrm{E}$ \\
100 & \\
88 & $\mathrm{~J}$ \\
0.9 & $\mathrm{~J}$ \\
91 & $\mathrm{~J}$ \\
240 & $\mathrm{E}$ \\
67 & $\mathrm{~J}$ \\
90 & $\mathrm{~J}$ \\
87 & $\mathrm{~J}$ \\
93 & $\mathrm{~J}$ \\
89 & $\mathrm{~J}$ \\
89 & $\mathrm{~J}$ \\
420 & \\
2 & $\mathrm{~J}$ \\
920 & \\
890 & \\
980 & \\
110 & \\
110 & \\
100 & \\
96 & $\mathrm{~J}$ \\
190 & \\
100 & \\
90 & $\mathrm{~J}$ \\
200 & $\mathrm{E}$ \\
2 & $\mathrm{~J}$ \\
180 & \\
150 & \\
200 & $\mathrm{E}$ \\
110 & \\
\hline & \\
\hline
\end{tabular}


Lab Name: PNNL.

Lab Code: PNNL

Case No.:

Matrix: (soil/water) SOLID

Sample wt/vol:

$0.5(\mathrm{~g} / \mathrm{mL}) \mathrm{G}$.

Level: (low/med) IOW

\% Moisture: not dec.

GC Column: DB-62475M X 2.55UM ID: 0.45 (mm)

Soil Extract Volume: (UL)
Contract : BNFL

SAS NO. :
EPA SAMPLE: NO.

AW-I0IMSD

SDG No.: 99021601

Lab Sample ID: 99-0650MSD

Lab File ID: 99021608

Date Received:

Date Analyzed: 02/16/99

(mm) Dilution Factor: 1.0

Soil Aliquot Volume:

(UL)

CAS NO.
COMPOUND
CONCENTRATION UNITS:

(ug/I or $\mathrm{ug} / \mathrm{Kg}$ ) UG/KG

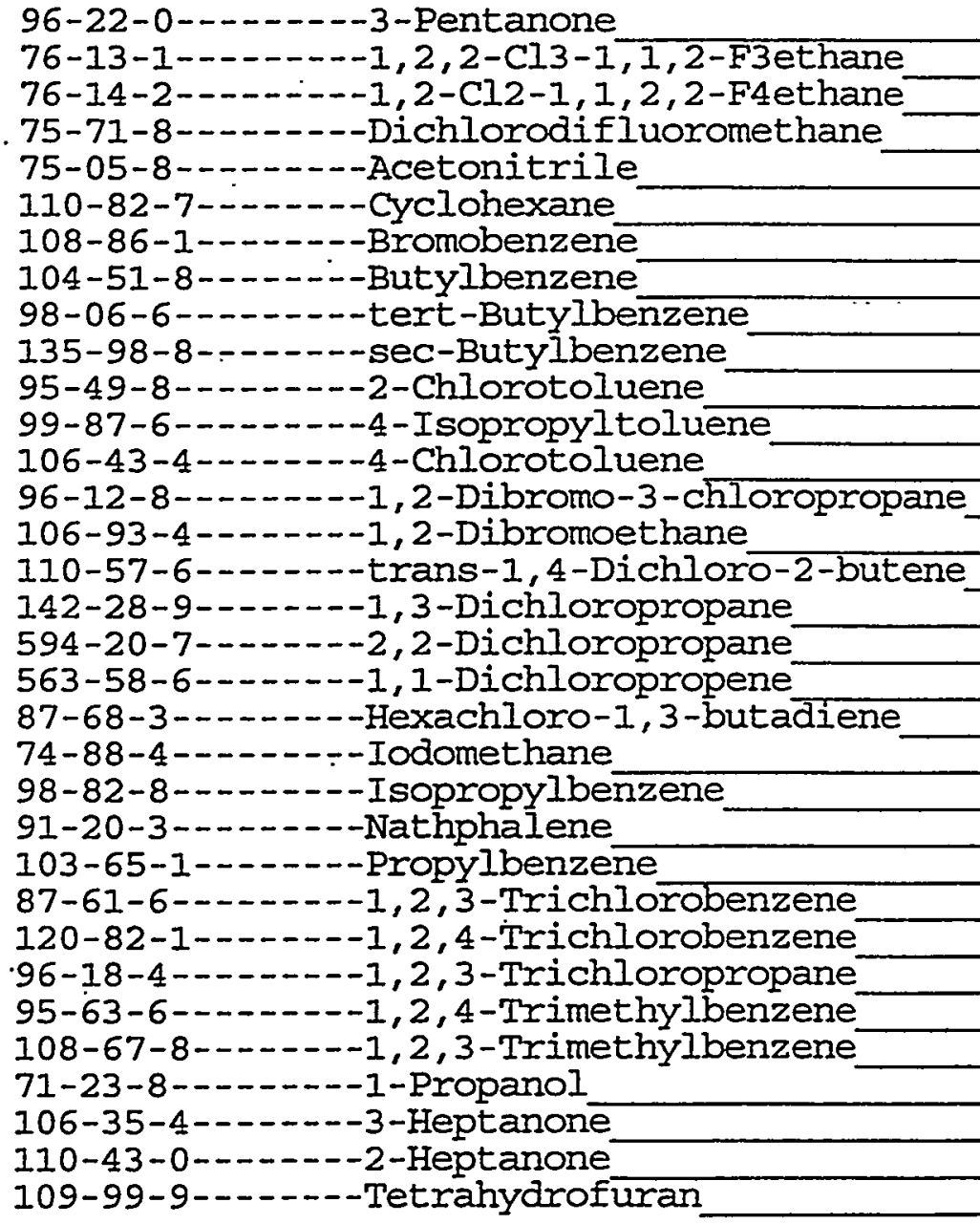

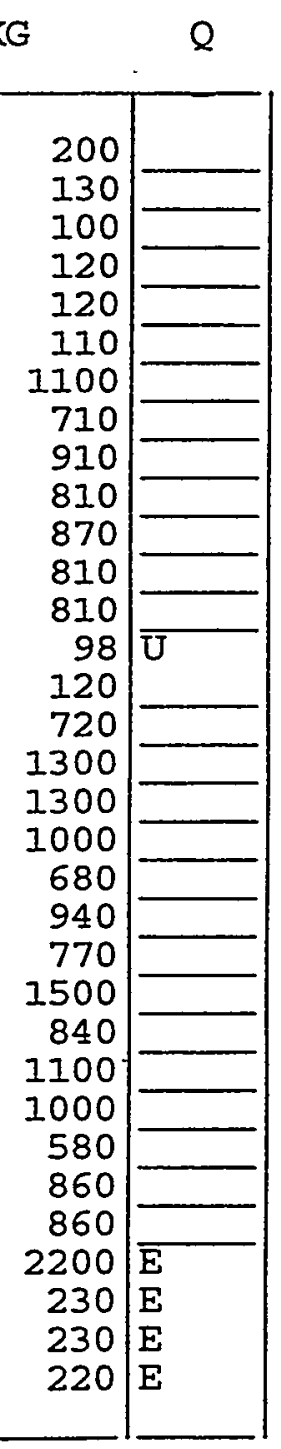


$1 \mathrm{~A}$

VOLATILE ORGANICS ANAIYSIS $\vdots$

DATA SHEET

Contract: BNFL

Case No.: SAS No.:
EPA SAMPLE NO.

AW-101MSD
Lab Name: PNNL

Lab Code: PNNL

Matrix: (soil/water) SOLID

Sample wt/vol:

$0.5(\mathrm{~g} / \mathrm{mL}) \mathrm{G}$

Level: (low/med) INW

$\div$ Moisture: not dec.

GC Column: DB-624 75M X 2.55UM ID: 0.45 (mm)

Soil Extract Volume: (uI)
CAS NO. $\frac{\text { COMPOUND }}{74-95-3------D \text { Dibromomethane }}$
Lab Sample ID: 99-0650MSD

Lab File ID: 99021608

Date Received:

Date Analyzed: 02/16/99

Dilution Factor: 1.0

Soil Aliquot Volume: (uL)
CONCENTRATION UNITS:

(ug/L or $\mathrm{ug} / \mathrm{Kg}$ ) UG/KG Q 
Lab Name: PNNL

Lab Code: PNNL

Case No.:

Matrix: (soil/water) SOLID

Sample wt/vol:

$0.4(\mathrm{~g} / \mathrm{mL}) \mathrm{G}$

Level: (low/med) LOW

$\%$ Moisture: not dec.

Contract: BNFL

SAS NO.:

Lab Sample ID: 99-065I

Lab File ID: 99021610

Date Received:

Date Analyzed: 02/16/99

GC Column: DB-624 75M X 2.55UM ID: 0.45 (mm)

Dilution Factor: 1.0

Soil Extract Volume: (u士)

Soil Aliquot Volume: (uL) CONCENTRATION UNITS:

CAS NO. COMPOUND (ug/I or ug/Kg) UG/KG

Q
1100

$110 \mathrm{U}$

1100

$110 \mathrm{U}$

$110 \mathrm{U}$

$110 \mathrm{U}$

$110 \mathrm{U}$

$100 \mathrm{~J}$

$110 \mathrm{U}$

$110 \mathrm{U}$

$110 \mathrm{U}$

$110 \mathrm{U}$

$110 \mathrm{U}$

$110 \mathrm{U}$

$110 \mathrm{U}$

$110 \mathrm{U}$

$110 \mathrm{U}$

$110 \mathrm{U}$

$110 . \mathrm{U}$

$110 \mathrm{U}$

$110 \mathrm{U}$

$110 \mathrm{U}$

$110 \mathrm{U}$

$110 \mathrm{U}$

$110 \mathrm{U}$

$110 \mathrm{U}$

$110 \mathrm{U}$

$80 \mathrm{~J}$

$110 \mathrm{U}$

$110 \mathrm{U}$

$110 \mathrm{U}$

$110 \mathrm{U}$

$110 \mathrm{U}$ 
Lab Name: PNNL

Lab Code: PNNL

Case No.:

Matrix: (soil/water) SOLID

Sample wt/vol:

$0.4(\mathrm{~g} / \mathrm{mL}) \mathrm{G}$

Level: (low/med) IOW

$\%$ Moisture: not. dec.

GC Column: DB-624 75M X 2.55UM ID: 0.45 (mm)

Soil Extract Volume: (UIs)

Contract: BNFL

SAS NO.:

Lab Sample ID: 99-0651

Lab File ID: $\quad 99021610$

Date Received:

Date Analyzed: 02/16/99

(mm) Dilution Factor: 1.0

Soil Aliquot Volume:

(uL)

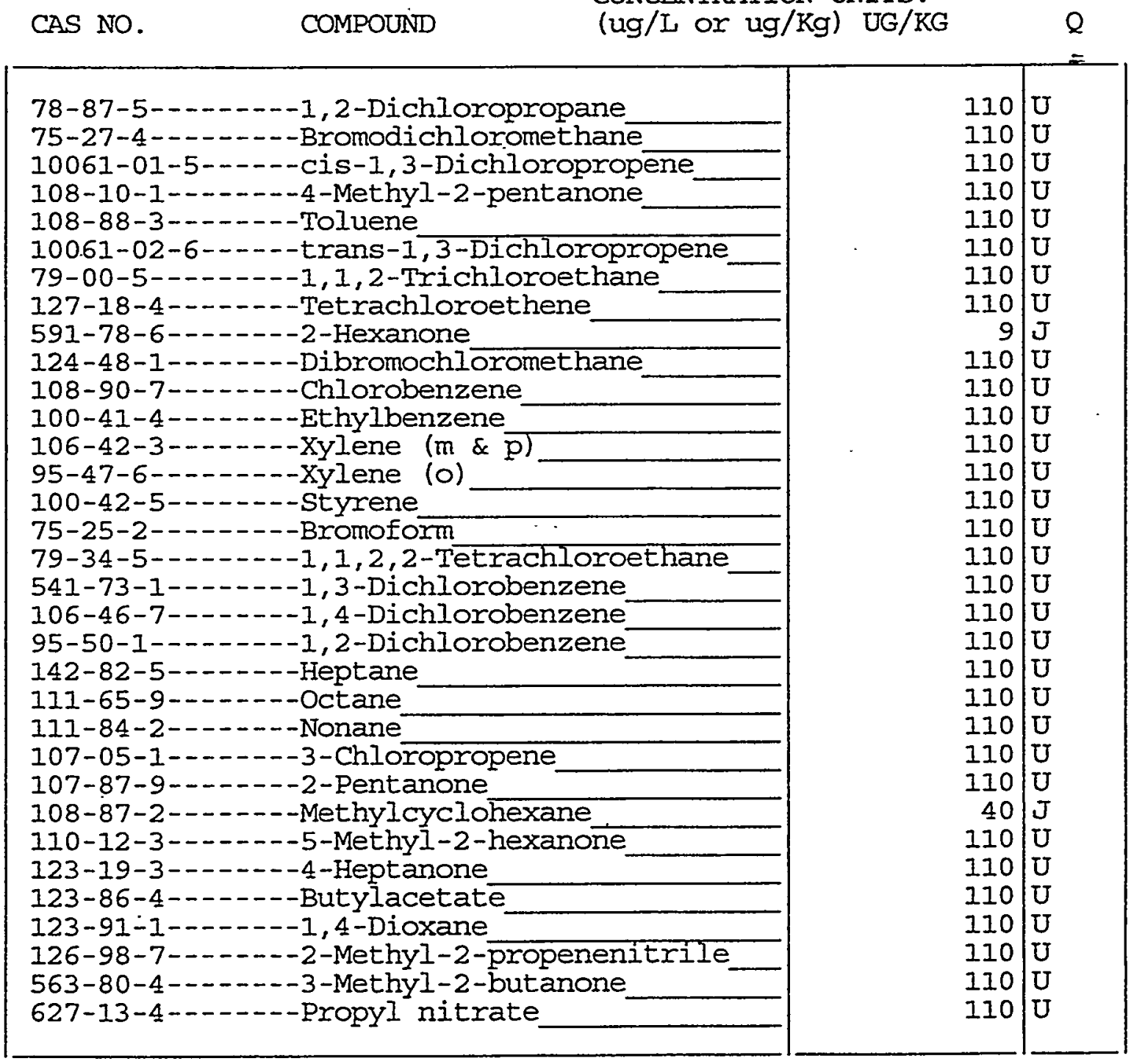


Lab Name: PNNL

Lab Code: PNNL

Case No.:

Matrix: (soil/water) SOLID

Sample wt/vol:

$0.4(\mathrm{~g} / \mathrm{mL}) \mathrm{G}$

Level: (low/med) IOW

$\%$ Moisture: not dec. GC Column: $\mathrm{DB}-62475 \mathrm{M} \times 2.55 \mathrm{MM}$ ID: 0.45 (mm) (uL)

Soil Extract Volume:
AN-107

SDG NO.: 99021601

Lab Sample ID: 99-0651

Lab File ID: $\quad 99021610$

Date Received:

Date Analyzed: 02/16/99

Dilution Factor: 1.0

Soil Aliquot Volume: (uL) CONCENTRATION UNITS :

CAS NO.

COMPOUND (ug/I or $u g / K g$ ) UG/KG

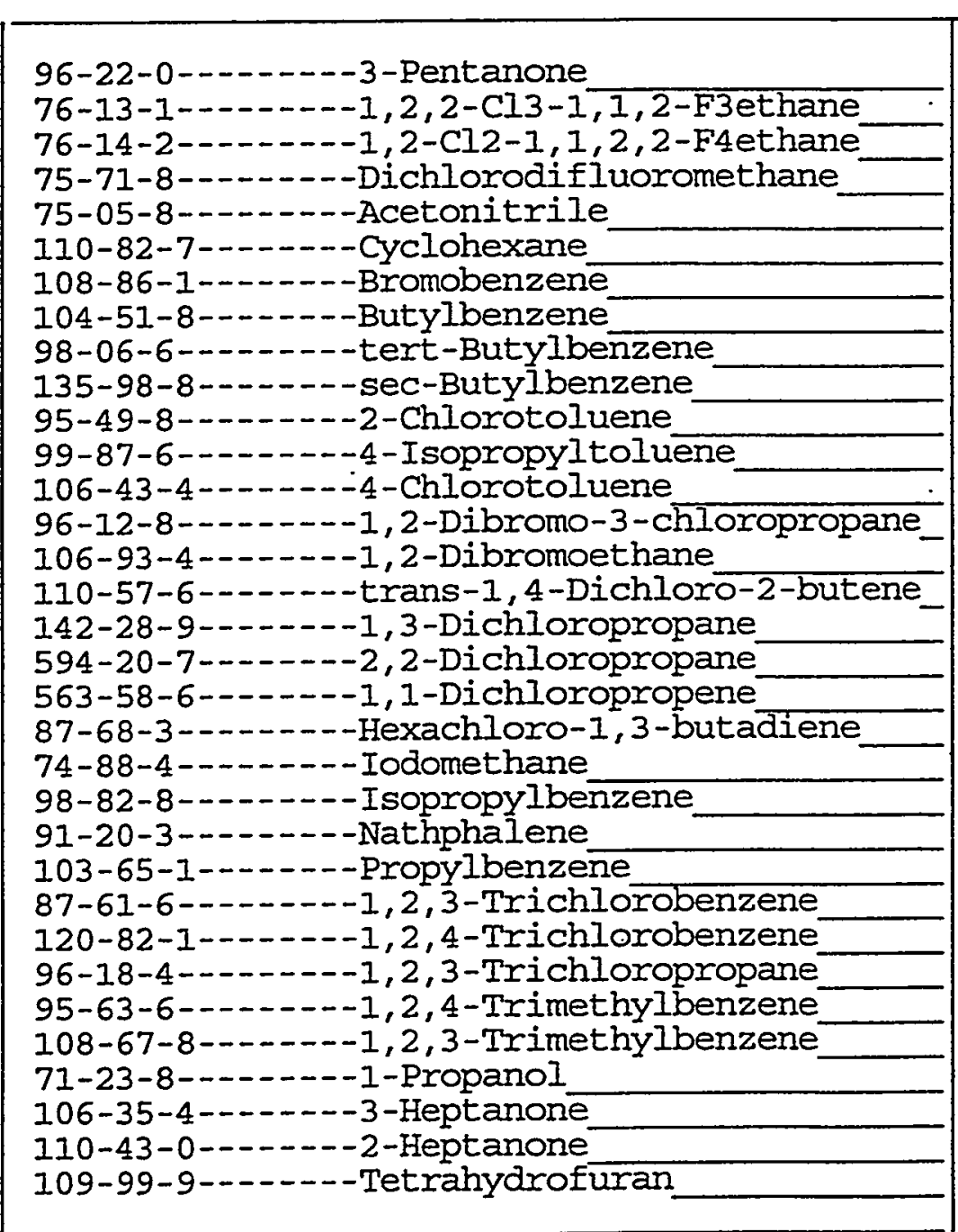

FORM I VOA
OIM03. 0 
$1 \mathrm{~A}$

VOLATILE ORGANICS ANALYSIS DATA SHEET

Lab Name: PNNL

Lab Code: PNNL

Case No.:

Matrix: (soil/water) SolID

Sample wt/vol:

$0.4(\mathrm{~g} / \mathrm{mI}) \mathrm{G}$

Level: (low/med) LOW

$\div$ Moisture: not dec.

GC Column: DB-624 75M X 2.55UM ID: 0.45

Soil Extract Volume:

(ư)

CAS NO.

COMPOUND

74-95-3--.---Dibromomethane
Contract: BNFL

SAS NO.: (mm)
EPA SAMPLE NO.

AN-107

SDG No.: 99021601

Lab Sample ID: 99-0651

Lab File ID: 99021610

Date Received:

Date Analyzed: 02/16/99

Dilution Factor: 1.0

Soil Aliquot Volume: (UL) CONCENTRATION UNITS:

(ug/L or ug/Kg) UG/KG Q

$110 \mathrm{U}$ 
Lab Name: PNNL

Lab Code: PNNL

Case No.:
Contract: BNFL

SAS NO.:
AN-107D

SDG NO.: 99021601
Matrix: (soil/water) SOLID

Sample wt/vol: $\quad 0.7(\mathrm{~g} / \mathrm{mL}) \mathrm{G}$

Level: (low/med) LOW

$\div$ Moisture: not dec.

GC Column: DB-624 75M X 2.55UM ID: 0.45 (mm)

Soil Extract Volume:
Iab Sample ID: 99-0651D

Lab File ID: $\quad 99021611$

Date Received:

Date Analyzed: 02/17/99

Dilution Factor: 1.0

Soil Aliquot Volume: (UI)

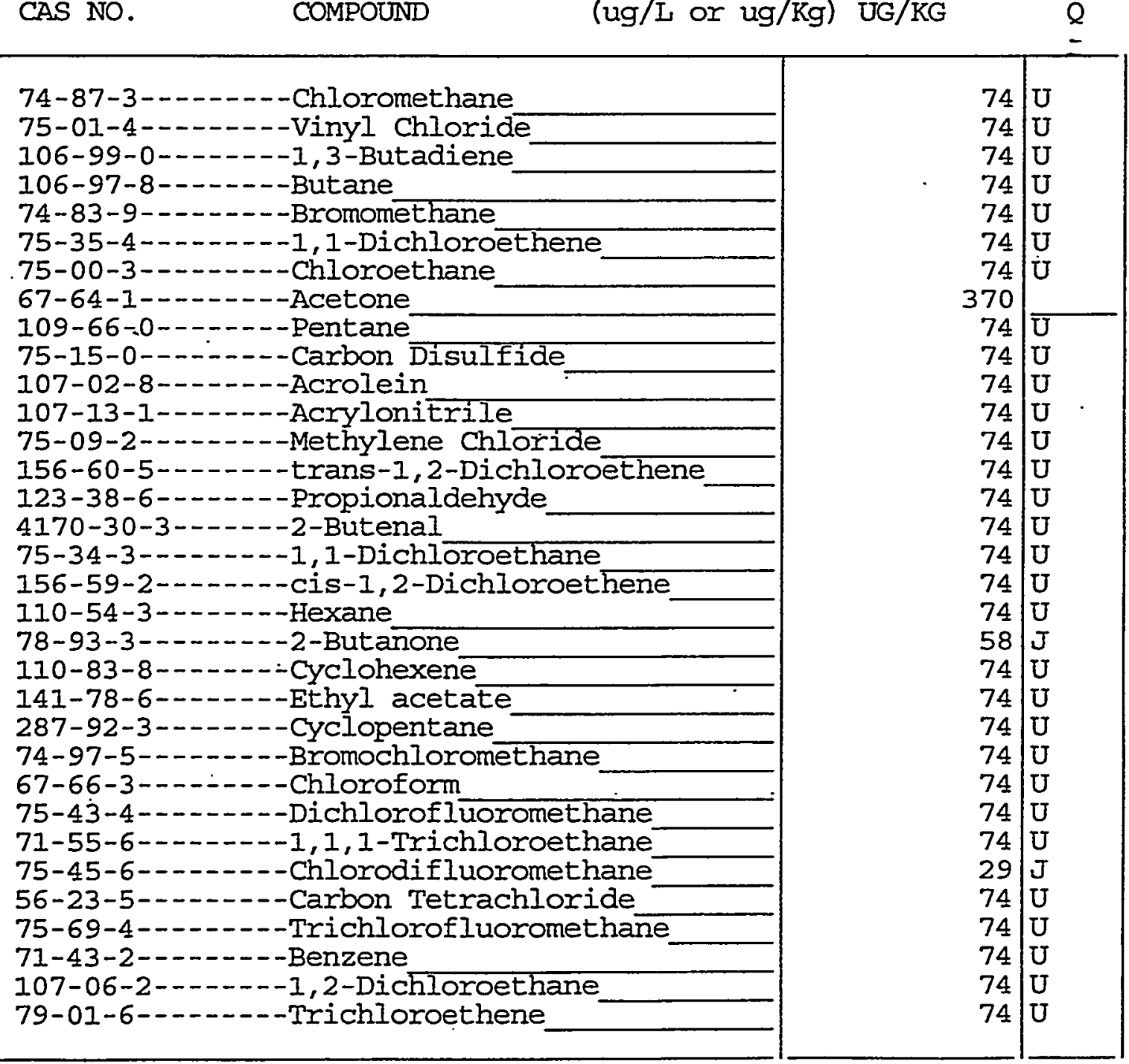


$1 A$

VOLATILE ORGANICS ANALYSIS DATA SHEET
Lab Name: PNNL,

Lab code: PNNL
Contract: BNFL

SAS NO.:
EPA SAMPLE NO.

AN-107D
Matrix: (soil/water) SOLID

Sample wt/vol:

$0.7(\mathrm{~g} / \mathrm{mL}) \mathrm{G}$

Level: (low/med) IOW

$\div$ Moisture: not dec.

GC Column: DB-624 75M X 2.55UM ID: 0.45 (mm)

Soil Extract Volume: (uI)
Lab Sample ID: 99-0651D

Lab File ID: 99021611

Date Received:

Date Analyzed: 02/17/99

Dilution Factor: 1.0

Soil Aliquot Volume:

(uL)

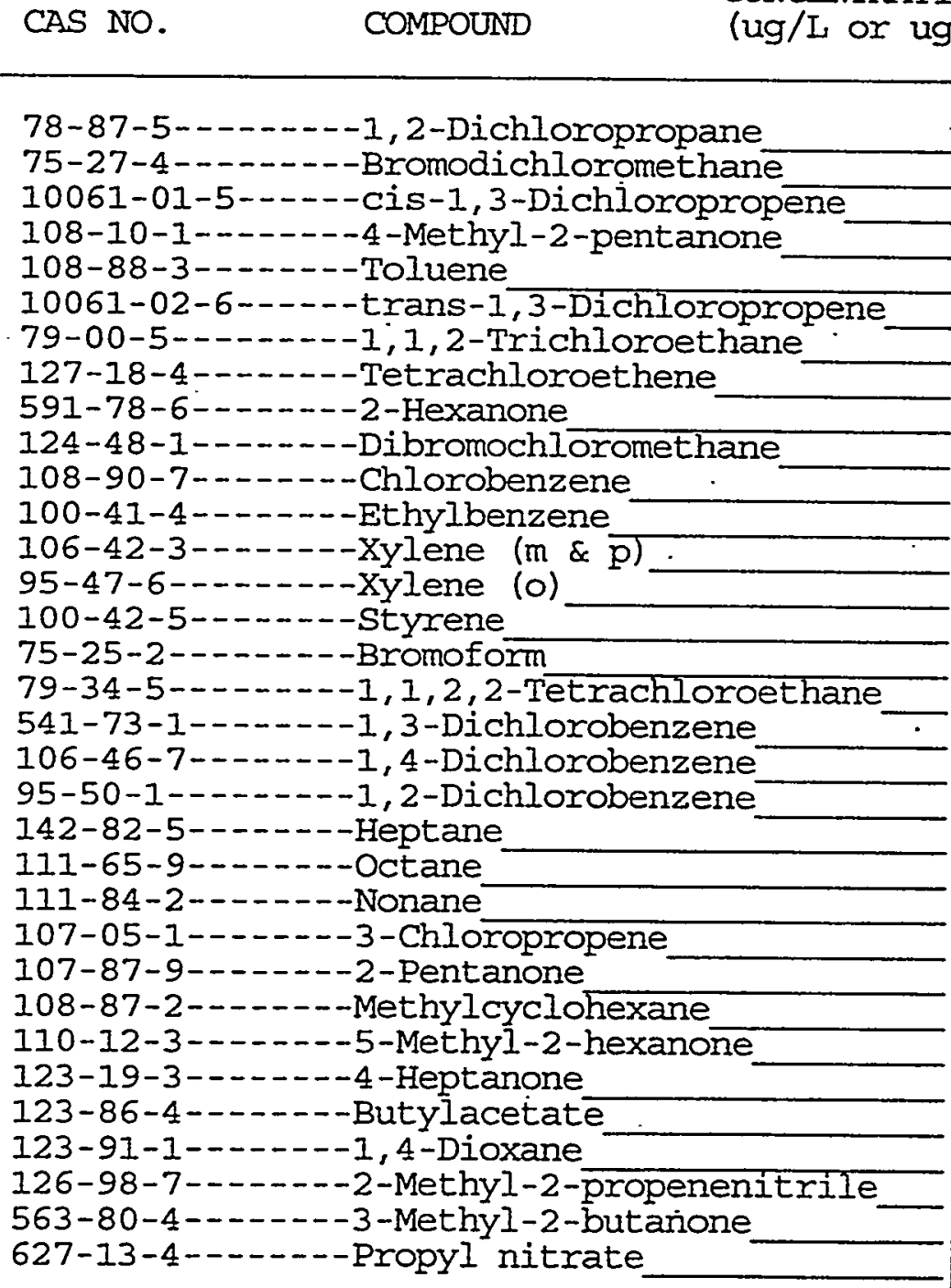

\section{NTRATION UNITS: \\ CONCENTRATION UNITS:}


Lab Name: PNNL

Lab Code: PNNL

Case No. :

Matrix: (soil/water) SOLID

Sample wt/vol:

$0.7(\mathrm{~g} / \mathrm{mL}) \mathrm{G}$

Level: (low/med) IOW

\% Moisture: not dec.

GC Column: DB-62475M X 2.55UM ID: 0.45 (mm)

Soil Extract Volume: (uI)
Contract: BNFL

SAS NO. :
EPA SAMPLE NO.

AN-107D

SDG NO.: 99021601

Lab Sample ID: 99-0651D

Lab File ID: 99021611

Date Received:

Date Analyzed: 02/17/99

(mm) Dilution Factor: 1.0

Soil Aliquot Volume: (ur)
CAS NO.
COMPOUND
(ug/L or ug/Kg) UG/KG

CONCENTRATION UNITS:

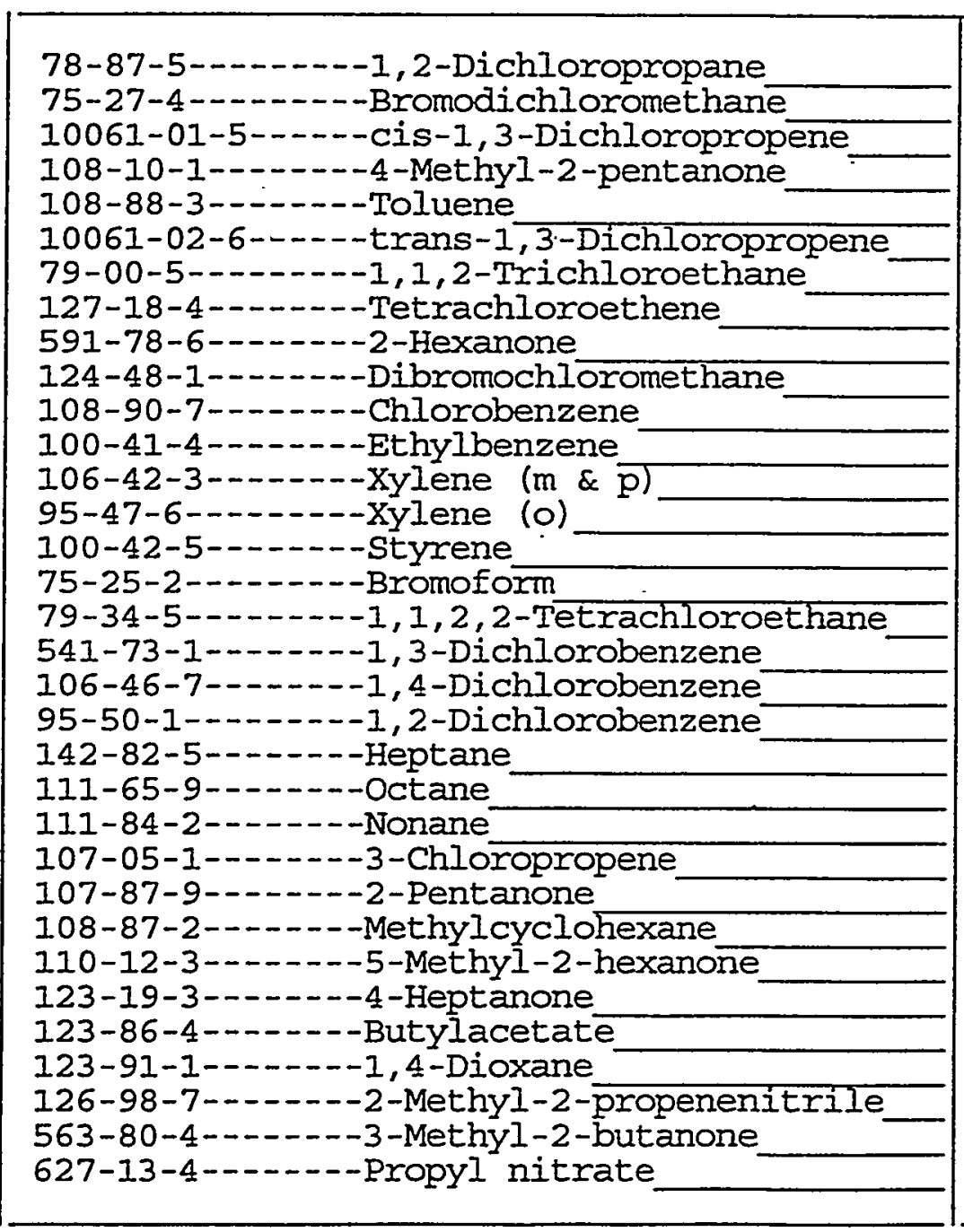


Lab Name: PNNL

Lab Code: PNNL
Contract: BNFL

SAS NO.:
AN-107D
Matrix: (soil/water) SOLID

Sample wt/vol:

$0.7(\mathrm{~g} / \mathrm{mL}) \mathrm{G}$

Level: (low/med) IOW

$\therefore$ Moisture: not dec.

GC Column: $\mathrm{DB}-62475 \mathrm{M} \times 2.55 \mathrm{UM}$. ID: 0.45 (mm) (uI)

Soil Extract Volume:

Lab Sample ID: 99-0651D

Lab File ID: $\quad 99021611$

Date Received:

Date Analyzed: 02/17/99

Dilution Factor: 1.0

Soil Aliquot Volume: (UI) CONCENTRATION UNITS:

CAS NO.

COMPOUND (ug/I or ug/Kg) UG/KG

\begin{tabular}{|c|}
\hline 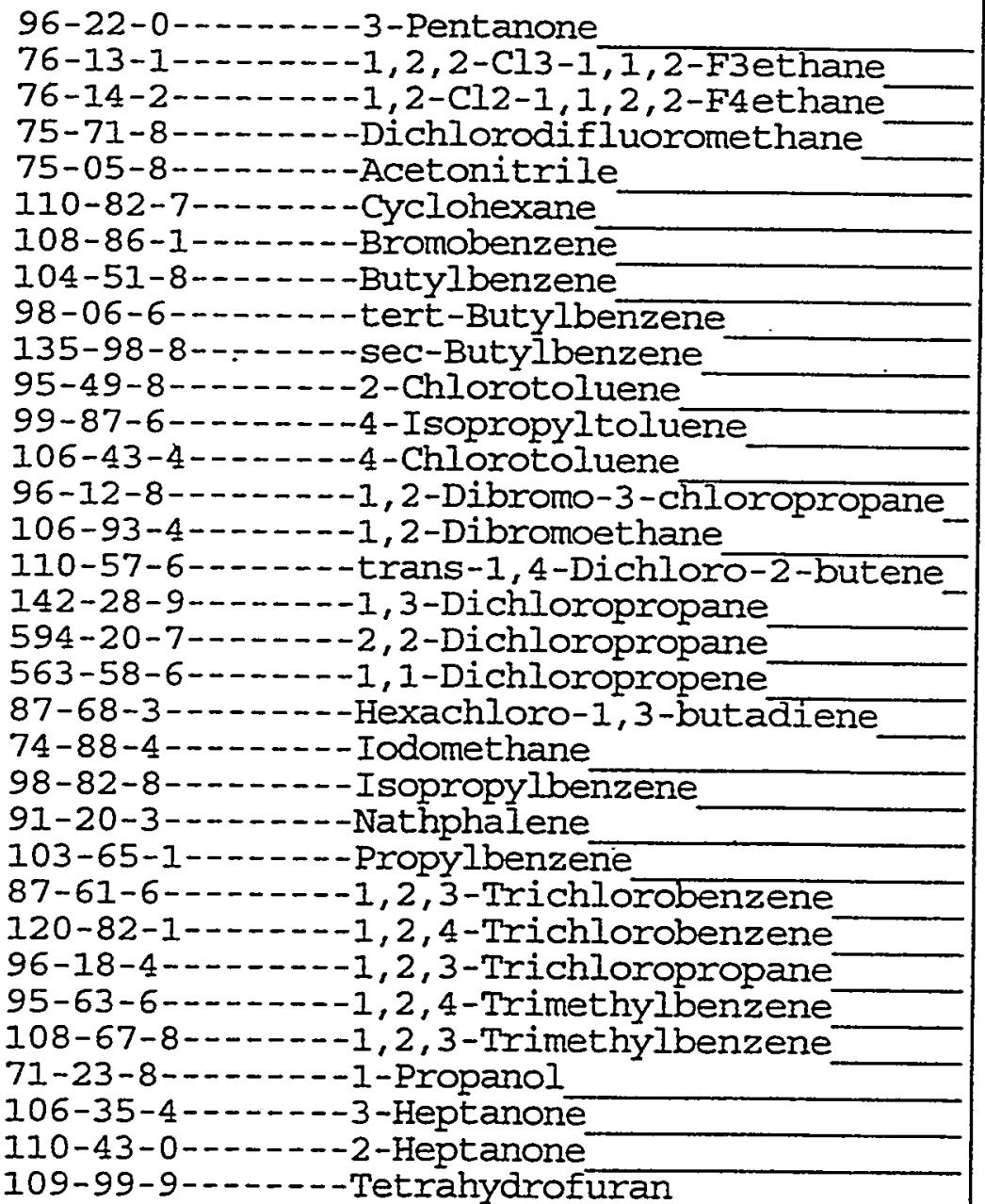 \\
\hline
\end{tabular}

\begin{tabular}{r|l}
\multicolumn{2}{l}{} \\
\multicolumn{1}{l}{} \\
\hline 74 & $\mathrm{U}$ \\
74 & $\mathrm{U}$ \\
74 & $\mathrm{U}$ \\
74 & $\mathrm{U}$ \\
89 & \\
74 & $\mathrm{U}$ \\
74 & $\mathrm{U}$ \\
74 & $\mathrm{U}$ \\
74 & $\mathrm{U}$ \\
74 & $\mathrm{U}$ \\
74 & $\mathrm{U}$ \\
74 & $\mathrm{U}$ \\
74 & $\mathrm{U}$ \\
74 & $\mathrm{U}$ \\
74 & $\mathrm{U}$ \\
74 & $\mathrm{U}$ \\
74 & $\mathrm{U}$ \\
74 & $\mathrm{U}$ \\
74 & $\mathrm{U}$ \\
74 & $\mathrm{U}$ \\
74 & $\mathrm{U}$ \\
74 & $\mathrm{U}$ \\
74 & $\mathrm{U}$ \\
74 & $\mathrm{U}$ \\
74 & $\mathrm{U}$ \\
74 & $\mathrm{U}$ \\
74 & $\mathrm{U}$ \\
74 & $\mathrm{U}$ \\
74 & $\mathrm{U}$ \\
74 & $\mathrm{U}$ \\
8 & $\mathrm{~J}$ \\
15 & $\mathrm{~J}$ \\
36 & $\mathrm{~J}$ \\
& \\
\hline
\end{tabular}


$I A^{\circ}$

VOLATİIE ORGANICS ANALYSIS DATA SHEET

Lab Name: PNNL

Contract: BNFL

Lab Code: PNNL

Case No.:

Matrix: (soil/water) SOLID

Sample wt/vol:

$0.7(\mathrm{~g} / \mathrm{mL}) \mathrm{G}$

Level: (low/med) IOW

SAS NO.:

Lab Sample ID: 99-0651D

Lab File ID: .99021611

Date Received:

Date Analyzed: 02/17/99

\% Moisture: not dec.

GC.Column: $\mathrm{DB}-62475 \mathrm{M} \times 2.55 \mathrm{UM}$ ID: 0.45 (mm)

Soil Extract Volume: (uL)

Soil Aliquot Vólume: (uI)
Dilution Factor: 1.0
$A N-107 D$

SDG NO.: 99021601
CAS NO. COMPOUND

74-95-3--..--Dibromomethane (ug/I or $u g / \mathrm{Kg}$ ) UG/KG 
Lab Name: PNNL

Lab Code: PNNL
Contract: BNFL

Case No.:
SAS NO. :
EPA SAMPLE NO.

AN-107MSD
Matrix: (soil/water) SOLID

Sample wt/vol:

$0.5(\mathrm{~g} / \mathrm{mI}) \mathrm{G}$

Level: (low/med) IOW

$\%$ Moisture: not dec.

GC Column: $\mathrm{DB}-62475 \mathrm{M} \times 2.55 \mathrm{UM}$ ID: 0.45 (mm) Soil Extract Volume: (UI)
Lab Sample ID: 99-0651MSD

Lab File ID: 99021612

Date Received:

Date Analyzed: 02/17/99

Dilution Factor: 1.0

Soil Aliquot Volume: (uL)
CAS NO. CONCENTRATION UNITS:

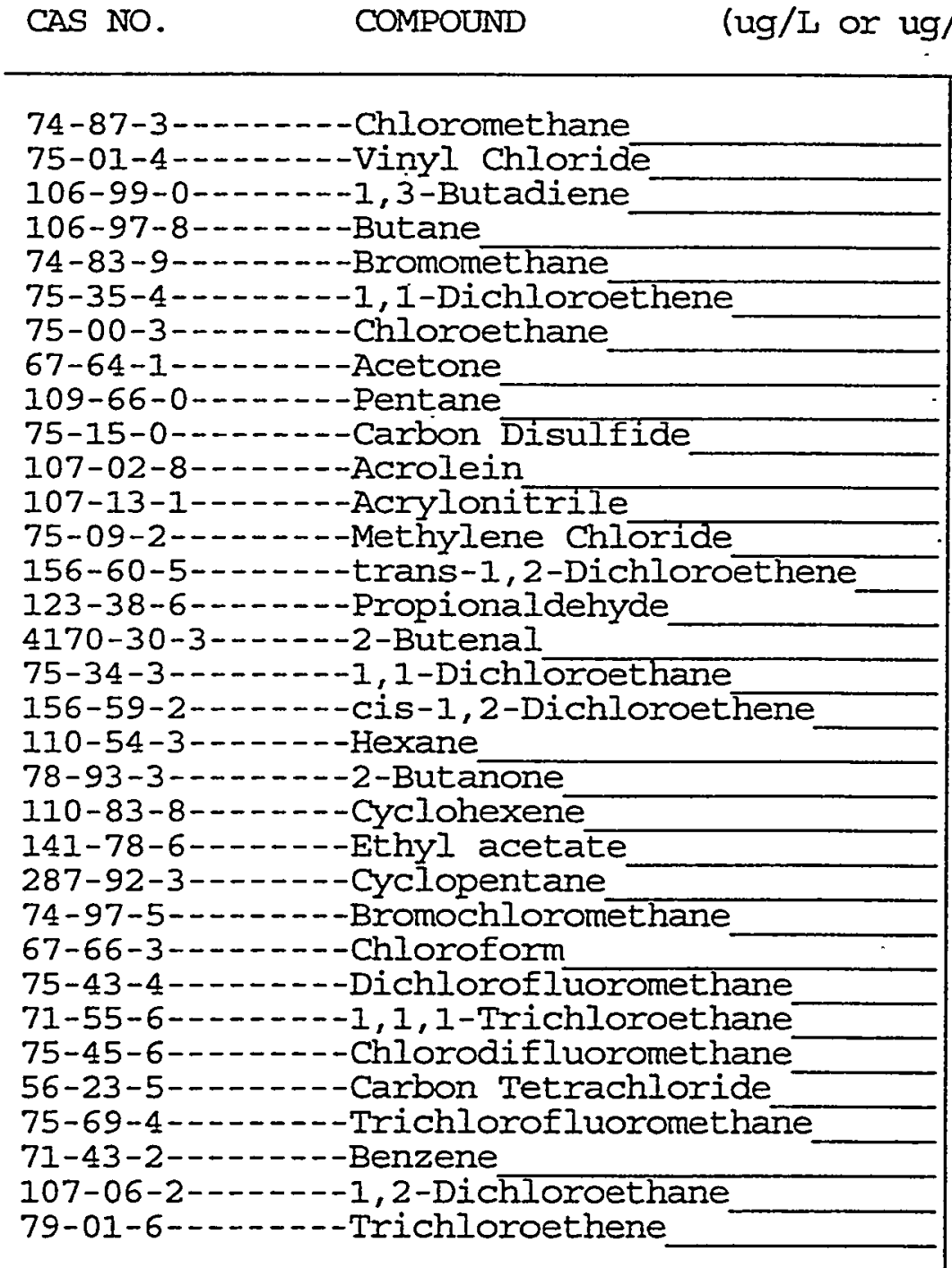


Lab Name: PNNL

Lab Code: PNNL

Case No.:
Contract: BNEL

SAS NO.:

AN-I07MSD

SDG No. : 99021601

Matrix: (soil/water) SOLID

Sample wt/vol:

$0.5(\mathrm{~g} / \mathrm{mL}) \mathrm{G}$

Level: (low/med) LoW

$\%$ Moisture: not dec.

GC Column: $\mathrm{DB}-62475 \mathrm{M} \times 2.55 \mathrm{UM}$ ID: 0.45 (mm)

Soil Extract Volume: (UI)
Lab Sample ID: 99-0651MSD

Lab File ID: 99021612

Date Received:

Date Analyzed: 02/17/99 (UI)

CAS NO.

COMPOUND CONCENTRATION UNITS: (ug/L or $\mathrm{ug} / \mathrm{Kg}$ ) UG/KG

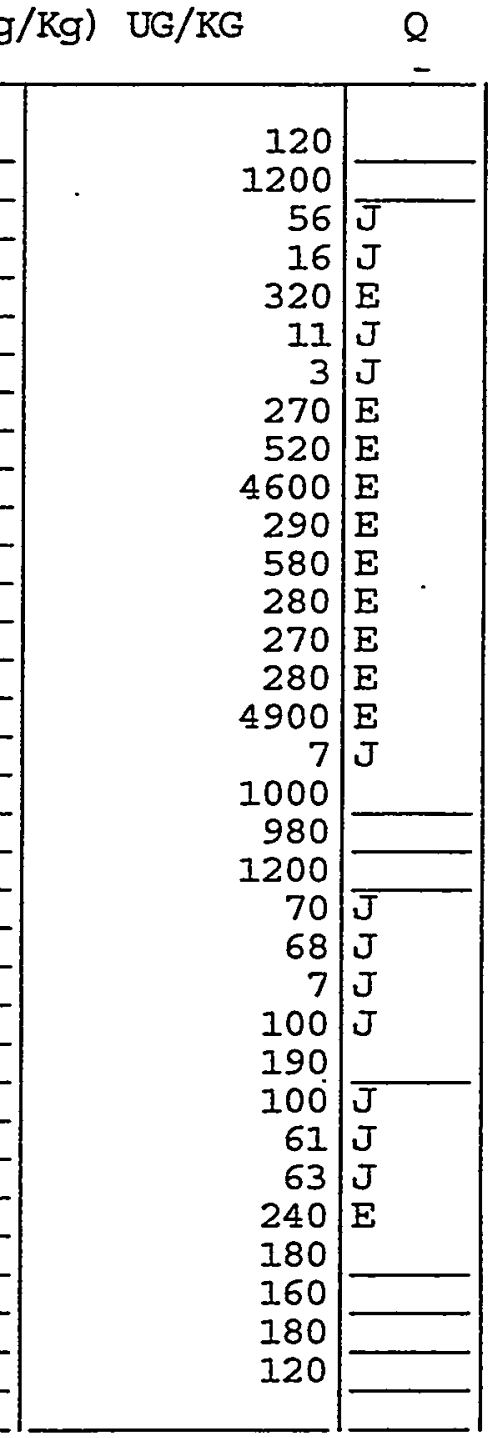

78-87-5------1, 2-Dichloropropane

75-27-4-1.-.--Bromodichloromethane 10061-01-5--.--cis-1, 3-Dichloropropene

108-10-1-..---4-Methyl-2-pentanone

108-88-3-.-.-.-Toluene

10061-02-6--.--trans-1,3-Dichloropropene

79-00-5--.-.-1,1,2-Trichloroethane

127-18-4--.----Tetrachloroethene

591-78-6-1----2-Hexanone

124-48-1-...-Dibromochloromethane

108-90-7-...---Chlorobenzene

100-41-4-...-.-Ethylbenzene

106-42-3-..---Xylene $(m \&$ p)

95-47-6-.....--Xylene (o)

100-42-5-...-.--Styrene

75-25-2-...-.-Bromoform

79-34-5--.----1, 1,2,2-Tetrachloroethane

541-73-1-.---1, 3-Dichlorobenzene

106-46-7--.--1, 4-Dichlorobenzene

95-50-1-..---1, 2-Dichlorobenzene

142-82-5--..---Heptane

$111-65-9-\ldots---0$-Otane

$111-84-2-\ldots-.-$ Nonane

107-05-1-...-3-Chloropropene

107-87-9-1.---2-Pentanone

108-87-2-.----Methylcyclohexane

110-12-3--.---5-Methyl-2-hexanone

123-19-3-...---4-Heptanone

123-86-4-.----Butylacetate

123-91-1--.---1,4-Dioxane

126-98-7-...--2-Methyl-2-propenenitrile

563-80-4-.----3-Methyl-2-butanone

627-13-4-..-.--Propyl nitrate 
Lab Name: PNNL

Lab code: PNNL

Matrix: (soil/water) SOLID

Sample wt/vol:

$0.5(\mathrm{~g} / \mathrm{mL}) \mathrm{G}$

Level: (low/med) Low

$\%$ Moisture: not dec.

GC Column: DB-624 75M X 2.55UM ID: 0.45 (mm)

Soil Extract Volume: (UL)

Contract: BNFL

SAS NO.:

EPA SAMPLE NO.

AN-107MSD

SDG NO.: 99021601

Lab Sample ID: 99-065IMSD

Lab File ID: 99021612

Date Received:

Date Analyzed: 02/17/99

(mm) . Dilution Factor: 1.0

Soil Aliquot Volume:

(uL)

CONCENTRATION UNITS:

CAS NO. COMPOUND (ug/L or $\mathrm{ug} / \mathrm{Kg})$ UG/KG

96-22-0--.----3-Pentanone

76-13-1-...--1, 2,2-Cl3-1,1,2-F3ethane

76-14-2-....-.-1,2-Cl2-1, 1, 2,2-F4ethane

75-71-8-...--Dichlorodifluoromethane

75-05-8-..-.-.-Acetonitrile

110-82-7-.....-. Cyclohexane

108-86-1-...---Bromobenzene

104-51-8-------Butylbenzene

98-06-6-------tert-Butylbenzene

135-98-8-------sec-Butylbenzene

95-49-8-------2-Chlorotoluene

99-87-6-...----4-Isopropyltoluene

106-43-4-...--4-Chlorotoluene

96-12-8-...--1, 2-Dibromo-3-chloropropane

106-93-4-...-1, 2-Dibromoethane

110-57-6-.----trans-1,4-Dichloro-2-butene

142-28-9-..--1, 3-Dichloropropane

594-20-7-..--2,2-Dichloropropane

563-58-6-...-1,1-Dichloropropene

87-68-3-..---Hexachloro-1, 3-butadiene

74-88-4-..-----Iodomethane

98-82-8--.-----Isopropylbenzene

91-20-3-..----Nathphalene

103-65-1-...--.-Propylbenzene

87-61-6-......-1, 2, 3-Trichlorobenzene

120-82-1---.--1,2,4-Trichlorobenzene

96-18-4--.---1,2,3-Trichloropropane

95-63-6-....--1, 2,4-Trimethyl benzene

108-67-8-..---1,2,3-Trimethylbenzene

71-23-8-..--.-1-Propanol

106-35-4-..----3-Heptanone

110-43-0-...--2-Heptanone

109-99-9-.---.-Tetrahydrofuran

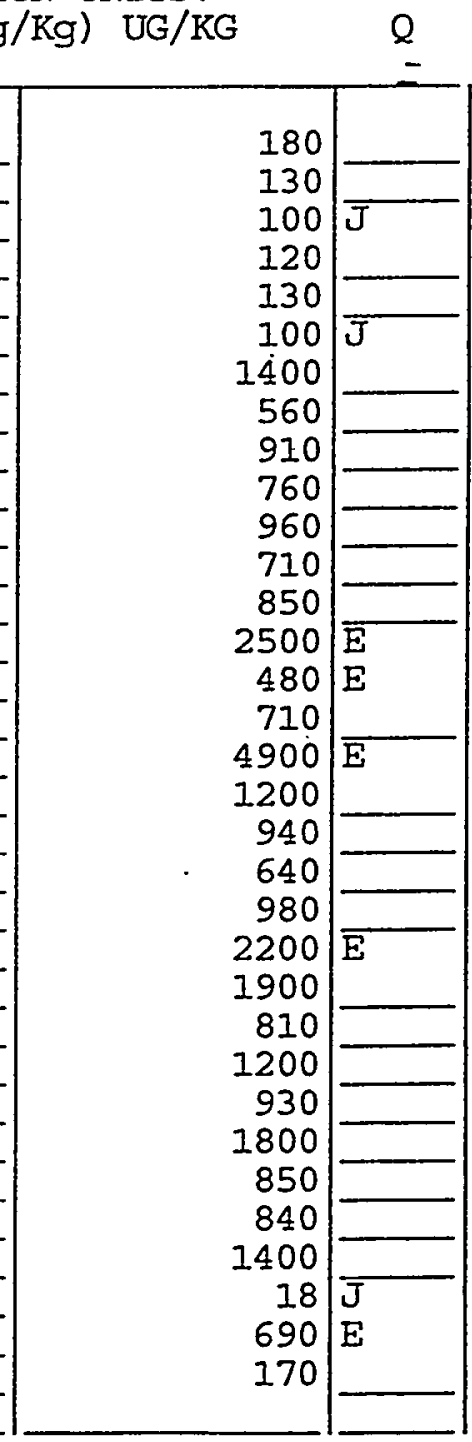




\section{VOLLATIIE ORGANICS ANALYSIS DATA SHEET}

Lab Name: PNNL

Iab Code: PNNL

Case No.:

Matrix: (soil/water) SOLID

Sample wt/vol:

$0.5(\mathrm{~g} / \mathrm{mL}) \mathrm{G}$

Level: (low/med) LOW

$\%$ Moisture: not dec.

GC Column: DB-624 75M X 2.55UM ID: 0.45 (mm) Soil Extract Volume: (UI)

Contract: BNFL,

SAS NO.:

SDG No.: 99021601

Lab Sample ID: 99-0651MSD

Iab File ID: 99021612

Date Received:

Date Analyzed: 02/17/99

. Dilution Factor: 1.0

Soil Aliquot Volume: (uL)

CAS NO. COMPOUND $\quad(u g / L$ or $\mathrm{ug} / \mathrm{Kg})$ UG/KG $Q$

\begin{tabular}{|c|c|}
\hline 74-95-3--n-Dibromomethane & 1300 \\
\hline
\end{tabular}


Lab Name: PNNL

Lab code: PNNL, Case No.:

Matrix: (soil/water) SOLID

Sample wt/vol:

$5.0(\mathrm{~g} / \mathrm{mL}) \mathrm{G}$

Ievel: (low/med) LOW

$\%$ Moisture: not dec.

GC Column: $\mathrm{DB}-62475 \mathrm{M} \times 2.55 \mathrm{UM}$ ID: 0.45 (mm) Soil Extract Volume: (UI)

Contract: BNFL

SAS NO.:

SDG NO.: 99021601

Lab Sample ID: VBLK03

Lab File ID: 99021604

Date Received:

Date Analyzed: 02/16/99

Dilution Factor: 1.0

Soil Aliquot Volume:

(uI) CONCENTRATION UNITS:

CAS NO. COMPOUND (ug/L or $u g / \mathrm{Kg}$ ) UG/KG

74-87-3-...---Chloromethane

75-01-4-....--Vinyl Chloride

106-99-0-.-.-.-1,3-Butadiene

106-97-8-...-.-Butane

74-83-9-...-.-Bromomethane

75-35-4-.-- - 1, 1-Dichloroethene

75-00-3.....-. Chloroethane

67-64-1-...-.-Acetone

109-66-0-..-.--Pentane

75-15-0-.-.--Carbon Disulfide

107-02-8-...--Acrolein

107-13-1-...--Acrylonitrile

75-09-2-...-.-Methylene Chloride

156-60-5--..--trans-1,2-Dichloroethene

123-38-6-------Propionaldehyde

4170-30-3-...--2-Butenal

75-34-3-..--1, 1-Dichloroethane

156-59-2-.----cis-1, 2-Dichloroethene

110-54-3-...-.-Hexane

78-93-3--.----2-Butanone

110-83-8-...--.-Cyclohexene

141-78-6-...-.-Ethyl acetate

287-92-3------Cyclopentane

74-97-5-...---Bromochloromethane

67-66-3-....-Chloroform

75-43-4--.----Dichlorofluoromethane

71-55-6-...--1, 1, 1-Trichloroethane

75-45-6-...-.-Chlorodifluoromethane

56-23-5--.----Carbon Tetrachloride

75-69-4-...-.-Trichlorofluoromethane

71-43-2-...---Benzene

107-06-2-..--1,2-Dichloroethane

79-01-6--.---Trichloroethene

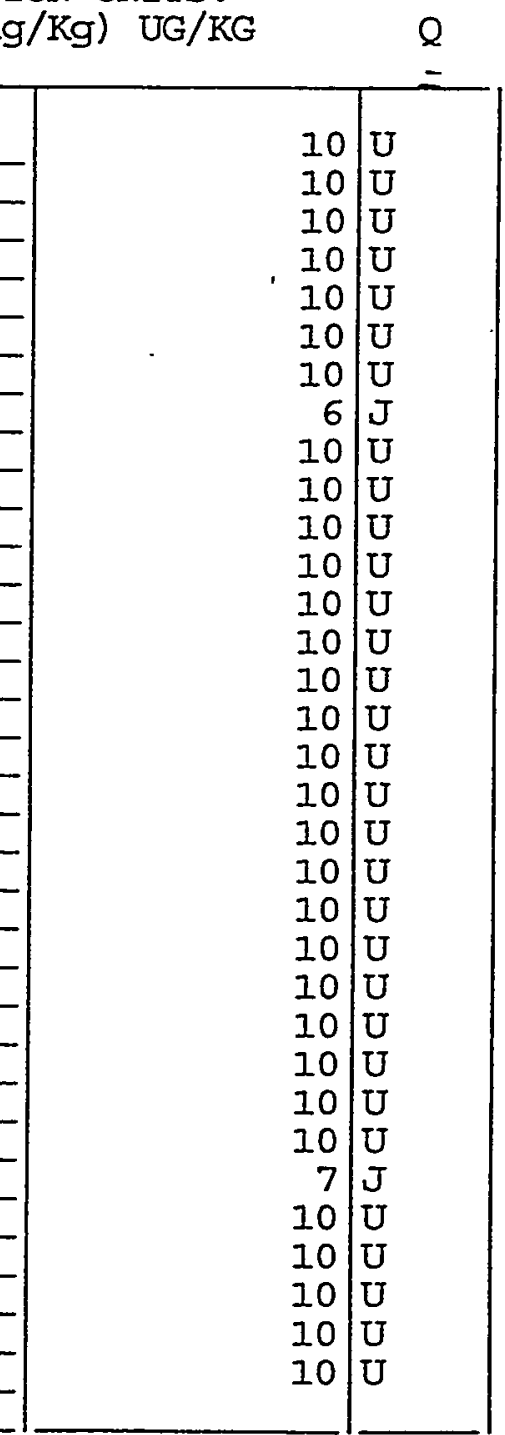


Lab Name: PNNL

Contract: BNFL

Lab Code: PNNL

Case No.:

Matrix: (soil/water) SOLID

Sample wt/vol:

$5.0(\mathrm{~g} / \mathrm{mL}) \mathrm{G}$

Level: (low/med) LOW

$\div$ Moisture: not dec.

GC Column: DB-624 75M X 2.55UM ID: 0.45 (mm)

Soil Extract Volume:
SAS NO.:
EPA SAMPLE NO.

\section{METHOD BLANK}

$\cdot 1$

SDG No.: 99021601

Lab Sample ID: VBLK03

Lab File ID: 99021604

Date Received:

Date Analyzed: 02/16/99

(mm) Dilution Factor: 1.0

Soil Aliquot Volume: (uL)

CONCENTRATION UNITS:

CAS NO.

COMPOUND (ug/L or $\mathrm{ug} / \mathrm{Kg}$ ) UG/KG

\begin{tabular}{|c|}
\hline 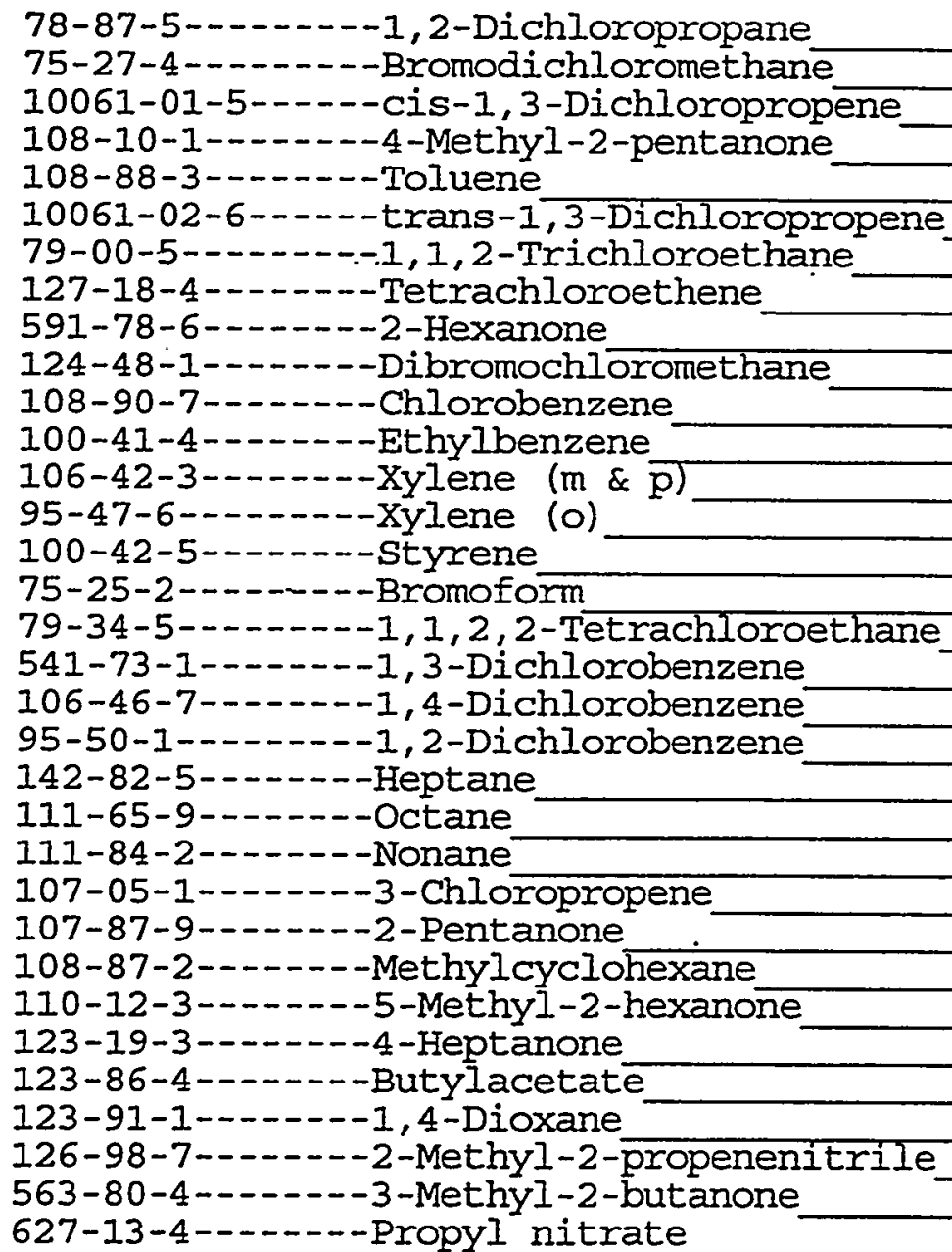 \\
\hline
\end{tabular}

FORM I VOA
OLM03.0 
Lab Name: PNNL

Lab Code: PNNL
Contract: BNFL

SAS NO.:
Matrix: (soil/water) SOIID

Sample wt/vol:

$5.0(\mathrm{~g} / \mathrm{mL}) \mathrm{G}$

Level: (low/med) Low

$\div$ Moisture: not dec.

GC Column: DB-624 75M X 2.55UM ID: 0.45 (mm)

\author{
(ur)
}

Lab Sample ID: VBLK03

Iab File ID: 99021604

Date Received:

Date Analyzed: 02/16/99
Soil Extract Volume:

COMPOUND
Dilution Factor: 1.0

Soil Aliquot Volume: (UI)
CAS NO.

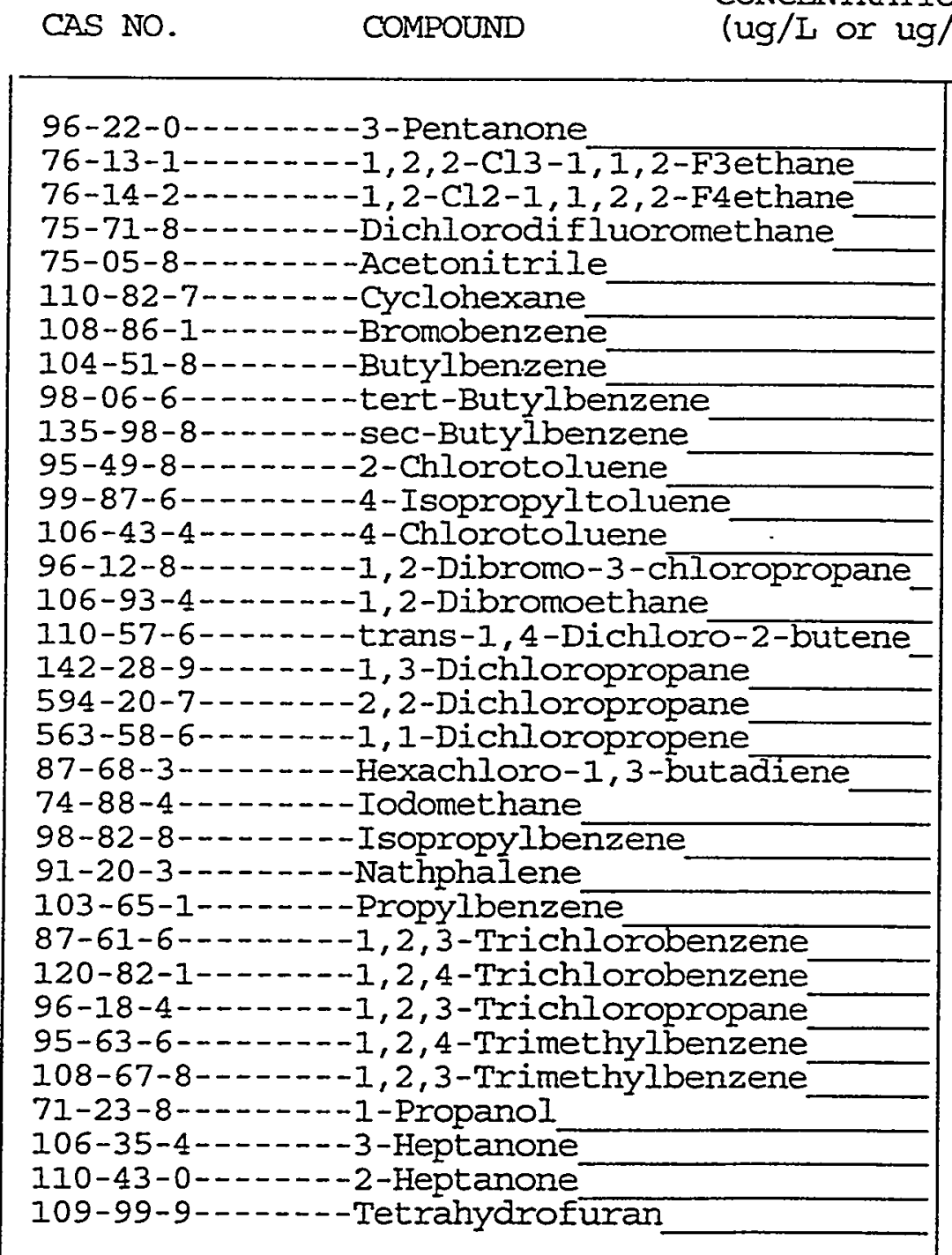
CONCENTRATION UNITS:
(ug/L or $u g / \mathrm{Kg}$ ) UG/KG

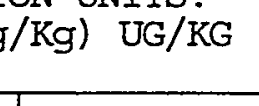


Lab Name: PNNL,

Lab Code: PNNL

Case No.:

Matrix: (soil/water) SOLID

Sample wt/vol:

$5.0(\mathrm{~g} / \mathrm{mL}) \mathrm{G}$

Level: (low/med) Low

$\%$ Moisture: not dec.

- GC Column: DB-624 75M X 2.55UM ID: 0.45 (mm) Soil Extract Volume: (UI)

\section{METHOD BLANK}

Contract: BNFL

SAS NO.:
SDG NO.: 99021601

Iab Sample ID: VBLK03

Iab File ID: 99021604

Date Received:

Date Analyzed: 02/16/99

\begin{tabular}{|c|c|c|c|c|}
\hline CAS NO. & COMPOUND & $\begin{array}{l}\text { CONCENTRATION UNITS: } \\
(\mathrm{ug} / \mathrm{I} \text { or } \mathrm{ug} / \mathrm{Kg}) \text { UG/KG }\end{array}$ & & \\
\hline $74-95-3$ & -Dibromomethane & & 10 & $\mathrm{~J}$ \\
\hline
\end{tabular}


1 A

VOLATILE ORGANICS ANALYSIS DATA SHEET
Lab Name: PNNL,

Lab Code: PNNL

Matrix: (soil/water) SOLID

Sample wt/vol:

$5.0(\mathrm{~g} / \mathrm{mL}) \mathrm{G}$

Level: (low/med) LOW

\% Moisture: not dec.

GC Column: DB-624 75M X 2.55UM ID: $0: 45$ (mm)

Soil Extract Volume: (uI)
Contract: BNEL

SAS NO.:
EPA SAMPLE NO.

VBLK

SDG No.: 99021601

Lab Sample ID: VBLK

Lab File ID: 99021603

Date Received:

Date Analyzed: 02/16/99

mm) Dilution Factor: 1.0

Soil Aliquot Volume: (uL) CONCENTRATION UNITS:

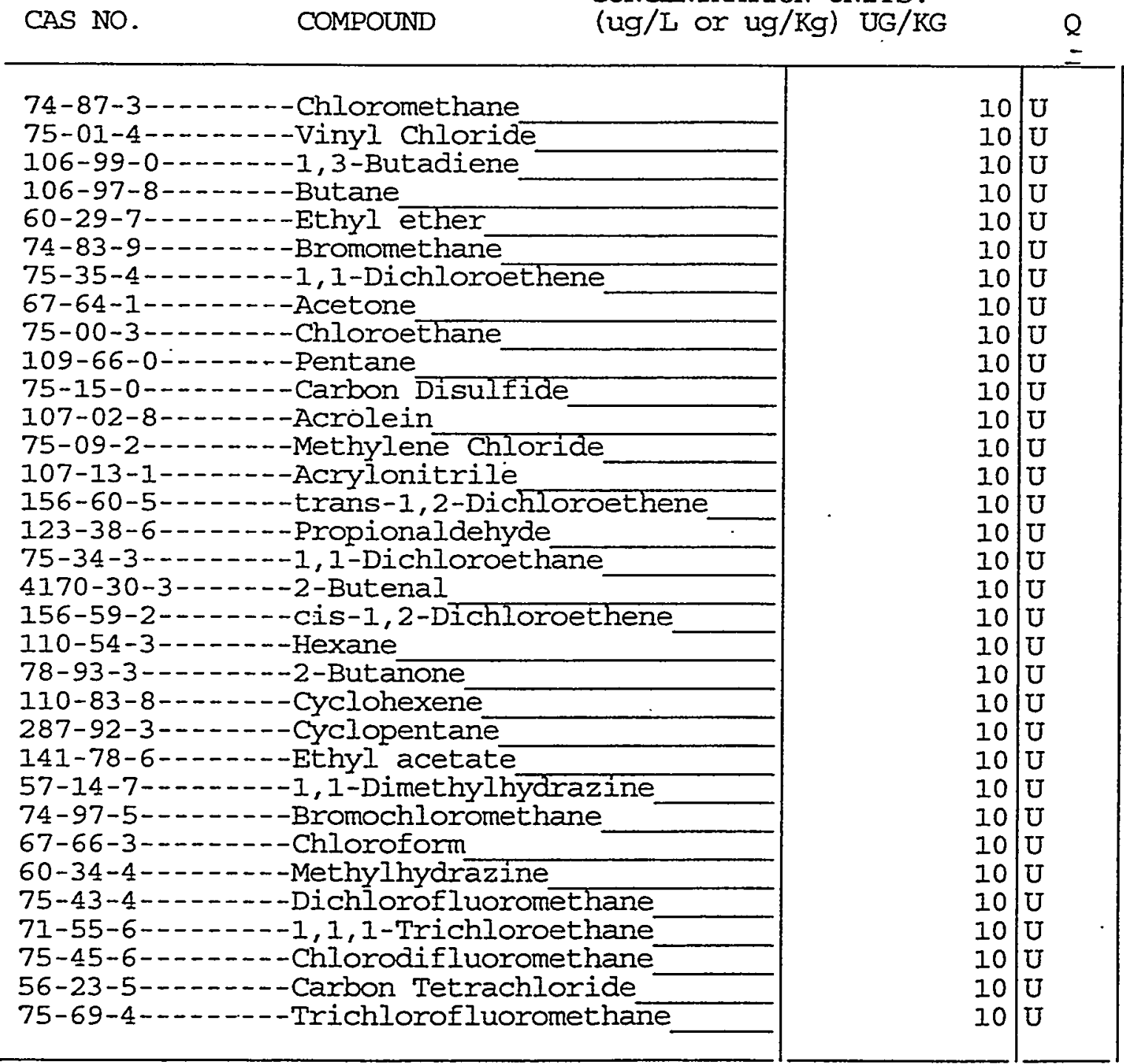


$1 A$

VÓLATILE ORGANICS ANALYSIS DATA SHEET

Lab Name: PNNL

Contract: BNFL

Lab code: PNNL

Case No.:

Matrix: (soil/water) SOLID

Sample wt/vol:

$5.0(\mathrm{~g} / \mathrm{mL}) \mathrm{G}$

Level: (low/med) LOW

$\%$ Moisture: not dec.

GC Column: DB-624 75M X 2.55UM ID: 0.45 (mm) (uI)

SAS NO. :
EPA SAMPLE NO.

VBLK

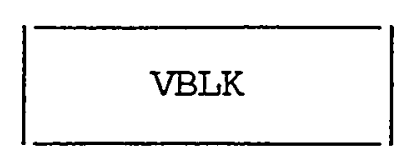

SDG NO.: 99021601
Soil Extract Volume:

CAS NO.

COMPOUND

Lab Sample ID: VBLK

Iab File ID: 99021603

Date Received:

Date Analyzed: 02/16/99

(mm) Dilution Factor: 1.0

Soil Aliquot Volume: (ut) CONCENTRATION UNITS:

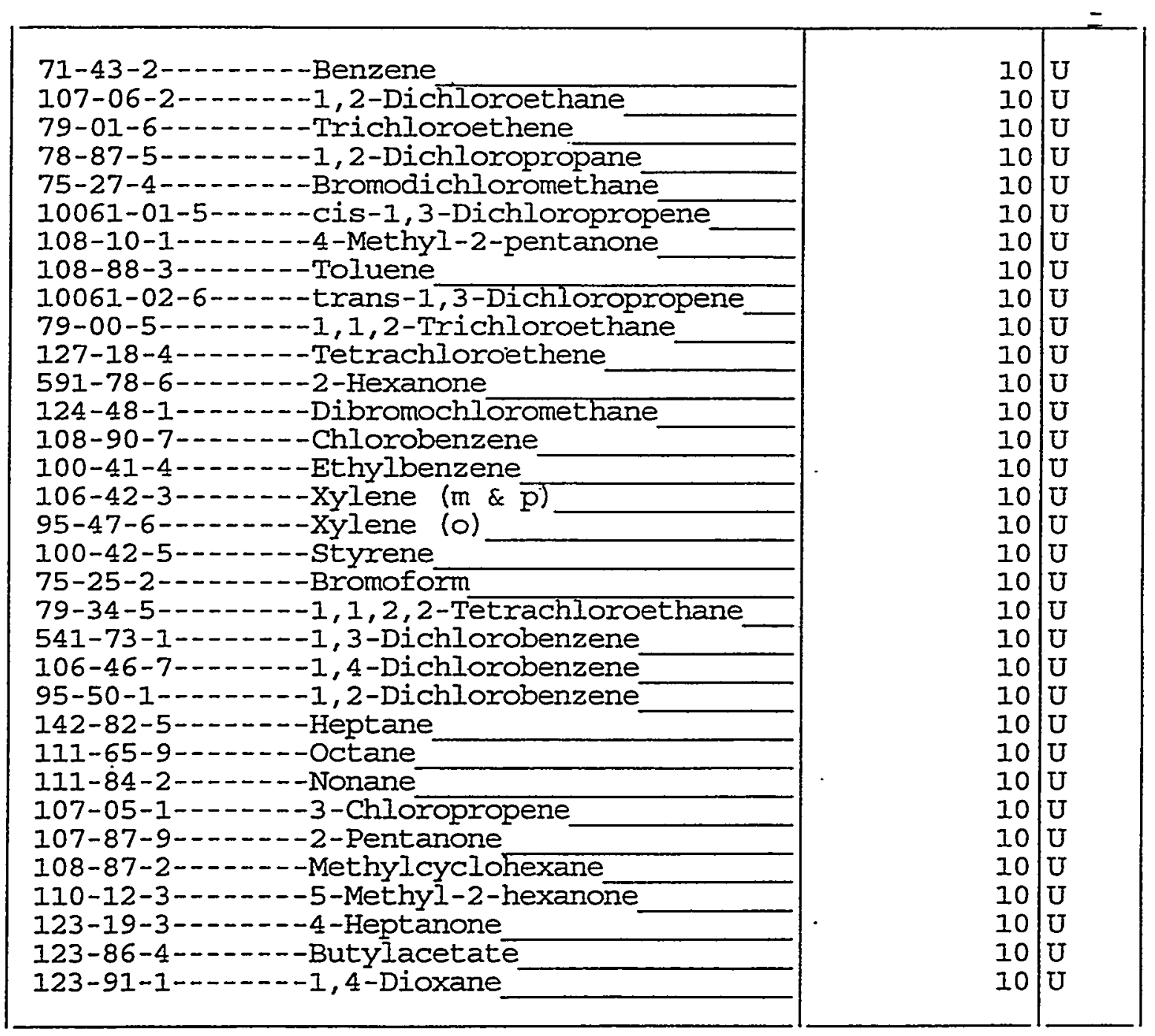


Lab Name: PNNL

Lab Code: PNNL Case No.:
Contract: BNFL

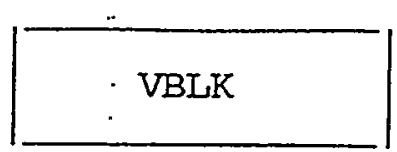

SDG NO.: 99021601
Matrix: (soil/water) SOLID

Sample wt/vol: $\quad 5.0(\mathrm{~g} / \mathrm{mL}) \mathrm{G}$

Level: (low/med) LOW

$\div$ Moisture: not dec.

GC Column: $\mathrm{DB}-62475 \mathrm{M} \times 2.55 \mathrm{UM}$ ID: 0.45 (mm)

Soil Extract Volume: (u山)
Lab Sample ID: VBLK

Lab File ID: 99021603

Date Received:

Date Analyzed: 02/16/99

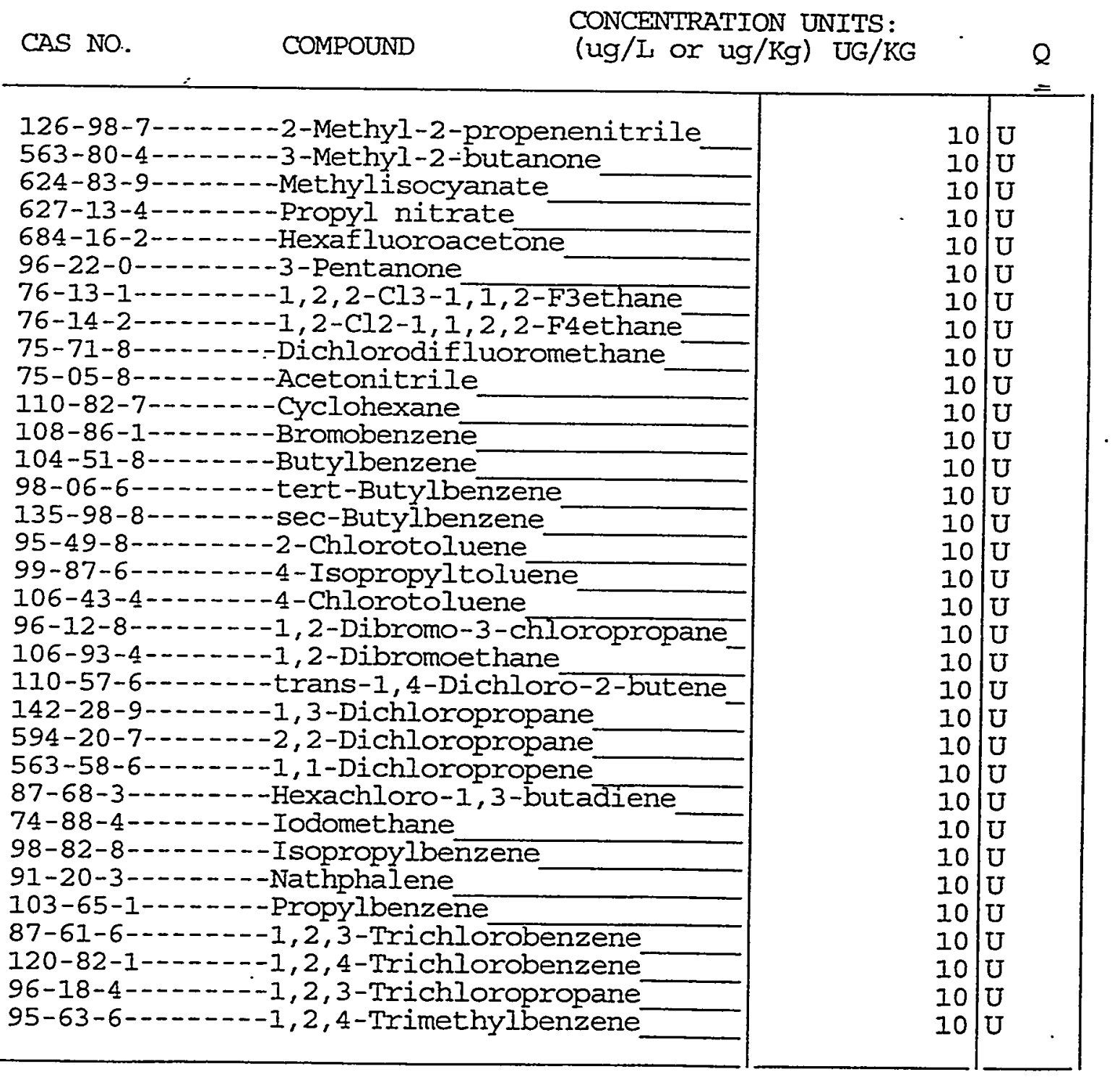


Lab Name: PNNL

Lab Code: PNNL
Contract: BNFL
EPA SAMPLE NOO.

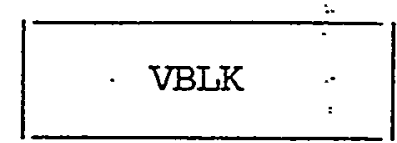

SDG NO.: 99021601
Matrix: (soil/water) SOIID

Sample wt/vol:

$5.0(\mathrm{~g} / \mathrm{mL}) \mathrm{G}$

Level: (Iow/med) LOW

$\div$ Moisture: not dec.

GC Column: DB-624 75M X 2.55UM ID: 0.45 (mm) Soil Extract Volume: (uL)
Lab Sample ID: VBLK

Lab File ID: 99021603

Date Received:

Date Analyzed: 02/16/99

Dilution Factor: 1.0

Soil Aliquot Volume: (UI)

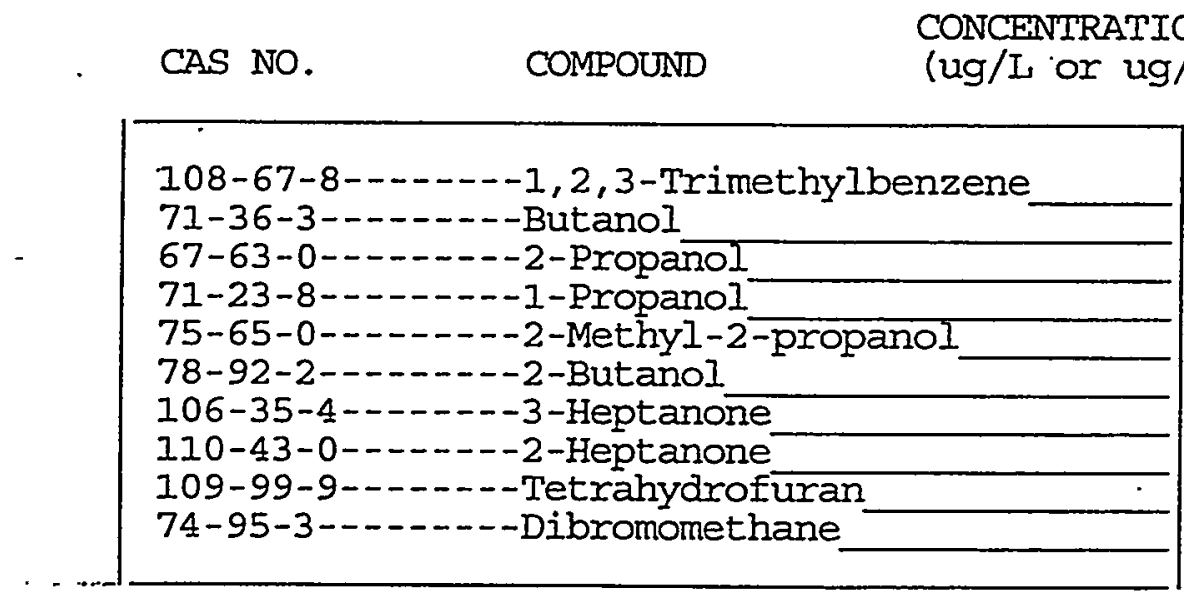


Lab Name: PNNL

Lab Code: PNNL
Contract: BNFL

SAS NO. :
AN-107

SDG No.: 99021601
Matrix: (soil/water) SOLID

Sample wt/vol:

$$
0.4(\mathrm{~g} / \mathrm{mL}) \mathrm{G}
$$

Level: (low/med) IOW

$\div$ Moisture: not dec.

GC Column: $\mathrm{DB}-62475 \mathrm{M} \times 2.55 \mathrm{MM}$ ID: 0.45 (mm) (UI)
Lab Sample ID: 99-0651

Lab File ID: 99021610

Date Received:

Date Analyzed: 02/16/99

Dilution Factor: 1.0

Soil Aliquot Volume: (uI)
Number TICs found: 3. .

(ug/L or $\mathrm{ug} / \mathrm{kg}$ ) ug/kg
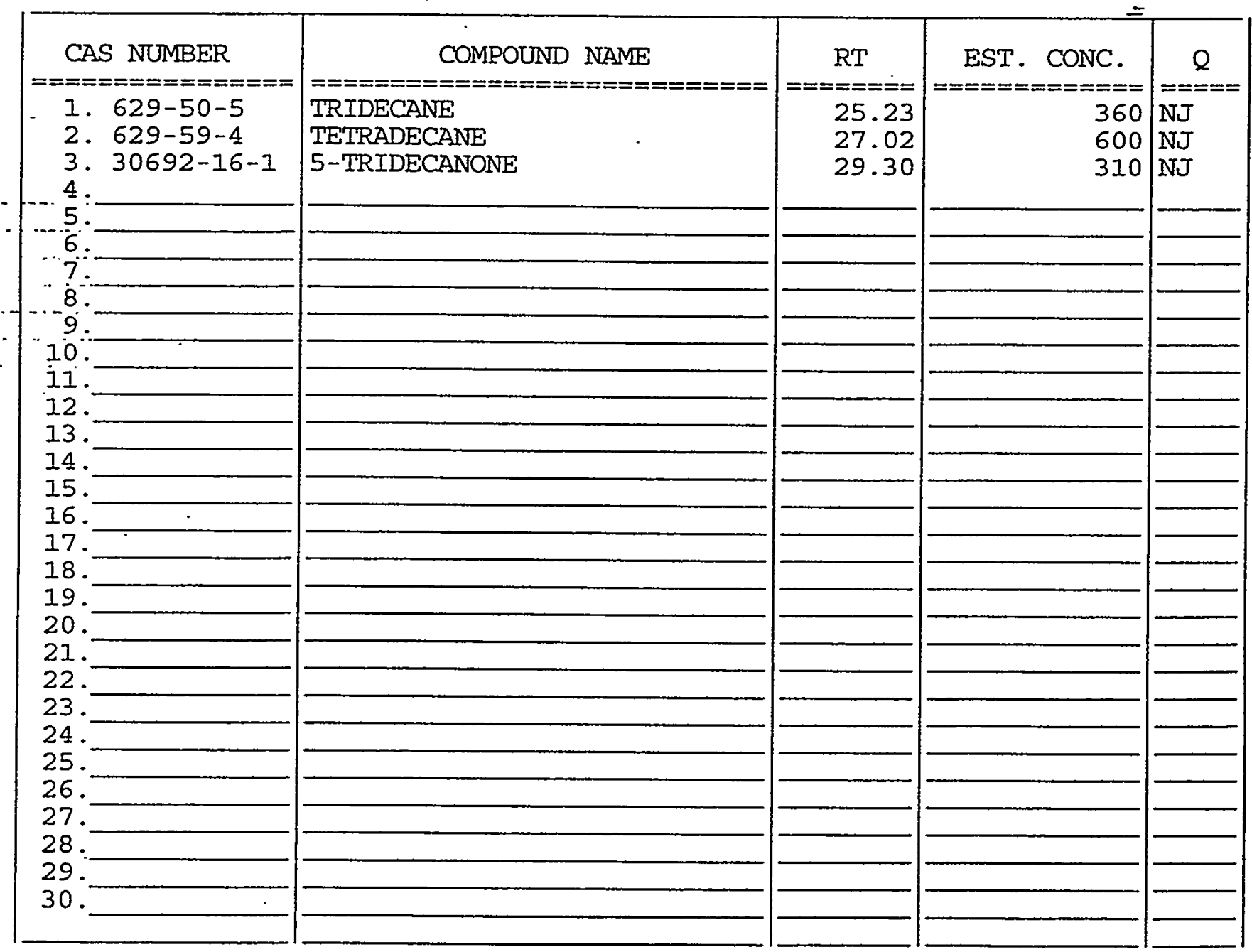
Lab Name: PNNL

Contract: BNFL

Lab Code: PNNL Case No.:

Matrix: (soil/water) SOLID

SAS NO. :
EPA SAMPLE NO.

AN-107D

SDG No.: 99021601
Sample wt/vol: $\quad 0.7$ (g/mI) G

Level: (low/med) IOW'

$\div$ Moisture: not dec.

GC Column: DB-624 75M X 2.55UM ID: 0.45 (mm)

Soil Extract Volume: (UI)
Iab Sample ID: 99-0651D

Lab File ID: 99021611

Date Received:

Date. Analyzed: 02/17/99

Dilution Factor: 1.0

Soil Aliquot Volume: (uL)

Number TICs found: 3 CONCENTRATION UNITS:

(ug/L or $\mathrm{ug} / \mathrm{Kg}$ ) $\mathrm{ug} / \mathrm{Kg}$

\begin{tabular}{|c|c|}
\hline 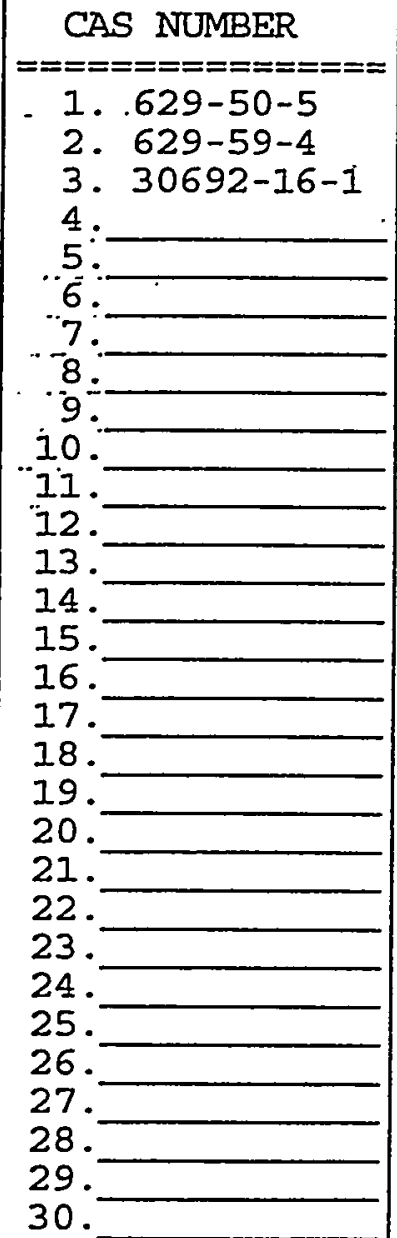 & $\begin{array}{l}\quad \text { COMPOUND NAME } \\
=========================== \\
\text { TRIDECANE } \\
\text { TETRADECANE } \\
\text { 5-TRIDECANONE } \\
\end{array}$ \\
\hline
\end{tabular}

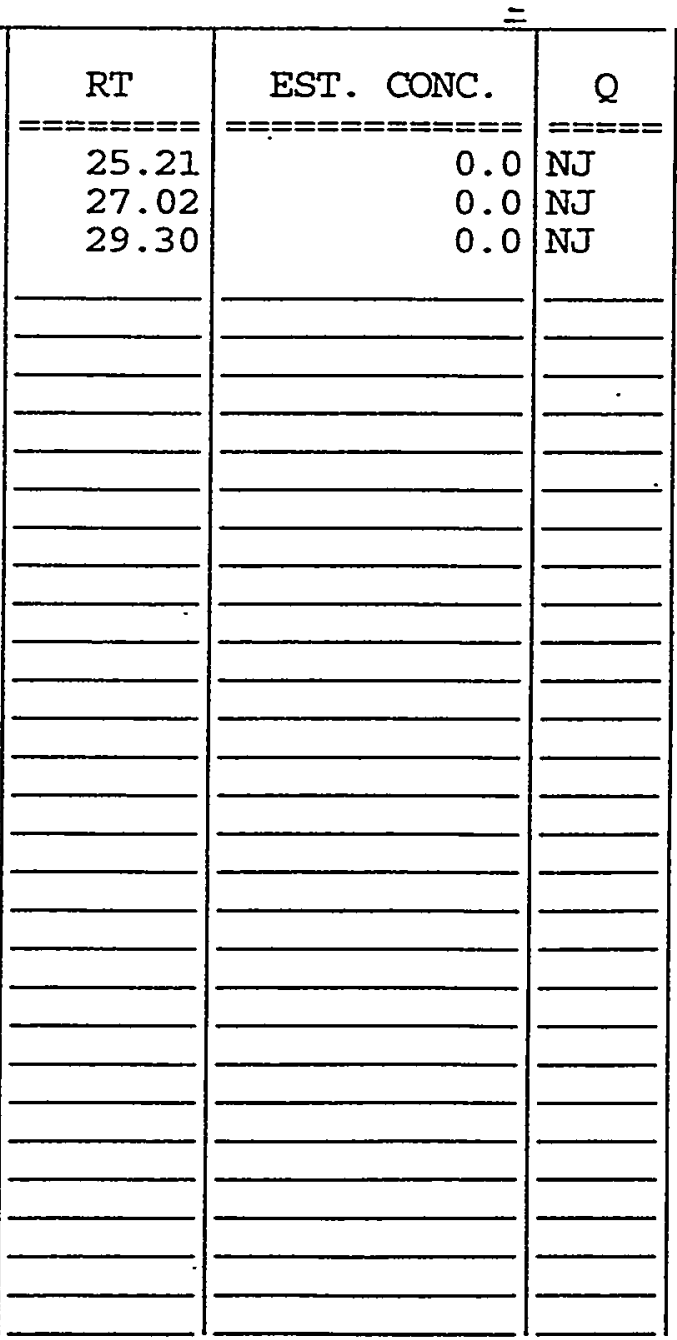


Lab Name: PNNL

Lab Code: PNNL,

Case No.:

Matrix: (soil/water) SOLID

Sample wt/vol:

$1.2(\mathrm{~g} / \mathrm{mL}) \mathrm{G}$

Level: (low/med) LOW

\% Moisture: not dec.

GC Column: DB-624 75M X 2.55UM ID: 0.45 (mm)

Soil Extract Volume: (uIi)

Number TICs found: 19
Contract: BNEL

SAS. NO. :
EPA SAMPLE NO.

AW-101

SDG No.: 99021601

Lab Sample ID: 99-0650

Lab File ID: 99021605

Date Received:

Date Analyzed: 02/16/99

Dilution Factor: 1.0

Soil Aliquot Volume: (uL)

CONCENTRATION UNITS:

(ug/L or $u g / \mathrm{Kg}) \mathrm{ug} / \mathrm{Kg}$

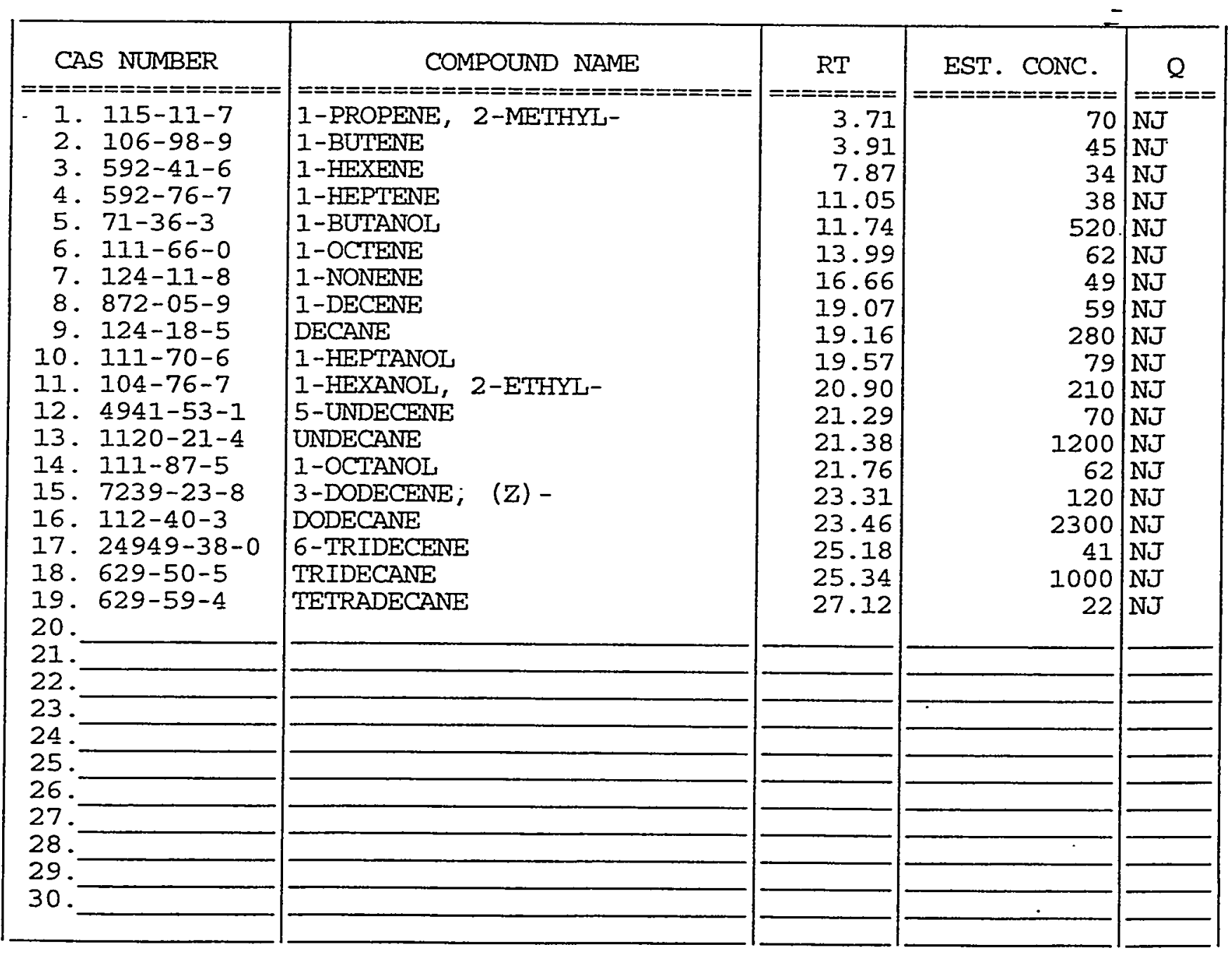


Lab Name: PNNL

Contract: BNFL

Irab Code: PNNL

Case No.:

Matrix: (soil/water) SOLID

Sample wt/vol:

$0.5(\mathrm{~g} / \mathrm{mU}) \mathrm{G}$

Level: (low/med) LOW

$\because$ Moisture: not dec.

GC Column: DB-624 75M X 2.55UM ID: 0.45 (mm)

Soil Extract Volume: (ut)

Number TICs found: 18
SAS NO. :
AW-101D

SDG No.: 99021601
Lab Sample ID: 99-0650D

Lab File ID: 99021606

Date Received:

Date Analyzed: 02/16/99

Dilution Factor: 1.0
Soil Aliquot Volume: (uL)
CONCENTRATION UNITS:

(ug/L or $\mathrm{ug} / \mathrm{Kg}$ ) ug/Kg

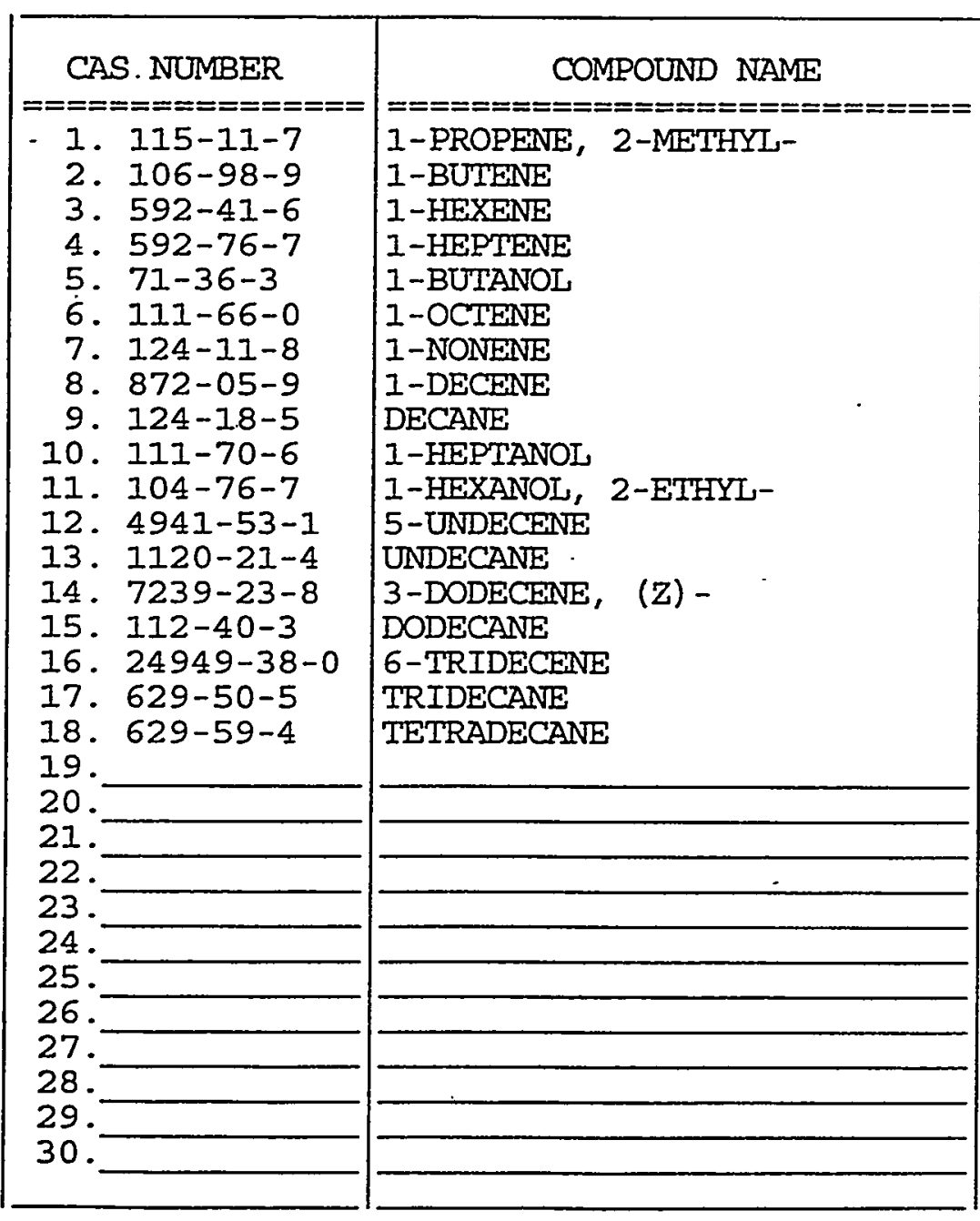

\begin{tabular}{|c|c|c|}
\hline 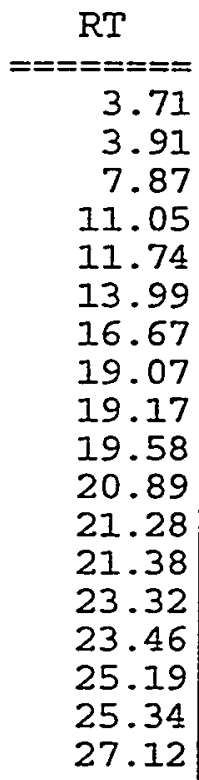 & 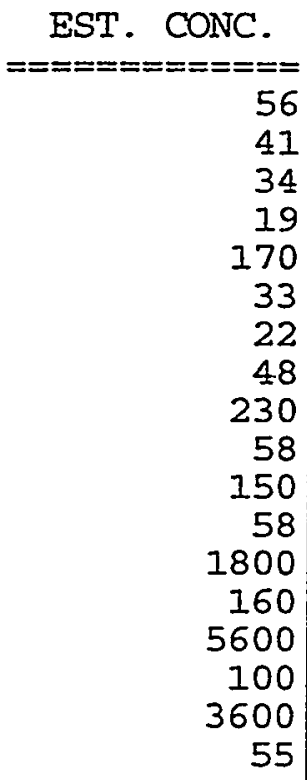 & 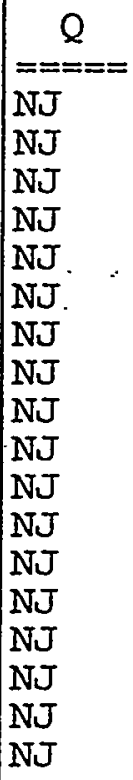 \\
\hline & & \\
\hline & & \\
\hline & & \\
\hline & & \\
\hline & & \\
\hline & & \\
\hline & & \\
\hline
\end{tabular}


Lab Name: PNNL

Contract: BNFL

Lab Code: PNNL

Case No.:

Matrix: (soil/water) SOIID

Sample wt/vol:

$5.0(\mathrm{~g} / \mathrm{mL}) \mathrm{G}$

Level: (low/med) LOW

$\div$ Moisture: not dec.

GC Column: DB-624 75M X 2.55UM ID: 0.45 (mm)

Soil Extract Volume:

Number TICs found: 0
EPA SAMPLE NO.

METHOD BLANK
SDG No.: 99021601

Lab Sample ID: VBLK03

Lab File ID: 99021604

Date Received:

Date Analyzed: 02/16/99

(mm) Dilution Factor: 1.0

Soil Aliquot Volume:

(uL)

CONCENTRATION UNITS:

(ug/I or $u g / \mathrm{Kg}$ ) ug/ $\mathrm{Kg}$

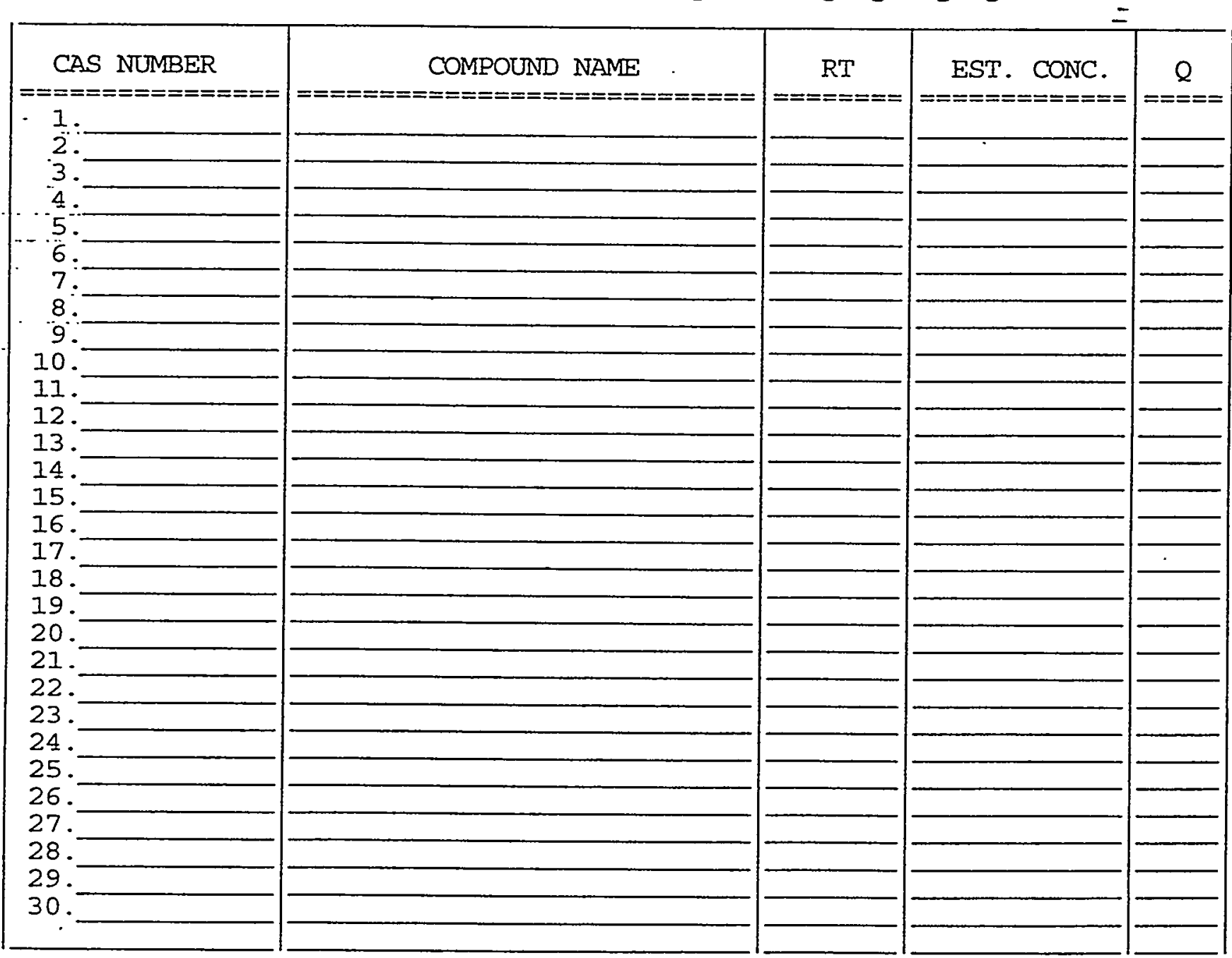


Lab Name: PNNL

Lab Code: PNNL

Case No.:

Matrix: (soil/water) SOLID

Sample wt/vol:

$5.0(\mathrm{~g} / \mathrm{mL}) \mathrm{G}$

Level: (low/med) LOW

\% Moisture: not dec.

GC Column: DB-624 75M X 2.55UM ID: 0.45 (mm)

Soil Extract Volume: (uL)

Number TICs found: 0
Contract: BNFL

SAS NO.:
EPA SAMPLE NO.

VBLK

SDG No.: 99021601

Lab Sample ID: VBLK

Lab File ID: 99021603

Date Received:

Date Analyzed: 02/16/99

Dilution Factor: 1.0

Soil Aliquot Volume: (uL)

CONCENTRATION UNITS :

(ug/I or $\mathrm{ug} / \mathrm{Kg}$ ) $\mathrm{ug} / \mathrm{Kg}$

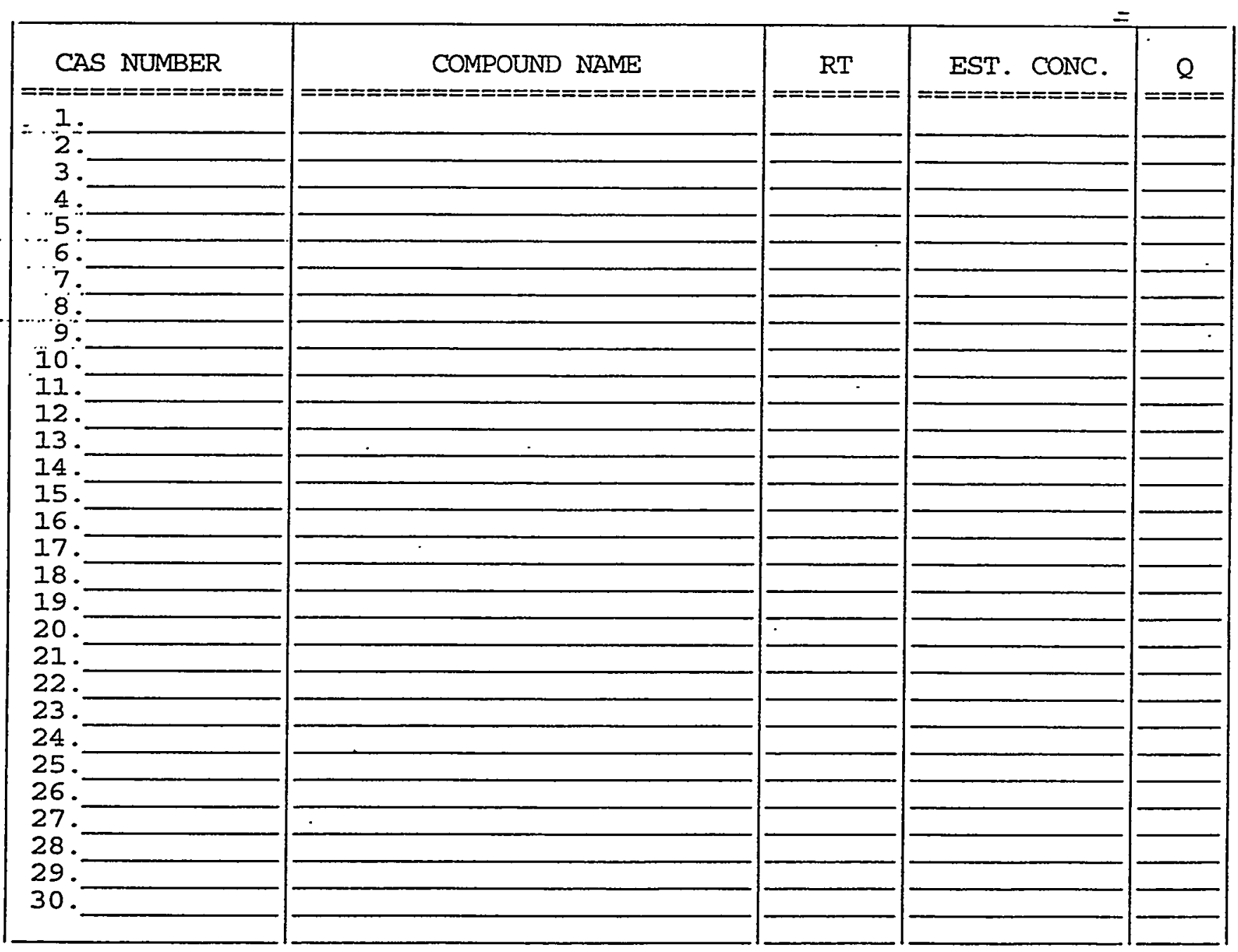


SOLID VOLATILE SYSTEM MONITORING COMPOUND RECOVERY

Lab Name: PNNL

Lab Code: PNNL,

Level: (low/med) LOW
Contract: BNFL

Case No.: $\quad$ SAS No.:
SDG No.: 99021601

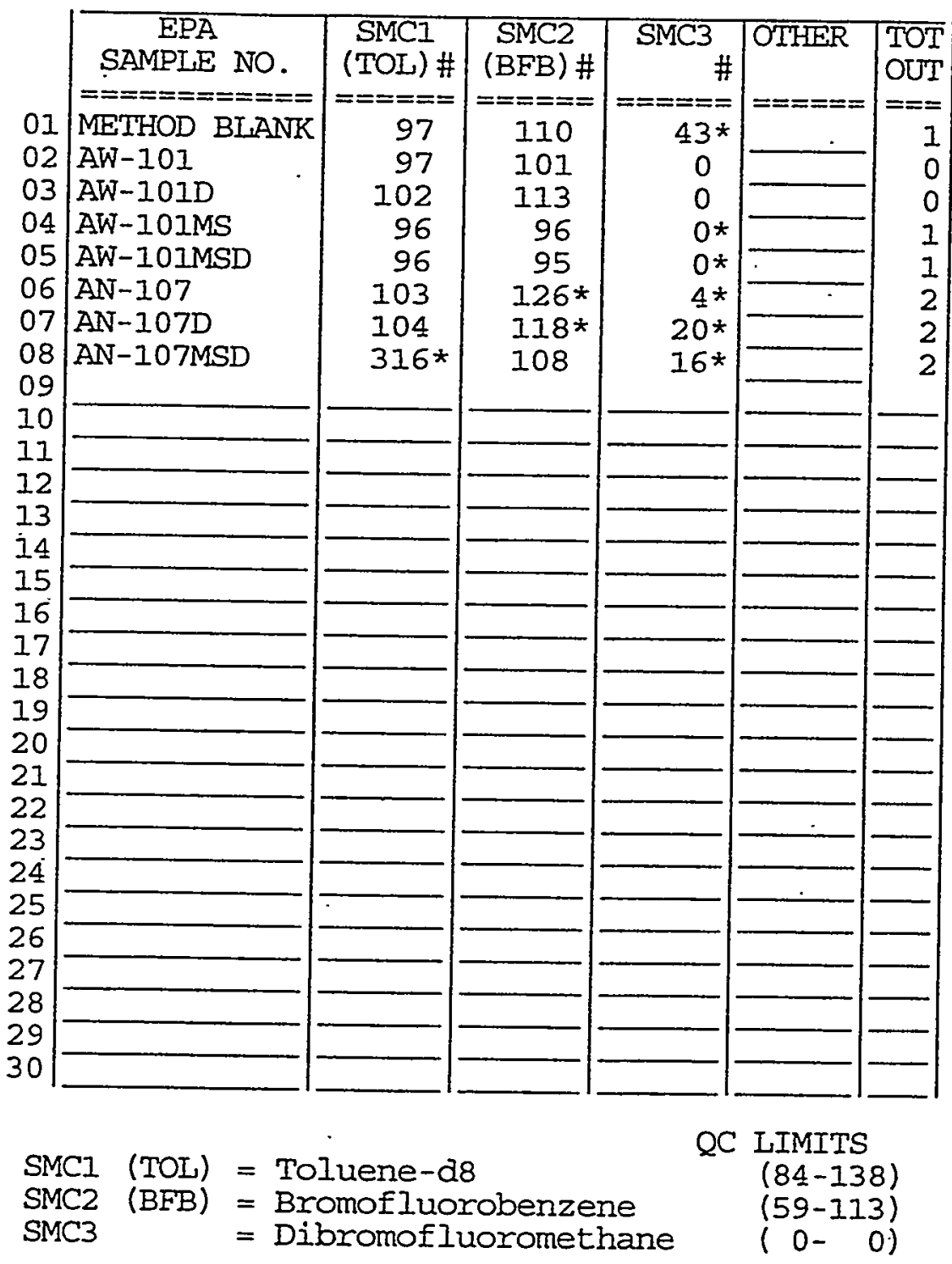

\# Column to be used to flag recovery values

* Values outside of contract required QC limits 
Lab Name: PNNL

Lab Code: PNNL
Contract: BNFL

SAS NO. :
SDG No.: 99021601

Matrix Spike - EPA Sample No.: AW-101 Level: (low/med) IOW

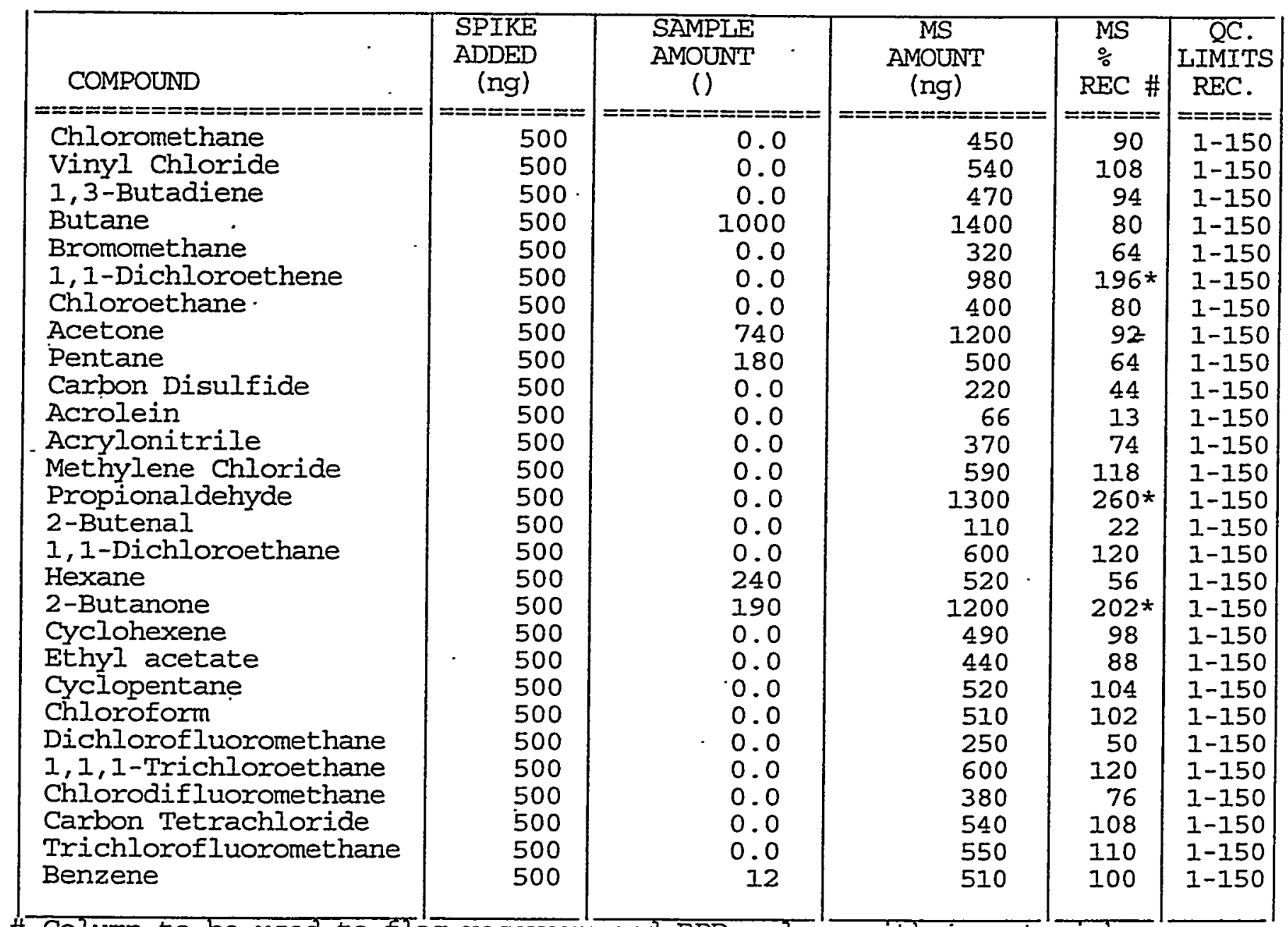

\# Column to be used to flag recovery and RPD values with án asterisk

* Values outside of QC limits

COMMENTS : 
Lab Name: PNNL

Iab Code: PNNL
Contract: BNFL

SAS NO.:
SDG No.: 99021601

Matrix Spike - EPA Sample No.: AW-10I Level:(low/med) LOW

\begin{tabular}{|c|c|c|c|c|c|}
\hline 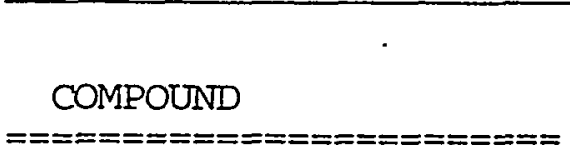 & $\begin{array}{l}\text { SPIKE } \\
\text { ADDED } \\
\text { (ng) } \\
========\end{array}$ & $\begin{array}{c}\text { SAMPLE } \\
\text { AMOUNI } \\
() \\
=====0=======\end{array}$ & $\begin{array}{c}\text { MS } \\
\text { AMOUNT } \\
(\mathrm{ng}) \\
============\end{array}$ & $\begin{array}{c}\text { MS } \\
\% \\
\text { REC \# } \\
=====\end{array}$ & $\begin{array}{l}\text { QC. } \\
\text { LIMITS } \\
\text { REC. } \\
=====\end{array}$ \\
\hline 1,2-Dichloroethane & 500 & 0.0 & 570 & 114 & $1-150$ \\
\hline Trichloroethene & 500 & 0.0 & 920 & $184 *$ & $1-150$ \\
\hline 1,2-Dichloropropane & 500 & 0.0 & 540 & 108 & $1-150$ \\
\hline cis-1,3-Dichloropropene & 500 & 0.0 & 330 & 66 & $1-150$ \\
\hline 4-Methyl-2-pentanone & 500 & 12 & 910 & $180 *$ & $1-150$ \\
\hline Toluene & 500 & 0.0 & 510 & 102 & $1-150$ \\
\hline trans-1,3-Dichloroprope & 500 & 0.0 & 460 & 92 & $1-150$ \\
\hline 1,1,2-Trichloroethane & 500 & 0.0 & 4 & 1 & $1-150$ \\
\hline Tetrachloroethene & 500 & 0.0 & 450 & 90 & $1-150$ \\
\hline 2-Hexanone & 500 & 47 & 970 & $185 *$ & $1-150$ \\
\hline Chlorobenzene & 500 & 0.0 & 460 & 92 & $1-150$ \\
\hline -Ethylbenzene & 500 & 3 & 450 & 89 & $1-150$ \\
\hline Xylene $(m \& p)$ & 500 & 7 & 490 & 97 & $I-150$ \\
\hline Xylene (0) & 500 & 3 & 470 & 93 & $1-150$ \\
\hline Styrene & 500 & 0.0 & 460 & 92 & $1-150$ \\
\hline $1,1,2,2$-Tetrachloroetha & 500 & 0.0 & 11 & 2 & $1-150$ \\
\hline Heptane & 500 & 250 & 490 & 48 & $1-150$ \\
\hline Octane & 500 & 230 & 530 & 60 & $1-150$ \\
\hline Nonane & 500 & 300 & 490 & 38 & $1-150$ \\
\hline 3-Chloropropene & 500 & 0.0 & 550 & 110 & $1-150$ \\
\hline 2-Pentanone & 500 & 0.0 & 880 & $176 *$ & $1-150$ \\
\hline Methylcyclohexane & 500 & 0.0 & 510 & 102 & $1-150$ \\
\hline 5-Methyl-2-hexanone & 500 & 0.0 & 390 & 78 & $1-150$ \\
\hline 4-Heptänone & 500 & 9 & 840 & $166 *$ & $1-150$ \\
\hline Butylacetate & 500 & 0.0 & 6 & 1 & $1-150$ \\
\hline 1,4-Dioxane & 500 & 110 & 770 & 132 & $1-150$ \\
\hline 2-Methyl-2-propenenitri & 500 & 0.0 & 680 & 136 & $1-150$ \\
\hline 3-Methyl-2-butanone & 500 & 41 & 860 & $164 *$ & $1-150$ \\
\hline
\end{tabular}

\# Column to be used to flag recovery and RPD values with an asterisk

* Values outside of QC limits

COMMENTS : 
SOLID VOLATILE MATRIX SPIKE/MATRIX SPIKE. DUPLICATE RECOVERY

Iab Name: PNNL

Lab Code: PNNL

Matrix Spike - EPA Sample No.: AW-101
Contract: BNFL

SAS NO.:

SDG No.: 99021601

Level: (low/med) IOW

\begin{tabular}{|c|c|c|c|c|c|}
\hline 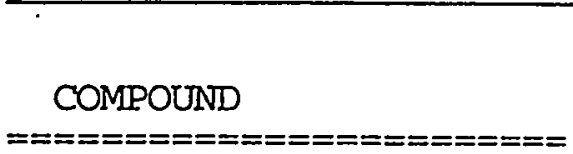 & $\begin{array}{l}\text { SPIKE } \\
\text { ADDED } \\
\text { (ng) } \\
=======\end{array}$ & $\begin{array}{l}\text { SAMPLE } \\
\text { AMOUNI } \\
() \\
============\end{array}$ & $\begin{array}{c}\text { MS } \\
\text { AMOUNT } \\
(\mathrm{ng}) \\
============\end{array}$ & $\begin{array}{c}\text { MS } \\
\frac{\%}{\circ} \\
\text { REC \# } \\
=====\end{array}$ & $\begin{array}{l}\text { QC. } \\
\text { I.IMITS } \\
\text { REC. }\end{array}$ \\
\hline $\begin{array}{l}\text { Propyl nitrate } \\
\text { 3-Pentanone } \\
1,2,2-C 13-1,1,2-F 3 \text { ethan } \\
\text {-1,2-Cl2-1,1,2,2-F4ethan } \\
\text { Dichlorodifluoromethane } \\
\text { Acetonitrile } \\
\text { Cyclohexane } \\
\text { 1,2-Dibromoethane } \\
\text { 3-Heptanone } \\
\text { 2-Heptanone } \\
\text { Tetrahydrofuran }\end{array}$ & $\begin{array}{l}500 \\
500 \\
500 \\
500 \\
500 \\
500 \\
500 \\
500 \\
500 \\
500 \\
500\end{array}$ & $\begin{array}{r}0.0 \\
0.0 \\
0.0 \\
0.0 \\
0.0 \\
49 \\
0.0 \\
0.0 \\
70 \\
70 \\
220\end{array}$ & $\begin{array}{l}550 \\
860 \\
560 \\
470 \\
530 \\
630 \\
520 \\
570 \\
950 \\
930 \\
920\end{array}$ & $\begin{array}{l}110 \\
172 * \\
112 \\
94 \\
106 \\
116 \\
104 \\
114 \\
176 * \\
172 * \\
140\end{array}$ & $\begin{array}{l}1-150 \\
1-150 \\
1-150 \\
1-150 \\
1-150 \\
1-150 \\
1-150 \\
1-150 \\
1-150 \\
1-150 \\
1-150\end{array}$ \\
\hline
\end{tabular}

\# Column to be used to flag recovery and RPD values with an asterisk

* Values outside of QC limits

COMMENTS : 
Lab Name: PNNL

Lab Code: PNNL
Contract: BNFL

SAS NO.:
SDG No.: 99021601

Matrix Spike - EPA Sample No.: AW-101. Level: (low/med) LOW

\begin{tabular}{|c|c|c|c|c|c|c|}
\hline & $\begin{array}{l}\text { SPIKE } \\
\text { ADDED }\end{array}$ & $\begin{array}{c}\text { MSD } \\
\text { AMOUNT }\end{array}$ & $\begin{array}{c}\text { MSD } \\
\frac{\circ}{0}\end{array}$ & $\%$ & \multicolumn{2}{|c|}{ QC LII } \\
\hline COMPOUND & (ng) & (ng) & REC \# & RPD \# & RPD & REC. \\
\hline 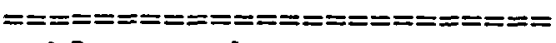 & $===== \pm== \pm$ & $=== \pm===0== \pm==$ & $======$ & $======$ & $======$ & $==\approx===$ \\
\hline Chloromethane & 500 & 460 & 92 & 2 & 40 & $1-150$ \\
\hline Vinyl Chloride & 500 & 560 & 112 & 4 & 40 & $1-150$ \\
\hline 1,3-Butadiene & 500 & 560 & 112 & 17 & 40 & $1-150$ \\
\hline Butane & 500 & 1100 & 20 & $120 *$ & 40 & $1-150$ \\
\hline Bromomethane & 500 & 280 & 56 & 13 & 40 & $1-150$ \\
\hline 1,1-Dichloroethene & 500 & 1000 & $200 *$ & 2 & 40 & $1-150$ \\
\hline Chloroethane & 500 & 380 & 76 & 5 & 40 & $1-150$ \\
\hline Acetone & 500 & 1400 & 132 & 36 & 40 & $1-150$ \\
\hline Pentane & 500 & 580 & 80 & 22 & 40 & $1-150$ \\
\hline Carbon Disulfide & 500 & 260 & 52 & 17 & 40 & $1-150$ \\
\hline Acrolein & 500 & 38 & 8 & $48 *$ & 40 & $1-150$ \\
\hline Acrylon: & 500 & 270 & 54 & 31 & 40 & $1-150$ \\
\hline Methylene Chloride & 500 & 660 & 132 & 11 & 40 & $i-150$ \\
\hline Propionaldehyde & 500 & 1600 & $320 *$ & 21 & 40 & $1-150$ \\
\hline 2-But & 500 & 120 & 24 & 9 & 40 & $1-150$ \\
\hline 1,1-Dichloroethane & 500 & 640 & 128 & 6 & 40 & $1-150$ \\
\hline Hexane & 500 & 680 & 88 & $44 *$ & 40 & $1-150$ \\
\hline 2-Butanone & 500 & 1400 & $242 *$ & 18 & 40 & $1-150$ \\
\hline Cyclohe & 500 & 510 & 102 & 4 & 40 & $1-150$ \\
\hline Ethyl acetate & 500 & 540 & 108 & 20 & 40 & $1-150$ \\
\hline Cyclopentane & 500 & 580 & 116 & II & 40 & $1-150$ \\
\hline Chloroform & 500 & 540 & 108 & 6 & 40 & $1-150$ \\
\hline Dichlorofluoromethane & 500 & 280 & 56 & 11 & 40 & $1-150$ \\
\hline $1,1,1$-Trichloroethane & 500 & 660 & 132 & 10 & 40 & $1-150$ \\
\hline Chlorodifluoromethane & 500 & 480 & 96 & 23 & 40. & $1-150$ \\
\hline Carbon Tetrachloride & & 560 & 112 & 4 & 40 & $1-150$ \\
\hline Trichlorofluoromethane & & 600 & 120 & 9 & 40 & $1-150$ \\
\hline Benzene & 500 & 520 & 102 & 2 & 40 & $1-150$ \\
\hline
\end{tabular}

\# Column to be used to flag recovery and RPD values with an asterisk

* Values outside of QC limits

COMMENTS: 
3B

SOLID VOLATILE MATRIX SPIKE/MÄTRIX SPIKE DUPLICATE RECOVERY

Lab Name: PNNL

Lab Code: PNNL

Case No.:
Contract: BNFL

SAS NO.:
SDG No.: 99021601

Matrix Spike - EPA Sample No.: AW-101 Level: (low/med) IOW

\begin{tabular}{|c|c|c|c|c|c|c|}
\hline & $\begin{array}{l}\text { SPIKE } \\
\text { ADDED }\end{array}$ & $\begin{array}{c}\text { MSD } \\
\text { AMOUNT }\end{array}$ & $\begin{array}{l}\text { MSD } \\
\%\end{array}$ & $\%$ & \multicolumn{2}{|c|}{ QC IIMITS } \\
\hline $\begin{array}{l}\text { COMPOUND } \\
\text { Co=" }\end{array}$ & $\begin{array}{c}\text { (ng) } \\
=========\end{array}$ & (ng) & REC \# & RPD \# & $\mathrm{RPD}$ & REC. \\
\hline 1,2-Dichloroethane & 500 & $===========$ & 118 & $====-=$ & $\begin{array}{r}==== \\
40\end{array}$ & $1-150$ \\
\hline Trichloroethene & 500 & 930 & $186 *$ & 1 & 40 & $1-150$ \\
\hline 1,2-Dichloropropane & 500 & 550 & 110 & 2 & 40 & $1-150$ \\
\hline cis-1,3-Dichloropropene & 500 & 280 & 56 & 16 & 40 & $1-150$ \\
\hline 4-Methyl-2-pentanone & 500 & 1100 & $218 *$ & 19 & 40 & $1-150$ \\
\hline Toluene & 500 & 520 & 104 & 2 & 40 & $1-150$ \\
\hline trans-1,3-Dichloroprope & 500 & 440 & 88 & 4 & 40 & $1-150$ \\
\hline $1,1,2$-Trichloroethane & 500 & 4 & 1 & 0 & 40 & $1-150$ \\
\hline Tetrachloroethene & 500 & 450 & 90 & 0 & 40 & $1-150$ \\
\hline 2-Hexanone & 500 & $\cdot 1200$ & $231 *$ & 22 & 40 & $1-150$ \\
\hline Chlorobenzene & 500 & 450 & 90 & 2 & 40 & $1-150$ \\
\hline - Ethylbenzene & 500 & 430 & 85 & 4 & 40 & $1-150$ \\
\hline Xylene $(m \& p)$ & 500 & 460 & 91. & 6 & 40 & $1-150$ \\
\hline Xylene (o) & 500 & 440 & 87 & 7 & 40 & $1-150$ \\
\hline styrene & 500 & 440 & 88 & 4 & 40 & $1-150$ \\
\hline $1,1,2,2$-Tetrachloroetha & 500 & 9 & 2 & 0 & 40 & $1-150$ \\
\hline Heptane & 500 & 540 & 58 & 19 & 40 & $1-150$ \\
\hline Octane & 500 & 550 & 64 & 6 & 40 & $1-150$ \\
\hline Nonane & 500 & 500 & 40 & 5 & 40 & $1-150$ \\
\hline 3-Chloropropene & 500 & 480 & 96 & 14 & 40 & $1-150$ \\
\hline 2-Pentanone & 500 & 970 & $194 *$ & 10 & 40 & $1-150$ \\
\hline Methylcyclohexane & 500 & 500 & 100 & 2 & 40 & $1-150$ \\
\hline 5-Methyl-2-hexanone & 500 & 450 & 90 & 14 & 40 & $1-150$ \\
\hline 4-Heptanone & 500 & 1000 & $198 *$ & 18 & 40 & $1-150$ \\
\hline Butylacetate & 500 & 12 & 2 & $67 *$ & 40 & $1-150$ \\
\hline 1,4-Dioxane & 500 & 880 & $154 *$ & 15 & 40 & $1-150$ \\
\hline 2-Methyl-2-propenenitri & 500 & 740 & 148 & 8 & 40 & $1-150$ \\
\hline 3-Methyl-2-butanone & & 1000 & $192 *$ & 16 & 40 & $1-150$ \\
\hline
\end{tabular}

\# Column to be used to flag recovery and RPD values with an asterisk

* Values outside of QC Iimits

COMMENTS : 
SOLID VOLATILE MATRIX SPIKE/MATRIX SPIKE DUPLICATE RECOVERY

Lab Name: PNNL

Contract: BNFL

Lab Code: PNNL Case No.:

SAS NO.:

SDG No.: 99021601

Matrix Spike - EPA Sample No.: AW-101 Level:(low/med) IOW

\begin{tabular}{|c|c|c|c|c|c|c|}
\hline & $\begin{array}{l}\text { SPIKE } \\
\text { ADDED }\end{array}$ & $\begin{array}{c}\text { MSD } \\
\text { AMOUNT }\end{array}$ & $\begin{array}{c}\text { MSD } \\
\%\end{array}$ & $\div$ & \multicolumn{2}{|c|}{ QC LIMITS } \\
\hline COMPOUND & (ng) & (ng) & REC \# & RPD \# & RPD & REC. \\
\hline 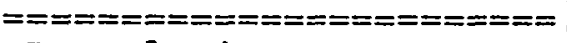 & $=========$ & 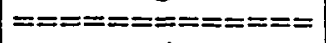 & $======$ & $======$ & $====\approx=$ & $=====$ \\
\hline Propyl nitrate & 500 & 570 & 114 & 4 & 40 & $1-150$ \\
\hline 3-Pentanone & 500 & 1000 & $200 *$ & 15 & 40 & $1-150$ \\
\hline $1,2,2-C 13-1,1,2-F 3 e t h a n$ & 500 & 640 & -128 & 13 & 40 & $1-150$ \\
\hline $1,2-\mathrm{Cl} 2-1,1,2,2-\mathrm{F} 4 \mathrm{ethan}$ & 500 & 520 & 104 & 10 & 40 & $1-150$ \\
\hline Dichlorodifluoromethane & 500 & 610 & 122 & 14 & 40 & $1-150$ \\
\hline Acetonitrile & 500 & 630 & 116 & 0 & 40 & $1-150$ \\
\hline Cyclohexane & 500 & 550 & 110 & 6 & 40 & $1-150$ \\
\hline I,2-Dibromoethane & 500 & 580 & 116 & 2 & 40 & $1-150$ \\
\hline 3-Heptanone & 500 & 1100 & $206 *$ & 16 & 40 & $1-150$ \\
\hline 2-Heptanone & 500 & 1100 & $206 *$ & 18 & 40 & $1-150$ \\
\hline Tetrahydrofuran & 500 & 1100 & $176 *$ & 23 & 40 & $1-150$ \\
\hline
\end{tabular}

\# Column to be used to flag recovery and RPD values with an asterisk

* Values outside of QC limits

RPD: 4 out of 67 outside limits

Spike Recovery: 26 out of 134 outside limits

COMMENTS: 
$4 \mathrm{~A}$

VOLATILE METHOD BLANK SUMMARY

Lab Name: PNNL

Contract: BNFI,

Lab Code: PNNL

Case No.:

Lab File ID: 99021604

SAS NO.:
EPA SAMPLE NO.

METHOD BLANK
Date Analyzed: 02/16/99

GC Column: DB-624 75M X 2.55UMID: 0.45 (mm)

Instrument ID: HPI
Lab Sample ID: VBLK03

Time Analyzed: 1845

Heated Purge: $(\mathrm{Y} / \mathrm{N}) \mathrm{Y}$

THIS METHOD BLANK.APPLIES TO THE FOILOWING SAMPLES, MS and MSD:

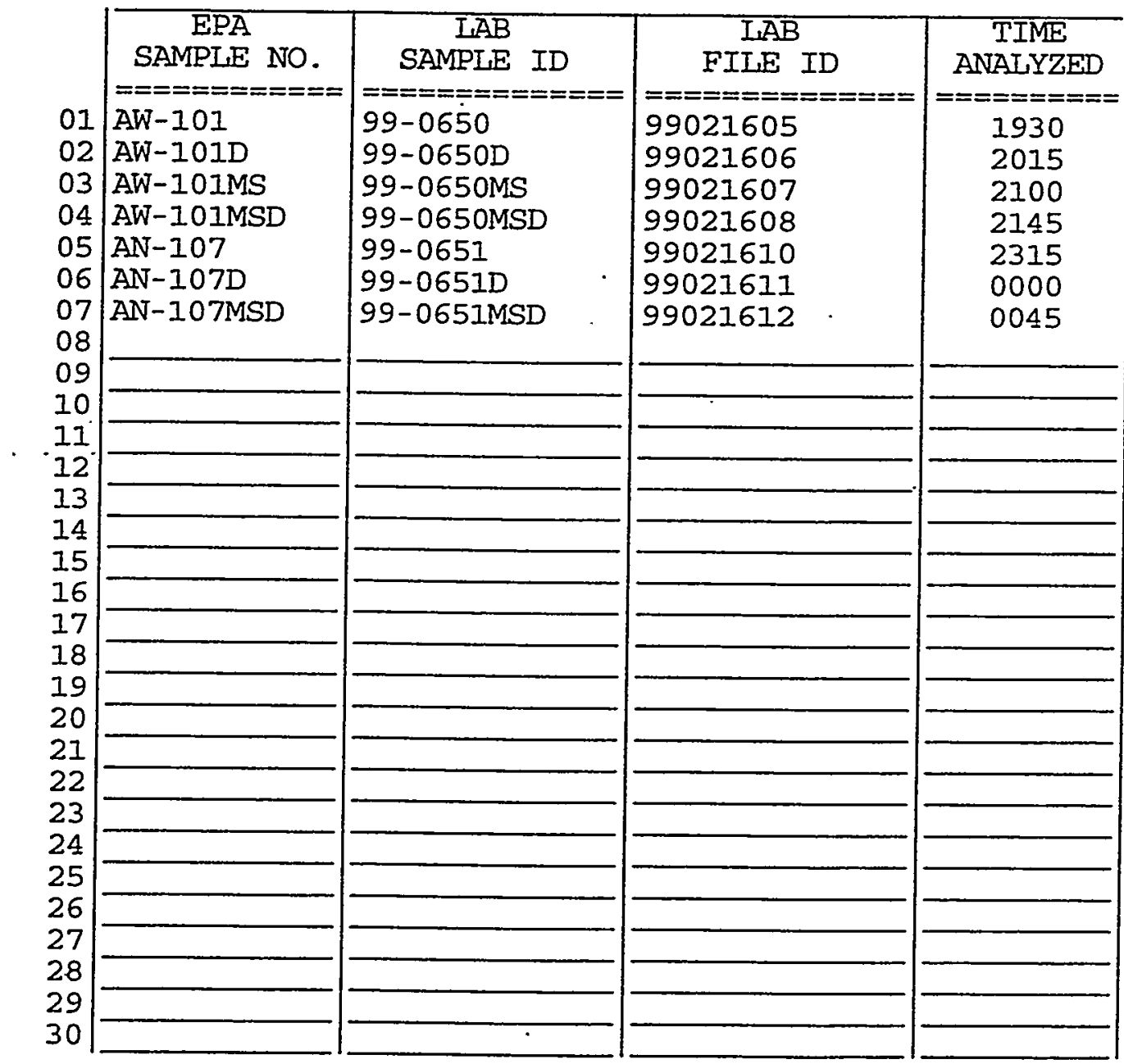

COMMENTS :

page 1 of 1 
VOLATILE ORGANIC INSTRUMENT PERFORMANCE CHECK BROMOFLUOROBENZENE (BFB)

Inab Name: PNNL

Iab Code: PNNL Case No.:

Lab File ID: 99021601

Instrument ID: HPI

GC Column:
Contract: BNFL

SAS NO.:
SDG No.: 99021601

BFB Injection Time: 1632

Heated Purge: $(\mathrm{Y} / \mathrm{N}) \mathrm{N}$

\begin{tabular}{|c|c|c|}
\hline $\begin{array}{l}\mathrm{m} / \mathrm{e} \\
=====\end{array}$ & 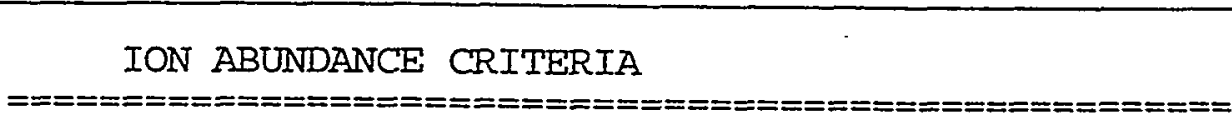 & $\begin{array}{c}\text { \% RELATIVE } \\
\text { ABUNDANCE } \\
============\end{array}$ \\
\hline 50 & $8.0-.40 .0 \%$ of mass 95 & 24.3 \\
\hline 75 & $30.0-66.0 \%$ of mass $9 \overline{5}$ & 42.4 \\
\hline 95 & Base Peak, 100\% relative abundance & 100.0 \\
\hline 96 & $5.0-9.0 \%$ of mass 95 & 6.7 \\
\hline 173 & Less than $2.0 \%$ of mass 174 & $0.0 T=0.0) 1$ \\
\hline 174 & $50.0-120.0 \%$ of mass 95 & 97.5 \\
\hline 175 & $4.0-9.0 \%$ of mass 174 & $8 . 8 \longdiv { ( 9 . 0 ) 1 }$ \\
\hline 176 & $93.0-.101 .0 \%$ of mass $\overline{174}$ & $94.9(97.3) 1$ \\
\hline .177 & $5.0-9.0 \%$ of mass 176 & $5.6(5.9) 2$ \\
\hline
\end{tabular}

THIS CHECK APPLIES TO THE FOLLOWING SAMPLES, MS, MSD, BLANKS, AND STANDARDS:

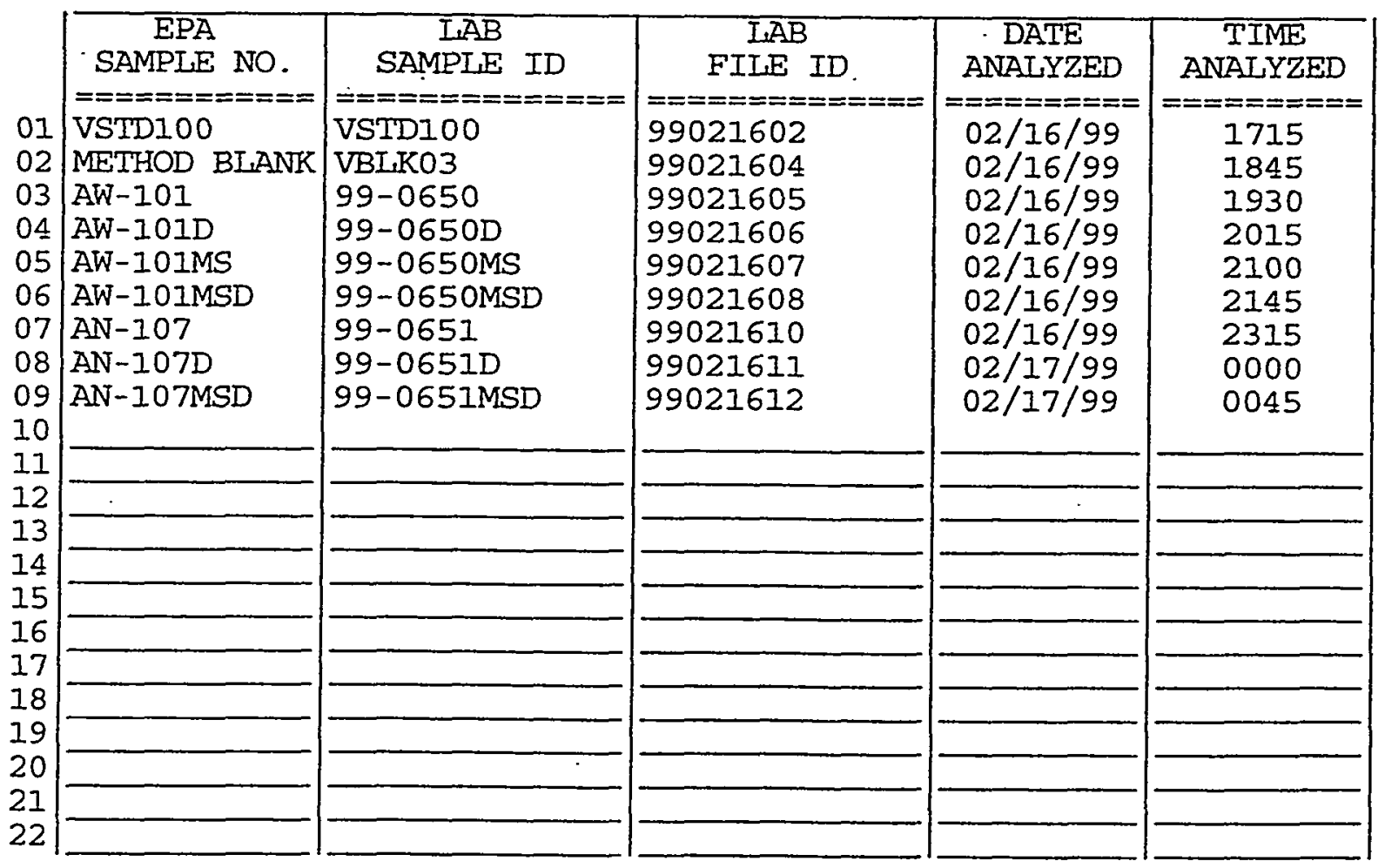

page 1 of 1 
$7 A$

VOLATILE CONTINUING CALIBRATION CHECK

Lab Name: PNNL

Contract: BNFL

Lab Code: PNNL

Case No.:

SAS NO.:

SDG No.: 99021601

Instrument ID: HP1

Calibration Date: 02/16/99 Time: 1715

Lab File ID: 99021602

Init. Calib. Date (s) : 02/11/99

$02 / 11 / 99$

Heated Purge: ( $Y / N) N$

Init. Calib. Times: 1249

1549

GC Column: DB-62475M X 2.55UM ID: 0.45 (mm)

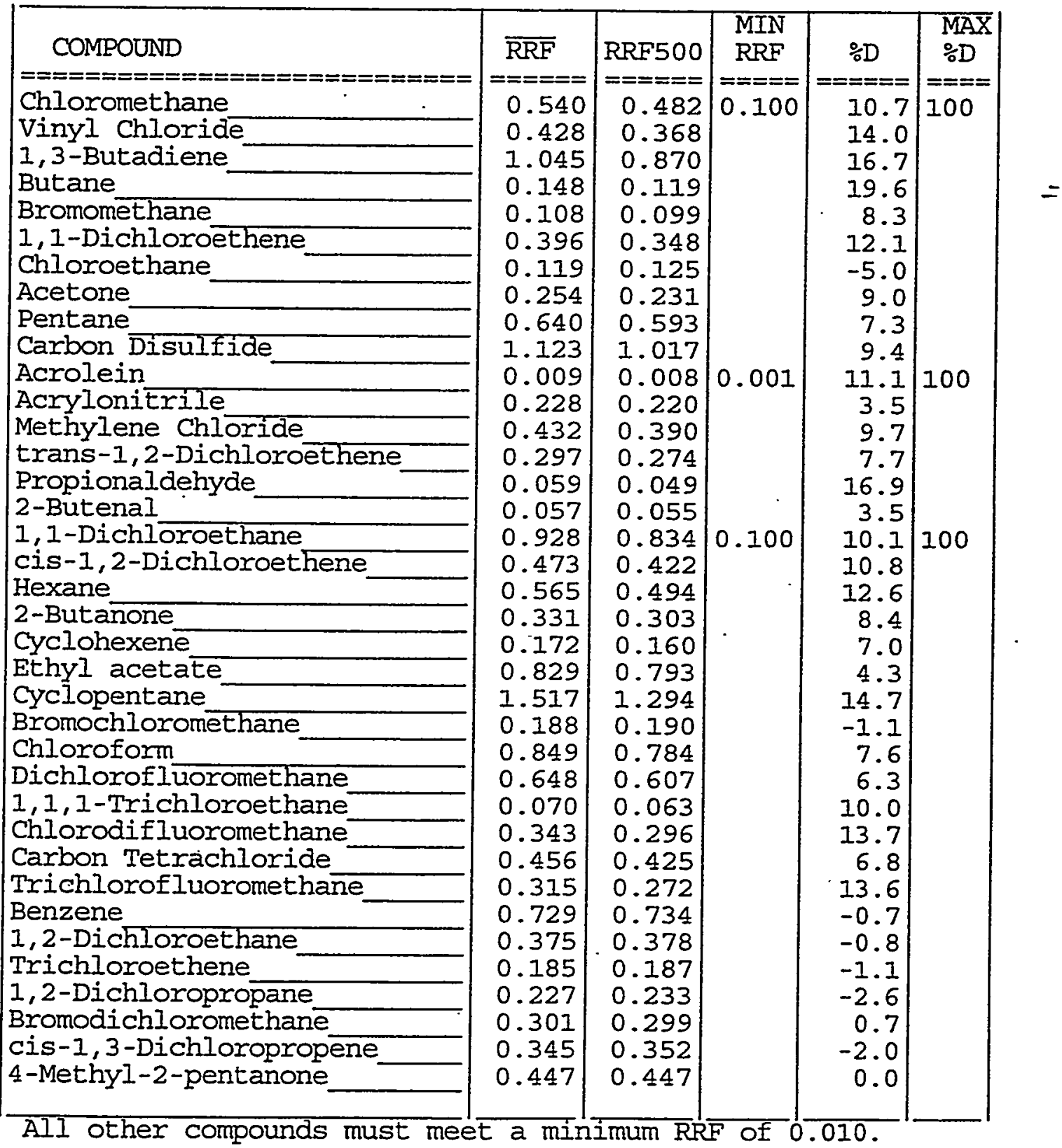

page 1 of 3 
VOTATIT:

Lab Name: PNNL

Contract: BNFL

Lab Code: PNNL

Case No.:

SAS NO.:

SDG NO.: 99021601

Instrument ID: $\mathrm{HPI}$

Calibration Date: 02/16/99 Time: 1715

Lab File ID: 99021602

Init. Calib. Date(s) : 02/11/99 02/11/99

Heated Purge: (Y/N) N Init. Calib. Times: $1249 \quad 1549$

GC Column: DB-624 75M X 2.55UM ID: 0.45 (mm)

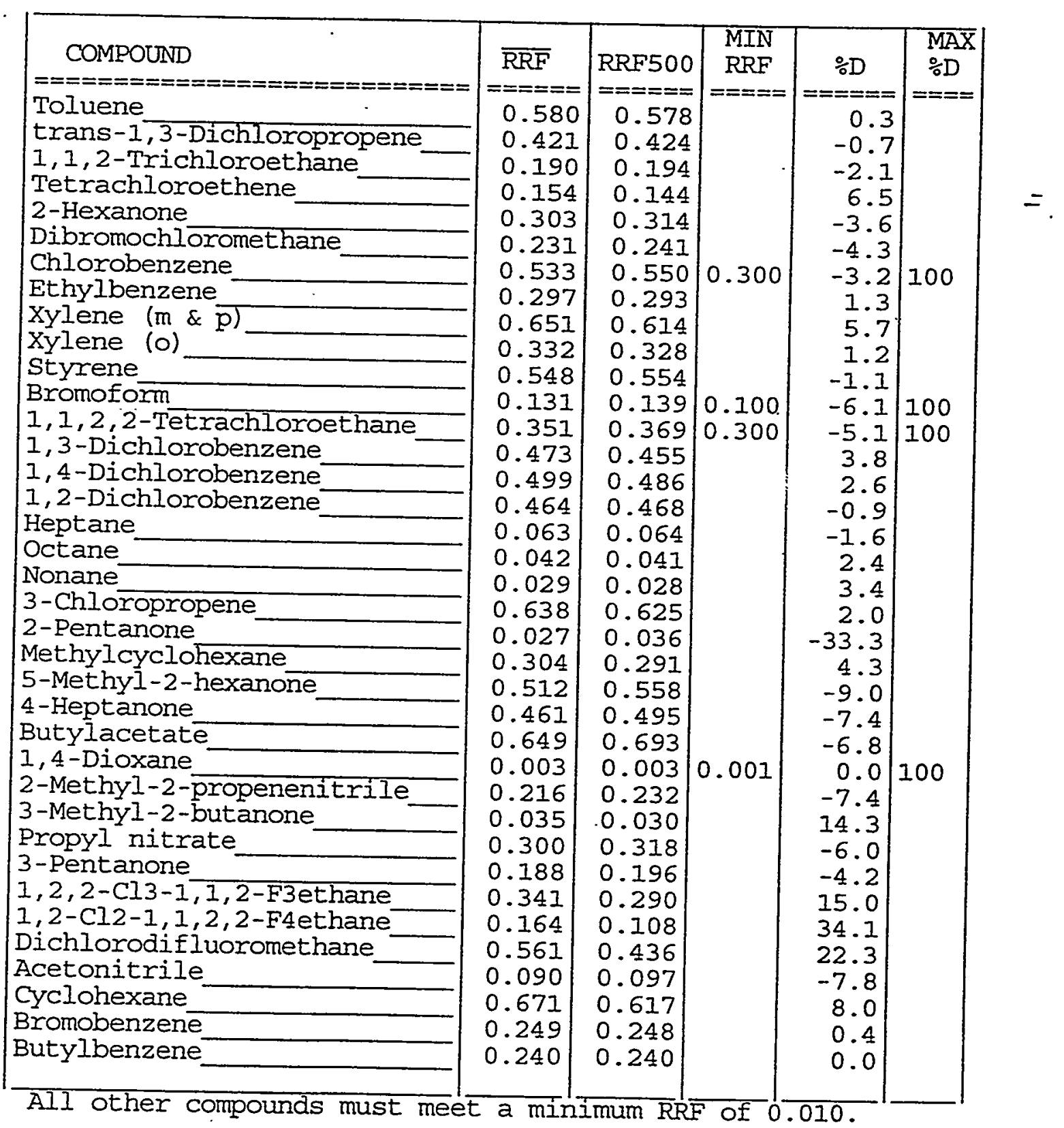

Jage 2 of 3 
Lab Name: PNNL

Lab Code: PNNL Case No.:
- Contract: BNFL

SAS No. :

SDG No.: 99021601
Instrument ID: HP1

Lab File ID: 99021602

Heated Purge: $(\mathrm{Y} / \mathrm{N}) \mathrm{N}$
Calibration Date: 02/16/99

Time: 1715

Init. Calib. Date (s) : 02/11/99

$02 / 11 / 99$

Init. Calib. Times: 1249

1549

GC Column: $\mathrm{DB}-62475 \mathrm{M} \times 2.55 \mathrm{UM}$ ID: 0.45 . (mm)

\begin{tabular}{|c|c|c|c|c|c|}
\hline 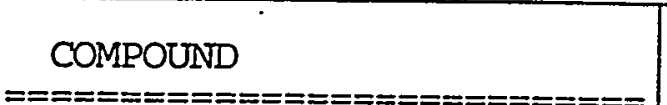 & $\overline{\mathrm{RRF}}$ & RRF500 & $\begin{array}{l}\text { MIN } \\
\text { RRF }\end{array}$ & $\div \mathrm{D}$ & $\begin{array}{l}\text { MAX } \\
\because D\end{array}$ \\
\hline $\begin{array}{l}============================ \\
\text { tert }- \text { Butylbenzene }\end{array}$ & $======$ & $=====$ & $====$ & $======$ & $===$ \\
\hline sec-Butylbenzene & $\begin{array}{l}0.824 \\
1.184\end{array}$ & $\begin{array}{l}0.816 \\
1.185\end{array}$ & & $\begin{array}{r}1.0 \\
-0.1\end{array}$ & \\
\hline 2-Chlorotoluene & 0.869 & 0.868 & & 0.1 & \\
\hline 4-Isopropyltoluene & 0.921 & 0.916 & & 0.5 & \\
\hline 4-Chlorotoluene & 1.000 & 1.009 & & -0.9 & \\
\hline $\begin{array}{l}\text { 1,2-Dibromo-3-chloropropane } \\
\text { 1,2-Dibromoethane }\end{array}$ & $\begin{array}{l}0.065 \\
0.205\end{array}$ & $\begin{array}{l}0.073 \\
0.213\end{array}$ & & $\begin{array}{rl}-12.3 \\
-3 & 9\end{array}$ & \\
\hline trans-1,4-Dichloro-2-butene & 0.157 & 0.175 & & -11.5 & \\
\hline 1,3-Dichloropropane & 0.375 & 0.393 & & -4.8 & \\
\hline 2,2-Dichloropropane & 0.332 & 0.297 & & 10.5 & \\
\hline $\begin{array}{l}\text { 1,1-Dichloropropene } \\
\text { Hexachloro-1,3-butadiene }\end{array}$ & $\begin{array}{l}0.640 \\
0.114\end{array}$ & 0.629 & & 1.7 & \\
\hline $\begin{array}{l}\text { Hexachloro-1,3-butadiene } \\
\text { Iodomethane }\end{array}$ & $\begin{array}{l}0.114 \\
0.484\end{array}$ & 0.119 & & $-4 \cdot 4$ & \\
\hline Isopropylbenzene & 0.824 & $\begin{array}{l}0.412 \\
0.843\end{array}$ & & $\begin{array}{l}14.9 \\
-2.3\end{array}$ & \\
\hline Nathphalene & 0.764 & 0.822 & & -7.6 & \\
\hline Propylbenzene & 1.347 & 1.355 & & -0.6 & \\
\hline $1,2,3$-Trichlorobenzene & 0.248 & 0.246 & & 0.8 & \\
\hline 1,2,4-Trichlorobenzene & 0.265 & 0.263 & & 0.8 & \\
\hline 1,2,3-Trichloropropane & 0.525 & 0.564 & & $-7 \cdot 4$ & \\
\hline $1,2,4$-Trimethylbenzene & 0.927 & 0.925 & & 0.2 & \\
\hline ,2,3-Trimethylbenzene & 0.903 & 0.907 & & -0.4 & \\
\hline 1-Propanol & 0.057 & 0.052 & & 8.8 & \\
\hline $\begin{array}{l}\text { 3-Heptanone } \\
\text { 2-Heptanone }\end{array}$ & 0.310 & 0.338 & & -9.0 & \\
\hline $\begin{array}{l}\text { 2-Heptanone } \\
\text { Tetrahydrofuran }\end{array}$ & $\begin{array}{l}0.450 \\
0.215\end{array}$ & 0.474 & & -5.3 & \\
\hline $\begin{array}{l}\text { Tetrahydrofuran } \\
\text { Dibromomethane }\end{array}$ & $\begin{array}{l}0.215 \\
0.134\end{array}$ & 0.214 & & 0.5 & \\
\hline 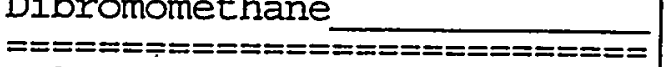 & $\begin{array}{r}0.134 \\
=====\end{array}$ & $\begin{array}{r}0.138 \\
======\end{array}$ & $===$ & $=====$ & $====$ \\
\hline Toluene- d8 & 0.592 & 0.599 & & -1.2 & \\
\hline Bromofluorobenzene & 0.368 & 0.370 & & -0.5 & \\
\hline Dibromofluoromethar. & 0.348 & 0.344 & & 1.1 & \\
\hline
\end{tabular}

All other compounds must meet a minimum $R R$ of 0.010 . 
$8 \mathrm{~A}$

VOLATIIE INTERNAL STANDARD AREA AND RT SUMMARY

Lab Name: PNNL

Lab code: PNNL
Contract: BNEL

Case No.:

Lab File ID (Standard): 99021602

Instrument ID: HP1

SAS NO.: $\quad$ SDG NO.: 99021601
Date Analyzed: 02/16/99

Time Analyzed: 1715

GC Column: DB-624 75M X 2.55UM ID: 0.45 (mm) Heated Purge: (Y/N) N

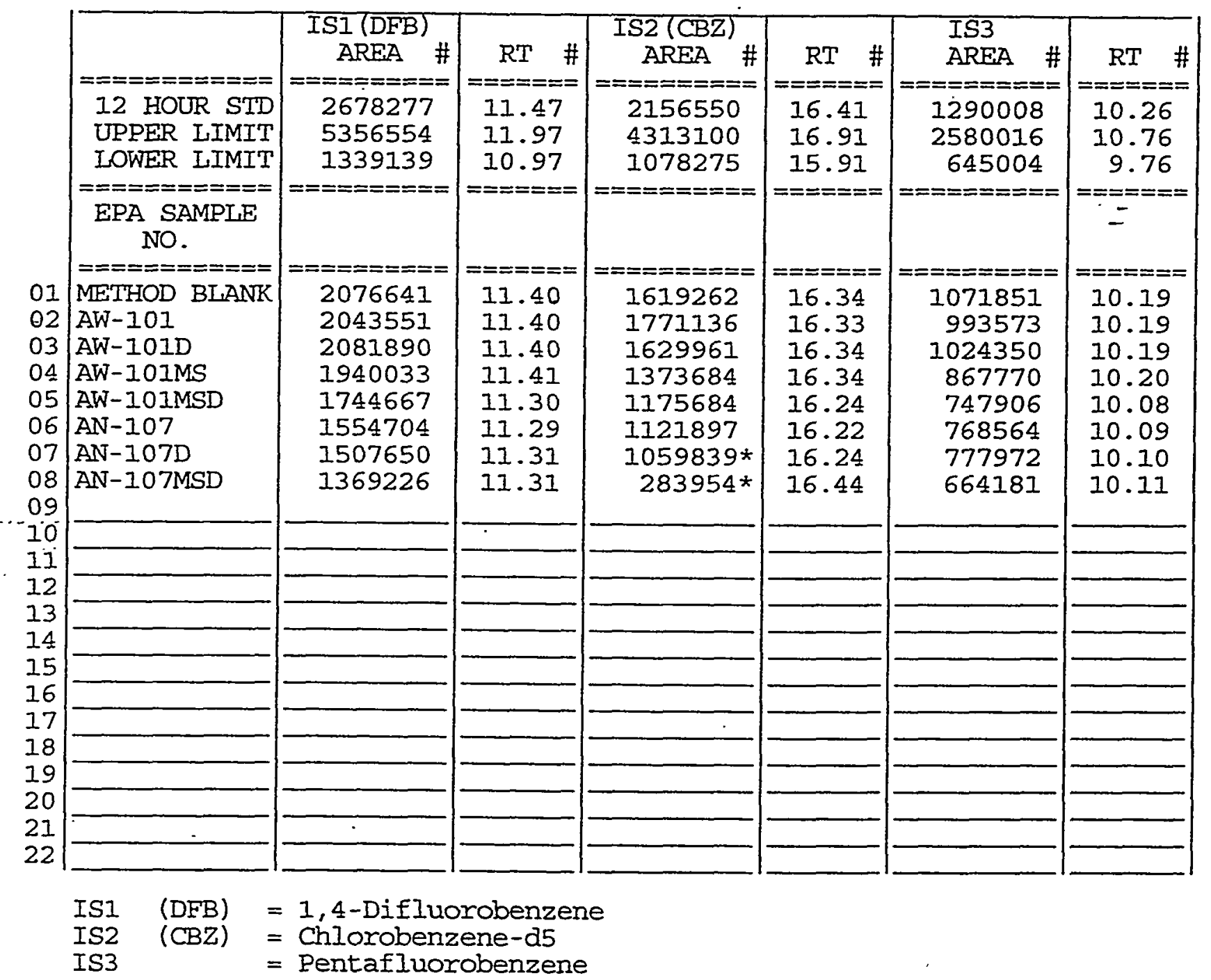

AREA UPPER IIMIT $=+100 \%$ of internal standard area

AREA IOWER IIMIT $=-50 \%$ of internal standard area

RT UPPER LIMIT $=+0.50$ minutes of internal standard RT

RT IOWER LIMIT $=-0.50$ minutes of internal standard RT

\# Column used to flag values outside QC limits with an asterisk.

* Values outside of QC Iimits.

Jage 1 of 2 
$8 \mathrm{~A}$

VOLATILE INTERNAT STANDARD AREA AND RT SUMMARY

Lab Name: PNN!

Lab Code: PNNL Case No.:

Lab File ID (Standard) : 99021602

Instrument ID: HPI

GC Column: $\mathrm{DB}-62475 \mathrm{M} \times 2.55 \mathrm{UM}$ ID: 0.45 (mm)
Contract: BNFL

SAS NO.:
SDG NO.: 99021601

Date Analyzed: 02/16/99

Time Analyzed: 1715

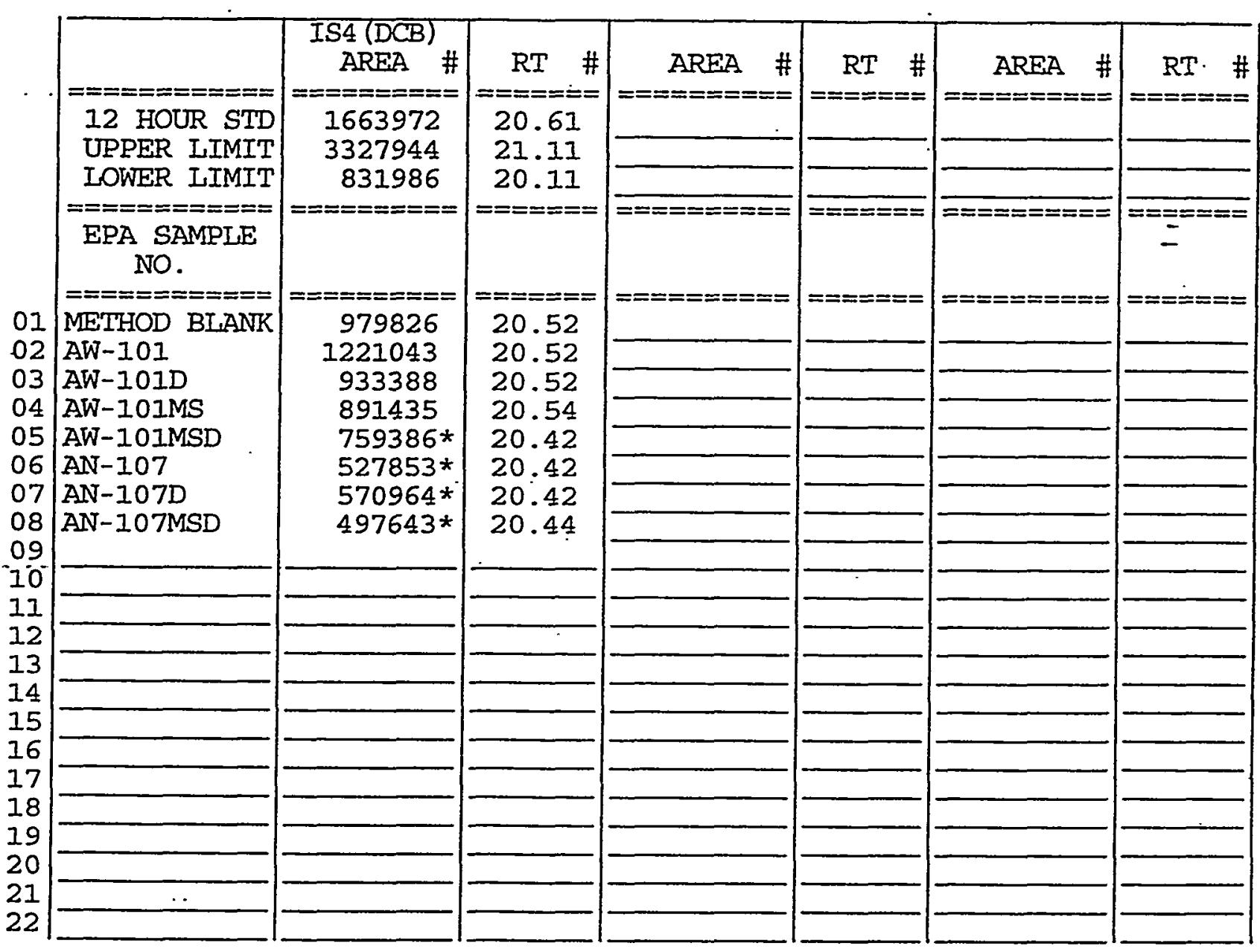

IS4 $(\mathrm{DCB})=1,4-\mathrm{Dichlorobenzene-d4}$

AREA UPPER LIMIT $=+100 \%$ of internal standard area AREA IOWER IIMIT $=-50 \%$ of internal standard area RT UPPER LIMIT $=+0.50$ minutes of internal standard RT RT LOWER IIMIT $=-0.50$ minutes of internal standard RT

\# Column used to flag values outside QC limits with an asterisk.

* Values outside of QC limits. 


\section{APPENDIX B}

\section{SEMI-VOLATILE ORGANIC ANALYSIS RESULTS}

AW-101 Supernatant and Solids - Forms 1B through 8C

AN-107 Supernatant and Solids -- Forms 1B through 8C 
Lab Name: PNNL,

AW-101

Iab Code: PNNL Case No.:

SAS NO.:

SDG No.: 990205

Matrix: (soil/water) SUPERNATAN

Sample wt/vol: $(\mathrm{g} / \mathrm{mL}) \mathrm{MI}$

Lab Sample ID: 99-0648

Level: (low/med) IOW

$\%$ Moisture:

decanted: $(Y / N)$

Lab File ID: 99020606

Date Received:

Concentrated Extract Volume: 2000 (uL)

Date Extracted:

Injection Volume: (uI)

Date Analyzed: 02/06/99

GPC Cleanup: $(\mathrm{Y} / \mathrm{N}) \mathrm{N} \quad \mathrm{pH}: 12.5$

Dilution Factor: 1.0

CONCENTRATION UNITS:

CAS NO. COMPOUND (ug/L or ug/Kg) UG/I

Q

\begin{tabular}{|c|}
\hline 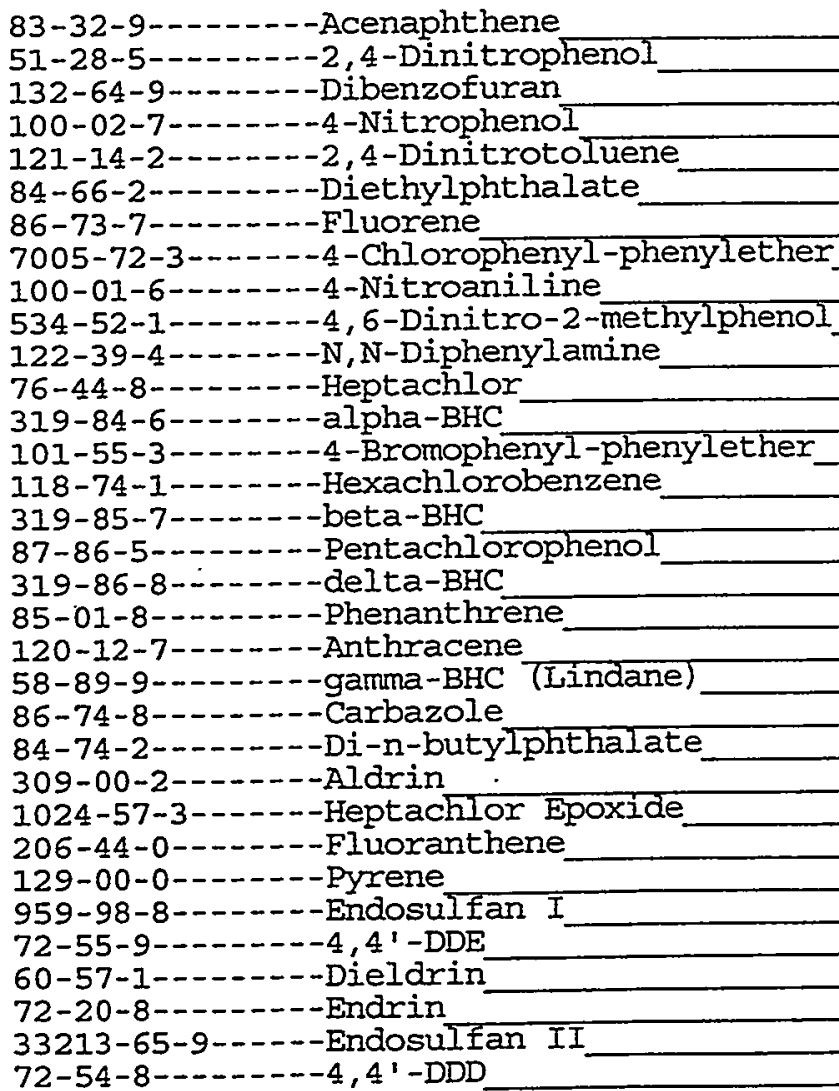 \\
\hline
\end{tabular}

$2 1 0 \longdiv { 0 }$

$37 \mathrm{~J}$

$210 \mathrm{U}$

$210 \mathrm{U}$

$210 \mathrm{U}$

$210 \mathrm{U}$

$210 \mathrm{U}$

$210 \mathrm{U}$

$210 \mathrm{U}$

$210 \mathrm{U}$

$210 \mathrm{U}$

$210 \mathrm{U}$

$210 \mathrm{U}$

$210 \mathrm{U}$

$210 \mathrm{U}$

$210 \mathrm{U}$

$210 \mathrm{U}$

$210 \mathrm{U}$

$210 \mathrm{U}$

$210 \mathrm{U}$

$210 \mathrm{U}$

$210 \mathrm{U}$

$210 \mathrm{U}$

$210 \mathrm{U}$

$210 \mathrm{U}$

$210 \mathrm{U}$

210

$210 \mathrm{U}$

$210 \mathrm{U}$

$210 \mathrm{U}$

210

$210 \mathrm{U}$

$210 \mathrm{U}$

FORM I SV-2

OLM03.0 
Lab Name: PNNL

Lab code: PNNL

Case No.:

Matrix: (soil/water) SUPERNATAN

Sample wt/vol: $(\mathrm{g} / \mathrm{mL}) \mathrm{ML}$

Level: (low/med) IOW

$\%$ Moisture: decanted: $(Y / N)$

Contract: BNFL

SAS NO.:
EPA SAMPLE NO.

AW-101

SDG No.: 990205

Concentrated Extract Volume: 2000 (uL)

Injection Volume: (uI)

Lab Sample ID: 99-0648

Lab File ID: 99020606

Date Received:

Date Extracted:

Date Analyzed: 02/06/99

Dilution Factor: 1.0

GPC Cleanup: $\quad(Y / N) N \quad p H: 12.5$

CONCENTRATION UNITS:

CAS NO. COMPOUND (ug/L or $u g / \mathrm{Kg}$ ) UG/L

$Q$

\begin{tabular}{|c|}
\hline 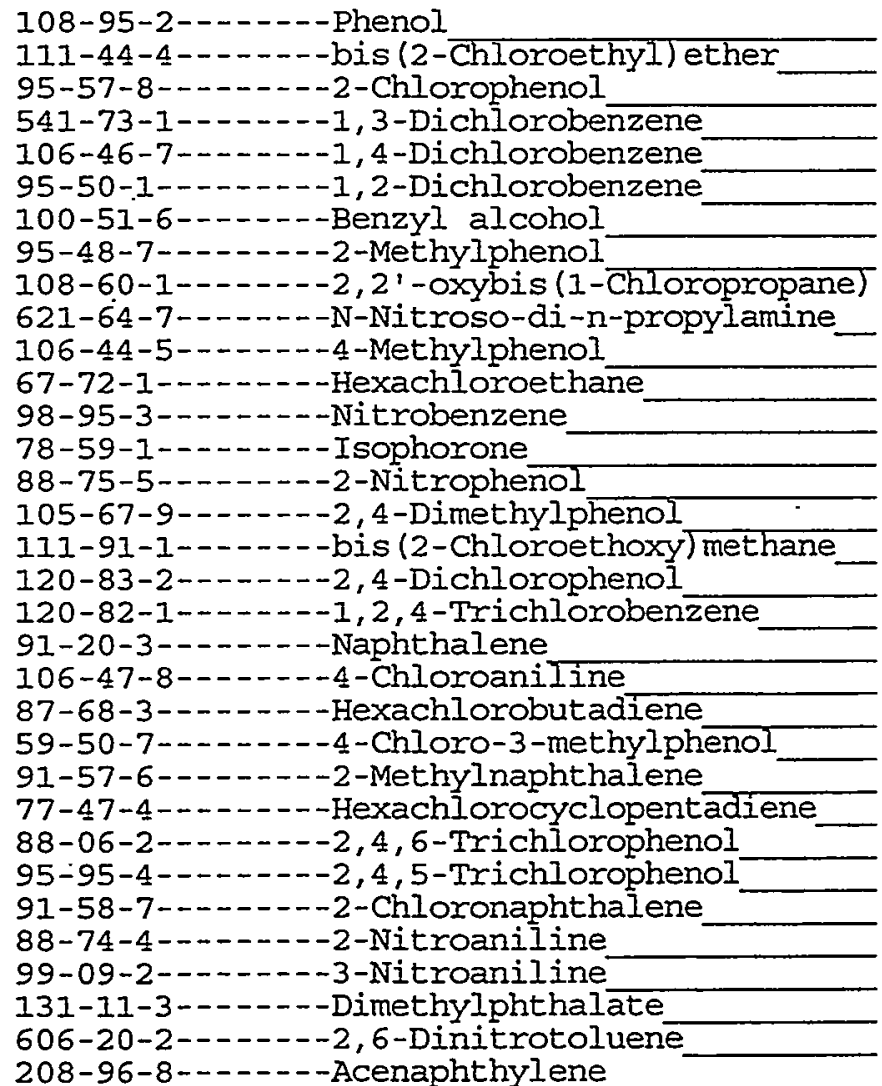 \\
\hline
\end{tabular}

\begin{tabular}{|l|l|l|}
210 & $U$ \\
210 & $U$ \\
210 & $U$ \\
210 & $U$ \\
210 & $U$ \\
210 & $U$ \\
210 & $U$ \\
210 & $U$ \\
210 & $U$ \\
210 & $U$ \\
210 & $U$ \\
210 & $U$ \\
210 & $U$ \\
210 & $U$ \\
210 & $U$ \\
210 & $U$ \\
210 & $U$ \\
210 & $U$ \\
210 & $U$ \\
210 & $U$ \\
210 & $U$ \\
210 & $U$ \\
210 & $U$ \\
210 & $U$ \\
210 & $U$ \\
210 & $U$ \\
210 & $U$ \\
210 & $U$ \\
210 & $U$ \\
210 & $U$ \\
210 & $U$ \\
210 & $U$ \\
210 & $U$ \\
& \\
\hline & \\
\hline
\end{tabular}

FORM I SV-1

OLMO3.0 
Lab Name: PNNL

Lab Code: PNNL

Case No.:

Matrix: (soil/water) SUPERNATAN

Sample wt/vol:

$(\mathrm{g} / \mathrm{mL}) \mathrm{ML}$

Level: (low $/$ med)

LOW

\% Moisture:

decanted: $(Y / N)$

Concentrated Extract Volume: 2000 (UT)

Injection Volume: (UI)
$\mathrm{pH}: 12 . .5$
GPC Cleanup
$(Y / N) N$

EPA SAMPLE NO.

AW-101

Contract: BNFL

SAS NO.:

SDG NO.: 990205

Lab Sample ID: 99-0648

Lab File ID: $\quad 99020606$

Date Received:

Date Extracted:

Date Analyzed: 02/06/99

Dilution Factor: 1.0

CONCENTRATION UNITS:

CAS NO.

COMPOUND

(ug/L or $u g / \mathrm{Kg}$ ) UG/L

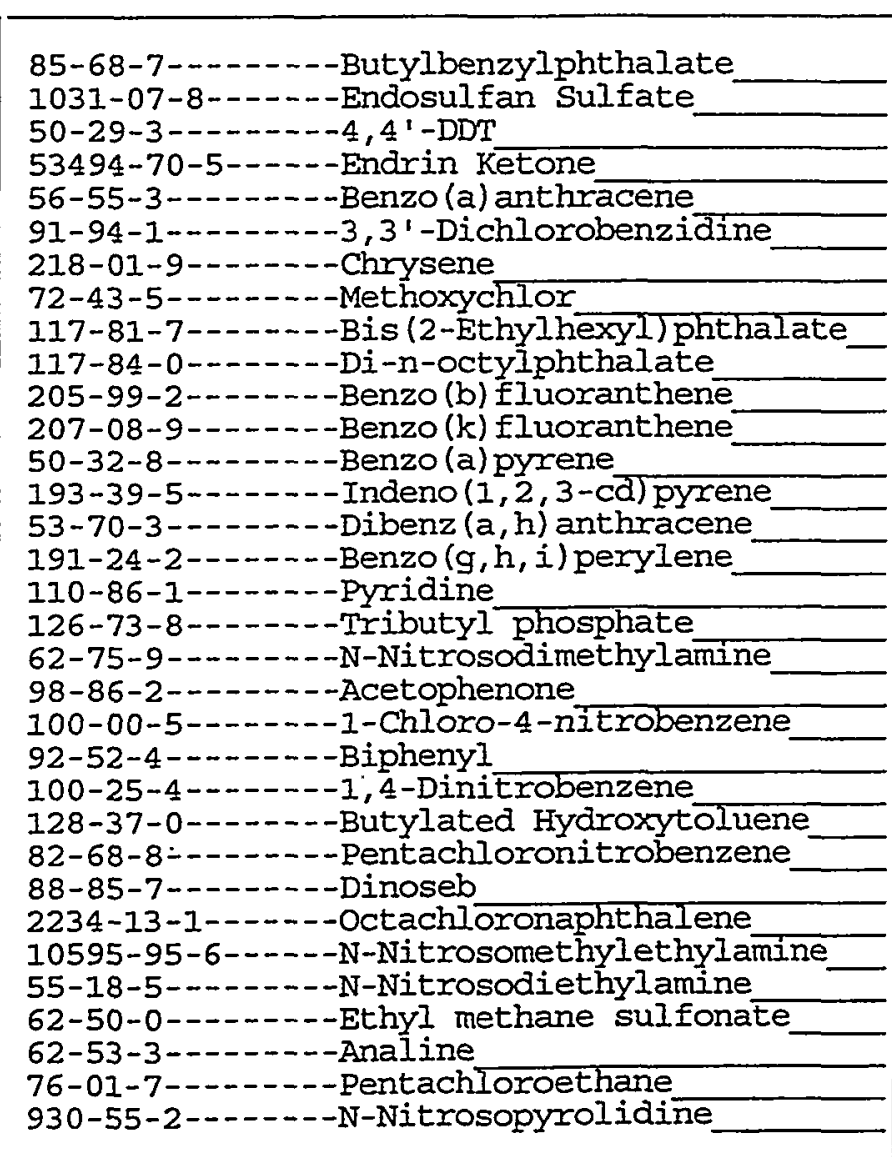

\begin{tabular}{|r|r|}
210 & $U$ \\
210 & $U$ \\
210 & $U$ \\
210 & $U$ \\
210 & $U$ \\
210 & $U$ \\
210 & $U$ \\
210 & $U$ \\
210 & $U$ \\
210 & $U$ \\
210 & $U$ \\
210 & $U$ \\
210 & $U$ \\
210 & $U$ \\
210 & $U$ \\
210 & $U$ \\
210 & $U$ \\
19 & $J$ \\
160 & $J$ \\
2 & $J$ \\
210 & $U$ \\
3 & $J$ \\
210 & $U$ \\
210 & $U$ \\
210 & $U$ \\
210 & $U$ \\
210 & $U$ \\
160 & $J$ \\
210 & $U$ \\
210 & $U$ \\
210 & $U$ \\
210 & $U$ \\
210 & $U$ \\
& \\
\hline & \\
\hline
\end{tabular}


Lab Name: PNNL

Iab Code: PNNL

Case No.:

Matrix: (soil/water) SUPERNATAN

Sample wt/vol: $(\mathrm{g} / \mathrm{mI}) \mathrm{MI}$

Level: (low/med) LOW

\% Moisture: decanted: $(Y / N)$

Concentrated Extract Volume: 2000 (uL) Injection Volume: (uL)

Contract: BNFL

SAS NO.:

SDG NO.: 990205
Lab Sample ID: 99-0648

Lab File ID: 99020606

Date Received:

Date Extracted:

Date Analyzed: 02/06/99

Dilution Factor: 1.0
EPA SAMPLE NO.

AW-101
GPC Cleanup:
$(\mathrm{Y} / \mathrm{N}) \mathrm{N}$
$\mathrm{pH}: 12.5$

CAS NO.

COMPOUND

CONCENIRATION UNITS:

(ug/L or $u g / \mathrm{Kg}$ ) UG/L

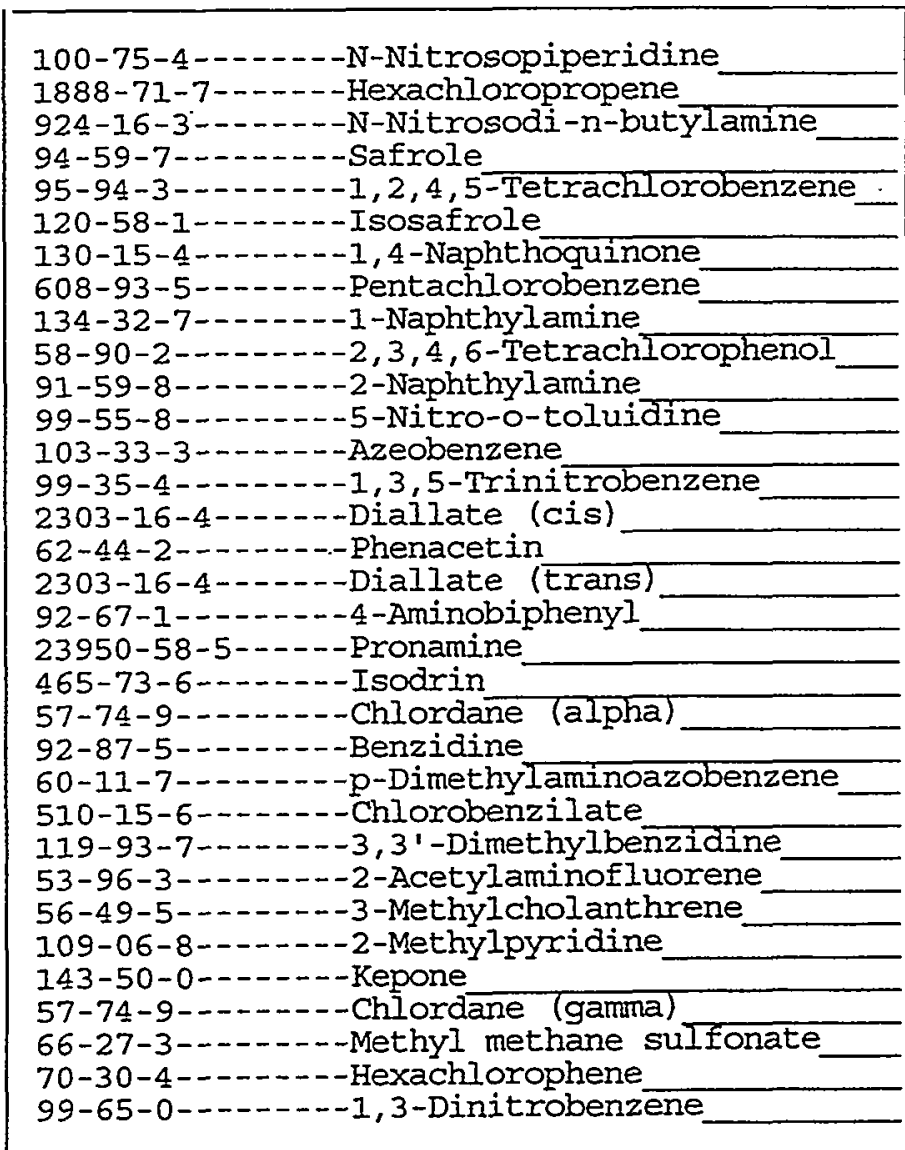


Lab Name: PNNL

Lab Code: PNNL

Case No.:

Matrix: (soil/water) SUPERNATAN

Sample wt/vol: $(\mathrm{g} / \mathrm{mL}) \mathrm{ML}$

Level: (low/med)

\% Moisture:

LOW

decanted: $(\mathrm{Y} / \mathrm{N})$

2000 (UL)

(uL)

Injection Volume:
Contract: BNFL SAS NO.: Lab Sample ID: 99-0648 Lab File ID: 99020606 Date Received:

Date Extracted:

Date Analyzed: 02/06/99

Dilution Factor: 1.0

$(\mathrm{Y} / \mathrm{N}) \mathrm{N}$

$\mathrm{pH}: 12.5$

GPC Cleanup:

CONCENTRATION UNITS:

CAS NO.

COMPOUND

(ug/L or ug/Kg) UG/L

Q

87-65-0---.---2, 6-Dichlorophenol

$210 \mathrm{U}$ 
Lab Name: PNNL

Lab Code: PNNL,

Case No.:

Matrix: (soil/water) SUPERNATAN

Sample wt/vol:

$(\mathrm{g} / \mathrm{mL}) \mathrm{ML}$

Level: (low/med) LOW

\% Moisture:

decanted: $(Y / N)$

Contract: BNFI

SAS NO.:
EPA SAMPLE NO.

AW-101D

SDG NO.: 990205

Concentrated Extract Volume: 2000(uI)

Injection Volume:

(uL)

Iab Sample ID: 99-0648D

Lab File ID: 99020607

Date Received:

Date Extracted:

Date Analyzed: 02/06/99

Dilution Factor: 1.0

GPC Cleanup: $(\mathrm{Y} / \mathrm{N}) \mathrm{N} \quad \mathrm{pH}: 12.5$

CONCENTRATION UNITS

CAS NO. COMPOUND (ug/L or $\mathrm{ug} / \mathrm{Kg}) \mathrm{UG} / \mathrm{L}$

$Q$

\begin{tabular}{|c|c|c|}
\hline 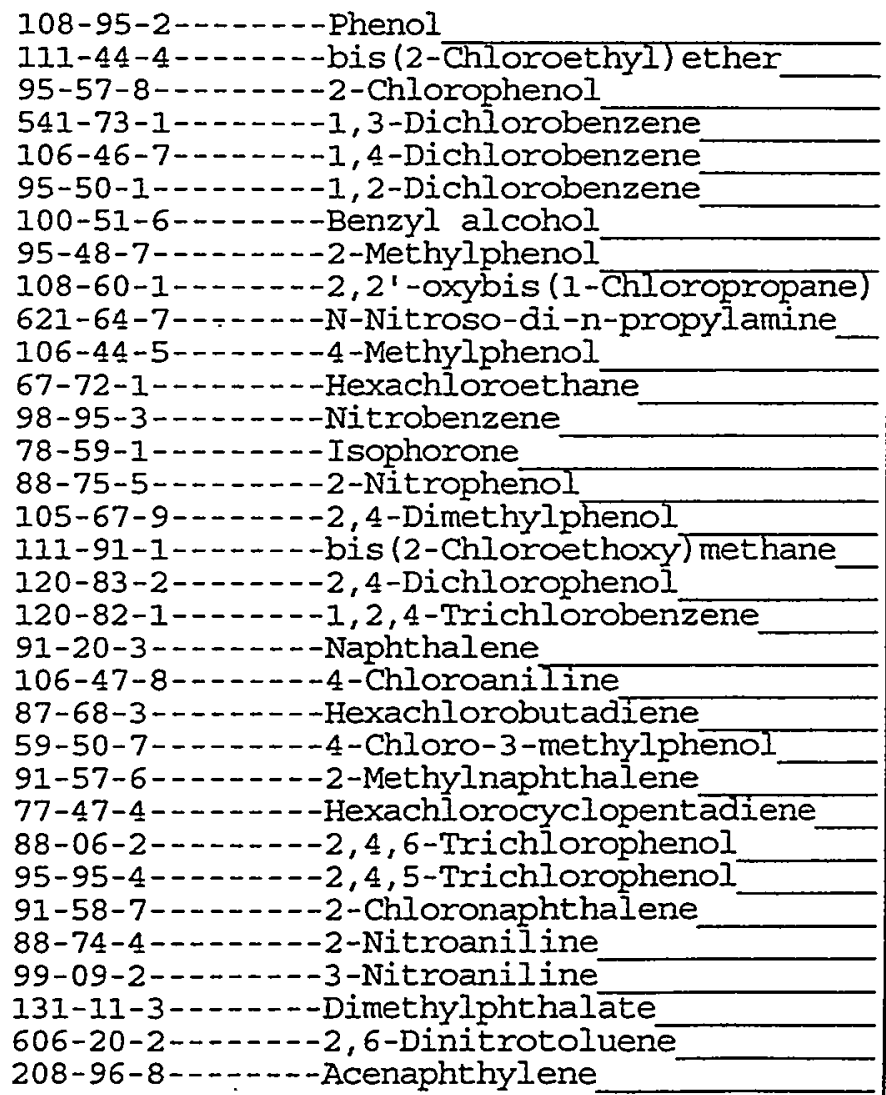 & $\begin{array}{l}200 \\
200 \\
200 \\
200 \\
200 \\
200 \\
200 \\
200 \\
200 \\
200 \\
200 \\
200 \\
200 \\
200 \\
200 \\
200 \\
200 \\
200 \\
200 \\
200 \\
200 \\
200 \\
200 \\
200 \\
200 \\
200 \\
200 \\
200 \\
200 \\
200 \\
200 \\
200 \\
200\end{array}$ & $\begin{array}{l}U \\
U \\
U \\
U \\
U \\
U \\
U \\
U \\
U \\
U \\
U \\
U \\
U \\
U \\
U \\
U \\
U \\
U \\
U \\
U \\
U \\
U \\
U \\
U \\
U \\
U \\
U \\
U \\
U \\
U \\
U \\
U \\
U\end{array}$ \\
\hline
\end{tabular}

FORM I SV-1

OLMO3.0 
Lab Name: PNNL

Lab code: PNNL,

Case No.:

Matrix: (soil/water) SUPERNATAN

Sample wt/vol: $(\mathrm{g} / \mathrm{mL}) \mathrm{ML}$

Level: (low/med)

LOW

\% Moisture: decanted: $(\mathrm{Y} / \mathrm{N})$

Contract: BNFL

SAS NO.:
EPA SAMPLE NO.

AW-IOID

SDG NO.: 990205

Concentrated Extract Volume: 2000 (uL)

Injection Volume: (ut)

Lab Sample ID: 99-0648D

GPC Cleanup: $\quad(\mathrm{Y} / \mathrm{N}) \mathrm{N}$

$\mathrm{pH}: 12.5$

Lab File ID: $\quad 99020607$

Date Received:

Date Extracted:

Date Analyzed: 02/06/99

Dilution Factor: 1.0

CAS NO.

COMPOUND

CONCENTRATION UNITS: (ug/L or $u g / \mathrm{Kg}$ ) UG/L

Q

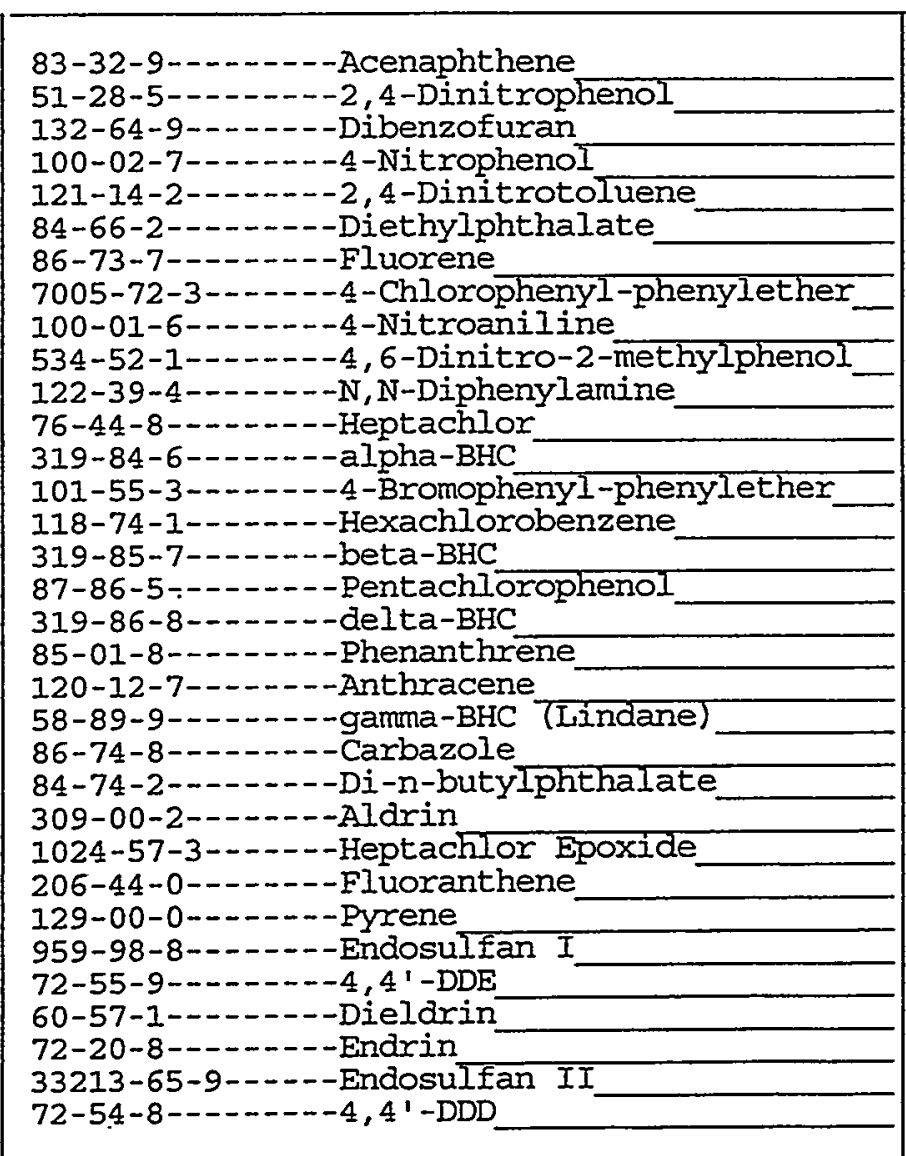

FORM I SV-2

OLMO3.0 
Lab Name: PNNL

Iab code: PNNL Case No.:

Matrix: (soil/water) SUPERNATAN

Sample wt/vol: $(\mathrm{g} / \mathrm{mL}) \mathrm{ML}$

Ievel: (low/med)

LOW

\% Moisture: decanted: $(Y / N)$

Contract : BNFL

SAS NO.:

Iab Sample ID: 99-0648D

Lab File ID: 99020607

Date Received:

Date Extracted:

Date Analyzed: 02/06/99

Concentrated Extract Volume: 2000 (UL) Injection Volume: (UI)

Dilution Factor: 1.0
AW-101D

SDG NO.: 990205

GPC Cleanup: (Y/N) N $\quad \mathrm{pH}: 12.5$

CONCENTRATION UNITS:

CAS NO. COMPOUND $\quad(u g / I$ or $u g / \mathrm{Kg}) \mathrm{UG} / \mathrm{L}$

$Q$

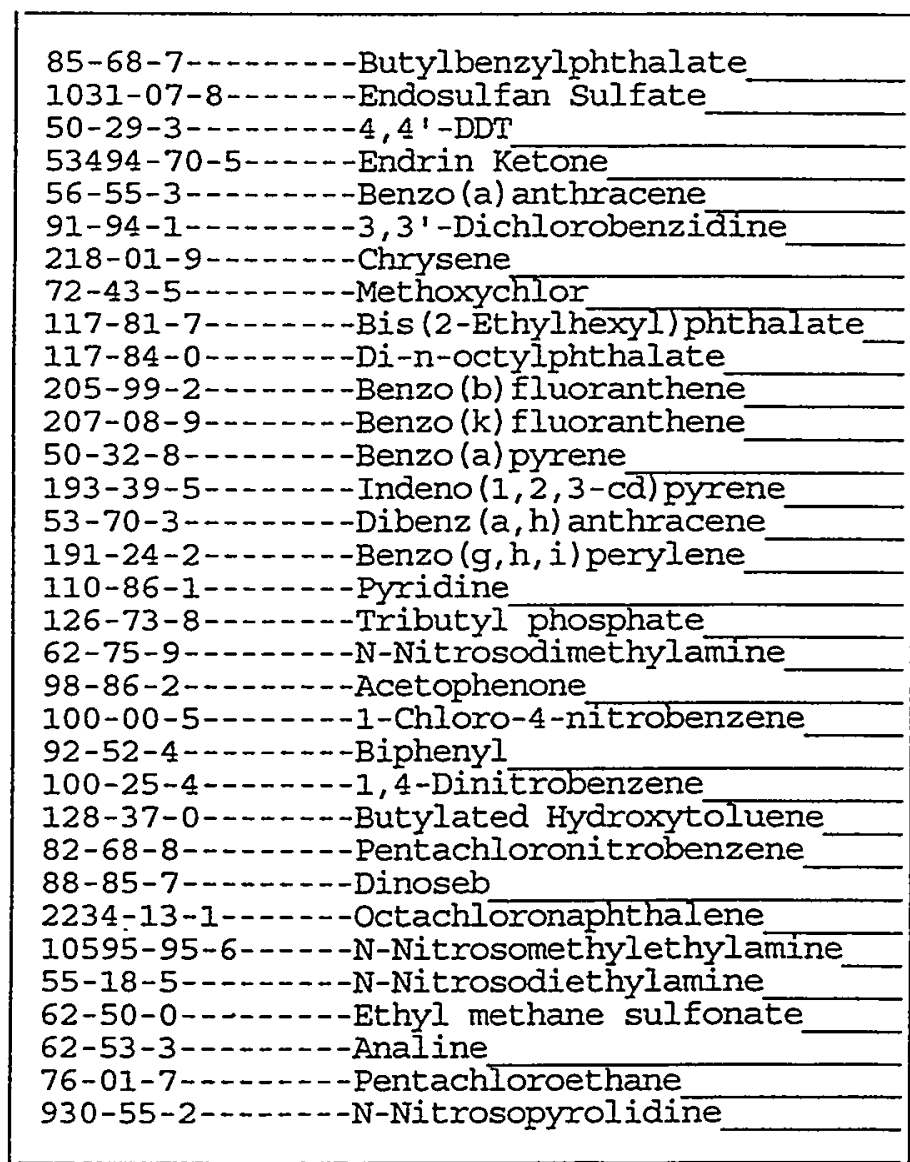

FORM I SV-3

OLM03.0 
Lab Name: PNNL

Lab Code: PNNL

Case No.:

Matrix: (soil/water) SUPERNATAN

Sample wt/vol: $(\mathrm{g} / \mathrm{mL}) \mathrm{ML}$

Level: (low/med) IOW

$\%$ Moisture: decanted: $(\mathrm{Y} / \mathrm{N})$

Concentrated Extract Volume: 2000 (ب) Injection Volume: (uI)

GPC Cleanup: $(Y / N) N$
Contract: BNEL

SAS NO. :

AW-101D

SDG No.: 990205

Lab Sample ID: 99-0648D

Lab File ID: 99020607

Date Received:

Date Extracted:

Date Analyzed: 02/06/99

Dilution Factor: 1.0

CAS NO.

COMPOUND

CONCENTRATION UNITS: (ug/L or ug/Kg) UG/L

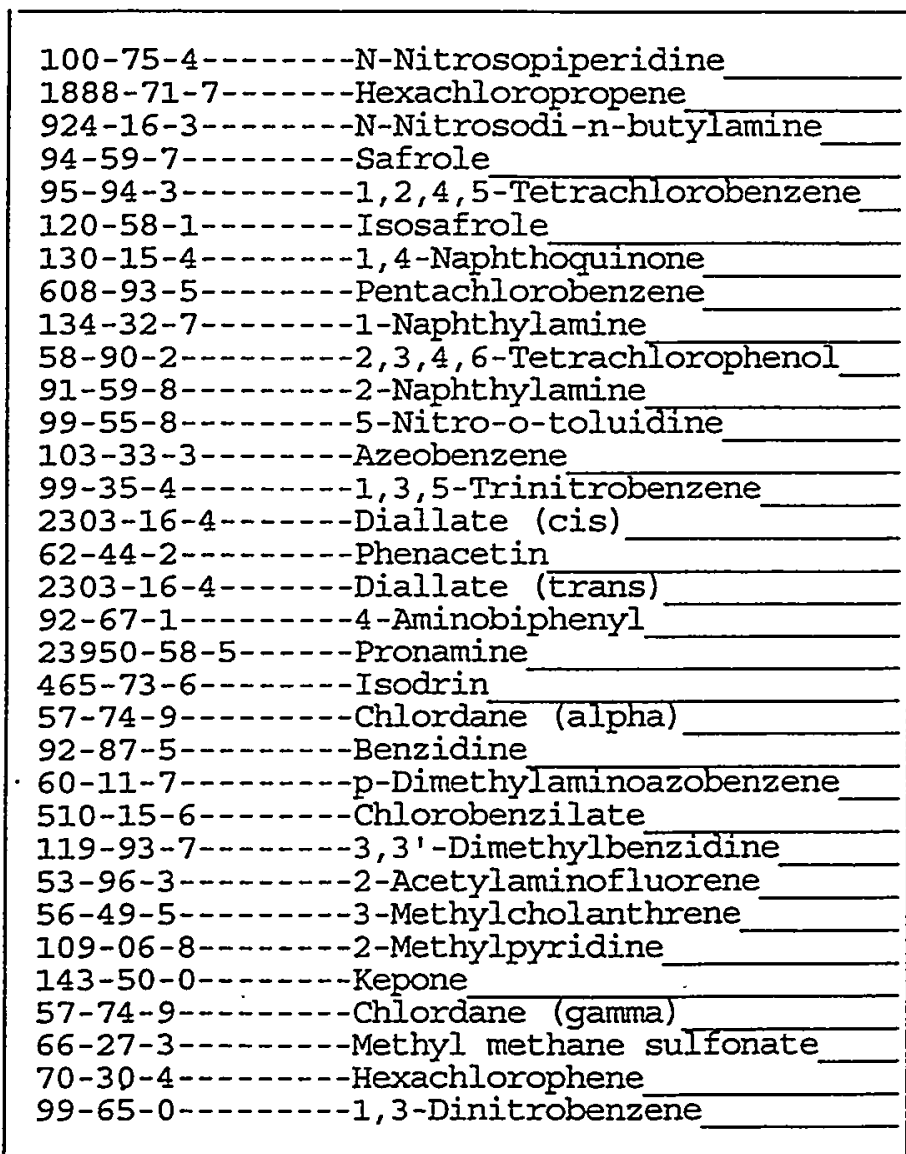

FORM I SV-4

\begin{tabular}{|r|r|}
\hline 200 & $U$ \\
200 & $U$ \\
200 & $U$ \\
200 & $U$ \\
200 & $U$ \\
200 & $U$ \\
200 & $U$ \\
200 & $U$ \\
3 & $J$ \\
200 & $U$ \\
200 & $U$ \\
200 & $U$ \\
200 & $U$ \\
200 & $U$ \\
200 & $U$ \\
200 & $U$ \\
200 & $U$ \\
200 & $U$ \\
200 & $U$ \\
200 & $U$ \\
200 & $U$ \\
200 & $U$ \\
200 & $U$ \\
200 & $U$ \\
200 & $U$ \\
200 & $U$ \\
200 & $U$ \\
200 & $U$ \\
200 & $U$ \\
200 & $U$ \\
200 & $U$ \\
200 & $U$ \\
200 & $U$ \\
& \\
\hline & \\
\hline
\end{tabular}

OIMO3. 0 
$1 \mathrm{C}$

SEMIVOLATILE ORGANICS ANALYSIS DATA SHEET

Lab Name: PNNL

Lab code: PNNL

Case No.:

Matrix: (soil/water) SUPERNATAN

Sample wt/vol: $(g / m U) ~ M U$

Level: (low/med)

LOW

decanted: $(Y / N)$

Contract : BNFL

SAS NO.:

Lab Sample ID: 99-0648D

Iab File ID: $\quad 99020607$

Date Received:

Date Extracted:

Date Analyzed: 02/06/99

Dilution Factor: 1.0

Injection Volume: (UI) 2000 (u)

GPC Cleanup: $(\mathrm{Y} / \mathrm{N}) \mathrm{N}$

$\mathrm{pH}: 12.5$

CAS NO.

COMPOUND

CONCENTRATION UNITS:

. (ug/L or $\mathrm{ug} / \mathrm{Kg}$ ) UG/L

Q

87-65-0--.----2,6-Dichlorophenol

$200 \mathrm{U}$


Lab Name: PNNL.

Lab Code: PNNL

Case No.:
Contract: BNFL

SAS NO.:

AW-101MS

Matrix: (soil/water) SUPERNATAN

Sample wt/vol: $(\mathrm{g} / \mathrm{mL}) \mathrm{ML}$

Level: (low/med) LOW

\% Moisture: decanted: $(Y / N)$

Concentrated Extract Volume: 2000 (uI)

Injection Volume:

(uL)

GPC Cleanup: $(Y / N) N \quad \mathrm{pH}: 12.5$
CAS NO.

COMPOUND
Lab Sample ID: 99-0648MS

Lab File ID: $\quad 99020608$

Date Received:

Date Extracted:

Date Analyzed: 02/06/99

Dilution Factor: 1.0

\begin{tabular}{|c|}
\hline 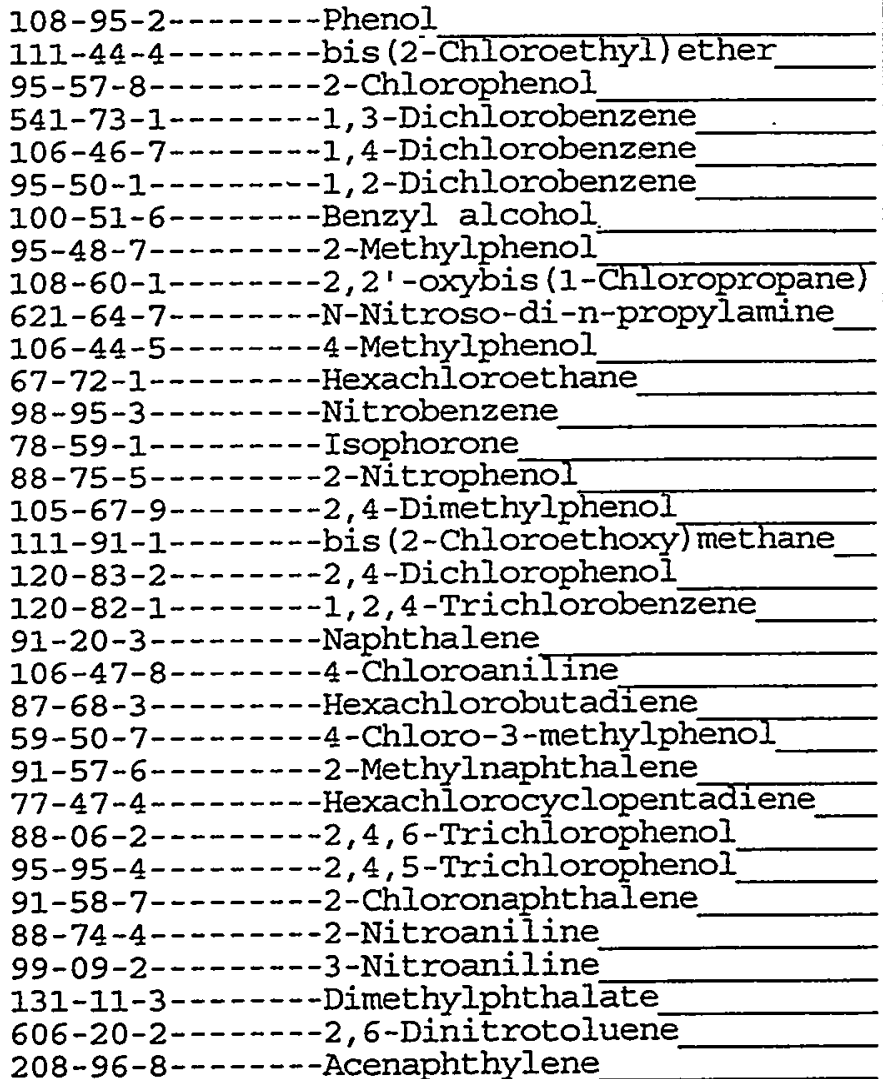 \\
\hline
\end{tabular}

FORM I SV-I
CONCENIRATION UNITS: (ug/L or $\mathrm{ug} / \mathrm{Kg}$ ) UG/L 
Lab Name: PNNL

Lab code: PNNL

Case No.:

Matrix: (soil/water) SUPERNATAN

Sample wt/vol:

Ievel: (low/med) LOW

$\div$ Moisture:

$(\mathrm{g} / \mathrm{mL}) \mathrm{ML}$

Concentrated Extract Volume: 2000 (uI)

Injection Volume:

(uI)
EPA SAMPLE NO.

AW-I0IMS

Contract : BNFL

SAS NO.:

SDG NO.: 990205

Lab Sample ID: 99-0648MS

Lab File ID: $\quad 99020608$

Date Received:

Date Extracted:

Date Analyzed: 02/06/99

Dilution Factor: 1.0

GPC Cleanup: $(\mathrm{Y} / \mathrm{N}) \mathrm{N} \quad \mathrm{pH}: 12.5$

CAS NO.

COMPOUND

CONCENTRATION UNITS:

(ug/I or $u g / \mathrm{Kg}$ ) UG/L

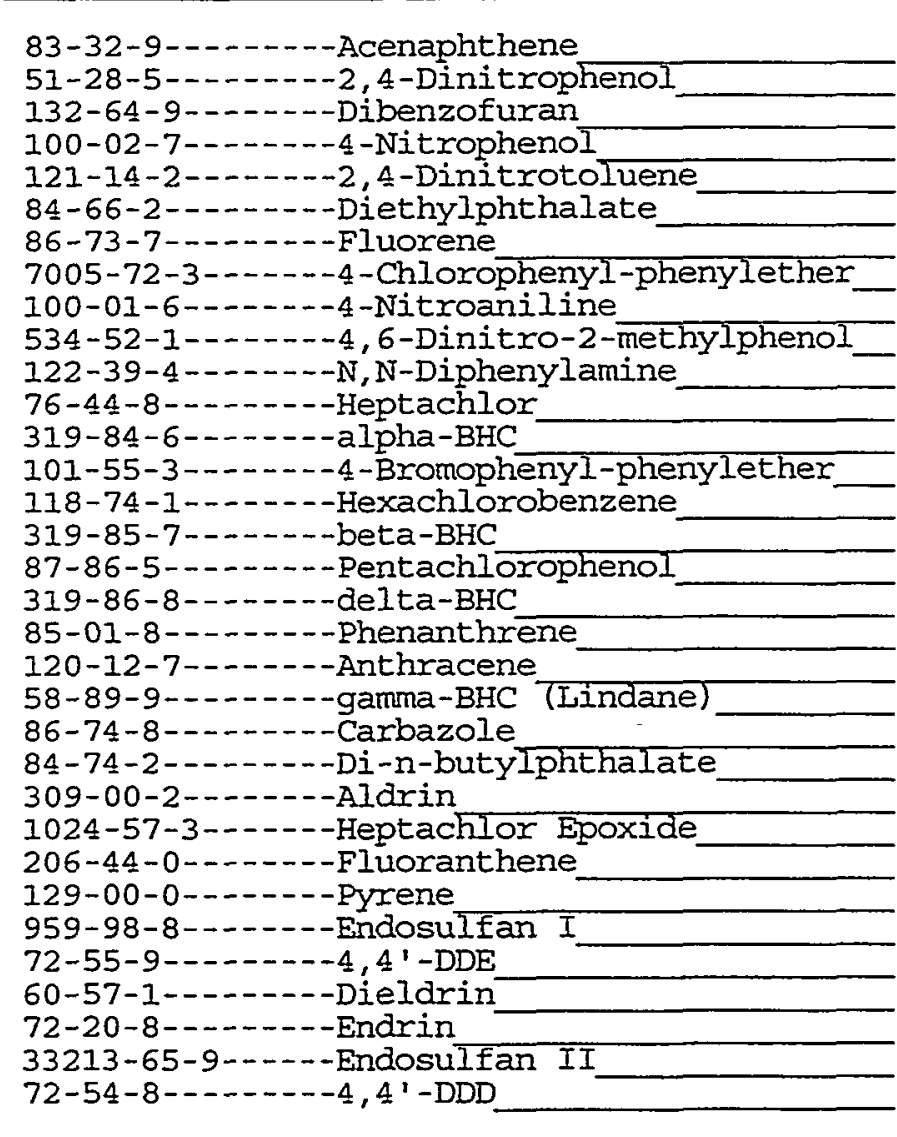

FORM I SV-2

\begin{tabular}{|l|l|}
770 & \\
300 & $\mathrm{U}$ \\
300 & $\mathrm{U}$ \\
840 & \\
550 & \\
300 & $\mathrm{U}$ \\
300 & $\mathrm{U}$ \\
300 & $\mathrm{U}$ \\
300 & $\mathrm{U}$ \\
200 & $\mathrm{U}$ \\
640 & \\
300 & $\mathrm{U}$ \\
300 & $\mathrm{U}$ \\
300 & $\mathrm{U}$ \\
300 & $\mathrm{U}$ \\
300 & $\mathrm{U}$ \\
920 & \\
300 & $\mathrm{U}$ \\
300 & $\mathrm{U}$ \\
300 & $\mathrm{U}$ \\
300 & $\mathrm{U}$ \\
300 & $\mathrm{U}$ \\
300 & $\mathrm{U}$ \\
300 & $\mathrm{U}$ \\
300 & $\mathrm{U}$ \\
300 & $\mathrm{U}$ \\
890 & \\
300 & $\mathrm{U}$ \\
300 & $\mathrm{U}$ \\
300 & $\mathrm{U}$ \\
300 & $\mathrm{U}$ \\
300 & $\mathrm{U}$ \\
300 & $\mathrm{U}$ \\
& \\
\hline & \\
\hline
\end{tabular}

OLM03.0 
Lab Name: PNNL

Lab code: PNNL

Case No.:

SAS NO. :

AW-101MS

Matrix: (soil/water) SUPERNATAN

Sample wt/vol: $(\mathrm{g} / \mathrm{mL}) \mathrm{ML}$

Lab Sample ID: 99-0648MS

Level: (low/med) LOW

Lab File ID: 99020608

\% Moisture: decanted: $(Y / N)$

Date Received:

Date Extracted:

Concentrated Extract Volume: 2000 (uU) Date Analyzed: 02/06/99

Injection Volume: (UI)

Dilution Factor: 1.0

GPC Cleanup: $\quad(\mathrm{Y} / \mathrm{N}) \mathrm{N} \quad \mathrm{pH}: 12.5$

$\begin{array}{ll}\text { COS NO. COMPOUND } & \text { CONCENTRATION UNITS: } \\ \text { (ug/L or ug/Kg) UG/L }\end{array}$

\begin{tabular}{|c|}
\hline 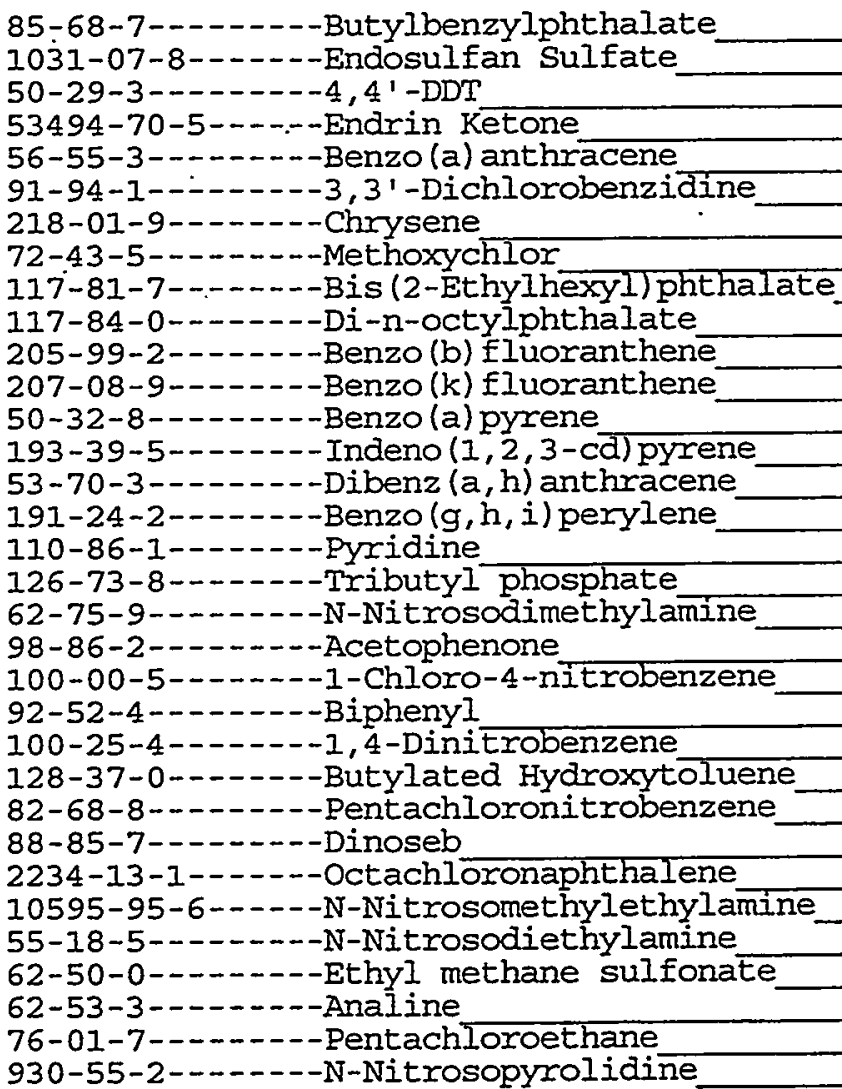 \\
\hline
\end{tabular}

FORM I SV-3

OLM03. 0 
Lab Name: PNNL

Lab code: PNNL

Case No. :

Matrix: (soil/water) SUPERNATAN

Sample wt/vol: $(\mathrm{g} / \mathrm{mL}) \mathrm{MI}$

Level: (low/med) IOW

\% Moisture: decanted: $(Y / N)$

Concentrated Extract Volume: 2000 (UI) Injection Volume: (uL)

AW-IOIMS

Contract : BNFI

SAS NO.:

SDG No.: 990205

Lab Sample ID: 99-0648MS

Lab File ID: 99020608

Date Received:

Date Extracted:

Date Analyzed: 02/06/99

Dilution Factor: 1.0

GPC Cleanup: $(Y / N) N \quad \mathrm{NH}: 12.5$

CONCENTRATION UNITS:

CAS NO. COMPOUND (ug/I or ug/Kg) UG/I

Q

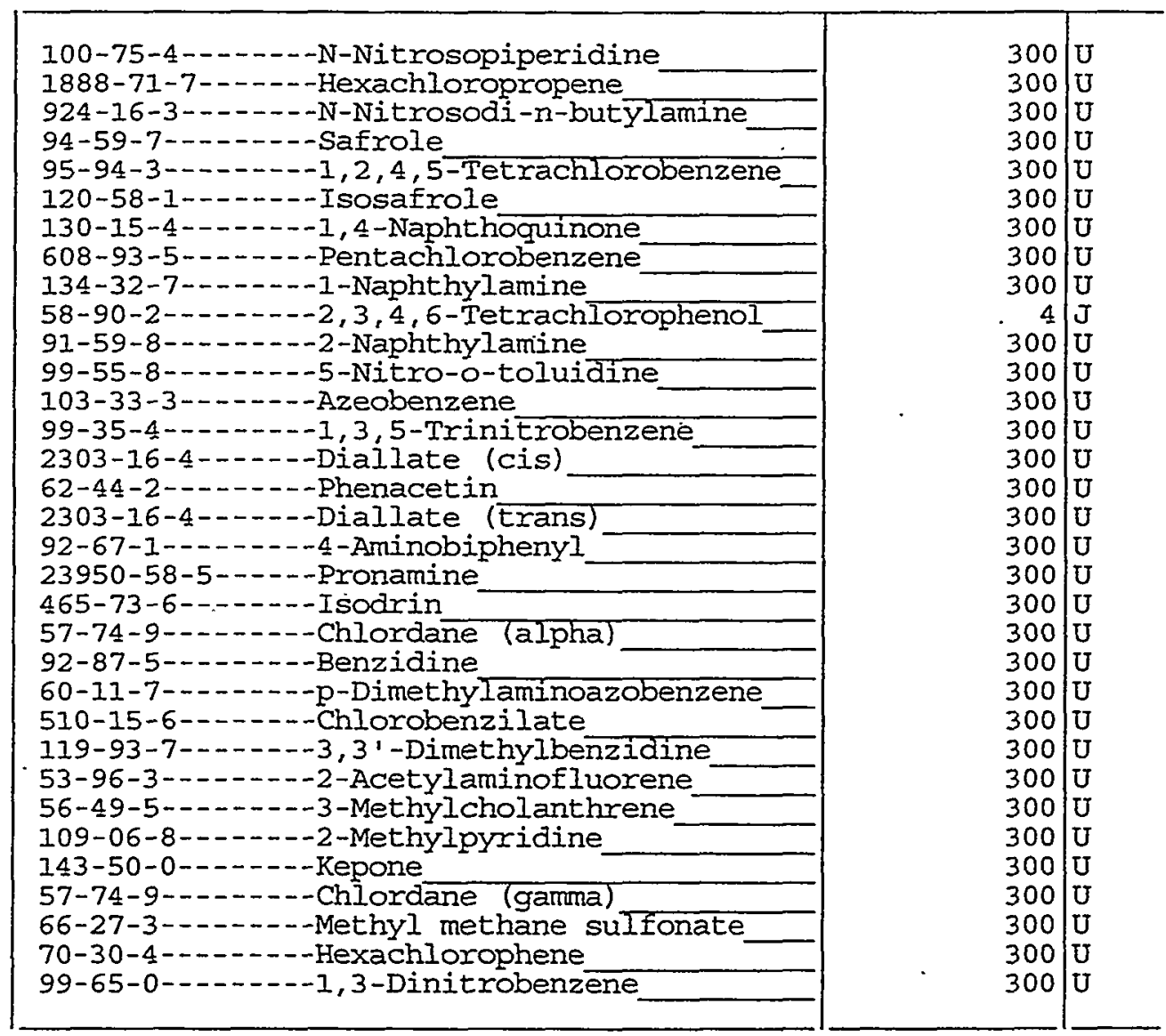


Lab Name: PNNL

Contract: BNFL

Iab Code: PNNL Case No.:

SAS Ño. :

AW-101MS .

Matrix: (soil/water) SUPERNATAN

Lab Sample ID: 99-0648MS

- Sample wt/vol: $(\mathrm{g} / \mathrm{mL}) \mathrm{ML}$

Lab File ID: $\quad 99020608$

Level: (low/med) IOW

Date Received:

$\%$ Moisture: decanted: $(Y / N)$

Date Extracted:

- Concentrated Extract Volume: 2000 (uI)

Date Analyzed: 02/06/99

Injection Volume: (uI)

Dilution Factor: 1.0

GPC Cleanup: $(Y / N)$ N

$\mathrm{pH}: 12.5$

$\begin{array}{ll}\text { CAS NO. } \quad \text { CONCENTRATION UNITS: } \\ \text { COMPOUND } & (\mathrm{ug} / \mathrm{L} \text { or } \mathrm{ug} / \mathrm{Kg}) \mathrm{UG} / \mathrm{I}\end{array}$


Lab Name: PNNL

Lab Code: PNNL - Case No.:

Matrix: (soil/water) SUPERNATAN

Sample wt/vol:

$(\mathrm{g} / \mathrm{mL}) \mathrm{ML}$

Level: (low/med) IOW

$\%$ Moisture:

decanted: $(\mathrm{Y} / \mathrm{N})$

Concentrated Extract Volume: 2000 (uI)

Injection Volume:

(uL)
Contract: BNFL

SAS NO.:
AW-101MSD

SDG No.: 990205

GPC Cleanup: $\quad(\mathrm{Y} / \mathrm{N}) \mathrm{N} \quad \mathrm{pH}: 12.5$

Lab Sample ID: 99-0648MSD

Lab File ID: 99020609

Date Received:

Date Extracted:

Date Analyzed: 02/06/99

Dilution Factor: 1.0

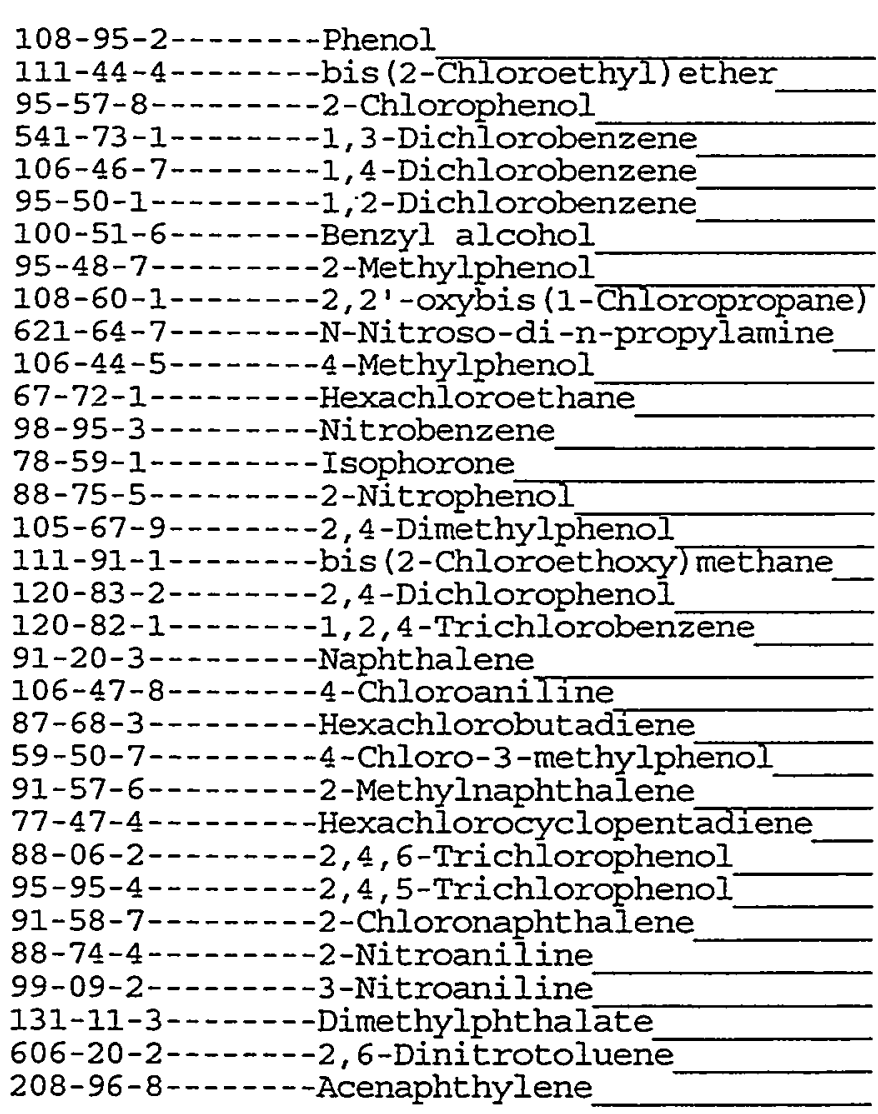

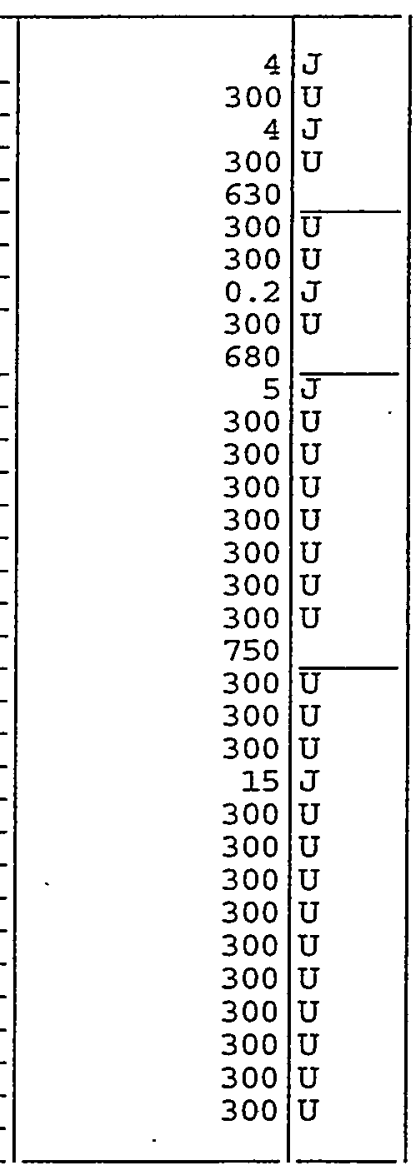

FORM I SV-I
OLMO3.0 
Lab Name: PNNL

Lab code: PNNL

Case No.:

Matrix: (soil/water) SUPERNATAN

Sample wt/vol:

$(\mathrm{g} / \mathrm{mL}) \mathrm{ML}$

Level: (low/med) LOW

\% Moisture: decanted: $(\mathrm{Y} / \mathrm{N})$

Concentrated Extract Volume: 2000 (uI)

Injection Volume: (UI)

Contract : BNEL

SAS NO.:

Lab Sample ID: 99-0648MSD

Lab File ID: 99020609

Date Received:

Date Extracted:

Date Analyzed: 02/06/99

Dilution Factor: 1.0

GPC Cleanup: (Y/N) N $\mathrm{NH}: 12.5$

CONCENTRATION UNITS :

CAS NO.

COMPOUND

(ug/L or ug/Kg) UG/L

$\bar{Q}$

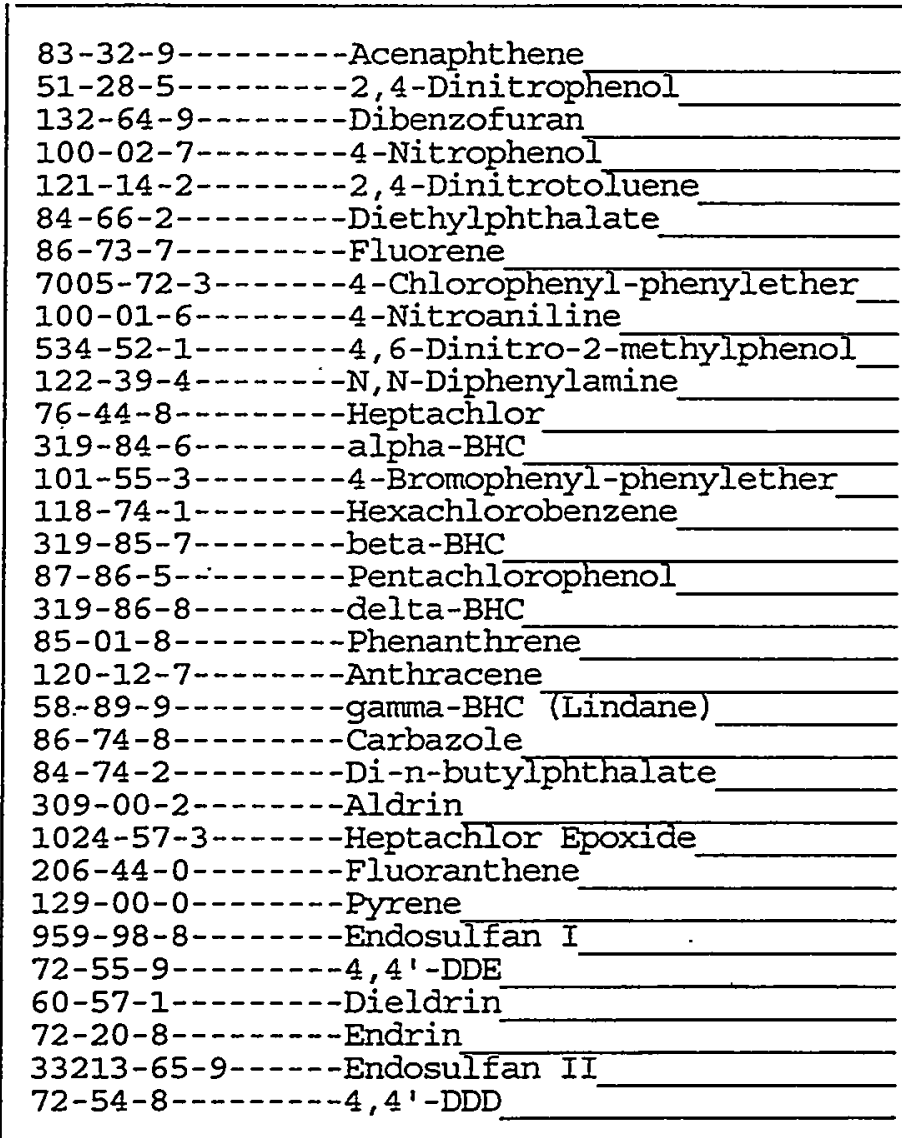

FORM I SV-2

OIMO 3.0 
Lab Name: PNNL

Lab Code: PNNL

Case No.:

Matrix: (soil/water) SUPERNATAN

Sample wt/vol:

$(\mathrm{g} / \mathrm{mL}) \mathrm{ML}$

Level: (low/med) LOW

$\div$ Moisture: decanted: $(\mathrm{Y} / \mathrm{N})$

Concentrated Extract Volume: 2000 (uI)

Injection Volume: (UI)

Contract: BNFL
EPA SAMPLE NO.

AW-101MSD

SDG No.: 990205

Iab Sample ID: 99-0648MSD

Lab File ID: 99020609

Date Received:

Date Extracted:

Date Analyzed: 02/06/99

Dilution Factor: 1.0

GPC Cleanup: $\quad(Y / N) N \cdot \quad$ pH: 12.5

\section{CONCENTRATION UNITS:}

CAS NO.

COMPOUND

(ug/L or $\mathrm{ug} / \mathrm{Kg}$ ) UG/L

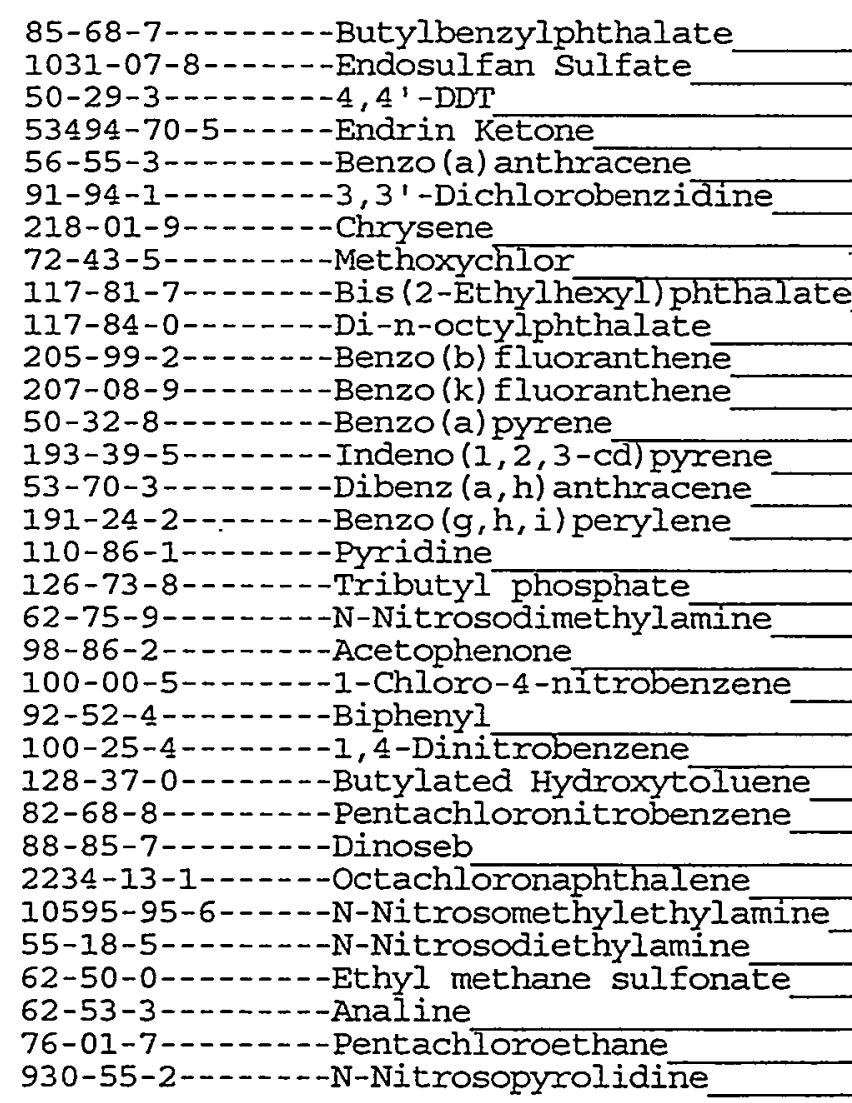

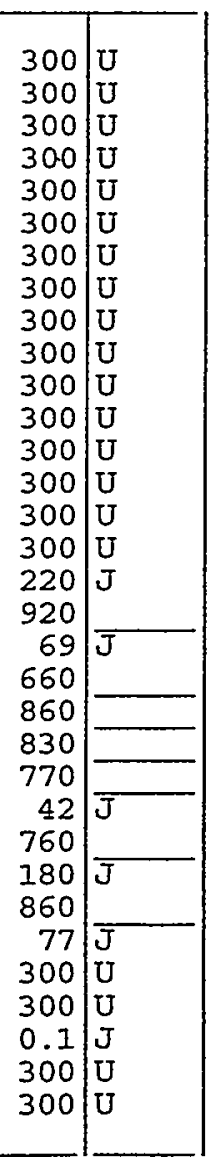

OLM03.0 
Lab Name: PNNL

Lab Code: PNNL

Case No.:
Contract: BNFL

SAS NO. :
EPA SAMPLE NO.

AW-IOIMSD

SDG NO. : 990205
Matrix: (soil/water) SUPERNATAN

Sample wt/vol: $(\mathrm{g} / \mathrm{mL}) \mathrm{ML}$

Level: (low/med)

LOW

$\div$ Moisture: decanted: $(Y / N)$

Concentrated Extract Volume: 2000 (uU)

Injection Volume: (UI)

GPC Cleanup: $(\mathrm{Y} / \mathrm{N}) \mathrm{N} \quad \mathrm{pH}: 12.5$
Lab Sample ID: 99-0648MSD

Iab File ID: 99020609

Date Received:

Date Extracted:

Date Analyzed: 02/06/99

Dilution Factor: 1.0

CAS NO.

COMPOUND

CONCENTRATION UNITS: (ug/L or ug/kg) UG/L

$\bar{Q}$

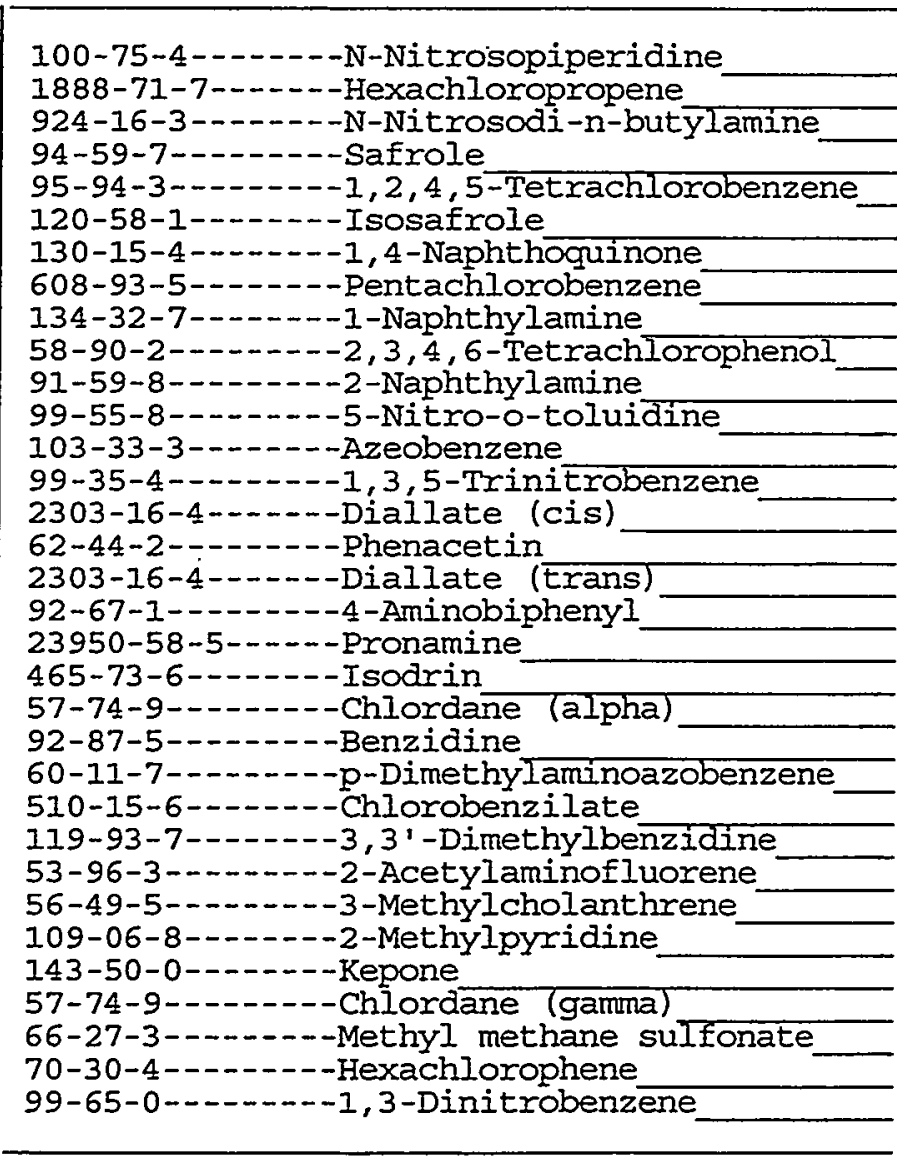


Lab Code: PNNL

Case No.:

SAS NO.:

AW-101MSD

Matrix: (soil/water) SUPERNATAN

Iab Sample ID: 99-0648MSD

Sample wt/vol:

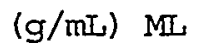

Lab File ID: 99020609

Level: (low/med) LOW

Date Received:

$\%$ Moisture: decanted: $(\mathrm{Y} / \mathrm{N})$

Date Extraçted:

Concentrated Extract Volume: 2000 (uI)

Date Analyzed: 02/06/99

Injection Volume: (ut)

Dilution Factor: 1.0

GPC Cleanup: $(\mathrm{Y} / \mathrm{N}) \mathrm{N}$

$\mathrm{pH}: 12.5$

\begin{tabular}{|c|c|c|c|c|}
\hline CAS NO. & COMPOUND & $\begin{array}{l}\text { CONCENTRATION UNITS: } \\
(\mathrm{ug} / \mathrm{L} \text { or } \mathrm{ug} / \mathrm{Kg}) \text { UG/L }\end{array}$ & & $\bar{Q}$ \\
\hline $87-65-0$ & 2,6-Dichlorophenol & & 300 & $U$ \\
\hline
\end{tabular}


Lab Name: PNNL

Lab code: PNNL

Case No.:

Matrix: (soil/water) SUPERNATAN

Sample wt/vol: (g/mU) MU

Level: (low/med)

LOW

$\because$ Moisture: decanted: $(Y / N)$

Concentrated Extract Volume: 2000 (uL)

Injection Volume: (uI)

Contract : BNFL

SAS NO.: Lab Sample ID: SBLK03 Lab File ID: 99020605 Date Received:

Date Extracted:

Date Analyzed: 02/06/99

Dilution Factor: 1.0

GPC Cleanup: $(Y / N) N \quad \mathrm{NH}: 7.0$

CONCENTRATION UNITS :

CAS NO.

COMPOUND

(ug/I or $\mathrm{ug} / \mathrm{Kg}$ ) UG/L

108-95-2-------Phenol

111-44-4--.---bis (2-Chloroethyl) ether

95-57-8-.......-2-Chlorophenol

541-73-1------1, 3-Dichlorobenzene

106-46-7------1, 4-Dichlorobenzene

95-50-1-...-1, 2-Dichlorobenzene

100-51-6-....--Benzyl alcohol

95-48-7-....--2-Methylphenol

108-60-1-....-2, $2^{\prime}$-oxybis (1-Chloropropane)

621-64-7--.---N-Nitroso-di-n-propylamine

106-44-5------4-Methylphenol

67-72-1_......-Hexachloroethane

98-95-3-.....-Nitrobenzene

78-59-1_.....-Isophorone

88-75-5-...-.-2-Nitrophenol

105-67-9-...-2, 4-Dimethylphenol

11I-91-1-....-bis (2-Chloroethoxy) methane

120-83-2-..--2,4-Dichlorophenol

120-82-1-.......-1, 2,4-Trichlorobenzene

91-20-3-------Naphthalene

106-47-8---1--4-Chloroaniline

87-68-3-...-.-Hexachlorobutadiene

59-50-7--.---4-Chloro-3-methylphenol

91-57-6-------2-Methylnaphthalene

77-47-4------Hexachlorocyclopentadiene

88-06-2-......-2, 4, 6-Trichlorophenol

95-95-4-...-.-2, 4, 5-Trichlorophenol

91-58-7-....-2-Chloronaphthalene

88-74-4-.-----2-Nitroaniline

99-09-2-.....-3-Nitroaniline

131-11-3------Dimethylphthalate

606-20-2--.---2,6-Dinitrotoluene

208-96-8-----Acenaphthylene
EPA SAMPLE NO.

METHOD BLANK

SDG No.: 990205 
Lab Name: PNNL

Iab code: PNNL

Case No. :
Contract: BNFL

SAS NO.:
EPA SAMPLE NO.

METHOD BLANK
Matrix: (soil/water) SUPERNATAN

Sample wt/vol: $(\mathrm{g} / \mathrm{mL}) \mathrm{ML}$

Level: (low/med) IOW

\% Moisture: decanted: $(Y / N)$

Concentrated Extract Volume: 2000 (uU) Injection Volume: (uL)
Lab Sample ID: SBLK03

Lab File ID: 99020605

Date Received:

Date Extracted:

Date Analyzed: 02/06/99

Dilution Factor: 1.0
GPC Cleanup:
$(\mathrm{Y} / \mathrm{N}) \mathrm{N}$
$\mathrm{pH}: 7.0$

CONCENTRATION UNITS:

CAS NO. COMPOUND $(u g / L$ or $u g / K g) U G / L$ Q

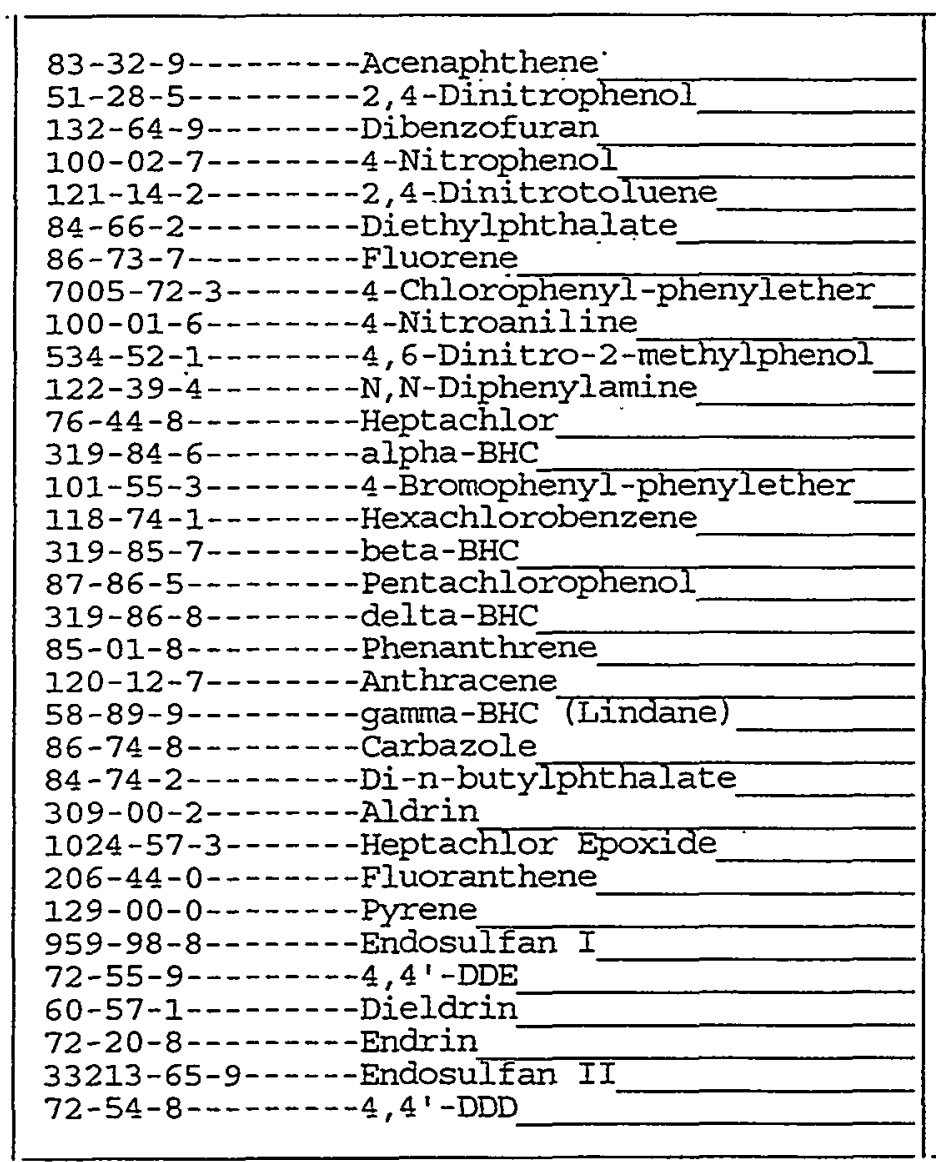


Lab Name: PNNL

Contract: BNFL

Lab Code: PNNL

Case No.:

SAS NO.:

METHOD BLANK

Matrix: (soil/water) SUPERNATAN

Sample wt/vol:

(g/mI) MI

Level: (low/med) LOW

: Moisture:

decanted: $(\mathrm{Y} / \mathrm{N})$

Concentrated Extract Volume: 2000 (uI)

Injection Volume:

(uI)

GPC Cleanup: $(\mathrm{Y} / \mathrm{N}) \mathrm{N} \quad \mathrm{pH}: 7.0$

GPC Cleanup: $(\mathrm{Y} / \mathrm{N}) \mathrm{N} \quad \mathrm{pH}: 7.0$
Iab Sample ID: SBLK03

Lab File ID: 99020605

Date Received:

Date Extracted:

Date Analyzed: 02/06/99

Dilution Factor: 1.0

CAS NO.

COMPOUND

CONCENTRATION UNITS

(ug/I or $u g / \mathrm{Kg}$ ) UG/I

\begin{tabular}{|c|}
\hline 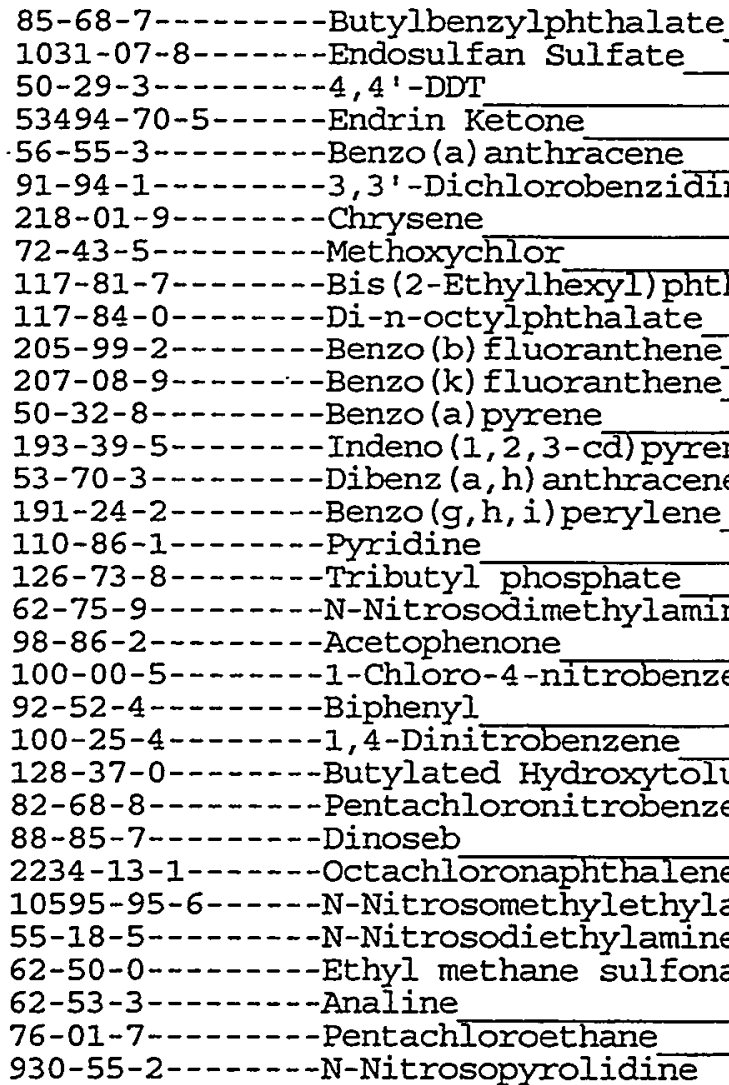 \\
\hline
\end{tabular}

FORM I SV-3 
Lab Name: PNNL

Lab Code: PNNL

Case No.:

Matrix: (soil/water) SUPERNATAN

Sample wt/vol:

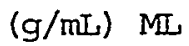

\% Moisture:

decanted: $(\mathrm{Y} / \mathrm{N})$

Concentrated Extract Volume: 2000 (uI)

Injection Volume:

(uL)

Contract : BNFL

SAS NO.:

Lab Sample ID: SBLK03

Lab File ID: 99020605

Date Received:

Date Extracted:

Date Analyzed: 02/06/99

Dilution Factor: 1.0

GPC Cleanup: $(Y / N) N \quad \mathrm{pH}: 7.0$

CONCENTRATION UNITS:

CAS NO.

COMPOUND

(ug/I or ug/Kg) UG/L

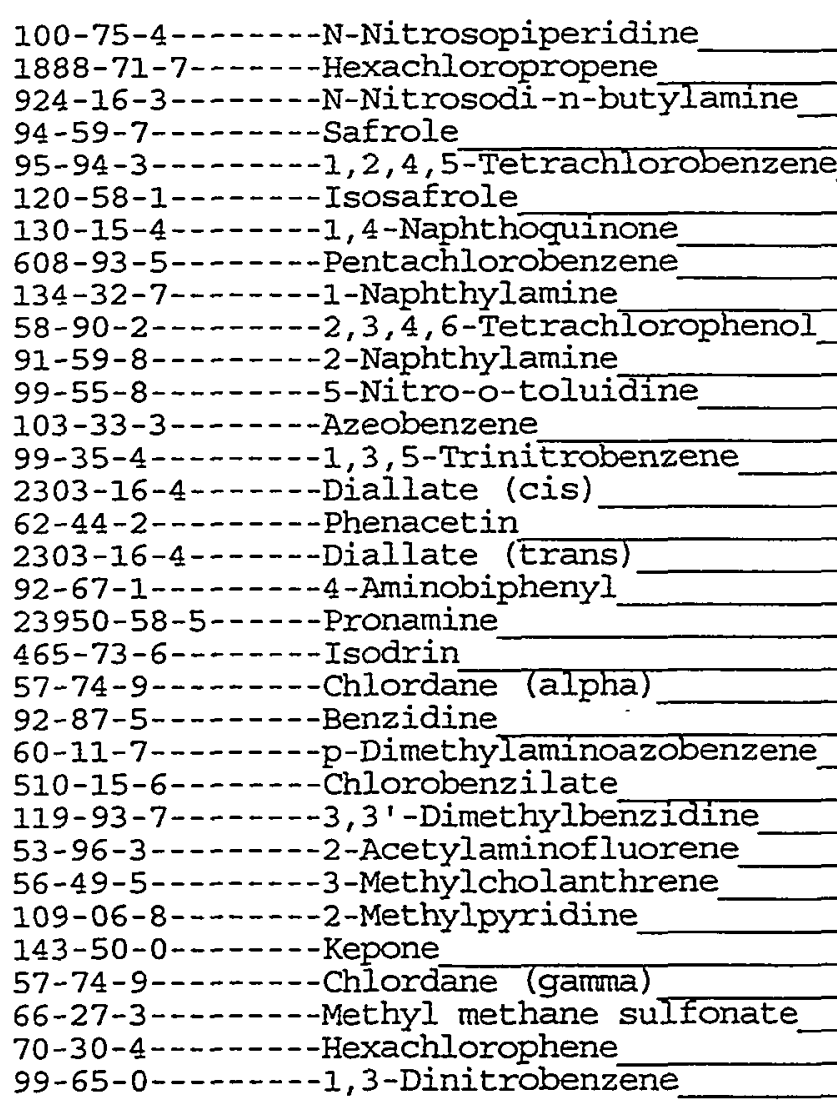

\begin{tabular}{|l|l|}
300 & $U$ \\
300 & $U$ \\
300 & $U$ \\
300 & $U$ \\
300 & $U$ \\
300 & $U$ \\
300 & $U$ \\
300 & $U$ \\
300 & $U$ \\
300 & $U$ \\
300 & $U$ \\
300 & $U$ \\
300 & $U$ \\
300 & $U$ \\
300 & $U$ \\
300 & $U$ \\
300 & $U$ \\
300 & $U$ \\
300 & $U$ \\
300 & $U$ \\
300 & $U$ \\
300 & $U$ \\
300 & $U$ \\
300 & $U$ \\
300 & $U$ \\
300 & $U$ \\
300 & $U$ \\
300 & $U$ \\
300 & $U$ \\
300 & $U$ \\
300 & $U$ \\
300 & $U$ \\
300 & $U$ \\
& \\
\hline & \\
\hline
\end{tabular}


Lab Name: PNNL Contract: BNFL EPA SAMPLE NO.

Lab Code: PNNL Case No.:

SAS NO.:

METHOD BLANK

Matrix: (soil/water) SUPERNATAN

Lab Sample ID: SBLKO3

Sample wt/vol: $(\mathrm{g} / \mathrm{mL}) \mathrm{ML}$ Iab File ID: 99020605

Level: (low/med) LOW

Date Received:

$\%$ Moisture: decanted: $(Y / N)$

Date Extracted:

Concentrated Extract Volume: 2000 (uI)

Injection Volume: (uL)

Date Analyzed: 02/06/99

GPC Cleanup: $(Y / N) N$

$\mathrm{pH}: 7.0$

Dilution Factor: 1.0

$\begin{array}{ll}\text { CAS NO. COMPOUND } & \text { CONCENTRATION UNITS: } \\ \text { (ug/L or ug/Kg) UG/L }\end{array}$

87-65-0---.---2, 6-Dichlorophenol

$300 \mathrm{U}$ 
Lab Name: PNNL

Lab code: PNNL

Case No.:
Contract : BNFL

SAS NO. :
EPA SAMPIE NO.

AW-101-

SDG NO.: 990205
Matrix: (soil/water) SOLID

Sample wt/vol:

$4.2(\mathrm{~g} / \mathrm{mL}) \mathrm{G}$

Ievel: (low/med) LOW

$\%$ Moisture: 0 decanted: $(Y / N) N$

Concentrated Extract Volume: 2000 (uI)

Injection Volume: (uL)
Lab Sample ID: 99-0650-

Lab File ID: 99020611

Date Received:

Date Extracted:

Date Analyzed: 02/07/99

Dilution Factor: 1.0

GPC Cleanup: $\quad(\mathrm{Y} / \mathrm{N}) \mathrm{N} \quad \mathrm{pH}: 12.5$

$\begin{array}{ll} & \text { CONCENTRATION UNITS: } \\ \text { CAS NO. COMPOUND } & \text { (ug/L or ug/Kg) UG/KG }\end{array}$

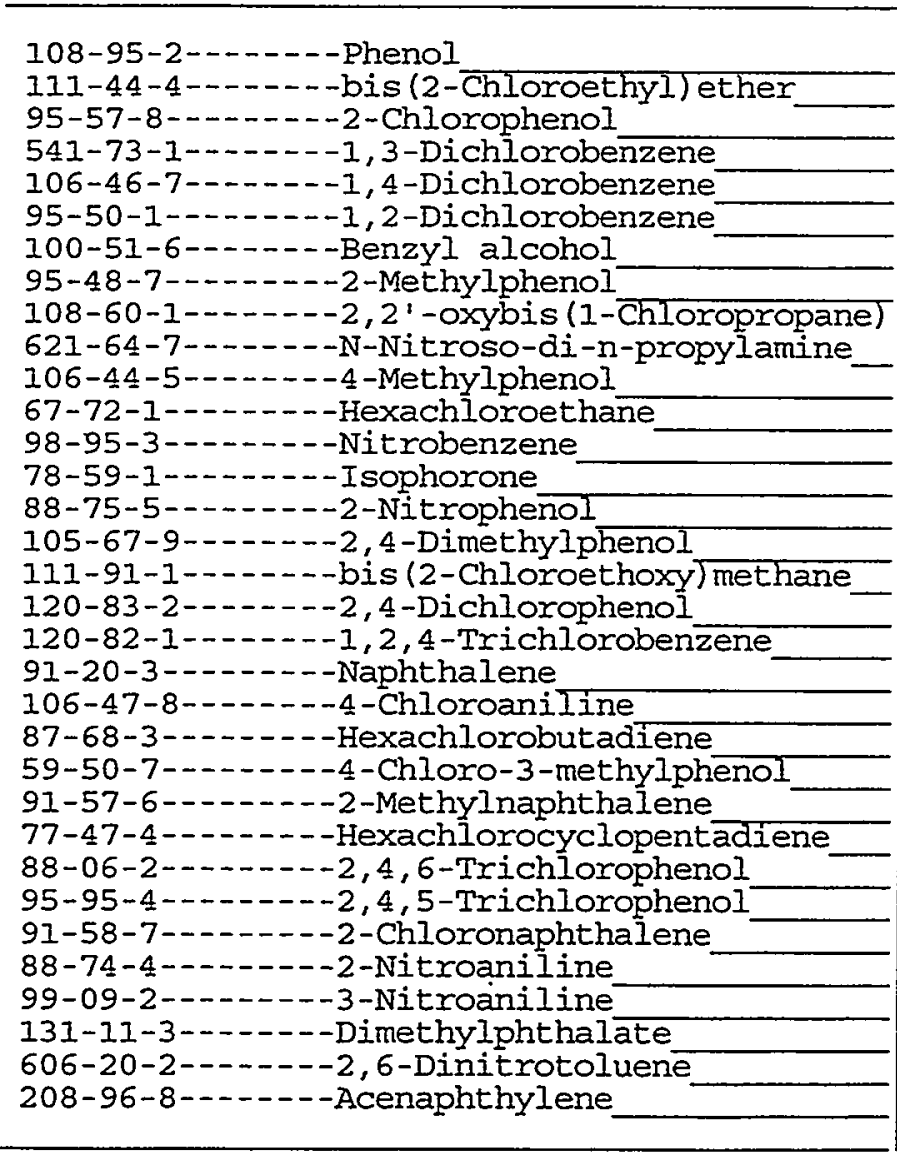

FORM I SV-I
$\bar{Q}$

\begin{tabular}{|l|l|l|}
4800 & $\mathrm{U}$ \\
4800 & $\mathrm{U}$ \\
4800 & $\mathrm{U}$ \\
4800 & $\mathrm{U}$ \\
4800 & $\mathrm{U}$ \\
4800 & $\mathrm{U}$ \\
4800 & $\mathrm{U}$ \\
4800 & $\mathrm{U}$ \\
4800 & $\mathrm{U}$ \\
4800 & $\mathrm{U}$ \\
4800 & $\mathrm{U}$ \\
4800 & $\mathrm{U}$ \\
4800 & $\mathrm{U}$ \\
4800 & $\mathrm{U}$ \\
4800 & $\mathrm{U}$ \\
4800 & $\mathrm{U}$ \\
4800 & $\mathrm{U}$ \\
4800 & $\mathrm{U}$ \\
4800 & $\mathrm{U}$ \\
4800 & $\mathrm{U}$ \\
4800 & $\mathrm{U}$ \\
4800 & $\mathrm{U}$ \\
4800 & $\mathrm{U}$ \\
4800 & $\mathrm{U}$ \\
4800 & $\mathrm{U}$ \\
4800 & $\mathrm{U}$ \\
4800 & $\mathrm{U}$ \\
4800 & $\mathrm{U}$ \\
4800 & $\mathrm{U}$ \\
4800 & $\mathrm{U}$ \\
4800 & $\mathrm{U}$ \\
4800 & $\mathrm{U}$ \\
4800 & $\mathrm{U}$ \\
& \\
\hline & \\
\hline
\end{tabular}

OIM03.0 
Lab Name: PNNL

Lab Code: PNNL

Matrix: (soil/water) SOIID

Sample wt/vol:

$4.2(\mathrm{~g} / \mathrm{mL}) \mathrm{G}$

Level: (low/med)

IOW

\% Moisture: 0 decanted: $(Y / N) N$

Concentrated Extract Volume: 2000 (uJ)

Injection Volume:

(uL)
$\mathrm{pH}: 12.5$
GPC Cleanup:
$(Y / N) N$

EPA SAMPLE NO.

AW-101-

Contract: BNEL

SAS NO.:

SDG No.: 990205

COMPOUND

CONCENTRATION UNITS:

CAS NO.

(ug/L or $u g / K g$ ) UG/KG

$\overline{\mathrm{Q}}$

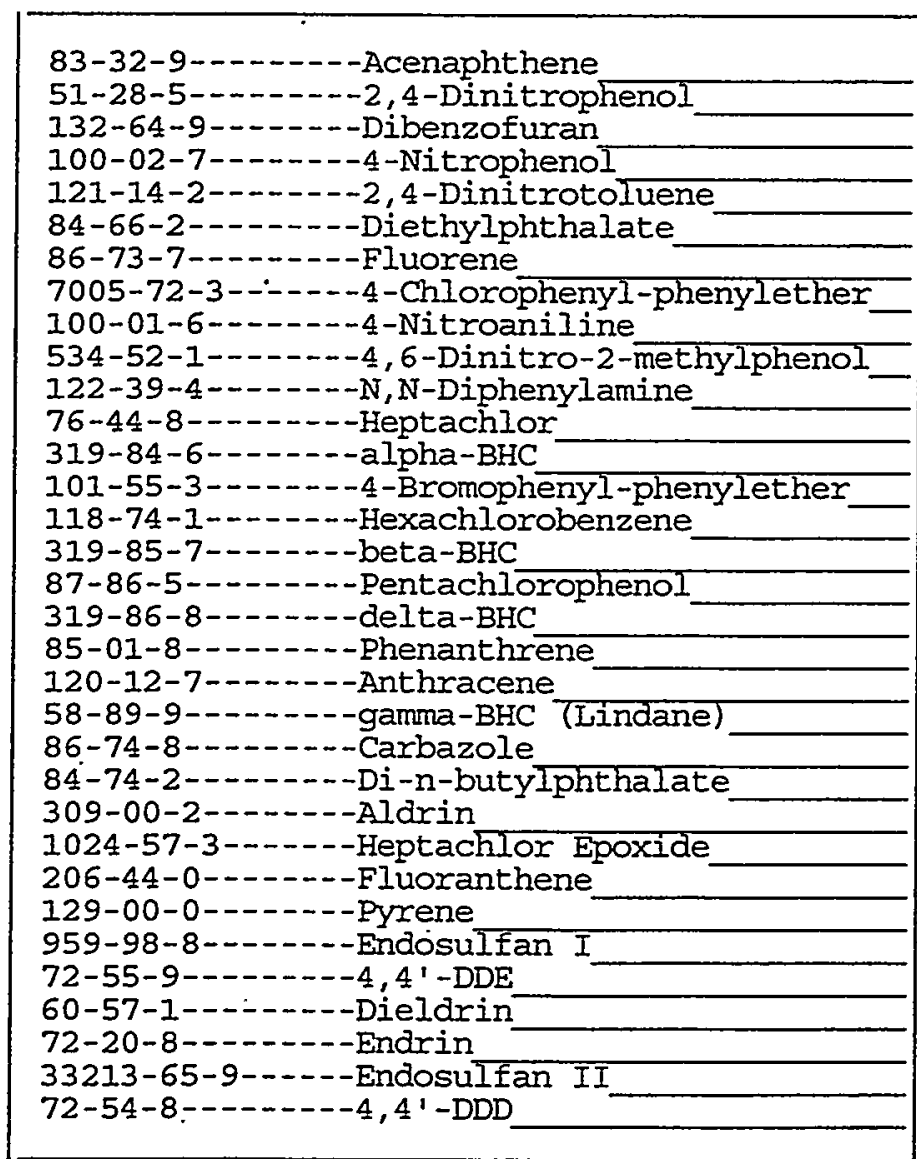

\begin{tabular}{|l|l|l|}
\hline 4800 & $\mathrm{U}$ \\
4800 & $\mathrm{U}$ \\
4800 & $\mathrm{U}$ \\
4800 & $\mathrm{U}$ \\
4800 & $\mathrm{U}$ \\
4800 & $\mathrm{U}$ \\
4800 & $\mathrm{U}$ \\
4800 & $\mathrm{U}$ \\
4800 & $\mathrm{U}$ \\
4800 & $\mathrm{U}$ \\
4800 & $\mathrm{U}$ \\
4800 & $\mathrm{U}$ \\
4800 & $\mathrm{U}$ \\
4800 & $\mathrm{U}$ \\
4800 & $\mathrm{U}$ \\
4800 & $\mathrm{U}$ \\
4800 & $\mathrm{U}$ \\
4800 & $\mathrm{U}$ \\
4800 & $\mathrm{U}$ \\
4800 & $\mathrm{U}$ \\
4800 & $\mathrm{U}$ \\
4800 & $\mathrm{U}$ \\
4800 & $\mathrm{U}$ \\
4800 & $\mathrm{U}$ \\
4800 & $\mathrm{U}$ \\
4800 & $\mathrm{U}$ \\
4800 & $\mathrm{U}$ \\
4800 & $\mathrm{U}$ \\
4800 & $\mathrm{U}$ \\
4800 & $\mathrm{U}$ \\
4800 & $\mathrm{U}$ \\
4800 & $\mathrm{U}$ \\
4800 & $\mathrm{U}$ \\
& & \\
\hline & \\
\hline
\end{tabular}

FORM I SV-2 
Lab Name: PNNL

Lab code: PNNL

Matrix: (soil/water) SOLID

Sample $w t /$ vol:

$$
4.2(\mathrm{~g} / \mathrm{mI}) \mathrm{G}
$$

Level: (low/med) LOW

\% Moisture: 0

decanted: $(Y / N) N$

Concentrated Extract Volume: 2000 (UI)

Injection Volume:

(uL)

contract: BNFL

SAS NO.:
EPA SAMPIE NO.

AW-10I-

SDG NO.: 990205
Lab Sample ID: 99-0650-

Lab File ID: $\quad 99020611$

Date Received:

Date Extracted:

Date Analyzed: 02/07/99

Dilution Factor: 1.0

GPC Cleanup: (Y/N) N $\mathrm{pH}: 12.5$

\begin{tabular}{|c|c|c|}
\hline $\begin{array}{l}\text { CONCENTRAT } \\
\mathrm{lug} / \mathrm{L} \text { or } \mathrm{u}\end{array}$ & $\begin{array}{l}\text { N UNITS: } \\
\mathrm{Kg}) \text { UG/KG }\end{array}$ & 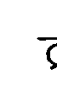 \\
\hline 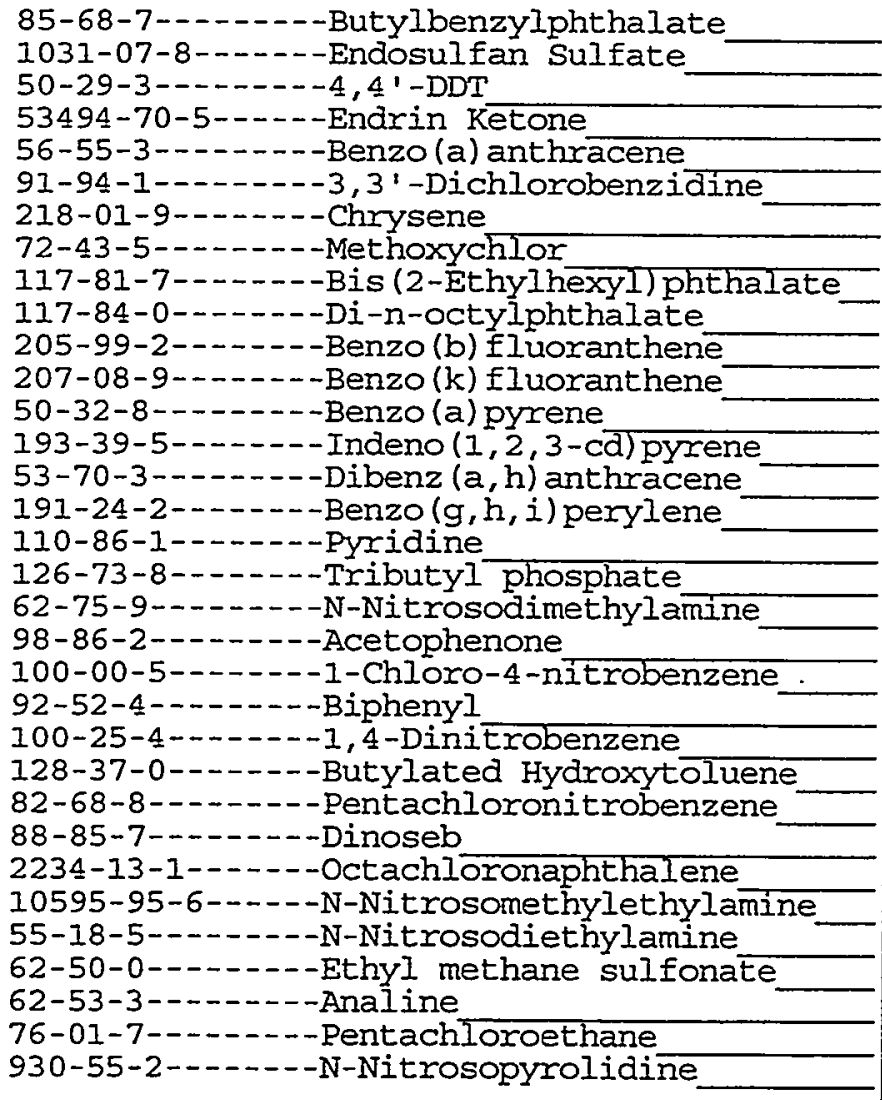 & $\begin{array}{r}4800 \\
4800 \\
4800 \\
4800 \\
4800 \\
4800 \\
4800 \\
4800 \\
4800 \\
4800 \\
4800 \\
4800 \\
4800 \\
4800 \\
4800 \\
4800 \\
4800 \\
900 \\
17000 \\
4800 \\
4800 \\
4800 \\
4800 \\
4800 \\
4800 \\
4800 \\
4800 \\
1800 \\
4800 \\
4800 \\
4800 \\
51 \\
4800\end{array}$ & $\begin{array}{l}U \\
U \\
U \\
U \\
U \\
U \\
U \\
U \\
U \\
U \\
U \\
U \\
U \\
U \\
U \\
U \\
U \\
J \\
U \\
U \\
U \\
U \\
U \\
U \\
U \\
U \\
J \\
U \\
U \\
U \\
J \\
U\end{array}$ \\
\hline
\end{tabular}


Lab Name: PNNL

Contract: BNFL

AW-101-

Lab Code: PNNL

Case No.:

SAS NO.:

SDG No.: 990205

Matrix: (soil/water) SOLID

Sample wt/vol:

$4.2(\mathrm{~g} / \mathrm{mL}) \mathrm{G}$

Level: (low/med)

LOW

\% Moisture: 0

decanted: $(\mathrm{Y} / \mathrm{N}) \mathrm{N}$

Concentrated Extract Volume: '2000 (UI)

(uL)

Injection Volume:

GPC Cleanup: $\quad(\mathrm{Y} / \mathrm{N}) \mathrm{N} \quad \mathrm{pH}: 12.5$

GPC Cleanup: $\quad(\mathrm{Y} / \mathrm{N}) \mathrm{N} \quad \mathrm{pH}: 12.5$
Lab Sample ID: 99-0650-

Lab File ID: 99020611

Date Received:

Date Extracted:

Date Analyzed: 02/07/99

Dilution Factor: 1.0

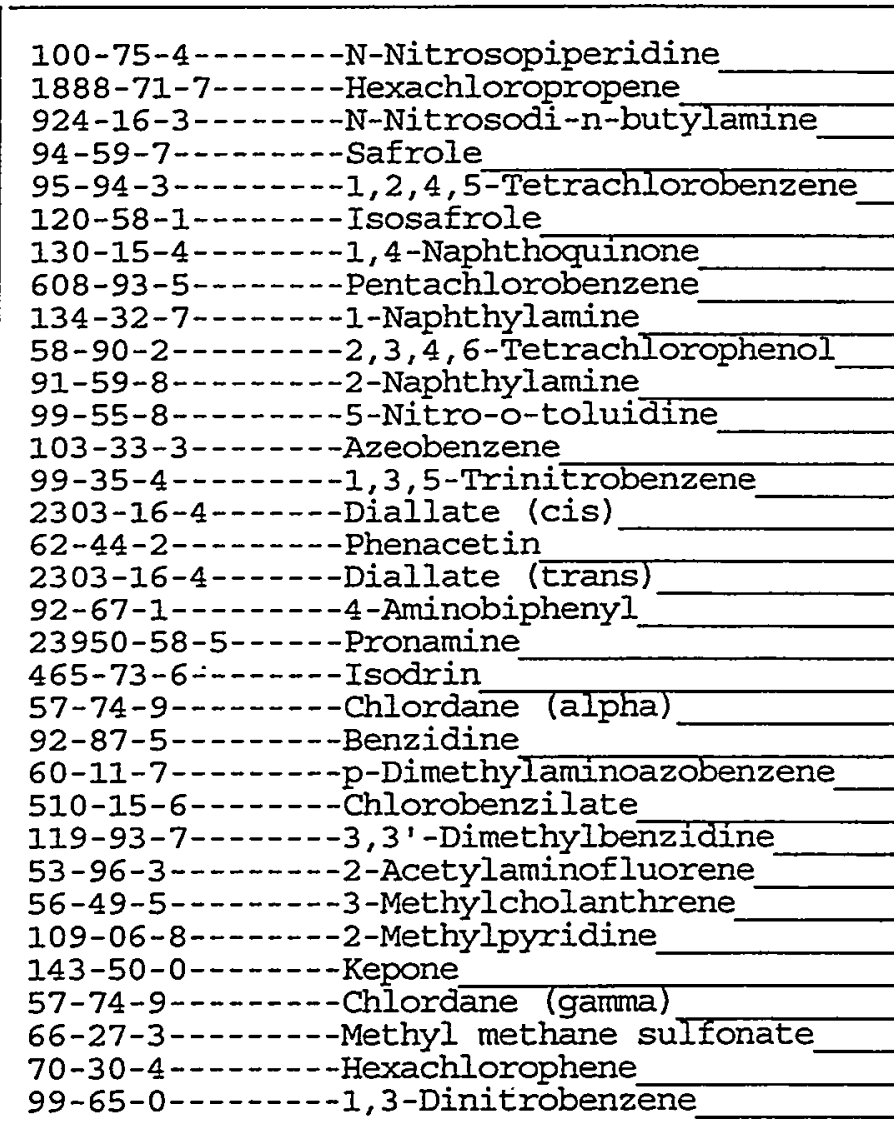

FORM I SV-4

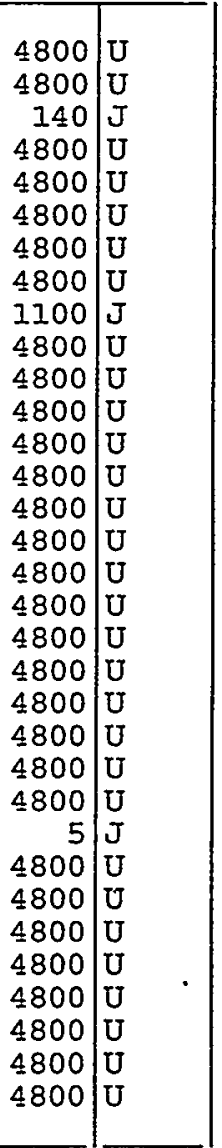

OLM03.0 
SEMIVOLATILE ORGANICS ANALYSIS DATA SHEET

Lab Name: PNNL,

Iab Code: PNNL

Case No.:
Contract: BNFL

SAS NO.:
AW-101-

SDG NO.: 990205
Matrix: (soil/water) SOLID

Sample wt/vol:

$$
4.2(\mathrm{~g} / \mathrm{mL}) \quad \mathrm{G}
$$

Level: (low/med) LOW

$\%$ Moisture: 0 decanted: $(Y / N) N$

Concentrated Extract Volume: . 2000 (uس)

Injection Volume: (uU)
Iab Sample ID: 99-0650-

Lab File ID: 99020611

Date Received:

Date Extracted:

Date Analyzed: 02/07/99

Dilution Factor: 1.0

GPC Cleanup: $(\mathrm{Y} / \mathrm{N}) \mathrm{N} \quad \mathrm{pH}: 12.5$

$\begin{array}{ll}\text { CAS NO. COMPOUND } & \text { CONCENTRATION UNITS: } \\ \text { Cug/L or } \mathrm{ug} / \mathrm{Kg}) \text { UG/KG } & -\overline{\mathrm{Q}}\end{array}$

87-65-0----.-2, 6-Dichlorophenol__ $\quad 4800 \mid \mathrm{U}$ 
Lab Name: PNNL

Lab Code: PNNL

Case No. :

SAS NO.:

SDG NO.: 990205

Matrix: (soil/water) SOLID

Lab Sample ID: 99-0650-D

Sample wt/vol:

$4.7(\mathrm{~g} / \mathrm{mL}) \mathrm{G}$

Lab File ID: 99020612

Level: (low/med) LOW

Date Received:

\% Moisture: 0 decanted: $(\mathrm{Y} / \mathrm{N}) \mathrm{N}$

Date Extracted:

Concentrated Extract Volume: 2000 (uU)

Date Analyzed: 02/07/99

Injection Volume: (ur)

Dilution Factor: 1.0

GPC Cleanup: $\quad(Y / N) \mathrm{N}$

$\mathrm{pH}: 12.5$

CAS NO.

COMPOUND

CONCENTRATION UNITS :

(ug/L or ug/Kg) UG/KG $-\bar{Q}$

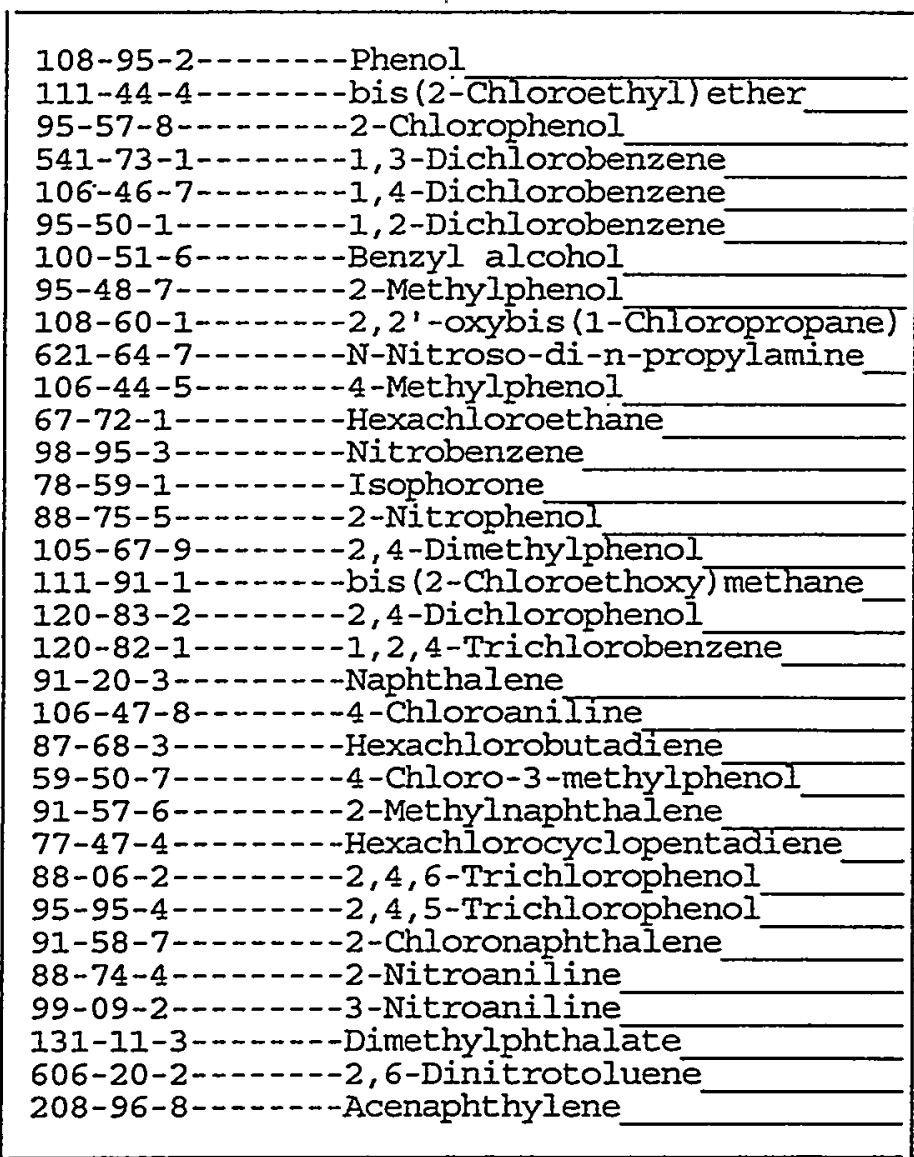

\begin{tabular}{|l|l|l|}
\hline 4300 & $\mathrm{U}$ \\
4300 & $\mathrm{U}$ \\
4300 & $\mathrm{U}$ \\
4300 & $\mathrm{U}$ \\
4300 & $\mathrm{U}$ \\
4300 & $\mathrm{U}$ \\
4300 & $\mathrm{U}$ \\
4300 & $\mathrm{U}$ \\
4300 & $\mathrm{U}$ \\
4300 & $\mathrm{U}$ \\
4300 & $\mathrm{U}$ \\
4300 & $\mathrm{U}$ \\
4300 & $\mathrm{U}$ \\
4300 & $\mathrm{U}$ \\
4300 & $\mathrm{U}$ \\
4300 & $\mathrm{U}$ \\
4300 & $\mathrm{U}$ \\
4300 & $\mathrm{U}$ \\
4300 & $\mathrm{U}$ \\
4300 & $\mathrm{U}$ \\
4300 & $\mathrm{U}$ \\
4300 & $\mathrm{U}$ \\
4300 & $\mathrm{U}$ \\
4300 & $\mathrm{U}$ \\
4300 & $\mathrm{U}$ \\
4300 & $\mathrm{U}$ \\
4300 & $\mathrm{U}$ \\
4300 & $\mathrm{U}$ \\
4300 & $\mathrm{U}$ \\
4300 & $\mathrm{U}$ \\
4300 & $\mathrm{U}$ \\
4300 & $\mathrm{U}$ \\
4300 & $\mathrm{U}$ \\
& \\
\hline & \\
\hline
\end{tabular}


Lab Name: PNNL

Lab Code: PNNL

Case No.:

Matrix: (soil/water) SOIID

Sample wt/vol:

$4.7(\mathrm{~g} / \mathrm{mL}) \mathrm{G}$

Level: (low/med) LOW

$\div$ Moisture: 0 decanted: $(Y / N) N$

Concentrated Extract Volume: 2000 (UI)

Injection Volume:

(uI)
Contract: BNFL

SAS NO.:
EPA SAMPLE NO.

$A W-101-D$

SDG No.: 990205

Lab Sample ID: 99-0650-D

Lab File ID: $\quad 99020612$

Date Received:

Date Extracted:

Date Analyzed: 02/07/99

Dilution Factor: 1.0

GPC Cleanup: $\quad(Y / N) N \quad \mathrm{pH}: 12.5$

$\begin{array}{ll} & \text { CONCENTRATION UNITS: } \\ \text { CAS NO. COMPOUND } & (u g / L \text { or ug/Kg) UG/KG }\end{array}$

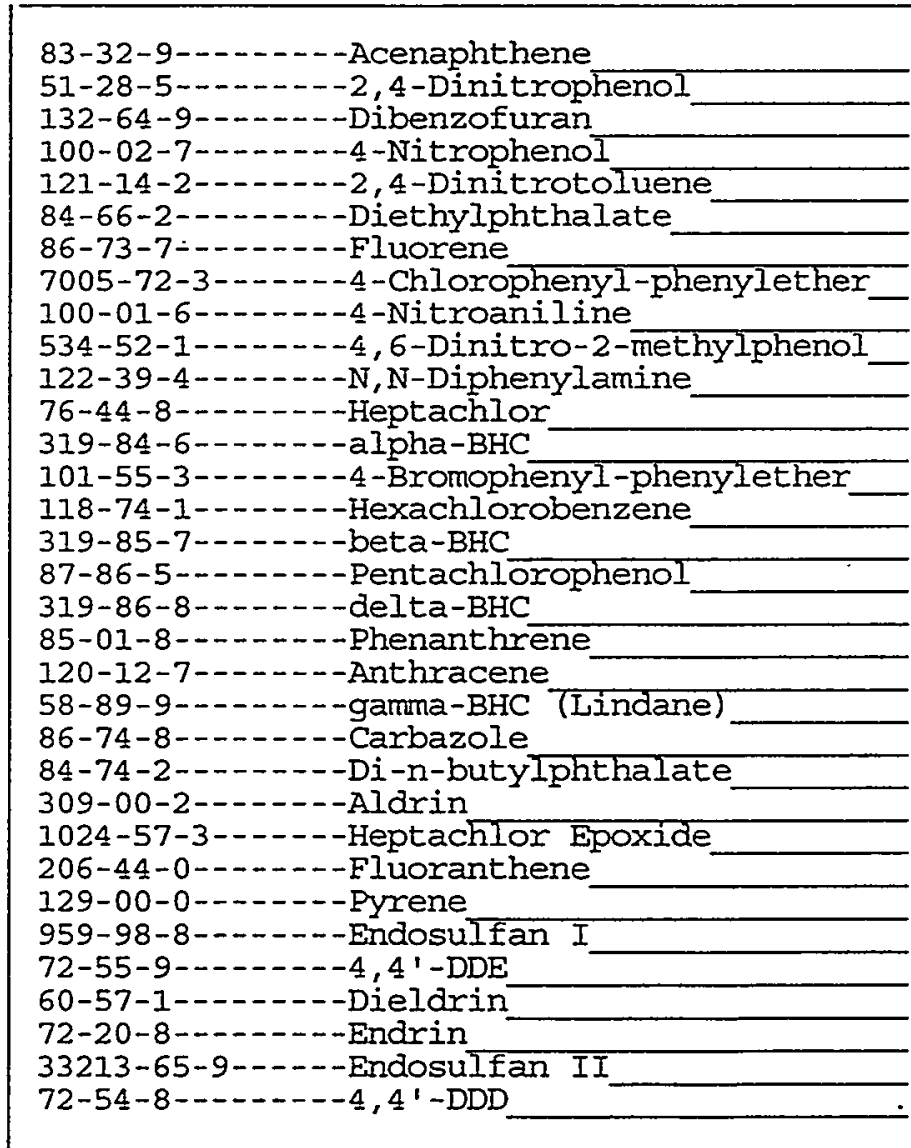

FORM I SV-2

\begin{tabular}{|c|c|}
\hline $\begin{array}{l}4300 \\
4300 \\
4300 \\
4300 \\
4300 \\
4300 \\
4300 \\
4300 \\
4300 \\
4300 \\
4300 \\
4300 \\
4300 \\
4300 \\
4300 \\
4300 \\
4300 \\
4300 \\
4300 \\
4300 \\
4300 \\
4300 \\
4300 \\
4300 \\
4300 \\
4300 \\
4300 \\
4300 \\
4300 \\
4300 \\
4300 \\
4300 \\
4300\end{array}$ & $\begin{array}{l}U \\
U \\
U \\
U \\
U \\
U \\
U \\
U \\
U \\
U \\
U \\
U \\
U \\
U \\
U \\
U \\
U \\
U \\
U \\
U \\
U \\
U \\
U \\
U \\
U \\
U \\
U \\
U \\
U \\
U \\
U \\
U \\
U\end{array}$ \\
\hline
\end{tabular}

OLM03. 0 
Lab Name: PNNL

Lab Code: PNNL

Case No.:

Matrix: (soil/water) SOLID

Sample wt/vol:

$4.7(\mathrm{~g} / \mathrm{mL}) \quad \mathrm{G}$

Level: (low/med)

LOW

\% Moisture: 0 decanted: $(Y / N) \mathrm{N}$

Concentrated Extract Volume: 2000 (Uل)

Injection Volume: (uL)
Contract: BNEL

SAS NO. :
AW-101-D

SDG No.: 990205

Lab Sample ID: 99-0650-D

Lab File ID: 99020612

Date Received:

Date Extracted:

Date Analyzed: 02/07/99

Dilution Factor: 1.0

$\mathrm{pH}: 12.5$

\section{CAS NO. \\ CONCENTRATION UNITS: \\ (ug/I or ug/Kg) UG/KG}

$(\mathrm{Y} / \mathrm{N}) \mathrm{N}$

\begin{tabular}{|c|}
\hline 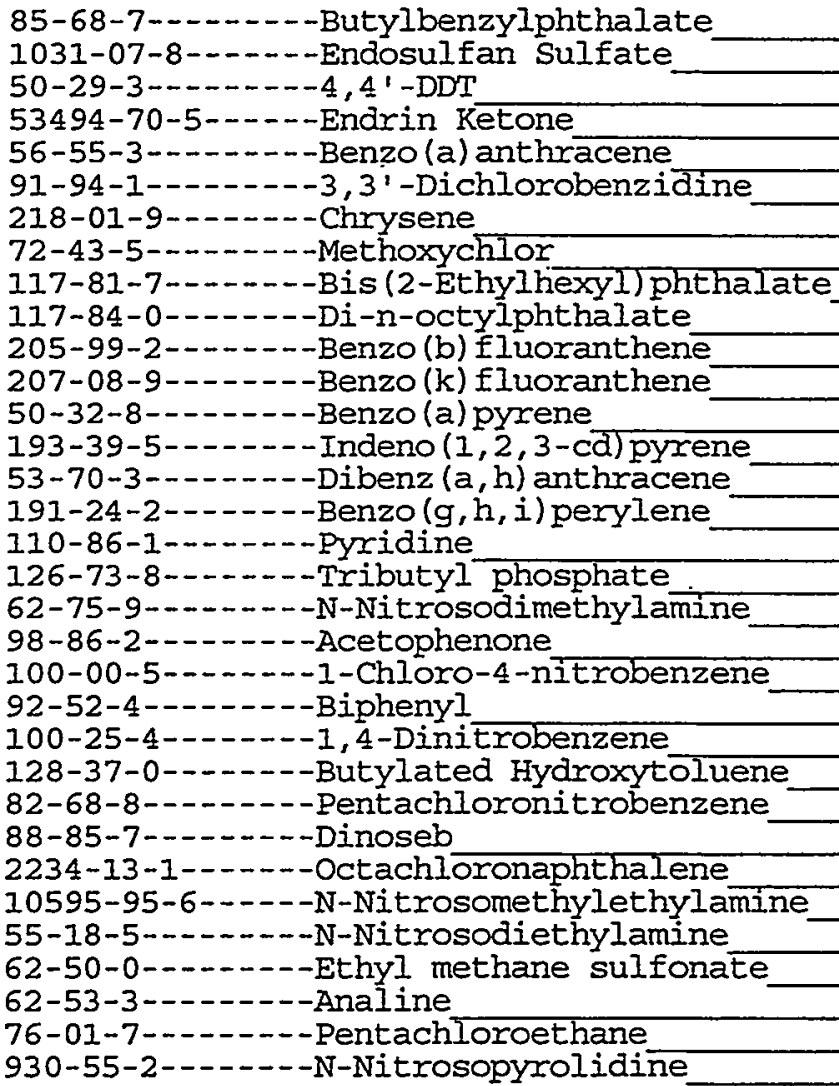 \\
\hline
\end{tabular}

FORM I SV-3

\begin{tabular}{|l|l|l|}
\hline 4300 & $\mathrm{U}$ \\
4300 & $\mathrm{U}$ \\
4300 & $\mathrm{U}$ \\
4300 & $\mathrm{U}$ \\
4300 & $\mathrm{U}$ \\
4300 & $\mathrm{U}$ \\
4300 & $\mathrm{U}$ \\
4300 & $\mathrm{U}$ \\
4300 & $\mathrm{U}$ \\
4300 & $\mathrm{U}$ \\
4300 & $\mathrm{U}$ \\
4300 & $\mathrm{U}$ \\
4300 & $\mathrm{U}$ \\
4300 & $\mathrm{U}$ \\
4300 & $\mathrm{U}$ \\
4300 & $\mathrm{U}$ \\
4300 & $\mathrm{U}$ \\
1800 & $\mathrm{~J}$ \\
4300 & $\mathrm{U}$ \\
4300 & $\mathrm{U}$ \\
4300 & $\mathrm{U}$ \\
4300 & $\mathrm{U}$ \\
4300 & $\mathrm{U}$ \\
4300 & $\mathrm{U}$ \\
4300 & $\mathrm{U}$ \\
4300 & $\mathrm{U}$ \\
4300 & $\mathrm{U}$ \\
4300 & $\mathrm{U}$ \\
4300 & $\mathrm{U}$ \\
4300 & $\mathrm{U}$ \\
4300 & $\mathrm{U}$ \\
96 & $\mathrm{~J}$ \\
4300 & $\mathrm{U}$ \\
& \\
\hline & \\
\hline & \\
& \\
4 & \\
4 & \\
4 & \\
4 &
\end{tabular}

OIM03. 0 
Lab Name: PNNL

Lab code: PNNL Case No.:

Matrix: (soil/water) SOLID

Sample wt/vol:

$4.7(\mathrm{~g} / \mathrm{mu}) \mathrm{G}$

Level: (low/med) IOW

\% Moisture: 0 decanted: $(\mathrm{Y} / \mathrm{N}) \mathrm{N}$

Concentrated Extract Volume: 2000 (UL)

Injection Volume: (UI)

GPC Cleanup: $(\mathrm{Y} / \mathrm{N}) \mathrm{N} \quad \mathrm{pH}: 12.5$
EPA SAMPLE NO.

$A W-101-D$

Contract: BNFI

SAS NO.:

SDG NO.: 990205
Lab Sample ID: 99-0650-D

Lab File ID: 99020612

Date Received:

Date Extracted:

Date Analyzed: 02/07/99

Dilution Factor: 1.0

$\begin{array}{ll}\text { CAS NO. COMPOUND } & \text { CONCENTRATION UNITS: } \\ \text { (ug/L or ug/Kg) UG/KG }\end{array}$

\begin{tabular}{|c|}
\hline 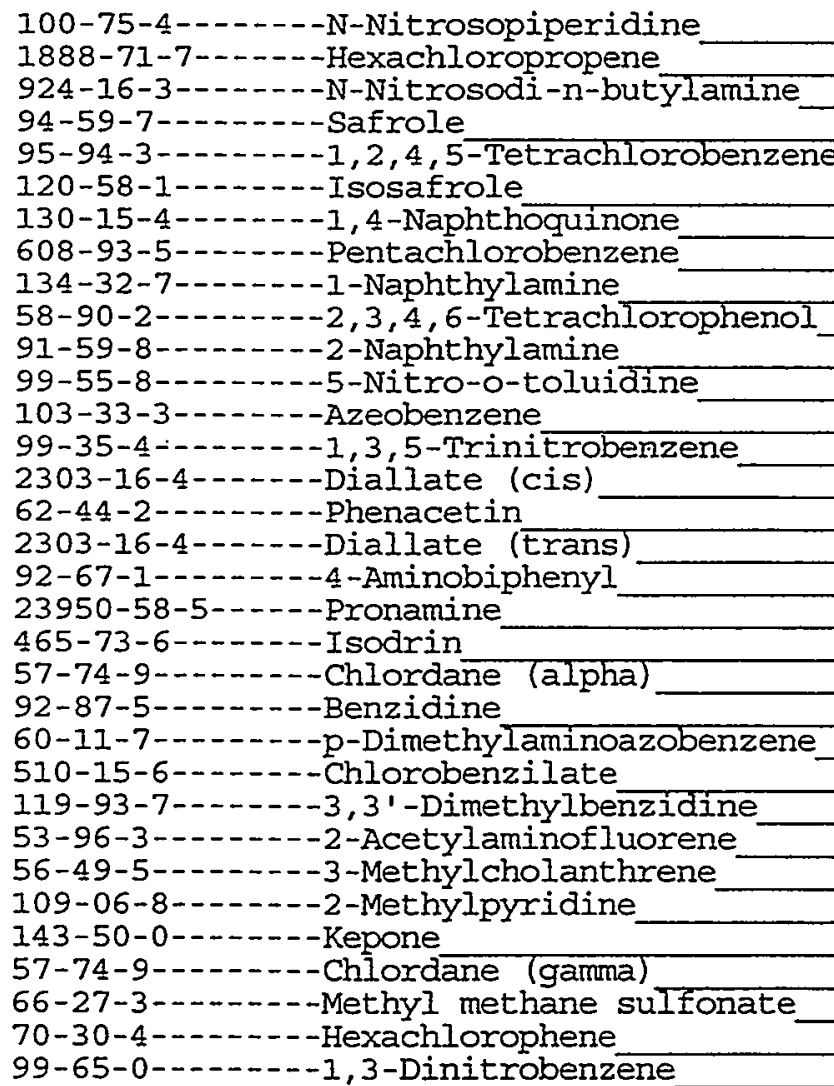 \\
\hline
\end{tabular}


Lab Name: PNNL

contract: BNFL

Iab code: PNML Case No.:

Matrix: (soil/water) SOIID

Sample wt/vol:

$4.7(\mathrm{~g} / \mathrm{mL}) \mathrm{G}$

Irevel: (low/med)

LOW

$\%$ Moisture: 0

decanted: $(Y / N)$ N

Concentrated Extract Volume: 2000 (uI)

Injection Volume: (ut)

GPC Cleanup: $(\mathrm{Y} / \mathrm{N}) \mathrm{N}$

$\mathrm{pH}: 12.5$
AW-101-D

SAS NO.:

SDG NO.: 990205

Lab Sample ID: 99-0650-D

Lab File ID: 99020612

Date Received:

Date Extracted:

Date Analyzed: 02/07/99

Dilution Factor: 1.0

\section{CAS NO.}

COMPOUND

CONCENTRATION UNITS: (ug/L or ug/Kg) UG/KG 
Lab Name: PNNL

Lab Code: PNNL

Case No.:

Matrix: (soil/water) SOLID

Sample wt/vol:

$2.8(\mathrm{~g} / \mathrm{mU}) \quad \mathrm{G}$

Level: (low/med) LOW

\% Moisture: 0 decanted: $(\mathrm{Y} / \mathrm{N}) \mathrm{N}$

Concentrated Extract Volume: 2000 (uU)

Injection Volume: (UI)
EPA SAMPLE NO.

AW-101-MS

Contract: BNFL

SAS NO.:

SDG No. : 990205

Lab Sample ID: 99-0650-MS

Lab File ID: 99020613

Date Received:

Date Extracted:

Date Analyzed: 02/07/99

Dilution Factor: 1.0

GPC Cleanup: $\quad(\mathrm{Y} / \mathrm{N}) \mathrm{N} \quad \mathrm{pH}: 12.5$

CONCENTRATION UNITS:

CAS NO.

COMPOUND

(ug/I or $\mathrm{ug} / \mathrm{Kg}$ ) UG/KG

$-\bar{Q}$

\begin{tabular}{|c|}
\hline 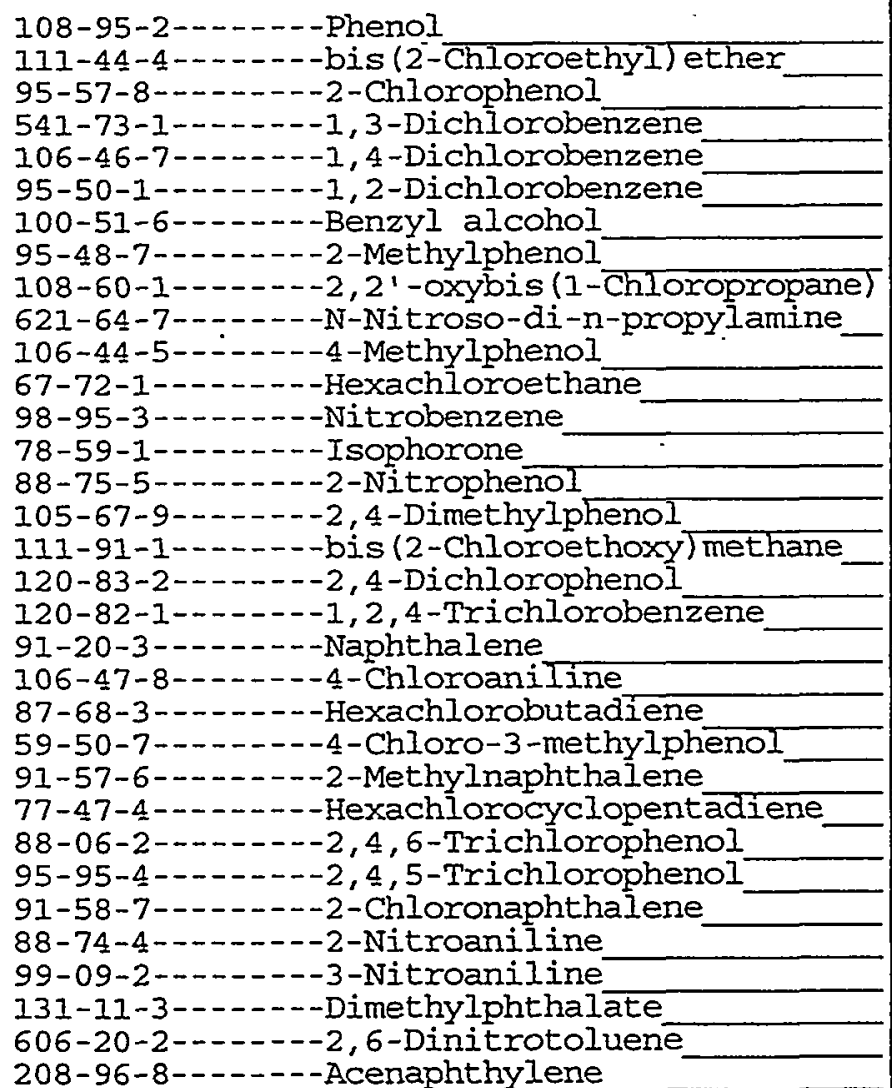 \\
\hline
\end{tabular}

6500

$7200 \mathrm{U}$

11000

7200

11000

7200

$7200 \mathrm{U}$

$110 \mathrm{~J}$

7200

16000

1300

$7200 \mathrm{U}$

7200 U

7200

14000

7200

$7200 \mathrm{U}$

$7200 \mathrm{U}$

17000

7200

$7200 \mathrm{U}$

7200 U

$3900 \mathrm{~J}$

$7200 \mathrm{U}$

$7200 \mathrm{U}$

$7200 \mathrm{U}$

$7200 \mathrm{U}$

$7200 \mathrm{U}$

$7200 \mathrm{U}$

$7200 \mathrm{U}$

$7200 \mathrm{U}$

7200 U

7200 
Lab Name: PNNL

Iab Code: PNNL

Case No. :

Matrix: (soil/water) SOIID

Sample wt/vol:

$2.8(\mathrm{~g} / \mathrm{mL}) \mathrm{G}$

Level: (low/med) Low

$\because$ Moisture: 0 decanted: $(\mathrm{Y} / \mathrm{N}) \mathrm{N}$

Concentrated Extract Volume: 2000 (uL)

Injection Volume: (uL)

Contract: BNFL SAS NO.:

AW-101-MS

SDG No.: 990205

Iab Sample ID: 99-0650-MS

Lab File ID: 99020613

Date Received:

Date Extracted:

Date Analyzed: 02/07/99

Dilution Factor: 1.0

GPC Cleanup: $(\mathrm{Y} / \mathrm{N}) \mathrm{N} \quad \mathrm{pH}: 12.5$

CONCENTRATION UNITS:

CAS NO. COMPOUND $\quad(u g / L$ or $\mathrm{ug} / \mathrm{Kg}) \mathrm{UG} / \mathrm{KG} \quad-Q$

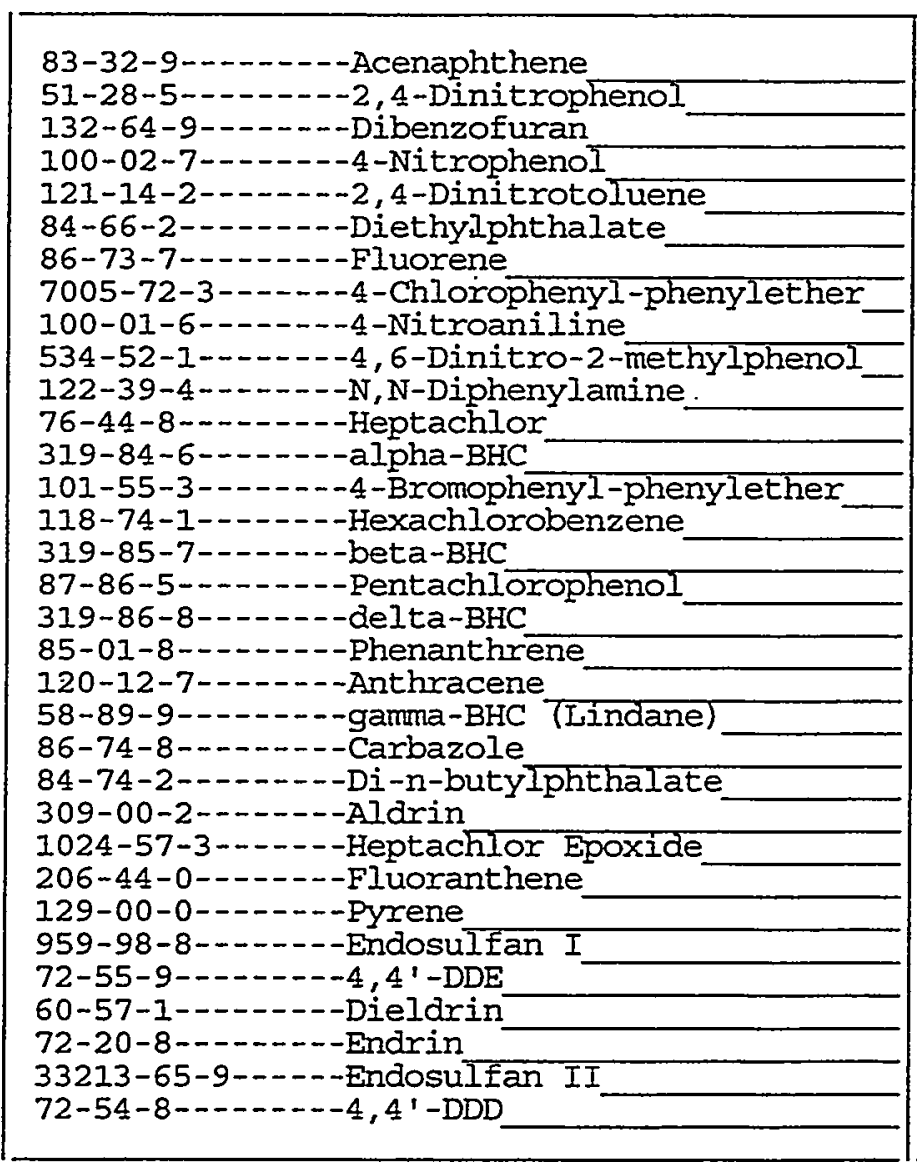

FORM I SV-2

\begin{tabular}{r|r|l|} 
& \\
20000 & \\
7200 & $\mathrm{U}$ \\
7200 & $\mathrm{U}$ \\
50000 & \\
590 & $\mathrm{~J}$ \\
1100 & $\mathrm{~J}$ \\
7200 & $\mathrm{U}$ \\
7200 & $\mathrm{U}$ \\
7200 & $\mathrm{U}$ \\
7200 & $\mathrm{U}$ \\
560 & $\mathrm{~J}$ \\
7200 & $\mathrm{U}$ \\
7200 & $\mathrm{U}$ \\
7200 & $\mathrm{U}$ \\
7200 & $\mathrm{U}$ \\
7200 & $\mathrm{U}$ \\
27000 & \\
7200 & $\mathrm{U}$ \\
7200 & $\mathrm{U}$ \\
7200 & $\mathrm{U}$ \\
7200 & $\mathrm{U}$ \\
7200 & $\mathrm{U}$ \\
7200 & $\mathrm{U}$ \\
7200 & $\mathrm{U}$ \\
7200 & $\mathrm{U}$ \\
7200 & $\mathrm{U}$ \\
24000 & \\
7200 & $\mathrm{U}$ \\
7200 & $\mathrm{U}$ \\
7200 & $\mathrm{U}$ \\
7200 & $\mathrm{U}$ \\
7200 & $\mathrm{U}$ \\
7200 & $\mathrm{U}$ \\
& \\
\hline & \\
\hline
\end{tabular}

OLM03.0 
Lab Name: PNNL

Lab Code: PNNL

Case No.:

Matrix: (soil/water) SOLID

Sample wt/vol:

$$
2.8(\mathrm{~g} / \mathrm{mL}) \mathrm{G}
$$

Contract: BNFL

Level: (low/med)

LOW

$\%$ Moisture: 0

decanted: $(\mathrm{Y} / \mathrm{N}) \mathrm{N}$

Concentrated Extract Volume: 2000 (uI)

Injection Volume: (UL)

SAS NO.:

SDG NO.: 990205

Lab Sample ID: 99-0650-MS

Lab File ID: $\quad 99020613$

Date Received:

Date Extracted:

Date Analyzed: 02/07/99

Dilution Factor: 1.0

GPC Cleanup: $\quad(\mathrm{Y} / \mathrm{N}) \mathrm{N} \quad \mathrm{pH}: 12.5$

CONCENTRATION UNITS:

CAS NO.

COMPOUND

(ug/L or $\mathrm{ug} / \mathrm{Kg}$ ) UG/KG

$-\mathrm{Q}$

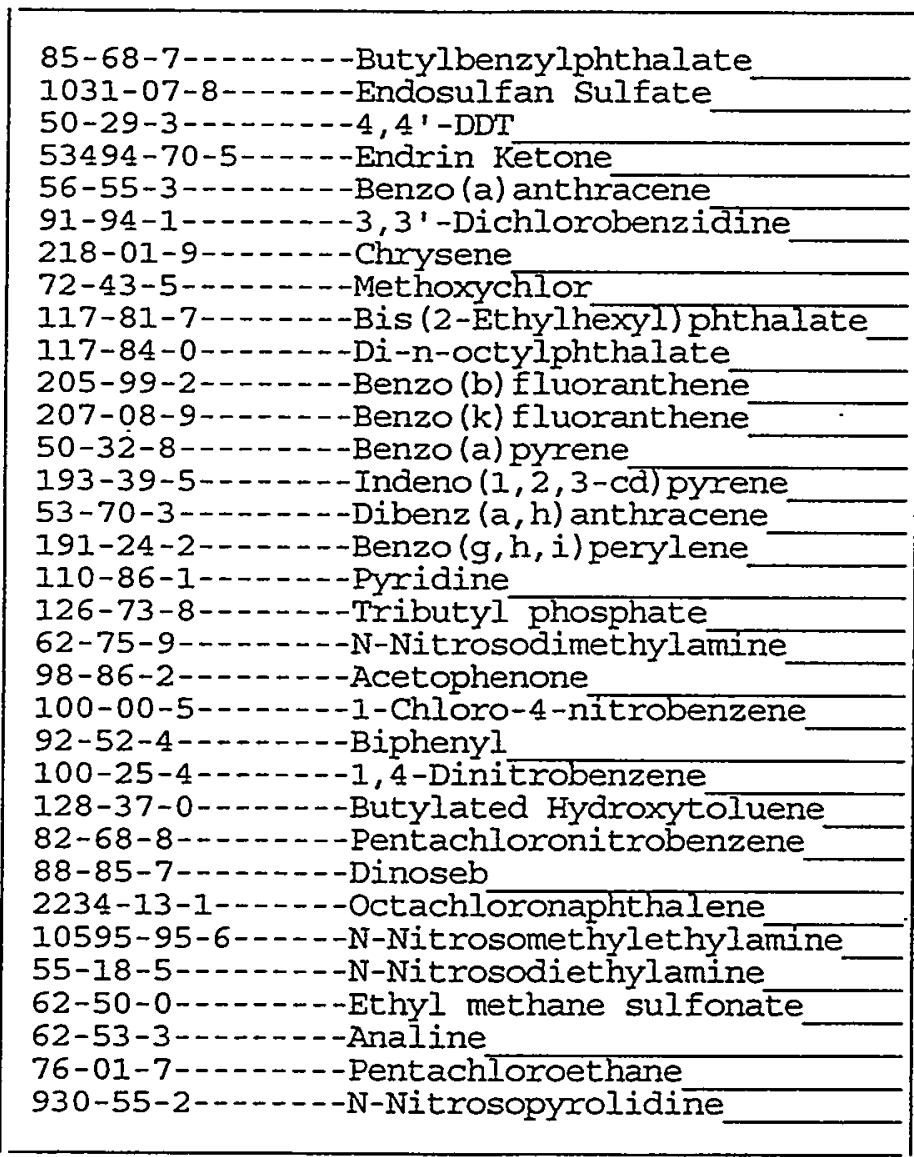

FORM I SV-3

OLMO 3.0 
Lab Name: PNNL

Lab Code: PNNL

Case No.:

Matrix: (soil/water) SOLID

Sample wt/vol:

$$
2.8(\mathrm{~g} / \mathrm{mL}) \quad \mathrm{G}
$$

Contract: BNFL

SAS NO.:

SDG No.: 990205

Lab Sample ID: 99-0650-MS

Lab File ID: 99020613

Date Received:

Date Extracted:

\% Moisture: 0 decanted: $(\mathrm{Y} / \mathrm{N}) \mathrm{N}$

Date Analyzed: 02/07/99

Concentrated Extract Volume: 2000 (uL)

Injection Volume: (uL)

Dilution Factor: 1.0

GPC Cleanup: $(\mathrm{Y} / \mathrm{N}) \mathrm{N} \quad \mathrm{pH}: 12.5$

$\begin{array}{ll}\text { CAS NO. } & \text { CONCENTRATION UNITS: } \\ \text { COMPOUND } & (u g / I \text { or ug/Kg) UG/KG }\end{array}$

100-75-4-...-N-Nitrosopiperidine

1888-71-7--.--Hexachloropropene

924-16-3-...-N-Nitrosodi-n-butylamine

94-59-7-......-. Safrole

95-94-3------1,2,4,5-Tetrachlorobenzene

120-58-1------Isosafrole

130-15-4------1,4-Naphthoquinone

608-93-5--.-.-Pentachlorobenzene

134-32-7--..--1-Naphthylamine

58-90-2 ......-2, 3,4,6-Tetrach lorophenol

91-59-8-------2-Naphthylamine

99-55-8-------5-Nitro-o-toluidine

103-33-3------Azeobenzene

99-35-4-------1, 3, 5-Trinitrobenzene

2303-16-4-..--Diallate (cis)

62-44-2--.-----Phenacetin

2303-16-4 -...-Diallate (Erans)

92-67-1-.----4-Aminobiphenyl

23950-58-5-...-Pronamine

465-73-6--.----Isodrin

57-74-9-.....-Chlordane (alpha)

92-87-5--.-----Benzidine

60-11-7-...---p-Dimethylaminoazobenzene

510-15-6------Chlorobenzilate

119-93-7--------3,3'-Dimethylbenzidine

53-96-3-2...-2-Acetylaminofluorene

56-49-5-------3-Methylcholanthrene

109-06-8 -...-2-Methylpyridine

143-50-0-....-.-Kepone

57-74-9-..---Chlordane (gamma)

66-27-3-....-Methyl methane sulfonate

70-30-4-....---Hexachlorophene

99-65-0-..--1, 3-Dinitrobenzene
EPA SAMPIE NO.

AW-101-MS

\author{
(19)
}


$1 C$

SEMIVOLATILE ORGANICS ANALYSIS DATA SHEET

Lab Name: PNNL

Contract: BNFL

Lab Code: PNNL Case No.:

Matrix: (soil/water) SOLID

Sample wt/vol:

$$
2.8(\mathrm{~g} / \mathrm{mL}) . \mathrm{G}
$$

Level: (low/med)

LOW

decanted: $(\mathrm{Y} / \mathrm{N}) \mathrm{N}$

Concentrated Extract Volume: 2000 (uL)

Injection Volume: (uI)

SAS NO.:

AW-101-MS

Lab Sample ID: 99-0650-MS

Lab File ID: $\quad 99020613$

Date Received:

Date Extracted:

Date Analyzed: 02/07/99

Dilution Factor: 1.0

$\mathrm{pH}: 12.5$

GPC Cleanup: $(Y / N) N$

$\begin{array}{ll} & \text { CONCENTRATION UNITS: } \\ \text { CAS NO. COMPOUND } & \text { (ug/L or ug/Kg) UG/KG }\end{array}$

87-65-0--.----2, 6-Dichlorophenol

( $7200 \mid \mathrm{U}$


Lab Name: PNNL

Lab code: PNNL

Case No.:

Matrix: (soil/water) SOLID

Sample wt/vol:

$2.6(\mathrm{~g} / \mathrm{mL}) \mathrm{G}$

Level: (low/med)

LOW

$\div$ Moisture: 0 decanted: $(\mathrm{Y} / \mathrm{N}) \mathrm{N}$

Concentrated Extract Volume: 2000 (uI)

Injection Volume: (UI)
Contract: BNFL

SAS NO.:
AW-101-MSD

SDG No.: 990205

GPC Cleanup: $\quad(\mathrm{Y} / \mathrm{N}) \mathrm{N} \quad \mathrm{pH}: 12.5$

CAS NO.

COMPOUND

CONCENTRATION UNITS:

(ug/L or $u g / K g$ ) UG/KG

${ }^{-} \mathrm{Q}$

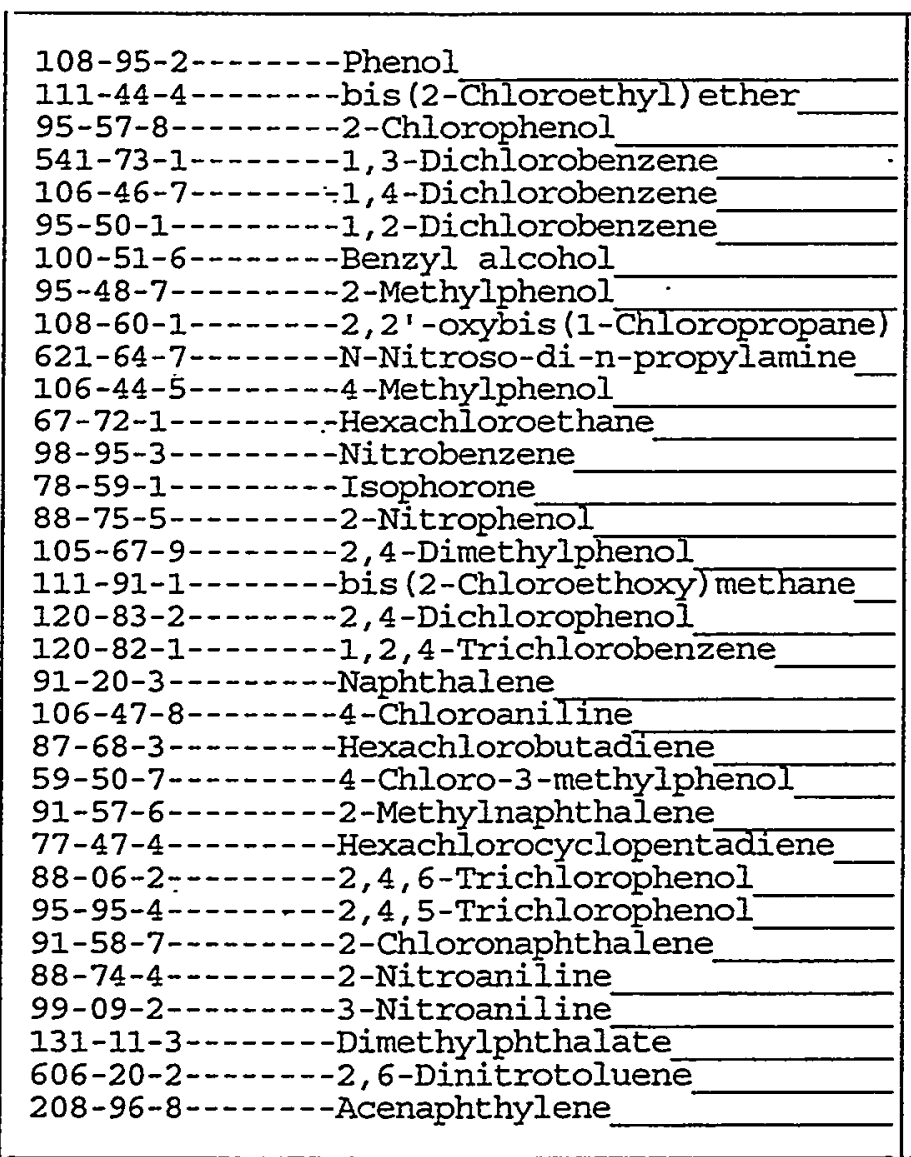

FORM I SV-1

OLM03.0 
Lab Name: PNNL

Iab Code: PNNL

Case No.:

Matrix: (soil/water) SOLID

Sample wt/vol:

$2.6(\mathrm{~g} / \mathrm{mL}) \mathrm{G}$

Contract : BNFL

Level: (low/med) IOW

$\%$ Moisture: 0 decanted: $(\mathrm{Y} / \mathrm{N}) \mathrm{N}$

Concentrated Extract Volume: 2000 (UT)

Injection Volume:

(uJ)

SAS NO.:

Lab Sample ID: 99-0650-MSD

Lab File ID: 99020614

Date Received:

Date Extracted:

Date Analyzed: 02/07/99

Dilution Factor: 1.0

GPC Cleanup: $\quad(Y / N) \mathrm{N} \quad \mathrm{pH}: 12.5$

CONCENTRATION UNITS:

CAS NO. COMPOUND $\quad(u g / I$ or $\mathrm{ug} / \mathrm{Kg}) \mathrm{UG} / \mathrm{KG} \quad Q$

\begin{tabular}{|c|}
\hline 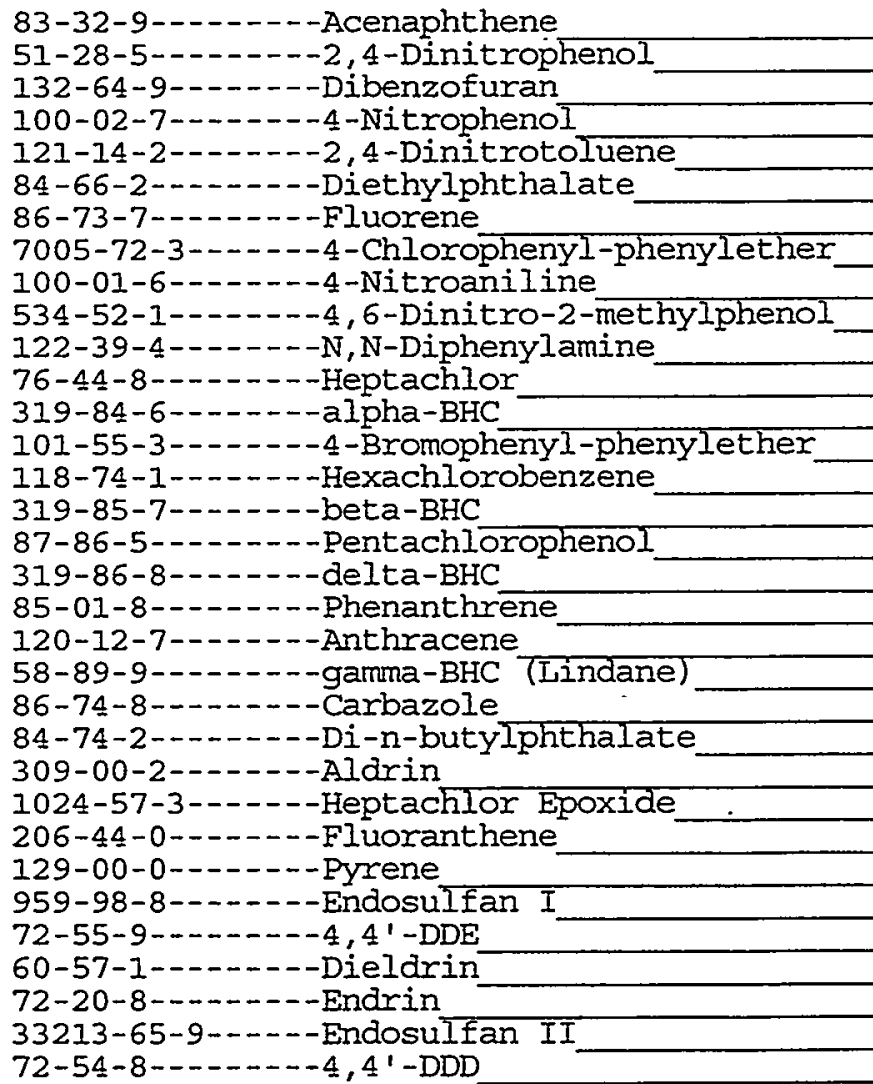 \\
\hline
\end{tabular}

FORM I SV-2

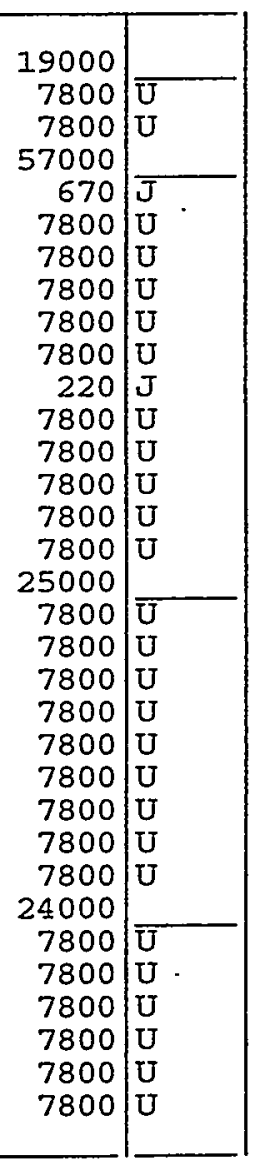

OLM03.0 
Lab Name: PNNL

Lab Code: PNNL

Case No.:
Contract: BNFL

SAS NO. :
EPA SAMPLE NO.

AW-101-MSD

SDG NO.: 990205
Matrix: (soil/water) SOLID

Sample wt/vol:

$2.6(\mathrm{~g} / \mathrm{mL}) \mathrm{G}$

Level: (Iow/med) LOW

$\%$ Moisture: 0 decanted: $(Y / N) \mathrm{N}$

Concentrated Extract Volume: 2000 (uI)

Injection Volume:

(uI)

GPC Cleanup:

$(\mathrm{Y} / \mathrm{N}) \mathrm{N}$

$\mathrm{pH}: 12.5$
Lab Sample ID: 99-0650-MSD

Lab File ID: $\quad 99020614$

Date Received:

Date Extracted:

Date Analyzed: 02/07/99

Dilution Factor: 1.0

CAS NO.

COMPOUND

CONCENTRATION UNITS: (ug/L or $\mathrm{ug} / \mathrm{Kg}$ ) UG/KG

$Q$

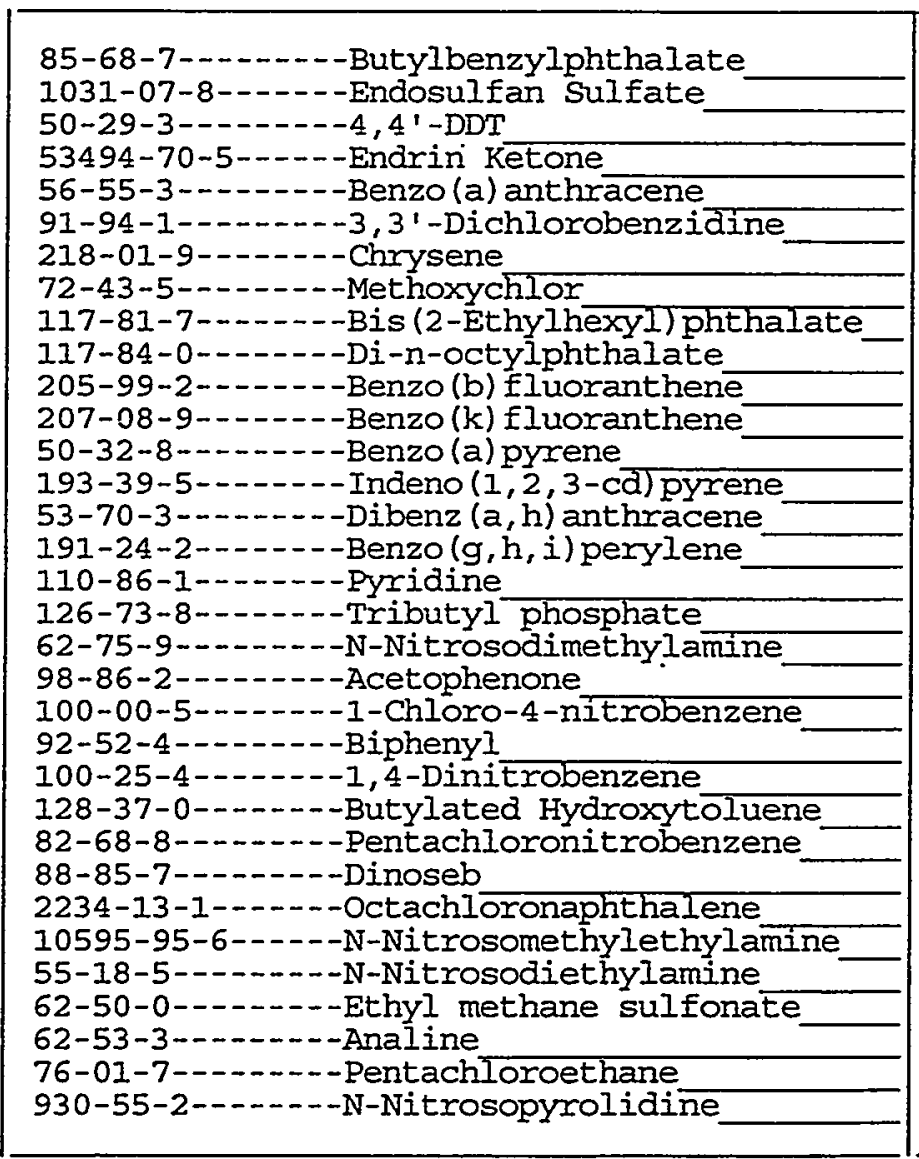


Lab Name: PNNL

Iab code: PNNL

Case No.:
Contract: BNFL

SAS NO.:
EPA SAMPIE NO.

AW-101-MSD

SDG NO.: 990205
Matrix: (soil/water) SOLID

Sample wt/vol:

$2.6(\mathrm{~g} / \mathrm{mL}) \mathrm{G}$

Level: (low/med) Iow

$\%$ Moisture: 0 decanted: $(\mathrm{Y} / \mathrm{N}) \mathrm{N}$

Concentrated Extract Volume: 2000 (uU)

Injection Volume:

(ur)

GPC Cleanup: $(\mathrm{Y} / \mathrm{N}) \mathrm{N} \quad \mathrm{pH}: 12.5$
Lab Sample ID: 99-0650-MSD

Iab File ID: 99020614

Date Received:

Date Extracted:

Date Analyzed: 02/07/99

Dilution Factor: 1.0

CAS NO.

COMPOUND

CONCENTRATION UNITS:

(ug/I or $u g / \mathrm{kg}$ ) UG/KG

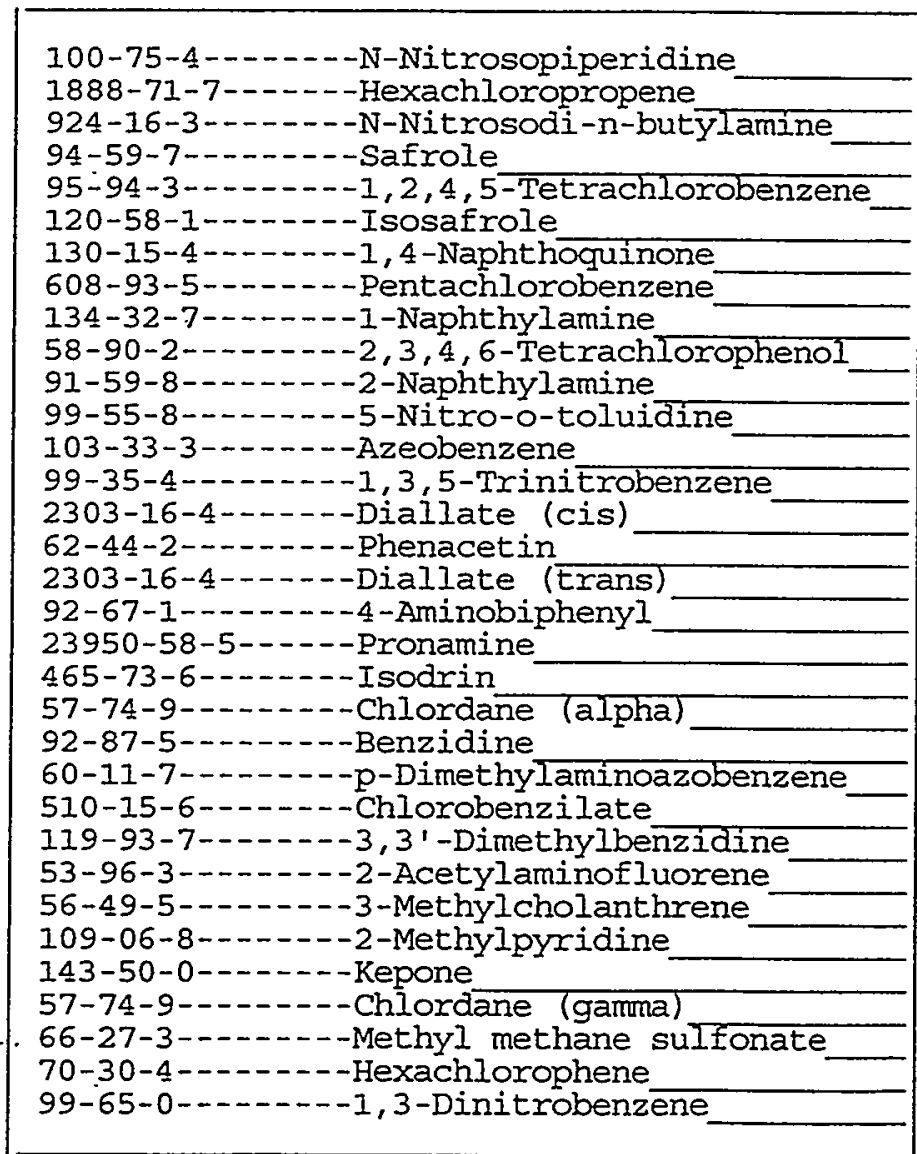

FORM I SV-4

OLM03.0 
Lab Name: PNNL

Lab Code: PNNL

Case No.:

Matrix: (soil/water) SOLID

Sample wt/vol: . $2.6(\mathrm{~g} / \mathrm{mL}) \mathrm{G}$

Level: (low/med) LOW

$\because$ Moisture: 0 decanted: $(\mathrm{Y} / \mathrm{N}) \mathrm{N}$

Concentrated Extract Volume: 2000 (UL)

Injection Volume: (uL)

GPC Cleanup: $(\mathrm{Y} / \mathrm{N}) \mathrm{N}$

$\mathrm{pH}: 12.5$
Contract: BNFL

SAS NO.:

Lab Sample ID: 99-0650-MSD

Lab File ID: 99020614

Date Received:

Date Extracted:

Date Analyzed: 02/07/99

Dilution Factor: 1.0

SDG No.: 990205

AW-101-MSD

CONCENTRATION UNITS:

CAS NO.

COMPOUND

(ug/L or ug/Kg) UG/KG

Q 
Lab Name: PNNL

Lab Code: PNNL

Case No.:
Contract: BNFL

SAS NO. :
EPA SAMPLE NO.

METHOD BLANK
Matrix: (soil/water) SOLID

Sample wt/vol:

$5.0(\mathrm{~g} / \mathrm{mL}) \mathrm{G}$

Level: (low/med)

IOW

$\%$ Moisture: 0

decanted: $(Y / N) N$

Concentrated Extract Volume: 2000 (uL)

Injection Volume: (UI)

GPC Cleanup: $(\mathrm{Y} / \mathrm{N}) \mathrm{N}$

$\mathrm{pH}: 7.0$
Lab Sample ID: SBLKO4

Lab File ID: $\quad 99020610$

Date Received:

Date Extracted:

Date Analyzed: 02/06/99

Dilution Factor: 1.0

CAS NO.

COMPOUND

CONCENTRATION UNITS:

(ug/I or ug/Kg) UG/KG

Q

\begin{tabular}{|c|}
\hline 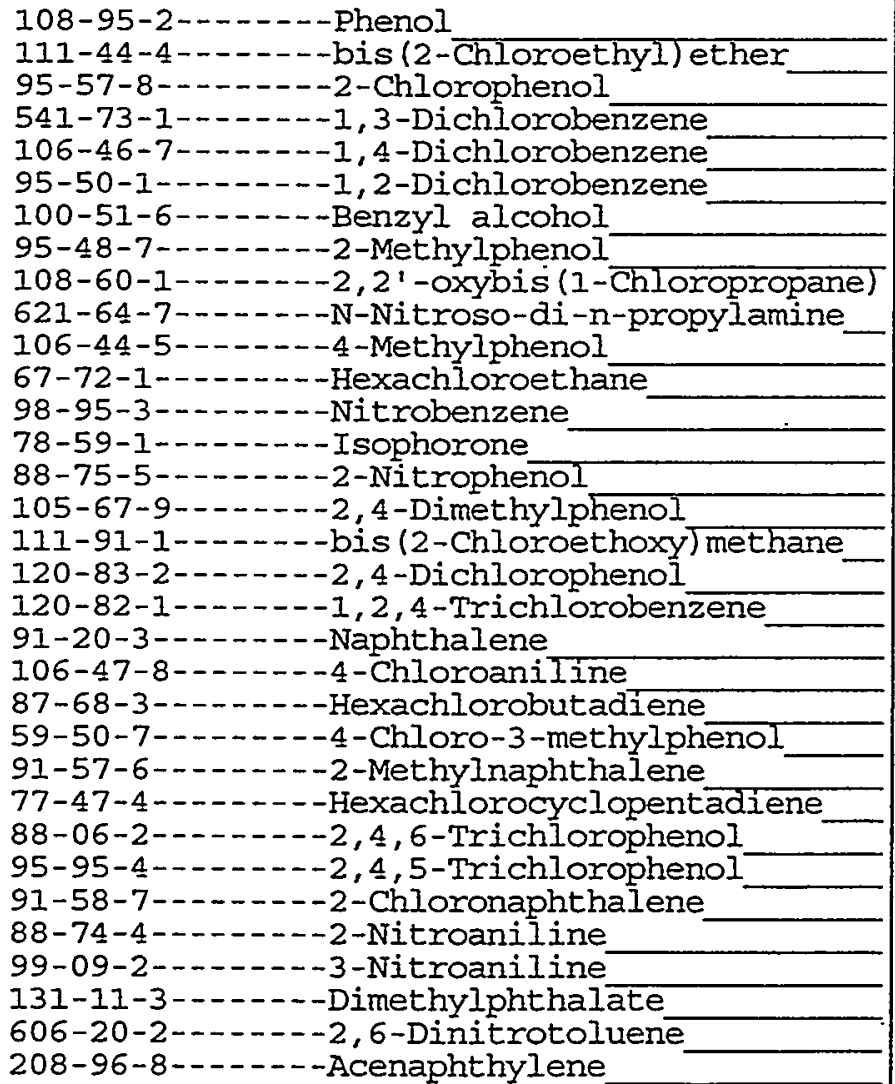 \\
\hline
\end{tabular}

FORM I SV-I

OLMO3. 0 
Lab Name: PNNL

Iab code: PNNL

Case No.:
Contract: BNFL

SAS NO.:
EPA SAMPLE NO.

METHOD BI.ANK
Matrix: (soil/water). SOIID

Sample wt/vol:

$5.0(\mathrm{~g} / \mathrm{mI}) \mathrm{G}$

Level: (low/med) LOW

\% Moisture: 0 decanted: $(\mathrm{Y} / \mathrm{N}) \mathrm{N}$

Concentrated Extract Volume: 2000 (uI)

Injection Volume: (uI)

GPC Cleanup: $(\mathrm{Y} / \mathrm{N}) \mathrm{N}$

$\mathrm{pH}: 7.0$
Lab Sample ID: SBLKO4

Lab File ID: 99020610

Date Received:

Date Extracted:

Date Analyzed: 02/06/99

Dilution Factor: 1.0

CONCENTRATION UNITS:

CAS NO. COMPOUND (ug/I or ug/Kg) UG/KG

Q

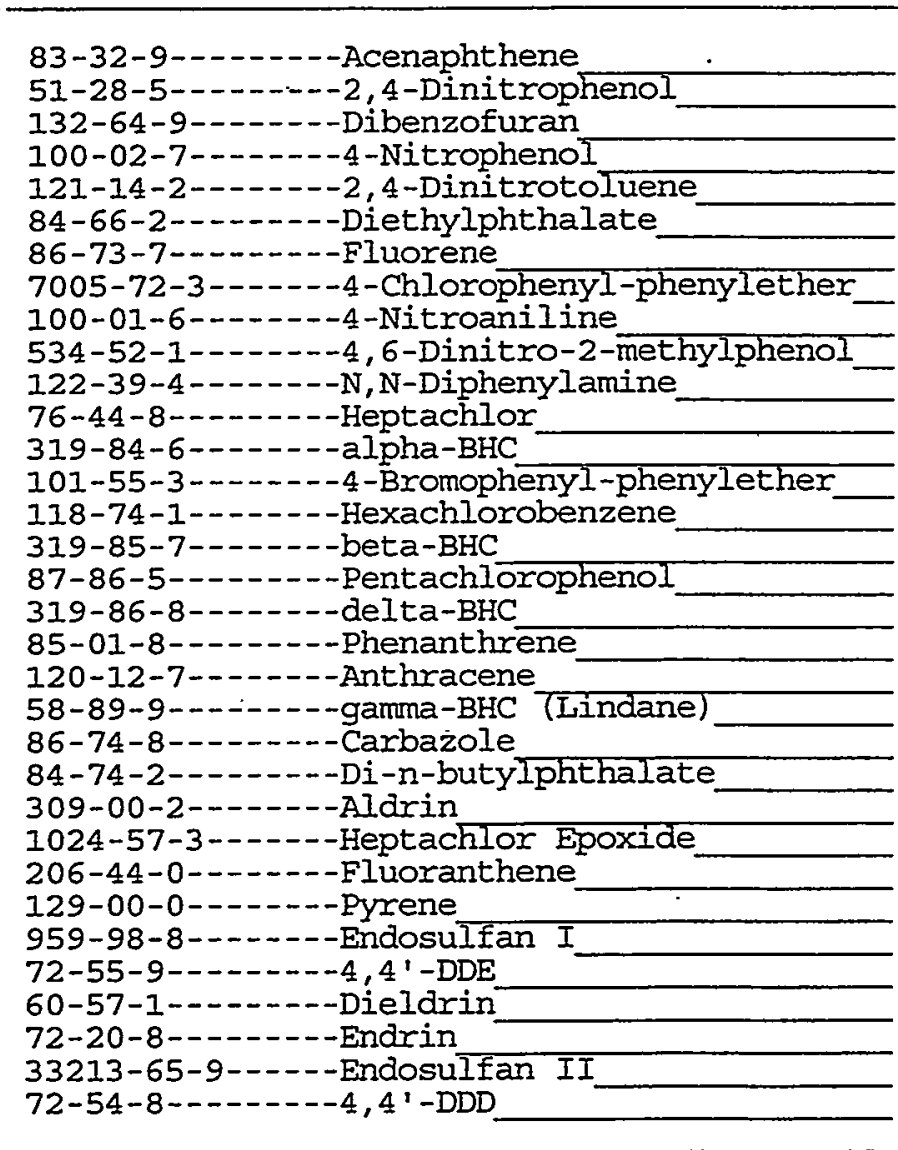

FORM I SV-2

OLM03.0 
Lab Name: PNNL

Lab code: PNNL

Case No.:
Contract: BNFL

SAS NO. :
EPA SAMPLE NO.

METHOD BLANK
Matrix: (soil/water) SOLID

Sample wt/vol:

$$
5.0(\mathrm{~g} / \mathrm{mL}) \mathrm{G}
$$

Level: (low/med) LOW

$\%$ Moisture: 0 decanted: $(\mathrm{Y} / \mathrm{N}) \mathrm{N}$

Concentrated Extract Volume: 2000 (uL)

Injection Volume:

(uL)
$\mathrm{pH}: 7.0$
GPC Cleanup
$(\mathrm{Y} / \mathrm{N}) \mathrm{N}$

Lab Sample ID: SBLKO4

Lab File ID: 99020610

Date Received:

Date Extracted:

Date Analyzed: 02/06/99

Dilution Factor: 1.0

CONCENTRATION UNITS:

CAS NO.

COMPOUND

(ug/I or $\mathrm{ug} / \mathrm{Kg}$ ) UG/KG

Q

85-68-7--.-.--Butylbenzylphthalate 1031-07-8-..---Endosulfan Sulfate 50-29-3--..---4,4'-DDT

$53494-70-5-\ldots . .-$-Endrin Ketone

56-55-3-...--Benzo (a) anthracene

91-94-1-...--3, 3'-Dichlorobenzidine

218-01-9-...-.-Chrysene

72-43-5-...-.-Methoxychlor

117-81-7--..-Bis (2-Ethylhexyl) phthalate

117-84-0--.--Di-n-octylphthalate

205-99-2-...--Benzo (b) fluoranthene

207-08-9-...--Benzo (k) fluoranthene

50-32-8-..----Benzo (a) pyrene

193-39-5-...-. Indeno $(1,2,3$-cd)pyrene

53-70-3-...-Dibenz $(a, h)$ anthracene

$191-24-2-\ldots-$ - Benzo $(g, h, i)$ perylene

110-86-1-...--Pyridine

126-73-8-..--Tributyl phosphate

62-75-9-...--N-Nitrosodimethylamine

98-86-2-.-.--Acetophenone

100-00-5-1.--1-Chloro-4-nitrobenzene

92-52-4-2.---.-Biphenyl

100-25-4-...-1, 4-Dinitrobenzene

128-37-0-...--Butylated Hyaroxytoluene

82-68-8-....-. Pentachloronitrobenzene

88-85-7-...-.-Dinoseb

2234-13-1-...-Octachloronaphthalene

10595-95-6--.-N-Nitrosomethylethylamine

55-18-5-n-n-N-Nitrosodiethylamine

62-50-0-....-.-Ethyl methane sulfonate

62-53-3-...---Analine

76-01-7-....--Pentachloroethane

930-55-2-.---N-Nitrosopyrolidine

\begin{tabular}{l|l|l}
4000 & $U$ \\
4000 & $U$ \\
4000 & $U$ \\
4000 & $U$ \\
4000 & $\mathrm{U}$ \\
4000 & $\mathrm{U}$ \\
4000 & $\mathrm{U}$ \\
4000 & $\mathrm{U}$ \\
4000 & $\mathrm{U}$ \\
4000 & $\mathrm{U}$ \\
4000 & $\mathrm{U}$ \\
4000 & $\mathrm{U}$ \\
4000 & $\mathrm{U}$ \\
4000 & $\mathrm{U}$ \\
4000 & $\mathrm{U}$ \\
4000 & $\mathrm{U}$ \\
4000 & $\mathrm{U}$ \\
4000 & $\mathrm{U}$ \\
4000 & $\mathrm{U}$ \\
4000 & $\mathrm{U}$ \\
4000 & $\mathrm{U}$ \\
4000 & $\mathrm{U}$ \\
4000 & $\mathrm{U}$ \\
4000 & $\mathrm{U}$ \\
4000 & $\mathrm{U}$ \\
4000 & $\mathrm{U}$ \\
4000 & $\mathrm{U}$ \\
4000 & $\mathrm{U}$ \\
4000 & $\mathrm{U}$ \\
4000 & $\mathrm{U}$ \\
4000 & $\mathrm{U}$ \\
4000 & $\mathrm{U}$ \\
4000 & $\mathrm{U}$ \\
& \\
\hline & \\
\hline
\end{tabular}

OLM03.0 
Lab Name: PNNL

Lab Code: PNNL

Case No.:

Matrix: (soil/water) SOIID

Sample wt/vol:

$$
5.0(\mathrm{~g} / \mathrm{mL}) \mathrm{G}
$$

Level: (low/med) IOW

\% Moisture: 0 decanted: $(\mathrm{Y} / \mathrm{N}) \mathrm{N}$

Concentrated Extract Volume: 2000 (uس) Injection Volume: (UJ)

GPC Cleanup: $\quad(\mathrm{Y} / \mathrm{N}) \mathrm{N}$ $\mathrm{pH}: 7.0$
Contract : BNFL

SAS NO.:

SDG NO.: 990205

METHOD BLANK

Lab Sample ID: SBLKO4

Lab File ID: $\quad 99020610$

Date Received:

Date Extracted:

Date Analyzed: 02/06/99

Dilution Factor: 1.0

CAS NO.

COMPOUND

CONCENTRATION UNITS:

(ug/I or $\mathrm{ug} / \mathrm{Kg}$ ) UG/KG

Q

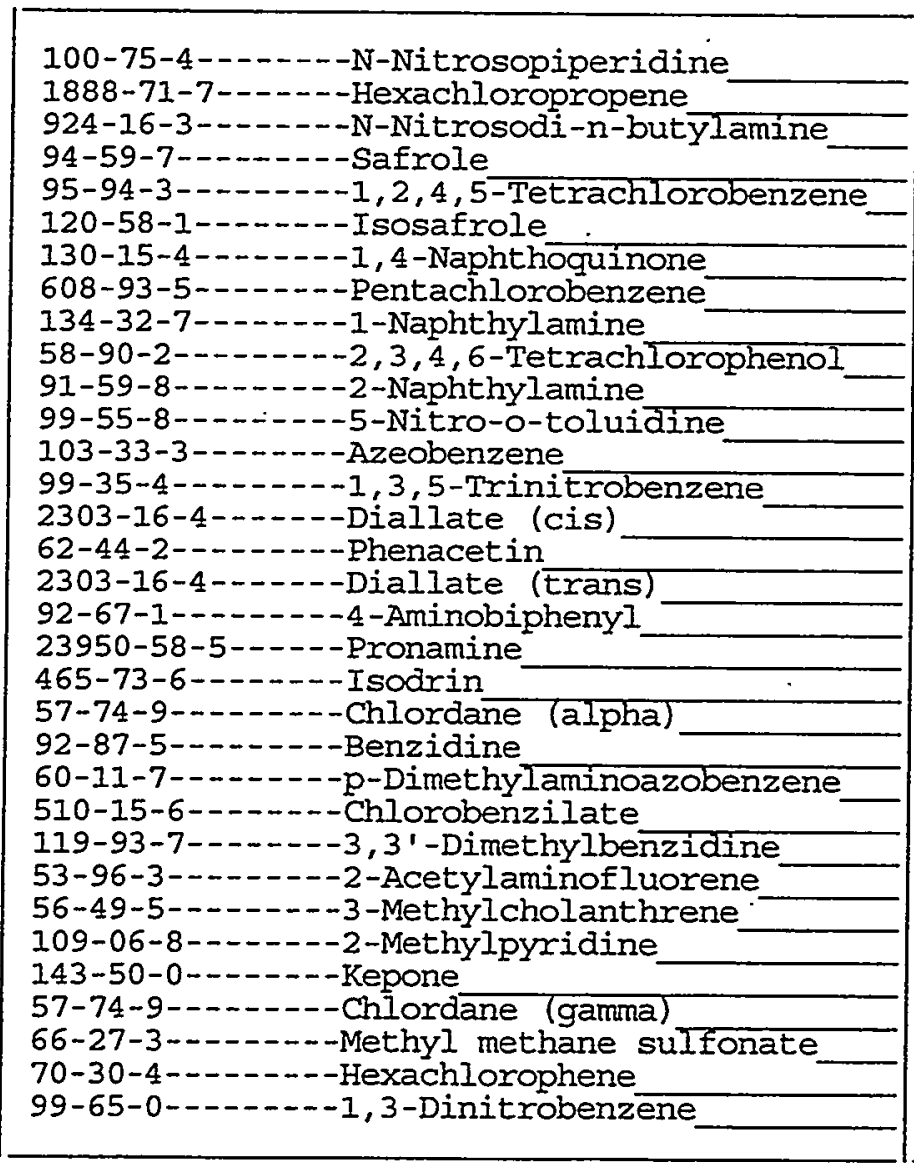


$1 C$

SEMIVOLATIIE ORGANICS ANALYSIS DATA SHEET

Lab Name: PNNL

Contract: BNFI

Case No.:

Matrix: (soil/water) SOLID

Sample wt/vol:

$5.0(\mathrm{~g} / \mathrm{mL}) \mathrm{G}$

Ievel: (low/med)

IOW

\% Moisture: 0

decanted: $(\mathrm{Y} / \mathrm{N}) \mathrm{N}$

Concentrated Extract Volume: 2000 (UL)

Injection Volume: (uL)

GPC Cleanup: $\quad(\mathrm{Y} / \mathrm{N}) \mathrm{N}$

$\mathrm{pH}: 7.0$.
SAS NO. :

Iab Sample ID: SBLKO4

Lab File ID: 99020610

Date Received:

Date Extracted:

Date Analyzed: 02/06/99

Dilution Factor: 1.0

METHOD BLANK

CAS NO:

COMPOUND

CONCENTRATION UNITS:

(ug/L or ug/Kg) UG/KG Q

$4000 \mathrm{U}$ 
Lab Name: PNNL

Lab code: PNNL

Matrix: (soil/water) WATER

Sample wt/vol:

Level: (low/med) LOW

$\%$ Moisture: decanted: $(Y / N)$

Concentrated Extract Volume: 2400 (uI) Injection Volume: (uL)

Contract : BNFL

SAS NO. :

Lab Sample ID: LCS

Lab File ID: 99020615

Date Received:

Date Extracted:

Date Analyzed: 02/07/99

Dilution Factor: 1.0

GPC Cleanup: $(\mathrm{Y} / \mathrm{N}) \mathrm{N} \quad \mathrm{pH}: 7.0$

CAS NO.

COMPOUND

CONCENTRATION UNITS :

(ug/L or $u g / \mathrm{kg}$ ) UG/L

\begin{tabular}{|c|}
\hline 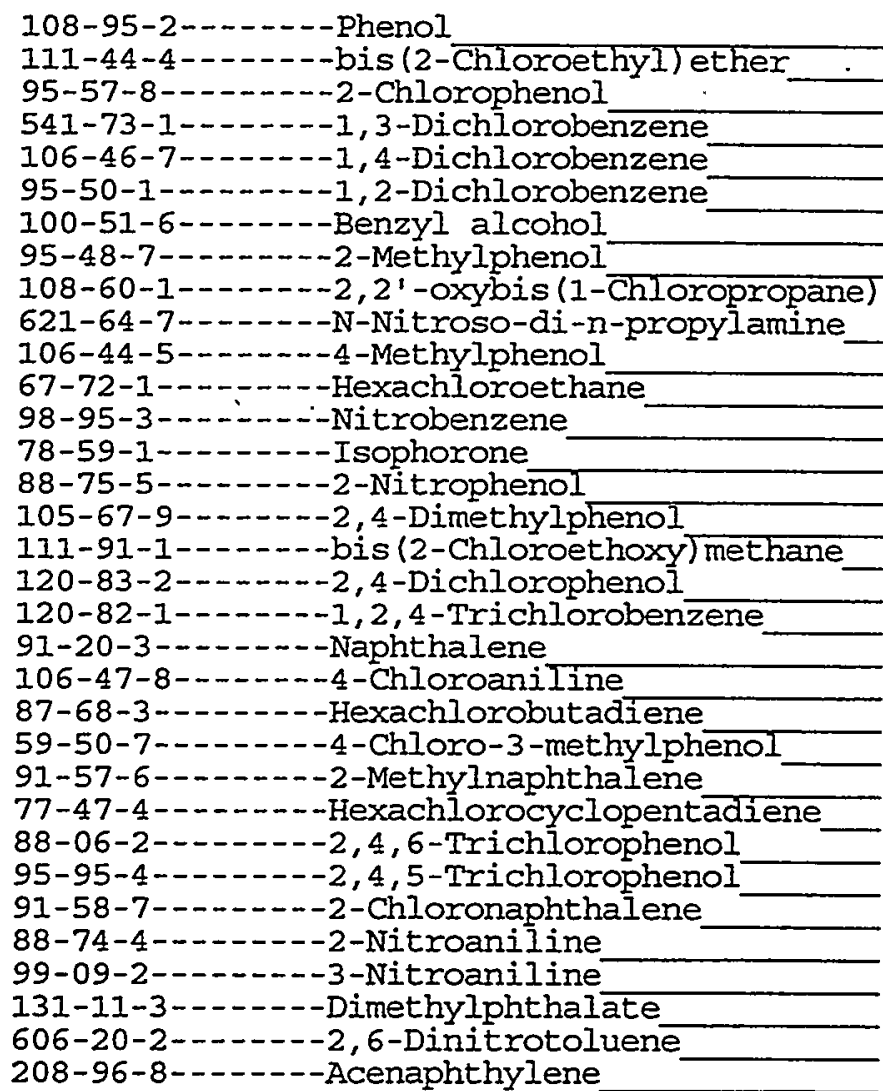 \\
\hline
\end{tabular}

FORM I SV-I

OLM03.0 
Iab Name: PNNL

Iab code: PNNL

Case No.:

Contract : BNFL

Matrix: (soil/water) WATER

Sample wt/vol: $(g / m L) M L$

Level: (low/med)

LOW

\% Moisture:

decanted: $(Y / N)$

Concentrated Extract Volume: 2400(uL)

Injection Volume:

(uL)

SAS NO.:

EPA SAMPLE NO.

LCS

SDG No.: 990205

Lab Sample ID: ICS

Lab File ID: 99020615

Date Received:

Date Extracted:

Date Analyzed: 02/07/99

Dilution Factor: 1.0

GPC Cleanup: $(\mathrm{Y} / \mathrm{N}) \mathrm{N} \quad \mathrm{pH}: 7.0$

CONCENTRATION UNITS:

CAS NO. COMPOUND (ug/I or ug/Kg) UG/I Q

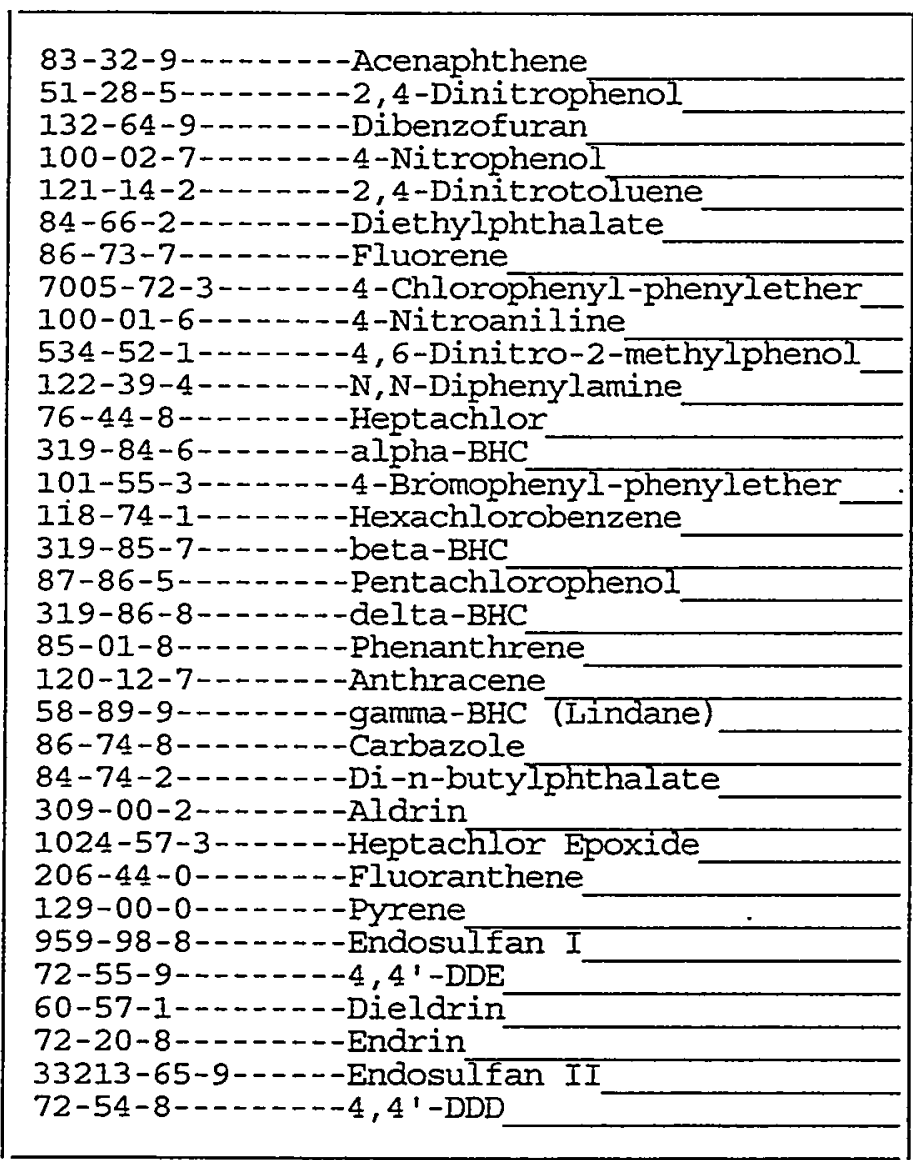


Lab Name: PNNL

Lab Code: PNNL
Contract: BNFL

SAS NO. :
EPA SAMPLE NO.

LCS
Matrix: (soil/water) WATER

Sample wt/vol: $(\mathrm{g} / \mathrm{mL}) \mathrm{ML}$

Level: (low/med)

LOW

\% Moisture: decanted: $(Y / N)$

Concentrated Extract Volume: 2400 (uI) Injection Volume: (ut)

GPC Cleanup: $(Y / N) N$

$\mathrm{pH}: 7.0$
Lab Sample ID: LCS

Lab File ID: $\quad 99020615$

Date Received:

Date Extracted:

Date Analyzed: 02/07/99

Dilution Factor: 1.0

CAS NO.

COMPOUND

CONCENTRATION UNITS: (ug/L or $u g / \mathrm{kg}$ ) UG/L

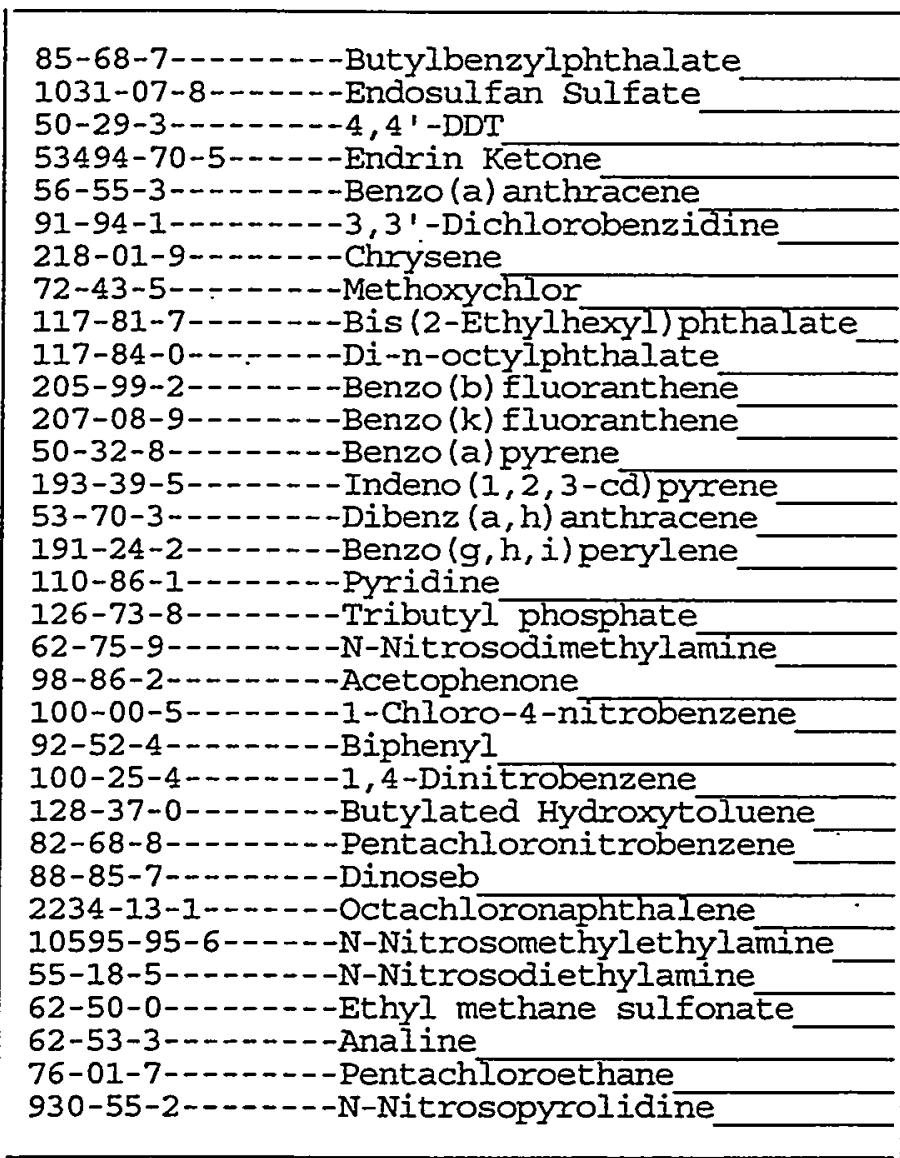

1100
1100
1100
1100
1100
900
1100
1000
1100
1000
980
980
1000
750
1000
1000
2100
1300
11000
2100
1400
1300
1500
1200
2300
1700
1200
1000
990
1000
900
950
9


Lab Name: PNNL

Contract: BNFI

Lab code: PNNL

Case No.:
SAS NO.:
ICS

SDG No.: 990205
Matrix: (soil/water) WATER

Sample wt/vol: $(\mathrm{g} / \mathrm{mL}) \mathrm{ML}$

Level: (low/med) IOW

\% Moisture: decanted: $(Y / N)$

Concentrated Extract Volume: 2400 (UL) Injection Volume: (uU)

GPC Cleanup: $\quad(\mathrm{Y} / \mathrm{N}) \mathrm{N} \quad \mathrm{pH}: 7.0$
Lab Sample ID: LCS

Lab File ID: $\quad 99020615$

Date Received:

Date Extracted:

Date Analyzed: 02/07/99

Dilution Factor: 1.0

CONCENTRATION UNITS: CAS NO. COMPOUND (ug/I or ug/Kg) UG/L

Q

100-75-4-1.-- N-Nitrosopiperidine

1888-71-7-...-Hexachloropropene

924-16-3-...-N-Nitrosodi-n-butylamine

94-59-7-.....-.-Safrole

95-94-3-..----1, 2,4, 5-Tetrachlorobenzene

120-58-1--.---Isosafrole

$130-15-4-\ldots---1,4-N a p h t h o q u i n o n e$

608-93-5-..---Pentachlorobenzene

134-32-7-...-.-Naphthylamine

58-90-2-..---2, 3,4, 6-Tetrachlorophenol

91-59-8-...--2-Naphthylamine

99-55-8-..-- - - Nitro-o-toluidine

103-33-3-..-.-Azeobenzene

99-35-4-...-1, 3, 5-Trinitrobenzene

2303-16-4-..--Diallate (cis)

$62-44-2-\ldots-\cdots-1$ - Phenacetin

2303-16-4-..--Diallate (trans)

92-67-1-...-4-Aminobiphenyl

23950-58-5-...-Pronamine

465-73-6---.---Isodrin

57-74-9-.....-Chlordañe (alpha)

92-87-5-....---Benzidine

60-11-7-...--p-Dimethy laminoazobenzene

510-15-6-1---Chlorobenzilate

I19-93-7-...---3,3'-Dimethylbenzidine

53-96-3--..-2-Acetylaminofluorene

56-49-5-..---3-Methylcholanthrene

109-06-8---.--2-Methylpyridine

143-50-0-...--Kepone

57-74-9--.---Chlordane (gamma)

66-27-3-...-.-Methyl methane sulfonate

70-30-4-...---Hexachlorophene

99-65-0-...-1, 3-Dinitrobenzene

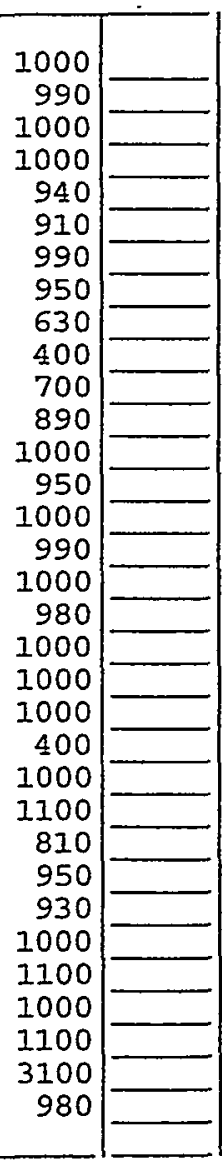

OLM03. 0

\begin{tabular}{rr}
1000 \\
990 \\
1000 \\
1000 \\
940 \\
910 \\
990 \\
950 \\
630 \\
400 \\
700 \\
890 \\
1000 \\
950 \\
1000 \\
990 \\
1000 \\
980 \\
1000 \\
1000 \\
1000 \\
400 \\
1000 \\
1100 \\
810 \\
950 \\
\hline \\
1030 \\
1100 \\
1000 \\
1100 \\
3100 \\
980 \\
\end{tabular}


Lab Name: PNNL

Contract: BNFL

Lab Code: PNNL Case No.:

SAS NO.:

ICS

Matrix: (soil/water) WATER

Lab Sample ID: LCS

Sample wt/vol: (g/mL) ML Lab File ID: 99020615

Level: (low/med) LOW

\% Moisture: decanted: $(\mathrm{Y} / \mathrm{N})$ Date Received:

Concentrated Extract Volume: 2400 (ur) Date Extracted:

Injection Volume: (UI) Date Analyzed: 02/07/99 GPC Cleanup: $\quad(\mathrm{Y} / \mathrm{N}) \mathrm{N} \quad \mathrm{pH}: 7.0$ GPC Cleanup: $\quad(\mathrm{Y} / \mathrm{N}) \mathrm{N} \quad \mathrm{pH}: 7.0$ Dilution Factor: 1.0 CAS NO. COMPOUND

CONCENTRATION UNITS: (ug/L or $\mathrm{ug} / \mathrm{Kg}$ ) UG/L Q 87-65-0-..--.--2, 6-Dichlorophenol 
Lab Name: PNNL

contract: BNEL

AW-101

Iab Code: PNNL Case No.:

SAS NO.:

SDG No. : 990205

Matrix: (soil/water) SUPERNATANT

Irab Sample ID: $99-0648$

Sample wt/vol: $\quad 0.000(\mathrm{~g} / \mathrm{mL}) \mathrm{ML}$

Lab File ID: $\quad 99020606$

Level: (low/med) IOW

Date Received:

$\%$ Moisture: decanted: $(\mathrm{Y} / \mathrm{N})$

Date Extracted:

Concentrated Extract Volume: 2000 (uU)

Date Analyzed: 02/06/99

Injection Volume: (uน)

Dilution Factor: 1.0

GPC Cleanup: (Y/N) N $\mathrm{pH}: 12.5$

CONCENTRATION UNITS:

Number TICs found: 22

(ug/L or $u g / \mathrm{Kg}$ ) ug/L

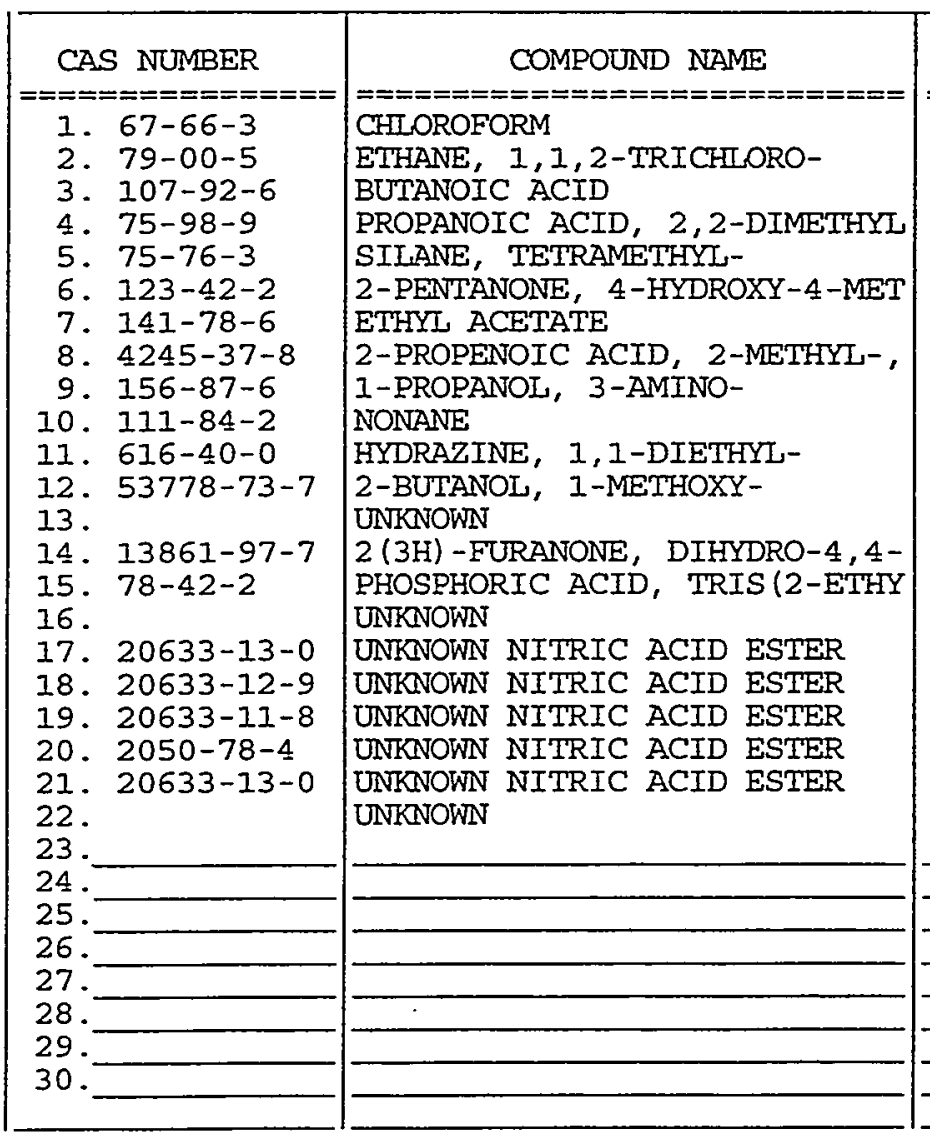

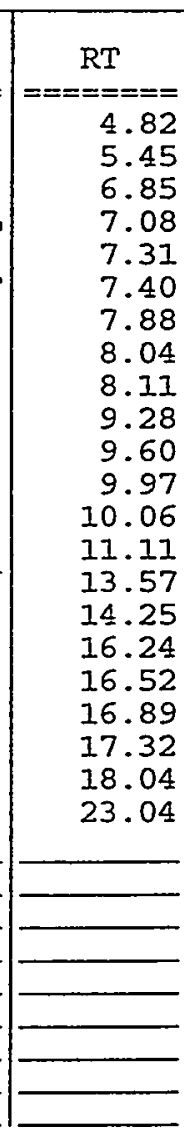

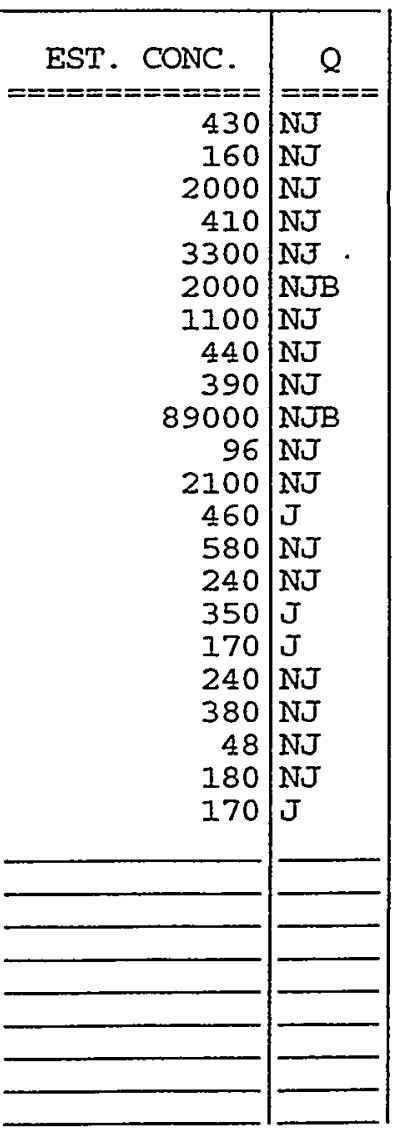

FORM I SV-TIC

OLM03. 0 
Lab Name: PNNL

Contract: BNFI

Lab Code: PNNL

Case No.:

SAS NO. :

Matrix: (soil/water) SUPERNATANT

Lab Sample ID: 99-0648D

Sample wt/vol:

$0.000(\mathrm{~g} / \mathrm{mL}) \mathrm{ML}$

Lab File ID: $\quad 99020607$

Level: (low/med) LOW

Date Received:

$\because$ Moisture: decanted: $(\mathrm{Y} / \mathrm{N})$

Date Extracted:

Concentrated Extract Volume: 2000 (UI)

Date Analyzed: 02/06/99

Injection volume: (uI)

Dilution Factor: 1.0

GPC Cleanup: $\quad(\mathrm{Y} / \mathrm{N}) \mathrm{N}$

$\mathrm{pH}: 12.5$

Number TICs found: 12

CONCENTRATION UNITS:

(ug/L or $u g / \mathrm{Kg}$ ) ug/I

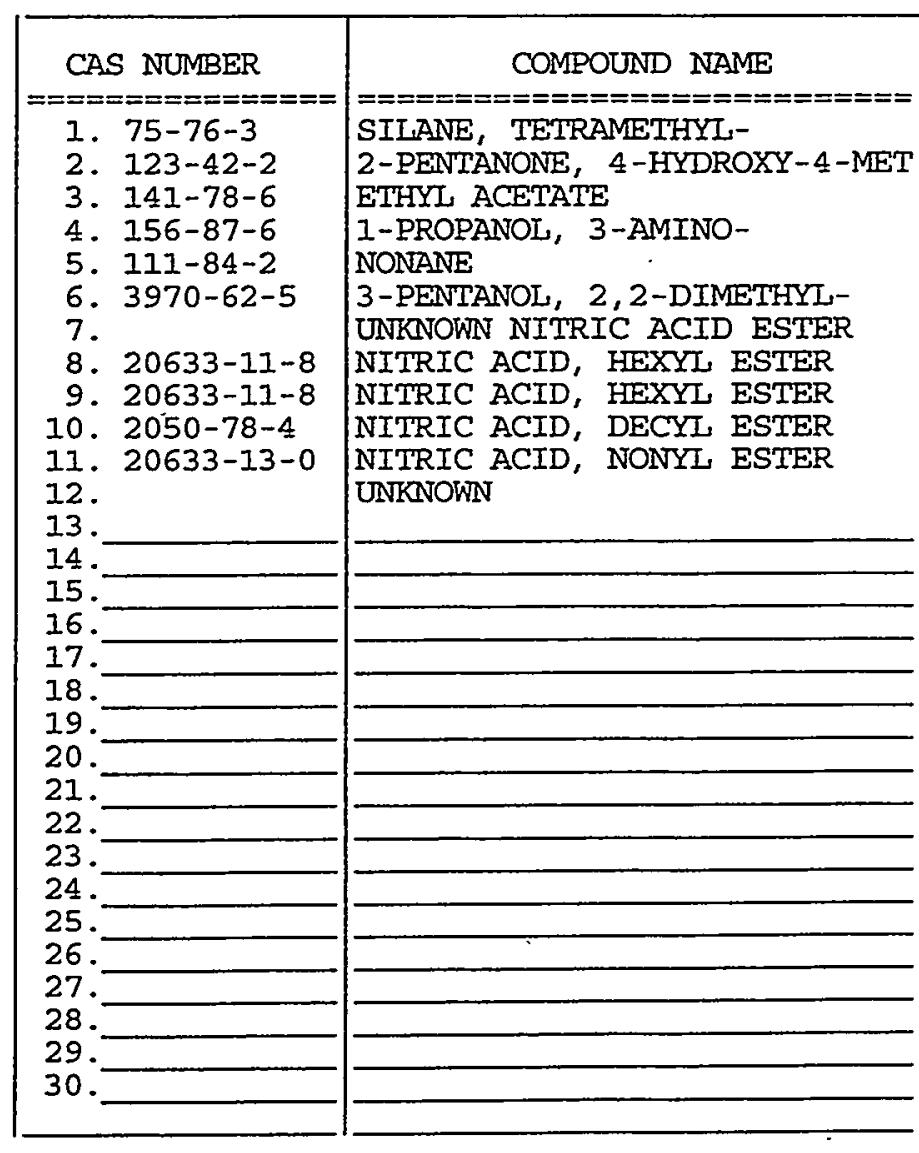

\begin{tabular}{|r|}
$R T$ \\
$======$ \\
7.30 \\
7.42 \\
7.88 \\
8.11 \\
9.28 \\
9.97 \\
16.23 \\
16.51 \\
16.88 \\
17.31 \\
18.04 \\
23.04 \\
\hline \\
\hline \\
\hline
\end{tabular}

$\begin{array}{r}===== \\ 7.30 \\ 7.42 \\ 7.88 \\ 8.11 \\ 9.28 \\ 9.97 \\ 16.23 \\ 16.51 \\ 16.88 \\ 17.31 \\ 18.04 \\ 23.04 \\ \hline\end{array}$
EST. CONC. $=============$ = $====$ $2300 \mathrm{NJ}$ 5200 NUB $1100 \mathrm{NJ}$ $510 \mathrm{NJ}$ 80000 NJB $3000 \mathrm{NJ}$ $130 \mathrm{~J}$ 200 NJ 300 NJ 66 NJ $150 \mathrm{NJ}$ $170 \mathrm{~J}$

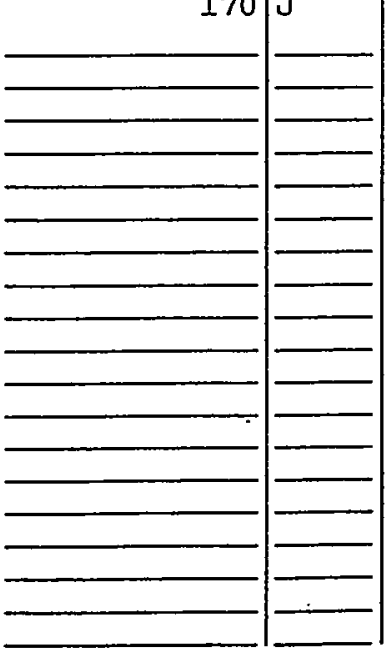


Lab Name: PNNL

Contract: BNFL

Iab Code: PNNL

Case No.:

SAS NO.:

EPA SAMPLE NO.

Matrix: (soil/water) SUPERNATANT

Sample wt/vol:

$0.000(\mathrm{~g} / \mathrm{mL}) \mathrm{ML}$

Lab File ID: 99020605

Level: (low/med) LOW

Date Received:

\% Moisture:

decanted: $(Y / N)$

Date Extracted:

Concentrated Extract Volume: 2000 (uU)

Date Analyzed: 02/06/99

Injection Volume:

(uL)

Dilution Factor: 1.0

GPC Cleanup: $(\mathrm{Y} / \mathrm{N}) \mathrm{N} \quad \mathrm{pH}: 7.0$

Number TICs found: 2

CONCENTRATION UNITS:

(ug/I or $u g / \mathrm{Kg}$ ) ug/L

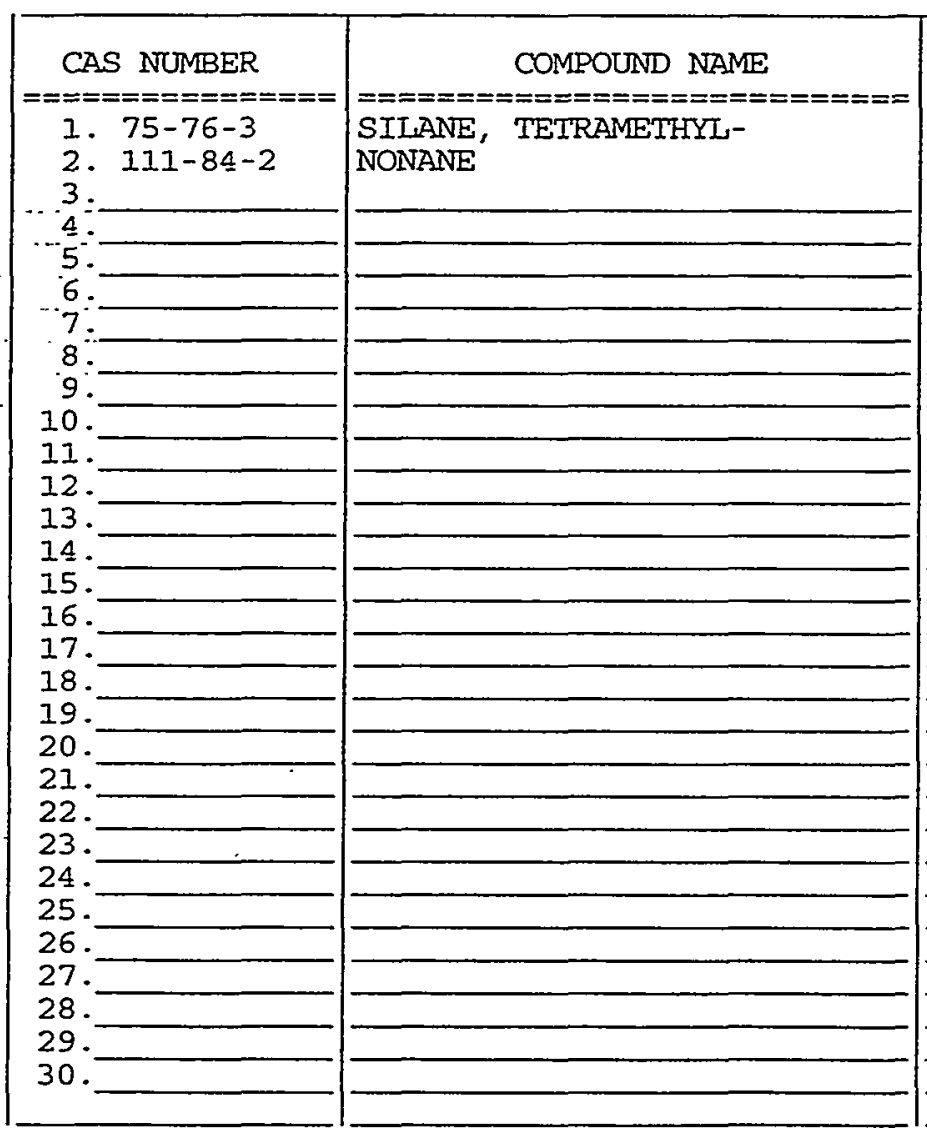

\begin{tabular}{|c|c|c|}
\hline$===\frac{\mathrm{RT}}{}==$ & $===========$ & $\frac{Q}{N===}=$ \\
\hline & & \\
\hline & & \\
\hline & & \\
\hline & & \\
\hline & & \\
\hline & & \\
\hline & & \\
\hline & & \\
\hline & & \\
\hline & & \\
\hline & & \\
\hline & & \\
\hline & & \\
\hline & & \\
\hline & & \\
\hline & & \\
\hline & & \\
\hline & & \\
\hline & & \\
\hline & & \\
\hline & & \\
\hline
\end{tabular}


Lab Name: PNNL

Contract: BNFI

Lab Code: PNNL

Case No.:

SAS NO.:
EPA SAMPLE NO.

AW-101-

SDG No.: 990205
Matrix: (soil/water) SOLID

Sample wt/vol:

$4.2(\mathrm{~g} / \mathrm{mL}) \mathrm{G}$

Level: (low/med) LOW

$\div$ Moisture: decanted: $(\mathrm{Y} / \mathrm{N})$

Concentrated Extract Volume: 2000 (uL)

Injection Volume: (uI)

GPC. Cleanup: $\quad(Y / N) N \quad \mathrm{pH}: 12.5$
Lab Sample ID: 99-0650-

Lab File ID: $\quad 99020611$

Date Received:

Date Extracted:

Date Analyzed: 02/07/99

Dilution Factor: 1.0

Number TICs found: 15

CONCENTRATION UNITS:

(ug/I or $u g / \mathrm{kg}$ ) ug/kg

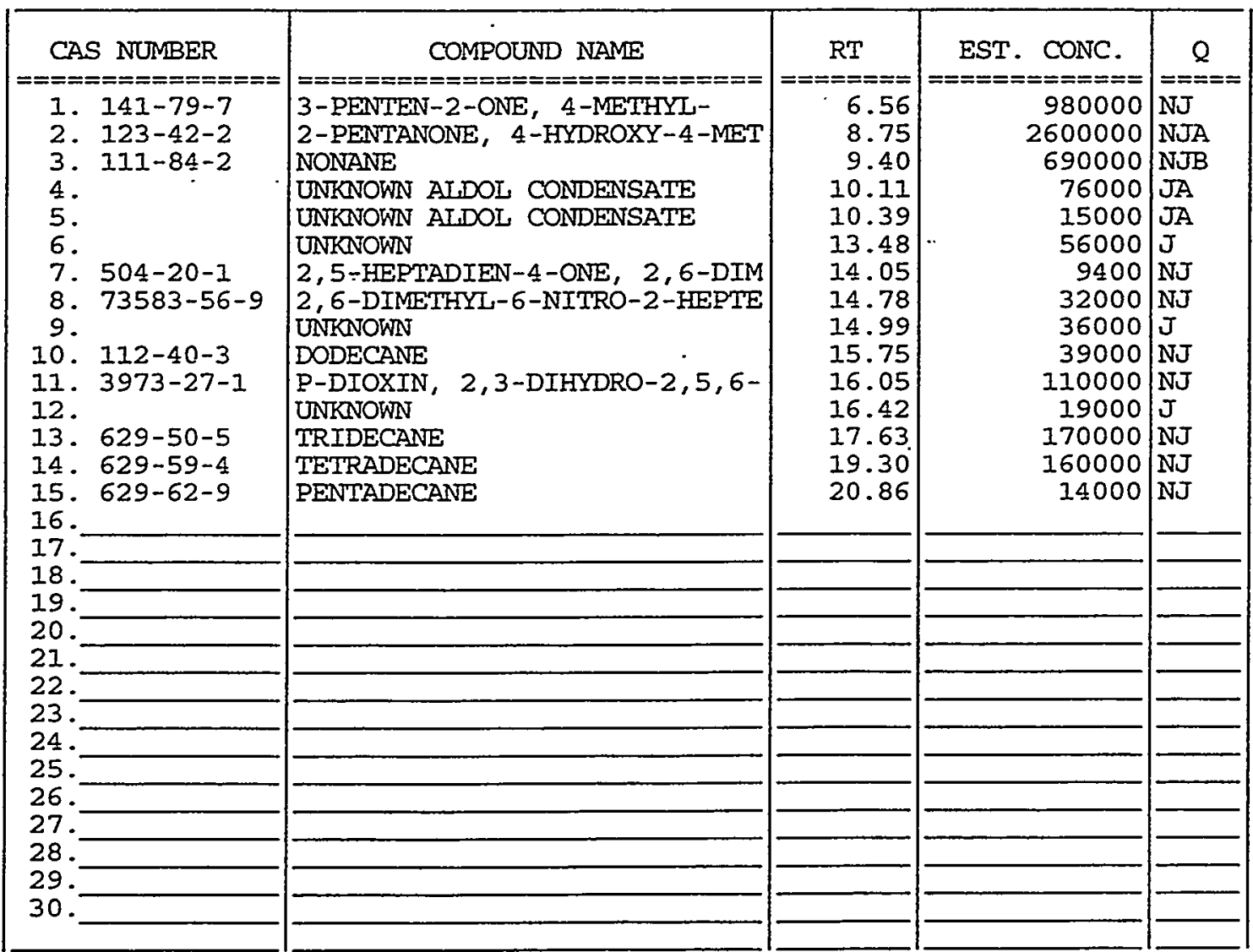


Lab Name: PNNL

Lab Code: PNNL

Case No.:
Contract: BNEL
EPA SAMPLE NO.

$A W-101-D$
Matrix: (soil/water) SOLID

Sample wt/vol:

$4.7(\mathrm{~g} / \mathrm{mL}) \mathrm{G}$

Level: (low/med) LOW

\% Moisture: decanted: $(\mathrm{Y} / \mathrm{N})$

Concentrated Extract Volume: 2000 (uI)

Injection Volume: (uI)
SAS NO.:

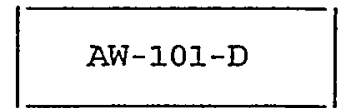

SDG No.: 990205

GPC Cleanup: $\quad(\mathrm{Y} / \mathrm{N}) \mathrm{N} \quad \mathrm{pH}: 12.5$

Lab Sample ID: 99-0650-D

Lab File ID: 99020612

Date Received:

Date Extracted:

Date Analyzed: 02/07/99

Dilution Factor: 1.0

CONCENTRATION UNITS: Number TICs found: 18

(ug/L or $\mathrm{ug} / \mathrm{Kg}$ ) ug/Kg

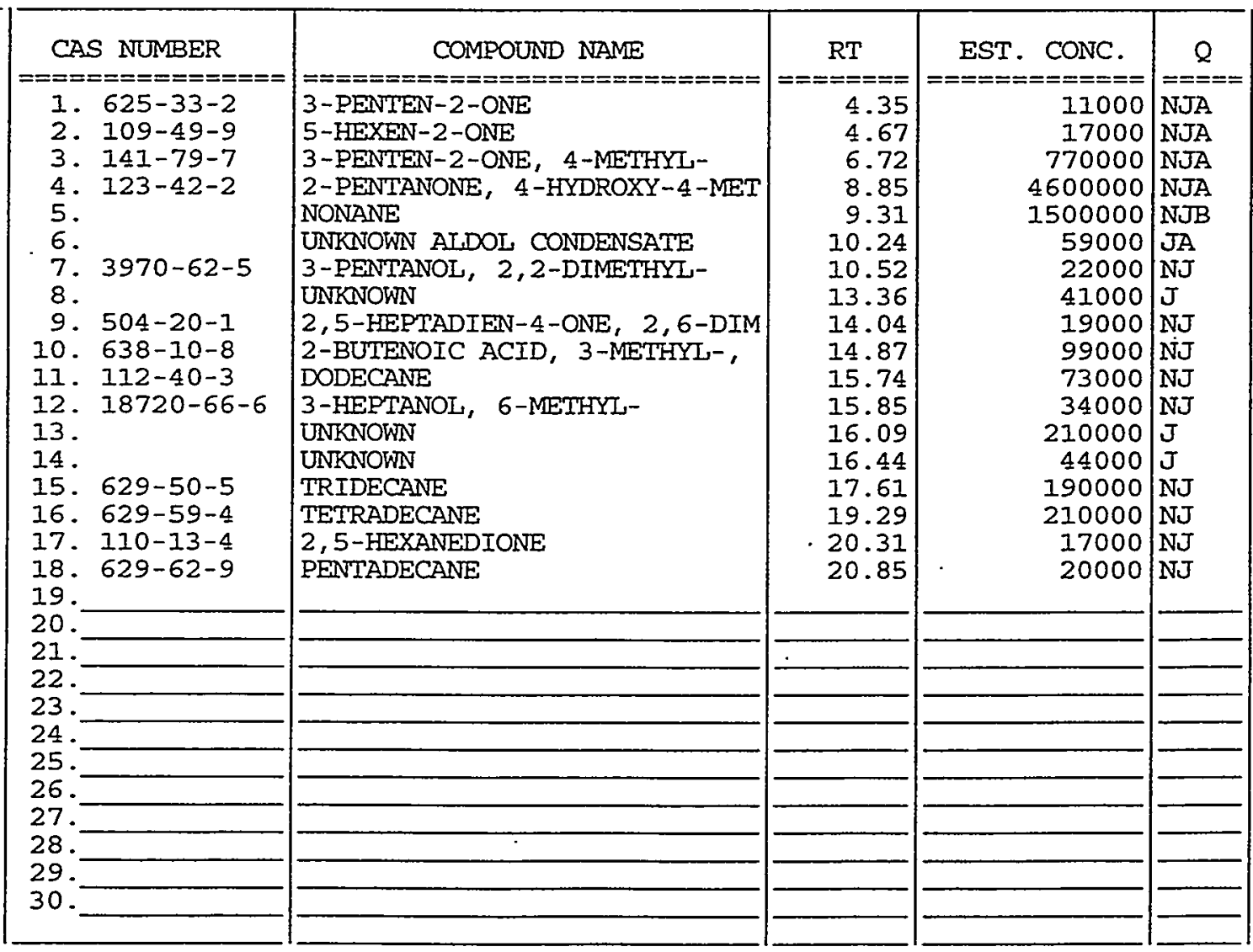


Lab Name: PNNL

Contract: BNFL

Iab Code: PNNL,

Case No.:

SAS NO.:

EPA SAMPLE NO.

Matrix: (soil/water) SOLID

Sample wt/vol:

$5.0(\mathrm{~g} / \mathrm{mL}) \mathrm{G}$

Lab Sample ID: SBLKO4

Level: (low/med) LOW

Lab File ID: 99020610

$\%$ Moisture:

decanted: $(Y / N)$

Date Received:

Concentrated Extract Volume: 2000 (uL)

Date Extracted:

Injection Volume: (uJ)

Date Analyzed: 02/06/99

GPC Cleanup: $(\mathrm{Y} / \mathrm{N}) \mathrm{N} \quad \mathrm{pH}: 7.0$

Dilution Factor: 1.0

CONCENTRATION UNITS :

Number TICs found: 3

(ug/L or $\mathrm{ug} / \mathrm{Kg}$ ) ug/ $\mathrm{Kg}$

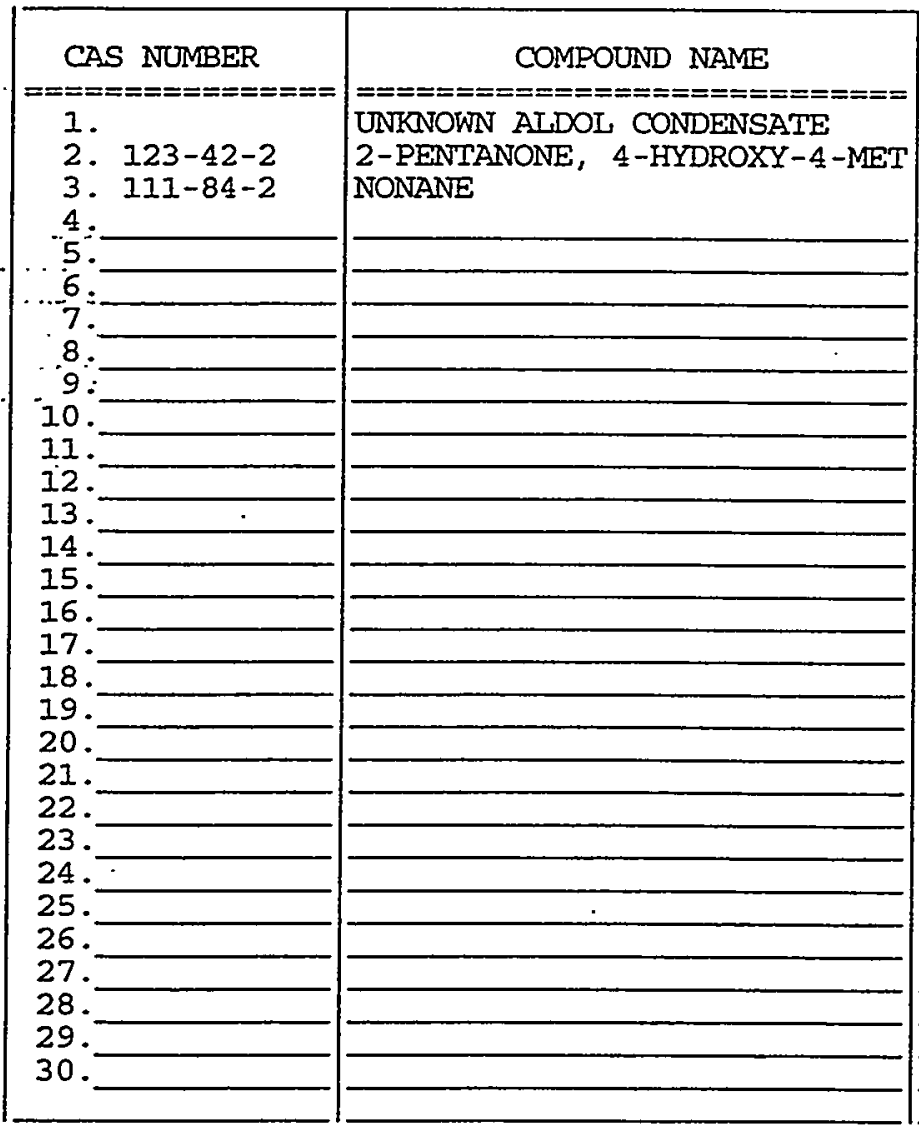

$\begin{array}{r}R T \\ \text { RT } \\ 6.64 \\ 7.50 \\ \hline .34 \\ \hline \\ \hline \\ \hline\end{array}$

EST. CONC. $=============0===$ .64

.50 $20000 \mathrm{JA}$

2. $123-42-2$ NONANE

FORM I SV-TIC

OLM03. 0 
Lab Name: PNNL

Iab Code: PNNL Case No.:

Level: (low/med) LOW
Contract: BNFI

SAS No.:

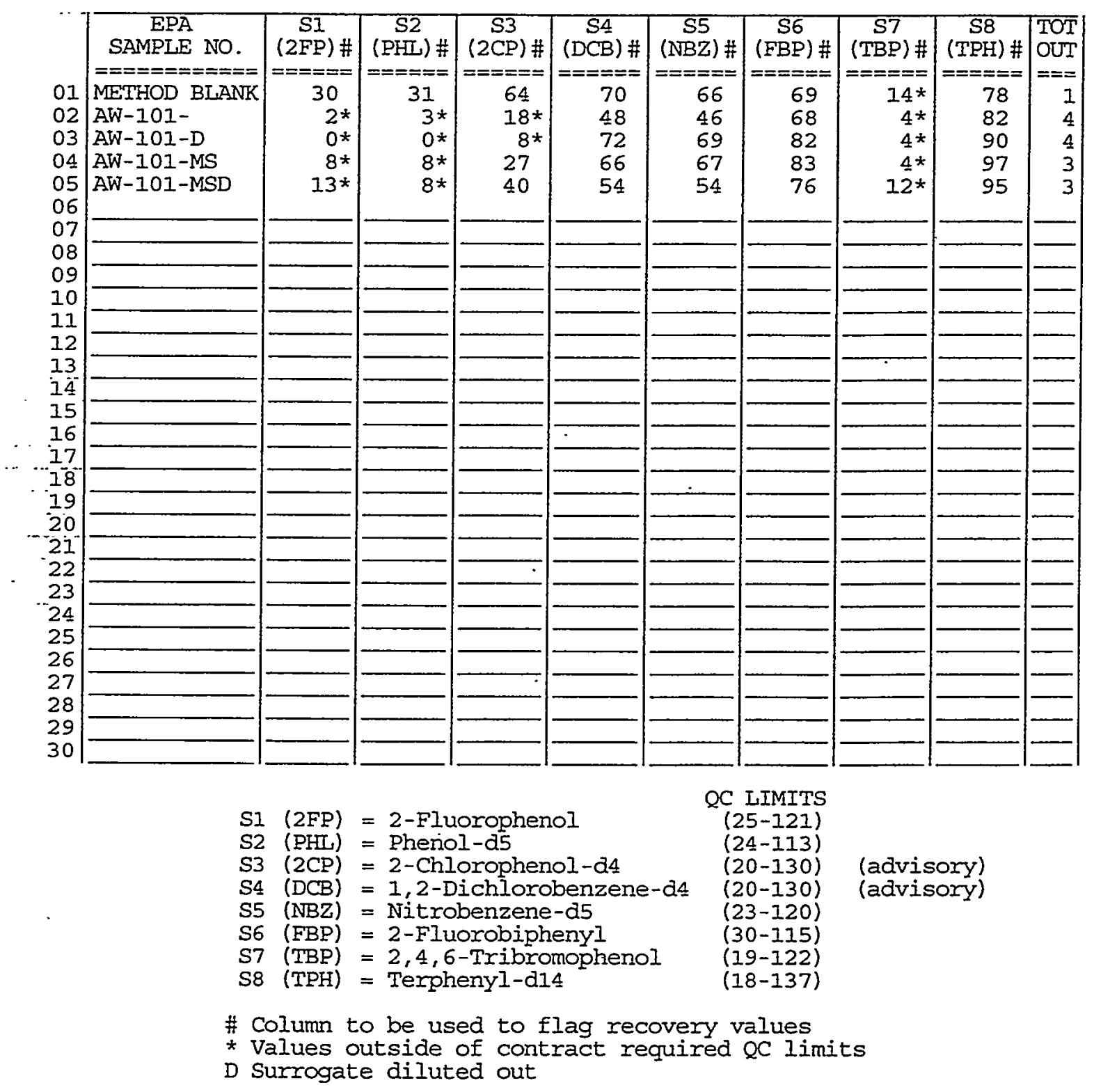

page 1 of 1

FORM II SV-2

OLM03. 0 
Lab Name: PNNL

Lab code: PNNL
Contract: BNFL

Case No. :
SAS NO.:

SDG NO.: 990205

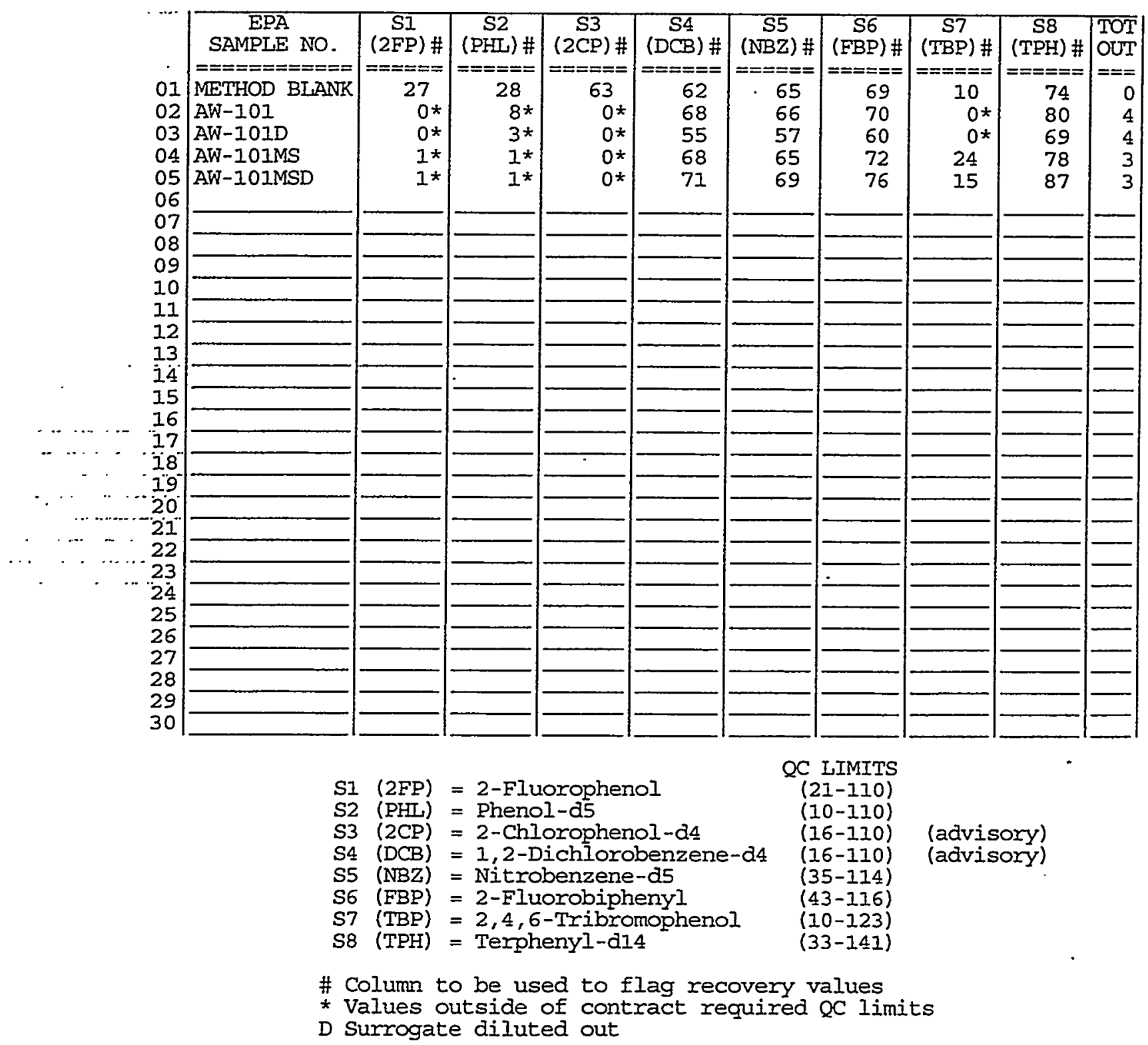


Lab Name: PNNL

Lab Code: PNNL
Contract: BNFL

SAS NO.:

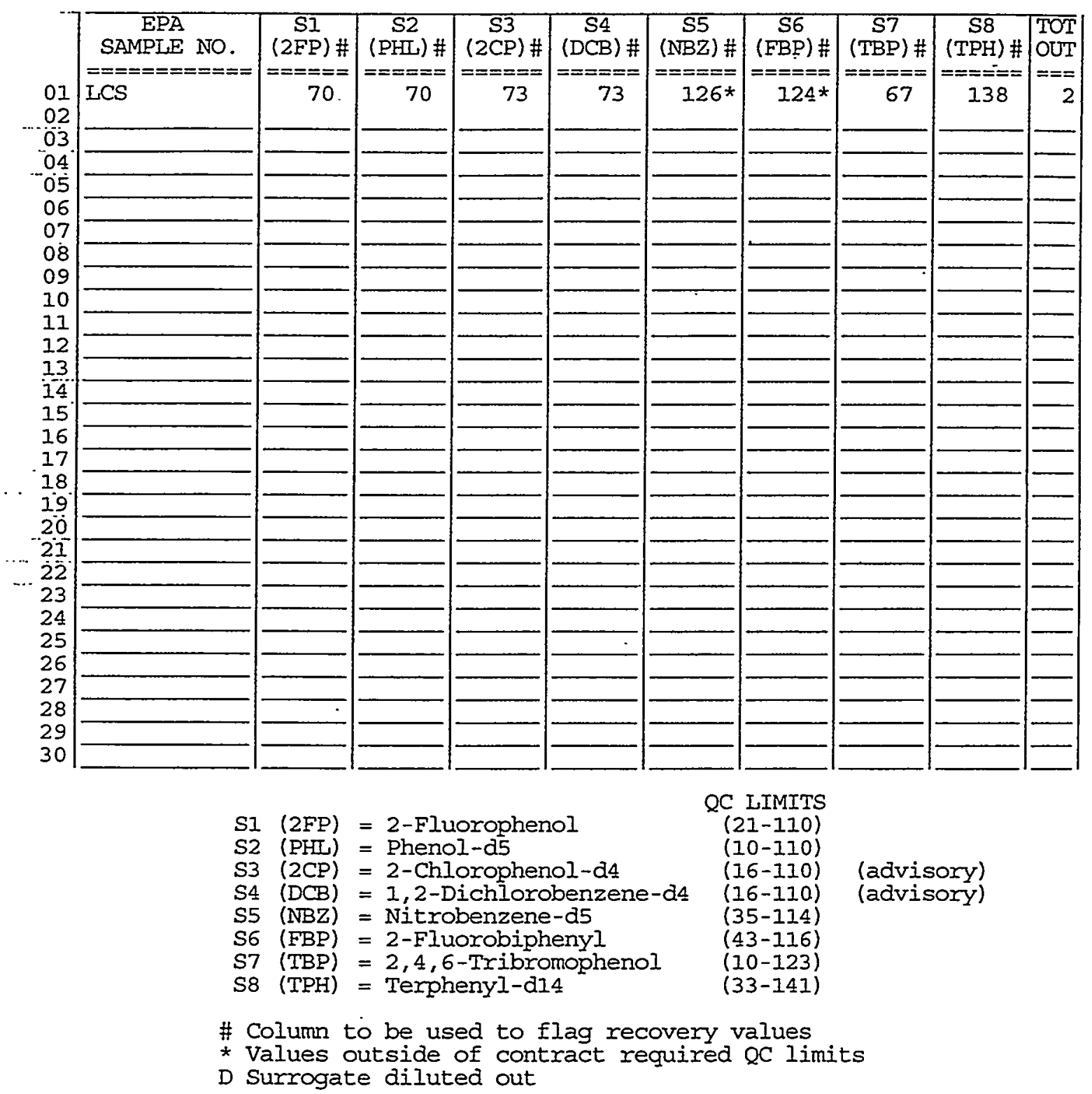


Lab Name: PNNL

Contract: BNFL

Lab Code: PNNL

Case No.:

SAS NO.:

SDG No.: 990205

Matrix Spike - EPA Sample No.: AW-101

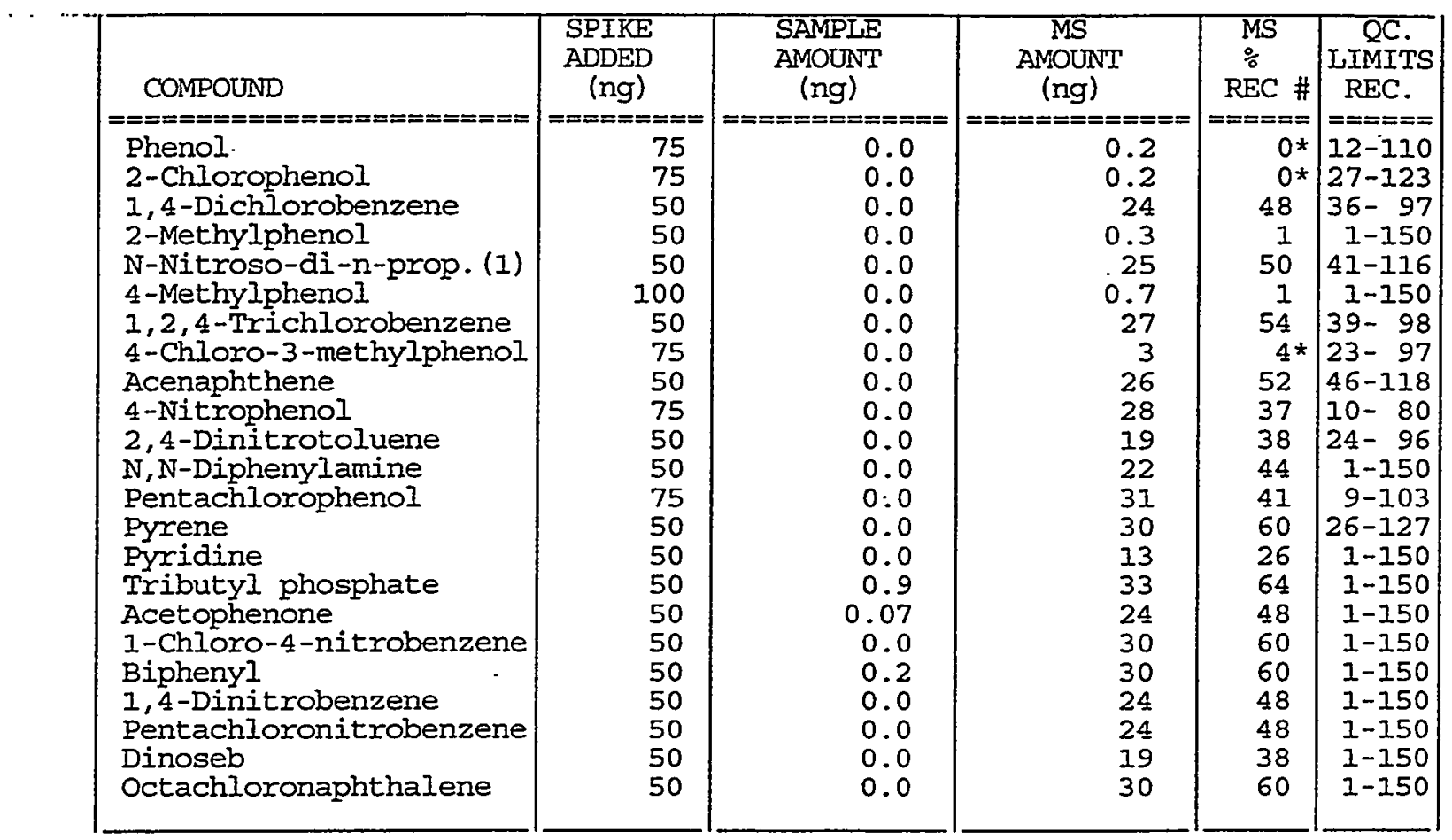

(1) N-Nitroso-di-n-propylamine

\# Column to be used to flag recovery and RPD values with an asterisk

* Values outside of QC Iimits

COMMENTS: 
Lab Name: PNNL

Lab Code: PNNL

Case No.:

Matrix Spike - EPA Sample No.: AW-101
Contract: BNFL

SAS NO.:

SDG No. : 990205

\begin{tabular}{|c|c|c|c|c|c|c|}
\hline & $\begin{array}{l}\text { SPIKE } \\
\text { ADDED }\end{array}$ & $\begin{array}{c}\text { MSD } \\
\text { AMOUNT }\end{array}$ & $\begin{array}{c}\text { MSD } \\
\%\end{array}$ & $\%$ & \multicolumn{2}{|c|}{ QC IIMITS } \\
\hline COMPOUND & (ng) & (ng) & REC \# & RPD \# & RPD & REC. \\
\hline 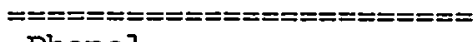 & & & & & & \\
\hline Phenol & 75 & 0.1 & $0 *$ & 999* & 42 & $12-110$ \\
\hline 2-Chlorophenol & 75 & $0 . \overline{1}$ & $0 *$ & $999 *$ & 40 & $27-123$ \\
\hline 1,4-Dichlorobenzene & 50 & & 42 & 13 & 28 & $36-97$ \\
\hline 2-Methylphenol & 50 & 0.008 & $0 *$ & $200 *$ & 50 & $1-150$ \\
\hline N-Nitroso-di-n-prop. (I) & 50 & 23 & 46 & 8 & 38 & $41-116$ \\
\hline 4-Methylphenol & 100 & 0.2 & $0 *$ & $200 *$ & 50 & $1-150$ \\
\hline $1,2,4$-Trichlorobenzene & 50 & 25 & 50 & 8 & 28 & $39-98$ \\
\hline 4 -Chloro-3-methylphenol & 75 & 0.5 & $1 *$ & $120 *$ & 42 & $23-97$ \\
\hline Acenaphthene & 50 & 24 & 48 & 8 & 31 & $46-118$ \\
\hline 4-Nitrophenol & 75 & 18 & 24 & 43 & 50 & $10-80$ \\
\hline 2,4-Dinitrotoluene & 50 & 18 & 36 & 5 & 38 & $24-96$ \\
\hline N,N-Diphenylamine & 50 & 20 & 40 & 10 & 50 & $1-150$ \\
\hline Pentachlorophenol & 75 & 27 & 36 & 13 & 50 & $9-103$ \\
\hline Pyrene & 50 & 28 & 56 & 7 & 31 & $26-127$ \\
\hline Pyridine & 50 & 7 & 14 & $60 *$ & 50 & $1-150$ \\
\hline Tributyl phosphate & 50 & 31 & 60 & 6 & 50 & $1-150$ \\
\hline Acetophenone & 50 & 22 & 44 & 9 & 50 & $I-150$ \\
\hline 1-Chloro-4-nitrobenzene & 50 & 29 & 58 & 3 & 50 & $1-150$ \\
\hline Biphenyl & 50 & 28 & 56 & 7 & 50 & $1-150$ \\
\hline 1,4-Dinitrobenzene & 50 & 26 & 52 & 8 & 50 & $1-150$ \\
\hline Pentachloronitrobenzene & 50 & 25 & 50 & 4 & 50 & $1-150$ \\
\hline Dinoseb & 50 & 6 & 12 & $104 *$ & 50 & $1-150$ \\
\hline Octachloronaphthalene & 50 & 29 & 58 & 3 & 50 & $1-150$ \\
\hline
\end{tabular}

(I) N-Nitroso-di-n-propylamine

\# Column to be used to flag recovery and RPD values with an asterisk

* Values outside of QC limits

RPD: 7 out of 23 outside limits

Spike Recovery: 8 out of 46 outside limits

COMMENTS: 
Lab Name: PNNL

Contract: BNFL

Lab Code: PNNL Case No.:

SAS NO.:

SDG NO.: 990205

Matrix Spike - EPA Sample No.: AW-101-. Ievel: (low/med) IOW

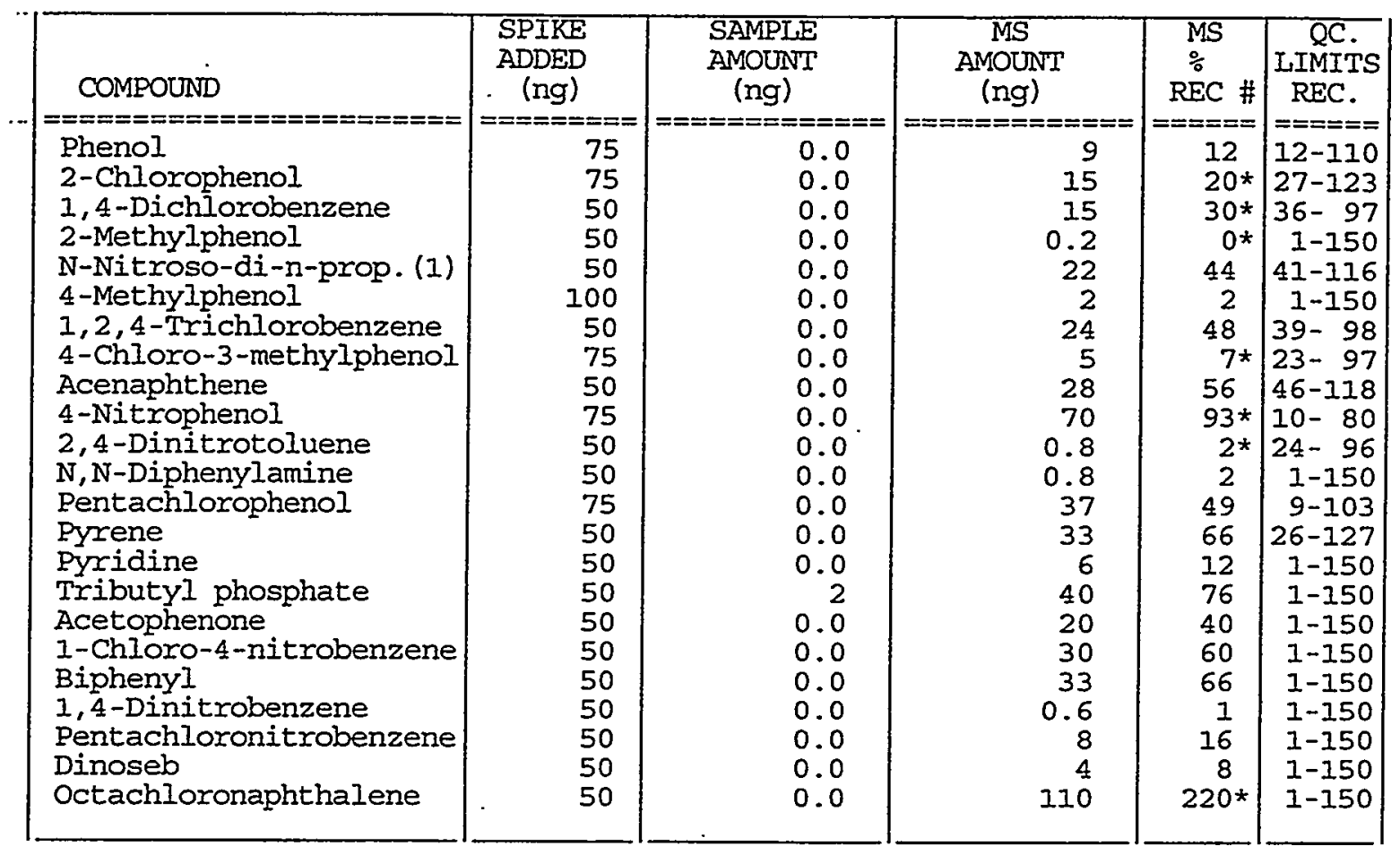

(1) N-Nitroso-di-n-propylamine

\# Column to be used to flag recovery and RPD values with an asterisk

* Values outside of QC limits

COMMENTS : 
3D

SOLID SEMIVOLATILE MATRIX SPIKE/MATRIX SPIKE DUPLICATE RECOVERY

Lab Name: PNNL

Contract: BNFL

Lab Code: PNNL

Case No. :

SAS NO.:

SDG No.: 990205

Matrix Spike - EPA Sample No.: AW-101- Level: (low/med) LOW

\begin{tabular}{|c|c|c|c|c|c|c|}
\hline & $\begin{array}{l}\text { SPIKE } \\
\text { ADDED }\end{array}$ & $\begin{array}{c}\text { MSD } \\
\text { AMOUNT }\end{array}$ & $\begin{array}{c}\text { MSD } \\
\vdots \\
\vdots\end{array}$ & $\div$ & \multicolumn{2}{|c|}{ QC IIMITS } \\
\hline COMPOUND & & (ng) & REC \# & RPD \# & RPD & REC. \\
\hline 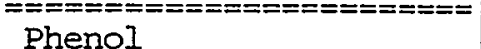 & $\begin{array}{c}== \pm===== \\
75\end{array}$ & $=======-===$ & $==-==-1$ & $=====$ & $=====$ & $\begin{array}{l}===== \\
12-110\end{array}$ \\
\hline 2-Chlorophenol & 75 & 22 & 29 & 37 & 40 & $\begin{array}{l}12-110 \\
27-123\end{array}$ \\
\hline 1,4-Dichlorobenzene & 50 & 16 & $32 *$ & 6 & 28 & $36-97$ \\
\hline 2-Methylphenol & 50 & 0.4 & 1 & $200 *$ & 50 & $1-150$ \\
\hline N-Nitroso-di-n-prop. (1) & 50 & 19 & $38^{*}$ & 15 & 38 & $41-116$ \\
\hline 4-Methylphenol & 100 & 2 & 2 & 0 & 50 & $1-150$ \\
\hline 1,2,4-Trichlorobenzene & 50 & 24 & 48 & 0 & 28 & $39-98$ \\
\hline 4-Chloro-3-methylphenol & 75 & 7 & $9 *$ & 25 & 42 & $23-97$ \\
\hline Acenaphthene & 50 & 25 & 50 & 11 & 31 & $46-118$ \\
\hline 4-Nitrophenol & 75 & 73 & $97 *$ & 4 & 50 & $10-80$ \\
\hline 2,4-Dinitrotoluene & 50 & 0.8 & $2 *$ & 0 & 38 & $24-96$ \\
\hline N,N-Diphenylamine & 50 & 0.3 & $i$ & $67 *$ & 50 & $1-150$ \\
\hline Pentach̆lorophenol & 75 & 32 & 43 & 13 & 50 & $9-103$ \\
\hline Pyrene & 50 & 30 & 60 & 10 & 31 & $26-127$ \\
\hline Pyridine & 50 & 0.5 & 1 & $169 *$ & 50 & $1-150$ \\
\hline Tributyl phosphate & 50 & 36 & $6 \overline{8}$ & 11 & 50 & $1-150$ \\
\hline Acetophenone & 50 & 18 & 36 & 10 & 50 & $1-150$ \\
\hline 1-Chloro-4-nitrobenzene & 50 & 29 & 58 & 3 & 50 & $1-150$ \\
\hline Biphenyl & 50 & 30 & 60 & 10 & 50 & $1-150$ \\
\hline 1,4-Dinitrobenzene & 50 & 1 & 2 & $67 \star$ & 50 & $1-150$ \\
\hline Pentachloronitrobenzene & 50 & 8 & 16 & 0 & 50 & $1-150$ \\
\hline Dinoseb & 50 & 4 & 8 & 0 & 50 & $1-150$ \\
\hline Octachloronaphthalene & 50 & 300 & $600 *$ & $93 *$ & 50 & $1-150$ \\
\hline
\end{tabular}

(1) N-Nitroso-di-n-propylamine

\# Column to be used to flag recovery and RPD values with an asterisk

* Values outside of $Q C$ limits

RPD: 5 out of 23 outside iimits

Spike Recovery: 13 out of 46 outside limits

COMMENTS: 
Lab Name: PNNL

Lab Code: PNNL

Case No.:

Lab File ID: 99020610

Instrument ID: HP1

Matrix: (soil/water) SOLID

Level: (low/med) LOW
Contract : BNFL

SAS NO. :

SDG No.: 990205

Lab Sample ID: SBLKO4

Date Extracted:

Date Analyzed: 02/06/99

Time Analyzed: 2328

THIS METHOD BLANK APPLIES TO THE FOLIOWING SAMPIES, MS and MSD:

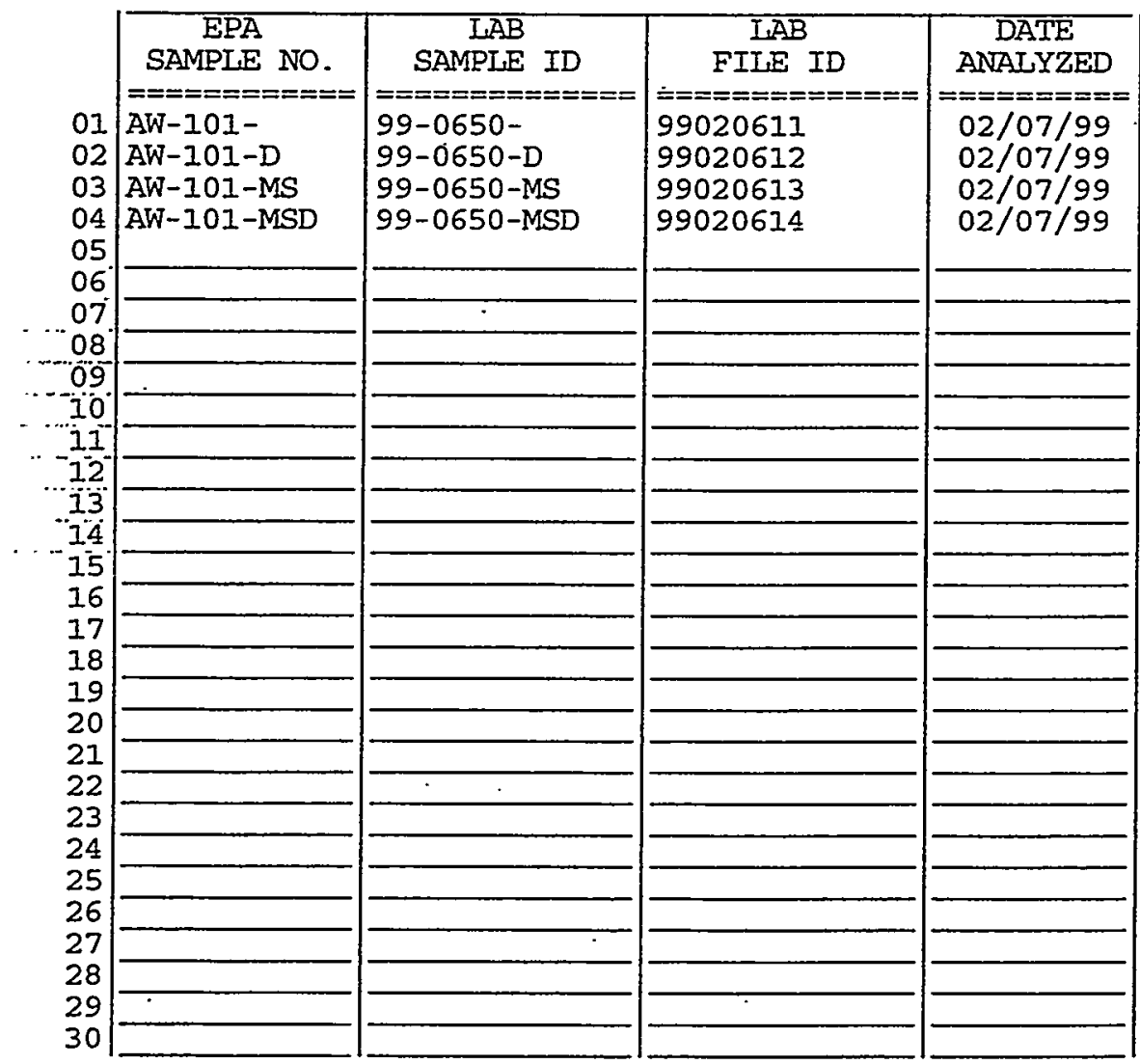

COMMENTS :

page 1 of 1 
Lab Name: PNNL

Lab Code: PNNL.

Caise No. :

Lab File ID: 99020605

Instrument ID: HP1

Matrix: (soil/water) SUPERNATANT

Level : (low/med) LOW
Contract: BNFL

SAS NO. :

Lab Sample ID: SBLK03

Date Extracted:

Date Analyzed: 02/06/99

Time Analyzed: 1903

THIS METHOD BLANK APPIIES TO THE FOLIOWING SAMPLES, MS and MSD:

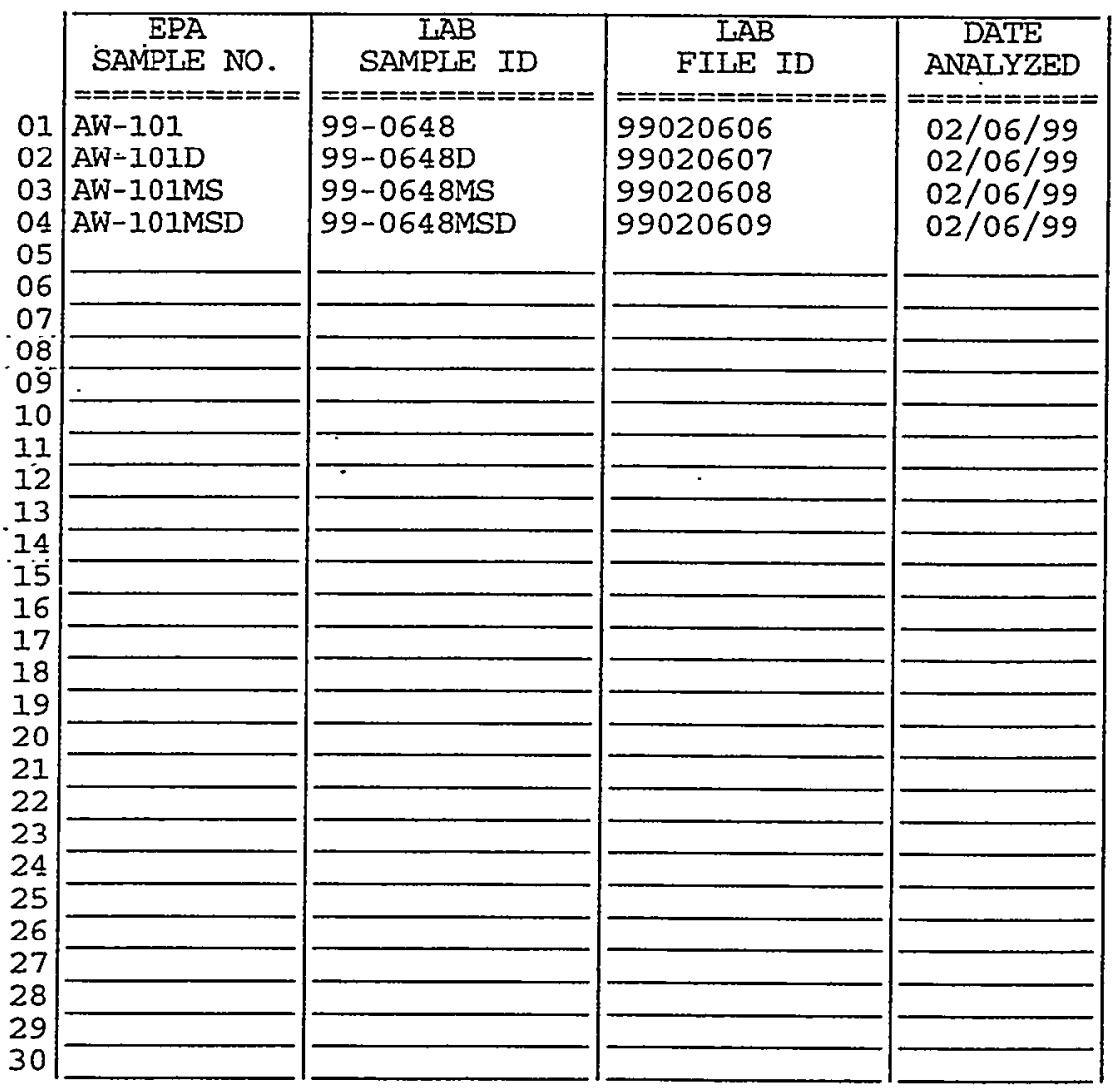

COMMENTS : 
SEMIVOLATILE ORGANIC INSTRUMENT PERFORMANCE CHECK DECAFLUOROTRIPHENYL PHOSPHINE (DFTPP)

Lab Name: PNNL

Lab Code: PNNL Case No.:

Lab File ID: 99020601

Instrument ID: HPI contract: BNFL

SAS NO. :

SDG NO.: 990205

DFTPP Injection Date: 02/06/99

DFTPP Injection Time: 1532

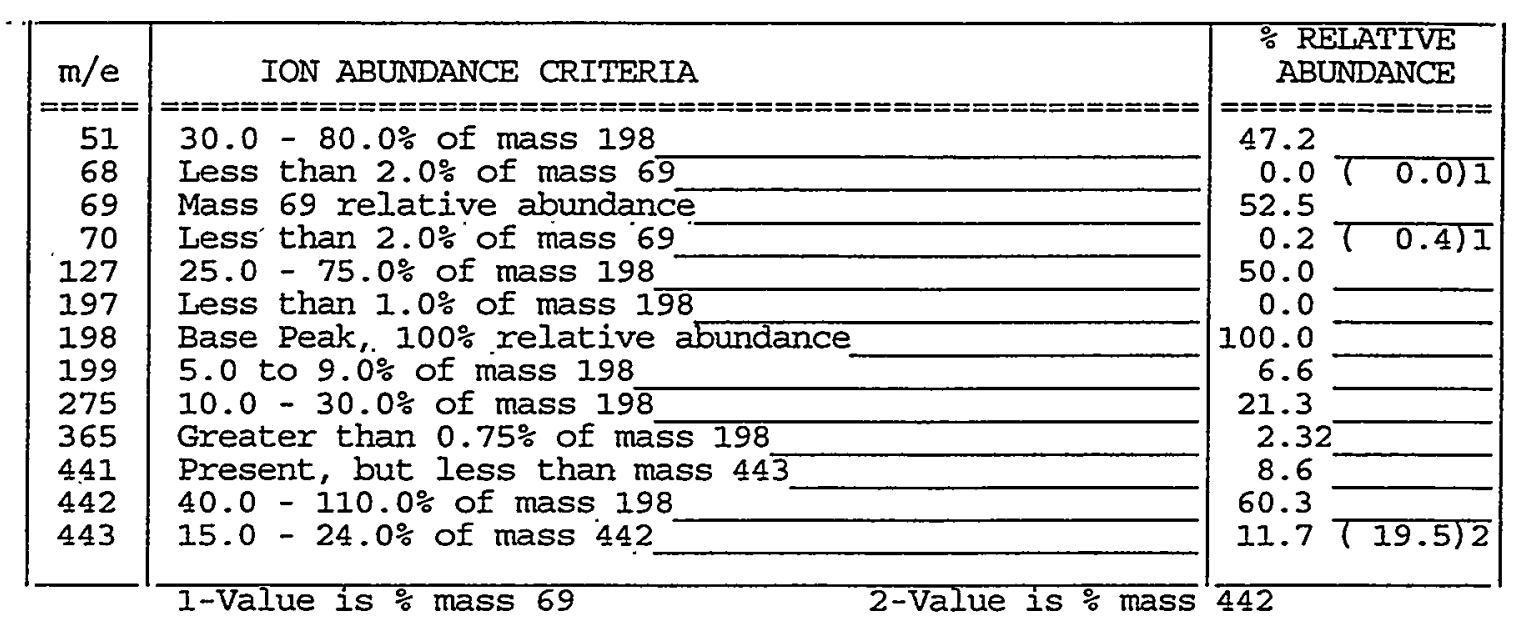

THIS CHECK APPLIES TO THE FOLLOWING SAMPLES, MS, MSD, BLANKS, AND STANDARDS:

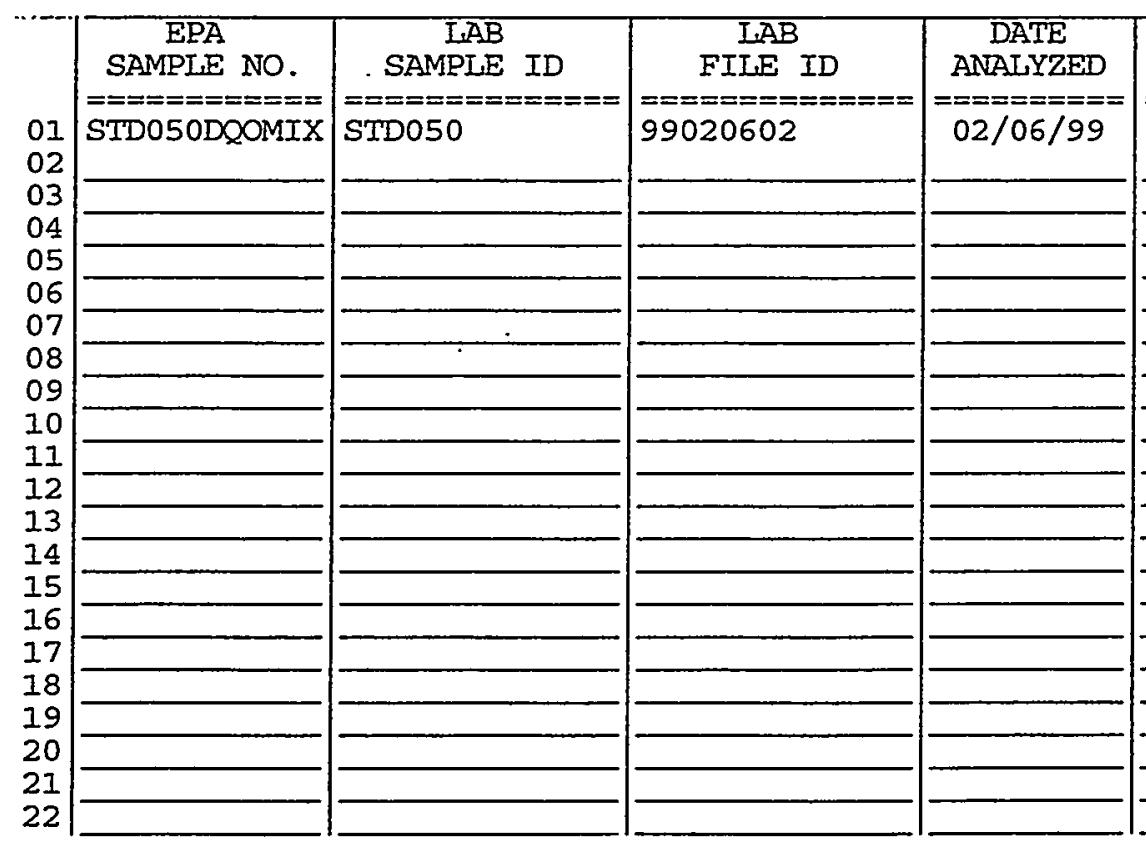

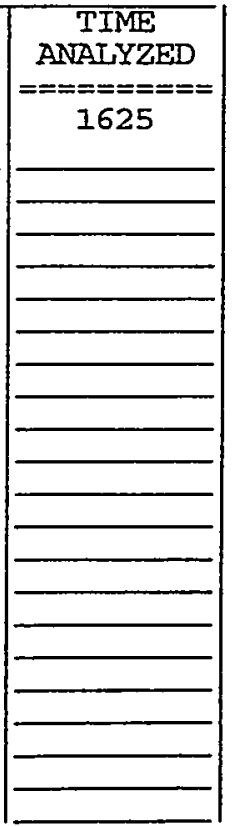

page 1 of 1 
Lab Name: PNNL

Lab Code: PNNL

Case No.:

Lab File ID: 99020603

Instrument ID: HP1
Contract : BNFL

SAS NO.:

SDG NO.: 990205

DFTPP Injection Date: 02/06/99

DFTPP Injection Time: 1718

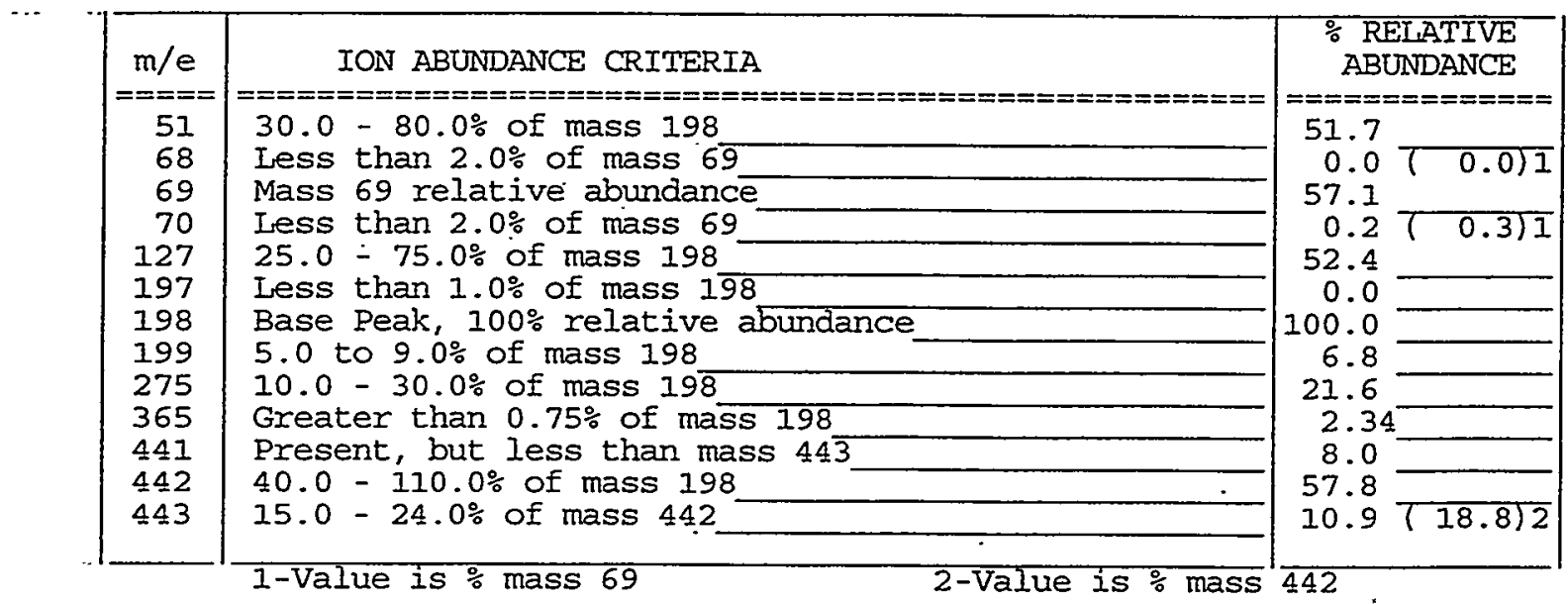

THIS CHECK APPLIES TO THE FOLIOWING SAMPLES, MS, MSD, BLANKS, AND STANDARDS:

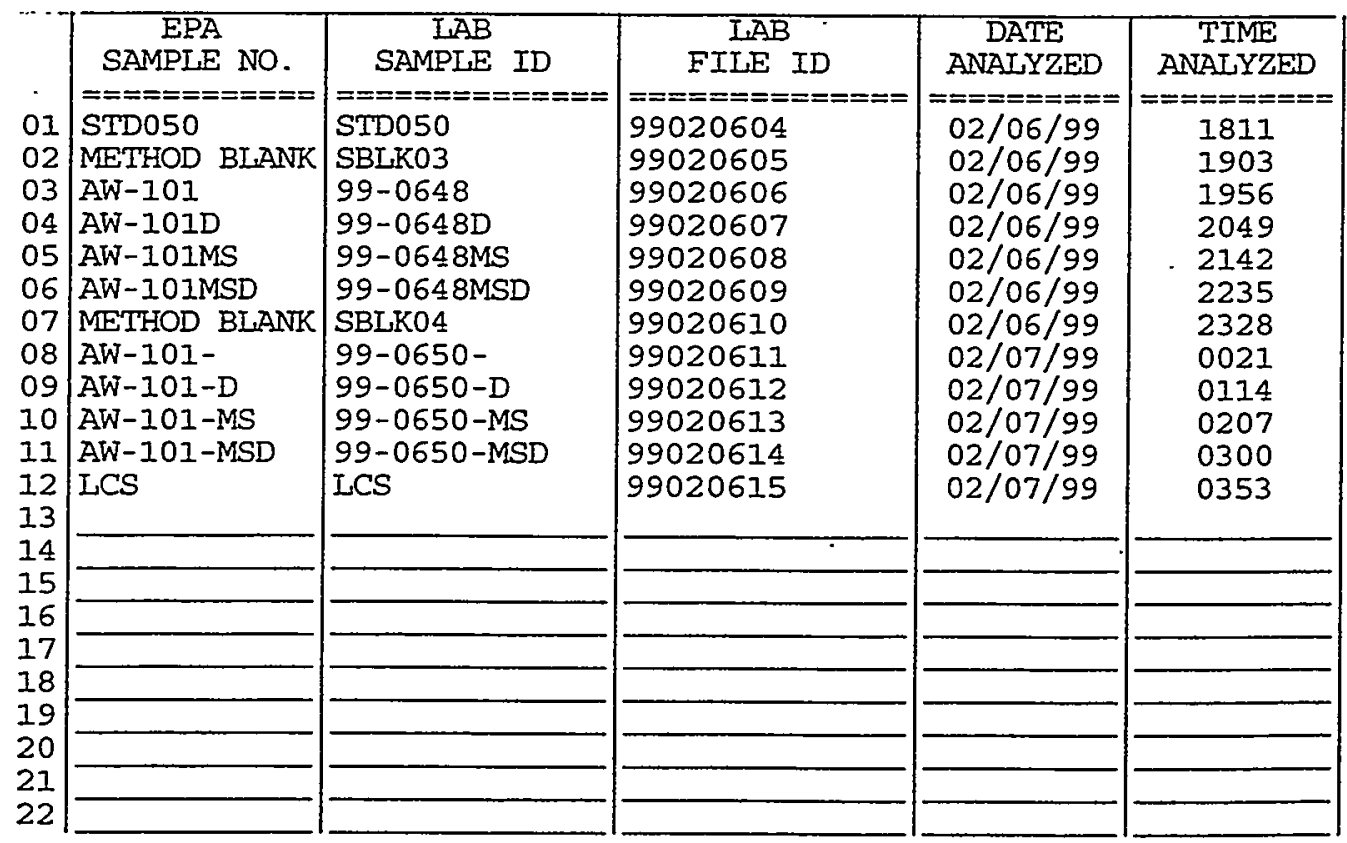

page 1 of 1 
Lab Name: PNNL

Lab Code: PNNL

Instrument ID: HP1

Iab File ID: 99020602
Contract: BNFL

SAS NO.:
Case No.:
Calibration Date: 02/06/99

Init. Calib. Date(s) : 02/04/99

Init. Calib. Times: 1820
SDG NO.: 990205
Time: 1625

\section{2}

$02 / 05 / 99$

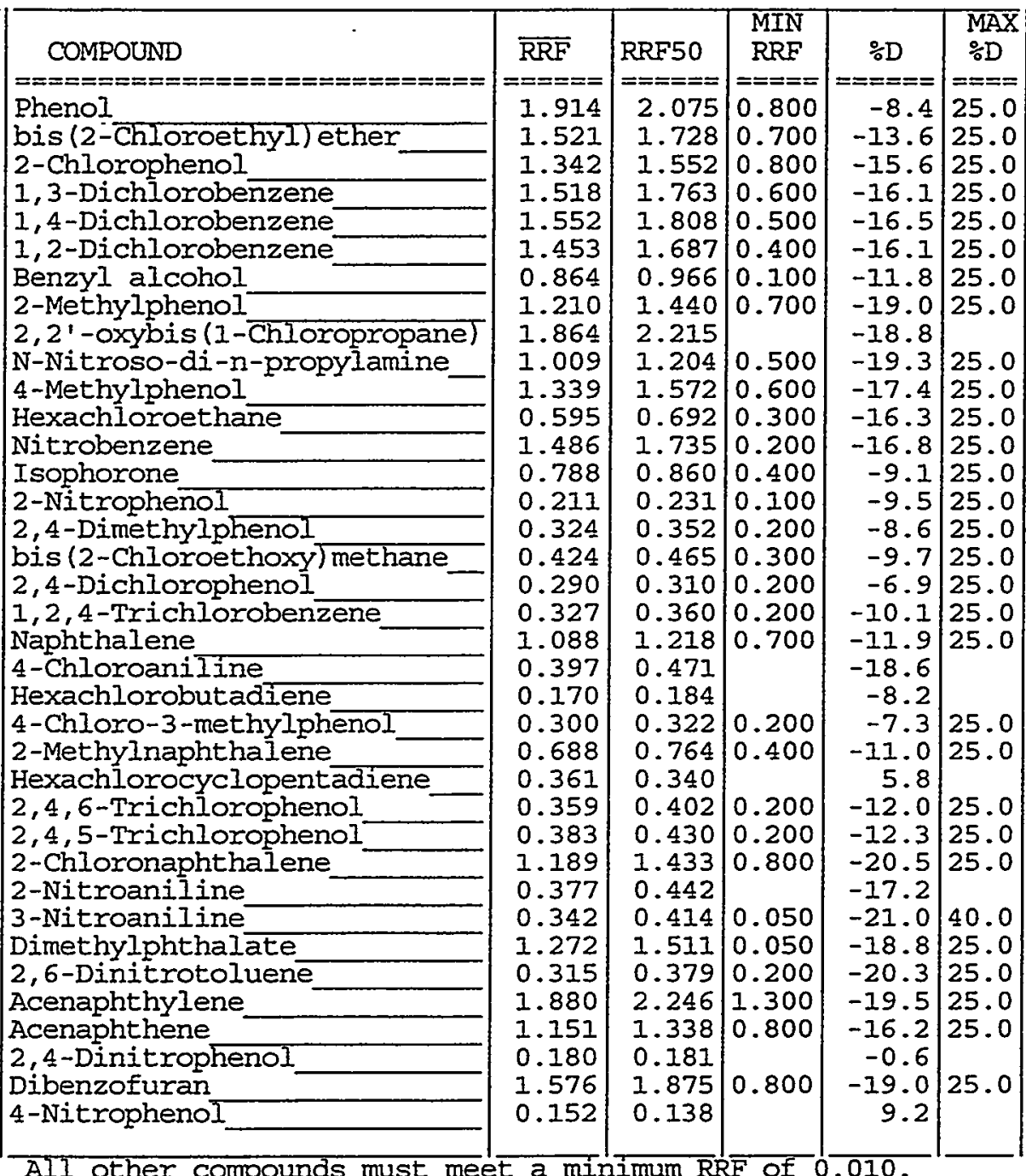

All other compounds must meet a minimum RRF of 0.010 .

\footnotetext{
a
} 
$7 C$

SEMIVOLATILE CONTINUING CALIBRATION CHECK

Lab Name: PNNL

Contract: BNFL

Lab Code: PNNL . Case No.: SAS No.:

SDG No.: 990205

Instrument ID: HPI

Calibration Date: 02/06/99 Time: 1625

Lab File ID: 99020602

Init. Calib. Date(s) : 02/04/99 02/05/99

Init. Calib. Times: 18201532

\begin{tabular}{|c|c|c|c|c|c|}
\hline 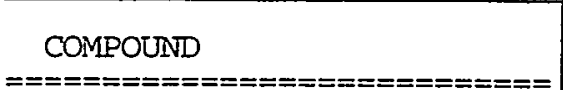 & $\begin{array}{l}\overline{R R F} \\
====\end{array}$ & $\begin{array}{l}\text { RRF50 } \\
=====\end{array}$ & $\begin{array}{l}\text { MIN } \\
\text { RRE } \\
=====\end{array}$ & $====$ & $\begin{array}{l}\text { MAX } \\
\because D \\
====\end{array}$ \\
\hline $\begin{array}{l}2,4 \text {-Dinitrotoluene } \\
\text { Diethylphthalate }\end{array}$ & $\begin{array}{l}0.398 \\
1.263\end{array}$ & $\begin{array}{l}0.486 \\
1.512\end{array}$ & 0.200 & $\begin{array}{l}-22.1 \\
-19.7\end{array}$ & 25.0 \\
\hline Fluorene & 1.253 & 1.484 & 0.900 & $-18 \cdot 4$ & 25.0 \\
\hline $\begin{array}{l}\text { 4-Chlorophenyl-phenylether } \\
\text { 4-Nitroaniline } \\
\text { 4,6-Dinitro-2-methylphenol } \\
\text { N, N-Diphenylamine }\end{array}$ & $\begin{array}{l}0.554 \\
0.337 \\
0.236 \\
0.722\end{array}$ & $\begin{array}{l}0.655 \\
0.401 \\
0.282 \\
0.767\end{array}$ & 0.400 & $\begin{array}{r}-18 \cdot 2 \\
-19.0 \\
-19.5 \\
-6.2\end{array}$ & 25.0 \\
\hline Heptachlor & 0.179 & 0.192 & 0.050 & -7.3 & 40.0 \\
\hline alpha-BHC & 0.155 & 0.165 & 0.050 & -6.4 & 40.0 \\
\hline 4-Bromophenyl-phenylether & 0.211 & 0.226 & 0.100 & -7.1 & 25.0 \\
\hline Hexachlorobenzene & 0.236 & 0.251 & 0.100 & -6.4 & 25.0 \\
\hline beta-BHC & 0.117 & 0.134 & 0.050 & -14.5 & 40.0 \\
\hline $\begin{array}{l}\text { Pentachlorophenol } \\
\text { delta-BHC }\end{array}$ & $\begin{array}{l}0.161 \\
0.125\end{array}$ & $\begin{array}{l}0.149 \\
0.132\end{array}$ & $\begin{array}{l}0.050 \\
0.050\end{array}$ & $\begin{array}{r}7.4 \\
-5.6\end{array}$ & $\begin{array}{l}25.0 \\
40.0\end{array}$ \\
\hline Phenanthrene & 1.139 & 1.216 & 0.700 & -6.8 & 25.0 \\
\hline Anthracene & 1.139 & 1.216 & 0.700 & -6.8 & 25.0 \\
\hline $\begin{array}{l}\text { gamma-BHC (Iindane) } \\
\text { Carbazole }\end{array}$ & $\begin{array}{l}0.112 \\
1.061\end{array}$ & $\begin{array}{l}0.116 \\
1.146\end{array}$ & 0.050 & $\begin{array}{l}-3.6 \\
-8.0\end{array}$ & 40.0 \\
\hline Di-n-butylphthalate & 1.376 & 1.512 & & -9.9 & \\
\hline Aldrin & 0.170 & 0.182 & 0.050 & -7.0 & 40.0 \\
\hline Heptachlor Epoxide & 0.057 & 0.062 & & -8.8 & \\
\hline Fluoranthene & 1.063 & 1.160 & 0.600 & -9.1 & 25.0 \\
\hline Pyrene & 1.485 & 1.626 & 0.600 & -9.5 & 25.0 \\
\hline EndosuIfan I & 0.052 & 0.056 & & -7.7 & \\
\hline $4,4,-D D E$ & 0.267 & 0.290 & 0.050 & -8.6 & 40.0 \\
\hline Dieldrin & 0.277 & 0.297 & 0.050 & -7.2 & 40.0 \\
\hline Endrin & 0.080 & 0.090 & 0.050 & -12.5 & 40.0 \\
\hline EndosuIfan II & 0.045 & 0.047 & & -4.4 & \\
\hline $\begin{array}{l}\text { 4, 4' -DDD } \\
\text { Butylbenzylphthalate }\end{array}$ & $\begin{array}{l}0.461 \\
0.767\end{array}$ & $\begin{array}{l}0.485 \\
0.837\end{array}$ & 0.050 & $\begin{array}{l}-5 \cdot 2 \\
-8 \cdot 3\end{array}$ & 40.0 \\
\hline $\begin{array}{l}\text { Butyloenzy phinalate } \\
\text { Endosulfan Sulfate }\end{array}$ & 0.076 & 0.081 & & -6.6 & \\
\hline $4,4^{\prime}-\mathrm{DDT}$ & 0.414 & 0.431 & 0.050 & -4.1 & 40.0 \\
\hline Endrin Ketone & 0.124 & 0.127 & 0.050 & -2.4 & 40.0 \\
\hline Benzo (a) anthracene & 1.370 & 1.484 & 0.800 & -8.3 & 25.0 \\
\hline 3, $3^{\prime}$-Dichlorobenzidine & 0.388 & 0.465 & & $-19 \cdot 8$ & \\
\hline Chrysene & 1.153 & 1.253 & 0.700 & -8.7 & 25.0 \\
\hline
\end{tabular}

page 2 of 4 
Lab Name: PNNL

Lab Code: PNNL
Contract: BNFI

Case No.:
SAS NO.:

SDG NO.: 990205
Instrument ID: HPI

Lab File ID: 99020602
Calibration Date: 02/06/99

Time: 1625

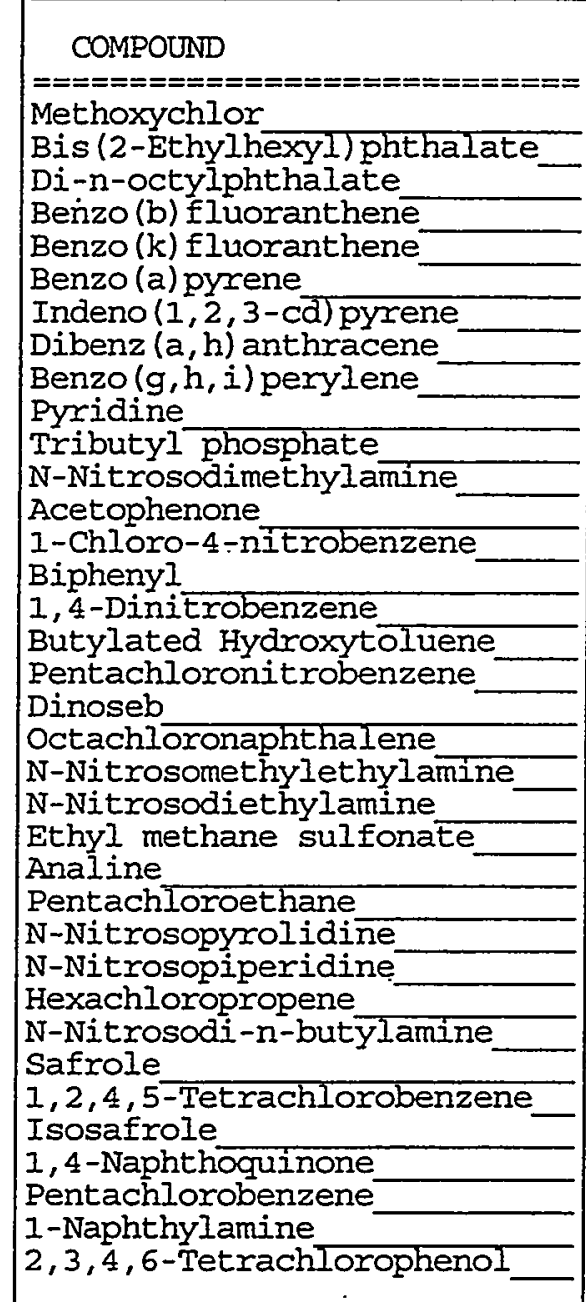

\begin{tabular}{|l|} 
RRF \\
$======$ \\
0.937 \\
1.049 \\
1.793 \\
1.275 \\
1.195 \\
1.034 \\
1.081 \\
0.907 \\
0.923 \\
0.985 \\
1.147 \\
0.603 \\
1.848 \\
0.123 \\
1.372 \\
0.218 \\
0.894 \\
0.090 \\
0.215 \\
0.099 \\
0.467 \\
0.712 \\
1.118 \\
2.065 \\
0.516 \\
0.703 \\
0.192 \\
0.200 \\
0.254 \\
0.266 \\
0.541 \\
0.547 \\
0.321 \\
0.439 \\
1.006 \\
0.278 \\
\end{tabular}

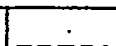
RRF50 1.026 1.127 1.975 1.367 1.367

1.360

1.149

0.962

0.947

1.112

1.176

0.660

2.158

0.123

0.123
1.419

0.207

0.889

0.095

0.229

0.095

0.508

0.822

1.292

2.399

0.589

0.834

0.834
0.211

0.212

0.282

0.296

0.636

0.605

0.375

0.516

1.254

0.308
: 02/04/99

1820
02/05/99

1532

All other compounds must meet a minimum RRF of 0.010 . 
Lab Name: PNNL

Lab Code: PNNL

Instrument ID: HPI

Lab File ID: 99020602 contract: BNFL

Case No. :
SAS NO.:

SDG No.: 990205

Calibration Date: 02/06/99 Time: 1625

Init. Calib. Date(s) : 02/04/99 02/05/99

Init. Calib. Times: $1820 \quad 1532$

\begin{tabular}{|c|c|c|c|c|c|}
\hline COMPOUND & $\overline{\mathrm{PDF}}$ & & MIN & & $\begin{array}{l}\mathrm{MAX} \\
\end{array}$ \\
\hline 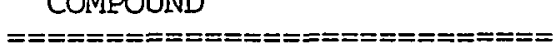 & $=== \pm==$ & $======$ & $====$ & $======$ & \\
\hline 2-Naphthylamine & 0.680 & 0.882 & & -29.7 & \\
\hline 5-Nitro-o-toluidine & 0.367 & 0.450 & & -22.6 & \\
\hline Azeobenzene & 1.028 & 1.062 & & -3.3 & \\
\hline $1,3,5$-Trinitrobenzene & 0.212 & 0.216 & & -1.9 & \\
\hline Diallate (cis) & 0.133 & 0.143 & & -7.5 & \\
\hline Phenacetin & 0.419 & 0.438 & & -4.5 & \\
\hline Diallate (Erans) & 0.312 & 0.335 & & -7.4 & \\
\hline 4-Aminobiphenyl & 0.580 & 0.720 & & -24.1 & \\
\hline Pronamine & 0.353 & 0.381 & & -7.9 & \\
\hline Isodrin & 0.128 & 0.137 & & -7.0 & \\
\hline Chlordane (alpha) & 0.103 & 0.112 & & -8.7 & \\
\hline Benzidine & 0.488 & 0.533 & & -9.2 & \\
\hline p-Dimethylaminoazobenzene & 0.483 & 0.525 & & -8.7 & \\
\hline Chlorobenzilate & 0.509 & 0.544 & & -6.9 & \\
\hline 3,3'-Dimethylbenzidine & 0.368 & 0.474 & & -28.8 & \\
\hline 2-Acetylaminofluorene & 0.274 & 0.302 & & -10.2 & \\
\hline 3-Methylcholanthrene & 0.551 & 0.593 & & -7.6 & \\
\hline 2-Methylpyridine & 1.046 & 1.161 & & -11.0 & - \\
\hline Kepone & 0.076 & 0.082 & & -7.9 & \\
\hline Chlordane (gamma) & 0.102 & 0.118 & & -15.7 & \\
\hline Methyl methane sulfonate & 0.411 & 0.470 & & -14.4 & \\
\hline Hexachlorophene & 0.032 & & & 100.0 & \\
\hline 1,3-Dinitrobenzene & 0.244 & 0.290 & & -18.8 & \\
\hline 2,6-Dichlorophenol & 0.296 & 0.314 & & -6.1 & \\
\hline 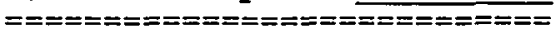 & $==== \pm=$ & $======$ & $=====$ & $======$ & $====$ \\
\hline 2-Fluorophenol & 2.703 & 3.049 & 0.600 & -12.8 & 25.0 \\
\hline Phenol-d5 & 3.439 & 3.917 & 0.800 & -13.9 & 25.0 \\
\hline 2-Chlorophenol-d4 & & & 0.800 & & 25.0 \\
\hline 1,2-Dichlorobenzene-d4 & & & 0.400 & & 25.0 \\
\hline Nitrobenzene-d5 & 1.446 & 1.683 & 0.200 & -16.4 & 25.0 \\
\hline 2-Fluorobiphenyl & 1.292 & 1.506 & 0.700 & -16.6 & 25.0 \\
\hline $2,4,6$-Tribromophenol & 0.223 & 0.232 & & -4.0 & \\
\hline Terphenyl-d14 & 0.950 & 1.029 & 0.500 & -8.3 & 25.0 \\
\hline
\end{tabular}


Iab Name: PNNL

Lab code: PNNL

Instrument ID: HP1

Lab File ID: 99020604
Contract: BNFL

Case No.:

SAS NO.:

SDG No.: 990205

Calibration Date: 02/06/99 Time: 1811

Init. Calib. Date(s) : 02/04/99 02/05/99

Init. Calib. Times: 1820

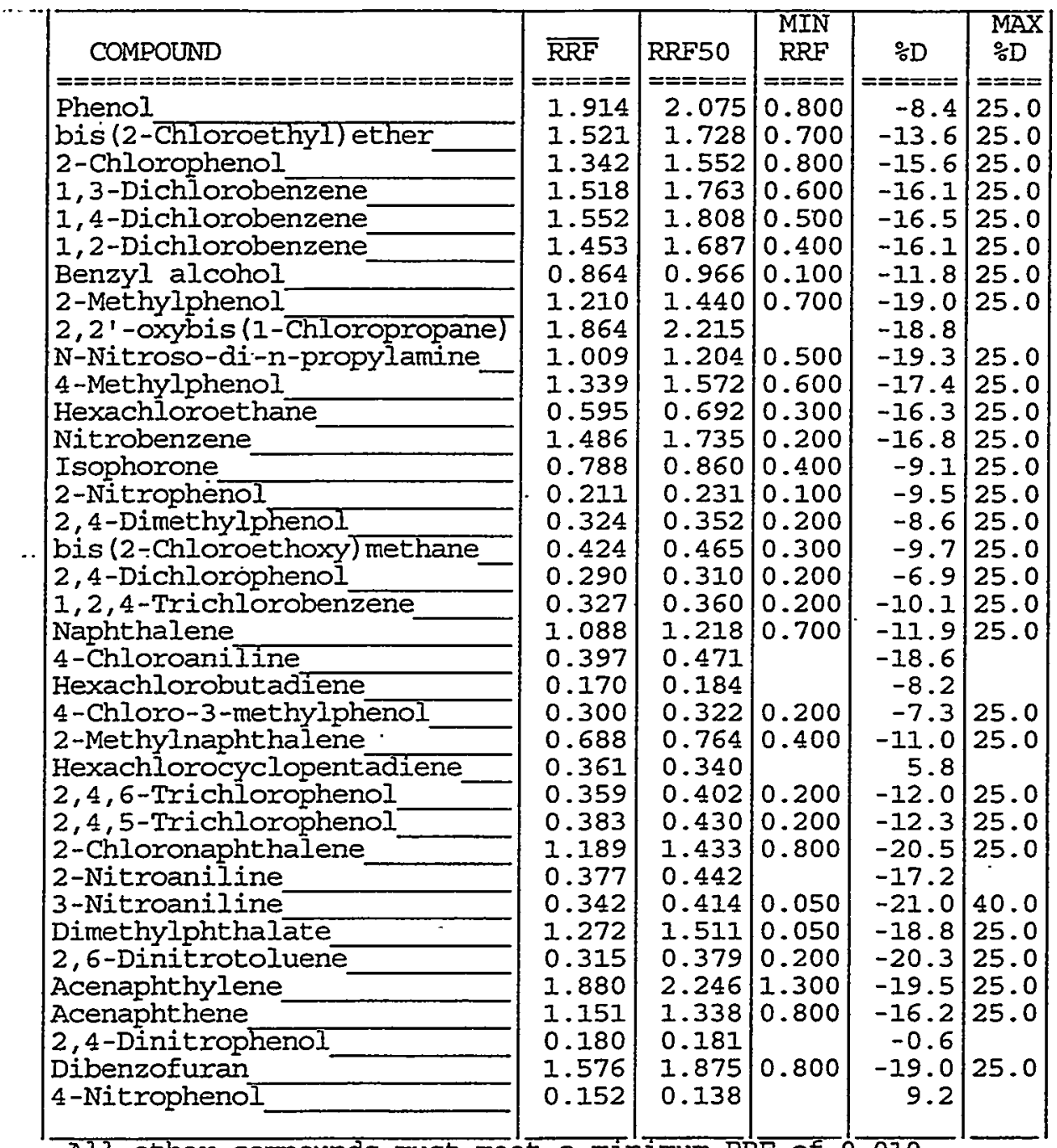

All other compounds must meet a minimum RRF of 0.010 . 
Lab Name: PNNL'

Lab code: PNNL
Case No.:
Contract: BNEL

SAS NO.:
SDG No.: 990205

Lab File ID: 99020604

Calibration Date: 02/06/99 Time: 1811

Init. Calib. Date(s) : 02/04/99 02/05/99

Init. Calib. Times: $1820 \quad 1532^{\circ}$

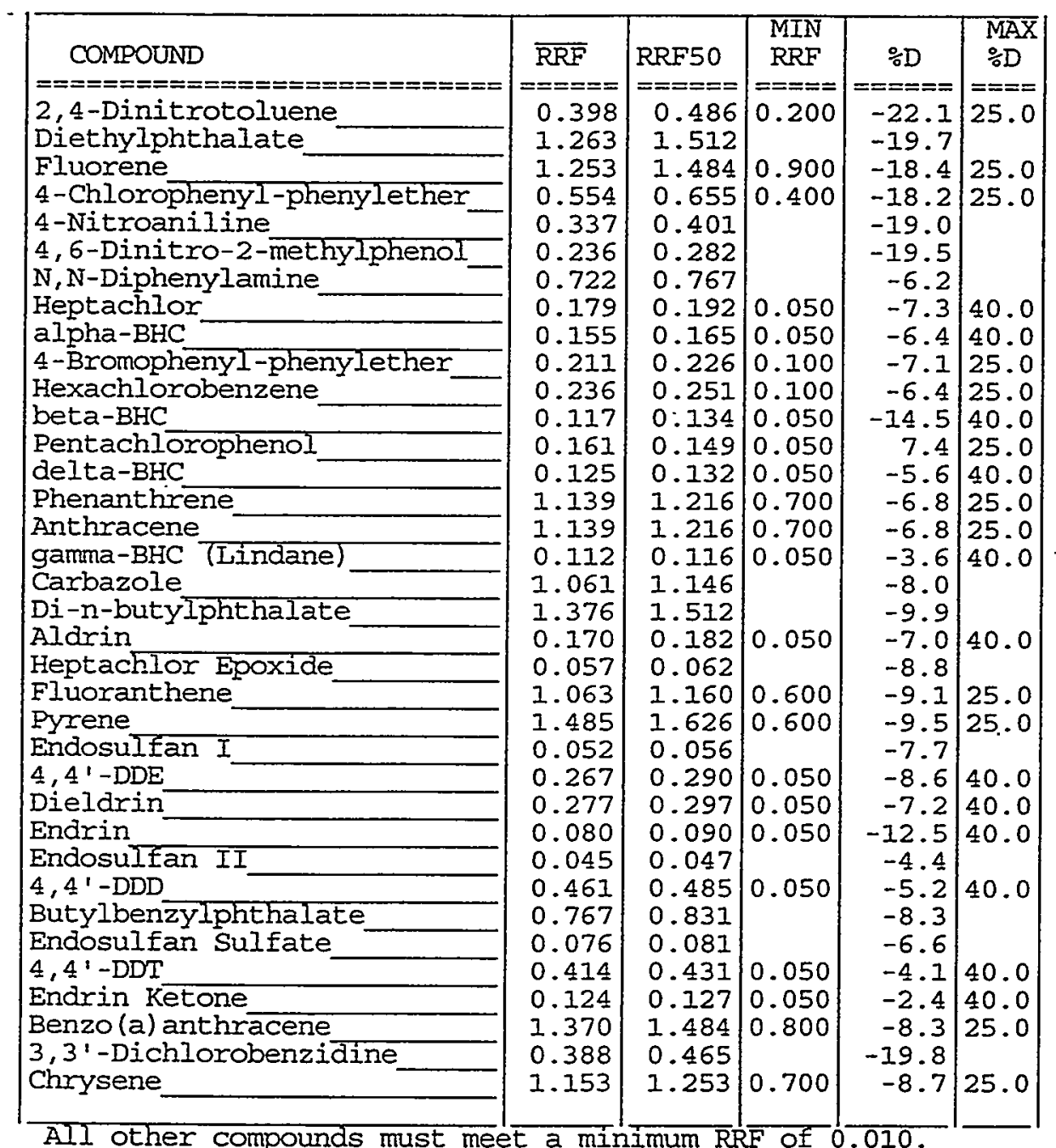

page 2 of 4 
Lab Name: PNNL

Lab Code: PNNL

Instrument ID: HPI

Iab File ID: 99020604
Contract: BNFL

SAS NO. :

SDG No.: 990205
Case No.:
Time: 1811

Init. Calib. Date(s): 02/04/99 02/05/99

Init. Calib. Times: 1820

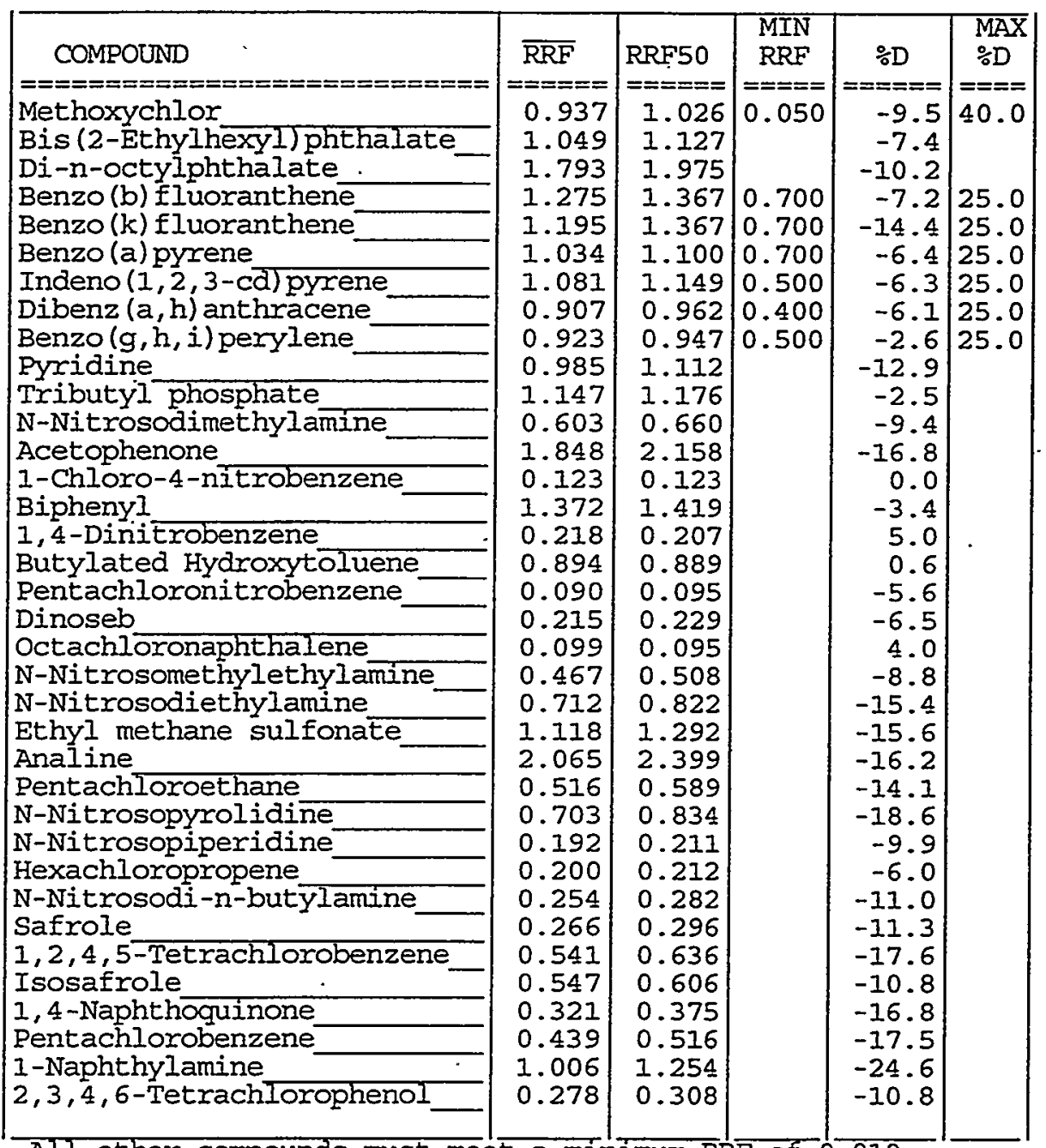

All other compounds must meet a minimum $R R \bar{F}$ of 0.010 . 
$7 C$

SEMIVOLATILE CONTINUING CALIBRATION CHECK

Lab Name: PNNL

Contract: BNFL

Lab code: PNNL,

Case No. :

SAS NO. :

SDG No.: 990205

Instrument ID: HPI

Calibration Date: 02/06/99

Time: 1811

Lab File ID: 99020604

Init. Calib. Date(s): 02/04/99 02/05/99

Init. Calib. Times: 1820

1532

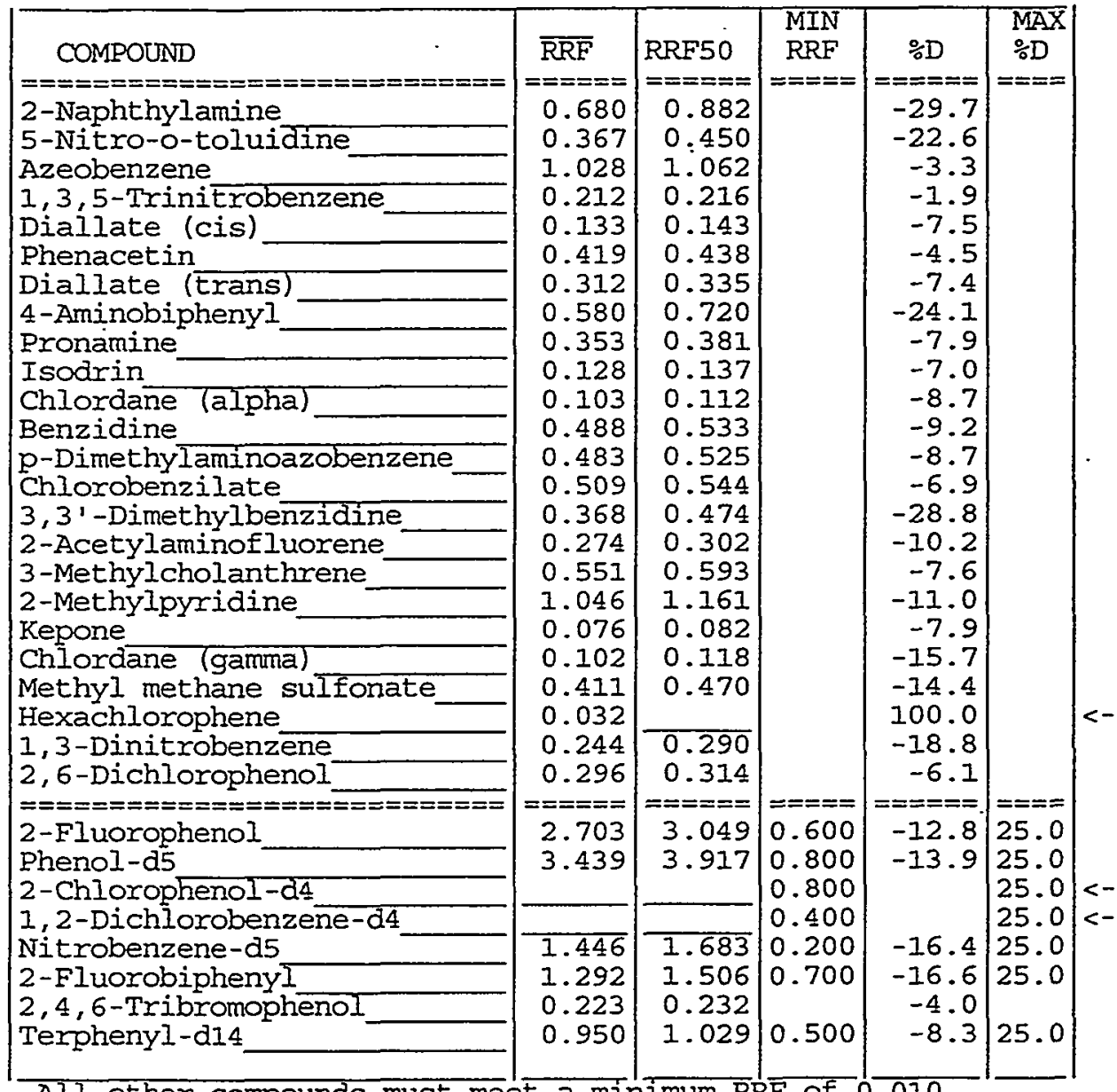

All other compounds must meet a minimum RRF of 0.010 . 
Lab Name: PNNL

Lab Code: PNNL

Case No. :

Lab File ID (Standard): 99020604

Instrument ID: HPI
Contract: BNFL

SAS NO.:

SDG No.: 990205

Date Analyzed: 02/06/99

Time Analyzed: 1811

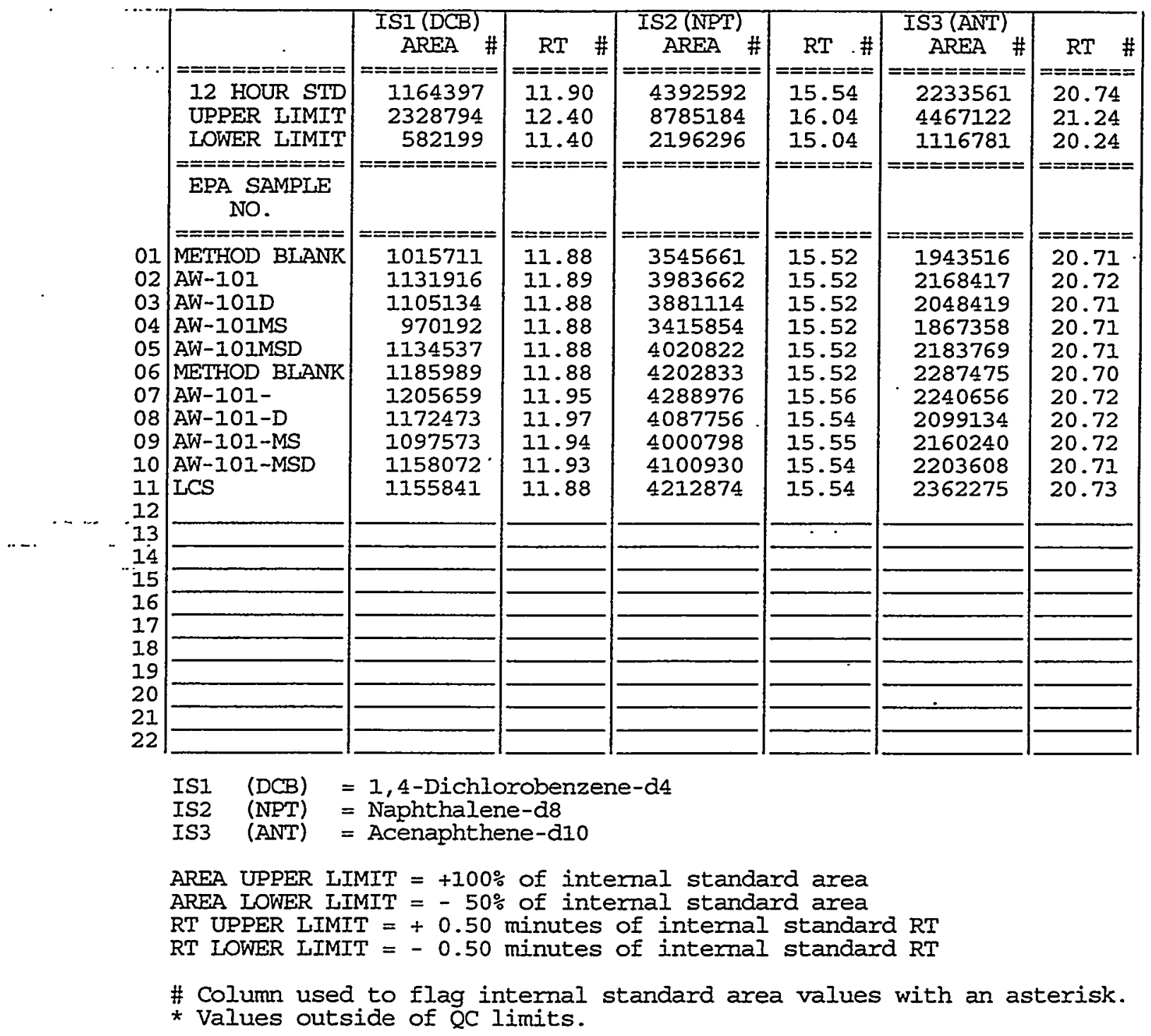

page 1 of 1
OLM03.0 
Iab Name: PNNL

Lab Code: PNNL Case No.:

Lab File ID (Standard): 99020604

Instrument ID: HPI
Contract: BNFL

SAS NO.:

SDG. No. : . 990205

Date Analyzed: 02/06/99

Time Analyzed: 1811

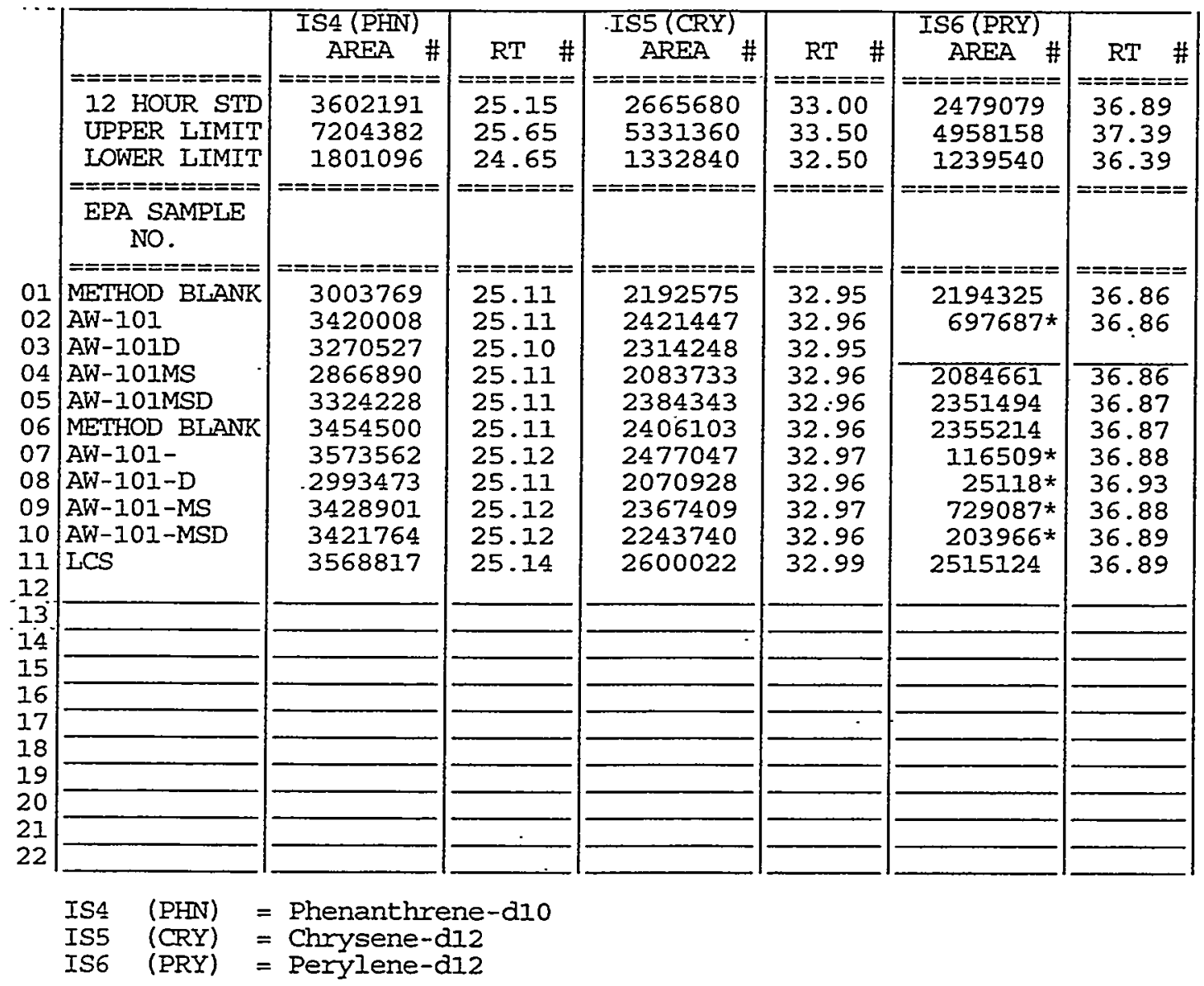

AREA UPPER IIMIT $=+100 \%$ of internal standard area AREA LOWER IIMIT $=-50 \%$ of internal standard area RT UPPER IIMIT $=+0.50$ minutes of internal standard RT RT IOWER LIMIT $=-0.50$ minutes of internal standard RT

\# Column used to flag internal standard area values with an asterisk. * Values outside of QC limits. 
Iab Name: PNNL

Lab Code: PNNL Case No.:

Lab File ID (Standard) : 99020604

Instrument ID: HPI
Contract: BNFL

SAS NO.:

SDG NO.: 990205

Date Analyzed: 02/06/99

Time Analyzed: 1811

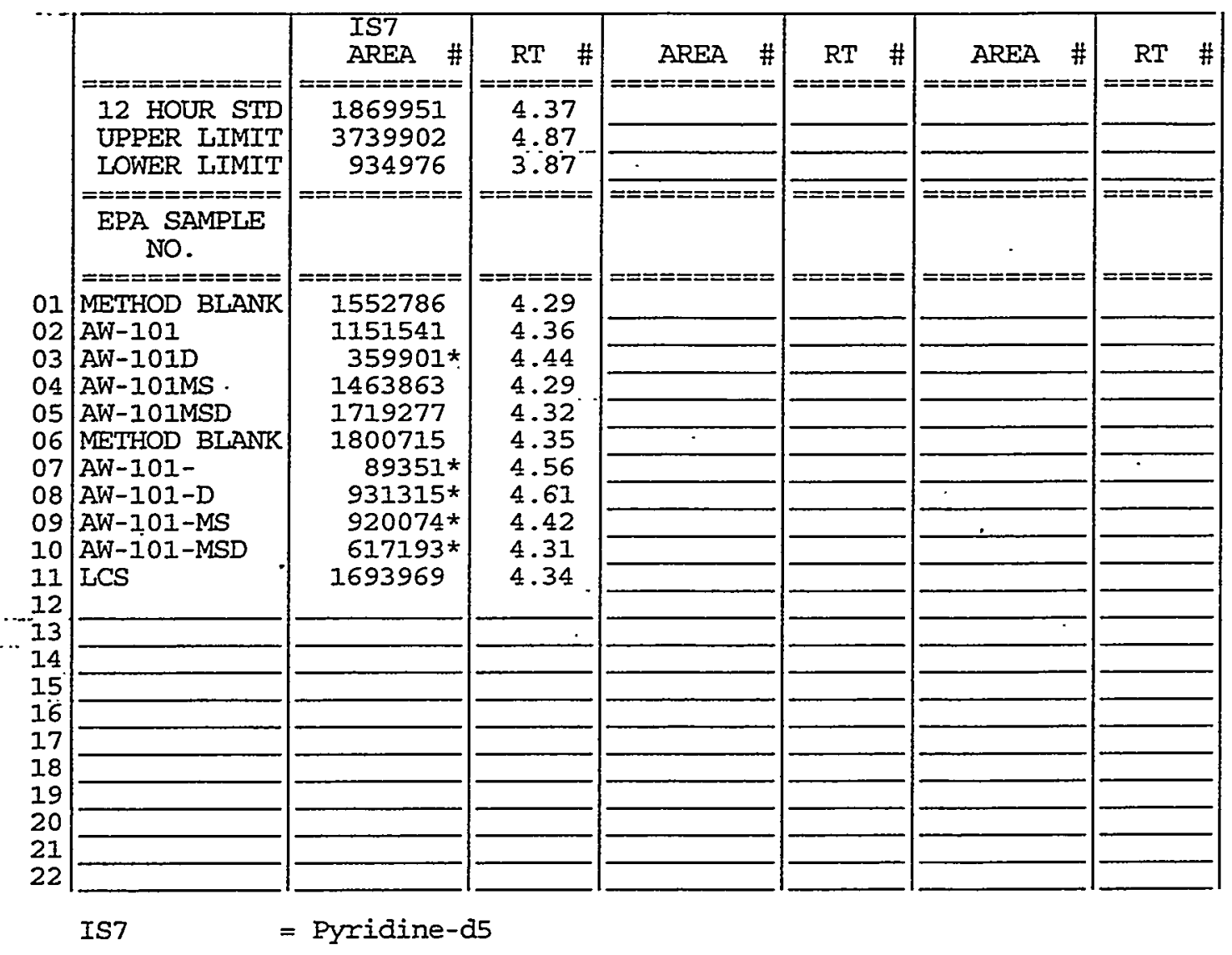

AREA UPPER LIMIT $=+100 \%$ of internal standard area AREA LOWER LIMIT $=-50 \%$ of internal standard area RT UPPER LIMIT $=+0.50$ minutes of internal standard RT RT LOWER LIMIT $=-0.50$ minutes of internal standard RT

\# Column used to flag internal standard area values with an asterisk. * Values outside of QC limits. 
Lab Name: PNNL

Irab Code: PNNL

Case No.:

Matrix: (soil/water) SUPERNATAN

Sample wt/vol: (g/mL) MI

Level: (low/med)

LOW

$\%$ Moisture: decanted: $(Y / N)$

Concentrated Extract Volume: 2000 (uI)

Injection Volume: (UI)

Contract: BNEL

SAS NO.:

Lab Sample ID: 99-0649

Lab File ID: 99010506

Date Received:

Date Extracted:

Date Analyzed: 02/05/99

Dilution Factor: 1.0

SDG NO.: 990205

GPC Cleanup: $\quad(Y / N) N \quad \mathrm{NH}: 11.0$

AN-107

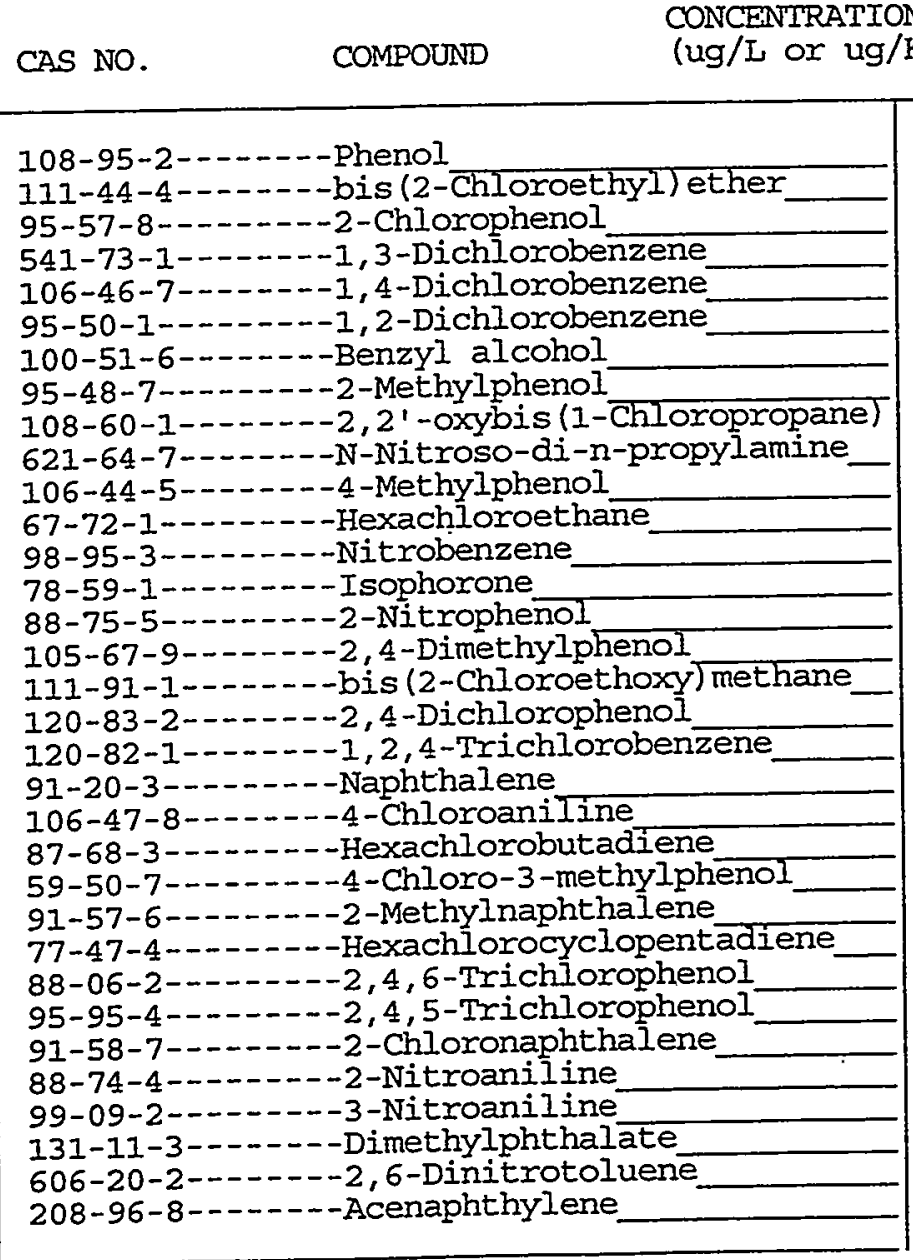


Lab Name: PNNL

Lab code: PNNL

Case No.:

Matrix: (soil/water) SUPERNATAN

Sample wt/vol:

$(\mathrm{g} / \mathrm{mL}) \mathrm{ML}$

Ievel: (low/med) LOW

\% Moisture:

decanted: $(Y / N)$

Concentrated.Extract Volume: 2000 (uI)

Injection Volume: (uI)

Contract: BNFL

SAS NO.:

Iab Sample ID: 99-0649

Lab File ID: 99010506

Date Received:

Date Extracted:

Date Analyzed: 02/05/99

Dilution Factor: 1.0

GPC Cleanup: $(\mathrm{Y} / \mathrm{N}) \mathrm{N} \quad \mathrm{pH}: 11.0$

\section{CONCENTRATION UNITS:}

\begin{tabular}{|c|c|c|}
\hline 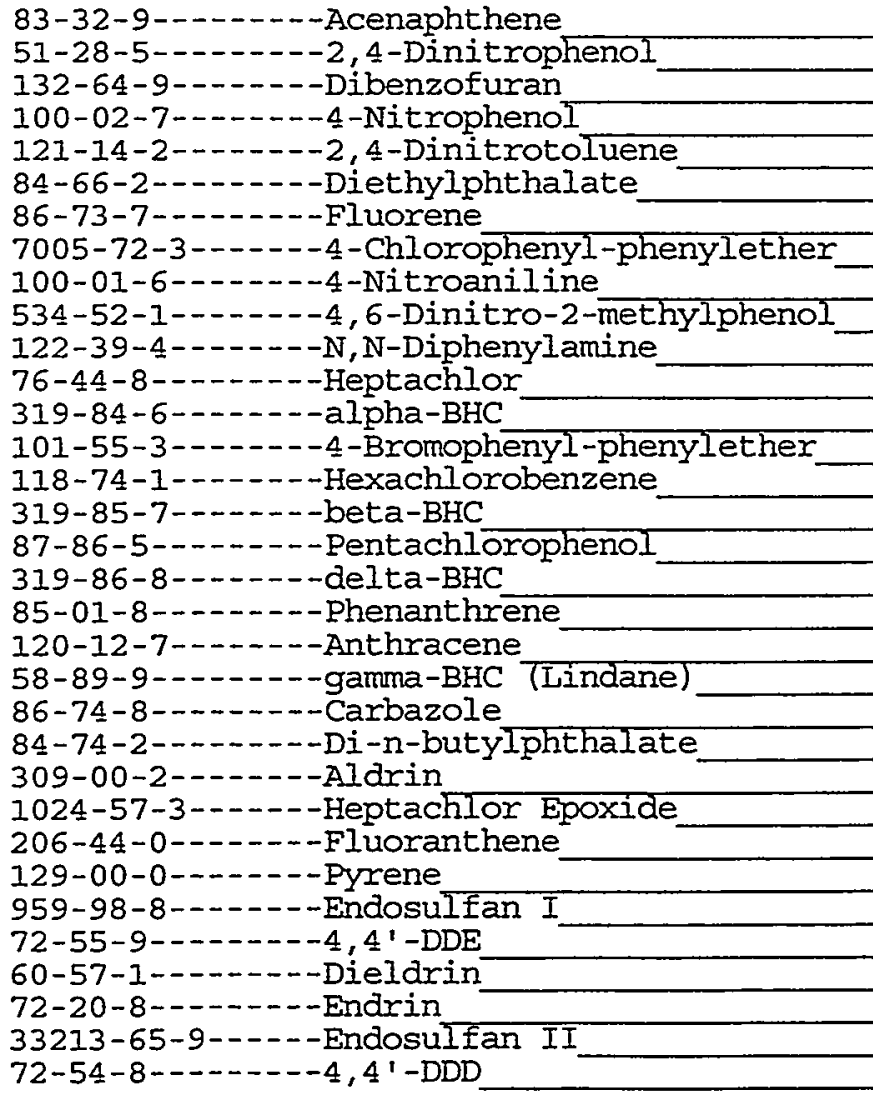 & $\begin{array}{l}310 \\
310 \\
310 \\
310 \\
310 \\
160 \\
310 \\
310 \\
310 \\
310 \\
310 \\
310 \\
310 \\
310 \\
310 \\
310 \\
310 \\
310 \\
310 \\
310 \\
310 \\
310 \\
310 \\
310 \\
310 \\
310 \\
310 \\
310 \\
310 \\
310 \\
310 \\
310 \\
310\end{array}$ & $\begin{array}{l}U \\
U \\
U \\
U \\
U \\
J \\
U \\
U \\
U \\
U \\
U \\
U \\
U \\
U \\
U \\
U \\
U \\
U \\
U \\
U \\
U \\
U \\
U \\
U \\
U \\
U \\
U \\
U \\
U \\
U \\
U \\
U \\
U\end{array}$ \\
\hline
\end{tabular}

FORM I SV-2 
Lab Name: PNNL

Lab Code: PNNL

Case No.:

Matrix: (soil/water) SUPERNATAN

Sample wt/vol:

$(\mathrm{g} / \mathrm{mL}) \mathrm{ML}$

Level: (low/med)

LOW

$\div$ Moisture:

decanted: $(Y / N)$

Concentrated Extract Volume: 2000 (uL)

Injection Volume:

(UI)

Contract: $\mathrm{BNFL}$

SAS NO.:

Iab Sample ID: 99-0649

Lab File ID: . 99010506

Date Received:

Date Extracted:

Date Analyzed: 02/05/99

Dilution Factor: 1.0

GPC Cleanup: $(\mathrm{Y} / \mathrm{N}) \mathrm{N} \quad \mathrm{pH}: 11.0$

CONCENTRATION UNITS:

CAS NO.

COMPOUND

(ug/L or $u g / \mathrm{kg}$ ) UG/L

Q

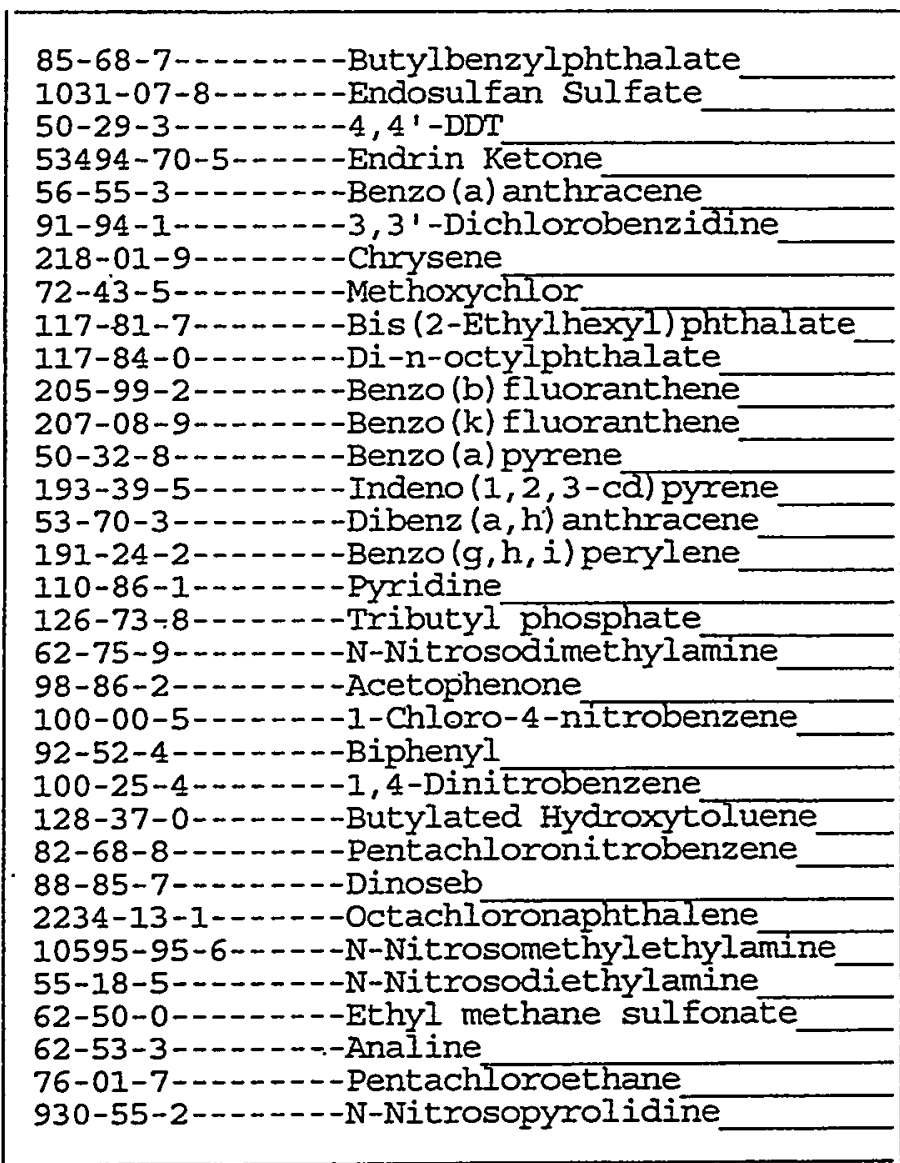

FORM I SV-3

OLM03.0 
Lab Name: PNNL

Irab Code: PNNL

Case No.:
Contract: BNFL

SAS NO.:
AN-107

SDG No.: 990205
Matrix: (soil/water) SUPERNATAN

Sample wt/vol:

$(\mathrm{g} / \mathrm{mL}) \mathrm{ML}$

Level: (low/med) IOW

\% Moisture:

decanted: $(Y / N)$

2000 (uL)

(UI)

Injection Volume:
Lab Sample ID: 99-0649

Lab File ID: 99010506

Date Received:

Date Extracted:

Date Analyzed: 02/05/99

Dilution Factor: 1.0

$(\mathrm{Y} / \mathrm{N}) \mathrm{N}$

$\mathrm{pH}: 11.0$

CAS NO.

COMPOUND

CONCENTRATION UNITS

(ug/L or ug/Kg) UG/L

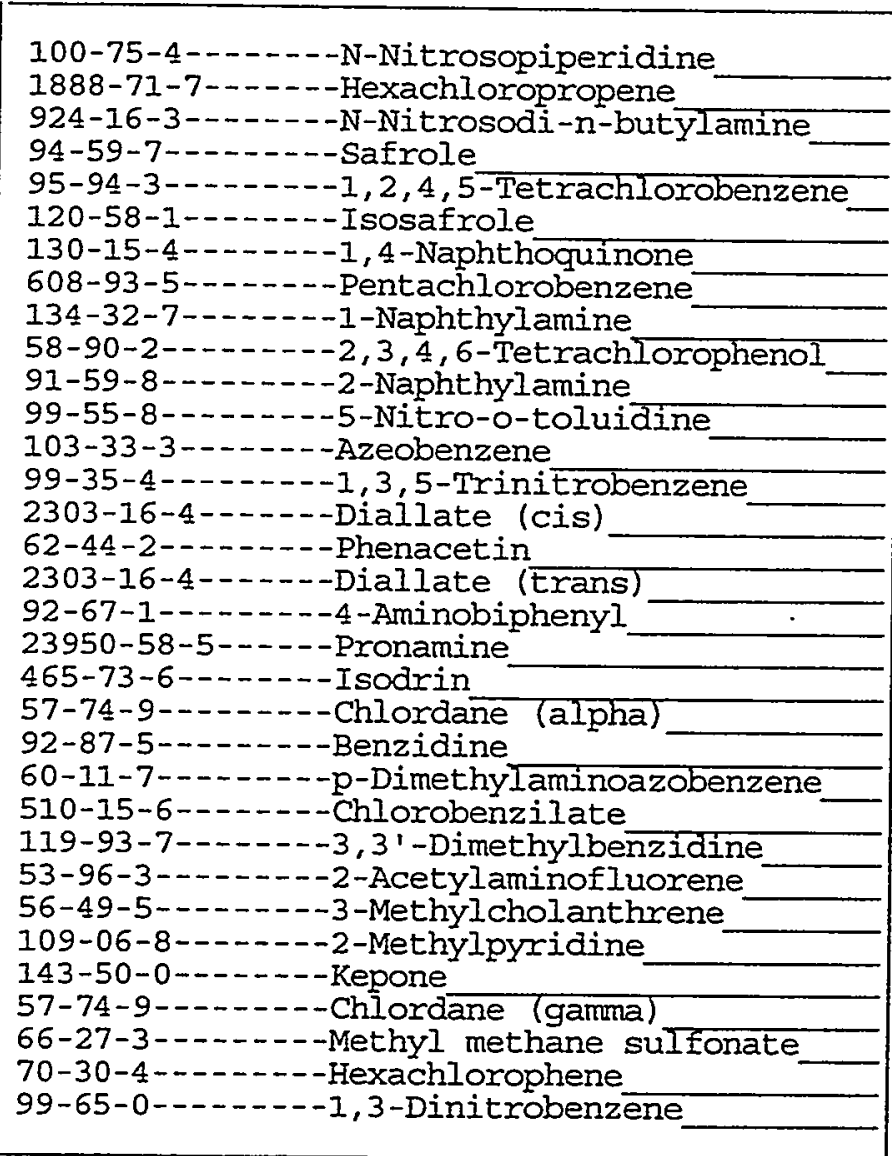

\begin{tabular}{|r|r|r}
310 & $U$ \\
310 & $U$ \\
310 & $U$ \\
310 & $U$ \\
310 & $U$ \\
310 & $U$ \\
310 & $U$ \\
310 & $U$ \\
22 & $J$ \\
310 & $U$ \\
310 & $U$ \\
310 & $U$ \\
310 & $U$ \\
310 & $U$ \\
310 & $U$ \\
310 & $U$ \\
310 & $U$ \\
6 & $J$ \\
310 & $U$ \\
310 & $U$ \\
310 & $U$ \\
310 & $U$ \\
310 & $U$ \\
310 & $U$ \\
310 & $U$ \\
310 & $U$ \\
310 & $U$ \\
310 & $U$ \\
310 & $U$ \\
310 & $U$ \\
310 & $U$ \\
310 & $U$ \\
310 & $U$ \\
& \\
& \\
\hline
\end{tabular}

FORM I SV-4

OLMO3.0 
Lab Name: PNNL

Lab Code: PNNL

Case No.:

SAS NO.:

SDG NO.: 990205

Matrix: (soil/water) SUPERNATAN

Lab Sample ID: 99-0649

Sample wt/vol:

$(\mathrm{g} / \mathrm{mL}) \mathrm{ML}$

Lab File ID: 99010506

Level: (low/med) LOW

Date Received:

\% Moisture:

decanted: $(\mathrm{Y} / \mathrm{N})$

Date Extracted:

Concentrated Extract Volume: 2000 (UI)

Date Analyzed: 02/05/99

Injection volume: (UI)

Dilution Factor: 1.0

GPC Cleanup: $\quad(Y / N) N$

$\mathrm{pH}: 11.0$

CAS NO.

COMPOUND

CONCENTRATION UNITS:

(ug/L or $u g / K g$ ) UG/L

$Q$ 
Lab Name: PNNL

Lab code: PNNL

Case No.:
Contract: BNFI

SAS NO.:
AN-107D

SDG No.: 990205
Matrix: (soil/water) SUPERNATAN

Sample wt/vol:

Level: (low $/ \mathrm{med})$

\section{IOW}

\% Moisture:

decanted: $(\mathrm{Y} / \mathrm{N})$

Concentrated Extract Volume: 2000 (ut)

Injection Volume: (uL)

(g/mL) ML

GPC Cleanup: $(Y / N) N$

$\mathrm{pH}: 11.0$
Lab Sample ID: 99-0649D

Lab File ID: 99010507

Date Received:

Date Extracted:

Date Analyzed: 02/05/99

Dilution Factor: 1.0

CAS NO. COMPOUND

CONCENTRATION UNITS: (ug/I or $u g / \mathrm{Kg}$ ) UG/L

Q

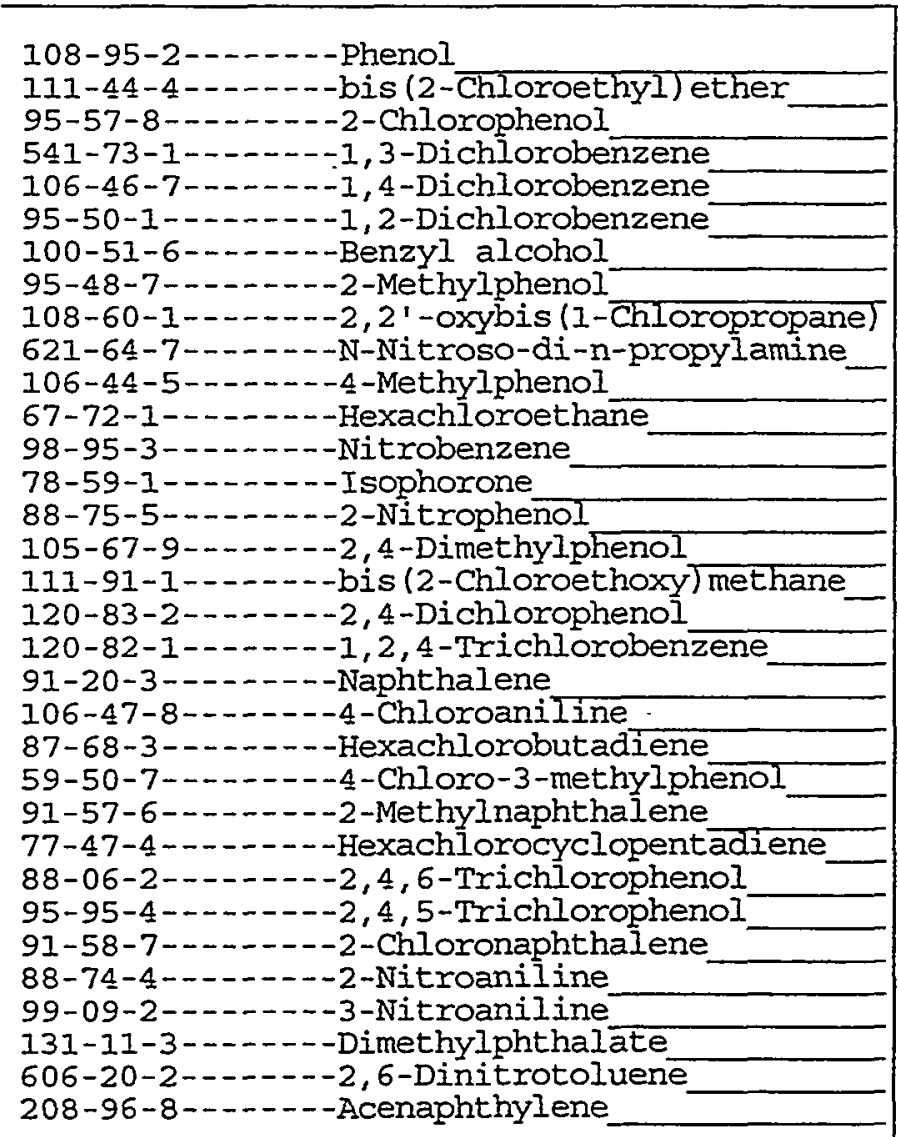

108-95-2-..---Phenol

111-44-4--..--bis (2-Chloroethyl) ether

106-46-7-------1,4-Dichlorobenzene-

95-50-1-...--1,2-Dichlorobenzene

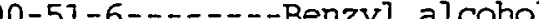

621-64-7-.....-N-Nitroso-di-n-propylamine

106-44-5-.-.---4-Methylphenol

$88-75-5-2-\cdots-2-N i t r o p h e n o$

105-67-9-...--2,4-Dimethylphenol

$111-91-1-\ldots . .-$ bis $(2-C h l o r o e t h o x y)$

120-82-1-...-1, 2,4-Trichlorobenzene

91-20-3-...--Naphthalene

$106-47-8-\ldots-.--4-C h l o r o a n i l i n e$

87-68-3-....-Hexachlorobutadiene

59-50-7-...---4-Chloro-3-methylphenol

88-06-2-.....-2, 4, 6-Trichlorophenol

95-95-4 .....--2, 4, 5-Trichlorophenol

91-58-7-.....--2-Chloronaphthalene

131-11-3-...-Dimethylphthalate

208-96-8-...--Acenaphthylene

\begin{tabular}{|l|l|}
\hline 310 & $U$ \\
310 & $U$ \\
310 & $U$ \\
310 & $U$ \\
310 & $U$ \\
310 & $U$ \\
310 & $U$ \\
310 & $U$ \\
310 & $U$ \\
310 & $U$ \\
310 & $U$ \\
310 & $U$ \\
310 & $U$ \\
310 & $U$ \\
310 & $U$ \\
310 & $U$ \\
310 & $U$ \\
310 & $U$ \\
310 & $U$ \\
310 & $U$ \\
310 & $U$ \\
310 & $U$ \\
310 & $U$ \\
310 & $U$ \\
310 & $U$ \\
310 & $U$ \\
310 & $U$ \\
310 & $U$ \\
310 & $U$ \\
310 & $U$ \\
310 & $U$ \\
310 & $U$ \\
310 & $U$ \\
& \\
& \\
3
\end{tabular}

OLM03.0 
Lab Name: PNNL

Lab Code: PNNL

Case No.:

Matrix: (soil/water) SUPERNATAN

Sample wt/vol: $(\mathrm{g} / \mathrm{mL}) \mathrm{ML}$

Level: (low/med)

IOW

$\div$ Moisture: decanted: $(Y / N)$

Concentrated Extract Volume: 2000 (uI)

Injection Volume: (uL)

Contract: BNEL SAS NO.:

Lab Sample ID: 99-0649D

Iab File ID: 99010507

Date Received:

Date Extracted:

Date Analyzed: 02/05/99

Dilution Factor: 1.0

GPC Cleanup: $\quad(\mathrm{Y} / \mathrm{N}) \mathrm{N} \quad \mathrm{pH}: 11.0$

$\begin{array}{ll}\text { CONCENTRATION UNITS: } & \text { COMPOUND NO. } \\ \text { CAg/L or ug/Kg) UG/L }\end{array}$

$Q$

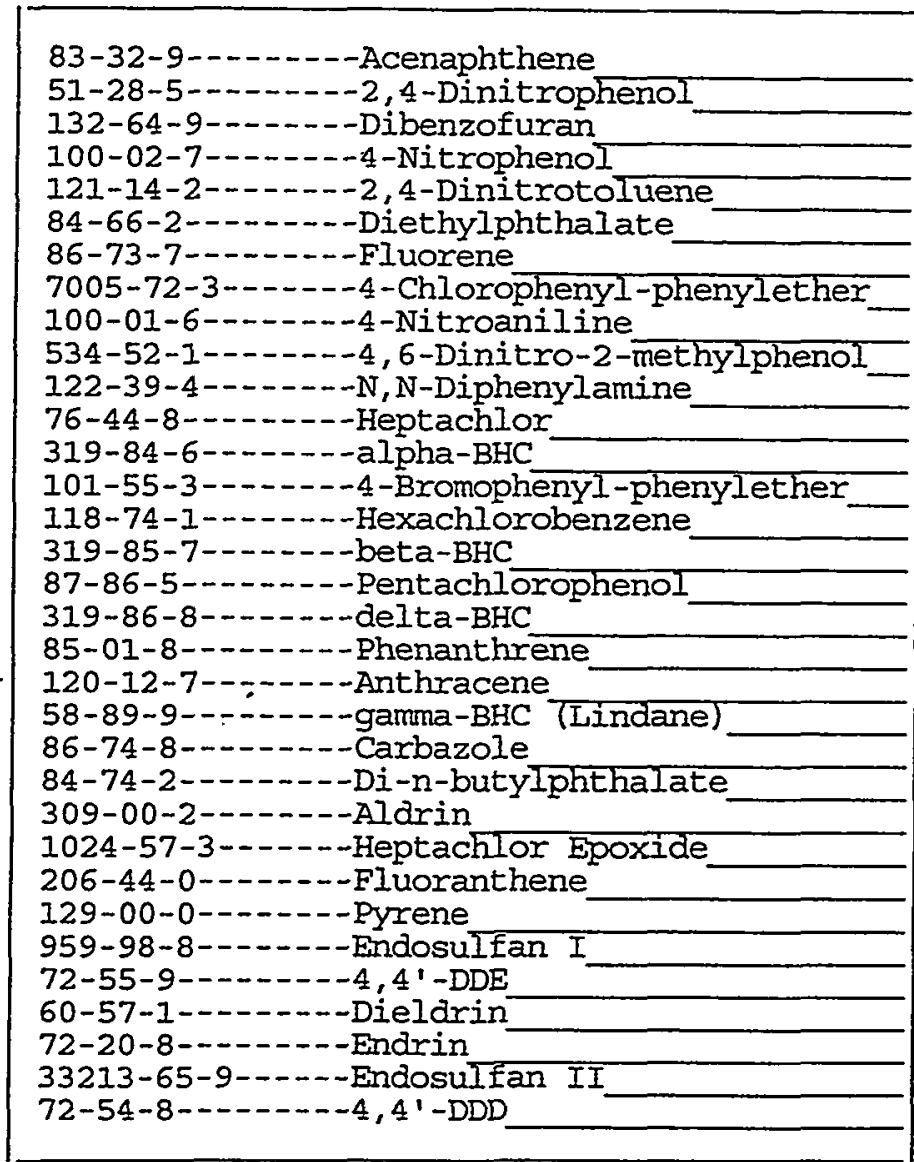

\begin{tabular}{|l|l|l|}
310 & $U$ \\
310 & $U$ \\
310 & $U$ \\
310 & $U$ \\
310 & $U$ \\
310 & $U$ \\
310 & $U$ \\
310 & $U$ \\
310 & $U$ \\
310 & $U$ \\
310 & $U$ \\
310 & $U$ \\
310 & $U$ \\
310 & $U$ \\
310 & $U$ \\
310 & $U$ \\
310 & $U$ \\
310 & $U$ \\
310 & $U$ \\
310 & $U$ \\
310 & $U$ \\
310 & $U$ \\
310 & $U$ \\
310 & $U$ \\
310 & $U$ \\
310 & $U$ \\
310 & $U$ \\
310 & $U$ \\
310 & $U$ \\
310 & $U$ \\
310 & $U$ \\
310 & $U$ \\
310 & $U$ \\
& \\
-
\end{tabular}

FORM I SV-2

OLM03.0 
Lab Name: PNNL

Lab code: PNNL

Matrix: (soil/water) SUPERNATAN

Sample wt/vol: $(\mathrm{g} / \mathrm{mL}) \mathrm{ML}$

Ievel: (low/med)

INW

$\%$ Moisture: decanted: $(\mathrm{Y} / \mathrm{N})$

Concentrated Extract Volume: 2000 (UI) Injection Volume: (UI)

Contract: BNEL

SAS NO.:

AN-107D

SDG No.: 990205

Iab Sample ID: 99-0649D

Iab File ID: 99010507

Date Received:

Date Extracted:

Date Analyzed: 02/05/99

Dilution Factor: 1.0

GPC Cleanup: $(\mathrm{Y} / \mathrm{N}) \mathrm{N} \quad \mathrm{pH}: 11.0$

CAS NO. COMPOUND $\quad$ CONCENTRATION UNITS:

Q

\begin{tabular}{|c|}
\hline 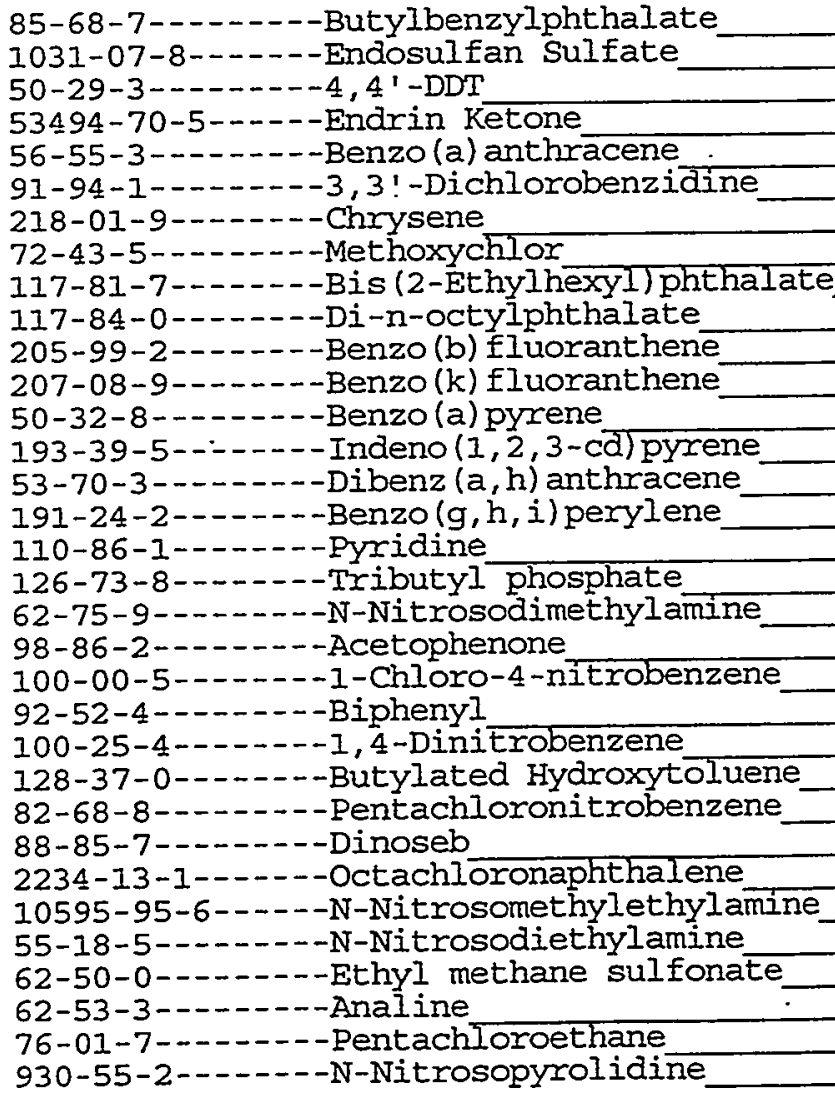 \\
\hline
\end{tabular}


Lab Name: PNNL

Lab code: PNNL

Case No. :

Matrix: (soil/water) SUPERNATAN

Sample $w t / v o l:$ $(\mathrm{g} / \mathrm{mL}) \mathrm{ML}$

Level: (low/med)

LOW

\% Moisture: decanted: $(\mathrm{Y} / \mathrm{N})$

Concentrated Extract Volume: 2000 (UU) Injection Volume: (UL)

Contract: BNFL SAS NO.:

Lab Sample ID: 99-0649D

Lab File ID: 99010507

Date Received:

Date Extracted:

Date Analyzed: 02/05/99

Dilution Factor: 1.0

GPC Cleanup: $\quad(\mathrm{Y} / \mathrm{N}) \mathrm{N} \quad \mathrm{pH}: 11.0$

$\begin{array}{ll} & \text { CONCENTRATION UNITS: } \\ \text { CAS NO. COMPOUND } & (\mathrm{ug} / \mathrm{L} \text { or } \mathrm{ug} / \mathrm{Kg} \text { ) UG/L }\end{array}$

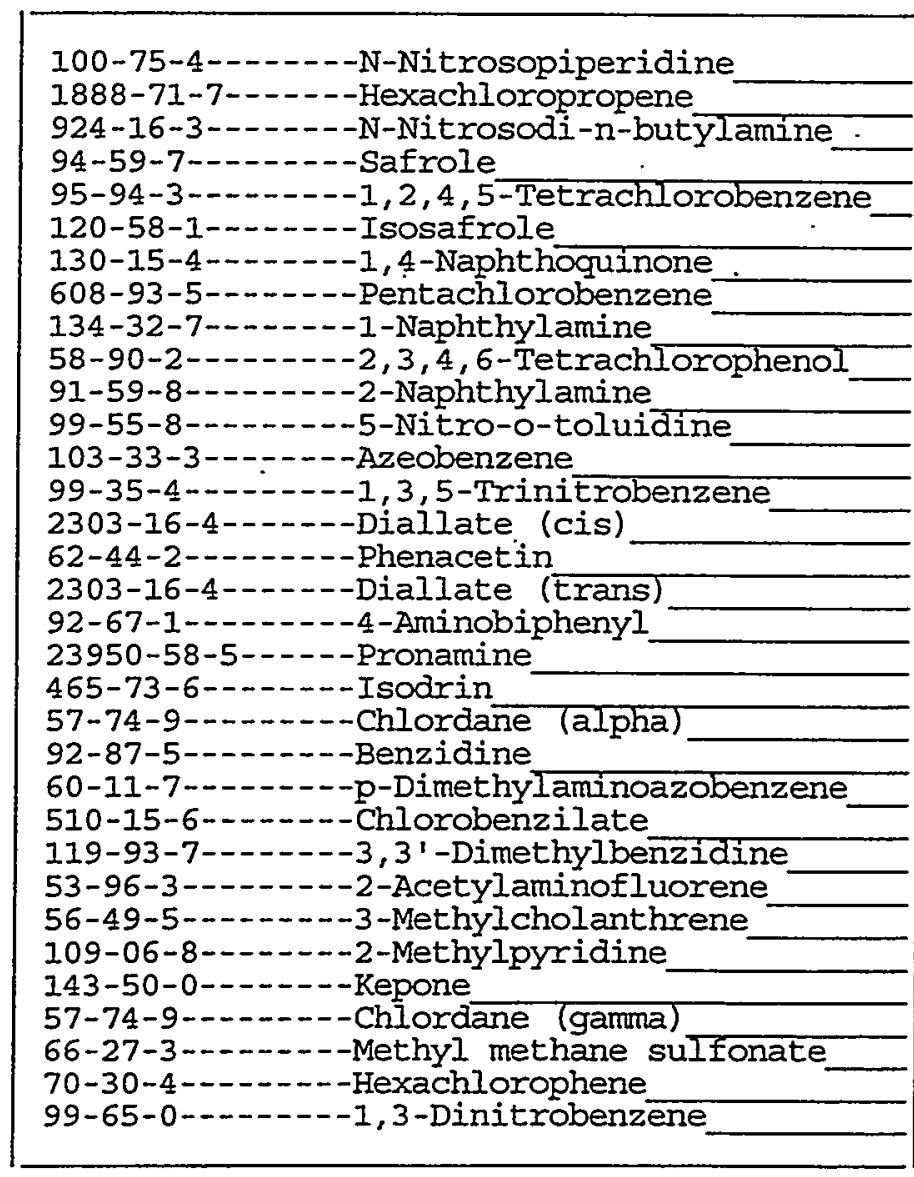

FORM I SV-4 
$1 \mathrm{C}$

SEMIVOLATILE ORGANICS ANALYSIS DATA SHEET

Lab Name: PNNL

Contract: BNFL

Iab Code: PNNL Case No.:

Matrix: (soil/water) SUPERNATAN

Sample wt/vol: $(\mathrm{g} / \mathrm{mL}) \mathrm{ML}$

Level: (low/med) IOW

\% Moisture: decanted: $(Y / N)$

SAS NO.:

Concentrated Extract Volume: 2000 (UI)

Injection Volume: (uI)

GPC Cleanup: $(\mathrm{Y} / \mathrm{N}) \mathrm{N}$

$\mathrm{pH}: 11.0$

CAS NO.

COMPOUND

CONCENTRATION UNITS: (ug/L or $u g / \mathrm{Kg}$ ) UG/L
Lab Sample ID: 99-0649D

Lab File ID: 99010507

Date Received:

Date Extracted:

AN-107D

Date Analyzed: 02/05/99

Dilution Factor: 1.0

\begin{tabular}{l|l|l|l}
\hline $87-65-0 \ldots-2,6-D i c h l o r o p h e n o l$ & 310 & $\mathrm{U}$ \\
\hline
\end{tabular}


Lab Name: PNNL

Irab Code: PNNL

Case No.:

Matrix: (soil/water) SUPERNATAN

Sample wt/vol:

$(\mathrm{g} / \mathrm{mI}) \mathrm{MI}$

Level: (iow/med)

IOW

\% Moisture:

decanted: $(Y / N)$

Concentrated Extract Volume: 2000 (uI)

Injection Volume: (uI)

Contract: BNFI
AN-107MS

SDG No. : 990205

GPC Cleanup: (Y/N) N $\mathrm{pH}: 11.0$

SAS NO.:

Lab Sample ID: 99-0649MS

Lab File ID: 99010508

Date Received:

Date Extracted:

Date Analyzed: 02/05/99

Dilution Factor: 1.0 .

$\begin{array}{ll} & \text { CONCENTRATION UNITS: } \\ \text { CAS NO. COMPOUND } & (u g / L \text { or ug/Kg) UG/L }\end{array}$

\begin{tabular}{|c|}
\hline 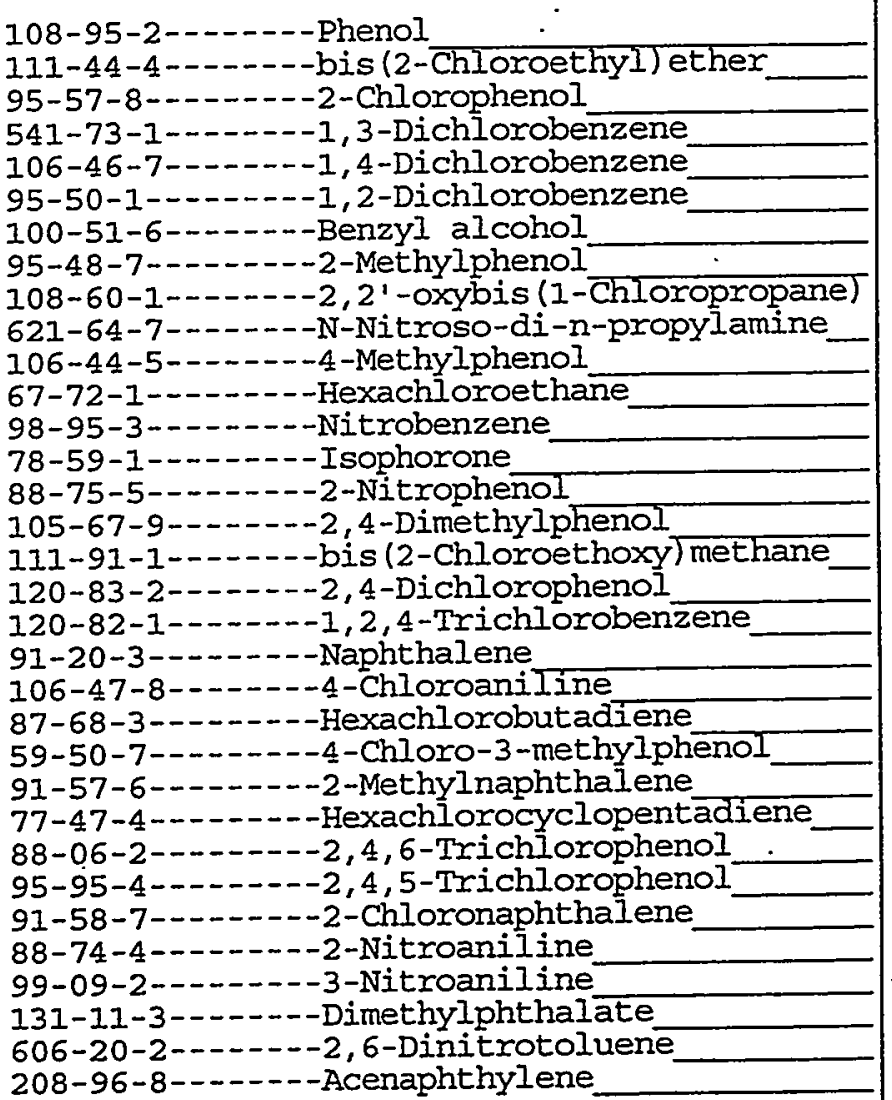 \\
\hline
\end{tabular}

FORM I SV-1

OLMO 3.0 
Lab Name: PNNL

Iab code: PNNL Case No.: SAS NO.:

SDG NO.: 990205

Matrix: (soil/water) SUPERNATAN

Sample wt/vol: $(\mathrm{g} / \mathrm{mL}) \mathrm{ML}$

Level: (low/med) LOW

$\div$ Moisture: decanted: $(Y / N)$

Concentrated Extract Volume: 2000 (ut) Injection Volume: (UI) $\mathrm{pH}: 11.0$
Lab Sample ID: 99-0649MS

Irab File ID: 99010508

Date Received:

Date Extracted:

Date Analyzed: 02/05/99

Dilution Factor: 1.0
GPC Cleanup: $(\mathrm{Y} / \mathrm{N}) \mathrm{N}$

CAS NO.
COMPOUND
CONCENTRATION UNITS: (ug/I or $\mathrm{ug} / \mathrm{Kg}$ ) UG/L
83-32-9-1-28-5-

132-64-9-.---Dibenzofuran

100-02-7--.---4-Nitrophenol

121-14-2-..--2,4-Dinitrotoluene

84-66-2-...--Diethylphthalate

86-73-7-------Fluorene

7005-72-3--.-4-Chlorophenyl-phenylether

100-01-6------4-Nitroaniline

534-52-1-.... - 4, 6-Dinitro-2-methylphenol

122-39-4-..- N, N-Diphenylamine

76-44-8-...-.-Heptachlor

319-84-6--.--alpha-BHC

101-55-3-1.--4-Bromophenyl-phenylether

118-74-1-.----Hexachlorobenzene

319-85-7--...--beta-BHC

87-86-5-...-.-Pentachlorophenol

319-86-8-----delta-BHC

85-01-8-....-. Phenanthrene

120-12-7--.---Anthracene

58-89-9-...-.--gamma-BHC (Lindane)

86-74-8--.-.-.-Carbazole

84-74-2-....-Di-n-butylphthalate

309-00-2-...-Aldrin

1024-57-3-....-Heptachlor Epoxide

206-44-0-.-.---Fluoranthene

129-00-0-.....-.-Pyrene

959-98-8-...--Endosulfan I

$72-55-9-\ldots-\cdots-4,41-D D E$

60-57-1-------Dieldrin

72-20-8-...-.-Endrin

33213-65-9-..--Endosulfan II

$72-54-8--\ldots-4,4^{\prime}-\mathrm{DDD}$

\begin{tabular}{r|r|l|}
2400 & \\
640 & $\mathrm{U}$ \\
640 & $\mathrm{U}$ \\
3100 & \\
2500 & \\
640 & $\mathrm{U}$ \\
640 & $\mathrm{U}$ \\
640 & $\mathrm{U}$ \\
91 & $\mathrm{~J}$ \\
640 & $\mathrm{U}$ \\
2100 & \\
640 & $\mathrm{U}$ \\
640 & $\mathrm{U}$ \\
640 & $\mathrm{U}$ \\
640 & $\mathrm{U}$ \\
640 & $\mathrm{U}$ \\
2200 & \\
640 & $\mathrm{U}$ \\
640 & $\mathrm{U}$ \\
640 & $\mathrm{U}$ \\
640 & $\mathrm{U}$ \\
640 & $\mathrm{U}$ \\
640 & $\mathrm{U}$ \\
640 & $\mathrm{U}$ \\
640 & $\mathrm{U}$ \\
640 & $\mathrm{U}$ \\
2500 & \\
640 & $\mathrm{U}$ \\
640 & $\mathrm{U}$ \\
640 & $\mathrm{U}$ \\
640 & $\mathrm{U}$ \\
640 & $\mathrm{U}$ \\
640 & $\mathrm{U}$ \\
& \\
\hline & \\
\hline
\end{tabular}

OLMO3.0 
Lab Name: PNNL.

Lab Code: PNNL

Case No. :
Contract: BNFL

SAS NO.:
AN-107MS

SDG No.: 990205
Matrix: (soil/water) SUPERNATAN

Sample wt/vol:

$(\mathrm{g} / \mathrm{mL}) \mathrm{MI}$

Level: (low/med)

LOW

$\div$ Moisture:

decanted: $(Y / N)$

Concentrated Extract Volume: 2000 (uL)

Injection Volume:

(uL)
Lab Sample ID: 99-0649MS

Lab File ID: 99010508

Date Received:

Date Extracted:

Date Analyzed: 02/05/99

Dilution Factor: 1.0

GPC Cleanup: $(\mathrm{Y} / \mathrm{N}) \mathrm{N} \quad \mathrm{pH}: 11.0$

CONCENTRATION UNITS :

CAS NO.

COMPOUND

(ug/L or $\mathrm{ug} / \mathrm{Kg}$ ) UG/I

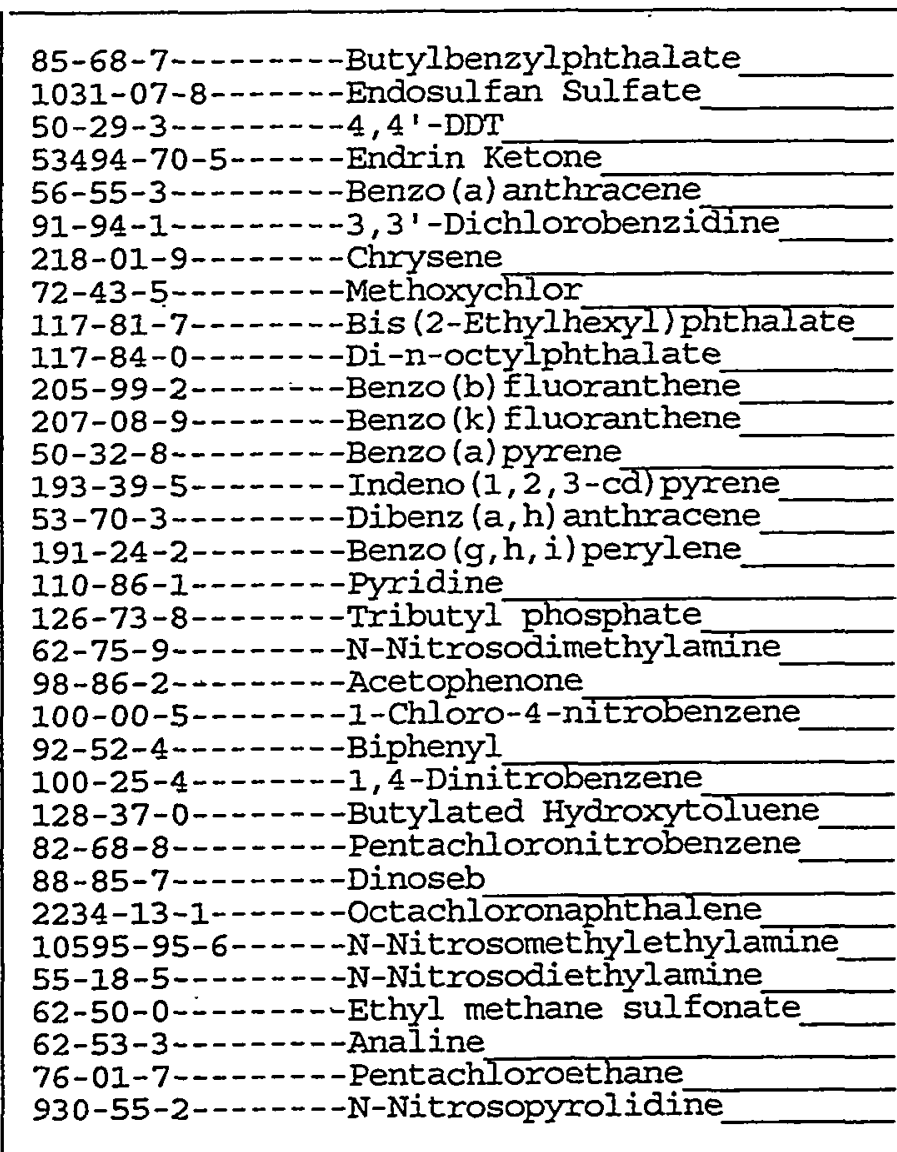

FORM I SV-3 
Lab Name: PNNL

Lab Code: PNNL

Case No.:

Matrix: (soil/water) SUPERNATAN

Sample wt/vol:

$(\mathrm{g} / \mathrm{mI}) \mathrm{MI}$

Ievel: (low/med) IOW

$\%$ Moisture:

decanted: $(Y / N)$

Concentrated Extract Volume: 2000 (UI)

Injection Volume:

(uI)
Contract : BNFL

SAS NO. :
EPA SAMPLE NO.

AN-107MS

SDG NO.: 990205

GPC Cleanup: $(\mathrm{Y} / \mathrm{N}) \mathrm{N} \quad \mathrm{pH}: 11.0$

Lab Sample ID: 99-0649MS

Lab File ID: $\quad 99010508$

Date Received:

Date Extracted:

Date Analyzed: 02/05/99

Dilution Factor: 1.0

CAS NO.

COMPOUND

CONCENTRATION UNITS:

(ug/L or ug/Kg) UG/L

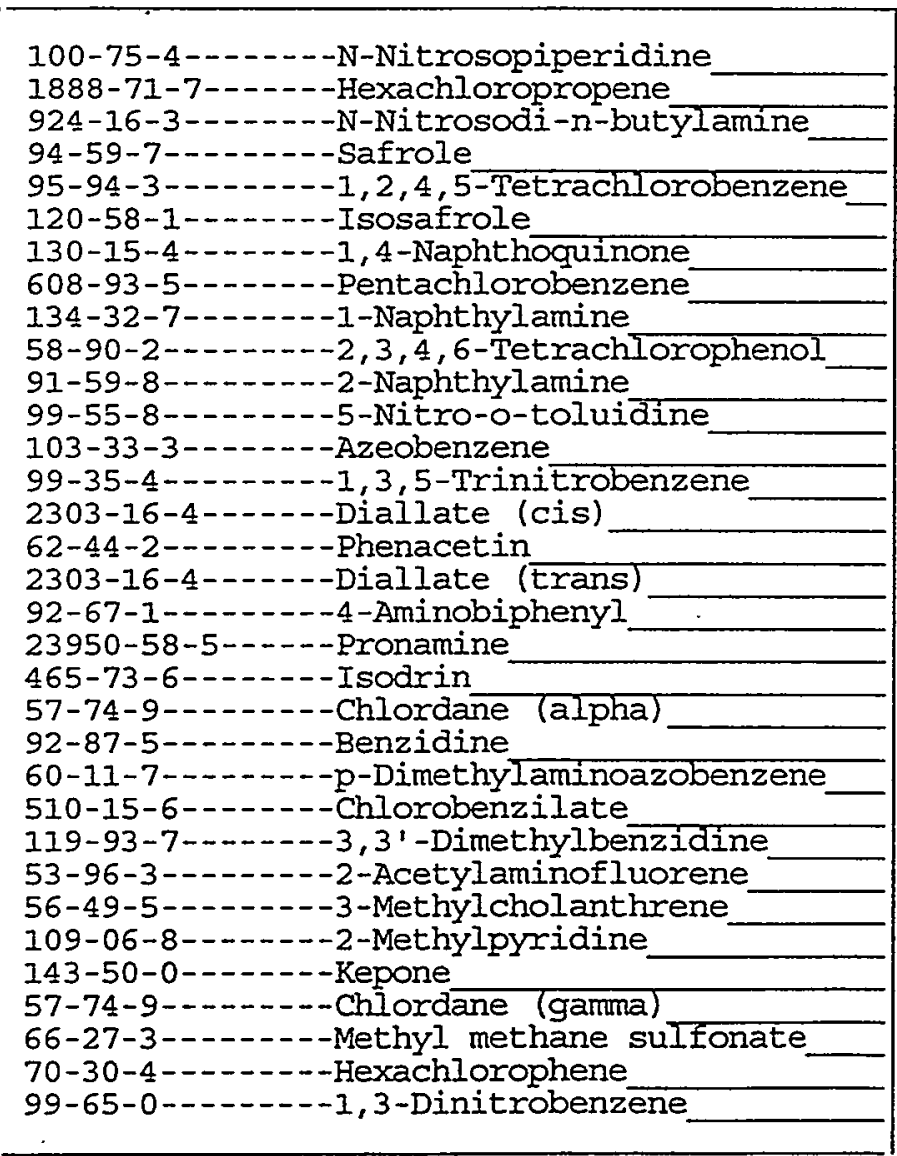

FORM I SV-4

OLM03. 0 
Lab Name: PNNL,

Contract: BNFL

Lab Code: PNNL

Case No. :

Matrix: (soil/water) SUPERNATAN

Sample wt/vol:

$(\mathrm{g} / \mathrm{mL}) \mathrm{ML}$

Level: (low/med) LOW

$\%$ Moisture:

decanted: $(Y / N)$

Concentrated Extract Volume: 2000 (uI)

Injection Volume: (uT)
AN-107MS

SDG No.: 990205 GPC Cleanup: $(\mathrm{Y} / \mathrm{N}) \mathrm{N} \quad \mathrm{pH}: 11.0$

CAS NO.

COMPOUND
SAS NO.:

Lab Sample ID: 99-0649MS

Lab File ID: 99010508

Date Received:

Date Extracted:

Dilution Factor: 1.0

\begin{tabular}{|c|c|c|}
\hline 87-65-0-n-- - 2, 6-Dichlorophenol & 640 & $\mathrm{U}$ \\
\hline
\end{tabular}


Lab Name: PNNL,

Lab code: PNNL,

Case No.:

Matrix: (soil/water) SUPERNATAN

Sample wt/vol:

$(\mathrm{g} / \mathrm{mL}) \mathrm{ML}$

Level: (low/med)

IOW

\% Moisture:

decanted: $(\mathrm{Y} / \mathrm{N})$

Concentrated Extract Volume: 2000 (uI)

Injection Volume:

(UI)
EPA SAMPLE NO.

AN-107MSD

SAS NO.:

SDG NO.: 990205

Lab Sample ID: 99-0649MSD

Lab File ID: 99010509

Date Received:

Date Extracted:

Date Analyzed: 02/05/99

Dilution Factor: 1.0

GPC Cleanup: $(Y / N) N \quad \mathrm{pH}: 11.0$

CONCENTRATION UNITS:

\begin{tabular}{|c|}
\hline 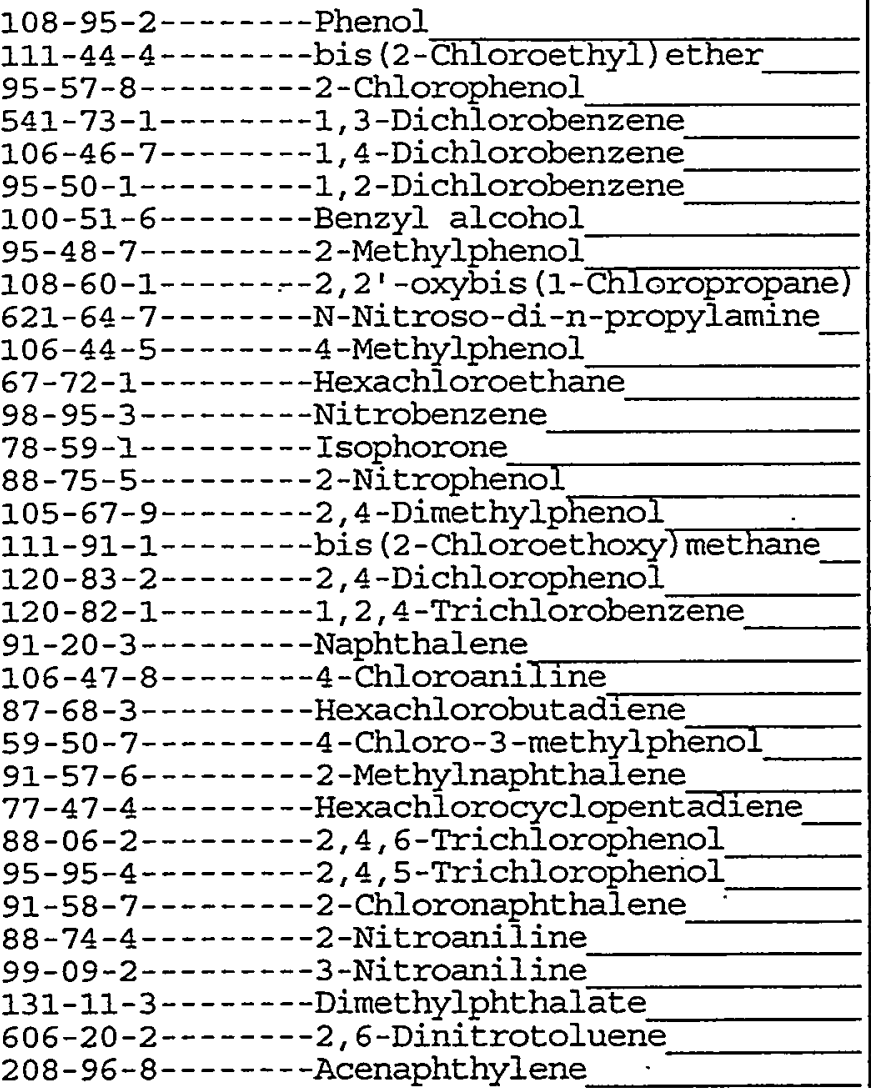 \\
\hline
\end{tabular}

FORM I SV-I

OLM03.0 
Lab Name: PNNL

Lab Code: PNNL,

Case No.:

Matrix: (soil/water) SUPERNATAN

Sample wt/vol:

$(\mathrm{g} / \mathrm{m \Psi}) \mathrm{ML}$

Level: (low/med). IOW

\% Moisture:

decanted: $(Y / N)$

Concentrated Extract Volume: 2000 (uL)

Injection Volume:

(ur)

Contract: BNFL
EPA SAMPLE NO.

AN-107MSD

SDG No.: 990205

Lab Sample ID: 99-0649MSD

Lab File ID: 99010509

Date Received:

Date Extracted:

Date Analyzed: 02/05/99

Dilution Factor: 1.0

GPC Cleanup: $\quad(Y / N) N$

$\mathrm{pH}: 11.0$

CAS NO.

COMPOUND

CONCENTRATION UNITS:

(ug/L or $\mathrm{ug} / \mathrm{Kg}$ ) UG/I

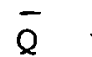

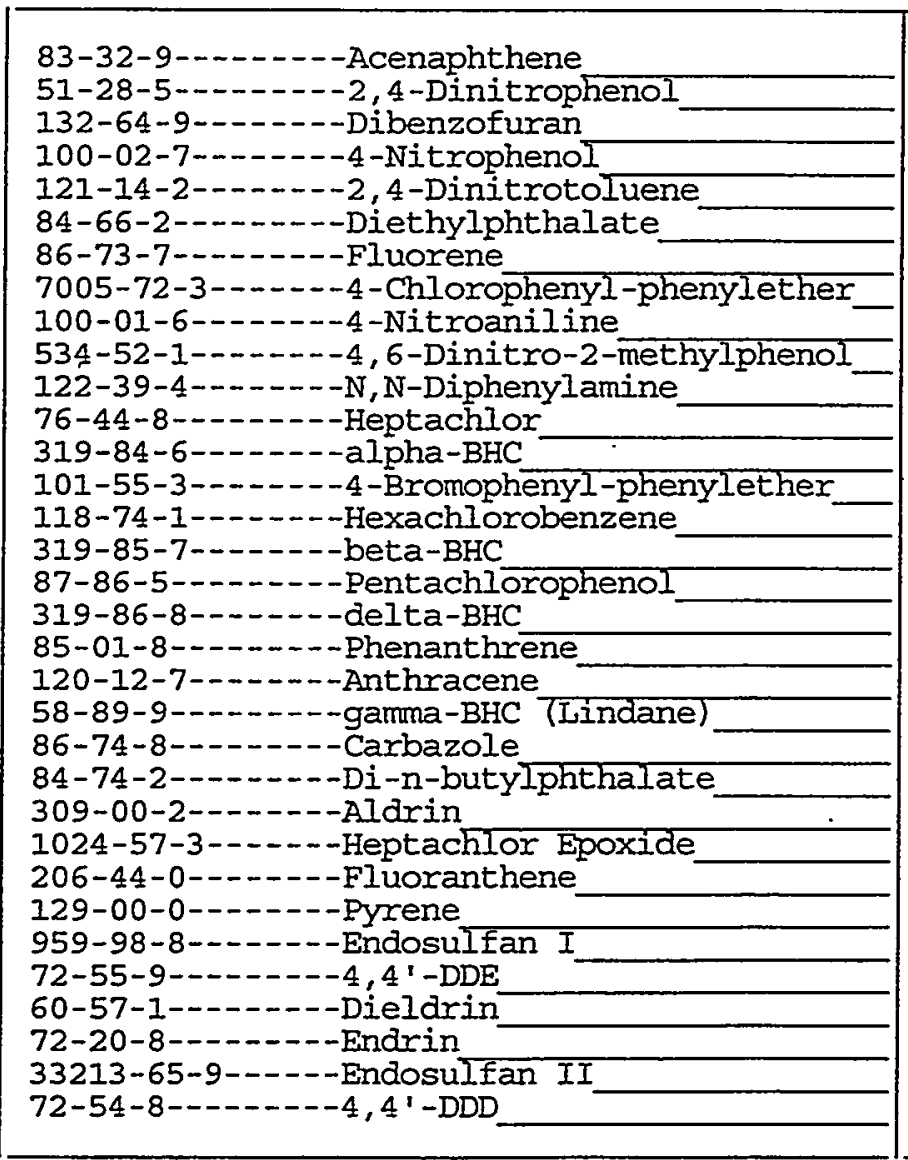

FORM I SV-2

OLM03. 0 
Lab Name: PNNL

Iab Code: PNNL

Case No.:

Matrix: (soil/water) SUPERNATAN

Sample wt/vol:

$(g / m L) M I$

\% Moisture:

decanted: $(\mathrm{Y} / \mathrm{N})$

Concentrated Extract Volume: 2000 (UI)

Injection Volume:

(UT)

Contract: BNFL

SAS NO. :

Lab Sample ID: 99-0649MSD

Lab File ID: 99010509

Date Received:

Date Extracted:

Date Analyzed: 02/05/99

Dilution Factor: 1.0

GPC Cleanup: $(\mathrm{Y} / \mathrm{N}) \mathrm{N} \quad \mathrm{pH}: 11.0$

CAS NO

COMPOUND

CONCENTRATION UNITS:

(ug/L or ug/Kg) UG/L

$\bar{Q}$

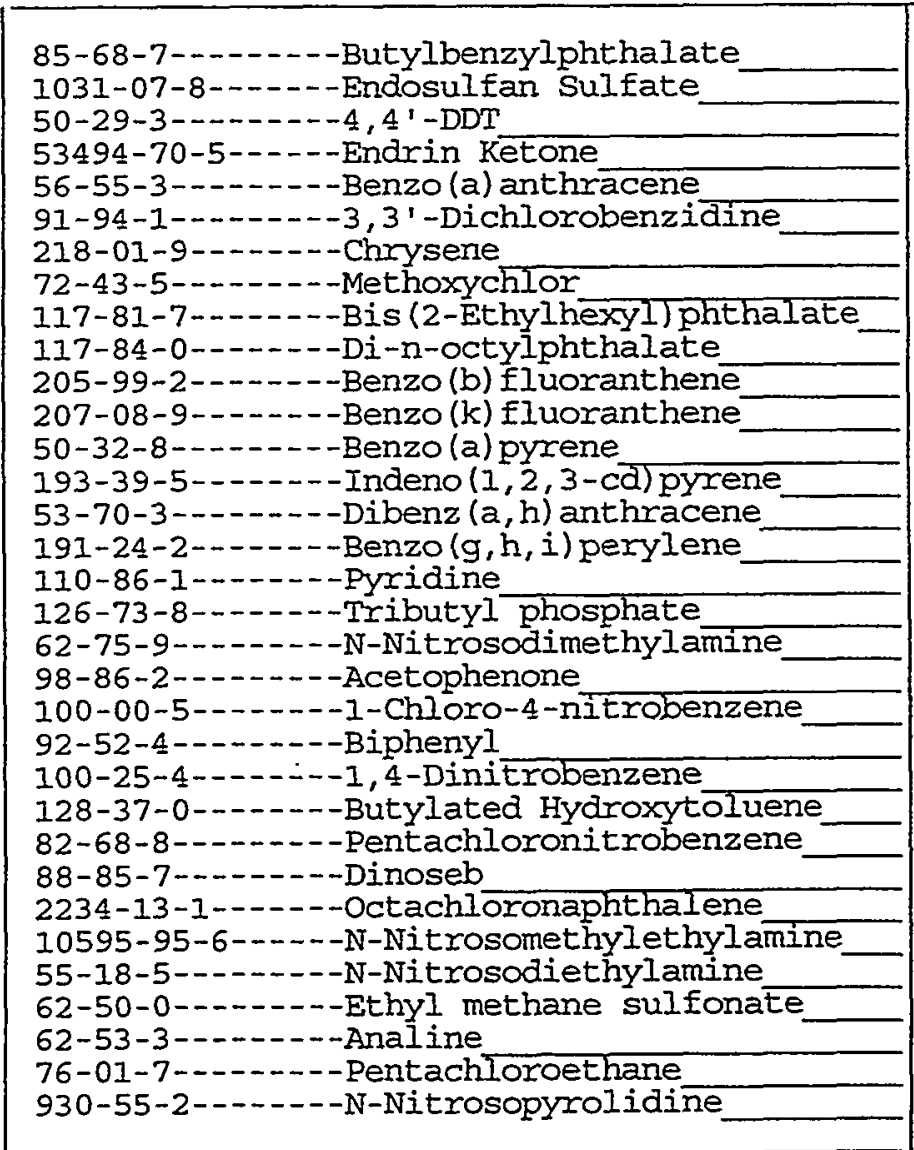

\begin{tabular}{r|l}
640 & $\mathrm{U}$ \\
640 & $\mathrm{U}$ \\
640 & $\mathrm{U}$ \\
640 & $\mathrm{U}$ \\
640 & $\mathrm{U}$ \\
640 & $\mathrm{U}$ \\
640 & $\mathrm{U}$ \\
640 & $\mathrm{U}$ \\
640 & $\mathrm{U}$ \\
81 & $\mathrm{~J}$ \\
640 & $\mathrm{U}$ \\
640 & $\mathrm{U}$ \\
640 & $\mathrm{U}$ \\
640 & $\mathrm{U}$ \\
640 & $\mathrm{U}$ \\
640 & $\mathrm{U}$ \\
2800 & \\
2700 & \\
230 & $\mathrm{~J}$ \\
3100 & \\
3000 & \\
2800 & \\
2900 & - \\
720 & \\
3500 & \\
62 & $\mathrm{~J}$ \\
2500 & \\
640 & $\mathrm{U}$ \\
640 & $\mathrm{U}$ \\
640 & $\mathrm{U}$ \\
640 & $\mathrm{U}$ \\
640 & $\mathrm{U}$ \\
640 & $\mathrm{U}$ \\
& \\
\hline & \\
\hline
\end{tabular}

FORM I SV-3

OLMO 3.0 
Lab Name: PNNL

Lab Code: PNNL

Case No. :

SAS NO.:

AN-107MSD

Matrix: (soil/water) SUPERNATAN

Sample wt/vol: $(\mathrm{g} / \mathrm{mL}) \mathrm{ML}$

Level: (low/med) LOW

$\%$ Moisture: decanted: $(\mathrm{Y} / \mathrm{N})$

Concentrated Extract Volume: 2000 (uL)

Injection Volume: (uU)

GPC Cleanup: $\quad(Y / N) N \quad \mathrm{pH}: 11.0$

GPC Cleanup: $(\mathrm{Y} / \mathrm{N}) \mathrm{N} \quad \mathrm{pH}: 11.0$

Lab Sample ID: 99-0649MSD

Lab File ID: 99010509

Date Received:

Date Extracted:

Date Analyzed: 02/05/99

Dilution Factor: 1.0

CONCENTRATION UNITS: (ug/I or $u g / \mathrm{Kg}$ ) UG/L

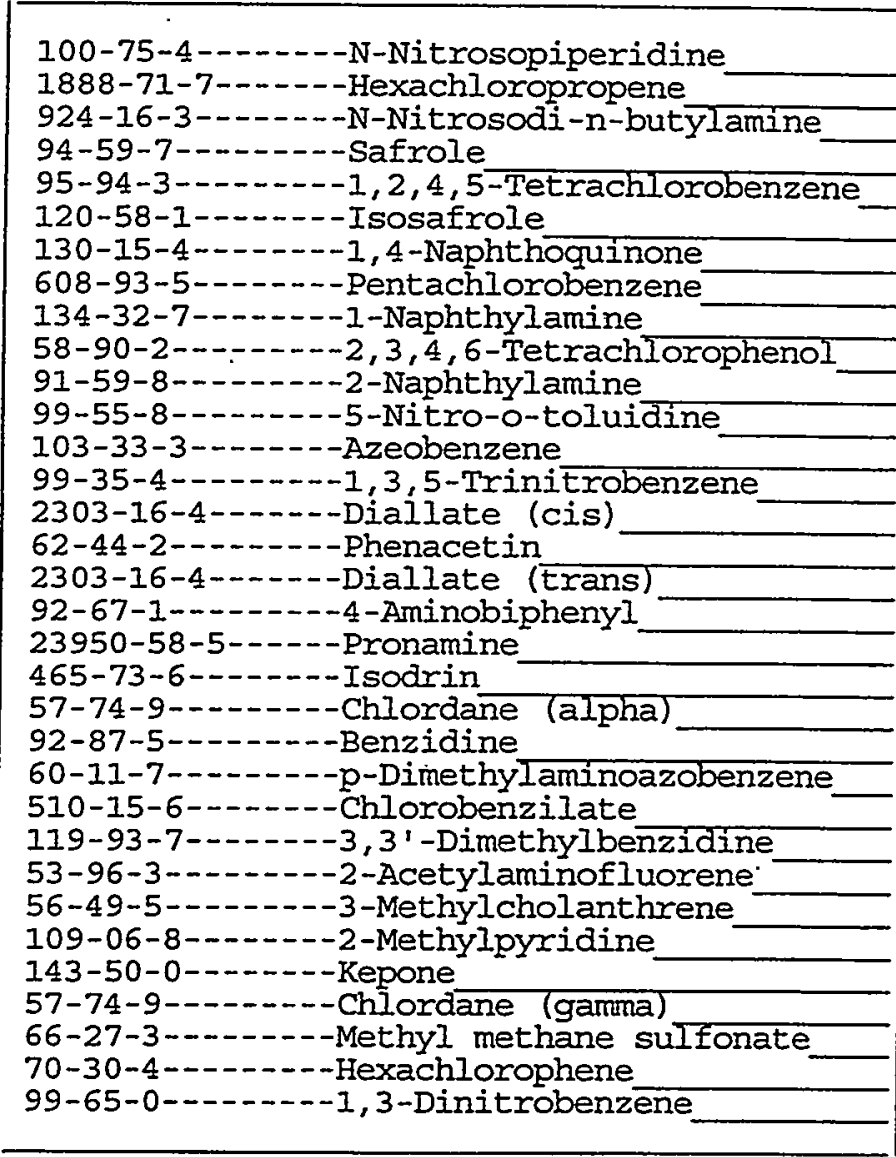


Lab Name: PNNL

Lab Code: PNNL

Matrix: (soil/water) SUPERNATAN

Sample wt/vol:

$(\mathrm{g} / \mathrm{mL}) \mathrm{ML}$

Level: (low/med) LOW

$\%$ Moisture:

decanted: $(Y / N)$

Concentrated Extract Volume: 2000 (uI)

Injection Volume: (UI)

GPC Cleanup: $\quad(Y / N) N$

$\mathrm{pH}: 7.0$
EPA SAMPLE NO.

Contract: BNFL

SAS NO.:

SDG No.: 990205

Iab Sample ID: SBLKOI

Lab File ID: 99010515

Date Received:

Date Extracted:

Date Analyzed: 02/06/99

Dilution Factor: 1.0

CAS NO.

COMPOUND

CONCENTRATION UNITS:

(ug/L or $u g / \mathrm{Kg}$ ) UG/I,

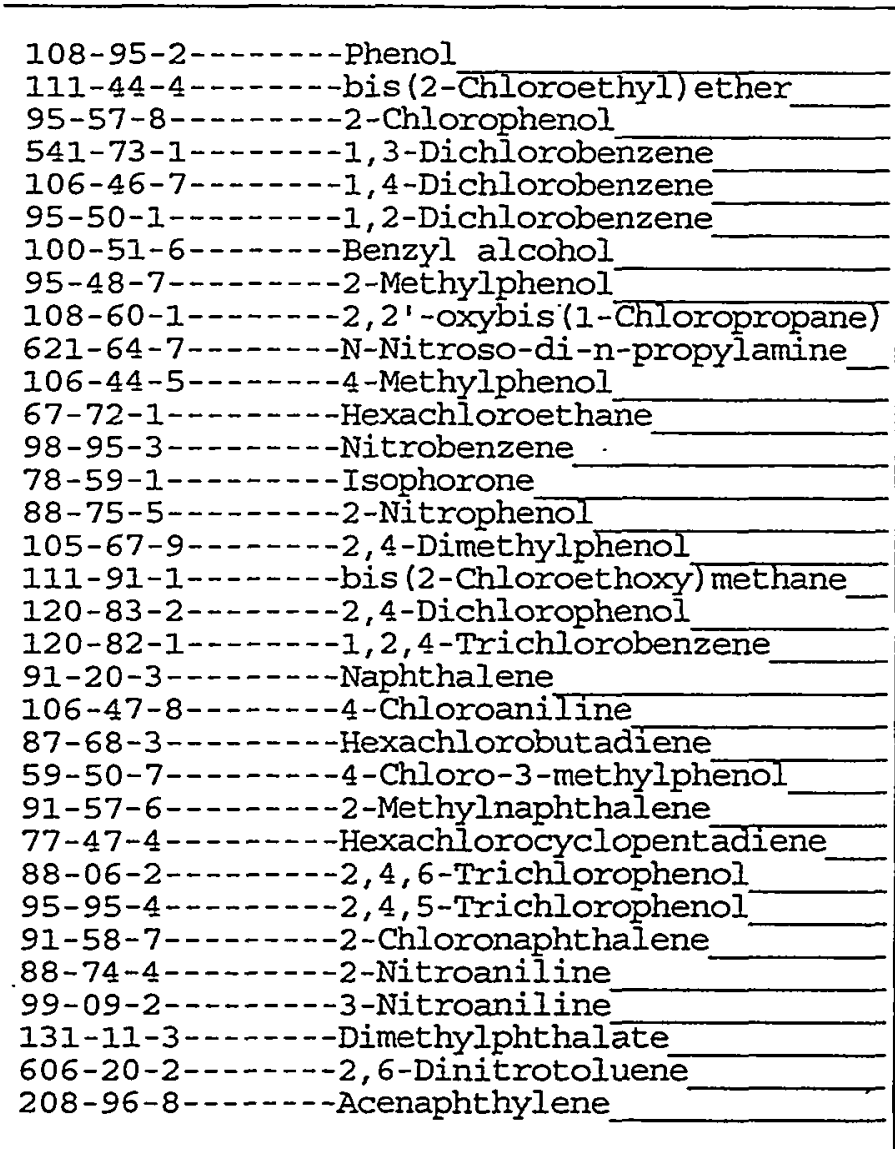

FORM I SV-1

\begin{tabular}{|l|l|l|}
\hline 200 & $U$ \\
200 & $U$ \\
200 & $U$ \\
200 & $U$ \\
200 & $U$ \\
200 & $U$ \\
200 & $U$ \\
200 & $U$ \\
200 & $U$ \\
200 & $U$ \\
200 & $U$ \\
200 & $U$ \\
200 & $U$ \\
200 & $U$ \\
200 & $U$ \\
200 & $U$ \\
200 & $U$ \\
200 & $U$ \\
200 & $U$ \\
200 & $U$ \\
200 & $U$ \\
200 & $U$ \\
200 & $U$ \\
200 & $U$ \\
200 & $U$ \\
200 & $U$ \\
200 & $U$ \\
200 & $U$ \\
200 & $U$ \\
200 & $U$ \\
200 & $U$ \\
200 & $U$ \\
200 & $U$ \\
& \\
\hline & \\
\hline
\end{tabular}

OLMO3 .0 
Lab Name: PNNL

Lab code: PNNL

Case No. :
Contract : BNFL

SAS NO.:
EPA SAMPLE NO.

METHOD BLANK
Matrix: (soil/water) SUPERNATAN

Sample wt/vol:

Ievel: (low/med)

$(\mathrm{g} / \mathrm{mL}) \mathrm{ML}$

$\%$ Moisture:

decanted: $(\mathrm{Y} / \mathrm{N})$

Injection Volume:

(uI)
Lab Sample ID: SBLKOI

Lab File ID: $\quad 99010515$

Date Received:

Date Extracted:

Date Analyzed: 02/06/99

Dilution Factor: 1.0

GPC Cleanup: $\quad(Y / N) N \quad \mathrm{pH}: 7.0$

CONCENTRATION UNITS :

CAS NO.

COMPOUND

(ug/L or $u g / K g$ ) UG/I

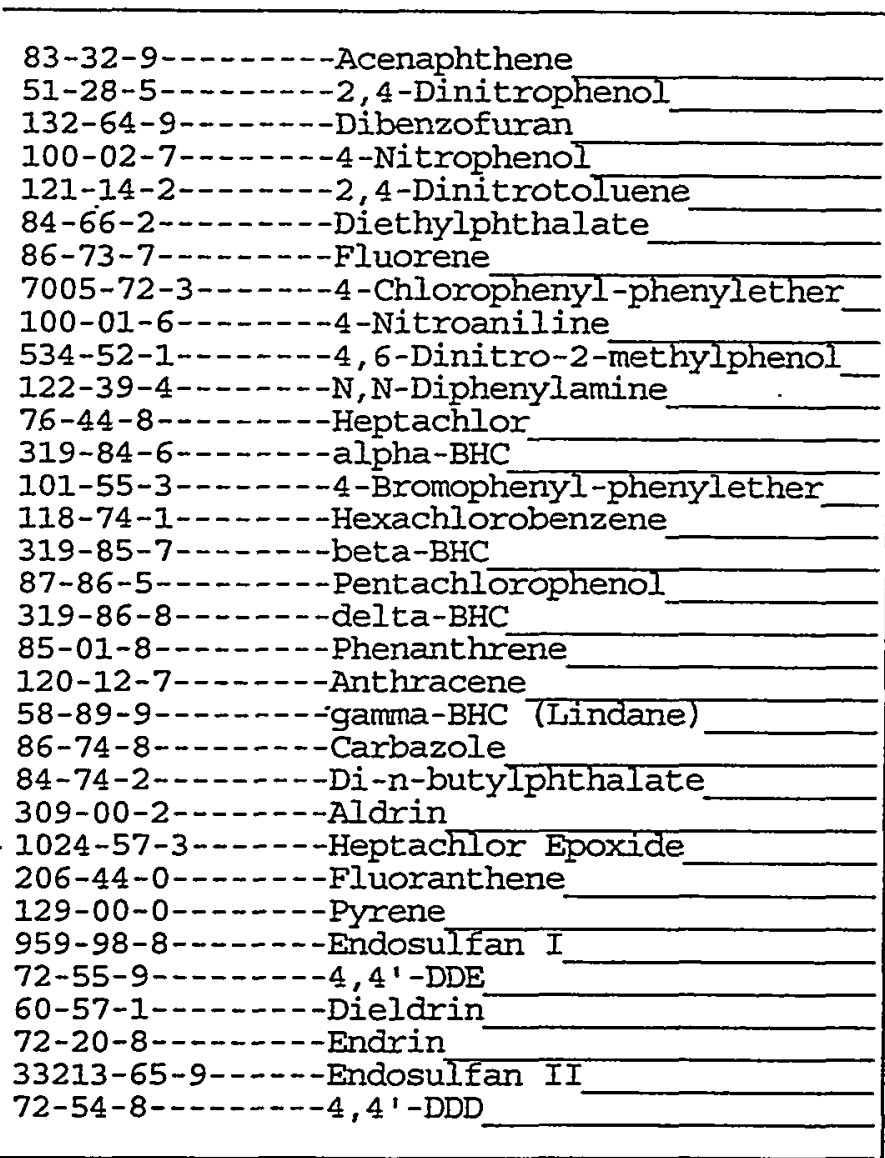

FORM I SV-2

\begin{tabular}{|l|l|l}
\hline 200 & $\mathrm{U}$ \\
200 & $\mathrm{U}$ \\
200 & $\mathrm{U}$ \\
200 & $\mathrm{U}$ \\
200 & $\mathrm{U}$ \\
200 & $\mathrm{U}$ \\
200 & $\mathrm{U}$ \\
200 & $\mathrm{U}$ \\
200 & $\mathrm{U}$ \\
200 & $\mathrm{U}$ \\
200 & $\mathrm{U}$ \\
200 & $\mathrm{U}$ \\
200 & $\mathrm{U}$ \\
200 & $\mathrm{U}$ \\
200 & $\mathrm{U}$ \\
200 & $\mathrm{U}$ \\
200 & $\mathrm{U}$ \\
200 & $\mathrm{U}$ \\
200 & $\mathrm{U}$ \\
200 & $\mathrm{U}$ \\
200 & $\mathrm{U}$ \\
200 & $\mathrm{U}$ \\
200 & $\mathrm{U}$ \\
200 & $\mathrm{U}$ \\
200 & $\mathrm{U}$ \\
200 & $\mathrm{U}$ \\
200 & $\mathrm{U}$ \\
200 & $\mathrm{U}$ \\
200 & $\mathrm{U}$ \\
200 & $\mathrm{U}$ \\
200 & $\mathrm{U}$ \\
200 & $\mathrm{U}$ \\
200 & $\mathrm{U}$ \\
& \\
\hline & \\
\hline
\end{tabular}

OLMO3.0 
Lab Name: PNNL

Lab Code: PNNL

Case No.:

Matrix: (soil/water) SUPERNATAN

Sample wt/vol: $(\mathrm{g} / \mathrm{mL}) \mathrm{ML}$

Level: (low/med)

LOW

$\%$ Moisture: decanted: $(\mathrm{Y} / \mathrm{N})$

Concentrated Extract Volume: 2000 (uL)

Injection Volume: (uU)
Contract: BNFL

SAS NO.:

Lab Sample ID: 99-0649MSD

Lab File ID: 99010509

Date Received:

Date Extracted:

Date Analyzed: 02/05/99

Dilution Factor: 1.0

GPC Cleanup: $(Y / N) N$ $\mathrm{pH}: 11.0$

AN-107MSD

SDG NO.: 990205

$\begin{array}{lll} & \text { CONCENTRATION UNITS: } & - \\ \text { CAS NO. COMPOUND } & (u g / I \text { or ug/Kg) UG/L } & Q\end{array}$

\begin{tabular}{|l|l|l|}
\hline $87-65-0-\ldots-2 .-2,6-D i c h l o r o p h e n o l$ & 640 & $\mathrm{U}$ \\
\hline
\end{tabular}


Lab Name: PNNL

Contract: BNFL

METHOD BL.ANK

Lab Code: PNNL

Case No.:

SAS NO.:

SDG NO.: 990205

Matrix: (soil/water) SUPERNATAN

Sample wt/vol: $(\mathrm{g} / \mathrm{mL}) \mathrm{ML}$

Level: (low/med) IOW

$\%$ Moisture: decanted: $(Y / N)$

Concentrated Extract Volume: 2000 (uت)

Injection Volume: (uI)

$\mathrm{pH}: 7.0$
Lab Sample ID: SBLKOI

Lab File ID: $\quad 99010515$

Date Received:

Date Extracted:

Date Analyzed: 02/06/99

Dilution Factor: 1.0
GPC Cleanup: $\quad(Y / N)$

CAS NO.
COMPOUND
CONCENTRATION UNITS: (ug/L or $u g / \mathrm{Kg}$ ) UG/L
$\bar{Q}$

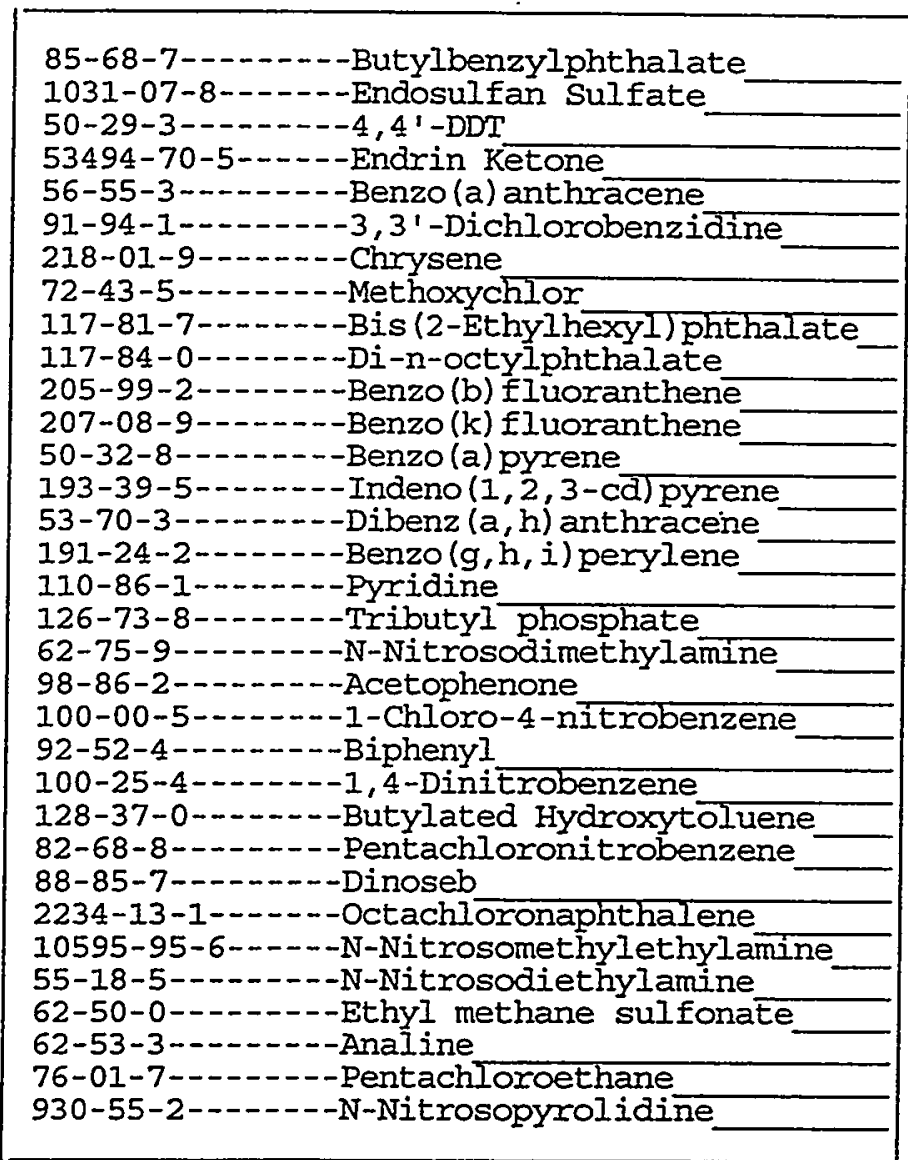

FORM I SV-3
OLMO3. 0 
Lab Name: PNNL

Iab Code: PNNL

Case No.: contract: BNEL

SAS NO.:
EPA SAMPLE NO.

METHOD BLANK
Matrix: (soil/water) SUPERNATAN

Sample wt/vol: (g/mL) ML

Level: (Iow/med) LOW

․ㅡㅇ Moisture: decanted: $(\mathrm{Y} / \mathrm{N})$

Concentrated Extract Volume: 2000 (uL) Injection Volume: (uI)

GPC Cleanup: $(\mathrm{Y} / \mathrm{N}) \mathrm{N} \quad \mathrm{pH}: 7.0$
Lab Sample ID: SBLK0I

Lab File ID: $\quad 99010515$

Date Received:

Date Extracted:

Date Analyzed: 02/06/99

Dilution Factor: 1.0

CAS NO.

COMPOUND

CONCENTRATION UNITS: (ug/I, or $u g / \mathrm{Kg}$ ) UG/L

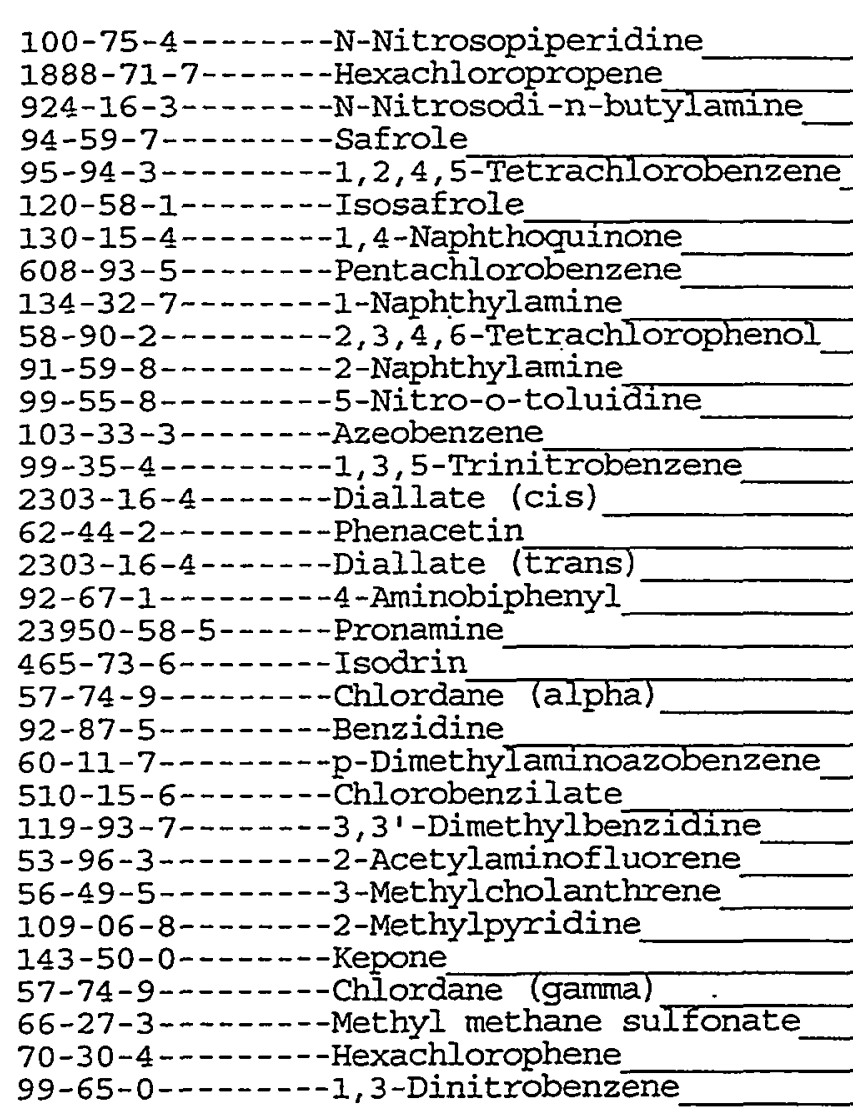


Iab code: PNNL

Case No.:

SAS NO. : METHOD BLANK

Matrix: (soil/water) SUPERNATAN

Iab Sample ID: SBLK0I

Sample wt/vol:

$(\mathrm{g} / \mathrm{mL}) \mathrm{ML}$

Lab File ID: $\quad 99010515$

Level: (Iow/med) IOW

Date Received:

\% Moisture: decanted: $(\mathrm{Y} / \mathrm{N})$

Date Extracted:

Concentrated Extract Volume: 2000 (UI)

Date Analyzed: 02/06/99

Injection Volume: (uJ)

Dilution Factor: 1.0

GPC Cleanup: $(Y / N) N$ $\mathrm{pH}: 7.0$

CAS NO.

COMPOUND

CONCENTRATION UNITS:

(ug/L or $u g / K g$ ) UG/L

$\bar{Q}$

87-65-0-- - - - 2-Dichlorophenol $200 \mathrm{U}$

FORM I SV-5

OLM03. 0 
Lab Name: PNNL

Lab code: PNNL

Case No.:

Matrix: (soil/water) SOLID

Sample wt/vol:

$4.6(\mathrm{~g} / \mathrm{mL}) \mathrm{G}$

Level: (low/med) LOW

\% Moisture: 0 decanted: $(Y / N) \mathrm{N}$

Concentrated Extract Volume: 2000 (UI)

Injection Volume: (Uน)

Contract: BNEL

SAS NO.:
SDG No.: 990205

EPA SAMPLE NO.

AN-107-

Iab Sample ID: 99-0651-

Lab File ID: 99010511

Date Received:

Date Extracted:

Date Analyzed: 02/05/99

Dilution Factor: 1.0
GPC Cleanup:
$(Y / N) N$
$\mathrm{pH}: 11.0$

CAS NO.

COMPOUND

CONCENTRATION UNITS:

(ug/I or ug/Kg) UG/KG

$-$

\begin{tabular}{|c|}
\hline 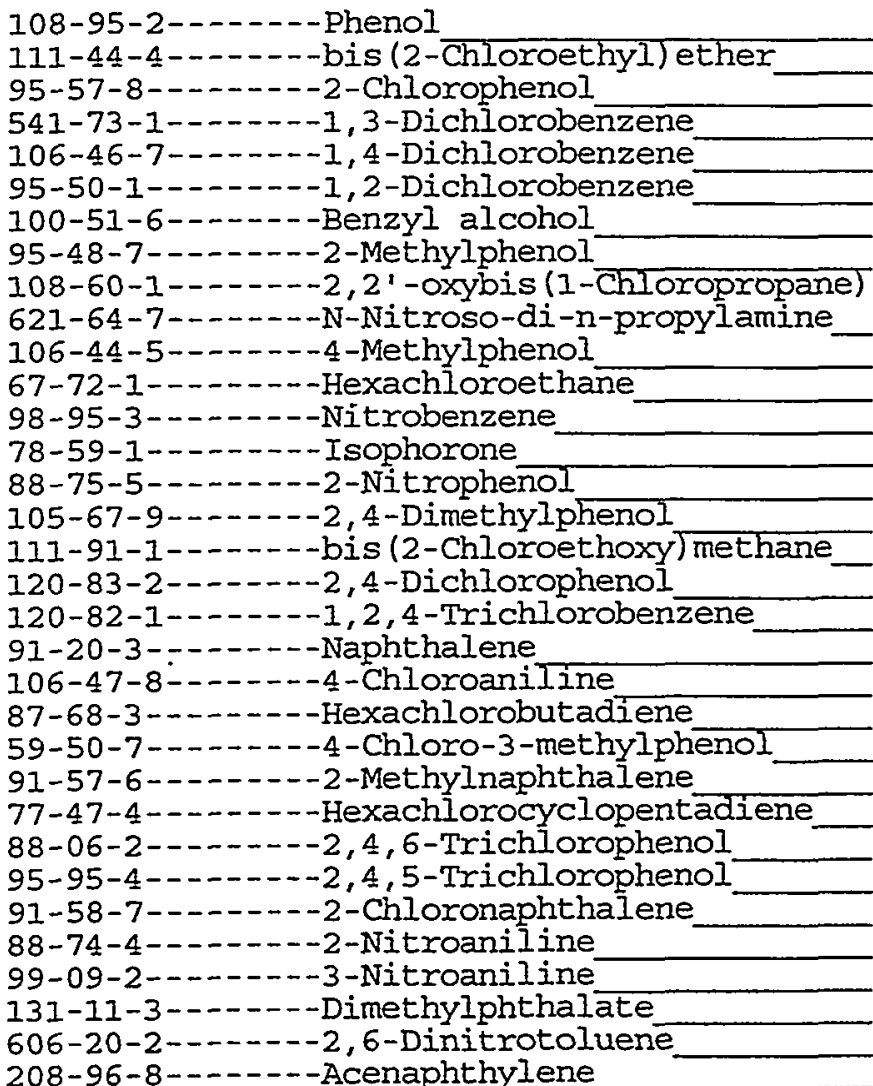 \\
\hline
\end{tabular}

\begin{tabular}{|l|l|l|}
\hline 4300 & $\mathrm{U}$ \\
4300 & $\mathrm{U}$ \\
4300 & $\mathrm{U}$ \\
4300 & $\mathrm{U}$ \\
4300 & $\mathrm{U}$ \\
4300 & $\mathrm{U}$ \\
4300 & $\mathrm{U}$ \\
4300 & $\mathrm{U}$ \\
4300 & $\mathrm{U}$ \\
4300 & $\mathrm{U}$ \\
4300 & $\mathrm{U}$ \\
4300 & $\mathrm{U}$ \\
4300 & $\mathrm{U}$ \\
4300 & $\mathrm{U}$ \\
4300 & $\mathrm{U}$ \\
4300 & $\mathrm{U}$ \\
4300 & $\mathrm{U}$ \\
4300 & $\mathrm{U}$ \\
4300 & $\mathrm{U}$ \\
4300 & $\mathrm{U}$ \\
4300 & $\mathrm{U}$ \\
4300 & $\mathrm{U}$ \\
4300 & $\mathrm{U}$ \\
4300 & $\mathrm{U}$ \\
4300 & $\mathrm{U}$ \\
4300 & $\mathrm{U}$ \\
4300 & $\mathrm{U}$ \\
4300 & $\mathrm{U}$ \\
4300 & $\mathrm{U}$ \\
4300 & $\mathrm{U}$ \\
4300 & $\mathrm{U}$ \\
4300 & $\mathrm{U}$ \\
4300 & $\mathrm{U}$ \\
& & \\
\hline & \\
\hline
\end{tabular}


Lab Name: PNNL

Iab Code: PNNL

Case No.:
Contract: BNFL
EPA SAMPLE NO.

AN-107-

SAS NO. :
Matrix: (soil/water) SOLID

Sample wt/vol:

$4.6(\mathrm{~g} / \mathrm{mL}) \mathrm{G}$

Level: (low/med) IOW

\% Moisture: 0 decanted: $(\mathrm{Y} / \mathrm{N}) \mathrm{N}$

Concentrated Extract Volume: 2000 (UT)

Injection Volume: (uI)

$\mathrm{pH}: 11.0$
Iab Sample ID: 99-065I-

Lab File ID: 99010511

Date Received:

Date Extracted:

Date Analyzed: 02/05/99

Dilution Factor: 1.0
GPC Cleanup: $(\mathrm{Y} / \mathrm{N}) \mathrm{N}$

CAS NO.
COMPOUND
CONCENTRATION UNITS: (ug/L or $\mathrm{ug} / \mathrm{Kg}$ ) UG/KG
$\bar{Q}$
83-32-9-- - - - Acenaphthene

51-28-5_......-2,4-Dinitrophenol

132-64-9--.-.--Dibenzofuran

100-02-7-..---4-Nitrophenol

121-14-2 - ..--2,4-Dinitrotoluene

84-66-2-....-Diethylphthalate

86-73-7--.-.---Fluorene

7005-72-3 - - - - Chlorophenyl-phenylether

100-01-6-...--4-Nitroaniline

534-52-1-...-4 6-Dinitro-2-methylphenol

$122-39-4 \ldots-\ldots, N-D i p h e n y l a m i n e$

76-44-8-...---Heptachlor

319-84-6-...-alpha-BHC

101-55-3-..--4-Bromophenyl-phenylether

118-74-1-...---Hexachlorobenzene

319-85-7-------beta-BHC

87-86-5-_...-. Pentachlorophenol

3I9-86-8--.---delta-BHC

85-01-8-....-Phenanthrene

120-12-7--..---Anthracene

58-89-9-....-.-gamma-BHC (Iindane)

86-74-8-------Carbazole

84-74-2-...-.-Di-n-butylphthalate

309-00-2-.-.-Aldrin

1024-57-3--.---Heptachlor Epoxide

206-44-0-..---Fluoranthene

129-00-0-..-.---Pyrene

959-98-8-...-.-EndosuIfan I

72-55-9-....--4, 4'-DDE

60-57-1-.-.--Dieldrin

72-20-8-....--Endrin

33213-65-9-...-EndosuIfan. II

$72-54-8-\ldots \ldots-4,4$ ' $-D D D$
$4300 \mathrm{U}$

$4300 \mathrm{U}$

$4300 \mathrm{U}$

$4300 \mathrm{U}$

4300 U

$4300 \mathrm{U}$

$4300 \mathrm{U}$

$4300 \mathrm{U}$

$4300 \mathrm{U}$

$4300 \mathrm{U}$

4300 U

$4300 \mathrm{U}$

$4300 \mathrm{U}$

$4300 \mathrm{U}$

$4300 \mathrm{U}$

$4300 \mathrm{U}$

$4300 \mathrm{U}$

$4300 \mathrm{U}$

$4300 \mathrm{U}$

4300 U

$4300 \mathrm{U}$

4300 U

4300 U

$4300 \mathrm{U}$

$4300 \mathrm{U}$

$4300 \mathrm{U}$

4300 U

4300 U

$4300 \mathrm{U}$

$4300 \mathrm{U}$

$4300 \mathrm{U}$

$4300 \mathrm{U}$

$4300 \mathrm{U}$ 
Lab Name: PNNL

Lab code: PNNL

Case No.:
Contract: BNFL SAS NO.:
EPA SAMPLE NO.

AN-107-
Matrix: (soil/water) SOLID

Sample wt/vol:

$4.6(\mathrm{~g} / \mathrm{mL}) \mathrm{G}$

Level: (low/med) Iow

$\%$ Moisture: 0 decanted: $(Y / N) \mathrm{N}$

Concentrated Extract Volume: 2000 (uL)

Injection Volume: (uL)

GPC Cleanup: $\quad(\mathrm{Y} / \mathrm{N}) \mathrm{N} \quad \mathrm{pH}: 11.0$

\begin{tabular}{|c|c|c|}
\hline S NO. & COMPOUND & $\begin{array}{l}\text { CONCENTRATION UNITS: } \\
(u g / L \text { or } u g / K g) \text { UG/KG }\end{array}$ \\
\hline
\end{tabular}

Lab Sample ID: 99-0651-

Lab File ID: 99010511

Date Received:

Date Extracted:

Date Analyzed: 02/05/99

Dilution Factor: 1.0

\begin{tabular}{|c|}
\hline 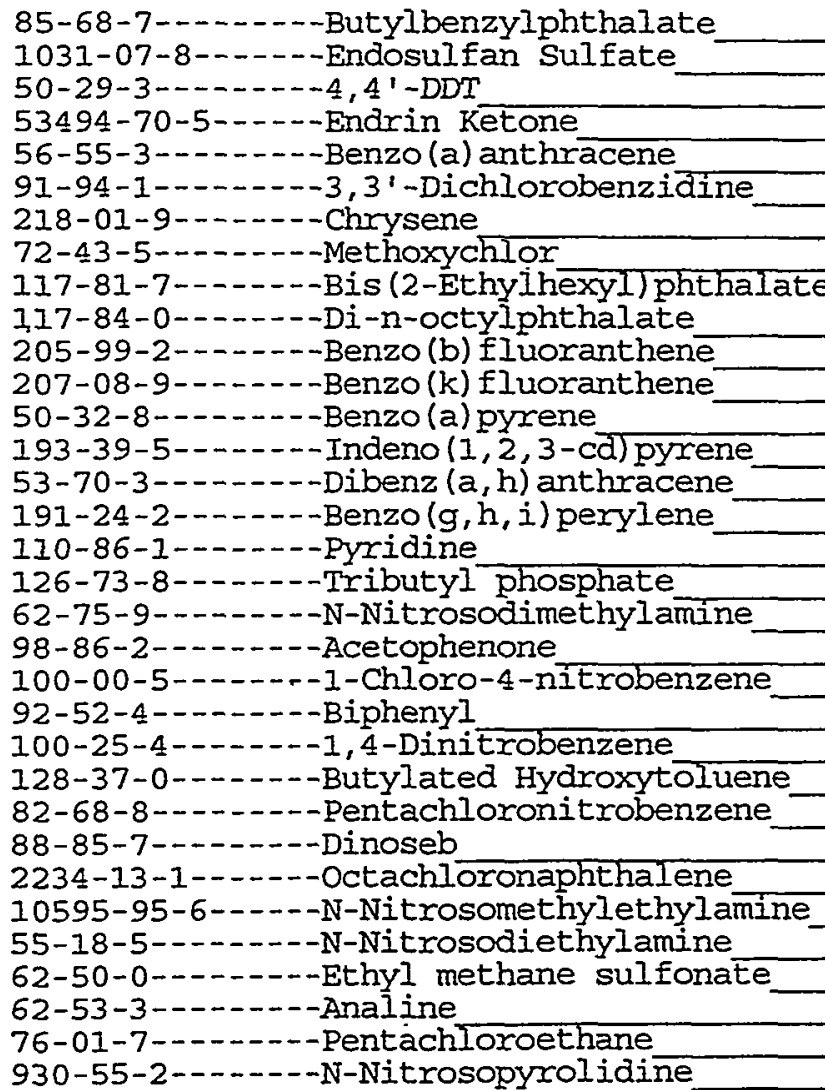 \\
\hline
\end{tabular}

FORM I SV-3 
Lab Name: PNNL

Lab code: PNNL

Case No.:

Matrix: (soil/water) SOIID

Sample wt/vol:

$4.6(\mathrm{~g} / \mathrm{mL}) \mathrm{G}$

Level: (low/med) IOW

\% Moisture: 0 decanted: (Y/N) N

Concentrated Extract Volume: 2000 (u山)

Injection Volume: (UI)
AN-107-

Contract: BNEL

SAS NO. :

SDG NO.: 990205

Lab Sample ID: 99-0651-

Iab File ID: 99010511

Date Received:

Date Extracted:

Date Analyzed: 02/05/99

Dilution Factor: 1.0

GPC Cleanup: $(\mathrm{Y} / \mathrm{N}) \mathrm{N} \quad \mathrm{pH}: 11.0$

CONCENTRATION UNITS:

CAS NंO.

COMPOUND

(ug/I or $u g / \mathrm{Kg}$ ) UG/KG

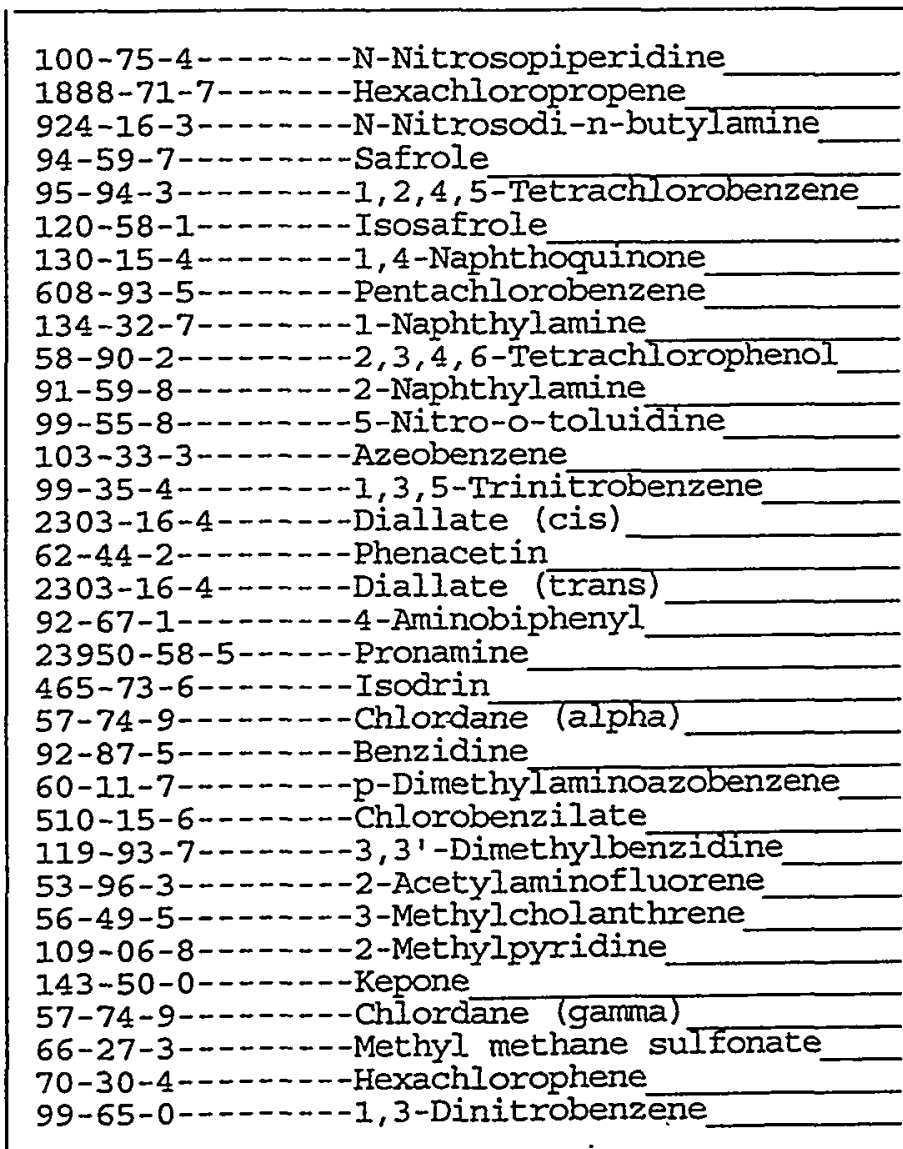

4300

4300

$4300 \mathrm{U}$

$4300 \mathrm{U}$

$4300 \mathrm{U}$

$4300 \mathrm{U}$

$4300 \mathrm{U}$

$4300 \mathrm{U}$

5400

$4300 \mathrm{U}$

$4300 \mathrm{U}$

$4300 \mathrm{U}$

$4300 \mathrm{U}$

$4300 \mathrm{U}$

$4300 \mathrm{U}$

$4300 \mathrm{U}$

$4300 \mathrm{U}$

$4300 \mathrm{U}$

$4300 \mathrm{U}$

$4300 \mathrm{U}$

$4300 \mathrm{U}$

$4300 \mathrm{U}$

$4300 \mathrm{U}$

$4300 \mathrm{U}$

$4300 \mathrm{U}$

$4300 \mathrm{U}$

$4300 \mathrm{U}$

$4300 \mathrm{U}$

$4300 \mathrm{U}$

$4300 \mathrm{U}$

$4300 \mathrm{U}$

$4300 \mathrm{U}$

$4300 \mathrm{U}$

FORM I SV-4

OLMO3. 0 
Lab Name: PNNL

Lab code: PNNL

Case No.:

Matrix: (soil/water) SOLID

Sample wt/vol:

$$
4.6(\mathrm{~g} / \mathrm{mL}) \mathrm{G}
$$

Level: (low/med)

LOW

decanted: $(Y / N) N$

\% Moisture: 0

Concentrated Extract Volume: 2000 (UI)

Injection Volume: (ut)

$\mathrm{pH}: 11.0$

GPC Cleanup: $(\mathrm{Y} / \mathrm{N}) \mathrm{N}$
EPA SAMPLE NO.

AN-107-

contract: BNFI

SAS NO.:

SDG No.: 990205

Lab Sample ID: 99-0651-

Lab File ID: 99010511

Date Received:

Date Extracted:

Date Analyzed: 02/05/99

Dilution Factor: 1.0

CAS NO.

COMPOUND

CONCENTRATION UNITS:

(ug/L or $u g / K g$ ) UG/KG

$\bar{Q}$ 
Lab Name: PNNL

Iab code: PNNL

Case No.:
Contract : BNFL

SAS NO.:
EPA SAMPLE NO.

$A N-107 D$

SDG No.: 990205

Matrix: (soil/water) SOLID

Sample wt/vol:

$4.8(\mathrm{~g} / \mathrm{mL}) \mathrm{G}$

Lab Sample ID: 99-065ID

Ievel: (low/med) IOW

$\%$ Moisture: 0 decanted: $(Y / N) \mathrm{N}$

Lab File ID: 99010512

Date Received:

Concentrated Extract Volume: 2000 (uL)

Date Extracted:

Injection Volume:

(uL)

Date Analyzed: 02/05/99

GPC Cleanup: $(\mathrm{Y} / \mathrm{N}) \mathrm{N} \quad \mathrm{pH}: 11.0$

Dilution Factor: 1.0

CAS NO.

COMPOUND

CONCENTRATION UNITS:

(ug/I or $\mathrm{ug} / \mathrm{Kg}$ ) UG/KG

$\bar{Q}$

\begin{tabular}{|c|}
\hline 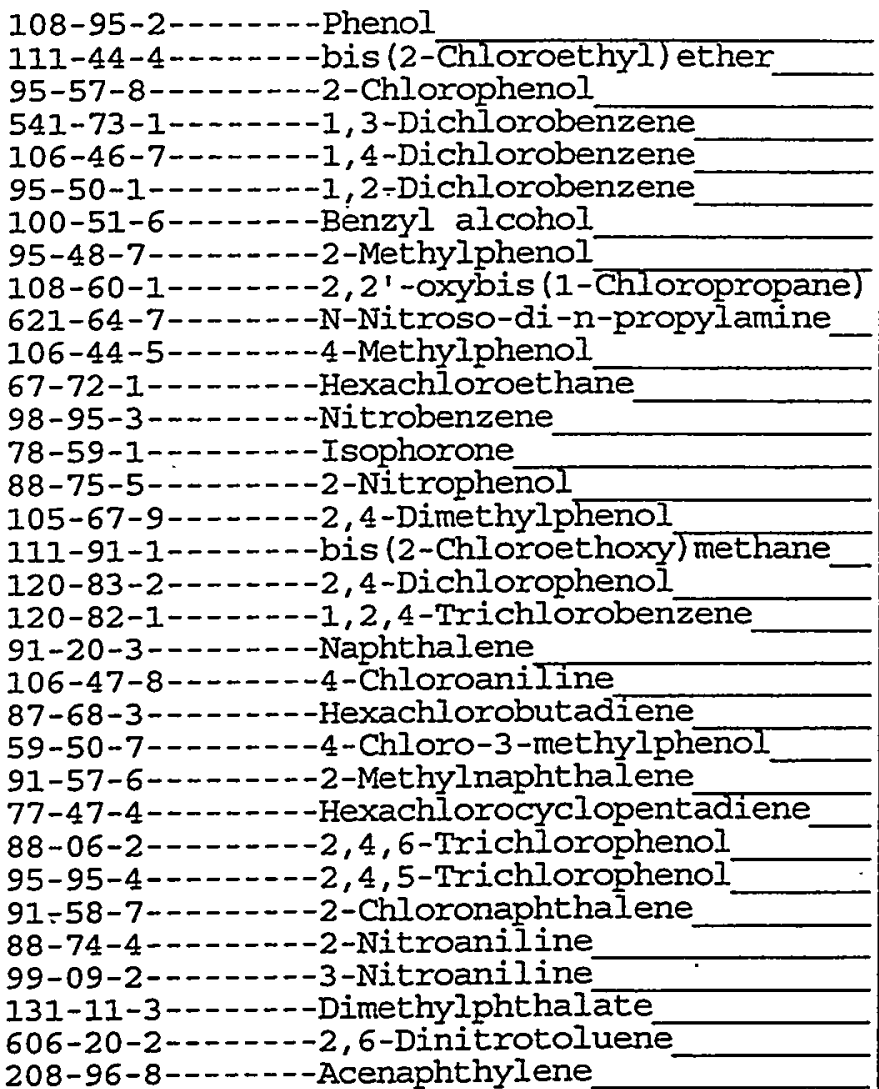 \\
\hline
\end{tabular}

FORM I SV-I

OIMO3. 0 
Lab Name: PNNL

Iab Code: PNNL

Case No. :
Contract: BNFL

SAS NO.:
EPA SAMPLE NO.

AN-107D

SDG NO.: 990205
Matrix: (soil/water) SOLID

Sample wt/vol:

$4.8(\mathrm{~g} / \mathrm{mI}) \mathrm{G}$

Level: (low/med) IOW

$\div$ Moisture: 0 decanted: ( $\mathrm{Y} / \mathrm{N}) \mathrm{N}$

Concentrated Extract Volume: 2000 (uL)

Injection Volume:

(uI)

GPC Cleanup: $(\mathrm{Y} / \mathrm{N}) \mathrm{N} \quad \mathrm{pH}: 11.0$
Lab Sample ID: 99-0651D

Lab File ID: 99010512

Date Received:

Date Extracted:

Date Analyzed: 02/05/99

Dilution Factor: 1.0

CONCENTRATION UNITS : CAS NO. $\quad$ COMPOUND (ug/I or ug/Kg) UG/KG

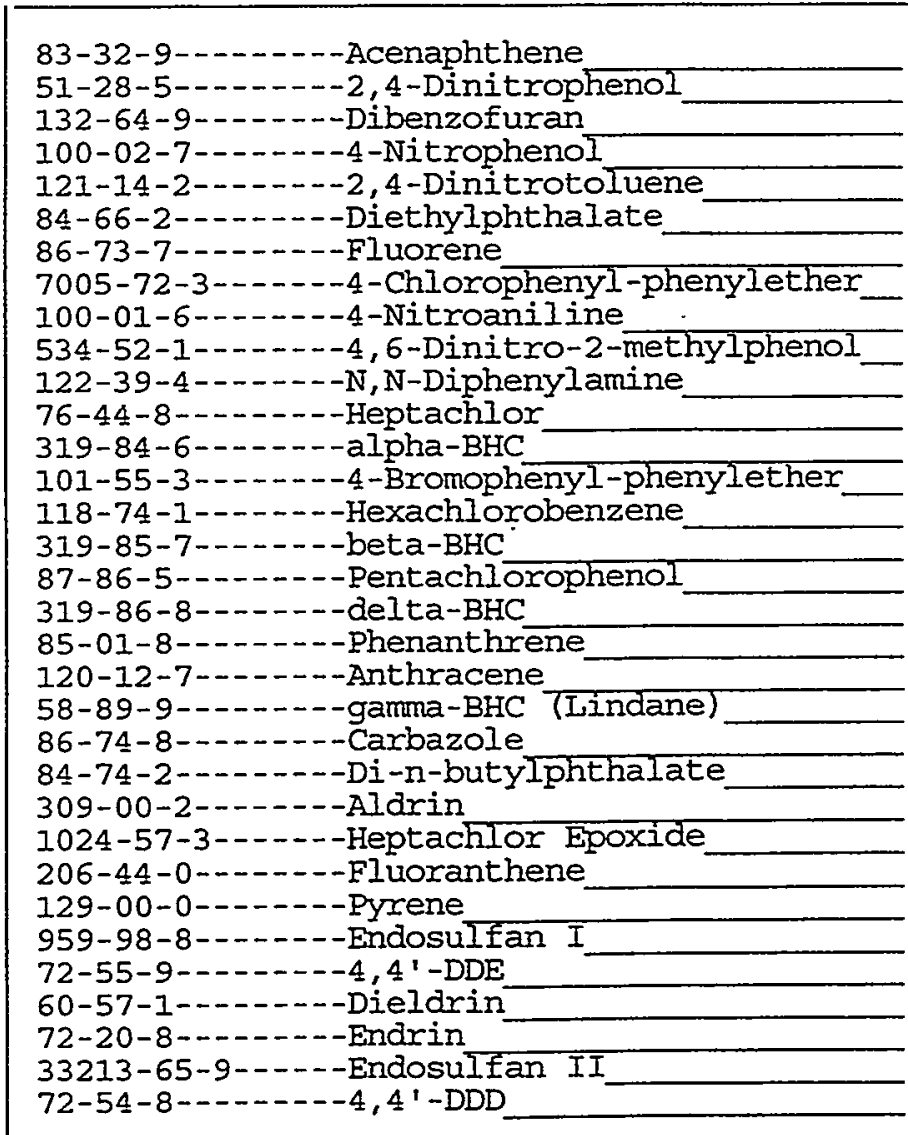

FORM I SV-2
OLMO3.0 
Lab Name: PNNL

Lab Code: PNNL
Contract: BNFL
EPA SAMPLE NO.

AN-107D
Matrix: (soil/water) SOIID

Sample wt/vol:

$4.8(\mathrm{~g} / \mathrm{mL}) \mathrm{G}$

Level: (low/med)

LOW

\% Moisture: 0

decanted: $(Y / N) N$

Concentrated Extract Volume: 2000 (uI)

Injection Volume: (uL)

GPC Cleanup: (Y/N) N $\mathrm{pH}: 11.0$
SAS NO.:

SDG No.: 990205

Lab Sample ID: 99-0651D

Lab File ID: 99010512 .

Date Received:

Date Extracted:

Date Analyzed: 02/05/99

Dilution Factor: 1.0

CAS NO.

COMPOUND

CONCENTRATION UNITS: (ug/L or $u g / K g$ ) UG/KG

\begin{tabular}{r|l|}
4100 & $\mathrm{U}$ \\
4100 & $\mathrm{U}$ \\
4100 & $\mathrm{U}$ \\
4100 & $\mathrm{U}$ \\
4100 & $\mathrm{U}$ \\
4100 & $\mathrm{U}$ \\
4100 & $\mathrm{U}$ \\
4100 & $\mathrm{U}$ \\
640 & $\mathrm{~J}$ \\
4100 & $\mathrm{U}$ \\
4100 & $\mathrm{U}$ \\
4100 & $\mathrm{U}$ \\
4100 & $\mathrm{U}$ \\
4100 & $\mathrm{U}$ \\
4100 & $\mathrm{U}$ \\
4100 & $\mathrm{U}$ \\
1400 & $\mathrm{~J}$ \\
260 & $\mathrm{~J}$ \\
2600 & $\mathrm{~J}$ \\
4100 & $\mathrm{U}$ \\
4100 & $\mathrm{U}$ \\
65 & $\mathrm{~J}$ \\
4100 & $\mathrm{U}$ \\
4100 & $\mathrm{U}$ \\
4100 & $\mathrm{U}$ \\
4100 & $\mathrm{U}$ \\
4100 & $\mathrm{U}$ \\
4100 & $\mathrm{U}$ \\
4100 & $\mathrm{U}$ \\
4100 & $\mathrm{U}$ \\
4100 & $\mathrm{U}$ \\
4100 & $\mathrm{U}$ \\
4100 & $\mathrm{U}$ \\
& \\
\end{tabular}

FORM I SV-3 
Lab Name: PNNL

Iab Code: PNNL

Case No.:
Contract : BNFL

SAS NO.:
EPA SAMPLE NO.

AN-107D

SDG NO.: 990205
Matrix: (soil/water) SOLID

Sample wt/vol:

$4.8(\mathrm{~g} / \mathrm{mL}) \mathrm{G}$

Level: (low/med) LOW

$\%$ Moisture: 0 decanted: $(Y / N) N$

Concentrated Extract Volume: 2000 (UI)

Injection Volume:

(uL)

GPC Cleanup: $(\mathrm{Y} / \mathrm{N}) \mathrm{N} \quad \mathrm{pH}: 11.0$

Lab Sample ID: 99-0651D

Lab File ID: 99010512

Date Received:

Date Extracted:

Date Analyzed: 02/05/99

Dilution Factor: 1.0

CONCENTRATION UNITS:

CAS NO. COMPOUND $(\mathrm{ug} / \mathrm{L}$ or $\mathrm{ug} / \mathrm{Kg}) \mathrm{UG} / \mathrm{KG}$

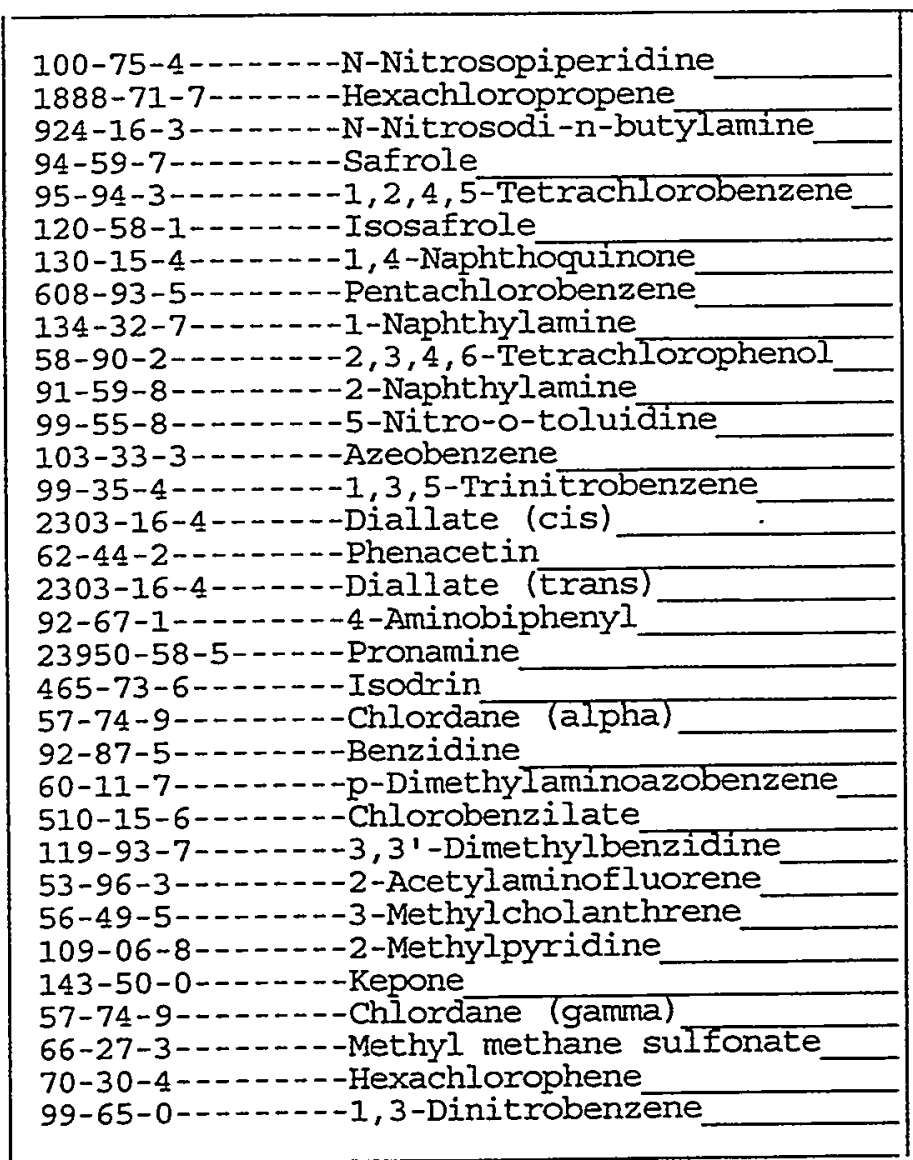

FORM I SV-4

OLMO3. 0 
Lab Name: PNNL

Lab Code: PNNL

Case No.:

Matrix: (soil/water) SOLID

Sample wt/vol:

$$
4.8(\mathrm{~g} / \mathrm{mL}) \quad \mathrm{G}
$$

Level: (low/med)

IOW

decanted: $(Y / N) N$

: Moisture: 0

Concentrated Extract Volume: 2000 (UL)

Injection Volume: (uIs)
Contract: BNFL

SAS NO. :

Lab Sample ID: 99-0651D

Lab File ID: 99010512

Date Received:

Date Extracted:

Date Analyzed: 02/05/99

Dilution Factor: 1.0

GPC Cleanup: $\quad(\mathrm{Y} / \mathrm{N}) \mathrm{N}$

$\mathrm{pH}: 11.0$

CAS NO.

COMPOUND

CONCENTRATION UNITS:

(ug/L or ug/Kg) UG/KG

$\bar{Q}$ 
Lab Name: PNNL

Lab code: PNNL

Case No.:
Contract: BNFL

SAS NO.:
EPA SAMPLE NO.

AN-107-MS

SDG No.: 990205
Matrix: (soil/water) SOIID

Sample wt/vol:

$2.7(\mathrm{~g} / \mathrm{mL}) \mathrm{G}$

Level: (low/med) LoW

$\because$ Moisture: 0 decanted: $(\mathrm{Y} / \mathrm{N}) \mathrm{N}$

Concentrated Extract Volume: 2000 (UI)

Injection Volume:

(uI)
Lab Sample ID: 99-0651-MS

Lab File ID: 99010513

Date Received:

Date Extracted:

Date Analyzed: 02/05/99

Dilution Factor: 1.0

GPC Cleanup: $(Y / N) \mathrm{N} \quad \mathrm{pH}: 11.0$

CAS NO.

COMPOUND

CONCENTRATION UNITS:

(ug/L or $u g / \mathrm{Kg}$ ) UG/KG

$\bar{Q}$

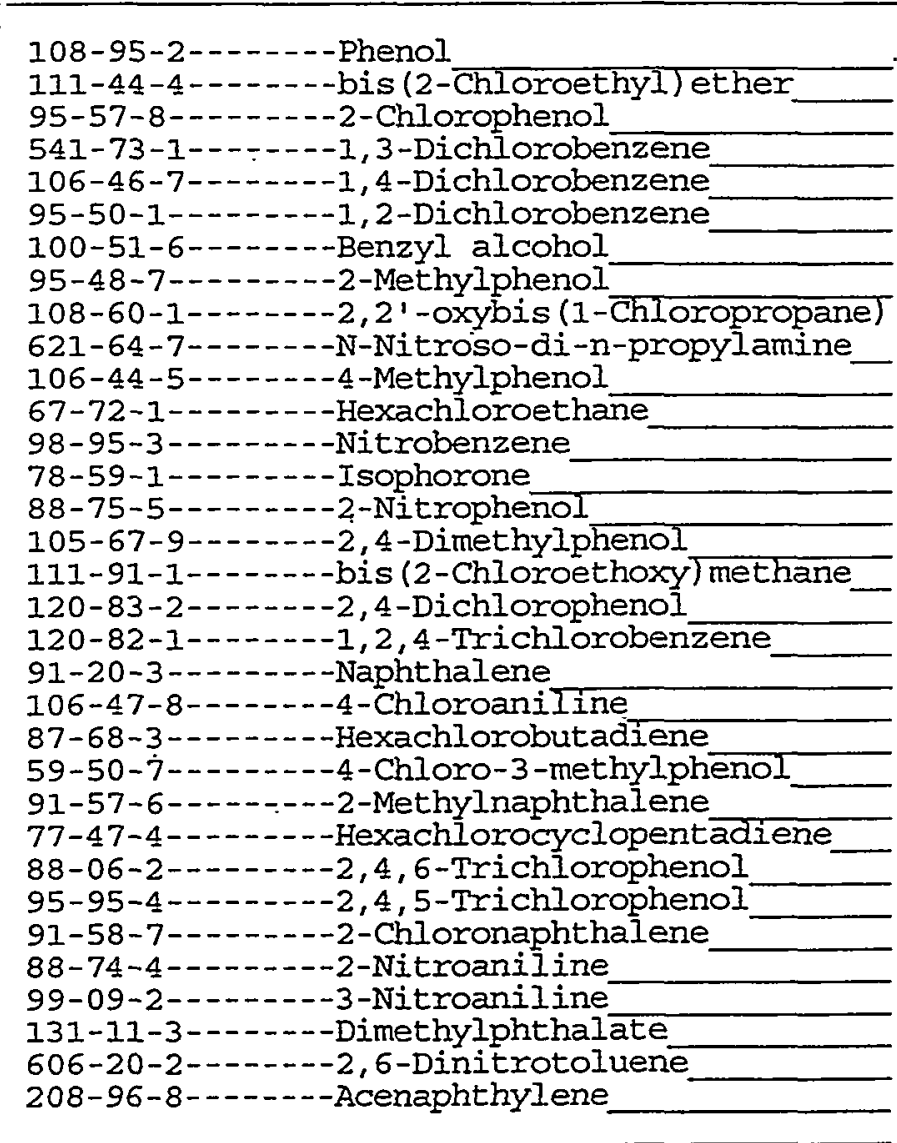

FORM I SV-I

\begin{tabular}{r|r|l|}
3100 & $\mathrm{~J}$ \\
7300 & $\mathrm{U}$ \\
14000 & \\
7300 & $\mathrm{U}$ \\
23000 & \\
7300 & $\mathrm{U}$ \\
7300 & $\mathrm{U}$ \\
7300 & $\mathrm{U}$ \\
7300 & $\mathrm{U}$ \\
22000 & \\
7300 & $\mathrm{U}$ \\
7300 & $\mathrm{U}$ \\
7300 & $\mathrm{U}$ \\
7300 & $\mathrm{U}$ \\
25000 & \\
7300 & $\mathrm{U}$ \\
7300 & $\mathrm{U}$ \\
7300 & $\mathrm{U}$ \\
26000 & \\
7300 & $\mathrm{U}$ \\
7300 & $\mathrm{U}$ \\
7300 & $\mathrm{U}$ \\
3700 & $\mathrm{~J}$ \\
7300 & $\mathrm{U}$ \\
7300 & $\mathrm{U}$ \\
7300 & $\mathrm{U}$ \\
7300 & $\mathrm{U}$ \\
7300 & $\mathrm{U}$ \\
7300 & $\mathrm{U}$ \\
7300 & $\mathrm{U}$ \\
7300 & $\mathrm{U}$ \\
7300 & $\mathrm{U}$ \\
7300 & $\mathrm{U}$ \\
& \\
\hline & \\
\hline
\end{tabular}

OTM03.0 
Lab Name: PNNL

Iab code: PNNL

Case No.:

Matrix: (soil/water) SOIID

Sample wt/vol:

$2.7(\mathrm{~g} / \mathrm{mL}) \mathrm{G}$

Level: (low/med)

LOW

\% Moisture: 0 decanted: (Y/N) N

Concentrated Extract Volume: 2000 (uU)

Injection Volume: (UI)

$\mathrm{pH}: 11.0$
EPA SAMPLE NO.

Contract: BNFL

SAS NO. :

SDG No.: 990205
GPC Cleanup: $(Y / N)$ N

CAS NO.
COMPOUND
Lab Sample ID: 99-0651-MS

Lab File ID: $\quad 99010513$

Date Received:

Date Extracted:

Date Analyzed: 02/05/99

Dilution Factor: 1.0

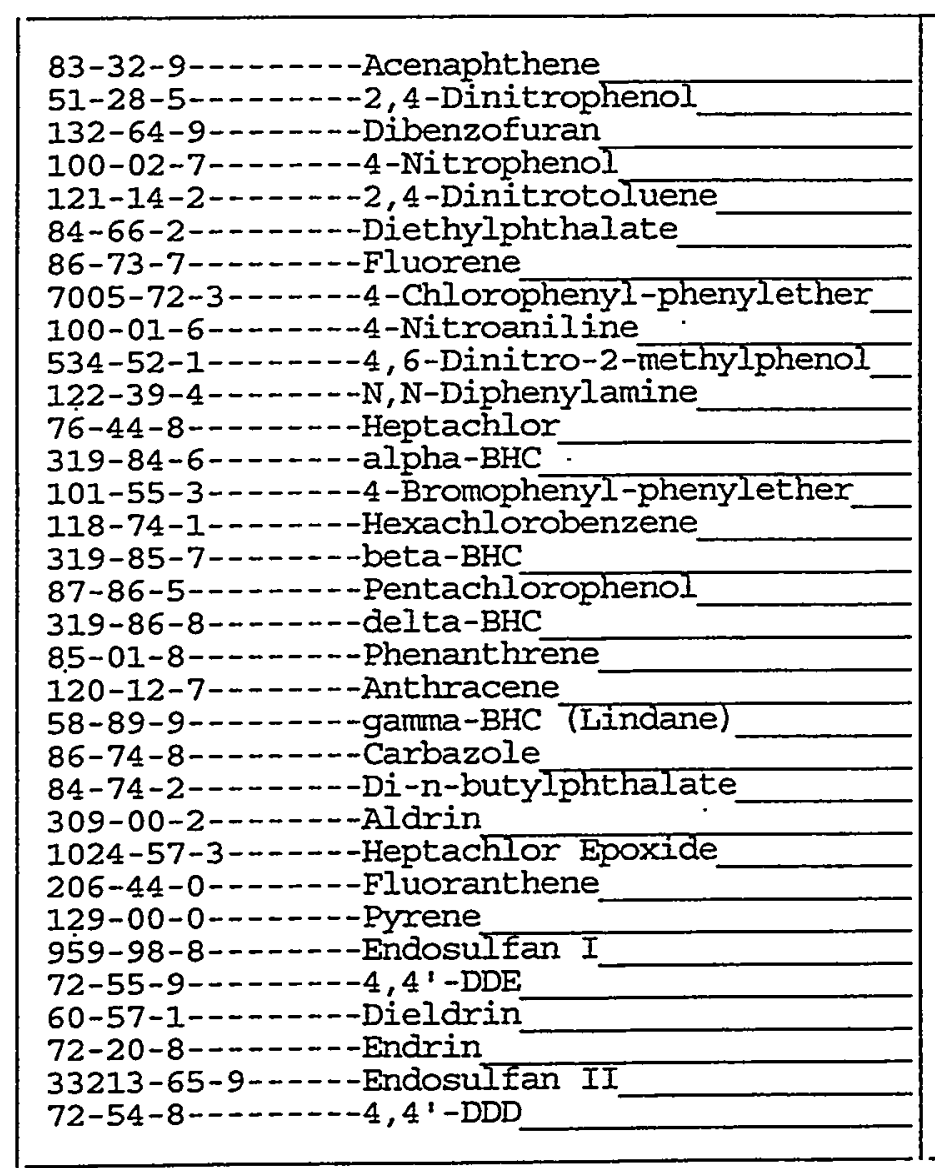

FORM I SV-2
CONCENTRATION UNITS: (ug/I or $u g / K g$ ) UG/KG 
Lab Name: PNNL

Iab code: PNNL
Contract: BNFL

SAS NO. :
EPA SAMPLE NO.

AN-107-MS
Lab Sample ID: 99-065I-MS

Lab File ID: 99010513

Date Received:

Date Extracted:

Date Analyzed: 02/05/99

Dilution Factor: 1.0

Injection Volume: (uI)

CONCENTRATION UNITS: (ug/I or $u g / \mathrm{Kg}$ ) UG/KG

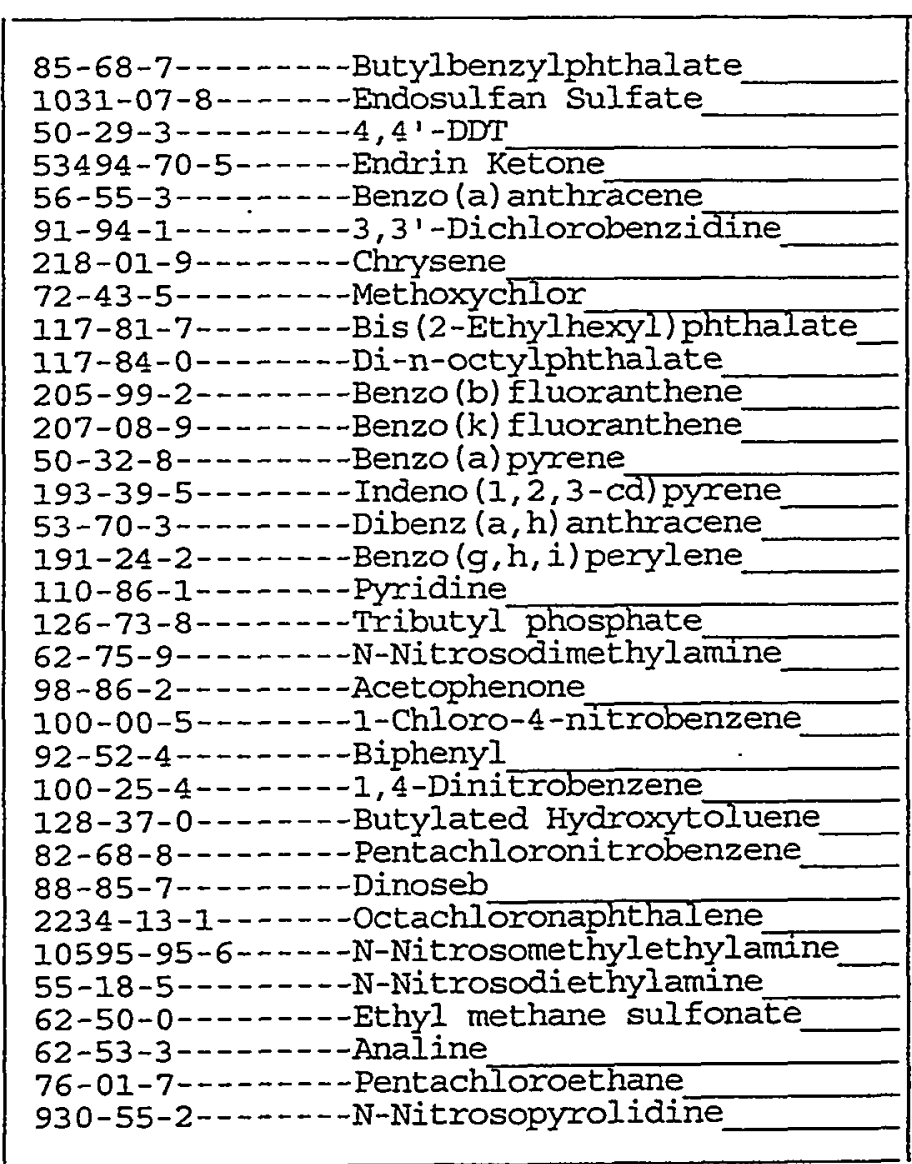

\begin{tabular}{r|l}
7300 & $\mathrm{U}$ \\
7300 & $\mathrm{U}$ \\
7300 & $\mathrm{U}$ \\
7300 & $\mathrm{U}$ \\
7300 & $\mathrm{U}$ \\
7300 & $\mathrm{U}$ \\
7300 & $\mathrm{U}$ \\
7300 & $\mathrm{U}$ \\
1200 & $\mathrm{~J}$ \\
7300 & $\mathrm{U}$ \\
7300 & $\mathrm{U}$ \\
7300 & $\mathrm{U}$ \\
7300 & $\mathrm{U}$ \\
7300 & $\mathrm{U}$ \\
7300 & $\mathrm{U}$ \\
7300 & $\mathrm{U}$ \\
19000 & \\
19000 & \\
530 & $\mathrm{~J}$ \\
23000 & \\
22000 & \\
\hline 20000 & - \\
24000 & \\
790 & $\mathrm{~J}$ \\
26000 & \\
\hline 22000 & \\
24000 & \\
7300 & $\mathrm{U}$ \\
7300 & $\mathrm{U}$ \\
7300 & $\mathrm{U}$ \\
29 & $\mathrm{~J}$ \\
7300 & $\mathrm{U}$ \\
7300 & $\mathrm{U}$ \\
\hline & \\
\hline
\end{tabular}

OIM03.0 
Lab Name: PNNL

Lab code: PNNL
Contract: BNET
EPA SAMPLE NO.

AN-107-MS

SDG No.: 990205

Matrix: (soil/water) SOLID

Lab Sample ID: 99-0651-MS

Sample wt/vol:

$2.7(\mathrm{~g} / \mathrm{mL}) \mathrm{G}$

Lab File ID: 99010513

Ievel : (low/med)

LOW

\% Moisture: 0 decanted: $(\mathrm{Y} / \mathrm{N}) \mathrm{N}$

Date Received:

Concentrated Extract Volume: 2000 (uس)

Date Extracted:

Injection Volume: (uL)

Date Analyzed: 02/05/99

Dilution Factor: 1.0

GPC Cleanup: $(\mathrm{Y} / \mathrm{N}) \mathrm{N} \quad \mathrm{pH}: 11.0$

CONCENTRATION UNITS:

CAS NO.

COMPOUND

(ug/L or $\mathrm{ug} / \mathrm{Kg}$ ) UG/KG

$\overline{0}$

100-75-4-...-.-N-Nitrosopiperidine

1888-71-7--.---Hexachloropropene

924-1:6-3-.----N-Nitrosodi-n-butylamine

94-59-7-.-.----Safrole

95-94-3--..-- 1, 2, 4, 5-Tetrachlorobenzene

120-58-1-...--Isosafrole

130-15-4-...-.-1, 4-Naphthoquinone

608-93-5-..---Pentachlorobenzene

134-32-7-.....-Naphthylamine

58-90-2--.----2,3,4, 6-Tetrachlorophenol

91-59-8-.--..-2-Naphthylamine

99-55-8-....--5-Nitro-o-toluidine

103-33-3-...-.-Azeobenzene

99-35-4-.-----1, 3, 5-Trinitrobenzene

2303-16-4-..-Diallate (cis)

62-44-2-.....--Phenacetin

2303-16-4-..--Diallate (Erans)

92-67-1------4-Aminobiphenyl

23950-58-5--.--Pronamine

465-73-6-...-..-Isodrin

57-74-9-..... Chlordane (alpha)

92-87-5-.-----Benzidine

60-11-7-..---p-Dimethylaminoazobenzene

510-15-6-...-Chlorobenzilate

119-93-7-...--3,3'-Dimethylbenzidine

53-96-3-..---2-Acetylaminofluorene

56-49-5--.-.-3-Methylcholanthrene

109-06-8-.---2-Methylpyridine

143-50-0-.-----Kepone

57-74-9-...--Chlordane (gamma)

66-27-3-...-.-Methyl methane sulfonate

70-30-4-...--Hexachlorophene

99-65-0-..----1, 3-Dinitrobenzene

\begin{tabular}{|c|c|c|}
\hline $\begin{array}{l}. \\
. \\
.\end{array}$ & $\begin{array}{r}7300 \\
7300 \\
7300 \\
7300 \\
7300 \\
7300 \\
26 \\
7300 \\
7300 \\
130 \\
7300 \\
7300 \\
7300 \\
570 \\
7300 \\
7300 \\
7300 \\
7300 \\
7300 \\
7300 \\
7300 \\
7300 \\
7300 \\
7300 \\
7300 \\
7300 \\
7300 \\
7300 \\
7300 \\
7300 \\
7300 \\
7300 \\
7300\end{array}$ & $\begin{array}{l}U \\
U \\
U \\
U \\
U \\
U \\
U \\
U \\
U \\
J \\
U \\
U \\
U \\
J \\
U \\
U \\
U \\
U \\
U \\
U \\
U \\
U \\
U \\
U \\
U \\
U \\
U \\
U \\
U \\
U \\
U \\
U \\
U\end{array}$ \\
\hline
\end{tabular}

OIMO3.0 
Lab Name: PNNL

Irab Code: PNNL

Case No.:

Matrix: (soil/water) SOLID

Sample wt/vol:

$2.7(\mathrm{~g} / \mathrm{mL}) \mathrm{G}$

Level: (low/med) LOW

\% Moisture: 0

decanted: $(\mathrm{Y} / \mathrm{N}) \mathrm{N}$

Concentrated. Extract Volume: 2000 (UL)

Injection Volume: (uI)
Contract: BNFL

SAS NO.:
AN-107-MS

SDG No.: 990205

GPC Cleanup: $(\mathrm{Y} / \mathrm{N}) \mathrm{N}$

$\mathrm{pH}: 11.0$

Lab Sample ID: 99-0651-MS

Lab File ID: 99010513

Date Received:

Date Extracted:

Date Analyzed: 02/05/99

Dilution Factor: 1.0

CAS NO

COMPOUND

CONCENTRATION UNITS:

(ug/L or ug/Kg) UG/KG

$\bar{Q}$

87-65-0--..--2, 6-Dichlorophenol

7300 
Lab Name: PNNL

Lab code: PNNL

Case No.:

Matrix: (soil/water) SOLID

Sample wt/vol:

$$
3.3(\mathrm{~g} / \mathrm{mL}) \mathrm{G}
$$

Level: (low/med) LOW

$\%$ Moisture: 0 decanted: $(\mathrm{Y} / \mathrm{N}) \mathrm{N}$

Concentrated Extract Volume: 2000 (UI) Injection Volume: (uU)
EPA SAMPLE NO.

AN-107-MSD
SDG No.: 990205
Contract: BNFL

SAS NO.:
Lab Sample ID: 99-0651-MSD

Lab File ID: 99010514

Date Received:

Date Extracted:

Date Analyzed: 02/05/99

Dilution Factor: 1.0
GPC Cleanup: $\quad(\mathrm{Y} / \mathrm{N}) \mathrm{N}$
$\mathrm{pH}: 11.0$

$\begin{array}{ll}\text { CONCENIRATION UNITS: } \\ \text { CAS NO. COMPOUND } & \text { (ug/L or ug/Kg) UG/KG }\end{array}$

108-95-2-.------Phenol

111-44-4-....--bis (2-Chloroethyl) ether

95-57-8-...-.-2-Chlorophenol

541-73-1-----1, 3-Dichlorobenzene

106-46-7-.....-1, 4-Dichlorobenzene

95-50-1--.-.-1, 2-Dichlorobenzene

100-51-6-.......-Benzyl alcohol

95-48-7---.-.-2-Methylphenol

108-60-1-..---2,2'-oxybis (1-Chloropropane)

621-64-7--...-N-Nitroso-di-n-propylamine

106-44-5-.---4-Methylphenol

67-72-1-..---Hexachloroethane

98-95-3--.----Nitrobenzene

78-59-1-...---Isophorone

88-75-5-......-2-Nitropheñl

105-67-9--.---2,4-Dimethylphenol

111-91-1--.---bis (2-Chloroethoxy) methane

120-83-2-------2,4-Dichlorophenol

120-82-1------1, 2, 4-Trichlorobenzene

91-20-3-.....-Naphthalene

106-47-8------4-Chloroaniline

87-68-3-.....-Hexachlorobutadiene

59-50-7-...--4-Chloro-3-methylphenol

91-57-6--.--2-Methylnaphthalene

77-47-4-...-- - Hexach Iorocyclopentadiene

88-06-2-...--2,4,6-Trichlorophenol

95-95-4-------2,4,5-Trichlorophenol

91-58-7-...---2-Chloronaphthalene

88-74-4-....--2-Nitroaniline

99-09-2-...--3-Nitroaniline

131-11-3-...--Dimethylphthalate

606-20-2-----2, 6-Dinitrotoluene

208-96-8-...-.-Acenaphthylene

\begin{tabular}{r|r|l}
29000 & \\
6100 & $\mathrm{U}$ \\
31000 & \\
6100 & $\mathrm{U}$ \\
21000 & \\
6100 & $\mathrm{U}$ \\
6100 & $\mathrm{U}$ \\
10000 & \\
6100 & $\mathrm{U}$ \\
21000 & \\
24000 & \\
6100 & $\mathrm{U}$ \\
6100 & $\mathrm{U}$ \\
6100 & $\mathrm{U}$ \\
6100 & $\mathrm{U}$ \\
6100 & $\mathrm{U}$ \\
6100 & $\mathrm{U}$ \\
6100 & $\mathrm{U}$ \\
24000 & \\
6100 & $\mathrm{U}$ \\
6100 & $\mathrm{U}$ \\
6100 & $\mathrm{U}$ \\
31000 & \\
6100 & $\mathrm{U}$ \\
6100 & $\mathrm{U}$ \\
6100 & $\mathrm{U}$ \\
6100 & $\mathrm{U}$ \\
6100 & $\mathrm{U}$ \\
6100 & $\mathrm{U}$ \\
6100 & $\mathrm{U}$ \\
6100 & $\mathrm{U}$ \\
6100 & $\mathrm{U}$ \\
6100 & $\mathrm{U}$ \\
& \\
\hline & \\
\hline
\end{tabular}


Iab Name: PNNL

Lab Code: PNNL

Case No.:

Matrix: (soil/water) SOLID

Sample wt/vol:

$3.3(\mathrm{~g} / \mathrm{mL}) \mathrm{G}$

Level: (low/med) IOW

$\div$ Moisture: 0 decanted: $(\mathrm{Y} / \mathrm{N}) \mathrm{N}$

Concentrated Extract Volume: 2000 (uU)

Injection Volume:

(U士)

Contract: BNFL

SAS NO.:

Iab Sample ID: 99-0651-MSD

Lab File ID: $\quad 99010514$

Date Received:

Date Extracted:

Date Analyzed: 02/05/99

Dilution Factor: 1.0

GPC Cleanup: $\quad(\mathrm{Y} / \mathrm{N}) \mathrm{N} \quad \mathrm{pH}: 11.0$

CONCENTRATION UNITS:

CAS NO.

COMPOUND

(ug/I or $u g / K g$ ) UG/KG

$\overline{\mathrm{Q}}$

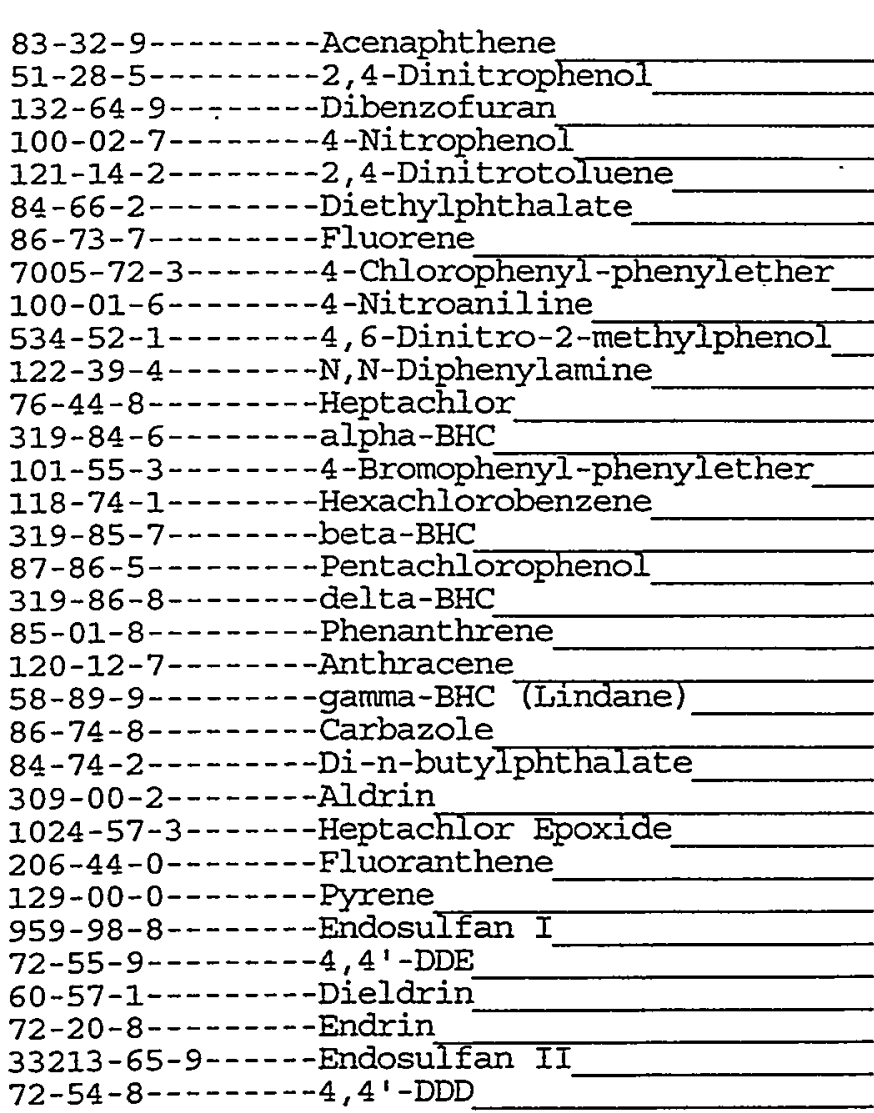

FORM I SV-2

OLM0 3.0 
Lab Name: PNNL

Lab Code: PNNL

Matrix: (soil/water) SOLID

Sample wt/vol:

$$
3.3(\mathrm{~g} / \mathrm{mL}) \mathrm{G}
$$

Contract: BNFL

SAS NO. :
EPA SAMPIE NO.

AN-107-MSD

SDG No.: 990205
Level: (low/med) LOW

$\%$ Moisture: 0 decanted: $(Y / N) N$

Concentrated Extract Volume: 2000 (UT)

Injection Volume:

(ur)

GPC Cleanup: (Y/N) N $\mathrm{pH}: 11.0$
Lab Sample ID: 99-0651-MSD

Lab File ID: $\quad 99010514$

Date Received:

Date Extracted:

Date Analyzed: 02/05/99

Dilution Factor: 1.0

CAS NO.

COMPOUND

CONCENTRATION UNITS:

(ug/L or $u g / \mathrm{Kg}$ ) UG/KG

$\bar{Q}$

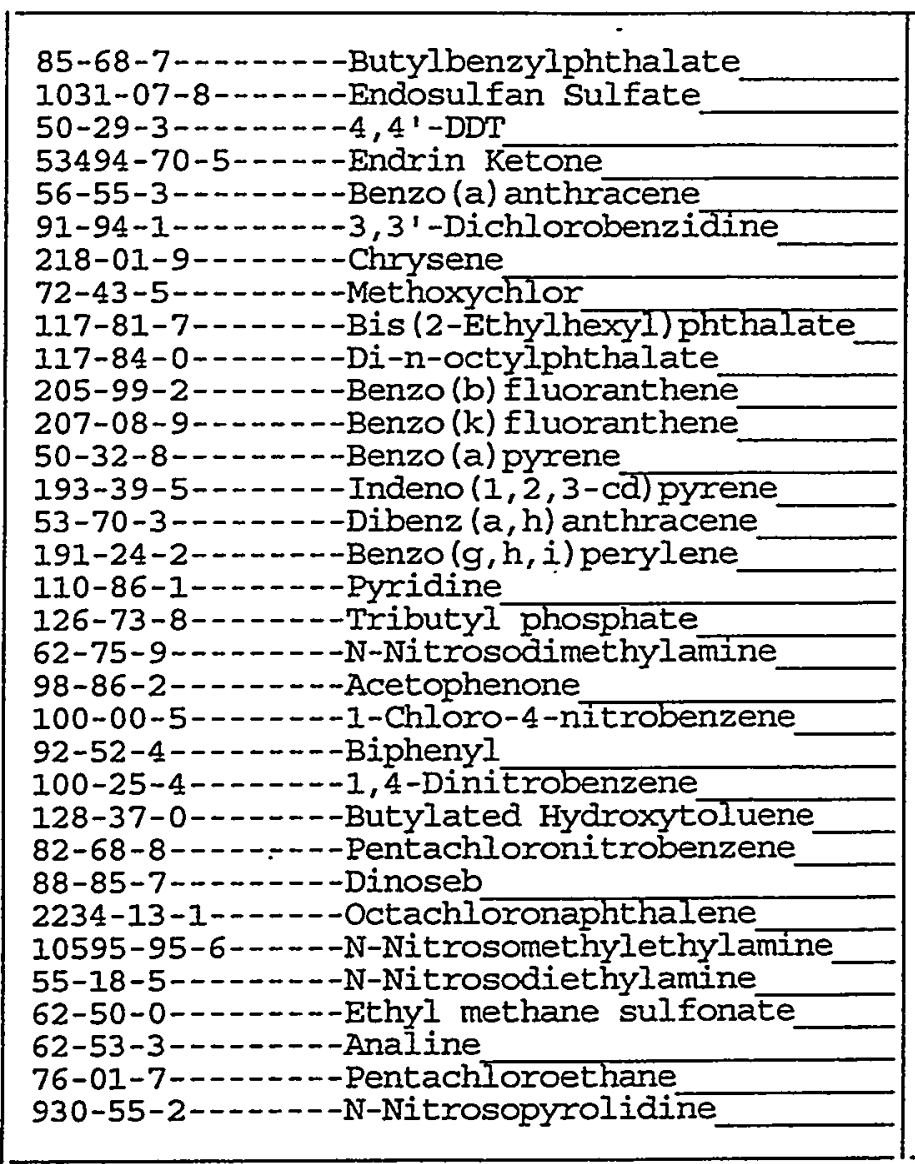

FORM I SV-3

OLM03. 0 
Lab Name: PNNL

Lab Code: PNNL
Contract: BNFL
AN-107-MSD

SDG NO. : 990205
Matrix: (soil/water) SoLID

Sample wt/vol:

$$
3.3(\mathrm{~g} / \mathrm{mI}) \mathrm{G}
$$

Level: (low/med)

LOW

\% Moisture: 0

decanted: $(\mathrm{Y} / \mathrm{N}) \mathrm{N}$

Concentrated Extract Volume: 2000 (uI)

Injection Volume:

(ut)

GPC Cleanup: $(Y / N) N \quad \mathrm{pH}: 11.0$
Lab Sample ID: 99-0651-MSD

Lab File ID: 99010514

Date Received:

Date Extracted:

Date Analyzed: 02/05/99

Dilution Factor: 1.0

CAS NO.

COMPOUND

CONCENTRATION UNITS:

(ug/L or ug/Kg) UG/KG

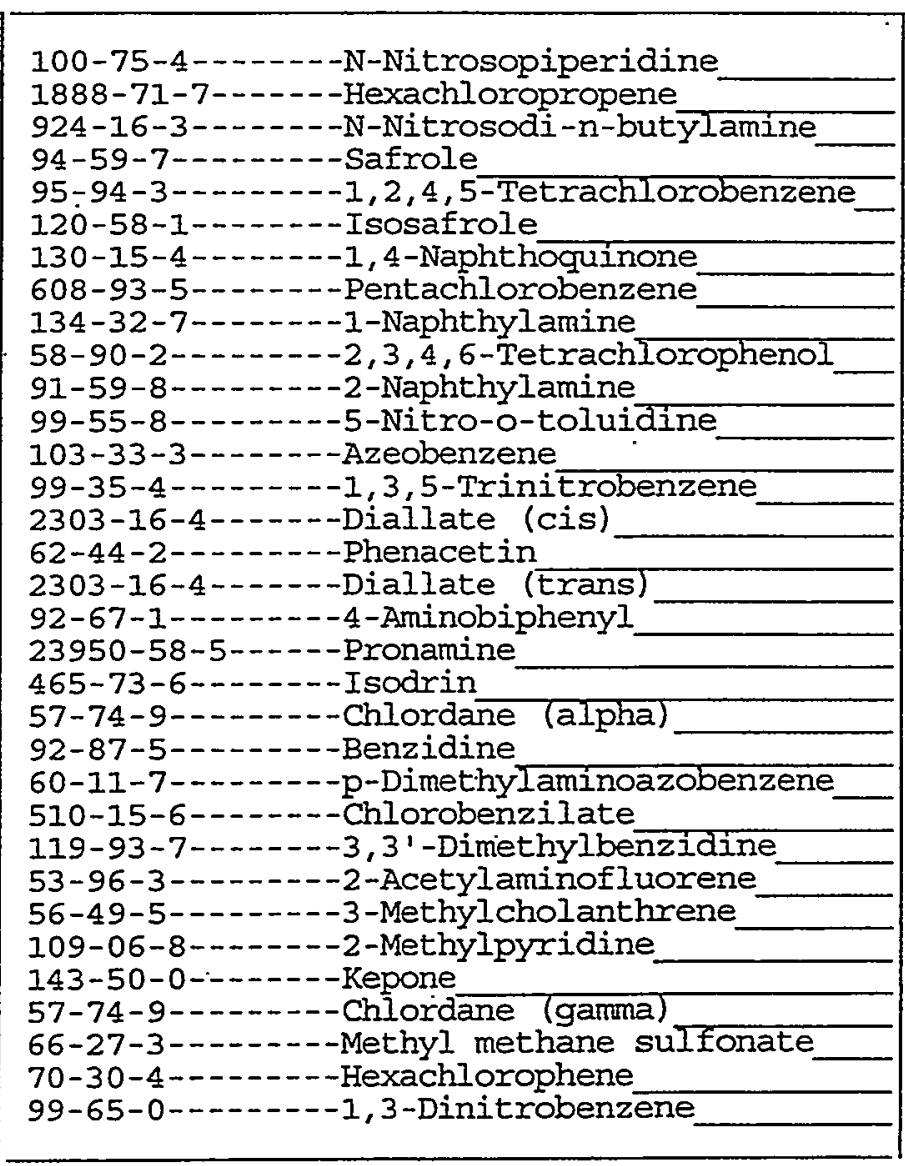

FORM I SV-4

OLM03.0 
Lab Name: PNNL,

Lab Code: PNNL

Case No.:

Matrix: (soil/water) SoIID

Sample wt/vol:

$3.3(\mathrm{~g} / \mathrm{mL}) \mathrm{G}$

Level: (low/med)

LOW

$\div$ Moisture: 0

decanted: $(Y / N) N$

Concentrated Extract Volume: 2000(uIs)

Injection Volume: (uL)

GPC Cleanup: $(\mathrm{Y} / \mathrm{N}) \mathrm{N}$

CAS NO.

$\mathrm{pH}:$ i1. 0
Contract: BNFL

SAS NO. :

Lab Sample ID: 99-0651-MSD

Lab File ID: 99010514

Date Received:

Date Extracted:

Date Analyzed: 02/05/99

Dilution Factor: 1.0

SDG NO.: 990205

AN-107-MSD

CONCENTRATION UNITS:

(ug/L or ug/Kg) UG/KG $\bar{Q}$ 
Lab Name: PNNL

Lab Code: PNNL
Contract: BNFL

Case No.:

SAS NO. :
METHOD BLANK
Matrix: (soil/water) SOLID

Sample wt/vol:

$5.0(\mathrm{~g} / \mathrm{mL}) \mathrm{G}$

Level: (low/med)

LOW

\% Moisture: 0

decanted: $(Y / N) N$

Concentrated Extract Volume: 2000 (ut)

Injection Volume:

(uI)

GPC Cleanup: $(\mathrm{Y} / \mathrm{N}) \mathrm{N} \quad \mathrm{pH}: 11.0$
Lab Sample ID: SBLK02

Lab File ID: 99010510

Date Received:

Date Extracted:

Date Analyzed: 02/05/99

Dilution Factor: 1.0

CONCENTRATION UNITS:

CAS NO.

COMPOUND

(ug/L or $\mathrm{ug} / \mathrm{Kg}$ ) UG/KG

$\bar{Q}$

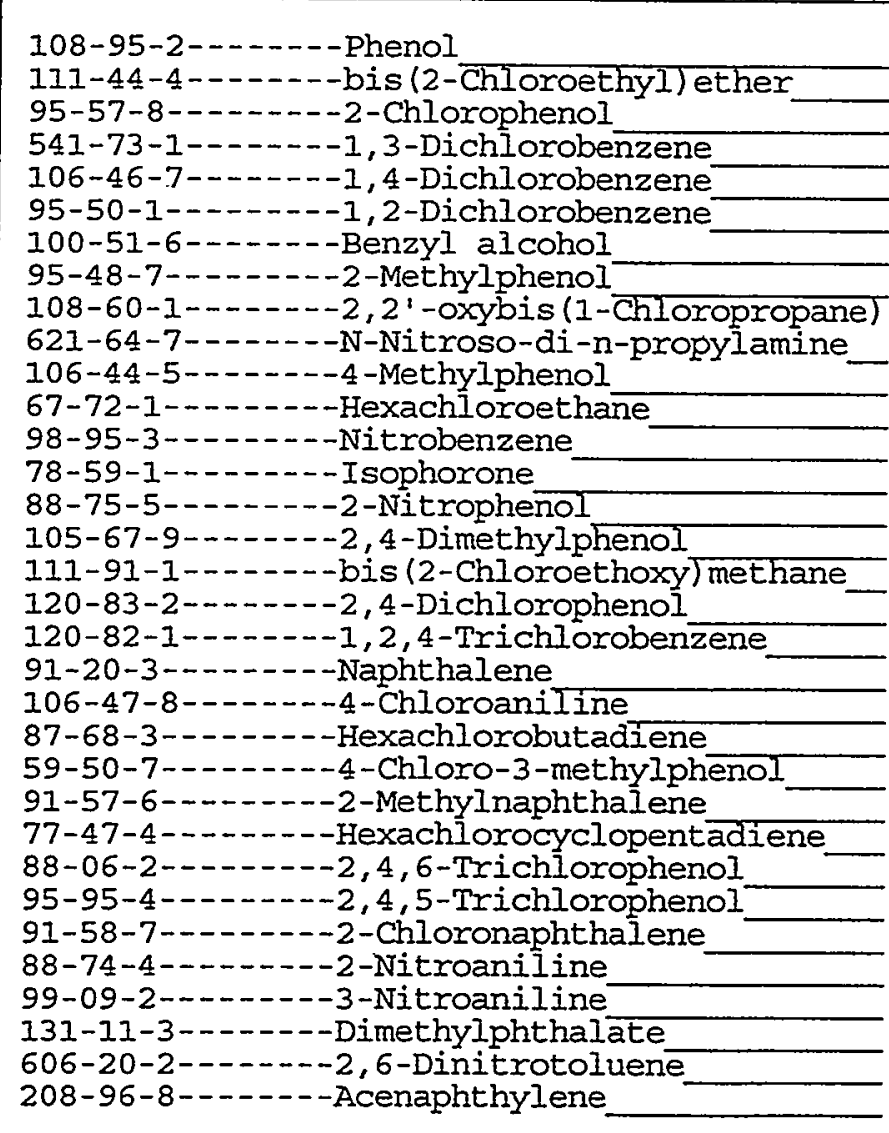

208-96-8-------Acenaphthylene

\begin{tabular}{|l|l}
4000 & $\mathrm{U}$ \\
4000 & $\mathrm{U}$ \\
4000 & $\mathrm{U}$ \\
4000 & $\mathrm{U}$ \\
4000 & $\mathrm{U}$ \\
4000 & $\mathrm{U}$ \\
4000 & $\mathrm{U}$ \\
4000 & $\mathrm{U}$ \\
4000 & $\mathrm{U}$ \\
4000 & $\mathrm{U}$ \\
4000 & $\mathrm{U}$ \\
4000 & $\mathrm{U}$ \\
4000 & $\mathrm{U}$ \\
4000 & $\mathrm{U}$ \\
4000 & $\mathrm{U}$ \\
4000 & $\mathrm{U}$ \\
4000 & $\mathrm{U}$ \\
4000 & $\mathrm{U}$ \\
4000 & $\mathrm{U}$ \\
4000 & $\mathrm{U}$ \\
4000 & $\mathrm{U}$ \\
4000 & $\mathrm{U}$ \\
4000 & $\mathrm{U}$ \\
4000 & $\mathrm{U}$ \\
4000 & $\mathrm{U}$ \\
4000 & $\mathrm{U}$ \\
4000 & $\mathrm{U}$ \\
4000 & $\mathrm{U}$ \\
4000 & $\mathrm{U}$ \\
4000 & $\mathrm{U}$ \\
4000 & $\mathrm{U}$ \\
4000 & $\mathrm{U}$ \\
4000 & $\mathrm{U}$ \\
& \\
\hline & \\
\hline
\end{tabular}

FORM I SV-I
OLM03. 0 
Lab Name: PNNL

Lab Code: PNNL
Contract: BNFL

SAS NO. :
EPA SAMPIE NO.

METHOD BLANK
Matrix: (soil/water) SOLID

Sample wt/vol:

$5.0(\mathrm{~g} / \mathrm{mL}) \mathrm{G}$

Ievel: (low/med)

LOW

$\%$ Moisture: 0

decanted: $(Y / N) N$

Concentrated Extract Volume: 2000 (uI)

Injection Volume: (uI)

GPC Cleanup: $(Y / N) N \quad \mathrm{NH}: 11.0$

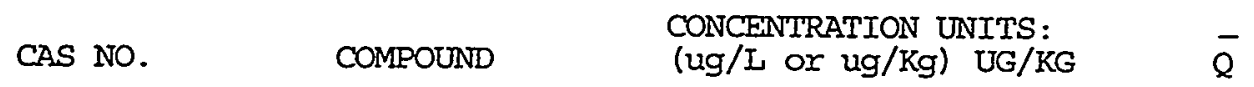

Iab Sample ID: SBLK02

Iab File ID: 99010510

Date Received:

Date Extracted:

Date Analyzed: 02/05/99

Dilution Factor: 1.0
$\bar{Q}$

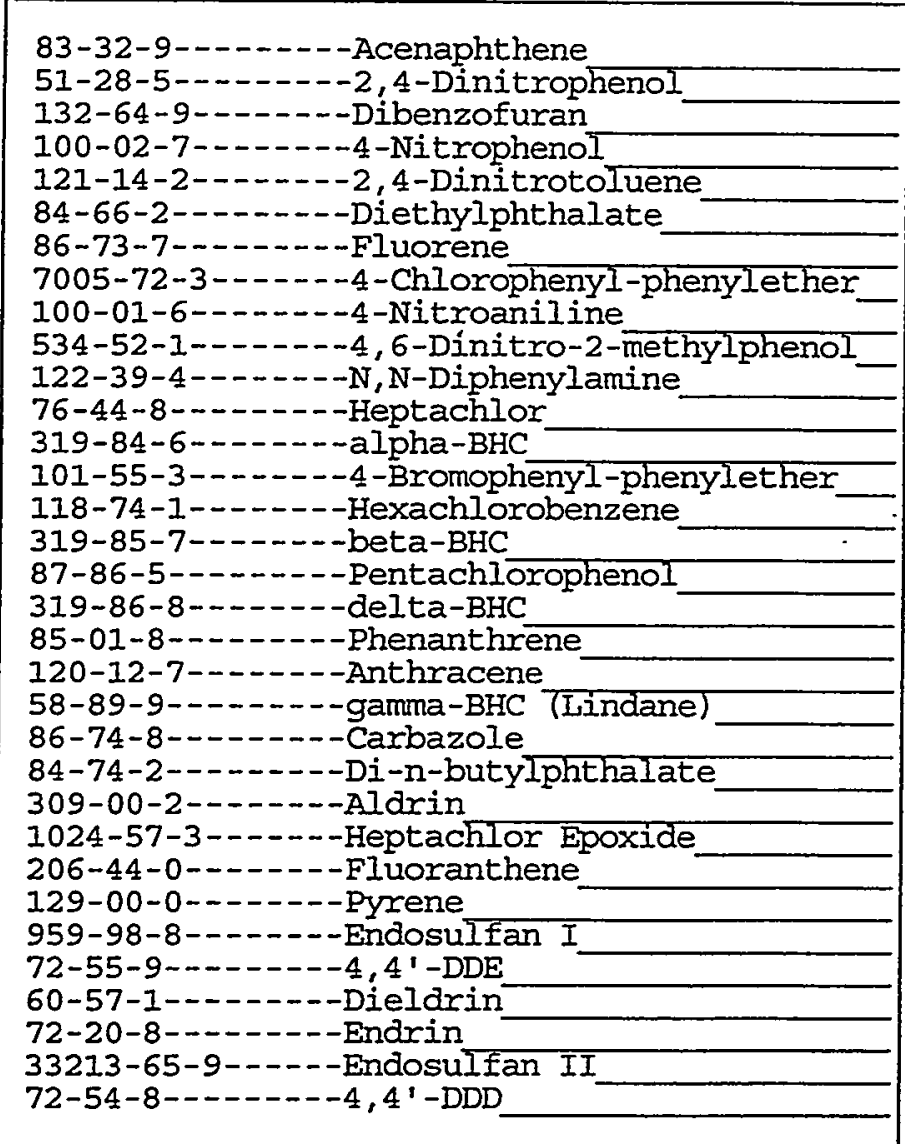

FORM I SV-2

\begin{tabular}{l|l}
\hline 4000 & $U$ \\
4000 & $U$ \\
4000 & $U$ \\
4000 & $U$ \\
4000 & $U$ \\
4000 & $U$ \\
4000 & $U$ \\
4000 & $U$ \\
4000 & $U$ \\
4000 & $U$ \\
4000 & $U$ \\
4000 & $U$ \\
4000 & $U$ \\
4000 & $U$ \\
4000 & $U$ \\
4000 & $U$ \\
4000 & $U$ \\
4000 & $U$ \\
4000 & $U$ \\
4000 & $U$ \\
4000 & $U$ \\
4000 & $U$ \\
4000 & $U$ \\
4000 & $U$ \\
4000 & $U$ \\
4000 & $U$ \\
4000 & $U$ \\
4000 & $U$ \\
4000 & $U$ \\
4000 & $U$ \\
4000 & $U$ \\
4000 & $U$ \\
4000 & $U$ \\
& \\
\hline
\end{tabular}

OLM03.0 
Lab Name: PNNL

Lab Code: PNNL

Case No.:

Matrix: (soil/water) SOLID

Sample wt/vol:

$5.0(\mathrm{~g} / \mathrm{mL}) \mathrm{G}$

Level: (low/med) Low

$\div$ Moisture: 0 decanted: $(Y / N) N$

Concentrated Extract Volume: 2000 (uI)

Injection Volume: (UT)

Contract: BNFL

SAS NO.:

Lab Sample ID: SBLK02

Iab File ID: $\quad 99010510$

Date Received:

Date Extracted:

Date Analyzed: 02/05/99

Dilution Factor: 1.0

GPC Cleanup: $(Y / N) N \quad \mathrm{pH}: 11.0$

$\begin{array}{lll}\text { CAS NO. COMPOUND } & \text { CONCENTRATION UNITS: } & \overline{\mathrm{Q}}\end{array}$

\begin{tabular}{|c|}
\hline 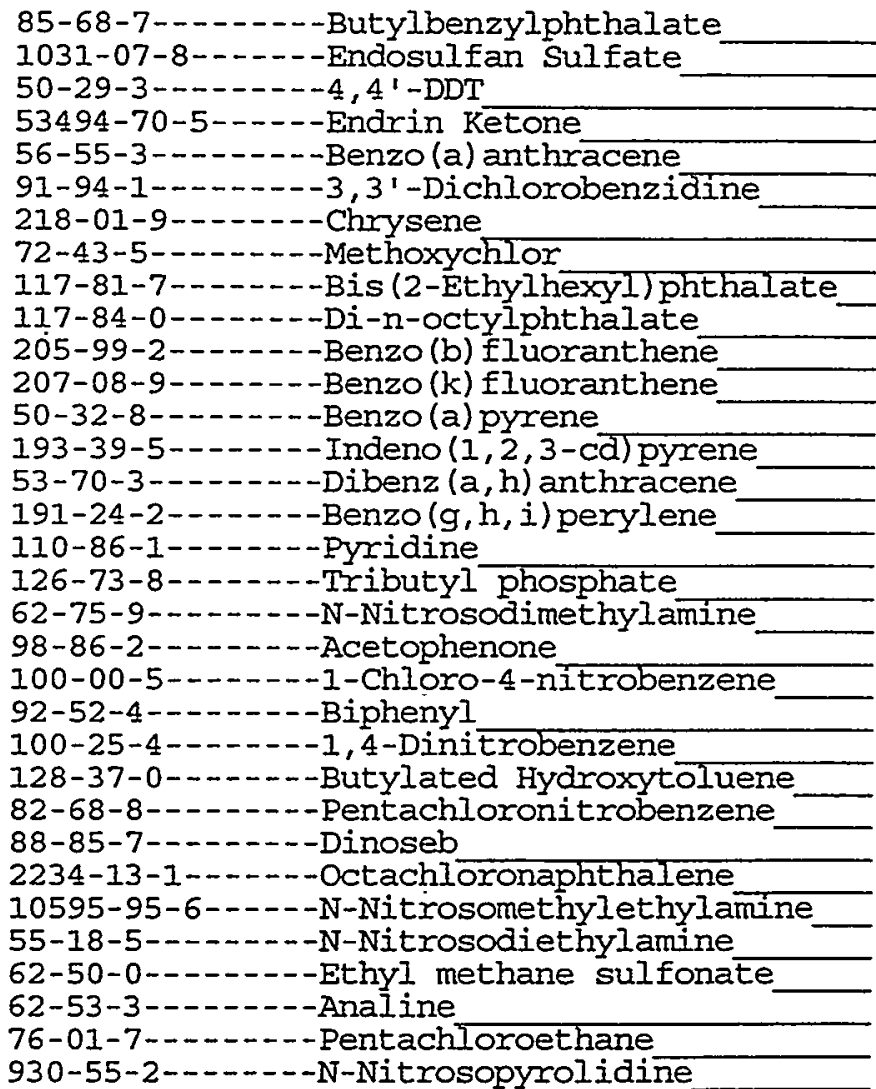 \\
\hline
\end{tabular}

FORM I SV-3

\begin{tabular}{l|l}
\hline 4000 & $U$ \\
4000 & $U$ \\
4000 & $U$ \\
4000 & $U$ \\
4000 & $U$ \\
4000 & $U$ \\
4000 & $U$ \\
4000 & $U$ \\
4000 & $U$ \\
4000 & $U$ \\
4000 & $U$ \\
4000 & $U$ \\
4000 & $U$ \\
4000 & $U$ \\
4000 & $U$ \\
4000 & $U$ \\
4000 & $U$ \\
4000 & $U$ \\
4000 & $U$ \\
4000 & $U$ \\
4000 & $U$ \\
4000 & $U$ \\
4000 & $U$ \\
4000 & $U$ \\
4000 & $U$ \\
4000 & $U$ \\
4000 & $U$ \\
4000 & $U$ \\
4000 & $U$ \\
4000 & $U$ \\
4000 & $U$ \\
4000 & $U$ \\
4000 & $U$ \\
& \\
\end{tabular}

OLM03.0 
Lab Name: PNNL

Lab Code: PNNL

Case No.:

Matrix: (soil/water) SOLID

Sample wt/vol:

5.0. $(\mathrm{g} / \mathrm{mL}) \mathrm{G}$

Level: (low/med) LOW.

$\because$ Moisture: 0 decanted: $(Y / N) N$

Concentrated Extract Volume: 2000 (UI)

Injection Volume: (uI)

Contract: BNFL SAS NO.:
EPA SAMPLE NO.

METHOD BLANK

SDG No.: 990205

Lab Sample ID: SBIKO2

Lab File ID: 99010510

Date Received:

Date Extracted:

Date Analyzed: 02/05/99

Dilution Factor: 1.0

CONCENTRATION UNITS: (ug/L or $\mathrm{ug} / \mathrm{Kg}$ ) UG/KG
$\bar{Q}$

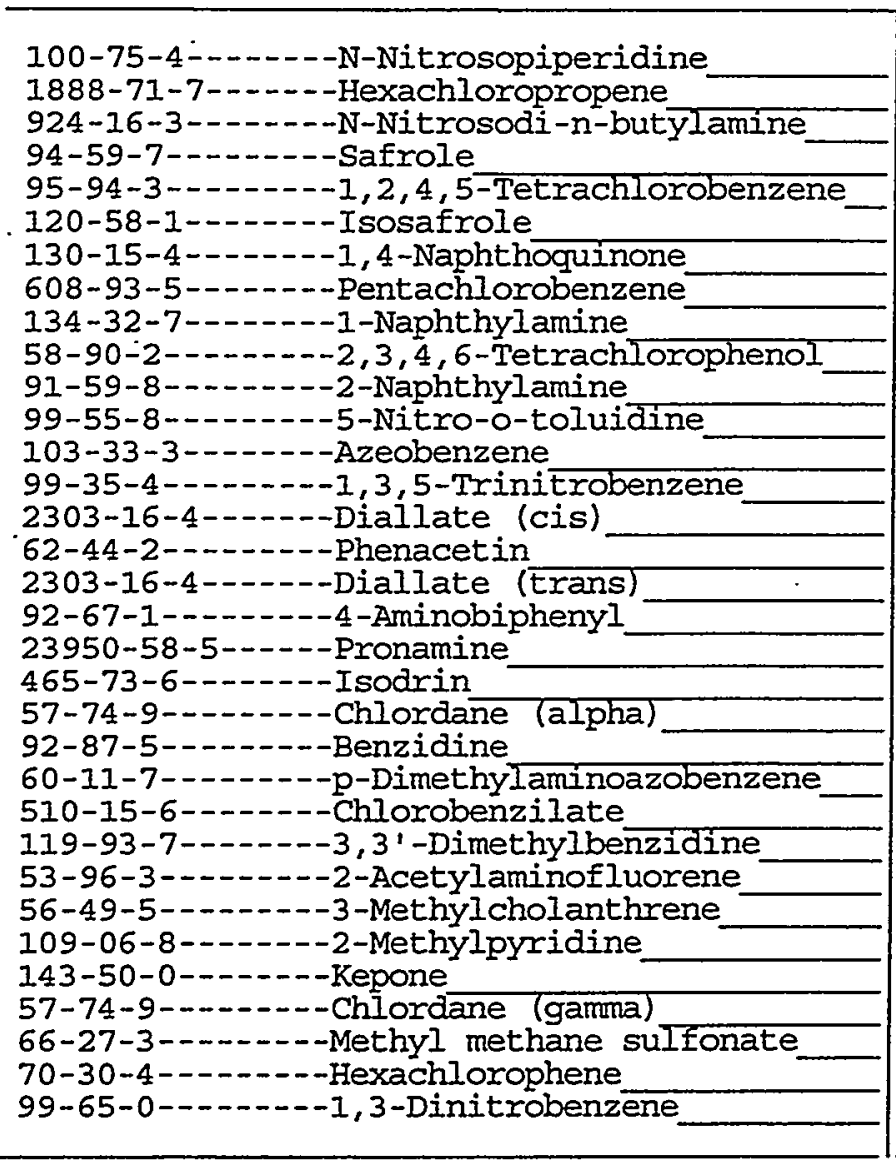

FORM I SV-4

\begin{tabular}{l|l|}
4000 & $U$ \\
4000 & $U$ \\
4000 & $U$ \\
4000 & $U$ \\
4000 & $U$ \\
4000 & $U$ \\
4000 & $U$ \\
4000 & $U$ \\
4000 & $U$ \\
4000 & $U$ \\
4000 & $U$ \\
4000 & $U$ \\
4000 & $U$ \\
4000 & $U$ \\
4000 & $U$ \\
4000 & $U$ \\
4000 & $U$ \\
4000 & $U$ \\
4000 & $U$ \\
4000 & $U$ \\
4000 & $U$ \\
4000 & $U$ \\
4000 & $U$ \\
4000 & $U$ \\
4000 & $U$ \\
4000 & $U$ \\
4000 & $U$ \\
4000 & $U$ \\
4000 & $U$ \\
4000 & $U$ \\
4000 & $U$ \\
4000 & $U$ \\
4000 & $U$ \\
& \\
\end{tabular}

OLM03.0 
Lab Name: PNNL

Iab code: PNNL

Matrix: (soil/water) SOLID

Sample wt/vol:

$5.0(\mathrm{~g} / \mathrm{mL}) \mathrm{G}$

Level: (low/med)

LOW

$\div$ Moisture: 0 decanted: $(\mathrm{Y} / \mathrm{N}) \mathrm{N}$

Concentrated Extract Volume: 2000 (u工)

Injection Volume: (uI)

Contract: BNFL,

SAS NO. :

Lab Sample ID: SBLK02

Lab File ID: $\quad 99010510$

Date Received:

Date Extracted:

Date Analyzed: 02/05/99

Dilution Factor: 1.0

GPC Cleanup: $(\mathrm{Y} / \mathrm{N}) \mathrm{N}$

$\mathrm{pH}: 11.0$

CAS NO.

COMPOUND

CONCENTRATION UNITS:

(ug/L or ug/Kg) UG/KG

$\bar{Q}$ 
Lab Name: PNNL

Iab code: PNNL,

Case No.:
Contract: BNFL

SAS NO. :
EPA SAMPLE NO.

AN- 107

SDG No.: 990205
Matrix: (soil/water) SUPERNATANT

Sample wt/vol:

$0.000(\mathrm{~g} / \mathrm{mL}) \mathrm{ML}$

Level: (low/med) LOW

\% Moisture: decanted: $(\mathrm{Y} / \mathrm{N})$

Concentrated Extract Volume: 2000 (uL)

Injection Volume: (UI)

Lab Sample ID: 99-0649

GPC Cleanup: $(Y / N) N \quad \mathrm{pH}: 11.0$

Number TICs found: 27

CONCENTRATION UNITS:

(ug/L or $u g / \mathrm{Kg}$ ) ug/L

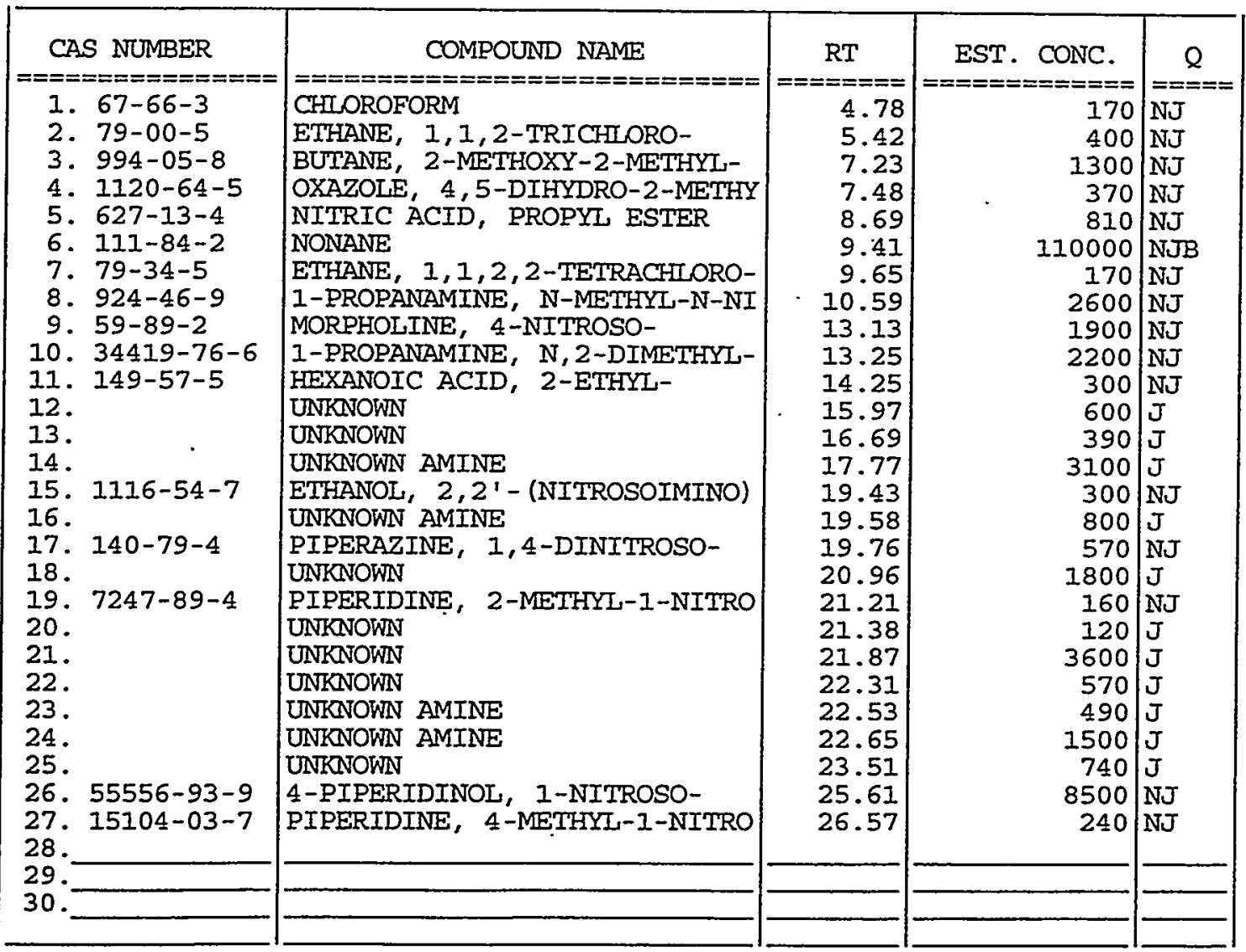


Lab Name: PNNL

Contract: BNFL

Lab Code: PNNI

Case No.:

SAS NO. :

Matrix: (soil/water) SUPERNATANT

Sample wt/vol:

$0.000(\mathrm{~g} / \mathrm{mL}) \mathrm{ML}$

Level: (low/med) LOW

$\div$ Moisture:

decanted: $(\mathrm{Y} / \mathrm{N})$

Concentrated Extract Volume: 2000 (uI)

Injection Volume: (uL)

$\mathrm{pH}: 11.0$
EPA SAMPLE NO.

AN-107D

SDG NO.: 990205

Lab Sample ID: 99-0649D

Lab File ID: $\quad 99010507$

Date Received:

Date Extracted:

Date Analyzed: 02/05/99

Dilution Factor: 1.0

CONCENTRATION UNITS:

Number TICs found: 23

(ug/L or ug/Kg) ug/t

\begin{tabular}{|c|c|c|c|c|}
\hline $\begin{array}{l}\text { CAS NUMBER } \\
=============== \\
1.67-66-3 \\
2 \cdot 79-00-5 \\
\text { 3. } 994-05-8 \\
4 \cdot 1120-64-5 \\
\text { 5. } 34075-28-0 \\
6 \cdot 625-58-1 \\
7 \cdot 111-84-2 \\
\text { 8. } 924-46-9 \\
9 \cdot 39884-53-2 \\
10 \cdot 59-89-2 \\
11 \cdot 111-14-8 \\
12 \cdot 149-57-5 \\
13 \cdot \\
14 \cdot \\
15 \cdot \\
16 \cdot 1116-54-7 \\
17 \cdot 3034-41-1 \\
18 \cdot 140-79-4 \\
19 \cdot \\
20 \cdot \\
21 . \\
22 \cdot 100-75-4 \\
23 \cdot 55556-86-0 \\
24 \cdot 1\end{array}$ & 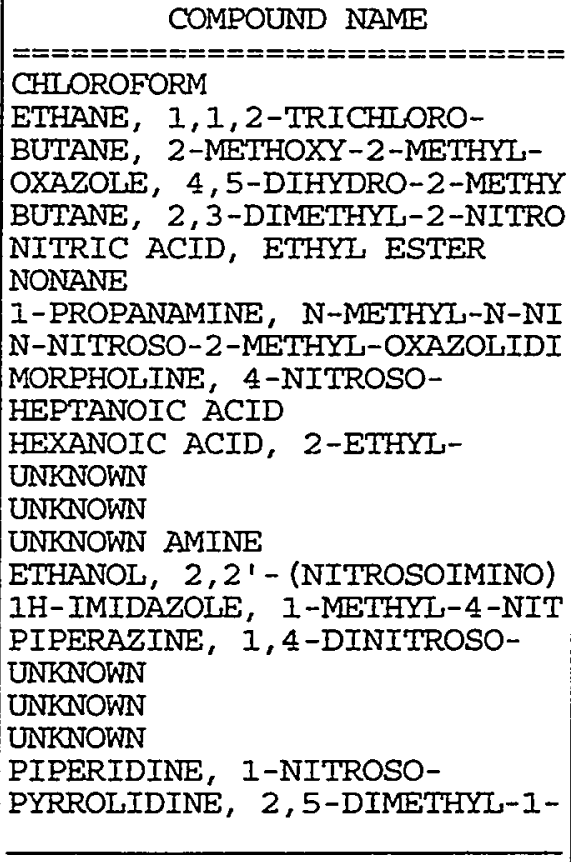 & $\begin{array}{r}R T \\
====== \\
4.79 \\
5.42 \\
7.24 \\
7.49 \\
7.98 \\
8.76 \\
9.43 \\
10.61 \\
11.67 \\
13.16 \\
13.45 \\
14.28 \\
14.44 \\
15.99 \\
17.77 \\
19.52 \\
19.64 \\
19.79 \\
20.76 \\
20.98 \\
22.32 \\
25.74 \\
26.68\end{array}$ & $\begin{array}{r}\text { EST. CONC. } \\
=========0 \\
400 \\
300 \\
1400 \\
470 \\
1200 \\
2400 \\
140000 \\
3800 \\
770 \\
4000 \\
9900 \\
780 \\
840 \\
660 \\
3400 \\
1100 \\
880 \\
1600 \\
790 \\
3300 \\
1100 \\
17000 \\
860\end{array}$ & \begin{tabular}{l}
\multicolumn{1}{r}{$Q$} \\
$=====$ \\
$N J$ \\
$N J$ \\
$N J$ \\
$N J$ \\
$N J$ \\
$N J$ \\
$N J$ \\
$N J$ \\
$N J$ \\
$N J$ \\
$N J$ \\
$N J$ \\
$J$ \\
$J$ \\
J \\
$N J$ \\
NJ \\
NJ \\
J \\
J \\
J \\
NJ \\
NJ
\end{tabular} \\
\hline $\begin{array}{l}25 . \\
26 \\
27 . \\
28 \\
29\end{array}$ & & & & \\
\hline
\end{tabular}


Lab Name: PNNL

Lab Code: PNNL

Case No.:

contract : BNFL

SAS NO.:
EPA SAMPLE NO.

AN-107-

Matrix:' (soil/water) SOLID

Lab Sample ID: 99-0651-

Sample wt/vol:

$4.6(\mathrm{~g} / \mathrm{mI}) \mathrm{G}$

Lab File ID:

99010511

Level: (low/med) IOW

Date Received:

\% Moisture:

decanted: $(\mathrm{Y} / \mathrm{N})$

Date Extracted:

Concentrated Extract Volume: 2000 (uL)

Date Analyzed: 02/05/99

Injection Volume:

(UL)

Dilution Factor: 1.0

GPC Cleanup: $(\mathrm{Y} / \mathrm{N}) \mathrm{N}$

$\mathrm{pH}: 11.0$

Number TICs found: 21

CONCENTRATION UNITS:

(ug/L or $\mathrm{ug} / \mathrm{Kg}$ ) ug/ $\mathrm{kg}$

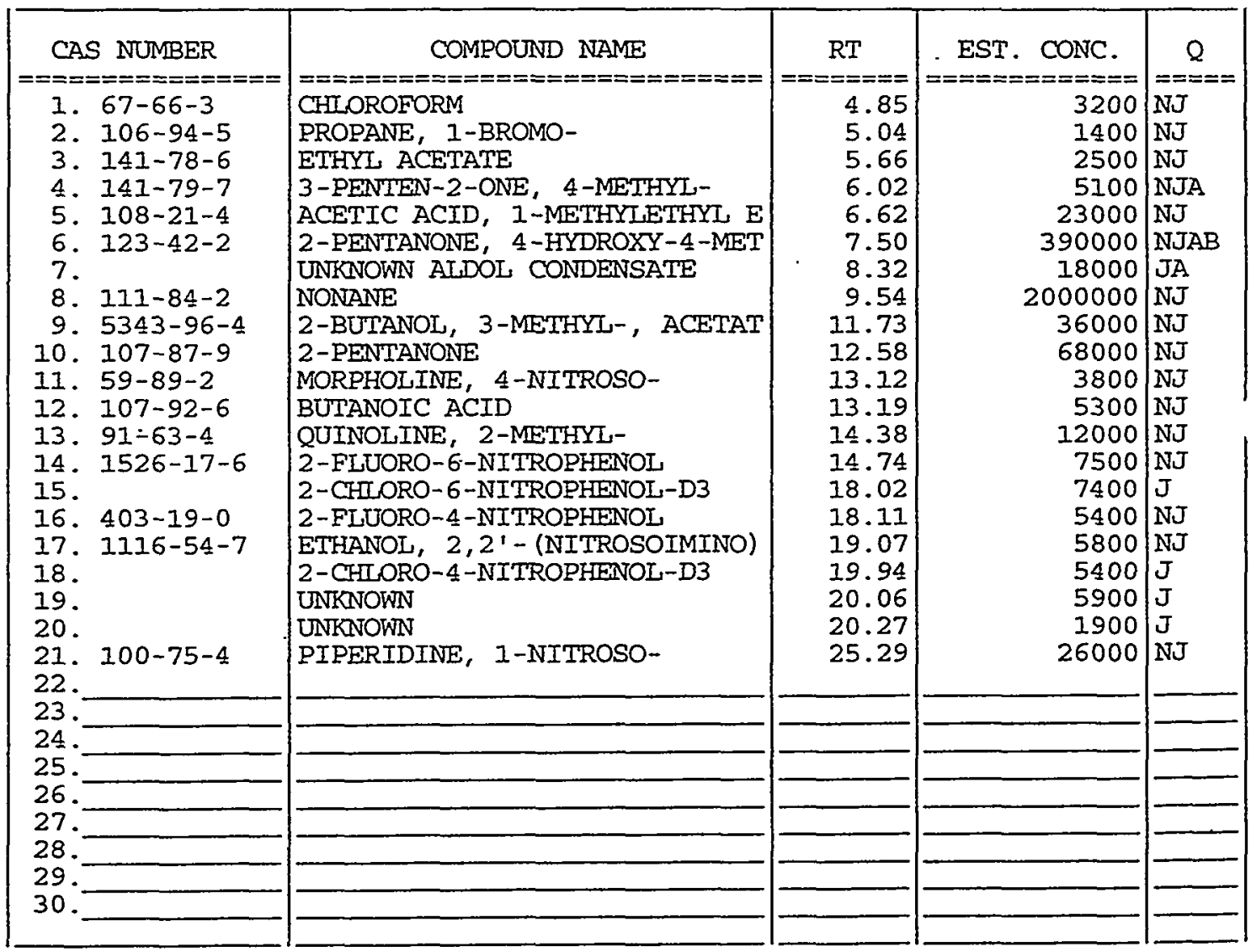

FORM I SV-TIC

OLM03. 0 
Iab Name: PNNL

Lab Code: PNNL

Case No.:

Matrix: (soil/water) SOLID

Sample wt/vol:

$4.8(\mathrm{~g} / \mathrm{mL}) \mathrm{G}$

Level: (low/med) LOW

$\div$ Moisture: decanted: $(Y / N)$

Contract: BNFI SAS NO.:
EPA SAMPLE NO.

AN-107D

SDG NO.: 990205

Concentrated Extract Volume: 2000 (UI)

Injection Volume: (uU)

Lab Sample ID: 99-0651D

GPC Cleanup:

$(\mathrm{Y} / \mathrm{N}) \mathrm{N}$

$\mathrm{pH}: 11.0$

Lab File ID: $\quad 99010512$

Date Received:

Date Extracted:

Date Analyzed: 02/05/99

Dilution Factor: 1.0

Number TICs found: 18

CONCENTRATION UNITS:

(ug/L or ug/Kg) ug/Kg

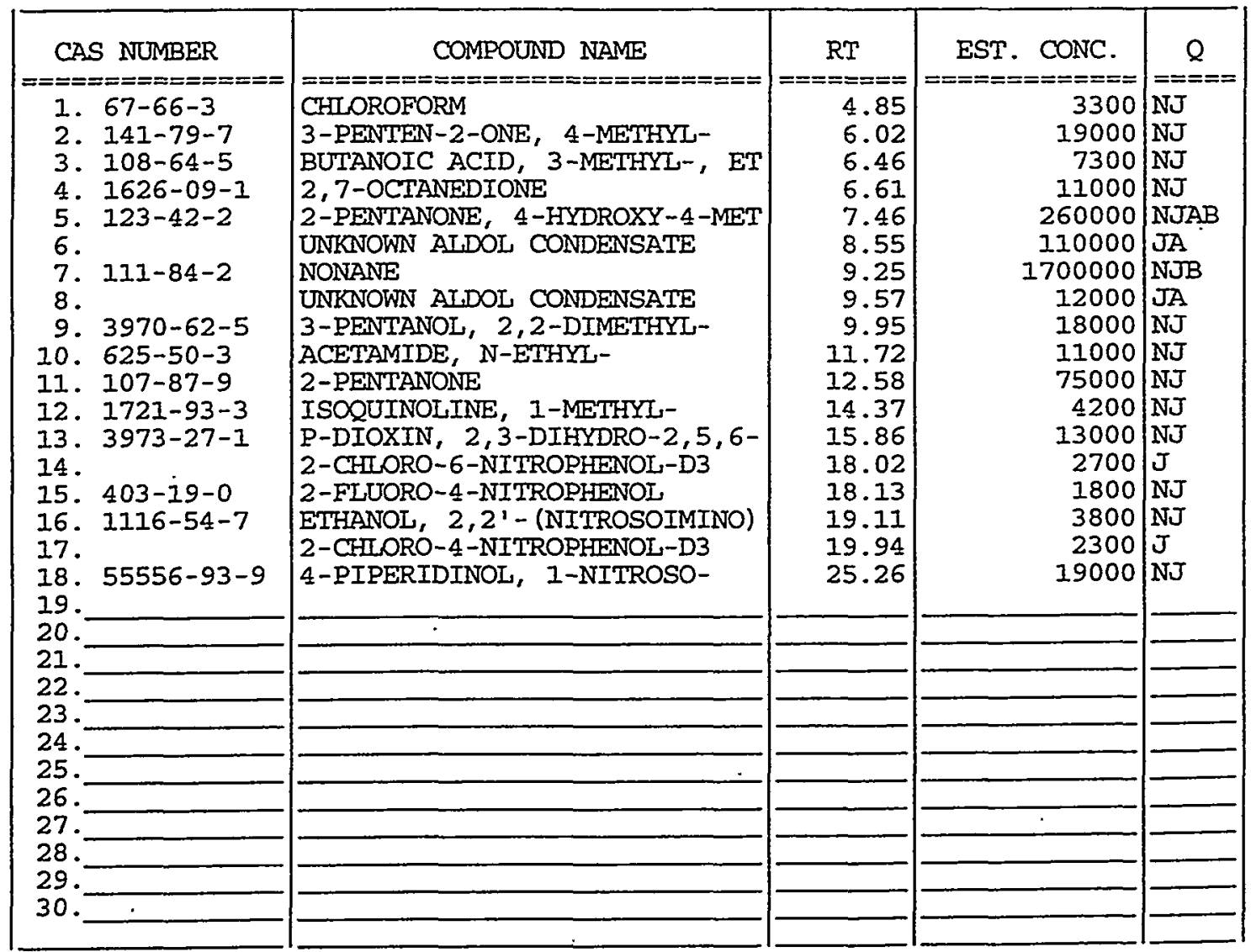


IF

SEMIVOLATILE ORGANICS ANALYSIS DATA SHEET TENTATIVELY IDENTIFIED COMPOUNDS

Lab Name: PNNL

Contract: BNFL

Lab code: PNNL

Case No.:

Matrix: (soil/water) SOLID

Sample wt/vol:

$5.0(\mathrm{~g} / \mathrm{mL}) \mathrm{G}$

Level: (low/med) LOW

$\div$ Moisture:

decanted: $(Y / N)$

SAS NO.:

Lab Sample ID: SBLK02

Lab File ID: 99010510

Date Received:

Date Extracted:

Concentrated Extract Volume: 2000 (uI)

Injection Volume: (ut)

GPC Cleanup: $\quad(Y / N)$ N

$\mathrm{pH}: 11.0$

Dilution Factor: 1.0
EPA SAMPLE NO.

\section{METHOD BLANK}

SDG NO.: 990205

Number TICs found: 3

CONCENTRATION UNITS :

(ug/L or $u g / \mathrm{Kg}$ ) ug/Kg

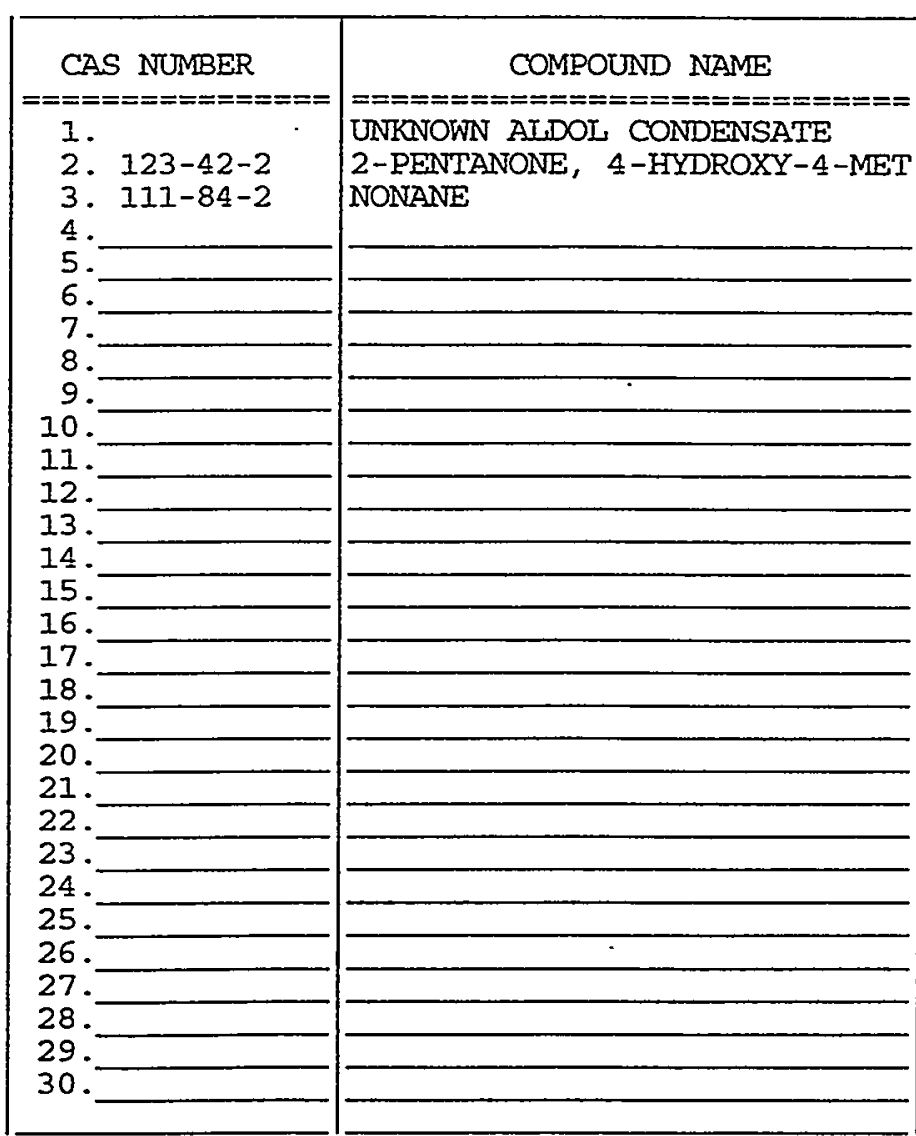

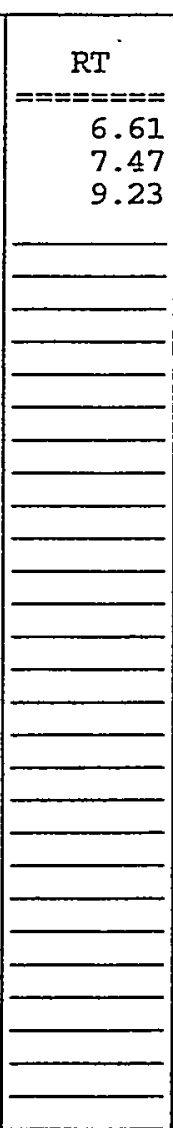

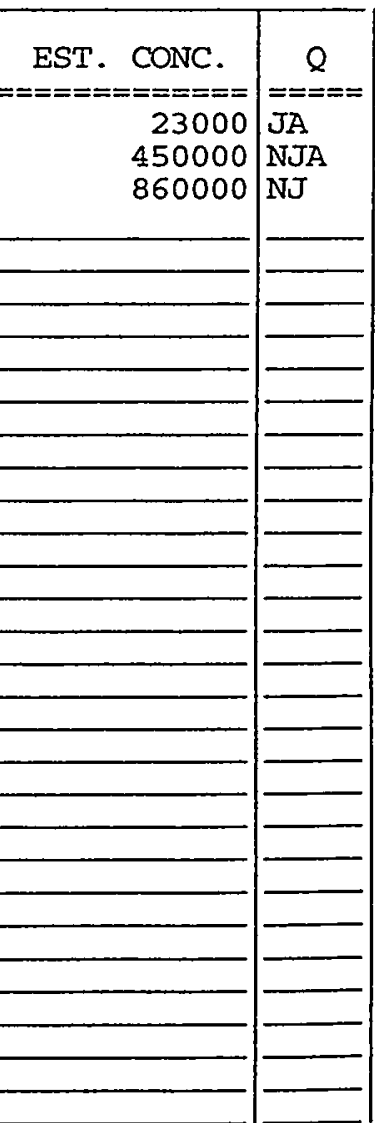


Lab Name: PNNI.

Contract: BNFL

Lab Code: PNNL

Case No.:

Matrix: (soil/water)

Sample wt/vol:

$0.0(\mathrm{~g} / \mathrm{mL}) \mathrm{G}$

Level: (low/med) IOW

\% Moisture:

decanted: $(Y / N)$

Concentrated Extract Volume:

(uIs)

SAS NO. :
EPA SAMPLE NO.

SOLVENT BLANK

SDG No.: 990205
Injection Volume:

$(\mathrm{Y} / \mathrm{N}) \mathrm{N}$

GPC Cleanup:

Number TICs found: 0
Iab Sample ID: SOLVENT

Lab File ID: 99010505

Date Received:

Date Extracted:

Date Analyzed: 02/05/99

Dilution Factor: 1.0

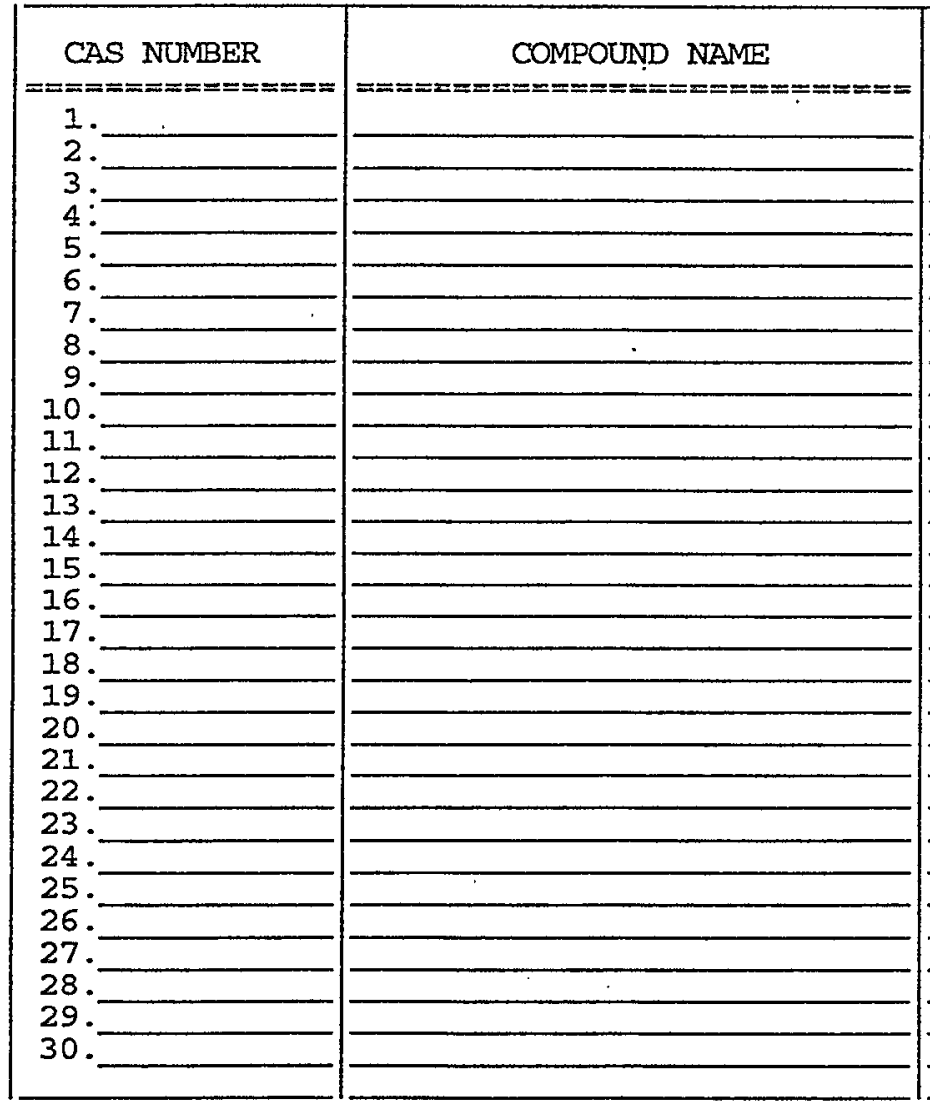

FORM I SV-TIC
CONCENTRATION UNITS:

(ug/L or $u g / \mathrm{kg}$ )
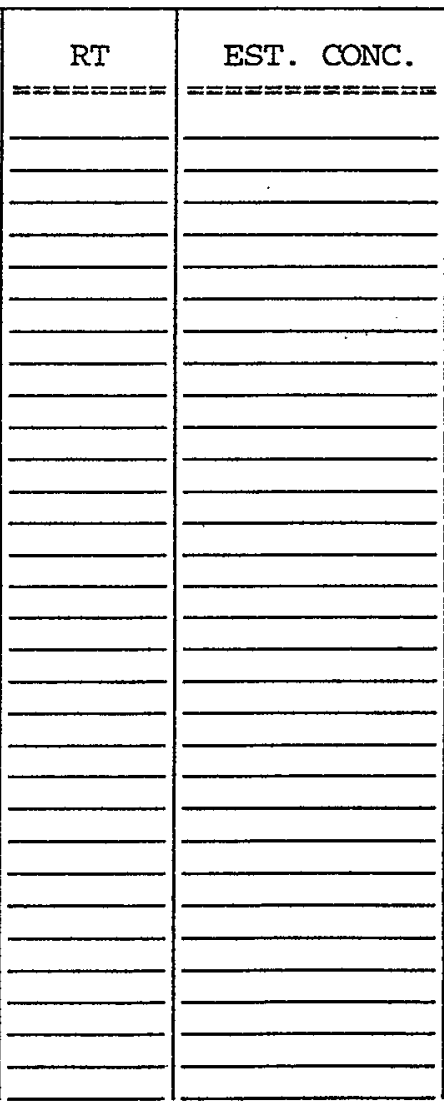

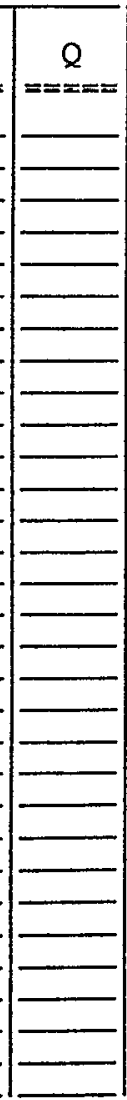

OLM03. 0 
Lab Name: PNNL

Lab Code: PNILL Level: (low/med) IOW
Contract: BNFL

SAS NO.:

Case No.:

EPA

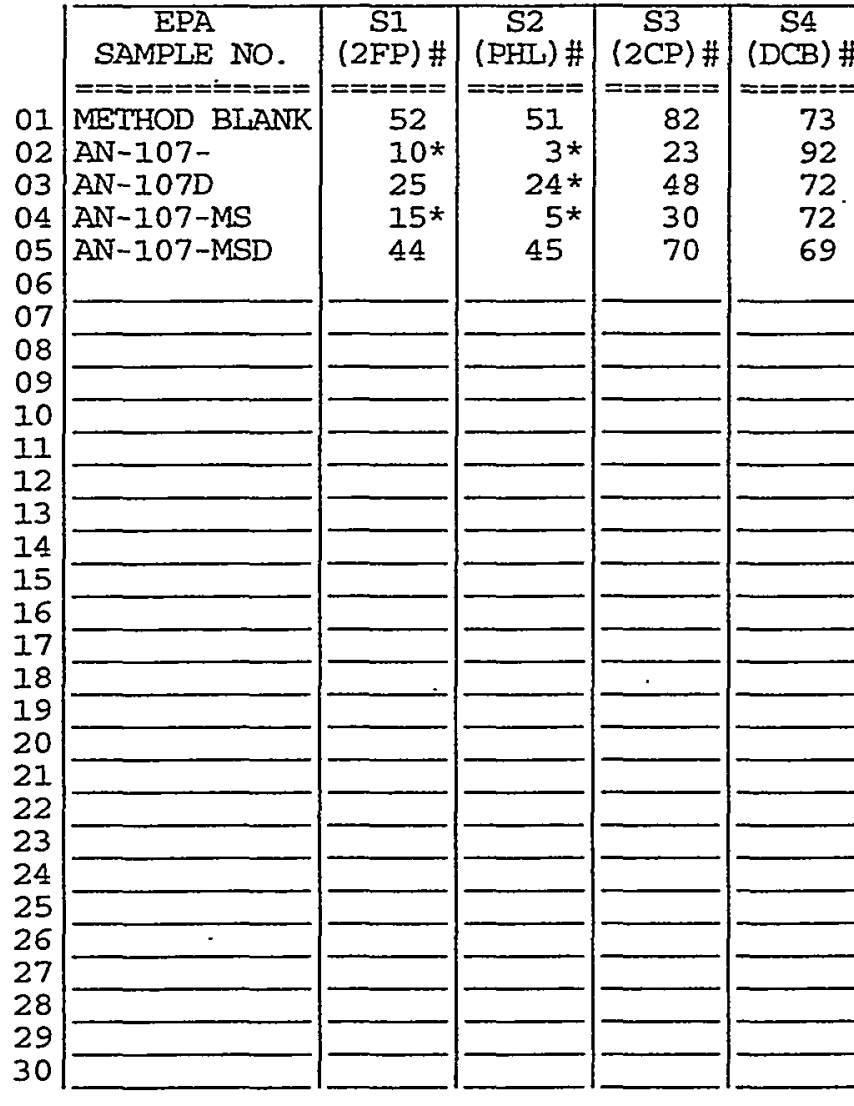

SI $(2 \mathrm{FP})=2-\mathrm{Fluorophenol}$

S2 (PHL) = Phenol-d5

S3 $(2 C P)=2$-Chlorophenol-d4

S4 $(D C B)=1,2-$ Dichlorobenzene-d4

S5 $(\mathrm{NBZ})=$ Nitrobenzene -25

S6 $(\mathrm{FBP})=2-$ Fluorobiphenyl

S7 $($ TBP) $=2,4,6-$ Tribromophenol

S8 $(\mathrm{TPH})=$ Terphenyl- $\mathrm{d14}$

\# Column to be used to flag recovery values

* Values outside of contract required QC limits

D Surrogate diluted out

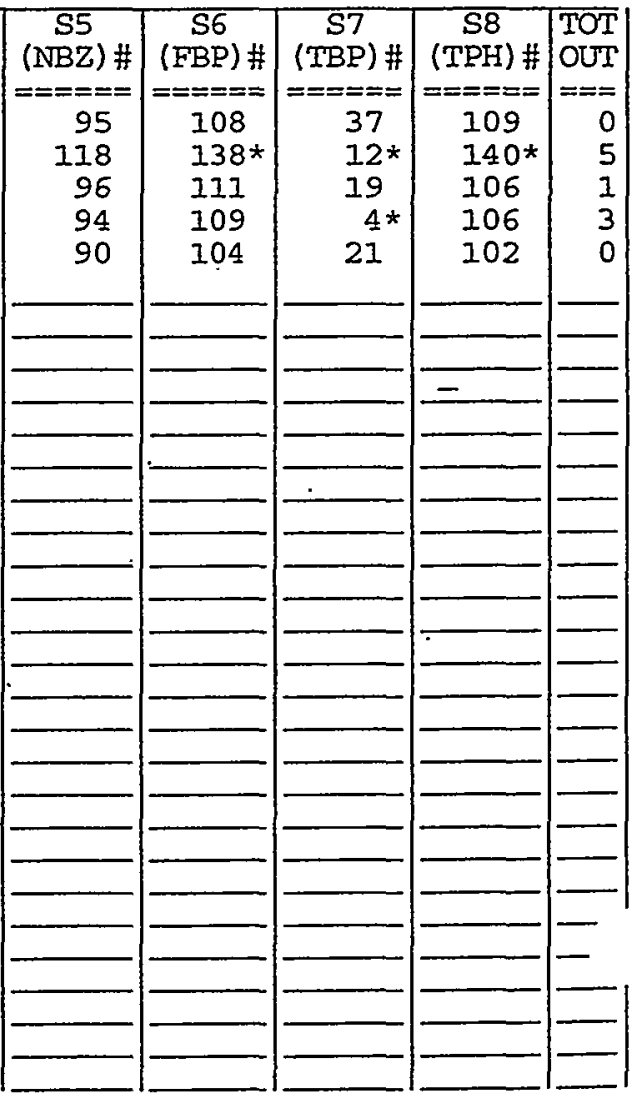

$(25-121)$

$(24-113)$

$(20-130)$

$(20-130)$

$(23-120)$

$(30-115)$

$(19-122)$

$(18-137)$ (advisory)

(advisory) 
Lab Name: PNNL

Lab code: PNNL
Contract: BNFL,

Case No. :
SAS NO. :

SDG No.: 990205

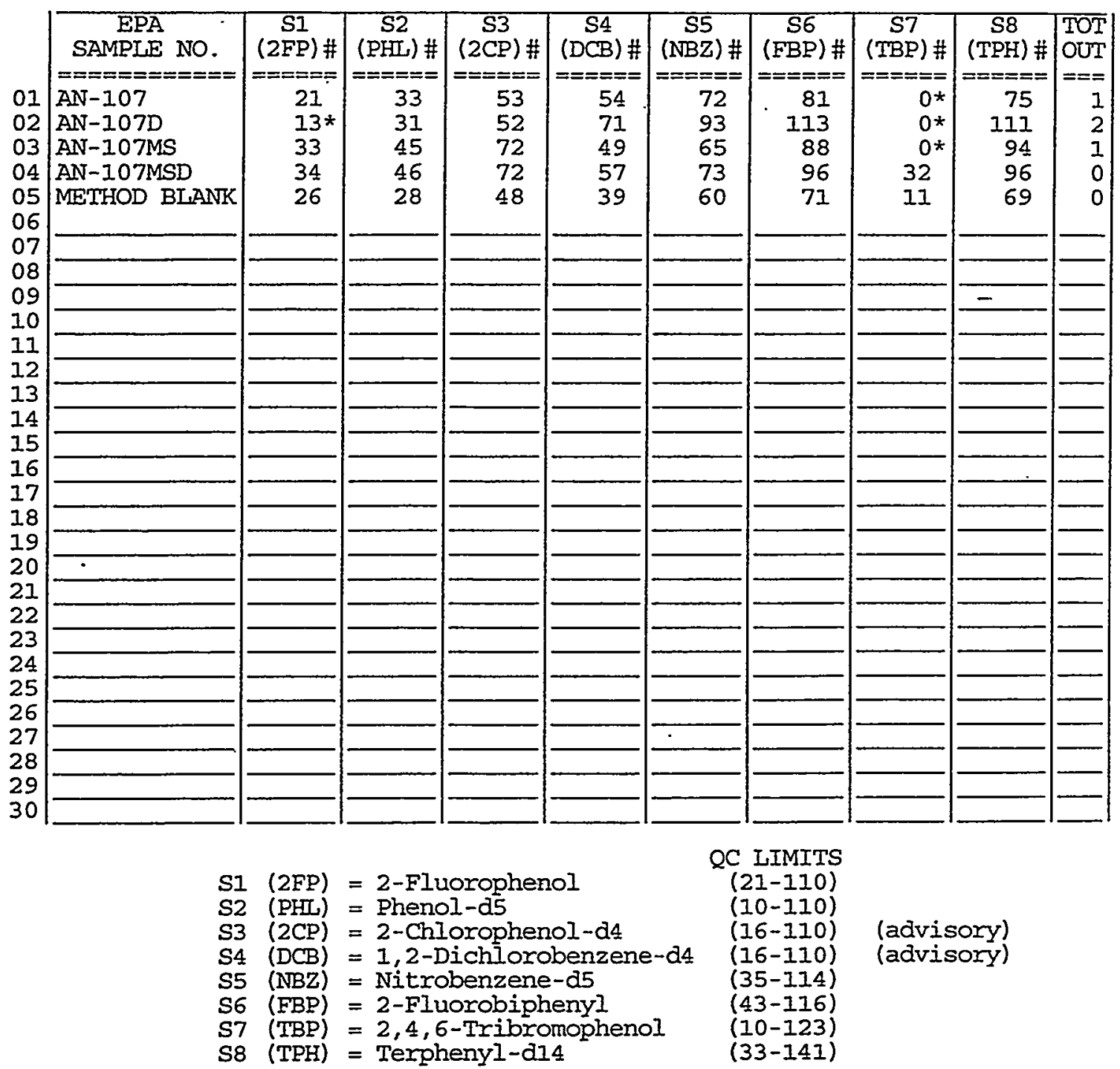

\# Column to be used to flag recovery values

* Values outside of contract required $Q C$ limits

D Surrogate diluted out 
Iab Name: PNNL

Lab Code: PNNL
Contract: BNFL

Case No.: $\quad$ SAS No.:

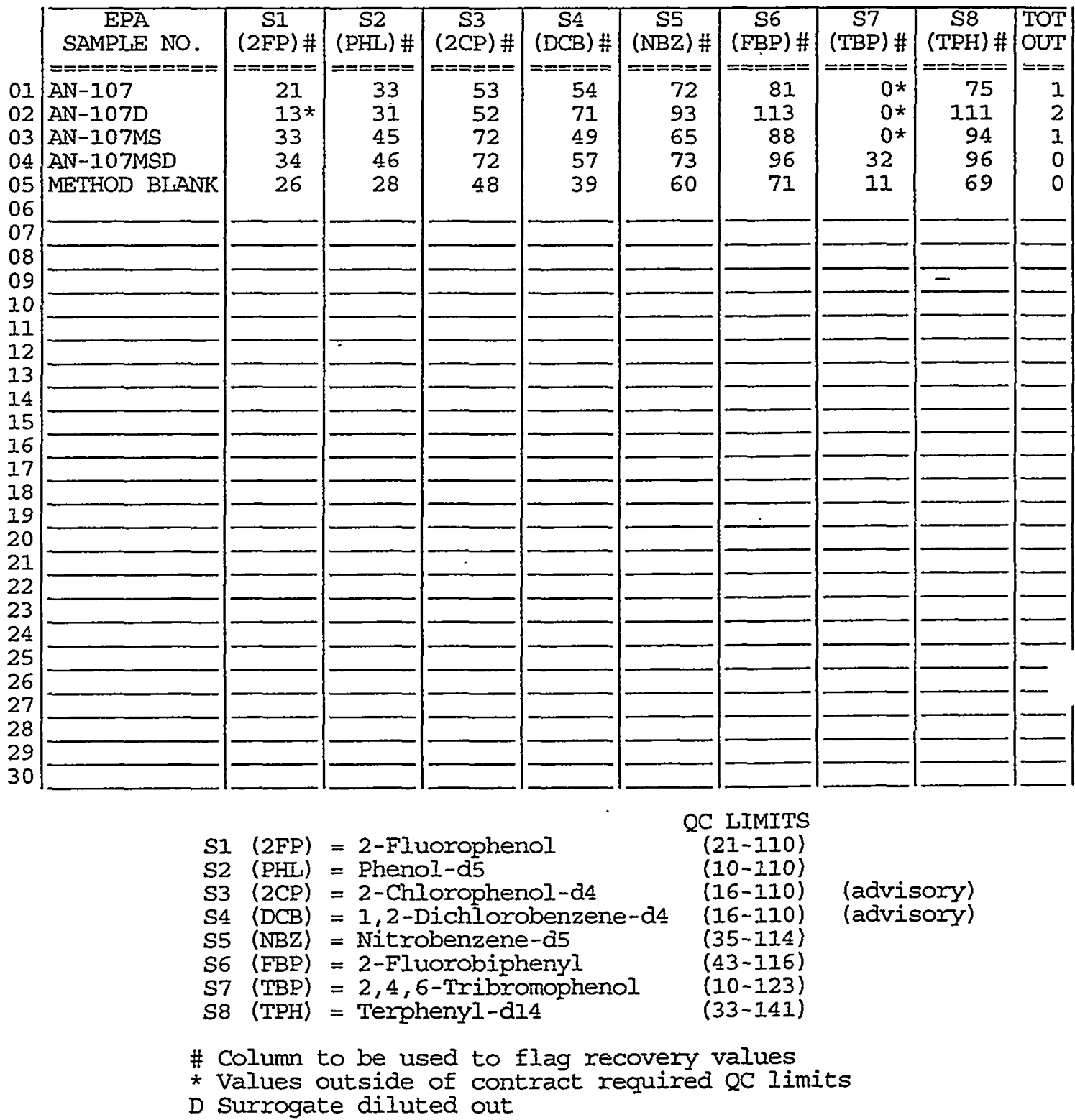


Lab Name: PNNL

Lab Code: PNNL

Matrix Spike
Contract: BNFL

Case No.:

SAS NO.:

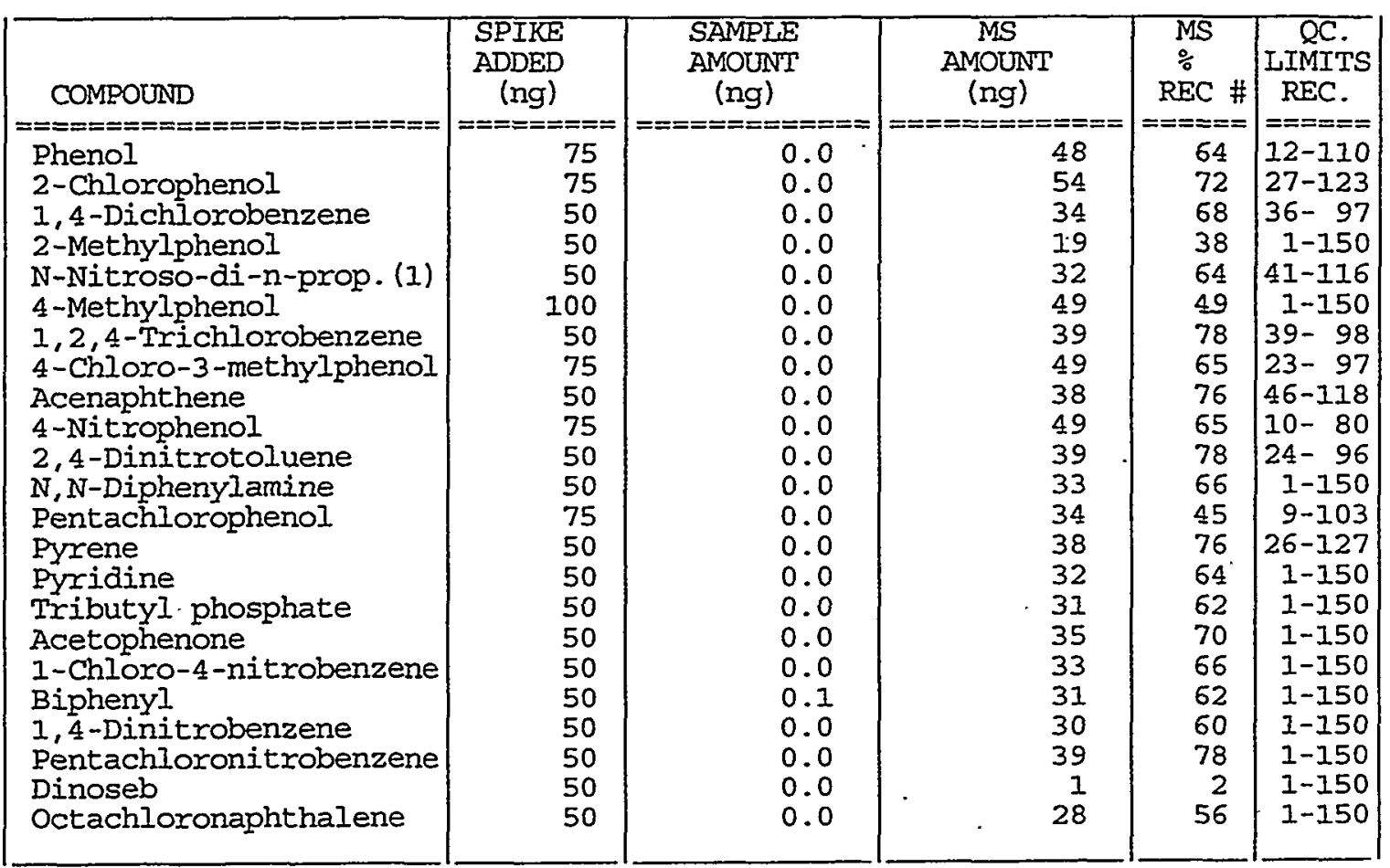

(1) N-Nitroso-di-n-propylamine

\# Column to be used to flag recovery and $R P D$ values with an asterisk

* Values outside of QC limits

COMMENTS:

page $I$ of 2

FORM III SV-I

OLM03.0 
Lab Name: PNNL

Contract: BNFI

Lab code: PNNL

Case No. :

SAS NO.:

SDG NO.: 990205

Matrix Spike - EPA Sample No.: AN-107

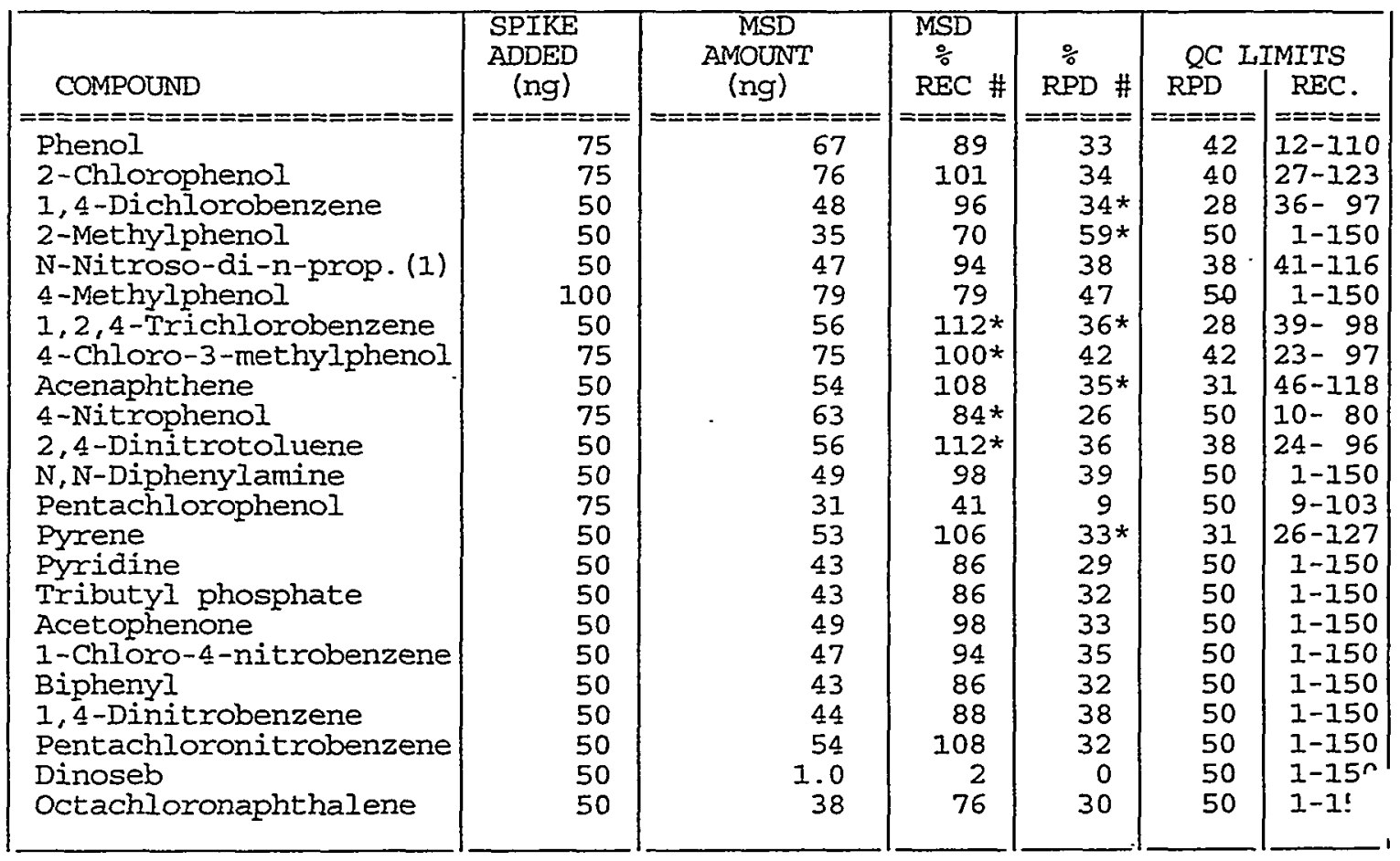

(1) N-Nitroso-di-n-propylamine

\# Column to be used to flag recovery and RPD values with an asterisk

* Values outside of QC limits

RPD: 5 out of 23 outside Iimits

Spike Recovery: 4 out of 46 outside limits

COMMENTS : 
Lab Name: PNNL

Contract : BNEL

Lab Code: PNNL

Case No.:

SAS NO.:

SDG NO.: 990205

Matrix Spike - EPA Sample No.: AN-107- Ievel: (low/med) LOW

\begin{tabular}{|c|c|c|c|c|c|}
\hline $\begin{array}{l}\text { COMPOUND } \\
====================x= \\
\text { Phenol } \\
\text { 2-Chlorophenol } \\
\text { 1,4-Dichlorobenzene } \\
\text { 2-Methylphenol } \\
\text { N-Nitroso-di-n-prop. (1) } \\
\text { 4-Methylphenol } \\
\text { 1,2,4-Trichlorobenzene } \\
\text { 4-Chloro-3-methylphenol } \\
\text { Acenaphthene } \\
\text { 4-Nitrophenol } \\
\text { 2,4-Dinitrotoluene } \\
\text { N,N-Diphenylamine } \\
\text { Pentachlorophenol } \\
\text { Pyrene } \\
\text { Pyridine . } \\
\text { Tributyl phosphate } \\
\text { Acetophenone } \\
\text { 1-Chloro-4-nitrobenzene } \\
\text { Biphenyl } \\
\text { 1,4-Dinitrobenzene } \\
\text { Pentachloronitrobenzene } \\
\text { Dinoseb } \\
\text { Octachloronaphthalene }\end{array}$ & 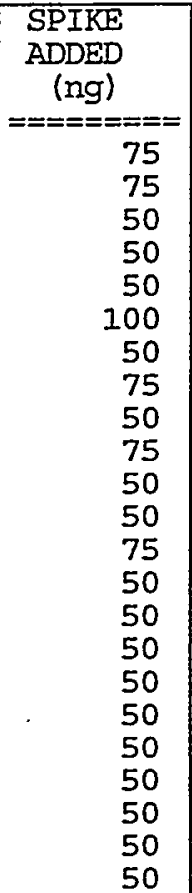 & 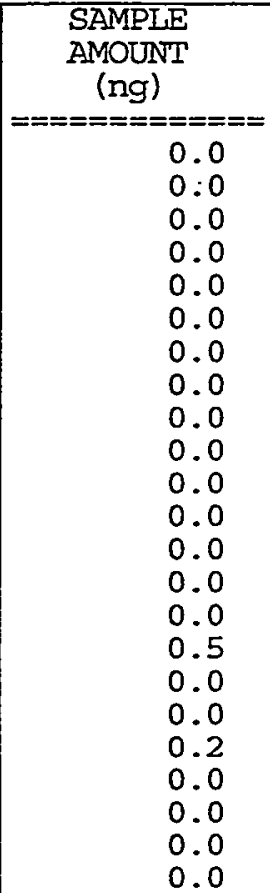 & \begin{tabular}{|c}
\multicolumn{2}{c}{$\begin{array}{c}\text { MSS } \\
\text { (ng) }\end{array}$} \\
$==========$ \\
4 \\
18 \\
31 \\
0.0 \\
31 \\
0.0 \\
35 \\
5 \\
33 \\
80 \\
38 \\
20 \\
36 \\
34 \\
26 \\
26 \\
32 \\
30 \\
28 \\
32 \\
36 \\
30 \\
33
\end{tabular} & $\begin{array}{c}\text { MS } \\
\% \\
\text { REC \# } \\
====== \\
5^{*} \\
24 * \\
62 \\
0^{*} \\
62 \\
\theta^{*} \\
70 \\
7 * \\
66 \\
107 * \\
76 \\
40 \\
48 \\
68 \\
52 \\
51 \\
64 \\
60 \\
56 \\
64 \\
72 \\
60 \\
66\end{array}$ & $\begin{array}{c}\text { QC. } \\
\text { IIMITS } \\
\text { REC. } \\
===== \\
12-110 \\
27-123 \\
36-97 \\
1-150 \\
41-116 \\
1-150 \\
39-98 \\
23-97 \\
46-118 \\
10-80 \\
24-96 \\
1-150 \\
9-103 \\
26-127 \\
1-150 \\
1-150 \\
1-150 \\
1-150 \\
1-150 \\
1-150 \\
1-150 \\
1-150 \\
1-150\end{array}$ \\
\hline
\end{tabular}

(1) N-Nitroso-di-n-propylamine

\# Column to be used to flag recovery and RPD values with an asterisk

* Values outside of QC limits

COMMENTS : 
Lab Name: PNNL Contract: BNFL

Lab code: PNNL

Case No.:

SAS NO. :

SDG NO.: 990205

Matrix Spike - EPA Sample No. : AN-107-

Level: (low/med) IOW

\begin{tabular}{|c|c|c|c|c|c|c|}
\hline $\begin{array}{l}\text { COMPOUND } \\
\text { ======================== } \\
\text { Phenol } \\
\text { 2-Chlorophenol } \\
\text { 1,4-Dichlorobenzene } \\
\text { 2-Methylphenol } \\
\text { N-Nitroso-di-n-prop. (I) } \\
\text { 4-Methylphenol } \\
\text { 1,2,4-Trichlorobenzene } \\
\text { 4-Chloro-3-methylphenol } \\
\text { Acenaphthene } \\
\text { 4-Nitrophenol } \\
\text { 2,4-Dinitrotoluene } \\
\text { N, N-Diphenylamine } \\
\text { Pentachlorophenol } \\
\text { Pyrene } \\
\text { Pyridine } \\
\text { Tributyl phosphate } \\
\text { Acetophenone } \\
\text { l-Chloro-4-nitrobenzene } \\
\text { Biphenyl } \\
\text { I, 4-Dinitrobenzene } \\
\text { Pentachloronitrobenzene } \\
\text { Dinoseb } \\
\text { Octachloronaphthalene }\end{array}$ & 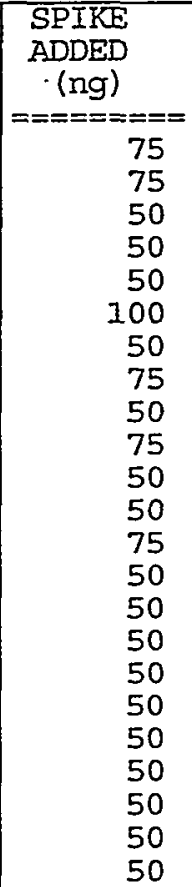 & \begin{tabular}{|c}
\multicolumn{2}{c}{$\begin{array}{c}\text { MSD } \\
\text { AMOUNT } \\
\text { (ng) }\end{array}$} \\
$===========$ \\
47 \\
51 \\
34 \\
17 \\
34 \\
39 \\
39 \\
51 \\
39 \\
61 \\
42 \\
11 \\
45 \\
39 \\
32 \\
31 \\
35 \\
33 \\
31 \\
36 \\
42 \\
34 \\
38
\end{tabular} & 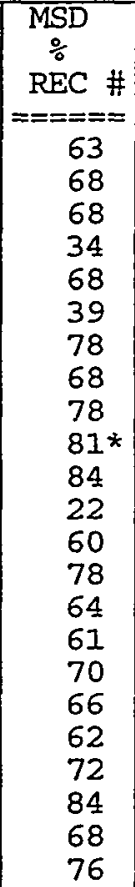 & $\begin{array}{c}\% \\
R P D \# \\
===== \\
170 * \\
96 * \\
9 \\
9 \\
11 \\
163 * \\
17 \\
28 \\
10 \\
58 * \\
22 \\
14 \\
21 \\
18 \\
9 \\
10 \\
10 \\
12 \\
15 \\
12 \\
14\end{array}$ & $\begin{array}{c}Q C L \\
R P D \\
===== \\
42 \\
40 \\
28 \\
50 \\
38 \\
50 \\
28 \\
42 \\
31 \\
50 \\
38 \\
50 \\
50 \\
31 \\
50 \\
50 \\
50 \\
50 \\
50 \\
50 \\
50 \\
50 \\
50\end{array}$ & $\begin{array}{l}\text { IMITS } \\
\text { REC. } \\
===== \\
12-110 \\
27-123 \\
36-97 \\
1-150 \\
41-116 \\
1-150 \\
39-98 \\
23-97 \\
46-118 \\
10-80 \\
24-96 \\
1-150 \\
9-103 \\
26-127 \\
1-150 \\
1-150 \\
1-150 \\
1-150 \\
1-150 \\
1-150 \\
1-150 \\
1-150 \\
1-1\end{array}$ \\
\hline
\end{tabular}

(1) N-Nitroso-di-n-propylamine

\# Column to be used to flag recovery and RPD values with an asterisk

* Values outside of QC Iimits

RPD: 4 out of 23 outside limits

Spike Recovery: 7 out of 46 outside limits

COMMENTS: 
Lab Name: PNNL

Lab code: PNNL Case No.:

Lab File ID: 99010510

Instrument ID: HP1

Matrix: (soil/water) SOLID

Level: (low/med) LOW
Contract: BNFI

SAS NO.:
EPA SAMPLE NO.

METHOD BLANK

SDG No.: 990205

Lab Sample ID: SBLKO2

Date Extracted:

Date Analyzed: 02/05/99

Time Analyzed: 1959

THIS METHOD BLANK APPLIES TO THE FOLIOWING SAMPLES, MS and MSD:

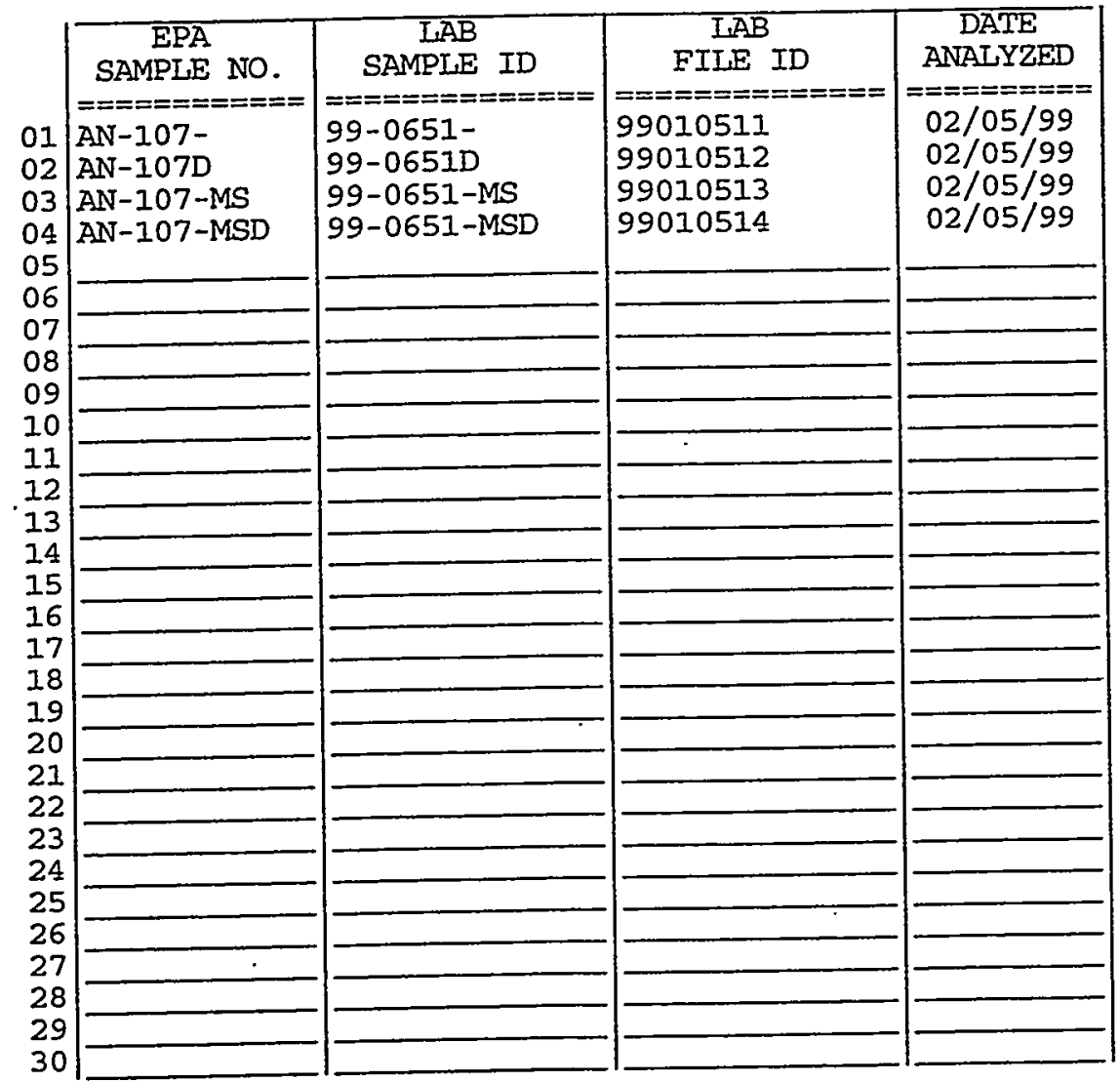

COMMENTS : 
SEMIVOLATIIE ORGANIC INSTRUMENT PERFORMANCE CHECK DECAFLUOROTRIPHENYLPHOSPHINE (DFTPP)

Lab Name: PNNL

Iab code: PNNL

Case No.:

Iab File ID: 99010501

Instrument ID: HPI
Contract : BNEL

SAS NO.:

SDG No.: 990205

DFTPP Injection Date: 02/05/99

DETPP Injection Time: 1200

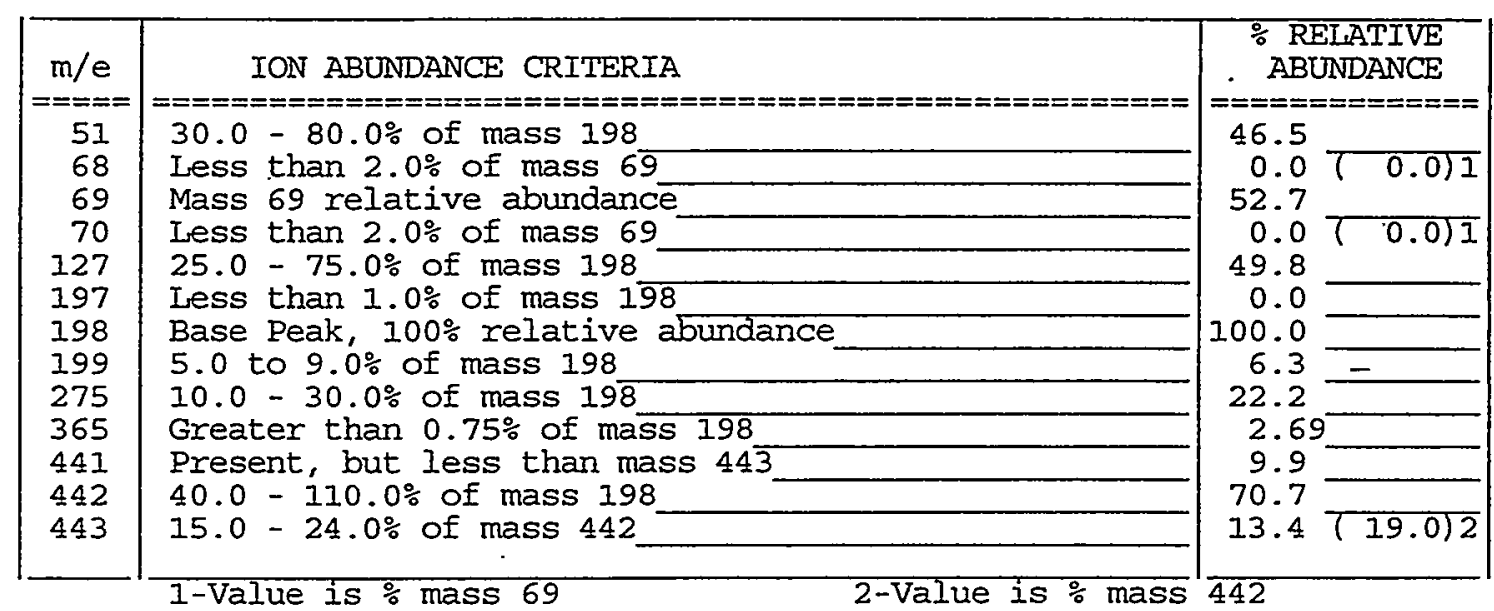

THIS CHECK APPLIES TO THE FOLIOWING SAMPLES, MS, MSD, BLANKS, AND STANDARDS:

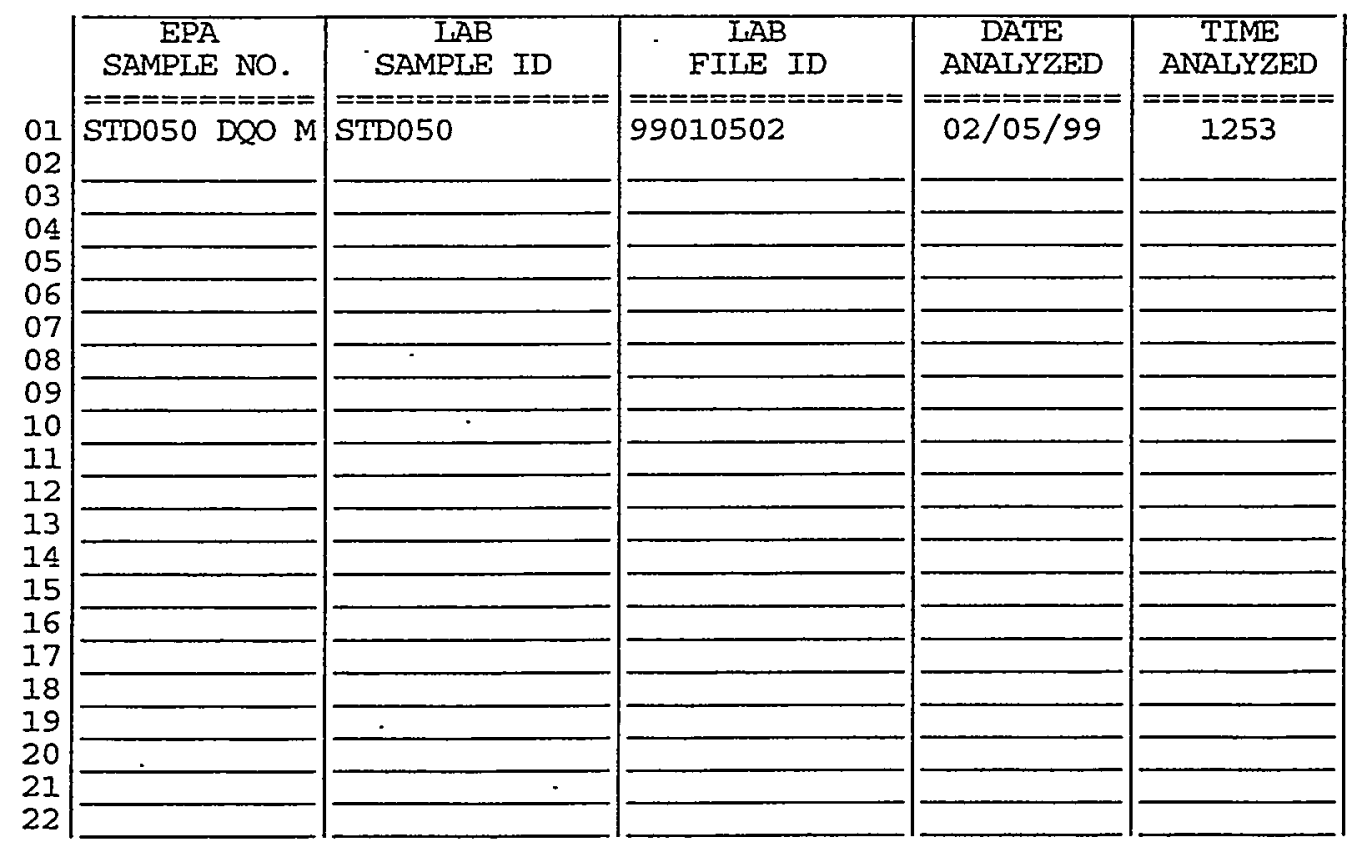

page 1 of 1 

DECAFLUOROTRIPHENYLPHOSPHINE (DFTPP)

Lab Name: PNNL

Lab Code: PNNL

Lab File ID: 99010503

Instrument ID: HPI
Contract: BNFL

SAS NO.:

SDG No.: 990205

\begin{tabular}{|c|c|c|}
\hline $\begin{array}{l}\mathrm{m} / \mathrm{e} \\
====\end{array}$ & 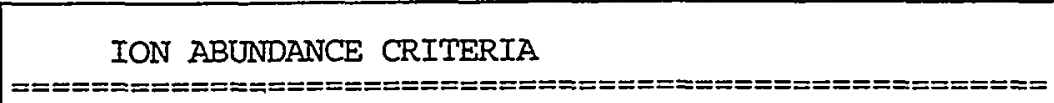 & $\begin{array}{c}\% \text { REIATIVE } \\
\text { ABUNDANCE } \\
============\end{array}$ \\
\hline 51 & $30.0-80.0 \%$ of mass 198 & 51.1 \\
\hline 68 & Less than $2.0 \%$ of mass $6 \overline{9}$ & $0.0 T$ \\
\hline 69 & Mass 69 relative abundance & 57.2 \\
\hline 70 & Less than $2.0 \%$ of mass 69 & 0.37 \\
\hline 127 & $25.0-75.0 \%$ of mass 198 & 52.8 \\
\hline 197 & Less than $1.0 \%$ of mass $1 \overline{98}$ & 0.0 \\
\hline 198 & Base Peak, $100 \%$ relative abundance & 100.0 \\
\hline 199 & 5.0 to $9.0 \%$ of mass 198 & $6.6=$ \\
\hline 275 & $10.0-30.0 \%$ of mass $19 \overline{8}$ & 21.5 \\
\hline 365 & Greater than $0.75 \%$ of mass 198 & 2.38 \\
\hline 441 & Present, but less than mass $44 \overline{3}$ & 8.2 \\
\hline 442 & $40.0-110.0 \%$ of mass 198 & $5 8 . 4 \longdiv { \square }$ \\
\hline 443 & $15.0-24.0 \%$ of mass 442 & $10.9718 .7) 2$ \\
\hline
\end{tabular}

THIS CHECK APPLIES TO THE FOLIOWING SAMPLES, MS, MSD, BLANKS, AND STANDARDS:

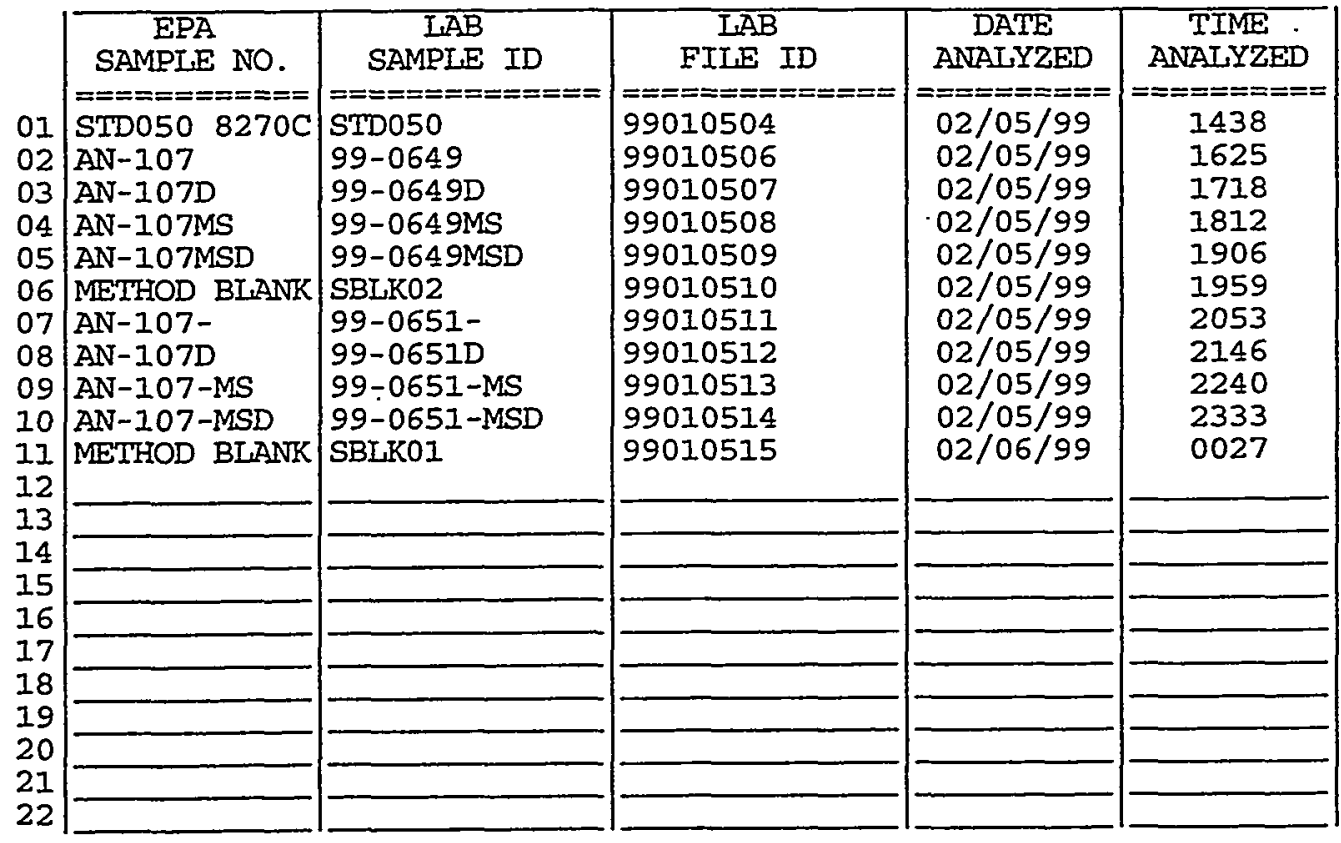

page 1 of 1 
7B

SEMIVOLATILE CONTINUING CALIBRATION CHECK

Lab Name: PNNL

Contract: BNFL

Lab Code: PNNL

Case No.:

SAS NO. :

SDG NO.: 990205

Instrument ID: HP1

Calibration Date: 02/05/99 Time: 1253

Lab File ID: 99010502

Init. Calib. Date (s) : 02/04/99 02/05/99

Init. Calib. Times: $1820 \quad 1532$

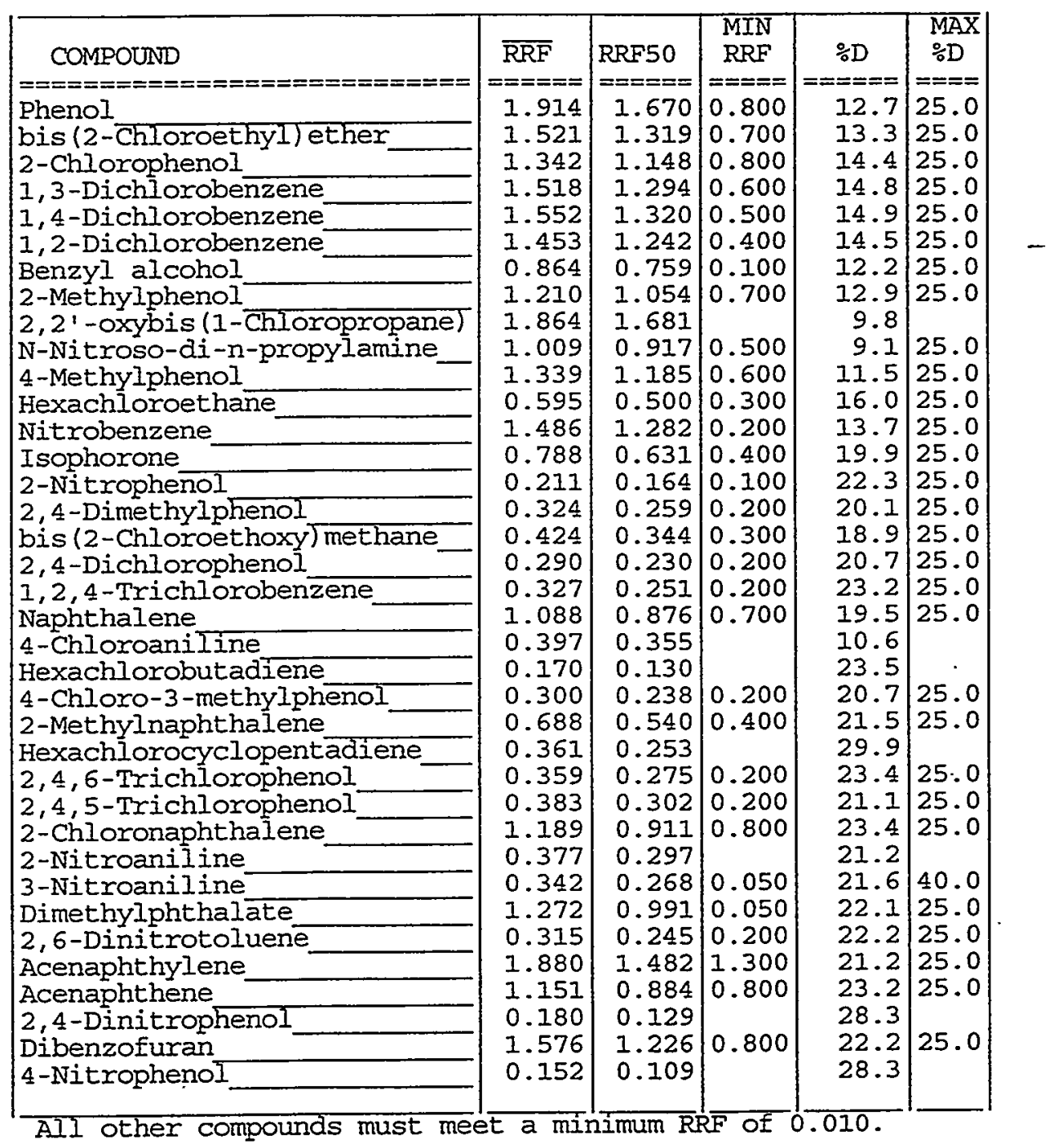

page 1 of 4

FORM VII SV-I

OIMO3. 0 
$7 \mathrm{C}$

SEMIVOLATILE CONTINUING CALIBRATION CHECK

Lab Name: PNNL

Contract: BNFL

Lab Code: PNNL

Case No.:

SAS NO.:

SDG No.: 990205

Instrument ID: HP1

Calibration Date: 02/05/99 Time: 1253

Lab File ID: 99010502

Init. Calib. Date (s) : 02/04/99 02/05/99

Init. Calib. Times: 1820

1532

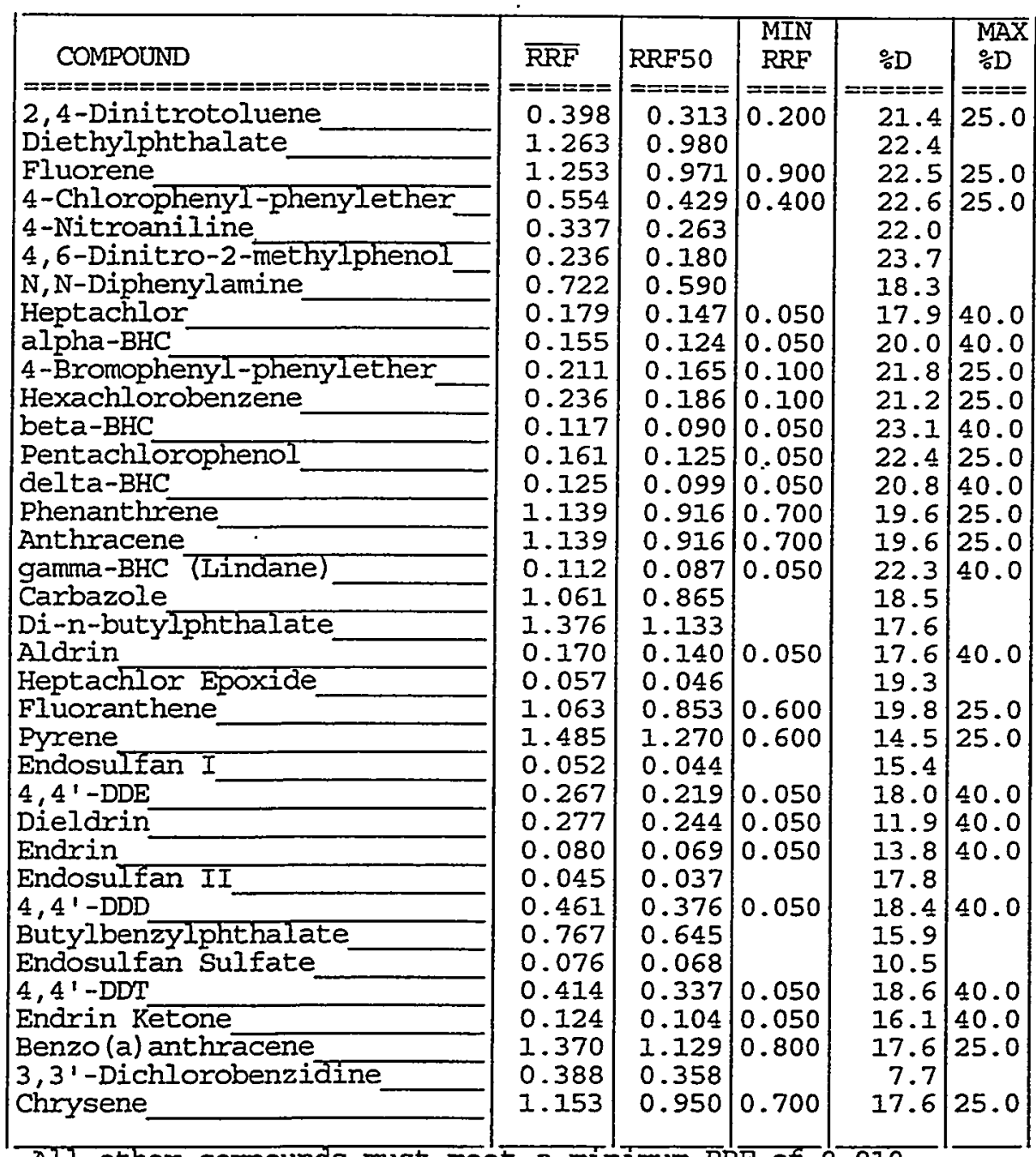

All other compounds must meet a minimum RRF of 0.010 . 
Lab Name: PNNL

Iab code: PNNL

Instrument ID: HPI

Iab File ID: 99010502
Contract: BNFL

Case No.:
SDG NO.: 990205

Time: 1253

$02 / 05 / 99$

1532

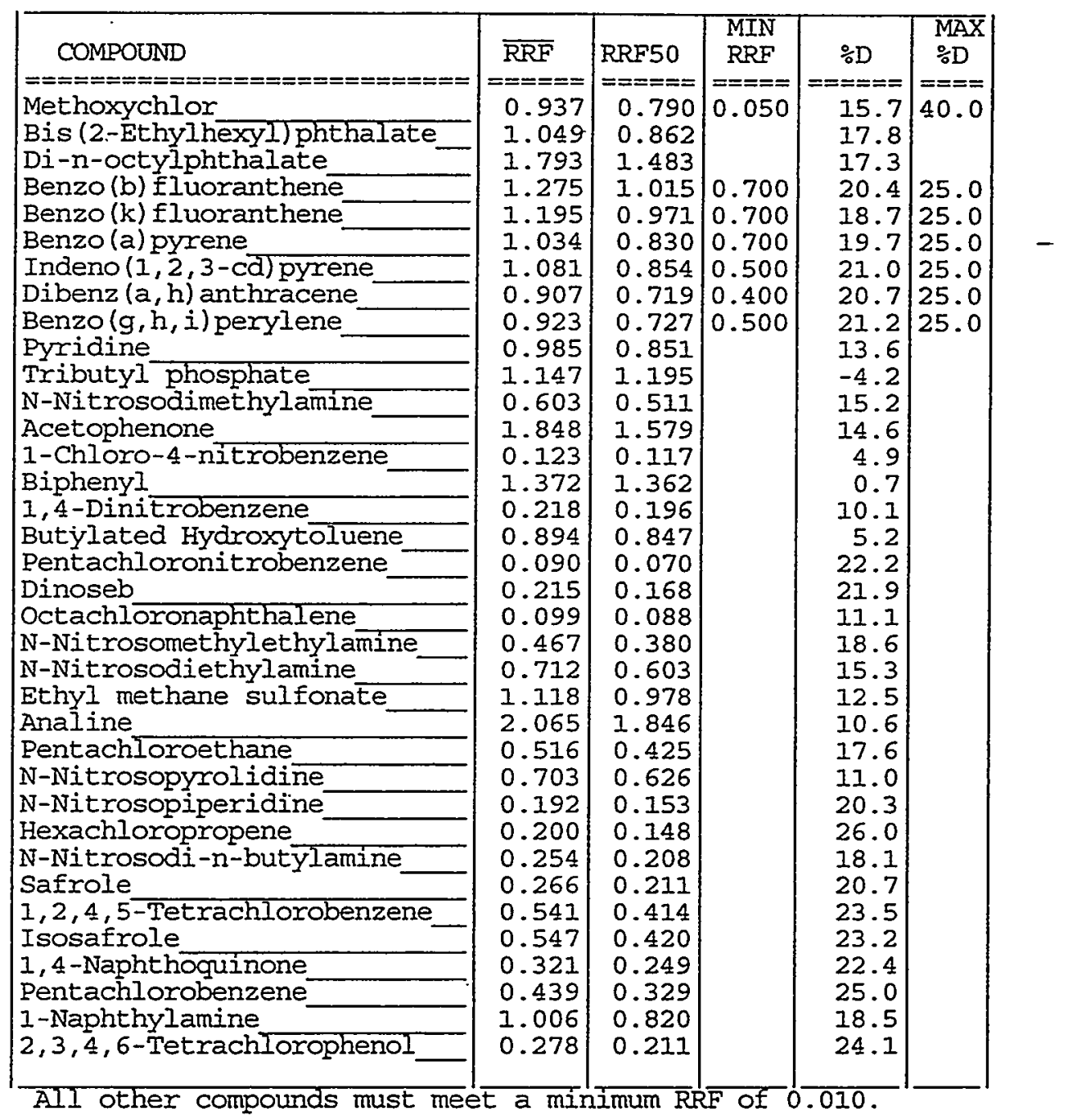

page 3 of 4

FORM VII SV-3

OLM03.0 
$7 \mathrm{C}$

SEMIVOLATILE CONTINUING CALIBRATION CHECK

Lab Name: PNNL

Lab Code: PNNL

Instrument ID: HPI

Lab File ID: 99010502
Contract: BNFI

SAS NO.:

SDG NO.: 990205
Case No.:
Time: 1253

\begin{abstract}
Init. Calib. Date(s) : 02/04/99 02/05/99
Init. Calib. Times: $1820 \quad 1532$
\end{abstract}

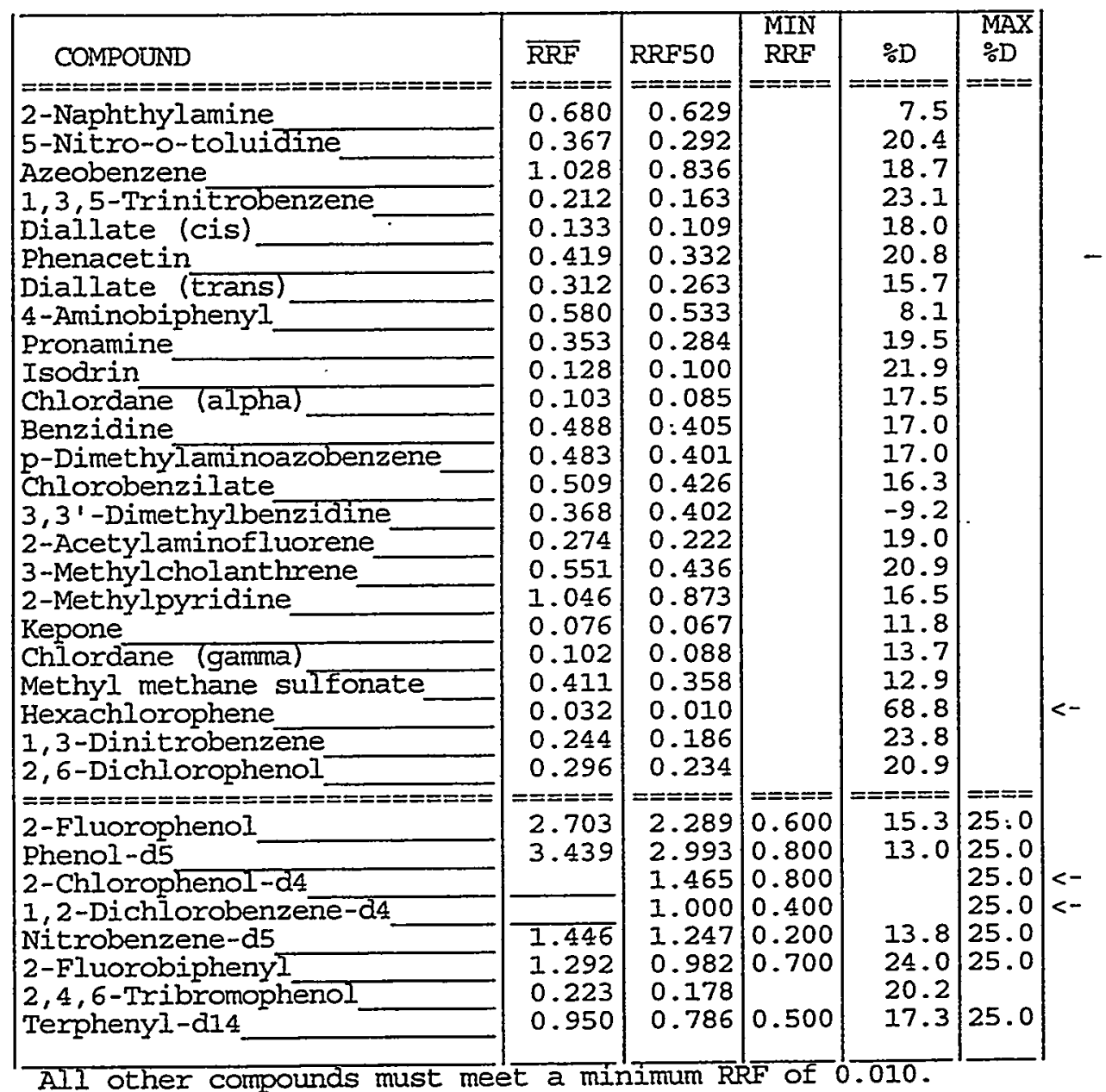


$7 B$

SEMIVOLATIIE CONTINUING CALIBRATION CHECK

Lab Name: PNNL

Lab code: PNNL,

Instrument ID: HP1

Lab File ID: 99010504
Contract: BNFL

Case No.: SAS No.:
SDG NO.: 990205

Time: 1438

Init. Calib. Date(s) : 02/04/99 02/05/99

Init. Calib. Times: $1820 \quad 1532$

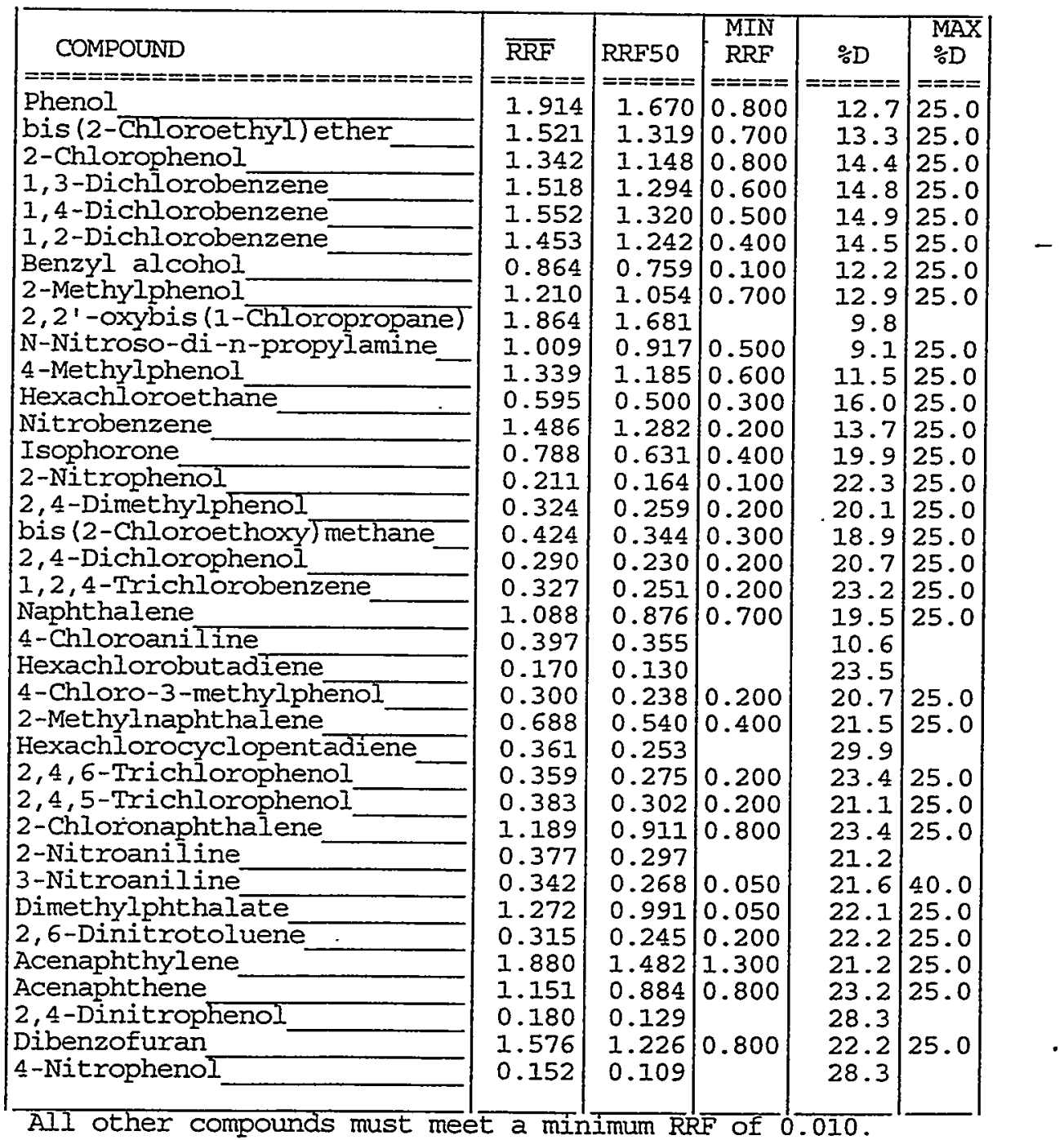

page 1 of 4 
$7 \mathrm{C}$

SEMIVOLATILE CONTINUING CALIBRATION CHECK

Lab Name: PNNL

Contract: BNFL

Lab Code: PNNL

Case No.:

SAS NO.:

SDG No.: 990205

Instrument ID: HPI

Calibration Date: 02/05/99 Time: 1438

Lab File ID: 99010504

Init. Calib. Date(s) : 02/04/99 02/05/99

Init. Calib. Times: $1820 \quad 1532$

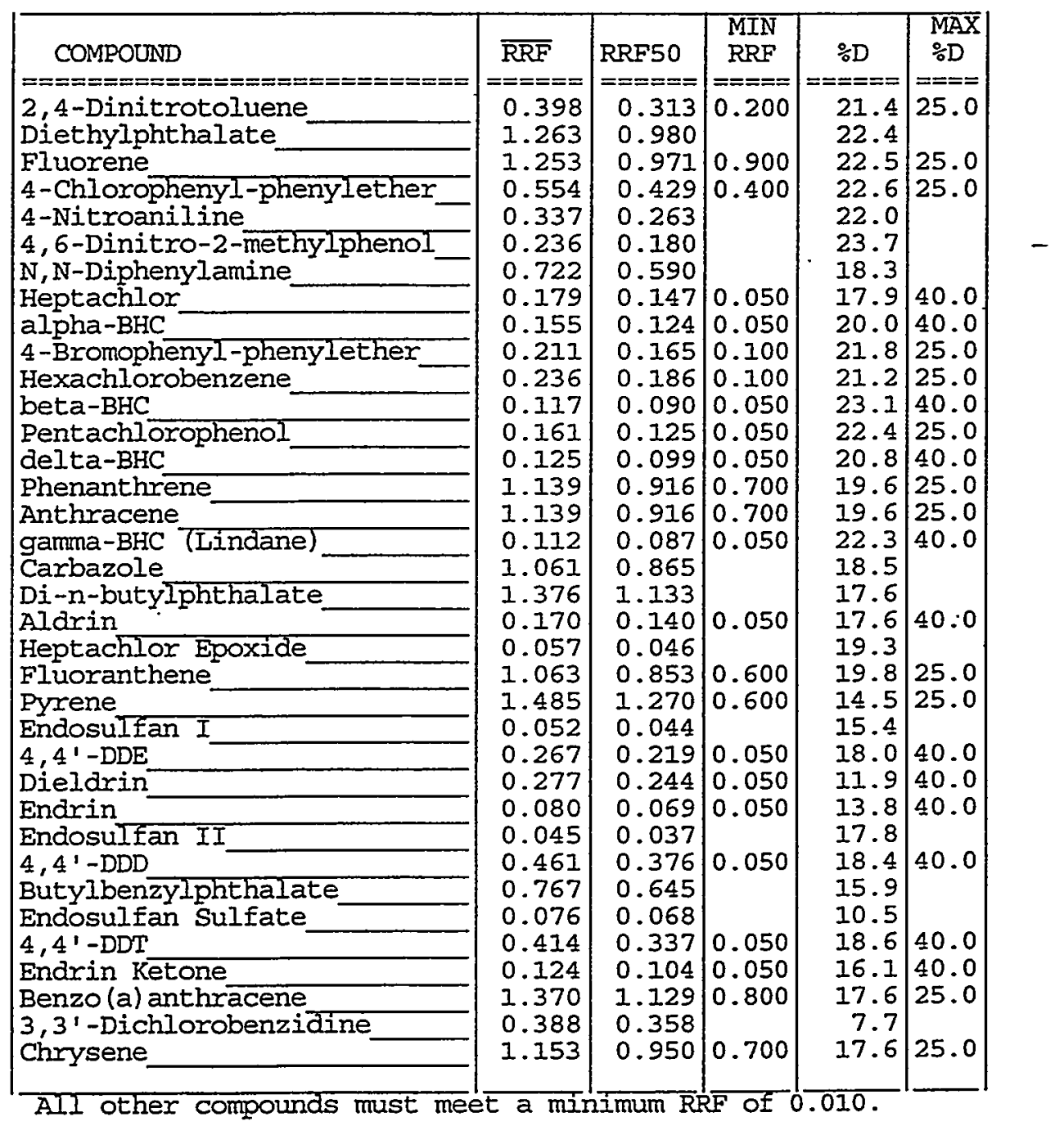

page 2 of 4

FORM VII SV-2

OLM03.0 
$7 \mathrm{C}$

SEMIVOLATILE CONTINUING CALIBRATION CHECK

Lab Name: PNNL

Lab code: PNNL

Instrument ID: HPI

Lab File ID: 99010504
Contract: BNFL

Case No.:

SAS NO.:

SDG NO.: 990205

Calibration Date: 02/05/99 Time: 1438

Init. Calib. Date(s): 02/04/99 02/05/99

Init. Calib. Times: 1820

1532

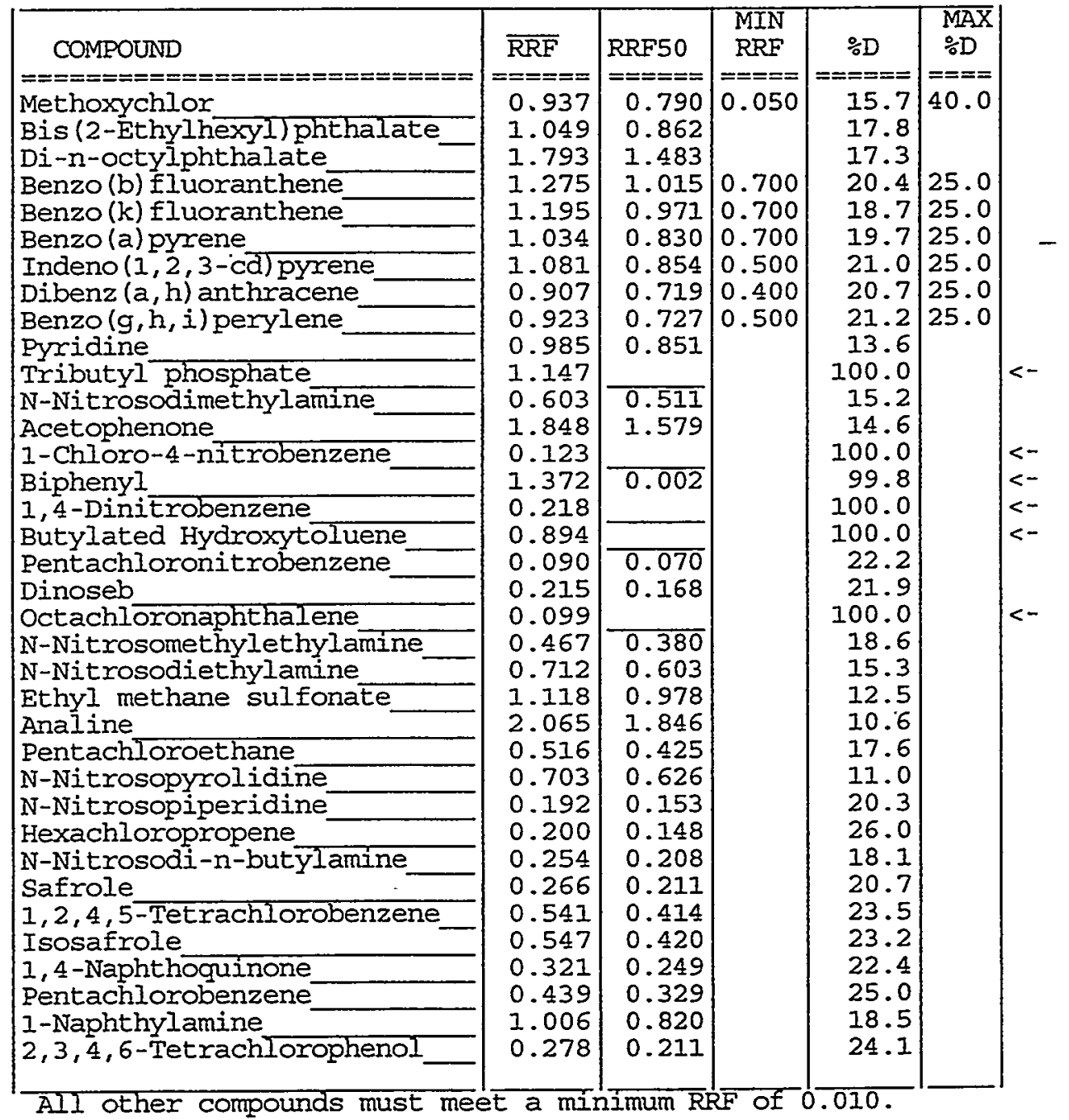

page 3 of 4

FORM VII SV-3

OLM03 .0 
Lab Name: PNNL

Lab code: PNNL

Instrument ID: HPI

Lab File ID: 99010504
Contract: BNFI

SAS NO.:

Init. Calib. Date(s) : 02/04/99 02/05/99

Init. Calib. Times: $1820 \quad 1532$

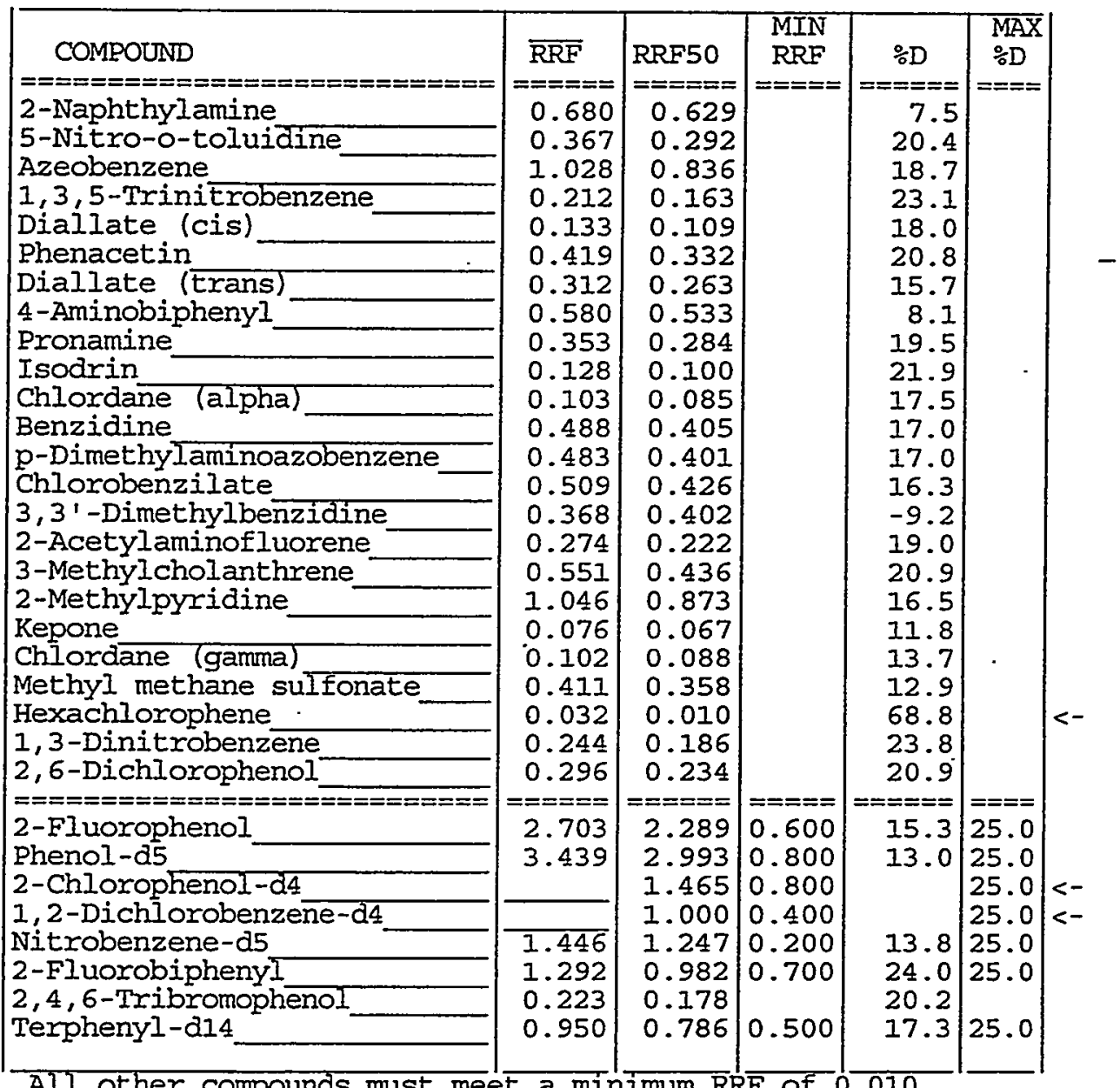

All other compounds must meet a minimum $R R \bar{F}$ of 0.010 
Iab Name: PNNL

Lab code: PNNL

\author{
Case No. :
}

Lab File ID (Standard) : 99010504

Instrument ID: HP1
Contract: BNFL

SAS No.: $\quad$ SDG No.: 990205

Date Analyzed: 02/05/99

Time Analyzed: 1438

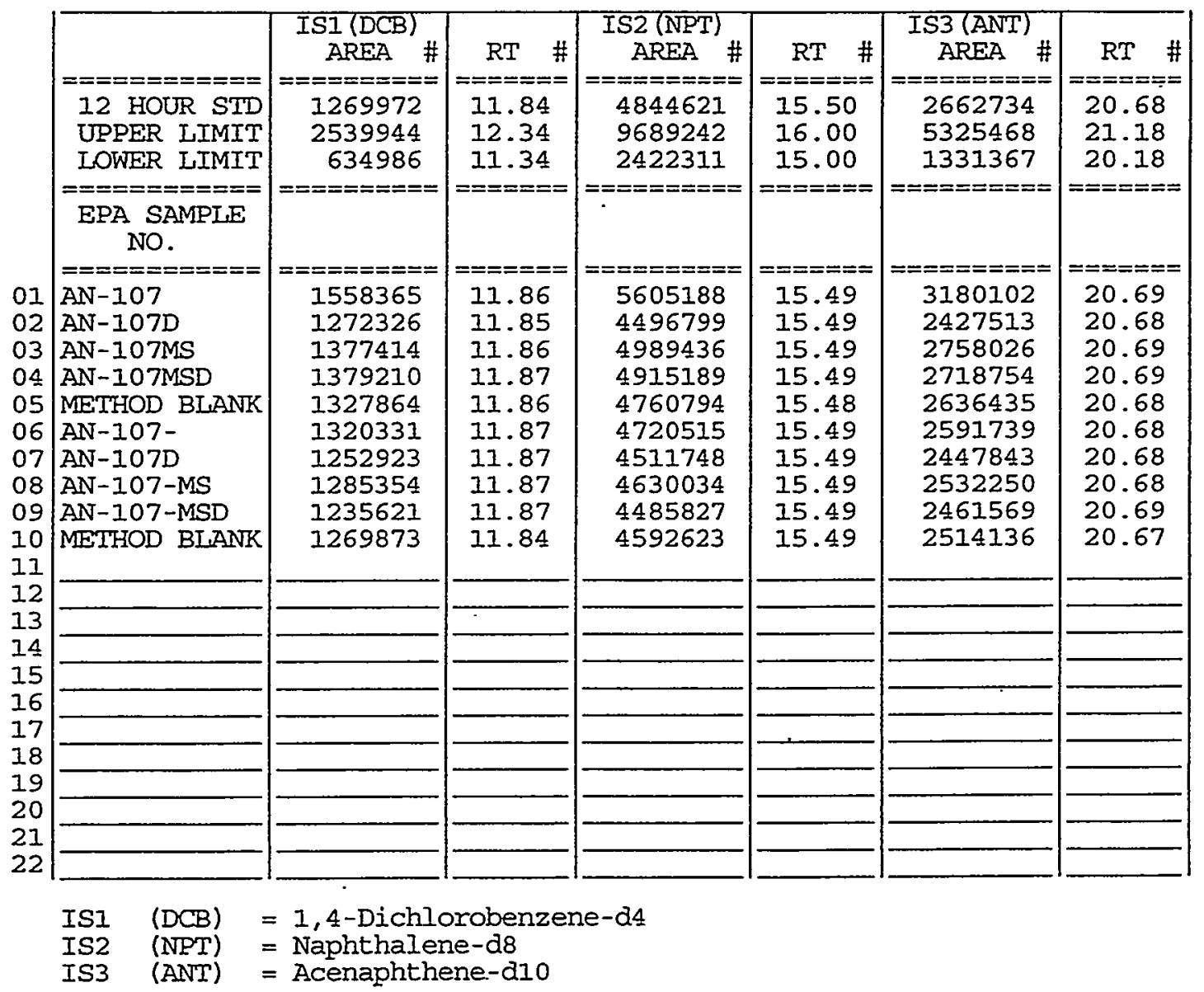

AREA UPPER LIMIT $=+100 \%$ of internal standard area AREA LOWER LIMIT $=-50 \%$ of internal standard area RT UPPER IIMIT $=+0.50$ minutes of internal standard RT RT LOWER LIMIT $=-0.50$ minutes of internal standard RT

\# Column used to flag internal standard area values with an asterisk. * Values outside of QC Iimits. 
Lab Name: PNNL

Lab Code: PNNL Case No.:

Lab File ID (Standard): 99010504

Instrument ID: HP1
Contract: BNFL

SAS NO.: $\quad$ SDG NO.: 990205

Date Analyzed: 02/05/99

Time Analyzed: 1438

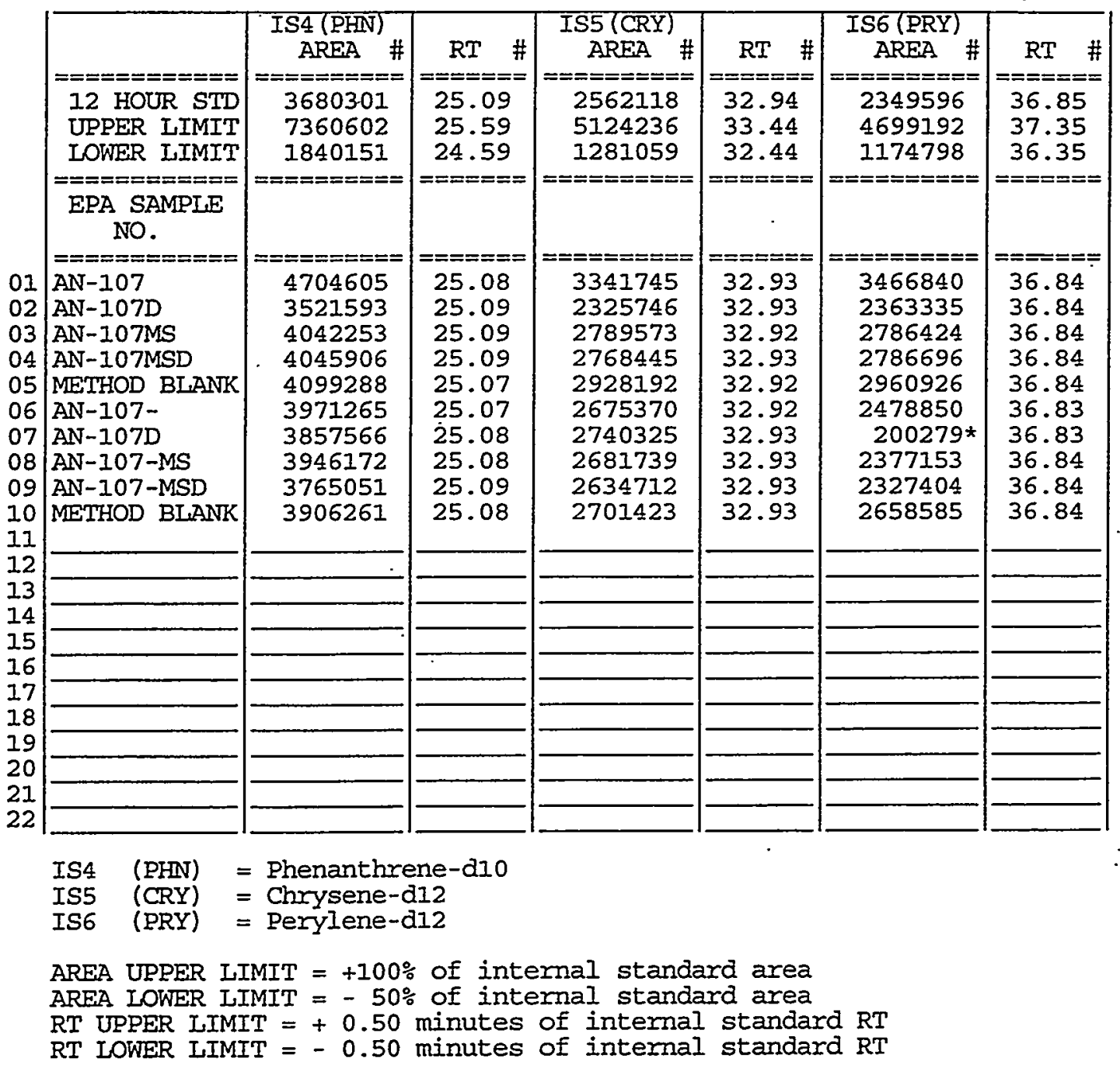

\# Column used to flag internal standard area values with an asterisk. * Values outside of QC limits. 
Lab Name: PNNL

Contract: BNFL

Lab Code: PNNL Case No.:

SAS NO.:

SDG NO.: 990205

Lab File ID (Standard): 99010504

Date Analyzed: 02/05/99

Instrument ID: HPI

Time Analyzed: 1438

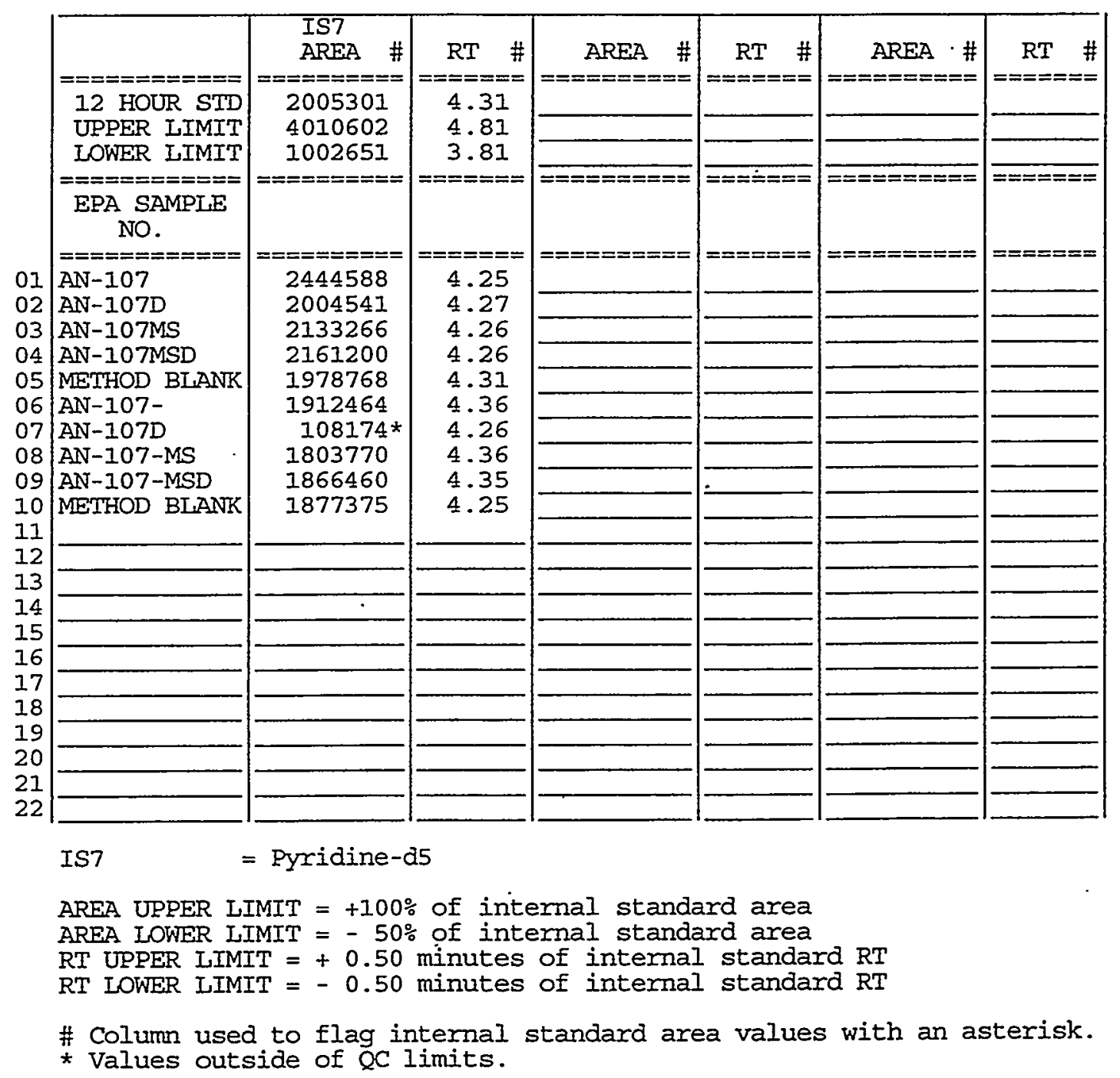

page 1 of 1 
Lab Name: PNNL

Irab Code: PNNL Case No.:

Lab File ID: 99030303

Instrument ID: HPI
Contract: BNFL

SAS NO. :

SDG No. : 990312

DFTPP Injection Date: 03/03/99

DFTPP Injection Time: 1858

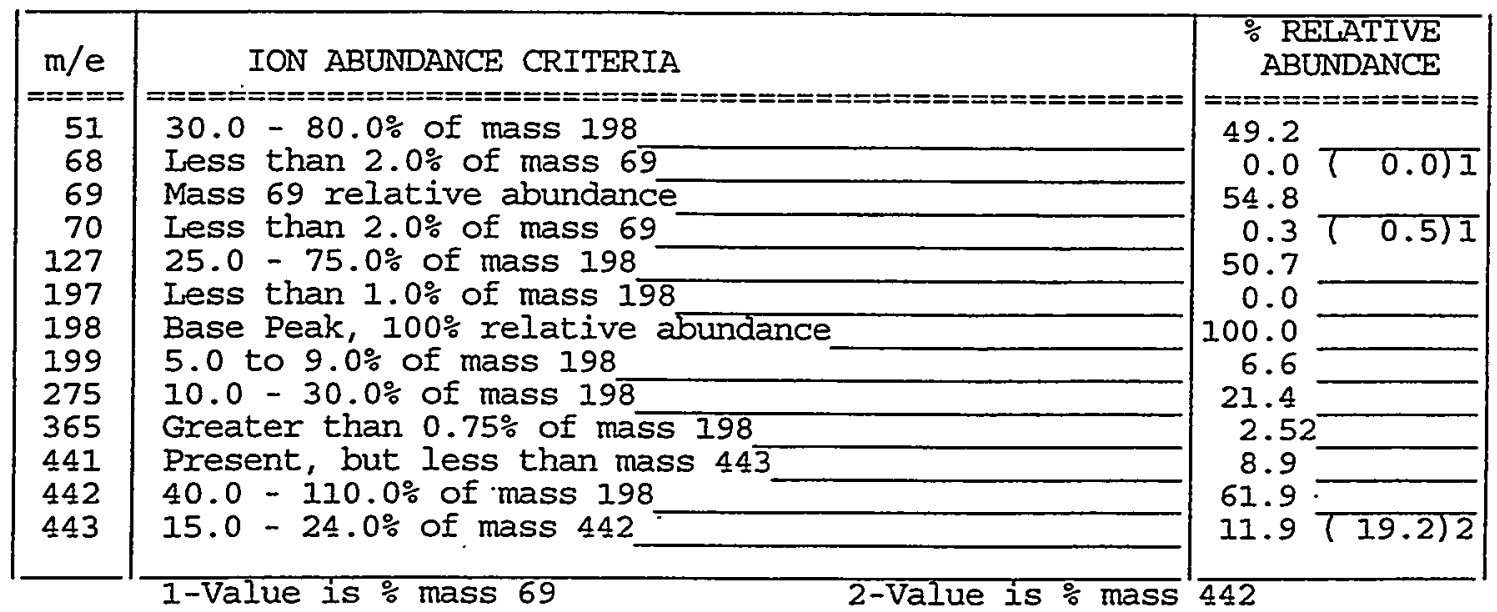

THIS CHECK APPLIES TO THE FOLLOWING SAMPLES, MS, MSD, BLANKS, AND STANDARDS:

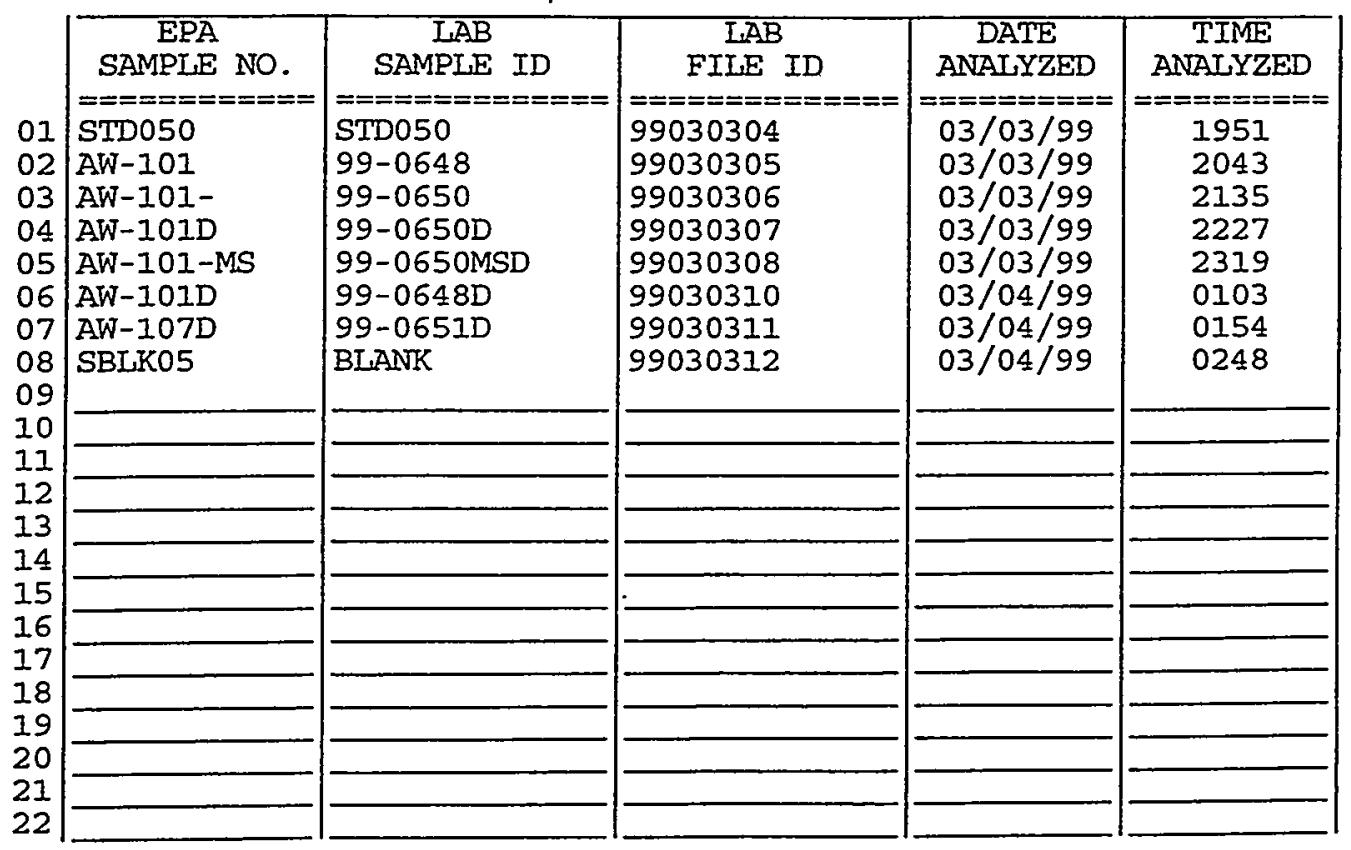

page 1 of 1 
Lab Name: PNNL

Iab Code: PNNL. Case No.:

Lab File ID (Standard): 99030304

Instrument ID: $\mathrm{HPI}$
Contract : BNFL

SAS NO. :

SDG No.: 990312

Time Analyzed: 1951 
Lab Name: PNNL

Lab Code: PNNL

Lab File ID (Standard): 99030304

Instrument ID: HPI
Contract: BNFL

SAS NO.:

SDG NO.: 990312

Date Analyzed: 03/03/99

Time Analyzed: 1951

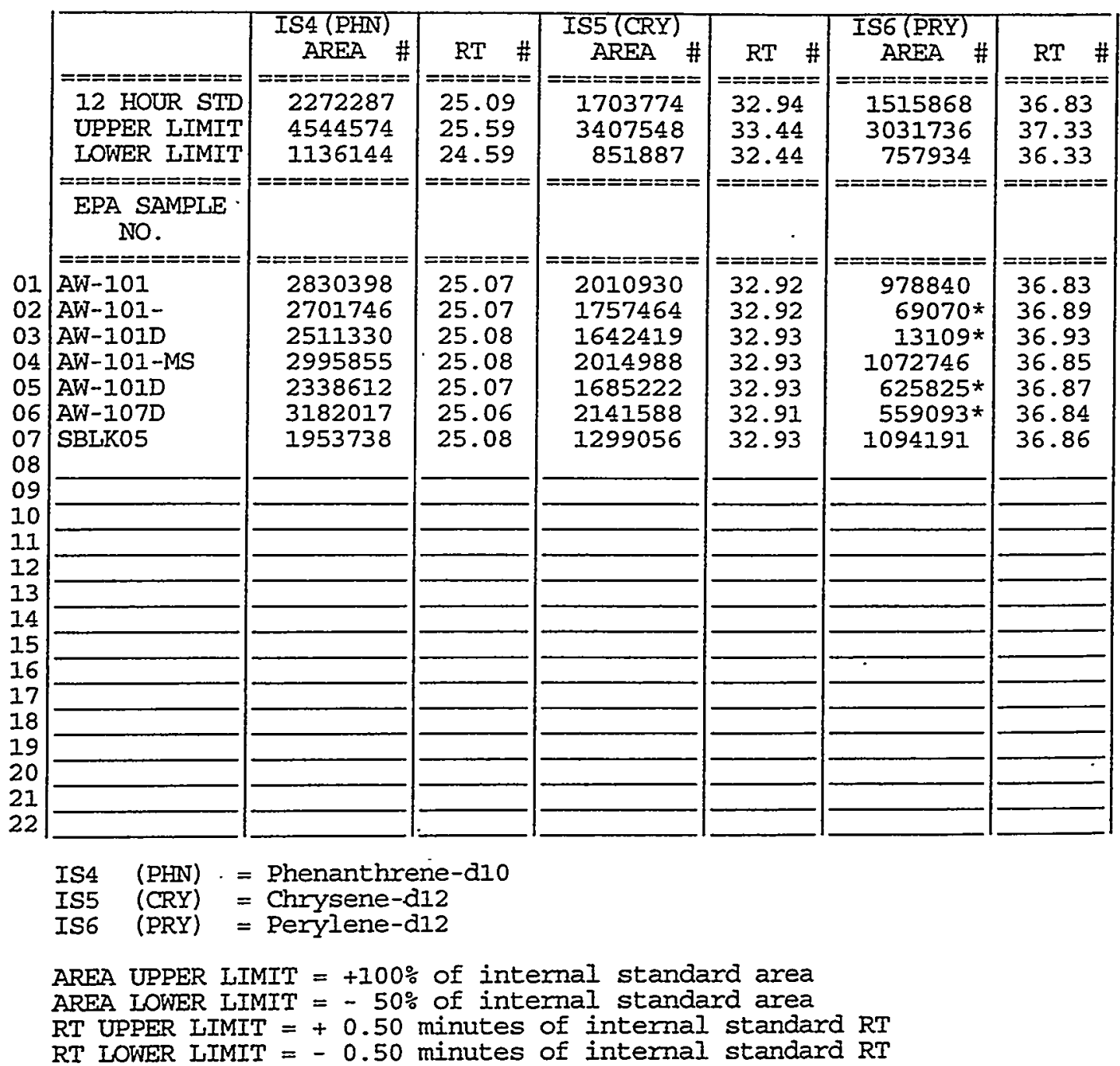

\# Column used to flag internal standard area values with an asterisk. * Values outside of QC limits.

page 1 of 1

FORM VIII SV-2

OLM03.0 
Lab Name: PNNL

Contract : BNFL

Lab Code: PNNL Case No.:

SAS NO. :

SDG No.: 990312

Lab File ID (Standard) : 99030304

Date Analyzed: 03/03/99

Instrument ID: HPI

Time Analyzed: 1951

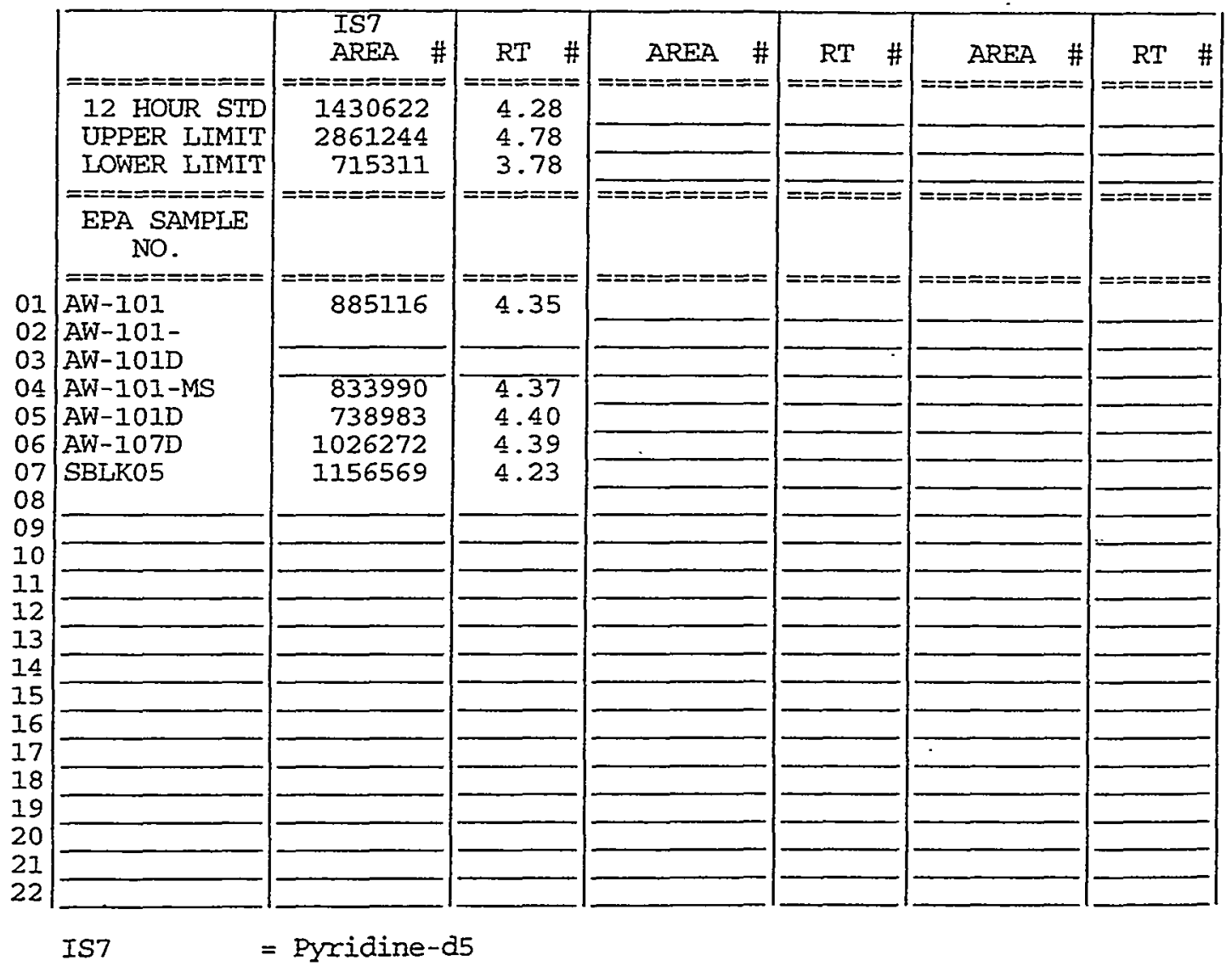

AREA UPPER IIMIT $=+100 \%$ of internal standard area AREA IOWER LIMIT $=-50 \%$ of internal standard area RT UPPER IIMIT $=+0.50$ minutes of internal standard RT RT LOWER IIMIT $=-0.50$ minutes of internal standard RT

\# Column used to flag internal standard area values with an asterisk. * Values outside of QC limits. 
SEMIVOLATILE ORGANIC INSTRUMENT PERFORMANCE CHECK DECAFLUOROTRI PHENYLPHOSPHINE (DFTPP)

Lab Name: PNNL

Lab Code: PNNL

Case No.:
Contract: BNFL

SAS NO.:

SDG No.: : 980221

DFTPP Injection Date: 02/04/99

DFTPP Injection Time: 1727

Instrument ID: HPI

\begin{tabular}{|}
$\%$ RETATIVE \\
ABUNDANCE \\
$============$ \\
59.4 \\
$0.0(0.0) 1$ \\
$64.9(0.4) 1$ \\
$0.3(0.3$ \\
56.3 \\
0.0 \\
100.0 \\
6.8 \\
20.6 \\
2.22 \\
7.6 \\
52.3 \\
10.3 \\
\\
\hline 442
\end{tabular}

THIS CHECK APPLIES TO THE FOLLOWING SAMPLES, MS, MSD, BLANKS, AND STANDARDS:

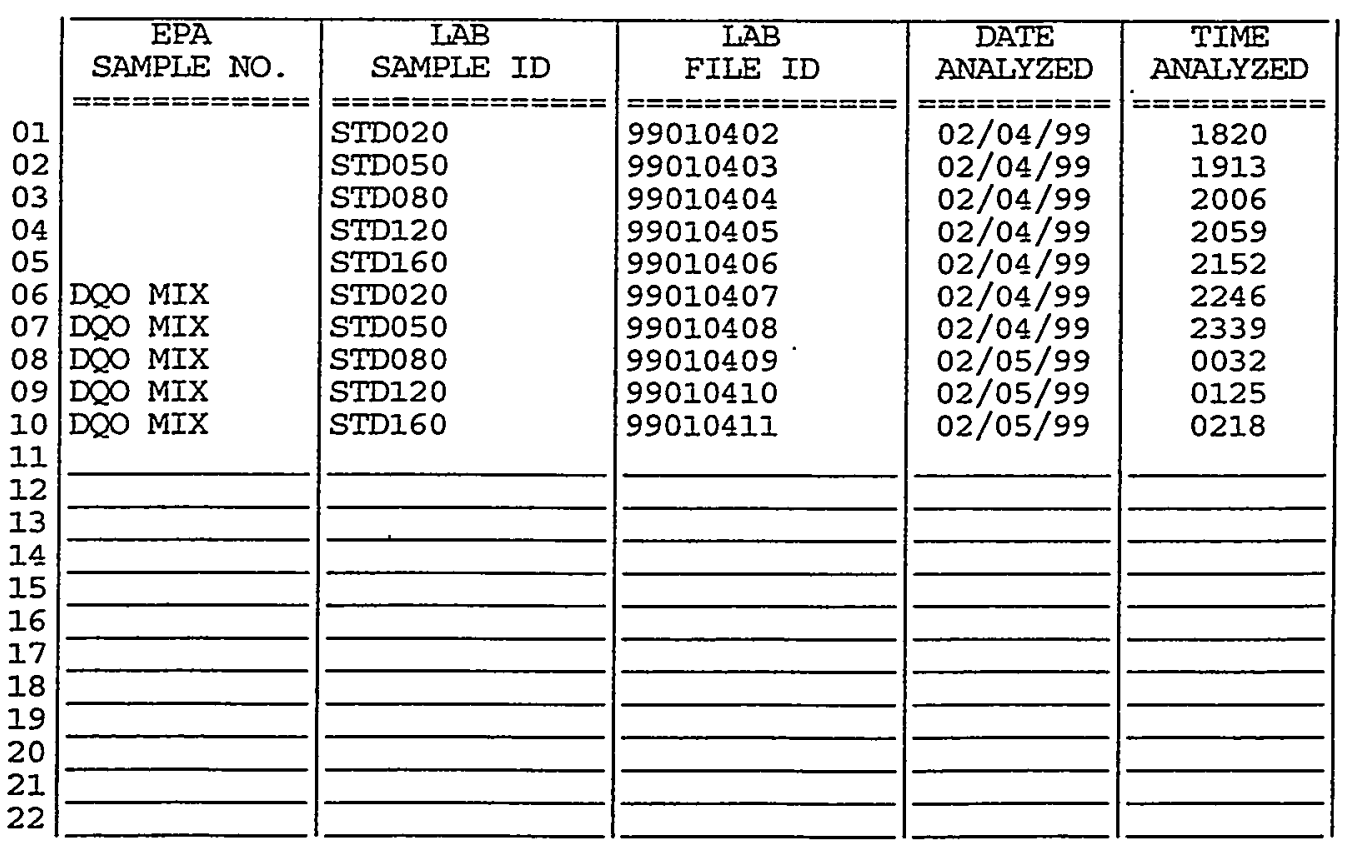

page 1 of 1 
$6 \mathrm{~B}$

SEMIVOIATILE ORGANICS INITIAL CALIBRATION DATA

Lab Name: PNNL

Lab Code: PNNL

Instrument ID: HPI
Contract : BNFL

SAS NO.:

SDG No.: 980221
$02 / 05 / 99$

0218

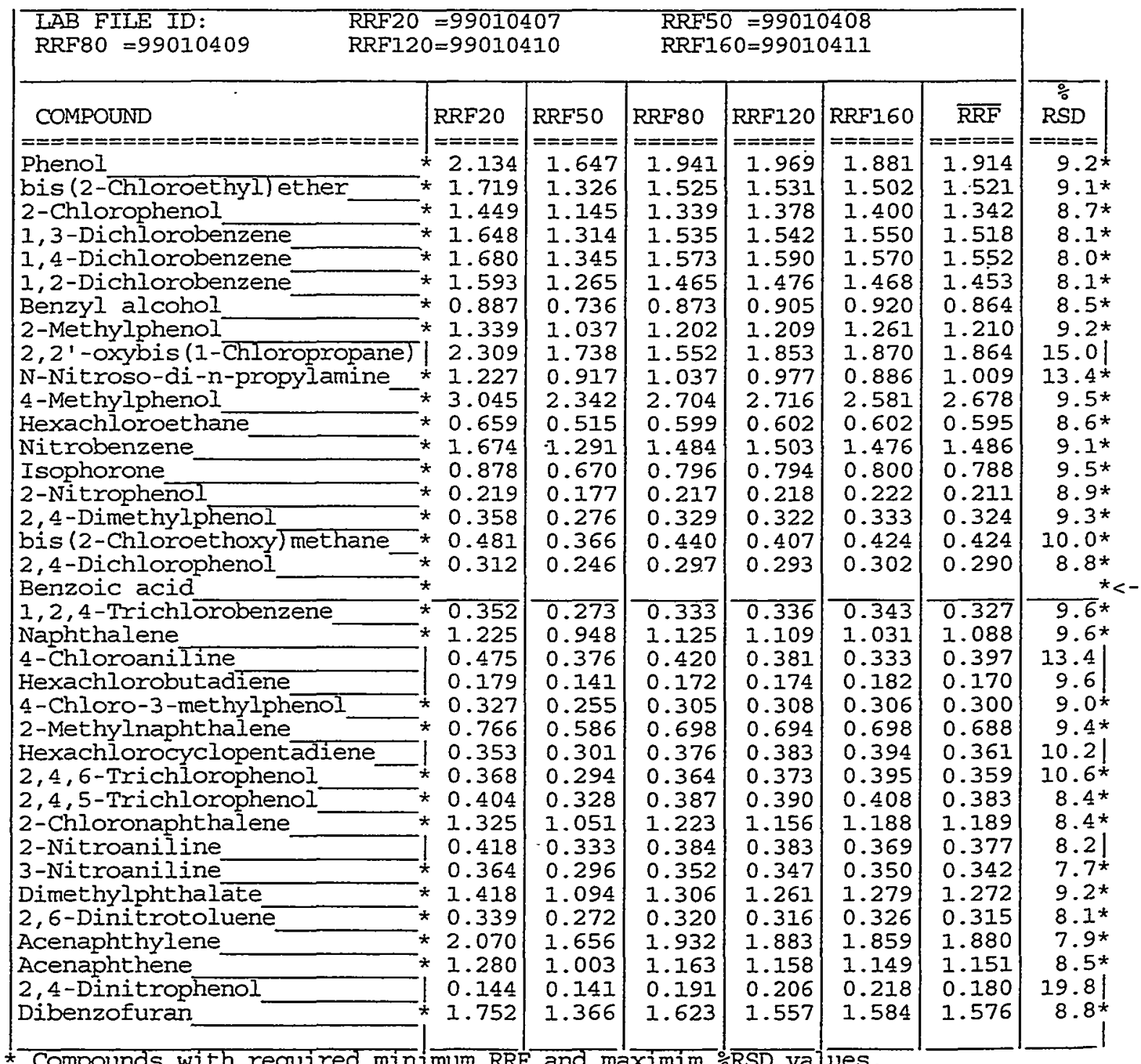

Compounds with required minimum RRF and maximim $\% R S D$ values

Al1 other compounds must meet a minimim RRF of 0.010 . 
Lab Name: PNNT,

Lab Code: PNNL

Instrument ID: HP1
Contract: BNFL

SAS NO.:

SDG NO.: 980221

$02 / 05 / 99$

0218

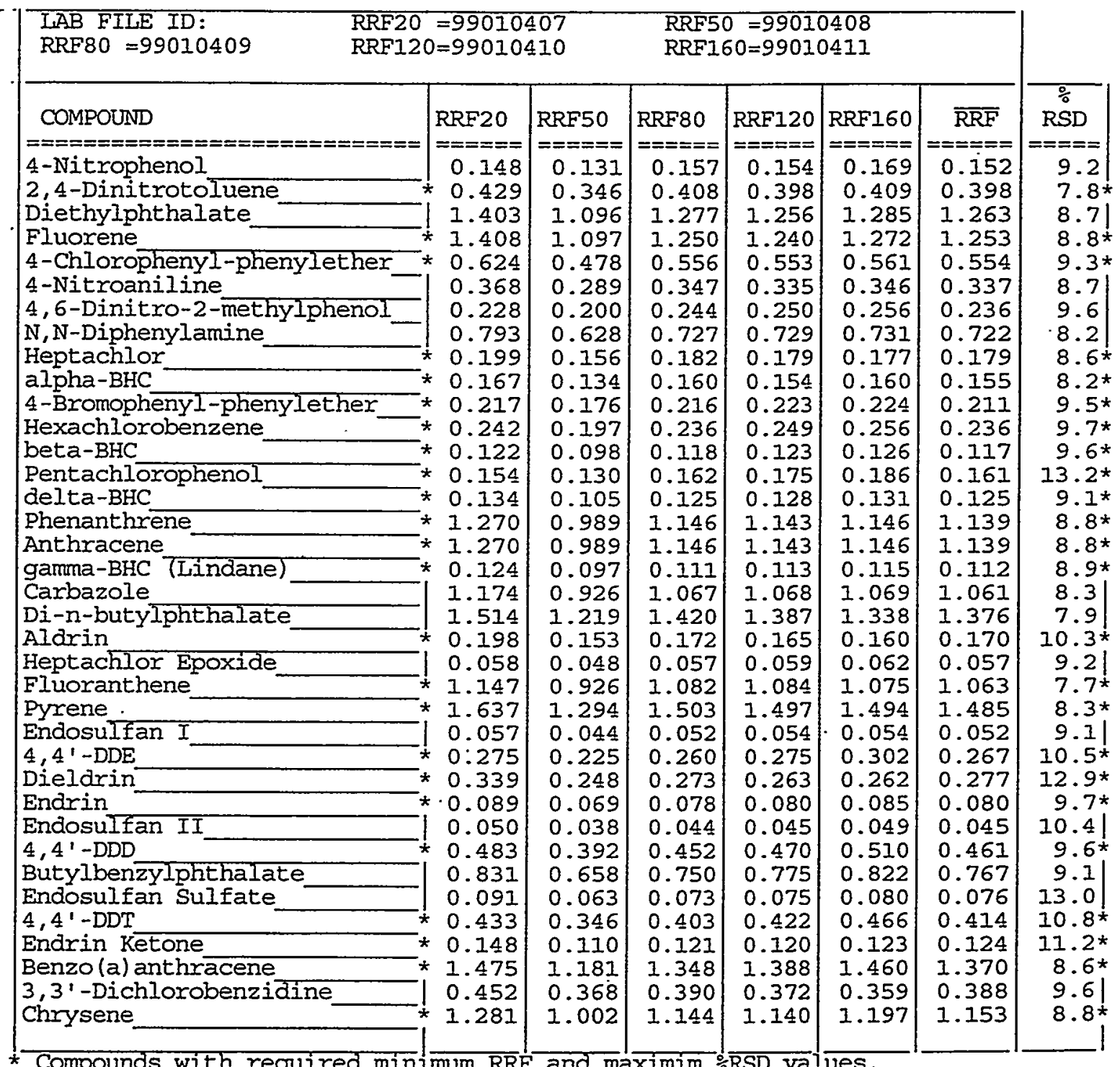

Compounds with required minimum $R R$ and maximim $: \overline{R S D}$ values

AlI other compounds must meet a minimim RRF of 0.010 . 
$6 \mathrm{C}$

SEMIVOLATILE ORGANICS INITIAL CAIIBRATION DATA

Lab Name: PNNL

Lab code: PNNL

Instrument ID: HPI contract : BNFL

Case No.:

SAS NO. :

SDG NO.: 980221

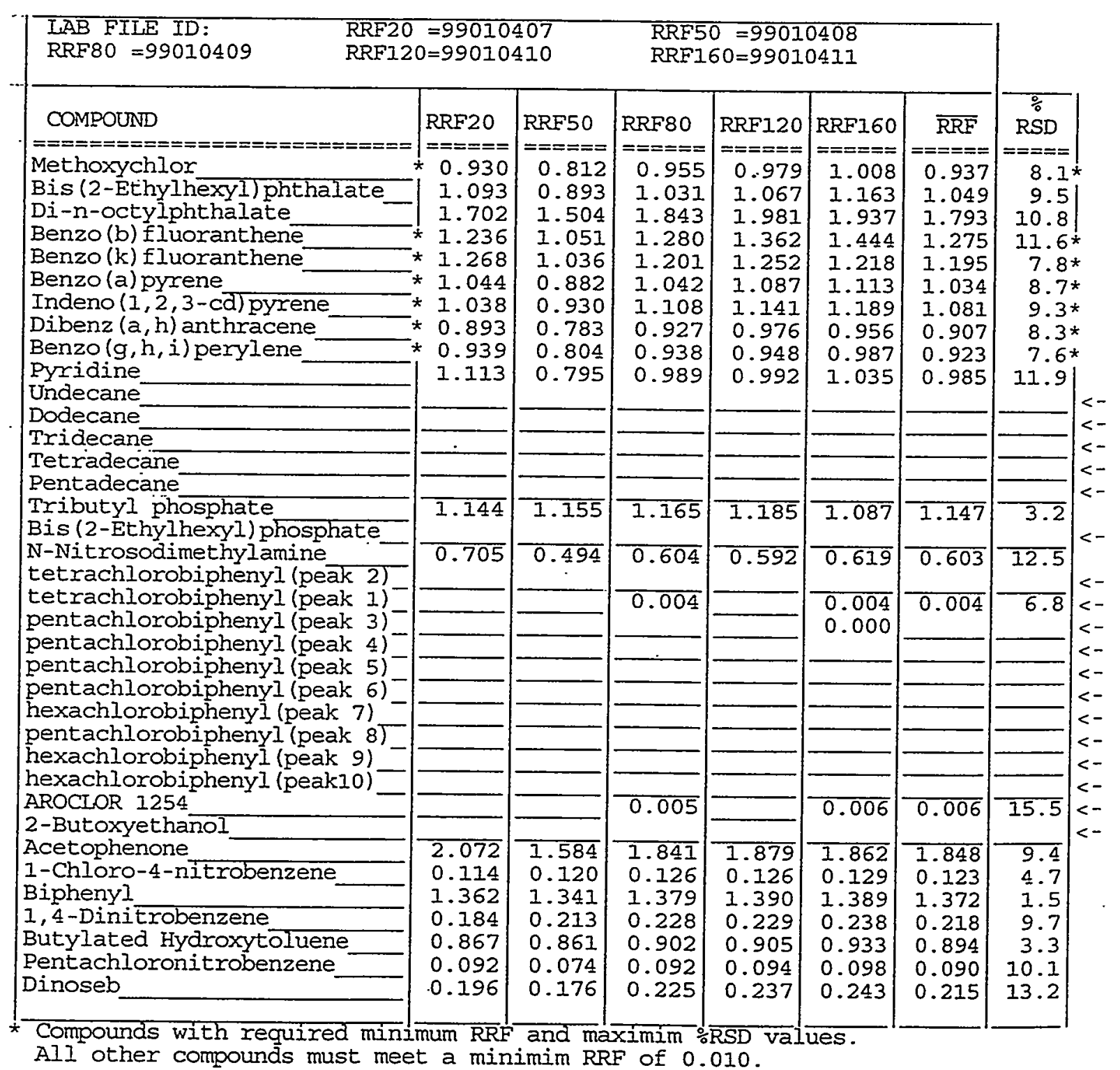

page 3 of 5 
Lab Name: PNNL

Lab Code: PNNL

Instrument ID: $\mathrm{HPI}$ contract: BNEL

Case No.:

SAS NO.:

SDG NO.: 980221

Calibration Date(s) : 02/04/99 02/05/99

Calibration Time(s): 1820

0218

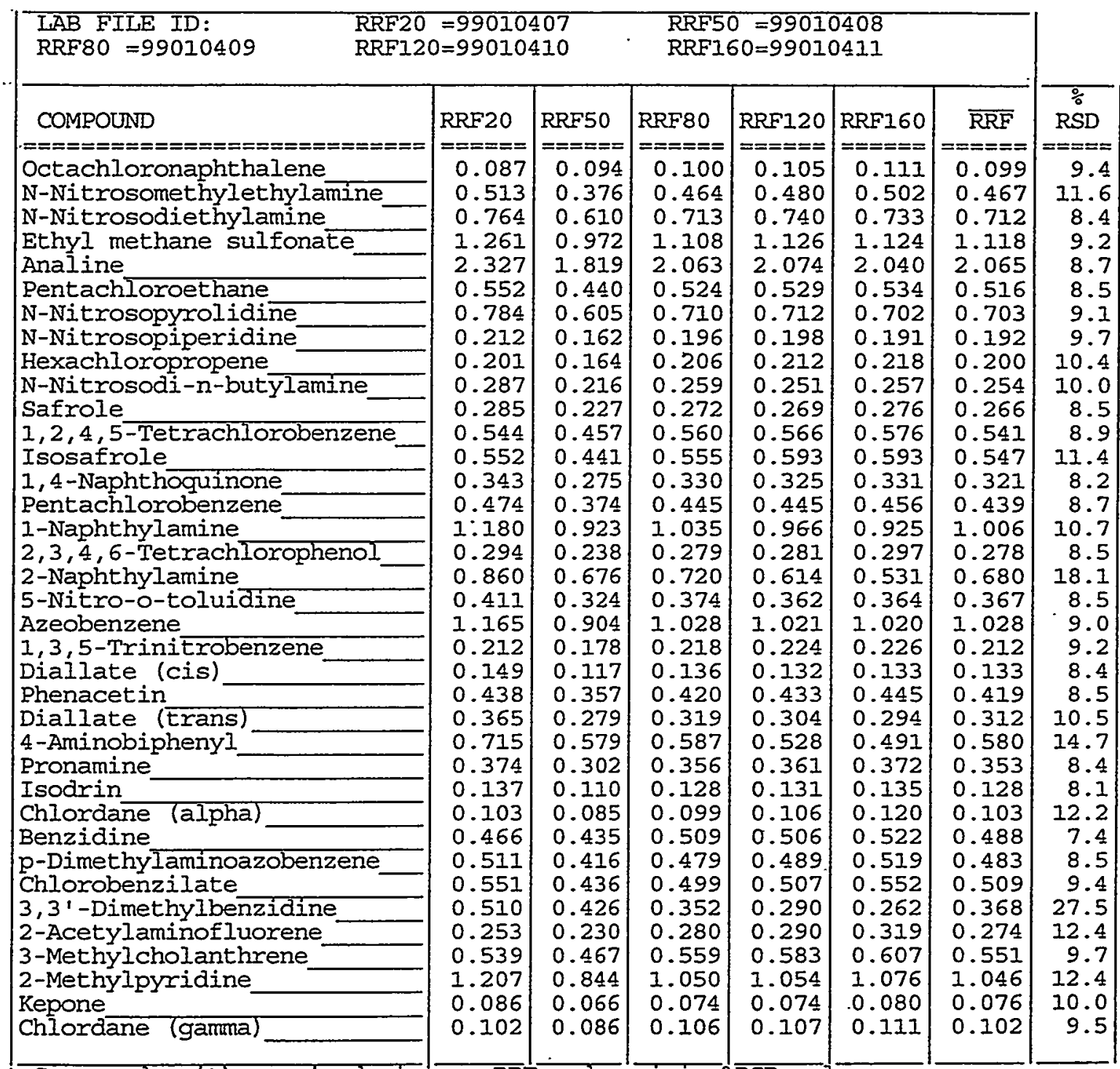

Compounds with required minimum $R R F$ and maximim $\% \overline{R S D}$ values.

Al1 other compounds must meet a minimim RRF of 0.010 . 
$6 \mathrm{C}$

SEMIVOLATILE ORGANICS INITIAI CALIBRATION DATA

Lab Name: PNNL

Lab Code: PNIL,

Instrument ID: HP1
Contract: BNFL

Case No.:
SAS NO.:

SDG No.: 980221

$\begin{array}{ll}\text { Calibration Date (s) : } 02 / 04 / 99 & 02 / 05 / 99 \\ \text { Calibration Time (s) : } 1820 & 0218\end{array}$

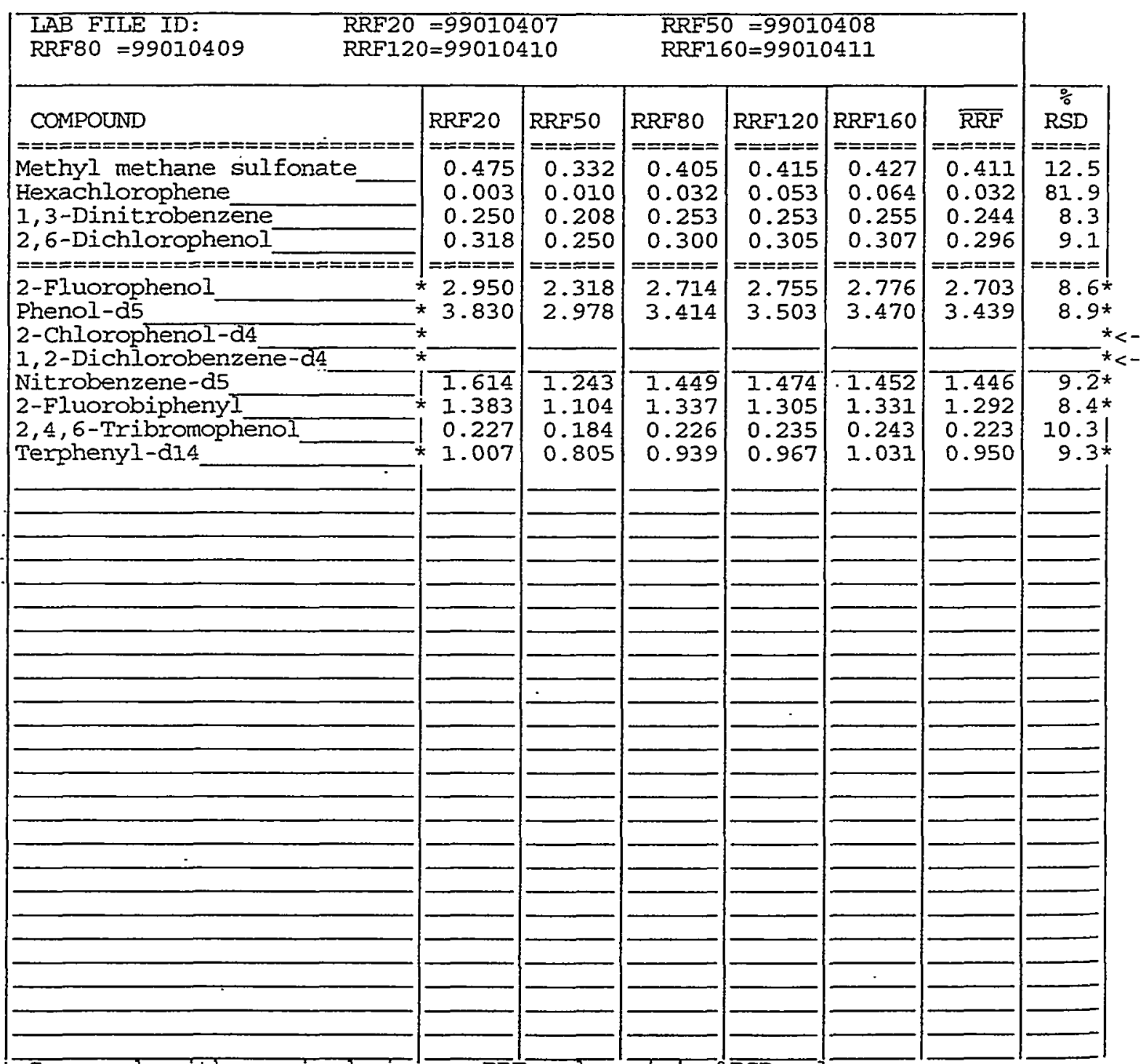

Compounds with required minimum RRF and maximim $\div \overline{R S D}$ values. All other compounds must meet a minimim RRF of 0.010 . 


\section{APPENDIX C: Dioxins/Furans Analysis}

Table C.1 Ion Abundance Ratios and Response Factors for Standards

Table C.2 Ion Abundance Ratios for Supernatant Matrix Spikes

Table C.3 Ion Abundance Ratios for Solids Matrix Spikes

Table C.4. Ion Abundance Ratios for Supernatant Samples, Duplicates, and Process Blanks

Table C.5 Ion Abundance Ratios for Solids Samples, Duplicates, and Process Blanks 
Table C.1. Ion Abundance Ratios and Response Factors for Standards

\begin{tabular}{|c|c|c|c|c|c|c|c|}
\hline Compound & $\begin{array}{c}\text { Native } \\
\text { Conc. } \mu \mathrm{g} / \mathrm{L}\end{array}$ & $\begin{array}{c}\text { Mass } \\
\mathrm{m} / \mathrm{z}\end{array}$ & $\begin{array}{c}\text { Native } \\
\text { Ion Ratio }\end{array}$ & $\begin{array}{c}\text { Labeled } \\
\text { Ion Ratio } \\
(x)\end{array}$ & $\mathbf{R R F}^{(2)}$ & $\begin{array}{c}\text { Average } \\
\text { RRF }\end{array}$ & \%RSD \\
\hline \multirow[t]{5}{*}{$\mathrm{HpCDD}$} & 2.5 & 424 & 1.02 & 1.12 & 1.03 & 0.95 & 14.1 \\
\hline & 10.0 & & $\frac{1.02}{1.06}$ & $\frac{1.12}{1.06}$ & 0.97 & & \\
\hline & 50.0 & & 1.01 & 0.99 & 1.05 & & \\
\hline & 200.0 & & 1.04 & 1.06 & 0.73 & & \\
\hline & 1000.0 & & 0.86 & 1.07 & $0.31^{*}$ & & \\
\hline & & & & & & & \\
\hline \multirow[t]{5}{*}{$\mathrm{HpCDD}$} & 2.5 & 426 & & & 1.14 & 0.97 & 16.1 \\
\hline & 10.0 & & & & 0.97 & & \\
\hline & 50.0 & & & & 1.03 & & \\
\hline & 200.0 & & & & 0.76 & & \\
\hline & 1000.0 & & & & $0.37^{*}$ & & \\
\hline & & & & & & & \\
\hline \multirow[t]{5}{*}{$\mathrm{HpCDF}$} & 2.5 & 408 & 0.97 & 1.00 & 1.08 & 1.07 & 13.8 \\
\hline & 10.0 & & 1.06 & 1.02 & 1.19 & & \\
\hline & 50.0 & & 1.00 & 0.99 & 1.14 & & \\
\hline & 200.0 & & 0.97 & 1.05 & 0.86 & & \\
\hline & 1000.0 & & 1.02 & 1.20 & $0.38^{*}$ & & \\
\hline & & & & & & & \\
\hline \multirow[t]{5}{*}{ HpCDF } & 2.5 & 410 & & & 1.12 & 1.08 & 9.9 \\
\hline & 10.0 & & & & 1.14 & & \\
\hline & 50.0 & & & & 1.13 & & \\
\hline & 200.0 & & & & 0.92 & & \\
\hline & 1000.0 & & & & $0.45^{*}$ & & \\
\hline & & & & & & & \\
\hline \multirow[t]{5}{*}{$\mathrm{HxCDD}$} & 2.5 & 390 & 1.39 & 1.28 & 1.19 & 1.12 & 4.9 \\
\hline & 10.0 & & 1.26 & 1.25 & 1.12 & & \\
\hline & 50.0 & & 1.26 & 1.27 & 1.11 & & \\
\hline & 200.0 & & 1.17 & 1.27 & 1.06 & & \\
\hline & 1000.0 & & 0.98 & 1.31 & $0.31^{*}$ & & \\
\hline & & & & & & & \\
\hline \multirow[t]{6}{*}{$\mathrm{HxCDD}$} & 2.5 & 392 & & & 1.10 & 1.12 & 2.5 \\
\hline & 10.0 & & & & 1.11 & & \\
\hline & 50.0 & 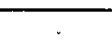 & & & 1.12 & & \\
\hline & 200.0 & & & & 1.15 & & \\
\hline & 1000.0 & & & & $0.42^{*}$ & & \\
\hline & & & & & & & \\
\hline \multirow[t]{5}{*}{$\mathrm{HxCDF}$} & 2.5 & 374 & 1.24 & 1.25 & 1.06 & 1.16 & 13.5 \\
\hline & 10.0 & & 1.23 & 1.27 & 1.10 & & \\
\hline & 50.0 & & 1.25 & 1.27 & 1.11 & & \\
\hline & 200.0 & & 1.18 & 1.29 & 1.40 & & \\
\hline & 1000.0 & & 1.12 & 1.38 & $0.50^{*}$ & & \\
\hline
\end{tabular}




\begin{tabular}{|c|c|c|c|c|c|c|c|}
\hline Compound & $\begin{array}{c}\text { Native } \\
\text { Conc. } \mu g / L \\
\end{array}$ & $\begin{array}{c}\text { Mass } \\
\mathbf{m} / \mathbf{z}\end{array}$ & $\begin{array}{c}\text { Native } \\
\text { Ion Ratio }\end{array}$ & $\begin{array}{c}\text { Labeled } \\
\text { Ion Ratio }\end{array}$ & $\mathbf{R R F}^{(2)}$ & $\begin{array}{c}\text { Average } \\
\text { RRF }\end{array}$ & $\%$ RSD \\
\hline \multirow[t]{5}{*}{$\mathrm{HxCDF}$} & 2.5 & 376 & & & 1.06 & 1.21 & 17.5 \\
\hline & 10.0 & & & & 1.14 & & \\
\hline & 50.0 & & & & 1.12 & & \\
\hline & 200.0 & & & & 1.53 & & \\
\hline & 1000.0 & . & & & $0.62^{*}$ & & \\
\hline & & & & & & & \\
\hline \multirow[t]{5}{*}{$\overline{\mathrm{OCDD}}$} & 5.0 & 458 & 0.97 & 0.90 & 0.88 & 0.96 & 11.7 \\
\hline & 20.0 & & 0.93 & 0.87 & 1.02 & & \\
\hline & 100.0 & & 1.01 & 0.93 & 1.08 & & \\
\hline & 400.0 & & 0.97 & 0.92 & 0.84 & & \\
\hline & 2000.0 & & 1.24 & 0.74 & $0.25 *$ & & \\
\hline & & & & & & & \\
\hline \multirow[t]{5}{*}{ OCDD } & 5.0 & 460 & & & 0.82 & 0.89 & 10.8 \\
\hline & 20.0 & & & & 0.96 & & \\
\hline & 100.0 & & & & 0.98 & & \\
\hline & 400.0 & & & & 0.80 & & \\
\hline & 2000.0 & & & & $0.30^{*}$ & & \\
\hline & & & & & & & \\
\hline \multirow[t]{5}{*}{ OCDF } & 5.0 & 442 & 0.98 & 0.90 & 1.18 & 1.11 & 13.2 \\
\hline & 20.0 & & 0.80 & 0.87 & 1.20 & & \\
\hline & 100.0 & & 0.84 & 0.93 & 1.18 & & \\
\hline & 400.0 & & 0.97 & 0.92 & 0.90 & & \\
\hline & 2000.0 & & 0.56 & 0.74 & $0.35^{*}$ & & \\
\hline \multirow{5}{*}{ OCDF } & 50 & 444 & & & 100 & 114 & $10 ?$ \\
\hline & $\frac{5.0}{20.0}$ & 444 & & & $\frac{1.09}{1.31}$ & 1.14 & 19.2 \\
\hline & 100.0 & & & & 1.30 & & \\
\hline & 400.0 & & & & 0.85 & & \\
\hline & 2000.0 & & & & $0.46^{*}$ & & \\
\hline & & & & & & & \\
\hline \multirow[t]{5}{*}{$\overline{\mathrm{PeCDD}}$} & 2.5 & 356 & 1.35 & 1.61 & 0.61 & 0.92 & 25.6 \\
\hline & 10.0 & & 1.43 & 1.68 & 0.95 & & \\
\hline & 50.0 & & 1.55 & 1.62 & 1.17 & & \\
\hline & 200.0 & & 1.36 & 1.62 & 0.95 & & \\
\hline & 1000.0 & & 1.39 & 1.64 & $0.31^{*}$ & & \\
\hline \multirow{5}{*}{ PeCDD } & 2.5 & 358 & & & 0.72 & 1.05 & 217 \\
\hline & 10.0 & & & & 1.11 & & \\
\hline & 50.0 & & & & 1.23 & & \\
\hline & 200.0 & & & & 1.13 & & \\
\hline & 1000.0 & & & & $0.36^{*}$ & & \\
\hline & & & & & & & \\
\hline \multirow[t]{5}{*}{ PeCDF } & 2.5 & 340 & 1.32 & 1.70 & 1.13 & 1.02 & 14.4 \\
\hline & 10.0 & & 1.42 & 1.36 & 1.04 & & \\
\hline & 50.0 & & 1.63 & 1.62 & 1.11 & & \\
\hline & 200.0 & & 1.34 & 1.68 & 0.81 & & \\
\hline & 1000.0 & & 1.30 & 1.57 & $0.31^{*}$ & & \\
\hline
\end{tabular}




\begin{tabular}{|c|c|c|c|c|c|c|c|}
\hline Compound & $\begin{array}{c}\text { Native } \\
\text { Conc. } \mu \mathrm{g} / \mathrm{L}\end{array}$ & $\begin{array}{c}\text { Mass } \\
\mathrm{m} / \mathrm{z}\end{array}$ & $\begin{array}{c}\text { Native } \\
\text { Ion Ratio }\end{array}$ & $\begin{array}{l}\text { Labeled } \\
\text { Ion Ratio }\end{array}$ & $\mathrm{RRF}^{(2)}$ & $\begin{array}{c}\text { Average } \\
\text { RRF }\end{array}$ & \%RSD \\
\hline \multirow[t]{5}{*}{ PeCDF } & 2.5 & 342 & & & 1.46 & 1.15 & 18.6 \\
\hline & 10.0 & & & & 1.00 & & \\
\hline & 50.0 & & & & 1.10 & & \\
\hline & 200.0 & & & & 1.02 & & \\
\hline & 1000.0 & & & & $0.37^{*}$ & & \\
\hline & & & & & & & \\
\hline \multirow[t]{5}{*}{ TCDD } & 0.5 & 320 & 0.68 & 0.76 & 1.68 & 1.79 & 11.4 \\
\hline & 2.0 & & 0.72 & 0.79 & 1.59 & & \\
\hline & 10.0 & & 0.87 & 0.71 & 2.04 & & \\
\hline & 40.0 & & 0.76 & 0.79 & 1.87 & & \\
\hline & 200.0 & & 0.90 & 0.79 & $1.99 *$ & & \\
\hline & & & & & & & \\
\hline \multirow[t]{5}{*}{ TCDD } & 0.5 & 322 & & & 1.92 & 1.82 & 7.3 \\
\hline & 2.0 & & & & 1.74 & & \\
\hline & 10.0 & & & & 1.68 & & \\
\hline & 40.0 & & & & 1.95 & & \\
\hline & 200.0 & & & & $1.72^{*}$ & & \\
\hline & & & & & & & \\
\hline \multirow[t]{5}{*}{ TCDF } & 0.5 & 304 & 0.74 & 0.86 & 0.90 & 1.06 & 11.8 \\
\hline & 2.0 & & 0.80 & 0.68 & 1.09 & & \\
\hline & 10.0 & & 0.87 & 0.81 & 1.19 & & \\
\hline & 40.0 & & 0.66 & 0.70 & 1.03 & & \\
\hline & 200.0 & & 0.70 & 0.92 & $0.65^{*}$ & & \\
\hline & & & & & & & \\
\hline \multirow[t]{5}{*}{ TCDF } & 0.5 & 306 & & & 1.04 & 1.04 & 7.9 \\
\hline & 2.0 & & & & 0.93 & & \\
\hline & 10.0 & & & & 1.10 & & \\
\hline & 40.0 & & & & 1.09 & & \\
\hline & 200.0 & & & & $0.86^{*}$ & & \\
\hline & & & & & & & \\
\hline
\end{tabular}

1) Concentration of labeled compounds: OCDD and OCDF $=200 \mu \mathrm{g} / \mathrm{L}$, all other labeled compounds at 100 $\mu \mathrm{g} / \mathrm{L}$

2) Relative response factors (RRF) marked with an asterisk not included in determining the average response factor 
Table C.2. Ion Abundance Ratios for Supernatant Matrix Spikes

\begin{tabular}{|c|c|c|c|c|c|c|c|c|c|c|}
\hline \multirow{4}{*}{$\begin{array}{r}\text { Sample-ID } \\
\text { Tank } \\
\text { Material } \\
\text { Injection \# } \\
\text { Ion Ratios }\end{array}$} & \multicolumn{4}{|c|}{ 99-0648'Supernatant } & \multicolumn{4}{|c|}{ 99-0649 Supernatant } & \multirow{2}{*}{\multicolumn{2}{|c|}{$\mathbf{L C S}$}} \\
\hline & \multicolumn{2}{|c|}{ AW-101 MS } & \multicolumn{2}{|c|}{ AW-101 MSD } & \multicolumn{2}{|c|}{ AN-107 MS } & \multicolumn{2}{|c|}{ AN-107 MSD } & & \\
\hline & \multicolumn{2}{|c|}{49} & \multicolumn{2}{|c|}{64} & \multicolumn{2}{|c|}{54} & \multicolumn{2}{|c|}{$\mathbf{5 5}$} & \multicolumn{2}{|c|}{61} \\
\hline & Native & Labeled & Native & Labeled & Native & Labeled & Native & Labeled & Native & Labeled \\
\hline \multicolumn{11}{|l|}{ Analyte } \\
\hline HpCDD & 1.04 & 0.99 & 1.05 & 1.11 & 1.06 & 1.05 & 1.06 & 1.08 & 1.05 & 1.04 \\
\hline $\mathrm{HpCDF}$ & 0.90 & 0.95 & 1.06 & 1.05 & 1.00 & 1.09 & 0.98 & 1.05 & 1.00 & 1.10 \\
\hline $\mathrm{HxCDD}$ & 1.19 & 1.26 & 1.21 & 1.23 & 1.26 & 1.23 & 1.23 & 1.14 & 1.25 & 1.26 \\
\hline $\mathrm{HxCDF}$ & 1.15 & 1.40 & 1.19 & 1.43 & 1.17 & 1.39 & 1.09 & 1.37 & 1.20 & 1.41 \\
\hline OCDD & 0.93 & 0.95 & 0.91 & 0.90 & 0.92 & 0.98 & 0.94 & 0.95 & 0.99 & 0.98 \\
\hline OCDF & 0.94 & 0.95 & 0.91 & 0.90 & 0.94 & 0.98 & 0.96 & 0.95 & 0.93 & 0.98 \\
\hline PeCDD & 1.65 & 1.37 & 1.54 & 1.55 & 1.66 & 1.67 & 1.35 & 1.72 & 1.55 & 1.64 \\
\hline $\mathrm{PeCDF}$ & 1.56 & 1.74 & 1.48 & 1.69 & 1.41 & 1.32 & 1.34 & 1.32 & 1.65 & 1.61 \\
\hline TCDD & 0.79 & 0.65 & 0.75 & 0.75 & 0.72 & 0.70 & 0.77 & 0.66 & 0.73 & $0.31^{*}$ \\
\hline TCDF & 0.65 & 0.67 & 0.77 & 0.81 & 0.72 & 0.73 & 0.78 & 0.88 & 0.83 & 0.72 \\
\hline
\end{tabular}

* Designates ion abundance ratio that does not meet QC criteria shown in Table 6.e.

Table C.3. Ion Abundance Ratios for Solids Matrix Spikes

\begin{tabular}{|c|c|c|c|c|c|c|c|c|}
\hline \multirow{4}{*}{$\begin{array}{r}\text { Sample ID } \\
\text { Tank } \\
\text { Material } \\
\text { Injection \# } \\
\text { Ion Ratios }\end{array}$} & \multicolumn{4}{|c|}{ 99-0648 Solids } & \multicolumn{4}{|c|}{ 99-0649 Solids } \\
\hline & \multicolumn{2}{|c|}{ AW-101 MS } & \multicolumn{2}{|c|}{ AW-101 MSD } & \multicolumn{2}{|c|}{ AN-107 MS } & \multicolumn{2}{|c|}{ AN-107 MSD } \\
\hline & \multicolumn{2}{|c|}{44} & \multicolumn{2}{|c|}{$\overline{45}$} & \multicolumn{2}{|c|}{56} & \multicolumn{2}{|c|}{63} \\
\hline & Native & Labeled & Native & Labeled & Native & Labeled & Native & Labeled \\
\hline \multicolumn{9}{|l|}{ Analyte } \\
\hline$\overline{\mathrm{HpCDD}}$ & 1.03 & 1.09 & 1.06 & 1.06 & 1.04 & 1.05 & 1.10 & 1.00 \\
\hline $\mathrm{HpCDF}$ & 0.91 & 1.09 & 1.04 & 1.08 & 1.03 & 1.06 & 1.06 & 1.04 \\
\hline $\mathrm{HxCDD}$ & 1.10 & 1.11 & 1.19 & 1.32 & 1.05 & 1.17 & 1.25 & 1.19 \\
\hline $\mathrm{HxCDF}$ & 1.07 & 1.32 & 1.20 & 1.32 & 1.24 & 1.36 & 1.24 & 1.42 \\
\hline OCDD & 0.95 & 0.93 & 1.02 & 0.86 & 0.85 & 0.89 & 0.97 & 0.94 \\
\hline OCDF & 0.97 & 0.93 & 0.94 & 0.86 & 0.93 & 0.89 & 0.90 & 0.94 \\
\hline PeCDD & 1.33 & 1.62 & 1.39 & 1.69 & 1.51 & 1.68 & 1.57 & 1.53 \\
\hline PeCDF & 1.33 & 1.36 & 1.46 & 1.58 & 1.45 & 1.32 & 1.57 & 1.72 \\
\hline TCDD & 0.79 & 0.69 & 0.78 & 0.67 & 0.81 & 0.70 & 0.79 & 0.73 \\
\hline TCDF & 0.81 & 0.78 & 0.76 & 0.80 & 0.73 & 0.78 & 0.79 & 0.82 \\
\hline
\end{tabular}


Table C.4. Ion Abundance Ratios for Supernatant Samples, Duplicates, and Process Blanks

\begin{tabular}{|c|c|c|c|c|c|c|c|c|c|c|c|c|}
\hline \multirow{5}{*}{$\begin{array}{l}\text { Tank } \\
\text { Sample ID } \\
\text { Injection } \\
\text { Ion Ratios }\end{array}$} & \multicolumn{6}{|c|}{ AW-101 Supernatant } & \multicolumn{6}{|c|}{ AN-107 Supernatant } \\
\hline & \multirow{2}{*}{\multicolumn{2}{|c|}{$\begin{array}{c}\text { 99-0648bl } \\
\text { ProcBlk } \\
\end{array}$}} & \multirow{2}{*}{\multicolumn{2}{|c|}{$\begin{array}{l}99-0648 \\
\text { Sample }\end{array}$}} & \multirow{2}{*}{\multicolumn{2}{|c|}{$\begin{array}{l}\text { 99-0648d } \\
\text { Duplicate }\end{array}$}} & \multicolumn{2}{|c|}{ 99-0649bl } & \multicolumn{2}{|c|}{$99-0649$} & \multicolumn{2}{|c|}{ 99-0649d } \\
\hline & & & & & & & & çBlk & & nple & Dul & licate \\
\hline & \multicolumn{2}{|c|}{67} & \multicolumn{2}{|c|}{68} & \multicolumn{2}{|c|}{48} & \multicolumn{2}{|c|}{51} & \multicolumn{2}{|c|}{52} & \multicolumn{2}{|c|}{53} \\
\hline & Native & Labeled & Native & Labeled & Native & Labeled & Native & Labeled & Native & Labeled & Native & Labeled \\
\hline \multicolumn{13}{|l|}{ Analyte } \\
\hline HpCDD & & 1.09 & & 1.08 & & 0.96 & & 1.05 & & 1.04 & & 1.05 \\
\hline$\overline{\mathrm{HpCDF}}$ & & 1.11 & & 1.06 & & 1.07 & & 1.02 & & 1.04 & & 1.05 \\
\hline HxCDD & & 1.27 & & $1.67^{*}$ & & 1.20 & & 1.14 & & 1.19 & & 1.21 \\
\hline $\mathrm{HxCDF}$ & & 1.13 & & 1.27 & & 1.38 & & 1.23 & & 1.27 & & 1.33 \\
\hline OCDD & & 0.95 & & 0.89 & & 0.90 & & 0.83 & & 0.88 & & 0.85 \\
\hline$\overline{O C D F}$ & & 0.95 & & 0.89 & & 0.90 & & 0.83 & & 0.88 & & 0.85 \\
\hline PeCDD & & $1.26^{*}$ & & 1.73 & & $1.82^{*}$ & & $1.84^{*}$ & & 1.64 & & 1.70 \\
\hline PeCDF & & 1.52 & & 1.41 & & 1.63 & & 1.58 & $\cdot$ & 1.63 & & 1.67 \\
\hline$\overline{T C D D}$ & & $\overline{0.67}$ & & $\overline{0.86}$ & & 0.71 & & 0.85 & & 0.68 & & 0.73 \\
\hline TCDF & & 0.82 & & 0.84 & & 0.83 & & $0.94^{*}$ & & 0.65 & & 0.78 \\
\hline
\end{tabular}

"*" = Ion abundance ratio does not meet QC criteria shown in Table 6.e.

"Blanks" = No signal or peak area detected; ion abundance ratio is zero $(0)$ or undefined.

Table C.5. Ion Abundance Ratios for Solids Samples, Duplicates, and Process Blanks

\begin{tabular}{|c|c|c|c|c|c|c|c|c|c|c|c|c|}
\hline \multirow{5}{*}{$\begin{array}{l}\text { Tank } \\
\text { Material } \\
\text { Sample ID } \\
\text { Injection \# } \\
\text { Ion Ratios }\end{array}$} & \multicolumn{6}{|c|}{ AW-101 Solids } & \multicolumn{6}{|c|}{ AN-107 Solids } \\
\hline & \multicolumn{2}{|c|}{$99-0650$} & \multicolumn{2}{|c|}{$99-0650$} & \multicolumn{2}{|c|}{ 99-0650d } & \multicolumn{2}{|c|}{ 99-0651pb } & \multicolumn{2}{|c|}{$99-0651$} & \multicolumn{2}{|c|}{$99.0651 d$} \\
\hline & \multicolumn{2}{|c|}{$\frac{\text { ProcBlk }}{46}$} & \multirow{2}{*}{\multicolumn{2}{|c|}{$\frac{\text { Sample }}{42}$}} & \multicolumn{2}{|c|}{ Duplicate } & \multicolumn{2}{|c|}{ ProcBlk } & \multicolumn{2}{|c|}{ Sample } & \multicolumn{2}{|c|}{ Duplicate } \\
\hline & & 46 & & & \multicolumn{2}{|c|}{43} & \multicolumn{2}{|c|}{60} & \multicolumn{2}{|c|}{59} & \multicolumn{2}{|c|}{58} \\
\hline & Native & Labeled & Native & Labeled & Native & Labeled & Native & Labeled & Native & Labeled & Native & Labeled \\
\hline \multicolumn{13}{|l|}{ Analyte } \\
\hline $\mathrm{HpCDD}$ & 0.97 & 1.12 & 0.96 & 1.09 & $0.79^{*}$ & 1.08 & 0.97 & 1.07 & $1.23^{*}$ & 1.05 & 1.14 & 1.08 \\
\hline $\mathrm{HpCDF}$ & 0.95 & 1.15 & 1.05 & 1.08 & & 1.06 & $1.69^{*}$ & 1.04 & & 1.07 & 1.08 & 1.07 \\
\hline $\mathrm{H} \times C D D$ & $0.43^{*}$ & 1.25 & $0.94^{*}$ & $0.98^{*}$ & $2.36^{*}$ & $0.98^{*}$ & & 1.30 & $1.68^{*}$ & 1.28 & 1.18 & 1.27 \\
\hline $\mathrm{HxCDF}$ & 1.31 & 1.39 & 1.36 & $1.54^{*}$ & $0.00^{*}$ & 1.20 & $0.77^{*}$ & 1.36 & & 1.28 & 1.30 & 1.32 \\
\hline OCDD & $1.33^{*}$ & 0.93 & $1.18^{*}$ & 0.87 & $1.84^{*}$ & 0.90 & 1.00 & 0.86 & & 0.90 & 0.85 & $\overline{0.82}$ \\
\hline$\overline{O C D F}$ & 0.76 & 0.93 & 0.86 & 0.87 & 0.92 & 0.90 & & 0.85 & & 0.90 & 0.89 & 0.82 \\
\hline PeCDD & $0.97^{*}$ & 1.58 & 1.28 & 1.48 & & 1.60 & & $1.82^{*}$ & & 1.61 & 1.52 & 1.65 \\
\hline PeCDF & $1.05^{*}$ & 1.60 & $4.33^{*}$ & 1.69 & & 1.64 & & 1.61 & & 1.61 & 1.49 & 1.66 \\
\hline TCDD & & $0.54^{*}$ & $1.50^{*}$ & 0.75 & & 0.82 & & 0.88 & & 0.69 & 0.67 & 0.73 \\
\hline TCDF & $0.60^{*}$ & & $0.60^{*}$ & $1.25^{*}$ & & 0.89 & & $0.97^{*}$ & & 0.77 & 0.72 & 0.85 \\
\hline
\end{tabular}

"Blanks" = No signal or peak area detected; ion abundance ratio is zero $(0)$ or undefined. 
PNWD-2461

BNFL-RPT-001, Rev. 1

\section{Distribution}

No. of

Copies

\section{OFFSITE}

2 DOE/Office of Scientific and Technical Information
No. of

Copies

\section{ONSITE}

5 British Nuclear Fuels, Limited

M. E. Johnson (4)

A. Thompson

BN-FL

BN-FL

14 Pacific Northwest National Laboratory

J. A. Campbell

E. W. Hoppe

P8-08

J. L. Huckaby

G. S. Klinger

K7-15

D. E. Kurath

P7-22

G. M. Mong

P7-28

E. V. Morrey

P8-08

M. W. Urie (2)

P7-28

Information Release Office (5) K1-06 